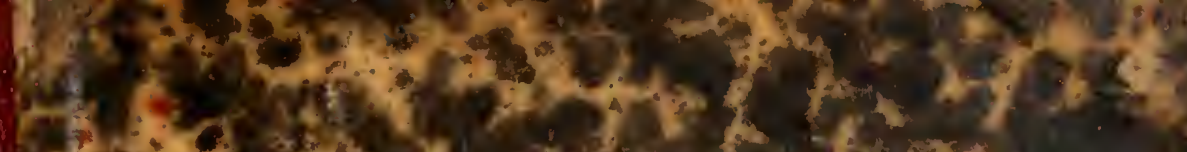
tay 4 rom $3 x+y)=0$ t $120.40104+2=5$ - 2 .

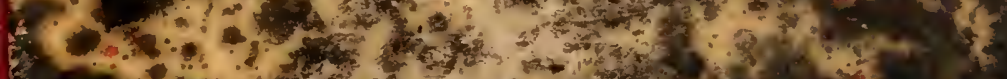

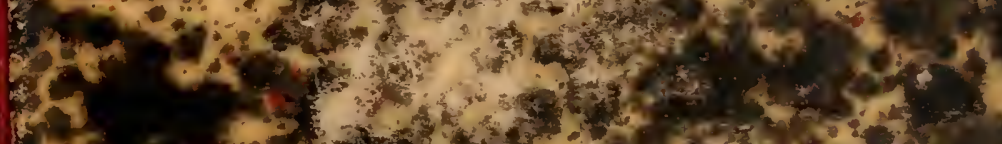

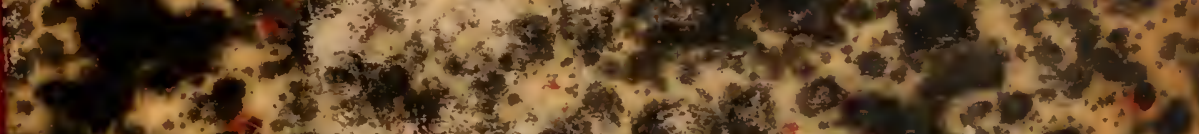

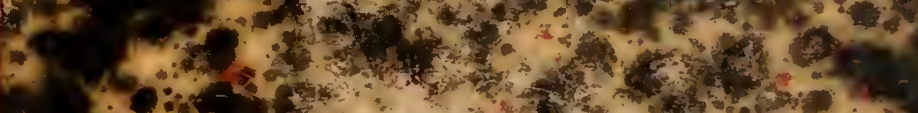
b.

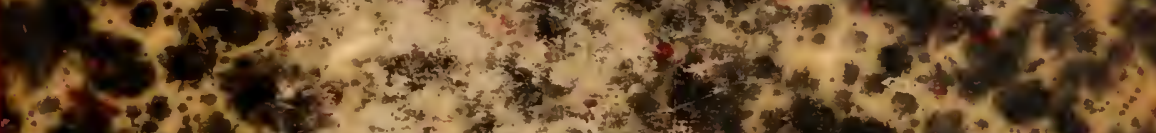

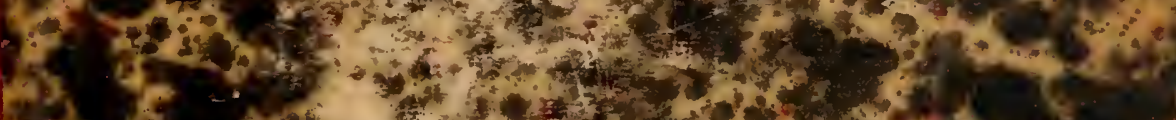

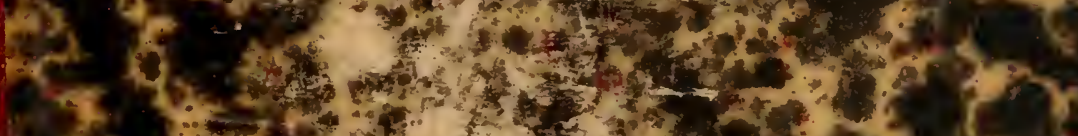

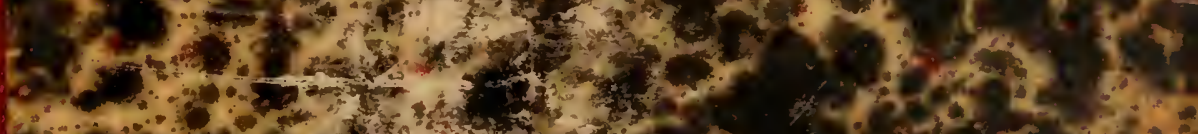

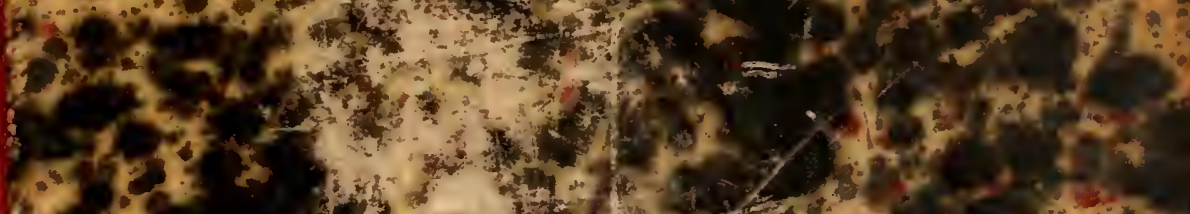

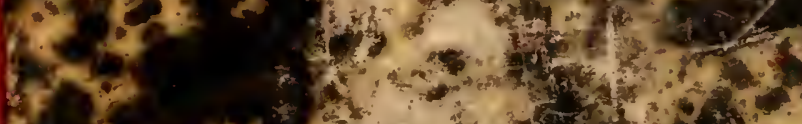

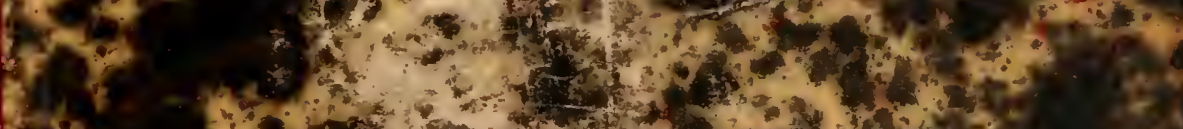

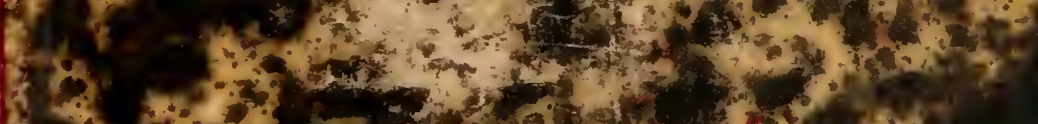

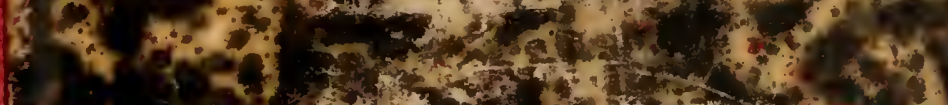

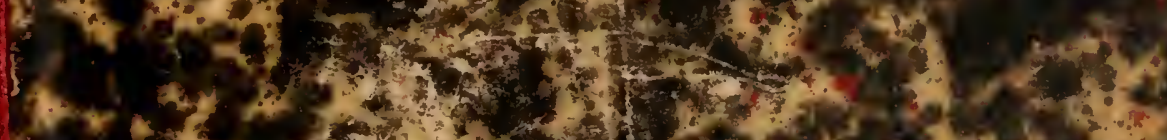

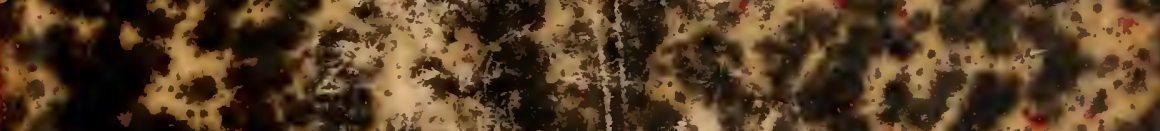

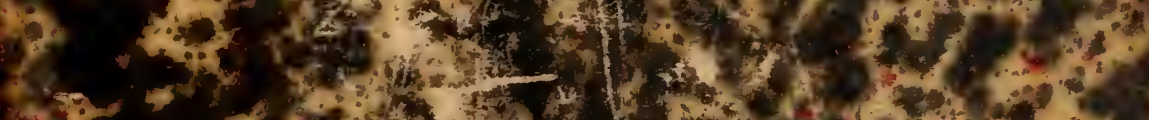

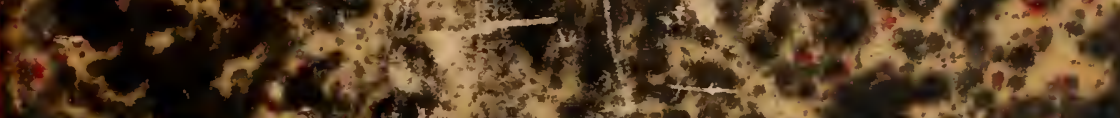

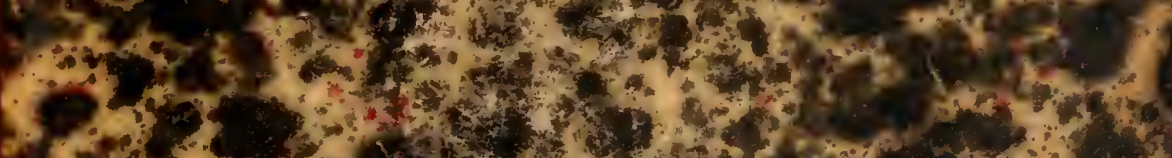

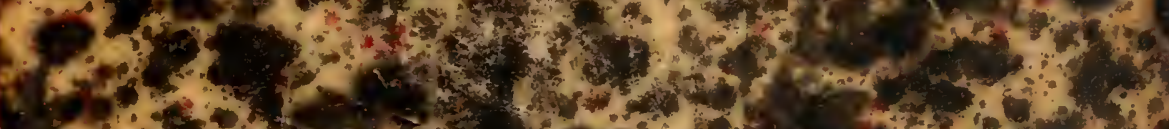
t. $3 x$.

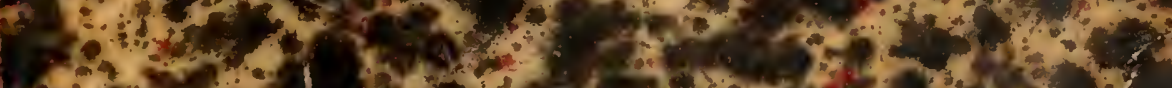

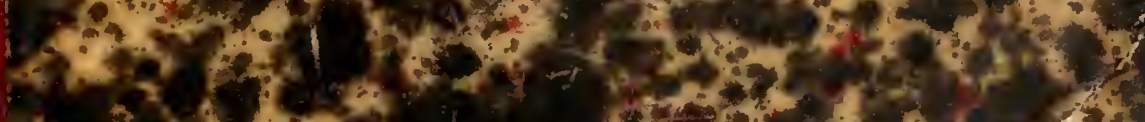

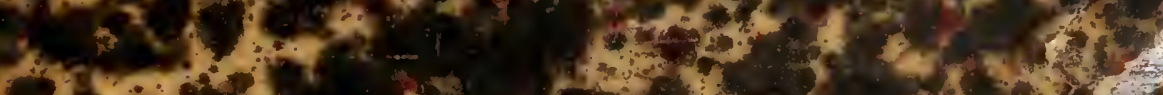

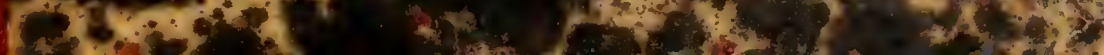




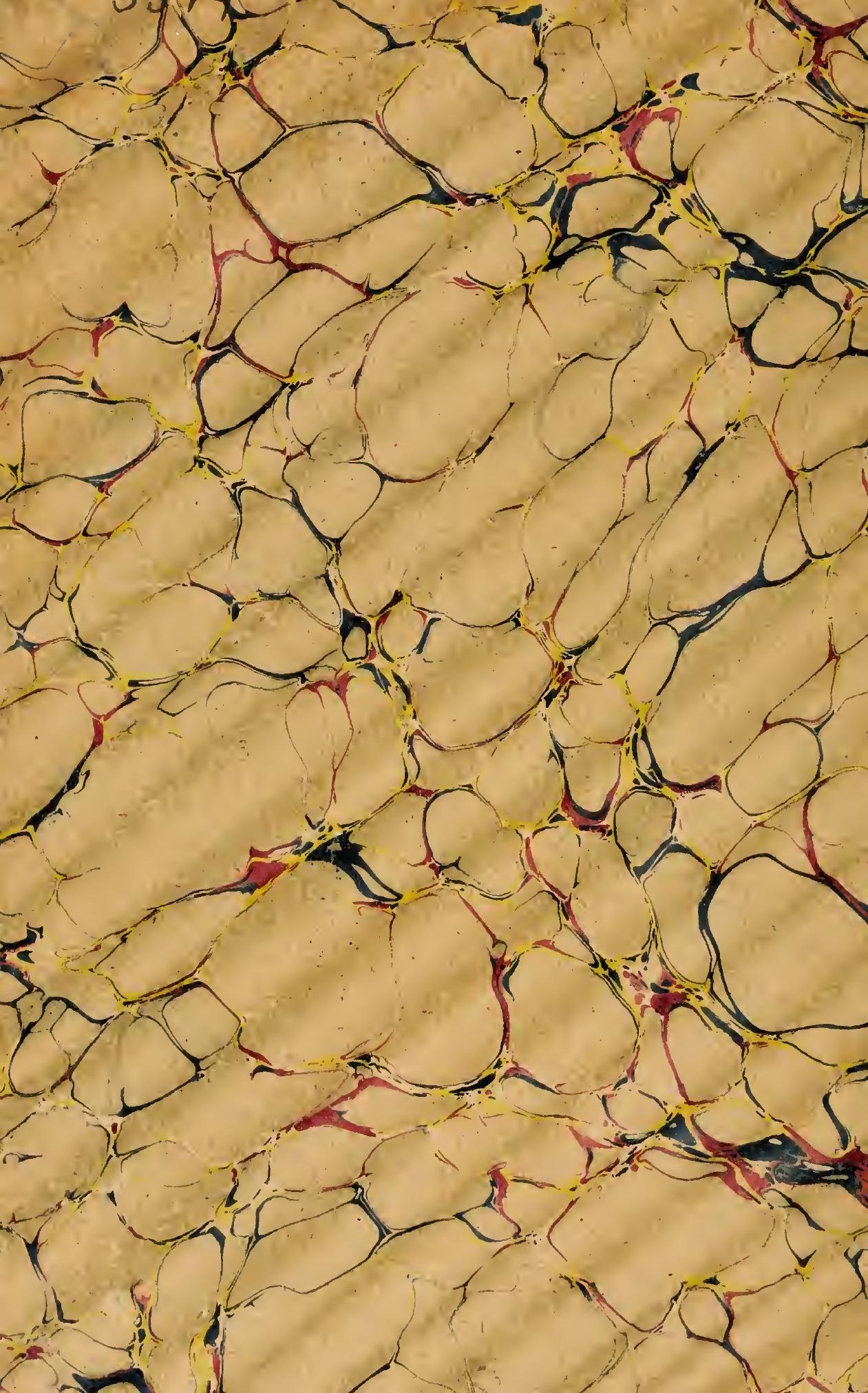




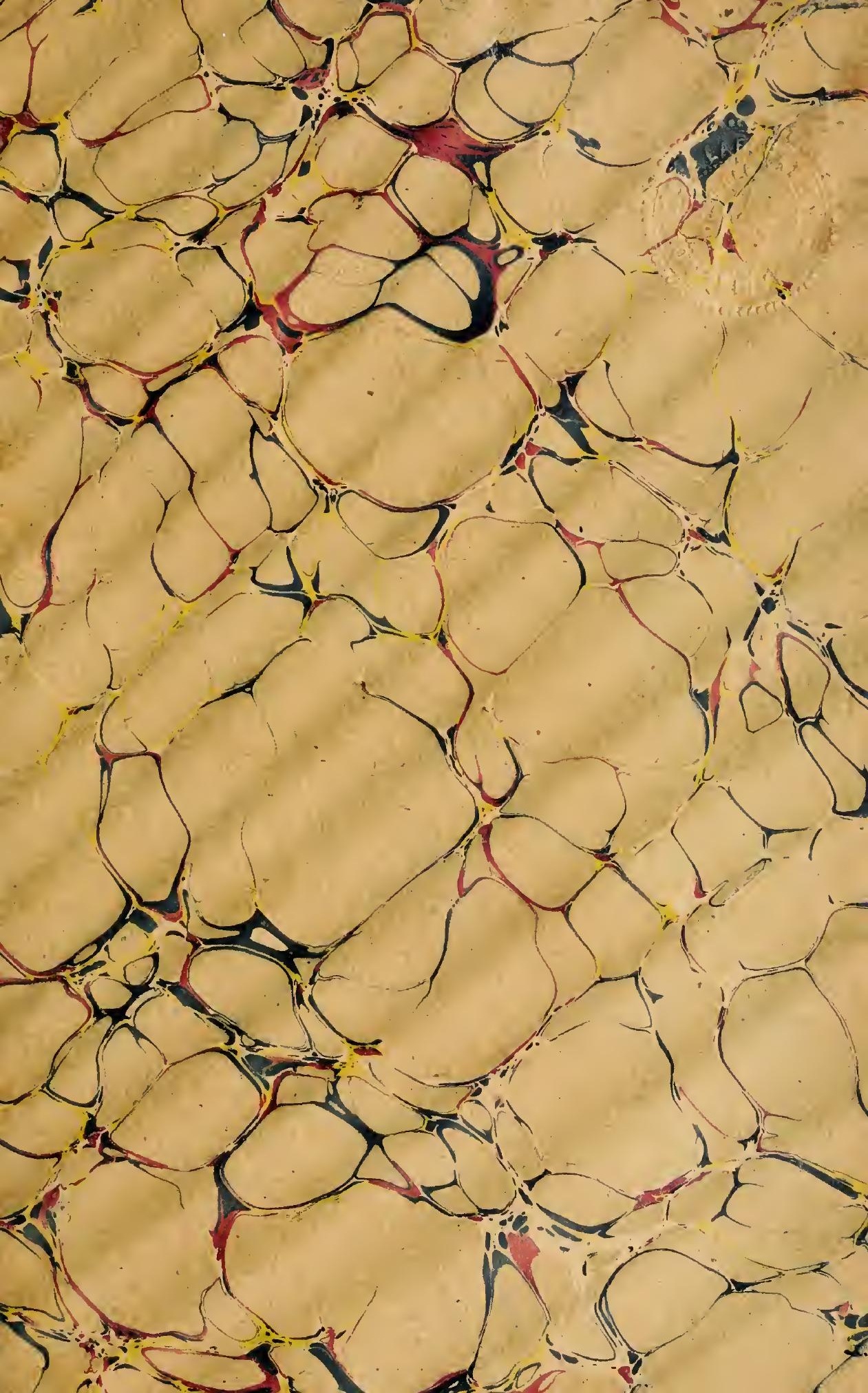




$$
(x-2)
$$








\section{TR A ITÉ}

DE

ZOOLOGIE CONGRETE 
IMPRIMERIE PAUL SCHMIDT 20, rue du Dragon, Paris

PHOTOGRAYRE DUCOLRTOUX ET HULLARD 


\section{TR A ITÉ \\ Ace. 3519.}

DE

\section{ZOOLOGIE}

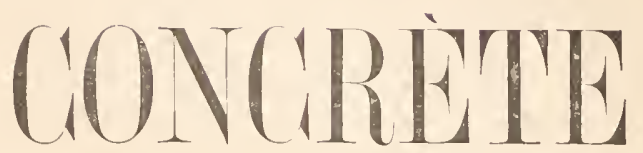

PA R

\section{Yves DELAGE}

PROFESSEUR

A LA FACCLTÉ DES SCHEXES DE P.ARIS

\section{Edgard HÉROUARD}

chllF Des trayaux de zoologere

I L. FACULTÉ DES SCIEYCES DE PAK1S

LEÇONS PROFESSÉES A LA SORBONNE

TOME I

LA CELLULE

ET

LES PROTOZOAIRES

AVEC 870 FIGURES DONT UN GRAND NOMBRE EN PLUSIEURS COULEURS

\section{P\RIS}

\section{LI'BRAIRIE C. REIN WALD}

SGILEICHER FRERES, EDITELRS

15, IUUE HES SAINTS-PIIIES, 15

1896 
921 


\section{A \\ H. DE LAGAZE-DUTHIERS}

AU FONDATEUR

DES ARCHIVES DE ZOOLOGIE EXPÉRIMENTALE

ET DES LABORATOIRES DE ROSCOFF ET DE BANYULS

\section{AU SAVANT}

QUI A CONSACRE SA VIE AU PROGRĖS DE LA ZOOLOGIE FRANÇAISE 



\section{PREFACE}

\section{Zoologie concrète?!}

Ce titre demande à être expliqué, ar on n'en saisit pas tout d'abord le sens. Et cependant il contient en lui seul toute lit justification de cet ourrage.

Cela semble insinuer que les précédents étaient. en général. des Traités de zoologie abstraite.

Ce n'est pas une insinuation, c'nst une affirmation positive ef nous espérons démontrer qu'elle est entièrement justifiée.

Il existe deux sciences seurs, la Zoologie et l'Anatomie comparée, qui, si on les compare telles qu'clles ŕtaient comprisis: il y a quelque cinquante ans. ont pour objet des études netlement distinctes.

L'Analomie comparée rnvisage les fonctions des êtres organisés et en particulier des animaux et elle étudie. non pas ces fonctions en elles-mèmes, ce qui est le propre de la Physiologie, mais les organes qui les remplissent, et romme ces organes sont d'autant plus semblables qur les ètres sont plus voisins, elle étudie la variation progressive dans la série animale des organes chargés de l'accomplissement de chaque fonction. Par rapport à l'organe elle est concrète puisqu' elle l'étudie en lui-même dans tous ses caractères; mais par rapport à l'animal elle est abstraite puisqu’elle fait abstration, dans chaque forme de la série, de tout ce qui n'est pas l'organe qu'elle étudie pour le moment. Et quand elle a passé en revue tuutes les fonctions et tous les organes, elle ne s'occupe point de réunir ces membres épars et de constituer des types d'ètres possédant tel ou tel arrangement d'organes pour accomplir l'ensemble de ces fonctions. Cela, c'est l'Anatomie comparée vraie, dont nous trouvons un superbe exemple dans l'ouvrage magistral de Ilenri Milne-Ebwards. 
La Zoologie. au contraire, nous parlons loujours do celle d'autrefois, est une science essentiellement concrète: clle montre l'animal lui-mêne. elle l'étudie comme personne entière ayant son individualité dans la nature. Cette individualité, il est vrai, n’est pas réelle, car la Zoologie met à la place des innombrables individus de l'espèce ou du genre, un individu idéal qui résume en lui toute l'espice ou tout le genre, mais cela n'altère en rieu son caractère concret, car il nous suffil de prendre en main un individu quelconque pour voir en lui toul ce su’elle nous décrit à l'occasion de l'espèce ou du genre auxquels il appartient. Par contre, au point de vue de la connaissance entière des animaux, clle est essentiellement incomplète, car ayaut pour but seulement de les nommer of de les classer, elle no lient compte que de re qui est nócessaire et suffisant pour ce but. éest-ì-dire des caractères extéricurs: ollo nóglige entièrement l'analomie interne, clle ignore les organes chargés des fonctions les plus essentielles.

Ces deux sciences sont done incompletes l'une el l'autre,

Lorsqu'on a voulu constituer, pour l'enseignement, des ouvragges où la science des animaux fùt traitée dans son ensemble, on a cru pouvoir les compléter l'une par l'autre en les associant. Or, on s'est en cela radicalement trompí.

C'est de l'Allemagne, dont nous arons élé si longtemps tribulaires pour les ouvrages destinés à l'enseignement supéricur, que nous est vemu le lype de ces ouvages mixks où, presque indiffírenment sous le titre de Zoologie ou sous celui d'Anatomie comparée, on troure le sujet laité de la manière suivante. Le liègne animal est tout d’abord divisé en grandes seclions (embranchement, phylum, ete.). Iclles que les Échinodermes, les Mollusques, les Vers, ele., qui sont étudiées séparément. Prenons une de ces sections, les Mollusques, par exemple. Le chapitre commence par des généralités sur le groupe: cost une pelite Aratomie comparée des Mollusques dans latpuelle on expose la varialion des fonctions el des organes dius a groupe. tont comme l'on ferail au chapitre Mollusperes d'un haile d'Anatomic compraréc tel quon le comprenail autrelois. Puis, on annonce que le groupe se divise en tant de elasses et immódiatement on alorde leur étude, on les examine séparénent, les unes a la suite des autres. Prenons celle des Gastéroprodes. On la 
traite comme on a fait de l'embranchement des Mollusques, c'est-i-dire que l'on écrit un petit chapitre d'Anatomie comparée tel qu'on le ferait pour une Anatomie comparée vraie, en se plagiant au point de vue de la rariation des fonctions et de leurs organes sans se préoccuper des animaux qui les possèdent. Puis on passe à la sous-classe, à l'ordre, au sous-ordre, toujours de la mème manière, et c'est seulement alor's que l'on change de plan. Li, brusquement, on tombe dans la Zoologie pure, c'est-ì-dire que l'on fait défiler sous les yeux, les familles. les genres principaux, roile mème les espices les plus importantes sans en faire connaitre autre chose que les caractères presque exclusivement extérieurs qui seuls les dislinguent.

Est-ce de la Zoologie?

Non!

Est-ce de l'Analomie comparée?

Pas davantage!

Ce sont des chapitres d'Anatomie comparéc emboités les uns dans les autres et dont le dernier de chaque groupe contient un clapitre de Zoologie pure.

Est-ce au moins une science mixte complétant l'une par lautre celles dont elle prend les titres?

Moins encore! Car, ce qui manque à l'Anatomie comparée pour une connaissance entière de l'etre et des ètres. c'est le lien de ces organes dícrits séparément, dans l’individu qui les posside; or, la partie zoologique ne le donne pas puisqu'elle ne définit plus que les caractìres extérieurs. Ce qui manque à la Zoologie, pour cette mème connaissance, c'est la conformation, la disposition, les rapporls des organes internes dans chaque forme; or, les chapitres anatomiques ne l'indiquent pas, restant toujours dans le vague et l'impersonnel.

L'étudiant arrive à la fin du chapitre des Mollusques, sans qu’on lui ait jamais expliqué comment est organisé, dans son ensemble, un quelconque de ces animaux. C'est cependant ce qu'il lui aurait fallu pour dissiper les brumes que laisse dans son cerveau le rague désespérant des descriplions abstraites. Il a besoin de grouper ces notions sans lien en un tableau où il puisse reposer sal voe sur un ensemble défini qui parle ì l’imagination, et. par suite, ait fuclque chance de rester dans la mémoire. 
Aussi l'avons-nous vu souvent se livrer au travail fastidieux de prendre un animal et de rechercher dans les chapitres anatomiques, tout ce que l'on dit de lui en citant son nom entre parenthèses à la suite de quelque courte indication, de maniò̀e à se constitner un type au moins sur lequel il puisse reposer son esprit. Mais jamais il n'y arrive, car colui que l'on cite ì propos de l'appareil digestif. n'est plus cité quand on passe au systène nerveux ou aux organes de la Reproduction. Il n'arrive jamais que le même soit pris pour exemple à propos de toutes les fonctions, et l'étudiant se résigne, de guerre lasse, à prendre les choses comme il les trouve et à rester dans le vague des abstractions. Ce travail yüil n’a pu farre, c'est à l'auteur à le fare pour lui. C'est à l'auteur à lui présenter les choses sous la forme où il le désire, où il a besoin qu'elles soient pour en aroir une notion précise et pour les retenir.

Le défaut que nous signalons est commun à tous les ouvrages allemands que nous avons pu examiner. Il se retrouve mème dans cette admirable encyclopédic, le Thier-lieich de Brons où une pléiadr d'auteurs de premier ordre onl fixé l'étal actuel de nos connaissances zoologiques. L’étudiant qui, par unc exception rare, se hasarte à fouiller dans ce rolumineux compendium y troure les matières exposées avec plus de détails. mais toujours suivant le même plan. Il lui faut pour trouver des notions concròtes, des descriptions anatomiques assises sur un être rócl, chercher dans les mémoires spéciaux, dans les monographies. Et rraiment il n'en a pas le lemps.

Nous arons longtemps vécu en France sur les traductions de ces ouvrages et cola n’a pas eu seulement l'inconvénient de nous imposer leurs défauts, mais celıi bien plus grave de nous les faire accepter. Nous sommes derenus les esclaves de ce plan défectueux et, sauf exception tout à fail rare, les onrages publiés en France sont conçus daus le neème esprit. On nous donne, sous le titre de Zoologie, des Anatomies comparées bàtardes, où l'Anatomic comparéce et la Zoologie sont simplement fragmentées el juxlaposérs.

Ce ne est pas à dire que ces ourrages ne puissent ètre fort bion faits. Il en est d'excellents diuns leur geme. Mais nous affirmons que ce genre ne convient pas pour apprendie. Cie sont des lives 
que l'on peut lire arec intérêt, consulter arec fruit, mais où on ne samrail apprendre quand on ne sait pas déjà. Nous n’aurions jamais pris la plnme si notre ambition eût pu ètre de faire mieux: dans la mème voie. car nous aurions pu ne pas réussir. Tandis que nous sonmes sùrs de rendre service on faisant autrement, en offrant à l'étudiant, comme nous Ie disions il y a un instant, les connaissances zoologiques sous la forme où il désire, où il a besoin. qui elles soient.

Nous arons ainsi défini notre but; il nous faut exposer mainlenant les moyens par lisquels nous espérons l'atteindre.

Le moyen le plus naturel serait évidemment de présenter mne série do tableamx monographiques des ères réels, c’est-à-dire des espices. Mais les espères ne diffirent que par des caractères extérieurs trìs secondaires. Les genres roisins sont eux-mèmes si semblables que leur organisalion intérieme diffère à peine. Il fiut aller au moins jusqu à la famille et, le plus souvent, jusqu'au sous-ordre pour trourer des différences d'organisation dignes d'ètre décrites dans mo ouvrage qui, nualgré son étendue, restera néanmoins b́ómentaire. Il semble qu’en choisissant dans chaque sous-ordre un itre bien caractéristique, en le décrivant en lui-mème complètement et en faisant connaitre. par leurs différences arec ce type essenticl, toutes les autres formes du sousordre qui méritent d'ètre signalées, on ait ì la fois les avantages d'une extension raisonnable, de descriptions précises des types essenticls servant de jalons, et d'mne connaissance sultisante des formes secondaires.

Ce plan est celui que nous avons toujours appliqué dans notre enseignement. Nous en avons pris le modèle dans Ies Iecons de notre maitre le professeur II. de LAcaze-Duthiers qui sait donner it ses descriptions de lypes un cachet si attrayant, gràce aux innombrables observations personnelles qu'il a recueillies au cours de ses campagnes seientifiques. Cela seul nous eùt fait un deroir de lui offrir cet ouvrage, mème en labsence des raisons majeures qu indique notre dédicace.

Nous arons done atoplé pour cel ouvrage le plan qui nous avait semblé fo meilleur pour l'enseignement oral. mais aree quelques modificalions. 
En cherchant dans chaque sous-ordre le type caractéristique à décrire à fond, nous nous sommes bien vite aperçus que souvent ce type nexiste pas. Ou bien il n'y a rraiment pas une forme réelle fondamentale dont les autres soient dérivées, ou bien il y en a plusieurs qui mériteraient à titre égal d'ètre choisies. En outre, il n'arrive pas toujours, tant s'en faut, que ces types aient été décrits entièrement par les auteurs. De l'un on n'aura éludié que lel ou tol système. de l'autre on ignore le développement. Il eùt fallu à chaque instant, sous peine de laisser la description incomplète. ce qui ne se pourait, metle dans le corps d'un animal quelque systìme d'organes qui n’a été décril que chez un autre plus ou moins lifférent. On eût en de la sorte. sous l'étiquette d'ètre réel. un ètre à deni idéal, nayant jamais existé dans la nature. Il valait bien mieux dès lors rejeter ces demimesures et conslituer de propos délibéré et toujours, pour chaque sous-ordre, un type tel qu”il se dessine dans l'esprit de celui qui a la connaissance du groupe entier, et qui résume en lui ce qui est commun à toutes les formes réelles de ce groupe, ou qui so présente comme une forme initiale simple, dont les autres dériveraient par des complications progressives.

Ciel être qui, idéal ou réel, représente en tout cas la forme fondamentale ì laquelle les autres se rattachent, nous l'avons appelé le Type morphologique. Nous l'avons décrit avec un soin particulier, faisant connattre à propos de lui tout ce qu’il est utile de savoir sur l'anatomie, la phrysiologie, l'embryogénie, du groupe dont il est te chef. Puis nous avons décrit, ì sa suite, les gemes composant le groupe.

La description des genres peut, en effel, se limiter le plus sourent aux caractères extérieurs. leurs caractìres splanchnologiques ne différant en général de ceux du type en rien d'essentiel. Nous n’avons pas mème cherché à donner tous les caractères différenticls extérieurs, ayant pour but non de fournir des diagnoses complìtes mais de montrer la variété des formes, el leur enchainement; et ce but, nous pensons l'avoir mieux atteint en nous allachant au critérium taxonomique, au caractère choisi pour ordonner le groupe et en montrant sa variation progressive à travers les genres qui le composent. Par contre. nous niarons pas crainl, toules les fois qu un genre présentait des particularilés 
anatomiques, physiologiques on embryogéniques d’un intérèt suffisant, de to faire connaitre avec tout lo dótail nécessaire.

Tel est lo principe qui nous a guidés.

Voyons comment l'application a pu en être faitr.

Il fallait définir non seulement los groupes inféricurs qui so décomposent immódiatement en genres, la famille ou le sous-ordre, mais aussi les groupes supérieurs, ordre, sous-classe, classe, qui, bien qü̈ls se divisent on catégories imaginées par l'homme ot non en ètres réels, n’on ont pas moins, cux aussi, leurs caractères, d'autant plus importants qu’ils sont plus généraux et d’autant plus difficiles ì dólinir qu’ils sont moins précis. Pour aux, plus encore que pour les groupes de genres, il fallait créer un Type morphologique, prosque forcément idéal, mais qui néanmoins donnât un corps et la vir ì ce qui, sans cola, fùt restó vague et abstrail. Nous avons done établi dans le règne animal un type morphologique pour chaque embranchement, dans l'embranchement un pour chaque classe, dans la classe un pour chaque sousclasse, dans la sous-classe 111 pour charpue ordro ot dans l'ordre un pour chaque sous-ordre; enfin dans chaque sous-ordre sont étudiés les genres qui le composent (').

Il no nous semble pas douleux que celte méthode est plus profitable pour l'étude que colle des ancions ouvrages, mais nous voyons bion l'objection qu me telle manicre de faire va susciler.

Vous reprochez aux autres, dira-t-on, de rester dans les abstractions et vous allez plus loin qu'eux encore en créant un lype idéal: vous revendiquez le mérite d’ètro concrets et rous ètes plus abstrails que reux à qui vous reprochez ce défaut.

Mais concrel ne veut pas dire réel. Un type peut ètre concret bien qu'il soit idéal. Qu'importe ì l'ćtudiant, lorsqu il lit une description précise arec l'indication de tous les organes et de leurs rapports, que l’ètre ainsi décrit existe récllement dans la naturo ou qu'il représento seulement la moyenne, nous dirions presque le porlrait composite d'un petit groupe d’ètres róels? Liidée

(1) Nous avons relégué les familles au second plan dans les notes, estimant qu'elles ne méritaient pas que l'on établit pour chacune d'elles un type morphologique et que les genres peuvent, au point de vue oủ nous nous sommes placés, se passer de ce groupement intermédiaire. 
qu’il se fera de l'être décril et plus tard du groupe entier noen sera ni moins précise ni moins justo.

Nous nous étions proposé d'abord de citer tous les genres. Mais il nous a fallu reconnaître que, pour ètre sùrs de n'en omettre aucun, au milieu de la foule immense de ceux qui sont épars dans les pelits mémoires descriptifs, nous aurions dù dépenser un temps énorme, hors de proportion avec le bénéfice que l'étudiant en retirerait. Ce sera l'affaire des éditions ultérieures de compléter sous ce rapport chaque volume, en même temps que de les corriger parallìlement au progrès incessant des découvertes. En attendant nous pouvons dire que la plupart, la très grande majorité des genres est ainsi signalée, et qu'en cherchant un nom de genre quelconque, à la lable alphabétique détaillée, qui terminera chaque volume et qui sera ensuite fondue avec les autres en une grande table unique à la fin de louvrage, presque toujours on le trouvera. En se reportant à la page indiquée on aura soit sa description. soit quelques mots de diagnose a son sujet, soit tout au moins l'indication de sa place au milieu des genres voisins: et toujours, en remontant au lype morphologique, on aura la description précise ef détaillée de sa conformation intérieure. sauf des différences secondaires qui n’altèrent point sa constitution essentielle et qu’indiquent les diagnoses par lesquelles on le fait dériver de ce type.

Entrons maintenant dans quelques détails sur l'exécution typographique, détails qui ne sont pas sans importance, car nous avons cherché avec grand soin à les combiner de manière à rendre facile la recherche de ce dont chacun a besoin.

Nous arons employé concurremment deux caractères, l'un large el espacé de lecture facile, pour le gros texte, l'autre plus lin of plus serré pour les notes. C'est l'application qui nous a paru la plus judicieuse pour lo cas présent de la méthode dont l'un de nous a montré ailleurs (') les avantages et qu’il voudrait voir appliquer partout.

1) Yves Detage : "Sur la manière d'écrire dans les sciences naturelles ». Préface d'un mémoire sur" "l'Embryogénie des éponges » in Arch. de zool. exple et gén'e, $2^{e}$ série, 1. $\lambda, 1892$. 
Gràce à ces dispositions, rien n'est plus facile au lecteur que de graduer la profondeur à lapuelle il veut pénétrer dans la connaissance des animaux: selon la catégorie i lapuelle il appartient ou suivant les besoins, il peut s'en tenir au type morphologique de la classe ou aller jusqu' à la sous-classe, à l'ordre ou au sous-ordre en lisant les indications complémentaires du texte fin ou en les laissant de còté; de là il peut pousser aux genres principaux décrits dans le gros texte ou aller enfin jusqu'au bout en lisant dans le petit texle des notes ce qui concerne les genres moins importants.

Par une innovation typographique qui nous parait très avantageuse, nous arous rendu saillante dans la marge. au lieu de la marquer comme dordinaire par un recul, la première ligne des alinéas conmençant par un nom de gente et nous avons marqué tous ces noms de genre par un alinéa. Cetle disposilion rend très fitcile ot tros rapide la recherche des noms de genre dans le corps de l'ouvrage lorsqu'on ne roudra pis recourir anx lables (").

Une aulre immoration, d'un ordre lout différent, consiste dans le remaniement de la nomenclature. Seule dans toutes les sciences. l'histoire naturelle manque de règles générales pour la formation des termes qu'elle crór pon désigner les objels de son étude. La confusion la plus complète règno dans nos vocabulaires taxonomiques. Aucun terme ne porle en lui quoi que re soil qui puisse faire reconnailre s’il désigne un embranchement, une classe, un ordre, une famille, ete. La mèmo désinence serl à dósigner pour les uns l'ordre, pour les aulres la famille. pour d'aulros un des groupements intermédiaires, et aucun auteur n’a cherché à établir une règle fixe pour l'ensemble des groupes successifs d'une taxonomie complite. Pour remédicr à cel étal do choses nous arons élabli la règle suivante. La désinence :
ia désigne la classe,
ida - l'ordie.
ina - la tribu,
ix désigne la sous-classe.
idie - le sous-ordre.
ince - la famille,
ea désigne les groupements intercalaires

(1) Cependant, lorsque nous donnons une liste de noms sans indication de caractère, nous la mettons sur deux ou trois colonnes. Dans ce cas, l'attention est immédiatement attirée sur les noms de genre qui ne sont pas à la ligne par les espaces blancs que produil cette disposition typographique. 
dont on peut aroir besoin éveutuellement, entre deux quelconques des groupes réguliers sus-indiqués (').

Nous n’avons pas parlé jusqu’ici de l’illustration de l'ourrage. Nous avons gardé pour la fin ce côté de la question pour le mieux mettre en relief en raison de son importance. C'est en effet une question capitale. Tous les étudiants sont unanimes à dóplorer la pénurie de figures dans les ouvrages qui sont entre leurs mains. Wème lorsqu’il y en a un grand nombre, il s'en faut encore de beaucoup qu’il y en ait assez. Un traité de Zoologie doit réserver dans ses pages presque aulant de place aux figures qu'au texte. si bonne que soit une description. elle esl toujours imparfaitement comprise et ne laisse dans l’imagination du lecteur qu'un tableau indécis lorsquelle n’est pas accompagnéc d'une figure, si simple et si sobre qu'elle soit. Ce n'est pas tout: pour qu'une figure soit utile, il faut quelle apprenne quelque chose et, pour celi, qu'elle ne soit pas une nourelle reprodurtion de celles que tous connaissent par cour pour les avoir déjà vues dans tous les ouvrages qui ont traité le même sujet. qu’elle ne soit pas ce que les étudiants désignent sous le nom expressif de vieux cliché. Mais ces vieux clichés s’ils chagrinent les lecteurs et aussi les auteurs. sont agréables aux éditeur's pour des raisons aisées à contprendre et ce sont eux qui les imposent aux uns et aux autres. Nous avons eu la bonne fortune de trouver un éditeur jeune ef intelligent, moins próoccupé de la question lucrative que du désir de faire bien, et qui nous a accordé un nombre illimilé de figures, toutes nouvelles. publiées dans le texte el en quatre couleurs.

Les Protozoaires. animaux simples, sans organes, sont de maurais exemples pour montrer tout le parti que l'on peut tirer de quatre couleurs londamentales et de leurs combinaisons pour

\section{(1) Pour les termes français il faudrait dire :}

ies pour les classes,
iés - sous-clisses, lides pour les ordres,
idés pour les groupes intercalaires de valeur quelconque.

Nous n'avons pas osé appliquer strictement cette rẻgle, retenus par la crainte de créer des termes trop clioquants en face de ceux consacrés par un long usage ou par leur emploi dans le langage non scientifique. Nous le regrettons un peu maintenant. Mais pent-ètre dans les volumes suivants nous dẹcciderons-nous à appliquer cette régle plus rigoureusement. 
illustrer l'analomic des animaux et nous prions le lecteur d'atlendre les volumes suivants avant de porter un jugement définitif à cot égard. L’un de nous (Yves Delage) s’est chargé plus spécialement du texle el l'autre (E. Hérouard) des dessins; mais nous n'en acceptons pas moins l'un et l'autre la responsabilité de l'ensemble, car c'est d'un nutuel accord que nous arons pris une délermination, quand, au cours de l'élaboralion du travail, un point présentait quelque difficulté spéciale.

Ciela nous amène à un aveu par lequel nous voulons terminer celte préface.

Nous avons dil les avantages de notre plan sans affectation de fausse molestie, arec celte franchise presque brutale qui est dans notre temprimament. Nous dirons done non moins franchement que l'ourage doit contenir des creurs. Dans le texle comme dans les figures, il en conlient inćvitablement.

Mais pouvions-mous les éviler?

Il est relativement facile de ne point engager sa responsabilité lorsque l'on se contente de résumer en un chapitre d'Inatomie comparée ce qu'ont dit les auteurs qui ont étudié les animaux dont il traite. Si ces aulours nont ótudié l’appareil digestif que dans tel lype, le système circulatoire que dans un second, les organes génilaux que dams un troisième, on se contente de dire que lappareil digeslif est ainsi fait clez cet animal, le systime circulatoire ainsi disposé dans cet autre. les organes génitaur ainsi conformés dans ce troisiome, ot lo lecteur s'arrange comme il peut de ces notions décousues. Autrement difficile élail notre tâche à nous qui nous sommes inposé de conslituer dans chaque description un type complet. En réunissant ainsi en un mème ètre ce qui n’a élé souvent vu que séparément chez plusieurs, parfois assez éloignés les uns des autres, nous nous exposons à établiz des connexions plus ou moins inexactes, à réunir des dispositions exclusives l'une de l'autre, ele., ete. Il faudrail avoir approfondi tous les groupes par des études personnelles pour éviler sùrement cet écueil.

Aussi liendrons-nous compte dans les édilions futures des avis qui nous scront donnés et mène des reproches qui nous seront faits. 
Mais il faut bien remarquer que, dans nos types morphologiques. pour avoir loccasion de décrire a leur sujet le plus grand nombre possible des dispositions anatomiques qui se rencontrent dans le groupe quils résument, nous arons sourent réuni à dessein des caractères qui s’excluent. Par exemple. nous donnerons à un Lamellibranche enfermé un pied bien musclé. voire même un byssus si cela nous convient; nous donnerons au type morphologique des Infusoires les cirres ventraux d'un Hypotrichide arec la musculature d'un llétérotrichide, etc.. ete. Ce ne sont pas là des fautes mais des avantages au contraire. Cela permet de constituer un type non réel. mais possible, et plus complet que les types réels : et, en continuant sa lecture, l'étudiant verra bientòt que les Lamellibranches enfermés ont, en général, le pied faible et jamais de byssus, que les Hétérotrichides nont pas de cirres ventraux, que les Iypolrichides n'ont pas de musculature cutanée, etc. L’avantage reste ct l’inconrénient disparait.

Le livre dont nous renons de délinir le bul et l'esprit est une neuvre de longur haleine et il ne nous faudra pas moins de huit années pour étudier en autant de rolumes lous les embranchements du Rìgne animal.

Puissions-nous avoir la force d'aller jusqu'au bout!

ter novembre 1893. 


\section{AVIS AU LECTEUR}

Dans toutes les descriptions anatomiques l'animal est supposé placé verticalement, la tète en haut, la face ventrale on avant. Les termes haut, bas, avant, arrière ont done, dans lourrage entice et sans exception, les significations qüimplique celle orientation. Les termes droit of gauche s'appliquent toujours à lanimal décrit saus tenir compte de la position de lobservaleur. Quand il y a arantage à rapporter l'orientation de quelque partie au rorps de l'animal plutot quaux dinensions dr l'asprace, nous employons les expressions dislal el proximal signifiant. velle-ci plus près du centre ef cellelà, plus pròs de la périphérir, cépllatique ou caudal signifiant plus près de la lète ou plus près do la queue. Pour désigner les plans principaux suivant lesquels l’animal peut ètre supposé coupé, ou sur lesquels on peut projeter des organes. nous employons les mots : sagitlal pour le plan médian-rertical, dorso-ventral, coronal ou frontal pour le plan vertical allant de droite à gauche et $1.0 \mathrm{cms}$ versal pour liun quelconque des plans horizonlaux perpendiculaires à l'axe vertical déterminé par l'intersoction des précédents.

Les dimensions sont exprimées. suirant leur malure en mètres, millimètres ou microns (millièmes de millimètres) représentés par les lettres ${ }^{m}$, ${ }^{\mathrm{mm}}$ ou $\mu$. Toutes les fois qu'aucune lettre ne précise l'unité employée c'est du millimètre qüil s'agit.

Les figures qui illustrent l'ouvage appartiennent à trois catégories. Les unes, et ce sont les plus nombreuses, sont des schémas drossés par nous de toutes pièces d'après les descriplions et les dessins des auteurs: elles sont marquées (Sch.). D’autres sont empruntées aux auleurs; nous l’indiquons par le nom de l'auteur précédé de l'abréviation (d’ap...) Ex. : (d’ap. Fronzel). D’autres, enfin, 
sont empruntées aussi à des auteurs mais morlifiées soit pour faire disparaitre dans la représentation d’un genre quelque caractère purement spécifique, soil pour meltre en lumière quelque trail de struclure qui nous semblait peru clait dans la figure originale. Nous ne pouvions ni prendre pour nous, ni infliger à l'auteur de la figure qui nous servait de modìle lia paternité de telles figures. Nous les avons indiquées en faisant précéder le nom de lauteur des lettres im. (Ex. : im. Carpenter) pour rappeler que nous avons imité, sans la copier tout à fait, la figure do cet anteur.

Nous arons mis partout les noms d'auteurs en toutes lettres ef entre parenthèses ayaut plusieurs fois constaté les obscurités qui résultent des autres manirres de laire. Les fermes taxonomiques non suivis d'une indication entre parenthèses sont ceux que nous proposons soit comme nouveaux, lorsque nous établissons un groupement auquel on navait pas songé, comme le sous-ordre de Scaiotrichides par exemple; d'autres, beaucoup plus nombreux, sont ceux des termes anciens dont nous avons modifié la désinence comme nous l'avons indiqué dans la préface.

L'onvrage contient, outer les tableaux synoptiques des pages 527 et suivautes qui pourront rendre des services pour les recherches, cinq tables, une méthodipue au rommencement et quatre ì la fin. De ces dernières me est londer billiographique auquel renvoie les chiffres entre crochets ì la suite des noms d'auteurs en petites capitales, la seconde est la lable des mots techniques, la troisième, celle des noms des hòtes des parasites, la derniore at la plus importante est l’index générique des Protozoaires.

Liusage des prenières se comprend sans explications.

Pour la dernière, quelques indications sont nécessaires pour en faciliter l'usige.

Celte table conlient deux sortes de caractires: l'un plus gros pour les noms de groupes, l'autre plus petit pour les noms de genres. Dans clacune des deux séries on trourera deux sortes de noms. Les uns, sans parenthìses, alignés au bord de la colonne, sont ceux des groupes aldoptés ou des genres décrits dans cel ouvrage. Les autres, entre parentheses el en recul sur l'alignement de la colonne, désignent les synonymes soil des groupes soil des genres décrits ef chaque synonyme est suivi d’un mol saus parenthisses qui est le nom du groupe ou du gente dont il est synonyme el pui est décrit 
dans l'ouvrage à la page indiquéc par le numéro qui suil son nom it sa place alphabétiqur.

Cela permel de trouver immédiatement les noms des genes et des groupes non acceptés dans cet ouvrage el relégués par nous en synonymie. Mais il fallait, en outre, faire l'opération inverse et indiquer, pour chacun des groupes et des gemres acceptés par nous, les noms synonymes admis par d'autres auteurs. D'ordinaire, c'est dans le corps du texte que se trouvent ces indications. Il nous a paru prélérable de les reléguer à la table et nous les avons placées entre parenthèses à la suite des noms acceptés par nous, après le numéro indiquant le renvoi au texte.

Dans la détermination des synonymes, nous niavons cité chaque terme qu'ume fois, à l'orcasion du genre avec lequel il se confond le plus complìlement, sans nous inquiéter sil a été aussi employé comme équivalent parliel de quelque autre genre. Notre but. en effet, était moins de donner une synonymie conplìte (ce qui est l'afficipe des ouvrages phis spéciaux) que de fournir au lecteur une liste de lermes aussi complète que possible, afin de ne jamais le laisser sans aucun renseignement sur lies noms de genres ou de groupes qu'il peut avoir occasion de chercher dans celte table.

Enfin, pour fomrnir une liste alphabétique des Prolozoaires parasites sans ajouter encore une table aux précédentes, nous arons, dans l’inder générique, marqué d’un astérisque les noms des genres parasites. Dans celte liste nons avons marqué de l'astérisque non seulement les parasites vrais permanents ou temporaires, mais aussi toules les formes commensales et celles qui ne demandent a thote quin support on un abri, estinant quil valait miens prendre le terme parasite daus son acception la plus large, laissant ì chaque lecteur le soin d'éliminer les formes dont il ne roudrail pas trnir compte au point de vue où il s'est placé. 



\section{TABLE DES MATIÈRES}

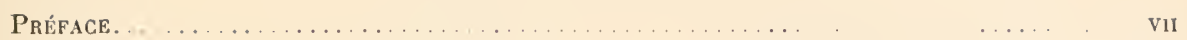

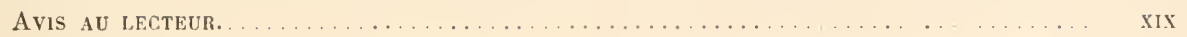

PREMIĹRE PARTIE

\section{LA CELLULE ET SES FONCTIONS}

I. - Structure de la Cellule.... _ . . . . . . . . . . . .

1. Le cytoplasma. . . . . . . . . . .

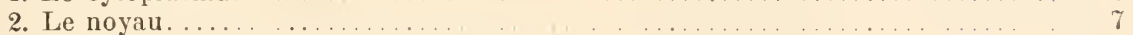

3. Le centrosome et la sphère attractive... ...................... 11

4. Les organes accidentels du cytoplasma ........................ 12

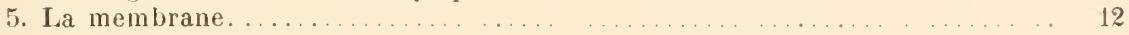

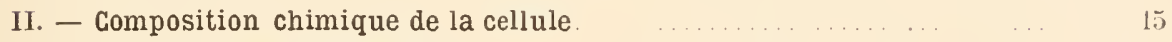

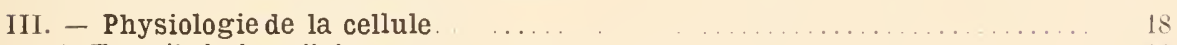

1. Travail de la cellule. . . . . . . . . . . . . . . . . . . . . . . . . . . 19

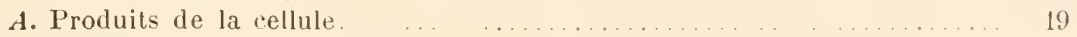

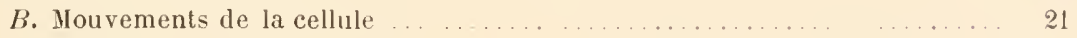

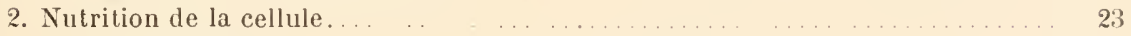

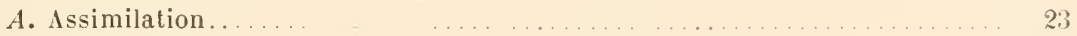

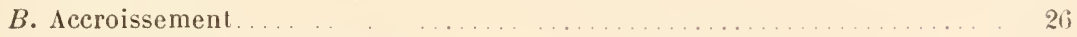

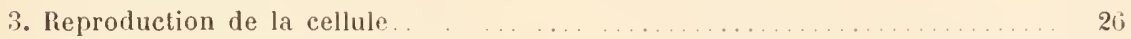

A. Division indirecte ou mitose . . . . ........................ 27

1. Division du noyaı. ... _ . . . . . . . . . . . 27

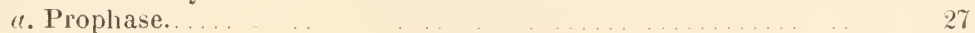

b. Mètaphase . $\quad$.... $\quad \ldots \ldots \ldots \ldots \ldots \ldots \ldots \ldots$

c. Anaphase ... .... .... . . . 32

Rapports des chromosomes avec les filaments. 35

Origine de filaments des fuseaux....... 35

Permanence des chromosomes. 36

2. Division du corps cellulaire. . 36

B. Division directe ou amitose... . . . . . . . . . . 37

Relation entre les divisions directe et indirecte... 37

Théories sur la division cellulaire...... 38

4. Conjugaison... . . . . . 40

A. Conjugaison totale.

$B$. Conjugaison nucléaire. 43

5. Fécondation............ . 44

Préparation et maturation des produits sexuels.

1. Division réductrice.................. . 45

a. Spermatogénèse et spermatozoïde ....... 45

b. Ovogénèse el øuf mùr..... $\quad 46$

2. Réduction chromatique....... ... ... is

3. Modifications cytoplasmiques........ . . . . . . . 49

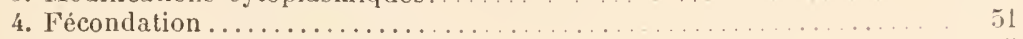

Théories des globules polaires . ........ . . . . . . . . 


\section{DEUXIENE PARTIE \\ LES PROTOZOAIRES}

$1^{\text {re }}$ Classe. - RHIZOPODES.

RHIZOPODIA

Type morphologique.

Structure.

Physiologie

La question des Monères

1re Sous-Classe. - Protéomyxés

Proteomyxiæ

fer Ordre. - Acystosporẻs.

Acyslosporida.

Type morphologique.

Genres

(6.)

66

66

66

2e Ordre. - Azoosporés.

Asoosporida.

Type morphologique

Genres

3e Ordre. - Zoosporés.

Zoosporida

Type morphologique

Genres.

2e Sous-Classe. - Mycétozoaires.

frordre. - Pseudoplasmodiès

Mycetozoariæ.

Pseudoplasmodida

Type morphologique.

Genres

2. Ordre. - Filoplasmodiès.

oul

Labyrinthulés.

Filoplusmodida

$\left.\begin{array}{c}\text { vel } \\ \text { Labyrinthulida }\end{array}\right\}$

Type morphologique.

Genres.

3e Ordre. - Euplasmodies

Euplasmodida (Myxomycètes)

Tyue morphologique.

Genres.

$3^{\text {e }}$ Sous-Classe. - Amœbiens.

fer Ordre. - Fymnammbiens

- Tupe morphologiqur.

\section{Amœbiæ}

Gymnamebida

\section{Structure}

Genres.

2. Ordre. - Thécamobiens

Thecampebilla.

Type morpholorique. Genres.

1er Sous-Ordre. - Gromides.

Gromidie.

2e Sous-Ordre. - Miliolides. .

Milinlida

3e Sous-Ordre. - Arènacés.

Arenarida. 
2e Ordre. - Perforès

Type morphologique.

Ler Sous-Ordre. — Lagénides ....... Layemirla'.

Type morylotogique. . .

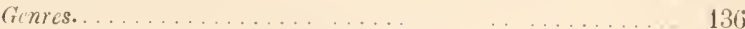

$2^{c}$ Sous-Ordre. - Chilostomellides. rhilostmmellitix. _ . . . . . . 138

Type morphologique ....................... 138

Genres.............................. 138

3e Sous-Ordre. - Textularides..... Textulurida. _............ 139

Type morphologique. .................... 139

Genres... . . . . . . .............. . . 139

$4^{\text {e }}$ Sous-Ordre. - Globigèrinilles... Gilobigerinirla . . . . . . . . . 141

Type morphologique........... . . ..... $14 \mathrm{~L}$

Genres... . . ..................... 14

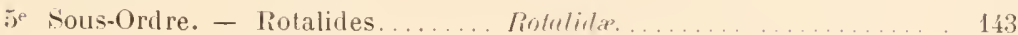

Type morphologique. . ......... . . . . ... 143

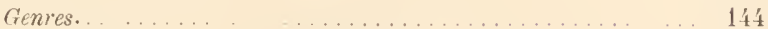

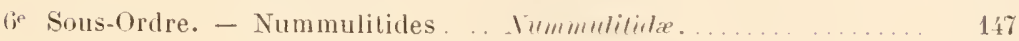

Type morphologique.... . . . . . . . . . . . 147

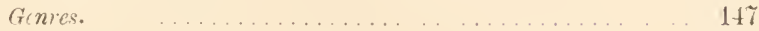

Appendice aux Foraminifères. . _ . . . . . $\quad \ldots \quad 153$

Stromatoporiens. . . ...... strometoproren . . . . . . . . . 15.3

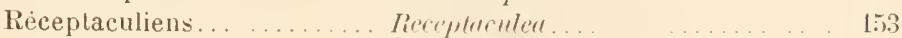

Testamobiformiens ........ Textnmmbifmmen . ........ 15́

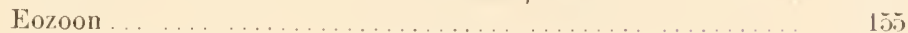

5e Sous-Classe. - Héliozoaires. ........ Heliozoariæ. ........... $156^{\circ}$

Type morphologique... . . . . . . . . . . . . . 156

Structure...... $\ldots \ldots \ldots \ldots 6$

Physiologie ...................... 158

1er Orilre. - Aphrothoracides.

Lhhiotherucidu............ 163

2e Ordpe. - Chlamydophorides... . Chlomydoyhmidn ......... . 166

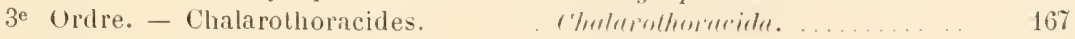

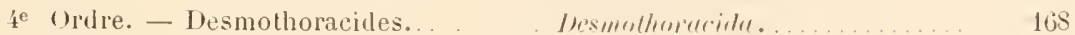

$6^{\mathrm{e}}$ Sous-Classe. - Radiolaires . . . . . . . Radiolariæ. . . . . . . . . . . . 169

Type morphologique... . _ . . . . . . ...... 169

Structure. . . . . . . . . . . . . . . 170

Physiologie. . . . . . . . . . . . . 172

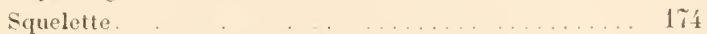

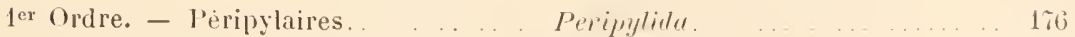

ler Groupe. - Monocyllaires. . Monoryllaren. .... ......... 176

Type morphologique.................. . 176

Ler Sous-()rdre. - Thalassicollides... Thalussicollida . . . . _ _... 177

Type morphologique. . .................... 177

Genres.............................. 177

2esous-Ordre. - Thalassosphèrides. Thalassosphxi ila...... _... 178

Type morphologique....................... 178

Genres............................. 178

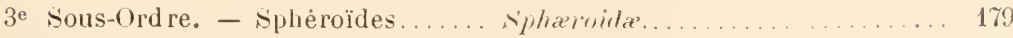

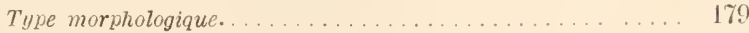

Genres.............................. 179

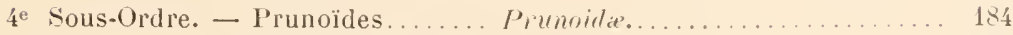

Type morphologique.................... 184

Genres.......................... 
5e Sous-Ordre. - Discoïdes.

Discoidie.

Type morphologique....................... 18 T

Genres.............................. 187

6e Sous-Ordre. - Larcoïdes........ Larcoideit ................... 191

Type morphologique. ....................... 191

Genres.............................. 192

2e Groupe. - Polycyttaires.... . Polycytlaren . . . . . . . . . . . . . 19.)

Type morphologique...................... 195

Structure....................... 196

Physiologie. . . . . . . . . . . . . . . . . . 197

Évolution........................ 197

Ler Sous-Ordre. - Collozoïdes...... Collozoidat. . . . . . . . . . . . . 201

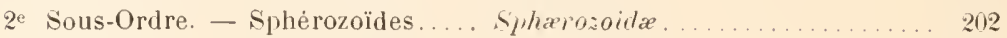

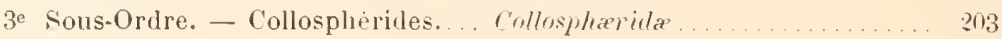

2e Ordre. - Actipylaires............ telip!lirla

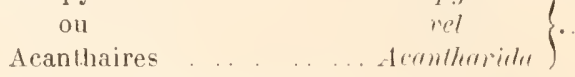

T!lpe morphologique..................... 20. 20 f

Ler Sous-Ordre. - Acanthonides... Acanthomirlat... . . . ....208

Type morphologique.................... 208

Genres................................ 20. 208

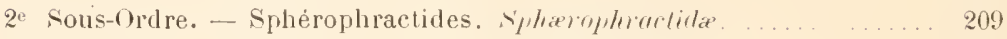

Type morrthologique. .................... 209

Genres................................ 210

3e Sous-ordre. - Prunophractides.. J'mophrofidit. . . . . . . 212

Type morphologique. . . ........ 212

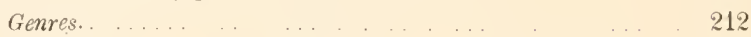

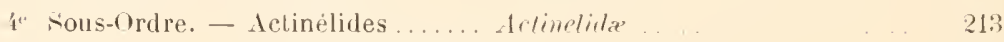

Type morphologique.... . . . . . . . 213

Genres. $\quad \ldots \ldots \ldots \ldots \ldots \ldots \ldots \ldots \ldots \ldots$

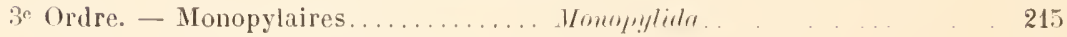

Type morphologique......... . . . . . . . 215

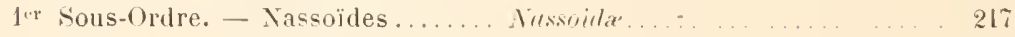

Type morphologique..................... 217

Genres............................ 217

2e Sous-Ordre. - Plectö̈des...... Plectnilæ. . .... .... 217

Type morphologique... .... ... _ . . . . . . 217

Genres.............................. 218

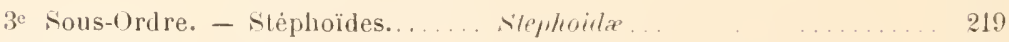

Type morphologique..... ... . 219

Genres.................... . . 219

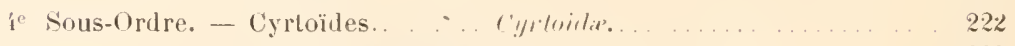

Type morphologique.................. 222

Genres.... . .................. 224

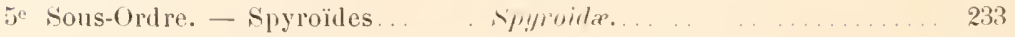

Type morphologique. .... . . . . . . . . . . . . . . 233

Genres..................... . . . 233

6 Sous-Oralre. - Botryoüdes...... Bolryuila . . . . . . . 235

Type morphologique...................... 235

Genres............................. ... 235

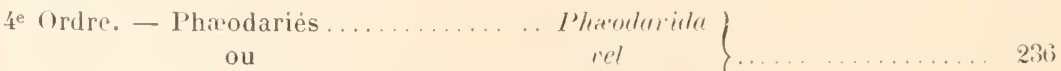

Cannopylaires ............ Camonylirla

Type moriholonique. 
1er Sous-Ordre. - Phaocystides.... Phæucystirl .._. . . 240

Type morphologique......... ... . . . . . . . . 240

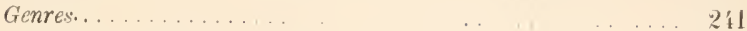

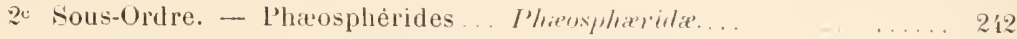

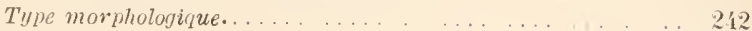

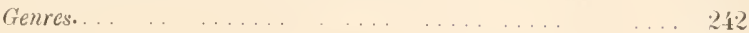

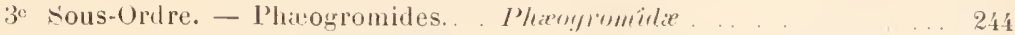

Type morphologique...... . . .... ... . . 241

Genres.................... 2ít

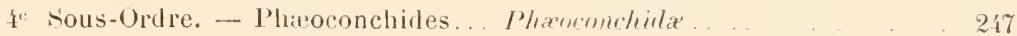

Type morphologique. . . .... . . . . 217

Genres.................... . 248.

Appendice aux Rihizopodes

T'axopodes.

Trixpuritere

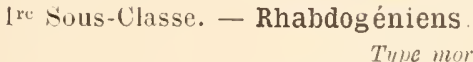

Type morphologique.

fro Tribu. - Céphalines

ou

l'olycystines.

¿ Tribu. - Acéphalines.

ou

Monocystines

Type morphologique.

C'ejhluelinre..

vel

Aephatina. vel

Jonoeystina...

Genres.

2 sous-Ordre. - Coccidides....... Curcillida.

Type morphologique.

Structure.

Physiologie...

Genres.

3e Sous-Ordre. - Hémosporides.... IIxmm:mirla.

Type morphologique.

Structure.

Physiologic.

Genres.

$\ldots$

.... 
2 e Sous-Classe. - Amœbogéniens. Urdre. - Nématocystides

Sous-Ordre. - Myxosporides

Type morphologique.

Struclure.

Physiologie

Genres.

Tubes parasites des Articules.

Amobosporidies.

Amøbiens de Sagilla.

Parasites de la vaccine, de la variole, de l'herpès zoster.

P'arasites de la fièvre du Texas... ... . . . . . . . . . . . . . 300

Parasites de l'hémoglobinurie des bestiaux ....... . . . . . 300

Parasiles du molluscum conlagiosum. ...................... 300

Parasites de la psorospermose folliculaire végètante ou maladie de Darier.

Parasites de la maladie de l'aget. .

Parasites trouvès dans certaines thoracentèses. . . . . . . . . . . . 300

Parasiles de certaines cirrhoses. _ ............ . . 300

Parasites des mélanosarcomes el des cirrhoses biliaires......... 301

Parasites du cancer.............................. 301

Sur le prétendu dimorphisme des Sporozoaires _.... . 302

$3^{e}$ Classe. - FLAGELLÉS.

FLAGELLIA. . .

303

303

303

305

318

319

319

Type morphologique.

1er Sous-Ordre. - Oligomastigides

Mingmastigidas.

320

1re Tribu. - Acraspédincs.

Acrespuedine

320

320

321

Genres.

C'loano-Flagellés.

Cresspeeline

2e Tribu. - Ćraspédines

ou

nel

(hoonoflagellina)

Type morphologique.

Genres.

2e Sous-Ordre. - Hétéromastigides. Helermmastiguda....

Type morphologitrue...

3e Sous-Ordre. - Polymastigides... P'nlymasligilde _. . . 337

Type morphologiquc.

l re Tribu. - Astomines.

Astominum

Type morpholoyique.

Genres.

2c Tribu. - Monostomines..... ILunostominu.

Type morphologique.

Gonres..................... 339

3c Tribu. - Distomines ....... Distomima... . . . . $3 \mathfrak{4 0}$

Type morluoloyique. 
$4^{\mathrm{e}}$ Tribu. - Trichonymphines.. Trichonymphina. . 342

Type morphologique.. . ......... 342

Genres.... . . . . . . . . . . . . . . .

Appendice aux 'l'richonymplina............... 344

2: urdre. - Euglénides... . . Emglenidu........ 345

Type morphologique................. 3 4.

Ire Tribu. - Astasines..... .. Astusina ........... 346

Type morphologique. . ............. 340

Genres...... . . . ............. 347

2* Tribu. - Euglenines... ..... Euglenima ........... . . . . 348

Type morphologique................. . . 348

Genres......... .................. . 349

3* Tribu. - Péranémines. ....Peranemina.......... ... 351

Type morphologique.................. 351

Genres.... . . . . . . . . . . . . . 351

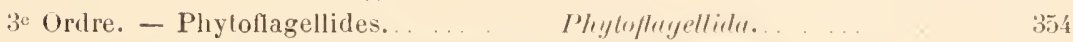

Ire Tribu. - Chloromonadines. Choromomadima..... 354

Type morphologique. . . . . . . . . . . . . 351

Genres.................. . . 3.5

2: Tribu. - Chromomonadines.. Chromomomalimu.... 355

Type morphologique. . . . . $\quad \ldots \quad \ldots \ldots . \ldots 35$

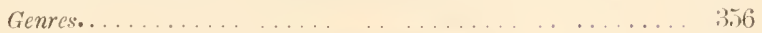

3 'Tribu. - Chlamydomonadines Chlamydomonarlina........ . . 360

Type morphologique. . ... . ............... . . 360

Genres........................... ... 362

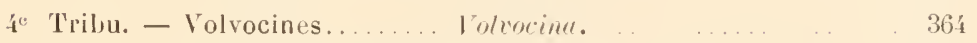

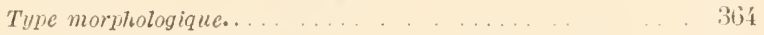

Strueture. . . . . . . . $36 \ldots \ldots \ldots \ldots \ldots \ldots \ldots$

Physiologrie . . . . . . . . . . . . . . . 365

Genres.......................... 367

20 Sous-Classe. - Silicoflagelles........ Silicoflagelliæ. ......... 371

Type morphologique................... 371

Genres............................ 372

3e śous-Classe. - Dinoflagellés ... . . . . . Dinoflagelliæ . . _.... . $\quad 373$

Type morphologique........ . ............ $3{ }^{2} 3$

Structure. ............ . . . 375

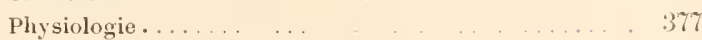

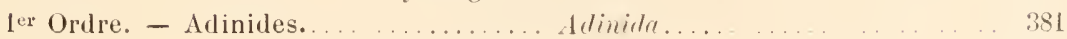

Type morphologique..................... 381

Genres................................... 381

2. Ordre. - Dinifèrides............ Diniferidı.... ... . . . 382

Type morphologique...................... 382

Genres................................. 382

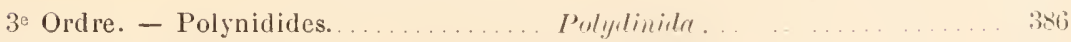

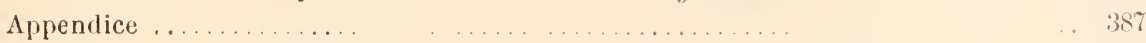

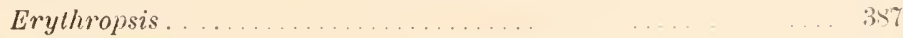

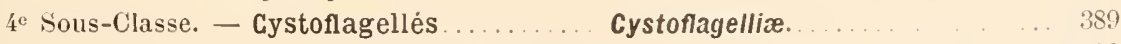

Type morphologique....................... 389

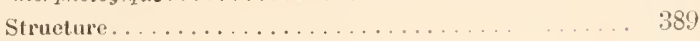

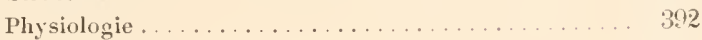

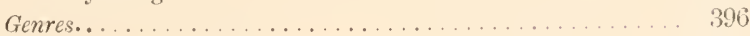

$5^{\mathrm{e}}$ Sous-Classe. - Catallactes ......... Catallactiæ .............. 398

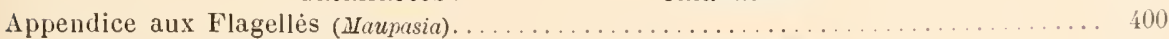

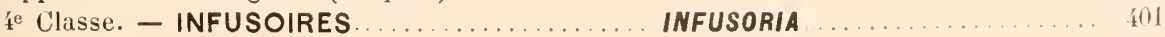


Physiologie................. 412

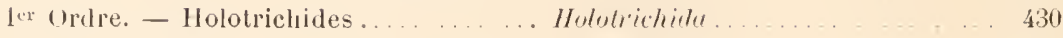

$1^{\text {er }}$ Sous-Ordre. - Gymnostomides . Figmmostomidat. . . . . . . . . . 431

Type morphologique. . .................. . . 431

Genres........................... 435

2e Sous-Ordre. - Hyménostomides. Hymenostomida. . . . _ ..... 444

Type morphologique. $\quad \ldots . \ldots \ldots \ldots 44$

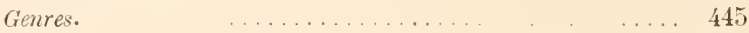

2u Ordre. - Hétérotrichides....... Heterntrichide... . 453

Type morphologique................. 453

1er Sous-Urdre. - Polytrichides.... Pulylrichidat...... . . 457

Type morphologique. . . . . . ...... 457

Genres...................... 458

2e Sous-Ordre. - Oligotrichides.... Oligotrichidie ...... . . 465

Type morphologique.... . . ....... . . 465

Genres......................... . 465

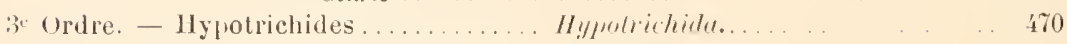

Tipe morphologique....... ... . . 470

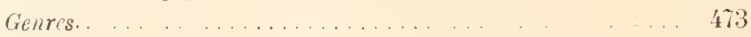

4' Ordre. - Péritrichides...........'Pritrichida....... 48

I*r Sous-Ordre. - Scaiotrichides ou Péri-

trichides senestres.... Scuiolithida..... $\$ 49$

2. Sous-ordre. - Dexiotrichides ou Pé-

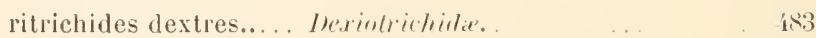

Type morphologique............. . . 483

Structure . . . . . . . . . . . . . 4 483

Physiologie.................... 486

Genres.......................... . . . . 489 2c Sous-Classe. - Tentaculifères

ou

Suceurs.

Type morphologique.

Tentaculiferiæ

vel

Structure.

Physiologie. .

Gienres.
Suctoriæ

500

500

500

503

508

11. - Caractères généraux des Protozonires.......
I1. - Tableaux symoptiumes de la classification des l'xotozoaixes

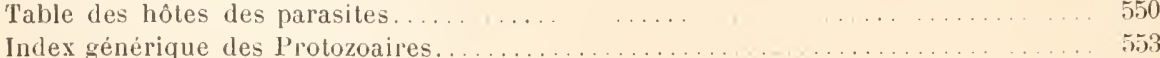




\section{LA CELLULE}

\section{LES PROTOZOAIRES}





\section{PREMIERE PARTIE}

\section{LA CELLULE ET SES FONCTIOAS}

RÉSUMÉ DE CYTOLOGIE GÉNÉRALE

Tout ce qui vit n'est que cellules.

Il n'est guère douteux qu'il y a eu autrefois, et il est possible qu'il existe encore aujourd'hui, les masses protoplasmiques vivantes, sans formes ni dimensions définies, non rncore différenciées en cellules. Mais, cela mis à part, on peut dire que la cellule est l'unité organique universelle. Nous proposons de la définir de la manière suivante qui nous paraîl bien rendre ce qu'il y a d'essentiel dans sa conception. La cellule est l'organe protoplasmique le plus simple qui, angant une forme propre el une taille délerminée, soil capable de vivre seul, ou n'ail liesoin de s'associer qu'à ses semblables pour former des étres capables de vie indépendante. Elle constitue à elle seule les èlres simples dits unicellulaires et, en se multipliant, elle forme les plus compliqués. Mème les parties qui, chez les uns et les autres, semblent le plus étrangères à sa nature dérivent d'elle. Nous montrerons, en temps et lieu, que les capsules, les coquilles, les masses gélatineuses où divers Protozoaires abritent leur corps, que la substance fondamentale du carlilage et des os des Métazoaires, et la partie liquide de leur sang, etc., que tout cela n'est que produits cellulaires de natures variées: en sorte que tout ce qui, chez les ètres vivants, n'est pas directement cellule dérive de la cellule.

On conçoit, dès lors, que l'étude de la cellule en général est le préliminaire obligé de tout ouvrage de zoologie.

Nous étudierons done d'abord la cellule et ses fonclions : mouvements, sécrétion, assimilation, accroissement, division, conjugaison. Après cela, nous serons en état d'aborder l'étude des Protozoaires. Mais pour les Hétazoaires, il n'en est pas tout à fait de mème, car chez eux les cellules s'associent en lissus et, pour cela, se différencient dans des sens très variés, se spécialisant de manière à mieux accomplir certaines de leurs fonctions générales, mais dégénérant d'un autre côté au point de ne pouvoir vivre seules, sans le secours des autres cellules de l'organisme. Ces différenciations spéciales, si utiles à l'ensemble, sont fatales aux 
éléments qui les subissent, en ce sens qu'elles suppriment la capacité de reproduction indéfinie qu'ils possédaient auparavant. Il en résulte qu'en se perfectionnant l'organisme cellulaire se condamne à mort, et ce serait en mème temps la mort de l'espèce si certains de ses éléments ne restaient indifférenciés et capables de survivre et de reproduire l'ètre entier, ou plutôt ne se différenciaient dans un sens tout particulier pour mieux assurer sa reproduction. C'est là l'origine des éléments reproducteurs dont les plus simples sont les spores.

D’autre part, c'est un fait général, presque universel chez les êtres vivants que, de temps en temps, deux individus se fusionnent en un seul. Toute la race acquiert, de ce fait, un regain de vie et d'activité. Chez les Protozoaires, cette fusion est facile puisqu'ils sont réduits à une seule cellule : elle constitue leur conjugaison. Chez les Métazoaires, la conjugaison des deux corps pluricellulaires, cellule à cellule, serait impossible; aussi prend-elle place, dans leur cycle évolutif, au moment où ils sont unicellulaires, c’est-à-dire représentés par leur élément reproducteur. La conjugaison des éléments sexuels devient la fécondation.

Ainsi nous devrions, pour ètre absolument méthodiques, décrire ici la conjugaison et n'étudier la fécondation qu'au moment d'aborder les Métazoaires. Mais ce serait séparer deux choses qui sont unies par des transitions insensibles et qui demandent à rester ensemble. Nous joindrons done l'histoire de la fécondation avec ses préliminaires, préparation et maturation des produits sexuels, à celle de la cellule, et cela constituera un chapitre assez complet de cytologie générale (*) qui sera une utile introduction à l'étude de la soologie.

\section{I. - STRUCTURE DE LA CELlule}

Plus encore que les formes animales appartenant ì un mème groupe naturel, la cellule est variable. La taille, la forme, la structure, les fonctions, tout varie en elle à un degré extrème. Aussi devons-nous appliquer dès maintenant à son étude la méthode d'exposition dont nous avons montré les avantages dans la préface de cet ouvrage. Nous allons prendre une cellule idéale, aussi complète que possible, et la décrire en elle-mème, quilte à indiquer dans les notes en quoi les diverses cellules réelles diffèrent de ce type.

Notre cellule est un petit corps, irrégulièrement arrondi, mesurant quelques centièmes de millimètres, translucide, très délicat, juste assez ferme pour conserver sa forme propre tant qu'il n'est pas comprimé (").

(1) La taille des cellules est extrêmement variable. Cerlains spermatozoïdes n'ont pas plus de $1 / 2$ :. . Le jaune de l'œuf des oiseaux n'est qu'une énorme cellule, celui

(*) Cependant, en raison de la nature de cet ouvrage, nos descriptions s'appliquent plus spécialement à la cellule animale. 
Un examen rapide au microscope nous la montre (fig. 1) composée des parlies suivantes que nous allons successivement étudier : lo une mince membrane prolectrice l'entourant de toutes parts; 20 un corps ou cytoplasma formant essentiellement sa masse; $3^{\circ}$ III noyau, glolule sphérique, pàle, plus réfrigent que le reste, situé au centre ou non loin de lui; $f^{\circ}$ un centrosome, globule beaucoup plus petit que le précérlent, qui ne se montre nettement et n'entre en activité qu'au moment de la division (").

\section{LE CYTOPLASHA.}

Examiné à un faible grossissement, le cytoplasma se montre sous l'aspect d'une substance homogène, demi-fluide, transparente $\left({ }^{2}\right)$.

Un examen plus minutieux et l'emploi de réactifs convenables permettent de distinguer, dans le crtoplasma en apparence homogène, diverses parlies fizurúes qui léterminent en lıi une víritable struclure. Ces parties figurées sont des fibrilles et des gromulations.

a. Fibrilles. - Les fibrilles sonl dr très fins filaments formés d'une substance plus dense, plus ferme que le reste du cytoplasma et qui sillonnent celui-ci dans tous les sens. Leur disposition rrair est l'objet de discussions qui ne sont pas encore tranchées.

Les uns croient qu'elles forment un réseau (fig. ?), c'est-à-dire qu'elles se ramifienl, anastomosenl leur's branches et les soulent aux points de rencontre on nouds du réseau. La sulıstance du réticulum constituerait le spongioplesma, celle qui occupe les mailles serait le hyaloplasma. Les autres assurent, au contraire, que ces filaments ne sont ni ramifiés, ni anastomosés, restent partout indépendants les uns des autres et forment de simples filrilles.

Ces fibrilles seraicnt contractiles et on assure les aroir rues parfois

d'Epiornis devait ètre gros comme une petite orange. D'ordinaire, la cellule est microscopique et mesure de $1 / 100$ à $1 / 10^{\circ}$ de millimètre. La forme est si rariable qu'on ne saurait presque rien dire de général à son sujet. On peut considérer une forme sphérique comme fondamentale et primitive. Mais celte splière se transforme en polyèdre jar pression réciproque dans divers épithéliums, en lamelles par tassement dans l'epiderme et les endothéliums, en fibres dans les nerfs et les muscles, etc., enfin, dans les leucocytes ou chez les Rhisopodes, elle devient tout à fait irrègulière par suite de prolongements rariés qu'elle émet dans tous les sens.

I) Chez les Protozuaires, le centrosome n'existe presque jamais. Il n'a été observé que dans un nombre de cas extrêmement restreint, chez les Noctiluques par exemple.

(2) Nous appelons ici cytoplasma le protoplasma du corps cellulaire pour l'opposer au nucleoplasma ou protoplasma du noyau. Les. Illemands ne font pas cette distinction et appellent protoplasma ce que nous appelons cytoplasme. 
se contracter dans la cellule vivante. Leur ensemble constiluerait la substance filaire ou mitome, et la substance dans layuclle elles serpentent serait le paraplasma. Ce paraplasma ol cotle substance filuire ne diffèrent done, le premier du hyaloplasma el la seconde du spongioplasma, que par l'ilée que se font de leur disposilion et de leurs proprićtés les auteurs qui ont proposé ces dénominations. Filaments du réseau ou fibrilles sont d'ailleurs d'une finesse extrème : leur épaisseur n'alleint pas li..

b. Granulations. - Le plasma homogène interposé aux filaments ou aux fibrilles est parsemé de granulations extrèmement nombreuses et très potites, qui ont, pour la pluparl, un diamètre inférieur à $I_{\not \prime .}$. Leur taille est d'ailleur's très inégale. Élles ont l'aspect de petites particules formées d'une substance plus dense que le reste du cytoplasma.

$c$. Vacuoles. - On olsserve généralement, dans le plasma qui haigne les filaments et les granulations, des vacuoles, c'est-i-dire de pretites cavités arrondies contenant, non du protoplasma, mais un simple liquide aqueux tenant en dissolution quelques matières albuminoüles el surtout des substances salines. Ces vacuoles sont extrèmement variables. D'ordinaire elles sont très visibles; parfois, quand elles semblent absentes, un examen plus minutieux les fait découvrir, mais si petites el si serrées qu’elles avaient par là échappé à la vue (').

(1) Les observateurs sont loin d'être d'aceord sur la signification de toutes ces parties. La structure du cytoplasma est un des sujets les plus chaudement controversès de la cytologie.

Quelques-uns le croient homogime, non qu'ils nient l'existence des granulations, des vacuoles ou même des fibrilles, mais ils ne voient dans ces organes que des particularités sans importanee et laissent à la substance amorphe interposée le ròle essentiel dans la manifestation des propriétès. C’est principalement Strasburger [8́1 qui a soutenu cette opinion et elle a encore beaucoup d'adhérents parmi les botanistes, ce qui tient à ce que, chez les plantes, le pliénomène si facile à constater de la colatiou du cytoplusma dans la cellule semble incompatible avee une strueture fixe quelconpue. Mais, dans ce cytoplasma homogène, Strasburger distingue deux parties, une d'importance secondaire, nutritive, le trophoplasma, et une active dans tous les phénomènes essentiels dont la cellule est le siege, le kinoplasma. C'est ce dernier 'jui, pentant la division, fournit les filaments des asters et du fuseru.

La théorie réliculaire (fig. 2) est due à IInitzalaxix [ 72$]$ el a lexplg [85]. C'est ce dernier qui a proposé les noms de spongioplasma et de hyaloplasma. Il se séprare de Heitzmann principalement en ce qu'il accorde au liyaloplasma amorphe le role essentiel que celui-ei attribuait aux filaments du réseau.

La première observation des fibrilles est due à Kupfrer [75], mais cest surtout Flemming [82] qui a généralisé la théorie de la strueture fibcillaire (fig. 3) et c'est lui qui a eréé les dénominations de Filarsubstans on Mitom et de paraplasua. Ces fibrilles seraient les agents de la contratilité du protoplasma.

Personne ne nie l'existence des granulations, car clles sont très faciles à voir et connues depuis fort longtemps. Mais. tandis qu'on les considère d'ortinaire comme des particules inertes, sins attributions bien importantes, certinins auteur's sont d'un avis tout différent et voient en elles la seule partie vraiment vivante de la cellule, la seule artive dans la manifestition des propriétés. Hivers réactifs, la fuchsine acide surtout, colorent ces parlicules et en font roir beaucoup plus qu'on n'en apercevrait sans cela. Le cytoplasma apparait alors sous un aspect tout à fait nouveau. Il se 


\section{LE NOYAU}

Le noyau (fig. 7) de notre cellule se présente sous l'aspect d'une vésicule pàle. arrondie, qui d'ordinaire occupe sensilılement le centre

montre formé de granulations qui constituent la presque totalitẻ de sa masse, le lıyaloplasma amorphe se réduit à une minime quantité de substance interposée entre elles, et les fibrilles elles-mêmes fig. 4) apparaissent comme n'arant pas d'existence réelle et formées de minimes granulations orientées à la file. Il semble qu'en présence le cet aspect, on soit autorisé i considérer la structure rétirulèe ou fibrillaire comme dépourvue de réalité objective. La granulation devient le seul èlément vivant du cyto-

Fig. 2.

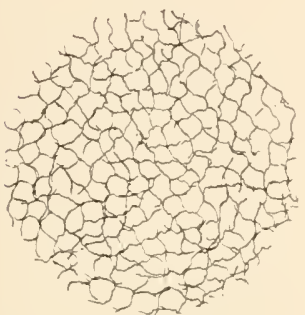

Structure réticulaire (Sch.).
Fig. 3 .

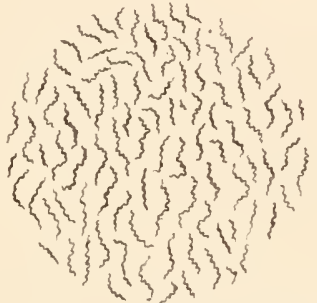

Structure fibrillaire (Sch.).
Fig. 't.

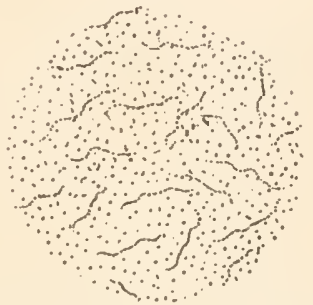

Structure granulaire (Sch.).

plasma et le facteur spécifique des propriétés cellulaires. C'est ainsi que MAGGI [7s] et surtout Altwank [9t], ainsi que ceux qui les ont suivis, considèrent les choses. Pour marquer plus nettement cette opinion, Altmann substitue au nom banal de granulations celui de granules. La structure granulaire est pour lui la structure vraie du cytoplasma.

Personne, non plus, ne nie l'existence des racuoles, mais, elles aussi, sont considérées en général comme des accidents de structure sans signification spéciale. Or, outre ces vacuoles banales, on arrive à découvrir en employant de très bons objectifs, que souvent la substance mème du cytoplasma, celle qui a l'air homogène, est en réalité criblée d'une multilude énorme de vacuoles extrêmement petites, régulières, arrondies ou subpolyédriques par pression réciproque (fig. 5). Ces racuoles élémentaires ont été découvertes par Kunstıer [82], mais elles ont été surtout étudiées par Bürschul [92] qui a généralisé leur existence, les a appelées alvéoles pour les distinguer

Fig. 5.

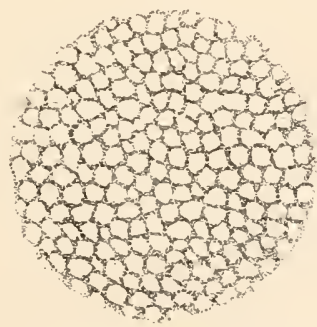

Structure alvéolaire Sch
Fig. 6.

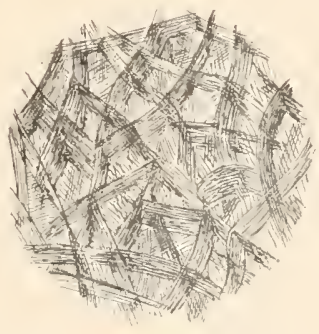

Structure a réolaire (Sch.).

des vacuoles banales et a conclu á une structure alćolaire générale du cytoplasma. Pour lui, le réticulum n'est que l'inagge négative du réseau des alvéoles, les fibrilles 
de celle-ci ("). Cette vésicule possèrle naturellement une membrane; sa

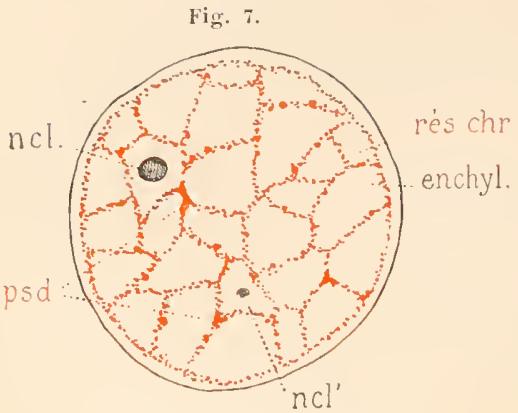

Noyau idéal (Seh.).

enchyl., enchylema; nel., nucléole; nel'., nucléole secondaire; psil., pseudo-nucléole; rés. elır., réseau chromatique.

laire, pour se reformer aussitôt après. cavité est occupée, non par une substance gélatineuse comparable au cytoplasma, mais par un liquide, te suc nucléaire, qui baigne trois sortes d'éléments figurés : le réseau de linine, la chromatine et le (ou les) nucléole.

a. Membrane nucléaire. - La membrane nucléaire est très mince, hyaline, parfaitement tendue sous la pression du suc nucléaire et sépare ce sue du cytoplasma; elle est constante et ne disparait que momentanément, pendant une courte phase de la division cellu-

n'existent pas ou sont des accidents de structure de la substance plasmatique interalvéolaire, les granulations enfin existent, mais à titre de particules inertes, non vivantes, logées aussi dans la substance interalvéolaire. Celle-ci est formée d'une matière albumineuse vivante complexe, tandis que le contenu des alvéoles est un simple liquide inerte, le chrlema.

Eisuond [90] soutient une théorie intermédiaire qui tient le milieu entre l'alvéolaire et la réticulaire. Il y aurait un réticulum formé, non de filaments, mais de lamelles protoplasmiques ramifiées et anastomosées, limitant des aréoles polygonales, communiquant entre elles par le fait que leurs parois sont partout incomplètes (fig. 6). L,es aréoles seraient occupées par un liquide comparable au chylema : c'est la théorie aréolaire.

Tous ces aspects sont très réels. Il est incontestable que le cytoplasma montre des fibrilles, des granulations et des alvéoles. La difficulté est de savoir laquelle de ces structures est caractéristique, el si l'une d'elles est essentielle et universelle, tandis que les autres ne seraient 'jue des aspe'ts sans réalité oljective ou des dispositions sans importance. Clracun tient a sa thicorie et montre des préparations très nettes, mais aucun n'est arrivé à pronver qu'une structure soit seule réelle, univer'selle ef essentielle à l'exclusion des autres. Il semble qúe les résultats dépendent autant des réactifs employés, que do la nature des cellules observées et il reste possible que la structure soit tantòt réticulaire, tantòt fibrillaire, tantòt alvéolaire. Uuant aux granules, ils existent incontestablement partout, mais leur signification reste, malgré tout, très problématique. A notre avis, il ny a rien d'essentiel dans ces dispositions direrses qui ne correspondent qu’à des fonctions speciales et locales: le vrai facteur des propriétès générales de la cellule est la substance chimi jue du protoplasma dans toulesses parlies, et les fibrilles, alréoles et granules sont, ì titre ėgal, des différenciations locales correspondant i des fonctions spéciales. Des observations récentes confirment cette opinion: la cellule nerveuse serait fibrillaire, la cellule glandulaire alvéolaire, etc.

(1) Cela n'est rrai que quand le cytoplasma est sensiblement lomogène. Quand il contient une suffisante abondance de matières non protoplasmiques, réserves nutritives, celles-ci se rassemblent vers un pôle, et le protoplasma vers l'autre, en sorte que la masse de chacune va en décroissant régulièrement en sens inverse d'un pôle a l'autre. O. IIERTwig [81] a montré que, dans ce cas, le noyau se rapproclie du point où il y a le plus de protoplasina. On peut dire qu'il occupe sensiblement le centre de gravité du cytoplasma pur de la cellule : c'est la loi de position dn noyan. 
b. Suc nucléaire. - Le suc nucléaire ou enchylema (enchyl.) est un liquide qui joue dans le noyau le mème rôle que le suc cellulaire dans les racuoles du cytoplasme. Il est, comme lui, formé d'une dissolution paurre en substances albumineuses et assez riche en sels: mais il est en proportions beaucoup plus grandes que ce dernier et forme la majeure partie de la masse du noyau. Ce suc. sulıstance accessoire, baigne les éléments figurés essentiels.

$c$. Réseau de linine. - Le réseau de linine ou réseau achromatique a été ainsi nommé parce que l'on a appelé linine sa substance, et que cette substance est incolorable par les teintures ordinaires. Il est constitué d'une manière semblahle au réseau filaire du cytoplasma. Ce sont des filaments extrèmement fins, ramifiés et anastomosés en réseau, c'està-dire dont les différents brins aloutissant à un mème point nodal sont soulés en ce point. Partout où une branche rencontre la membrane nucléaire. elle se soude à elle et s'y termine.

Cependant, on n'est pas bien sìr que cette lisposition réticulée soit l'expression réelle de la nature des choses. Dans un réseau vrai, tous les brins ont la mème valeur. Or il est possible qu'il n'en soit pas le mème ici. On voit, en eflet (fiģ. S), au moment de la division du noyau, le réseau se rompre en certains points de maniere ì laisser un filament continu auquel restent appendus, comme de petites ramifications courtes et simples, les restes de brins qui se sont rompus. On est donc en droit de se demander si le réseau ne serait pas formé d'un long filament continu, pelotonné, avec de petites hranches secondaires établissant des anastomoses temporaires entre ses différentes sinuosités. Mème, dans certains

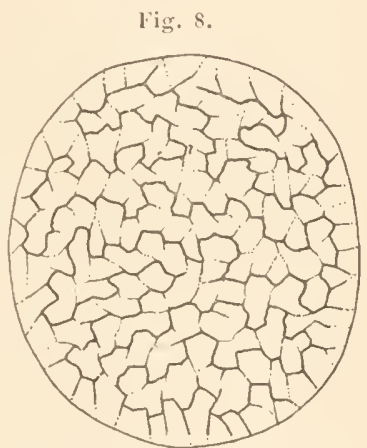

Spireme en formation (Sch.). cas, ce travail préparatoire te la division isole, non pas un long filament unique, mais plusieurs filaments dejà reconrlés en anse pour constituer les futurs chromosomes. Dans ce cas, if faudrait listinguer dans le réseau ces anses et les filaments secondaires des anastomoses temporaires (").

(1) Toutes ces questions sont trés liligieuses. Nous donnons la l'opinion la plus ordinaire, d'après laruelle il y aurait, sous l'apparence de réseau parfait, les novaux à filaments uniques et des noyaux constituant plusieurs anses, distinctes. Ces derniers sont appelés noyaux de liabl. Dans ees noyaux fig. ?), les anses sont orientées et ont toutes leur convexité tournée vers un des pôles du noyau oì elles respectent un espace oecupé par une substance claire et appelé champ polaire.

Strasburger [84], Carror [8́'], ete., ont émis l'opinion que le réseau n’a pas d'existenee réelle. Le filament serait vraiment unique et continu d'un bout à l'autre sans ramifications; là

Fig. !.

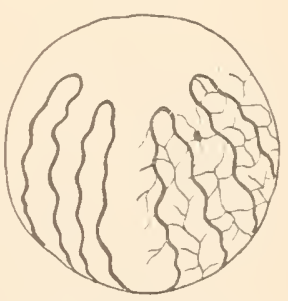

Structure du noyau d'apr. Rabl (Sch.). 
d. Chromatine. - Sur le réseau de linine sout disposés (rés. chr.) de petits grains, formés d'une substance très avide de matière colopante el qui a reçu pour cela le nom de chromatine. Les petits grains eux-mèmes sont appelés quelquefois mucléomicrosomes pour les distinguer des microsomes ou granulations du cyloplasma. Souvent, sur les points nodaux du réseau, la chromatine s'accumule en masses un peu plus considérables que l'on a appelées les corps nucléiniens ou pseudo-nucléoles (ncl'.).

Les rapports exacts des grains de chromatine avec les filaments de linine ne sont pas très nettement élucidés. Souvent les premiers paraissent ètre simplement accolés aux seconds, mais souvent aussi ils sont nettement dans leur épaisseur, et il se pourrait bien qu’il en fùt toujours ainsi. Leur diamètre est bien supérieur à celui des filaments, mais ceux-ci se renfleraient au niveau des grains pour les revètir d'une mince couche de leur substance.

e. Nucléoles. - Libres dans le suc nucléaire, à l'intérieur des mailles du réseau el sans allache avec lui, se trouvent un ou plusieurs globules arrondis constituant le (ou les) nucléole ( $n c l$.). Quand il y en a plusieurs, il y en a, d'ordinaire, un de taille prédominante. aussi a-t-on cru qu’il étail unique. Lorsqu'ils sont multiples, petits et à peu près de même taille, on donne plutòt à leur ensemble le nom de corps nucléolaire.

Cetle structure compliquée est aujourd'hui admise par la presque universalité des histologistes. Il n’y a, croyons-nous, que Altmaxx qui la combatte pour élendre au noyau sa théorie de cytoplasma (").

où l'on croit voir une ramification, il n'y aurait, en rẻalité, que deux anses faisant partie du même filament continu, situẻes dans des plans différents, et se croisant sans se toucher, ou du moins sans se souder. D’après C. Schxeider [91], au contraire, non seulement le réseau serait réel, mais il se continuerait, à travers la membrane nucléaire, avec les fibrilles du cytoplasma.

(1) Altmans [\%] considère les petits illots contenus dans les mailles du réseau de linine comme des granules, ef ce réseau avec les grains de chromatine comme une substance intergranulaire sans importance. Il est parvenu, en effet, à colorer exclusivement le suc nucléaire et à $y$ faire apparaitre ale petites masses arrondies ou polyédriques indépendantes. La plupart des auteurs pensent qu'il n'y a la qu'un artifice de préparation par lequel il domne le relief d'images positives à ce qui forme le fond du tableau, mais lui assure que ce sont ses adversaires qui font cette erreur. La question, thérifuement, est assez cmbarrassante: si on vous présente un damier, pouvez-vous dire s'il est fait de cases noires sur un fond blanc ou de cases blanches sur un fond noir? On pourrait aussi considėrer qu'il y a des cases blanches et des noires sur un fond entièrement couvert, et donner ainsi une demi-satisfaction aux deux parties. Il est possible que, dans le cas présent, la vérité soit là.

l'resque tous les histologistes s'accordent aussi à attribuer à la chromatine et au nurlèole les ròles essentiels dans les fonctions du noyau.

On admet aussi, très génẻralement aujourd hui, que le noyau est un organe constant et nécessaire de li cellule. Pendant longtemps, on a attachè une grande importance aux cytodes et aux Monires de lläckel, qui appelait ainsi les formeo sans noyau, cellules de tissu ou organismes inférieurs. Pour la question des Nonères, voir au type morphologique des lihizopodes). Mais on s'est aperçu que celte prétendue absence s'expliquait souvent par l'imperfection des méthodes ou des instruments. Après avoir découvert un noyau chez la plupart des Monires et des eytodes et mème chez les 


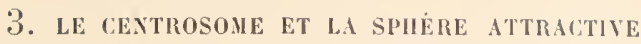

Tant que notre cellule ast à l'état de repos, éest-à-dire en dehors du moment où elle se prépare à se diviser, on ne voit ténéralement pas, dans son cytoplasma, l'organe dont il est question ici. Mais pendant la division, on l'aperçoit assez facilement.

Il se compose de trois parties (fig. 10) : au centre, un globule plus dense, le centrosome (ctrs.), colorable d'une façon intense par certains réactifs; autour de lui, une zone de protoplasma différencié, la sphlère athactive ou archoplasma ; enfin, partant de la sphère comme les rayons d'un astre lumineux el s'étendant plus ou moins loin dans le protoplasma ambiant, des stries divergentes qu'on appelle l'aster (ast.).

Quand la cellule repasse à l'état de repos, l'aster s'évanouit complètement (fig. 1) : il n'est qu'un aspect dû̀ à un état des parties, qui cesse quand ces parties relombent dans l'inaction. Le centrosone et la sphère

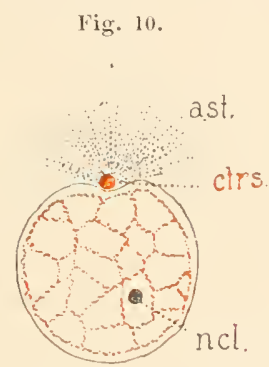

Noyau avee le centrusone et la sphère altractive ì l'intéricur (Sch.).

ast., aster; etrs., rentrosome; ne1., nucliole.

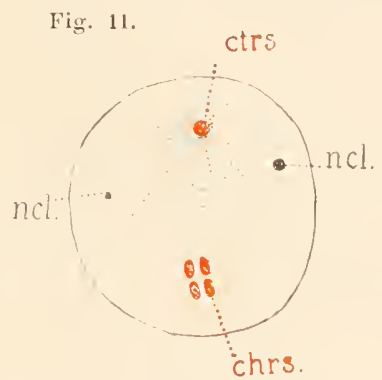

Noyau avec le centrosome it l'intérieur (Sch.).

cIrr., chromosomis ; ctrs., centrosome; nel.. nel., nucleoles.

semblent aussi disparaître mais, en réalité, ils ne font que se carher et on peut généralement les retrouver logés dans une petile dépression de la membrane nucléaire (fig. 10), d'où ils sortirout de nouveau au moment d'une division nourelle, ponr reformer un nouvel aster.

Mais dans d'antres cas, on n'arrive pas à les lécéler à celte place et l'on trouve, à l’intéricur mème du noyau (fig. 11), un corpuscule (ctrs.) qui lui ressemble à tel point, que certains autenrs, Braver [93] par exemple, affirment que c'est lui qui se retire dans le noyau mème, pendant le repos de la cellule, pour en sortir à chaque division (").

Bactéries, on a, par une induction à notre avis un peu hàtive, nié l'existence d'organismes sans noyau. Il semble cependant peu probable que la cellule se soit constituée d'emblée avec lous ses organes. La nucléine a dù exister dans la cellule avant de se condenser dans un organe différencié de celle-ci. Une expérience de Kirasser [85] semble bien démonstrative à cet égard. Cet auteur a extrait, par des procédés chimiques, de la nucléine des cellules de Levure, chez lesquelles on n’a jamais pu constater l'existence du noyau.

(1) D’autres auteurs, en particulier Jubis [93], remarquant que le nucléole disparait 


\section{LES ORG.INES ACCIDENTELS DU CYTOPLASMA}

Enfin, dans le cytoplasme de nolre cellule, on peut trouver, mais cela n'est pas constant: des vacuoles pulsatiles ou non; des réserves nutritives accumulées dans la cellule pendant les phases de nutrition exubérante pour faire face aux besoins d'un jeûne éventuel; enfin, une multitude très variée de produits de sécrétion ou d'excrétion solides, que nous aurons à énumérer plus fard, en éludiant la physiologie de la cellule.

\section{丂. LA MEMBRANE}

La cellule est sourent nue, c'est-à-dire n'a d'autre paroi que la surface externe de son cytoplasma. Hais, daus ce cas, on observe toujours que celle surface se dispose de manière à séparer, le plus netfement possible, le corps cellulaire du milieu ambiant. La substance fondamentale hyatine forme seule la surface, el s'y lermine par un bord continu d'un dessin absolument pur, sans permettre aux parties

au moment où le eentrosome se montre, et inversement, sont d'avis que ce dernier n’a pas d'existence indépentante et ne serait autre que le nucléole qui, pendant le repos de la cellule, prendrait place lans le noyau, pour présider aux fonctions végétatives de celui-ci et qui, pendant la division, en sortirait pour diriger celle opération. Hais Gugnard [91] a formellement ru le nuclèole et le centrosome coexister sans interruption, pendant toute la durée du cycle évolutif d'une cellule. Cela prouve que la théorie du nuctiote-centrosome est fausse dans beaucoup de cas, el il reste peu

Fig. 12.

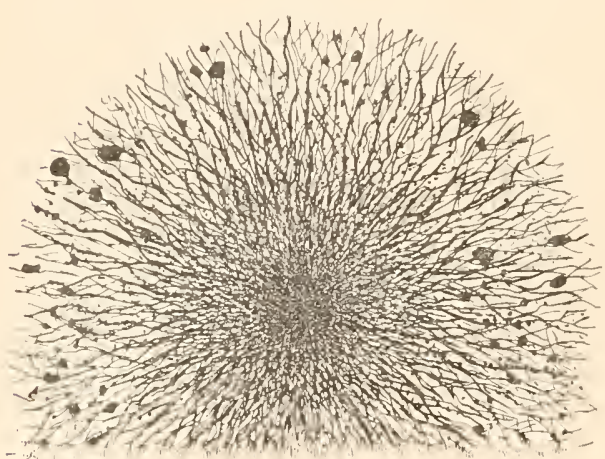

Cytoplasma el centrosome d'ap. EIsuoni).

Le ecutre presque noir ost le rentrosome, la zone moyenue sombre est la splure attractive et la porlion priphlurique claipe est le eyloplasme. de chances pour qu'elle soit vraie dans les autres.

Enfin, l'existence mème du centrosome et de la sphère, en tant que formations indèpendantes, a ètè mise en question. Ersmond [90, 94], étendant à cela sa théorie sur la structure du cytoplasma, ne voit dans ces organes qu'un point du cytoplasme où les aréoles sont si petites et si serrees quelles lonnent l'illusion l'un corps oparfue (fig. 12); cet état des areoles serait dù à ce fu'en ce point les échanges nutritifs seraient minima ou nuls.

Chez les plantes, il y a momentanément, cote à cote, deux centrosomes et deux splıeres; nais cette difference n'a aucune importance, car les centrosumes, comme nous le verrons, se reproduisent par dirision avant la division du noyan. Guand on en lrouve deux, cela tient seulement à ce que leur division est trés précoce et se fait dès que la cellule entre en repos à la suite de la division nucléare précédente.

On le voit, la question n'est pas mùre, on ne peut décider, en toute assurance, si le centrosome et la sphère sont permanents ou non, ni s'ils viennent du noyau ou du 
qu'elle baigne de s'étendre jusque-là. Jamais on ne voit une vacuole à demi ouverte à la surface, une granulation à moitié saillante au dehors. Toujours un enduit de substance hyaline, si mince qu'il soit, revèt la surface et, en raison de sa tension superficielle, tend à s'arrondir sur lıi-mème, à se refermer sur son contenu. Souvent, cette couche superficielle prend une fermelé particulière et assure un rôle protecteur plus efficace. Néanmoins, tant que la cellule n'aura d'autre revètement que son cytoplasma, sans rien de plus, nous dirons qu'elle est nue, sans membrane. La différenciation de la partie superficielle du cytoplasme en ectoplasme, que nous rencontrerons souvent chez les Protozoaires, ne constituera pas non plus pour nous une membrane, parce que cet ectoplasme, malgré sa densité plus grande, n’est que du cytoplasma pur.

D'aulres fois et très souvent, la cellule a un revètement particulier, que l'on appelle membrane, cuticule, pellicule, périplaste, capsule, coque, coquille, etc, etc. La plus grande confusion règne dans celle nomenclature à laquelle on ajoute sans cesse de nouveaux termes parce que l'on sent le manque de précision des anciens, augmentant ainsi le mal au lieu d'y porter remède. Nous allons tàcher de mettre un peu d'ordre dans tout cela.

Le premier pas dans la constitution d'une membrane consiste dans le fait que des substances particulières, plus résistanles aux aggents physiques el chimiques que le protoplasma, se déposent dans ses couches superficielles et les transforment en une enveloppe protectrice. Mais, on le voit, celte enveloppe est parlie intégrante de la cellule, vivante comme elle; elle ne saurait s'en séparer par suite d'une mue et, au moment de la division, elle se divise avec le corps cellulaire. Ilistochimiquement, ce genre d'enveloppe se distingue par l'action de la pepsine qui la digère en partie, mais en partie seulement, dissolvant sa portion protoplasmique et respectant les substances additionnelles. La putréfaction agit dans le mème sens, détruisant la première et laissant les secondes qui sont relativement imputrescibles, en sorte qu'après la mort de la cellule, son enveloppe persiste plus ou moins longtemps. Nous appellerons celte sorte d'enveloppe membrane cellulaire vraie ou simplement membrane cellulaire. C'est ce que Kíters appelle périplaste ehez les Flagellates et Bütscnu pellicule chez les Ciliés.

Si ces mèmes substances ou d'autres, résistantes, solides, imputrescibles, non digestibles dans la pepsine, au lieu de se déposer dans les couches superficielles du cytoplasma, sont sécrétées par lui, à sa

cyloplasma. Ce qui semble le plus probable pour le moment, c'est que le centrosome est un organe réel, permanent, et que la sphère attractive estune sorte particulière de protoplasma (archoplasma de Boveri, kinoplasma de Strasburger), disposé autour de lui en une zone sphérique, et se continuant en dehors avec les filaments qui forment le réseau filaire du cytoplasma. 
surface, en dehors de lui mais à son contact, elles constituent une membrane dans laquelle il n’y a rien de protoplasmique, rien de vivant : nous l'appellerons alors cuticule. Cela n'empêche pas, d'ailleurs, que la culicule puisse s'accroître par intussusception, aussi bien que par dépôt de couches nouvelles à sa face profonde, l'intussusception étant un phénomène physique et non exclusivement physiologique ('). La cuticule n'est pas, d'ailleurs, forcément une substance solide et résistante : sa seule caractéristique absolue est son origine, sa siluation par rapport au cytoplasma. Ainsi, nous rencontrerons sourent, chez les Protozoaires, des enveloppes gélatineuses qui, par leur nature, ne sont que des cuticules. Les membranes kystiques, si fréquentes chez les Protozoaires, sont des cuticules; la membrane vitelline de l'œuf en est une aussi, comme le prouvent sa formation rapide après la fécondation et le fait qu'elle ne prend pas part à la segmentation.

Enfin, si nous supposons que la cuticule, au lieu d'adhérer au corps cellulaire, se solilifie à quelque distance de lui, ou plutôt que la cellule, aussitôt après sa sécrétion, se rétracte de manière à laisser un espace libre entre elle et son enveloppe, nous dirons que cette enveloppe est une capsule. Le plus souvent, la capsule n'est pas continue : elle s'ouvre en un ou plusieurs points, sur des surfaces plus ou moins étendues. Il en résulte que, malgré rette protection, la cellule est en rapport, par sa surface, avec le milieu ambiant et qu'elle peut, dans sa capsule, se munir d'une cuticule ou d'une membrane. Les logettes que se sécrètent beaucoup de Protozoaires comme Metacineta parmi les Tentaculifères, Cothurnia et Tintinnoides parmi les Ciliés, Jinobryon et Salpingæca parmi les Flagellates, enfin la coquille chilineuse ou calcaire des Foraminifères, sont des capsules ( $\left(^{\mathbf{2}}\right)$.

(1) Ainsi, un cristal ealcique déposé dans une solution magnésienne finit par se transformer en eristal magnésien sans, que sa forme ait été modifiée, par substitution interne de moléeules de magnésie aux molécules de ehaux. A l“inverse de la membrane, la euticule peut être muée et elle ne prend pas part à la division de la eellule.

${ }^{(2)}$ Ici, eomme partout, les distinetions absolues vont à l'eneontre de la réalité des faits. Il peut arriver qu'à la surface d'une vraie membrane cellulaire se déposent, par sécrétion extérieure, des couches cuticulaires, sans que rien ne marque la distinction entre ces deux produetions. Il semble en être ainsi pour la membrane eellulosique des eellules végétales. Les membranes jeunes eontiennent une substanee albumineuse (dermatoplasma de WIEsser), tandis que les couches superficielles des membranes vieilles et épaisses semblent bien ne eontenir que de la eellulose plus ou moins lignifiée et encroùtée de substances minérales. La menurane végétale ne prend pas part à la division, la nouvelle cloison s'ajoutant simplement aux parties aneiennes, mais elle n'est jamais muée : elle est membrane en dedans. cuticule en dehors et ces deux parties ne se séparent jamais. Dans ee cas, il peut arriver de voir une enveloppe que l'on avait appelée membrane, être rejetie partiellement par une mue, et la ecllule apparaître au dehors avec une nembrane nouvelle. Rien n'empêehe, non plus, dlans une vraie membrane, que les eouehes les plus aneiennes et les plus éloignées du eentre trophique ne meurent et ne soient éliminées comme une cutieule, tandis que les eouehes plus jeunes forment une membrane bien vivante 


\section{II. - COMPOSITION GIIMIOLE DE IA CELLULE.}

On ne sait rien de la composition chimique des minces membranes des cellules animales de lissu. Dès qu'elles s'épaississent, on peut reconnaitre en elles la présence de substances diverses, parmi lesquelles la chitine $\left(\mathrm{C}^{60} \mathrm{I}^{100} \mathrm{Xz}^{8} \mathrm{O}^{38}\right)$, la liératine, la cellulose $\left(\mathrm{C}^{6} \mathrm{II}^{10} \mathrm{O}^{5}\right)$ et ses variétés, etc., jouent le rôle principal. Ces mêmes substances se retrouvent dans les cuticules et les capsules : la membrane vitelline de l'auf est formée de liératine, substance sulfurée, non phosphorée dont la formule rationnelle n'a pas été établie.

Le cytoplasma comprend dans sa constitution des nucléo-allumines, des globulines, de la lécithine, de la cholestérine et, en fait le substances minérales: du fer, sans doute en combinaison organique avec la nucléoalbumine, des chlorures et des phosphates de potassium, de sodium, de magnésium et de calcium.

Les deux substances albumineuses que renferme le cytoplasma ne sont pas indifféremment mélangées en lui : les nucléo-albumines forment les parties figurées (fibrilles, yranules, spongioplasma), les globulines forment la partie amorphe (luyaloplasma ou paraplasma): cela semble indiquer que les premières ont une importance plus grande que celles-ci.

Dans le noyau, la membrane nucléaire est formée d'amplipyrénine, et le réseau nucléaire est fail de linine ou parachromatine; les grains chromatiques sont formés de chromatine, et le nucléole est contitué pill de la paranucléine ou pyrénine. Enfin le suc nucléare, comme le suc cellulaire des racuoles dn cyloplasma, est un liquide aqueux, contenant en dissolution des sels et quelques substances allıminoïdles.

Il semblerait, d'après cela, que la composition chimique de toutes les parties de la cellule soit bien comnue, puisque toutes ont recu des noms de substances chimiques. Mais ce n'est là qu'une apparence tout à fait trompeuse.

De ce que l'on a donné à toutes ces substances des noms à désinence en ine, il ne s'ensuit pas que toutes soient sur le mène pied, et il y a grande utilité í établir une distinction nette entre ces diverses substances à désinence semblable. Les unes nous sont complìtement inconnues dans leur composition chimique; on ne sait ni leur formule détaillée, ni leur formule brute; on ne pent dire si elles sont simples ou si elles

au-dessous d'elle. Nous n’ignorons pas non plus que Bürschur a trouvé jusque dans la carapace de l'Ecrevisse, qui d'après nos définitions est une cuticule typique, une structure qui rappelle celle du protoplasma.

Tout cela prouve qu'il faut tenir compte des transitions, les exceptions et des réserves; mais les trois types l'enveloppe existent certainement el il vaut mieux les accepter, pour l'enseignement du moins, dùton un peu forcer les choses, que te tout laisser, sous prétexte de rigueur scientifique, daus l'élat de confusion où cela est dlans la plupart des ouvrages. 
sont des mélanges de substances définies différentes. Disons le mot: ce . ne sont pas des substances chimiques. De ce nombre sont la linine, la pyrénine ou paranucléine, l'amphipyrénine, elc.; on les a nommées et distinguées d'après la maniere dont elles se comportent en présence de certaines matières colorantes, mais sans rien savoir des réactions qui se passent dans la fixation de la couleur.

Nous voulons dire par là, non qu'on ne sait rien de la composition chimique du nucléole, par exemple, ou du réseau achromalique, mais qu'en les disant formés de pyrénine ou de linine, on ne fait pas une réponse ayant un sens chimique précis. Pyrénine, linine, ctc., signifient seulement: substances reconnaissables à tel aspect microscopique, à telle manière de se comporter en présence de telle matière colorante.

Au contraire, la liératine, la nucléine, la globuline, la plastine, etc., sont des substances chimiques vraies, dont on sait plus ou moins selon les cas, mais qui méritent de prendre place dans un ouvrage de chimie pure (').

Cela lien compris, examinons celles de ces substances dont la constitution chimique ne nous est pas lout à fait inconnue.

La kératine est une substance albuminoüde, c'est-ì-dire allıumineuse incomplète et dépourvue en outre de phosphore, mais riche en soufre.

Les nucléo-allumines sont des sulsstances albumineuses $\left(^{*}\right)$ vraies, légèrement phosphorées. Elles sont solubles dans le suc gastrique qui les décompose en peptone restant en solution et en acide nucléique qui se précipite. Ces sulsstances sont, sans doute, multiples et rariées dans le cytoplasma, mais on n'est pas en état de distinguer, ni mème de dénombrer celles qui font partie de chaque cytoplasma en particulier.

Les globulines sont aussi des substances albumineuses complètes, mais non phosphorées, insolubles dans l'cau pure, solubles dans les solutions salines à $̋$ à $10 \%$, d'où l'eau pure les reprécipite.

La lécilline est une graisse phosphorée, soluble dans l'alcool.

La cholestérine est un alcool monoatomique solide, cristallisant en tahlettes, solubles dans l'éther et dans l'alcool bouillant.

La chromatine est formée de cholestérine unie à de la nucléinc qui en forme presque toute la masso. Celte nucléine est elle-mème une substance richement phosphorée, insoluble dans le suc gastrique.

(1) Un exemple fera bien comprendre cette distinction. Iien que chromatine et nucléine: soient presque synonymes, la chromatine appartient au premier groupe, et la nucléine au second, et l'on peut lire que la chromatine des histologistes est une des variélés de la nucléine des chimistes. Rien n'empêclıe, d'ailleurs, que ces substances passent de la première catégorie dans la seconde. C'est ce qui est arrivé, dans une certaine mesure, pour la plastine de Reinke [81] à la suite des recherches de ZaCHARIAS [83].

(') Substances albumineuses est pris ici dans le sens fixé par Danilevski, par opposition à substances albuminoüdes. Les premières ont une composition analogue à celle de l'albumine de l'œuf; les secondes en diffèrent en ce qu'il leur manque un ou plusieurs des groupes chimiques essentiels, nècessaires pour constituer les premières. 
La pyrénine et la linine ou parachromatine semblent ètre des combinaisons d'albumine avec de la plustine, substance analogue à la nucléine, mais beaucoup moins riche en phosphore (').

(1) Toutes ces notions semblent bien décousues, et elles le sont, en effet, si on s'en tient a ees données expérimentales. Mais elles deviennent beatroup plus claires et mieux lices entre elles, si on les envisage à la lumière d'une théorie, qui n'est peutêtre pas a l'abri de toute objection. mais qu'il est bon l'accepter. au moins provisoirement, en raison les commodites quelle procure. Voici cette théorie, telle quelle s'est degagée peu à peu des nombreux travaux récents, en particulier de ceux de Kossel [81, 82], re Zacharias [83], d'Altiraxi [89], de Liliexfeld [92, 93], ete.

Les substances constituant la partie fondamentale du eytoplasma ou des organes du noyau seraient toutes des combinaisons, en proportions variées, liune seule substance phosphorée, l'acide uncléique, avec des substances protéiques non phosphorées. L'acide nncléique est un corps chimique défini que l’on a préparé et isolé. Il est riehe en phosphore el eorrespond à la formule brute $\mathrm{C}^{29} \mathrm{II}^{19} \mathrm{Az} \mathrm{Pl}^{3} \mathrm{O}^{22}$, ce qui lui donne environ $14 \%$ de cette substance. 11 eonstitue, à l'élat presque pur, la tête des spermatozoïdes, formée comme on sait des parties essentielles du noyau et du eytoplasma. Ini aux matières protéiıues. il forme des nncléines dont il existe des espèces nombreuses et qui, prises au sens large, peuvent comprendre toutes les substances protéiques phosphorées de la cellule. llus elles sont aeides et riches en phosphore, et plus aussi leur ròle semble important. Lit chromatine est une nucléine ordinaire, très riche en acide nucléique et, par conséquent, en plosphore, et franchement acile; la plastime l'est sensiblement moins; la limine et la prónine le sont moins eneore, puisqu'elles sont formées par l'union de la plastime avec une nouvelle matière albumineuse, non phosphorée. Enfin, diuns les mucléo-albmmines du cytoplasma, la proportion d'aeide nueléique derient trés faible; la teneur en phosphore tombe a $1 / 2$ ou $1 \%$, et la substance devient eneore moins acide.

Quant aux autres substances, phosphorées ou non, que l'on reneontre dans le cytoplasma ou dans le noyau (lécithime, cholestérine, sels minéraux', elles sont, en quelque sorte, additionnelles et ne doivent pas être considèrées comme faisant partie intégrante de la molécule albumineuse. C'est par ces substances additionnelles que le cytoplasma peut ètre rendu, dans certaines cellules, beaucoup plus riche en plosphore total que le noyau, bien que sa matière albumineuse constituante le soit beaneouj, moins. Si l'on ne tient compte que de cette dernière, les iléments de la eellule se classent ainsi, par ordre décroissant d'aeidité et de rieliesse en phosphore : 10 la chromatine; 20 le nueliole et les substances achromatiques du noyau; $3^{\circ}$ les portions figurées du cyloplasma. Toutes ces substances sont acides; le suc nucléaire et le lyyaloplasma du cytoplasme sont basiques. Pris en masse, le noyau est acide parce qu'il contient une quantité dominante de substances acides et le corps cellulaire est basirue parce que la substanee dominante en lui, non pour l'importance mais par sa masse, est la globuline basique du hyaloplasma.

Cette acidité differente et l'affiniti differente pour les couleurs bisigues ou acides qui en est la conséquence sont la principale cause de l'ẻlectivité des diverses couleurs par les diverses parties de la cellule. Mais á cette cause s'en joignent d'autres qui nous sont encore absolument inconnues, et c'est pour eela que l'histoehimie des couleurs n'est encore qu'une techmiqne, un recueil de formules empiriques et non une science. Cette science, si elle était connue, serait d'un intérêt extrème pour la biologie, car elle nous permettrait d'avaneer dans la connaissance de la constitution du protoplasma. 


\section{III. - PIISSIOLOGIE DE IA CELLULE}

Notre cellule travaille, elle se nourrit, elle se divise. Sa vie se résume dans ces trois fonctions essentielles dont les autres ne sont que des cas particuliers.

10 Elle travaille, c'est-à-dire qu'elle fabrique continuellement des substances nouvelles, au dépens de celles qui la constituent à l'état de repos. Ces substances sont toujours, sinon individuellement, du moins dans leur ensemble, plus oxydées que celles dont elles dérivent. Aussi, pour les former, la cellule doit-elle consommer de l'oxygène qui lui est fourni par la respiration; et, bien que les choses se passent en réalité tout autrement, le résultal final est le mème que si les prorluits provenaient d'une oxydation directe du protoplasma. Ces réactions chimiques développent de la chaleur et, par conséquent, mettent en liberté une certaine quantité de force vive qui se dépense sous la forme de travail physique, c'est-à-dire de mourement: de là, la motilité.

2 Mais notre cellule n'est pas un simple appareil physique que les forces ne fassent que traver'ser et qui doive, à chaque instant, rendre à un bout toute l'énergie qu'il a reçue par l'autre; elle est vivante et emmagasine les énergies qui lui sont fournies, pour les dépenser irrégulicrement et selon qu'elle est sollicitée ì le faire par les diverses excitations, d'où l'excitabilité.

$3^{\circ}$ En fournissant les produits de son industrie, la cellule a, soit dépensé in toto, soit modifié dans sa composition une partie de son protoplasma; elle s'est usée : c'est la désassimilation. Elle doit donc emprunter aux lipuides alimentaires qui la baignent de quoi refaire sa substance, de quoi se reconstituer dans son étal initial. Mlais ces aliments ne sont pas formés de substances semblables à celles qu'elles doivent remplacer; il leur faut subir une transformation qui les rende semblables à celles-ci. C'est ce qu'exprime admirablement le mot assimilation. Unie à la fonclion précédente elle constitue la nutrition.

$4^{\circ}$ On pourrait conceroir un organisme qui accomplirait indéfiniment la série de phénomènes que nous venons d'énumérer, car ils constituent un cycle fermé, a la seule condition que l'assimilation soit juste égale à la désassimilation. En fait, cela n’a jamais lieu. Toujours la première l'emporte et il en résulte un accroissement. Enfin, comme la cellule a cette propriété générale et absolue de tous les organismes vivants d'avoir une limite de taille, elle doit, après s'ètre accrue au delà l'un certain degré, se réluire par division : c'est ainsi qu'elle se multiplie, se reproduit. Et, après s'ètre ríduite par division, elle doil reprendre sa taille primitive, en sorte que la reproduction entraine l'accroissement, comme l'accroissement entrante la division.

Voilà comment toutes les propriétés et fonctions secondaires de la 
cellule se résument dans son cycle érolutif, aux trois principales que nous arons données conme essentielles:

Io Travail, comprenant: ( ) fabrication de substances dont certaines sont oxydées el entrainent la respiration; (b) production des monvements provoqués par l'excitabilité el entrainant la désassimilation;

2o Assimilation réparant les pertes produites par le travail et dépassant le but, de manière à produire l'accroissement;

$3^{\circ}$ IVivision, à la fois cause el effel de l'accroissement el constituant la reproduction de la cellule.

Nous allons les éludier successivement.

\section{TRAVAIL DE LA CELLULE.}

Le travail de la cellule, avons-nous vu, se divise en deux parties: une fabrication de substances et une production de mouvements, quil faut éludier séparément.

\section{A. PRODUITS DE LA CELLULE.}

Il nous faut ici, de toute nécessité, ahandonner la description monographique d'une cellule idéale, tant est grande la variété des phénomènes qui peuvent se présenter.

Les substances produites par la cellule peurent ètre divisées en deux catégories, selon qu'elles restent ì son intérieur ou qu'elles sont rejetées au dehors et, dans ces deux catégories, il y a à distinguer: les produits d'excrétion, nuisibles à l'organisme, engendrés non en vue d'eux-mèmes, mais comme conséquence inévitable de la production les substances utiles ou des mouvements, et les produits de sécrétion uliles à l'organisme. Enfin, lorsqu'on aura divisé ces produits en solides, liquides et gazeux, on aura élabli toutes les catégories nécessaires pour metre un peu d'ordre dans celte nomenclature.

a. Les produits d'excrétion externes sont: au premier rang l'acide carbonique el la vapeur d'eau, produits ultimes de désassimilation puisqu'ils comportentle relour à des substances minérales très simples el très stables, puis l'acile urique, l'urée, l'acide hippurique, la guanine, elc., etc. (').

$b$. Les produits d'excrétion internes sont rares, car la cellule n’a aucun avantage à conserver en elle des produits nuisibles. Cela arrive cependant quelquefois, par exemple, dans les cellules rénales des Mollusques, Acéphales et Gasléropodes, el de quelques Crustacés et Vers inférieurs, dans le corps adipeux péricardiaque des Insectes, etc. La substance excrélée est à l'étal solide, sous la forme d'une concrélion, d'une sorte de caleul urinaire contenu dans une vacuole. Là, elle peut ou len-

(1) Nous n'avons nullement l'intention de faire ici une énumération complète de ces substances, voulant seulement donner une idée générale des faits. 
tement se dissoudre et finir par être rejetée, ou grossir de plus en plus, comprimer le noyau, amincir la cellule et enfin la détruire, et, désormais extra-cellulaire, rester inerte daus les tissus qui la supportent tant bien que mal.

$c$. Les produits de sécrétion internes sont extrèmement nombreux et de natures très diverses.

Les uns comme l'huile, le glycogène sont des réserves alimentaires destinées à être reprises au moment du besoin lorsque l'alimentation ne pourra faire face aux frais de la dépense, soit pendant la vie de l'adulte, soit pendant la reproduction, au moment où l'ètre devra se nourrir sans avoir encore les moyens nécessaires pour recueillir les aliments et les digérer. Au nombre de ces derniers sont les substances alimentaires connues sous le nom le lécithiques que l'on appelle protolécithe ou dentolécithe, selon qu'elles sont disposées dans l'œuf non segmenté ou dans les cellules de l'embryon.

D'autres jouent un ròle passif lans l'organisme, comme la myéline des fibres nerveuses, les squelettes intracellulaires de certains Zoophytes (spicules des Éponges, coquilles des Rarliolaires, etc.), les pigments inertes destinés simplement à protéger des organes trop sensibles (pigrments chorö̈liens) ou à colorer les téguments.

D'autres encore jouent un rôle chimique comme le pigment rétinien, l'hémoglobine des globules sanguins nucléés ou les ferments divers que contiennent les cellules, indépendamment de ceux qui sont émis au dehor's par des cellules glandulaires spéciales (').

D'autres enfin constituent de véritables appareils intracellulaires destinés à accomplir ou perfectionner un phénomène mécanique actif : tels sont les sarcoblastes', petits prismes qui, par leur alignement en longueur et en largeur, forment les filırilles musculaires striées, et qui sont formés principalement de musculine.

$d$. Les produits de sécrétion externes ne sont ni moins variés ni moins intéressants. Au premier rang, viennent les produits liquides élaborés par les glandes sécrélrices, salive, suc gastrique, larmes, mucus nasal, etc., puis viennent les membranes, les cuticules (cuticule chitineuse des Insectes, Vers, elc., ele.) incrustées ou non ultérieurement de calcaire (carapace des Crustacós), les capsules, la coquille des Mollusques, etc. Enfin prend place ici une formation que l'on avait envisagée jusqu'à ces dernières années d'une tout autre manière, c'est la substance intercellulaire des tissus de la famille conjonctive, fibre conjonctive et élastique, masse fondamentale du cartilage et de los.

Cetle manière de concevoir les choses élargit et simplifie beaucoup la conception des organismes supérieurs. Elle permet le ne voir en eux que des agrégats de cellules à constitulion typique et de com-

(1) Peut-être fant-il ranger ici la técithine et la chotesthérine, que nous avons vues toujours unies à la substance albumineuse dans le protoplasma. 
prendre la signification de tout ce qui, en eux, n'est pas cellule et de tout ce qui, dans leurs cellules, n'est pas cyloplasme ou noyau (").

\section{B. MOUVEMENTS DE LA CELLULE}

Toul ce qui vit étant formé de cellules, tout mouvement d'organisme vivant est mouvement rellulaire. Hais la plupart des mouvements des ètres vivants sont dus à des contractions qui se produisent dans des cellules différenciées ou dans des parties différenciées de cellules. Tels sont les mouvements musculaires, tels sont ceux des cils, flagellums, membranelles, etc. Nous devons les laisser provisoirement de còté pour ne nous occuper que de ceux de la cellule dépourvue d'organes moteurs spéciaux, en un mol, de la cellule en général. Ces mouvements ont toujours leur siege dans le cyloplasma; le noyau n'y prend aucune part. lls sont de quatre sorles:

$I^{\circ}$ Des mouvements intéricurs se produisanl seulement au moment de la division pour opérer les séparations, groupements nouveaux, remanicments quelconques que nécessile cette importante opéralion. -

(1) Nous avons rangé dans la même ratégorie lous les produits de la cellule, qu'ils soient sécrétés ou exrétés, internes ou externes, destinés à rester en elle ou à en être expulsés. Tous ces probluits sont, en effet, lomologues au point de vue morphologípe. Mais, sous d'autres rapports, ils sont profourlément différents. Nous avons établi une distinction physiologique entre cux en les divisant en secreta uliles et excreta nuisibles. Il faut montrer, en outre, en quoi ils different les uns des autres à un point de vue elimique, d’ailleurs lrès générat.

Le plus grand nombre de ees prorluits provient de dédoublements du protoplasma, opérés avec hydratation ef sans oxydation, pent-être même, d'alp'ès Gautıer [9x́], avee réduction. Non seulement les substances dérivées immédiatement de l'albumine (prismes musculaires, ferments des glantes digestives, lı́moglobine du sang), les alcools (cholestérine), les liydrates le carbone sucre, glycogène, amilon et la longue serrie de corps gras'; mais aussi les anides comme l'urée, el mème les substanees (adenine, guanine) appartenant au groupe de l'acide urifue, se forment, de cette manière, sans oxydation et par conséquent avee peu ou point de dégagement de chaleur. C'est seulement lorsque ees corps, surtont les graisses et les sucres, se transforment en prorluits plus simples, parmi lesquels l'acide carbonique et l'eau sont les plus importants, que l'oxygène intervient, ef c'est alors surtout que se produit la chaleur d'ou dérive la foree vive nécessaire à la production du mouvement. Il ne faudrait pas conclure de la que l'oxygène se fixe directement sur les produits qu'il est chargé de brùler, il est absorbé par le protoplasma, et lì, sans toute, s'accouplissent, dans des réactions simultanées complexes, les phénomènes que nous dissocions pour les saisir plus clairement.

C'est pour cette oxydation des produits ultimes et pour la prodnction de la chaleur ou du mouvenent que l'oxygène est nécessaire. Il est aussitòt dépensé que reçu, il ne s'accumule pas et, dès 'fu'il cesse d'ütre fourni, Jes réactions normales de la cellule sont arrètées. Les autres aliments, au contraire, s'aceumulent dans la cellule et sont cmployés peu à peu, et šils cessent d'être apportés, la cellule continue néanmoins à fonetionner normalement pendant un temps assez long. C'est pour cela que la respiration constitue, sous un certain rapport, une fonetion distincte de l'assimilation des aliments. 
Nous les laissons de côté pour les décrire et en chercher l'explication au moment où nous nous occuperons de la livision cellulaire - ;

20 Des inourements intérieurs de circulation rotaloire continue des parties centrales du cytoplasma par rapport aux parties périphériques immobiles de la cellule - Ces mouvements ne changent pas la forme extérieure de la cellule; ils portent le nom de circulation du protoplasma ou cyclose; on les observe surtout dans les lufusoires et chez les plantes -;

$3^{\circ}$ Des mouvements extérieurs dans lesquels c'est la cellule entière qui se déforme, émetlant et rétractant ses prolongements appelés pseudopodes;

$4^{\circ}$ Des transports de la cellule in toto sans déformation apparente par attraction ou répulsion exercée par un agent physique ou physiologique : ce sont les tactismes.

Il n'est presque pas d'agents naturels qui ne puissent exercer un tactisme fort ou faible, positif (attraction) ou négatif (répulsion) sur une cellule, pourvu que celle-ci soit suffisamment mobile pour lui obéir. La pesanteur', la lumière, la chaleur, l'humillité, les agents chimiques (oxygène, acide carbonique, sels minéraux en solution), enfin le voisinage d'autres cellules semblables ou différentes (biotactisme) sont pour la cellule un excitanl qui l'altire vers la source d'excitation on la repousse loin d'elle. Laattraction les lencocytes par l'oxygène, observée par Raxver, est un des exemples les plus frappants de ce genre d'actions.

Il faut considérer ces mouvements comme de nature toute physique et comparables à c'eux qu'exécutent de petits objets électrisables en présence de corps chargés d'électricité.

Tout autres sont les mouvements d'émission des pseudopodes et de circulation protoplasmique. Ceux-là semblent être spontanés el résulter d'une manifestation de la vie aussi incompréhensible que la vie elle-même. On a cherché à les expliquer cepenulant, c'est-ì-dire à les ramener à des phénomènes physiques; mais on n’est arrivé encore qu’à des conclusions hypothétiques (").

(1) Ces questions sont si importantes qul elles méritent de nous arrèter un instant. Quand un pseudopode s'allonge, il se montre formé exclusivement de protoplasma hyalin; si une exeitation queleonque le fait rétracler, aussitót il devient trouble el, dans sa masse, apparaissent de minimes granulations et vacuoles qui n'y existaient pas l'instant d'auparavant. Cela montre qu'il s'est produit en lui une brusque modification physico-chimique qui a eu pour conséquence le retrait de cel organe.

Comment une modificalion physico-chimique peut-elle avoir celle conséquence?

Berthorn [86] pense qu'il peut en être ainsi si cellemodificalion retentit sur l'intensité de la tension sıperficielle. Lorsqu une cellule placée dans un liquirle, dans l'eau, se mainlient arronlie, e'est que l'allraction des molécnles de sa surfare, les unes pour les autres, est plus forte que l'attraction de ces mèmes molécules pour l'eau ambiante. S'il en était autrement, la cellule se désagrégerait aussitot. Dans l'eau jure, la différence d'attraction est très grande et par suite la tension superficielle de la cellule est très forte. Mais l'eau ne reste pas pure autour de la cellule: les échanges osmoliques out 


\section{NUTRITION DE LA CELLULE}

\section{A. ASSIMILATION.}

Pour former les produils de son travail, produits matériels ou mouvements, la cellule dépense les substances dont elle est composée.

lieu, qui la chargent de substances salines dissoutes. Celles-ci diffusent sans cesse dans le liquide, mais pas instantanément, en sorte qu il s'étalılit autour de la cellule une série de couches concentriques où le degré de concentration va en diminuant de dedans en dehors. La couche qui confine immédiatement à la cellule est assez. concentrée pour que la différence d'attraction soit très faible et que très faible, par conséquent, soit aussi la tension superficielle. Dans ces conditions, il suffit des moindres variations dans lhomogénéité de la cellule pour que la tension superficielle soit vaincue en un point. Là, aussitit, se forme un pseurlopode. Le pseudopode n'est done pas poussé par la cellule, il est aspiré par le milieu ambiant. Dés que se prorluit en lui, sous l'influence d'une excitation yuelconque, une modification physico-chimique qui a pour effet d'augmenter sa tension superficielle, aussitôl celle-éi redevient supérieure á la succion exercée par le liquide et il se rétracte.

Cette modification phrsieo-chintique déterminée jar l'excitant et que manifeste l'apparence trouble que prend le pseuiloporle avant de se rélracter, Berthold n'a pas cherché à la déterminer. Verwors 92 a tenté, sinon de la déterminer, du moins de la deviner, et il propose l'lypothese suivante.

Les molécules formant la masse intérieure du cytoplasma sont toutes, mais à des degrés différents, aviles d'oxygène. Aussi se précipitent-elles sans cesse vers la surfare et lì se saturent le ce gaz. Sous cet état l'oxydation, leurs attractions mutuelles se trouvent trés réduites, aussi la tension superficielle de la cellule est-elle très faible, et, dans les points oì elle est minima, se forment des pseudoporles. Mais ces molécules très oxydées sont devenues, on se chargeant d'oxygène, très instables et aptes, à la manière des explosif's, a se décomposer brusquement sous l'action des moindres excitants. Aussi, dès qu'une excitation atteint le pseudopode, toutes ses molécules se décomposent et abandonnent de l'acide carbonique, de l'acide lactique, ete., qui se dégagent aussilòt dans l'eau. Par le fait mème de rette décomposition, les molécules se trouvent dans une nouvelle condition chimique dans laquelle leur tension superficielle est beauroup plus forte et leur chimiotactisme pour l'oxygène beaucoup moindre. Par suite de cela, le pseurlopode se rétracte et les molécules déchargées rentrent dans la profondeur du cytoplasma. Là, elles s'unissent à des substances excrétées par le noyau et repassent au premier étal, où leur chiniotactisme pour l'oxygène est, le nouveau, positif et très fort. On remarquera que cette théorie explique en mème temps les mourements des pseudopodes et le mourement vital lui-même qui est la source de la force déployée.

Quixcke [s8] a observé qu'une goutte d'huile, placée dans une solution de carbonate de sourle, s'agite de mourements qui rappellent singuliẻrement l'émission et le retrait des pseudopodes. Ces mouvements tiennent à ce qu'il s'est formé à sa surface un savon soluble qui diffuse dans l'eau et, en diffusant, entraine un peu la masse d'huile sous-jacente. Aussitit dissous ces savons se reforment et le phénomène dure tant qu'il $y$ a de l'huile. Mans une solution albunineuse, la chose est la mème, gràce à un savon albunineux qui se forme et se comporte de la mème façon. Quincke admet et croit démontrer que tout protoplasma est entouré d'une couche huileuse assez mince pour ne pas gèner les phénomènes osmotiques, et suffisante pour donner lieu aux phénomènes ei-dessus décrits. En un point, la pellicule se trans- 
Elle doit done les reformer sans cesse. C'est le but de sa nutrition. Or, c’est là pour elle un problème difficile, car elle doit tirer de ses aliments, qui sont différents d'elle, de quoi reformer sa substance à elle, non seulement sa substance, mais ses substances, car tout s'use et se dépense en elle. Le plasma dans lequel baignent les innombrables cellules du corps d'un animal supérieur est à peu près le mème pour toutes, et cependant elles sont, elles, de natures différentes. Elles doivent, chacune selon sa nature, tirer de ce fond nutritif commun des

forme en savon d’albumine, se dissout; aussitòt, en ce point, la tension superficielle se trouve diminuée el un pseudopode se forme; mais, dès que la couclıe huileuse s'est reformée, la tension reprend sa valeur première et le pseudopode se refracte.

En combinant ses idées sur la structure alvéolaire du cytoplasma, arec la théorie de Quincke, Bütschu [92] est arrivé à une explication des mouvements pseudopodiques, nolablement differente de la précédente, quoique fondèe sur le mème principe. Bütschli broie de l'huile avee dn carbonate de polasse finement pulvérisé, et place dans l'eau un fragment de la pâte ainsi obtenue. L'eau diffuse à travers l'huile et va lissoudre les parcelles de sel polassique, remplaçant chacune d'elles par une gouttelette d'une solution du mème sel. Les goutlelettes d'eau alcaline saponifient une parlie de la paroi luileuse qui se dissout. Si alors on ajoute de la glycérine diluée, celle-ci se dissout dans le savon et l'on a, en définitive, une masse composée de goulteleftes extrêmentent fines, formées de savon polassique dissous dans une solution aqueuse de glycérine, el noyées lans une masse d'huile qui forme les cloisons de séparation entre les gouttelettes. En oulre, une couche d'huile confinue forme la limite externe. l'ensemble forme une émulsion fine qui reproduit d'une manière saisissante la structure alvéolaire fue ce savant attribue au protoplasma. Si l'on met sous le mieroseope une goutte de celte émulsion, on la voit se mettre en marelie aree toufes les allures d'une Amibe. Le mouvement lure 24 lieures et plus; il est excité par la clıaleur qui peut le faire reprendre quand il est arrêté; entre les électrodes d'une pile, l'Amibe artificielle se dirige vers le pôle négatif. Bütschli explique ce mouvement de la manière suivante. Quelque part, à la surface, pour une cause accidentelle quelconque, quelques alvéoles viennent à se rompre et laissent écouler leur contenu savonneux qui, en ce point, vient former la surface. Comme la tension superficielle de ce liquirle est moindre dans l'eau que celle de l'huile, il se forme en ce point une saillie. Pour la former, les alvéoles superficiels se portent en avant et font derrière eux te la place; les alvéoles profonds s'avancent pour la combler, et ainsi, de proche en proche, jusque dans la profondeur. Quand la voussure superficielle est formée, le mouvement devrait sarrêter, mais de nouveaux alvèoles crèvent à la surface el le phénomène continue indéfiniment et provoque un déplacement d'ensemble, une trinslation de l'Amibe artificielle. Bülschli voit là l'explication des mouvements du protoplasma, disant que, puisifue le protoplasua ei les nousses savonucuses ont la même structure, la cause des mouvements de eelles-ci doil être aussi la cause des mouvements de celui-là. Cette conclusion n’est pas légitime, car on pourail renverser la proportion et lire: les mouvements du protoplasma el des Amibes arlificielles sont très semblables, mais ils ne peuvent êfre dus anx utemes eauses, la constilution chimique des deux objets ètant absolument diffèrente.

En somme, ces explications des nouvements du profoplasma sont toutes très liypothétiques et la dernière est mùme très invraisemblable. 11 ne faut y voir encore qu'une teulative louable pour ramener il des causes physiques, un phénomène qui ne sera vraiment expliqué que lorsqu'on y aura réussi. Pour le moment c'est la thèorie de Verworn qui, par la nature des phẻnomènes qu'elle met en jeu, nous semble la moins éloiguèe de la vérité. 
substances dillérentes. La cellule nerveuse n'en tirera pas les mèmes élémeuls que la cellule glandulaire, ni la cellule grlautulaire les mèmes que la collule musculaire. De plus, chaque cellule doit cu tirer des substances différentes : de la nucléine, de la linine, de l’amphipyrénine pour son noyau, des globulines et des uucléo-alhumines pour son cytoplasma, ete.; et non pas une nucléine, une globuline, une nucléo-albumine quelconques, mais celles qui entrent dans sa constitution spéciale et qui sont différentes des substances homonymes des cellules des autres tissus.

On conçoit combien ce travail est difficile dans sa délicate précision. On arrive cepentant à se rendre compte qüil soit possible en considérant les choses de la manière suivante.

Deux ordres de phénomènes bien distincts collaborent au résultat final : des phénomènes osmotiques et des phénomènes chimiques. Les phénomènes osmotiques opèrent un triage de substances; ils ont pour instruments les membranes. Quand il existe une vraie membrane cellulaire, celle-ci joue son rôle mais, d̀ son défaut, la membrane protoplasmique est là, qui n’admet dans le cytoplasma que les substances qui doivent y entrer. De mème la membrane nucléaire n'admet dans le noyau que certaines des substances qui ont pénétré dans le cytoplasma. Le phénomène semblerait s'arrèter là. Mais s’il est vrai, comme le pensent quelques histologistes, en particulier de V'rass, qu'il n'est pas une fibrille, un microsome, un globule cytoplasmique ou nucléinien, qui ait, sinon une membrane prople, du moins une couche superficielle plus ou moius différenciée en membrane, il est possible alors que ce triage osmotique de substances aille beaucoup plus loin et apporte son concours à la nutrition des moindres particules intracellulaires.

Les phénomènes chimiques jouent un rôle non moins important. Toute substance nouvelle ayant franchi la harriere d'une membrane trouve, derrière celle-ci, des sulıstances préexistantes avec lesquelles elle se combine, par réaction réciproque, étant modifiée par elles et les modifiant. Ainsi les substances qui entrent, les ingesta, sont modiliées successivement, à chaque membrane qu'elles lraversent, par un triage osmotique et, entre deux membranes successives, par des réactions chimiques; les substances qui sortent, les egesta, font de mème. La cellule est donc sans cesse traversée par deux courants inverses, l'un d'entrée, l'autre de sortie, se croisant dans tous ses points, el la composition chimique de chaque particule dépend des réactions qui se passcut à son niveau sous l'influence de ce double courant.

Il est extrèmement probahle que les ingesta se rapprochent de plus en plus de la nature des substances qu'ils doivent remplacer. Isolé dans le liquille où vit la cellule, un noyau ne saurait vivre et se nourrir. Le cytoplasma est son milieu nutritif obligé : cela prouve que le cytoplasma fait subir anx substances dont se nourrit le noyau, une préparation qui rend ces substances plus semblables à celles qui conslituent 
le noyau. le mème. une cellule privée de novau ne peut s'accroître et vivre dans le milieu nutritif, non parce qu il lui manque une direction supérieure comme on le dit, mais parce que les substances cytoplasmiques ne subissent plus l'élaboration que produisaient en elles les substances déversées par le noyau dans le cyloplasma. De tout cela résulte:

$1^{\circ}$ (que l'assimilation se fail par approximations successives, la particule nutritive se rapprochant progressivement de la nalure de la parlicule qu'elle doit remplacer, à chaque triage osmotique et à chaque réaction chimique qu'elle subil lepuis son entrée dans la cellule jusqu'à son arrivée au but. L'assimilation ne pouvait ètre mieux nommée, elle est une ad-similation progressive;

- Que le noyau n’est pas le supéricur hiérarchique du cytoplasma, et que la vie de la cellule résulte d'un consensus fonclionnel entre ces deux organes. Elle est la résultante des forces physiques et des réactions chimiques dont les composantes résident dans le cytoplasma aussi bien que dans le noyau.

\section{B. ACCROISSEMENT}

L’assimilation pourrait à la rigueur ne faire que réparer exaclement les pertes dues à l'usure. Le plus sonvent il n'en est pas ainsi; l'assimilation dépasse le lut, apporte plus de matériaux que le travail n’en détruil: il en résulte que la cellule s'accroit. Or, le fait général qu’il faut ici conmaître, c’est que partout et toujours, l’accroissement se fail par intussusception.

Pour toutes les parties liquides ou mème très molles et précipilables, le phénomène se comprend sans difficulté, les liquides imbilıent toutes les parties et se précipitent au sein de leur masse en molécules solides qui déterminent leur accroissement. Mais pour les parties denses, comme les grains d'amilon, los membranes, on a longlemps cru cela impossible et admis l'accroissement par juxtaposition. L’intussusception cependant s'applique aussi à ces organes et voici comment elle est possible.

l'accroissement des parties intérieures produit une lurgescence de la cellule qui distent la membrane, écarte ses molécules et permet un dépôt de nolécules nouvelles qui maintient, sans effort de distension, le volume qui tout à l'heure n'úlail obtenu que gràce à ret effort. liès lors, une nouvelle turgescence peut délerminer une nouvelle distension, en sorte que te phínomine peut continner.

\section{:3. REPRODLCTION DE LA CELLILE}

L'aceroissement do la cellule ne continue pas indéfiniment.

Tous lis ètres vivants ont une limite de laille propre à leur espìcr, et, fund colle limile est alluinto, un nouvel accroissoment élant inpossible, un phénomene nouveau se reproduit. C"est la division, par lanjuclle la rellule se reproduit. 
La division est une fonction capitale dans la vie de la cellule. Elle est son senl mode de reproduction. Sur elle repose, non seulement la reproduction de tous les ètres unicellulaires, mais aussi la formation du corps des organismes pluricellulaires, puisque tous ont pour point de départ une cellule unique. Elle se fail suivant deux modes: la division direcle ou amitose et la division indirecte appelée aussi milose ou liaryoliynèse, ce dernier terme signitiant plus spécialement division indirecte du noyau.

\section{A. DIVISION INDIRECTE OU IITOSE}

Dans la mitose, la division du noyau précède toujours celle du corps cellulaire. C'est donc par elle que nous devons commencer.

\section{DIVISIOX DU NOYAU}

Les phénomènes de la division nucléaire se passent les uns dans le cytoplasma, les autres dans le noyau; ils débutent à peu près simultanément et, selon tonte apparence, indépendamment dans ces deux organes. Ils ont élé divisés en trois phases : l'une de désagrégation du noyau maternel, c'est la prophase, l'autre de constitution des leux noyaux filles, c'est l'anaphase et, entre les leux, un stade intermédiaire tròs court u’appartenant pas à l'une plutôt qu’à l'autre, la métaphase.

\section{a. Prophase}

\%. Dans le noyau. - On se rappelle qu'an stale repos (fig. 1:3), la clıromaline est disposće sous la forme de grains ou de pretites masses irrégulières

Fig. 13 .

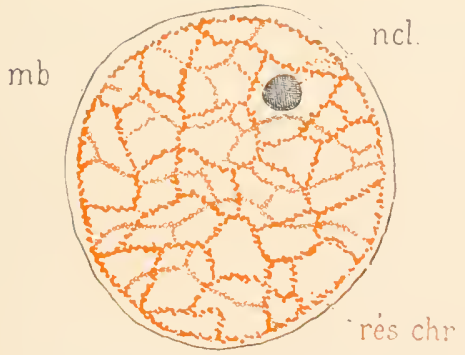

Prophase au début Seh

mo., membrane nuchaire; me1., nucliole; rés. chr.., riseau ehromatique. lig. If.

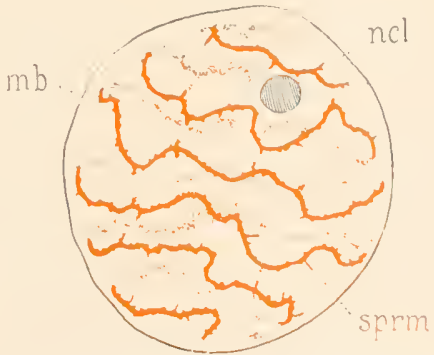

Prophase. Siade de peloton serré (Sch.)

mo., nombrane nucleaire; net.. nuclesle; sprum., spirème.

le long des filaments achromaliques très fins dont la disposition, an moins en apparence, est celle l'un réseau.

Le premier phénomène qui se produit est une modification de ce résean, en place duquel on trouve, au bout de quelque temps, un 
filament continu, fin et très long, contourné en peloton irrégulier. Sur ce filament, toute la chromatine s'est distribuée plus régulièrement de manière ì le revètir tout entier.

C'est la phase de spirème ou pelolon et plus particulièrement celle de pelolon serré (fig. 1 i).

l'arfois on peut constater que le filament, au lieu d'ètre continu d'un bout à l'autre, est formé de quelques longs segments disposés bout à bout; mais en lout ras, chaque segment est filiforme, continue l'enroulement du segment précédent, et jamais ne se ramifie ni ne se soulle à ses voisins (').

Le second phénomène est un raccourcissement du filament qui s'épaissit en conséquence et se transforme en un cordon. Par suite de cette diminulion de longueur, les anses du peloton s'écartent les uns des autres: c'est la phase du spirime dite de peloton lâche (fig. 1S). En mème

(1) Tout le monde est d'accord sur ces dispositions, mais, naturellement, les avis diffèrent sur la manière dont elle est obtenue, selon l'idée que l'on se fait de la disposition rraie des filaments de linine sur le réseau apparent du noyau au repos. Ces idées, nous l'avons vu (p. 6 et suiv.), peuvent se ramener à trois.

10 La linine forme un réseau vrai (figr. 15$)$ avec ramifieation et soudure des filaments

Fig. 15.

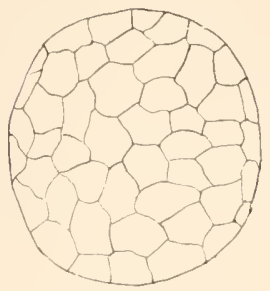

Réseau de linine (Sch.).
Fig. 16.

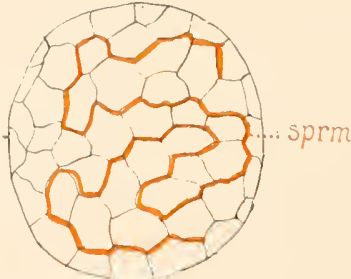

Spireme (sprm.) commengant à šindividualiser (Scli.).
Fig. 17.

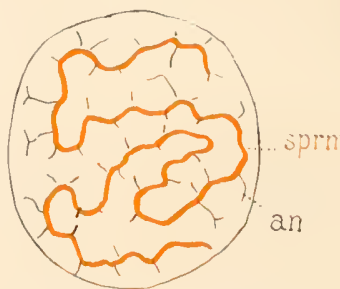

Spireme (sprm.) achevant de s'individualiser (Seh.). all., anastomoses.

aux points nodaux (Flemmis . - Dans ce eas les mailles se rompraient en les points précisément nécessaires pour ne laisser qu'un long filament eontinu, pelotonné (fig. 16 et 17). Le long de ce filament sont appendus les petits bouts (an.) qui fermaient les mailles coupées. Ces petits bouts se rétractent peu à peu et linissent par disparaitre. On rencontre effeetivement (fig. 17, sprm.) des figures de noyau i ce stade, montrant un lilament déchiqueté el comme hérissé de petiles épines molles qui correspondent bien à ee que l'on est en droit d'attendre dans eette théorie.

20 La linine formerait un filament unique, continu, jamais ramifié ni soudé à luimême, mais très irrégulièrentent eontourné et entreeroisant ses sinuosités qui, sans cesse, passent l'une sur l'autre. mais suns se souder aux points de croisement (Carnor [8'], Strasburger [8'] première opinion). - Dans ce cas, la plase du peloton serré s'obtient par un simple arrangement de sinuosités qui se disposent un peu plus régulièrement.

$3^{\circ}$ La linine forme des anses principales indépendantes, reliées seconılairement par un réseau de filaments beancoup plus fin (RABL [85]). - Il sulfit alors que les filaments de ce réseau seeondaire se coupent et soient résorbés par les anses prineipales. 
temps, le cordon devient plus homogène, son apparence granuleuse, déchiquetée, fait place à une forme cylindrique régulière, due à une répartition plus uniforme de la chromatine le long de lui.

A la phase de peloton làche succìde celle de peloton segmenté (fiğ. 19).

Fig. 18.

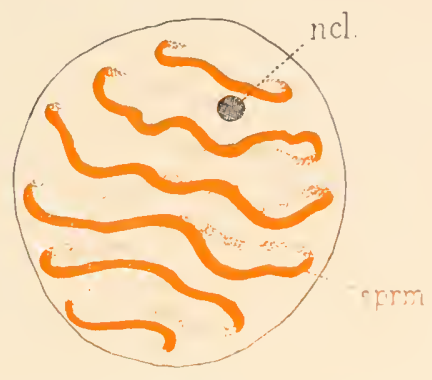

Prophase.

Stade de peloton lache (Sch.).

ncl., nucléole; sprm., spirime.
Fig. 19.

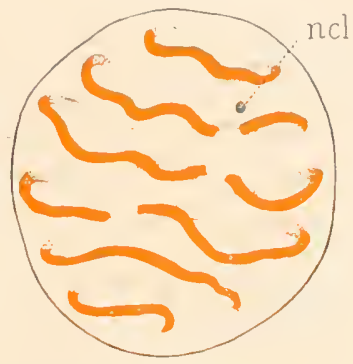

Prophase.

Segmentation transversale (Sch.).

ncl., nucléole.

Elle consiste en ce que le filament se coupe transversalement en un certain nombre de segments, ordinairement 12 a 2 . chez les animaux, plusieurs dizaines chez les plantes, appelés segments nucléaires ou anses chromatiques ou chromosomes (").

Dès que les segments sont formés, on constale qu'ils ne sont plus simples, mais composés chacun de deux filaments parallèles étroitement rapprochés (fig. 20).

Une fine ligne claire les sépare seulement l'un de l'autre. Cela provient de ce qu'ils ont subi une segmentation longitudinale, phénomène d'importance capitale, qui a pour effet de répartir d'une manière rigoureusement égale la chromatine du noyau mère entre les deux noyanx filles $\left({ }^{2}\right)$.

Pendant la formation du peloton, les nucléoles ont peu à peu diminué le volume et finalement disparu. Nous arons indiqué plus haut (p. 11, note) ce qu'ils semblent devenir. A ce moment aussi, lorsque les chromosomes sont bien individualisés, la membrane nucléaire

(1) Cette phase ne prend place ici que dans les cas Fig. 20.

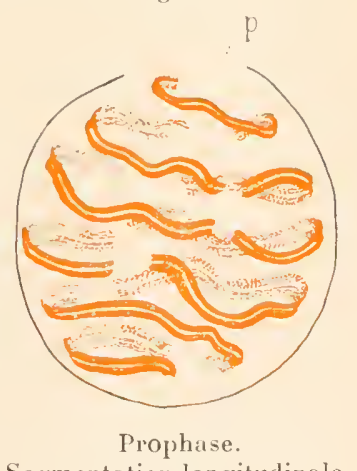

Segmentation longitudinale (Sch.).

p., région où la membrane cellaire commence à disparaitre. (ou théories), oì il y a dans le noyau au repos un réseau ou un filament continu. Dans les noyaux de liabl (fig.y) les anses primitives constituent les futurs chromosomes et ne se segmentent pas de nouveau.

${ }^{(2)}$ Le moment où elle débute est difficile à déterminer et sans doute variable. Parfois, elle a lieu sur les chromosomes déjà séparés; plus souvent, elle paraît débuter sur le peloton làche. 
commence à se résorber et disparaît peu à peu, laissant le contenu du noyau en libre communicalion avec le cytoplasina.

३. Dans le cytoplasma. - Pendant ce temps, des phénomènes non moins importants se sont produits dans le cytoplasma. Prenons comme typique le cas où le centrosome ost unique à ce moment (fig. 21).

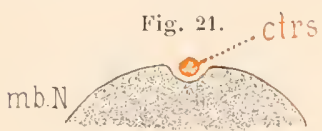

A
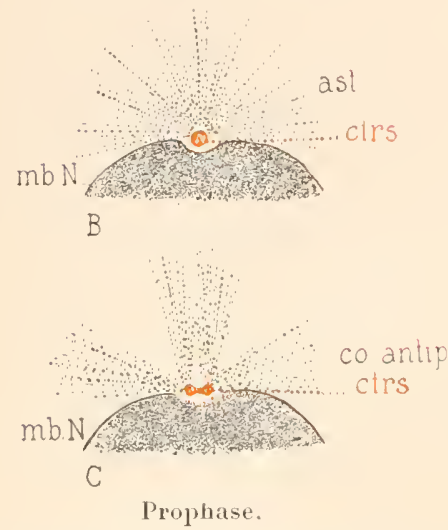

Phènomènes cytoplasmiques (Sch.). $A$, état de repos; $B$, formation de l'aster; $C$, division du centrosone. ast., aster; co. antip., cines antipodes: ctrs., centrosome: mb. N., membrane nucleaire.

Nous avons vu qu'à l'état de repos, il est logé dans une petite masse de protoplasma hyalin, la vésicule attructive, limitée parfois extérieurement par une bordure un peu plus dense appelée couche corticale. Dans le cyloplasma ambianl, on ne remarque, à ce moment, rien de particulier $(A)$. llais, pendant que se forme le peloton nucléaire, on voit se dessiner autour de la vésicule attractive de fines stries rayonnantes disposées comme les rayons l'un astre lumineux et constituant l'aster (ast., en $B)$. L'aster est d'abord tout petit et, la vésicule étant au contact du noyau, c'est seulement du côté opposé à sa paroi que se montrent les rayons; à mesure que la division progresse, la vésicule s'écarte du noyau eíles rayons devienuent plus grands et plus accentués. Bientôt, dans la vésicule attractive encore impaire, le centrosome se dédoulle en deux petits granules arjacents $\left(C^{\prime}\right)$; bientôt après, la vésicule s'élire en biscuit et se divise à son lour. Les deux pelites vésicules conliguës se séparent lentement l'une de l'autre et, dès qu'elles se sont un peu écarlées, on voit entre elles le premier rudiment d'un fuseau. C'est le fuseau central (/us. ctrl., fig. 20) formé de fins filaments pâles (achromatiques) qui se portent d'une vésicule à l'autre, lessinant deux côtues ałlossés par leur's bases. C'est à ce moment que la membrane nucléaire commence à se détruire par résorption, d'abord au niveau de la fossette où était logée la vésicule allractive el, de là, en tous sens ver's le pôle opposé. Elle a ainsi bientôt complètement disparu et il n'y a plus dès lor's de distinction entre les phénomènes intra et extranucléaires.

Pendant ce temps (fig. 22), les deux vésicules, chacune munie de son centrosome (ctrs.) et entrainant son aster, continuent de s'écarter pour se placer en deux points diamétratement opposés, allongeant entre elles le fuseau qui les réunit (/us. ctrl.). Les asler's forment alor's aux pôles du fuseau deux figures appelées coines antipodes (co. antip.). Bien avant ce stade, on voit se dessiner des filaments achromatiques (co. attract.) qui partent des résicules et vont se jeter sur les chromosomes (uns. chrt.). Fila- 
ments et chromosomes sont d'abord tous d'un mòme côté du fuseau mais, peu à peu, ils se disposent en cercle autourde lui, d’une façon très régulièrc et constituent le fuseau périphérique Fig. 22. formé lui aussi de deux cònes d'attraction (co. attr., fig. 23). On a atteint la nisétaphase.

\section{b. Métaphase.}

La métaphase, très courte, ne comporte pas, comme les deux autres phases, une série de plıénomèıes successifs : c'est un état, appelé parfois stade de métaliynèse. A ce inoment (fig. 23), la figure nucléaire se compose de yuatre parties : Io les pôles,

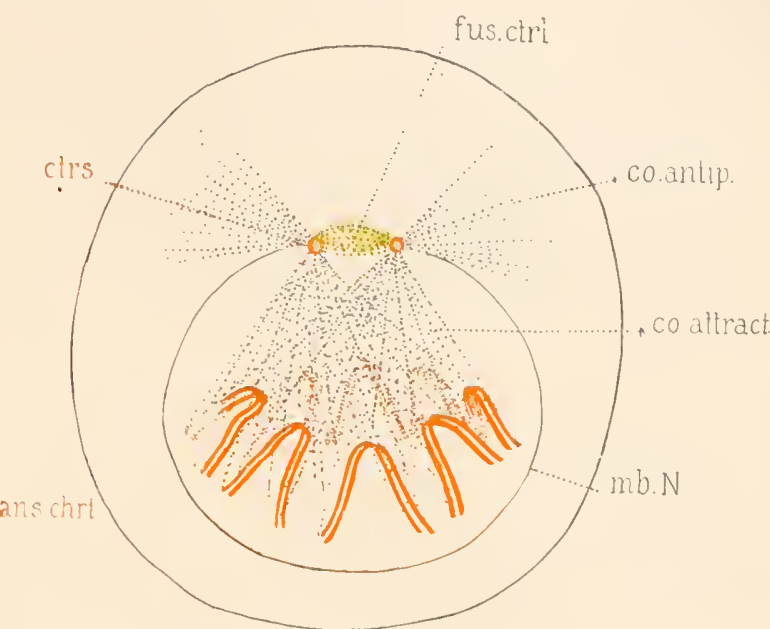

Prophase (Sch.). Formation des fuseaux.

auø. (hrt., anses chromatirues : co. antip., cones antipodes co. attract., cònes d'altraction ou fuseaux piriphèriques: fus. ctrl., fuscan ecutral; mb. N., membrane uncléaire. comprenant chacun un centrosome (ctrs.), une vésicule directrice et un aster ou conne antipode (co. antip.) 'pui rayonne dans le cyloplasma dans la région opposée au fuseau. Ia figure constituée par cet aster double constitue l'amplicaster;

Fig. 23. 23

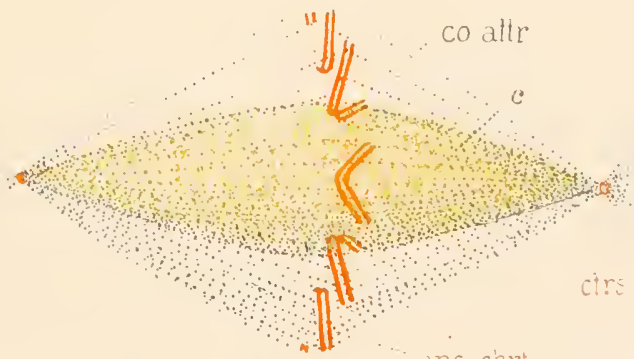

co. antip

$$
\text { Metaphase (Sch.). }
$$

ans. chrt, anses chromatiques; c., fuseau central; co. antip., cònes antipodes; co. attr., cònes d'attraction ou fuseau périphirique; etrs., cenlrosomes.

Qo le fuseau central (c.) (Herusv), formé de cequ'on a appolé les filaments unissants qui vont sans interruption d'un pôle à l'autre; $3^{\circ}$ les chromosomes ou anses chromatiques (ans.chrt.), disposés en cercle régulièrement autour de l'équateur du fuseau central et en dehors de lui. Ils ont pris 
une forme en anse régulière et sont orientés, sans exception, le sommet de l'anse vers l'axe du fuseau et les branches divergentes en dehors. Dans cet état, ils forment ce que l'on a appelé la plaque équatoriale ou plaque nucléaire; $4^{\circ}$ enfin, les cônes d'attraction (co. attro) formés par les filaments périphériques qui partent des pôles et se jettent chacun sur un des chromosomes.

On se rappelle que ceux-ci sont, depuis longtemps déjà à ce moment, divisés longitudinalement en deux cordons parallèles. Ces cordons ou anses jumelles sont disposés de manière à regarder chacun un des pôles. On est tenté de crọire que les filaments périphériques issus d'un mème pôle s'attachent précisément sur celle des deux anses qui est tournée vers lui. Mais on n’a jamais pu s'assurer de ce détail.

\section{c. Anaphase.}

Tout se passe alors comme si les filaments attachés aux chromosomes se contractaient et entrainaient les drux moitiés de ceux-ci chacun ver's l'autre des pôles (fig. 29). On voit, en effet, les deux anses

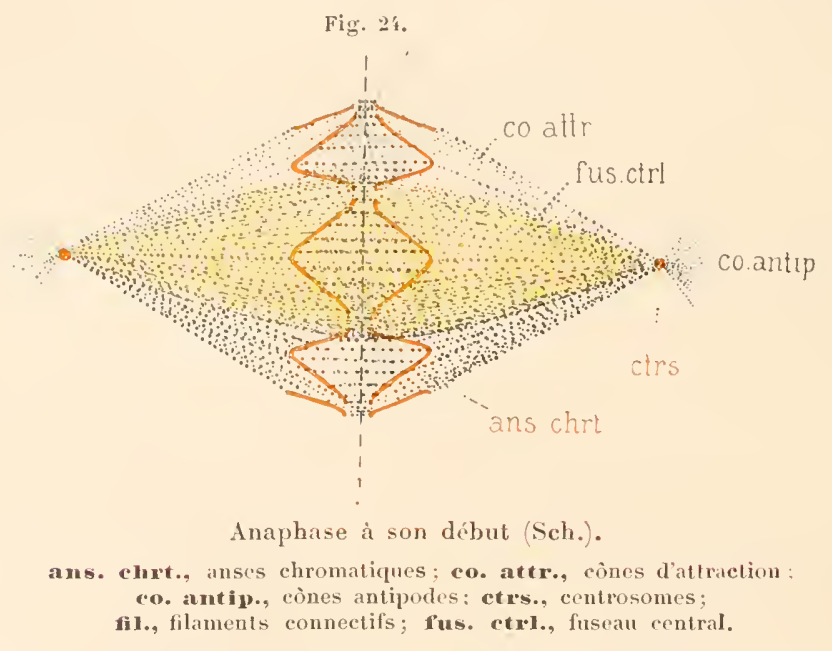

jumelles (ans. chrt.) de chaque chromosome, s'écarter l'une de l'autre, en commençant prar le milieu, de manière à former ensemble : d'abord une ellipse allongée transversalement, puis un cercle, puis une ellipse à grand axe dirigé comme celui du fuseau. Les deux moiliés se liennent encore prar les bouts, mais ces bouts se séparent à leur tour et elles sont entraînées chacune ver's un des pôles.

Elles s'en rapprochent beaucoup, mais ne l'atteignent pas tout à fait (fig. 20 ); il reste, entre leurs sommets qui n’arrivent pas au contact et la vésicule directrice qu'elles n'atteignent pas, un petit espace appelé le champ polaire. 
Pendant toute la durée de ce mouvement, les anses jumelles (ans. chrt.) sont restées unies par des filanents connectifs (fil.uniss.) qui s'étendent entre elles, d'autant plus longs qu'elles sont plus écartées.

Les phénomènes qui suivent constituent la reconstitution du noyau à l'état de repos, semblable à l'état primitif, sauf qu'il y a deux noyaux au lieu d'un. 1 chacun des pôles (fig. 26), les chromosomes perdent leur forme et leurs dispositions réFig. 25 .

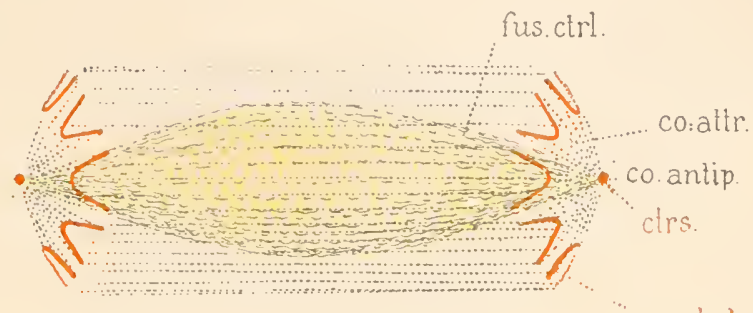

ans chrt

Anaphase.

Formation des noyaux filles (Sch.).

ans. chrt., anses chromatiques; co., antip., cònes antipodes ; co. antr., fuseau périphérique; etrw., centrosounes;

fil. uniss., filaments connectils; fus. etrl., fusean central.

gulières; leurs branches se contournent, leur anse s'ourre, ils s'allongent et finalement s’arrangent en un ensemble irrégulier qui rappelle, it peu près, le stade de peloton segmenté; puis ils se rapprochent, deviennent moins distincts les uns des autres et forment, plus ou moins nettement, le peloton liche (fig.27) el enfinle peloton serré. C'est là le stade dispireme (fig. 28). Enfin, la forme du (ou des) Fig. 26.

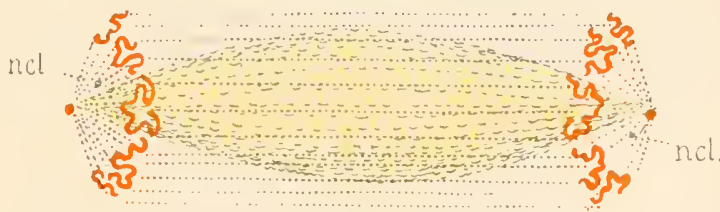

Anaphase.

Formation des spirèmes filles (Sch.). ne1., nucléoles.

cordon devient elle-mème irrégulière, déchiquetée, comme si de fius filaments poussaient sur les côtés et, sans qu'on ait bien vu comment, le stade de réseau au repos se trouve rétabli. Fig. 27. La membrane $\left(m b . N^{\top}\right.$.), en mème temps, se reconstitue peu à peu, finit par enfermer complètement le noyau, et les nucléoles (ncl.) réap paraissent, petits d'abord, puis avec leur volume normal. Le fuseau

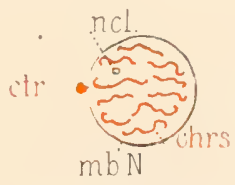

Anaphase. Formation de la membrane des noyaux filles (Sch.).

chrs., chromosomes; ctr., centrosomes; mb. N., membrane nucléaire; ncl., nucléoles. central, très net et intact pendant que les chromosomes se mouvaient

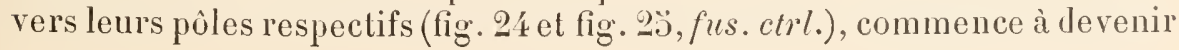


moins distinct à mesure que l'on approche du stade dispirème et, quand la membrane commence à se former, il achève de disparaître. L'aster lis-

Fig. 28.
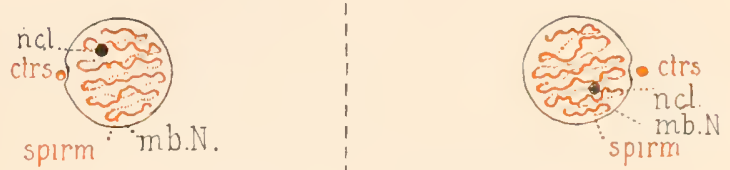

Anaphase.

Aebèvement des noyaux filles.

ctrs., centrosomes; mb. $\mathbf{x}$., membrane nucléaire; ncl., nucléole; sprm., spirc̀me.

Mais comment se forment les nucléoles des noyaux filles que l'on voit apparaître à nouveau dans ceux-ci, tandis que ceux du noyau mère avaient disparu au commencement de la division?

(1) Plaque cellulaire et plaque intermédiaire de Flemming. - Dans les cellules végétales, il y a quelque chose de plus. Avant que le fuseau central ne disparaisse, on voit apparaître, sur chacun des filaments interposés aux anses jumelles, exactement dans

Fig. 29.

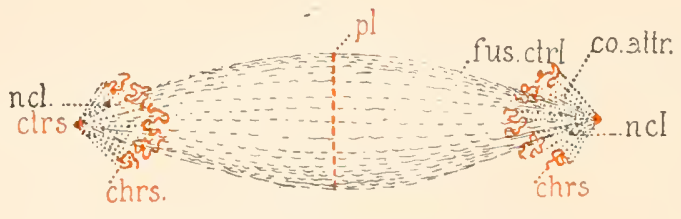

Plaque cellulaire vue de profil (Sch.). pl., plaque cellulaire. Autres lettres comme dans les figures précédentrs. paraît alor's aux pôles, la vésicule attractive avec son centrosome (ctrs.) devient moins distincte aussi et, sans changer de place, se trouve logée dans une dépression de la membrane nucléaire (").

Nous voyons par là comment se déiloublent les centrosomes et les éléments nucléiniens du noyau. 
On admet généralement qu'ils sont formés aux dépens des nouveaux chromosomes. Les uns croient qu'ils disparaissent complètement pour former le centrosome (V.p. II, note) et sont reformés par les chromosomes en tolalité el ì nouveau. Les autres, plus nombreux, pensent, en se fondant sur quelques aspects histochimiques, qu'ils abandonnent leur substance chromatique aux chromosomes et se reforment ensuite par réagglomération de cette même substance (fig. 26, ncl.) (').

(1) Zimmermann [93], au contraire, assure que, chez les plantes, ils se dissocient, se répandent sous la forme de petits grains chromatiques dans le cytoplasma et s'agglomèrent de nouveau pour former les nucléoles de nouvelles cellules.

Tels sont les phénomènes principaux de la division nucléaire indirecte. De crainte d'obscureir une description en somme assez compliquée, nous nous sommes astreints à négliger les exceptions, variantes, divergences de faits ou d'opinions, innombrables en ces matières. Mais il est deux points sur lesquels il est nécessaire de s'expliquer ici. Ce sont les rapports des chromosomes avec les filaments et l'origine du fuseau lui-même.

Rapports des chromosomes avec les filaments. - La description donnée ci-dessus s'applique au noyau du type de liabl. Sa caractéristique (fig. 22) est l'apparition d'un fuseau tout petit, en dehors du groupe des chromosomes et la distinction entre un fuseau périphérique lié aux chromosones et un fuseau central indépendant d'eux. Or, dans bien des eas, la chose semble se passer d'une tout autre manière (fig. 32). Le centrosome (avec sa sphère attractive) se divise, ses deux moitiés s'écartent et se portent aux deux extrémités d'un mème dianètre du noyau en glissant sur la membrane intacte de celuici et, pendant tout ce temps, il n'y a pas trace de fuseau. Les centrosomes s'écartent alors un peu du noyau et un espace clair apparait entre eux et le noyau et tout autour de celuici; en même temps, la membrane nucléaire semble se flétrir, colmme si elle avait laissé suinter du sue nucléaire pour former la zone claire en question. Bientòt, on voit se dessiner, à partir des pôles, un fuseau complet (co. attr.) qui s'avance peu à peu vers le noyau, et l'englobe dans ses filaments. Alors seulement, la membrane nucléaire disparait et les chromosomes (chr.) entrent en rapport avec le fuseau et se soudent à ses fils de la manière décrite précédemment. On con-

Fig. 32.

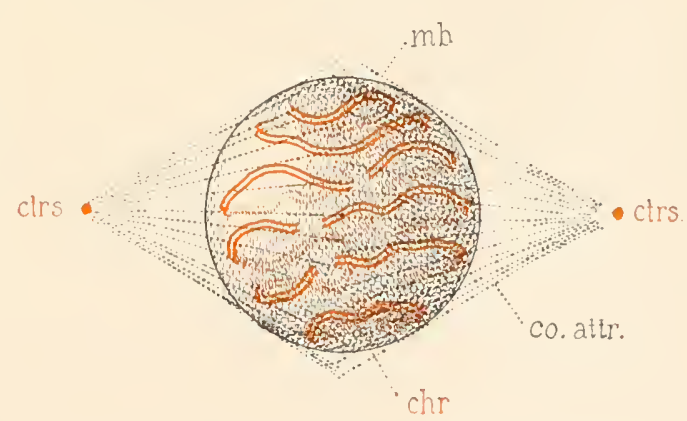

Aulre mode de formation du fuseau (im. Vialleton).

ehr., anses chromatiques; ctrs., centrosomes ;

co. attr., cònes d'attraction; mb., membrane nucléaire. coit qu'il n'y a pas ici de distinction entre fuseau central et filaments périphériques.

Origine des filaments des fuseaux. - Sur cette question, trois opinions principales sont en présence: $1^{\circ}$ Strasburger [84], Guignard [91], et avec eux la plupart des botanistes et, parmi les zoologistes, Bover1 [s8], Hennegux [91], II. Fol, BoBRETzki, ete., admettent que tous les filaments du fuseau sont d'origine extranucléaire. Ils émaneraient des sphères attractives, ou se différencieraient dans le cytoplasma voisin ; 2 o Bütschli, R. Hertwig, Pfitzner, Gruber, Garnox, Rabl, Zacharias, Cheviakor [87], 0. Hertwig [94], les font provenir exclusivement de la linine du réseau 


\section{DIVISION DU CORPS CELLULAIRE}

La division du corps cellulaire est aussi simple que la division nucléaire est compliquée. Elle commence pendant l'anaphase au moment où les anses jumelles atteignent les pôles. Pendant qu’à leurs dépens le spirème se reforme et que le noyau se reconstitue, se montre à la surface de la cellule, exactement daus le plan équatorial du fuseau, un sillon. Ce sillon commence en un point ct s'élend rapidement en cercle toul autour de la cellule. Au moment où les asters disparaissent, le cercle est complet. Il s'approfondit alor's peu à peu et finit par couper la cellule mère en deux cellules filles dont chacune contient un des noyaux filles issus de la division nucléaire.

Dans ce mode de division, le noyau est coupé en deux parties égales. Mais il s'en faut de beaucoup qu’il en soit toujours de mème pour le cytoplasma (').

nucléaire. Ils s'appuient surlout sur le fait que, dans certains cas, le fuseau tout entier peut se trouver à l'intérieur du noyau lorsque la membrane nucléaire est encore intacte; 30 enfin, Ed. Van Beneden [83], Platner, Hernany, Flemmixg [91], Prenant [91], Mirrophanof [94], leur attribuent avec beaucoup dapparence de raison une double origine. Ce que nous avons appelé le fuseau central ou, dans le cas d'un fuseau unique, la portion polaire de ce fuseau, semble indubitablement provenir de la substance mème des vésicules attractives ou du cytoplasma ambiant, tout comme les rayons de l'aster. Mais la portion équatoriale du fusean périphérique, ou de l'unique fuseau s'il n'y en a qu'un, proviendrait des filaments de linine du réseau mucléaire disposés ad hoc el urris aux filaments venus les pòles. Malgré tant d'efforts dépensés à la solution de ces ruestions depuis ruelques années, on voit que bien des points restent encore obscurs sur l'origine lu fuseau et sur ses relations exactes avec les chromosomes.

Permanence des chromosomes. - Une autre question litigieuse et Irès importante, comme on le verra plus tarl, est celle de la permanence des cliromosomes. Il y a sur ce point deux opinions principales : $1^{\circ}$ les chromosomes sont constants en nombre, mais nullement en substance; le filament se recoupe n'importe comment (O. HerTwg [90]); 20 ils sont permanents, soit ru'ils ne perdent à aucun moment leur individualité (RABL [89]), soit que le filament se recoupe au même point, (Boveri [92]).

Mais ce qui est bien plus mystérieux encore c'est la cause de ces phénomènes. Cela donne l'impression d'une troupe de marionnettes jouant une petite pièce muette mais très compli đuée, avec une merveilleuse précision de mouvements, et rentrant dans la coulisse pour recommencer à la division suivante; nous comprenons le but de l'action, e'est le partage équitable des substances et organes du noyau mère entre les deux noyaux filles. Mais nous sommes bien loin de voir tous les mourements et de comprendre toute leur signification.

(1) Nous avons vu plus haut (p. 24) que le noyau dans la cellule oceupe, daprès la loi de position de 0 . Hentwig $[84]$, le centre de gravité du eytoplasma. Le mème anteur a cherché à formuler une loi de direction du plan de division, indiquant la place de ce plan dans la cellnle ou, ce fui revient au môme, la direction du fuseau, car le plan de division n'est autre que le plan équatorial du fuseau prolongé. Sa loi est que laxe du fuseau se dirige comme sil étail une aiguille amantée, et que le cytoplasma pur fùt du fer, landis que les matières non protoplasmiques seriant inertes. Il se place parallèlement à la direction de la plas grande masse du protoplasma. II vaudrait mieux dire symétriquement par rapport à celte masse, car 


\section{B. DIVISION DIREGTE OU AMITOSE}

Ce mode de division, beaucoup plus simple que le précédent, a été connu bien avant lui et, pendant lien longlemps, on a cru qu'il étail le seul. Le signe caractéristirjue de celte division était le noyau en biscuit. On sait aujourd'hui qu'elle est, au contraire, bien plus rare que l'autre, et heaucoup d'histologistes voudraient la réduire à un processus d'altération morbide, de dégénérescence ou de stérilité cellulaire.

Le cas typique (fig. 33) se réduit à ceci : le noyau s'allonge, s'étire el se coupe, le corps cellulaire en fait autant, et bientôt, au lieu d'une cellule, il y en a deux. Il n'y a là ni intervention des centrosomes, ni fuseau, ni formation de chromosomes, ni disparition de la membraue nucléaire $(m b . V$.). Le noyau garde l'aspect qu'il avait à l'état de repos. Le sort du centrosome est assez oloscur. Souvent, on ne le voit pas se diviser. Dès lors, il doit manquer à l'une des deux cellules au moins, qui devient, par là, incapable dé-

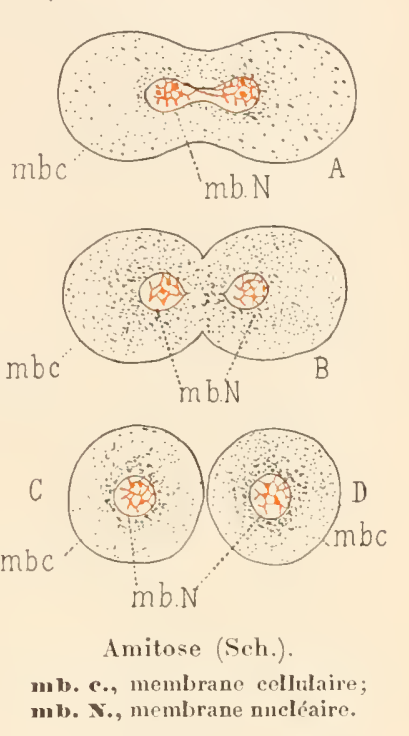
sormais de division indirecte. Parfois on l'a vu se diviser et mème former un petit fuseau (').

Ce mode de division est beaucoup moins fixe que la mitose. On a cherché à voir dans certaines de ses formes un intermédiaire entre elle et la mitose, mais sans trouver rien de démonstratif.

\section{RELATION ENTRE LES DIVISIONS DIRECTE ET INDIRECTE}

Quelle peut ètre la signification relalive de ces deux modes de division?

dans la segmentation des œufs il arrive souvent que, pour une cellule riche en vitellus, le plan de division sépare une étroite calotte au pôle animal. Ce qui montre que le fuseau était perpendiculaire au gâteau de cytoplasma qui formait ce pôle.

(1) Dans quelques cas, il semble prendre une part active à la division nucléaire ; Meves [91] l'a vu dans les spermatogonies de la Salamandre s'étirer en un ruban qui se met en croix avec le point qui réunit les deux moitiés dlu noyau en biscuit, puis se souder en anneau autour de ce point comme pour l'étrangler, et aider à la division. Ilais il n’a pu suivre le phénomène. Anxhold [88] a décrit sous le nom de fragmentation nucliaire, une division dans laquelle le noyau se fragmente en molrceaux sans disposition régulière. Enfin Göppert [91] a vu cette fragmentation se faire par un processus très bizarre. Le noyau se perce l'un trou en son centre et se transforme ainsi en un anneau qui s'ouvre, puis se fragmente en deux ou plusieurs morceaux. 
Trois hypothèses principales ont été émises à ce sujet, mais aucune n’est suffisamment appuyée:

$1^{\circ}$ L'amitose est un procédé de division primitif en train de disparaître, pour laisser la place au procédé plus perfectionné de la mitose. - Cette hypothèse est la plus naturelle, elle a cependant beaucoup moins de partisans que la suivante;

$2^{\circ}$ Elle est au contraire plus jeune phylogénétiquement que la mitose. Elle se produit uniquement chez des cellules en dégénérescence ou arrivées presque au terme de leur puissance reproductrice. - D'apr'ès les uns, elle condamne à mort la cellule où elle s'est produite une fois, en limitant à zéro ou à un très petit nombre, ses divisions ultérieures. D'où le nom expressif de glas funère de la cellule qui lui a été donné par Rabl [91]. D'autres pensent qu'une mitose peut intervenir et régénérer en quelque sorte la cellule;

$3^{\circ}$ L'amitose est un procédé de division spécial qui se produit dans des conditions déterminées.

Les recherches ultérieures pourront seules nous dire laquelle de ces suppositions est la vraie.

\section{THÉORIES SUR LA DIVISION CELLULAIRE}

Quelques tentatives intéressantes ont été failes pour tàcher de découvrir les causes de la division cellulaire, soit ses causes mécaniques, soit ses causes déterminantes.

En ce qui concerne les causes mécaniques deux forces ont été invoquées: d’une part la contraction des filaments du fuseau, de l'autre une attraction chimiotactique exercée par les centrosomes sur les chromosomes. La première opinion a été émise par E. Vax Bexeden [87]. D'après lui, les filaments émanés des sphères attractives s'attacheraient direclement sur les anses jumelles et les attireraient vers les pôles, en se contractant. Cette traction serait même la cause de la division longitudinale des chromosomes. Cette ilée a été admise par un grand nombre d'auteurs: Boveri, O. Hertwig, Bergil, Rawitz, C. Scinxeider, Rabs, et ce dernier a fourni une explication très complète de la manière dont se passe les phénomènes (').

(1) Rabu admet que les filaments des asters et "lu fuseau sont déja fout formés dans la cellule au repos, mais qu'on ne les voit pas, parce yu’à ce moment ils sont très fins et non tendus. Ils partent tous de la sphère attractive qui est le centre mécanique de la cellule (fig. 34). Les uns serpentent dans le cyloplasma, les autres pinètrent dans le noyau par un trou percé dans sa membrane au fond de la dépression où la sphère est logée. Ils ront s'altacher anx anses chromatiques (ans. chrl.) qui, thans sa théorie (V. p.9) sont déja individualisees pendant le repos du noyau. Les phénomènes nutritifs amènent les filaments, qui sont tous contractiles, ì un état où ils n'attendent qu'une excitation interne ou externe pour se contracler. Ceux du cyloplasma entrent en jeu les premier's. Ils se raidissent, deviennent plus courls, plus gros et rertilignes, toutes conditions qui concourent à les rendre visibles et donnent l'image le l'aster. En continuant à se contracter, ils tirent sur le centrosone el la vésicule en sens inverse, et 
Mais Flemung [91] et après lui Hermann ont opposé une objection capitale à celte théorie séduisante : ils ont fait remarquer que la division longiludinale des chromosomes précède sourent celle des asters et du centrosome. La théorie peut ètre vraie en partie, mais elle est fausse en certains points, insuffisante dans d'autres. Cerlainement, la division du centrosome et celle des chromosomes sont deux phénomènes, connexes peut-être, mais non unis entre eux par la relation de cause à eflet.

La seconde cause a été invoquée par Strasburgen [93]. Ce savant pense que les anses glissent seulement sur les filaments, attirées par une force chimiotactique (il vaudrait mieux dire biolaclique) émanant des sphères attractives (').

déterminent leur division. Leur écartement a pour effet de fendre en long les filaments (p.) qui vont aux chromosomes, en sorte qu'après cette scission longitudinale achevée, en chacun des points où un filament s'attachait au chromosome, il s'en

Fig. 3't.

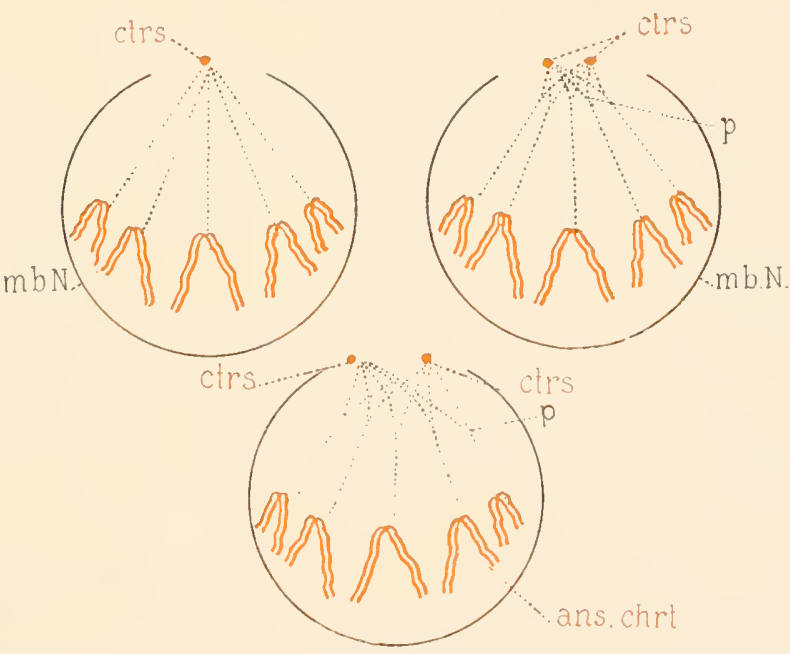

Formation du fuseau, d'après les idées de Rabl (Sch.).

ans. chrt., anses chromatiques; ctrs., centrosomes; mb. N., membrane nucléaire; p., points ou les filaments se séparent.

attache maintenant deux qui se rendent chacun à l'un des centrosomes. Quand les centrosomes sont lout it lait écartés et que les filaments se contractent, ils prennent la disposition des éléments du fuseau, amènent les chromosomes dans le plan équatorial et, tirant sur eux en sens inverse, les dédoublent en long. Cette contraction, en se continuant, sépare les chromosomes en deux groupes et les entraîne vers les pôles.

(1) Il se fonde principalement sur le fait que, chez les Liliacees, dans les cellules mères du pollen, il n'y a pas de fibres s'attachant aux chromosomes.

Mais Flemang [94] trouve cette alsence insuffisamment démontrée. Iläcker [94], au contraire, confirme l'opinion de Strasburger, en constatant que, chez Sida cristallina, au moment où les anses vont se déplacer', il se produit dans les centrosomes 
Bien plus hypothétiques encore sont les forces invoquées comme causes déterminantes de la division.

On sait que Suexcer [64] a, depuis longtemps, attiré l'attention sur ce que, lorsqu'un être organisé s'accroìt, sa surface croit comme le carré de ses dimensions et son volume comme le cube. L'assimilation doit ètre proportionnelle au volume et, comme elle ne se fait que par la surface, il en résulte que plus l'ètre s'accroìt, plus sa nutrition devient difficile. Von Rees [87] appliquant ces consilérations à la cellule, croit y trouver les raisons de sa division. Mais, montrer qu'une chose est avantageuse, u'est pas expliquer pourquoi elle se fait. Il faudrait montrer comment la gène de la nutrition devient l'excitant physiologique de la division (").

Toute cellule, en vivant, accumule en elle des produits usés qu'elle doit rejeter. Elle s'en débarrasse d'ordinaire en les excrétant. Lexdu [90] pense qu'elle a pu aussi y arriver, en séparant d'elle-mème la partie de son corps où ces produits, qu’il appelle ballast, sont accumulés. La division aurait alors pour origine l'excrétion dont elle ne serait qu'un mode particulier $\left(^{2}\right)$.

\section{CONJUGAISON}

La conjugaison est un phénomène inverse de la division dans lequel deux cellules distinctes se fusionnent en une seule. Elle a pour effet de constituer un individu cellulaire nouveau, formé des substances de deux cellules différentes. La race y gagne un regain de vie qui se montre chez les cellules issues des divisions consécutives à la conjugaison, se conserve plus ou moins longtemps, et s'épuise peu à peu à mesure que les générations agames se succèdent, jusqu’à ce qu’il soit renouvelé par une conjugaison nouvelle $\left({ }^{\mathbf{3}}\right)$.

un changement de constitution que les réactifs colorants meltent en évidence. Au lieu de former une masse pleine logée dans la sphère attractive, ils deviennent vésiculeux et laissent diffuser autour d'eux un liquide colorable. Ce liquide serait l'agent de l'attraction chiniotactique des chromosomes.

(1) ReEs cherche à montrer par quelques exemples que cette action excitante de la pénurie nutritive est un fait. Chez les Protozoaires, les conditions défavorables provoquent la division.

OrR a elıerehé à trouver dans l'asphyxie relative due à la diminution de la surface respiratoire relativement au volume la cause du mouvement qui opère la division.

${ }^{2}$ ) Dès lors, des deux cellules nées de la division, l'une serait toujours plus pure que l'autre et ce seraient les cellules de la lignée la plus pure qui continueraient la vį de l'espèce.

(3) Cette décrépitude de la race conséculive à une trop longue série de divisions a été admirablement observée clrez les Ciliés par Maupas qui lui a lonné le nom expressif de dígénérescence sénile. En voyant que la conjugaison est la condition indisjensable de la reproduction scissipare indéfinie chez tous les êtres où elle existe, on serait tenté de généraliser et de la croire indispensable à tous sans exception. Mais 
La conjugaison n'est pas une fonction des cellules organisées en tissus; elle ne se rencontre que chez les cellules constituant des ètres capables de vie indépendante, c'est-à-dire chez les ètres unicellulaires ou pluricellulaires homoplastides (*), ou chez les héléroplastides à la phase unicellulaire de leur cycle évolutif.

On est convenu d'appeler gamètes les cellnles qui se conjuguent.

Il y a deux sortes de conjugaison. Dans l'une, les gamètes se fondent complèlement l'un dans l'autre; ils perdent entièrement leur individualité dans l’élément qui résulte de leur union: nous l'appellerons conjugaison totale. Dans l'autre, ils se rapprochent, se soudent temporairement, échangent une moitié de leur noyau, puis se séparent: nous l'appellerons conjugaison partielle ou nucléaire ( ${ }^{\mathbf{}}$ ).

\section{A. CONJUGAISON TOTALE}

La conjugaison totale n’est pas un simple mélange des substances des deux gamètes. Les noyaux se fondent complètement l'un dans l'autre, et les cytoplasmas, en se mêlant, subissent une contraction qui rappelle tout à fait celle qui se produit dans la combinaison chimique. Tandis que, dans ces associations superficielles où les cellules nues se soudent en un syncytium (comme daus les Ilyxomycètes, certains Iléliozoaires et aussi dans quelques Eponges) la colonie a un volume égal à la somme de ceux de ses composants, ici la cellule issue de la conjugaison a toujours un volume moindre que celui des deux gamètes avant la conjugaison; mème, si l'un d'eux est nolablement plus petit que l’autre, le volıme final peut ètre inférieur à celui du gamète le plus gros.

La conjugaison totale se rencontre surtout chez les plantes. Mais elle a été observée aussi chez quelques Protozoaires. On en doit distinguer deux sortes : l'isogamie et l'hétérogamie. Dans la première, les deux gamètes sont identiques et l'on ne pent dire que l'un soit màle et l'autre

bon nombre d'Algues et la plupart des Champignons se reprorluisent exelusivement par spores asexuelles. Il y a done des êtres chez lesquels, sùrement, la conjugaison n'existe pas; mais pour beaueoup d'autres, c'est seulement qu'elle n'a pas eneore été observee, et le progrès des reeherches diminue leur nombre tous les jours.

(1) Il y a sans donte, dans ee dernier eas, aussi éehange de parties du eytoplasma par des eourants qui s'établissent entre les deux eellules, mais la conjugaison est incomplète puisque les gamètes reprennent leur individualité, et nous l'appelons nucléaire parce que l'échange des moiliés de leurs noyaux en est le phénomène le plus apparent sinon mème le plus important Les Allemands appellent la eonjugaison totale copulation réservant le nom de conjugaison à eelle cui est partielle. Celte dénomination est aussi inpropre que possible, car s'il est un acte où il n'y ait pas fusiou rles individus qui se rapprochent, e'est bien la copralation.

$\left.{ }^{*}\right)$ On uomme homoplastides les êtres formés de plusienrs cellules, mais toutes semblables entre elles, et hétéroplastides ceux qui sont formés de cellules différenciées en divers sens. 
femelle. Dans la seconde, l'une des deux est plus ou moins assimilable à un élément femelle, l'autre à un élément mâle.

l. isogamie pure est assez rare. Dans ce mode de conjugaison, les gamètes identiques peuvent avoir deux formes. Tantôt ils sont, l'un et l'autre, des cellules ordinaires grosses, immobiles et toutes semblables à leurs voisines qui ne se conjuguent pas. Cela s'observe chez Zygogonium, Closterium et quelques autres Algues et, parmi les animaux, chez divers Sporozoaires, en particulier les Grégarines. Tantôt, les gamìtes sont des zoospores qui ne diffèrent en rien, pour l'aspect et la constitution apparente, des zoospores stériles de la reproduction asexuelle. Acetabularia, Bothrydium, Ulothrix et d'autres Algues inférieures en fournissent des exemples (').

Dans l'hétérogamie, la différence entre les deux gamètes peut offrir divers degrés.

Il y en a trois principaux.

Dans le premier, les deux gamètes ne se distinguent en rien, à l'origine, mais la manière dont ils se comportent montre en eux une différence. Chez Spirogyra, très voisin de Zygogonium, des deux gamètes, d'aspect identique et conformés comme des cellules ordinaires, l'un reste immobile dans sa loge et l'autre quitte la sienne pour passer dans celle du premier; il y a là un faible indice de sexualité, l'un des éléments se rapprochant ile l'cuf prar son inertie, l'autre du spermatozoïde par sa mobilité. Il semble y avoir quelque chose de semblable chez certains Foraminifères. Chez Ectocarpus, Giraudia et quelques autres Algues phœosporées, les deux gamètes ont l'aspect de zoospores et sont d'aboril également mobiles, mais bientôt l'un s'arrête et se fixe, tandis que l'autre reste mobile et vient se souler à lui.

Dans un second cas, les gamètes sont distincts dès l'origine, mais par leur taille seulement. Ils sont tous deux immobiles et en forme de cellules ordinaires, comme chez Dictyota, ou tous deux mobiles et en forme de zoospores, l'une grosse, macrospore, l'autre petite, microspore, comme chez Zanardinia qui est une Algue phoosporée et, parmi les animaux, chez divers Radiolaires $\left(^{\mathbf{2}}\right)$.

(1) Celte isogamie pure a un grand intérêt théorique. Elle nous montre que la fusion de deux protoplasmas d'où est dérivée la reproduction sexuelle n'est, dans sa condition primitive, qu'un accroissement brusque et considérable des substances le la cellule. La plupart des auteurs admettent, entre les gamètes isogames, ure différence invisible. Ils vont au delà des résullats de l'observation, et sans nécessité, car on peut très bien concevorr qu'une augmentation violente des substances de la cellule suffise à accroitre son énergie vitale comme fail, avec plus de modération, l'assimilation des aliments.

(2) Chez spirogrra, on voit en général les cellules successives de deux filaments parallèles se conjuguer ainsi toutes ensemble et, presque toujours, toutes celles d'un même fríament sont mâles ou femelles. Le premier se vide lans le second. Mais parfois un filament se ploie et les cellules d'une de ses moitiés se conjuguent avec celles de l'autre. Cela semble indiquer quejles différences de constitution entre 
Enfin le plus haut degré de l'hélérogamie est atleint lorsque les deux gamètes diffèrent, a la fois, par la taille et par la conformation. Ce n’est déjà plus de la conjugaison el on pourrait tout aussi bien décrire ces cas comme appartenant à la génération sexuelle. Cela serait d'autant phus légitime que celte conjugaison a deux formes qui sont calquées, l'une sur la reproduction sexuelle des animaux supérieurs, l'autre sur celle des plantes phanérogames. Chez Fucus, Volvox, il y a un vérilable ouf, gros, sphérique, immobile et des zoospores mâles qui ne diffèrent des spermatozoïdes que par le nom; et chez Peronospora et quelgnes autres champignons voisins, l'ouf ayant le même aspect, le gamète mâle a la forme l'une pelite cellule qui se soude à lui et lui instille son contenu protoplasmique comme fait un grain de pollen avec son boyan pollinique.

\section{B. CONJUGAISON NUCLÉAIRE}

Celle sorte de conjugaison ne diminue pas le nombre des individus comme faisait la précédente, mais elle ne l'augmente pas non plus et elle est, comme celle-ci, la condition nécessaire de lenr multiplication par livision. Ici, la chose a mème été démontrée rigoureusement pour les Infusoires par M.mpas [88]. His dans l'impossibilité de retremper leur énergie vilale dans la conjugaison, les lufusoires meurent fatalement, incapables de contimuer à se diviser. Celle forme appartient à l'isogamie pure, et ne se rencontre que chez les animanx. On l'a olsservée chez presque tous les Ciliés. Jeux individus identiques, mais alleints de dégénérescence sénile, se rapprochent, se soudent par leurs membranes; un orifice se perce par où des courants s'élablissent entre les cytoplasmas, puis les noyaux se divisent dans chaque

les cellules màles el les femelles ne sont pas absolues mais relatives, de même qu'un corps peut être électro.positif par rapport à un autre et électronégatif par rapport à un troisième. Chez les Foraminifères, le phénomène est mal connu et son interprétation n'est pas cerlaine. On voit souvent deux individus d'aspect identique s'accoler et se séparer ensuile sans paraître avoir rien échangé de leur substance. Chez Arcella, on a vu, pendant ce rapprochement, le contenu de l'un des deux indivirlus passer tout entier lans la loge de l'autre et laisser la sienne vide. Che\% Eclocarpa, Giraudiu, Scylosiphon. l'une des zoospores se caractèrise comme femelle par le fait qu'elle se fixe par un de ses deux flagellums et rétracte l'aulre dans son corps protoplasmique. La figure ci-contre montre la conjugaison chez Zanardinia. Chez les Radiolaires, on n'est pas très bien fixé sur la signification relative des diverses spores que l'on voit se former. Branum pense qu'il y en a qui sonl de vraies zoospores asexuelles (spores à crislaux) Fig. 35 .

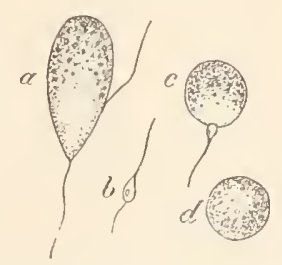

Conjugaison de Zanardinia (d'ap. Retnke).

a., l'oosphere;

b., l'anthirozoide:

c., conjugatison ;

d. produit de la conjugaison. et d'autres qui sont des gamètes, de deux tailles différentes macrospores et microspores). Mais on ne connaît pas leur évolution ultérieure. 
individu séparément, l'un des deux demi-noyaux de chacun d'eux passe dans le conjoint et se joint au demi-noyau resté en place pour former le noyau mixte définitif. Les deux conjoints se séparent alors et leur produit de division n'a plus aucune trace de la dégénérescence sénile dont ils étaient alteints (').

Il existe nettement, chez les Infusoires, dans la conjugaison, un phénomène de réduction chromatique ( $\mathrm{V}$. p. $4 S$ pour l'explication de ces mots). Dans beaucoup de cas de conjugaison totale, on a observé quelque chose d'analogue, quoique sous une forme très simplifiée, et il semble que l’expulsion préalable d’une certaine quantité de chromatine. soit un phénomène général. Mais ce fait est beaucoup mieux connu chez les ètres qui ont une reproduction sexuelle; aussi l'étudierons-nous seulement à propos de ceux-ci.

\section{FÉCONDATION}

La fécondation est la conjugaison avec hétérogamie, poussée jusqu’à la transformation des gamètes en produits sexuels, cuf ou ovule, spermatozoïle, anthérozö̈de ou grain de pollen. Elle n'a lieu que chez les ètres pluricellulaires. Elle est l'acte essentiel et décisif de la reproduction sexuelle, mais elle est précédée d'une série de phénomènes qui, pour ètre moins frappants, n'en sont pas moins d'une importance capitale. Ces phénomènes sont ceux de la préparation des produits sexuels. Relativement à la première qui ne dure qu'un instant, ils sont très longs. Ils sont à la fécondation ce que la charge de l'arme est au coup de fusil.

\section{PRÉPARATION ET MATURATION DES PRODUITS SEXUELS.}

La maturation les produits sexuels n'est pas seulement ce phénomène par lequel toute cellule loit grandir et devenir adulte pour ètre apte à ses fonctions. Il y a ici quelque chose le plus. Nous avons expliqué dans un précédent chapitre que le nombre des chromosomes reste fixe dans la division cellulaire. Or, dans la fécondation comme dans la conjugaison, la cellule initiale de l'organisme futur est formée de la réunion des cellutes sexuelles màles et femelles, et l'œuf fécondé contient tous les chromosomes réunis de l'ovule el du spermatozoüle; si done ceux-ci en contenaient le nombre normal de l'espèce, ce nombre irait en se doublant à chaque génération. Or, il est fixe dans chaque espiece. Il faut donc, pour qu’il se maintienne invariable, qu’à un moment donné il dimiume de moitié. Ce moment se rencontre précisément pentant la maturation des produits sexuels et la dimi-

(1) On trouvera dans ce volume nème au chapitre de la reproduction des Infusoires ciliés une description de ces phénomènes. 
nution se fait par un processus qui a reçu, de Weismans [91], le nom de division réductrice. Il nous faut étudier cette maturation et en particulier la division réductrice dans les deux éléments sexuels.

\section{DIVISION RÉDUCTRICE}

a. Spermatogénèse et Spermatozoïde. - La spermalogénèse est surtout bien connue chez Ascaris megalocephala grâce aux recherches de Vax Beneden et Jubin [84], O. Hertwig [90], Boveri [87, 92], Brauer [93], etc.

Au fond du cul-de-sac testiculaire, on trouve, comme toujours, des éléments jeunes, que l'on peut nommer cellules germinales. Ce sont les éléments primitifs d'où doivent dériver les éléments sexuels.

Leur transformation progressive se fail en quatre phases : une de mulliplication, une d'accroissement, une de réduction et une de maturation.

Les cellules germinales commencent par se diviser un très grand nombre de fois el se multiplient beaucoup en diminuant de volume. En cet état, elles constituent les spermatogonies. Arrivées à un certain degré de petitesse, les spermatogonies cessent de se diviser et se mettent à grossir considérablement; elles se transforment ainsi ell un nombre égal de spermatocytes, dits de premier ordre. Ces spermatocytes sont les cellules grand'mères des spermatozoïdes; ils se divisent exactement deux fois : leurs filles se nomment les spermatocyles de deuxième ordre et leurs pelites-filles les spermatides (ou spermatozoïdes non mùrs) qui se transforment chacune en un seul spermatozoïde mùr, sans se diviser et par une simple modification dans la forme, le volume et l'arrangement de ses parties constituantes.

Ces spermatides sont des cellules d'aspect ordinaire, mais elles ont ceci de particulier que, chez elles, le nombre de chromosomes se trouve réduit de moitié. Nous verrons bientôt par suite de quoi il en est ainsi. Le spermatozoïde mùr diffère beaucoup de la spermatide par l'aspect ef la constilution. Sous sa forme typique la plus complète, il comprend (fig. 36) les parties suivantes : en avant une téte, effilée antérieurement, obtuse en arrière où elle donne insertion à un long llagellum, la queue; à la pointe de la tète un petit globule clair (ctrs.); entre

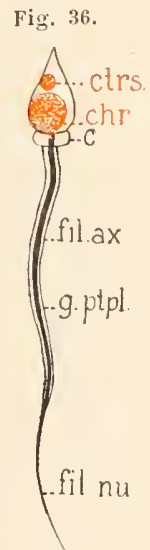
la tète et la queue, une zone étroite, le segment intermédiaire (c.). La queue se compose d'un long filcment axile (fil.ax.) souvent strié en long, entouré dans sa partic supérieure d'une gaine protoplasmique $(g \cdot p t p l$.) qui laisse en arrière le filament axile à nu (fil. $n u)$. Ce filament traverse 
le segment intermédiaire el va s'attacher directcment à l'extrémité obtuse de la tète. Le spermatozoïde, lorsqu'il est mobile, progresse la tète en avant, poussé par les ondulations de son flagellum.

Où sont dans cette structure les parties de la spermatide?

Les chromosomes tassés en une masse compacte forment la majeure partie de la tète. Le centrosome est toujours présent, mais les uns le croient représenté par le segment intermédiaire, les autres par le globule céphalique antérieur. Cetle dernière opinion semble la plus justifiée. Dans ce cas, le segment intermédiaire serait le représentant du cytoplasma. Dans la queue, la gane est sûrement d'origine eytoplasmique, tandis que le filament axile est d'origine eytoplasmique pour les uns, nucléaire pour les autres. D'ailleurs cela a peu d’importance car, dans la fécondation, la tête et le segment intermédiaire entrent seuls dans l'œuf. Ainsi dans la partie qu'utilise la fécondation, sont représentés sùrement les chromosomes, sùrement aussi le centrosome et très probablement le cytoplasma.

b. Ovogénèse et œuf mûr. - L'ovogénèse est calquée sur la spermatogénèse. Dans Ascaris megalocephala que nous prendrons encore comme type, les cellules germinales qui occupent le fond du culde-sac de l'ovaire donnent, en se divisant, de pelites cellules, les ovogonies, qui n'ont aucun caractère spécial et se multiplient beaucoup en diminuant de volume. A un moment donné, la phase de multiplication s'arrête, les ovogonies se mettent à grossir, beaucoup plus mème que les spermatogonies à ce stade, parce qu'elles se chargent, en outre, de réserves alimentaires abondantes et passent à l'état l'ovocytes de premier ordre. Ces ovocytes de premier ordre sont ce que les histologistes appelaient les ovules et qu'ils caractérisaient par leur volume, leur forme sphérique el leur noyau (vésicule germinative) gros, central, bien rond, réfringent. En cet état, ce ne sont pas cependant les vrais ovules capables d'ètre fécondés, ce sont leurs cellules grand'mères et, comme dans la spermatogénèse, il faut encore deux divisions pour leur donner naissance. L'ovocyte de premier ordre se divise done en deux ovocytes de deuxième ordre et chacun de ceux-ci en deux cellules finales, ovules mîrs, qui sont les homologues des spermalides.

Mais, pendant celte phase de réluction, l'ovogénèse présente, avec la spermatogénèse, des différences sinon essentielles, du moins très remarquables. Les deux divisions des ovocytes de premier ordre ne sont pas égales. Des deux cellules filles, l'une, très grosse, continue la lignée de l'ouf, l'autre, très petite, est un produit de rebut que l'on appelle le premier globule polaire. L'une et l'autre sont cependant sœurs et représentent les ovocytes de deuxième ordre. Dans la division suivante, le gros ovocyle de deuxième ordre se divise de mème très inégalement en deux cellules scurs représentant les spermalides du màle, l'une grrosse, l'ovule mùr avec un nombre de chromo- 
somes réduit de moitié et l'autre toute petite, qui est le second globule polaire. Le premier globule polaire qui est, si l'on peut dire ainsi,

Fig. 37.

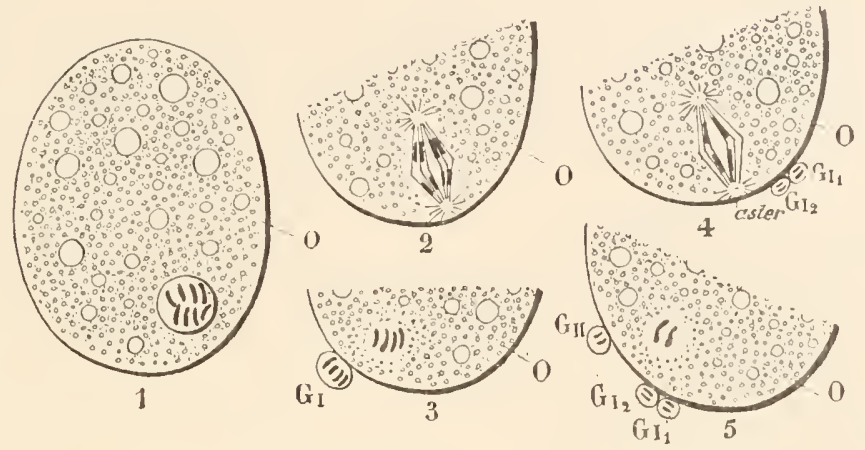

Émission des globules polaires (im. Weismann).

l'oncle du second se divise comme son frère, l'ovocytededeuxième ordre, en deux autres, et disparaît aussi laissant à sa place deux globules polaires, frères entre eux et cousins du second globule. En sorte que, finalement, on a un œuf bien développé et trois globules polaires, cellules naines, incapables d'évolution ultérieure.

Le cas décrit ici est le plus complet, mais le moins fréquent. Il s'observe chez les Mollusques, par exemple. Mais d'ordinaire, le premier globule polaire ne se divise pas et persiste à côté du second.

L'ouf, en ce moment entièrement mùr, est prèt à être fécondé; il n’y a pas ici cette phase distincte de maturation qui, dans la spermatogénèse, était nécessaire pour transformer les spermaFig. 38.

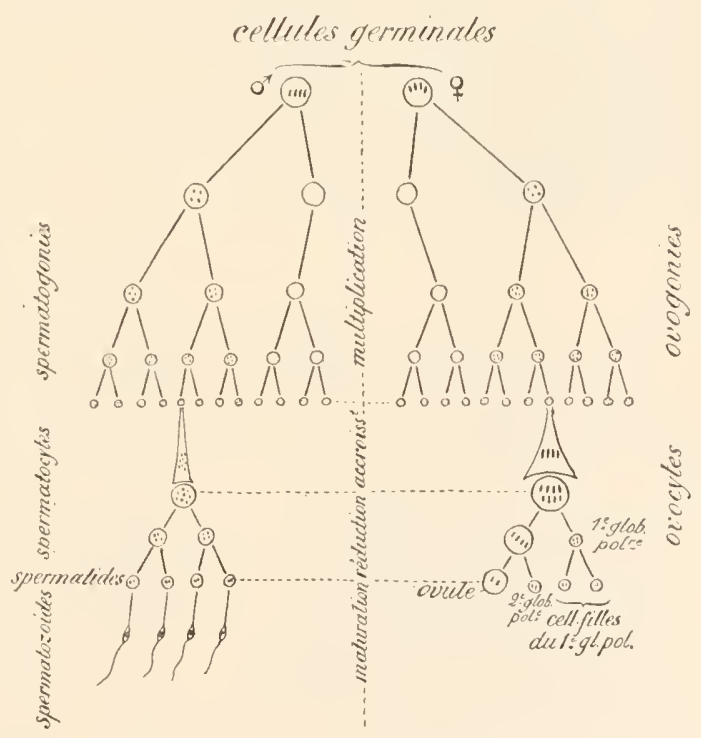

Maturation des produits sexuels (Sch. d'ap. MAUPAs). tides en spermatozö̈des. Le tableau ci-dessus (fig. 38) montre le parallélisme de ces deux évolutions (').

(1) Les phénomènes de l'ovogénèse ont été vérifiés chez un grand nombre d'animaux. 


\section{RÉduction Cirromatique}

Nous avons expliqué que le phénomène principal de la maturation était la réduction des chromosomes à un nombre moitié moindre, et nous arous vu que cette réduction était en effet obtenue. Mais nous n'arons pas dit comment elle l'était. Voici comment les choses se passent.

Les cytes de premier ordre (ovocytes ou spermatocytes de premier ordre) présentent un nombre de chromosomes qui semble moitié moindre que le nombre normal mais qui, en réalité, est deux fois plus grand, chacun de ces chromosomes étant formé de quatre autres, réunis en un petit groupe. Ainsi, dans Ascaris où il y a normalement quatre chıromosomes, les cyles de premier ordre n'en contiennent que deux, mais ces deux sont formés chacun de quatre réunis en un pelit groupe appelé groupe quaterne (Vierergruppe).

Chacun de ces groupes quaternes n'est autre chose qu'un chromosome déjà préparé pour deux divisions qui vont avoir à se faire très rapidement.

La première de ces divisions, celle qui donne naissance aux cytes de deuxième ordre (spermatocytes de deuxième ordre, ovocytes de deuxième ordre et premier globule polaire), va donc simplement dédoubler ces deux groupes quaternes, en deux groupes binaires, et la deuxième, celle qui donne naissance aux produits définitifs (spermatide, ovule mùr, deuxième globule polaire et produit de la division du premier globule), va dédoubler ces deux groupes binaires en deux chro-

Fig. 39.

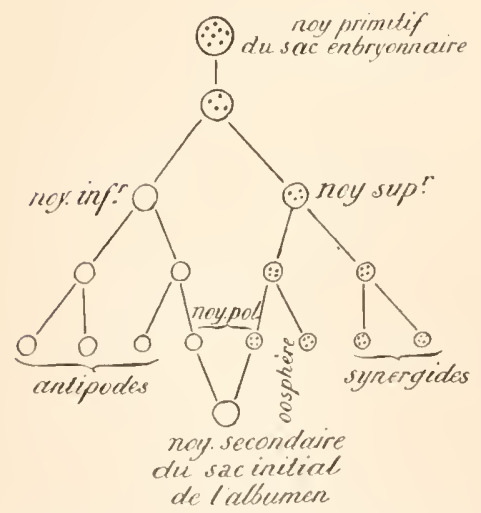

Féeondation chez les plantes (Sihéma d'ap. les descriptions de Guignard). mosomes simples; ainsi se trouvera effectuée la réduction des chromosomes à un nombre moitié moindre. Quant aux groupes quaternes, leur origine n'est pas connue, on les troure déjà dans les dernières gonies, mais on ne sait, ni quand, ni comment les chromosomes, normaux comme nombre et disposition des gonies précédentes, se sont disposés ainsi en groupes, moitié moins nombreux mais formés chacun

Ils sont semblables à eux-mêmes, partout où l'on a rencontré des globules polaires. Même chez les Infusoires, on a retrouvé quelque chose de tout à fait analogue. On n'a guère ètudié ì ce point de vue les ovules des plantes inférieures, mais, chez les Phanérogames (fig. 39), on sait, grace aux recherches de Guignard โ92|, qu'il existe des phẻnomènes tout à fait comparables. 
de quatre chromosomes élémentaires qui sont déjà ceux des éléments définitifs (").

Le schéma ci-contre (fig. 40) rend compte du phénomène.

Ainsi l'essentiel de la réduction ne se passe pas comme on le croyait pendant les divisions réductrices. Celles-ci ne font qu'achever une besogne qui s'est préparée, on ne sait trop à quel moment, pendant les nombreuses divisions qui domnent naissance aux dernières gonies. Cette circonstance rend bien plus difficile la solution des importantes questions théoriques qui se rattachent à la division réductrice car, s’il était déjà mal aisé de surveiller les deux divisions réductrices, il devient presque

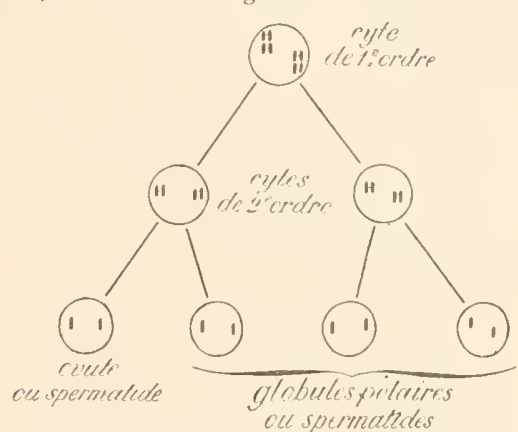

Réduction chromatique par les groupes quaternes (Sch.). impossible de saisir un phénomène qui se passe, on ne sait plus où. Ce mode de division réductrice n'est pas universel, et souvent il subit des modifications importantes, mais il parait bien être le plus typique, celui dont les autres sont sans doute dérivés $\left({ }^{2}\right)$.

Dans la parthénogénèse où, le plus souvent sinon toujours, le deuxième globule polaire ne s'élimine pas, la réduction de nombre des chromosomes n'a plus sa raison d'ètre puisqu'il n’y a pas fécondation; elle n'a pas lieu d'ailleurs, leur nombre élant d'abord une fois doublé, puis réduit une seule fois de moitié $\left({ }^{3}\right)$.

\section{MODIFICATIONS CYTOPLASMIQUES}

Les éléments sexuels mûr's ne diffèrent pas seulement par les chromosomes des cellules somatiques ou des gonies et des cytes qui leur ont donné naissance.

Dans le spermatozoïde (fig. 36), il n'y a, outre les chromosomes condensés en une masse compacte, qu'un centrosome et un peu de ce cytoplasma spécial et actif que Strasburger [92] a appelé linoplasma par

(1) La découverte des groupes quaternes, due à Boveri, est de date assez récente $[\mathrm{s} 7,88,90]$. On croyait auparavant que le cyte de deuxième ordre avait le nombre normal de chromosomes et que ceux-ci, sans se diviser longitudinalement, passaient par moitié dans les produits de la dernière division. C'est ainsi que les choses sont décrites dans les ouvrages remontant à quelques années.

(²) RückEnt [94́] l'a retrouvé mème chez les Vertébrés, mais assez fortement modifié, il est rrai, et surtout moins net.

$\left.{ }^{3}\right)$ Il est probable que l'unique globule ne produit aucune modification qualitative, Weishaxn [91] a montré qu'il pouvait cependant en être ainsi, mais son explication est hypothétique. 
opposition au trophoplasma nutritif et qui, en outre du centrosome, formerait la sphère attractive, le réseau filaire el les filaments du fuseau et des aster's.

Dans l'œuf (fig. 41), on trouve tous les éléments d'une cellule ordinaire; mais y sont-ils bien au complet et dans les proportions normales?

Nous verrons en étudiant la fécondation que, dans bien des cas et en particulier chez l'Ascaride, l'œuf mùr ne contient pas de centrosome en sorle que cet organe qui existait cerlainement dans les gonies a dù disparaître à un certain moment, mais on ne sait ni où, ni comment (').

D'autre par', les globules polaires n'entrainent avec leur's chromosomes qu'une quantité négligeable de cytoplasma, en sorle que, dans l'wuf mùr, celte substance devient fortement prédominante par rap'port à la substance nucléaire. On sait en outre que, le plus souvent, l'œuf se

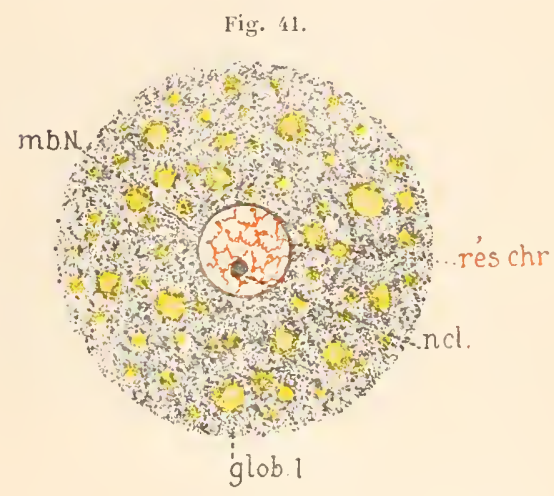

Euf mûr.

glob. 1., globule licithique;

mb. N., membrane nucléare;

nel., nuclèus; rés. chr., résean chromatique. charge de substances nutritives lécithiques (glob. l.), parfois en quantité énorme, qui diminuent encore la masse relative du noyau. En sorle que les deux éléments sexuels se caractérisent lorsqu'ils sont mùrs: $1^{\circ}$ par une parfaite similitude de constitution de leur noỵau; $2^{\circ}$ par une différence aussi grande que possible dans leurs parties cyloplasmiques. Le spermatozoïde est, en effel, absolument dépourvu de cytoplasma nutritif (trophoplasma de Strasburger) el d'éléments mutritifs lécilhiques, et bien muni an contraire de cytoplasma aclif (kinoplasma de Strasburger); l'auf', au contraire, est riche en éléments trophiques (lécilhe el trophoplasma) et pauvre en linoplasma.

C'est pour cela que te premier ne peut se nourrir el que le second ne peul se segnnenter. On roil, par là, d'avance, que le but de la fécondation sera de constituer par leur rémion une cellule complète apte à se segmenter et à vivre de ses propres ressources jusqu'à ce qu'elle ait formé les organes qui permettront à l'embryon de tirer sa nourriture du dehors.

(1) Gette absence de centrosome dans l'œuf tend à se généraliser. Nous n’osons cependant pas encore affirmer que cela soit général. (V. plus loin, p. đ33, à propos du quadrille de For.) 


\section{FÉCONDATION}

Il y a seulement une vingtaine d'années, la fécondation élait définie: la pénétration el la fusion de l'élément sexucl mâle dans l'élément sexuel femelle. Réduite à cela, la fécondation est connue chez un très grand nombre d'ètres vivants et elle est identique chez tous. Mais on a aujourd'hui pénétré plus avant dans l'ensemble du phénomène et trouvé nombre de faits nouveaux extrèmement importants. Nalheureusement ils ne sont connus que dans un petit nombre de cas et ne sont pas partout semblables à eux-mèmes. Aussi, pour laisser au texte principal sa netteté et sa sobriété, nous allons décrire un cas imaginaire très complet, renvoyant aux notes pour les applications, exemples, réserves et exceptions.

Lorsque l'wuf mùr est placé dans un liquide où nagent les spermatozoïdles mûrs, ceux-ci (sperm., fig. 42) s'approchent de lui, poussés par les ondulations de leur flagellum, et bientòt un ou plusieurs le rencontrent. Cette rencontre n'est pas le simple effet du hasard. Il y a une véritable attraction ì distance des éléments l'un par l'autre, mais le spermatozoïde seul en manifeste les effets, car la masse de l'œuf est trop considérable pour ètre déplacée.

Quand un spermatozoïde est arrivé assez près de la surface de l'œuf, l'attraction devient assez énergique pour déplacer, non pas l'œuf, mais une partie de son vitellus qui s'élève en un cône d'attraction à la surface de l'auf, juste en face du spermatozoïle qui est dirigé vers lui la tête en avant. Le cône s'allonge, la tète s'avance, les deux parties

Fig. 12.
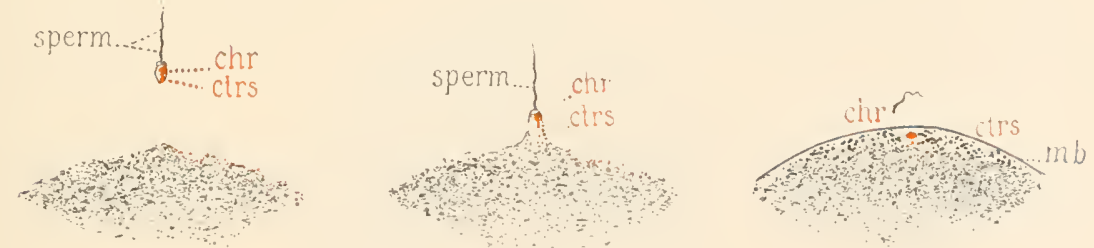

Pénétration du spermatozoide dans l'ouf (im. Fol).

chr., noyau de chromatine ou pronucléus mâle; etrs., centrosome ou spermocentre; mb., membrane vitelline; sperm., spermatozoïde.

s'accolent l'une à l'autre et le cône, rentrant dans le vitellus, entraîne le spermatozoïle avec lui. La queue se détache et n'entre pas dans l'oeuf ou reste à la surface et, en tout cas, paraîl ne jouer aucun ròle daus les phénomènes ultérieurs. La fécondation externe est accomplie. Aussitôt une mince membrane vitelline $(m b$.) se forme autour de l'œuf à partir du point où le spermatozö̈le a disparu, et oppose une barrière aux autres spermatozoïles. D'ailleurs, l'attraction sexuelle diminue peu 
à peu, et bientôt se disperse la foule de spermatozoïdes qui assiégeaient l'œuf quelque temps auparavant. Dès que le vitellus est refermé audessus d'elle (fig.43), la lète du spermatozoïle se divise en deux éléments essentiels, le centrosome (ctrs.) et le noyau de chromatine (chr.) que nous

Fig. 43.
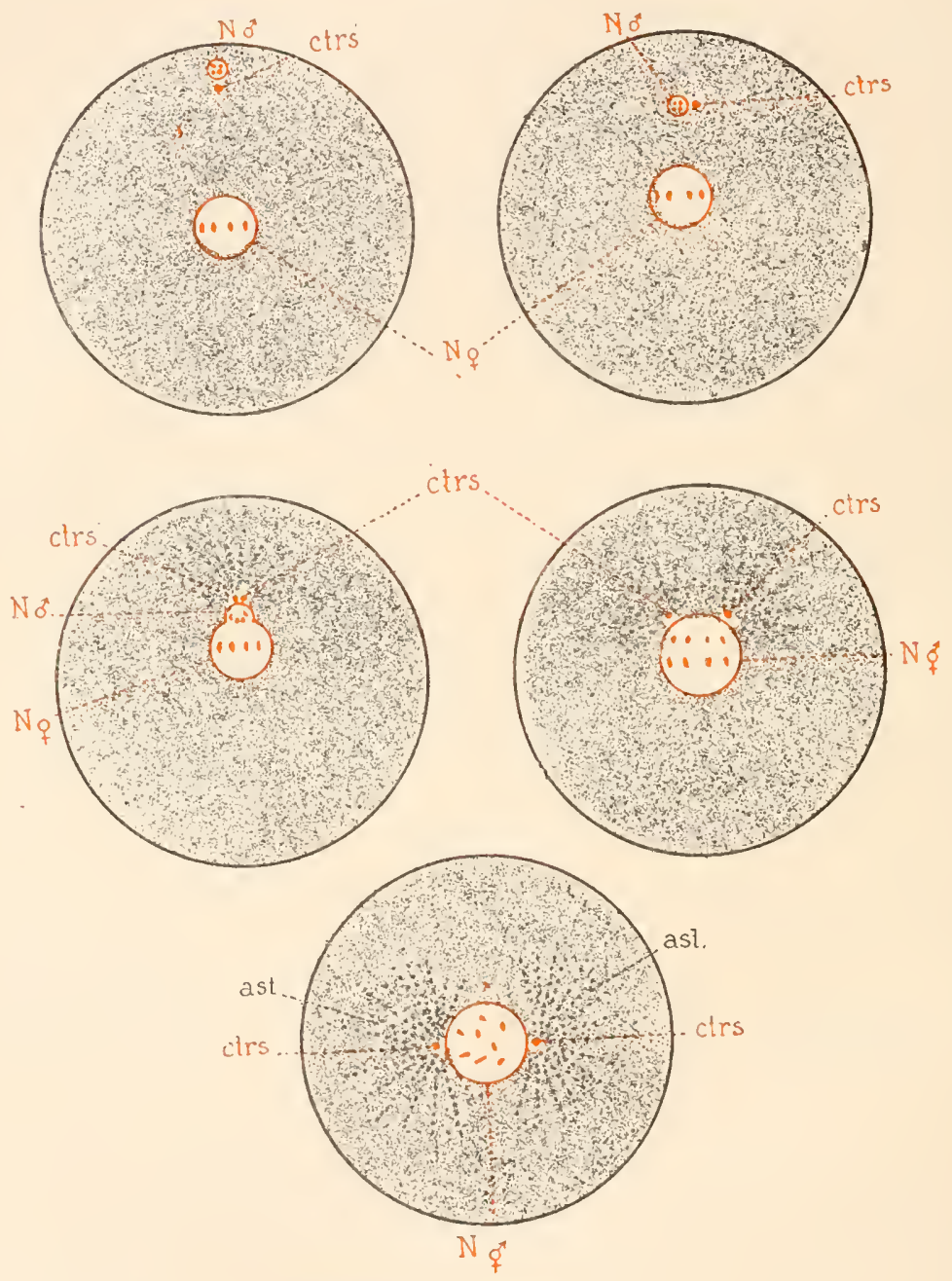

Fécondation sans ovocentre (Sch.).

appellerons, celui-ci promucléus mâle avec E. Van Bexenex [s3], celui-là spermocentre avec II. Fou [91], qui se dirigent l'un et l'autre vers le centre de l'ceuf, le premier en avant du secont. Là, au centre, se trouve le noyau de l'wuf ou pronucléns femelle ( $\boldsymbol{N}$. P). Les deux pronucléus se dirigent l'un vers l'antre, comme par l'effet d'une attraction réciproque, 
continuation de l'attraction sexuclle, et hientôt ils arrivent à se joindre, mais non loin du centre, car l'élément $\left(X . \sigma^{-1}\right)$ màle continue d'ètre plus actif, plus mobile et fait la majeure partie du chemin. La différence d'aspect entre les deux pronucléus est au début très grande. Celui de l'œuf est gros, clair, et montre ses chromosomes distincts, comme à la fin d'une division, qui vient en effet d'avoir lieu pour l'ćlimination du second globule polaire. Celui du spermatozö̈de, an contraire, est petit, opaque, à la manière d'une matière très condensée. Penlant ce court royage, il se gonfle, devient à peu près aussi gros que le pronucléus femelle, s'éclaircil et montre bientòt à son intérieur des chromosomes distincts qui sont ceux qu'il contenait à l'état de spermalide et s'étaient tassés et condensés pour occuper moins de place. Les chromosomes sont donc en nombre juste égal à celui qui se trouve dans le pronucléus femelle. Quand les deux noyaux se sont rencontrés, ils se fusionnent en un seul, constituant un novau unique, le noyau de segmentation. Ce dernier se place au centre de l'cuf. Il contient exactement deux fois plus de chromosomes que les noyaux sexuels. La fécondation a done effacé l'effet provisoire de la division réductrice. Pendant ce temps, le spermocentre (ctrs.) se divise en deux centrosomes qui se placent anx deux pôles dı noyau de segmentation et serviront à ses divisions ultérieures.

Cela montre: $I^{\circ}$ que le noyau de l'wuf fécondé est formé de la fusion des pronucléus, c’est-à-lire des noyaux màle et femelle, et que, par suite, les noyaux de toules les cellules de l'êlre futur seront formés de la mème façon; 2o que le centrosome de l'ouf fécondé, et par suite celui de toutes les cellules du futur animal, provient du spermocentre, c'est-à-dire du centrosome paternel (').

(1) Nous avons récrit ainsi les choses pour tenir compte, comme c'était notre devoir, des découvertes les plus récentes. Mais nous ne pouvons nous résigner à laisser de côté une série d'observations qui ont, les premières, il n'y a que peu d'années, jeté une vive lumiẻre sur les phénomènes de la fécondation et qui n'ont peut-être pas été aussi complètement renversées qu’on veut bien le dire.

Nous voulons parler de celles de II. For.

D'après ce savant (fig. 4'), l'cuf a un centrosome, l'ovocentre, tout aussi bien que le spermatozoïde. Le spermocentre et l'ovocentre sont situés, pendant la fécondlation, derrière les pronucléus dont ils dépendent et, après la fusion de ceux-ci, se trouvent placés en deux points diamétralement opposés du noyau de conjugaison et non loin de sa paroi. Là, ils se divisent de la même manière que dans la division cellulaire: leurs deux moitiés, glissant autour du noyau, se placent chacune à 90 degrés de leur position initiale; elles se rencontrent par conséquent et, en deux nouveaux points diamétralement opposés, se trouvent réunis un demi-spermocentre et un demi-ovocentre. Ces deux demi-centrosomes se fusionnent entre eux, comme ont fait les pronucléus et constituent les deux centrosomes de l'œuf fécondé, déjà en position pour effectuer la première division nucléaire qui va se faire presque aussitòt. Dans tous leurs mouvements, les centrosomes sont accompagnés d'un aster dont ils occupent le centre, tout comme dans la division cellulaire.

Cet ensemble de mouvements si admirablement combinés, si singulièrement symétriques a reçu de H. Fol [91] qui l'a découvert le nom expressif de quadrille des centres. Ce savant considère la part des centrosomes comme aussi essentielle que celle 
En somme, si l'on jette un coup d'œil général sur l'ensemble des phénomènes qui constituent la fócondation on voit que, chez deux individus semblables entre eux sauf le sexe, une cellule de l'organisme aple à la reproduction de l'espèce el destince à celte fonction s'est d'ahord rendue incapalle de la remplir en se dépouillant d'une partie des substances qui lui eussent été nécessaires pour cela et qu’elle possédait à l'origine. Chez le mâle, ce sont les sulıstances nutritives et le trophoplasma; chez la femelle, c'est le centrosome formé de kinoplasma; chez l'un et l'autre, c'est en outre une moitié des chromosomes. Ces deux cellules incomplètes se sont rendues ainsi complémentaires l'une de l'autre, chacune ayant garlé, accumulé en elle, ce qui manque à l'autre. En se fusionnant, elles forment donc une cellule complìte qui offre, en outre, celle particularité de rémnir en elle les substances de deux individus distincts.

des noyaux dans la fécondation et définit cette dernière : la fnsion de deux demi-
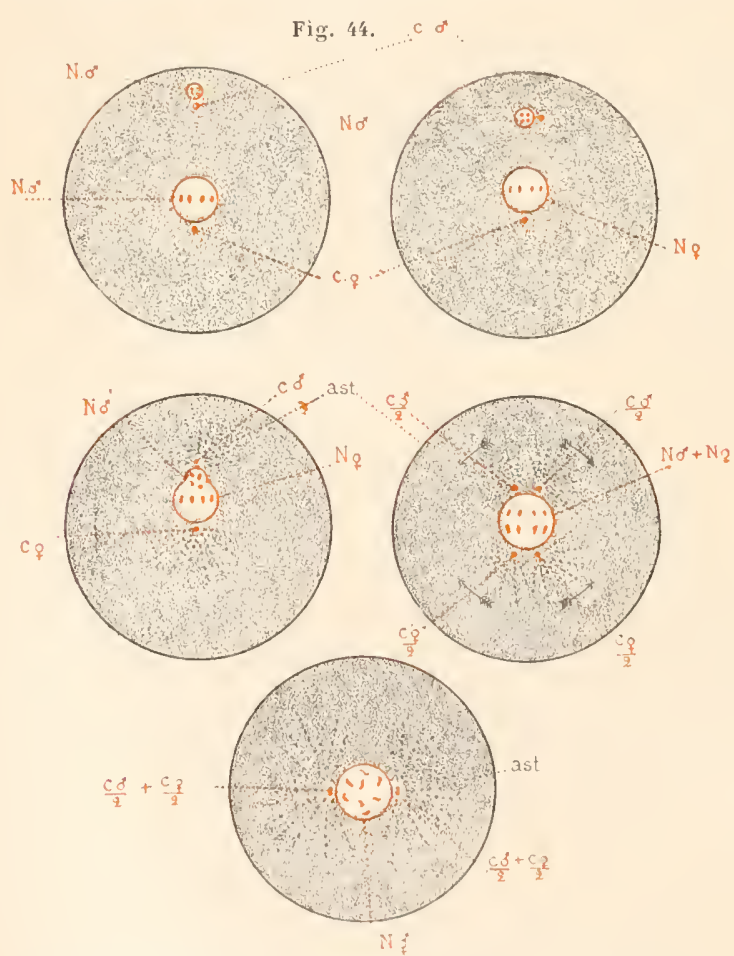
noyamx et de quatre demicentrosomes, prosenant d'sléments de sexe opposé, en un moyan et deux centrosomes formés, par parties égales, des smbstances des deux parents. Ces observations ont été faites sur des Echinodermes et, pendant quelques années, on a cru que les choses se passaient suivant deux modes : le mode d'Ascaris, sans ovocentre, et le mode des Echinodermes avec ovocentre ot quadrille des centres. On tendail seulement à considérer ce dernier comme un processus exceptionnel, ancestral peut-être et tendant à disparaître. Mais voilà que MATHEws [95] vient de faire voir que le prétendu ovocentre des Eehinodermes n'esl qu'un second spermocentre qui se trouve la seuloment dans le ras do dispermie, c'est-ì-dire de fécontation par deux spermatozoides, cas assez fréquent chez ces animaux. Mais peutètro ne faut-il pas trop se

hater de conclure sur des observalions encoro si récentes et de rejeter l'ovocentre et le quarlille avant que de nouvelles recherches aient tout à fail tranché la question. 


\section{THÉORIE DES GLOBULES POLAIRES}

Il est parfaitement établi queles globules polaires sont, au point de vue morphologique, des ovules abortifs et, au point de vue physiologique, des substances de rebut. Mais sur la question de savoir quelle est cette substance, les opinions les plus disparates ont été et sont encore admises (").

Tant que l'on n’a pas connu le détail des phénomènes de la fécondation, on a pu trouver très plausible l'opinion que la division réductrice sert à rendre le noyau de l'œuf moins prédominant par rapport à la masse du spermatozoïde. Mais aujourd'hui que l'on sail qu'il y a autant de choses essentielles, en qualité et en quantité, dans cette tête de spermatozoïde que dans la vésicule germinative, il faut chercher ailleurs une explication.

Strasburger [84] voit dans le rejet des globules une épuration de la substance nucléaire nécessaire pour lui permettre son évolution ultérieure. Il combat l'idée que se font Mlixot et Vax Bexeden sur la nature de cette épuration par d'excellents arguments, mais n'en fournit pas une meilleure.

Boverı [90], ayant remarqué dans l'wuf féconlé d'Ascaris megalocephala deux chromosomes de trop, les considère comme représentant ceux du premier globule incomplètement éliminés et, avant constaté qu'il ne troublent point le développement, conclut que la substance de ces chromosomes ne diffère en rien de celle des chromosomes conservés. C'est une conclusion illégitime, car ces chromosomes sont peut-être suffisamment éliminés lorsqu'ils sont rejetés du noyau et empèchés de se joindre au reste du corps nucléinien.

Depuis que l'on connait la constance de nombre des chromosomes, on s'accorde à reconnaître l'élimination d'une moitié d'entre eux comme indispensable. C'est sùrement là une des fonctions de la division réductrice. Mais elle ne s'applique qu'au deuxième globule, et on peut s'étonner que la réduction de nombre ne se fasse pas simplement par segmentation du filament nucléaire en un nombre moitié moindre de fragments, et la réduction de masse par une diminution de l'accroissement nutritif.

O. Iertwig [90], qui est aussi d'avis que la substance éliminée n'a point quelque qualité spéciale, a trouvé néanmoins le moyen d'expliquer d’une manière fort ingénieuse la nécessité de son expulsion. D’après lui, l'ovocyte de premier ordre se divise deux fois pour donner quatre ovules, mais de ces quatre ovules un seul garde tout le cytoplasma; dès lors les globules polaires sont des ovules sacrifiés, des frères cadets

(1) Il n'y a pas à compter comme opinion digne d'être discutée celle que définit la dénomination de globules directeurs donnée autrefois aux globules polaires. Il est vrai que le premier plan de segmentation passe par le point où ils confinent à l'œuf, mais c'est uniquement parce que ce plan, en passant par là, se trouve perpendiculaire au plan de la division précédente. Les globules polaires n'ont aucune action directrice sur les segmentations de l'œuf fécondé. 
déshérités au profit d'un seul ainé qui a gardé tout l'héritage de cytoplasma. L'émission des grlobules servirait, non à épurer le noyau de l'ovule, mais à enrichir son cytoplasma. I.es faits, en somme, ponrraient se résumer ainsi: chez le màle, les divisions qui s’intercalent entre le spermatocyte de premier ordre ef les produits mùrs n’a pour effet que de réduire dàns ces produits le nombre de chromosomes à la moitié, et la quantité de chromatine au quart, de la valeur qu’ils avaient chez le premier; chez la femelle, les divisions homologues ont, d'une part ce mème effet, d'autre part colui de porter au quadruple la quantité de cytoplasma par rapport à ce qu'elle aurait été si la division de l'ovocyle de premier ordre avait donné naissance ì quatre ovules de mème valeur. Nais tandis que, chez le màle, la chromatine du spermatocyte de premier ordre se divise en quatre portions également utilisables, chez la femelle, trois de ces portions sont purement rejetées pour laisser à la quatrième tout le cytoplasma qui aurail dù les accompagner.

Il doit y avoir du vrai dans cette remarqualble théorie, mais elle n'explique pas tout. Si les chromosomes avaient tous la mème valeur, il n'y aurait aucune raison pour qu'une division longitudinale si précise attribuât à chaque cellule fille, exactement une moitié lle chacun d'eux. Ils pourraient se rendre les uns d'un còté, les autres de l'autre et les deux groupes destinés aux deux cellules filles pourraient être composés de n’importe quelles parties, pourvu qu’ils fussent égaux en nombre. La division longiludinale n'a sa raison d'ètre que si les chromosomes ne sont pas identiques entre eux, et sil en est ainsi les chromosomes rejetés représentent autre chose que ceux qui sont conservés.

La question est de savoir ce qu’ils représentent.

Une des explications les plus anciennes et les plus célèbres en mème temps est celle de Mıхот [77] à laquelle Batfour el Vax Brneden ont aussi attaché leurs noms. Elle peut se résumer ainsi : l'ơuf fécondé est liermaphrodite; comme il répartit également son plasma nucléaire entre les produits de sa division, les deux premiers blastomères le sont aussi ; ceux-ci se comportent de mème et ainsi de suite tant qu'il se passe des divisions dans le corps de l'animal. Toute cellule du corps est done, par essence, hermaphroulite et l'ouf non fécondé n'y fail pas exception. Il doit, à sa maturité, pour derenir fécondable, développer en lui une polarité femelle et, pour cela, éliminer sa partie môle. La fécondation lui rend son hermaphroditisme un instant perdu. Mais celle théorie n'est pas soulenable. Strasburger [84], Köluher [85], Ilalbez [86], Weisuaxn [87] ont fait remarquer avec raison que l'wuf n'élimine pas la substance mâle qu'il tient de son père, puisque le produit pent assumer des caractères des ancètres mâles de la femelle. Si cette théorie était vraie un enfant ne pourrait ressembler an père de sa mère, ni à aucun des ancêtres de son père, ce qui est évidemment faux.

Il serait trop long d'exposer el de discuter ici toutes les opinions qui ont été émises sur cette importante question. Nous ne pouvons que 
renvoyer les personnes que cela intéresse à l'ouvrage de l'un des auteurs où elles sont exposées tout au long $\left({ }^{*}\right)$.

Nous terminerons done ici ce chapitre en émettant une hypothèse qui nous semble, malgré ses allures paradoxales, mieux rendre comple des faits que celles qui ont été proposées jusqu’ici.

Elle n'est pas d'ailleurs nouvelle en tous points, mais elle combine diverses idées d'une manière à laquelle on n'avait point songé.

Les organismes les plus simples sont susceptilhtes de sé reproduire indéfniment par division; ils n'ont besoin ni de conjugaison ni de réduction chromatique. Mais chez ceux où l'organisation et les phénomènes chimiques de la vie sont plus compliqués, nous constatons qu’il en est autrement et que la fusion périodique de deux individus entre eux est une condition indispensable de la survie indéfinie de l'espèce. Celte fusion comporte deux phénomènes, la réduction chromatique et la fécondation (ou coujugaison).

On considère en général cette dernière comme étant la partie essentielle du phénomène et la première comme n'étant qu'un pliénomène accessoire destiné à rendre l'autre possible. Ce serait le mélange des substances des deux conjoints qui rendrait possible la réalisation d'une nouvelle série de divisions agames; et la réduction chromatique ne serait destinée qu’à rendre possible ce mélange, qui constitue la fécondation, en mettant les gamètes en élat de se fusionner.

D'après notre hypothèse c'est l'inverse qui est vrai.

Le phénomine essentiel est la réduction chromatique, et la fécondation est une addition avantageuse mais non indispensable.

Les organismes les plus simples ont un cycle métabolique fermé, c'est-à-dire qu'après une durée de vie quelconque, ils se retrouvent identiques à ce qu’ils étaient auparavant, les substances éliminées étant parfaitement équivalentes à celles qu'ils ont incorporées. Aussi n'y a-t-il aucune raison pour que la division qui leur a été possible une fois ne le soit pas indéfiniment. Mais, à mesure que l'organisation se complique, le cycle nutritif se déforme, les egesta ne correspondent plus rigoureusement aux ingesta et il s'accumule lentement dans l'organisme des substances qui le détériorent, altèrent toutes les fonctions et en particulier la faculté de division. La cellule est condamnée à mourir.

Les cellules de tissus n'ont aucun moyen de se sauver, mais les ètres unicellulaires ou plutôt les homoplastides et les cellules reproductrices des liétéroplastides savent rejeter en bloc, en une seule opération, ces substances, s'épurer et rederenir ainsi, d'emblée, capables d'une nouvelle série de divisions agames.

Nous concevons toute une période du développement phylogénétique des êtres, pendant laquelle la réduction, il faudrait dire l’épu-

* Voyez: Y. Delage : La structure du protoplasma et les théories sur l'hérédité et les grands problemes de la biologie générale. Grand in-8o, XVI-878 p. avec fig. Paris, Reinwald et Cie, 1895. 
ration chromatique, était le seul phénomène périodique qui coupât la série des générations agames. Il doit y avoir encore des ètres représentant ce stade. Labré a observé chez les Coccidies une réduction chromatique non suivie de conjugaison qui suffit à assurer la perpétuité de leur évolution. Il y en a d'autres, sans doute, que l'on découvirira avec le temps. Celte épuralion chromatique est représentée chez les êtres supérieurs par le premier globule polaire. La reproduction parthénogénétique est un mode primitif dans lequel tout se borne ì cette épuration. Si l'on pourait empêcher la sortie du deuxième globule, tous les ètres se développeraient parthénogénéliquement, comme il arrive chez ces Crustacés où le deuxième globule, après s'ètre formé, vient se refondre dans le noyau de l'ieuf.

Mais, chez la plupart d'entre eux, la fécondation s'est ajoutée au fait essentiel de l'épuration chromalique, comme épiphénomène apportant avec lui l'avantage d'un nucléoplasma plus varié, par suite plus plastique, apte à se plier à des conditions érolutives plus diverses. C'est un peu, ainsi qu'on l'a dit (à supposer que les aptitudes acquises soient héréditaires), comme un homme dont tous les ancètres auraient exereé la même profession, comparé à un autre dont les ancètres auraient élé artisans dans divers métiers. Dans la lutte pour l'existence, le premier n'aurait qu'une corde à son are, le seconıl en aurait plusieurs, et pourrait trouver à gagner sa vie là où l'autre mourrait de faim.

Ainsi, il y aurait à distinguer parmi ces phénomènes : $1^{\circ}$ un acte essentiel et parfois unique, l'épuration chromatique (réduction chromatique totale de certains organismes inférieurs, globule polaire unique des formes parthénogénétiques, premier globule des êtres à reproduction sexuelle amphimixique); $2^{\circ}$ un acte secondaire, l'émission du deuxième glolule polaire, réduisant de moitié la quantité de chromatine et le nombre des chromosomes el arrêtant, par cela mème, toute possibilité de développement ultérieur, à moins que la demi-cellule ainsi affamée ne trouve à s'unir à une autre et à redevenir cellule complète, capahle de se diviser de nouveau, et joignant à l'avantage de son épuration celui d'une constitution nucléoplasmatique plus variée (").

(1) D’aillcurs la succession philogénétique des plıénoınèncs a pu n'être pas toujours la même, et il a pu arriver yue la conjugaison s'établisse chez certains êtres assez simples pour n'avoir pas encore éprouvé le besoin d'épuration chromatique; e'est alors que l'on observerait la conjugaison ou même la fécondation sans réduction chromatique, comme chez les Spirogyres, les Volvoces et divers autres. 


\section{DEUTIEYUE PARTIE}

\section{LES PROTOZOAIRES}

C'est seulement lorsque nous aurons étudié les Protozoaires qu'il nous sera possible de les définir et de dire en quoi ils diffèrent des végétaux d'une part et des Métazoaires de l'autre. Disons seulement, pour fixer les idées du lecteur, que nous allons étudier sous ce nom les êtres qui sont unicellulaires ou formés de cellules plus ou moins nombreuses mais similaires et non disposées en feuillets emboìtés ni différenciées en tissus, et qui, pendant leur période d’accroissement, sont doués de mobilité.

Les Protozoaires constituent un embranchement et se divisent en quatre grandes classes: les Rinzopodes, les Sponozodires, les Fiageliés et les INATSORES.

\section{I ${ }^{\text {re }}$ Chasse \\ RHIZOPODES. - RIIIZOPODIA \\ [RIIZOPODA (DUIARDIN)]}

Les Rhizopores sont des Protozoaires formés d'une cellule nue et munie, en fait d’appendices locomoteurs et préhenseurs, de prolongements non permanents appelés pseudopodes.

\section{TYPE MORPHOLOGIQUE}

(FIG. 45)

L'ètre qui résume en lui les caractères généraux des Rhizopodes est. au sens le plus large de ce mot, l'amibe: non pas le genre Amceba, mais l’amobien en général, forme idéale, que nous appellerons, pour abréger amile, au féminin, sans capitale et sans ilalique pour le distinguer du genre Amibe (Amœba) pris, comme d'ordinaire, au masculin.

C'est une simple cellule. A ce titre, il possède les parties habiluelles de la cellule: le cytoplasma et le noyau, avec leur structure essentielle et leurs propriétés ordinaires. Avec cela sans rien de plus, la cellule de tissu d'un Nétazoaire peut vivre, parce qu’une partic au moins de ses 
fonctions lui est facilitée par les autres cellules associées à elle pour former l'organisme. Ici, il n'en est plus de mème. La cellule doit vivre seule, isolée; elle doit par conséquent se suffire à clle-même pour toutes

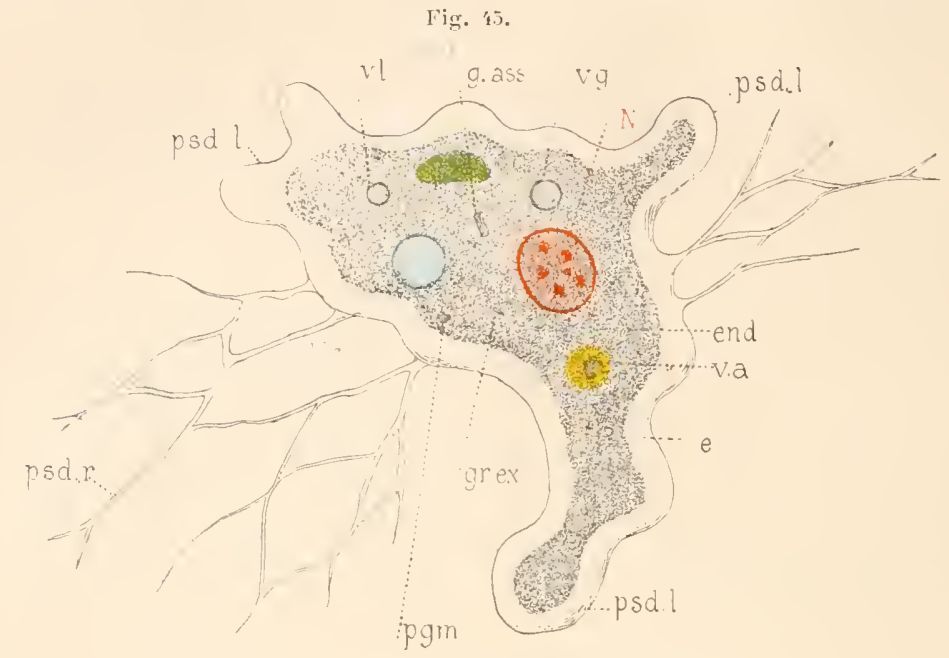

Rhizopode (Type morphologique). (Sch.).

chrp., chromoplaste; end., endoplasme; e., ectoplasme; gr. ex., grain d'exerétion ; g. ass., grains d'assimilation; $\mathbf{x}$., noyan; psd. 1., psendopodes lubés ; psd. r., psendopodes réticulés; pgnu., pigment; v. a., vacuole alimentaire; v. g., vacuole à gaz; v. 1., vacuole à liquide; v. ‘., vésicule pulsatile.

les fonctions essentielles d'assimilation, d'excrétion, de reproduction, de défense contre les intempéries, les agressions, etc.... Aussi doit-elle, et cette remarque peut s'appliquer à l'ensemble des Protozoaires, sans exceller en rien, être apte à tout. Celte nécessité va nous donner la raison des particularités de sa structure à la fois rudimentaire et compliquée, et de sa physiologie à la fois simple et complète.

\section{Structure.}

Noyau. - Le noyau $(N$.) est loin d'avoir, on du moins de laisser voir, la structure compliquée que nous lui avons trouvée dans la cellule en général. Il a une membrane et renferme un suc cellulaire; mais, en fait de parties solides intranucléaires, il ne montre guère que quelques masses chromatiques dans lesquelles on ne peut discerner en général nii chromosomes ni nucléoles.

Le cytoplasme ne renferme pas de centrosome. Il est différencié en deux parties, une masse centrale, l'endoplasme (end.), et une couche périphérique, l'ectoplasme (e.).

Endoplasme. - L'endoplasme a la structure essentielle de tout cytoplasma. On y reconnait une substance fonlamentale hyaline, chargée de minuscules granulations élémentaires et, le plus souvent, parsemée 
de vacuoles. Tout ce que l'on y trouve en outre est logé en lui sans faire partie de sa structure. Sans parler du noyau, il contient: la vésicule pulsatile ( $\boldsymbol{V} . c_{\text {. }}$ ), vacuole permanente et contractile, dont nous aurons à parler surtout à propos de sa physiologie; des vacuoles alimentaires (v.a.), contenant des particules nutritives flottant dans une goutlelette de liquide; des vacuoles fécales qui sont les mèmes que les précédentes après l'action des sucs digestifs; des vacuoles simples, ne contenant que du liquide $(v . l$. ou des gaz (v.g.); des grains d'assimilation (g.ass.) yui sont des états sous lesquels se condense la substance alimentaire avant d'ètre utilisée et qui réunissent sous une mème rulrique des particules de nature très diver'ses, grains d'amidon, de paramylon, gouttelettes huileuses, ete.; des grains d'excrétion (gr.ex.) qui, à l'inverse des précédents, sont des substances usées destinées à ètre éliminées et qui ont revètu provisoirement l'état solide; souvent des pigments (pgm.) qui doivent avoir une origine analogue; parfois enfin des corps de nature spéciale, comme des chromoplastes (chrp.), petites masses protoplasmiques chargées de chlorophylle, de diatomine ou de substances analogues; et bien d'autres choses encore, à titre d'éléments accillentels, fixes peut-ètre dans chaque espèce en particulier, mais accidentels en ce qui concerne le type général. Tout cela se déplace dans l'endoplasme très mobile, au hasard des déformations incessantes de l'animal.

Ectoplasme. - L'ectoplasme a essentiellement la mème constitution que l'endoplasme, mais sa structure physique est un peu plus ferme; il est hyalin, homogène, ne contenant aucune des inclusions qui chargent l'endoplasme, et prend pour lui les fonctions de la vie de relation, proteclion du corps, mouvements, capture des aliments, etc. Son ròle protecteur, extrèmement sommaire, consiste, en temps ordinaire, à maintenir l'endoplasme trop diffluent. Il a mème parfois pour cela, mais pas toujours, sa couche superficielle un peu plus ferme encore que le reste. Pour la capture des aliments et les mouvements, il forme l'organe caractéristique de la classe: le pseudopode.

Pseudopodes. - Les pseudopodes sont des expansions protoplasmiques qui s'étendent, prennent les formes les plus diverses et peuvent à chaque instant rentrer dans le corps sans laisser aucune trace de leur existence, tandis que d'autres se forment ailleurs pour disparaìtre à leur tour un peu plus tard et ètre remplacés par d'autres encore.

Malgré leur diversité extrème, on en peut distinguer deux sortes. Les uns sont gros, obtus, peu ou point ramifiés, semblables à des lobes saillants, d'où leur nom de pseudopodes lobés (psd.l.). Leur caractère essentiel est de ne pas se souder entre eux quand ils arrivent à se rencontrer, et de rentrer toujours dans le corps au point mème où ils ont pris naissance. Peut-ètre sont-ils formés par l'ectoplasme, mais comme celui-ci conserve à peu près sur eux la mème épaisseur que sur le reste du corps, il en résulte que, pour peu qu’ils soient gros, l'endoplasme pénètre à leur intérieur. Les autres, au contraire, sunt plus 
minces, très ramifiés et, lorsqu'elles se rencontrent, leurs branches se soudent volonlier's entre elles. Il en résulle qu'elles arrivent à former un réseau, d'où leur nom de pseudopodes réticulés (psd.r.). A chaque instant, dans ce réseau, des mailles se coupent, d'autres se forment, en sortc 'jue le cytoplasma sorti du corps en un point par un pseudopode peut fort bien rentrer dans le corps par un autre point. Il y a d'ailleurs entre les deux sorles de nombreux intermédiaires, les uns, bien que longrs et filiformes s'agglutinent à peine, d'autres, bien que lout à fait réticulés, sont aussi larges à leur base que des lobés.

\section{Physiologie.}

Mouvements. - L'animal se déplace par le moyen de ses pseudopodes d'une manière très singulière. Il émet un ou plusieurs de ces prolongements vers le point où il veut aller, puis, au lieu de les faire rentrer dans son corps, fait l'inverse pour ainsi dire, faisant lluer sa substance vers eux, en sorle que, peu à peu, tout le corps se trouve transporté au point où d'abord il n'y avait qu'une faible partie de sa masse. Le mouvement continue ainsi et, de la sorte, l’animal se déplace, mais on le conçoil, d'une manière très paresseuse.

Alimentation. - L'ètre n'a point de bouche, mais tout point de son corps peut en faire fonction. Qu'une parcelle nutritive vienne à rencontrer la paroi du corps en un point quelconque, l'ecloplasme excité par son contact s'élève tout aulour d'elle, l'enserre et finit par l'englober en se refermant au-dessus d'elle, emprisonnant en mème temps une petite quantité de l'eau ambiante. La parcelle alimentaire se trouve ainsi, d'emblée, contenue dans une gouttelette liquide qui constitue une vacuole alimentaire. Mais l'ordinaire, ce sont surtout les pseudopodes, principalement les réticulés, qui servent à la capture des aliments. Rien ne leur est plus facile puisqu'à clıaque instant ils se séparent et s'aggrlutinent dans de noureaux points. Il leur suffil de s'écarter en face de la particule à saisir et de se refermer derrière elle pour l'englober.

Digestion. - La vacuole alimentaire dont nous avons vu le mode de formation se déplace dans l'endoplasme selon les déformations que subit le corps dans ses mouvements et est promenée ainsi de tous côtés. Le liquide qui entoure la particule alimentaire est d'abord de l'eau pure, mais des échanges osmotiques ont lieu avec celui qui imbibe l'endoplasme, et bientôt il devient acide. La présence de ferments n’a pu y c̀lre décélée directement, mais elle est démontrée par le résultat physiologique qui est la dissolution de la particule ou du moins de ses parties alibiles. Le liquide digestif se trouve ainsi transformé en une sorte de chyle qui repasse en sens inverse dans l'endoplasme, el le résidu inutilisable est expulsé par une série de phénomènes exactement inverses de ceux de la capture. Il n'y 
a aucune place prédestinée à cette issue : tout point du corps peut servir d'anus aussi bien que de houche. L'animal ne paraît guère en état de faire un choix entre ses aliments, et souvent il absorbe des particules qu'il doit rejeter tout entières sans en avoir tiré aucun profit.

Respiration et excrétion. - La vésicule pulsatile est une vacuole permanente, sans parois propres, située dans l'endoplasme et qui, rythmiquement, se contracte, disparait un moment, puis reparait à la mème place. Nous verrons plus tard que, chez d'autres Protozoaires plus élevés en organisation, les Ciliés et mème les Flagellés, la vésicule communique avec le dehor's, au moins au moment où elle se contracte, et expulse le liquide qu'elle contient. Ce liquide a été introduit daus l'organisme, soit avec les aliments, soit par diffusion par toute la surface et, en traversant le corps, il a pu céler au cytoplasme son oxygène et se charger de l'acide carbonique et des produits le désassimilation solubles de celui-ci. Il pourvoit donc aux fonctions respiratoire et excrétrice à la fois. Chez les Rhizoporles, il est extrêmement probable qu'il en est de mème, mais la chose est beaucoup moins nelte, et bien des observateurs dignes de foi assurent que le lìuide chassé par la systole se répand dans la couche superficielle du corps, pour produire une sorte decirculation plutôt que pour exercer les fonctions indipuées plus haut. On ne voit pas bien en quoi cette circulation serait nécessaire, mais il faut bien admettre aussi que les autres fonctions attribuées à la vésicule peuvent s'accomplir sans elle par des échanges osmotiques s'étendant à toute la surface du corjs, car beaucoup de Rhizopodes sont alssolument privés de cet organe (").

En somme, la question réclame de nouvelles recherches.

Association, colonies. - Cette facilité du protoplasma des Rhizopodes à se souder à lui-mème se manileste non seulement entre les piseudopodes d'un même individu, mais aussi entre ceux d’individus voisins. Le plus souvent, deux Rhizopodes de mème esjòce qui se rencontrent passent l'un contre l'autre, puis s'écartent, tout comme d'un autre objet quelconque. Mais d'autres fois (cela dépend des espòces et de l'état des individus) on les roit se souder et rester unis. L'union peut se limiter aux pseudopodes ou aller jusqu'à la fusion complìte des cytoplasmes. Ce n'est pas là d'ailleur's un acte sexuel, car les novaux ne se fusionnent pas, le nombre des individus ainsi réunis est variable et parfois très considérable, et enfin il n'en résulte aucun phénomène de reproduction. C'est une simple association coloniale, végétative, qui a pour but la défense ou l'attaque, l'animal pouvant ainsi, gràce à son volume, échapper à des ennemis trop petits, ou englober des proies plus volumineuses. Le plus souvent, d'ailleurs, ces associations ne sont que temporaires, mais quand la fusion a été complète, il est presque certain que les indi-

(1) Il est possible que la vésicule soit nécessaire seulement chez ceux oủ les produits excrémentitiels se trouvent être très peu solubles. 
vidus ne se reconstituent pas avec leur protoplasma primilif, et se forment d'une masse équivalente du mélange.

Enkystement. - Dans des condilions encore mal déterminées, l'animal se met momentanément à l'abri des influences du monde extérieur', en rélractant ses pseudopodes, s'arrondissant et sécrétant autour de lui une capsule résistanle et peu perméable sous laquelle il n'a plus rien à craindre. C'est une phase de vie presque latente. Il en ressort en dissolvant ou rompant son kyste et reprend sa vie ordinaire. Mais c'est là un phénomène rare, relalivement à sa fréquence chez les autres Prolozoaires.

Reproduction. - Le mode essentiel de reproduction de notre Rhizopode est la division simple ì l'état libre. Le noyau s'allonge, prend la forme en biscuit, puis se divise; le cytoplasma s'étrangle puis se divise, lui aussi, en deux portions qui se séparent, emportant chacune un noyau. L'animal n’a pas même jour' cela rentré ses pseudopodes ni changé rien à ses allures, et les deux individus filles ont d'emblée les caraclères de leur parent. Parfois, cette division se fait suivant le procédé classique de la mitose ou selon celui de l'amilose. Mais le plus souvent, on observe une sorte de mitose rudimentaire, incomplète, sans centrosome, sans anses jumelles bien netles, avec une vague indication de fuseau. Bien que les études les plus récentes aient montré des karyokinèses nettes dans certains cas où on n'avait vu auparavant qu'une division directe, il semble bien probable que la mitose n'est pas générale el que, chez ces ètres inférieurs, elle s'estlentement établie par un perfectionnement progressif dont ils nous montrent encore quelques phases (').

Ces divers caractères de notre type morphologique ne sont, bien entendu, pas du tout absolus, et nous allons, en entrant dans le détail de notre étude, les voir subir des restrictions et des extensions assez considérables. La distinction entre l'endoplasme et l'ectoplasme est fréquemment nulle. Les pseudopodes sont ou lobés ou réliculés et non les uns

(1) Dans quelques eas cependant, mais fort rares, le phénomène se eomplique, soit par le fait d'un enkystement, soit par celui d'une eonjugaison. Voiei en quelques mots ee qui se passe dans ees deux eas.

Division sous un kyste. - Parfois, c'est au réveil d'un enkystement proteeteur que l'animal se divise. Il ne s'était pas enkysté pour ce but, mais il arrive pendant la vie ralentie sous son kyste à eet état de maturité qui précède la division et il utilise son état d'enkystement pour se multiplier sous cet abri. Mais le plus sourent, il s'enkyste spécialement pour se diviser. Ln tout eas, il est rare qu'alors il se divise seulement en deux. Il se segmente, par division répétée, en un nombre plus ou moins considérable de petits fragments qui eonstituent des sortes de spores et se transforment, après l'éelosion, chacun en un individu nouveau, simplement en poussant des pseudopodes et en grandissant.

Conjugaison. - Plus rare encore est la eonjugaison. Ce phénomène eonsiste en un rapriroehement de deux individus qui échangent une partie de leurs substanees ou se fusionnent eomplètement. Mais e'est à peine si, elıez les Rhizopodes, on a une ou deux observations d'échanges vraiment sexuels entre deux individus soudés. 
lobés, les autres réticulés sur le même individu, et leur irrégularité ne dépasse pas, dans chaque espèce, une limite qui permet à celle-ci de conserver un certain faciès presque aussi caractéristique que celui des ćtres à forme fixe. La vésicule pulsatile est fréquemment absente, parfois multiple. L’animal sécrète souvent des capsules ou des coquilles de forme parfois extrêmement compliquée. Enfin le noyau lui-mème n'est pas constant et les ètres qui en manquent sont réduits à de simples petites masses de protoplasma.

Toutes ces variations seront étudiées à leur place, au fur rt à mesure que nous les rencontrerons (').

(1) La question des Monères. - Nous rlevons cependant ici dire quelques mots d'une fuestion sur laquelle les avis des naturalistes sont encore trés partagés. Ciest celle des Mlonères.

On trouve, chez les Protozoaires, principalement chez les Rhizopodes, des formes dépourvues de noyau. Ce ne sont pas de vraies cellules, mais des cytodes. C'estlà un fait d'importance capilale au point de vue de la physiologie de la cellule et de sa phylogénése.

Iläclies, qui a surtont étudié ces cytodes et a découvert une bonne partie des Nonéres, a réuni ces dernières en un groupe qu'il a placé au degré inférieur de l'échelle des êtres vivants. Ilais, comme les observations d'Iläckel et des antres parrains des Monères remontent á une date assez reculée oú la technique microscopique elail encore fort imparfaite, il est arrivé que des observations ultérieures ont montré un noyau chez beaucoup de ces prétendus cylodes, les Famprefles, les Protamibes, et d'autres cheore, $y$ compris mème les liactéries, et l'on s'est demandé si tous reux oú on n'en a pas encore trouvé n’en aurdient pas un également, mais plus difficile encore a deceler. La chose n'est pas probable et justu'à preuve du contraire, on II a pas le droil de se refuser á adnettre les Honères. L'existence de res èlıes est d'aillenrs très malurelle, presque certaine mène " priori, l'ètre cellulaire complet ayant presque nécessairement été précédé de formes phylogénétipues d’un degrè inféricur d'organtusation. Mais ule lá à réunir les Monères, comme le vondrait Iläcliel, en une classe, il y a loin; car il n’est pas prouvé que les divers groupes de Protistes $\left(^{*}\right)$ descendent les uns des autres, et plusieurs ont des formes monéres á leur base, en sorte que éest violer les affinilés zoologicpues que d'aller prendre les Honères partout où il s'en trouve el le les réunir en un groupe unique. Nous laisserons done les Monères oi elles sont, parmi les Foraminiféres, les Myxomycétes, les l'rotéonyxés, etc., les considérant simplement comme des formes inférieures de cliacun de ces groupes et nous contentant de les sigualer au passage comme dépourvues de noyau.

Voici la liste tes Monères avec leur place lans la classification adoptée dans cet ouvrage.

Protogenes: l'rotéomyxé acystosporé.

Cymmopluys: -

Iyxodiclyum: -

sichizogenes: -

balleglius :

Protobathybius: -

Areluerince :

Vampyrella Gomphonematis: Protéomyxe

azoosporé (").

Protamalor : Amobien nu.
Gloïdinm : Amœbien nu.

Mycetomyxa : -

Chromatella: $\quad$ -

Gringa :

Lieberkühuia I'agneri (d'après Verworn': Foraminifère imperforè.

Monobia : Iléliozoaire

sans que les obser-
vateurs nient for-

Hieckelina : $\quad-\quad \mid \begin{aligned} & \text { mellement sa pré- } \\ & \text { sence. }\end{aligned}$

(*) HïKE appelle ainsi l'ensemble des formes inférieures sans noyau, qu'il réunit, animanx et végrétaux, dans un groupe dont il fait un RE(iNE : 
Nous diviserons les Rhizopodes en six sous-classes:

Proténomés, formes très inférieures à caractìres négalifs;

HYCÉTOZOAREs, ayant une tendance à former des plasmorles;

A Now BIENs, à pseudoporles lobés non anastomosables;

Foramiviféres, à preudopodes ramifiés el s'anastomosant en réseau;

HéLIOzoAIREs, à pseudopodes fins, rayonnants, avec: fiłament axile;

Radolarres, caractérisés par une sorte de coquille interne, la capsule centrale.

\section{$1^{\text {re }}$ Sous-Cilasse \\ PROTÉOMYXÉS. - PROTEOMYXIA \\ [Proteomya (Ray Lankester)]}

Dans ce groupe ont été réunies des formes assez nombreuses qui ne constituent guère un ensemble naturel, mais qui n’ont pas non plus d'affinités bien nettes avec les autres groupes de Rhizopodes et qui ont l'inconvénient de détruire l'homogénéité de ceux-ci, lorsque l'on cherche à les répartir entre eux. Nous le diviserons en trois ordres:

Acrstosponés qui se reproduisent par simple division, sans formation de kystes, ni de spores:

Azoospones, qui se reproduisent par des kystes d'où sortent des jeunes qui ont d'emblée la forme d'amibes;

ZoosrorÉs dont les kystes donnent naissance à des petits ètres flagellés, comparahles à des zoosprores et qui, plus tard seulement, se transforment en amibes.

$\mathcal{L}^{\mathrm{er}}$ OR DIR E

$$
\text { ACSTOSPORES. - ACYTOSTORID. }
$$

[Aмçв.ia reticllosa (Bülschli)]

\section{TYPE MORPHOLOGIQUE}

(FIG. 46)

Fig. 46

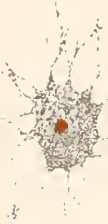

A.yolesporé

(Type morpholegies) (sch.

Le type de ces itres serait une amilue à pseudopodes réticulés, et réduite à une struclure extrèmement simple. Le corps est absolument nu, il n'y a point de distinction en ectoplasme et endoplasme, il n'y a pas de vésicule pulsatile, sonrent pas de noyau, entin la forme est extremement changeante. Lanimal ne s'enkiste jamais et se reproduit simplement et indéfiniment par division directe.

Malheureusement, la plupart de ces formes ont éló insulfisamment éludićes, leur cycle évolutif n'a pas élé assez longtemps suivi, en sorte qu'il se pourrait bien que de nouvelles études vinssent changer leur position systématique. 


\section{GENRES}

Le plus simple parmi ceux du moins dont l'existence est ccrlaine est Protogenes (Hïckel) (fig. 47) dont il n'existe qu'une espèce, $P$. primordialis (Ilickel), trouvée par cet observateur en 186í dans la Médilerranée, près de Nice. Il est remarrjuable par ses fins peudopodes rayonnants très nombreux ne formant pas de réseaux compliqués. II se nourrit de Protozoaires parfois presque aussi gros que lui, qu'il capture avec ses pseudopodes $(0,1$ à 0,2 . Mer $)$.

Gymnophrys (Cienkovsky) (fig. 4S) diffère du précédent par le fait yu’il n’émet ses pseudopodes, d'ailleurs plus longs el plus réticulés, yue par un, deux ou trois points de sa surface $(0,0 \%$ sans les pseudopodes. Mer) (').

Boderia (Wright) pourrait ètre défini un Gymnoplurys pourvu de un ou plusieurs noyaux (Mer).

Pontomyxa (Topsent) (fig. 49) est min ètre singulier qui forme, en lig. 4 i.

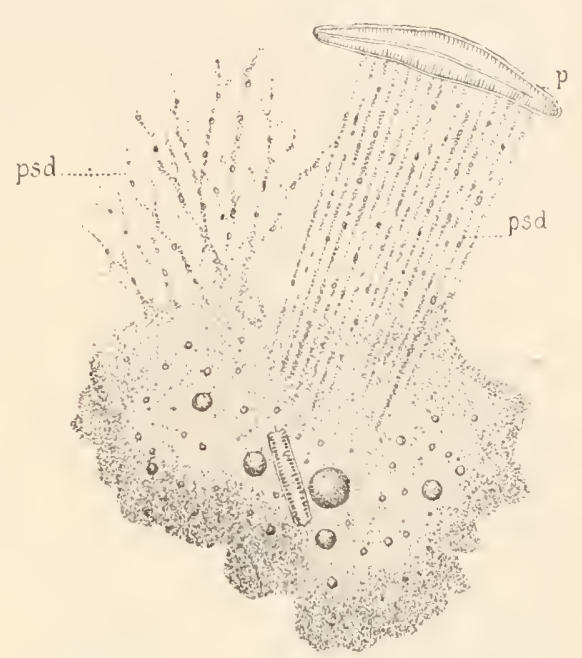

Protogenes ( $P$. primordialis) (im. 1lärkel). psad., psendopodes; p., proie.

s'étendant, un réseau si irrégulier que l’on ne peul dire ce qui appartient au corps el ce qui dépend des psendopodes. Il est coloré en jaune d'or
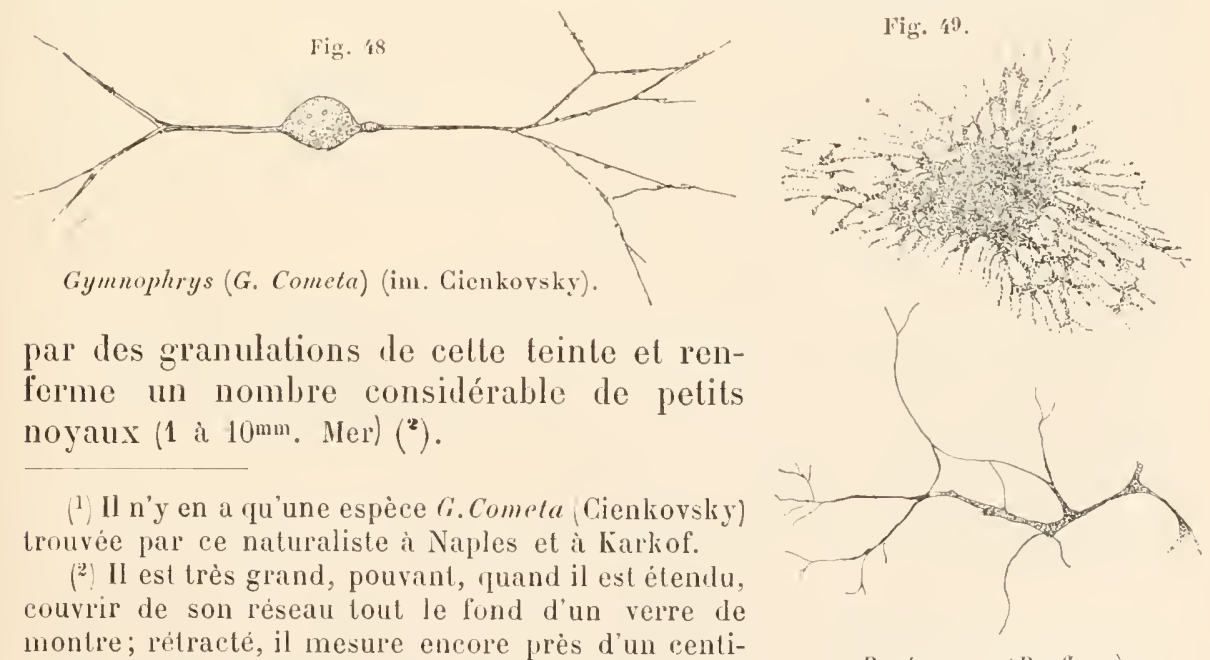

par des granulations de celte teinte et renferme un nombre considérable de petits noyaux (1 i $10 \mathrm{~mm}$. Ner) (')

(1) Il n'y en a qu'une espèce C.Comela (Cienkovsliy) trouvée par ce naturaliste a Naples et à Karkof.

(2) Il est très grand, pouvant, quand il est étendu, couvrir de son réseau tout le fond d'un verre de montre; rétracté, il mesure encore près d'un centimetre carré. Mais ce sont lí les grands échantillons, car il en est de toute taille et n'importe quel fragment

Pontomy.re (P. flava)

(d ap. Topsent). séparé avec des ciseaux peut vivre et grandir absolument comme un individu intact. 
Myxodictyum (Iläckel) entin (fig. 50), est remarquable jar l'absence de

Fig. 50 .

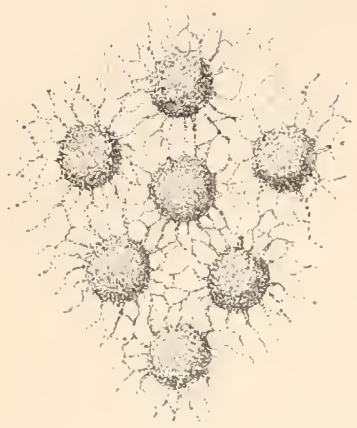

Myxodictyum (im. Häckel). noyau el par ses pseudoporles formanl un riche réseau qui s'unit ì celui d'individus voisins, en sorte que l'animal forme des colonies où Häckel a compté jusqu’à soixante-dix individus. Il y a lá un acheminement vers la condition caractéristique des Mycétozoaires, et peutitre pourrait-on le placer parmi ces animaux si son cycle évolutif n'était entièrement incomnu (Mer') (').

(1) Il n'en existe qu'une seule espèce ( $M$. Sociale, Iläckel) trouvẻe une seule fois en 1867, à Gibraltar.

Nous placerons ici, en appendice, deux formes dont l'existence en tant qu'organismes réels et indépendants peut ètre mise en doute. La première est

Schizogenes (Pouclıct) trouvé par ce naturaliste dans la cavité viscérale de divers Ostracodes et Cladocères d'eau douce. Ce sont des masses de proloplasma entièrement hyalin, sans vacuoles ni noyau, de taille extrèmement variable, de forme absolument indéterminée, sans pseudopodes, yui se meuvent par une reptation indécise et sans cesse se percent de fentes quí, lorsqu'elles sont assez étendues, détaclient des lambeaux qui constituent de nouveanx individus $(0,01$ à 0,03 . Eau douce).

La seconde est te célèbre

Bathybius (Huxley) (fig. Sl). Cet être serait formé de substance protoplasmique absolument amorphe, sans taille déterminée, sans noyaux, sans trace aucune d'organisation, sans autre caractère d’être vivant que sa composition chimique protoplasmique el sa faculté de se mouvoir et de saccroître en se nourrissant. Il n'aurait pas de reproduction véritable, élant susceptible d'aceroissement indéfini et ne se multipliant que par désagrègation en fragments quelconques sous l'aclion des trammatismes accidentels. Il a été trouvé d'abord en 1868, par IIUxcer, dans un limon ramené onze aus auparavant des grandes profondeurs par les sondages pour le câble transallantique et conserve dans l'alcool; il a ensuite été observé vivant et mobile par Wrvil.le Thompson et Calipenter en 1868 dans les produits des dragages du Porcupine dans le golfe de Gascogne et par Bessels en 1872-73, dans ceux des dragages du Polaris. Mais le Challenger ne l'a trouvé nulle part. Ses partisans admetlent yu'il tapisse des surfaces considérables du fond de la mer dans Thtlantique Nord, étendant son immense réseau dans les interstices des particules sans nom qui forment le fond vaseux de ces abìmes. Mais son existence a été rendue très douteuse, par l'examen minutieux des faits. Les analyses n’ont décélé en lui que $3 \%$ de substances albunineuses, tandis que des substances ininérales (acide silicique ou sulfate de chaux) forment la plus grande partie de sa masse. En versant de l'alcool fort dans de l'eau de mer, on délermine un précipité de sulfate calcique qui a tout l'aspect de l'organisme en question. Même les petites particules calcaires de forme régulière que l'on avait cru faire partie de son organisation: Coccosphères Fig. jl.

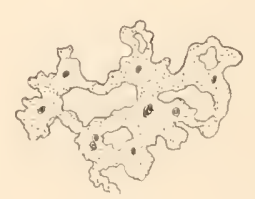

Bathybius. el Coccolihes, Rhabdosphères et Rhabdolilhes lui sout étrangères et sont sans doute ntême le simples précipités. II arting a pu les reproduire avec leur forme caractéristique en versant du carbonate le clatux dans une solution albunineuse.

Batlıybius étant caractérisé par ces nodules calcaires, Bessels a appelé

Protobathybius (Bessels) la gelée protoplasmique fondamentale, sans ces particules étran- 
$\underline{Q}$ O ORDRE

\section{AZOOSPORE - 1ZOOSTURIDA}

[Movidisa AZOOSporea (Kopf)]

\section{TYPE MORPHOLOGIQQUE}

(FIG. 52 ET 53)

Ce type est une simple amilie, toute semblable it celle du type prérédent. mais qui ne manque de noyau qu'exceptionnellement, et possède parfois une résicule pulsatile. Cette amibe vit en parasite sur des Algues inférieures (alg.) dont elle dévore le contenu en perçant leur menbrane et absorbant leur protoplasma et leur chlorophylle, dont les produits de désassimilation lui communiquent les couleurs rariées. Elle peut se reproduire aussi par division, mais son principal procédé de reproduction consiste daus un enkystement ( $I B)$ suivi d'une division sous le kyste en un petit nombre de petites masses protoplasmiques (emliny.) qui sortent du kystern perçant sa paroi et prennent immérliatement la forme de leur parent pour mener ì leur tour la mème vie.

Parfois ces petites masses s'enkystent (fig. "̋3) pour se mettre à l'abri des

\section{Fig. 53.}

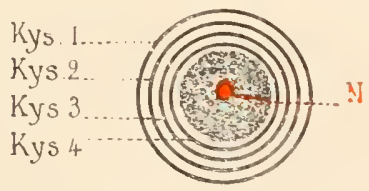

Kystes de protection (Sch.).
Fig. 52.
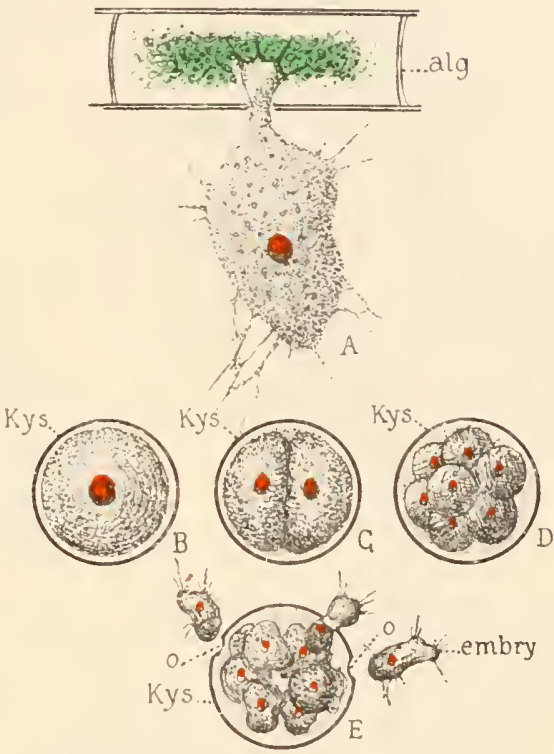

Azoosporé (Type morphologique) (Seh.).

alg., algue; embry., embryon; kys., kiste; o., orifiees. inlluences nocives sous une membrane plus épaisse, souvent double, triple ou mime quadruple (Kys. 1 it $K y s .4)$, mais il est rare que sous ce kiste épais elles se divisent; le plus souvent, elles en sortent simples, sans s'ètre nultipliées. On ılonne, un peu abusivement, le nom de spores au contenu de ces kistes épais. L’animal a une certaine tendance à s'unir à ses voisins en un plasmode. 


\section{GENRES}

Le représentant le plus complet de ce groupe et aussi le mieux connu est le genre

Vampyrella (Cienkovsky)(fig.: 花). Ici, l’amilse(A) a une forme assez régulière. Elle émel autourd'ellede lins pseudopodes rayonnants peu réticulés qui lui donnent un air d'llóliozoaire et qui partent d'un ectoplasmeincolore hyalin formant une couche mince autour de l'endoplasme granuleux, vacuolaire el coloré. Elle possède un noyau et une vésicule pulsatile $(0, ;$ it 0,7 . Her et eau douce) (').

Leptophrys (Ilertwig el Lesser)

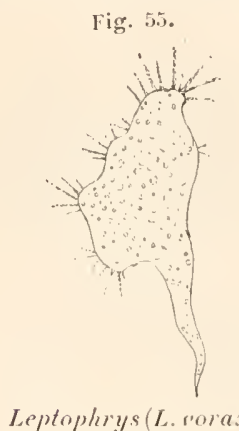

Leptophrys (L. rorax) (im. Zoph).

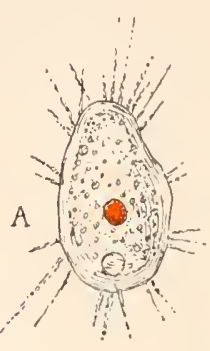

Fig. 5'.
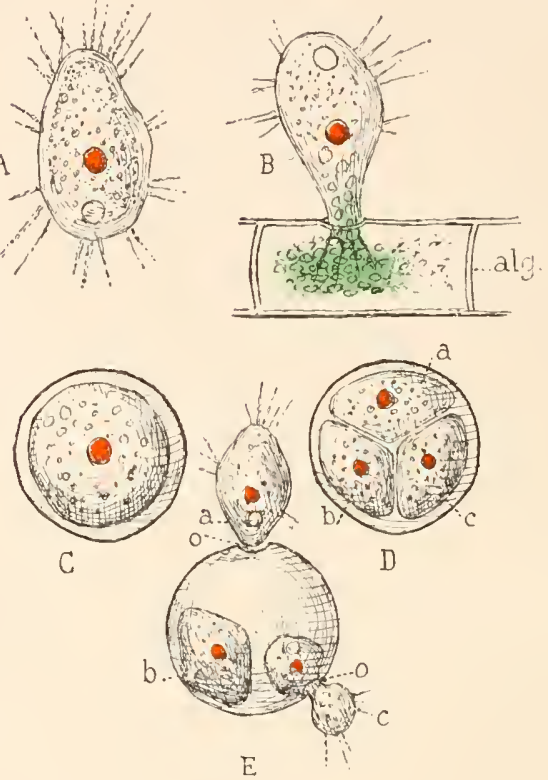

Tampyrella (Sch.).

(fig. :3i) n’est qu’une Vampyrelle polynucléée. Il est remarquable, en outre, par la présence de paramylon dans son endoplasme $0,1 \%$. Lau donce!.

(1) La Vampyrelle est en général colorée en rouge. Elle vit sur des $A$ lğues ( $B: a l_{g}$.). rampe sur elles, perce leur paroi d'un trou rond et, par cetrou, introduit des pseudopodes qui digerent le contenu sur place ou l'aspirent du dehors sans y pénétrer. D'autres fois, si la proie est assez petite (kysle d'Eugline), elle l'enveloppe tonte entière et la digire ainsi. Parfois, elle s'unit ì deux ou trois autres individus en un plasmodium : on voit les deux individus se joindre, se sourler, se fondre entierement l'un dans l'autre; mais ce r'est pas là un acte sexuel, car les noyaux paraissent quelquefois rester indépendants. Dans certaines circonstances, elle s'enkyste $(C)$ sous de nombreuses el épaisses enveloppes qu'elle sécrète en se contractant chaque fois un peu plus. On ne connait pas le sort de ces kystes de protection. Elle se reproduit par division; mais ce mode de multiplicalion n'est pas fréquent. Le plus sonvent, elle se reprodnit après s'ètre enkystée, soit pour digérer à l'aise une nourriture abondante, soit pour s'abriter kyrstes de repos et kystes de protection), e'est-à-dire qu'elle rentre ses pseudopodes, s'arrondit, évaeue ses résidus 
Endyonema (Zopf) (fig. ÖO) est une forme roisine, mais polynuclée, qui vil et s'enlivste dans la cavití cellulaire de diverses Nostocacées $(0,04$ ì 0,05 . Eau douce).

Fig. 56.

Vampyrellidium (7opf) (fig. : :7) ressemble aussi lieancoup aux Vampyrelles, mais la division est son mode unique de reproduction (10 à 12 p... Eau douce' ("). Haplococcus (\%opf) (fig. :is) est remarpualile par son parasitisme. Il habile entre les fibres musculaires du Cochon et de fuelques autres animanx ( $\left.{ }^{*}\right)$.

Bursulla (Sorokin) (fig. :in) vit sur lo crottin de cheral Fig. 57.

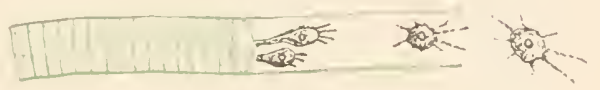

Vampyrellidium (V. wagans) (d'ap. Zupf). où ses amiles de couleur rosée rampeut el se nourrissent aver leurs pirmlopoles rarrs, et forment parfois des plasmodes de derix it quatere inlivilus. 10 : $12 \%(3)$.

alimentaires, ibeint sa vésicule pulsatile et sécrìte auturur d'elle une membrane. Sols refte memlsrane, elle se divise en un pelit nombre de fragments (delx it qualre) $(D: a, b, c)$, , fui sortent

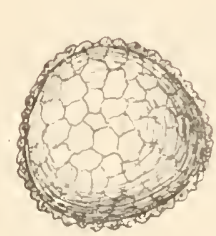

A

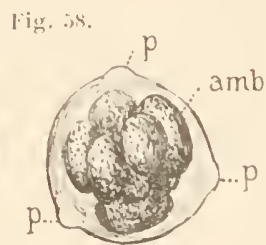

B

$$
\text { IIaplococeus (im. Zopl }
$$

1. Le livsle. Vue superfieielle pour montrer los seulptures.

B. Kisle monlant les amibes am lo. qui so forment à son incerienr: p., pores de sortie.

lig. 5\%.

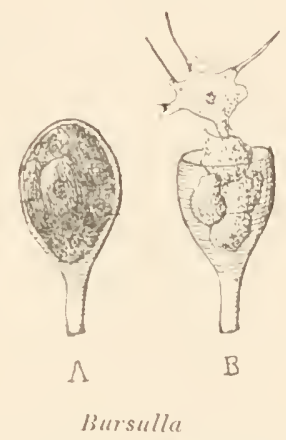

(B. crystallina) im. Zopf).

A. Lo kysle. Vue superficielle.

B. Le mine ouverl montrin! les amibes qui s'en (iehappent. en pereant la paroi du livsle $(E: a, b, c)$, en général par autant de trous $(o)$ qüils ourrent charun en face de lui, el se montrent imméliatement avec les caractères de petites lamprrlles. Les residus indigirés, s’il en est, restent dans te liyste. Cihez une seule espece, T. Comphonematis (Jäcliel), le novau selalil absent. Celle espèce serait done une Momimer.

Monadopsis (Klein), n'est qu'une espère de Vampyrelle.

(1) De ces liysles, que leur proi soit mince ou épaisse, il sort toujours sans s'êlre divisé; il n'a pas de pigment rouge.

() Ià, il forme rles livstes de 20 y environ de riamètre, à paroi ornée de seulp-

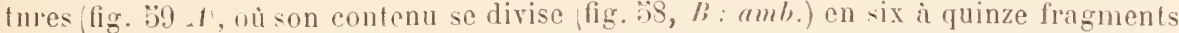
qui sortent par des orifices p.; et l'on sernit tenté de voir en lui un Sporozoaire, si ces liagments, une fois sortis, ne se nontraient sous la forme rle viaies amibes. Il ne parait pas nuire à son hoite.

3) Les Plasmores s'enliystent et se divisent sous Je liyste en luuit porlions qui donneront autant d'amibes. Il r a aussi un enlirstement de protection d'oì le corps protoplasmique sort sans sietre divisé, mais aussitot forme un liyste de division. 
Enteromyxa (Cienkorsky) (fig. 60) est une amilie qui forme, en se

Fig. 60 .

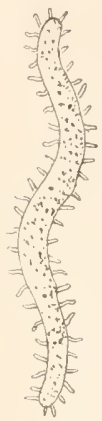

Enteromy.ra.

(E. palmolosa)

(im. Zop)

$3^{\circ}$ () RIDRE

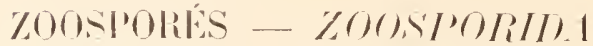 \\ [MOX.ADL:LOOSPORE. (Zopf $)]$}

\section{TYPE MORPHOLOGIQUE}

(FIG. 61)

L'amilse $(A)$ ne diforre point de celle du type précédent. Elle est touFig. 61 .
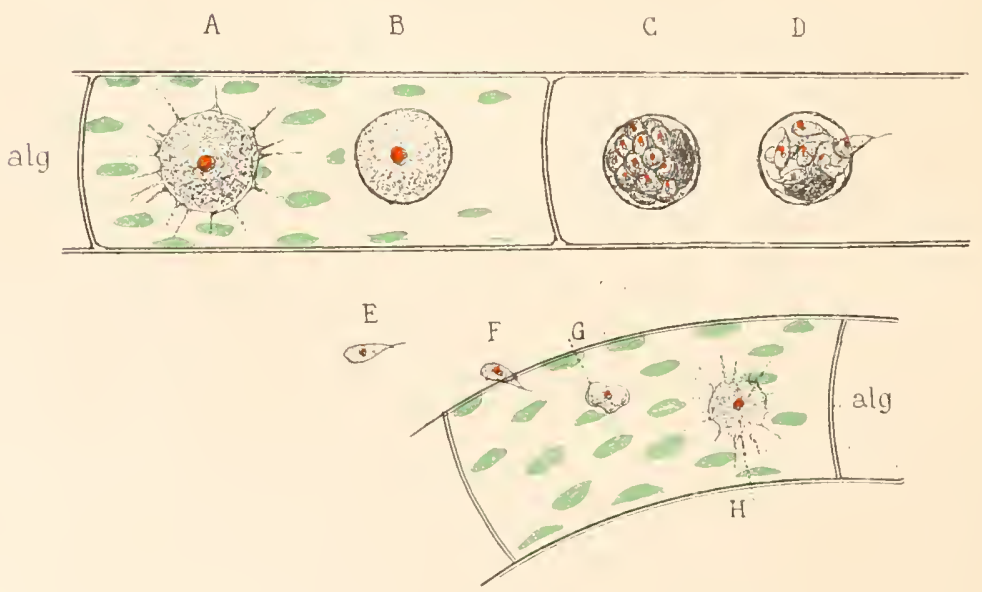

Zoosporé (Type morphologique) (Seh.).

jours petite, le plus souvent nucléée, rarement pourvue d'une vésicule

1) Co genre est placé par Zopif parmi les Mysomycètes.

On pourrait peut-être placer ici le genre $W_{j} x$ ttstrum (Häckel) que nous avons mis parmi les Hycélozoaires (1. 8.3). 
pulsatile et presque toujours munie de pseudopodes rayonnants non réticulés que lıi donnent une certaine ressemblance avec Actinophrys. Elle montre une faible différenciation de sa couche superficielle en ectoplasme livalin. Il y a sourent plusieurs racuoles dont certains observateurs ont ru parfois l'mne ou l'autre se contracter, mais il y a loin de là à la vésicule pulsatile permanente si nelte chez les Rhizopiodes.supérifur's. C'ertaines espèces, mème dans les genres où d'ordinaire les pseudopodes existent, en sont dépourvues et nont que des lobes amoboïdes.

Cetle amibe vit en parasite dans la cavité cellulaire mème de certaines Algues inférieures (alg.) (Diatomées. Spiroggres, etc.) dont elle dérore le contenu, chlorophylle et protoplasma. Elle grossit ainsi beaucoup et il n'est pas rare qu'elle se fusiomne arec les autres individus de son espèce, si clle en rencontre dans la mème cellule, en un plasmodium. Hais ce plasmode ne réunit jamais qu un petit nombre d'individus. Quand elle a assez grossi, elle s'enkrste (IB). l'our cela, elle rentre ses psrudopodes. s'arrondit et se sécrète une cuticule plus on moins épaisse. Sous ce lirste, elle se divise en un certain nombre de pelits fragments nuclérs $(C)$ qui sarroulissent el, à maturité, sortent du lịste (II) où reste un reliquat de segmentation. Mais voici où gì la caractéristigue de l’ordre. Ces jeunes, au lieu de prendre immédiatenent la forme d'amiles comme leurs pareuts, se montrent sous l’aspect de petiles moniales que l'on décore du nom assez mal approprii de soospores $(E)$. lls sont formés d'une pelite masso de proloplasma pourve d’un noyau. somvent d'une résicule pulsatilu, et prolongée en ivaut en mu long llagellum. Ce flagellum, d'ailleurs, u'est pas, comme celui des rraies llonales, neflement distinct ì sa baso: le corps se prolonge insensilhement en larme batavique, et ce flagellum u’est guère qu'nn psembopole mieux ilessiné et plus lixe. Il ne constitue qu'un apprareil moteur bien imparfait, car l'animal a bean le manourrer, il s’agite sur place plus qu’il ne fait de chemin. Yéanmoins, il tinit par pencontrer une Algue de l'espice qui lui convient, perce sa memlirane $(F)$, pénetre dans sa cavité, perd son llag̣ellum (G), se transforme en amibe (II) et recommence la mème érolution (").

Dans certaines circonstances, l'animal forme un liysle durable. II sécrète alors une memlıane beaucoup plus épaisse, apròs s’ètre délorrassé de tous ses résidus alimentaires, el vil lì un temps si long que la plupart ،lu temps on n’a pu suive son évolution ultérieure. Il semble que d'ordinaire il en ressorte sans s'itre morlifié. Mais quelquelois il se livise sous ce kisste qui ahoutit en léfinitive à la mème érolution que ceux précédemment lécrits $\left(^{2}\right)$.

(1) La transition de la zoospore a lamibe se fait par gradation. La zoospore, à tout moment, est amoboüde; ufuand elle a pris place dans la cellule, elle le devient davantage, parfois résorbe son fouet, puis le reforme quelque temps aprés, jusıu’a ce qu'enfin elle le perde définitivement et devienne tout à fait amoboüde.

(2) Zopr etablit une différence tranchée entreces kystes qu'il appelle sporocystes et les premiers quil déxigne sous le nom de zoocystes. Mais ils ne different en somme 


\section{GENRES}

Pseudospora (Cienkovsky) représente à peu près notre type morphologique. Il est caraclérisé génériquement par l'absence de plasmodium (Zoospore 8 à 10 [.. Eau douce) (').

Colpodella (Cienkovsky) (fig.62) se distingue parl'absence de phase amilie (').

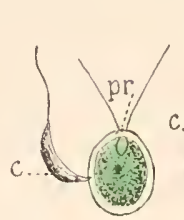

A
Fig. 62.

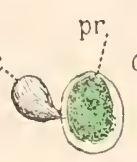

B

Colpodella.

(C. pugnax) (im. Zopr).

c.. Colpodella:

pr., proie (Chlamidomonas) attaquéc par. les colpodella.
Fig. 63.

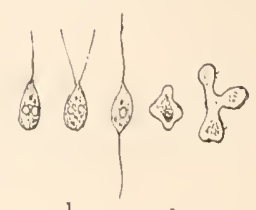

$a \quad b \quad c \quad d \quad c$

Protomonas

(im. Zopf).

a. b, e, zoospores;

d, e, amibes.

Protomonas (Cienkovsky) (fig. 63), au contraire, a une phase plasmodium ( $\left.{ }^{3}\right)$.

Diplophysalis (Zopf) (fig. 64) n'est, par la plupart de ses caractères, qu'un Ṕseudospora, mais il est remarquable par la forme

Fing. Gí.

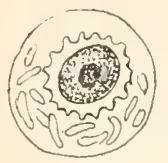

Diplophysalis

(D. Stagnalis)

(d'ap. Zopf). étoilée de la membrane interne dans les kisstes durahles. II se reproduit par des kystes lont le contenu se segmente et donne des zoospores.

Toutes les formes précélentes avaient eeci de particulier yue, arant de s'enkyster, elles ne rejettent pas leurs résidus alimentaires, que l'on retrouve dans le kiste, au milieu ou à côté de la masse protoplasmique. C'est linverse dans les quatre genres suivants qui épurent soigneusement leur substance avant de s'enkyster (“).

que par le nombre et J'épaisseur des membranes protectrices et par la présence d'une membrane autour du produit de la division des seconds (spores). Nous rencontrerons dans les Protozoaires supérieurs de ces enkystements de repos oul de protection aboutissant parfois à une division, sans que les auteurs aient songè à voil Jà un morle spécial de reprorluction.

(1) La zonspore a un noyau el une vésicule pulsalile, elle peut avoir un ou deux flagellums. L'amibe est parfois dépourvue de pseudopodes. On a observé des liystes durables, mais sans division subséquente et à ŕvolution inconnue.

(2) Les zonspores (fig. $63 a$ à e) de la seule espece connue, $r$. pugnax (Cienkovsiy) s'attaruent á leur proie ( $m$.), un Chlamidomonas, par Jeur extremité postérieure qui est effilée comme l'extrémité antérieure flagellifère et aspirent son contenu. Elles perilent alors Jeur fonet (B) ef ressemblent à un Colpode. Bientòt l'animal s'enkyste, sous deux membranes et se divise. A la maturité, la membrane interne sort de la coque du liste comme un sac herniaire el, en se gèlifiant, met les zoospores en Jiberté. On a observé aussi un liyste durable dont l’èvolution n'a pu être suivie.

${ }^{(3)}$ La zoosprore a un ou deux cils, snit còte à còte au mème pòle, soit aux deux pòles npposés. Les amibes qui naissent d'elle, peuvent s'enkyster et se reproduire isolement, mais le plus sourent elles s'missent d'abord en un plasmodium.

(4) D’après ce caractère, les premiers sont réunis en une famille tes Pszcdospo- 
Gymnococcus (Zopf) est, sauf la différence que nous renons de signaler, toui semblable à Pseulospora. Wais en outre il forme volontiers un plasmodium arec les autres individus de son espèce qui peuvent se rencontrer dans les mèmes cellules hospitalières ('). Aphelidium (\%opf) se distingue par sa reproduction qui a lieu exclusirement aux moyens de ses kivstes durables, munis d'un orifice operculé $\left({ }^{2}\right)$. Protomyxa (Häckel) (fig. 6:̈) est une énorme amilıe à pseudopodes extrèmement ramifiés el réticulés pouvant atteindre jusqu’ì $\mathrm{Imm}$. Mais sous cetle forme, elle représente un plasmodium, l'amilıe originelle étant de taille ordinaire

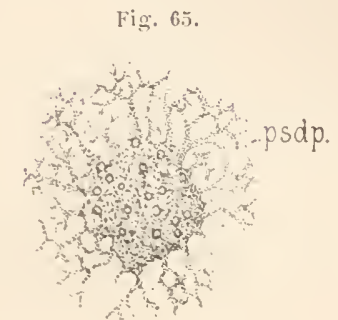

Protomyxa

P. aurantiaca) (d'ap. Haickel. purilp., psendopodes. et se fusionnant si complètement ì l'autres individus de sa race, que l'amile résultante est toute semblalile et n'en diffore que par la taille. Ni che\% l'amile, ni che\% lo plasmode il n'y aurait de noyau; ce serail donc une Jonipe (Ier) ( $\left.{ }^{3}\right)$.

Pseudosporidium (Zopf) (fig. 6i6) est remarquahle par le fait quilil ne forme pas de livste. Cela lient ì ce qu'occupant toute la cavité de la cellule hospitalière, il se contente de la paroi de cette cellule, comme protection pour les produits de sa division. Ses zoospores sont à 111 dans la cavilé de Fig. 66.

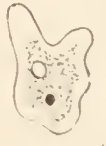

A

I'seudospuridium

'r. Brassanium, d'ap. Zopif). la cellule qui l'héberge. (Tronvé par lirass dans des inlusions régétalles).

Ce Pseudosporidium nous conduit naturellement au genre

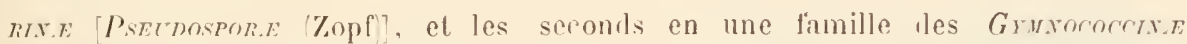
Gomocoret: (Zopl']. Zopf itablit eneore entre ees familles celle diffirence que. dans la première, les spores durables sont sous un lịste. lamilis que dans la ceeonde. elles sont nues, er qui reut dire simplement qu’il y a dans le premier eas sérétion d'une seeonle membrane sous la premiere, apres retrait du eorjes protoplasmique.

(1) La zoospore a tleux fouets. On a observé la division transversale et l'enkystement durable.

(2) Ia senle espece du genre, 1. deformans (Zopf), a une zoospore extrimement petite 2 a $3(2)$ munie d'un seul fonet. Elle alonne une anihe guire plus grosse, que l'on trouve installée dans la cellule d'une Confervacée, Coleochirte, sans savoir si c'est elle ou la zoospore qui y a fait effraction. Lis, elle dévore le contenu de la cellule qui s'lypertrophie a mesure, grossit et se divise sans s'enkyster en un nombre immense de minuseules zoospores.

(3) P. auriantaca (Hïekel), seule espèce du genre, a été trouvée en 1867 par ILÄcket. aux Canaries sur les eoquilles de śpirula oi it forme des taches orangées visibles à l’ail nu. Il n'a pas èté retrouvé depuis. Ciràce à sa taille, le plasmode peut capturer de grosses proies, en partieulier des l’éridiniens. Il forme des liystes de 0,2 , d'où sortent des zoospores munies d'un gros fout, qui se transforment presque aussitot en amibes.

Nous placerons iei provisoirement deux formes eneore insuffisamment connues, Eclobiella et Pseudamphimonas, dont la place réelle ne pourra èlre exaetement 
Plasmodiophora (Voronin) (fig. 6s), qui ne forme pas non plus de kystes, mais dont les produits de division, au lieu d'otre nus, sont munis d'une membrane à la manière de spores, ce qui rapproche ce genre des Hycétozoaires que nous allons hientòt étulier. Le corps protoplasmique se divise en innombrables petites masses enkystées séparément et réunies entre clles seulement par une cerlaine quantité de substance interstitielle non utilisée. Ici encore. lia chose s'explique par le fait que les tissus de l'hote foumissent à ces spores une protection suffisante, jusqu'au moment où elles donnent naissance à une zoospore munie d'un cil antérieur et d'une vésicule pulsatile (').

Tetramyxa (Gïbel) est un genle roisin chez lequel l'amibe, avant de sporuler', se divise, se segmente en fragments dont chacun produit quatre spores seulment. Il vit en parasite sur direrses plantes aquatiques sur lesquelles il détermine les productions de galles $\left(^{*}\right)$.

déterminée que lorsque l’on connailra plus complètement leur mode de reproduclion. Ectobiella (de Bruyne) (fig. 67) est représentée par une zoospore à deux cils antérieurs qui

Fig. 6i7.

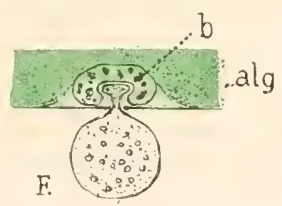

Eetubiella. (E. Plateani) (d'in. de Bruyne). se transforme en une petite amibe parasite de certaines Diatomées qu'elle perce et dont elle se nourrit ì la manière des Colpodella, pour pénẻtrer à leur intérieur. Elle insinue un court pseudopode lans la cavité de la Diatomée $\left(a l_{g}\right.$. $)$ et assimile sa substance sur place par ce pseudopode. Elle n'englobe pas, comme les autres, des parcelles solides, mais dissout le protoplasma de l'hote et l'absorbe par sa surface, ì l'ètat liquide $(b)$. Par le reste de sa surface clle n'èmet point de pseudopodes ou parfois fait saillir un ou deux lobes courts et oblus. A un moment donné, elle s'enkyste et l'on ne sait pas ce qui se passe ensuite.

Pseudamphimonas (de Bruyne) dont on ne connait que la zoospore à un ou deux cils et l'amibe qui vit en parasite sur les racines de Caulerpa et forme un plasmorlium.

${ }^{1}{ }^{1}$ ) Cette zoospore (fig. 68, I, I) très petite $(2$ a 3 p.) comme celle de Pseudosporidium, se rend vers les jeunes radicelles des Brassica Clioux), et se transforme en une petite amibe $C$ ) qui s'introduit ( $D$ ) dans une cellule épidermique, en dévore le contenu, passe à une cellule voisine el ainsi de suite, en s'enfonçant dans le parenchyme cortical. C'est dans le tissu mème, qu'elle forme, après avoir heaucoup grossi, ses spores en quantité immense $E$. Celles-ci sont mises en liberté par destruction de la plante avec le concours de Bacillns amylobacter. Le nombre considèrable des parasites qui attaquent une même plante détermine par irritation des nodosités lypertrophiques el le chou finit par périr. En 1869 la moitié des Cloux fut détruite en Russie par ce parasite. La maludie se nomme vulgairement la hernie du Chou.

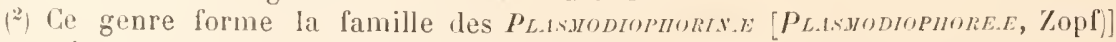
caractėrisee par ses spores et l'absence de kyste. 
2 SOUS-CLASSE

\section{MYCE'TO\%OAIRES - MYCETOZOARIE}

[Mriomycètes (Aucl.) + L.1BYRTNTILLE.1 (IÏ̈̈ckel)]

Ce sont des amibes qui ont pour caractère le se réunir, à un moment donné de leur cycle évolutif, en colonies temporaires ou permanentes. Nous les divisons, en suivant à peu près les idées de \%opif en trois ordres:

l'seudorlasmodoA qui sont les Acrasiées de Van Tieghem;

Filoplasmodina qui sont les Labyrinthulés de Cienkorsliy;

Euplasmodida qui sont lirs Myxomycetes des auteurs.

Ces trois ordres se caractérisent nettement par le fait que, dans le premier, les individus se groupent sans se souder (pseudoplasmorlium); dans le dernier, ils se soudent et se fusionnent lemporairenent, mais entierement (plasmodium vrai) : dans le second, cntin, la soudure n a lieu que par les ramifications des peudopodes (plasmodimn filamenteux).

Ler () R D RE

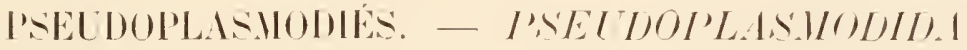

[Acrusiés (Van Tieghem)]

\section{TYPE MORPHOLOGIOUE}

(FIG. 69)

A l'état adulte, l'ètre est une pelite anibe (.1) de forme pas trop irrégulière, qui émel de gros piseudopodes rares, lobés, obtus. Il possede un noyau et une vésicule pulsatile. Il vit sur des matieres animales humides, se meut, se nourril, se multiplie par simple division. Après avoir assez longtemps mené cette vie, et généralement quand la nourriture se faitrare, lesindividus, lorsqu'ils se rencontrent, au lieu de se séparer sans s'occuper l'un de l'autre, restent unis $(B)$ et ainsi, peu ì peu, tous ceux d'une mème pelite région arrivent à se grouper en une seule masse $(C)$. Ce sont donc, généralement quoique pas forcément, des

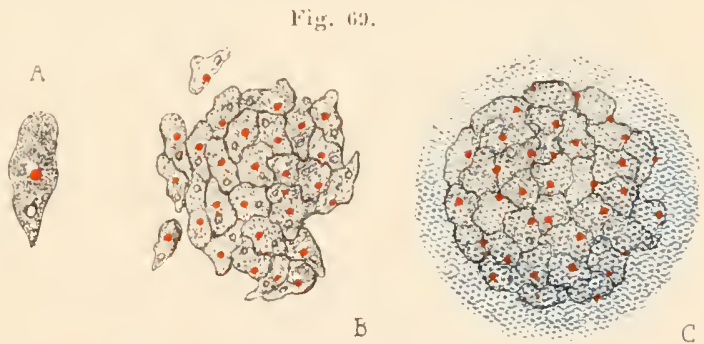
individus nés d'un parent commun. Une fois réunies, les petites amibes 
s'arrondissent, sécrètent une substance gélatineuse qui comble leurs intervalles et enveloppe leur ensemble, et chacune en outre s'enkyste sous une (II), deux, ou même trois enveloppes concentriques. Les kịstes mesurent 10 \% environ. En cet élat, la petite masse peut attendre sans danger le relour des conditions favorables. Alors, les corps protoplasmiques sortent de leurs enveloppes $(E)$ et se retronvent semblables it l'anibe qui nous a servi de point de départ; ils se dispersent et recommencent chacun le mène cycle évolutil" (').

\section{GENRES}

Pour faire de nolre type morphologique un

Guttulina (Cienkorsky), nous n'aurions qu’à préciser la forme de la colonie de liystes qui est sphérique el sessile, ou en massue fixée par le manche, parfois divisée en quelques tubérosités, et forme des taches d'apparence laiteuse (Sur le crottin de cheval et autres substances analogues).

Copromyxa ('\%opf) (fig. 70) semble n'ètre qu'une espèce du précédent.

Dans les autres genres (c'est le cas le plus fréquent chez ces êtres) certains individus restent sté-

Fig. 70 .

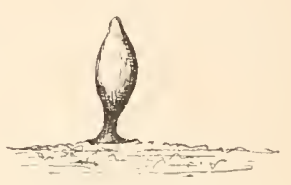

Copromyxa.

(C. protea) (im. Zopf). riles et se liquéfient sous la membrane épaisse de leur kyste; ils se soudent en outre fortement entre eux après s'ètre disposés en pédoncules de formes variées. Par ce processus les colonies de kystes arrivent à prendre des formes diverses qui permettent de distinguer les genres. Chez

Acrasis (Van Tieghem) le pédoncule est filiforme et porte à son sommet les liystes disposés en chapelet. Chez

Dictyostelium (Brefeld) le pédoncule est épais, formé de plusieurs files cellulaires et les kystes forment un petit renflement sphérique au sommet.

Polysphondylium (Brefelı) est d'abord un Dictyostelium, mais en grandissant il ramifie son pédoncule. Enfin chez

Cœnonia (Van Tieghem) le pédoncule, ramifié ou non, se termine par une capsule à bord denté qui supporte la masse sphérique des liystes.

( $\left.{ }^{1}\right)$ Il est à remarquer que cet enkystement n'est pas un phénomène reproducteur, puisque le nombre des individus n'augmente pas par son fait. C'est un enkystement de protection. On ne doit donc pas donner le nom de spores aux petites amibes enliystées. On le fait cependant en général, et cela est légitime dans une certaine mesure, car nous verrons, dans des formes assez voisines ayant un cycle paralléle, le contenu se diviser sous le kyste; et, d'autre part, tous ees indivirlus qui se réunissent pour s'enkyster sunt en général descendants d'un même parent. C'est donc comme si celui-ci s'ètait divisé sous son krste pour leur donner naissance et les avait ensuite mis en liberté pour qu ils puissent ehereher leur nourriture. Il u'y a done qu'une anticipation d'un stade du cycle sur ceux qui auraient dù le précéder. 


\author{
Qe ORDRE \\ FILOPLASUODIES. - FILOPLASIUODID. 1 \\ iel

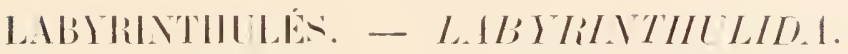 \\ [L.HBTRTTIILLEA (IIäckel)] \\ TYPE MORPHOLOGIQUE
}

(FIG. 71 A 73)

Ce groupe ne contenant que deux ou trois genres dont un seul bien conmu el certain, Labyrinthula (Cienkorsky), nous décrirons ce genre comme type du groupe.

\title{
Structure.
}

Chez ce Labyrinthula, l'amilse n’est jamais isolée. Elle vil réunie en colonie avec un grand $n o m b r e$ de ses semblables. Elle a une forme

Fig. 71.

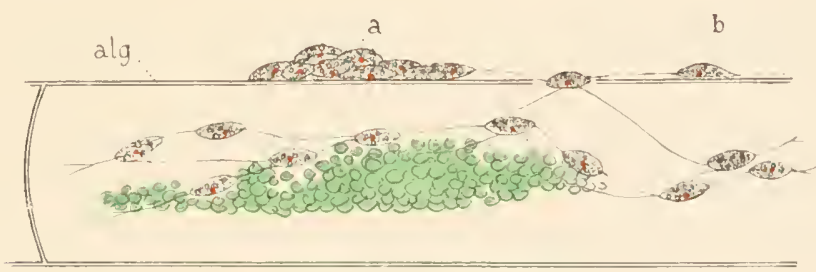

Filoplasmodie: Type morphologique. (Labyrinthula Cientiossiyi) (Sch.). alg., algue; a, $\mathbf{b}$, amibes.

régulière, celle d'un fuseau (b) de $S$ à 10 ou $1: 3$;. de long et elle ne se déforme guère que pour s’allonger ou se raceourcir plus ou moins.

Elle est formée d'un endoplasma légèrement granuleux contenant le noyau et quelques racuoles et d'une mince couche superficielle d'ectoplasma hyalin qui forme aussi les pseudoporles.

Elle émel à chacun de ses pôles un pseudopode long, fin, raide, qui s'unit à ceux des cellules roisines et forme arec eux un réseau délicat et très irrégulier. Assez souvent, on roit en outre un ou deux pseudopodes naissant des parties latérales du corps ot se jetant sur le réseau four en former une nouvelle maille. l'ar le moven le ce réseau, lous les individus sont donc réunis en un plasmodium, mais de nature spéciale, conslitué seulement par des pseudopodes filiformes, d'où le nom de plasmodium filamenteux (nous dirons filoplasmodium) qui lui a élé donné. 


\section{Physiologie.}

Locomotion. - Les anilıes semblent moliles sur ce réseau, comme si elles pouraient glisser sur lui et le parcourir tout entier. C'est mème ainsi que leur mouvement avait élé d’abord interprété par Cienovsky, qui voyail dans le réseau une sécrétion filamenteuse destinée à leur servir de guile. Mais \%opf [92] a montré que le réseau était formé simplement par l'ensemble des psirudoporles filiformes anastomosés el que

Fig. 72.

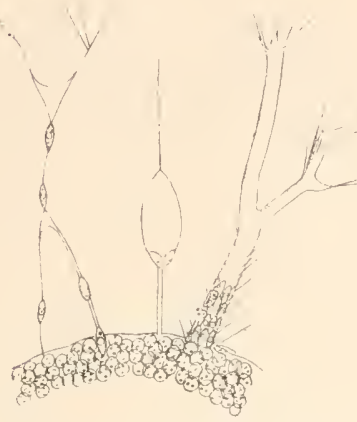

Filoplasmodie. Type morpholog. Labyrinthula

(L. ritellina) (im. Cienkorsliy)

Fragment de la colonie rétracté montrant des individus commencant it se mettre en marche. les amibes, pour se déplacer, contractent el rentrent le pseudopode situé du cóté où elles vont, allongent, an contraire, celui qui est du ròté dont elles s’áloignent, ce qui donne alssolument l'illusion du mouvement aupuel Cienkorsky avait cru. La vilesse est d'ordinaire $20 \%$ par minute. Elle est donc très faible.

La colonie a done dans son ensemble une forme incessamment variable el tris irrégulière. Elle comprend d'ordinaire (fị. 72) une masse centrale de figure quelconque formée par des individus un peu contraclés ef arrondis, simplement juxtaposés, sans fusion aucune de leur protoplasma. Dans cellemasse, les individus superficiels émeltent sculs quelines psimlopodes qui les rattachent au réseau extérieur. Ce ríseau cxtérienr est formé comme nous l'avons décril et les individus qui sont sur lui so diplacent sans cesse, les uns rentrant dans l'amas central, tandis yne d'aulres, de l'amas central, se lancent dans le réseau. Dans quelques circonstances, tous les individus sont groupés en une seule masse et il n'y a ni réseau, ni pseudoporles. Mais, à un moment donnć, les individus les plus superficicls émetlent leurs pseruloporles, les inclinent de lous côtés jusqu’à ce qu'ils en aient rencontré un autre auquel ils se soulent, commençant ainsi le réseau sur lequel ils commencent à se mouvoir. Arrivés à la périphérie, ils font de mème et auğmentent ainsi peu à peu le réseau sur lequel les autres individus se lancent ì leur suite.

Division. - Les amibes se reproduisent (fig. 73) par division transversale et les deux moiliés du fuseau divisé restent unies par un filament pui devient un pseudopode commun s'étendant de l'un à l'antre. La nourriture est capturér par les pseuloprodes. Les amibes pénètrent dans l’intérieur d’Mlgues inférieures (Diatomées, Spirogyros) et les vident lentement peu à veu on voil les pelites parcelles lig. $\pi 3$.

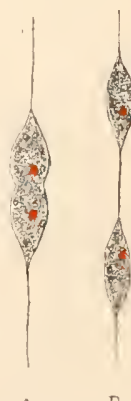

A $B$

Division des liloplasnoudiés (Seh.). 
capturées rouler lentement le long des pseudopodes, entrainées sans doute par quelque courant protoplasmique invisible, et arriver aux anibes qui sans doute les retiennent.

Enkystement. - Dans certaines circonstances, les amibes rentrent peu à peu leurs pseudopodes et se réunissent en une masse dense sans trace de réseau. Elles grossissent el s'enkistent individuellement. Au sortir de l'enkystement, elles reprennent leur forme de fuseau et se metlent tout de suite à former des pseudopodes et ì constituer le réseau. Toutes celles de la colonie s'rnkystent d'aillour's à peu près en mème temps et sortent aussi ensemble de leurs krstes. Ce n'est évidemment, dans ce cas observé chez L. Cienkovskyi par Zopif, qu'un enkystement de repos, puisque le nombre das individus n'augmente pas par ce processus (").

Mais dans d'autres cas, comme celui de L. macrocystis (Cienliovsky), toute la masse s'enferme en outre sous une écorce sécríce en commun, les individus s'enliystent séparément sous celte enveloppe et, dans leur kyste, subissenl une division en qualre pelites masses nucléées qui, au sorlir de l’enkystemenl, donnent aulant d'anibes completes. C'est alor's une vérilablic reproduction $\left(^{2}\right)$.

\section{GENRES}

Labyrinthula (Cienkorsky), 'lue nous renons de décrire comme lype morphologique, est le seul représentant bien aréré de l’ordré, les genres Ihiplophrys el Chlamydomyxa ( $\left.{ }^{3}\right)$ élant encore, celui-ri trop insuffisam-

(1) \%opf n'est cependant pas sìr qu'il n'y ait pas une division en deux dans le kyste.

( ${ }^{2}$ L'habitat de ces èlres est variable. L. vilellina (Cienkovsky) et L. marrocylis (Cienkovsky) ont èté trouvés par cet auteur sur les pilotis du port d'Odessi, un peu hrors de l'eau ou complèlement immergés. De Bruyne [99? a retrouvé la seconde à Naples et l'a vue se nourrir de Diatonées dont les valves vidées etaient remplies par ses amibes. Leurs colonies sont visibles à l'wil nu sous l'aspect de petiles taches rouge brique ou jaune pile.

Kopf a trouvé L. Cienkos'ski (Kopf) vivant en parasite sur des Spirogyra d'Allemagne dont il pergait les cellules avec ses pseudopodes et dont il vidait le contenu ajrès avoir pénétré a leur intérieur.

Lorsque l'on croyait que le ríseau était une sécrétion, la position systématique des Labyrinthulés ètait des plus embarrassantes. Mais aujourd'hui leurs relations avec les Myxomycètes, et en particulier avec les Acrasiees, sont devenues fort claires. Zopf les réunit à ces dernières sous le nom de Sorophorées.

(3) Faut-il placer ici le genre

Diplophrys (Barker) placé ordinairement parmi les Foraminifères?

liig. it.

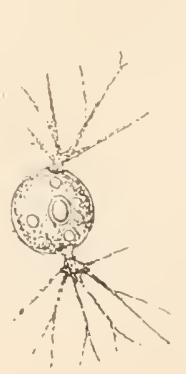

\section{Diplophrys}

(D. Archeri)

(in. Hertwig u. Lesser).
Fig. is.

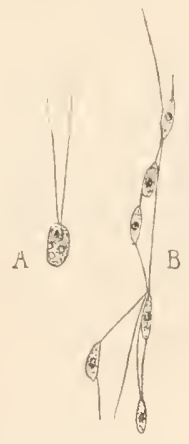

\section{Diplophrys}

(D. stercorea)

(d'ap. Cienkorsky).

Si l'on considère D. Archeri (Barker) (fig. 74) qui vit dans l'eau douce, il semble 
ment connu, celui-là trop hétérogène pour pouvoir ètre raltachés avec certitude aux Labyrinthulés (8 à $1: 3$ p. Mer et eau douce).

évident que cet être, arec sa membrane formant presque une coquille, sa vie isolẻe à l'àge adulte, est un Foraminifère monothalame voisin d'Amplitrema. Nous le présenterons de nouveau en étudiant les Gromides (V. p. 116).

Mais si l'on examine $D$. stercorea (Cienkovsky) (fig. 7ö), qui vit sur le crottin de cheval, la ressemblance est frappante avec les labyrinthulès. L'amibe est semblable avec son corps nu, son noyau, ses deux ou trois vésicules pulsatiles, ses deux minces pinceaux de fins pseudopodes raides, insérés aux deux pòles $(A)$, et surtout avec son groupement colonial $(B)$ et ses mourements presque identiques avec ceux des Labyrinthnla.

On est peut-êlre en droit de se demander si ces deux espèces ne conslitucraient pas deux genres très différents apparlenant, l'un aux Foraminifères, l'autre aux Labyrinthulés. Il faudrait pour se prononcer, les étıdier au noureau point de vue proposé par Zopf et présenté par Cienkorslíy lui-même.

Une question non moins délicate se pose au sujet du genre

Chlamydomyxa (Archer) (fig. 76). Cet être qui a reçu le nom significatif de $C$. lab)rinthuloides (Arclier) a, à l'élat d'extension, la forme d'un Foraminifère dont le corps serail contenu dans une sorle de capsule cellulosique ouverte en fente au sommet et laisserait sortir un gros trone protoplasmique bientôt ramifié en pseudopodes réticulés absolument irréguliers. Le tout, en èlat d'extension, mesure environ un millimètre. Il n'y a point de noyau. mais des vésicules pulsatiles se rencontrent éparses cà et là dans la partie inférieure. Il se nourrit de proies solides qu'il absorbe à la manière d'une amibe. Plus souvent on le rencontre complètement renfermé dans son enveloppe cellulosique, et en cel état il peut aussi se rlévelopper.

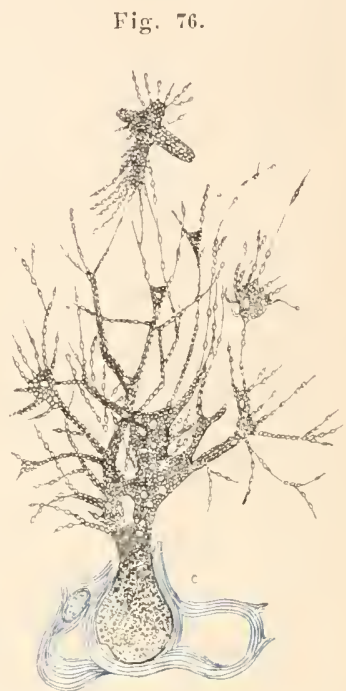

Chlamydomy.ra

(C. Tabyrinthuloüles)

(iim. Archer).

c., capsule cellulosique.

Jusqu'ici rien de bien étonnant. Mais, si l'on examine le réseau, on roit qu il est parcouru par de petits corps proloplasmiques, non nuclées, qui semblent glisser sur de fins filaments et ces fins filaments sont protoplasmiques et ressemblent absolument à ceux de Lalyrinthula.

Mais alor's que signifient ces résicules pulsatiles au sein de la masse protoplasmique? Que signifient ces particules englobées comme par une amibe? Tout cela est extérieur au corps rrai des êtres formant la colonie, et si les particules nutritives peuvent à la rigueur se comprendre, les vésicules ne le peuvent pas. Ray Lankester a suggéré l'idée que les petits fuseaux mobiles pourraient n'ètre que des noyaux. Il faut de nouvelles études avant de se prononcer (Colonie $1 \mathrm{~mm}$. Eau douce). 
3e () RDRE

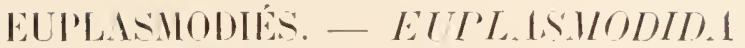 \\ [MYTOUYTETES (AUCt.)]}

TYPE MORPHOLOGIQUE

(FIG. 77 A 79)

Ici, comme dans le cas précédent, l'ètre, il l'élat adulte et isolé, est une simple annibe lobée el nucléce. Celte amile $($ fig. 77,1$)$ vilaumilieu des substances régélales altérées, hois pourri, lan, feuilles mortes, ele., circulant daus los interstices avee ses pseudopodes ol se nourrissint des particules assimilahles qu'elle rencontre. Linsi elle s’accroìl, se divise el se mulliplie avec arlivilé. Si les conditions leviennent défavorables, s’il survient par exemple de la sécheresse ou du froid, elle s'enkyste sous une membrane de cellulose. Au retour de conditions meilleures, elle sort de son kyste el reprend son mode de vie. Quand elle rencontre une autre amilse de mème espèce, elle passe indifférente, lant que la nourrilure est abondante. Nais quand celle vie a assez. duré, et principalement lorsune

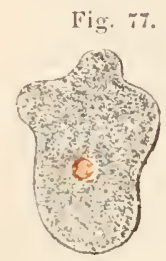

A
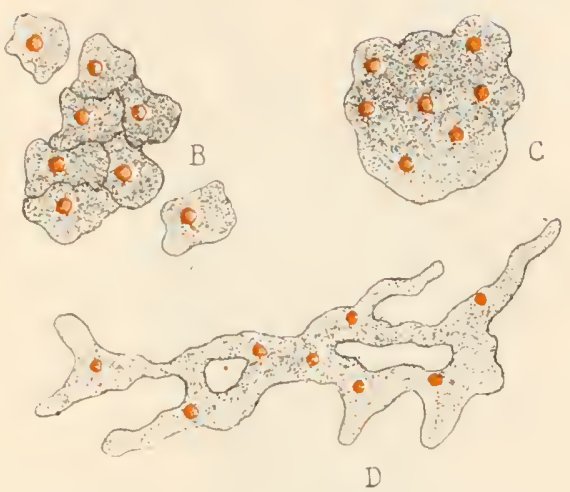

Euplasmodic (Type morphologique) (Sch.).

la nourriture devient rare, les amibes qui se rencontrent restent ensemble $(B)$ et, au lieu de s'accoler simplement comme celles des l'seudoplasmorlićs, se fondent entièrement en me amibe plus grrosse à deux noyaux. Cela continuant ainsi, les amibes arrivent à former les masses considérables $(C)$, reconvrant parfois plusieurs centimètres ou mème décimètres carrés. Leur vie n’est pas changée pour cela, el cet immense plasmode continue à vivre comme les amibes qui ont servi à le former. Il conlinue à se mouvoir alliré par la lumière failıle et l'humidité modérée. Quand il rencontre un obstacle, il s'ouvre devant lui, l'englobe, puis, quand il arrive à le dípasser, s'ouvre en arrière pour se dégager de lui. Cela lui donne une forme réliculée $(D)$ absolument variahle et irrégulière. Dans les mèmes condilions que les amibes isolées, les plasmodes s'enkystent. Mais ce n'est pas un enkystement in toto. La masse s'arrète, se condense, s'arrondit, puis se découpe en 
autant d'amilses qu'il y a de noyaux, et ce sont ces amilses qui s'enkystent côte à còte. Au sortir de l'enkystement elles se fusionnent de nouveau et

Fig. 78.

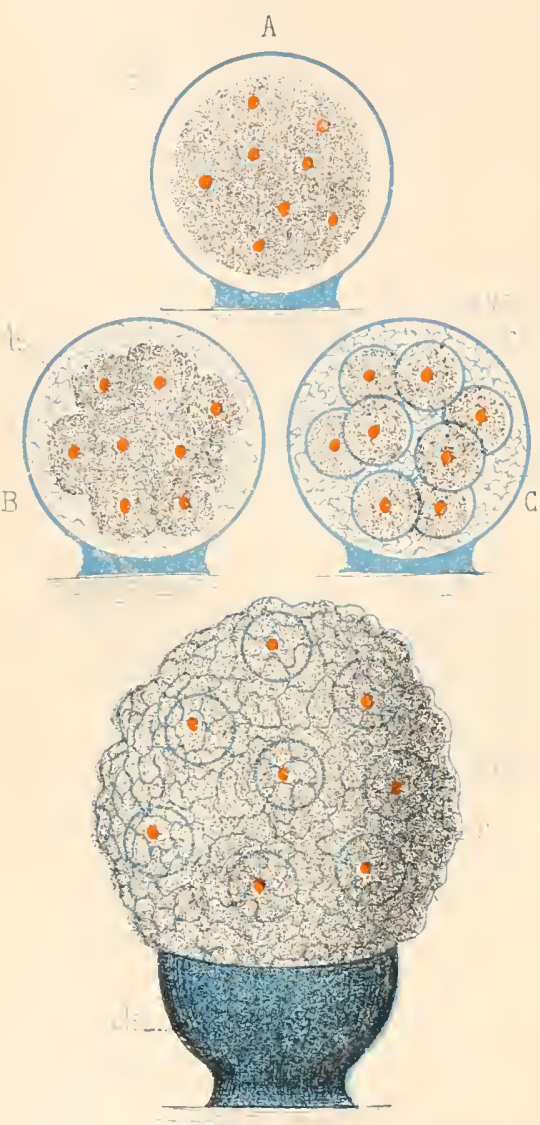

D

Euplasmodie : enkystement et sporulation (Sch.).

$A$ ì $D$, Stades sucessifs de la sporulation; els., kyste cellulosique commun; lxyr., kystes individucls; r., réseau.

(Dans ees fignres, on a supposé pour plus de clarté 'Iu'il n'existait qu'un trés petit nombre d'amibes fusionnés et par conséquent de spores). la masse reprend sa vie interrompue. Cet enlistement accidentel est de durée très variable. On en a observé qui avaient luré vingt ans.

A còté de cela, il y a un enkystement régulier (fig. 7s) auquel on donne encore le nom de sporulation, bien que la multiplication des individus nait lieu qu'au sortir de la prétendue spore. Pour cela, le plasmode commence par s’élever le plus haut possible sous l'action d'un géotropisme négatif accentué. Arrivé au point qui lui convient, il so concentre, prend une forme régulière $(A)$ et sécrète autour le lui une épaisse cronte collulosique $(c l s$.$) , souvent incrustée de cal-$ caire, qui se soude solidement au support. A l'intérieur, la masse se décompose, comme dans le cas jrécédent, en pelites amibes uninucléées ( $B)$ qui s'enkystent individuellement sous une membrane cellulosique ( $C$ : liys.), et la masse de ces kystes ou spores remplit la cavité de la coque cellulosique générale. Mais, comme cette épraisse coque serait plus lard trop résistante pour permettre aux spores de s'échapper, les amibes, avant de s'enkyster, sécrètent entre elles des filaments cellulosiques ramifiés qui forment dans la cavité un vaste réseau $(C: r$.$) dans les mailles du-$ quel les spores sont logées. Ces filaments prennent appui comme des ressorts sur la paroi interne du kyste général. L'humidité les relàche en les portant à s'enrouler, la sécheresse les bande en les étendant. Aussi lorsque le lyste ast mùr, sous l'influence de la sécheresse, ils se détendent, finissent par rompre la paroi devenue friable et, de la sorte, les spores sont mises en liberté. On donne le nom de capillitium $(D)$ à 
l'ensemble de cet appareil de filaments destiné à produire la rupture du kyste.

Sous l'influence de l'humidité, les spores (fig. 79, a) mises en liberté laissent échapper leur contenu qui sort sous la forme d'une petite masse d'abord inerte (b), mais qui lientôt pousse un court flagellum à l’extrémité qui porle le novau, forme une vésicule pulsatile à l'extrémité opposíe el se met à nager el à ramper dans le liquide $(c)$. Ju bout de peu de temps, le flagellum disparait et l'amibe riduite i ses pseudoporles recommence la vie que nous avons lécrite.

$$
\text { Fig. } 79 .
$$
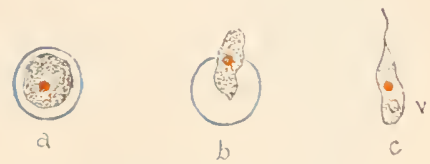

Euplitsmodié.

Formation de l'amibe (Sch.).

a, spore; b, sortie de lanibe; c, l'amibe pourvue diun flagellum et dune vésicule pulsatile v.

\section{GENRES}

Ce qui est variable ici, c'est surtoul la forme des livstes et le capillitium. Ciest principralement sur ces caraclères que les genres sont élalilis.

Lycea (Schrader) n’a pas de capillitium et la double membrane de son kyste se délruit entièrement pour donner issue aux spores. La forme des kystes est aussi simple que possible, elle représente celle du résean plasmodique vivant, mais arrèté et enlonré d’une double membrane.

Tubulina (Persoon) (fig. 80), a un kyste qui forme des petits

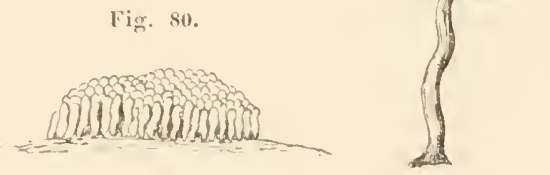
boullins implantés côle à còte sur un support qui lépend de lui. Limbladia (Fries) est un genre roisin.

Cribraria (l'ersoon) (fig. SI) n'a pas non plus de capillilium, mais le kyste spliérique, pédonculé, au lieu de s'ourrir par destruction totale de sa paroi, ne se détruit que dans les mailles d'un réseau dont les filaments persistent. Cela tient ì ce que la paroi lystique est parcourue en dedans par un réseau de banles d'épaississement qui résistent quand les parties minces siluées entre elles se ilétruisent à la malurité. On a assimilé, sans grande apparence de raison, ces bandes d'épaississement ì un caprillitium qui serail soudé à la paroi du kyste.

Dictydium (Schrader) (fig. Sz),

Enteridium (Ehrenlierg) et

Clathroptychium (Rostafinslii) présentent le mòme caractère. Nous placerons ici le genre

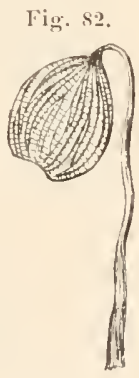

Dictydium

(D. cernuum) (im. Zopf). 
Myxastrum (Häckel) (fig. 83) qui se sépare les l'rotéomyxés par son mode de reproduction. Il est représenté par une amibe à pseulopodes rayon-

Fig. 83.

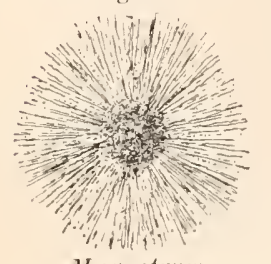

Myxastrum

(d’ap. Ilickel) nants et, l'après Iläckel, non nucléée. Les individus se réunissent en plasmodes pourant atteindre un demimillimètre de diamètre, ce qui leur permet d'absorber des proies plus grosses (Péridiniens, Diatomées, etc.). A un moment donné. ce plasmorle s'enkịste el divise son contenu en une cinquantaine de portions disposées radiairement. Ces parties se revêtent d'une épaisse membrane kystique el, par là, se révèlent comme des spores qui, mùres, s'ouvrent au sommet pour laisser échapper leur contenu sous la forme l'une amilie (').

Partout ailleurs il existe un vrai capillitium.

Cet organe se présente sous sa forme la plus simple chez

Trichia (Haller) (fig. S4) où il est formé de simples filaments libres en forme de tubes creux arec des épaississements spiraux. Cliez

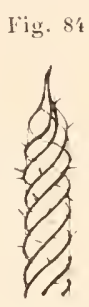

Trichia
Fig. 85.

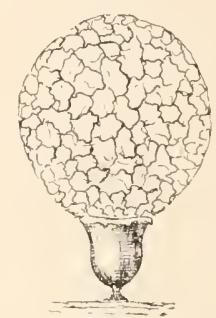

Areyria (1. incarnata) (im. de liary).

Arcyria (llill) (fig. 8̈3), au contraire, le capillilium forme un réseau soudé, au point où il la rencontre, avec la paroi interne du kyste. Celui-ci est pédonculé el s'ouvre en pyxide (*).

I la suite de ces genres, se présentent quelques formes qui ressemblent fondamentalement à Arcyria, mais qui s'en dislinguent par le caractire suivant: les noeuds lu réseau du capillitium sont volumineux et remplis de petits nodules calcaires, et des nodules semblables se rencontrent dans l'épaisse paroi du liyste. Tels sont:

Craterium (Trentepol) (fig. S6), dont le liyste a la forme l'une praile pédonculée et s'ouvre de la mème manière, et dont les spores violelles occupent, comme d'ordinaire, les mailles du réseau $\left({ }^{*}\right)$; ch

Fuligo (Haller), yui est le plus célèbre des Myxomycèles.

Fig. 86.

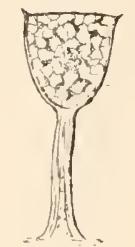

Craterium (C. sulgare)

(im. Zupf). C'est lui qui, le premier, a attiré les regards par ses limensions consi-

(1) Cet être a élé observé en 1867 par Häckel à l'ìle Lancerotte, aux Canaries. La seule espèce est $\boldsymbol{M}$. radians (IIäcliel). Les spores sont mises en liberlé par simple rupture du kyste sans capillitium.

(2) Le capillitium est conformé d'une manière analogue dans les genres

Hemiarcyria (Koslafinshi),

Reticularia (Bulliard),

(3) Genres voisins :

Physarum (l'ersoon),

Tilmadoche (Iries),

\section{Cornuvia (Rostafinsti),}

Lyoozala (Chrenherg),

Badhamia (Berkeley),

OEthaliopsis (Zopil).
Perichæna (Fries),

Lachnobotus (Files). 
dérables. Il forme i la surface des amas de tan ces fleurs du tan qui ont jusqu'à deux à trois lécimètres de large et deux à trois centimètres l'épaisseur. Ses kystes discö̈rles sont protégés par une épaisse croùte remplie de petits nodules calcaires semblables à ceux les nouds du capillitium.

Une derniere complication du capillitium résulte du prolongement du pédoncule dans la cavité du kịste en unc sorte d'axe central appelé columelle d'où partent les branches du réseau pour s’insérer d'autre part ì la paroi interne du kyste.

Stemonitis (Gleditsch) (fig. 87), donne une bonne idée de cette disposition. Ici, les spores redevienment claires et les nodules calcaires sont alisents ('). Chez

Amaurochete (Rostafinski), les columelles deviennent nonlreuses et putites, en sorte quielies se distinguent ì peine du réseau et lisparaissent presque. On pourrait donc placer ce genre à côté d'Aremria.

Combinons maintenant les caraclères de s'temonilis avec ceux de Croterium, c'est-it-dire une columelle it no!lules calcaires avec les spores violettes et nous aurons: soit

Didymium (Schrader) (fig. Ss), soit

Chondrioderma (Rostilinski), it colunelle courte et renflée $\left({ }^{*}\right)$.

Il faut faire une place it part pour le genre

Ceratium (Alberlini el Schweinit\%) (fig. 91), qui mériterail de s'opposer comme genre unipue d'un groupe des Exosporés à tous les autres Euplasmodiés formant ensemble un groupe des Endosporés. lei, en effol, la sécrélion durcie qui correspond au kiste est centrale et sert seulement de support aux spores qui sont libres à sa surfacr. Cette sécrétion est gélatineuse. Elle forme une sorte de gitteau (A) sur lequel s'élèvenl, soit des lamelles, soit de petites tiges (B). Tout ce minime édifice squelettique est revètu du plasmodium qui l'a sécrélé et qui forme i

(1) Il en est de mème chez Comatricha (Preuss) (fig. 88), Lamproderma (Rostafinsky), Enerthenema (Bowman).

(2) Soit un des genres: Lepidoderma (de Bary) (fig. 90), | Spumaria (Bulliard),

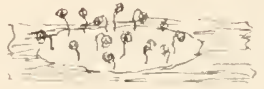

A

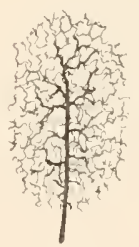

B

Comatricha (. Friesiana) (im. Zopf).

$A$, l'ensemble; $B$, un capillitium.
Fig. 88.

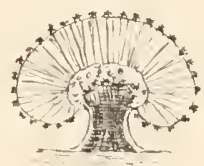

Didyminm

(D. farinaceum)

(im. Kopf).
Fig. 87.

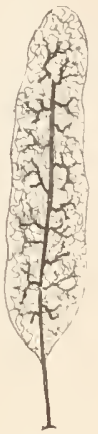

Stemonitis 5. formginea) (iin. $\%$ opl) 
sa surface une simple couche protoplasmique parsemée de noyaux. Quand tout cela est prêt, le plasmodium se divise, comme d'ordinaire, en autant de parcelles uninucléces qu’il y a de noyaux: comme d'ordinaire, ces parcelles s'enkystent sous une enveloppe cellulosique et constituent les prélendues spores; mais charune de ces spores, avant de s'enkyster, sécrète encore un minime péloncule gélalineux, au bout duquel elle reste appendue, comme un petit fruit (C). On trouve des kystes exosporés gros de quelqnes millimètres, sur le bois mort des Conifères. A la maturité, et lorsque la spore a acquis sa taille définilive $(I I)$, tout le squeletle gélatineux vient ì être dissous par une goutte d'eau et les spores tombent $(E)$. Quand elles sont mirres ellesmêmes, elles laissent échapper leur contenu sous la forme l'une petite amibe $(F)$. Mais celle-ci, au lieu de commencer tout de suite la vie errante habituelle, se divise successivement trois fois $\left(r_{i}, I I, l\right)$ et

Fig. :11.

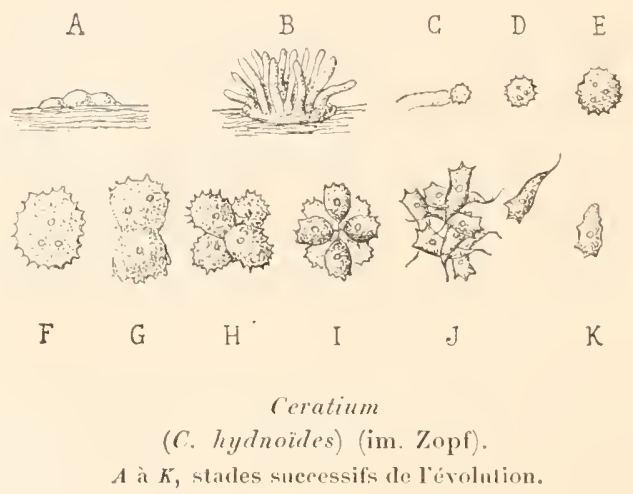

donme ainsi huit petites zoospores munies d'un flagellum (.J). Mais lientô, comme chez les Pseuloplasmorliés, ce llagellım lisparait et les petiles amibes $(K)$ se dispersent en quète de nomriture, et se multiplient par division simple jusqu’à ce que soit arrivé pour olles le moment le s'unir, comme d'ordinaire, on un véritable plasmodium qui sécrète de nouveau un support le gélatine et recommence la série de phénomènes déjà décrite.

Il y a ici, peul-ètre, un acheminement vers la constitulion de véritaliles spores auxquelles il ne manque, pour míritre toul à fait ce nom, que de se diviser sous leur enveloppe an lieu de ne le faire qu'apries. 
$3^{\text {e SOUS-Citasse }}$

AMCEBIENS. - A.MCEBI.E

[A.иezis.s (Desardix, emend.)]

Les Amorbiens, caractérisés essentiellement par leurs pseudopodes lobés, obtus, non anastomosables, se divisent en deux ordres:

liruvanosblexs ou Amobiens nus,

THECAUHEREs ou Amobiens à carapace.

$1^{\text {er }}$ ORDRE

A.MEBIENS NIS. - GIMNIMUEBIENS. - GYMIIMURID.

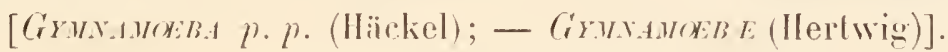

TYPE MORPHOLOGIQUE

(FIG.92 A 102)

Il se troure ici que le type morphologique est représenté par le genre riel Amolya. L'Amibe n'est pas le plus simple des Rhizopodes, mais, parmi les formes simples, il est une des plus typiques. Aussi méritet-il d’otre étudié avec quelque détail.

\section{Structure.}

Constitution générale. - L'Amilse (fiğ. 92) est un pelit ètre microssopique ou à peine visible à l'wil nu, incolore el si admirablement transparent quil a l'aspect d'un fragment de cristal. Sa forme est irrégulière el on ne saurail la préciser, car elle change à chaque instant. Cependant ces changements ne sont pas quelconques et, au milieu de ses variations, elle garde un facies qui permet de la reronnaitre. Sa forme dérive toujours d'un sphérö̈đe déformé par des lobes irréguliers et olslus, peu ou point ramiliés, qui partent des divers points de la surfaco. Ces lobes sont les pseudopodes (Iseudop.).

L'ètre se compose simplement d'un Fig. 92.

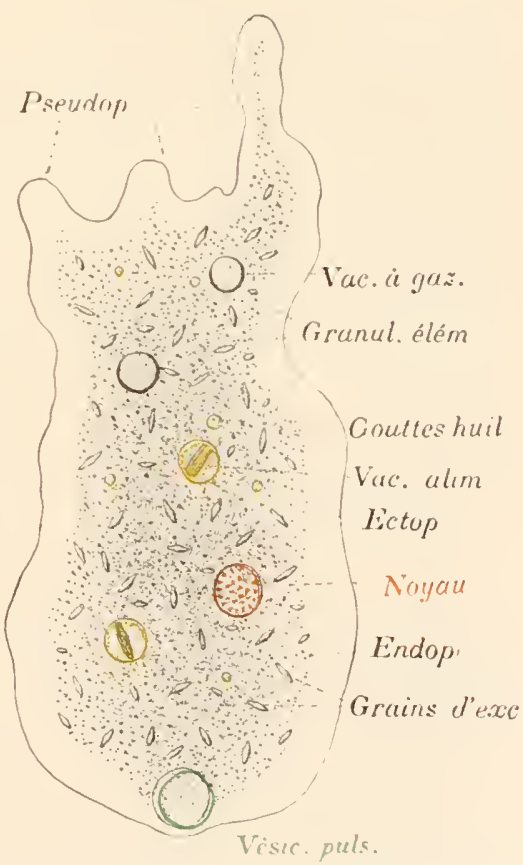

Gymnamobien. - Type morphologique. (Amaba) (Sch.).

Les lettres se passent d'explication. noyau (Noy.) el d'une petite masse nue de cytoplasma. Mais, dans ce 
cytoplasma, on peut distinguer deux courhes: un ectoplasma hyalin (Ectop.) et un endoplasma granuleux (Endop.) contenaut le nombreuses inclusions. Dans l'endoplasme, mais tout près de la surface, est une vésicule pulsatile (I'́sic.puls.).

Noyau. - Le noyau n'offre rien de bien particulier daus sa structure. Il est conforme, en ses traits essentiels, à celui du tyje général des Rhizopodes que nous avons précédemment décrit (').

Endoplasme. - L'endoplasme est formé d'un protoplasma fluide parsemé de granulations élémentaires ou microsomes (Granul. élém.), tels que nous les avons décrils dans la premicre partie de ce volume en parlant le la structure Ju protoplasma en géméral (p. 6). Il contient, en oulre, des parties très varićes désignées en hloc sous le nom l'inchsions endoplasmiques. Ce sont ici: des grains d'excrétion (Grains d'exc.), des vacuoles alimentaires (I'ac. alim.), des globules graisseux (Goultes huil.) et des vacuoles ì gaz (Vac. ì gaz).

Los grains d'excrétion sont beaucoup plus volumineux que les granulations élémentaires. Ils sont foncés, de couleur Jrunc ou verdàtre, de forme variable, souvent cristalline, et paraissent formés de pramides groupées ensemble. Ils ne sont pas de nature graisseuse, car ni l'alcool ni l'éther ne les dissolvent. Bïtscul les croit formés d'acide oxalique, Extz, d’acile urique. Is semblent ètre, non des résidus de la digestion, mais des excreta dus à la désassimilation. Ils sont naturellement très variables comme nombre el position ct peuvent mème manquer tout à fait à cerlains moments.

Les vacuoles alimentaires ne sont naturellement ni fixes ni constantes. Elles se forment autour des particules alimentaires au moment où celles-ci sont englobées, et disparaissent lorsfue leur digestion est achevée. Elles sont notablement plus grandes que l'aliment qu'elles renferment, contenant en outre un suc digestif dans lequel flotte celui-ci. Leur volume est donc variable ainsi que leur nombre et souvent on peut n'en rencontrer aucune.

Les globules graisseux, reconnaissables à leur solubilité dans l'alcool

(1) Le noyau n'est pas constant chez les Amobiens. Quelques genres (Prolameba, Gloidium) sont complés parmi les Monires. D'autre part, certains Imibes, renferment plusicurs noyaux et même un grand nombre. Ce nombre est très variable en lui-mème mais, dans chaque espéce, il est fixe, lu moins tant qu'il n'est pas trop élevé. Quand un Amibe est normalement uninuclée, il n'a jamais qu'un noyau; ceux qui en ont deux ou trois ne varient guére non plus sous ee rapport; mais au delit, il n'y a point de fixité: A. alba en a de 10 à 20, A. fibrillosa de 20 à 200 ; ehez une forme géante trés voisine des Amibes, Petomra riridis qui atteint 2 millimètres, Boukxe [91] en a trouvé jusıuá 1000. En général, il y en a d'autant plus que l'animal est plus volumineux. Le nombre de noyaux est dicté par la nécessité d'un rapport assez fixe entre leur masse el celle lu cytoplasma el, d'aprés Bounse, ce rapport serait d'environ 160 comme dans les eufs des Manmifères. Hans les formes polynuclées, les individus jeunes n'ont d'abord qu'un noyau qui se multiplie à mesure que l'animal grandit. 
et l'éther, sont très petits ( 1 à 2 \%.). Ce sont des produits de réserve, variables comme sont loujours les substances de cette nature. Souvent on n'en trouve pas trace; d'autres fois ils sont très nombreux.

Les vacuoles à gaz sont des lacunes adventives qui se forment dans le cytoplasma, là où une substance s'est légagée à l'élat gazeux. Leur gaz est l'acide carbonique comme le montre son absorption par les alcalis. Cet acide est, comme toujours, un produit d'excrétion respiratoire.

Vésicule pulsatile. - La vésicule pulsalile est, au contraire, une formation permanente. On ne l'a pas trouvée chez toutes les espèces, mais il en est peu che\% qui elle manque. Elle est rolumineuse, plus grosse que le noyau, ordinairement unique, parfois multiple, mais jamais ii n'y en a un très grand nombre. Elle est située clans la région du corps qui est postérieure dans la marche, et forme souvent une saillie it la surface. Elle appartirut à l'endoplasme mais, en raison de sa position superficielle, elle déprime l'ectoplasme qui est for't aminci à son niveau. Elle est animíe de deux mouvements inverses, l'mn de diastole lente pendant lequel elle se remplit, l'autre de systole brusque où elle disparait lout à fait pour un instant.

On admet généralcment qu’elle communique avec le dehors, soil par un pore permanent, soit par une mullitude de petits orifices ultramicroscopiques persant sa paroi externe comme une écumoire, soit par une rupture de celle paroi qui se ressouderail aussitôt. Mais il faut reconnaitre que, le plus souvent, on ne roil d'orifice à aucun moment, en sorte que sa communication arec le dehor's, si évilente chez les Infusoires ciliés, est ici un peu problématique. l)'apress quelques observaleurs, la vésicule, en se contractant, se viderait non au dehors, mais dans l'endoplasme. Nous aurons à revenir sur ces interprétations en parlant de la physiologie de l'animal.

Nous ne ferons que signaler les corpuscules brillants (') et autres

(1) Les corpuscules brillunts (fig. 93) (Glanzkörperchen), découverts d'abord par Greerf chez une forme voisine des Amibes, Pelomyar ont été retrouvés par Péxaro [no] dans quelques Amibes (.1. Proteus) (fig.9'). Mais ils sont loin de se rencontrer chez toutes. Ce sont des globules brillants, plus gros que les granules d'excrétion, formés d'une enveloppe et d'un contenu finement granuleux. Les petits granules arrondis ou en forme de bitonnets cylindriques arrondis au bout, que l'on croyait faire partie de leur structure, sont simplement des Bactéries aceolées à leur surface (Go[LD [9] ). P'arfois, les corpuscules brillants sont en biscuit comme s'ils itaient au moment de se diviser. On les considère connme Fig. 94. étant peut-être des spores et nous verrons (p. 98, note) 'juel róle on voudrait leur fatire jourer dans la reproduction. 
parties accidentelles qui peuvent se rencontrer dans le cytoplasme : particules étrangères, résiłus alimentaires, grains de chlorophylle (').

Ectoplasme. - L'ectoplasme forme une hordure continue sur toute la surface de l'animal. Il se distingue de l'endoplasme, non seulement par l'absence de granulations qui a pour conséquence son aspect hyalin, mais aussi par une structure plus ferme $\left(^{*}\right)$. Il ne montre dordinaire ancune structure même aux plus forts grossissements $\left(^{\mathbf{3}}\right)$. On n'y retrouve mème pas les microsomes habiluels. C'esł en lui cependant que semblent résider principalement la contractilité et le pouvoir d'émettre les pseudopodes. Ceux-ci (fig. 9:i) sont des prolongements en forme de lobes obtus peu ou point ramifiés, non anastomosables, se formant en un

(1) Des graius de sable ou des particules étrangères quelconques sont souvent absorbés par l'Amibe, en quelque sorte par erreur, ou pour tirer parti de quelque minime parcelle organique digestible qui s'y tronvait accolée. lci, éest bien par accident que ces corps étrangers sont incorporés, ear ils sont évacués ensuite comme des fèces et abandonnés, mais nous verrons que d'autres Rhizopodes se servent de ces particules pour se constituer une enveloppe protectrice.

Les fèces (carapaces de lliatomées, résidus divers) peuvent être cités dans la même catégorie.

Les grains de chlorophylle proviennent de fragments de cellules végétales ingérés à titre d'aliment. Les grains d'amidon que l'on trouve quelquefois ont une origine semblable. On ne voit pas, ici comme chez d'autres Rhizopodes, des Zoochlorelles, petites Algues vertes unicellulaires (Palmellacées sans doute) continuer á vivre dans le cytoplasma et peut-être y former de l'anidon, montrant ainsi un cas de symbiose intracellulaire.

(2) D'ordinaire, il n’est lans toute sa masse qu'une couche périphérique, moins fluide que la substance intérieure; mais parfois, il se condense en une pellicule qui a tout l'aspect d'une vraie membrane très délicate, bien qu'elle ne soit en réalité qu'une membrane protoplasmique plus individualisée que d'ordinaire.

Ce dernier cas se rencontre surtoul chez les Amibes terrestres (GrenfF [91]) et cela se concoit, car un animal terrestre est exposé a de plus rudes contacts que celui qui vit dans l'eau. Mème, cette membrane deviendrait parfois, chez ces Amibes terrestres, une vraie cutirule, ear Greeff dit l'avoir vue rejetée par une mue.

(3) Cliez les formes aruatiques, à peudopodes fluides, l'absence de toute structure fixe parait démontrée par le mode de formation des pseudopodes comme nous le verrons plus loin. Cliez les formes terrestres, Greeff aurait vu dans l'ectoplasme une multitude de petites fibrilles contractiles disposées radiairement, insérées aux surfaces externe et interne de l'ectoplasme et chargées de le mouvoir et de former les pseudopodes. Ces fibrilles, n'ayant jamais été vues avant l'intervention des réactifs, pourraient bien n'être qu'un produit artificiel. Mais il se peut aussi qu'il y ait là une différenciation réelle, les formes fluides ayant un ectoplasme sans structure, les formes terrestres ayant de vraies fibrilles contractiles, et les formes arquatiques fermes présentant une structure intermédiaire.

llans certains Amibes, surtout les terrestres à ectoplasma ferme, la partie superficielle de l'endoplasme se montre plus dense, moins mobile que la masse centrale et semble former une couche ì propriélés physiques et physiologiques intermédiaires. Cette couche a recu le nom de mésoplasme; elle correspond sans doute a ce que nous étudierons plus tard chez les lnfusoires sous le nom de plasma cortical. Mais elle manque le plus souvent et il ne semble pas, en somme, y avoir bien grand intérêt à en tenir compte. 
point quelconque, puis rentrant dans le corps sans laisser de traces. (Quand ils sont petits, peu épais, ils sont formés par l'ectoplasme seul. Mais quand ils sont plus larges, l'ectoplasme ne forme, comme ailleurs, que leur bordure périphérique, et l'endoplasme a accés à leur intérieur, d'une manière d'ailleur's toute passive. Ces formations constituent le trait le plus caractéristique de l'animal. Mais nous ne pourrons faire bien comprendre leur manière d'ètre et leur mode de formation qu'en étudiant leur physiologie.

\section{Physiologie.}

Locomotion. Pseudopodes. - La locomolion a lieu par les pseudopodes. D'ordinaire, le piseudopode (fig. 9̈̈) n'est qu'un prolongement de l'ectoplasme qui se déforme pour le constituer, mais sans modifier l'aspect de sa substance ni rompre sa continuité. Il s'élève en une saillie qui s'étrnd de plus en plus du côté où l'animal veut s'avancer ('). Tantôt il est long, élroit, digitiforme et formé par l'ectoplasme seul; tantôt il est large et, dans sa cavité, l'endoplasme a plus ou moins largement accès $\left({ }^{2}\right)$. Quand il se rétracte, il ne rentre pas d’ordinaire dans le corps, car alors celui-ci ne serait pas entrainé. C'est le corps qui s'avance vers le pseudoporle et sı fond avec lui. De là résulte un déplacement en masse. Puis un autre pseudoporte se forme qui se comporte de mème, et ainsi de suite. Mais ces phénomènes sont simultanés et non successifs et il y a, à la fois, plusieur's piseudopodes à différents étals, et progression plus ou Fix. 45. moins continue de la partie postérieure du corps $\left({ }^{3}\right)$.

(i) Les fibrilles de Gressr, si clles sont réelles, jouent sans doute un ròle dans ce mouvement.

(i) Il en est ainsi chez 1 . princeps (fig. 95). D'autres fois, chez 1. Limax (fig.96), par exemple, le pseudopode est aussi large que le corps luimême en sorte qu'il parait ne plus exister et que le corps semble s'avancer par une rep-

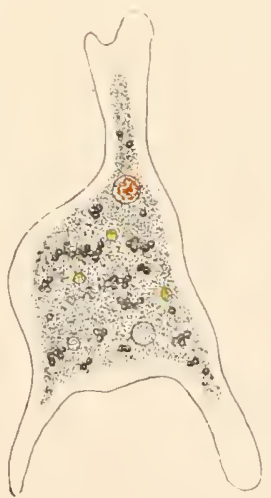

Amaba princeps (Sch.).

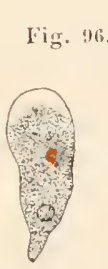

lig. 97.

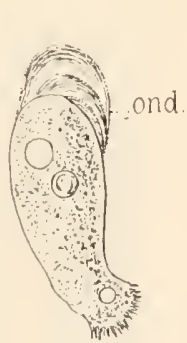

tation d'ensemble.

$\left({ }^{3}\right)$ Ciette sorte de pseudopode se rencontre toujours chez les formes terrestres et souvent chez les aquatiques. Mais d'autres fois, chez ces derniers, le mode de formation est tout autre. Il a été bien observé et décrit par P'évaro [90] chez A. undosa (fig. 97). $\Lambda$ la partie antérieure, l'ectoplasme se ramollit brusquement et, par ce point de moindre 
i.es pseudoporles ont en outre des mourements indépendants, ils peuvent s’infléchir de tous côtés. Le corps lui-mème peut so lordre de diverses façons.

Tous ces mouvements sont très lents et leur vitesse est fort variable selon les espèces of l'élat d'excitation de l’indivilu.

La direction du mourement est constante, au moins pendant quelpue temps, en sorte que l'on peut distinguer chez l'Imibe une partie antérieure où se forment les pseudoporles et qui détermine le mourement, et une postérieure passive qui se laisse entrainer. Mais ces parties n'ont aucune différence proformle entre elles, rar souvent la dirretion du mouvement change par le seul fail que c'est un autre point du corps qui devient le lieu de formation des pseudopodes.

Pendant que l'Amibe est en marche el que les parties antérieure el postérieure sont bien distinctes, on olsserve généralement à l'extrémilé postérieure une particularité singulière. Cette partie du corps est plus glutineuse que le reste et sourent elle entraine, collés à elle, des déhris de nature variée; elle se colle au sol et s'en délache lorsque son adhérence est vaincue par la traction de l'extrémité antérieure; elle est ainsi entraînce par petites secousses saccadées (').

resistance, sous l'influence de la pression intérieure, une vague de cy toplasma liquirle fait éruplion au dehors et s’épanclıe á la surface externe de l'Amibe. Mais, presque instantanément, a u contact de l'eau ambiante, ee eytoplasma lịuide se solidifie et en mème temps se sourle i l'eetoplasme qu'il recourre et se confond si bien avee lui qu'on ne peut plus l'en distinguer; en sorte que tout se retrouve dans le mème élat qu'au-

Fig. 98.

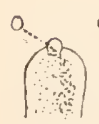

A.

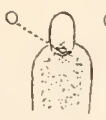

$B$

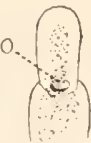

C

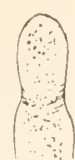

D
Formation d'un pseudopode chez A. Proteus (im. Pinard). paravant, sauf que l'Amibe s'est un peu avaneé. Mais bientôt une autre vague fait éruption, se eomporte de mîme, et ainsi de suite. Le corps s'arance par vagues successives projetėes et solidifiées.

D'autres fois, comme chez 1. Proteus (fig. 98), c'est le même phénomène mais, au lieu d'une large vague s'épanchant par une large fente, e'est une fuséc de liquide faisant éruption par un petit trou.

Péxard a nettement vu ee petit trou $(\mathrm{A}, \mathrm{B}, \mathrm{C}: 0)$ se former et rester quelques instants visible dans l'ectoplasme ferme; mais, dès que la fusée liquide s'est solidifiée en un pseudopode allongé, la soudure esl si intime au point d'union que toute trace de ectle disposition transitoire disparaît complètement (1)).

Blochmann [9'] a observé (ehez $A$. secunda et chez Pelomyxa) un autre mode de mourement dans lequel le eorps glisse par une sorle d'éeoulement continu de sa substance le long du support. Les moléeules de protoplasme de la couche superficielle du corps se jortent darrière en avant, ee qui prorluit un déplacement total de la surface sur le support. L'animal parcourt ainsi jusiu’a $0^{\mathrm{mm}}$ 弓̆ par minute. Il y a, enoutre, un mouvenent de l'endoplasma qui circule d'arriére en arant dans ses parlies centrales et d'arant en arrière dans sa eouche superficielle. Blockmann l'interprète romme une sorte de cyclose qui n'aurait rien de commun avee le mouvement préeédent, landis que PenAkD, ainsi que nous allons le voir dans un instant, le considère comme déterminé par eelui-ci.

(1) C'est à cela peut-être qu'est due la formation i cette extrémité de la houppe 
Dans les mourements de l'Amibe, l'endoplasme est passif. Mais il n’est pas immobile pour cela. En avant, où les pseudopodes en s'arançant font le vide derrière eux, il se précipite pour combler ce vide, et on arrière il est sans cesse poussé par la progression de la partie postírieure. Ce mourement est rendu visible par celui des granulations ef surtout des grains d'excrétion plus faciles à voir (fig. 99). Il est naturellement beaucoup plus accentué chez les espèces les plus fluiles. Chez 1. proteus, par exemple, on voit ces grains ainsi lancés d'arrière en avant, rehontir contre la paroi antérieure et attendre là, immobilrs, que l'extrémité postérieure les ait rejoints pour les lancer en avant de nouveau.

Fig. 99.

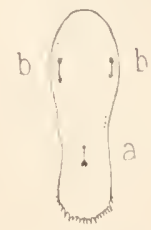

Monvemenls de lendoplasma chez 1. Proteus (im. Pénard).

C'est aussi vers l’extrémité inféricule que le noyau et surtout la résicule pulsatile se trouvent, le plus sourent sinon toujours, relégués.

Préhension des aliments. - Les pseudopodes des Amibes ne sont pas, comme cenx de tant d'autres Rhizopodes, les agents de la capture des aliments. Il peut leur arriver de saisir une proie, mais cela est rare. Leur fonction normale est la locomotion. Quant à la préhension des aliments elle se fait sclon le mode que nous arons décrit clez lo lype général des Rhizoporles, et par lous les points de la surface, priucipalement dans celtr région postérienre grhlineuse dont nous venons de parler (").

Digestion. - La digestion se fait encore suivant li mode que nous avous décrit à propos du type général des Rhizopoles. C’est ici principalement que Metchxisof et Li: Daxtec ont ru le liyuide de la vacuole devenir acide el la particule se dissocier et se liquéfier lentement. Au hout d'un certain temps, parfois plusieurs jours si la proie est grosse et indigeste, il ne reste plus qu’un résidu inutilisable que l'animal rejette par une série d'opérations qui répètent en sens inverse celles dr la capture. I'endant le temps de la digestion, la racuole alimentaire circule dans l'endoplasme

postriteur (fig. 100, p.), sorte de chevelu ectoplasmique qui semble formé de petils pseudopodes filiformes, mais jiseurloportes passifs, en quelque sorte, formés comme par un étirement de la substance réluctante il l'entrainement. D'autres voient dans cette houppe une formation active intermédiaire à des pseudopodes filiformes et à des flagellums.

(1) Cliez les Amilses à ectoplasme mou, le phénomène se comprend sans difficulté. Mais dans les cas oǹ il est différencié en membrane, Fig. 100. ou lor'sqüil y a une vraie cuticule comme chez les Amibes terrestres observés par Greetf, que se passe-t-il? Il est probable que, dans ce cas, la culicule se perce, la membrane se gélifie au conlact de la proie el sans doute sous son influence, et ne reprend ses caractères qu'après l'avoir laissée passer. La formation des pseudopodes chez $A$. proteus (p. 93, nole 3 nous a montré un phénomène analogue. 
comme les autres granulations, mais ne s'avance janais bien loin vers la région antérieure.

La nomrriture habituelle des Amibes consiste en régétaux, en Infusoires ou Rhizopodes de petite taille, et en débris de toutes sortes, animaux ou végétaux.

Les granules graisseux sont des produits d'ume alimentation exubérante mis en réserve pour les périodes de jeùne. Les grains d'excrétion sont des produits de désassimilation.

Respiration. - Cette fonction s’accomplit sans organes spéciaux par toute la surface du corps. Peut-ètre cependant la vísicule pulsatile contribue-t-elle, ici comme clıez les Ciliés, à fournir de l'oxygène aux parties profondes du corps.

Circulation. Excrétion. Vésicule pulsatile. - Ces fonctions sont encore le sujet de discussions, car ici les avis sont partagés, les auteurs voyant dans la résicule pulsalile, les uns un appareil circulatoire, les autres, ull organe excréteur.

Nous avons vu que la résicule se gonlle par une lente diastole et se ville par une systole brusque.

Mlais où évacue-t-elle son contenu?

Les uns pensent qu'elle l'épanche à l'intérieur', sous l'ectoplasme et constitue ainsi une sorte d'appareil circulatoire. Les autres admettent qu’elle le répand au dehors el sert ì expulser les produits usés. s゙il en est ainsi, elle loit aussi servir à la respiration car l'eau qu'elle évacue, venue du dehors (par osmose et avec les aliments), était chargée d'oxygù̀ne lorsqu'elle est entrée dans l'organisme. Apròs beaucoup de discussions et d'observations contradictoires, il semble s'útablir que la vésicule, ici comme chez tous les autres Protozoaires, s'ourre simplement à l'extérieur. Mais il n'y a pas d'orifice permanent. A chaque systole la paroi se déchire et se reconstitue par soudure parfaite imnédiatement apriss (').

(1) D'ordinaire, on admet que la vésicule se videà l'extérieur et constitue un organe excréteur : l'eau pénètre par osmose dans l'Amibe par lous les points de sa surlare, se charge de tous les produits de dénutrilion en les dissolvant et est expulseie rhythmiquement par la vésicule. Cette opinion s'appuic sur des observations où l'on a vu (ou cru voir) la vésicule se vider au dehors, et sur une remarque de Brandt qui a vu l'hématoxyline absorbée par l'animal arriver dans la vésicule et y prentre la teinte brume que lui communinuent les acides.

l'our Crapartide el Lachnanx, au contraire, la vésicule se vide dans l'endoplasme et constitue un appareil circulatoire. GreErF, PÉNand sont du mème avis. Celui-ci constate fue l'on ne voit jamais, au moment de la systole, une projection les frarticules flottantes lu liquide ambiant, projection qui ne manquerait pas de se produire dans ces circonstances. Le premier lait remarquer que la vésicule a souvent, clıez les Amibes terrestres, un volume égal au quarl de celui du corps el se vide tous les ruarts l'heure, en sorle que, si elle se vidait au dehors, l'exerition éliminerait toutes les heures un volume de lippuide igal à eclui de l'Amibe, ce qui. d'après lui, serait inadmissible. L'un ef l'autre ont vu yu'au moment où la visicule a rlisparu, à la 
Reproduction. - L'Amibe se reproduit essentiellement par division tantôt directe, tantôt indirecte, tantôt intermédiaire ì ces deux modes (').

En outre de ce mode de reproduction, habitucl et incontestahle, on a observé l'enkystement et la conjugaison, phénomènes qui, d'ordinaire. chez les P'rotozoaires, sont le prélude d'une division, mais qui, ici, ne semblent pas avoir cetle signification.

Enkystement. - Ce phénomène consiste d'ordinaire dans la sécrétion d'une membrane forte sous laquelle l'animal, arrondi en sphère, s'abrite pour se reposer, digérer à l’aise, ou se soustraire à des influences

suite de la systole, on observe dans l'endoplasme de nombreuses petites vésicules dissẻminées. Ces vésicules disparaissent peu à peu à mesure que la grosse se forme. Quand celle-ci est bien pleine (fig. 101, A), elle fait une forte saillie et n'est sépareje du dehors que par une paroi très mince; mais quand elle se contracte, elle ne fait pas éclater celte paroi, elle se vide en exprimant son contenu dans le cytoplasma ambiant, et c'est ce contenu qui, circulant dans tout le cytoplasme, se rassemble peu à peu dans les petites résicules disséminées, qui sont en

Fig. 102.

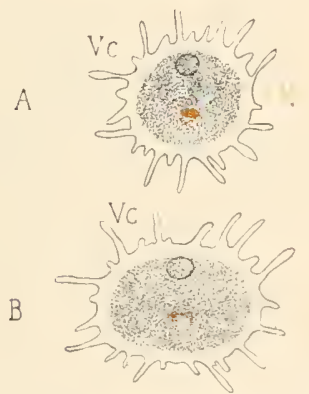

$c$

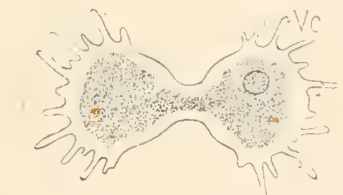

D

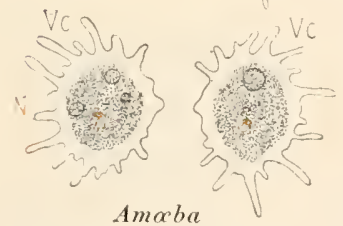

(Dactylosphara) (D. polypodia) (im. F.-E. Schulze).

$A$. B. $C, D$, phases sucecssives de la division amitosique.

Ve., Vésicule pulsatile. au pôle correspondant. Enfin, Mooke [93] a observé des mitoses alternant avec des divisions directes.

1. B. C. états successifs de la vésicule pulsatile ve. chez Amoba proteus

(im. Pénard). vésicule disparaitre progressivement par envahissement de sa cavité par le cytoplasme sous-jacent et a pu dessiner une phase de ce phénomène $(\boldsymbol{B}$ et $C$ ). Greefr assure que, chez les Amibes terrestres, qui ont une cuticule, la vésicule se vide sous la culicule en sorte que son contenu s'épanche sous la surface, ce qui est une bonne condition pour la fonction respiratoire. S'il en était ainsi, la résicule serait un organe non excréteur mais respiratoire et circulatoire. Mais les observations récentes de Büтschu, confirmées par Blocirmaxix [9'], nous ramènent à l'opinion la plus naturelle. Ce dernier a nettement vu chez un Amibe, la vésicule s'ouvrir au dehors à chaque systole.

(1) La division directe a été observée chez Amoba (Dactylosphire) polypodir par F.-F. Schulze, dontl'observation est devenue classique (fig. 102). C'est une division directe typique. Elle dure environ dix minutes. Son existence a été récemment confirmée chez A. crystalligera (Gruber) par Schaudive [9']. Ce même auteur $[95]$ a observé chez une autre espèce, $A$. binucleata (Gruber), une division intermédiaire à l'amitose et à la mitose, mais tenant davantage de cette dernière. C'est, en somme, une mitose mais sans centrosome, ni fuseau, dans laquelle les corps chromaliques, d'abord irréguliers de figure et de position, prennent la forme I'haltères et se disposent en plaque équatoriale, puis se coupent en deux moitiés dont chacune se rend

Fig. 101 .

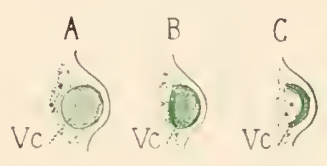


nocives (putréfaction, évaporation de l'eau), ou pour se diviser. Ici, l'enkystement est très sommaire: l'Amibe s'arrondit et reste immobile sous son ectoplasme devenu plus ferme el plus résistant, mais il n'y a pas de véritahle kyste. Sous l'influence d'excitations modérées, l'animal ramollil sa surface, élend des pseudopodes el reprend sa vie active, sans avoir rejelé aucune enveloppe; el on ne l'a jamais vu se diviser ni pendant ni immédiatement après celle phase de repos.

Conjugaison. - Il en est le même pour la conjugaison. Elle n'a été observée que très rarement et ne semble pas avoir ici la signification importante qu'on lui connaît chez les Infusoires par exemple (').

Habitat. - Les Amibes habitent la mer, l'eau douce ou la terre humide. Les terrestres se dislinguent par une texture plus ferme de leur ectoplasme el peut-ètre par la présence d'une cuticule. Quelques-uns vivent en parasiles dans le corps des animaux. A. intestini vulgaris se trouve dans le tube digestif de l'llomme où il parait d'ailleurs ne causer aucun dommage. Amøba coli (Lösch) (fig. 103) a élé trouvé pullulant dans le gros intestin atteint d'inflammations ulcéreuses.

Fig. 103.

\section{GENRES}

Amœba (Auct., emend. Bütschli) vient d'ètre décrit comme type morphologirque des Gymnamobiens (Ner et eau douce).

$A$ côté des Amilies, se trouvent quelques autres formes qui vont nous montrer la différenciation progressive

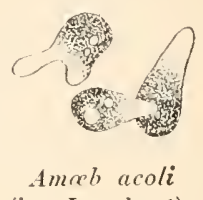

(im. Leuckart).

(1) D'ordinaire, quand des Amibes se rencontrent, ils se séparent sans adhérer l'un à l'autre aux points ou ils sont entrés en contact. Parfois cependant, on les a vus se fusionner deux ensemble, mais aucun phénomène particulier n’a suivi celte fusion; il se pourrait done qu'elle n'ait aucune signification sexuelle. Cela est d'autant plus admissible que, d'après les observations de Ṕ́xard [90], la conjugaison ressemble fort à une lulle dans laquelle l'indivilu le plus gros mange le plus pelit à la suile d'une fuite et d'une résistance énergique de celui-ci.

GreefF a décrit, chez une forme très voisine des Amibes, Pelomix a, une évolution des globules développés dans les corps brillants (fig. 93) en petiles masses qui sortiraient du corps de la mère et se transformeraient en jeunes individus semblables à celle-ci. Son interprétation est repoussée par Bütschl, mais confirmée par Konot xef [so] et par Weldon chez la mème Pelomy:x, et par Pérard [9o] chez un vrai lmibe, A. proteus. Konotxef [80] a vu ces corps brillants poursuivre l'évolulion suivante (fig. 108). Le cor'ps brillant, d'abord sphérique, se ereuse (.1) d'une petile cavilè dans laquelle pénètre le eytoplasma (nnais non ses alvéoles). Celte cavité s'agrandit de plus en plus, à la manicre de celte d'une gastrula embolique, et finit par réduire la partie réfringente à une capsule pleine de cytoplasma. Mème, l’orifice se ferme et la capsule devient une vésicule close, à prá épaisse $(B)$. De la paroi de la capsule naît une petile saillie $(C: t)$ qui s'avance $r n$ se pédonculant dans la cavité, puis se détache et forme a son intérieur un globule libre $(D: I)$, qui est de mème nature que la paroi et devient le corps brillant initial de la spore. A cóté, se forme lans le cytoplasma une résicule pulsalile $(I): V e$.$) . Finalenent, la coque anincie disparait, et il reste un globule muni$ 
de ces organismes. Au bas de la série nous trouvons le genre Protamœba (Häckel) (fig. 104) qui se distingue par l'absence de noyau el de vacuole pulsatile. L'absence du noyau, si elle est réelle, constitue un caractère d'infériorité important qui fait de ce genre une Monère (Mer et eau douce).

Gloidium (Sorokin) est un Protamilje pourvu d'une vésicule pulsatile. Mais, pas plus que celui-ci, il n'a de noyau; c'est donc encore une Monère (Eau douce).

Gringa (Frenzel) (fig. 10马) est aussi une Monère et diffère

Fig. 104.

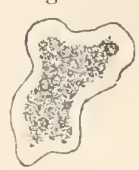

Protamaba

(P. agilis)

(d'ap. Häckel). fort peu du précédent (50 $\mu$. Lagunes).

Chætoproteus (Stein), avec son corps et ses pseudopodes hérissés de pelits prolongements spiniformes, n'est guère qu'une espèce du genre Amibe (Eau douce).

Trichosphærium (Schneider) de mème que

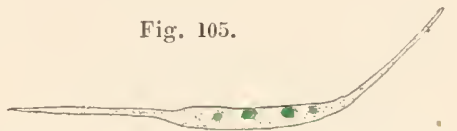

Gringa (G. filiformis) (d'ap. Frenzel).

Pachymyxa (Gruber) a une enveloppe de petites épines radiaires de nature indéterminée.

Hyalodiscus (Hertwig et Lesser) ne diffère d'Amœba en rien d'essentiel. On l'en a séparé pour

les formes qui, comme A. guttula (aujourd'hui Hyalodiscus)progressent par un unique pseudopode ayant toute la largeur du corps, en sorte que l'animal semble rampersans pseudopodes (V. p. 93) (Eau douce). De mème,

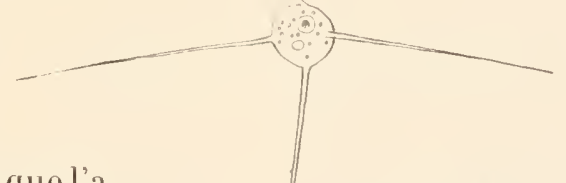

Dactylosplacrium (D. radiosum) (d'ap. Dujardin).
Fig. 107 .

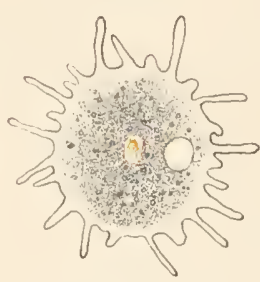

Dactylosphara

(D. polypodia)

d'ap. M. Schultze).

Dactylosphærium ou Dactylosphæra (Ilertwig et Lesser) (fig. 106, 107) a été

d'un corps brillant et d'une vésicule pulsatile qui est le point de départ d'un petit

Fig. 108.

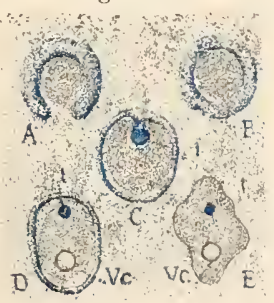

Érolution des corps brillants (im. Korotnef).

Voir dans le texte l'explication des lettres.
Amibe $(E)$ qui n'aura plus qu'à grandir. L'évolution du noyau n'a pas été suivie. Péxard n'a pas vu la formation des spores, mais il les a trouvées mùres dans l'organisme maternel réduit à elles el les a vues éclore. Il semble done permis d'admettre que certains Amibes se reproduisent par des spores formèes dans ces corps réfringents issus eux-mêmes du noyau. Mais nos connaissances manquent de précision sur ces points.

Enfin, Carter et Waldisch ont décrit chez $A$. radiosa une vraic reproduction sexuclle. Ils auraient vu des oufs et des spermatozoïdes dans des vésicules spéciales issues du noyau. Ces éléments se répandraient dans le cytoplasme et produiraient de jeunes Amibes. Mais il semble bien qu'il n'y a lit yu'une fausse interprélation de noyaux mulliples, peut-être

de parasites, ou même, d'après Rumblizr [95], de petiles pelotes de matières fécales. 
séparé du genre Amibe simplement à cause de ses pseudopodes digitiformes. Nous en avons parlé (p. 97, note) sous le nom d'A. polypodia. Il peut parfois rentrer ses longs pseudopodes digitiformes peu moljiles et en former d'autres, courts, oblus, très vifs, au moyen desquels il se déplace rapidement. Cela suffit à prouver le peu de valeur de la forme des pseudopodes comme caractère générique (Eau douce).

Chromatella (Frenzel) (fig. 109) diffère du précédent par ses pseudopodes servant au toucher et à la préhension des aliments, mais pas à la locomotion. Il a une vésicule pulsatile mais pas de noyau (5 a $6 \mu$. Lagunes).

Stylamœba (Frenzel) (fig. 110) en diffère par la possession d'un pédoncule; il a un noyau (visible seulement par les réactifs) et pas de vésicule (110 $\mu$. Lagunes).

Saltonella (Frenzel) a une forme polygonale, un noyau, pas de vésicule el, en place de pseudopodes, aux angles du polygone un petit bouton hyalin. On ne comprend guère comment, si mal armé pour la locomotion, il peut se déplacer comme il le fait, par petits sauts d'une largeur égale à son diamètre (12 p. Lagunes).

Eikenia (Frenzel) (fig. 111), bien qu’à peu près semblable, ne se déplace qu'en rampant (16 a $20 \mu$. Lagunes).

Plakopus (F.-E. Schulze) n'est aussi qu'un Amibe à pseudopodes membraniformes (Eau douce).

Pelomyxa (Greeff) (fig. 112) est un très gros Amilbe, muni de courts pseudopodes oblus, privé (à ce qu'il semble) de vésicule pulsatile et pourvu d'un très grand nombre de noyaux. Il est caractérisé par la possession d'un grand nombre de corps brillants très évidents (2mm. Eau douce) (").

Amphizonella (Greeff) (fig. 113) est remarquable par la présence d'une enveloppe gólatineuse relativement épaisse que traversent les courts psendopodes digitiformes hyalins. Par ce caraclère il se rapproche des Thécamœbiens (Eau douce et terre humide).

(1) Nous avons exposé plus haut (p. 98) les discussions

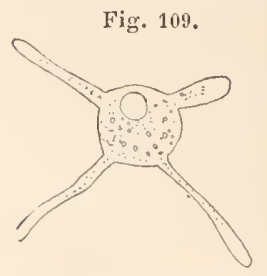

Cleromatella (C.argentina) (d'ap. Frenzel). Fig. 111.

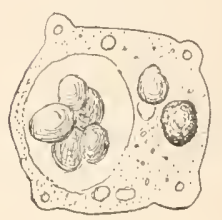

Eilenia (E. rotunda) (d'ap. Frenzel).

lig. 112.

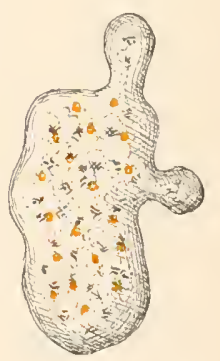

Pclomyxa (I'palustris) (im. Greeff).

Fig. 113.

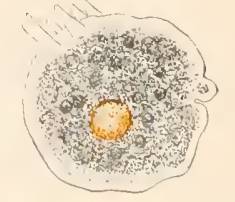

Amplizonella (A.violacea) (d'ap. Greeff). relatives à la signification de ces corpuscules et a leur róle dans la reproduction. 
Podostoma (Clarapède et Lachmann (fig. 114 ) est remarquable à un autre titre.

C'est comme un Dactylosphxrium qui, outre ses pseudopodes ordinaires $(p)$, en aurait un ou quelques-uns $\left(p^{\prime}\right)$ terminés par un prolongement long et mince, très mobile, rétractile, glutineux, servant à capturer les proies (Eau douce). Enfin

Arcuothrix (Hallez) (fig. IIJ̆) montre une différenciation encore plus avancée. Il a un seul pseudopode d'Amibe ordinaire et ce pseudopode, situé en avant dans la progression, a une réelle individualité en ce sens qu'il est toujours présent ou, quand par hasard il rentre dans le corps, se reforme à la même place et pareil à lui-même. A la partie postérieure se trouvent dleux très longs et très fins filaments pseudopodiques, bifides au bout et parsemés de petits renflements. Ces filaments sont glutineux et servent à retenir les proies. On ne les a pas vus rentrer à l'intérieur du corps (20 i 2 't $\mu$. Dans les cultures d'Ascaris megalocephala (').

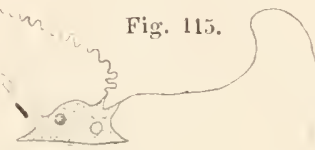

Podostoma

( $P$. filigerum)

(im. Clarapède et

Lachmann).

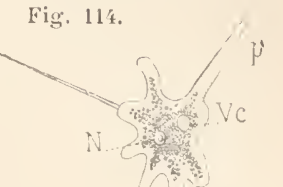

\section{OrdRe \\ TIÉCAMOEBIENS. - THECAMOEMIDDA \\ [AMUEiens T'estacés]}

Arcuothrix

(A. Balbianii) (im. Hallez).

\section{TYPE MORPHOLOGIOUE}

(FIG. 116)

Ce type peut se définir en trois mots : c'est un Amile muni d'une capsule.

Le corps mou de l'animal ne diffère de cehui du type morphologique

Fig. 116.

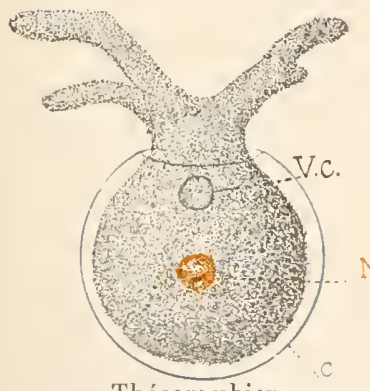

Thécamobien précédent qu'en deux points : 10 étant suffisamment protégé par la capsule, il n'a pas besoin de différencier à sa surface une couche protectrice, aussi ne montre-t-il pas d'ectoplasme; $2^{\circ}$ dans tous les points où il est en contact avec la capsule, il ne peut ni émettre des pseudopodes ni capturer d'aliments, aussi ces deux fonctions se limitent-elles à la région qui est en face del'ouverture de la capsule. Cette capsule (c.) est une mince enveloppe formée de chitine ou d’une substance analogue, sécrétée par la sur(Type morphologique) (Sch.). e., capsule; n., noyau; ve., vésieule pulsatile. face du cytoplasme. Elle est continue en ce sens qu'elle n’a pas de pores microscopiques, et est munie d'une simple large ouverture appelée bouche, par où sort une partie du corps qui émet des pseudopodes.

(1) Mycetomyxa (Zacharias) (fig. 118) nous parait devoir prendre place ici. Il se compose d'une 
L'animal rampe sur la bouche de sa capsule. Il peut, à l'occasion, se retirer complètement à son intérieur. Il arrive aussi quelquefois, mais très exceptionnellement, qu'il peut en sortir ('). Il ne lui est donc uni par aucun lien organique. Mais normalement il ne la quitte point. Elle s'accroît avec lui par intussusception et il la conserve jusqu'à la mort. Pour se reproduire il se retire à l'intérieur, se divise et l'un des individus filles garde l'ancienne capsule, tandis que l'autre en sort et s'en sécrète une nouvelle.

\section{GENRES}

Cochliopodium (Hertwig et Lesser) (fig. 117) est une des formes les plus simples. Son corps a la forme d'une marmite. Il est $A$, l'animal étendu; $B$, le même rétracté; revêtu d'une cuticule si mince et si souple Fig. 117.

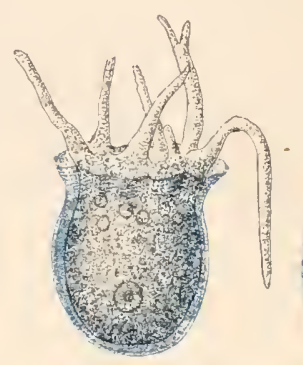

A

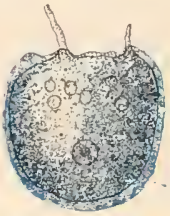

B

Cochliopodium (Sch.). qu'elle participe à ses déformations et se voit difficilement. A la bouche, qui est très large, cette cuticule se prolonge un peu sur la base des pseudopodes. Ceux-ci sont modérément longs, étroits, à peine ramifiés, quelque peu anastomosables à leur base (Eau douce) $\left({ }^{2}\right)$.

cellule fusiforme émettant par chacune de ses extrémités un prolongement pseudopodique ramifié un peu rigide. Certaines ramifications de ces prolongements se terminent par un ou plusieurs petits appareils formés, selon toute apparence, de filaments entortillés terminés par une sphérule verte qui n'est rien autre chose qu'une colonie fille de Pandorina morum. Ces sphérules sont en partie décolorées et représentent évidemment des proies capturées et déjà attaruées. Mais la nature des appareils terminaux est impossible à déterminer d'une manière tout à fait certaine et il reste possible qu'ils soient formés de petits segments fusiformes placés bout à bout. Dans la. cellule centrale, est une vésicule, mais qu'on n'a pas vu se contracter, et l'on n'a pas pu distinguer un noyau. Ce serait donc une Monère.

Fig. 118.

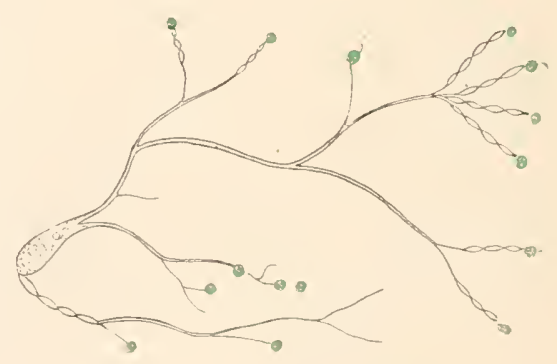

Mycetomy.xa

(M. Zopfii) (d'ap. Zacharias).

Cet être singulier appartient évidemment aux Rhizopodes, mais a quelques traits frappants de ressemblance avec certains Hycétozoaires ou Champignons inférieur's tel que Rhizidium ou Spirophora (26 «. Eau douce, lac de Plön).

(1) Rhumbler [91] a observé le fait chez Arcella, lorsque l'eau est trop chargée de Bactéries.

$\left.{ }^{2}\right)$ La cuticule est d'aspect chitineux, mais formée d'une substance différente de la chitine et plus résistante aux réactifs. Elle est ornée de ponctuations orientées suivant deux systèmes de lignes presque rectangulaires. Il y a un seul noyau, deux ou plusieurs vésicules pulsatiles. 
Arcella (Ehrenbery) (fig. 119 à 12I et 12 \& à 126) possìde une cuticule plus épaisse, très visible, véritable capsule ou coquille $(c)$ qui a la forme d'un verre de montre très profond dont l'onverture serail rétrécie par un diaphragme plan percé d'un trou central. Le corps ne la remplit pas

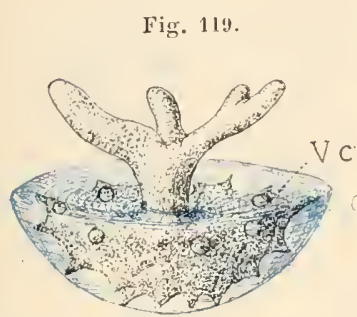

Areella

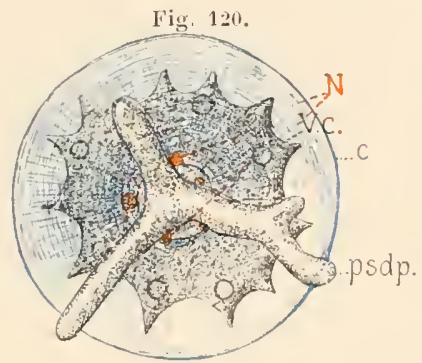

Areella

(1. sulgaris) (Sch.).

(1. sulgaris) lu de dessus (Sch.).

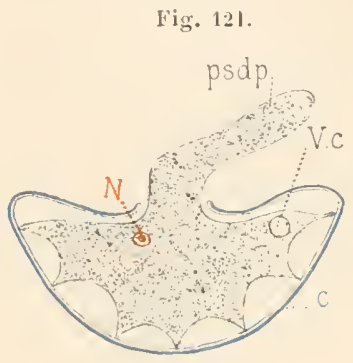

sreelle.

Goupe (sch.).

toute entière et lui est raltaché seulement par quelyues brides entre lesquelles sont des espaces courbes serrant de chambres incubatrices.

La partie qui confine à l'ouverture déhorde par là au dehors el émel qurlyues rares et gros psentopodes digitiformes ( $p s d p$.). Dans le corps, on remarque de nombreuses résicules pulsaliles ( $V_{c} c$.) el de nombreux (1 ì 32) noyaux $(N$.). Les mus et les autres sont disposés en cercle non loin du bord de la capsule, mais ceux-ci sont assez profonds, landis que celles-lì sont très superficielles. Ciruber [92] a vu les noyaux se multiplier par mitose sans disparilion de la membrane. Il y a, en outre, de nombreuses el grosses vacuoles it gaz servant de flotlenr it l'animal, qui semble prouvoir les former et les résorber selon qu’il veut flotter ou s'enfoncer.

La coquille est formée d'une mince couche anhiste interne et d'une couche externe de pelits prismes hexagonaux disposés côte à côle radiairement el soudés par une mince couche de substance agglulinante. Pour grossir, l'animal fait éclater sa coquille, la disjoint en quelques places et la répare sous ses nouvelles dimensions (Eau douce) (").

Pseudochlamys (Claparède el Lachmann) (fig. 122) s'en distingue par un diaphragme péribuceal purement membraneux.

Pyxidula (Ehrenberg) en diffère par sa surface hérissée de minimes pointes. L'un et l'autre diffèrent a peine du précédent, et Bütschli se demande s’ils ne repré-

Fig. 122.

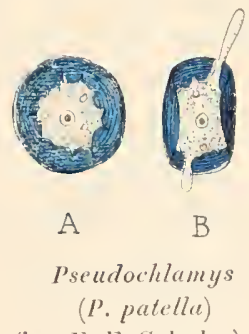

(iin. F.-E. Schulze). sculeraient pas des formes jeunes du genre Arcella (Eau douce).

(1) L'animal se reproduit par division : une partie du eorps sort de la coquille et se sécrète une coquille nouvelle, formant ainsi une nouvelle Arcelle jeune, collée 
Hyalosphenia (Stein) (fig. 1־3) en diffère par sa coquille anhiste et sa forme allongée suivant l'axe et fortement aplatie parallèlement à ce mème axe (Eau douce).

Difflugia (Leclerc) (fig. 127, 128, 130, 133, 131) a une capsule en forme de bouteille sans col. Le corps, qui est loin de la remplir, émet par l'orifice un petit nombre de longs pseudopodes digitiformes. Cette capsule n'est pas, comme chez les Arcelles, formée uniquement de substance sécrétée. Elle est, pour la majeure partie, composée de particules étrangères, fragments de quartz, carapaces de Diatomées, etc. Mais, dans chaque individu, elle est assez uniforme: si elle est formée de carapaces de Diatomées, elle ne contient que cela ; (im.F.-E. Schuze). si elle est bàtie de grains de sable, ceux-ci sont triés de taille et de forme assez homogènes. Ces particules ne sont pas aggglutinées du

bouche à bouche à l'ancicnne. Puis les deux individus se séparent. C'est done plutôt unc sorte de bourgeonnement qu'une division.

On a observé (fig. 124) une conjugaison de deux individus, parfois trois, quatre ou

Fig. 12'.

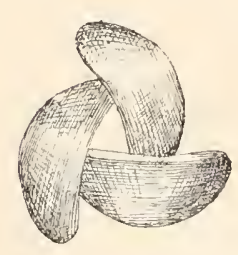

Arcella. Conjugaison (Sch.).

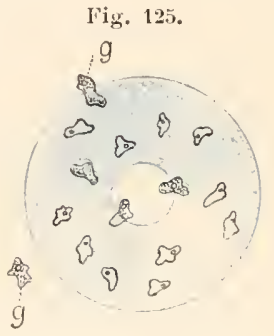

Arcella. Reproduction (Sch.). cinq, qui se soudent bouche à bouche et ćcliangent des courants protoplasmiques. Mais, comme on n'a vu ni les noyaux y participer, ni la division ou quelque antre reproduction se produire a la suite, on n'est pas sitr que ce soit là un acte sexuel.

La reproduction par bourgeons a èté nettement constatèc (fig. 125). On voit de petites masses (jusqu'à neul) de protoplasma logées dans le cytoplasma de l'animal mère. Ces petites masses $(g)$ se munissent d'un royau et d'une vacuole, se detachent, cheminent dans le cytoplasme, sortent de la coquille par la bouche, s’éloignent de la mère et se munissent d'une coquille. Malheureusement on ne sait pas comment se forment le noyau et la vacuole du bourgeon.

Pénard [90] aurait vu toute la masse de la mère se transformer en sortes de

Fig. 120 .

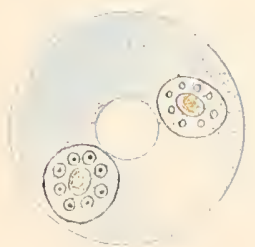

Arcella. Reproduction (Sch.). spores que l'on trouve, au nombre d'une douzaine, munies chacune d'un noyau et d'unc vacuole, attachées au fond de la coquille vide et fermè a l'orifice par un feutrage de détritus.

Busk [78] a décrit une formation de petites masses nucléées (fig. 126) qui se segmentent, formant une sorte de morula et finalement se désagrègent en fines particules dont il n'a pu suivre l'èvolution.

Les miloses observées par Gruber [92] dans les noyaux maternels semblent se produire pour une multiplicalion en vuc de la formation de ces spores ou bourgeons.

L'mliystement a èté observi. Le corps se contracte, se ramasse en boule et se fixe sous la bouche de la coquille, protége en outre par nue membrane kystique mince, de nouvelle formation. Quant it une reprobluction sexuelle affirmie iei aussi par Greefr, elle n'est pas mieux établie ici que chez les Amibes (V. p. 97). 
dehors. Elles ont été incorporées comme des aliments, rejetées à la surface et maintenues là, agglutinées par une minine quantité de sécrélion. Mème certains globules formés de toutes pièces à titre d'excreta entrent dans sa composition au mème titre que ces particules étrangères. Dans le corps, on trouve un nombre de noyaux qui varie tepuis quelques-uns jusqu’à 2.00 (BLANG [92]). Il y a aussi des vacuoles contractiles en nombre très variable (").

Quadrula (F.-F. Schulze) (fig. 129) est tout à fail

Fig. 129 .

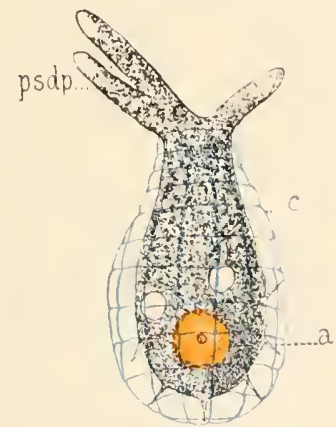

Quadrula (Q. symmetrica)

(im. F.-E. Sehulze).

Fig. 12s.

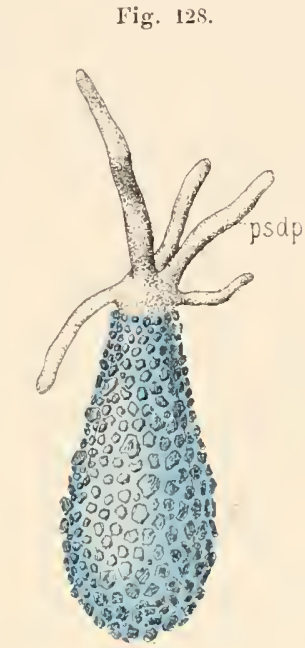

Difllugia (Sch.).
Fig. 127.

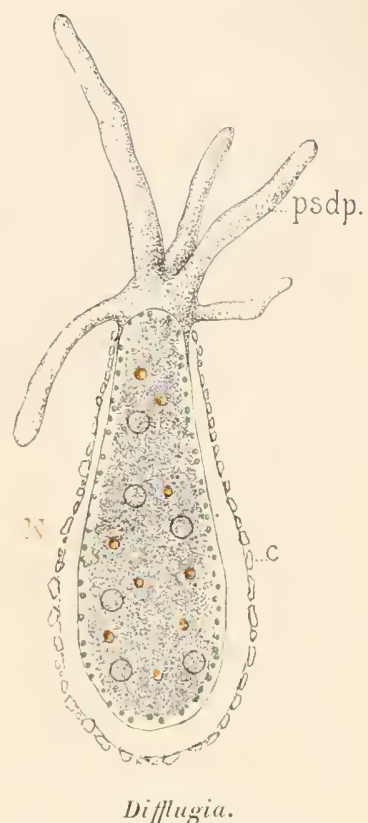

Coupe longitudinale (Seh.).

comparable au type précédent et s'en distingue principalement par sa coquille plus renflé et formée de plaquettes siliceuses carrées, transparentes (Eau douee).

(1) Le eorps est souvent hourré de grains le chlorophylle (fig. 130) eontenant ehacun un grain d'amidon; on y trouve aussi quelques Micrococcus verts.

Cela donne à ees individus une teinte verte très aeeentuée. Ces grains verts pourraient ètre des aliments, des parasites ou des commensaux. C'est eelte dernière interpretation qui est la plus probable, ear: 10 ils sont inaltérés; 2" ils paraissent, d'après Pésako [92], logés exelusivement dans l'eetoplasme, ee qui n'arrive jamis aux aliments et s'explique aisément chez des organismes vivants doués d'un taetisme positif pour la lumière; 30 enfin, les individus qui en possèdent supportent si

Fig. 130 .

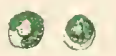

Grains de chlorophylle avec amidon au centre (im. Pinard). bien le jeùne qu'on a émis l'idie exagérée qu'ils pouvaient se passer d'aliments.

Les Difflugies se reproduisent par division, à la manière des Arcelles. L'un des deux produits sarde la coquille ancienne el l'autre doit s'en fabriquer une; mais on a constaté qu'avant ee moment le protoplasma se bourre de partieules destinées à former la eoquille nouvelle.

On a fréquemment observé (fig. 133) la conjugaison, même à plusieurs comme 
Nebela (Leidy) diffère du précédent par sa corquille fortement comprimée, piriforme de profil, à bouche bilabiée formée de plaquettes siliceuses, arrondies ou irrégulières, fixées sur une membrane de nature également siliceuse (Eau douce) (').

Heleopera (Leidy) s'en dislingue par sa coquille piriforme, à peu près lisse vers le haut, garnic au bas de grains de sable (Eau douce).

Lecquereusia (Schlumberger) (fig. 131) peut ètre considéré comme un Iniffugia dont la capsule, toute garnie do grains de sable, aurait déjeté sa bouche sur le côté et fait, en s'accroissant, un demi-lour de spire (lau douce).

Est-ce bien ici qüll convient de placer les deux genres ci-dessous qui sont insuffisamment étuliés? Le premier est

Petalopus (Claparède et Lachmann) (fig. 132) qui a, comme tous les précédents, ses pseudopodes limités à une partie du corps, sans que cela s'explique par la présence d'mne capsule. Mais l'absence de capsule est mise en doute par Bütschli. Elle pourrait exister et être si mince qu'elle aurait passé inaperçue, et l'unique espèce du genre n'a jamais été revue depuis sa lécouverte (Eau douce) $\left({ }^{2}\right)$. Le second est

Arcellina (du Plessis) dont la capsule, sphérique ou ovoüde, chitineuse, serait percée de tins pores s'ouvrant au dehors sur de petites tubérosités. L'animal est polynucléé et possède des corps brillants (Eau douce).

chez les Arcelles, et Javororsky [92] a vu pendant ce temps les noyaux se multiplier, s'entourer chacun d'un peu de protoplasma et se transformer en zoospores.

II. BLANC [92] a vu les noyaux s'isoler avec une portion du cytoplasma et se transformer en spores (fig. 131) qui sortiraient par la bouche pour levenir de petiles Dilflugies.

L'animal peut s'enligster sous une membrane de nouvelle formation protégée en outre par la coquille bouchie a lorifice par des delritus (fig. 134).

On l'a vu aussi abandonner sa coquille par une sorte de mate.

(1) Il y a peut-être exagération à èlever au rang de genre cetle forme que d'autres auteurs considirent comme une simple espece de Iriffugia ou de (anadrula.

Fig. 133

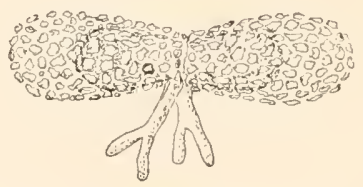

Diflugia

Corjugaison (im. Pénatd).
Fig. 131.

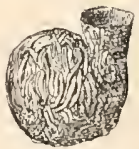

Lecquereusia

(L. spiralis) (im. Wallich).

Fig. 132.

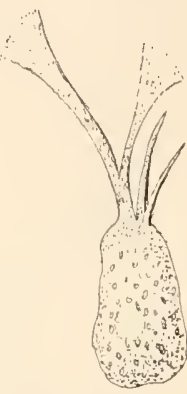

Petalopus

(I'. Diflupens) (d'ap. Claparide et

Lachmami).

(2) Pseudopodes aplatis au bout; noyau et vésicule pulsatile inconnus. 


\section{$4^{e}$ Sous-Chasse \\ FORAMINIFÈRES. - FORAMINIFERIE \\ [ForAMINIFERA (d'Orbigny, emend.)]}

Les Foraminifères se distinguent des Imobiens, auxquels on les réunit souvent, par un caractère capital : ils ont toujours des pseudopodes fins, ramifiés et anastomosables, en un mot réticulés, formant, en dehors du corps proprement dit de l'animal, un riche réseau de forme irrégulière (fig. 133̈). lls sont, en outre, toujours pourvus d'une capsule qui, ici, est si généralement encroûtée de substances minérales dures (calcaire, silice) qu'elle mérite bien le nom de coquille qu'on lui donne habituellement.

On divise les Foraminifères en deux ordres :

IMPERForés, à coquilles dépourvues de pores;

Perforés, à coquille percée, en outre de la bouche, de fins pores par où sortent des pseudopodes (").

$$
\text { 1er ORDRE }
$$

\section{IMPERFORÉS. - IMPERFORIIA \\ [IMPERForATA (Carpenter)]}

\section{TYPE MORPHOLOGIQUE \\ (FIG. 135)}

L'ètre est essentiellement constilué par un corps de Rhizopode réliculé renfermé dans une coquille. Cette coquille, dont la forme dérive de la sphère, est continue, percée seulement ì la partie supérieure d'une assez large ouverture, la bouche. Elle est constituée par du carlonate calcaire uni à une minime quantité d'un substratum chitineux qui sert de ciment aux molécules inorganiques. Le corps protoplasmique renfermé dans cette capsule n'offre rien de bien particulier. Il ne montre pas de différenciation en ectoplasme et endoplasme. 11 possède un noyau $(N$.) et probablement une résicule pulsatile $(V c$.$) . On y troure, à titre de résidus$ alimentaires, des carapaces de Diatomées et autres particules du mème genre ( $p m^{\circ}$.). Quand l'animal est au repos, il est entièrement renfermé dans sa coquille. Mais quand il est en

Fig. 135.

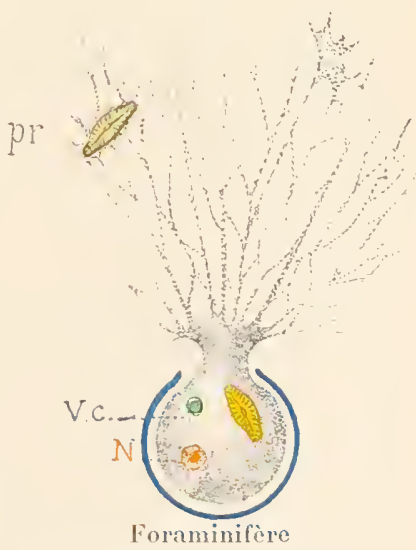

(Type morphologique) (Sch.).

N., noyau; pr., proie ;

Ve., vésicule pulsatile.

(1) La question du dimorphisme des Foraminifères sera traitée à l'occasion des 
état d'activité, principalement pour la recherche de la nourriture, il s'épanche au dehors et, en quelque sorte, déborde de sa coquille. Cette masse protoplasmique extérieure forme une sorte de bouchon irrégulier et c'est de lui, uniquement, que partent les pseudopodes. Ceux-ci sont larges à la base mais, en se ramifiant, deviennent rapidement plus fins. Ils s'anastomosent entre eux en un vaste réseau extrèmement irrégulier. Ce réseau de prolongements toujours en mouvement sert it la reptation et à la capture des aliments qui sont englobés par eux et entraìnés dans le corps ou digérés sur place. Le long des pseudopodes, mème les plus fins, on observe le curieux spectacle de la circulation du protoplasme rendue manifeste par le mouvement des granulations que l'on voit rouler, en direction centrifuge le long d'un bord, et en sens opposé le long de l'autre, courir ici vite, là plus lentement, hésitant un instant aux anastomoses avant de se décider ì suivre l'une ou l'autre des deux voies qui lui sont offertes.

L'animal se reproduit principalement par division (V. p. 120, la reprorluction des Miliolides). Pour cela, il se retire dans sa coquille et divise son corps protoplasmique, soit longiludinalement, soit transversalement; l'une des deux moitiés reste dans la coquille ancienne où elle continue à grandir à l'aise, tandis que l'autre en sort et se sécrète une coquille nouvelle (").

La coquille que nous avons attribuée à notre type morphologique est la plus simple comme forme el la plus caractéristique comme composition. Hais il faut savoir que cet organe est extrèmement varié chez les Foraminifères el que ses variations constituent le principal critérium dans la distinction des groupes grands et petits.

Nous distinguerons trois sous-ordres dans l'orlre des Imperforés :

Gromides, à coquille continue, chitineuse;

Miliolides, à coquille continue, calcaire; et

Arénacés, à coquille discontinue, formée de grains de sable.

A propos de chacun d'eux, nous exposerons l'importante question de l'accroissement de la coquille, qui diffère selon sa constitulion physique et chimique.

Miliolides et des Perforés au sujet desquels elle se pose le plus nettement (V. p. 118).

(1) La coquille, par son opacité, oppose de sérieuses difficultés à l'étude de l'organisation intérieure. On peut la dissoudre par les réactifs, mais l'animal ne peut plus être observé vivant. La présence dı noyau peut être aisément constatée après la mort. On s'est assuré ainsi qu'il y en avait toujours au moins un et sourent plusieurs, ou même un grand nombre. Quant à la résicule pulsatile, on n'a pu s'assurer de sa présence que chez les formes à coquille mince et transparente, mais pour les autres, on reste dans l'ignorance à son sujet. Chez les premières, on a souvent constaté l'existence de plusieurs vésicules, mais chez les formes à coquille opaque on ne sait rien de leur existence.

La division du noyau est, ici encore, fort embarrassante à définir. La plupart des observations anciennes signalent une simple dirision directe. Dans certains cas, on 
$1^{\text {er }}$ Sous-ORdRE

GROMIDES. - GROMIIDE

[GROMIDE. (Claparède et Lachmann)]

TYPE MORPHOLOGIOUE

(FIG. 136)

Ce type ne diffère de celui que nous avons esquissé pour l'ensemble des Imperforés en rien de ce qui concerne la forme de la coquille ou la constilution du corps. Nous le caractériserons d'un mot en disant que sa coquille est mince, formée d'une membrane chitineuse continue, douée d'une certaine souplesse, el toujours monothalame, c'est-à-dire à une seule loge.

Son accroissement se fait, comme chez Arcella el autres analogues, par intussusception, el doil être compris de la manière suivante. Elant un peu élastique elle se laisse distendre, ce qui entraine un écartement de ses molécules constiluanles. Eint’e les molécules ainsi écartées, de nouvelles molécules peuvent se déposer par précipilation au sein du liquide qui les baigne. Lorsqu'elles sont formées, elles remplissent les vides produits

Fig. 136.

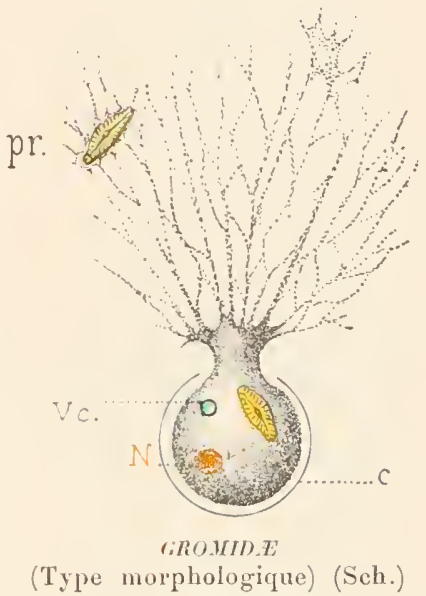
par la distension. Dès lors il n'y a plus distension et la coquille accrue

aurait une division indirecte plus ou moins réduite. Puis, brusquement, dans un type étudic a fond avec les ressources de la technique moderne, on découvre une mitose typi fue. En sorte que l'on se demande si les autres genres, étudiés de la mème manière, ne fourniraient pas des faits analogues. Nais nėanmoins on liésite a généraliser. Ici c'est Cheviakof [8s] qui a dicouvert cette mitose chez Euglypha. Le noyau au repos comprend une membrane, un nucléole et un réseau chromatique. Le réseau s'èpaissit, puis se coupe et l'on observe successivement les phases classiques de spirème, de peloton liche, de peloton segmenté, l'orientation des chromosomes, la division longitudinale et la disparition progressive du nucléole. A ce moment, se montrent deux centrosomes, venus on ne sait d'où, aux deux pôles du noyau, arec leurs asters; les anses jumelles se séparent, se portent aux deux pôles, le noyau s'allonge en biscuit, se coupe, les deux centrosomes disparaissent, et les deux noyaux filles repassent à l'ètat de repos. La membrane nucléaire persiste pendant toule la durée du phẻnomène.

En ce qui concerne la disision the l'animal, ajoutons que souvent, surtout quand la division est longitudinale, les deux jeunes abandonnent la coquille ancienne et s'en forment chacun une nouvelle. Quand elle est transversale, on a vu l'individu situé au fond sortir et laisser à l'autre la libre possession de la coquille ancienne.

Les prétendus corps reproducteurs signalès chez divers types ne sont, ainsi que l'a montré Rhumber [93], que des pelotes fẻcales que l'animal n'a pu éliminer à cause de leur volume et qu'il a isolèes en les entourant d'une membrane.

Dans quelques cas très rares (Iyalopus), on a observé la conjugaison de zoospores 
occupe sans effort la mème étendue qu'auparavant sous cet effort ('). Elle est donc susceptible d'une extension nouvelle et le phénomène recommence.

\section{GENRES}

Nous pouvons, dans ces Gromides, distinguer trois groupes, ayant pour chefs de file : l'un Euglypha, forme de transition entre les lobés et les réticulés, l'autre Crromia, franchement réticulé, avec une seule bouche, le troisième $D i$ plophrys, à deux bouches diamétralement opposées.

Euglypha (Dujardin) (fig. 137 à 140) peut servir de forme de transition entre les Amobiens testacés el les Foraminifères. Les pseudopodes sont en effet d'ordinaire fins, allongés, ramifiés, comme chez ceux-ci, mais anastomosables, à leur base seulement, et fort peu. La coquille (Test) (fig. 137) a la forme d'un ovoïde dont la petite extrémité serait largement tronquée par l'orifice buccal. Elle est formée de plaquettes siliceuses arrondies qui, en s'imbriquant par leurs bords, déterminent des dessins hexagonaux. Ces plaquettes sont sécrétées par le protoplasma et déposées à la surface du corps où elles sont soudées par un ciment chitineux $(0,1$ à 0,2 . Eau douce) $\left({ }^{2}\right)$.

flagellées, produites par division simultanée de tout le corps mou de l'animal, mais jamais on n'a pu suivre l'évolution du produit de la conjugaison.

(1) Parfois cependant le phénomène est plus brutal. Il se forme sous la pression du cytoplasma turgescent de petites déchirures de la coquille, qui se réparent parla sécrétion I'un nouveau ciment. Le fait a été constaté chez Arcella, comparable sous ce rapport aux animaux dont nous nous occupons en ce moment.

(2) Souvent l'orifice Juceal est denté el souvent aussi la coquille est ornée, surtout ver's le bas, de longues épines. Le noyau (Noy) gros et unique, est au centre de la partic inférieure du corps. Une résicule pulsutile (Tésic. puls.) se montre un peu plus laut. Le cytoplasma forme lrois assises: une supérieure (Zone alvéol.) très vacuolaire d'où partent les pseudopodes, une moyenne granuleuse (Zone granul.) où s'accumulent les aliments et résidus digestifs. et une inférieure (Zone hyal.) périnucleaire, hyaline. Euglypla présente une particularité rare chez les fíraminil’ères, c'est celle de 
L'Einglyphe et les quelques genres secondaires (') qui st rattachent i lui en raison de leur's pseurloporles fins, il est vrai, el aples à se ramifier, mais peu ou point anastomosables, ne sont pas de vrais fo-

s'enkyster. Pour cela (fig. 138), il commence par fermer sa coquille (c.) au moyen d'un diaplragme d'Nlgues et de Diatomées agglutinées, puis il se contracle et se r'éduit a une masse sphérique qui en oceupe le fond. Lit, il sécrète un premier kyste ovö̈de (kys.) formé de plaques contigués comme la coquille, puis, après un nouveau retrait, un second kyste sphérique plus petil (b), formé de petits grains arrondis, et suspendu dans le premier par un cordon.

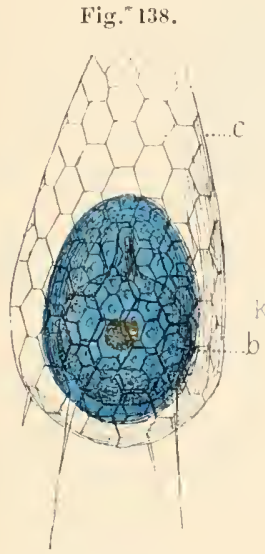

Euglyplia. Enkystement (Sch.).
Fig. 139 .

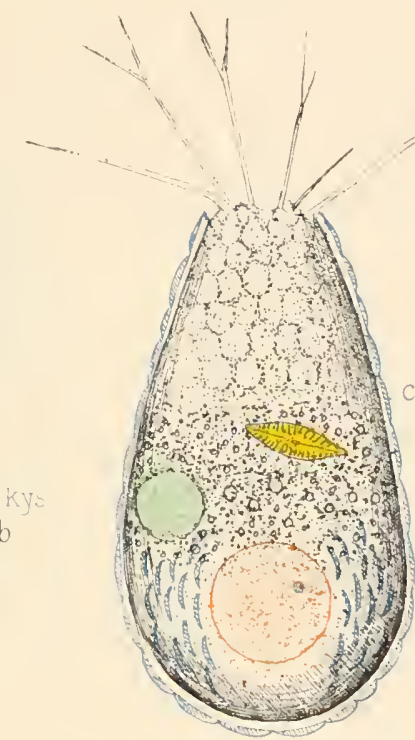

Euglypha. Division (im. Cheriakof). c., coquille; p., plaques de la nouvelle corr.
Fig. 1'il.

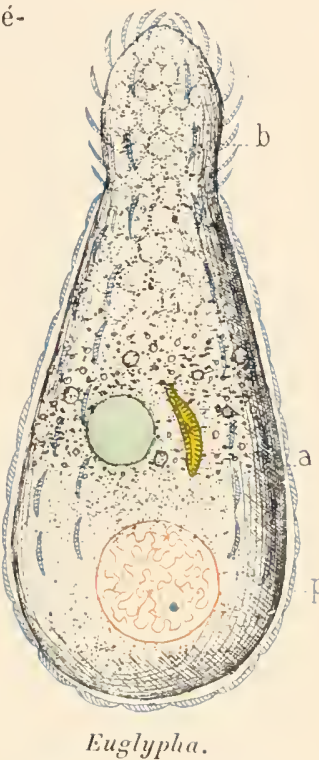

Division (im. Cheviakof).

a., individu primitif; to., nouvel individı; p., migration des plaques allant former la coquillo du nouvel individu.

La division (fig. 139, 140) a été lrès bien observée par Cheviakof [ss] et mérite de nous arrêter un instant. Quand l'Euglyphe se prépare à se diviser, elle sécrète d'abord de nombreuses petites plaquettes squelettiques, identiques à celles qui forment sa eoquille. Ces plaquettes (p.) se rassemblent dans le protoplasma hyalin périnueléaire, concentriquement autour du noyau. l'uis son protoplasma commence a faire hernie à l'orifice buccal oủ il forme un bouchon (b.) qui grossit progressivement jusqu'à constituer une masse igale à celle qui remplit la coquille. C'est d'abord le protoplasma alvéolaire qui sort, puis le protoplasma granuleux; la portion hyaline périnucleaire restant dans la eoquille. En même temps, les plaquettes squelettiques se rendent toutes dans la masse extérieure, et se disposent à sa pẻriphérie, en une conche continue, formant a cette masse fille, une coquille complète et normale d'Euglyphe. On a alors un être double, formé de deux Euglyphes soudés par la bouche, mais un seul d'entre eux possẻde un noyau. Ce noyau entre alors en division et donne naissance, par le processus indiqué plus haut (V. p. 109) ì un noyau fille qui se porte dans l'individu fille, entrainant avec lui une partie du protoplasma lyyalin périnucléaire. Les deux individus se séparent alors, forment des pseudopodes et il ne reste aucune trace de ce qui s'ètait passè.

Buocumaxy [8ז] a observé chez les Euglyphes une conjugaison suivie d'enkystement. (1) Voici, rapidement caractérisés, ces genres qui forment, avec Euglypha, la 
raminifères réticulés. On pourrait aussi lien les réunir aux Difflugies. Arec les genres suivants, au contraire, nous abordons les Foraminifères normaux.

Gromia (Dujardin) (fig. 141) possède une petite coquille chitineuse flexible, ovoïde, et le cytoplasma non seulement la remplit tout entière, mais déborde par l'orifice et forme tout autour d'elle une couche irrégulière. De tous les points de ce revêtement protoplasmique extérieur partent des pseudopodes ( $p s d p$.) anastomosés en un riche réseau irrégulier; mais c'est surtout en face de la bouche qu'ils sont nombreux el ramifiés (Mer et eau douce) (').

Des formes assez nombreuses se raltachent à ce type principal et plusieurs s'en distinguent par des caractires assez impor-

famille des ErolyphIx.E [Euglyphina (Bütschli)] :

Sphenoderia (Leidy) et

Placocysta (Leidy), qui ne sont guère que des espèces d'Euglypha;

Trinema (I)ujardin) (fig. 142), qui peutêtre défini un Euglypha, dont la bouche serait rejetée de côté (Eau douce);

Assulina (Ehrcnberg), qui est un Euglypha très aplati à bouchc irrégulièrcment dentée. (Eau douce);

Cyphoderia (Schlumberger) (fig. 143), qui est plus allongé et a sa coquille formée de plaquettes chitineuses plus petites (Eau douce et mer;

Fig. 1'3.

Discella (Nemec), qui se distingue par une coquille rudimentaire formée de petits disques brillants, indépendants et mobiles, et qui émet des pscudopodes seulement parses partics nues (Parasitc cntrc les lamelles branchiales de Ligidium);

Paulinella (Lautcrborn), lagénilorme, à coquille formée de cinq rangées verticales de plaquettes siliceuses hexagonales, à pscudopodes lorgs ct minces non anastomosables $(0,2$ a 0,3 . Eau douce;

Campascus (Leidy), 'Jui est un Cyphoderia dont la coquille serait incrustée dc corps étrangers et serait ornée en bas d'appendices spiniformes (Lau douce).

( $\left.{ }^{1}\right)$ L'animal ne possède pas de s'ésicule pulsatile. Quand il est jeune, il n'a qu'un noyu, mais les individus àgés en ont jusqu'à une soixantainc.

Fig. 112.

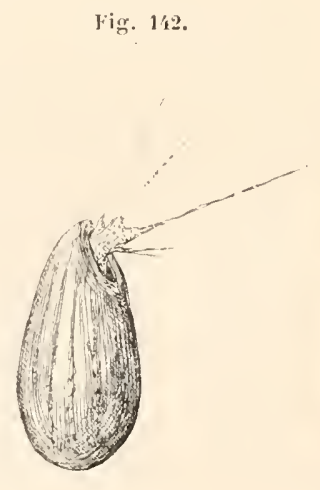

Trinema

(im. Dujardin).
Fig. 111.

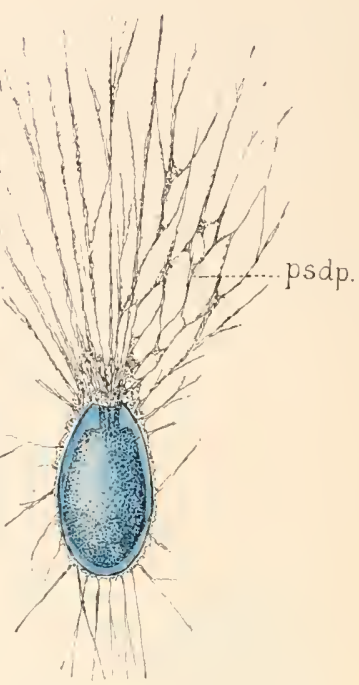

Gromia (Cx. osiformis)

(im. Dujardin).

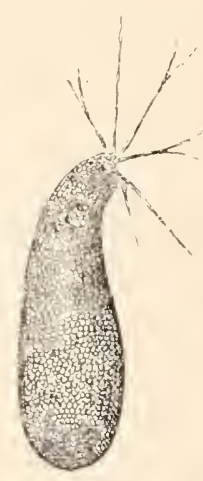

Cyphoderia

(C. margaritacea)

(im. Schulze).

Plagiophrys Claparède et Lachmann) a été créé pour les espèces de Cíomia sans coquille, si vaiment il en cxiste ce qui nest pas bien démontré; ce genre reste donc douteux. 
tants. Liextension du cyloplasma sur toute la face externe de la coquille, en particulier, est spéciale à la Gromie el ne se rencontre pas ailleurs. Hyalopus (Schaudinn) est un genre créé pour recevoir une espèce du genre Gromia, G. Dujardinii, qui se distingue des autres par ses pseudopodes entièrement hyalins non anastomosables, par ses courants de granulations, par la présence de certains grains réfringents brunàtres dans le corps et, fait plus inléressant, par la formation de zoospores isogames qui se conjuguent deux à deux (").

Lieberkuhnia(Claparède et Lachmann)(fig.144 et 14.3$)$ diffère dle la Gromie par sa coquille ovoïde ou piriforme $(c)$ dont l'ouverture est située dans une dépression latérale de la grosse extrémité qui est plus ou moins quadrilobée. En outre, le protoplasma ne forme pas un revêtement extérieur complet, mais émet un seul gros tronc pseudopodigue $(o)$ d'où se détachent de nombreuses ramifications anastomosées en réseau. On ne lui a pas trouvé de vésicule pulsatile $\left(0,4\right.$. Eau douce) $\left({ }^{\mathbf{2}}\right)$.

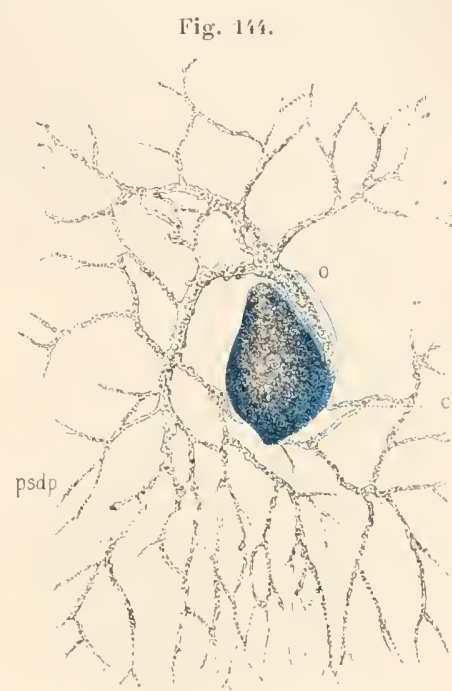

Lieberkühnia (L. Wagneri) (d'ap. Claparède et Lachmann).

Microgromia (R. Ilertwig) (fig. 146 à 149 ), forme très petite, est remarquable par son mode singulier de reproduction et par les colonies auxquelles elle donne naissance.

(1) Schaudinn a pu observer le retrait du corps protoplasmique dans la coquille et le morcellement du corps en petites masses nucléées (sans participation des grains bruns qui tombent au fond) qui se munis-

Fig. 145.
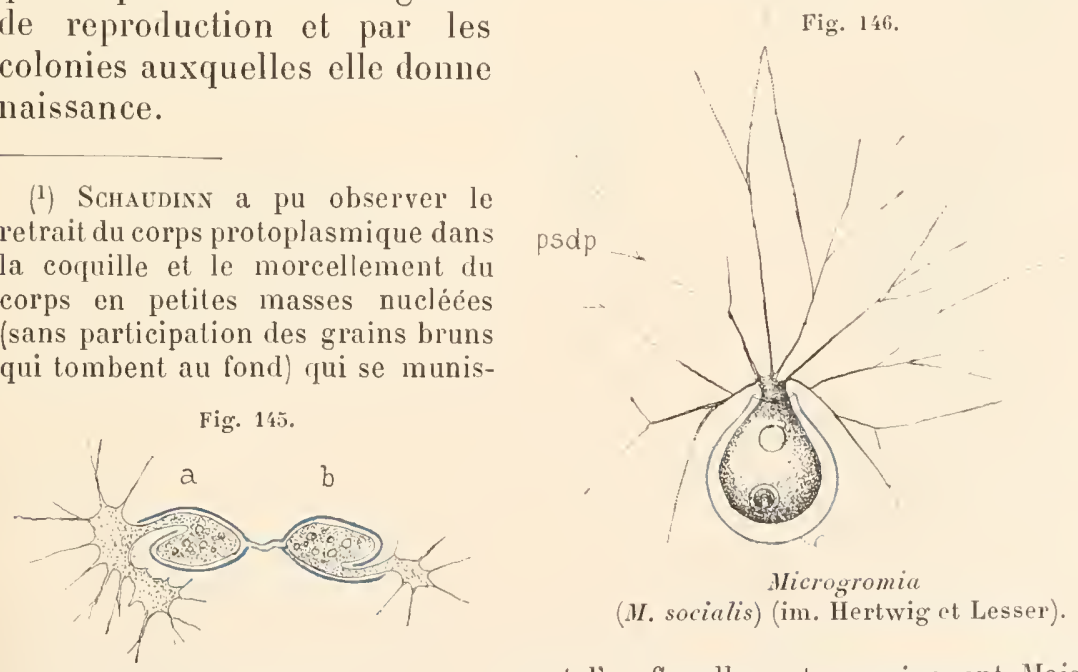

Lieberkühnia (L. paludosa). Division (im. Cienkorsky).

sent d'un flagellum et se conjuguent. Nais il n'a pu observer le sort ultérieur du produit de la conjugaison.

(2) L'animal se reproduit par division de la manière suivante (fig. 14.3). Une seconde bouche s'ouvre au fond de la coquille, et par là sort un second trone pseudopodique 
L’individu isolé (fig. 146) a une coquille (c) d'apparence chitineuse, en forme d'urne ouverte au sommet. Le corps protoplasmique contient un gros noyau unique etune petite vésicule pulsatile. Il n'émet au dehors qu'une petite masse de substance d'où partent seulement quelques fins pseudopodes peu ramifiés (').

La formation des colonies résulte (fig. 147, 148) d'une division

Fig. 148.

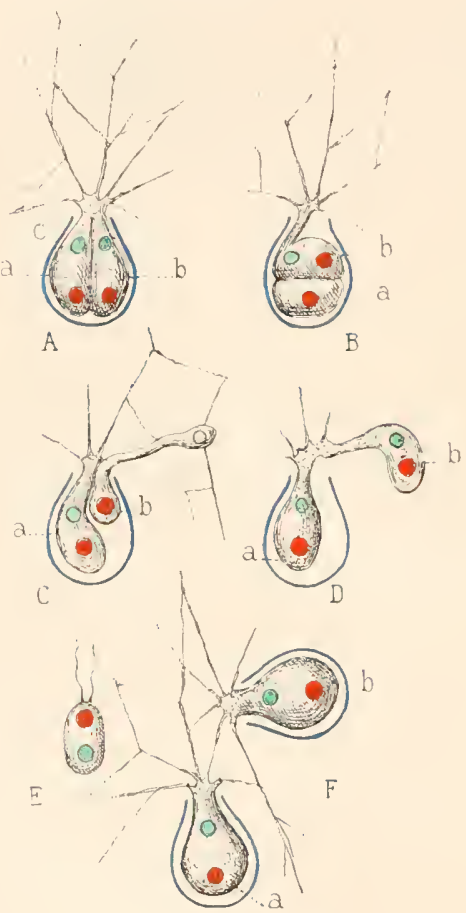

Microgromia.

Division (im. Hertwig et Lesser).

$A$, division longitudinale; $B$, division transversale; $C, D$, sortie d'un des deux individus; $E$, sa transformation en zoospore; $F$, son union à l'autre individu pour former une colonie. a., l'individu qui reste dans la coquille ancienne; $\mathbf{b . ,}$, celui qui en sort.

semblable au premier. La coquille se creuse à son équateur d'un sillon circulaire qui s'approfondit de plus en plus; elle finit par se couper; le corps mou continue à s'étrangler et l'isthme de réunion s'allonge, en sorte qu'on a deux individus $(a, b)$ réunis par un cordon. Enfin ce cordon se coupe et les deux individus se séparent.

(1) A un moment donné(fig. 148), le corps protoplasmique se divise, soit longitudi- 
incomptète. L'individu né d'une division ( $b$, dans $A$ à $D$, fig. 14S) sort bien comme d'ordinaire de la coquille; mais il reste attaché à l'ancien habitant (a) par quelques filaments pseudopodiques et se munit d'une coquille $(F)$. C'est là le commencement d'une colonie qui s'accroîtra par la répétition du même processus $\{40 \mu$. Eau douce) (").

Fig. 150 .

Pamphagus (Bailey) est un Vicrogromia à coque souple, suivant plus ou moins les mouvements du corps (Eau douce).

Lecythium (Hertwig et Lesser) n'est guère qu'une espèce du précédent (Eau douce).

Lecythia (Wright), que l'on place ici avec doute, serait un Lecythium porté sur un pédoncule (Mer).

Platoum (F.-E. Schulze) (fig. 150) forme aussi des colonies: c'est un Microgromia à bouche rétrécie, à coquille un peu souple et un peu trop vaste pour

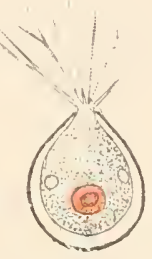

Platoum

(P. stercoreum)

(im. Cienkorsky). le corps qui l'occupe (Eau douce, terre humide et substances putréfièes). Plectophrys (Entz) ne diffère du précédent que par la structure de sa coquille (Marais salés de ḱlausenburg, Ilongrie).

Pseudodiffugia (Schlumberger) (fig. 13̈l) ne diffère de la Gromie que par sa coquille qui admet des particules étrangères comme celle de la Difflugie (Eau douce et stagnante).

Diaphoropodon (Archer) (fig. 152) a sa coquille entièrement formée de particules étrangères (Diatomées, etc.). Comme ì l'ordinaire, de la bouche Fig. 151.

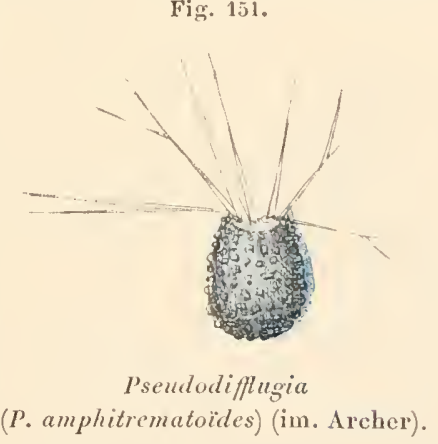
sortent des pseudopodes branchus, mais en outre de fins pseudopodes filiformes non réticulés passent dans les vides laissés entre les

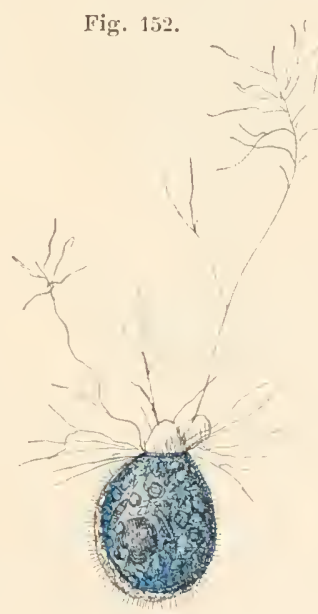

Diaphoropodon (D. mobile) (in. Archer). particules constituantes de la coquille sur toute la surface du corps.

nalement $(A)$ et alors les deux individus ont accès à la bouche, soit traversalement $(B)$ et dans ce cas l'un des deux est relégué au fond. Bientòt l'un des deux (b) (dans le cas de division transversale, c'est celui du fond) rampe hors de la coquille, reste attaché quelque temps à l'autre par quelques filaments $(C, D)$, mais s'en sépare sous la forme d'une zoospore à deux flagellums $(E)$ qui s'éloigne en nageant. Cette zoospore est sans doute destinée à se transformer en une pelite Gromie, mais on n'a pas suivi son évolution.

(1) Les individus de la colonie peuvent s'écarter les uns des autres (fig. 147) en 
Ces caractères lui donnent quelque analogie avec un Perforé, mais surtout une étroite ressemblance avec les Arénacés et il semble que l'on pourrait tout aussi bien le placer parmi ces derniers.

Les genres précédents étaient caractérisés, outre leur coquille mince et généralement chitineuse formée d'une seule loge, par leur bouche unique.

Les quelques suivants ont au contraire pour trait distinclif leur bouche double. La coquille est percée aux deux pôles opposés de deux orifices semblables par où sortent également des bouquets de pseudopodes réticulés('). Diplophrys (Barker) (fig. 13̈3) a ainsi une coquille sphérique ou fusiforme, percée d'une bouche arrondie en deux points diamétralement opposés. Mais ce genre à affinités douteuses pourrait aussi bien, comme nous l'avons vu page 81 , être placé parmi les Labyrinthulés (20 p. Eau douce et sur les excréments $\left({ }^{\mathfrak{2}}\right)$.

Ditrema (Archer) en diffère par sa coquille plus épaisse et ses bouches à bord un peu reployé en dedans (Eau douce). Amphitrema (Archer) (fig. 154) a, au contraire, la bouche

Fig. 153.

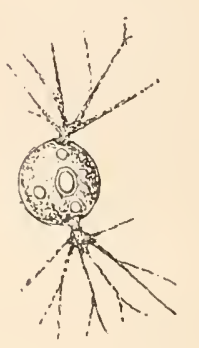

Diplophrys

(D. Archeri) (im.

Hertwig et Lesser) un peu saillante et la coquille incrustée de corps étrangers (Eau douce).

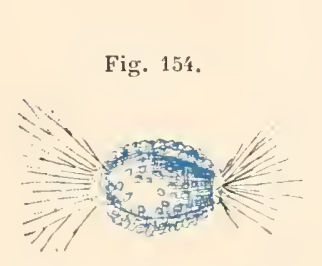

Amphitrema (1. Wrightianum) (im: Archer).

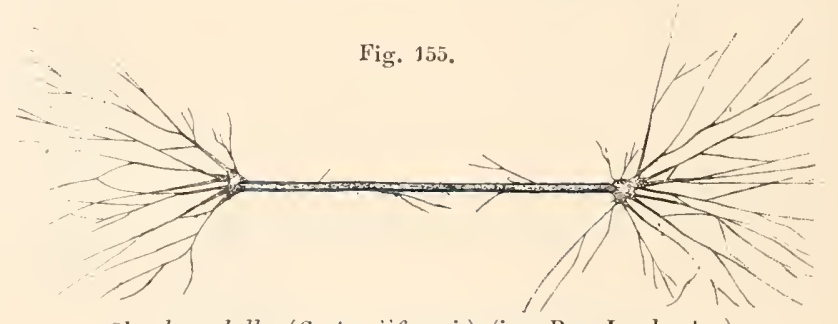

Shepheardella (S. txniiformis) (im. Ray Lankester).

Shepheardella (Siddal) (fig. 1亏弓̆), a une coquille hyaline membraneuse en forme de long tube rétréci aux deux bouts $\left\{\mathrm{g}^{\mathrm{mm}}\right.$. Mer $\rangle\left\langle{ }^{\mathbf{3}}\right)$.

allongeant leur's pseudopodes, ou se rapprocher en les rétractant (fig. 149). Ils arrivent parfois à former, en se collant les uns aux autres, des amas compactes émettant quelques pseudopodes par la périphérie. Dans cet état, ils ont été décrits par Archer comme un genre distinct sous le nom de Cystophys. On ne peut s'empêcher de remarquer une certaine ressemblance entre ces colonies et celles de certains Labyrinthulés (V. p. 81).

(1) Les précédents formaient la famille des Gromx.e [Gromidea (Clarapède et Lachmann)]; ceux-ci vont former celle des AMPIISTOMIV.E [Amphistomata (Hertwig et Lesser)]. Les uns et les autres étaient réunis par Brady dans son ordre de Gromidea.

() Cette coquille est d'ailleurs une simple membrane si mince que sa présence est douteuse. Il y a un noyau, plusieur's petites résicules pulsatiles et un ou deux globules graisseux de couleur orangée.

$\left({ }^{3}\right)$ Le protoplasma qui remplit le tube et fait saillie aux deux bouts pour former les 
2。 Sous-ORdRE

MILIOLIDES. - MILIOLIDE

[.MILIOLIDA (Carpenter emend.)]

\section{TYPE MORPHOLOGIQUE}

(FIG. 156 A 161)

Ici encore, le corps mou de l'animal ne diffère en rien de celui des autres Imperforés et c'est seulement dans la coquille que résident les caractères. Cette coquille est solide, calcaire, porcelainée, formée d'une substance calcaire sécrétée par le protoplasma en même temps qu'une matière chitineuse qui lui sert de ciment. Normalement, elle est simplement sphérique ou ovö̈de avec une large bouche à un pôle (').

Accroissement de la coquille. - Comment s'accroît-elle dans ce cas?

Le processus qui expliquait l'aceroissement des coquilles chitineuses n'est plus applicable ici, puisqu'elle est inextensible. Malheureusement on manque de matériaux pour résoudre cette imporlanle question.

Tout ce que l'on sait, c'est qu'elle s'accroît, que celle des adultes est plus grande que celle des jeunes : ce n'est donc pas comme chez les Coléoptères, par exemple, qui ont d'em-

Fig. 156 .

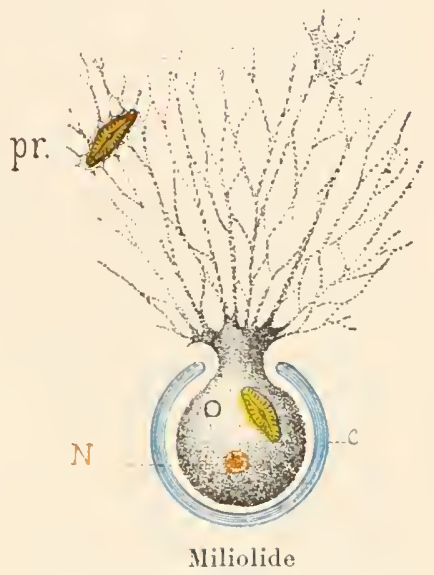

(Type morphologique) (Sch.).

N., novau; pr., proie; vc., vésicule pulsatile. blée leur taille définitive. On sait aussi qu'elle n'est pas rejetée et remplacée par une autre: ce n’est donc pas comme chez les Crustacés qui muent. On en est dès lors réduit à l'hypothèse de Max Schutz:e [54]: la coquille se résorberait par sa face interne, en mème temps qu'elle s'aceroîtrait, en épaisseur et par conséquent en surface, par le dépôt de nouvelles couches à sa face externe. La réalité de ce dépôt est d'ailleur's certaine, car e'est ainsi que se fait l'accroissement en épaisseur et que se forment, à la surface, les saillies, côtes, épines, qui ornent certaines coquilles. Le protoplasma qui déborde par la bouche pour former les pseudopodes s'étend, par moments au moins, sur toute la surface et dépose la substance minérale nécessaire pour les former.

pseudopodes, circule rapidenent dans le tube d'un bout à l'autre, entrainant le noyau qui roule sur lui-même en se déplaçant.

(1) La présence de ce ciment est rendue évidente par le fait que, dans certaines conditions de pénurie alimentaire, l'animal ne sécrète plus de calcaire et se forme une coquille chitineuse renfor'ée seulement de quelques grains de sable. Dans les grands fonds on a trouvé des coquilles formées d'une trame siliceuse. 
Voilà pour les coquilles monothalames, c'est-à-dire formées d'une

Fig. 157.

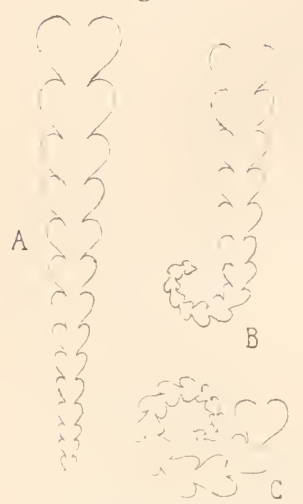

Miliolides.

Coquilles polythalames (Seh.).

$A, B, C$ différents modes d'agencement des loges. seule loge.

Mais nous pouvons dire dès maintenant que la plupart des Miliolides ont des coquilles polythalames, c'est-à-dire à plusieurs loges et, dans ce cas, la question se simplifie singulièrement.

Quand le corps s'est trop accru pour tenir dans la loge primitive, il se porte au dehors et en forme une seconde un peu plus grande, qui communique avec l'ancienne par' la bouche de celle-ci ouverte au fond de la seconde, et ainsi de suite. Il n'abandonne pas pour cela la loge ancienne, mais les occupe loutes les deux.

Les loges successives peuvent se disposer à la suite les unes des autres (fig. 157) de manières très différentes: en ligne droite $(A)$, en spirale $(C)$, en hélice, en crosse $(B)$, etc., etc., ce qui, joint à leurs différences individuelles, explique l’infinie variété de formes que peuvent revètir ces Foraminifères.

Dimorphisme. - Un autre facteur vient encore accroître cette diversité. Dans beaucoup de coquilles enroulées, on trouve, à l'origine de l'enroulement (fig. 158, $A$ et $B$ et $159, A)$, une loge initiale sphérique (A) appelée mégasphère, plus grande que les suivantes. Cette loge communique par un canal $(c)$ avec la première loge spirale qui, tout de suite, prend la disposition d'enroulement caractéristique de l'espèce. Or, chez d'autres individus de la même espece, on trouve (fig. $159, B$ ), en place de celte grande loge initiale, plusieurs petites loges (en général six) dont une initiale lrès petite, appelée Fig. 1 5 .

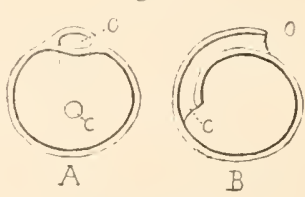

Mégasphère

(d'ap. Munier-Chalmas).

$A$, de face; $B$, en coupe.

Le eanal communique on $c$ avee la mégasphere, en o avec la 1 re loge spiralée.

microsphère, au centre, et les autres groupées autour d'elle en spirale, sans souci de l'arrangement spécial Fig. 159. des loges suivantes.

Ces deux formes ont été désignées: la première, sous le nom de mégaspliérique ou forme $\mathbf{A}$, la deuxième sous celui de mierosphérique ou forme B (').

(1) C'est a MM. Munier-Cinalmas el SculumBerger que l'on doit l'intéressante découverte de ce polymorphisme. Nous ne manquerms pas de signaler au passage les genres oì il
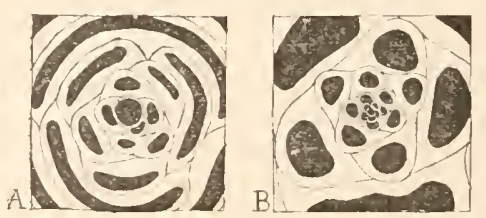

Purlion centrate de la coquille (d'ap. Nunier-Chalmas).

1, daus la forme mégaspherique; $b$, dans la forme microsphéripue. se rencontre. Voiri la liste le ceux oì il a élé observé parmi les lmperforés: Biloculina, Dillina, Fabularia, Lacasina, Friloculina, Trillina, (Muingusloculina, Pentellina, Inetrillina, Orbitoliles, Al'colina. Il exisle aussi chez divers Perforés. Nous le signalerons quand nous trailerons de ce groupe. 
Les relations mutuelles de ces deux formes ont été beaucoup discutées et l'on n'est que tout récemment, et seulement pour quelques genres, arrivé à une conclusion certaine. Deux opinions principales étaient en présence. D’après l'une, les deux formes seraient les états successifs d'un même individu. Les jeunes naîtraient tous de la forme A. Beaucoup d'entre eux vivraient et mourraient sans se modifier, mais quelques-uns, à un moment donné, résorberaient leur mégasphère et bourgeonneraient, à sa place et en sens inverse de l'accroissement à la bouche, une courte spire de quelques loges. D'ailleurs ces formes microsphériques, en se reproduisant, engendreraient de nouveau des formes A. Mais VAx Der Brock [93] et d'autres ont constaté que les loges voisines de la région affectée par le dimorphisme ne sont pas disposées de la même manière dans les deux formes, en sorte qu'il faudrait qu'une région importante de la coquille se résorbât et fût reformée à nouveau. Or ce travail prendrait un temps assez long et l'on devrait pouvoir surprendre des individus en voie de transformation, ce qui n'arrive jamais.

La seconde opinion est que ce dimorphisme est inilial, que les individus naissent et meurent dans la forme quileur est propre et que les deux formes résultent d'une alternance de génération, ceux de la forme $\mathbf{A}$ engendrant ceux de la forme B, et réciproquement. Cette opinion repose sur des faits certains, observés, il est vrai, chez un tout petit nombre de formes, mais il semble bien qu'on est en droit de les généraliser (').

(1) Lister [95] et Schaudinn [95] sont les observateurs auxquels on doit cette importante découvertc. Leur's observations, concordantes dans leurs traits généraux bicn qu'entreprises séparément, inspirent toutc confiance.

D’après Lister, qui a étudié a la fois les Imperforés (Orbitolites) et les Perforés (Polystomella), les individus a se reproduisent d'abord sous leur forme et par le procédé habituel. Les petites masses destinces à former les jeunes (V. p. 120), se munissent d'une coquille sphérique et constituent la loge initialc d'un jeunc de la forme $\mathbf{A}$. Celui-ci forme, en une heure, une deuxième loge, en vingt-quatre heures une troisième, etc, et devient peu à peu adulte. Cela continue ainsi pendant plusieurs générations; mais, à un moment donné, le processus change, l'animal se rétracte dans sa coquille et fragmente tout son contcnu en nombreuses petites masses arrondies qui se munissent d'un flagellum et constituent autant de zoospores. Ces zoospores (fig. 160), rui mesurent 4 p., sc conjugueraient et formeraient une petite masse de 6 a $13 \mu$ qui serait la loge initiale l'un individu de la forme B. Celui-ci grandirait mais, au moment de

Fig. 160.

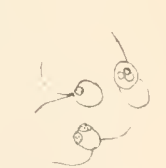

Polystomella Spores flagellées (d'ap. Lister). se reproduirc, donnerait naissance, non a des zoospores ni à des formes $\mathbf{B}$, mais à des formes $\mathbf{A}$, selon le procéde décrit il y a un instant pour celles-ci. Ainsi il y aurait alternance périodique cntre une série de générations ágames $\mathbf{A}$ et une génération sexuée $\mathbf{B}$.

l'après Śchaudinn, 'fui a étudié seulement Polystomella, la forme A ne se reproduit qu'exceptionnellement par elle-mêmc. La forme B est caractériséc par la présencc de nombreux noyaux répandus dans toutes les loges et provenant de la division d'un noyau initial unique $V$. ci-dessous, fig. 161). Son protoplasme sort de la coquille et sc morcelle autour des noyaux, chaque fragment devenant une loge 
Reproduction. - La reproduction par division, si facile à comprendre chez les formes monothalames ou à coquille souple, ne se conçoit plus aussi bien avec les coquilles calcaires à loges très nombreuses et très entortillées que nous allons maintenant rencontrer. Ici se présente un mode particulier de reproduction qui tient du bourgeonnement et de la division successive. Dans l'intérieur de la coquille, le noyau unique se divise en un grand nombre de petits noyaux qui se répandent dans toutes les loges ('). Ces noyaux condensent autour d'eux des petites masses de protoplasma qui prennent la forme d'un jeune individu, se munissent d'une coquille et sortent, soit par la bouche (Ammodiscus), soit par rupture de la coquille. Chez Miliolina le corps protoplasmique maternel sort en masse de la coquille et se divise en jeunes $\left({ }^{2}\right)$.

initiale macrosphérique d'individu A. Celui-ci est d'abord polynucléé, mais ses noyaux se fusionnent bientôt en un, en sorte qu’il est désormais uninucléé. Quand il est devenu adulte, son noyau se morcelle en fragments qui se répandent dans toutes les loges, y subissent une division mitosique et, se partageant le cytoplasme, donnent naissance à autant de zoospores qui se conjugueraient avant de se développer en individus de la forme $\mathbf{B}$.

Il faut noter que la conjugaison des zoospores et le sort ultérieur du produit de cette conjugaison hypothétique n'ont jamais étė observés. Schaudinn [94] a, il est vrai, vu la conjugaison des zoospores chez une Gromie (Iyalopus), mais les Gromies sont bien loin des Polystomelles et il n'a pu suivre l'érolution du produit conjugué.

"Dans leurs travaux sur le dimorphisme des Foraminifères, MU. Munier-Chalmas et Schlumberger avaient admis que la forme microsphérique dérivait par modifi-

Fig. 161.
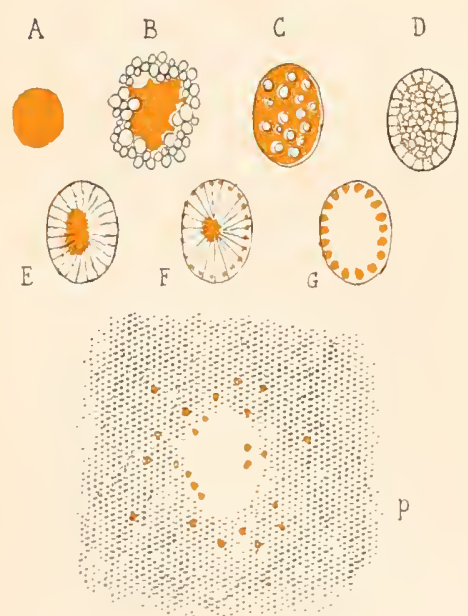

H

Miliolide (Type morphologique). Division du noyau (im. Schaudinn). cation interne de la forme macrosphérique. Aujourd'hui M. Munier-Chalmas pense que, contrairement à cette opinion, tous les faits observés sont en faveur de la seconde hypothèse qui accorde une origine distincte à chacune des deux formes $\left({ }^{*}\right) . » M$ M. Schlumberger s'est également rallié à l'opinion de Schaudinn et de Lister.

(1) Le mode de division du noyau a été étudié par Schaudinn [94]. Le noyau (fig. 161) est d'abord formé (A) par une masse chromatique homogène sans membrane. A un moment donné, il devient très vacuolaire $(B)$ au moyen de liquide cytoplasmique qu'il absorbe, grossit beaucoup et se munit l'une membrane $(C)$. Le réseau intervacuolaire se montre formé d'une substance achromatique sur laquelle sont semées des granulations chromatiques $(D)$. La chromatine s'accumule d'abord au centre $(E)$, puis se porte à la périphérie le long de filaments achromatiques radiaires $(F)$ et s'y accumule en petites masses disposées régulièrement $(G)$. Enfin la membrane se détruit et les petites masses mises en liberté $(I T)$ constituent les noyaux filles qui se dispersent dans le cytoplasme $(p)$.

(2) Chez Orbitolites, Brady [88] a trouvé des jeunes réduits à leur loge initiale au centre de la coquille. Il a vu aussi [92] que

$\left({ }^{*}\right)$ La note entre guillemets nous est communiquée par M. Munier-Chalmas. 


\section{GENRES}

Calcituba (Roboz) (fig. 162 à 166), par la simplicité de sa coquille et de son

Fig. 162.

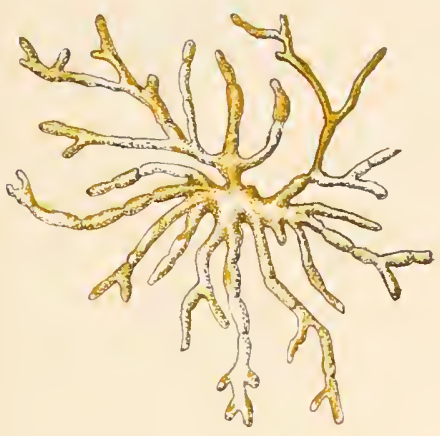

Calcituba (C. polymorpha) montrant la ehambre centrale et les tubes ramifiés qui en partent (d'ap. Schaudinn).
Fig. 163
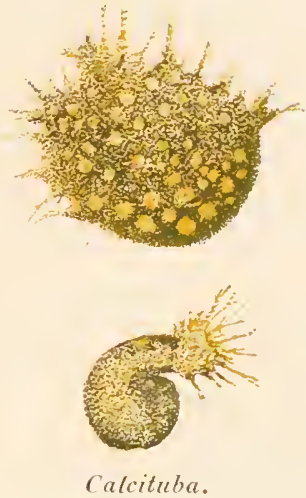

Formation de la loge centrale (d'ap. Schaudinn).
Fig. I64.

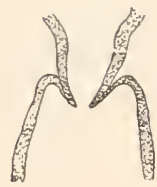

Calcituba. Septum séparant 2 chambres consécutives (d'ap. Schaudiun).

mode d'accroissement, se place à la base des Miliolides. A l'étal le plus parfait, c'est une coguille extrèmement mince, laissant voir la couleur rose du protoplasma sous-jacent, et formée simplement de grains calcaires sourés entre eux, mais pas assez noyés de ciment pour donner la structure porcelainée. Elle est formée (fig. 162) d'une grande chambrecentrale irrégulière d'où partent en tous sens des tubes ramifiés par dichotomie irrégulière. La paroi est imperforée, les tubes sont ouverts au bout, ce qui fait autant de bouches que de ramifications, enfin des septa incomplets assez espacés (fig. 164) les segmentent en chambres reconnaissables du dehors à un léger étranglement (10 à $12^{\mathrm{mm}}$. Mer) (').

l'animal, quand il est adulte, forme sur son bord de grandes chambres incubatrices tapissées d'une mince couche de protoplasma; le protoplasma des parties centrales $y$ arrive et $y$ forme de nombreux individus réduits à leur loge initiale avec un noyau, qui sont mis en liberté par résorption des parois de la chambre incubatrice. Schlumberger [88] fait remarquer que tous ces individus

Fig. 166.
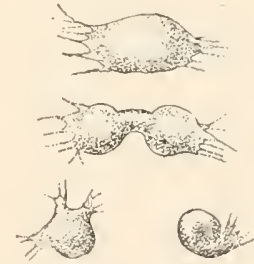

Calcituba.

Division des amibes (d'ap. Schandinn).
Fig. 165 .

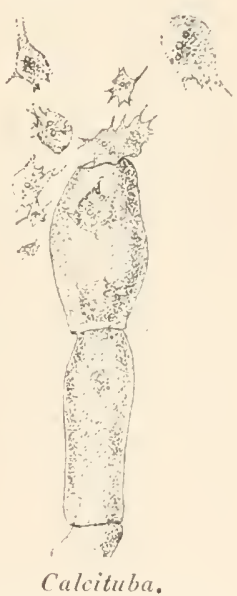

Sortie des prtites amibes (d'ap. Schaudinu). jeunes ont pour loge initiale une macrosphère. Il y a ici encore dimorphisme

( $\left.{ }^{1}\right)$ L'évolution de cette forme a été récemment étudiée par Schaudins [95] et 
Squamulina (11. Schultze) (fig. 167), représente le type morphologique du groupe it l'état monothalame, mais sa forme est plan-convexe; il est fixé par sa face plane et porte, excentriquement sur la face convexe, une bouche arrondie assez large (Vivant et peut-être aussi fossile).

Nubecularia (Defrance) (fig. 168), est polythalame, formé de loges fixées aussi par une face. Ses premières logges sont en spirale, mais les suivantes deviennent si irrégulièrement disposées

Fig. 168.

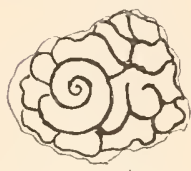

Nubccularia (Sch.). que la coquille n'a point de for-

Fig. 167.

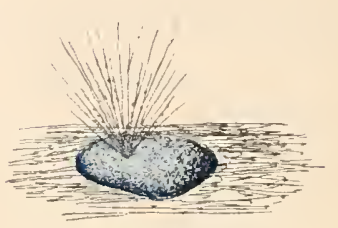

Squamulina

(S. lizvis) (d'ap. M. Schultze). me définie; elle est en outre souvent incrustée de sable.

Ce caractère monothalame, ou polythalame à disposition irrégulière des loges constitue la caractéristique assez peu nette de cette première série de genres (').

Bien mieux caractérisée est „la série suivante qui a pour type l'ancien genre

Miliola (Lamarck) (fig. 170, 171). La coquille est polythalame et les loges se succèdent en formant une spirale plane; chacune forme exactement un demi-tour et porte la bouche à son extrémité, en sorte que cet orifice

mérite de nous arrèter un instant. L'animal, constitué comme nous venons de le voir, est fixé sur quelque Algue marine qu'il mange. Il pousse sans cesse par la périphérie. Quand le support est mangé. la partie centrale se rompt en fragments qui tombent au fond tandis que les bouts des tubes restent sur l'Algue où ils continuent à grandir. Ces bouts, en s'accroissant, continuent à se ramifier, mais toujours ils grandissent par l'extrémité distale. tandis que l'extrémité proximale se rompt par fragments successifs qui tombent aussi au fond. Ces fragments délachés (que ce soient ceux du début ou les autres), s'ils sont tombés sur une $A$ lgue, peuvent y trouver de la nourriture et grandir. Sinon, ils sont affamés et alor's, ou bien s'isolent en fermant leurs orifices par une lamelle de chitine et attendent quelque chance de rencontrer des aliments à leur portée, ou bien ils s'égrènent en petites amibes qui sortent du tube (fig. 165), rampent et s'éloignent pour manger. Mais il reste toujours dans le tube une importante portion du protoplasma continu qui, lui, est destiné fatalement à mourir. Cies amibes peuvent, si elles sont assez grosses, se diviser (fig. 166), mais en tout cas elles mangent, grossissent et reconstituent peu à peu l'individu primitif. La forme de celui-ci résulte (fig. 163) de ce que, au début, elles ne forment pas de pseudopodes, restent contractées (montrant parfois une tendance à l'enroulement spiral) el secretent du carbonate de chaux. Ainsi se forme la première chanbre, puis elles émeltent de gros pseudopodes qui, à leur tour, se convrent de calcaire. Ceux-ei, des lors, ne croissent plus que par le bout, se dicholomisent, et ainsi s'explique la forme de l'animal. Le cycle est terminé.

Il y a là plusieur's noyaux par chambre. Ceux-ci ne se nultiplient jamais par division simple. Lem mode de multiplication est conforme it celui que nous arons décrit à la page 120.

(1) Cetle première série de genres constitue la famille des NubEculins:E [Nubecularina (Brarly)].

La serie suivante forme la famille des MiLroLs.e [Miliolinina (Brady)]. 
est transporté alternativement aux deux extrémités d'un même diamètre. Son entrée est rétrécie par une dent saillante à sa face interne ou par une plaque criblée. Si les loges sont peu ou point embrassantes, chacune s'ajoute aux précédentes sans les cacher, en sorte qu'il est facile de les compter toutes. Mais, dans le cas contraire, elles cachent tout ou partie des loges anciennes de manière à n'en laisser voir qu'un certain nombre et on en compte en dehors beaucoup moins qu'il n'y en a en réalité. C'est d'après cet aspect extérieur que l'on a établi divers genres dont le nom rappelle ce que l'on voit et nullement ce qui est (').

Supposons une Miliolide qui, constituée à l'état jeune comme un Miliola (Lamarck), c'est-à-dire avec un enroulement spiral régulier et deux loges par tour, change ensuite cette disposition et prenne alors trois ou quatre loges an plus à chaque tour, nous aurons un

Planispirina (Seguenza) (fig. I69) (Vivant et fossile).

Supposons maintenant qu'avec un début semblable, les loges, au lieu de continuer

(1) Voici ces genres :

Spiroloculina (d'Orbigny) (fig. 170), laisse voir toutes ses loges (Vivant et fossile);

Fig. 169.

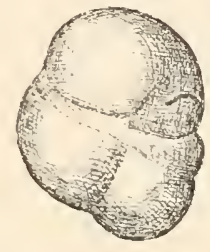

?.

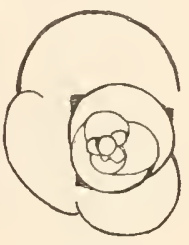

A

Planispirina (im. Schlumberger).

$A$, en coupe; $B$, entier.

Quinqueloculina (d'Orbigny) est un peu embrassant et laisse voir quatre loges d'un côté et trois de l'autre, mais comme deux se voient des deux còtés, il n'en reste que eing différentes visibles du dehors, d'où le nom (Vivant et fossile);

Pentellina (Munier-Chalmas), Massilina (Schlumberger) et Adelosina (Schlumberger) (fig. 171) sont des genres voisins; dans ce dernier, la loge initiale est complètement renfermée dans la suivante (Vivant);

Fig. 170 .

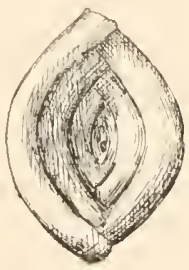

spiroloculina

(Sch.).
Fig. 171

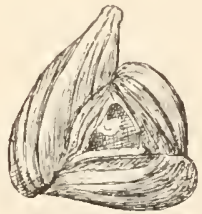

A

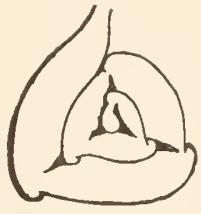

b
Adelosina (im. Sehlumberger). $A$, entier : $B$, en eoupe.

Triloculina (d'Orbigny), est plus embrassant et ne laisse roir que les trois dernières loges. (Vivant et fossile);

Trillina (Munier-Chaluas et Schlumberger) et

Linderina (Schlumberger) sont des genres voisins;

Biloculina (l'Orbigny) ne laisse plus roir que deux loges (Vivant et fossile);

Fabularia (Defrance) est un genre voisin à chambres cloisonnées. Enfin

Uniloculina (l’Orbigny), n’en laisse plus voir qu'une, la dernière (Vivant).

Malheureusement, cette simplicité élémentaire ne correspond pas à la réalité des fails, car les loges deviennent de plus en plus embrassantes à mesure que l'animal s'accroìt, et la Quinqueloculine devient plus tard Triloculine, en sorle que ces deux genres ne sont pas toujour's distincts et on a proposé de les réunir dans 
leur enroulement spiral, s'écartent pour se disposer en ligne droite suivant la tangente, et nous aurons un

Vertebralina (d'Orbigny) (fig. 172) (Vivant et fossile) (').

Peneroplis (Montfort) (fig. 173) a, comme les précédents, un enroulement différent suivant l'àge: les premières loges sont disposées en spirale, tandis que les suivantes, sans se disposer tout à fait en ligne droite, deviennent de moins en moins courbes. Mais ici, mème dans la partie jeune, le nombre des loges par tour est indéterminé et ne suit pas la loi des Milioles. Ce dernier caractère est celui d'une série

Fig. 172.

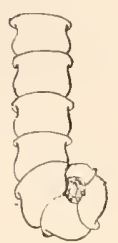

Vertebralina (im. Carpenter).
Fig. 173.

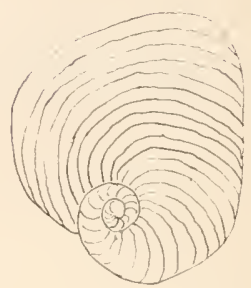

Peneroplss (im. Brady) de genres dont celui-ci est le type $\left(^{*}\right)$. On peut ajouter que les loges ne sont jamais embrassantes en sorte que la coquille reste très plate $\left({ }^{\boldsymbol{3}}\right)$ :

le genre Miliolina (Williamson). Bien plus, Munier-Chalmas et Schlumbergen [85] ont montré qu'une espéce, classée antérieurement comme Triloculine et comme Quinqueloculine selon son àge, était successivement constituée comme ces deux genres, puis comme une Biloculine et enfin comme une Uniloculine. Ils en ont fait le genre

Idalina (fig. 174) (Fossile). Genres voisins :

Periloculina Munier-Chalmas et Schlumberger, à chambres pourvues de côtes longitudinales saillantes intérieurement (Fossile), et

Lacazina (Munier-Chalmas), à chambres pourvues de piliers (Fossile).

Il résulte de là que les formes bi-, tri-, quinqueloculinaire ne caractérisent pas les genres de nom semblable, mais ceux-ci n'en sont pas moins très réels par l'ensemble de leurs caractères et méritent d'être conservés.

(1) Ces deux genres sont les types de la famille

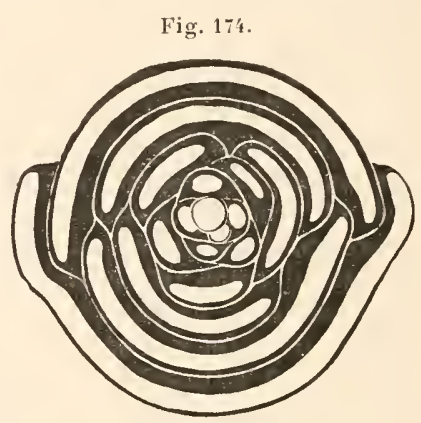

Idalina au stade biloculine (d'ap. Llunier-Chalmas et Schlumberger).

des HAUERINA [Hanerina (Brady)], caractérisés par ee début en coquille de Miliole et cette variation dans le nombre des loges ou le sens de leur succession. Dans la mème famille sont les genres :

Hauerina (d'Orbigny), qui diffère du premier par le fait que les tours précédents ne sont point, comme dans celui-ci, cachés par des expansions aliformes du dernier tour (Vivant et fossile);

Articulina (d'Orbigny), constitué à fort peu près comme le second (Vivant et fossile);

Ophthalmidium Kübler; , en tube spiral à cavité d'abord libre puis cloisonnée, el

Sigmoilina (Sclllumberger), genre dėdoublé des Spirillines.

(2) Ces genres constituent la famille des IDERLopLIxE [Peneroplidu (Brady)].

(3) Quant au détail de la disposition des loges, il est variable. Ici, il y a une courte partie spirale suivie d'une partie qui se détache suivant la tangente et s'élargit en éventail. La bouche est fermée par une cloison percée d'une unique rangée de trous et toutes les cloisons de séparation des loges ont le même caractère. 
Orbiculina (Lamarck) (fig. 175) a ses loges formées suivant la mème loi que Peneroplis, mais il se forme en outre de petites cloisons radiaires perpendiculaires aux faces de la coquille et aux cloisons concentriques séparant les loges. Ces cloisons divisent chaque loge en nombreuses logettes disposées comme les degrés d'une circonférence. Elles sont, de plus, percées de trous qui permettent aux logettes d'une mème loge de communiquer ensemble, tandis que les trous des cloisons circulaires leur permettent de communiquer avec celles des loges contiguës (Atteint $19 \mathrm{~mm}$. Vivant et fossile) (').

Fig. 175.

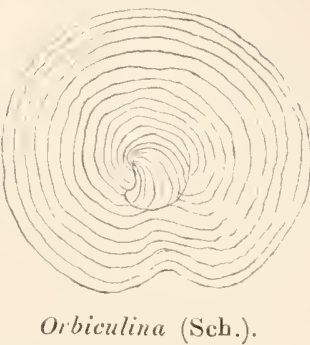

Orbitolites (Lamarck) (fig. 176) diffère du précédent princijalement par le fait que son enroulement est spiral dès l'origine et devient toujours et rapidement cyclique: il y a la loge centrale, puis deux loges faisant un deuxième tour et, dès le tour suivant, chaque loge fait un tour complet. Les loges s'épaississent en grandissant, en sorte que l'ensemble prend la forme d'un disque plus épais au bord qu'au centre; elles sont subdivisées en logettes de la mème manière que chez Orbi-

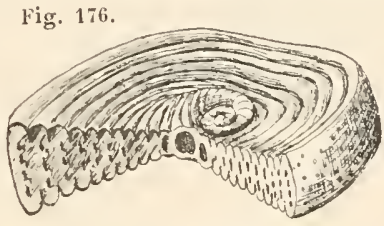

Orbitolites (Sch.). culina, et alternent d'un cycle à l'autre (Atteint 0 mm9. Vivant et fossile) $\left({ }^{*}\right)$.

(1) Une autre complication peut s'ajouter à la précédente et elle se présente dans les formes les plus typiques du genre. Que l'on suppose les dernières loges continuant à s'accroître en éventail cliez un Peneroplis; elles circonscriront peu à peu la partie ancienne de la coquille et finiront par l'entourer complètement. Les loges deviendront alors circulaires et formeront ehacune un tour complet. A partir de ce moment, l'aceroissement continuera de la même manière, chaque loge formant un tour entier, concentrique aux précédents. Dès lors, la bouche formera toute la circonfèrence de la coquille. Comme ehez Peneroplis, la bouche étant percée d'une rangée de trous, quand une nouvelle loge se formera, cette ancienne bouche deviendra la eloison circulaire de séparation entre les deux dernières loges, cloison percée aussi d'une rangẻe de trous pour les faire communiquer ensemble. Il peut aussi y avoir plusieurs rangẻes parallèles de trous, lor'sque la loge est assez épaisse.

(2) C'est à cela que se borne la complication dans les plus simples Orbitolites dont on a fait le sous-genre

Sorites (Ehrenberg). Mais dans les vrais

Orbitolites (Lamarck, $s$. str.), les logettes se subdivisent, non tout de suite, mais dans les cycles qui sont à quelque distance du centre, en trois parties superposées, une centrale et deux superficielles. La centrale, plus grande, plus élevée que les deux autres, conserve les mêmes rapports et multiplie simplement ses communications avee les logettes voisines de même ordre à nresure qu'elle augmente de hauteur. Dans les parties anciennes où elle est peu élevée, elle communique par un seul eanal avee ses deux voisines du même cyele et par deux canaux avec les voisines des eycles limitrophes avec lesquelles elle alterne. Mais dans les parties épaisses de la coquille, il y a, pour chacune de ces communications, plusieurs canaux superposés. Les périphèriques forment sur les deux faces du disque une couche de logettes spéciales. Celles-ci ne 
Cornuspira (Max Schultze) se distingue de tous les autres par le fait qu'il est monothalame étant formé d'un tube sans cloisons, contourné en longue spirale plane (Vivant et fossile) (").

Alveolina (d'Orbigny) (fig. 177), au contraire, au lieu de former une coquille plate, à axe d'enroulement très court, devient ovoüle ou fusiforme, à axe d'enroulement au moins aussi long et souvent plus que toutes les autres dimensions du corps. Cela tient à ce que les loges, d'ailleurs toujours enroulées en spirale régulière, sont très basses, mais très larges, et complètement embrassantes, chacune s'étendant d'un pôle à l'autre de la coquille et recouvrant complètement la partie correspondante du tour précédent. Comme elles sont

Fig. 177 .

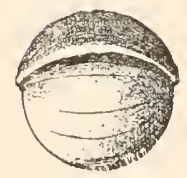

Alveolina

(im. Brady). très peu élevées dans le sens de l'enroulement, il en faut un grand nombre pour faire un tour. Les cloisons qui les séparent s'étendent parallèlement à l'axe d'enroulement, d'un pôle à l'autre, mais elles sont très basses et ne forment qu'une forte côte au plafond des loges, laissant celles-ci communiquer largement entre elles, au niveau de leur plancher. La dernière loge s'ouvre naturellement au dehors par une longue bouche qui va aussi l'un pôle à l'autre. Indépendamment de ces loges et cloisons primaires parallèles à l'axe, s'en trouvent de secondaires et même de tertiaires (15 à $75^{3} \mathrm{~mm}$. Vivant et fossile) ${ }^{(2)}$.

communiquent pas entre elles, mais seulement avec les logettes centrales sousjacentes, n'alternent pas d'un cycle à l'autre comme ces dernières, et enfin reculent un peu vers le centre de manière à être à cheval sur la cloison de séparation du cycle dont elles dépendent et du cycle précédent; et elles communiquent avec les logettes sous-jacentes de ces deux cycles. Ajoutons que les logettes périphériques sont, dans chaque cycle, plus nombreuses que les centrales, il y en a souvent trois ou quatre pour une de ces dernières.

(1) A ces genres ajoutons, comme faisant partie de la famille des Peneroplinx: Archiacina (Munier-Chalmas) qui est un Peneroplis sans còtes, décomposé en deux sousgenres:

Brœckina (Munier-Chalmas) et

Brøekella (Munier-Chalmas).

Le genre Cornuspira mériterait de former une famille à part.

(2) Les eloisons secondaires sont disposées suivant des plans parallèles à l'équateur du fuseau et, comme les cloisons primaires sont très peu développées, elles s'élendent sans interruption depuis la bouche jusqu'à l'origine de la coquille. Bien entendu, elles ne vont pas jusqu'à l'origine même de la coquille, car les loges anciennes très courtes ne peuvent être recoupées par autant de cloisons secondaires que les jeunes beaucoup plus grandes. Elles s'arrètent à des niveaux différents. Elles découpent la fente buccale eu une série d'orifices juxtaposés. Ces cloisons sont complètes, percées seulement dans claque loge primaire d'un ou deux orifiees qui font communiquer entre elles les loges secondaires qu'elles séparent. Enfin, dans cerlaines espèces, il s'ajoute encore ì cela des cloisons teriaires déterminant des loges de troisième ordre. Ces cloisons sont parallèles à la surface de la coquille. 11 y en a de deux à cinq dans chaque compartiment secondaire des loges primaires. Elles s'insèrent sur les cloisons secondaires, mais ne s'étendent, dans le sens de l'enroulement, que sur une partie de la lon- 
Keramosphæra (Brady) a une coquille sphérique arec une multitude de logettes de forme plus ou moins irrégulière disposées en courbes concentriques (Vivant) (").

\author{
$3^{\text {e SOUS-ORDRE }}$ \\ ARÉNACÉS. - ARENACIDA \\ [AsTRORIIZIDE (Brady) + ARENACEE (Bütschli)]
}

TYPE MORPHOLOGIQUE

(FIG. 178)

Ici encore, l'animal est fort semblable à celui que nous avons décrit pour notre type morphologique d'Imperforé, et c'est par la constitution de sa coquille qu'il se caractérise. Celte coquille $(c)$ est formée, en effet, de particules étrangères (grains de sable, carapaces de Diatomées, spicules d'Eponges, etc.), tantôt simplement agglutinées contre la surface du corps par le protoplasma superficiel, tantôt plus ou moins fortement cimentées entre elles par une substance organique qui d'ordinaire est, ou semble ètre, de la vraie chitine, mais parfois se montre avec les réactions spéciales de la matière cornée. Lorsqu'il y a un ciment chitineux complet formant un vernis continu à la face interne de la coquille, celle-ci est aussi imperforée que celle des groupes précédents, mais lorsqu'il en est autrement, il reste entre les particules mal associées qui forment la coquille de petits espaces par lesquels le cytoplasma sous-jacent peut émettre des prolongements mobiles analogues à des pseudopodes, et il y a là une condition qui rapproche ces êtres des Foraminifères perforés.

L'accroissement de la coquille se comprend aisément, soit que ses particules soient indépendantes les unes des autres, auquel cas il se conçoit sans explication, soit Fig. 178.

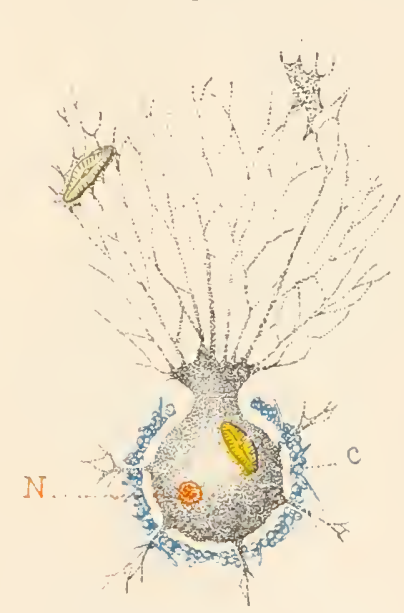

Arénacé (Type morphologique)

(Sch.).

c., coquille. qu'elles soient réunies par un ciment chitineux. Dans ce cas, les choses

gueur de la loge primaire de manière a laisser communiquer entre eux tous les compartiments qu'elles déterminent. Elles ont pour effet de recouper la sẻrie unique d'orifices buccaux en deux à cinq séries parallèles superposées. (Les fossiles seuls arrivent à la taille de $75^{\mathrm{mm}}$; les vivants atteignent $15^{\mathrm{mm}}$ seulement.) (Brady)].

Alseolina est le seul représentant de la famille des ALVEOLINLAE [Alveolininx

(1) On fait de ce seul genre la famille des KERA MOSPHARLNE [Keramosphærina (Brady)]. 
se passent pour cet enduit chitineux comme pour la coquille des Gromides. Quant au dépôt de nouvelles particules étrangères pour combler les lacunes produites par l'écartement des anciennes, il se fait au moyen des pseudopodes, ou de petits prolongements pseudopodiformes nés du cytoplasma dans les hiatus de la coquille, qui saisissent des particules et les accolent. L'animal ne prend d'ailleurs pas au hasard : il choisit la grosseur et la nature des matériaux, triant des grains de sable d'une certaine taille, ou des spicules d'Éponges. Dans bien des cas, il semble, en outre, que des résidus alimentaires, grains de sable, carapaces de Diatomées, etc., sont ajoutés à la coquille par le dedans, conformément ì ce qui a été observé ailleurs (chez Diffugia par Verworn) (").

On peut distribuer les Arénacés en deux tribus :

A STRORIIZINA, à grandes coquilles asymétriques, le plus souvent monothalames et en forme de tubes simples ou associés entre eux ou à une chambre centrale commune;

LITUOLINA, à coquilles régulières, le plus souvent polythalames, mais à loges séparées par des cloisons imparfaites, labyrinthiques; ces êtres représentent les isomorphes arénacées des Miliolides et des Perforés les plus simples.

1re Tribu

ASTRORIIZINES. - ASTRORIIZIAA

[AstroRHIZID.E (Brady)]

\section{GENRES}

Astrorhiza (Sandahl) (fig.l 79) a une coquille formée d'une épaisse couche de grains de sable et de vase, simplement accolés par le protoplasma ou légèrement cimentés. Cette coquille, parfois fusiforme, a ordinairement la forme d'un disque aplati et muni sur son bord de prolongements radiaires. Ces prolongements sont ouverts au bout et, par leurs extrémités, sortent autant de bonquets de pseudopodes réticulés $(10 \mathrm{~mm}$ et plus. Mer).

A Astrorhiza se rattachent quelques genres qui n'en diffèrent que par des caractères secondaires $\left({ }^{2}\right)$.

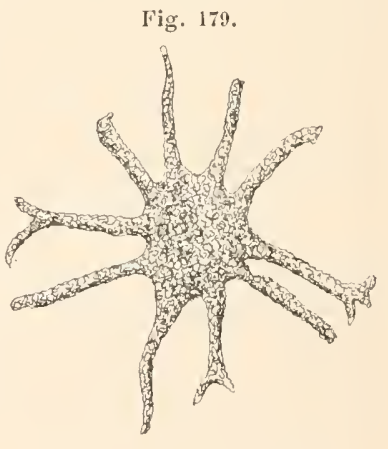

Astrorhisa (im. Brady).

(1) La coquille est ordinairement monothalame et de grande taille, souvent branchue ou radiée. Parfois sa cavité est subdivisée en compartiments par des constrictions de la paroi, mais ce ne sont là ni de vrais septa ni de vraies loges, et ces formes polythalames sont toujours asymétriques.

$\left.{ }^{2}\right)$ Ces genres, au nombre de einq, sont les suivants :

Pelosina (Brady) (fig. 182), qui a sa coquille formée seulement de chitine et de vase, avec 
Pilulina (Carpenter) (fig. I80) a si coquille, ici encore, monothalame et de forme ronde, formée uniquement de spicules d'Éponges ou de sable fin, sans ciment calcaire ou autre. La bouche est fusiforme, courbe Mer. Vivant).

Il en est de mème chez

Technitella (Norman), de forme ovale el cylindrique, ì bouche arrondie (Mer. Virant).

Saccammina(Sars) (fig. 181) est, selon les espèces, mono- ou polythalame. Les formes polythalames, toutes fossiles, sont formées de loges monolhalames unies, soit latéralement de manière à respecter la bouche, soit en série linéaire par leur tube buccal, le tube de la précédente élant soulé au pôle aboral de la loge suivante. Les formes vivantes sont monothalames sphériques, ont une carapace lisse formée de gros grains de sable soulés par un fort ciment corné qui forme en outre un enduit intérieur continu. La louche est an sommet d'un petil prolongement conique (Vivant et fossile) (').

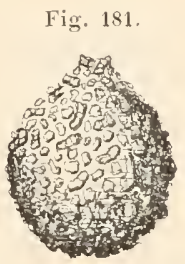

Saccammina (im. Brady).

une bouche au sommet d'un prolongement ehitineux, ce qui lui donne la forme d'une bouteille (Mer);

Storthosphæra (Brady) (fig. 183), 'jui est subgloljuleux, très irrégulier, garni de noubreuses protulérances terminées clacune par un orilice luceal $\mathbf{M e r}$;

Dendrophrya (Wright), qui peut êtle défini: un Astrurhiza à disque plus élevé et pourvu de prolongements ramifiès, dressés ou ranprants. Sa conuille est aussi formée de chitine el le vase(1;mm. Mer);

Syringammina (lirady), qui est au contrilie constilué de gros grains de sable lichement agglomérés, et formé d'une masse globuleuse de tubes branclus disposés radiairenent, arrangés plus nu moins distinctement en coucles superpusées (Her. Vivant;

Fig. 182.

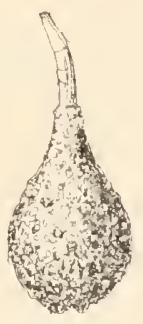

Pelosinu

(im. Brady). liig. 183.

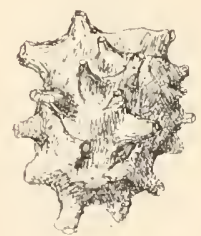

Storthosphapre

(im. Brady).

Julienella (Schlumberger), à test épais, à loge centrale cloisonnée et it tubes courts et irreguliers (Vivant).

Ces genres forment avec Astrorthiza la lamille des Astrominzm.L [Astrorhizine (Brady)].

(1) on ne sait rien de jlus au sujet des formes polythalames fossiles, mais RHunben $[9$, qui a fait une élude très détaillée de ce Foraminifère a fait connaitre plusieurs particularites intéressantes des formes vivantes monothalames. Quand il est jeune, saccanmiua a une coquille primitive le $0 \mathrm{mml}$ formée de tout petits grains avec des spicules d'Eponges el droursims. Mais il s'en forme bientot, en dehors de eelle-ci, une seeonde qui grossit beaueoup plus, en sorte que la première est accolée à elle comme une petite tubérosité. Cette seconde coquille n'est pas encore la eoquille définitive : elle n’a, en effel, pas le bouche el les grains de salıle yui la forment sont disposés sans aucune régularité en sorte qu'elle est très raboteuse. L'animal ne communique avec le dehors que par des expansions pseudopodiques qui passent dans les interstices des grains de sable. Fn cet état il a été décrit comme un genre spécial sous le noin de Psammosplixe (F.-L. Schulze).

Mais peu à peu, l'animal remanie sa eoquille, oriente ses grains de sable de manière 
Sorosphæra (Brady) est monothalame et n’a, à son unique loge, aucun orifice notable méritant le nom de bouche; ses pseudopodes sortent des orifices interstiliels, mais sa coquille comprend plusieurs chambres directement unies entre elles (Mer. Vivant) (').

Jusqu'ici, nous n’avons trouvé, comme éléments de formation de la coquille, que des grains de sable ef, s’il s'y trouvait mèlés des spicules d'Eponges, ce n'était qu’à titre exceptionnel.

Dans les Arénacés qui nous restent à citer, les spicules d'Éponges font régulièrement partie de la coquille et sont fortement unis entre eux et à des grains de sable pour la constituer. En outre, la forme de la coquille dérive de tubes cylimlriques directement associés entre eux $\left({ }^{*}\right)$. Jaculella (Brady) (fig. 181) forme un simple tube conique ourert à la grosse extrémité (atteint près de $10^{\mathrm{mm}}$. Mer. Vivant).

Bathysiphon (Sars) est en forme de tube légèrement conique, non cloisonné, dont la paroi, d'épaisseur notable (0mm:̈), contient des spicules d'ÉponFig. 18 '. ges agglutinés que l'on peut mettre en évidence en dissolvant la partie calcaire $\left(20^{\mathrm{mm}}\right.$. Mer. Vivant et fossile).

Marsipella (Normann) (fig. 18.i) est un tube cylindrique ou r'enllé en fuseau au milieu et ouvert aux deux houts (6mun. Mer. Vivant).

Rhabdammina (Sars) (fig. 186) est formé de tubes radiaires droits ou ramifiés, ouverts à l'extrémité, et sourent s'insérant par l'autre sur une partie centrale renflée qui joue le rôle de chambre commune (2:3mm. Mer. Vivant).

ì se former une surface tout à fait lisse, el se forme une bouche au sommet d'un prolongement conique. Dès lors, les wrifices par où passaient les pseudoporles se ferment.

l'animal contient un noyau avec plusieurs prétendus nucléoles qui se dissolvent peu à peu (sans donte quand l'animal se ulispose à se diviser) et fournissent la subslance d'un réseau chromatique qui se dessine progressivement.

Rhumbler decrit ici cinq nouveaux genres de Protozoaires ou Protophytes dont il indique a peine les affinités et dont nous ne ferons que eiter les noms en indiquant les groupes avec lesugels il leur trouve des ressemblances:

Rhynchogromia Rhumbler), Dactylosaccus (Rhumbler), Rynchosaccus (Rhumbler), Ophiotuba (Rliumbler) et

Dentrotuba (Rhumbler).

Il les a trouvés dans la corjuille de sa Saccammina. Il croit reconnaître des affinités chez le premier avec les fromies, chez le deuxième avec les Grégarines, rhez le troisième ef le cinquième peut-ĉtre avec les Characées, chez le quatrième avec les Iraliphigsema.

(1) Ces genres forment la fanille des SuccamM.E: [Saceamminince (Brarly)].

(2) Hs constituent la famille des RHABDAMMLE [Rhaldammininx (Brady)]. 
Hyperammina (Brady) pourrait se définir un Rhaldammina réduit à un tulse unique ayant les caractères de l'un des tubes du genre précédent Mer. Vivant).

Aschemonella (Brady) (fig. IS7), n'était la constiFig. 187. tution de sa coquille conforme à ce que nous venons d'indiquer, ne serait qu'une espèce d'Astrorhiza. Cependant, il faut noter que les tubes peuvent être moins nombreux et que, parfois, ils se soudent par leur extrémité au bout des tubes d'individus voisins, de manière à former un organisme polythalame (Ner. Vivant).

Rhizammina (Brady) (fig. ISS) est formé d'une masse libre de tubes flexibles,

Fig. 188 .

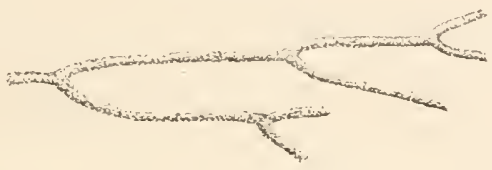

Rhizammina (im. Brady).

irrégulièrement ramifiés (25mm. Iler. Vivant). Sagenella (Brady) (fig. 189) est assez semblable, mais ses tulies sont fixés sur un supporl (pierre ou coquille) (Ier. Vivant).

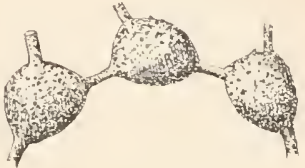

Aschemonella (im. Brady).

Botellina (Carpenter) est en forme de tube cylindrique dont l'intérieur porte des saillies irrégulièrement contournées, déterminant un cloisonnement incomplet. Il paraìt ètre fixé à une extrémité, tanclis que l'autre laisse passer les pseudoporles par des interstices (2:3mm. Mer. Virant).

Haliphysema (Bowerbank) (fig. 190) a une coquille constituée comme toujours de particules étrangères, mais qui sont ici presque exclusivement des spicules d'Éponges. La forme est celle d'une sorte de verre à pied long, étroit, irrégulier, dont le pied discoüle sert à fixer l'animal. Par l'orifice évasé, sortent les pseudopodes, longs, minces, irréguliers, très réticulés Mer. Vivant) (').

(1) Le corps contient de nombreux noyaux et des globules énigmatiques qui sont peut-être des sortes de spores.

Cet ètre singulier avait èté pris par Bowerbaxk pour une Éponge, et par Häckec $i \tau$. pour un ètre extrêmement simple à deux feuillets, le prétendu Enterozoon (Häckel)

Fig. 182.

Fig. 189.

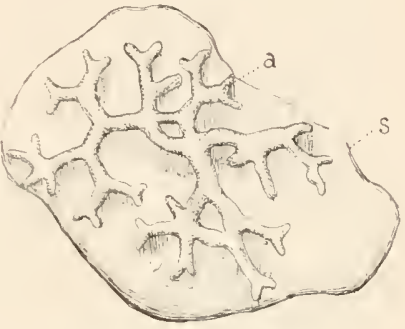

Sagenella (im. Brady).

a., lanimal; s., strpport. dont il faisait le type dune classe des Physémaires. Ses beaux dessins oủ est repré- 
2е TRि1в

\section{LITUOLINES. - LITLOLINA \\ [LITLOLID.F (Brady)]}

Indépendamment des caractères indiqués plus haut, la coquille présente, chez beaucoup de Lituolines, un caractère singulier. L'animal, après l'avoir formée, comme nous l'avons vu, de particules étrangères, l'accroît intérieurement par le dépòt, à la face interne, de nouvelles particules qui dessinent des lames irrégulières se coupant de la manière la plus variée, de facon à combler presque entièrement la cavité avec une substance réticulée labyrinthique. Les cloisons de séparation des loges polythalames se perdent plus ou moins dans cet ensemble. C'est ce caractère que nous désignerons sous le nom de chambres labyrinthiques chaque fois que nous le rencontrerons.

Les Lituolines sont souvent perforés et font ainsi le passage à l'ordre des Perforida (').

\section{GENRES}

Lituola (Lamarck) est une forme libre, polythalame, à loges labyrinthiques disposées en spirale régulière, sauf les dernières qui peurent prendre la direction de la tangente. La coquille est épaisse, rugueuse, grossière, formée de gros grains de sable (Vivant et fossile) $\left({ }^{\mathbf{2}}\right)$.

sentée l'organisation de cet être avec des feuillets épithéliaux réguliers à belles cellules nucléées ne correspondent à rien de réel.

${ }^{(1)}$ Bërschli [80-82] n’admet pas le groupe des Lituolines, préférant répartir ses genres parmi les Perforès et les Imperforés avec lesquels ils présentent le plus d'affinités.

Au point de vue de l'enseignement, cetle manière de faire a l'inconvénient de troubler la pureté des caractères des groupes où l'on fait entrer ces Lituolines. C'est pour cela que nous ne l'avons pas adoptée. Hais, au point de vue des affinités, elle est très admissible, la famille des Lituolines ne contenant guère de formes propres et étant surtout formée de représentants arénacès des types hyalins ou porcelainés des autres groupes tels que Comuspira, Miliolina, Peneroplis, Lagena. Nodosaria, Cristellaria, Globigerina, Rotalia, Nonionina, etc.

$\left(^{2}\right)$ Ce caractère de structure de la coquille est celui d'une série de genres qui constituent la famille des LITtoLINE [Lituolinx (Brady]) dont la Lituole est le type.

Ces genres sont les suivants:

Haplophragmium (Reuss) (fig. 191), qui est un Lituola à loges non labyrinthiques (Fossile);

Haplostiche (Reuss), qui est un Lituola dont les loges sont disposées en ligne droite ou un peu courbe. mais non spirale; il est parfois monothalame (Vivant et fossile);

Rheophax (Montfort), qui est un Ilaplostiche à loges non labrinthiques (Vivant et fossile);

Coskinolina (Stache), qui est un Haplophragmium dont les dernières loges vont en diminuant rapidement;

Bdelloidina (Carter), qui représente, avec la structure

Fig. 191.

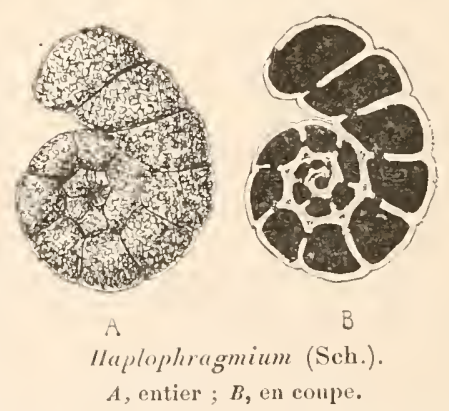

de coquille particulière aux Lituolines, un Peneroplis; il est fixé par une face; la 
Trochammina (Parker et Jones, emend. Brady) (fig. 192), au contraire, a sa coquille mince, lisse, par le fait qu'elle est formée de très petits grains de sable, fortement soudés par un ciment chitineux et calcaire. L'intérieur des chambres est lisse, non labyrin thique (Vivant et fossile)('). Endothyra(Phillips)(fig. 193) dissimule plus encore que les précédents son caractère arénacé par la prédominance du ciment calcaire sur les grains de sable (Fossile) $\left(^{2}\right)$.

Fig. 192.

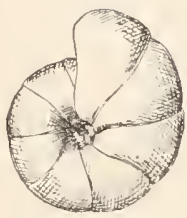

Trochammina (im. Brady).
Fig. 193.

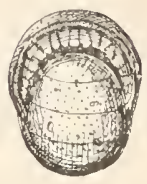

Endothyra

(E. crassa)

(im. v. Möller).

partie supérieure des loges est labyrinthique; le test serait perforé, les septa de séparation des loges sont percés d'une série de trous qui font communiquer les loges entre elles (Vivant, non fossile);

Placopsilina (d'Orbigny), qui représente le précédent sans perforations ni cloisons labyrinthiques (Vivant et fossile).

(1) Ces caractères se retrouvent dans toute une série de genres constituant la famille des TRochAMMx. [Trochamminine Brady)], dont Trochammina est le type. Génériquement celui-ci est caraclérisé par ses loges multiples enroulées à la manière de celles d'un Nautile ou d'un Trochus. Il est libre o'l fixé (Vivant).

Voici les autres genres de la famile :

Thurammina (Brady) (fig. 194), qui est monothalame, formé d'une seule loge sphérique, avec ou sans bouche principale à un pôle, mais avec plusieurs orifices accessoires au sommet de petites éminences ( Vivant);

Thuramminopsis (Häusler), qui n'est qu'un sous-genre du précédent;

Fig. 19\%

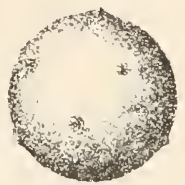

Thurammina (T. papillata) (im. Brady).

Hippocrepina (Parker), qui est aussi monothalame, mais à loge allongée, r'enflée à un bout, étroite à l'autre avec une bouche unique, de forme variable (Vivant);

Hormosina (Brady), qui est, pour la forme, un Rheophax a loges plus arrondies, pour la structure, un Trochammina (Vivant);

Ammodiscus (Reuss) (fig. 195), qui est libre et a une forme spirale ou héliçö̈dale plus ou moins irrégulière: il est néanmoins monothalame, sa coquille n'ayant point de cloison intérieure (Vivant et fossile);

Carterina (Brady), qui est polythalame, et représente un Trochammina avec spicules calcaires lui appartenant en propre (Vivant);

Webbina (d'Orbigny), qui est fixé, formé d'une ou plusieur's loges, réunies dans ce dernier cas en série irrégulière par des tubes de jonetion(Vivant).

${ }^{(2)}$ Il est le type de la famille des ExDotmrar.e [Endothyrinx (Brady)] caractérisée par cette structure de la coquille ainsi que par la netteté des cloisons qui séparent les loges. Celles-ci ne sont point labyrinthiques. La plupart de ces formes sont perforées et font le passage aux Perforida.

Voici les genres de cetle famille :

Nodosinel/a (Brady), qui est un Nodosaria (V. p. 133) pour la forme, un Endothyra pour la structure (Fossile);

Polyphragma (Reuss), qui a la forme d'un tube fixé par une extrémité, formé de courts segments cylindriques superposés, labyrinthique à l'intérieur et terminé par une bouche criblée (Fossile);

Involutina (Terquem), qui a un enroulement nautiloïde, mais à tours si embrassants que 
Loftusia (Brady) (fig. 196) est caractérisé par une structure arénacée si dissimulée par la finesse des grains et l'abondance du ciment calcaire qu'elle a pu être mise en doute. En outre, la structure intérieure des loges est compliquée par le développement d’un tissu labyrinthique abondant qui obstrue, en grande partie, la cavité de la coquille. Cette forme est perforée et fait, comme les précédentes, passage aux Perforida (Fossile) (').

Parkeria (Carpenter) est de forme sphérique et ses chambres très basses n'ont pas de rraies cloisons, mais sont soutenues par des piliers creux qui s'ouvrent dans la chambre située au-dessus et établissent ainsi une communica-

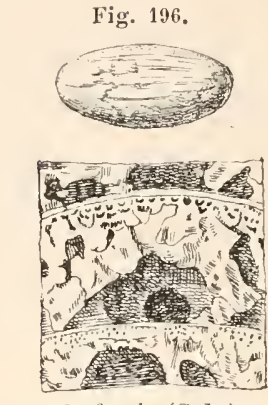

Loftusia (Sch.).

Ensemble de l'animal et coupe frontale. tion. Il n'y a pas de bouche. Les prétendues chambres initiales centrales, orientées suivant un rayon de la sphère, ne sont peut-être que la place du support primitif englobé par la coquille (Fossile).

l'ombilic est caché et que l'ensemble ne forme plus qu'une masse lenticulaire où l'on ne distingue pas les différents tours (Vivant et fossile);

Bradyina (Möller), qui est un Endothyra à bouche criblée (Fossile);

Stacheya (Brady), qui est fixé et dont l'enroulement est rendu irrégulier par la présence du support (Fossile).

(1) Que l'on se figure une grande coquille nautiloïde $(0 \mathrm{~mm} 8)$ à tours embrassants jusqu'à l'ombilic, de sorte que le dernier cache toujours tous les autres. Ces lours, très nombreux, jusqu'à vingt-cinq et plus, sont très peu élevés, mais très larges, en sorte que la coquille gagne plus en épaisseur, e'est-d-dire dans le sens de l'axe passant par l'ombilic el perpendiculairement à l'enroulement, que dans le sens de l'enroulement, el la coquille devient ainsi ovoïde ou même fusiforme, le grand axe passant par les onbilics. En outre, au lieu d'un cloisonnement régulier, avec des septa bien nets, séparant des loges bien libres, on trouve une disposition très différente. Plaçons-nous, par l'imagination, dans l'intérieur de la coquille en un point quelconque, les pieds tournés vers l'axe et la figure ver's l'ouverture. Nous avons pour plancher la lamelle qui nous sépare du tour précédent, pour plafond celle qui nous sépare du tour suivant. A droite et à gauche, nous pourrions atteindre jusqu'à l'ombilic. Le plancher est lisse, mais le plafond est garni d'une épaisse couche de tissu labyrintliqque qui rétrécit de moitié au moins l'espace déjà si restreint entre le plancher et la voùte. De distance en distance, sont les cloisons qui séparent les chambres. Ces cloisons sont obliques, complètes, mais formées du même tissu labyrinthique qui est à la voute, en sorte qu'elles laissent communiquer les loges entre elles. Enfin, entre les cloisons, s'étendent des tubes calcaires qui suludivisent la loge en loges secondaires. On voit que la cavité de la coquille est aux trois quarts remplie par ces productions squeletliques : les loges anciennes le sont même tout à fait.

La famille comprend seulement un autre genre:

Cyclammina (Brady), moins compliqué, simplement nautiloüde (Vivant). 
$2^{\circ}$ ORDRE

\section{PERFORES. - PERFORIDA \\ [PERForata (Carpenter)]}

\section{TYPE MORPHOLOGIQUE}

( FIG. 197)

Le corps mou n'offre, ici encore, aucune parlicularité différente de celles des Imperforés. La coquille $(c)$ est calcaire et, sous sa forme la plus simple, sphéroïdale. Elle est munie d'un large orifice qui est la bouche $(o)$, par où sortent des pseudopodes réticulés. Mais, en outre, elle est percée, sur toute sa surface, d'une multitude de fins pores qui donnent issue ì des pseudopodes filiformes rayonnants réticulés. Il existe un noyau mais, pas plus ici que chez les Perforés à coquille opaque, on ne sait s'il y a une vésicule pulsatile.

Comme chez les Imperforés, cette coquille simple, monothalame, réalise la forme primitive fondamenFig. 197 .

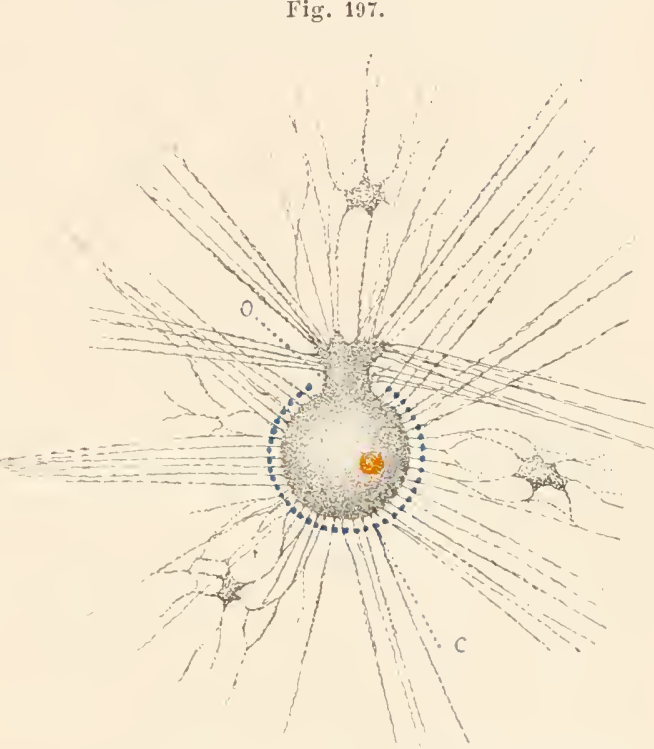

Perforé (Type morphologique). tale; mais, comme chez eux aussi, cette forme peut se compliquer par l'adjonction de nouvelles loges qui s'unissent entre elles de façons très variées. Nous retrouverons ici les mêmes complications progressives que chez les Imperforés, et l'autres encore (').

(1) Le caractère essentiel du Perforé est la perforation de la coquille. Nous avons trouvé dans le sous-ordre des Aréxacés des formes qui émettaient aussi des prolongements protoplasmiques sur la surface du corps mais, la plupart du temps, ces prolongements étaient simplement destinés à la capture des éléments de la coquille; ce n'étaient pas de vrais pseudopodes réticulés comparables à ceux qui sortaient par, la bouche. Dans les quelques cas où cette distinction n'eût pas été justifiée, il restait au moins ceci, que les pores par où ils sortaient étaient de simples liatus entre les éléments dissociés de la coquille et nous ne les avons jamais rencontrés dans des coquilles compactes. La présence de pores définis, donnant issue à de vrais pseudopodes à travers une coquille compacte est donc le caractère essentiel des PERFoRIDA. Tout ce que nous avons dit de l'accroissement de la coquille à propos des Perforés à coquille calcaire (V.p. 109 et 117) s'applique naturellement ici. 
Un Dimorphisme semblable à celıi des Imperforés existe aussi chez beaucoup de Perforés. Il n'y a qu'à appliquer ici tout ce que nous avons dit en discutant cette question à propos des Miliolides (V. p. IIS) (").

Nous diviserons les Perforés en six sous-orilres :

LAGENIDE, à pores fins et sans enroulement vrai;

CIILOSTOMELLID. à pores fins et enroulement de Miliolide;

TEXTLLARIDE, à pores fins et enroulement en hélice;

GLOBIGERINIDE. à pores larges ct cnroulement en spire obscure;

ROTALIDE, à pores larges et enroulement spiral régulier asymétrique;

NUMMILITID E, à pores fins et enroulement spiral régulier symétrique.

\section{$1^{\text {er }}$ Sous-OrdRe \\ LAGÉNIDES. - LAGENIDA \\ [LAGENID.E (Carpenter)]}

\section{TYPE MORPHOLOGIQUE}

Le type de ce sous-ordre serait une forme à perforations très fines, polythalame, à loges unies les unes aux autres en série linéaire, et agencées de telle façon que la partie supérieure de la précédente forme le fond de la suivante, les parois communes étant formées d'une seule lame calcaire qui appartient tout entière à la plus ancienne des deux. La forme de la série est, soit rectiligne, soit courbe, ou se complique d'autre manière. Enfin, il peut n'y avoir qu'une seule loge. De la combinaison de ceux de ces caractères qui sont variables, état monothalame ou polythalame et forme de la colonie dans le cas de polythalamie, résultent de nombreuses formes que l'on peut rattacher à quatre genres types.

\section{GENRES}

Lagena (Walker et Boys) (fig. 198) est monothalame et a une forme sphérique ou ovö̈le ou en fuseau; la bouche est à un pôle, généralement portée par un col allongé. La coquille est compacte, brillante, percée de pores très fins (Vivant et fossile) $\left({ }^{\mathbf{2}}\right)$.

Nodosaria (Lamarck) (fig. 199) est formé de plusieurs loges de Lagena disposées en ligne droite. La bouche est roude (Vivant et fossile).

(1) On l'a conslate dans les gentes suivants : Nodosaria, Dentalina, Siphogenerina, Rotalia, Truncatulina, Calcarina, Polystonella, Amphistegina. Nummulites et Assilina.

(2) Dans ce genre on a taillé des sous-genres que l'on peut considérer comme de
Fig. 199.

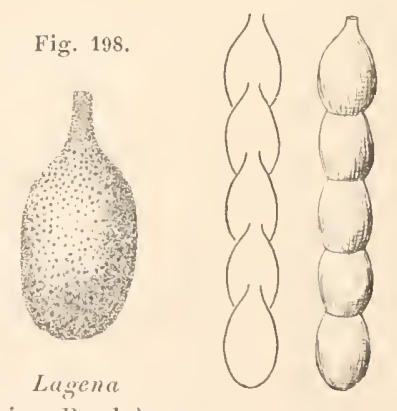

(im. Brady). 
Ce genre est le chef de file d'une longue série de genres dérivés ('). Polymorphina (fig. 200) (d'Orbigny)justifie son nom par ses loges de forme très variable, disposées sur deux rangs ou en spirale, mais toujours d'une façon peu nette, et plus ou moins embrassantes, en sorte que les nouvelles cachent un nombre variable des anciennes (Vivant et fossile) $\boldsymbol{(}^{\boldsymbol{2}}$ ).

Ramulina (Rupert Jones) (fig. 201) est formé, dans ses premières loges, comme une Polymorphine, mais continue par des loges armées de tubulures, les unes longitudinales servant à les

Fig. 200.

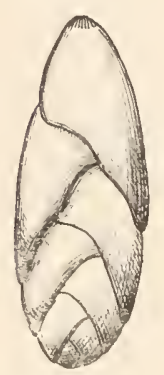

Polymorphina

(im. Brady).
Fig. 201.

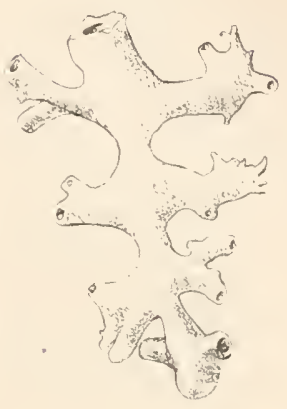

Ramulina

(d'ap. Schlumberger).

simples espèees. Ils forment avee Lagena la famille des LAGEVIV.E [Lageninx (Brady)]. Entosolenia (Ehrenberg) à col invaginé,

Fissurina (Reuss) à bouche fissiforme,

Capitellina (Marsson) à eol étranglé,

Sycidium (Sandb.), genre douteux, la structure de son test étant ineonnue.

(I) Voici, rapidement résumés, les caractères de ces genres :

Dentalina (d'Orbigny), semblable à Nodosaria, mais légèrement courbe (Vivant et fossile); Rhabdogonium (Reuss), semblable au mème, mais à loges earénées, triangulaires ou qua-

drangulaires sur la section transversale (Vivant);

Orthocerina (d'Or'bigny), à loges indistinetes à la surfaee;

Vaginulina (d'Orbigny), comprimé d'un eôté (Vivant et fossile);

Marginulina (d'Orbigny), en erosse (Vivant et fossile);

Planularia (Defrance), semblable, mais comprimé latéralement;

Cristellaria (Lamarek), parliellement ou tout à fait spiral (Vivant et fossile);

Lingulina (d'Orbigny), comme Nodosaria, mais eomprimé et à bouehe fissiforme;

Rimulina (d'Orbigny), Vagiuuliua à bouche fissiforme (Vivant et fossile);

Robulina (d'Orbigny), Cristellaria à bouche fissiforme (Vivant et fossile);

Conulina (d'Orbigny), simple sous-genre du précédent;

Glandulina (d'Orbigny), Nodosaria à loges embrassantes recouvrant la majeure partie des précédentes (Vivant et fossile);

Psecadium (Reuss), sous-genre de Robulina:

Frondicularia (Defrance), comprimé en feuille et ì logres triangulaires embrassantes, cachant les précédentes (Tivant et fossile);

Flabellina (dOrbigny), semblable au préeédent, mais à premières loges enroulées en spire comme chez Cristellaria (Fossile);

Amphimorphina (Neugeboren), commenesant en Frondicularia et finissant en Nodosaria: Dentalinopsis (Reuss), commençant en Rhabdogonium et finissant en Nodosaria:

Amphicoryne (Schlumberger), commengant en Cristellaria et finissant en Vodosaria(Vivant); Lingulinopsis (Reuss), commençant en Cristellaria et finissant en Lingulina, dont il ne constitue guère qu'un simple sous-genre.

Tous ces genres forment, avec leur chef de file, la famille des NoDositixa [genre Nodosariua (Carpenter)].

(2) Genres voisins :

Dimorphina (d'Orbigny), rui commence en Polymorphiua et finit en Vodosaria (Fossile); 
unir aux loges précédente et suivante, les autres latérales qui servent à fixer l'animal tandis que la loge elle-même est libre (Vivant)(').

ge Sous-Ordre

\section{CIILOSTOMELLIDES. - CHILOSTOMELLIDA \\ [CIILOstomellide (Brady); - CRyptostegia (Reuss)]}

\section{TYPE MORPHOLOGIQUE}

On peut s'en faire une idée en imaginant un Perforé polythalame à fins pores dont les loges sont disposées suivant la loi des Miliolides, c'est-à-dire formant chacune un demi-tour et transportant la bouche alternativement d'un pôle à l'autre de la coquille. Comme chez les Miliolides, elles peuvent revêtir l'apparence bi-, tri- ou uniloculinaire.

\section{GENRES}

Allomorphina (Reuss) (fig. 202) est construit comme une Triloculine, c'est-à-dire formé de loges embrassantes sur trois rangs, chaque loge nouvelle cachant la plus ancienne des trois précédentes et transportant la bouche fissiforme à son extrémité (Vivant et fossile).

Chilostomella (Reuss) est un Allomorphina, mais construit comme une Biloculine, sauf que les loges sont plus enveloppantes, en sorte que l'on ne voit qu'une faible partie de la précédente. La bouche est successivement transportée aux deux extrémités de l'axe (Vivant et fossile).

Fig. 202.

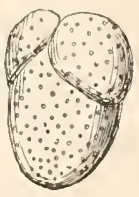

Allomorphina (im. Brady).

Ellipsoidina (Seguenza) est complètement embrassant et ne

laisse voir que la dernière loge comme les Uniloculines. Mais, à l'intérieur, toutes les loges ont la bouche dirigée dans le même sens et sont réunies entre elles par une petite colonne insérée près de la bouche fissiforme (Vivant et fossile) $\left(^{\boldsymbol{z}}\right)$.

Uvigerina (d’orbigny) qui a ses loges disposėes sur trois rangs en spire hélicoïdale et la bouche prolongée en col (Vivant et fossile);

Sagrina (d'Orbigny) qui commence en Uvigerina et se termine en Nodosaria (Virant et fossile).

Ces genres forment avec leur genre type la famille des POLYMORPINE [Polymorphinime (Brady)].

(1) Genre unique de la fanille des RaMrLTNA [Ramulininx (Brady)].

(2) Cel arrangement des loges qui rappelle celui des Globigérines. sauf qu’il n’est pas spiral comme chez celles-ci, donne la raison pour laquelle ces ètres avaient été réunis sous le nom de Cryptostegia (Reuss) aux Globigérines; mais celles-ci s'en distinguent, outre le caractère sus-indiqué, par la grandeur deleurs prores. 


\section{$3^{\text {e SOUS-ORDRE }}$ \\ TEXTULARIDES. - TEYTULARIDE \\ [TEXTULARIDE (Brady)]}

\section{TYPE MORPHOLOGIQUE}

C'est un Perforé polythalame à fins pores, à test hyalin chè les petites formes, recouvert chez les grandes d'une couche arénacée, ayant ses loges disposées toujours en hélice, mais de telle façon que, souvent, l'hélice portant juste deux ou trois loges par tour, celles-ci ont l'air disposées sur deux ou trois rangées parallèles non spirales. Souvent l'enroulement varie avec l'àge.

\section{GENRES}

Textularia (Defrance) (fig. 203) est formé de loges arrondies, assez peu embrassantes, disposées sur deux rangées contiguës. Les nouvelles loges appartiennent alternativement à l'une et à l'autre rangée et portent la bouche, en fente verticale, à leur bord interne presque sur le prolongement de la ligne de suture des deux rangées. La coquille est calcaire et finement poreuse (Fossile) (').

(1) A ce genre se rattachent de nombreuses formes dont beaucoup pourraient être comptées comme de simples sous-genres :

Plecanium (Reuss), formé de particules arénacées (Vivant et fossile); Venilla (Gümbel), qui commence en Plecanium et finit en Liguline (Fossile);

Grammostomum (Ehrenberg), à bouche terminale et corps déprimé d'avant en arrière (Vivant et fossile);

Schizophora (Reuss), Grammostomum finissant par des loges unisériẻes (Fossile);

Gemmulina (d'Orbigny), qui diffère du précédent par sa bouche ronde (Vivant);

Cuneolina (d'Orbigny), á corps comprimé en sens inverse de Grammostomum et autres, c'est-a-dire ayant les deux séries de loges aplaties l'une contre l'autre (Vivant et fossile);

Verneuilina (d'Orbigny), á coquille arẻnacée, à loges sur trois rangs donnant á la coquille la forme d'une pyramide triangulaire, et à bouche de Textularia (Vivant et fossile);

Tritaxia (Reuss), Verneuilina à bouche centrale (Vivant et fossile);

Reussia (Schwager), Verneuilina à coquille calcaire (Fossile);

Valvulina (d'Orbigny), semblable à Verneuilina, à coquille arénacée comme chez celui-ci, mais á grains très fins et á bouche recouverte d'une lèvre (Vivant et fossile);

Bigenerina (d'Orbigny), commençant en Textularia et finissant par des loges unisériées en ligne droite, avec une bouche ronde terminale (Vivant et fossile);

Siphogenerina (Schlumberger) (fig. 204), différant de Bigenerina par la présence d'un canal inférieur qui réunit toutes les loges et ne Fig. 20'

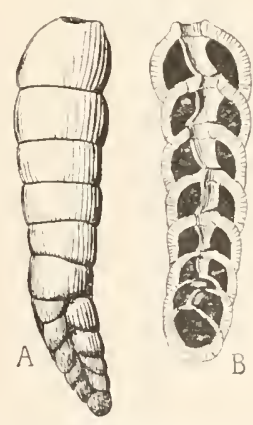

Siphogenerina (d'ap. Schlumberger). $A$, entier; $B$, en coupe. leur permet de communiquer que par une fente dont il est percé au-dessus de chaque bouche (Vivant et fossile); 
Bulimina (d'Orbigny) (fig. 205) est construit sur un autre plan. Ses loges sont enroulées en hélice et forment une coquille turbinée. La bouche, en virgule, est placée sur le côté de la dernière logge (Vivant et fossile) ("). Cassidulina (d'Orbigny) (fig. 206) peut ètre défini une Textulaire dont la coquille, à deux séries de loges, serait enroulée en spirale; la bouche est latérale, en fente (Vivant et fossile) ( $\left.{ }^{2}\right)$.

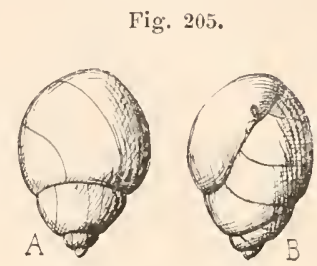

Bulimina

(im. Carpenter).
Fig. 206.

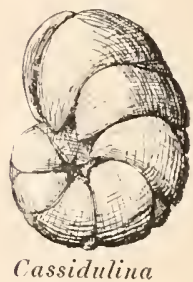

(Sch.).

Pavonina (d'Orbigny), semblable à Bigenerina, mais la partie qui, chez celui-ci, est droite devenant ici courbe, aplatie et en éventail, et la bouche étant cribriforme (Vivant);

Spiroplecta (Ehrenberg), différant de Textularia par ses premières loges enroulées en spirale (Vivant et fossile);

Gaudryina (d’Orbigny), à loges d'abord trisériées, puis bi- ou unisériées et à bouche en fente (Fossile);

Plectina (Marsson), représentant un Gaudryina à bouche ronde (Fossile);

Clavulina (d'Orbigny), à loges d'abord trisériées, puis unisériées, à coquille arénacée et à bouche valvulée (Vivant);

Climacammina (Brady), à loges disposèes à peu près comme chez une Textulaire, mais à bouche très large et fermée d'une plaque cribreuse, et à coquille calcaire revêtue d'une couche extérieure arénacée à ciment calcaire (Fossile);

Tetrataxis (Ehrenberg, emend. Nöller), à loges disposíes en hélice conique à axe creux communiquant toutes avec cette cavité columellaire par leur bouche tournée en dedans, et à coquille formée de deux couches, comme chez le précédent, mais avec la couche arénacée en dedans (Fossile);

Chrysalidina (d'Orbigny), qui peut ètre défini une Textulaire trisériée à bouche de Climacammina (Vivant et fossile).

Tous ces genres forment avec la Textulaire la famille des TExTuLarive [Textularidx (Carpenter)].

(1) Genres roisins :

Robertina (d'Orbigny) n'est qu'un sous-genre de Bulimina Vivant et fossile);

Virgulina (d'Orbigny), dont le pas de l'hélice est tel que les loges tendent à reprendre une disposition irrègulièrement bisériée (Vivant et fossile);

Bolivina d'Orbigny), tout à.fait bisérié, mais que sa bouche de Bulimine empèche de joindre aux Textulaires (Vivant et fossile);

Pleurostomel/a (Reuss), à loges bisériées aussi, mais à bouclıe de Bulimine très élargie et entaillée à son bord inférieur (Vivant et fossile);

Bifarina (Parker et Jones), commençant en hélice et se terminant par des loges unisériées.

Ces genres forment la famille des BULIMIN

(2) Genres voisins :

Orthoplecta (Brady), qui n'est qu'un sous-genre du précédent;

Ehrenbergia (Reuss), qui pourrait être défini : une Cassiduline déroulée.

Ces trois genres forment la famille des CASsID LISE [Cassidulina (Brady)]. 
$4^{\text {e Sous-Ordre }}$

GLOBIGÉRINIDES. - GLOBIGERTNID.E

[GLOBIGERINID E (Brady)]

\section{TYPE MORPHOLOGIOUE}

C'est un Perforé formé d'un petit nombre de loges sphériques, percées de gros et rares pores et disposées en spirale obscure.

\section{GENRES}

Globigerina (d'Orbigny) (fig.207 et 208) est une forme poly thalame très petite, formée de loges sphériques soudées les unes aux autres en spire confuse. Les loges nes'ouvrent pas les unes dans les autres : la spire réserve une sorte de cavité ombilicale dans laquelle toutes les bouches s'ouvrent plus ou moins profondément, ou bien cette cavité ombilicale est effacée et la dernière loge seule s'ouvre par la

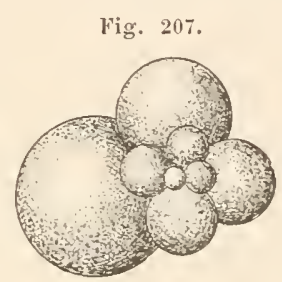

Globigerina (Sch.). Vu du cóté dorsal.
Fig. 208.

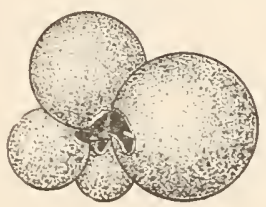

Globigerina (Sch.). lu du cóté ventral. bouche, à côté de la dépression qui la représente (Vivant et fossile) ("). Orbulina (d'Orbigny) (fig. 209 à 21 l) est très petit aussi, formé d'une coquille calcaire entièrement sphérique, avec deux sortes de perforations, les

Fig. 209.

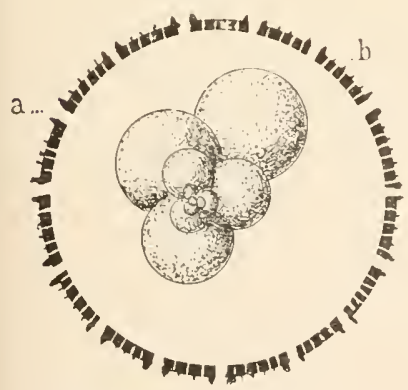

Orbulina (im. Schlumberger).
Fig. 210.

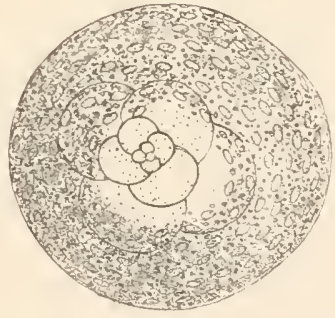

Orbulin" (lire d arihrence ve de fircel (im. Rhumbler).
Fig. 211.

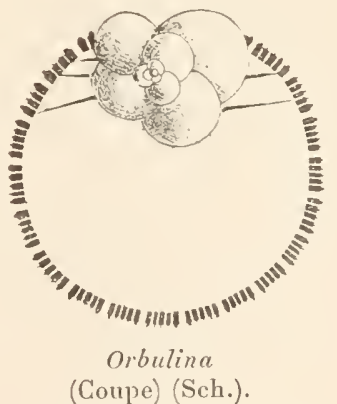

unes $(a)$ larges, espacées, les autres (b), petites, serrées.Il n’y a que rarement, en outre de cela, une ouverture plus large que l'on puisse considérer comme bouche. Les individus très adultes sont tous réduits à cela et sont, par conséquent, monothalames. Mais les autres ont, en

(1) Le test des formes pélagiques est toujours hérissé d'épines; ce genre est commun dans toutes les mers, pélagique ou dans les grands fonds. 
outre, à leur intérieur, une masse très semblable à une Globigérine, soudée par sa plus grande loge et par les extrémités de ses épines à la face interne du test de l'Orbuline (').

Les recherches récentes de Rucubler [94] ont montré que les choses se passent de la manière snivante. Les Gilobigérines sont de deux sortes, les unes à test épais, les autres à test extrêmement mince. Les premières constituent le genre Globigerina, les secondes ne sont que des jeunes d'Orbulina encore dépourvus de la loge sphérique caractéristique. Les deux sortes de Globigérines grandissent avec des caractères très semblables (sauf l'épaisseur du test) et se munissent des mèmes grandes épines. Si certaines ont été décrites comme étant dépourvues de ces appendices, c’étaient des Globigérines minces (futures (0rulines) dont les épines avaient été rompues par l'engin de pêche, en raison même de la fragilité de leur test. Quand les Globigérines minees arrivent à posséder douze à quatorze loges, elles se sécrètent une grande loge sphérique emboîtante et les voilà Orbulines avec Globigérine incluse. A ce moment la Globigérine occupe environ les $2 / 3$ de la cavité de l'Orbuline, et c'est à ce stade qu'appartiennent les formes présentant à peu près le rapport de taille indiqué. Mais, après un certain temps, la Globigérine incluse commence à entrer en légénérescence : son calcaire se résorbe, ses loges se réduisent à des lamelles membraneuses qui s'affaissent les unes sur les autres et finissenl par lisparaitre tout à fait. Toutes les Orbulines de grande taille sont dans ce cas. Elles contiennent parfois deux ou trois loges sphériques, incluses, concentriques, mais jamais de spire globigérinienne $\left(^{2}\right)$.

(1) On avait cru jusqu'ici que toutes les Orbulines étaient d'abord dépourvues de Globigérine, que certains individus en restaient dépourvus pendant toute leur vie, et que d'autres (dans la même espèce) en formaient une à leur intérieur, à un certain moment de leur existence, par bourgeonnement inlerne à l'intérieur de la loge périphérique. Il y aurait eu li dimorphisme comme chez les Milioles, existence simultanée des deux formes l'une $\mathbf{B}$ avec, l'autre $\mathbf{A}$ sans spire globigérinienne interne. avec cette différence qu'ici la loge initiale, loge sphérique orbulinienne, est à la périphérie et que les loges spiralées de la forme $\mathbf{B}$ poussent à son intérieur.

${ }^{(2)}$ Cette remarquable modification de la forme avec l'àge serait due à l'adaptation, les Globigérines étant pélagiques, celles à test mince sont exposées à être endommagées par l'action des vagues et prendraient, lorsque leur taille et le nombre de leurs loges deviennent assez grands pour que le danger soit réel, cette forme spliérique si avantageuse en pareil cas. C'est là évidemment une hypothèse. Mais le fait que l'évolution ontogénétique se passe comme il vient d'être dit repose sur des observations très démonstratives. Rhumber a trouvé, en effet, des Orbulines chez lesquelles la spire globigérinienne, au lieu d'ètre soudée à la face interne du test, faisait partie de sa surface sur une certaine étendue; et l'on voyait (fig. 210, 211), sur le test à trous plus grands et de deux sortes de l'Orbuline, une région perforée comme chez les Globigérines de trous beaucoup plus petits et tous égaux. Cela est impossible à expliquer si l'on admet que la loge orbulinienne est la première formée, et s'explique au contraire très aisément si c'est la spireglobigérinienne qui a formé la loge orbulinienne: la loge enveloppante a laissé libre une portion plus ou moins étendue de la spire enveloppée. Enfin, la présence des loges membraneuses flétries est la preuve formelle 
Hastigerina (Wyville Thompson) (fig. 204) a une coquille nautiloüde à parois minces, percées de pores fins et armés de longues épines. La bouche est grande, en croissant. Toute la coquille est noyée au sein d'une masse de protoplasma racuolaire qui ímet de lins pseudopodes réticulés. Les longues épines calcaires sont creuses et remplies de protoplasma (Une seule espèce, vivante, pélagique). Mikrocometes (Cienkorsky) est remarquable par sa coquille chitineuse percée de une à cinq ouvertures représentant de gros pores, et par son habitat: c'est, avec le genre Entzia (I)aday), le seul

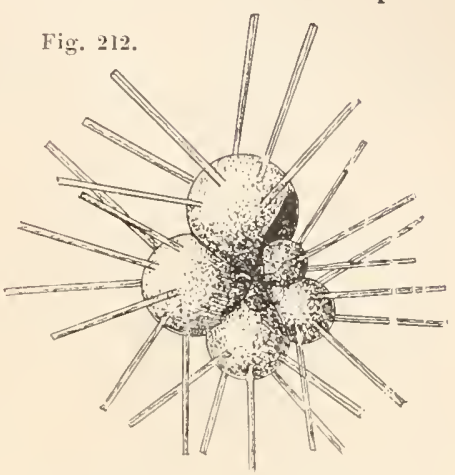

Ilastigerina (im. Brady). l'erforé qui ne soit pas marin (Eau douce et étangs salés) (").

¿i SOUS-ORDRE

\section{ROTALIDES. - ROTALIIIE \\ [Iotalif) l: (Brady)]}

\section{TYPE MORPHOLOGIQUE}

(FIG. 213)

C'ist une coquille polythalame, enroulée en spirale régulière, et plus Fig. 213. embrassante à la face inférieure qu'à la supérieure $I^{\prime}$, en sorte que celle-ci est concave et laisse roir toutes

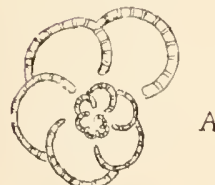

A

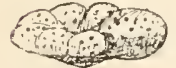

$B$

Rotalide (Sch.). les loges de la coquille, tandis que l'inférieure, légèrement ronvexe, ne laisse voir que celles du derniertour. C'est elle qui porte la bouche fissiforme. Le test est percé de larges pores. Les parois communes ne sont pas doubles, mais formées seulement par la paroi de la plus ancienne des deux $A$. Les loges communiquent entre elles par des orifices fissiformes $\left({ }^{2}\right)$.

d'une diginérescence de la portion glohigérinienne à l’intrieur de l'Orbuline et est incompatible, au contraire, avec l'hypothise de loges globigiriniennes à l'intirieur de l'orbuline.

(1) A res genres principaux s'ajoutent les quelques genres serondaires suivants: Pullenia (Parker et Jones), formant une spire à plusieurs tours, mais à loges très embrassantes, en sorte qu'on n'en voit qu'une partie; toutes les loges communiquent entre elles. Les perforations sont très fines, la bouche est au ras du tour précédent (Vivant et fossile)

Sphæroïdina (d'Orbigny), à loges plus embrassantes encore, en sorte qu'on ne voit que les trois a quatre dernieres (Vivant et fossile);

Candeïna (dOrbigny), a enroulement trochoïle et à bouche remplace par des orifices percés le long des sutures (Virant).

$\left.{ }^{2}\right)$ Mais parfois les parois sont doubles et il peut arriver que la convexité soit à la 
Le mode de reproduction par bourgeonnement interne, y compris le mode de division du noyau décrit rhez les Miliolides (V.p. 120) a été observé aussi chez quelques Rotalides (I)iscorbina). Les jeunes sortent par rupture de la coquille maternelle.

\section{GENRES}

Spirillina (Ehrenberg) (fig. 214) est conformé comme un Cornuspira, sans divisionsintérieures; on ne la rallache à ce sous-ordre, et arec quelques doutes, qu'en raison de son test perforé de gros canaux Vivant el fossile) (').

Discorbina (Parker el Jones) (fig. 913) retrace presque exactement notre type morphologique, mais la face inférieure n’est pas concave, étant remplie par un dépót secondaire de calcaire non poreux (Vivant el fossile).

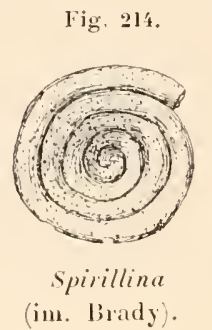

Fig. 215.

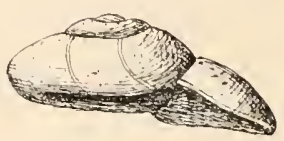

Discorbina

(im. Brady).

Rotalia (Lamarck, emend. Parker et Jones) (figg. 2l6) a des pores très fins, un enroulement héliçö̈lal el, ehez les grandes espèces du moins, les cloisons communes des loges sont doubles el comprennententre elles un espace vide d'où partent vers la surface des canaux qui se bifurquent avant de l'atteindre. Vivant et fossile: $\left({ }^{2}\right)$.

face infirieure, bien que celle-ci reste plus embrassante, el que les parois des loges soient doubles et pareourues par un srstime de canaux du test, mais cela est exceptionnel dans

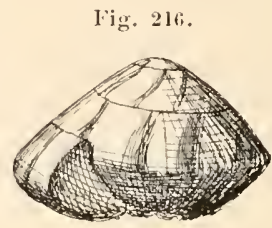

Rotalia (Seh.). ce sous-ordre.

(1) Il forme it lui seul la famille des SPIRILLAx. [Spirillinina ,Brady)].

(2) Genles voisins:

Asterigerina (d'Orbigny\}, simple sous genre du précédent;

Planorbulina (d'Orbigny), fixé par sa face supérieure devenue plane et laissant voir à peu près toutes les loges des deix còtis Vivant et fossile?;

Truncatulina (d'Orbigny), fortement convexe en dessous, plan ou concave en dessus (Vivant et fossile);

Anomalina (Parker et Jones), à tours de spire marqués trés fortement et à peu pres aulant en dessus qu'en dessous Vivant et fossile;

Planulina (d'Orbigny), tries plat, presque symelrique Vivant ef fossile)

(Ces trois genres ne sont guère que des sous-genres de Planorbulina);

Pulvinulina (Parker et Jones), biconvexe, at ombilic souvent comble; lest à pores fius (Vivant et fossile);

Cribrospira Möller, en luélice, à dernier tour seul visible, à orifice terminal cribreux, à cluisons simples et à pores larges fiossile ;

Cymbalopora (Itagenof) it loges enroulies dabord en hiclice, puis en cercle, formant un còne surbaissé à axe creux dans lequel sourrent les eavilis des luges (Vivant et fossile);

Carpenteria (Gray), disposé conme le précédent, mais moins regulierement ef fixi par la base du cone dont le sommet tronque, servant de butuche commune, se prolonge parfois en un tube simple ou dendritique (Vivant); 
Calcarina (d'Orbigny) (fig. 217) est constitué comme Rotalia, mais est recouvert d'un dépôt calcaire concrétionné appelé intersquelette, qui garnit

Fig. 217.

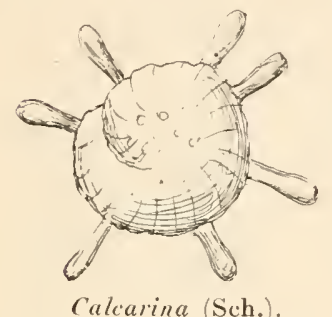
toute la surface, comble toutes les dépressions, tous les intervalles, ne laissant apercevoir qu'une faible partie du test (les dernières loges à la face inférieure) et des épines très développées, insérées sur le test et traversant cet intersquelette. Celuici se dépose pendant toute la vie, en sorte qu'il existe jusqu'au centre de la masse séparant les tours de spire; mais il augmente d'épaisseur arec l'àge, en sorte que la coquille rraie, avec ses loges à paroi propre mince, y est complètement noyée. Ces parois propres sont percées des gros pores habituels; l'intersquelelte, au contraire, est parcouru d'un r'iche système de fins canaux ramifiés et anastomosés qui viennent s'ourrir à la surface (Vivant et fossile) (").

Tinoporus (Carpenter) (fig. 218) a une forme variant de la sphère au cône et à la lentille, souvent arec de gros tubercules très saillants; il est formé de loges cuboïdes, toules semblables, disposées en strates horizontaux et verticaux séparés par des lames calcaires de mème direction; les parois des loges sont simples, généralement sans canaux daus leur épaisseur; les horizontales sont percées de nombreux et fins pores, les verticales d'un petit nombre d'orifices plus larges, en sorte qu'elles communiquent toules ensemble; les superficielles communiquent par leurs pores avec le dehors, mais il n’y a pas de grand orifice méritant le nom de bouche. Quand on examine avec soin la disposition des loges, on voit qu'il y en a au centre un certain nombre enroulées en une spire plane régulière, tandis que les autres sont orientées radiairement. Ces loges spirales constituent la coquille primitive et les autres sont des loges accessoires, développées comme celles de la carité axiale des l'atellines dans un squelette secondaire abondant qui s’est rapidement développé autour de la coquille primitive, et a empèché son développement ultérieur. L'n système de canaux, s'ourrant, d'une part dans les chambres, d'autre part au dehors se montre dans ce squelette secondaire et en particulier dans ses protubérances spiniformes (Vivant et fossile) ( ${ }^{\mathbf{z}}$ ).

Rupertia (Wallich), dont la coquille a un enroulement spiral irrégulier déterminant une masse sphérique irrégulière où les loges ne sont pas visibles et qui est fixée par une colonnette dépourvue de pores (Vivant);

Patellina (Williamson), enroulé conme le précédent, mais à cavité axiale remplie de loges irrégulièrement empilées communiquant avec celles de la surface, qui sont incomplètement divisées en logetles par des septa radiaires (Vivant et fossile).

(1) Tous ces genres, depuis el y compris Discorbina, forment la famille des Rotallat [Rotalina (Brady)].

$\left({ }^{2}\right)$ «M. Munier-Chalmas pense que certains genres de foraminifères se comportent 
Polytrema (Risso) (fig. 219) est formé d'une base encroùtante dans laquelle on reconnaît, au centre, un groupe de loges à arrangement spiral qui représente la coquille primitive. Mais ì ces loges primitives s'en ajoutent d'autres, beaucoup plus nombreuses, qui s'entassent d'alord en couches irrégulières, puis forment des tubes dressés ramifiés, creux. Cela donne à l'animal la forme d'un petit Polypier et, comme il est ordinairement rouge, il a été longtemps pris pour une sorte de Corail. Les loges de ce système secondaire n'ont pas de pores ordinaires, et leurs communications entre elles et avec le dehors s'établissent par des sortes d'enfoncements en forme de pilier's creux qui partent du plancher des loges, et descendent dans la loge sous-jacente, la traversent et se fixent à son plancher. La cavilé de ces piliers s'ouvre en haut à plein canal dans la loge supérieure, et en bas, par des orifices latéraux, dans la sous-jacente. Pour les loges superficielles, le tube s'ouvre distalement en dehors (').

comme s'ils représentaient des colonies dérivées par blastogénèse de types simples. Dans cette hypothèse, les loges sériées qui se développent sur le pourtour ou sur les côtés d'un individu central ou médian, représenteraient un développement blastogénétique; ainsi Tinoporus serait une colonie dérivée d'un type simple analogue à Calcarina. Il en serait de même des rapports d'Orbitö̈des et de Cycloclypens. Dans ce même ordre d’idées Dicyclina représenterait deux individus d'Orbitolina accolés par la face qui ne porte pas le réseau externe».

Cette note nous est communiquée par II. Munier-Chalmas.

Nous exprimons à notre collègue nos remerciements pour l'obligeance avec laquelle il a bien voulu nous donner la primeur de ses idées nouvelles. Mais il nous semble difficile d'interpréter comme un phénomène blastogénétique la formation des loges secondaires quand les loges de la coquille primitive se forment par un phénomène d'aceroissement.

Qui dit blastogénėse, en effet, dit formation d'un nouvel individu par bourgeonnement. Or chez ces êlres, l'individu comporte au moins un noyau : une loge sans noyau n'est pas un indivitu. Chez la Polystomelle macrosphérique, par exemple, la formation des nouvelles loges est un phénomène d'accroissement et non de blastogénèse : cela est démontré par le fait que le noyau reste unique pendant leur formation. Pour que l'on put dire que chez Tinoporas, les loges de la coquille primitive se sont formées par accroissement el celles de la coquille secondaire par blastogénèse, il faudrait que l'on ait constaté que le noyau reste unique pour la formation des loges de la première et se divise pour former les loges de la seconde. Or personne n'a constaté cela et nous sommes convaincus qu'en cherchant à le vírifier on reconnaitrait que les choses ne se passent pas ainsi. Très probablement, ici comme ailleurs, le noyau reste unique pendant l'accroissement de l'individu et se divise seulement à certains moments pour sa reproduction.

(1) Genres roisins:

Gypsina (Carter), parfois fixé et encroùtant, grossièrement perforé, sans système de 
$6^{\text {e SOUS-ORdRE }}$

\section{NLMULITIDES. - NUMULITIIIE.}

[Nimulaid.i (Carpenter, emend. Brady)]

\section{TYPE MORPHOLOGIQUE}

C'est un Perforé libre, à pores fins, polythalame, à enroulement régulièrement spiral et symétrique. Mlais les parois des loges sont doubles, en ce sens que, lorsqu'une nouvelle loge se forme, clle ne se contente pas, là où elle confine aux loges anciennes, de la paroi de celles-ci, mais en sécrète une autre qui la louble. Dans l'espace entre ces deux parois contiguës, nait un syslème compliqué de canaux. Enfin, un squelette supplémentaire et perforé se développe dans divers points. Mais il n’est guère possible desýstématiser les dispositions de ces diverses parlies; elles seront indiquées dans la description des genres.

\section{GENRES}

Fusulina (Fischer de Waldheim) (fig. 200). La coquille a l'aspert d'un petit fuseau de 10 à 12 millimètres de long. Pour la disposition intérieure des loges primaires et de leurs cloisons, c'est alssolument une Alvéoline.

Fig. 220.

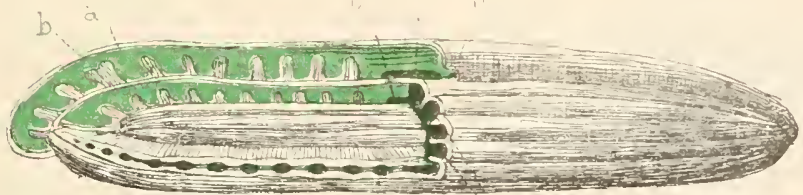

Fiusulina im. Carpenter
Ce sont les mèmes loges, basses et restant basses malgré les progrès de la croissance, courtes, en sorte qu'il en faut beaucoup pour faire un tour, mais larges et augmentant de

plus en plus en largeur à mesure que la coquille s’accroit, de manière à ètre toujours absolument embrassantes, s'étendant d'un pôle à l'autre de la coquille dans toute la longueur du méridien 'correspondant. La bouche forme une longue fente méridienne. Les cloisons primaires

canaux ni protubérances (Vivant et peut-ètre fossile, mais cela n'est pas certain);

Aphrosina (Carter), finement perforé, fixé, encroùtant (livant,

Thalamopora Römer), en forme de tigelle ramifiée, fixée par sa base et dipourvuc de bouche (Fossile);

Flabelliporus (Dervieux), caractérisé par le fait que les loges de la coquille initiale sont laissées tout au bord de la coquille par les logettes secondaires qui sont plus petites, d'abord semi-lunaires, puis rhomboïdales.

Ces genres forment la famille des Trooportre [Tinoporinx (Brady)] qui a pour caractère cette combinaison de loges primitives régulièrement spirales et de loges secondaires tout autrement disposées que l'on retrouve chez tous. 
sont simples comme chez l'Alvéoline. Mais le test est perforé de très fins pores extrèmement serrés. Il y a, en outre, des différences dansle détail de la structure. Ici, en eflet, les cloisons primaires sont complètes et séparent entièrement les loges $(l$. $)$, sauf un assez large orifice fissiforme $(o)$ au ras du plancher, dans le plan équatorial de la coquille. Tous ces orifices forment donc un canal spiral ininterrompu qui traverse toutes les loges en leur milieu. Il n'y a ni cloisons secondaires, ni cloisons tertiaires; mais il y a cependant des loges secondaires produites de la manière suivante. Les cloisons ne sont pas des lames planes. Elles sont planes seulement dans $1 / \ddot{3}$ environ de leur hauteur, le long du plafond $(a, b)$; mais dans les quatre autres cinquièmes, elles sont fortement onduleuses, plissées, disons même gaufrées. Les plis sont disposés perpendiculairement à la hauteur de la cloison, s'avançant alternativement dans les cavités des deux loges que la cloison sépare. Ils sont si saillants qu'ils arrivent à se rencontrer d'une cloison à l'autre, divisant aussi la loge en logettes $(c)$. Mlais ces logettes ne règnent, comme les plis, que dans les $1 / 3$ de la hauteur de la loge et communiquent ainsi toutes avec elle le long du plafond de celle-ci (Fossile) ${ }^{\mathbf{1}}$ ).

Polystomella (Lamarck) (fig. 2201, 2922 et 2023). La coquille a un enroulement nautilö̈de symétrique. Les loges sont nombreuses à chaque tour et très embrassantes, mais pas complètement, en sorte qu'à l'ombilic on devrait,

Fig. 221.
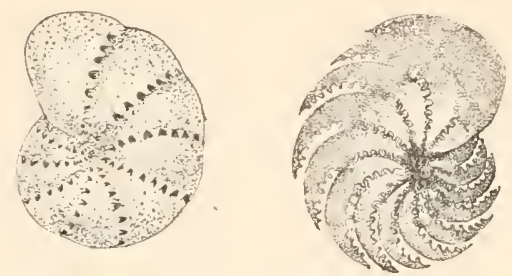

Polystomella.

Entier (d'ap. Brudy

et décalcifié (d’ap. Carpenter). le chaque côté, voir tous les tours précédents. On les voit en effet sur l'animal décalcifié. Mlais, le plus souvent, l'ombilic est comblé par un tissu squelettique secondaire, en sorte que l'ensemble devient lenticulaire. Les cloisons de séparation des loges sont simples et perforées d'une rangée de trémas disposés sur une seule ligne un peu au-dessus du plancher. 11 en est de mème pour l'orifice buccal. Enfin, les loges envoient en arrière des prolongements en cul-de-sac

(1) Genres voisins :

Hemifusulina, Möller) n'est guère qu'un sous-genre des Fusulines, mais ses cloisons sont formees de deux lamelles entre lesıuelles règne un espace qui s'ourre dans la loge par une fente située au-dessus de l'orifice de communication des loges. Dans cel espace se trouve,en outre, un système de canaux ramifiès (Fossile);

Fusulinella (Iöller) considéré par les uns comme un sous-genre des Fusulines serait pour les autres un Inperforé voisin d'Alseolina (Fossile);

Schwaggerina (IIöler) diffère de la Fusuline en ce que ses cloisons, dans la plus grande partic de leur ètendue, ne sont pas plissees; mais, en approchant de l'axe, elles deviennent brusquement trés sinteuses, se ramifient, anastomosent leurs ranifications et forment lá un tissu irrégulièrement réticulé, à peu prés comme thez les Nummulites (V.p. 150) (F'ossile).

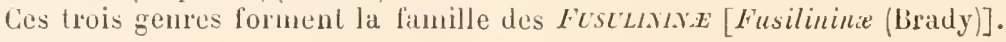


qui font saillie sur son hord postérieur. En outre de cela, il existe un système de canaux interseptaux et, de chaque ciité, un canal spiral qui suit la spire ombilicale (').

Ces deux canaux (fig. 222) sont réunis par les canaux méridiens ( $c l$.) qui suiventla suture de chaque cloison avec le plafond de sa loge. Ces canaux méridiens émettent : $1^{\circ}$ des canaux divergents (cd.) qui so portent alternativement en avant et en arrière, lans le plafond de la loge suirante

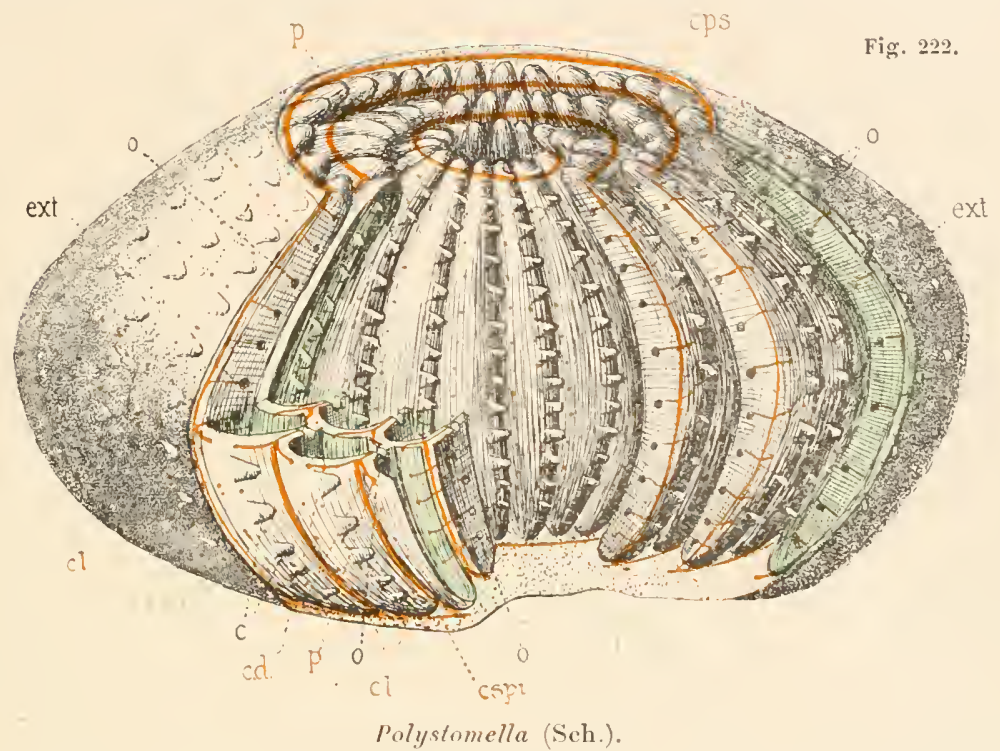

Test coupé pour moutrer la disposition des loges et des canaux interseptaux.

c., euls-de-sac des loges; edl., eanaux divergents; el., eanaux míridiens; eps., eanal héliçoildal supérieur; espi., canal hélicödal inférieur; ext., surface externe du test; 1.o.1., loges eoupées longitudinalement; 1.o.t., loges coupées transversalement; o., o., trémas faisant communiquer les loges entre elles: p., pores terminaux des eanaux divergents.

et dans colui de la précédente, el, très courts, se terminent presque immédiatement à la surface externe de ce plafond. Au dernier tour, ces canaux s'ouvrent donc au dehors par deux rangées de pores $(p$.$) parallèles à la$ suture; mais aux tours précédents, ils s'ouvient dans les loges siluées au-dessus d'eux ét établissent ainsi une communication entre ces loges et le système des canaux. Les canaux méridiens émettent, en outre, des canaux convergents qui descendent dans l'épaisseur le la cloison sous-

1) Pour bien comprendre cette structure, supposons enlevis le bouchon calcaire qui comble l'ombilic de chaque còti. Les fours n'étant pas completement embrassants, on voit de chacun de ceux qui précèdent le dernier, une chtroite bordure. Cette bordure dessine un petit trottoir hélicoidal qui ra, en descendant, de la bouche au centre de l’ombilic. Le long de rette hélice règne, de chaque côté, un eanal. Ce canal est logé exactement dans l'angle entre les deux fours, sous le bord extrême du plus jeune des deux, étant formé par un espare que laisse chaque nouvelle loge en se soudant à la loge correspondante dı tour précédent. 
jacente el vont se jeter dans les trémas (o) de communication entre les loges. Les deux masses calcaires qui comblent les ombilics, sont percées de canaux à peu près rectilignes, partant du canal spiral sous-jacent et débouchant au dehors par de fins pores. Tous les canaux sont occupés par des prolongements du protoplasma comparables aux pseudopodes (Vivant et fossile).

Les Polystomelles sont dimorphes. La forme $\mathbf{B}$, beaucoup plus rare que l'autre ne se rencontre guère qu'une fois sur quarante. C'est ì elle surtout, parmi les Perforés, que s'applique la théorie de Lister du Dimorphisme initial et des zoospores. Nous renvoyons pour le détail à l'exposé que nous en avons donné à propos des Miliolides (V. p. IIS) (').

Nummulites (Lamarck) (fig. 29-4, 290). La coquille est lenticulaire; elle a donc un axe très court. Malgré cela, elle est construite essentiellement comme une Fusuline, et la différence de forme tient seulement ì ce que les parties latérales des

Fig. 223.

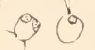

(8)

l'olystumellu. Spores flagellées (d'ap. Lister). loges sont si plates qu'elles n'augmentent, ì chaque tour', l'épaisseur de la coquille que l'une quantité très faible relativement à son augmentation de diamètre dans le plan équatorial. Les loges sont donc, comme celles de la Fusuline, étendues d'un pôle à l'autre tout le long des méridiens; mais, au lieu d'ètre à peine concaves vers le centre de la coquille, elles sont formées de deux moitiés qui se joignent au bord de la coquille sous un angle très aigu. Il y a un grand nombre de tours et

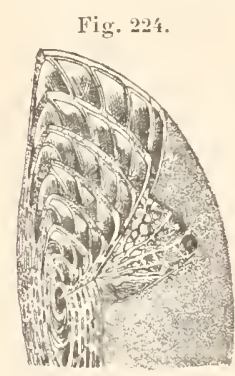

Nummulites.

Structure (Sch.).

Les cloisons sont doubles ainsi que toutes les parois communes un grand nombre de loges par tour. Le dernier tour est circulaire, il se ferme donc sur lui-mème, en sorte qu'il n'y a pas de bouche. Les cloisons sont complètes, sauf un orifice fissiforme au ras du plancher dans le plan équatorial, comme chez la Fusuline. Elles sont convexes vers la bouche et régulièrement disposées dans toute leur partie moyenne équatoriale, mais dans ces prolongements latéraux dirigés vers les pôles ou centre de la coquille, elles deviennent très irrégulières, se ramifient, s’anastomosent entre elles et donnent naissance à une sorte de réseau irrégulier.

(1) Le noyau toujours unique de la forme $\mathbf{A}$ est constamment silué dans une des deux ou trois dernieres loges de l'avant-dernier tour. L'un de nous a observé comment il s'élire pour passer, au fur et à mesure de l'accroissement, d'une loge dans la suivante, par quelqu'un des orifices de communication beaucoup plus étroits que lui. Nonionina (d'Orbigny) diffère du prócédent par sa bouche fissiforme, ses septa percés d'une fente unique dans le plan équatorial, l'absence des prolongements en cul-de-sac des loges et le faible développement du système de canaux du test (Vivant et fossile).

Ces deux genres forment la famille des PoLstomelux: [Polystomellinie (Brady)]. 
des loges el, entre ces parois, s'étend un système de cananx du test qu'il faut maintenant décrire. Il y a d'abord deux canaux spiraux, comme chez les Polystomelles, mais autrement situés : au lieu d'ètre relégués à la partie la plus externe de la loge, ce qui, ici, les coufondrait au pôle, ils sont tout près de l'équateur, séparés l'un de l'autre seulement par l'orifice fissiforme qui perce les cloisons pour faire communiquer les loges; ils sont contenus entre le plafond de la loge d'au-dessous et le plancher de la loge d'au-dessus. De ces canaux partent des ramifications qui se répandent dans l'épaisseur des cloisons et y forment tout un réseau. De nombreuses liranches de ce réseau s'ouvrent dans les loges et établissent la communication avec celles-ci, pour permettre au protoplasma de les envahir. En outre, il existe, le long du bord dorsal des loges, exactement dans le plan équatorial, une bande calcaire imperforée constituée par un épaississement de la paroi en ce point. Cette bande, appelée cordon dorsal suit naturellement tous les tours de spire dans le plan équatorial. Le cordon dorsal est parcouru dans loute sa longueur par quatre canaux plus petits que les canaux spiraux, mais qui donnent, comme eux, des branches qui se répandent dans toute l'épaisseur du cordon dorsal et se mettent en communication avec le réseau des cloisons, en sorte que tout le systime est continu.

Fig. 225 .

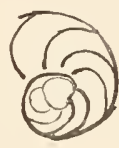

A.

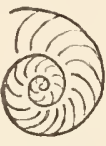

B
Nummulites. Formes A et B (iim. Schlumberger).

Il y a ici aussi dimorphisme, avec deux formes A et B (fig. 29.3) ('). Cycloclypeus (Carpenter) (fig. 226) est une grande coquille lenticulaire, contenant dans son plan équatorial une seule couche de loges d'abord spirales, puis circulaires. Tout le reste de l'épaisseur est formé par une épaisse masse calcaire seconlaire, perforée, dont les pores, développés ici en longs canaux en raison de l'épaisseur de la masse, s'ouvrent

(1) Le genre Nummulites (Lamarck) a été dédoub]'́ par d'Orbigny en deux sous-genres :

Nummulina (d'Orbigny), qui est la forme que nous avons décrite sous le nom de Nummulites (Lamarck) et

Assilina id'Orbigny), qui se distingue de Nummulina par

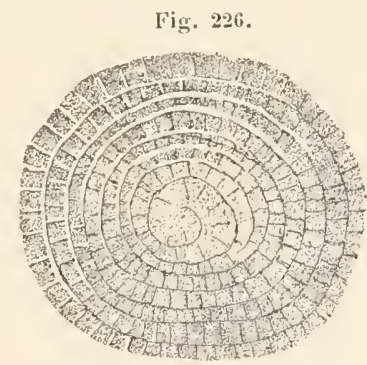

rycloclypeus (im. Brady). le fait que la portion des loges qui recouvre les tours précédents est si mince et si plate qu'elle laisse voir tous les tours de spire.

Genres voisins :

Operculina (d'Orbigny), Nummulite à tours d'abord non embrassants, puis embrassants; croissant rapidement en hauteur, ne devenant pas cirrulaires et laissant une bouche fissiforme au ras du plancher (Vivant et fossile);

Heterostegina (d'Orbigny) a un enroulement semblable à celui du précédent, mais a ses loges suhdivisées par des cloisons secondaires perpendiculaires aux cloisons primaires, et la bouche formėe d'une rangée d'orifices (Vivant et fossile);

Amphistegina (d'Orbigny) est très transparent, plus convexe d'un côté que de l'autre; 
au plafond des loges. Les loges d'un même tour ne communiquent pas entre elles, mais avec celles des deux tours contigus, avec lesquelles elles alternent, par de grosses perforations de leurs parois circulaires. Dans l'épaisseur des parois des loges et de leurs cloisons circule un riche système de canaux du test qui s'ouvrent fréquemment lans les loges et établissent une communication générale de toutes les parties de l'animal. L’épaisse couche du squelette secondaire est traversée par des enclaves de substance non poreuse, en forme de lames verticales (la coquille étant à plat), qui continuent jusqu’à la surface les parois des loges. Certaines sont minces, mais renflées en certains points en forme de cônes qui se terminent à la surface par une papille saillante représentant leur base. Ces enclaves sont formées de calcaire non perforé, mais sont traversées par des prolongements des canaux du test qui viennent s'ouvrir à la surface au niveau de leur base (50 à $60 \mathrm{~mm}$. Vivant et fossile).

Orbitoïdes (d'Orbigny) (fig. 227) se déduit aisément du précédent en ajoutant que la masse de calcaire secondaire est creusée de plusieurs couches de loges secondaires communiquant entre elles. Ces loges, dans une

Fig. 227.

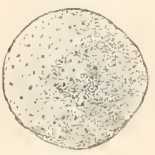

Orbitoïdes (im. Brady). même rangée verticale, communiquent entre elles, avec la loge principale correspondante et avec le dehors, par les perforations de la masse poreuse dans laquelle elles sont creusées; elles communiquent avec celles des rangées voisines, avec lesquelles elles alternent, par des canaux obliques allant aux deux qui lui correspondent un peu plus haut et un peu plus bas. Enfin les loges principales contiguës d'un même cycle communiquent entre elles par un canal percé dans leur cloison de séparation. Le système des canaux du test et des enclaves de substance non [roreuse, canaliculée, est semblable en ses traits essentiels à celui de Clycloclypeus (Fossilc) (").

des deux côtés ses loges se prolongent presque jusqu’à l'ombilic mais, du côté le moins convexe, elles restent simples dans leur partie latérale, tandis que, du côté le plus convexc, elles donnent naissance, par une bifurcation accessoire, chacune à une loge secondaire (Vivant et fossile);

Hemistegina (Kaufmann) est plan convexe, à loges atteignant l'ombilic du còté convcxe, effacées du côté plat par accolcment de lcurs parois en une masse calcaire feuilletée (Fossile);

Archædiscus (Brady), lenticulaire, formé d'un tube simple, sans cloisons, à parois percées de fins pores, et pelotonné sur lui-mème en nombreux tours spiraux alternativement contigus entrc eux ct séparés, noyés dans une masse calcaire finement tubulée qui comble tous les intervalles ct revêt la surface d'une couche uniforme Fossile).

Tous ces genres forment la famille des NumuLtTINe [Nummulitinip (Brady)].

(1) Gümber divise Orbitö̈des en cinq sous-genres :

Discocyclina (Gümbel),

Phipidocyclina (Gümbel).

Asterocyclina (Gümbel), Lepidocyclina (Gümbel),

Astinocyclina (Gümbel),

qu'il distingue par des caractères de forme et par quelques détails d'arrangement des loges principales.

Tous ces genres forment la famille des CrcLoctrpers.e [Cycloclypeine (Brady)]. 


\section{APPENDICE}

Aux Foraminifères se rattachent, avec doute, certaines formes dont les affinités ou mème la nature animale sont fort discutées. Nous les présenterons ici rapidement, sans vouloir prejuger par là de leur vraie nature, et uniquement pour donner au lecteur quelques renseignements ì leur sujet.

\section{STROMATOPORIENS. - STROMATOPORE.I}

[STROMATOPORID.1 (Nicholson et Murie)]

On désigne sous ce nom des formes, toutes fossiles, qui se présentent (fig. 228) sous l'aspect de lames calcaires onduleuses superposées parallèlement en masses feuilletées. Les espaces compris entre ces lames sont imparfaitement divisés en loges par de petites colonnes calcaires qui, d'ordinaire, s'étendent d'une lamelle à l'autre, mais souvent s'arrêtent à moitié route. Ces lamelles sont, en outre, percées de pores et ornées de papilles. Tout le système est traversé par des canalicules entrecroisés généralement à angle droit, qui sont répandus dans l'épaisseur des lames et passent de l'une à l'autre par les colonneltes. Il y a là cerlainement un ensemble de dispositions qui fait penser aux Foraminifères et en particulier aux genres $P a r$ leria et surtout (Bütschli) Polytrema; mais Rosen [6it] a décrit ces canilicules comme des fibres cornées el considéré tout le système comme fibreux. D'autre Fig. 22s.

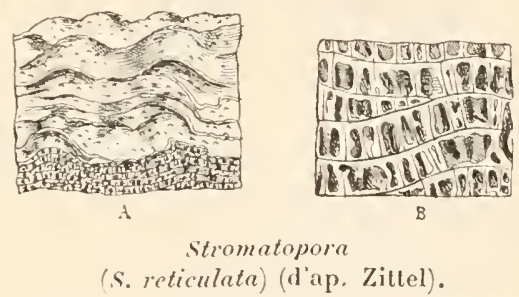
part, on observe parfois des canaux verticaux à parois propres et, à la surface des lamelles, des sillons éloilés groupés autour d'une papille saillante qui, jointes à d'autres particularités de la structure, font penser à certains Hydroïdes du groupe des Iydrocorallines Murie et Nicholson [78]). On hésite done, pour ces ètres, entre les Foraminifères, les Eponges et les Hydrocorallines. On en a décrit de nombreux genres dont nous ne donnerons que les noms:

Stromatopora (Goldfuss, emend. Nicholson et Murie) (fig. 228),

Stylodictyon (Nicholson et Murie), Chlathrodictyon (Nicholson el Murie), Pachystroma (Nicholson et Murie), Dictyostroma (Nicholson et Murie),
Ellipsactinia (Steinmann), Caunopora (Phillips),

Stromatocerium,Hall, emend. Nicholson et Muric).

Labechia (Lonsd.)

\section{RECEPTACULIENS. - RECEPTACULEA}

\section{[REYEPTACLLITIDE (Pümer)]}

Non moins douteuse est la signification des Receplaculiens, formés des débris de la grande famille des Dactyloporida dont les autres genres Dactylopora, Thyrsoporella, Gyroporella, Uteria, Petrascula, Osulites, etc.', ont été rendus par Munier-Chalmas aux Algues calcaires). Le genre type 
Receptaculites (Defrance)(fig. 229) est une grande coquille (100mm), en forme de coupe évasée, à parois épaisses. Ces parois sont revêtues, en dedans et en dehors, de plaquettes losangiques marquées de lignes diagonales. L'espace compris entre les dcux lames de plaquettes, et qui forme la plus grande partie de l'épaisseur de la paroi de la coupe, est divisé par des colonnettes radiaires qui s'appuient par leurs bases sur les plaquettes. Tout le système est traversé par de fins canalicules (Fossile).

Fig. 229.
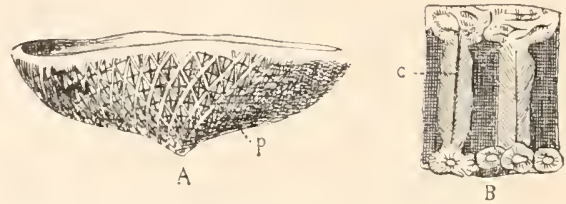

Receptaculites (R. Neptumi) (d’ap. Gümbel). A. l'ensemble; $B$, coupe transversale. Les autres genres, tous fossiles aussi, sont:

Ischadites (Murchison), Cyclocrinus Eichwald, Pasceolus (Billings), Polygonosphærites (Römer),
Dictyocrinus (IIall), Sphærospongia (Pengelly)?, Tetragonis (Eichwald)?,
Archæocyathus (Billings), Archæocyathellus Ford.', Protocyathus (Ford.).

\section{TESTAMOEBIFORMIENS. - TESTAMOERIFORMEA}

\section{[TESTAMOEBIFORMIA (Carter)]}

Nous signalerons ici encore trois formes vivantes dont la place précise n'est pas déterminée faute de renseignements suffisants à leur égard:

Ceratestina (Carter) (fig. 230), formé de chambres chitineuses, subglobuleuses réunies par un tube stolonifère (Vivant);
Fig. 230.

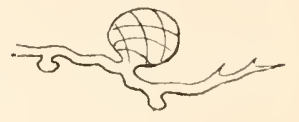

Ceralestina

(C. tessellata)

(d'ap. Carter).

Fig. 231.

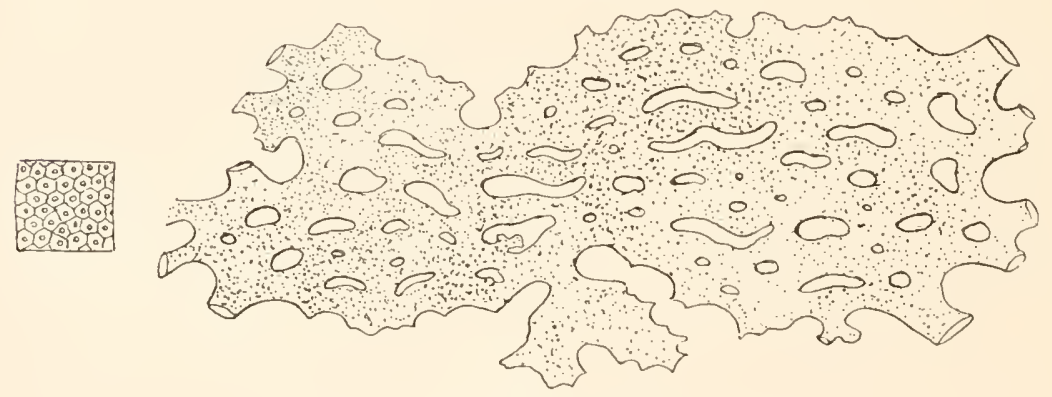

Cycleodictyina (C. compressa) (d'ap. Carter)

montrant. l'ensemble et le détail de la structure de la surface.

Cycleodictyina (Carter) (fig. 231), à coquille calcaire formant un réseau rampant sur le support et à surface uniformément ponctuée (rivant); 
Holocladina (Carter) (fig. 232), à coquille calcaire ramifièe à surface couverte de papilles marquees d'un point central (Vivant).

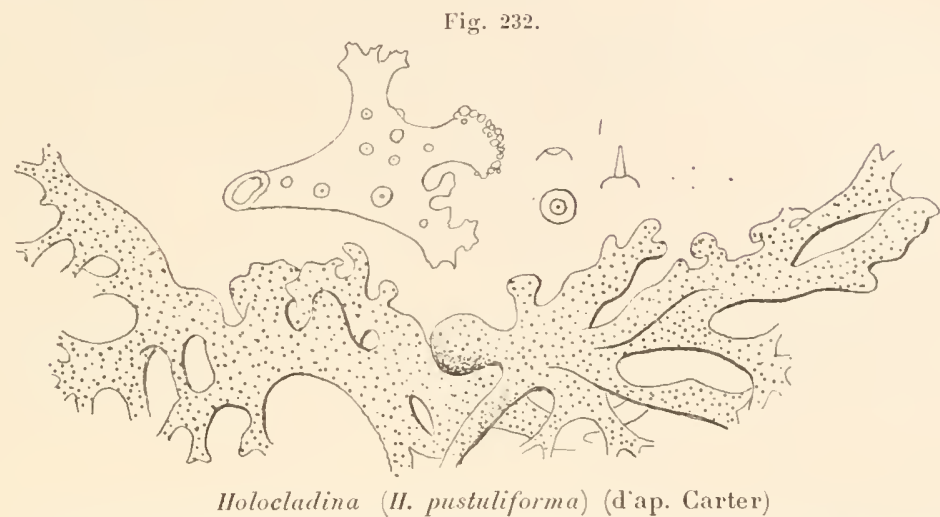

montrant l'ensemble, une branche plus grossie et le détail des tubercules de lit surface.

On ne sait rien des parties vivantes. Les formes de ces corquilles rappellent celles des Amobiens et Brady se demande si ees ètres ne seraient pas plutôt à rapprocher des Foraminiféres lobés testacés ou Thécamobiens.

\section{Eozoon}

Enfin reste le fameux

Eozoon (Jawson) (E. canadense) (fig. 233 qui serait, s'il est rraiment un animal, le plus aneien fossile counu, ayant véeu dans les gneiss laurentiens d'Amérique et d'Europe. Ce sont des rognons irrógulièrement lı́misplıeriques, gros comme la tète ou le poing, lormes de lamelles onduleuses alternantes de calcaire et de serpentine. Dans quelques échantillons, considérés comme mieux conservés, on observe que les lamelles de serpentine ne sont pas planes, mais forment des nodosités splériques soudées entre elles par leurs bords. La surface de ces nodosités se dessine conme une paroi propre, transparente, formée de chrysolite, d'asbeste, de fines fibres de serpentine, et l'on observe des canaux, remplis aussi de serpentine, qui se ramifient dans les lames calcaires ou réunissent deux lames serpentines voisines. D’aprés Dawson et Carpenter, la partie occupée par la serpentine serait un système de loges, communiquant entre elles, munies d'une paroi propre, et les parties remplies

Fig. 233.

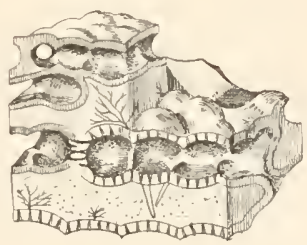

Eozoon (E. Canadense) (d'ap. Carpenter). de calcaires représenteraient un intersquelette. Dans ce cas, l'animal aurait eu, aprés sa mort, toutes ses cavitès remplies par la serpentine qui aurait comblé les parties occupées par le protoplasme. Nais la question est de savoir s'il n'y a pas lit une simple injection de serpentine dans des calcaires avec suffusion de celui-ci par celle-là. Le débat semble se trancher dans ce sens par les dernières recherches de Möвıus [78], mais les partieularités de forme des lames de serpentine restent inexpliquẻes.

Archœosphœrina (Dawson) serait un genre voisin.

Ajoutons enfin que IIAHs considère l'Eozoon comme un végétal et en fait le genre Eophyllum. 
$\because$ ¿ SOLS-CLASSE

\section{HÉLIOZOAIRES. - HELIOZOARLE \\ [HELIOZO.1 (Hïckel)] \\ TYPE MORPHOLOGIQUE \\ (FIG. 234 A 243)}

\section{Structure.}

L'animal est très petit, il atteint à peine un demi-dixième de millimètre el, comme il est très transparent, on ne peut guère l'apercevoir à l'oil nu dans l'eau où il vit. Il est, comme un Amibe, formé d'une

Fig. 23't.

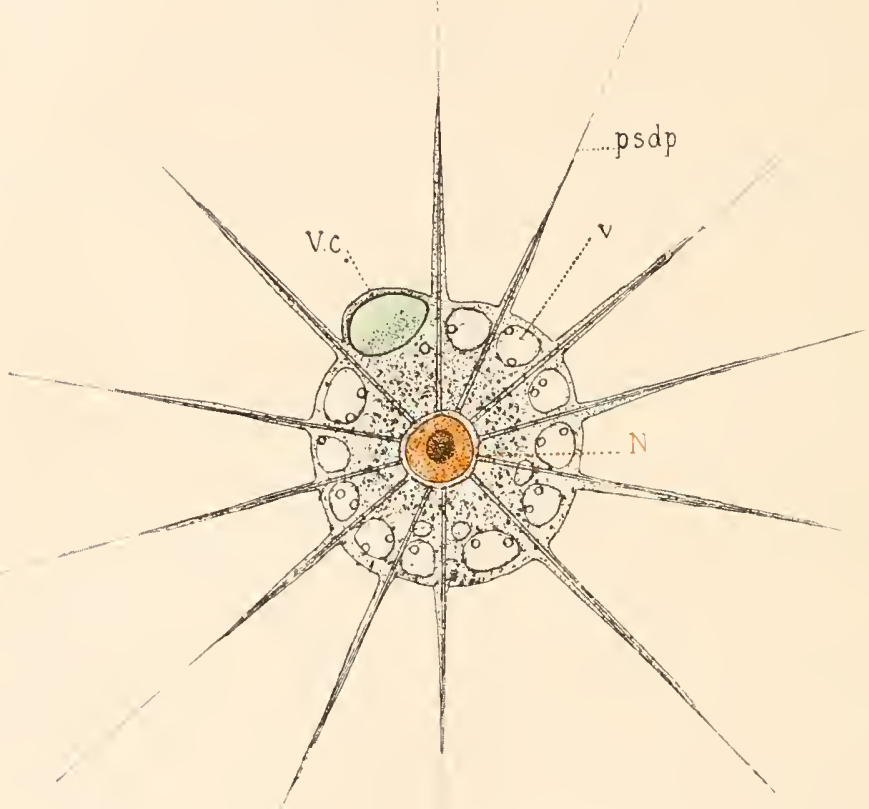

Hélioznaire (Type morphologique) (Sch.).

x.. noyau; psalp., pseudopodes; v., vacuole; V. c., vésicule pulsatile.

masse de protoplasme dans laquelle on distingue un endoplasme et un ectoplasme, pourvue d'un noyau, d'une vésicule pulsatile et de pseudopodes qui rayonnent autour d'elle. Mais toutes ces parties ont ici des caractères spéciaux qu'il faut faire connaitre.

Le noyau est central, grand, vésiculeux, formé d'une membrane et d'un suc nucléaire abondant. Il renferme un gros corps chromatique central que l'on appelle nucléole, bien que sans doute il corresponde 
plutôt à l'ensemble des parties chromatiques du noyau typique ('). L'endoplasme occupe la partie centrale du corps, autour du noyau, et forme environ la moitié de la masse totale. Il est hyalin, sans vacuoles, ni grauulations spéciales; les aliments ne pénètrent pas à son intérieur; il se continue avec l'ectosarque par une transition graluelle $\left({ }^{\boldsymbol{*}}\right)$.

L'ectoplasme forme à la surface du corps une épaisse couche périphérique. ll est formé d'un protoplasma granuleux et extrèmement vacuolaire.

Ces vacuoles $(V$.) sont remplies de liquides et si nombreuses qu'elles se compriment réciproquement et rendent cette région spumeuse $\left({ }^{\boldsymbol{3}}\right)$.

Les granulations sont souvent brillantes et probablement de la nalure des grains d'excrétion que nous avons rencontrés déjà chez les Amibes. Il y en a parfois dans le liquide des vacuoles.

La vésicule pulsatile (V.c.) est contenue dans l'ectoplasme. Elle est très grosse et tris superficielle, en sorte que souvent elle détermine une saillie volumineuse. Elle bat environ une fois par minute. Malgré sa grosseur et la netteté de ses contractions, nous retrouvons ici les mêmes divergences d'interprétation que nous avons rencontrées déjà au sujet de savoir si elle se vide en dehors ou au dedans. Il semble bien probable que la question se tranchera ici, comme d'ordinaire, dans le sens de l'ouverture au delıors. En tout cas, il n'y a pas d'orifice préformé et l'expulsion se ferait, d'après Zenkir, par une rupture fissiforme dont les bords se réagglutineraient aussitôt (").

(1) Pénard [89] considère ce eorps central eliez Actinophrys comme constituant à lui seul le vrai noyau (fig. 235), et voit dans la membrane, qu'il appelle capsule nucliaire,

Fig. 235 .

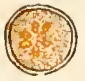

Noyau d'Actinophrys (im. Pénard). un équivalent de la capsule centrale des Radiolaires; il décrit celte membrane conme percée de pores et revèlue extérieurement d'une seeonde membrane continue. Cette interprétation parait d'autant moins justifice que parfois le corps chromatique central est multiple.

On ne distingue pas en ginerai de ríseau, mais Brauer [94] en a trouvé un dans les noyaux d'Actinospharium, et il est bien possible que l'on trouvât la même chose daurs d'autres genres si on les étudiait de la mème façon. Le noyau est normalement unique ehez les formes sans squelette, le plus souvent multiple chez celles pourvues d'un squelette.

() GreEf decrit une nemblane qui le séparerait de l'ectoplasme et serait l'équivalent de la eapsule centrale des Radiolaires. Mais cette membrane ne parait pas réelle.

(3) Les vacuoles sont beaucoup moins abondantes dans les formes pourvues d'un squelette que dans les nues.

(4) Pénard [89] est cependant bien affirmatif dans le sens contraire et donne pour preuve de son opinion que celte contraetion, quoique violente, ne ehasse jamais les minimes particules qui se trouvent en face d'elle. La paroi prend, pendant la systole, un aspect déchiqueté (fig. 236) veFig. 236.

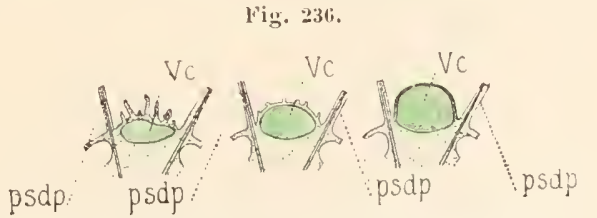

Héliozoaires. Contraction de la résicule pulsatile (Vc); psdp., pseudopodes (im. Pénard). llant de ce qu'elle ne peut se fondre dans les parties voisines aussi vite que le nécessiterait la rapidité du retrait. C'est l'ancienne opinion de Cíaparède el Lachanan. 
Les pseudopodes ( $p s d p$., fig. 234 et 2:37) sont, de beaucoup, l'organe le plus caractéristique. C'est par eux qu'il se listingue des autres types de Rhizopodes. Ils sont longs, atteignant au moins le diamètre du corps, très fins, rectilignes, rayonnants de tous côtés. Ils sont le siège de courants de granulations très nets; ils sont glutineux, peuvent se coller soit aux objets, soit entre eux, mais usent peu de cette dernière faculté et ne forment point de réseau, si ce n'est par exception et en quelque point limité, comme autour d'une proie. Enfin, ils possident une tigelle centrale appelée filament axile extrêmement fine, relativement solide, élasti-

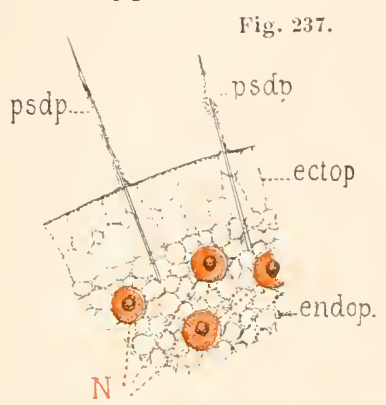

Héliozoaires. Type morpholog. (Actinospharium)

(im. Hertwig et Lesser).

ectop., ectoplasma; endop., endoplasma; $\mathbf{N}$., noyau psdp., psendopodes. que, qui règne dans toute leur longueur. Le protoplasma du pseudopode forme autour du filament axile une gaine mince et un peu irrégulière. A l'insertion sur le corps, il se perd immédiatement dans l'ectoplasme, tandis que le filament axile coutinue et se poursuit jusqu'au centre du corps où il se joint à ceux de tous les autres pseulopodes de l'animal. Le noyau étant central, il se trouve que les filaments axiles le traversent, mais il faut bien se rendre compte que ce n’est pas là un rapport fondamental car, dans certains genres où le noyau n'est pas central, les filaments ne se réunissent pas moins ainsi au centre du corps. D'ailleurs, ces filaments ne sont nullement des parties squelettiques, comparables à des spicules, par exemple. Ils sont formés uniquement de protoplasma d'une structure physique plus ferme et nous allons voir, en étudiant la physiologie des pseudopodes, qu'ils peuvent à chaque instant se fondre dans le protoplasma anbiant et se reformer tout aussi aisément (").

\section{Physiologie.}

Mouvements. - L'animal est ordinairement libre. Il se meut au moyen de ses pseudopodes en se halantsur eux, car ceux-ci, à volonté, deviennent glutineux au bout pour' se fixer, et cessent de l'ètre pour licher prise. Généralement, la chose se passe de la manière suivante. L'animal repose sur ses

(1) C'est le cas pour diverses formes it squelelte el pour quelques-unes, en particulier pour Grmnosphara (fig. 238). D'après SAssaki [93], cet lléliozoaire a de nombreux noyaux disséminés dans son ectoplasme el, au centre de son corps, se trouve une vésicule, munie d'une membrane, que l'auteur apFig. 238. pelle centre de rayonnement et que tous les filaments axiles traversent pour se ríunir à son centre. 11 compare ce centre de rayonnement au centrosome et à l'archoplasma car, dans la division, il se divise a la maniere de ces organes. Dans d'autre cas, les filaments axiles semblent s'arrèter à la surface du noyau ou même de l'ectoplasme. 
pseudopodes comme un Oursin sur ses piquants. Pour avancer, il en fixe un (ou quelques-uns) par le bout, un peu en avant et, le contractant pendant que ceux d'arrière làchent prise, il se déplace dans le sens voulu par une sorte de rotation très lente. Souvent, il reste fixé par quelques pseudopodes pour attendre des proies. D’autres fois, on le voit voguer en pleine eau et, sans doute, se diriger quelque peu par le mouvement

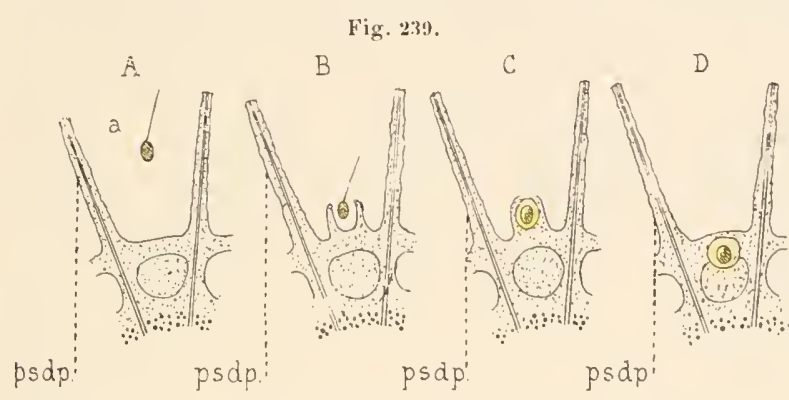

Héliozoaires. Type morphologique. Capture d'une petite proie (Sch.).

a., la proie; psip., psendopodes radiaires. de ses pseudopodes. Ceux-ci, gràce à leur filament axile, sont flexibles et souples. Ils peuvent s'allonger, s'incliner en divers sens, s'accoler momentanément aux voisins, se rétracter et mème rentrer tout à fait dans le corps et disparaitre, puis se reformer plus tard.

Alimentation, - Pour manger l'animal a deux procédés. Si la proie est petite (fig. 239) et est parrenue jusqu'à soll corps, il l'absorbe à la manière d'un Amibe en l'englobant avec une petite masse d'eau qui forme d'emblée une vacuole alimentaire. Si elle est très volumilleuse ( $a$, fig. $240, A)$, quelques pseudopodes s'attachent à elle et la fixent en devenant subitement glutineux $(B)$; ils dissolvent leur filament axile, deviennent gros, épais et, le long d'eux, la substance protoplasmique du corps s'arance ver's elle en quantité suffisante pour la cerner ( $C$ ' à $E$ ) et l'englober complètement. Elle est alor's facilement entraînée dans le corps et les sucs sécrétés par le cytoplasma forment autour d'elle la vacuole alimentaire. Les résidus fécaloüıles, toujours contenus dans une vacuole, sont poussés vers un point quelconque de la surface où la vacuole éclate et expulse son contenu.

Fig. 2 'to.
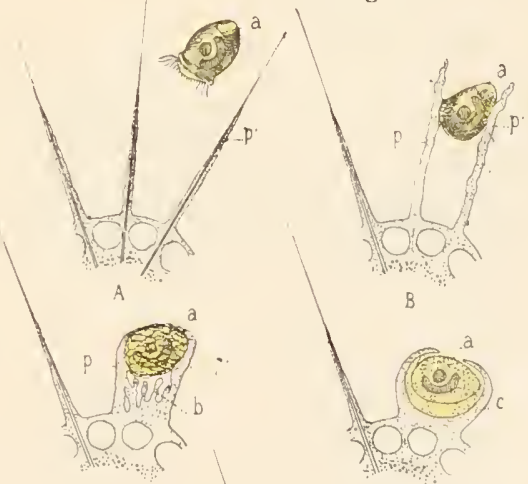

D

Héliozoaires.

Type morphologique.

Capture d'une grosse proie (Sch.).

$A, B, C$, la proie a est saisie par les psendopodes: $D$, $E$, stades plus avancés et vus en coupe pour móntrer la formation de la vacuole a; b., pseudopodes occasionnels saisissant la proie; p., p'., psendopodes radiaires.

L'alimentation est purement animale et consiste surtout en Protozoaires, 
parfois en Rotifères, souvent très volumineux. Jamais l'animal ne mange de Diatomées ou d'autres substances végétales.

Association. - Le plus souvent, quand deux individus se rencontrent, ils entremèlent leurs pseudopodes, mais sans se souder, et bientôt ils s'éloignent l'un de l'autre. Mais parfois (fig. 241), ils se fusionnent de la manière suivante. Quelques pseudopodes de l'un se soudent à ceux de

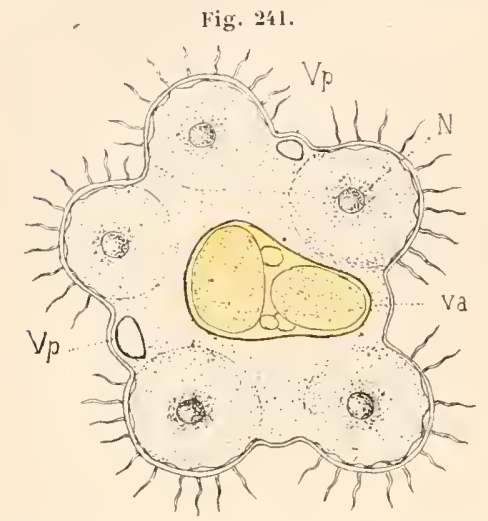

Héliozuaires. Type morphologique (Sch.). Association.

N., noyau; va., vacuole alimentaire;

vp., vésicules pulsatiles. l'autre individu. Les pseudopodes ainsi réunis dissolvent leur filament axile, se ramollissent, s'épaisissent, se raccourcissent, entrainant les deux individus l'un vers l'autre; les ectoplasmes, puis les endoplasmes, se joignent, se soudent. Mais la chose s'arrète là : les noyaux restent indépendants, et mème la fusion des corps n'est pas telle qu'on ne reconnaisse à la forme bilobée que l’individu ainsi formé est double. Puis un troisième, un quatrième individu ou un plus grand nombre se réunissent de la mème manière et l'on a ainsi une colonie temporaire, une association qui a pour but de constituer une individualité plus volumineuse, capable de capturer et de digérer de grosses proies, c'est une société de consommation. On trouve, en effet, presque toujours, dans ces individus multiples, de grosses proies plus ou moins digérées. Après quelque tempss de vie commune, les individus se séparent de nouveau et reprennent leur vie indépendante. On a souvent décrit ces associations, lorsqu’elles ne comportent que deux membres, comme des conjugaisons. Mais elles n'ont pas de signification sexuelle (").

Enkystement. - 1 l'approche le l'hiver et sans doute dans d'autres conditions où il éprouve le besoin de se proléger, l'animal s'enkyste. Pour cela, il ramollit, juis rétracle complètement tous ses pseudopodes, résorbe ses vacuoles et sa vésicule pulsatile, et se sécrète un kyste siliceux auquel s'adjoint souvent une couche gélatineuse. Au retour des conditions normales, il absorbe de l'eau, fait éclater son kyste, en sort el, en quelques heures, reforme sa résicule, ses vacuoles, ses pseudopodes, et reprend son aspect habituel.

L'enkystement est fondamentalement un acte de protection. Mais, ici comme dans tant d'autres cas, lanimal profite de cet état pour se diviser

(1) Cependant Grober dit avoir vu une fois un noyau unique dans une association de deux individus. Le même auteur a décrit chez lctinophrys une conjugaison entre un indivilu normal et un tout petit dépourvu de noyau. Mais ces observations sont restées isolies el l'interprétation, et peut ètre le fait mème, restent douteux. 
et l'enkystement devient, secondairement, le préliminaire d'un acte de reproduction. On le voit alors (fig. 24:3) se diviser sous son kyste en un nombre varié de fragments et, ce qui sort du kyste, ce sont plusieurs individus au lieu d'un (').

Division. - La division est sans doute le procédé essentiel de reproduction; mais elle a été rarement vue et ne présente rien de particulier $\left({ }^{2}\right)$.

Bourgeonnement. - Parfois (fig. 242), au lieu d'une division égale, il

(1) La chose a été bien observée par Brauer [94⿴囗 chez Actinosphxrium, Héliozoaire nu, polynucléé. L’animal, comme d'ordinaire, ramollit et retracle ses pseudopodes, et résorbe ses vacuoles avant de s'enkyster; mais en outre, il sécrète dans son endoplasme un grand nombre de granulations d'une substance vitelline destinée à nourrir au début les jeunes individus, nés de sa division. Il sécrète aussi (fig. 243) une enveloppe gélatineuse épaisse $(K y s$.) et forme dans son intérieur d'innombrables petites scutelles siliceuses $(A: s c t$.) qui se portent à la périphérie sous l'enveloppe gélatineuse. En mème temps, ses nombreux noyaux se fusionnent par petits groupes, ce qui réduit dans une forte proportion leur nombre total. On ne saurait voir, d'ailleurs, dans cette fusion, un acte sexuel puisque tous ces noyaux sont frères, descendant du noyau primitivement uni-

Fig. 2ะx.

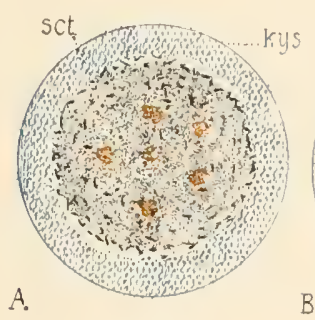

B
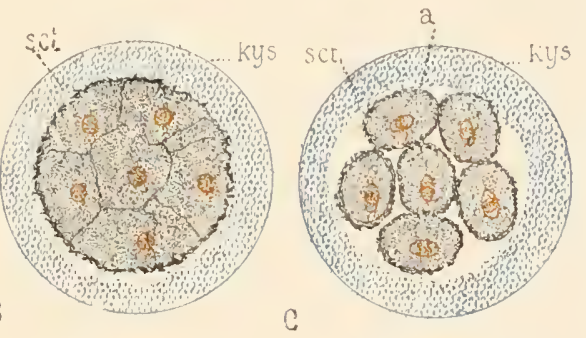

C
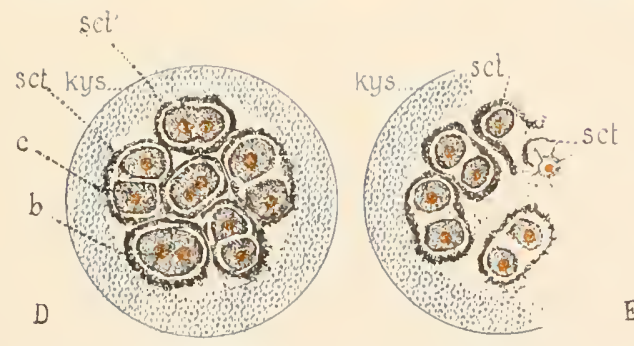

E
Fig. 242.

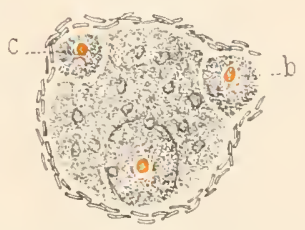

Héliozoaire (Type morphologique). ljourgeonnement (im. Hertwig). b., c., jeunes bourgeons. que de l'individu. Autour de ces nouveaux noyaux, le cytoplasme se divise en autant de masses arrondies (B) qui s'isolent sous une couche de ces scutelles siliceuses réunies à leur surface. Cela donne autant de liystes secondaires siliceux reunis sous une enveloppe gilatineuse commune $(C: s c t$. $)$. Ces liystes se divisent, a leur tour après division de leur noyau, en deux ou parfois quatre liystes tertiaires (D:c.), ayant chacun une couche de scutelles siliceuses (D et $E$ : sct.) sous

Héliozoaire (Type morphologique). Division pendant l'enkystement (Sch.). $A$, formation des scutelles dans le kyste de premier ordre. $B$, moreellement du protoplasma dans le kyste. $C$, formation des kystes de deuxiéme ordre. $D$, division dans les kystes de deuxieme ordre. $E$, sortie de l'embryou.

kys., enveloppe lystique de premier ordre; set., sct'., seutelles.

leur enveloppe commune de scutelles. Au printemps, ces kystes uninuclés donnent naissance chacun, par rupture des enveloppes $E)$, à autant de jeunes detinosphirium uninucléés qui, rapidement, multiplient leurs noyaux et prennent les autres caractères de l’individu norınal.

(2) La division du noyau est décrite comme directe. Mais chez Actinosphrerinm, 
y a séparation d'une ou plusieurs masses filles sensiblement plus petites. C'est, si l'on veut, un bourgeonnement, mais qui ne mérite guère ce nom, vu qu'il ne diffère de la division en rien d'essentiel (').

Sporulation. - Dans beaucoup de cas, l'animal, sans que l'on sache comment, émet des zoospores de 5 à $10 \%$, à deux cils dirigés l'un en avant, l'autre en arrière, munies d'un noyau et d'une vésicule pulsatile, qui nagent, puis perdent leurs cils, poussent des pseudopodes l'abord amœboïdes puis filiformes, et prennent l'aspect de petits Ciliophrys; mais peu à peu elles se transforment en individus normaux ${ }^{*}{ }^{*}$.

Conjugaison. - Enfin, on a vu parfois deux individus se conjuguer sous un kyste gélatineux. Le phénomène commence par une fusion des cytoplasmas (plastogamie), puis chacun des deux noyaux émet un globule polaire et enfin a lieu la fusion des noyaux (liaryogamie) $\left(^{\mathbf{3}}\right)$.

Ces caractères du type morphologique sont assez uniformes dans la série des genres. Les variations portent principalement sur deux points: le noyau peut être multiple et surtout des enveloppes de constitution variée s'ajoutent à l'organisme pour le protéger.

C'est en se fondant sur les caractères fournis par cette enveloppe, que l'on a divisé les Héliozoaires en ordres baptisés de noms bien barbares et qui mériteraient, au plus, de constituer des sous-ordres:

APHROTIORACIDA, comprenant toutes les formes nues, entièrement dépourvues de squelette;

C'ILAMYOPIIORIDA, chez lesquels le corps est recouvert d'une simple enveloppe gélatineuse;

CIIALAROTIORACIDA, ayant un revètement de spicules isolés;

DESMOTIORACIDA, ayant une véritable coquille continue.

En raison du peu d'importance de ces différences si faciles à résumer en quelques mots, nous nous abstiendrons de décrire un type morphologique pour ces groupes (').

dans la division des kystes, Brauer [9; a ohservé des mitoses Irés nettes et il est bien possible que ce processus soil plus répandu qu'on ne croit.

(1) Il a élé constaté entre autres dans le genre Acanthocystis.

(2) Parfois, on a vu sortir ces petits individus ciliophrydiformes, tout formés, du corps de leur parent et l'on a rattaché cela à un fait de bourgeonnement.

(3) Lobservation a été faile sur Actinophrys par Schaudinn [96] dont le mémoire nous parvient pendant la correction des épreuves.

(4) Il existe plusieurs Protozoaires qui possèdent à la fois des pseudopodes et un flagellum, et la question de savoir s'il convient de les réunir aux Rhizopodes en particulier aux IIéliozoaires) ou aux Flagellés a été résolue dans l'un etl'autre sens par les divers auteurs. Nous avons rangé parmi les Flagellés ceux-là seuls qui ont un flagellum permanent, et parmi les llẻliozoaires ceux dont le flagellum ne se montre que pendant les phases jeunes de leur vie. Nous pensons, en effet, que cet organe n'est caractéristique du Flagellé qu'à l’àge adulte et non quand il se présente temporairement comme organe de dissémination d'une forme larvaire.

I)'ailleurs nous ne manquerons pas de signaler chaque fois les affinités nrultiples de ces êtres à situation indécise. 
$1^{\text {er ORDRE }}$

\title{
APHRO'TIORACIDES. - APIIROTIIORACIDA
}

\author{
[APIROTIORACA (R. Hertwig)]
}

\section{GENRES}

Nuclearia (Cienkovsky) (fig. 244) diffère des formes normales par son corps amoboïde, souvent vacuolaire, sans distinction entre ectoplasme et endoplasme, par ses noyaux et vésicules pulsatiles multiples, par ses pseudopodes souvent bifurqués au bout, enfin par la présence accidentelle d'une enveloppe gélatineuse (Eau douce) (").

(1) A ce Nuclearia, à affinilés un peu douteuses, que d'autres rapprochent des Vampyrelles, mais qu'il semble plus naturel de considérer comme une forme inférieure des lléliozoaires nus, on peut rattacher un certain nombre de genres à affinités un peu contestables aussi que nous décrivons ci-dessous.

Arachnula (Cienkovsliy) est une forme assez mal connue, voisine de la précédente dont elle se distingue par son aspect rubané (Placé par d'autres à còté de Gymnophrys).

Archerina (Ray Lankester) est un curieux organisme constitué Fig. 2ut. comme un petit Nuclearia avec une grande vacuole non contractile et, en place de noyaux, deux corpuscules teintès en vert par de la chlorophylle. En cet état il ne mesure que 10 ou $15 \%$. Il se nourrit de Bactéries, à la manière d'un Actinophrys, s'accroît beaucoup et, à son intérieur, ses corpuscules verts se multiplient par division en 4, en sorte qu'il forme de petits amas de quatre grains ou de multiples de 4. Quand l'animal ne trouve plus à manger, il se sépare en autant de parties qu'il contient de grains, c'est-à-dire des centaines, chacun de ceux-ci entrainant une part du protoplasme. Ces individus s'enkystent, mais non pour se diviser, la multiplication résultant de la dissociation consécutive à la multiplication des corpuscules que nous venons le décrire. Ray Lankester place ces êtres à côté des Vampyrelles avec lesquelles ils ont en effet des rapports, mais leur mode de reproduction nous oblige à les en séparer. Ces corpuscules verts qui dominent si singulièrement la biologie de l'animal pourraient n'être que des noyaux colorés, mais leur division par 4 semble le contredire. Ce sont peut-être de vraies Algues vivant en symbiose avec un Rhizopode sans noyau (Eau douce).

Ciliophrys (Cienkovsky) est très semblable à Nuclearia, mais en diffère par le fait que, de temps à autre, on le voit rentrer ses pseudopodes, prendre une forme ovoïde, pousser un ou deux fouets à la grosse extrémité, se transformer, en un mot, en un Flagellé et se lancer à la nage, à la manière de ces animaux, le fouet en avant. Puis, à un autre moment, par une série de phénomènes inverse, il reprend sa constitution d'Iléliozoaire. Il y a là une singulière ressemblance avec ce que nous trouverons plus lard cliez certains Flagellates de la famille des Rhizomastigina. C'est au point que bien des auteurs, en particulier Iütschli, le placent parmi les Flagellés. Mais on peut aussi bien considérer ce changement d'état comme un retour à une condition larvaire différente, à celle de zoospore, pour les besoins de la locomotion. D'autre part, il ressemble tant aux jeunes Actinophrys que l'on s'est demandé s'il n'est pas simplement une forme jeune de ce genre (Eau douce).

Pythelios Frenzel a une conformation a peu pres semblable, mais s'en distingue par 
Actinophrys (Ehrenberg) (fig. 243̈) représente, ì peu de choses près, notre lype morphologique et nous n'aurons guìre à ajouter ì la description de celui-ci pour caractériser celui-là. Il est de forme sphé-

Fig. 2't5.

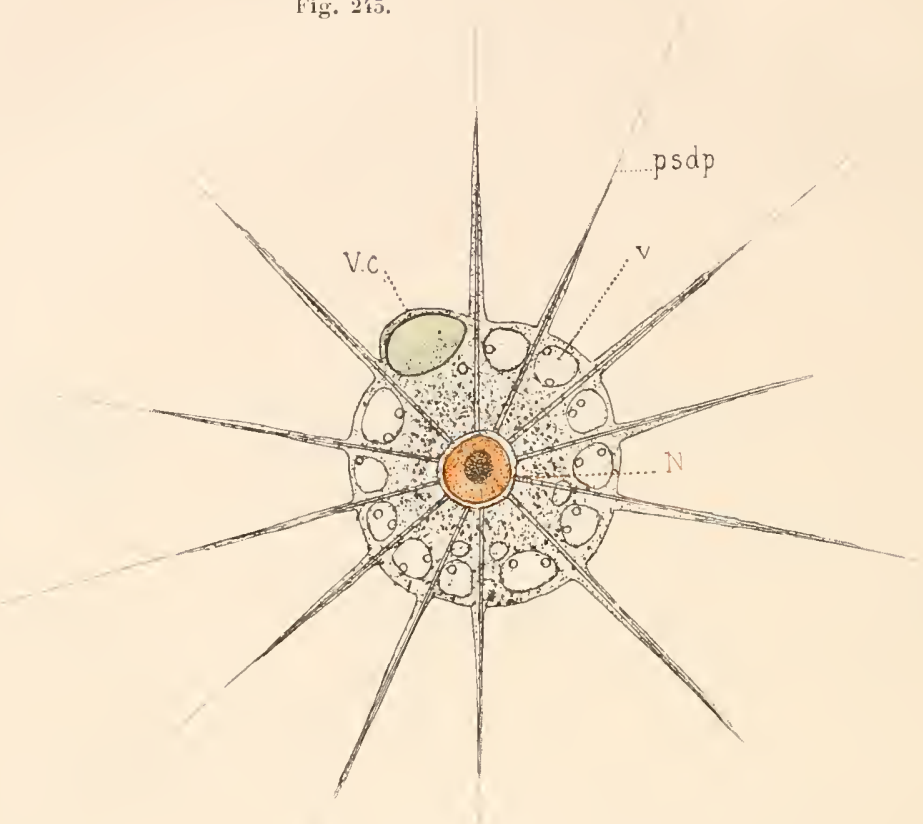

Actinophrys (Sch.).

rique, les vacuoles $(v$. ) de son ectoplasme sont très grandes, orientées radiairement, disposées en une seule couche périphérique, sauf parfois d'autres vacuoles irrégulièrement disposées au-dessous des précédentes. L'ectoplasme est peu distinct de l'endoplasme, réduit aux parois limitantes de ses vacuoles et les pseudopodes naissent sur le prolongement

un gros corps chlorophyllien qui lui permet une alimentation holophytique, c'està-dire semblable à celle des plantes (10 u. Lagunes).

Estrella (Frenzel) ressemble à Nuclearia, mais ne présente qu'un noyau et une seule vésicule pulsatile (כ̆, à à 12 . Lau douce).

Actinomonas (Kent) ressemble à Ciliophrys à son état Iléliozoaire, mais est fixé par un long filament pseudopodique. Il n'a pas d'ètat flagellé (Mer).

Monobia (Aimé Schneider) rappelle anssi un Ciliophrys à son état Héliozoaire, mais qui n'aurait ni noyau ni vésicule pulsatile. Ses pseudopodes portent des renflements fusiformes. La division laisse persister un pont protoplasmique entre les individus filles, de nonveaux ponts sétablissent et ainsi se forment de petites colonies qui peuvent comprendre jusiu'a huit individus Lau douce et terre humide). 
des cloisons de séparation. Le noyau unique est central et très grand, le vésicule pulsatile $\left(V_{c}\right.$.) est unique, très grande, très saillante (50 Mer et eau douce) (').

Actinosphærium (Stein) (fig. 2't6), nous ramèneaux formes normales des Héliozoaires. Il diffère de l'Aclinophrys par la multiplicité de ses noyaux, dont il y a jusqu’à 100 , logés dans l'endoplasme et pourvus d'un réseau chromatique très net. L'endoplasme est aussi vacuolaire mais à un bien moindre degré que l'ectoplasme, et il y a plusieurs vésicules pulsatiles (10 à 15 ) saillantes à la surface du corps $1 \mathrm{~mm}$. Eau douce) $\left({ }^{2}\right)$.

(1) Ce sont là du moins les caractères de l'espèce principale $(\boldsymbol{A} . \mathrm{sol})$, sinon unique, du genre. La forme d'eau douce est celle que nous avons décrite. La forme marine en diffère par la réduction considérable du système vacuolaire et par l'absence (si générale chez les Rhizopodes marins) de résicule pulsatile. Gruber [89] a fait l'observation intéressante que la Fig. 216. forme marine peut s'adapter à l'eau douce et y prend la structure vacuolaire de la forme d'eau douce, pour la reperdre quand on la met dans l'eau salée. Mais l'absence de vésicule pulsatile reste à titre de caractère différenliel permanent que Gruber considère conme de valeur spécifique.

(2) Nous avons indiqué plus haut les particularités de son enkystement.

Genres voisins:

Gymnosphæra (Sassaki). (V. à la page 15s les particularités de son centre de rayonnement) (Mer);

Actinolophus (F.-E. Schulze) pédonculé, à endoplasme et à noyau excentriques, avec une enveloppe gélatineuse très transparente (Mer);

Hæckelina (Mereschkovsky) serait un Actinolophe sans noyau (Mer); Actinosphæridium (Zacharias) (fig. 247) ne se distingue d'Actinolophus que par des caractères dignes à peine d'en faire un sous-genre de celui-ci (10 à $12 \mu$. Lau douce);

Zooteira (Wriglıt) est un Actinolophus à pédoncule très contractile (Ier).

Malgré la présence d'une faible couche gélatineuse chez quelques-uns d'entre eux tous ces genres forment l'ordre des Aphrothoraca de Hertwig.

Nous y ajoutons avec quelques réserves le genre

Camptonema (Schaudinn) [94], forme peut-être un peu aberrante et dont son auteur ne précise pas la position. Il se caractérise par son corps nu, ses pseudopodes à filament axile se prolongeant jusqu'au contact de l'un des nombreux (50 et plus) noyaux. L'endoplasme ne diffère de l'eetoplasme que par une strueture plus granuleuse. L'animal se déplace, non seulement en roulant sur ses pseudopodes, mais aussi en rampant au moyen de légères déformations amœboìdes (0,1ว̆. Mer). 


\section{$2^{\text {e }}$ ORDRE}

\section{GILAMYDOPIORIDES. - CIL AM YDOPIIORIDA \\ [CHLAMYDOPHORA (Archer)]}

\section{GENRES}

Heterophrys (Archer) (fig. 24S) représente un Nuclearia qui posséderait une épaisse enreloppe gélatineuse hyaline dans ses couches profondes, granuleuse en dehors, et qui émet de petits prolongements spiniformes entre lesquels passent les pseudopodes (Mer et eau douce) ( ${ }^{\mathbf{2}}$ ).

(1) Genres voisins :

Lithocolla (F.-E.Schulze) diffère de Nuclearia par un revêtement simple de petits grains de sable. Noyau et vésicule pulsatiles inconnus (Mer);

Elæorhanis (Greeff) a un revètement analogue avec des carapaces de Diatomées concurremment avec les grains de sable et, en outre, des granules de graisse colorée (Eau douce).

(Hais ces particules étrangères sont, Fig. 248. dans ces deux genres, adventices, directement accolées par les pseudopodes, sans sécrétion spéciale de ceux-ci);

Lithosphærella (Frenzel) ne diffère de Lithocolla qu'en ce que son revêtement est formé de plusieurs couches (25 a $32 \mu$. Mer et eau douce);

Chondropus (Greeff), au contraire, est revêtu d'une couche granuleuse de couleur jaune qui pourrait bien, si elle n’est pas protoplasmique, n'ètre qu'une enveloppe gélatineuse séerétée, comparable à celle de Chlamydophora auquel il faudrait alors le joindre (Eau douce);

Sphærastrum (Greeff) a aussi une enveloppe gélatineuse mais sans prolongements spiniformes et irréguliers; les pseudopodes sont souvent dirigés d'un seul côté et l'enreloppe se prolonge plus loin te ce côté que des autres. L'animal forme souvent des associations de nombreux individus dont les tuniques gélatineuses se fusionnent tandis que leurs corps sont unis seulement par des ponts protoplasmiques (Eau douce) ;

Astrodisculus (Greeff, emend. Archer) a, au contraire, son enveloppe gélatineuse régulière, et les pseudopodes rares et régulièrement rayonnants (Eau douce).

Certains auteurs placent ici le genre Mastigophrys (Zenker) qui représente un Actinomonas, mais libre et pourvu d'une enveloppe gélatineuse. Mais en raison de la permanence de son flagellum, nous préférons le joindre aux Flageliés (V. p. 322). 


\section{CHALAROTIIORACIDES. - CIIALAROTIIORACIDA \\ [CIIALAROTIORACA (IIertwig et Lesser)]}

\section{GENRES}

Acanthocystis (Carter) (fig. 249), pour l'ensemble de la structure, est notre type morphologique, mais avec quelques additions et particularités remarquables. Le corps est protégé par une enveloppe gélatineuse ferme et, audessous de lui, par une couche hyaline fluide qui semblerait appartenir à cette enveloppe, mais qui en réalité est protoplasmique, car si la couche périphérique se rompt, celle-ci fait éruption et forme une hernie qui émet des pseudopodes. Dans l'enveloppe gélatineuse sont implantés de longs spicules siliceux radiaires qui Fig. 2๕9.

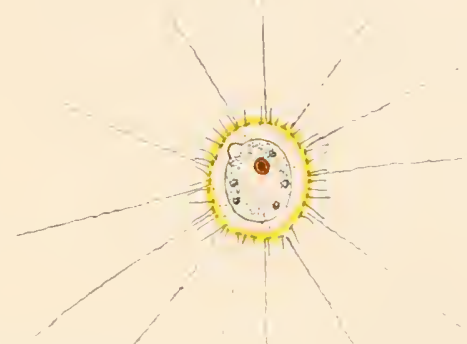
s'appuient à leur hase sur de petites baguettes tangentielles (40 à 50 (u) (') .

(1) L'ectoplasme peu distinct le l'endoplasme, tris vacuolaire, contient des corpuscules bleuatres réfringents qui sont des grains d'amidon, représentant une réserve nutrilive. Il contient souvent des Zoochlorelles. Pour capturer une proie, il rabat ses spicules comme fait un Oursin de ses piquants et les écarte ainsi que les baguettes tangentielles qui sont à leur base, car loul cela est absolument mobile dans la couche gélatineuse. Celle-ci se déprime alors, se creuse et finalement met à nu le protoplasma sous-jacent qui englobe la proie á la manière d'une amibe; puis tout reprend sa place. On a vи l'animal muer et rejeter son enveloppe squeletlique. Rappelons enfin le bourgeonnement qui a été observé ici (fig. 242). (Cette figure, appliquée au type morphologique, représente plus spécialement un Acanthocystis) (Eau douce et mer).

Ce squelette formé de pièces éparses est caractéristique de l'ordre dont l'Acantho-

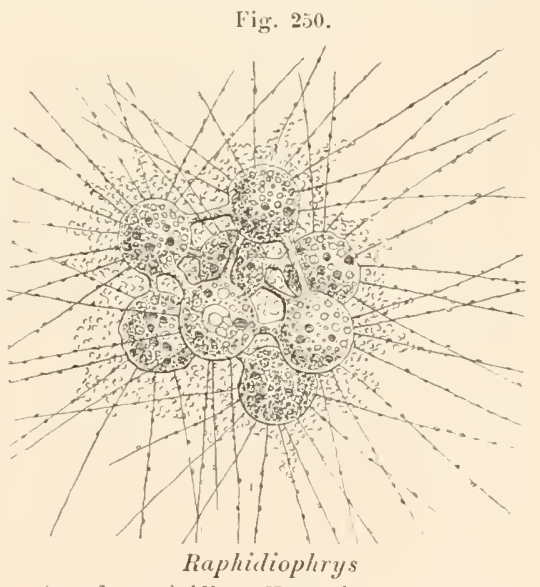

(R. elegans) (d'ap. Hertwig et Lesser). cystis est le type. Nous le retrouvons donc sous des formes variées dans les genres suivants qui appartiennent au même groupe :

Cienkowskya (Schaudinn) présente des petites plaques siliceuses perforées (12 $\mu$. Mer);

Raphidiophrys (Archer) (fig. 2:0) a son squelette formé de spicules siliceux en forme d'aiguilles droites ou comrbes disposées tangentiellement. Cependant, autour de la base des pseudopodes, elles se relèvent souvent en cònes et donnent à l'animal une forme 
$4^{\text {e ORDRE }}$

\title{
DESMOTHORACIDES. - DESWOTIORACIDA
}

\author{
[DESMOTHORACA (Hertwig et Lesser)]
}

\section{GENRES}

Chlathrulina (Cienkovsky) (fig. 2:31) est, en somme, conformé comme notre type morphologique, mais on n'observe pas chezlui de distinction en endoplasme et ectoplasme. Il a un seul noyau central; ses p'seudopodes sont un peu plus anastomosables que d'ordinaire; il est protégé par une coquille siliceuse sphérique et continue, mais percée de trous; il ne remplit pas complètement sa coquille et peut se déplacer à son intérieur. Cette coquille est portée au sommet d'un pédoncule creux, long et grêle de même substance (').

étoilée. Le noyau est simple ou multiple. Enfin, les individus forment fréquemment des associations dans lesquelles ils fusionnent leurs squelettes et forment une enveloppe commune, tandis que leurs corps ne sont unis que par des ponts protoplasmiques (Eau douce);

Pompholyxophrys (Archer) a son squelette formé de simples petites sphérules sur plusieurs couches entourant le corps a distance. Pseudopodes sans courants de granulations, souvent dichotomes au bout (Eau douce);

Pinacocystis (Hertwig et Lesser) a le sien formé d’une couclıe de petites plaquettes rondes contiguës (Mer). Chez

Pinaciophora (Greeff), les plaquetles sont en forme de petites feuilles lancéolées (Eau douce); Diplocystis (Pénard) présente des éléments squelettiques de deux formes 30 à 35 p. Lau douce);

Artodiscus (Pénard) a ses pseudopodes très élargis à leur base (15 à $30 \mu$. Eau douce);

Wagnerella (Merechliovsky) est formé d'une sphère fixée par un pédoncule élargi à la base; le tout est recouvert d'une enveloppe membraneuse dans laquelle sont des aiguilles siliceuses qui, sur le pédoncule, sont couchées, tandis que sur la tête, elles sont plus longues et dressées radiairement. Le noyau serait dans la partie évasée du pédoncule ( $1 \mathrm{~mm}$ de haut $\mathrm{y}$ compris le pied. Mer).

Fig. 252.

(1) Pour se reproduire, l'animal se divise à la manière ordinaire dans sa coquille et les leux individus filles (ou un seul) sortent de la coquille, se fixent, se sécrètent un pédoncule et, alor's seulement, forment leur squelette. II se reproduit aussi par spores flagellées qui se forment au nombre de deux dans la coquille et en sortent. Enfin, il sait aussi s'enkyster sous la coquille (0,1. Eau douce).

Genres roisins:

Hedriocystis (Hertwig et Lesser) (fig. 20̈2) ne diffère du précédent, outre la taille plus petite, que par sa coquille dont les trous sont percés au sommet de proéminences coniques (20 à $30 \mu$. Eau douce);

Orbulinella (Entz) n'a pas de pédoncule et a les orifices de sa coquille dilatés en entonnoir (Etangs salés);

Elaster (Grimm) n'a pas non plus de pédoncule, mais a les orifices

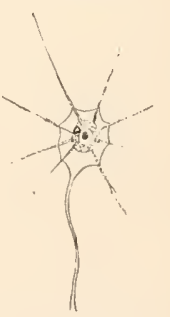

Hedriocystis

(II. pellucida)

(d'ap. Hertwig et Lesser). de sa coquille semblables à ceux de la Chlathruline $(20 \mu$. Eau douce). 
$6^{e}$ Sous-Cilasse

\section{RADIOLAIRES. - RADIOLARIA \\ [R.1DIOL.1RI.A (Häckel)]}

TYPE MORPHOLOGIQUE

(FIG. 253 A 255)

Bien qu’il soit rncore unicellulaire, l'animal présente ici, gràce à la présence de parties additionnelles, une complication qui ne laisse pas que

Fig. 253.

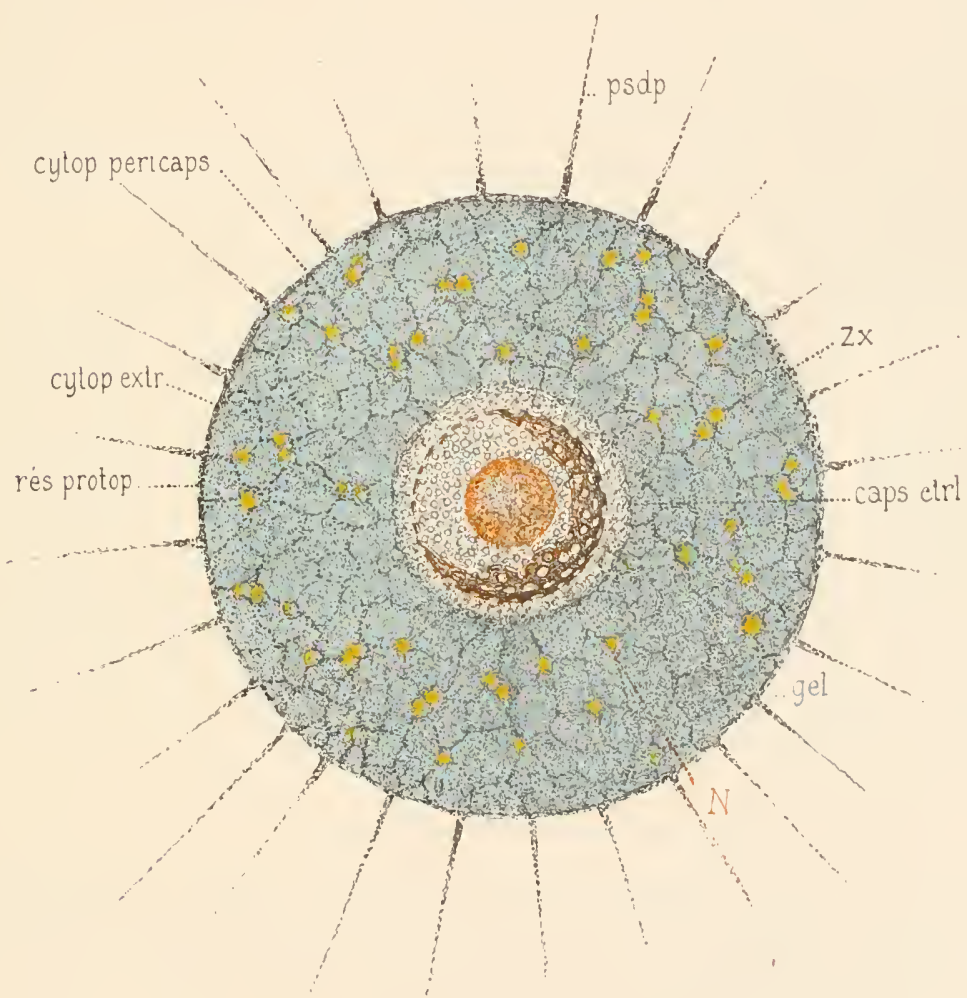

Radiolaire. Type morphologique (Sch.).

caps. ctrl., eapsule eentrale; cytop. extr., eytoplasma périphérique; cytop. pericaps., cytoplasma péricapsulaire; gel., gelée on calymina; $\mathbf{x}$., noyau; psdp., pseudopodes; rés. protop., réseau protoplasmique; $\mathbf{Z x}$., zooxanthelles. (Les pseudopodes devraient ètre réticulés. Ce caraetere, omis sur eette figure, se voit bieu sur la figure $376, \mathrm{p} .237$.

de rendre un peu difficile au premier abord son assimilation avec la cellule typique. Il est sphérique, te taille assez grande, se mesurant par millimètres ou tout au moins par dixièmes de millimètres. Au centre 
est un noyau $(N$.) et, au tour de lui, une couche protoplasmique entourée ellemème d'une membrane; il semblerait que nous ayons là la cellule entière arec sa membrane, son cytoplasma et son noyau. Cependant cet ensemble appelé ici capsule centrale (caps. ctrl.) ne forme qu'une partie de l'organisme. En dehors de cela, nous avons une très épaisse masse extracapsulaire entourant la capsule centrale et formée d'un réseau protoplasmique très riche (rés. protop.) dont les mailles sont remplies par une substance d'apparence et de consistance gélatineuses que nous appellerons la gelée (gel.) (Hícker lui donne le nom de calymna) et qui émet à la périphérie les pseudopodes $(p s d p$.$) . Il n'y a jamais de vésicule pul-$ satile.

Nous allons reprendre avec plus de détails la description de toutes ces parties. Mais nous devons dis maintenant montrer comment cet ensemble peut représenter la cellule ordinaire ou plutôt le Rhizoporle normal. Rien n'est plus aisé à comprendre, dès que l'on sait que la membrane de la capsule centrale est percée de trous qui font communiquer ensemble les cytoplasmes intra- et extracapsulaire. Or nous avons déjà rencontré des Rhizopodes où le cytoplasme forme deux masses incomplètement séparées par une barrière squelettique : les Gromies par exemple (V. p. 112). Supposons, an lieu de la Gromie, un Foraminifère perforé et nous aurons, comme ici, un Protozoaire dont le cytoplasme sera séparé en deux parties concentriques ne communiquant entre elles que par d'étroites perforations. Nous avons vu d'autre part que souvent, chez les lléliozoaires par exemple, le corps sécrète à sa surface une enveloppe gélatineuse. Supposons que cette sécrétion se dépose entre les mailles d'un riche réseau de pseudopodes réticulés, de manière à ne laisser libre que la partie périphérique du réticulum, et nous aurons absolument la représentation d'un Radiolaire. C'est ainsi que nous devons consilérer le Radiolaire pour ramener sa constitution, en apparence si aberrante, à celle d'un Rhizopode normal. Passons maintenant à la description détaillée de ses parties.

\section{Structure.}

Noyau. - Le novau ( $\boldsymbol{T}^{\text {. }}$.) ne présente rien de bien particulier. Il est central, gros, vésiculeux el montre, dans son sue nucléaire, un réseau serré et de nombreux granules chromatiques`qui ont l'aspect de nucléoles sans en avoir la signification cytologique (').

Gapsule centrale. - L’intérieur de la capsule (caps. ctrl.) est occupé par un cytoplasma intracapsulaire ordinaire lans lequel sont suspendues des inclusions de diverses sortes. On y rencontre: 10 des vacuoles, le tailles variées, mais petites si on les compare à celles que nous trouverons tout à l'heure en dehors de la capsule; $2^{\circ}$ des gouttelettes d'ume graisse colorée, le tailles très diverses aussi; $3^{\circ}$ des cristaux qui sont

(1) On le désigne parfois sous le nom de sésicule interne. 
formés d'une substance alhumineuse et représentent des réserves nutritives; to $^{\circ}$ enfin souvent du pigment (').

La membrane capsulaire est fort mince et constituée par une substance homogène, élastique, qui semble ètre la chitine. Elle est percée de trous qui font communiquer les cytoplasmes situés en dehors et en dedans d'elle $\left({ }^{2}\right)$.

Cytoplasme extracapsulaire. - Ce cytoplasme forme, avons-nous dit, un vaste réseau très riche, concentrique à la capsule centrale, el dont les mailles sont occupées par la gelée. Celle-ci est si transparente qu'on ute l'aperçoit pas sur le vivant $\left({ }^{\mathbf{3}}\right)$.

Mais la gelée ne forme pas toute la surface, et n'arrive pas tont à fait à la capsule. Il a donc, en réalité, trois régions en dehors de la capsule :

10 Une couche mince de cytoplasma péricapsulaire continue (cytop. péricaps.);

2o Un réseau (rés. protop.) à mailles occupées par la gelée (gel.);

-30 Enfin, un mince réseau de cytoplasma périphérique (cytop. extr.) couché à plat sur la gelée et laissant voir celle-ci dans ses mailles. De ce réseau périphérique partent les pseudopodes ( $p s d p$.$) . Dans le cyto-$ plasme extracapsulaire, mais principalement dans sa région moyenne réticulée, se trouvent diverses inclusions : des gouttelettes de graisse colorée, du pigment, de petites vacuoles (").

Les pseudopodes sont fins, rayonnés comme ceux des Héliozoaires, mais beaucoup plus anastomosables, formant un réseau, et lépourvus de filament axile (").

Dans les cordons du réseau protoplasmique répandu dans la gelée, se rencontrent, d'une manière constante, des Algues jaunes commensales (fig. 2马3, $Z x$ et fig. 23 i), ces mèmes Zooxanthelles que l'on retrouve vivant en symbiose avec tant d'autres animaux colorés. Elles sont là, très nom-

Fig. 254.

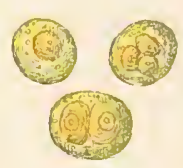

Zooxanthelles (Sch.) breuses, vivant et se reproduisant pour leur compte, à leur manière, par division, échangeant arec le Radiolaire des services dont nous

(1) Ces vacuoles ont élé décrites sous les noms de granulations vitellines ou de sphérules hyalines: elles contiennent en réalité un simple liquide.

(2) Dans quelques rares genres, elle manque complètement, dans d'autres moins rares elle se forme tardivement.

(3) La gelée est fréquemment creusée de nombreuses et grosses s'acuoles. Mais pas assez souvent cependant pour que ce caractère puisse prendre rang au nombre de ceux du type morphologique.

(") Les auteurs appellent souvent endoplasme et ectoplasme les protoplasmas intraet extra-capsulaire, mais nous ne savons pas du tout si l'assimilation avec les parties homonymes des autres Rhizopodes est légitime. Notre cytoplasma péricapsulaire est le Pseudopodienmutterboden des Allemands. 11 y aurait bien d'aulres dénominations a indiquer. Nous préférons les laisser de còté, et ne prendre partout que le nom le plus simple.

(5) Sauf certains pseudopodes dits axopodes des Acanthaires qui ont un filament axile (V. p. 207). Le caractère réticulé, omis sur la figure 233, se voit bien sur la figure 376, p. 237. 
allons parler. Elles possèdent une membrane de cellulose et contiennent un noyau, des grains d'amidon (et de paramylon) et sont colorées par des grains de diatomine qui jouent chez elles le mème rôle que joue la chlorophylle chez les Algues vertes (').

\section{Physiologie.}

\section{L'animal est essentiellement marin et pélagique $\left(^{2}\right)$.}

Mouvements. - Il flolle au gré des vagues sans faire aucun effort pour se diriger. Mais il n'est pas inerte pour cela : il peut agiter ses pseudopodes, se contracter et, par ce dernier moyen, agir sur son équilibre hydrostatique. Les contractions, dues à la simple contractilité générale du protoplasma, déterminent un mouvement exosmotique des liquides qui, étant plus légers que les autres sulistances, augmentent le poids spécifique et font plonger le corps. Quand cesse la contraction, le corps absorbe de nouveau du liquide et reprend son volume et sa densité primilifs qui le ramènent à la surface. L'animal peut aussi modifier sa forme par des contractions locales $\left({ }^{3}\right)$. Tombé au fond, il peut ramper lentement avec ses pseudopodes. Enfin, pour la capture des aliments, ses pseudopodes se comportent comme ceux des Rhizopodes réticulés.

Alimentation. - Il se nourrit, comme les Foraminifères, de particules saisies avec ses pseudopodes et digérées dans le Protoplasma extracapsulaire. Des courants protoplasmiques font ensuite circuler la sulsstance assimilée à travers la capsule jusqu'au noyau. Il se nourrit aussi de l'amidon formé par ses Xanthelles au moyen de l'acide carbonique qu'il leur fournit (').

(1) Elles sont constantes dans les espèces où elles existent normalement et qui sont de beaucoup plus nombreuses, mais non constantes pour l'ensemble des liadiolaires, car bien des espéces en sont privées. Dans le groupe des Phioodariées, elles paraissent manquer et dans celui des Acunthaires elles sont intracapsulaires.

(2) Ce caractère n'admettant aucune exception, nous nous abstiendrons dans l'étude des genres d'indiquer l'habitat.

(3) Eвerth [87] et d'autres ont décrit des sortes de fibres musculaires dans le protoplasma extracapsulaire de Thalassicola, mais cela ne semble pas pouvoir être généralisé.

On a avancé que ces contractions locales, éloignant sa forme de celle de la splière, avaient pour effet d'augmenter sa densité, en disant que la forme sphérique, étant celle qui réunit le plus grand volume sous la plus faible surface, correspondait à un maximum de densité. Nais c'est une erreur; la surface n'a ici aucune influence. j'renez une sphère de pâte à modeler et plongez-la dans l'eau. Vous aurez beau en la pétrissant la transformer en un cube ou en une lame, vous ne diminuerez en rien son poids dansl'eau. N'ayant modifié ni sa masse, ni la nature de sa substance, vous ne pouvez avoir atteint sa densité.

(4) Les auteurs s'accordent à admettre cela et cependant on ne voit pas pourquoi la matière amylacée sortirait du corps de l'Algue. Cela n'a pas lieu pour celles qui vivent en pleine eau et on ne voit pas comment il pourrait en être autrement iei, tant que l'Mlgue est intacte. Fanintzin [88] a observé une chose beaucoup plus rationnelle, c'est la digestion de l'amidon et de l'N]gue elle-même, tout entière, par le Radiolaire, lorsqu'il est affamé; ses sucs prennent alors, sans doute, une activité qui triomplie de la résistance de l'Nlyue. 
Hais cette alimentation n’est jamais exclusire.

$\mathrm{Vu}$ l'ahsence de vésicule pulsatile, l'excrétion et la respiration se font par échanges superficiels. La présence des Zooxanthelles rend cette dernière très aisée et peut-ètre les produits d'excrélion azolés sont-ils aussi, partiellement au moins, utilisés par ces Algues.

Reproduction. - La division n’est plus ici le procédé principal de reproduction comme il l'était dans les autres Rhizopodes el comme nous verrons qu’il l'est de nouveau dans le reste des Protozoaires (").

La sporulation est le procédé principal sinon exclusif de mulliplication. Le noyau commence par se diviser en un nombre de plus en plus grand de petits noyaux qui se répandent dans la capsule centrale. Chacun d'eux concentre autour de lui une portion du protoplasmo intracapsulaire et forme une petite masse allongée qui se munit d'un ou deux flagellums. L'ensemble constitue alors une zoospore qui a tout l'aspect d'un petit Flagellé. On voit ces zoospores s'agiter dans la capsule centrale qui ne contient plus qu'elles, puisque tout son contenu a été utilisé $\left({ }^{2}\right)$.

Pendant la formation des zoospores, l'animal a rentré ses pseudopodes, retiré à l'intérieur de sa capsule une bonne partie de son protoplasma extracapsulaire et est tombé au fond, immobile. Quand les zoospores sont mùres, la membrane capsulaire se rompt, les zoospores s'échappent et le reste du corps achère de se dissocier et périt.

Le sort de ces zoospores n'a jamais pu ètre suivi. Híckel ponse qu'elles prennent la forme d'un Ciliophrys et sécrètent ensuite la capsule centrale et la gelée caractéristiques. Cela est infiniment prohable, mais n'a pas élé observé.

Cependant les Zooxanthelles, organismes indruendants de celui qui a

(') La division n’a guère été observée d'une manière un peu eerlaine que dans le groupe des Phæodariées. Ailleurs, on a rencontré des individus à capsules en biscuit ou mème doubles, mais on n’a pas observe la suite du phénomène. La division est en somme un procédé de reproduction, rare chez tous les groupes, et absent peut-être ehez le plus grand nombre. Il faul faire exception cependant pour les Polycyttuires, formes coloniales où la colonie résulle de la division incomplète d'un individu primitif multiple. La formation de la colonie elle-mlème n'est pas un proédé de reproduclion, mais elle eontribue cependant à la multiplication, ear souvent la colonie se scinde en colonies filles qui continuent i s'accoitre. Les Polycytaires d'ailleurs, comme les Monocyttaires, possèdent la sporulation comme proérlé principal de reproduction.

(2) Lor'squ'il y a, ce qui est l'ordinaire, des cristaux et des gouttes d'huile dans la capsule, chaque zoospore prend un cristal et une ou plusieurs gouttes d'luuile. Il y a en effet précisément autant de cristaux que de petits noyaux. Dans la zoospore, le noyau est en avant, le cristal et les gouttes d'huile en arrière. Ces zoospores ainsi munies d'un crislal relativement très gros et placé asymétriquement à leur intérieur, faisant saillie hors d'elles, sont très curieuses. On les nomme spores à cristaux pour les distinguer de eelles qui n'en ont pas. Mais cette particularilé ne parait pas avoir une grande importance. Ce cristal, fait d'une matière albuminö̈de, est en effet une simple réserve nulritive, comparable au lécilhe d'un cuf et les spores qui en sont dépourvues ont peut-ritre aussi des matières de réserve sous une aulre forme. En tous cas, elles ne diffèrent pas plus des précédentes qqu'un ouf maigre d'un œuf gras. 
atteint ainsi le terme de son existence, ne sont pas nécessairement condamnées à périr avec les parties extracapsulaires qui les contiennent. Ici, comme dans le cas où elles sont mises en liberté par la destruction accidentelle de leur hôte, elles se protègent d'abord sous leur enveloppe cellulosique épaissie et gélifiée et se divisent sous cet abri. Mises alors en contact avec l'eau, elles se munissent d'un flagellum et se lancent à la nage. Il est probable que, sous une forme ou sous l'autre, elles ne peuvent vivre que si elles ont la chance d'être capturées par un Radiolaire jeune où elles se multiplient à l'aise. C'est sans doute ainsi que se fait l'infection des jeunes ( $\left.{ }^{1}\right)$.

\section{Squelette.}

Nous avons décrit comme type morphologique une forme sans squelelle. C'est qu'en effet les formes sans squelette sont, dans tous les groupes de Radiolaires, les plus primitives, celles dont les autres peuvent être considérées comme dérivées.

Nous verrons, en étudiant les genres, quelles sont les variations du squelette, critérium de première importance pour la caractéristique des groupes de tout ordre. Mais nous devons dès maintenant faire connaitre ce qu'est en général ce squelette, où il se forme, et comment.

Fig. 255.
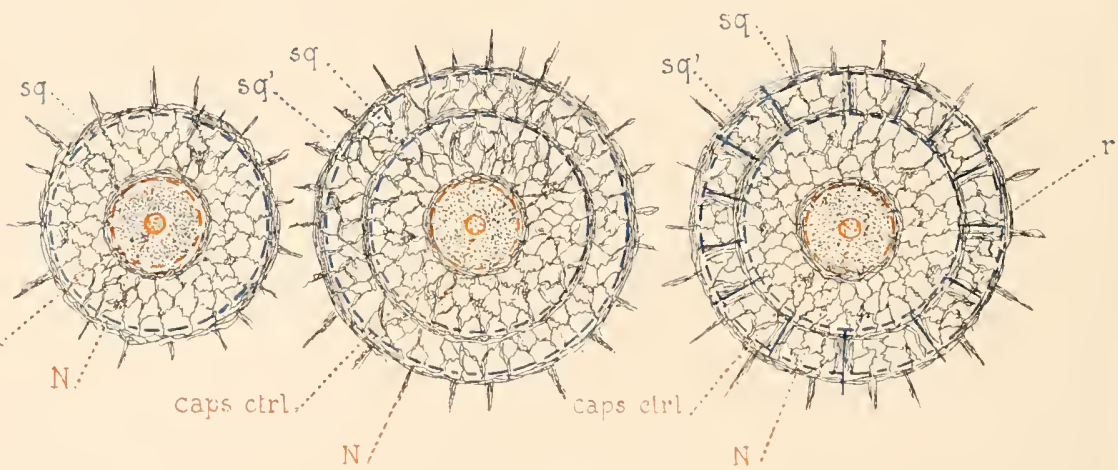

Squelette des Radiolaires (Sch.).

x., noyau; caps. ctrl., capsule centrale; sq., premiere enveloppe squelettique; sq.., seconde enveloppe r., rayons squelettiques.

Reprenons notre type morphologique el supposons que le réseau proloplasmique périphérique couché à plat à la surface te la gelée vienne à sécréter le la silice; il se formera ainsi, à la surface le la gelée,

(1) Régénération. - VER Wonn a runstalio que la capsule centrale isolée peut régénérer tout le reste du corps. Hais, privé du noyau, l’animal meurt fatalement.

Phosphorescence. - L'animal est quelquetois phosphorescent grace ì ses goultelettes d'huile culorée. 
un réseau siliceux qui sera la reproduction exacte du réseau protoplasmique. Considéré dans son ensemble, ce réseau formera une coquille en forme de sphère percée de trous (fig. 2马̈弓̆, $s q$ ). Selon la forme du réseau protoplasmique, ces perforations seront irrégulières ou régulières, de forme simple ou compliquée. Quel qu’il soit, le ressin de la coquille se trouve d'avance expliqué par celui du réseau protoplasmique.

Souvent les choses n’en restent pas là. L'animal sécrète en dehor's de son réseau protoplasmique, entre les bases de ses pseudopodes, une nouvelle couche de gelée qui peut ètre très épaisse. Cette gelée se trouve d'enıblée parcourue dans son épaisseur par un réseau protoplasmique et d'emblée revètue d'un réseau protoplasmique superficiel couché à plat sur sa surface, formés l'un et l'autre pas le réticulum des pseudopodes. Et la chose pourra continuer ainsi plusieurs fois, donnant ainsi naissance, en dehors de la gelée, du réseau radiaire et du réseau superficiel primitifs, à des assises de gelée, à des réseaux radiaires, à des réseaux concentriques, étagés les uns sur les autres, le dernier de ceux-ci constituant la réseau superficiel d'où naissent les pseudopodes. Mais ces réseaux protoplasmiques peuvent former des squelettes siliceux. Les réseaux concentriques couchés à plat sur les dépôts successifs de gelée donnent des coques grillagées, sphériques, concentriques, et les réseaux radiaires contenus dans l'épaisseur le ces mèmes assises de gelée donnent des tigelles radiaires $(r)$ qui unissent les coques grillagées successives entre elles et à la coquille primitive (').

D'ailleurs ces coquilles grillagées ne sont pas toujours complètes; elles peuvent ètre réduites à des spicules isolés et souvent à de simples épines, insérées tangentiellement sur les tigelles radiaires, aux points où celles-ci traversent leur niveau.

Si l'on ajoute à cela que la forme primitive de notre type devient, dans la réalité, souvent ovoïde ou lenticulaire ou déformée de mille autres façons, on se rend compte de l'infinie rariété de formes que peuvent prendre les coques grillagées et par la suite l'ensemble de la coquille.

Ajoutons enfin que la capsule centrale (caps. ctrl.) peut, en s'accroissant, atteindre un diamètre tel que la coquille primitive ou mème plusieurs coques grillagées concentriques parmi les plus internes, lorsqu’il y en a, passent dans sa cavité et, par suite de ce phénomène, on constate que, dans une mème espèce, les individus àgés ont une ou plusieurs coques concentriques intracapsulaires, tandis que les jeunes n'en ont point de telles $\left(^{*}\right)$.

(1) Nous appellerons coques les sphères grillagées concentriques, pour les distinguer de la coquille qui comprend l'ensemble des formations squelettiques.

$\left.{ }^{(2}\right)$ Nous laissons de còte, dans cette vue d'ensemble, les squelettes des teanthaires formées d'aiguilles radiaires d'acanthine (substance organique) qui se forment au centre rle la capsule et s'accroissent en direction centrifuge a partir de cette origine. Ciest un cas spécial qui sera étudié avec ce groupe. 
La sous-classe des Radiolaires se divise en quatre ordres caractérisés principalement par la grosseur, le nombre et la disposition des orifices de leur capsule centrale :

$I^{\circ}$ PERIPYLIDA, à capsule centrale percée de pores très nombreux et très fins répartis sur toute sa surface;

20 ACTIPYLID.A ou ACANTHIDA, à capsule percée de pores fins, nombreux, disposés symétriquement suivant des dessins réguliers. Leur squelette est composé d'aiguilles radiaires formées d'une substance organique, l'acanthine, et partant du centre de la capsule;

$3^{\circ}$ MoNOPYIDA, à capsule percée d'une seule ouverture très large fermée par un clapet percé de fins pores;

$4^{\circ} C^{\prime} A N O P Y L I D A$ ou PHEODIDA, à capsule percée d'une seule ouverture principale, pas très grande, prolongée en tube et accompagnée ou non de deux autres ouvertures secondaires plus petites, symétriquement placées. Dans leur gelée extracapsulaire se trouve une masse spéciale, forlement pigmentée appelée phrodium (').

$$
\text { Ler ORDRE }
$$

\section{PÉRIPYLAIRES. - PERIPYLIII.I}

[Peripylea (Ilertwig); - Peripylaria (Hïckel); - Splaeldara (Hiickel)]

Dans cet ordre, et dans lui seul, nous distinguerons, avant de passer aux sous-ordres, deux groupes les

MoNoCYTTAREA contenant les formes isolées et les

PoLYCYTTAREA contenant les formes coloniales ( $\left.{ }^{\boldsymbol{z}}\right)$.

$$
\text { 1er GROUPE }
$$

\section{MONOCYTTAIRES. - MONOCYTTAREA}

\section{[MOYOCYTTARIA (Häckel 1862 non 1887)]}

\section{TYPE MORPHOLOGIQৃUE}

(FIG. 256 ET 257)

Conforme de tous points au type morphologique général précédemment décrit, il n'a de particulier que le mode de perforation de sa capsule centrale. Au lieu des pores

(1) Sauf en ce qui concerne les Polycyttaires, nous avons suivi dans ce qui suit la classification créée par IIäckel dans sa magistrale monographie du Challenger où il donne une révision complète de toutes les espèces de la classe. Nous ne nous sommes pas interdit cependant des modifications de détail lorsqu'elles nous paraissaient apporter quelque clarti ou (quelque simplification.

(2) Dans les autres ordres, il n'y a pas de formes coloniales, aussi cette sublivision intercalaire ne se rencontrera pas. [IAAckEL] avait lui-même élabli celle distinction. Il a préféré ensuite répartir les Polycyltaires dans les groupes de Péripylaires 
assez gros et modérément nombreux que nous avions attribués à celui-ci,

Fig. 257

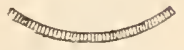

IIonocyttaire

(Type morphologique) Paroi de la capsule.

(Sch.).

il a une capsule uniformément criblée de pores très nombreux et très fins (fig. 2:7). Par rapport aux Polycyttaires, il est caractérisé par son individualité monozoïque, c'est-à-dire par l'absence de colonie.

D’après la présence oul'ahsence desquelette et, dans ce dernier cas, l'après sa forme et la constitution physique de ce squelette, les Monocyttaires se divisent en six sous-ordres:

TIIALASSICOLLIDA, sans squelette;

THALASSOSPHARID E. à squelette formé de spicules indépendants; SPIIEROIDE, à coquille en forme de sphère;

PRUNo IDE, à coquille en forme d'ellipsoüle;

DISCO ID.E, à coquille discoüle ou lenticulaire;

LARCOIDE, à coquille en forme d'ellipsoïde aplati parallèlement au grand axe.

$$
\text { Ler SOLS-ORDRE }
$$

$$
\text { THALASEICOLLIOES. - TIIALASSICOLLIDAE }
$$

\section{[TIILALASSICOLLIDA (Iläckel)]}

\section{TYPE MORPHOLOGIQUE}

(FIG. 258)

Tous les autres sous-orlres de ce groupe étant composés de formes pourvues d'un squelette, celui-ci est déjà caractérisé suffisamment par l’alisence de squelette, en quoi il est conforme au type morphologique rles Péripylaires monocytlaires. Son type morphologique est représenté exactement par le genre Thalassicolla.

\section{GENRES}

Thalassicolla (Huxley) (fig. 2:3) se caraclérise génériquement par la constitution vacuolaire de sa gelée. La gelée est en effet creusée de grandes racuoles (vac.) si nombreuses, si serrées, qu'elles se touchent presque et réduisent cette substance aux minces lames THAlssscotLiD.E (Type morphologique) constituant les parois des vacuoles. Celles-ei sont remplies d'un liquide hyalin Atteint $5 \mathrm{~mm}$ de diamètre (').

auxquels ils se rattachent par la constitution

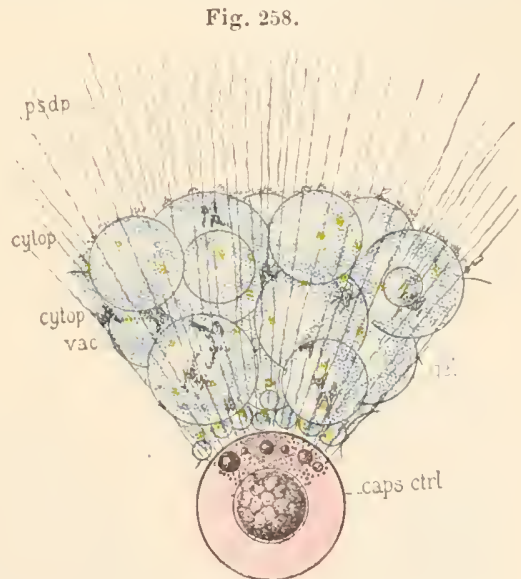

(Thalassicolla). Un secteur de l'ensemble de l'animal (Sch.).

caps. etrl., eapsule centrale contenant le noyau et des gouttes de graisse; cytop., cytoplasma; gel., gelatine; N., noyau; psdp., pseudopodes; vac., vicuoles.

de leur squelette. Mais Brandr [85] trouve, avec raison, que ces formes coloniales ont de grandes affinitès et préfère les laisser réunies. Nous nous rallions à son opinion.

(1) L’animal est donc très gros, sa capsule centrale a plus de $0 \mathrm{~mm}$ ¿̈ et son noyau prés 
2 Sous-OrdRE

THALASSOSPHÉRIDES. - THALASSOSPH.ERID.E

[THALASSOPIIERIDA (Iläckel)]

\section{TYPE MORPHOLOGIQUE}

(FIG. 259)

C'est notre type morphologique de Thalassicollide avec, en plus, des spicules siliceux $(s q$.$) , épars dans sa$ gelée, nullement soudés entre eux, et par suite ne constituant pas une coquille qui se tienne après la mort de l'animal.

\section{GENR:ES}

Thalassosphæra (Hïckel) est la réalisalion de ce type $(0,1$ à 0,2$)$ (")

de $0 \mathrm{~mm}$ 1. Cela a permis à Verwors les expériences curieuses auxquelles nous avons fait allusion (p.174). Il a pu réaliser l'èchange des capsules centrales entre deux individus et constater que la capsule peut régénérer le reste du corps tandis que si on enlève le noyau, ni celui-ci, ni le reste du corps ne peuvent continuer à vivre.

Fig. 259.

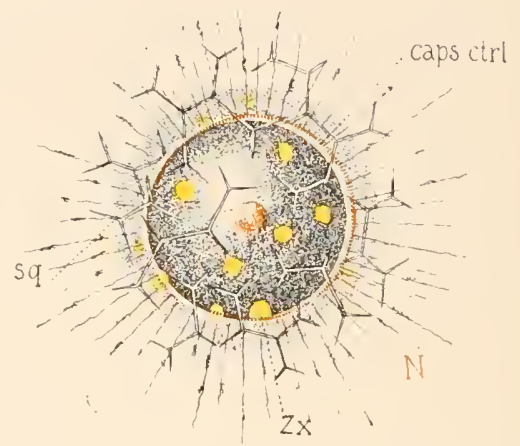

THALASSOSPHARIDE

(Type morphologique) (Sch.).

caps. ctrl., capsule centrale; N., noyau;

sq., squeletle; Zx., Zooxanthelles.

Genres voisins :

Thalassophysa (IIäckel) a un noyau branchu ou couvert de gibbosités ( 1 à $4 \mathrm{~mm}$ );

Thalassolampe (Häckel) (fig. 260) est un Thalassicolla sans vacuoles dans la gelée, mais a nombreuses vacuoles intracapsulaires $(0,5)$;

Thalassopila (Häckel) (fig. 261 est un Thalassolampe à noyau de Thalassophysa (引̈mm); Actissa [Iäckel) (fig. 262 n'a d'alvéoles nulle part. Il réalise à la fois notre type morphologique général et la forme la plus simple et la plus primitive de Radiolaire $(2 \mathrm{~mm})$.

Fig. 260.

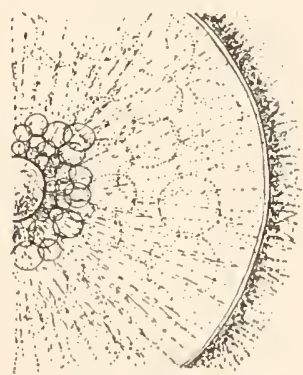

Thalassolampe

(im. lläckel).
Fig. 261 .

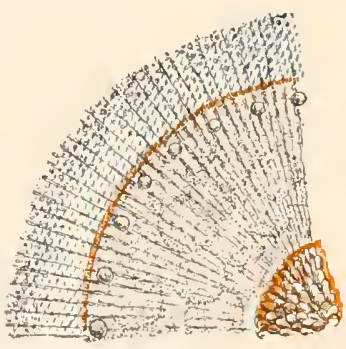

Thalassopila

(im. Häckel).
Fig. 262.

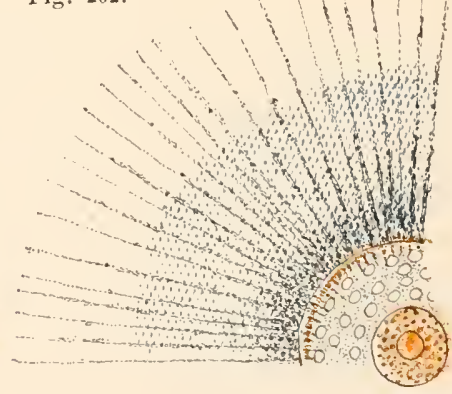

Actissa

(im. Häckel).

Ces genres forment la famille des ThuLAsstcoltix.e [Thulassicollida (Häckel)].

(1) Genles voisins :

Thalassoxanthium (IÏ̈ckel) est un Thulussospliere à spieules branchus $(0,2$; 
$3^{\text {e SOUS-ORDRE }}$

SPHÉROÏDES. - SPHAROIDA

[SPILEROIDEA (Häckel)]

\section{TYPE MORPHOLOGIQUE}

(FIG. 263 A 265)

C'est notre type morphologique général avec, en plus, une coquille formée d'une simple sphère siliceuse percée de trous. Celle-ci est évidemment une coquille primaire déposée à la surface de la gelée.

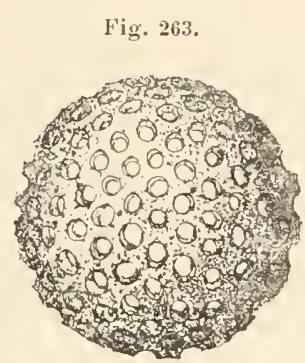

SPIIEROID.E

(Type morphologique) (Sch.).
Fig. 264.

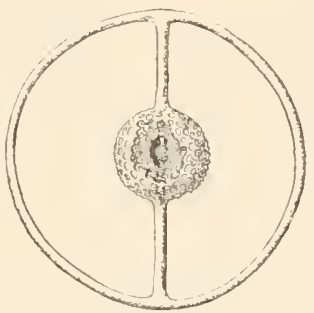

SPII.EROII).E.

Autre forme générale (Sch.).
Fig. 265 .

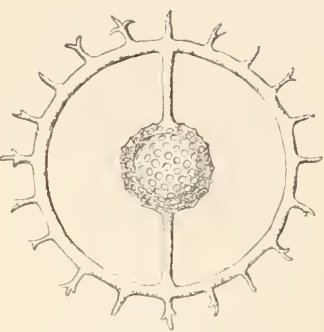

SPII.EROII.E.

Autre forme générale (Sch.).

\section{GENRES}

Cenosphæra (Ehrenherg) est la réalisation de ce lype 0,1 à 0,3$\rangle$.

Il existe une longue série de genres qui présentent les mèmes caractères, mais chez lesquels des coques grillagées secondaires s’ajoutent à la primaire et sont situées concentriquement en dehors d'elle (').

Physematium (Meyen les a simples, mais a re larges alvéoles intracapsulaires (6 à 12); Thalassoplancta (Itäcliel), de même (fig. 266), mais ses alvéoles, sont extracapsulaires (3 à 4);

Lampoxanthium (IIäckel) est pareil au précédent, mais a des spicules branchus ( 1 à $2 \mathrm{~mm}$;

Ces genres forment la famille des TIILILISSOSPIIERIN.E [Thalassosphierida (Häckel]);

Fig. 266.

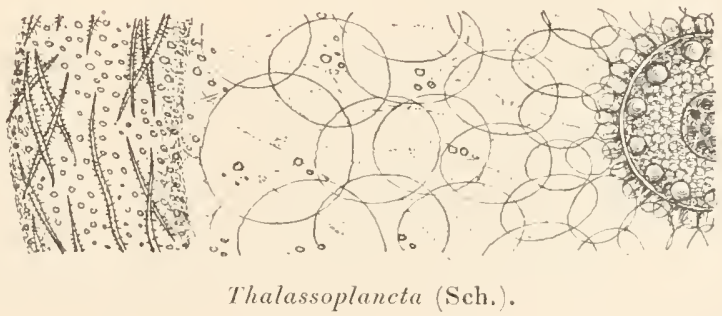

Stigmosphæra (Häcliel) possède, en plus, des spicules radiaires allant jusqu'au centre $(0,15$; 
Xyphosphæra (Häckel) (fig. 267) est semblable au précédent, mais s'en distingue par deux longues épines radiaires égales qui s'ajoutent à la coquille grillagée et sont disposées sur les prolongements d'un même diamètre $(0,1$ à 0,2$)$.

Il existe toute une série de genres qui diffèrent de celui-ci par des coques concentriques grillagées additionnelles qui s'ajoutent à la coque primitive et qui représentent par conséquent, avec le caractère de la double épine en plus, la série de genres dépendant de Cenosphara (').

Ethmosphæra (Häckel) (fig. 268), a les trous de la coquille prolongés en petits tubes saillants en dehors $(0,1$ à 0,2$)$;

Sethosphæra (Häckel) a les mêmes tubes, mais prolongés en dedans $(0,15$ à 0,2$)$;

Carposphæra (Häckel) a deux coques concentrique réunies par des trabécules radiaires et dont l'interne devient intracapsulaire $(0,1$ à 0.2$)$;

Liosphæra (Häckel) a aussi deux coques, mais l'une et l'autre extracapsulaires $(0,15$

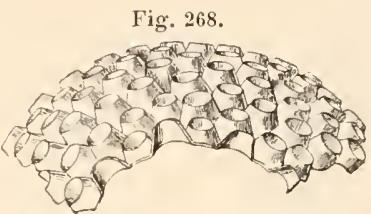

Ethmosphæra (im. Häckel).
Fig. 267.

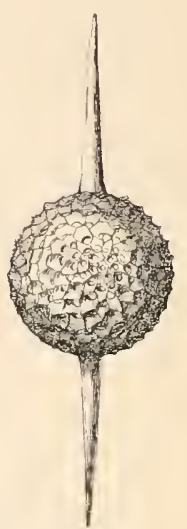

Xyphosphiera

(X. Ienus)

(im. Häckel). iो 0,3$)$;

Thecosphæra (Häckel) a trois coques concentriques dont deux intracapsulaires $(0,1$ à 0.4 ;

Rhodosphæra (Häckel) en a trois aussi, mais dont deux sont extracapsulaires $(0,2)$;

Cromyosphæra (Häckel) en a quatre dont deux en dedans et deux en delıors de la capsule $(0,1$ à 0,2$)$;

Caryosphæra (Häckel), en a cinq ou plus, deux en dedans et trois ou plus en dehor's $\{0,1$ à 0,2 !;

Spongodictyon (Häckel) en a deux seulement, l'une et l'autre intracapsulaires mais, en dehors de cela se trouve une sorte de tissu spongieux formé de tigelles entrecroisées en tous sens $(0,2$ à 1$)$;

Spongoplegma (Häckel) est semblable, mais n'a qu'une coque grillagée au lieu de deux $(0,2)$;

Plegmosphæra (Häckel) n'en a plus du tout. Il n'y a qu'une sphère de tissu spongieux avec une cavité au centre $(0,2$ à 1$)$;

Styptosphæra (Häckel) enfin, est dans le mème cas, mais la sphère est envahie jusıu'au centre par ce tissu spongieux 0,2 a 0,4 .

Ces genres forment la famille des Liospukzw. [Liospherida (Häcliel)].

(1) Voici ees genres :

Xiphostylus (Häckel) a ses denx épines inégales ou de forme différente $(0,07$ à 0,14$)$;

Saturnalis (lläckel) a ses deux épines réunies par un anneau $(0,07$ à 0,09$)$;

Stylosphæra (Ehrenherg) est un Iyphosphiera à deux coques grillagées $(0,1$ à 0,2$)$;

Sphærostylus (11äickel est un Typhostylus à deux sphères $(0,08$ à 0,14$)$;

Saturnulus (Häckel) est un Saturnalis à deux sphères $(0,08$ à 0,1$)$;

Amphisphæra (IIäckel) a trois sphères et les épines d’un Typhosphiera $(0,11$ à 0,2);

Amphistylus (IIäckel) a trois sphères et les épines d'un .ryphostylus $(0,11$ i 0,10$)$;

Saturninus (Häeliel) est un Saturnulus à trois sphères $(0,12)$;

Stylocromyum (Häckel) est un .ryphosphrra a quatre sphères $(0,16$ à 0,28$)$;

Cromyostylus (Häckel) est un Typhostylus a quatre sphères $(0,24)$;

Caryostylus (Häckel) a ciny splières ou plus el les épines comme Xyphostylus (0,3); 
Staurosphæra (Hïckel) (fig. 269) est encore tout semblable à Cenosphæra mais, au lieu d'avoir comme Xyphosphara seulement deux épines diamétrales, il en a quatre égales, dis- Fig. 269. posées en croix $(0,08$ à 0,2$)$.

lci de nouveau, la forme se complique par l'adhlition de coques grillagées concentriques à la coquille primitive, et parquelques autres caractères, lans une série de genres dont celui-ci est le chef de file, et qui ont tous commelui les quatre épines en croix (').

Hexastylus (Häckel) (fig. 270) dérive encore de Cenosphara, mais a six

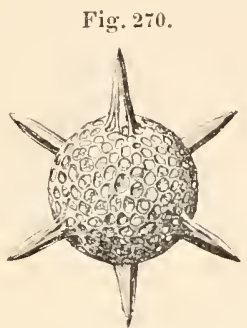

Ilexastylus (im. Hïckel).

Staurosphxra (S. Philippi) (im. Häckel). épines sur les prolongements de trois diamètres perpendiculaires. Les épines sont simples el égales et la coquille se compose d'une simple coque grillagée $(0,03$ à 0,3$)$.

Ici encore, se présente une série de formes dérivées qui se compliquent par le nombre des coques emboitées et les caractères des épines $\left(^{2}\right)$.

Spongostylidium (IIäckel) a la coquille spongieuse d'un spongodyctium avec les deux épines des genres précédents $(0,16)$;

Spongostylus (Häckel) diffère du genre spongoplegma par ces mêmes épines 0,1 à 0,5 ) enfin ;

Spongolonche (IIäckel) a res mêmes épines sur une coquille de styptosphrer $\langle 0,1$ à 0,5$)$.

Ces genres forment la famille des STYLOSPHARTXE [Stylospharida (Häckel)].

(1) Voici ces genres :

Staurostylus (IIäckel) a les épines semblables par paires seulement $(0,15$ à 0,2 '́);

Stylostaurus (Häckel) a une épine plus grande rue les trois autres $(0,1$ à 0,2$)$;

Staurolonche (Häckel) a deux coques et les quatre épines égales, simples $(0,08$ à 0,27$)$; Staurancistra (Häckel) a deux coques et les quatre épines égales, mais branchues $\{0,16\}$; Staurolonchidium (Häckel) a deux coques et les quatre épines égales par paires seulement $(0,12$ à 0,16$)$;

Stauroxyphos (Häckel) a deux coques et une des quatre épines plus' grande que les trois autres $(0,12)$;

Stauracontium (Häckel) a trois coques el les quatre épines simples et égales $(0,01$ à 0,27$)$; Staurocromyum (Häckel) a quatre coques et les quatre ípines simples et égales $(0,25)$; Cromyostaurus (IIäckel) a quatre coques et les quatre épines égales, mais branchues $(0,26)$; Staurocaryum (Häcliel) a cinq coques ou plus et les quatre épines égales $(0,22)$;

Staurodoras (Häckel) a, avec ses quatre épines, une coquille spongieuse jusqu'au centre, sans coques grillagées spéciales $(0,14$ à 0,25$)$.

Ces genres forment la famille des STLtRospIIERINE [Staurosphærida (IIäckel)].

$\left(^{2}\right)$ Voici les genres de cette série :

Hexastylarium (Häckel) a une paire d'épines différente des deux autres $(0,1$ à 0,18$)$; 
Acanthosphæra (Ehrenberg) (fig. 2Z1) dérive toujours du même Cenosphæra, mais en diffère par ses épines multiples, en nombre indéterminé, hérissant toute la coquille. Ici, celle-ci est formée d'une simple coque grillagée mais, comme dans les cas précédents, ce nombre augmente en mème temps que les caractères secondaires varient dans une série de genres qui dérivent de celui-ci $(0,06$ à 0,35$)$ (").

Hexastylidium (Häckel) a les trois paires d'épines différentes $(0,12$ à 0,2$)$;

Hexalonche (IIäckel) a deux coques et les épines simples et égales $(0,1$ à 0,23$)$;

Hexancistra (Häcliel) a deux coques et les épines égales, mais branchues $(0,1$ à 0,17$)$;

Hexaloncharium (Häckel) a deux coques, les épines simples, une paire différente des deux autres $(0,12$ à 0,16$)$;

Hexalonchidium (Häckel) a deux coques, les épines simples, mais les trois paires différentes $(0,12)$;

Hexacontium (Häcliel) a trois coques et les trois paires d'épines simples et égales $(0,1$ à 0,22$)$;

Hexadendron (IIäckel) a trois coques et les trois paires d'épines ègales, mais branchues $\langle 0,12$ à 0,15$\rangle$;

Hexacontarium (IIäckel) a trois coques, paire d'épine différente des deux autres $(0,15$ à 0,16$)$; Hexacromyum (IIäcliel) a quatre coques et ses trois paires d’épines simples el égales $(0,2$ à 0,32$)$; Cubosphæra (Häckel) a cinq coques ou plus et les trois paires d'épines simples et égales $\langle 0,2$; Hexacaryum (Häckel) a cinq coques ou plus et les trois paires d'épines égales, mais branchues $(0,22)$;

Hexadoridium (Häckel) (fig. 272) a deux coques intracapsulaires et, en dehor's, une sphère de tissu spongieux; les trois paires d'épines sont simples et égales $(0,2)$;

Fig. 272.

Hexadoras (IIäckel) est semblable au précedent, mais avec une seule coque $(0,15$ à 0,5$)$;

Cubaxonium (Iläckel) est scmblable au même, mais sans coque grillagée $(0,10$ à 0,20$)$.

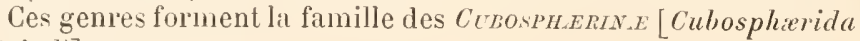
[Häckel)].

(1) Voici ces genres:

Heliosphæra (IIäckel) a ses épines de tailles différentes $(0,09$ à 0,30$)$;

Conosphæra (Häckel) a ses èpines creuses à paroi poreuse $(0,16$ à 0,25$)$;

Coscinomma (Häckel) a, entre ses épines simples, les pores de la coque grillagée prolongés en tubes creux $(0,16$ à $0,2: 3)$;

Cladococcus (J. Müller) a ses épines pourvues de ramifications latérales $(0,05$ à 0,16$)$;

Elaphococcus (Häckel) a ses épines dichotomes $(0,07$ à 0,15$)$;

Haliomma (Elırenberg) a deux coques, une intracapsulaire, l'autre extracapsulaire, et toutes ses épines simples et égales $(0,08$ à 0,42$)$;

Heliosoma lläckel) est semblable au précédent, mais avec des épines de deux sortes $(0,1$ à 0,25$)$; Elatomma (Häckel) est semblable encore au même, mais a ses épines branchues $(0,12$ a 0,2$)$; Leptosphæra (Iläckel) a deux coques, l'une intra, l'autre extracapsulaire $(0,3$ à 0,66 ; 
Sphæropyle (Dreyer) (fig. 273) a une coque grillagée (ou plusieurs (sq, sq') concentriques, réunies par des tigelles radiaires) et munie, en outre, d'un grand orifice ou pylome $(o)$. (Quand il y a plusieurs coques, la dernière seule en est munie) $(0,7$ à 0,24$)\left({ }^{1}\right)$.

Diplosphæra (IIäckel) est comme le précėdent, mais porte, en outre, des épines accessoires sur la coque interne $(0,36$ á 0,6 ;

Drimosphæra (Iläckel) de même, mais ses épines accessoires sont externes $(0,32$ à 0,4$)$.

Artrosphæra (Häckel), avec ses épines accessoires internes et spharopyle(im. Dreyer). externes, combine les deux genres précédents $(0,32$ à 0,7$)$;

Actinomma (IIäckel) a trois corques concentriques et toutes ses épines égales $\langle 0,08$ à 0,4$\rangle$;

Echinomma (Häckel) a aussi trois coques, mais possède des épines accessoires plus petites entre ses épines principales igales $\{0,09$ à 0,22$)$;

Pityomma (Häckel) est un Actinomma, à épines fourchues ou branchues $(0,1$ a a 0,28$)$;

Cromyomma (Iläckel), a quatre coques et ses épines simples et égales $(0,09$ a 0,22$)$;

Cromyechinus (Häckel) a quatre coques et de petites épines arcessoires entre les principales $(0,12$ à $0,2 \partial)$;

Cromyodrymus (Häckel) a quatre coques et ses épines fourchues ou branchues $(0,16$ à 0,22$)$;

Caryomma (Häckel) a cinq coques ou plus, deux intracapsulaires et trois ou plus extracapsulaires $(0,2 \partial$ à 0,3$\rangle$;

Arachnopila (Häckel) a toutes ses coques extracapsulaires d'un tissu arachnoïdien, à mailles triangulaires, sans tigelles entre les coques $(0,08$ à 0,15$)$;

A rachnopegma (IIäckel) est semblable, mais a des tigelles entre ses coques $(0,25$ à 0,28$)$;

Arachnosphæra (Häckel) est semblable encore, mais les mailles de ses coques sont polygonales et ses tigelles sont branchues $(0,1$ à 0,3$)$;

Spongosphæra (Etrenberg) a deux coques grillagées intracapsulaires et, en dehor's, une enveloppe sphérique de tissu spongieux partant de la coque externe $(0,2$ à 0,6$)$;

Rhizosphæra (Häckel) est semblable, mais a son enveloppe spongieuse séparée de la coque grillagée voisine $(0,25$ i 0,3$)$;

Spongopila (IIäckel) est comme spongosphrera, mais n'a qu'une coque grillagée intracapsulaire $(0,3$ à 0,6$)$;

Rhizoplegma (IÏ̈ckel) offre la même diffèrence par rapport à Rhizosphǐra $(0,2$ à 0,7$)$;

Lychnosphæra (Häckel) est comme le prẻcédent, mais a, en outre, des épines accessoires intracapsulaires $(0,6)$;

Centrocubus (Häckel) est un Spongopila dont la coque grillagée serait cubique $(0,6$ à 0,9$)$; Octodendron (IÏ̈ckel) offre la même variation par rapport à Thizoplegma $(0,2$ à 1,4); Spongiomma (Ilïckel) n'a pas de coque grillagée et est réduit à sa coquille spongieuse s’ètendant jusqu'au centre; ses épines sont simples $(0,16$ à 0,4$\rangle$;

Spongodrymus (Iläckel) est semblable, mais a ses épines branclıues 0,09 à 0,3$)$;

Spongechinus (Häckel) a ses épines simples, mais son tissu spongieux réserve une cavité centrale 0,2 à 0,50$)$;

Spongothamnus (Häckel) est dans le même cas, mais a ses épines branchues $(0,3$ à 0,4$)$.

Ces genres forment la famille des ARTRosPHeRA [Artrosphierida (Häckel)].

(') Dreyer fait de ce genre une famille des SPHEROPYLYXE [Sphixropylida (Dreyer)], dans laquelle il place aussi le genre Prunopyle (Dreyer) qui, selon le critérium de IIäckel, appartient au sous-ordre suivant, et le genre Stomatosphxra (Driesch) où il réunit, comme espèces, des formes appartenant à ces deux sous-ordres et qui est caractérisé par deux pylomes diamétralement opposés. 
4 e Sous-Ordre

PRUNOÏDES - PRUNOID.E

[PRUNOIDEA (Häckel)]

TYPE MORPHOLOGIQUE

(FIG. 274)

Revenons à notre type morphologique général et faisons-lui subir celle simple modification que la capsule centrale, au lieu l'être sphérique, prenne la forme d'un ellipsoïle, el nous aurons le type de ce nouveau groupe. C'est là un caractère plus important qu'il ne semblerait au premier abord, car il indique un accroissement inégal, plus rapide suivant un axeque suivant les autres. Cette forme ellipsoïde ne se limite pas d'ailleurs ì la capsule centrale, elle s'étend à la gelée et par suite à la coquille qui se moule sur elle. C'est là le caractère essentiel du groupe, mais nous verrons comment, par des modifications accessoires, il donne naissance i une grande variété de formes.

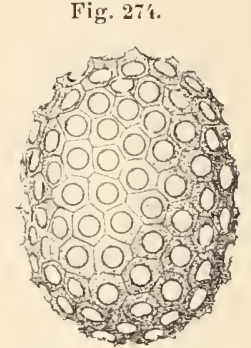

PRLYOID.E.

Type morphologique (Seh.).

\section{GENRES}

Cenellipsis (Häckel) est notre type morphologique sans autre morlification $(0,12$ à 0,36$)\left({ }^{*}\right)\left({ }^{2}\right)$.

Druppula (Häcliel) (fig. 273) est un Cenellipsis pourvu d'une seconde coque de mème forme. La plus interne est intracapsulaire $(0,07$ is 0,22$)\left({ }^{2}\right)$.

(1) Genres voisins:

Axellipsis (Iäckel) possède en plus une tigelle qui traverse la coquille suivant son axe $(0,1$ aे 0,12$)$;

Ellipsidium (IÏckel) est un Cenellipsis à coquille garnie d'epines rallaires

Fig. 275 .

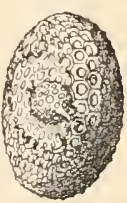

Druppula (im. Hïckel). $(0,12$ à 0,16$)$;

Ellipsoxiphus (Dunikovsky) a sa coruille comme Cenellipsis, mais surmontée a rhatue pôle a'une forte épine pleine $(0,08$ à 0,16$)$;

Axoprunum (Iäckel) ressemble au précédent, mais a dans sa coquille deux tigelles en croix 0,14$)$;

Ellipsostylus (Iäckel) ressemble au mème, mais a ses deux épines inégales 0,0 ว ì 0,18 ; Lithomespilus (Iäckel) a une de ses épines polaires remplacée par un petit buisson d'épines 0,08 à 0,12 ;

Lithapium (IIïckel) n’a qu'une épine ì un seul pôle $(0,12$ i 0,$1 ;$;

Pipetella (llïckel) a ses épines polaires creuses, à parois fenestrées $(0,15$ à 0,2 .

Ces genres forment la famille des ELLIPSTYE [Ellipsida Häckel)].

${ }_{(2)}^{2}$ Les genres voisins suivants ont de même une seconde cơtue ou même plusieurs:

Druppocarpus (Häcliel) est un Druppula à surface épinense 0,1 ì 0,17;

Prunulum (IIäckel) a trois coques dont deux intracapsulaires, sa surfare est lisse $(0,1$ ì 0,17$)$;

$\left({ }^{\bullet}\right)$ Ces dimensions sont celles du grand axe de la eoquille. 
Spongoxiphus (Häckel) (fig. 276) a deux coques grillagées intracapsulaires el, en dehors, une couche de tissu spongieux; il possède en outre les deux épines polaires $(0,18$ à 0,26$)(')$.

Prunopyle (Dreyer) (fig. 277) a soit une seule coque grillagée, soit plusicurs concentriques, mais possède une large ouverture ou pylome percée au pôle inférieur. (Quand il y a plusieurs coques, la dernière seule possèrle un pylome) $(0,1$ a 0,22$\rangle\left(^{2}\right)$.

Artiscus (Hiickel) (fig. 278) peut ètre défini un Cenellipsis dont la coquille est divisée par un étranglement équatorial en drux moitiés superposées $(0,11$ à 0,15$)\left({ }^{3}\right)$.

Prunocarpus IIäckel) est un Prunulum à surface épineuse $(0,1$ ' 1 a 0,18$)$;

Cromyodruppa IIäckel est lisse et a quatre coques ou plus, dont dleux intracapsulaires $0, \underline{2}$ à 0,3 ä;

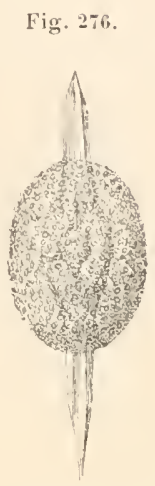

Spongoxiphus

(im. Ilickel).
Fig. 277.

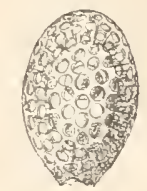

Prunopyle (iim. Dreyer).

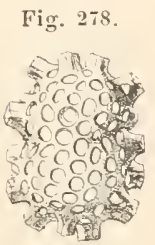

Artiscus

(im Häckel).

Cromyocarpus Häckel) est le prẻé̉lent avec une surface épineuse 0,2 ;

Lithatractus (IIäckel) a leux coques seulement, lont une inlracapsulaire, mais possède deux épines polaires égales $\{0,07$ à 0.17 ;

Druppatractus (Itäekel) ressemble au précídent, mais avee des épines polaires inégales $(0,07$ à 0,18$)$;

Stylatractus (IIäckel) a trois corues dont deux intracapsulaires et ileux épines polaires égales $(0,12$ à 0,3$)$;

Xiphatractus (11äckel) ressemble au précédent, mais avee ses ipines inégales 0,01 à 0,15 ); Cromyatractus (IIäckel) a quatre coques ou plus dont deux intraeapsulaires el deux épines polaires igales $(0,18$ à 0,37$)$;

Pipetta Häckel a deux coques dont une intracajrulaire et deux épines polaires, mais creuses à parois fenestrées 0,13 i 0,16$\}$;

Pipettaria (Iläckel)est semblable, mais a trois coques dont deux intracapsulaires 0,12 à 0,25 ;

Ces genres forment la famille des DretPetrIsE [Druppulida (IÏ̈ckel)].

(1) Genres voisins :

Spongoliva lläckel) est semblable, mais n'a pas les deux épines polaires 0,18 i 0,3$)$;

Spongatractus Häkel n’a qu’une coque et les deux épines 0,16 a 0,25 ;

Spongodruppa (IÏ̈ckel) n'a aussi qu'une corpue, mais pas d'épines $(0,12$ a 0,29$)$;

Spongoprunum (IIäckel) n'a plus que sa coquille spongieuse s'étendant jusqu'au centre, et a les deux épines $0,-2)$;

Spongocore (Ilickel) n’a pas d'épines, mais a un revètement superficiel grillagé (0.2 à 0 , î; ; Spongurus lläckel n’a ni revèlement ni épine $(0,11$ a 1$), 3$;

Spongellipsis Häckel n’a pas non plus d'épines et sa coquille spongieuse est vide au centre 0,2 à 0,36 .

Ces genres forment la fantil]e des SPOAGrRINE [Sponguridu Häckel)].

(2) Ce genre forme pour Dreyer la famille SPIL.EROPYLIV.E [Spharopylidu (Dreyer)] où il place aussi le genre spheropyle qui, pour nous, apparlient au sous-ordre précédent et le genre Stomatosphiera Dreyer) où il réunit comme espèces des formes appartenant à l'un et à l'autre sous-ordre et qui est caractèrisé par deux pylomes, un à chaque pôle.

(3) Genres voisins:

Stylartus (IIäckel) s'en dislingue par une forte épine à chaque pòle $(0,14$ à 0,18$)$. 
Panartus (Häckel) (fig. 279) peut être défini un Cenellipsis à coquille livisée en quatre parties superposées par trois étranglements dont un équatorial et deux parallèles à l'équateur. La capsule centrale est divisée de la même manière et il y a trois coques dont deux intracapsulaires $(0,18$ a 0,34 ) (').

Ommatocampe (Ehrenberg) (fig. 280) dérive de la même manière de Cenellipsis par iles étranglements transversaux de la coquille, mais ceux-ci sont au nombre de cinq ou plus et déterminent six chambres ou plus superposées $\left(s q^{\prime}, s q^{\prime \prime}, s q^{\prime \prime \prime}\right)$. Il y a leux coques intracapsulaires et une extracapsulaire et point d'épines ou de tubes polaires $(0,15$ à 0,30$)\left({ }^{2}\right)$.

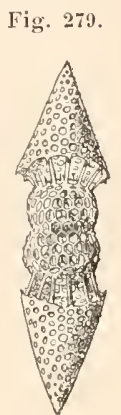

Panartus

(im. Häckel).
Fig. 280 .

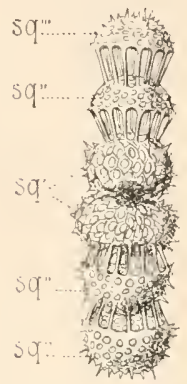

Ommatocampe (im. Häckel).

Cannartus (IIäckel) a les mêmes épines, mais creuses et à parois fenestrées $(0,11$ à 0,16$)$.

Ces trois genres forment la famille des A ITTLSCL.E [Artiscida (Iäckel)].

Cyphanta (Häcliel) (fig. 281) est un Arliscus à deux coques grillagées concentriques dont l'une intracapsulaire $(0,10$ à 0,15$)$;

Cyphonium (Häckel) est semblable, mais a trois coques dont deux intracapsulaires $(0,1$ ì 0,15$)$;

Cypassis Iäckel) en a quatre, deux intra, deux extracapsulaires 0,2 a 0,21 ;

Cyphocolpus (Häckel) en a cinq dont deux intracapsulaires $\langle 0,26$ à 0,3 ;

Cyphinus Ifäckel n'en a que deux, une intra et une extracapsulaire, mais possède deux épines ou faisceaux d'épines polaires 0,18 à 0,20 );

Cyphinidium (Häekel) est comme le précédent, mais a trois coques dont deux intracapsulaires $(0,12$ à 0,14$)$;

Cannartisous (Häckel) est un Cyphimus dont les deux épines sont creuses

Fig. 281.

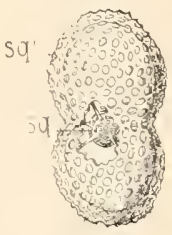

ryphanta

(im. Häckel). et à paroi fenestrée $(0,14$ à 0,17$)$;

Cannartidium (Häckel) est semblable au précédent, mais a trois corues dont deux intracapsulaires $(0,11$ à 0,15$)$;

Ces genres forment la famille des CYPIINTIx [Cyphinida (IIäckel)].

(1) Ces caractères se retrouvent dans la série de genres ci-dessous.

Peripanartus (Häckel) a deux ou trois coques extracapsulaires au lieu d'une $(0,25$ a 0,30$)$;

Panicium Häcliel est un Panartus avec deux épines ou groupes d'épines polaires 0,2 à 0,22 ;

Pepipanicium IIäckel) est un Peripanartus avec deux épines ou groupes d’épines polaires $(0,25$ i 0,27$)$;

Panarium (Hücliel) est un Panicium dont les deux épines polaires sont creuses et à parois fenestrées 0,16 à 0,26$)$;

Peripanarium (Häckel) est un Peripanicium dont les épines polaires ont le mème caractère que dans le précédent $(0,23$ à 0,28$)$.

Ces genres forment la famille des P.1.tRTINE [Panartida (Häckel)].

(2) Cette annulation et ces deux coques intracapsulaires se retrouvent dans les genres ci-dessous :

Ommatartus (IIäckel) semblable au précédent, mais avee deux tubes polaires fenestrés $0,2$ ' a 0,28$)$;

Desmocampe Häckel) est un Ommatocampe à quatre coques dont deux intracapsulaires $(0,16$ à 0,27$)$; 
כe Sous-Ordre

\section{DISCOÏDES. - DISCOID.E \\ [IISCOIDEA (Häckel)]}

\section{TYPE MORPHOLOGIQQUE}

(FIG. 282)

Revenons encore au type morphologique général des Radiolaires, et

Fig. 282.

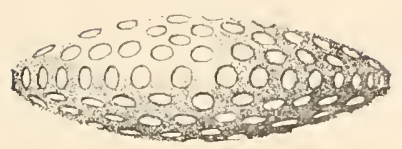

DISCOLD.E supposons qu'il y ait, iri comme chez les Prunoidx, un axe suivant lequel l'accroissement soit différent de celui des autres axes. Chez les Prunoïles, cet axe imprair était allongé et il en résultait un ellipsoïlle. Ici, il est raccourci et il en résulte une lentille ou un disque. Aussi la capsule centrale et la coquille grillagée sont très plates, discoüdes ou lenticulaires.

\section{GENRES}

Cenodiscus (Hïckel) est la simple représentation de ce type 0,15 à 0,20$)$ ('). Sethodiscus (Hïckel) est un Cenodiscus muni, en dedans de sa coque lenticulaire qui est extracapsulaire, d'une coque grillagée intracajsulaire sphérique, reliée à l'autre par des tiggelles radiaires $(0,1$ ì 0,26$)\left({ }^{*}\right)$.

Desmartus (Häckel) est un Ommatarlus i quatre coques dont deux intracapsulaires $(0,23$ i 0,25 ;

Zygocampe (HäckeI) est un Ommatocumpe à cinq chambres dont deux intracapsulaires $(0,17$ à 0,25$)$

Zygartus (Häckel) est un Ommatartus ì cinı chambres dont deux intracapsulaires $(0,25)$.

Ces genres forment la fanille des ZyGatRTIX [Zygartida (Hackel)].

(1) Genres voisins :

Zonodiscus (Häckel) est muni en outre d'une ceinture équatoriale siliceuse solide $(0,25)$;

Stylodiscus Häckel) a deux épines équatoriales raliales, diamétralement opposées $(0,15$ à 0,25$)$; Theodiscus (Häckel) a trois épines équidistantes au lieu de deux $(0,06$ à 0,12$)$;

Crucidiscus (Häckel) en a quatre, suivant deux diamètres perpendiculaires $(0,12$ a 0,20$)$;

Trochodiscus (IIäckel) en a toute une ceinture (10 à 20) plus ou moins irrégulièrement disposés $(0,15$ à 0,25$)$.

Cies genres forment Ia famille des CENODISCIxE [Cenodiscida (Häckel)].

() Genres voisins:

Periphæna (Ehrenberg) a, en outre, une ceinture équatoriale hyaline $(0,2$ à $0,20 \%)$;

Sethostylus (Häckel) porte, à l'équateur du disque, deux épines radiales diamétralement opposées $(0,13$ à 0,3$)$;

Triactiscus (Hïckel) en a trois $(0,15$ à 0,16$)$;

Sethostaurus (Häckel) en a quatre $(0,14$ à 0,4$)$;

Distriactis (Häckel) en a $\operatorname{six}(0,12$ à 0,2$)$;

Heliosestruin (IIäcliel) en a huit $(0,11$ a 0,2$)$;

Heliodiscus (Häckel) en a toute une ceinture $(0,12$ à 0,25$)$; 
Lithocyclia (Iläckel) (fig. 283) dérive du précédent par l'addition d'une ceinture équatoriale épaisse livisée par de petites cloisons en chambrettes disposées en zones circulaires concentriques et superposées $(0,13$ à 0,22$)$ ('). Archidiscus (Hïckel) (fig. 284). Supposons un Sethodiscus dont la coque grillagée externe soit franche-

Fig. 284

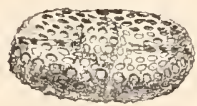

drehidiscus

im. Hiickel), ment discoïde et d'une hauteur ì peine égale au diamètre de la coque grillagée sphérique qu'elle contient. Il en résultera que les deux coque se confondront dans la partie moyenue du dis-

Fig. 283.

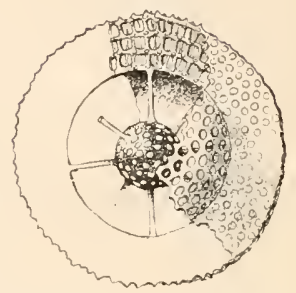

Lithocyclia (im. Häckel). que, et que l'espace entre les deux coques sera réduit i une ceinture circulaire. La capsule centrale renferme la coquille interne et reste en dedans de l'externe. Supposons maintenant cette ceinture circulaire incomplètement divisée en chambretles par des cloisons ou plutôt par de simples tigelles, comme dans la zone équatoriale le Lythocyclia et nous aurons un Archidiscus, forme importante, chef de file

Heliodrymus (Iläckel) de même, mais toutes, ou en partie, branchues $(0,15$ à 0,2$)$;

Phacopyle (Dreyer) est un Sethodiscus avec un pylome 0,12 ;

Phacodiscus (Häckel) (fig. 285) représente un Sethodiscus ì deux

Fig. 285.

coques grillagées sphériques intracapsulaires $(0,2$ à $0,40 \%)$;

Avec la même diffirence :

Perizona (Häckel) représente un Periplizena $(0,2: 3)$;

Phacostylus (Häckel) représente un Selhoslylus 0,12 i 0,1 ;

Phacostaurus (Häckel) représente un Selhostaurus $(0,10$ ì 0,22 ;

Astrosestrum (IIäckel) représente un Heliosestrum 0,12 it 0,2 ;

Astrophacus (Häckel) représente un Holiodiscus $(0,12$ à 0,3$)$.

Ces genres forment la famille des PHAcodsctr.E [Plıacodiscida [IIäckel)].

(1) Genres voisins :

Stylocyclia (Ehrenberg) a, en outre, le fond du disque armé de tleux épines radiales liamé-

tralement opposèes $(0,23$ à $0,20 \hat{)})$;

Trigonocyclia (IIäckel) a trois épines $\left(0,10^{\circ}\right)$;

Staurocyclia (lläckel) en a (quatre en croix 0,2 à $0,2 \overline{7}$ );

Astrocyclia (Iäckel) en a dix ou plus $(0,13$ it 0,25$)$;

Coccodiscus (IIäcliel) est un Lillocyclia à deux coques grillagèes intracapsulaires 0,20 i 0,23 .

Avec la mème différence:

Amphicyclia (IIäckel) représente Slylocyclia $(0,22$ il 0,27 ;

Coccocyclia (IIickel) représente Astrocyclia $(0,3$ it 0,32$)$;

Diplactura (Häckel) revient à Lithocyclia, nuais possède en plus deux bras éruatoriaux, radiaires, diamétralement opposés, creux, divisés en clrambrettes $(0,80$ ì 0,92$)$;

Trigonactura (IIäckel) en a trois équidistants 0,08 à 0,11 ;

Astractura (IIäckel) en a quatre en croix $(0,08$ a 0,11$)$;

Pentactura (IIäckel) en a cing régulièrement placés $\langle 0,09$ ì 0,1 ;

Amphiactura (Iäckel) est un Diplactura dont les bras équatoriaux sont réunis par une

lame à la manière d'un patagium $(0,0 y)$.

La même différence distingue seule:

Stauractura IIäckel) de Astractura 0,09 ì 0,13$)$; 
d'une longue série de genres qui en dérivent par des variations successives $(0,04$ à 0,08$)(')$.

Les plus importantes de ces variations consistent dans le nombre des chambrettes équatoriales qui peuvent former plusieurs anneaux au lieu d'un, et dans le développement d'épines équatoriales ou mème de pro-

Hymenactura (IIäckel) (fig. 286) de Trigonactura $(0,08$ à 0,11; ; Fig. 286.

Echinactura (Häckel) de Pentactura $(0,09$ à 0,12$)$.

Ces genres forment la famille des CoCCODISCINE [Coccodiscida (Häckel)].

(') Axodiscus (Häckel) n'a comme Archidiscus qu'un cercle de chambrettes équatoriales, mais porte des épines équatoriales radiales $\{0,04$ a 0,06 ;

Porodiscus (IIäckel), au contraire, a plusieurs rangées concentriques de ces chambrettes. Les premiers tours peuvent ètre spirıux $(0,1$ à 0,22$)$.

Tous les suivants sont dans le mème cas; ils en ont trois à six rangées en général:

Perichlamydium (Ehrenberg) est muni, en outre, d'une mince ceinture équatoriale poreuse 0,11 iे 0,13 ;

Ommatodiscus (Stöhr) est percé à l'équateur d’une large

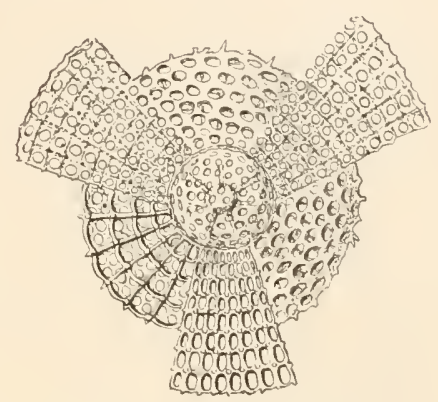

Iymenactura (im. Hackel). ouverture marginale entourée diune couronne dirpines 0,15 i 0,18 ;

Stomatodiscus (Häckel) en a deux diamétralement opposies 0,12 à 0,18; ;

Xiphodictya (Häckel) a le hord équatorial entier, continu, mais muni de leux épines équatoriales $(0,14$ à 0,17$)$;

Tripodictya(Iläckel) est de même, mais a trois épines cipuillistantes $(0,11$ ì 0,2$)$;

Staurodictya (Iläckel) en a quatre en croix $(0,1$ à 0,3$)$;

Stylodictya (Ehrenberg) en a cinq ou plus, irrégulièrement disposées $(0,11$ à $0,2: 3)$;

Stylochlamydium (IIäckel) est de mème, mais a, en plus, une mince ceinture équatoriale poreuse $(0,1$ i 0,2$)$;

Amphibrachium (IIäckel) est prolongé au bord íquatorial du disque en deux bras radiaires, creux, divisés en chambrettes $\{0,22$ à 0,3$\rangle$;

Amphymenium (Häckel) est semblable, avec un, patrgium en plus, c'est-à-dire une laıne, une palmature qui les réunit et qui est elle-même divisée en chambrettes $\{0,1$ à 0,2$)$;

Amphirropalum (Häckel) a ses deux bras ou l'un d'eux au moins) bifurqués et pas de patagium $(0,15$ a 0,18 ;

Amphicraspedum (Häckel)a ce patagium en plus $\{0,18$ i $0,2:)$;

Dictyastrum (Erhenberg) a trois bras simples réguliers $\{0.12$ a 0,23$)$;

Rhopalastrum (Ehrenberg) est de même, mais avec une symètrie bilatérale $(0,1$ a 0,33$)$;

Hynoniastrum (Ehrenberg) représente Duictyastru avec un patagium en plus $(0,02$ a 0,2$)$ et

Euchitonia (IIäckel) represente Rhopalastrum avec ce même patagium en plus $(0,14$ à 0,22$)$;

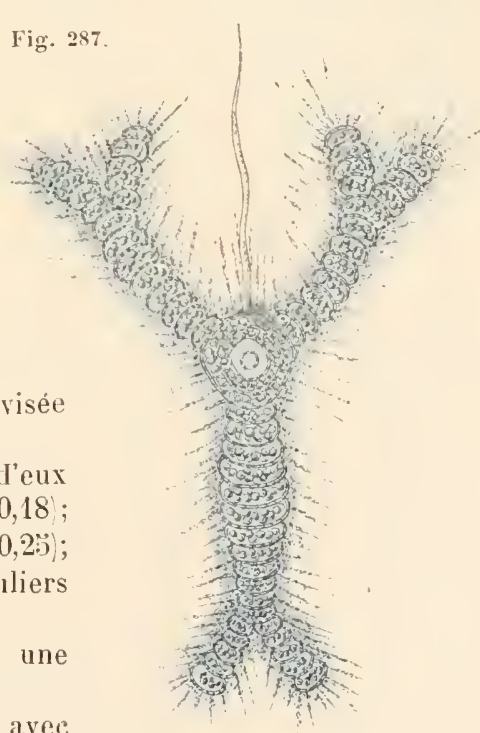

Chitonastrum.

(im. Häckel).

Chitonastrum (Häckel) (fig. 287) a ses trois bras bifurquès 0,16 a 0,24 . 1)ans une espèce 
longements équatoriaux creux et divisés eux-mêmes en chambrettes. Triolena (Häckel) (fig. 2SS) est un Archidiscus dont la ceinture grillagée serait interrompue par trois larges ouvertures latérales intéressant à la fois le plancher, le plafond et le bord équatorial $(0,0$ ' tà 0,06$)$ (') .

Fig. 288.

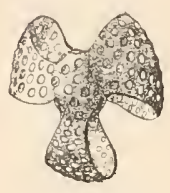

Triolena

(im. IIäickel).

Trigonastrum (Häckel) est de même avec un patagium en plus $(0,13$ à 0,24$)$; Stauralastrum (Häckel) a quatre bras simples équidistants $(0,12$ à 0,8$)$;

Hagiastrum (IJäckel) les a disposés en deux paires délerminant une symétrie bilatérale 0,2 à 0,4 ;

Histiastrum (Ehrenberg) est un Stauralastrum avee un patagium en plus $(0,15$ à $0,20 \%)$; Tesserastrum (IIäckel) est un IIagiastrum avec un palagium aussi en plus $(0,22$ à 0,3 ;

Stephanastrum (Elirenberg) a, avec ses quatre bras, une ceinture patagiale marginale $(0,2$ a 0,25$)$;

Dicranastrum (Häckel) (fig. 289)) a ses t juatre bras fourchus et équidistants $(0,12$ à 0,4$)$;

Myelastrum (Iäekel) a aussi quatre bras fourchus, mais disposès bilatéralement en deux paires $(0,36$ i 0,7 ;

Pentalastrum (Häekel) a cinq bras $(0,14$ à 0,5 ;

Pentrisastrum (Häckel) en a cinq aussi, mais avec un patagium $(0,14$ à 0,18$)$;

Pentophiastrum (Häckel) en a cinq fourchus $(0,25$ à 0,50$)$; Hexalastrum Häckel) en a six simples $(0,2$ à 0,4$)$, et

Hexinastrum IIäckel) en a six aussi, simples également, mais avee un patagium $(0,15)$.

Ces genres forment la famille des PoromISCIN [Porodiscida (Häckel)].

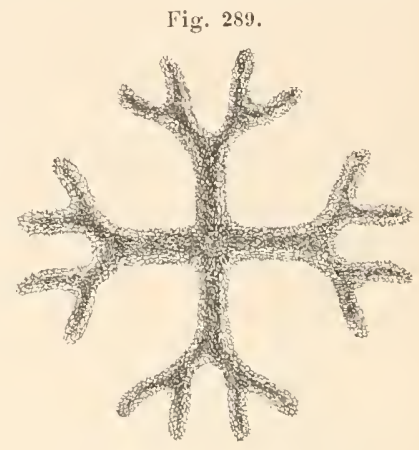

Dicranastrum (im. 1Iäckel).

(1) C'est l'origine d'une nouvelle série de variations caractéristiques d'une nouvelle sèrie de genres dans lesquels ces ouvertures deviennent plus nombreuses et se compliquent de diverses façons:

Triopyle (IIäckel) a ses ouvertures barrées en dehor's par la ceinture équatoriale qui reste complète, en sorte qu'elles sont dédoublées en trois paires intéressant chacune le plancher et le plafond seulement $(0,04$ a 0,06$)$;

Triodiscus (IIäekel) a ces mêmes trois paires d'ouvertures, mais partiellement ou entièrement recouvertes par une lame de tissu grillagé $\left(0,0_{1}^{\prime}\right.$ à 0,05$)$;

Pylolena (IIäckel). Supposons un Triolena qui ait une deuxième enveloppe grillagée qui répète exactement par rapport ì la coquille entière de Triolena la disposition de la ceinture grillagée de celui-ci par rapport à sa corque sphéritue centrale, et nous aurons un Pylolena. Cette partie externe de la coquille est seule extracapsulaire. Il rísulte évidemment de celte disposition quaux trois paires d'ouvertures de Triolena s'ajoutent trois autres paires toules semblables, mais plus grandes et situejes plus en dehors $(0,15$ à 0,20$)$;

Hexapyle (IIäckel) dérive de Triopyle par la mème modification $\langle 0,15$ à 0,20$\rangle$;

Pylodiscus (IIäckel) dérive de la mème manière de Triodiscus $(0,15$ à 1,20;

Discozonium (Häcliel) est un Pylodiscus muni en plus d'une ceinture équatoriale divisée en chambrettes et passant en dehor's des ouvertures $(0,2$ i 0.24$)$;

Discopyle (IIäckel) est un Discozonium chez lequel la ceinlure éłuatoriale en question est percée d'une ouverture borlée d'ẻpines comme celle d'Ommatodiscus $(0,15)$.

Ces genres forment la famille des PrLODISCINE [Iylodiscida IIäckel)]. 
Spongodiscus (Ehrenberg) (fig. 290) dérive de Cenodiscus comme tous les

Fig. 290.

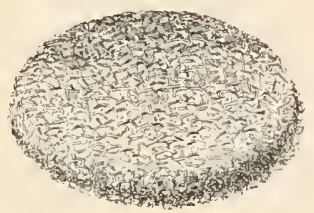

Spongodiscus (im. Häckel). précédents, mais d'une autre manière : ce qui s'ajoute ici à la coquille grillagée centrale, devenue sphérique comme dans les genres précédents, ce qui prend la forme discoüle ou lenticulaire, c'est une masse de tissu spongieux formée, comme toujours, de baguettes siliceuses soudées en un réseau serré el irrégulier (').

\section{$6^{\mathrm{e}}$ Sous-Ordre}

\section{LARCOIDDES. - LARCOID E \\ [LARCOIDEA (Häckel)]}

\section{TYPE MORPHOLOGIQUE}

(FIG. 291)

Dans les deux sous-ordres précédents, l'axe principal du corps était seul différent des deux autres et la forme générale était l'ellipsoïde de révolution ou le disque (cylindre très bas). Ici, les trois axes sont inégaux. L'axe vertical ou principal $(a)$ est le plus long des trois, comme dans les Prunoïdes; et, des deux axes liorizontaux, l'antéro-postérieur $(c)$ est plus court que le transversal (b). En sorte que, quel que soit le plan suivant lequel on coupe le corps, on obtient une ellipse. C'est un ellipsoïde lenticulaire. Et cela est vrai aussi bien pour la capsule centrale que pour la coquille. C'est le corp's tout entier dont l'accroissement est différent suivant les trois directions de l'espace.

Fig. 291.

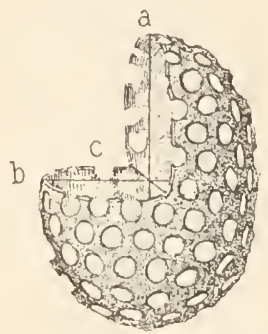

LARCOID.E (Type morphologique) (Sich.)

(i) Voici ces genres :

Spongophacus (IIäckel) possède en outre une ceinture éruatoriale solide ou porense $(0,2)$; Spongolonche (IIäckel) n'a pas cette ceinture, mais deux épines radiales, diamétralement opposées, dans le plan équatorial $(0,16$ i 0,2$)$;

Spongotripus (Häckel) en a trois $(0,12$ i $0,20 j$;

Spongostaurus (HäckeI) en a quatre en croix 0,1 i 0,16$)$;

Stylotrochus (IIäckel) en a cin'l ou plus $(0,12$ a 0,25$)$;

Spongotrochus (Iäckel), outre celles du genre précédent, en a sur les bords des deux faces du disque $(0,16$ à 0,24$)$;

Spongolena (Häckel)a, non des épines, mais deux prolongements de tissu spongieux disposés comme les épines de spongolonche $(0,1$ it 0,2$)$;

Rhopalodictyum (Ehrenberg) a trois de ces prolongements $(0,11$ a 0,30$)$;

Spongasteriscus (Iäckel) en a quatre en croix $(0,1$ a 0,2$)$;

Spongobrachium (IIäckel) représente Spongolenu avec un patagium en plus $(0,12$ à 0,16$)$;

Dictyocoryne (Ehrenberg) offre la même différence par rapport à Rhopalodict $\}$ um $\{0,1$ i 0,2 ;

Spongaster (Ehrenberg) offre la mème encore par rapport it spongusteriscus 0,08 à 0,2 ); 


\section{GENRES}

Cenolarcus (Häckel) (fig. 292) est la réalisation la plus simple de ce type. ll a une capsule centralr lentelliptique et une coquille grillagée extracapsulaire, simple, de mème forme $(0, \mathrm{C5}$ à 0,15$)\left({ }^{1}\right)$.

Larnacilla (Häckel) (fig. 293) peut ètre défini un Cenolarcus dont la coquille lentelliptique extracapsulaire renferme une coquille intracapsulaire semblable à celle d'Archidiscus. Dans la région équatoriale, la coque externe se confond avec la inoyenne de la mème façon que l'interne s'unit à la moyenne suivant le plan sagittal, et de là résulte l'union des trois corques entre elles $(0,06$ à 0,10$)\left({ }^{\boldsymbol{z}}\right)$. Monozonium (Häckel (fig. 29.4) est un Cenolarcus dont la coque extracapsulaire lentelliptique serait largement ouverte en haut et en bas, au point l'être réduite à une large ceinture équatoriale. Celte ceinture contient

Spongopyle (Dreyer) est un Spongodiscus avec une large ouverture ì

Fig. 292 .

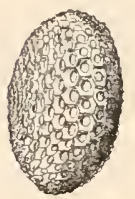

Cenolarcus

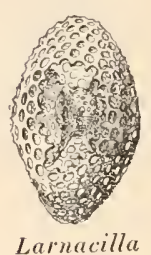

(im. Ḧ̈ickel).

Fig. 29i.

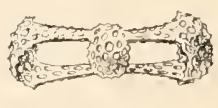

Monosonium

(im. H:ickel). la coquille (pylome) en plus $(0,1$ à 0,4$)$.

Ces genres forment la famille des SPoxgodscrixe [Spongodiscida (Häckel)].

(1) Genres voisins :

Larcarium (IIäckel) est un Cenolarcus muni d'épines ralliaires dans le plan équatorial $(0,09$ à 0,15$)$;

Coccolarcus (Häckel) a deux corjues grillagées, concentriques, semblables, de cette même forme lentelliptique, l'une intracapsulaire, l'autre extracapsulaire, et point d'épines $(0,09$ à 0,17$)$;

Larcidium (Iläckel) a, en outre, des épines radiaires $(0,12$ à 0,18$)$;

Spongolarcus (Häckel) n'a pas de coque grillagée, mais une coquille lentelliptique formée de tissu spongieux et réservant au centre une cavité vide de même forme $(0,12 \text { a } 0,2:)^{\prime}$;

Stypolarcus Häckel est semblable, mais le tissu spongieux envahit jusqu'au centre $(0,2)$.

Ces genres forment la famille des L.1PC.tRT:E [Larcarida (Iläckel)].

Larcopyle (Dreyer) est une Larcarine avec une large ouverture à la coquille (prlome) en plus $(0,13$ a 0,2$)$. Il forme pour Dreyer la famille les Luteroplas [Larcopylida (Dreyer)].

(2) Genres voisins :

Larnacidium (Häckel) est un Laruacilla avec des épines radiales dans le plan équatorial $(0,11$ à 0,14$)$;

Larnacalpis Häckel). Si, dans la coquille de Larnacilla, nous remplaçons la coque sphérique interne par une corpulle complète de Larnacilla, nous aurons une Larnacalpis. La corque la plus externe est seule extracapsulaire $(0,10$ it 0,14$)$;

Larnacantha (Iläckel) ressemble au précédent avec des épines équaloriales radiales en plıs $\{0,11$ i 0,18$\}$;

Larnacoma (Häckel) possède une deuxieme coque extracapsulitie en dehors d'une coquille identique à celle de Larnaralpis. reliée à celle-ci par des tigelles radiaires $(0,16$ à 0,27$)$;

Larnacospongus (II äclieljest un Larmacoma à corpe extracapsulaire spongieuse $\{0,17$ ì 0,22 ; Larnacostupa Iläckel) est un Iarnacospongns à épines équatoriales radiales $(0,15$ à 0,22$)$.

Ces genres forment la famille des I.AR.YArT.E [Lamacida (IIäckel']. 
une coque grillagée sphérique intracapsulaire qui lui est soudée le long de la partie moyenne des faces antérieure et postérieure $(0,02$ à 0,03$)\left({ }^{*}\right)$.

Tholartus (Häckel) (fig. 293̈) est un Cenolarcus dont la coquille lentelliptique est flanquée, aux extrémités de son axe transversal, de deux diverticules en forme de chambres arrondies. Il en résulte trois loges communiquant ensemble, deux latérales et une centrale. Tout cela est extracapsulaire, il n'y a pas de coque intracapsulaire $(0,1$ i 0,15$)\left({ }^{2}\right)$.

Fig. 295.

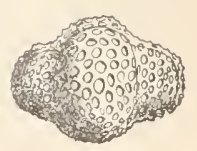

Tholarlus

(im. Häckel).

(I) Genres voisins:

Dizonium (IIäckel) a sa coque externe plus complète et percée, les deux ouvertures du précédent étant coupées cliacune en deux autres occupant les parties supéricure et inférieure des faces dorsale et rentrale par un pont de substance grillagée qui complète la coque suivant les mèridiens latéraux droit et gauche; parfois mème ces ouvertures sont recoupées comme chez Octopyle $(0,03$ ì 0,1$)$;

Trizonium (Häckel) l'a encore plus complète, chacune des quatre ouvertures précédentes étant encore recoupée en deux par un pont de substance grillagée qui complète la corque suivant les méridiens sagittaux antérieur et postérieur $(0,06$ a 0,12$)$;

Amphipyle (Iläckel) est un Trizoninm qui posséderait, en dehors de sa coquille devenue intracapsulaire, une corue incomplète extracapsulaire semblable a celle qui forme la ceinture de Monozonium $(0,09$ à 0,2$)$;

Tetrapyle (J. Müller) est comme le précédent, mais sa roque externe est semblable à celle d'un Dizonium $(0.12$ à 0,24$)$;

Octopyle (IIäckel) est comme le précédent, mais ses quatre ouvertures sont recoupées chacune en deux par une tigelle verticale 0,11 à 0,24$)$;

Pylonium (IIäckel) est semblable, mais sa coque exlerne est celle d'un Trizonium 0,15 à 0,18 );

Amphipylonium (Iäckel) est un Pylonium qui possède, en dehors de sa coquille, une coque incomplète semblable à celle de Monozonium $(0,24$ à 0.3$)$;

Tetrapylonium (Iäckel) est semblable, mais sa corfue externe est celle d'un Dizonium $(0,16$ à $0,2 \tilde{u})$;

Pylozonium (Iläckel) est semblable, mais sa coque externe est celle d'un Trizonium $\{0,24\}$.

Ces genres forment la famille des PrLoxwd [Pylonida (Iläickel)].

(2) Crenres voisins:

Tholodes (Häckel) est formé de deux coquilles de Tholartus concentriques et reliées par des tigelles radiales $(0,1)$;

Amphitholus Häckel peut être défini un Tholartus dont la loge centrale serait formée d'une coquille entière de Larnacilla, intracapsulaire $\{0,13$ à 1,16$\}$;

Amphitholonium (Iä̈kel) est un Amphitholus dont la coque la plus externe est enveloppée d'une seconde corpue, concentrique, toute semblable $\{0,16$ à 0,2 ;

Tholostaurus (IIäckel) est un Tholartus, mais muni de diverticules aux extrémités de ses axes transversal et longitudinal 10,12 à 0,16$)$;

Tholoma (Iläckel) est semblable avec double coque externe comme Tholodes ou Amphitolonium $(0,1$ ' à 0,26$)$;

Staurotholus (Iläckel) est un Tholostomrus avec une coquille intracapsulaire de Larnacilla en plus $(0,11$ i 0,16$)$;

Staurotholonium (IIäckel est un Staurotholus avec double coque externe 0,15 à 0,2 );

Tholocubus (Häckel) est encore un Tholartus, mais avec des diverticules aux extrémités de ses trois axes 0,15 a 0,16$\}$;

Tholonium (Häckel) est semblable avec double coque $(0,15$ à 0,16$)$; 
Zonarium (Iläckel) (fig. 296) est formé d’une coque intracapsulaire conslituée comme une coquille entière de Larnacilla ot d'une coque extracapsulaire lentelliptique, divisée en quatre loges diagonales par deux constriclions perpendiculaires, l'une dans le plan sagittal, l'autre dans le plan équatorial $(0,15$ à 0,22$)$ (').

Spirema (Hiickel) (fig. 997) a autour d'une coque grillagée intracapsulaire sphérique, une coque extracapsulaire lentelliptiqur (ou subsphérique) formée de tours

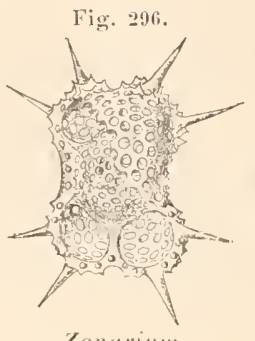

Zonarium (im. Häckel).
Fiig 297.

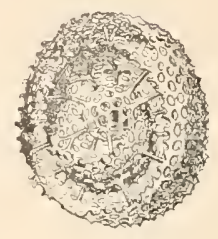

spirema

(im. Iläckel). spiraux comme celle d'un Nautile ou d'une Polystomelle $(0,16$ a 0,24$)\left({ }^{2}\right)$. Streblonia (Ilïckel) (fig. 298) est un Spirema à spire ascendante, c'est-ì-dire transformée en une hélice $(0,11$ is 0,4$)\left({ }^{3}\right)$.

Phorticium (Häckel) (fig. 299) a une coque intracapsulaire semblable à une coquille de Larmacilla, mais entourée d'une coque extracapsulaire grillagée à accroissement irrégulier $(0,12$ à 0,2$)\left({ }^{\mathbf{}}\right)$.

Cubotholus IIIäckel est un Tholoculius avec une

Fig. 298.

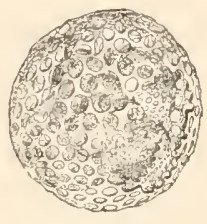

streblomia (ink. Hiicliel). coquille intracapsulaire de Larucilla an centre $(0,15$ i 0,16 ;

Fig. 29!.

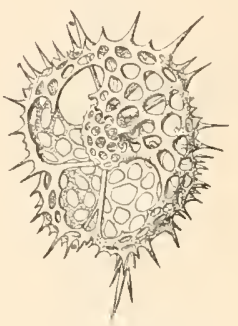

Phorticium

(iim. Häckel).

Cubotholomium Häckel est semblable, avec double coupe exlerne 0,2 a 0,28 );

Ces genres forment la famille des THoLost.de [Tholouida Uäckel)].

(1) Genres voisins :

Zoniscus (Itickel) a sa conpe externe à six loges determinées par trois constrictions, une sagittale et deux parallèles au plan équatorial 0,12 à 0,18 ;

Zonidium (IÏ̈ckel) l'a divisée en huil logres par quatre constrictions, trois comme celles du genre précédent el en plus une équatoriale $(0,13$ à 0,18$)$.

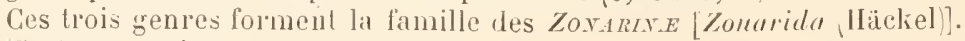

2) Genres roisins:

Lithelius (Itackel) est semblable, mais couvert de nonbreuses épines radiales $(0,12$ a 0,2$)$; Larcospira(Hacliel) a, comme coque intracapsulaire, une conuille entière de Laruacilla, et la spire de la coque externe fourne dans le plan équatorial de la coque interne 0,18 : $0,2 \%$;

Pylospira Häckel) est semblable, mais la spire est dans le plan frontal 0,18 i 0,22 );

Tholospira IIäckel) est semblable, inais la spire est dans le plan sagittal $(0,2$ à 0,27$)$ :

Spironium Häckel) a deux spires prenant origine cliacune d'un cóté et tournant en sens contraire de manière is dessiner un $8,0,12$ i 0,2 .

Ces genres forment la lamille des LIxHELINE [I,ithelida (IIäckel)].

(3) Genres voisins:

Streblacantha (Ilickel) est un streblonia épineux $(0,1$ i i 0,24$)$;

Streblopyle (hackel) est un streblonia contenant, a titre de coque interne intracapsulaire au lieu d'une simple sphère grillagée. mne corjuille le Larnacilla 0,2 ' a 0,27 ).

Ces trois genres forment la famille des streblowste [streblonida (Hackel)].

(1) Genre voisin:

Spongophortis IIackel) est un Morlicium it ropuille externe spongiense 0.15 i 0.25 . Cies deux genres forment la famille des Phortax. [Plorticida (Hackeli]. 
Soreuma (Hä̈ckel) (fig. 300) a romme prarlie intracapsulaire de sa coquille une simple sphère grillagée entourée, en guise de coque axterme extracapsulaire, d’une couche de chambreltes agglomérées salrs ordre $(0,17$ à 0,3$)$ ("). taires.

Avee ce gemre prend fin le groupe des Monocyt-

\section{בiROLPE}

Fig. 300.

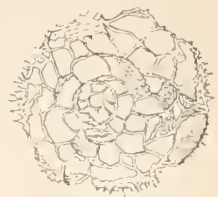

Soreuma

(im. Häckel

\section{POLVCTTMIRES - POLYCYTTAREA}

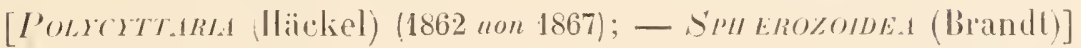

\section{TYPE MORPHOLOGIOQUE}

(FIG. 301 A 306)

L'individu zoologique est ici une colonie. Il est formé de plusieur'

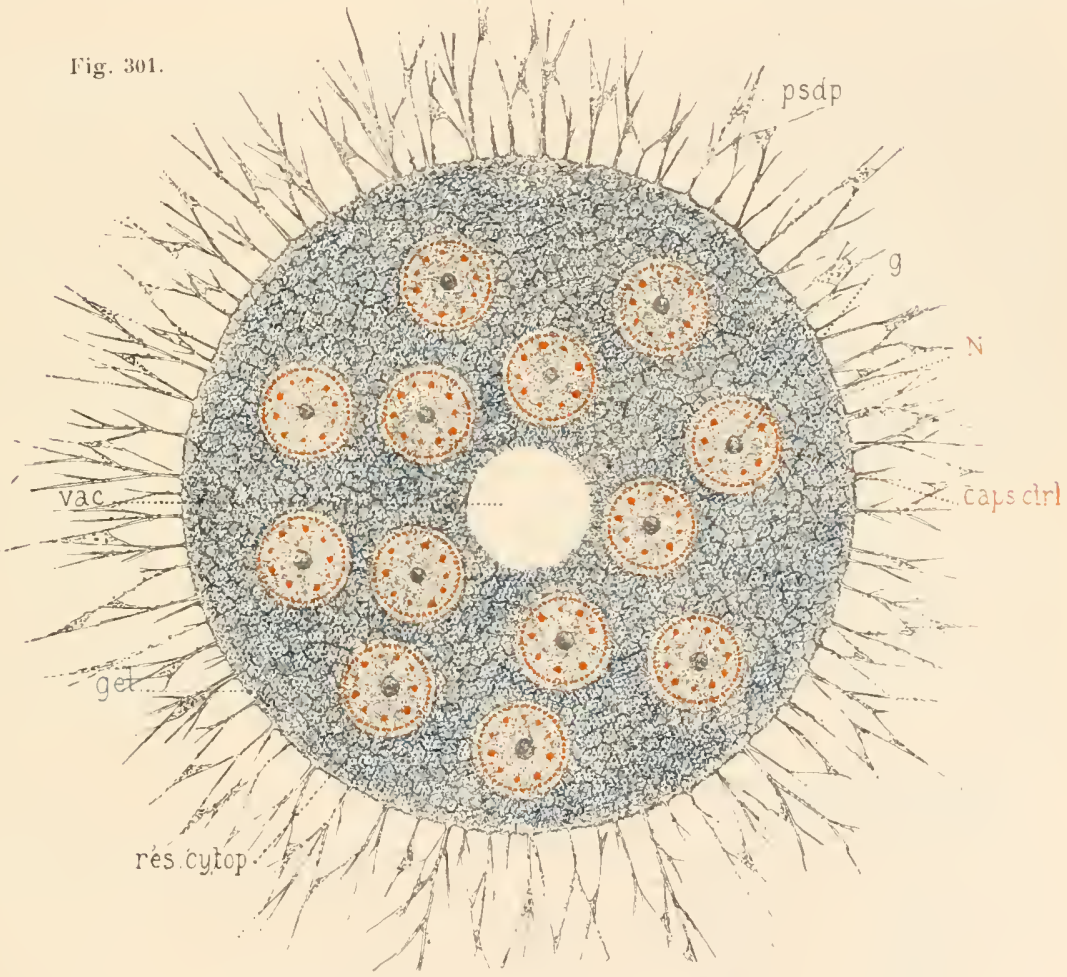

Polycyttaire (Type morphologique) (Seh.).

caps. ctr1., capsules centrales; $灬$. gonttes de graisse; gel., gélatine; $\mathbf{x}$., noyaux; psdp., pseudopodes; rés. eytop., réseau cy toplasmique.

1) Genre voisin :

Sorolarcus (Häckel) est semblable mais a, pour coque intracapsulaire, une coquille de I.arnacilla $(0,17$ i 0,23$)$.

Ces deux genres forment la famille des SorEUMLA [Soreumida Iäckel)]. 
individus morphologiques (caps. ctrl.) plus ou moins semblables à celui qui constitue à lui seul les autres types et de taille analogue; il est donc beaucoup plus gros. Il forme une masse sphérique de $100020 \mathrm{~mm}$ de diamètre, gélatineuse, demi-transparente. Pour nous faire une idée de sa constitution, représentons-nous de nombreux individus analogues à celui que nous avons décrit comme type morphologique de Radiolaire, groupés de manière à se confondre en une masse commune. Toutes les parlies extracapsulaires périphériques, gelée (gel.), pseudopodes $(p s d p$.$) , réseau cyloplasmique (rés. cytop.) avec ses inclusions y compris$ les Zooxanthelles, tout cela est confondu en une masse commune à toute la colonie et, dans celte masse, sont logées les capsules centrales (caps. ctrl.) avec leur contenu et mème leur couche protoplasmique péricapsulaire, qui ont gardé toute leur individualité (').

\section{Structure.}

La gelée commune, le vaste réseau proloplasmique qui la traverse, le réseau protoplasmique superficiel, les pseudopodes, les cellules jaunes, tout cela est identique à ce que nous avons décrit chez le Radiolaire simple. On doit seulement ajouter que la gelée (gel.) est toujours vacuolaire, tandis que dans les types simples, elle ne l'était que fréquemment, et une grosse vacuole centrale (vac.) occupe d'ordinaire le centre de la colonie.

Mais les capsules centrales présentent une particularité remarquable qui est le principral caractire sur lequel on se fonde pour faire des Polycyllaires un groupe à part au lieu de les répartir, comme simples formes coloniales, parmi des formes solitaires avec lesquelles elles présentent le plus d'affinités. Chaque capsule centrale présente à son centre un corps sphérique $(g)$ que l'on serait tenté de prendre pour le noyau et qui est une grosse goutle d'huile colorée; et les noyaux, pelits et nomhreux $(N$.$) , se trouvent logés dans la couche sphérique du protoplasma$ intracapsulaire; ils sont formés d'une masse de chromatine homogène (du moins en apparence) et disposés sur une ou deux couches, en cercle régulier.

Les capsules sont donc polynucléaires.

Elles sont d'ailleur's constituées comme celles des Péripylaires, c’est-à-dire percées de très fins pores uniformément répartis sur toute leur surface.

(1) Il faut sans doute considérer' les individus comme ayant gardé l'individualité, non seulement de leurs capsules el de leur proloplasma péricapsulaire, mais mèıne de tout ce qui constitue l'individu primaire, avec sil gelée et son réseau protoplasmique jusques et y compris celui qui est couché à plat à la surface de la gelée primaire et qui émet les pseudopodes. Car, dans les formes nunies d'une coruille grillagée, nous verrons que ces corquilles restent indépendantes comme les capsules centrales. íout se passe comme si c'rtait seulement la gelée secondaire avec les portions correspondantes du réseau protoplasmique qui se fusiomnaient. 


\section{Physiologie.}

L'animal vit absolument comme celui des formes solilaires. Il est comme lui pélagique et marin, comme lui ballotté passivement par les vagues, ne sachant que modifier quelque peu son poids spécifique pour s'enfoncer ou monter à la surface; il se nourrit comme lui, digère, respire, excrète comme lui. Il est de mème parfois phosphorescent, et le sioge de la lueur est la surface de contact entre la gontte l'huile centrale des capsules et le protoplasma ambiant.

\section{Évolution.}

Formation de la colonie. - Dans son évolution, le Polycyttaire pré-

Fig. 302 .

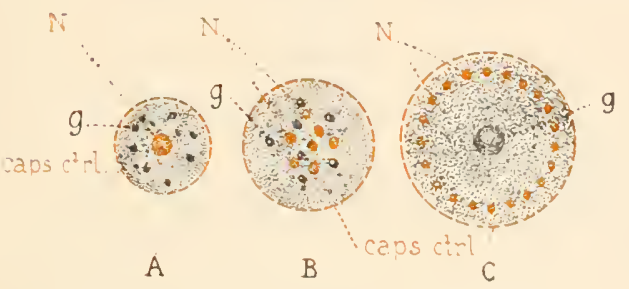

Polycytlaire. Déreloppement (Sch.)

$A, B, C$, états successifs des phénoménes qui se passent dans la capsule centrale; caps. ctrl., capsule centrale; g., gouttes d'huile; $\mathbf{x . , ~ n o y a u x . ~}$ sente diverses particularités remarquables, les unes, conséquence naturelle de sa constitution coloniale, les autres qui sont des caractères spéciaux.

Pas plus ici que chez les formes solitaires, on ne connait les transformations qui séparent la zoospore de l'animal constitué. (On sait seulement que celui-ci, à son état le plus jeune, est un in lividu simple, entièrement formé comme serait un tout petit Rarliolaire non colonial. 11 a, en effet, dans sa capsule centrale (fig. 302) 111 unique noyau central $(A: N$.), et quelques gouttes d'huile éparses $(A: g$.) dans le protoplasma intracapsulaire. Mais bientôt son noyau se divise en plusieurs autres qui viennent se ranger contre la paroi interne de la capsule, tandis que les gouttelettes d'huile se fusionnent en une grosse goutte centrale $(C: g$.$) et le caractère essentiel du Po-$ lycyttaire se trouve acquis.

Un deuxième phénomène caractéristique (fig. 303), est la multiplication des capsules $(A)$ par division incomplète, de manière à ce qu'elles restent unies par leur substance extracapsulaire et constituent pru à peu la colonie. Cette division a lieu tout simplement par étirement en liscuit $(B)$, séparation progressive des deux moi-
Fig. 303 .
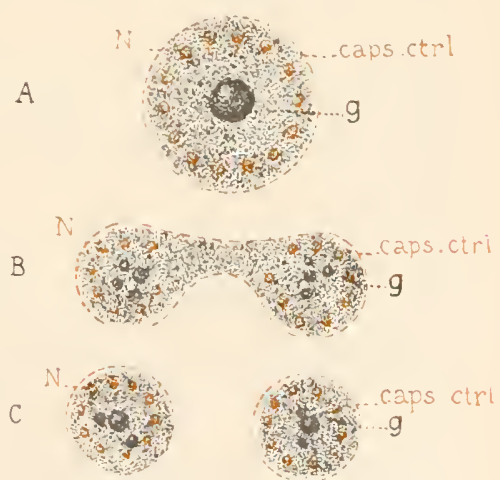

Polycyttaire. Multiplication des capsules centrales (Sch.).

$A, B, C$, états successifs. caps. ctrl., capsule centrale; $\mathbf{m}$. gouttes d'huile; N., noyall.

tiés et partage entre les deux (C) des éléments consécutifs dle la capsule, 
proloplasme(y compris lacouche extracapsulaire, goulted'huile el noyaux). Ainsi la division est ici beaucoup plus fréquente que chez les formes simples, mais elle survirail principalement à l'accroissement des colonies.

Reproduction par division. - Les colonies se reproduisent fréquemment par simple fragmentation en colonies plus petites qui grossissent par mulliplication des capsules jusqu'à ce qu'elles soient devenues ellesmèmes en état de se diviser.

Reproduction par isospores (lig. 301). - La formation des spores ne diffère en rien d’essentiel de ce que nous avons décrit à propos du lype général. Nais, les noyaux étant déja multiples, le travail de leur multiplicalion est déjà fait à moitié. La goutte d'huile centrale ( $A$ et $B: g)$ se

Fig. 30\%. .

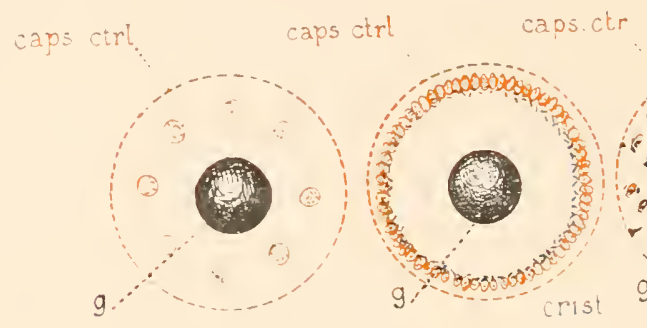

A

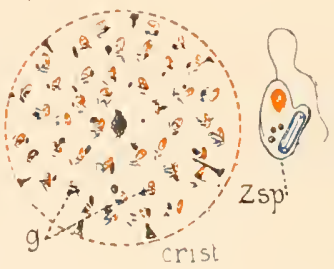

D

Polyryttaire. Reproduction par isospores (Sch.).

$\boldsymbol{A}$, capsule centrale contenant la goutte d'huile centrale et les noyaux; $\boldsymbol{B}$, les noyaux se sont divisés; $C$, la goutte d'huile centrale s'est dissocíe en gouttelettes qui s'associent aux noyaux et aux cristalloüdes; $D$, la zoospore contenant un novau, un cristalloüde et des gouttelettes huileuses, caps. etrl., capsule centrale contenant les noyaux; crist., cristalloïde; g., gouttes d'huile: Zsp., Zoospore.

divise en gouttelettes qui viennent prendre place à côté des noyaux $(C)$; il se forme des cristalloïdes (crist.) d'une substance de réserve albunineuse qui prennent place aussi à côté des noyaux. Ceux-ci se montrent netlement biréfringents. In noyau, un ou quelques cristaux, el un petil lot de goultelettes d'luile servent à constituer, avec une pelite masse du protoplasma ambiant, une zoospore $(I): Z s p$.$) (').$

Reproduction par anisospores (fig. 30\%). - Dans d'autres cas, les spores se forment d'une manière un peu différente el assument un autre caractère. Les noyaux se rémnissent par groupes autour d'un petit lot de gouttelelles d'huile issues de la grosse goultr centrale $(A)$, et une petite masse de proloplasma $(a)$ s'indivilualise autour de l'ensemble. Dans chacun de ces groupes, les noyaux se multiplient activement $(C: N$. $)$ et l'ensemble prend la forme d'une petite masse en roselte $(D)$ aver les

(1) Ici comme dans le cas général, ces zoospores sont mises en liberté par destruction de la capsule et quand les capsules sont viclées, le reste de la colonie se contratete. tombe au fond et meurt. 
noyaux à la périphérie et les gouttelettes d'huile au centre. Puis la rosette se dissocie en autant de secteurs qu'il y a de noyaux $(E)$ et chacun de ces secteurs formé d'une petite masse protoplasmique, d'un noyau et d'un amas de gouttelettes d'huile devient une spore réniforme chez laquelle on n’a réussi à voir qu'un seul flagellum. Mais ce qui est plus

Fig. 305 .
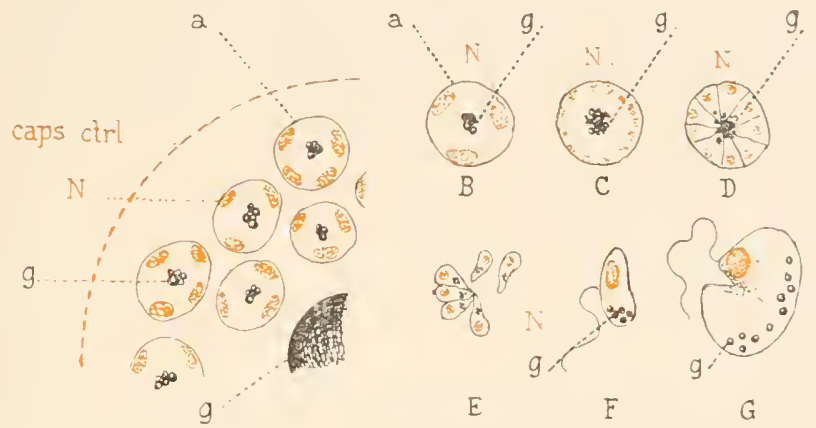

A

Polycyttaire. Reproduction par anisospores (Sch.).

A, une portion de la capsule centrale dans l'interieur de laquelle s'individualisent des petites masses de protoplasma contenant des noyaux et des gonttelettes graisseuses; $B, C, D, E$, états successifs d'une de ces petites masses protoplasmiques aboutissant à la formation des spores; $F$, microspores; $G$, macrospores. a., petite masse protoplasmique; caps, ctri., capsule ceutrale; $₫$, goutte d'huile; N., noyaux. singulier, c'est que ces spores sont de deux tailles, Les unes sont grosses, avec un noyau clair, un réseau chromatique fiı, délicat, làche : ce sont les macro$\operatorname{spores}(G)$; les autres sont plus petites environ de moitié, ont un novau foncé (après l'action des teintures), à réseau grosetserré: cesont les microspores $(F)$. Les unes etles autres sont lésignées sous le nom d'anisospores, par opposition aux isospores qui sont de taille uniforme. De plus, elles n'ont pas toujours des cristallö̈les, tandis que les isospores en ont sans exception. Brand [85] admet, sans aroir pu l'ohserver, que ces anisospores sont sexuées, les grosses étant femelles el les petites màles, et qu’il y a une certaine alternance, quoique non régulière, entre cette génération el la génération asexuelle par isospores. Après l'émission des anisospores la colonie se détruit (").

Pas plus ici que pour les autres Radiolaires on n'a pu suirre l'érolution des spores.

Corps extracapsulaires (fig. 306). - Indépendamment de ces divers éléments reproducteurs, il existe des formalions particulières qui semblent jouer un ròle soit dans l'accroissement, soit dans la multiplication de la colonie, et au sujet desquelles règnent les plus grandes incertitudes, ce sont les corps extracapsulaires. Ils sont formés chacun par une petite masse de protoplasma nucléée, arrondie. Ils sont situés hors des capsules, mais sourent dans leur voisinage immédiat, reliés par quelques tractus protoplasmiques au réseau général. D’après Braxd [55] ils naitraient par bourgeonnement des capsules déjà formées, et de

(1) Nous verrons que, selon les genres, les macrospores et microspores se forment tantòt dans les mêmes capsules, tantôt dans des capsules séparées. 
la manière suivante. La capsule se prépare comme pour former des zoospores, c'est-à-dire qu'elle garnit son protoplasma intracapsulaire de peliles ggoultes d'huile qui émanent de la grosse goutte centrale el viennent se ranger à côté des noyaux. La membrane capsulaire (caps. ctrl.) for'me des diverticules $(a, b)$ dans chacun desquels passe un petit groupe de noyaux $(N$.) et de gouttes d'huile $(g$.$) avec une petite masse du protoplasma$ intracapsulaire ambiant. Ces diverlicules se détachent et forment autant de corps extracapsulaires $(c)$. Parfois, ces corps pourraient se transformer en zoospores et quitter la colonie. Mais, le plus souvent, ils resteraient dans la colonie, y prendraient place et se transformeraient en nouvelles capsules centrales. Ce serail, pour les colonies jeunes, le principal procédé d'accroisse-

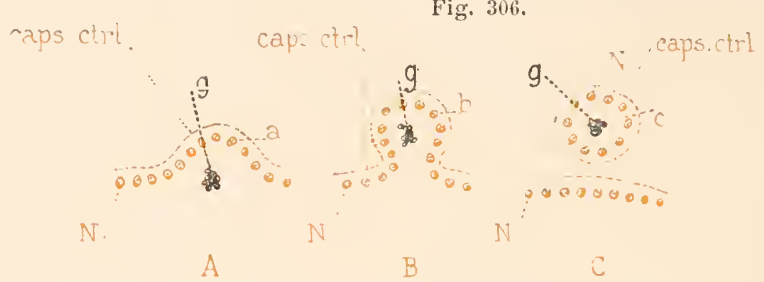

Formation des corps extracapsulaires (Sch.).

$A, B, C$, trois états successifs. a, bourgeon conmencant à se former; 1), bourgeon s'individualisant; $c$, bourgeon individualisé représentant le corps extracapsulaire; caps. etri., capsule centrale; g., gouttes de graisse; $\mathbf{N}$, noyanx.

ment et le principal mode de multiplication de leurs capsules.

Mais Fanixtzix [88] interprèle toul autrement les choses. Pour lui, les corps extracapsulaires sont des éléments sexués homologues aux macrospores dont ils ne sont qu'une modification; ils ne se transforment pas en capsules centrales, mais (probablement car il n'a pas vu tout cela) quittent la colonie et se transforment en nouveaux individus. Le cycle évolutif serait le suivant. Les petites colonies que l'on trouve au printemps grossissent pendant tout l'été et donnent des isospores et des anisospores qui servent évidemment à en former de nouvelles (bien qu'on n'ait pu suivre leur évolution); à l'automne, elles sont très grosses et se fragmentent en petites colonies qui sont celles que l'on trouve en hiver. C'est dans ces dernières qu'a lieu la formation des corps extracapsulaires. Elle ne se fait point par une sorte de bourgeonnement de la capsule. Celle-ci se prépare ahsolument comme pour la formation des anisospores el loute sa masse superficielle se transforme en sortes de macrospores qui sont les corps extracapsulaires, tandis que la masse résiduelle centrale correspond aux microspores. Les corps extracapsulaires formés, en somme, chacun d'une pelite masse arrondie de protoplasma et d'un noyau, rampent avec des pseudoporles le long du réseau et s'éloignent de leur lieu d'origine, entrainant parfois une cellule jaune. Famintzin n'a pu voir ce qu'ils deviement, mais il pense qu'ils doivent subir quelque conjugaison avec la parlie représentant les microspores (car parfois on les a trouvés approchés de celles-ci), et se comporter ensuite comme les anisospores ordinaires. Un voit que toute la question est encore bien olsscure. 
Les Polycyttaires se divisent en trois sous-ordres très aisés à distinguer aux caractères suivants:

$1^{\circ}$ Collozorde, dépourvus de squelette;

20 SPHEROID.e à squelette formé de spicules indépendants disposés tangentiellement autour des capsules centrales;

$3^{\circ}$ Colcosprerid.e. qui ont, autour de chaque capsule centrale, une coque grillagée continue.

Ces caractéristiques sont si netles qu’elles nous dispensent d'établir pour ces sous-ordres un type morphologique. Il suffit de prendre celui de l'ordre et d'y ajouter la caractéristique indiquée * (").

$$
\begin{gathered}
\text { fer Sous-Ordre } \\
\text { COLLOZOÏDES. - COLLOZOID E } \\
\text { [COLLOZOIDA (Häckel)] }
\end{gathered}
$$

Ce sous-ordre contient le seul genre :

Collozoum (Iläckel) (fig. 307,308). L’animal est for'mé essentiellement comme

Fig. 307.

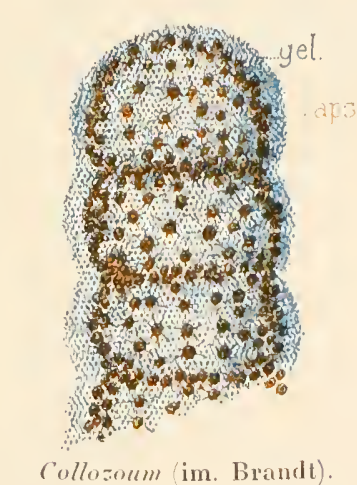

I'ue extrémité de la colonie.

caps., capsules centrales; gel., gelée.

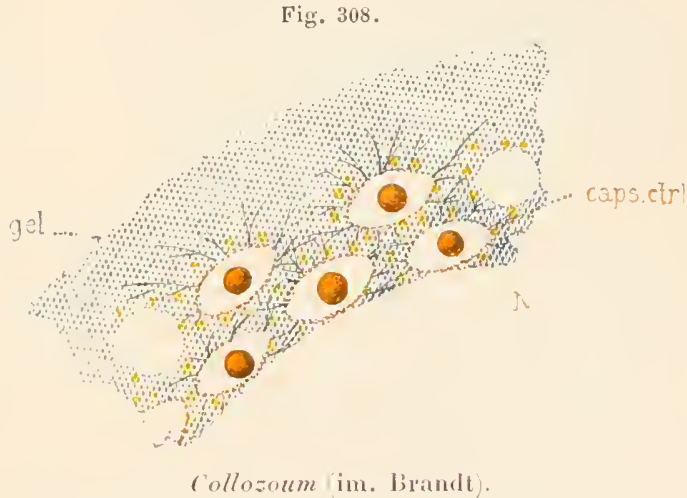

Detail de la constitution de la colonic.

notre lype général de Polycyltaires. Il n'a done pas de squelette. Mais la constitution des colonies est plus complexe en ce sens que les petites

(1) Brandt réunit les deux premiers groupes sous le nom de Sphrozoidea (Brandt) qu'il caractérise par leur squelctte nul ou formé d'aiguilles indépendantes et surtoul leurs vacuoles pleines d'un simple liquide aqueux et leur's macrospores et microspores à cristalloïdes petits ou nuls et naissant dans le mème individu. Il oppose á ce groupe les Collospharida (Brandt), qu'il caractérise par leurs vacuoles pleines d'une geléc liquide, leurs macrospores et microspores naissant dans des individus distinets et pourvues toujours de gros cristalloïdes, et leur squelette formé de sphères grillagées nul ilans le seul genre Iyxosphiera (Brandt).

(*) Dans le groupe de Polycyttaires, les dimensions indiquées sont celles des capsules centrales un des coquilles des individus. 
colonies sphériques se groupent en boudins moniliformes (fig. 308) de forme variée qui alteignent plusieurs centimètres de long $\{0,04$ à 0,5$)\left({ }^{\mathbf{I}}\right)$.

\author{
$2^{\text {e }}$ Sous-Ordie \\ SPIIEROZOIDDES. - SPIIEROZOID E \\ [SPHEROIDEA (Häckel non Brandt)]
}

Sphærozoum (Iläckel) (fig. 309) ne diffère de Collozoum que par la présence de spicules $(s q$.$) indépendants,$ branchus ou radiés, disposés à quelque distance autour des capsules (caps. ctrl.), tangentiellement, sans grande régularité. La forme et la taille sont analogues $(0,4$ à 0,3$)\left({ }^{2}\right)$.

(1) Dans une espèce du Challenger C. serpentinum (IIäckel), les capsules centrales sont allongées en cordons sinueux qui atteignent 20 à $40 \mathrm{~mm}$ de long et contiennent chacune une longue série de gouttes d'huile et sont bourrées de noyaux, comme si de nombreuses capsules spliériques s'étaient soudées pour les former. Dans d'autres les capsules sont elliptiques ou discoïdes comme dans les Prunoida ou les Discoidx parmi les Péripylaires

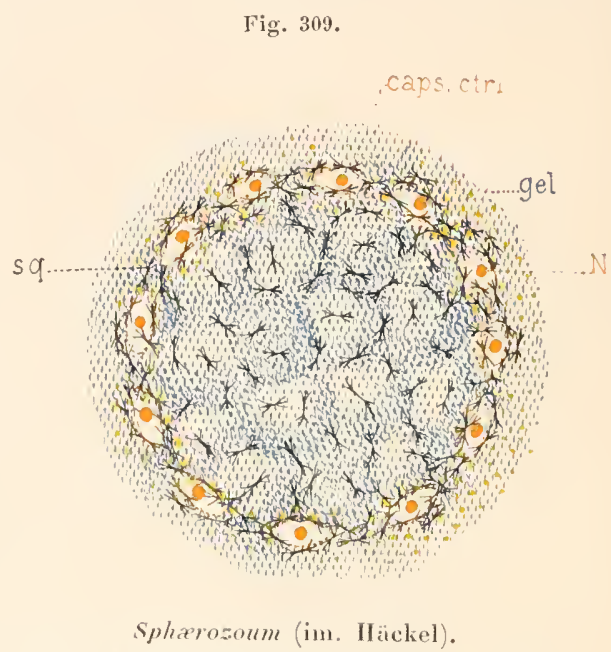

caps. ctrı., capsules centrales; gel., gelatine; N., noyau; sq., spicules squelettiques. Honocyttaires.

Häckel prévoit que les espèces dont il fait des sous-genres

Colloprunum (Häcliel),

Collodiscus (Häckel), etc., pourrontbien devenir des genres. Et, de fait, il forme des genres dans les autres groupes avec des caractères de moindre valeur.

Brandt fait un genre ì part de $C$. coruleum (Häckel) sous le nom de

Myxosphæra Brandt) et le place même dans sa famille des Collozoidea parce qu'il a les deux ou trois caractères de cette famille: vacuoles pleines de gelée liquéfiée, macrospores et microspores à gros cristaux et provenant d'individus distincts.

Ce genre forme pour Iläckel la famille des Collozoida (Iäckel), du sous-ordre des Colloidea (Häckel) dans l'ordre de Collodaria (Häckel).

${ }^{2}$ Genres voisins:

Belonozoum (IIäckel) a ses spicules simples, en forme d'aiguilles $(0,08$ à 0,3$)$;

Raphidozoum (IIäckel) les a de différentes sortes, les uns simples, les autres branchus $(0,03$ à $0,33 ̈)$.

Les spluxrozoida sont pour IIïckel une simple famille des Beloida (Häckel) dans l'ordre des Collodaria (Häckel). 


\section{COLLOSPHÉRIDES. - COLLOSPII.ERIID.E

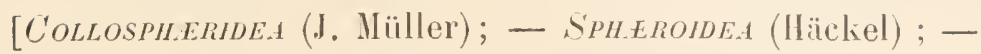 \\ Collospheride. (Brandt emend.)]}

Collosphæra (J. Müller) (fig. 3li(1) est un Sphærozoum dont les corquilles péricapsulaires seraient formées d'une coque grillagée continue ou, si l'on préfère, un Cenosphara colonial. Ces coquilles sont notablement plus grandes que les capsules el contiennent, par conséquent, une certaine quantité de substance extracapsulaire et, par leurs pores, passent des tractus protoplasmiques (res. cyt.) qui unissent les réseaux extracapsulaires (cyt.) intérieurs aux coquilles au réseau commun extérieur à ces coquilles. La colonie est sphérique, occupée au centre par une énorme vacuole remplie d'une gelée liquide et tous les individus sont disposés à lat périphérie $\left(0,1\right.$ à 0,21 ( $\left.{ }^{2}\right)$.

(1) Genres voisins :

Tribonosphæra (Iläekel) est un Collosphixra à cortuilles épineuses en dedans $(0,1$ à 0,12 );

Pharyngosphæra (IIäckel) est un Collosphix a a coquille munie en dedans de tubes centripètes fermés $(0,11$ à 0,12$)$;

Buccinosphæra (Häekel) a les mèmes tubes, mais fenestris $(0,1$ à 0,14$)$;

Acrosphæra (Iläckel) est un Collosphiera a coupilles garnies en dehors d'épines irrégulièrement distribuées $(0,0$ à à $0,20 ̋$;

Odontosphæra (Iläckel) est semblable au précedent, mais a de grands et de petits trous à sa corpuille et, regulièrement, une épine pour charfue grand trou $(0,1$ is 0,14$)$;

Chœnicosphæra (IIäckel) est semblable au mème, mais a pour chaque trou une couronne d'épines 0,1 ì 0,2$)$;

Siphonosphæra (J. Müller) a les grands trous de sa cotfuille prolongés extériemrement en tubes lisses, a parois continues, tronqués au bout $(0,04$ à 0,24$)$;

Mazosphæra (Ehrenberg) a, de plus que le précédent, une forte dent à l'orifice de chaque tube $(0,08$ a $0,1: 2)$;

Trypanosphæra (Iäckel) a une couronne de dents à l'orifice de chaque tube $(0,08$ à 0,150$)$;

Caminosphæra (Häckel) a aussi des tubes comme Siphonosphrra, mais ramifiés $(0,05$ à 0,15$)$;

Solenosphæra (Häckiel) a ses coquilles garnies de tubes semblables à Siphonosphitra, mais régulièrement disposés et à parois fenestrées 0,07 ì 0,18$)$; 


\author{
$2^{e}$ ORdRE \\ ACTIPYLAIRES. - ACTIPYLIDA \\ vel \\ ACINTIIAIRES. - ICANTIIARIDA \\ [ActipylaA (Hiickel); - AcantianaAa (Häckel); - \\ Acanthometrea (R. Hertwig)]

\section{TYPE MORPHOLOGIQUE} \\ (FIG. 311 A 314)
}

Prenons pour point de départ le type général des Radiolaires et

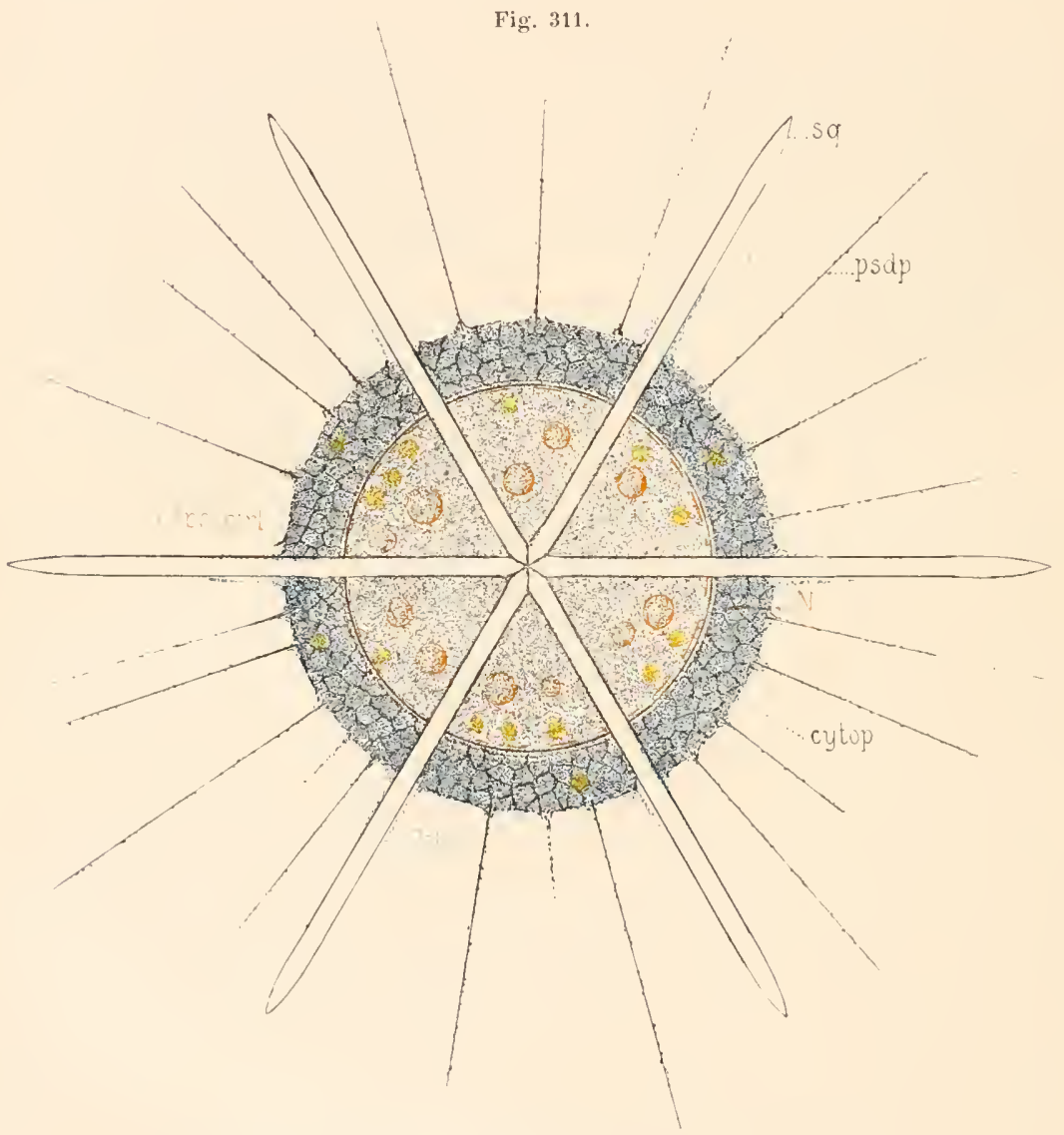

Acanthaires. Type morphologique (Acanthrometron) (Sch.).

caps. ctrl., capsule centrale: extop., crtoplasma ; gel., gelée ou calymna ; $\mathbf{N}$., noyaux psdp., psendoportes; sq., mayons du squelette.

Otosphæra (IIäckel) est de même, mais a une forte dent à l'orifice de ses tubes $\langle 0,1$ ì 0,2 ; Coronosphæra (Häckel) a, en plus, une rouronne de dents i chaque orifice 0,08 i 0,3 ; 
voyons par quelles modifications celui de cet ordre va en dériver. Deux points principaux sont à signaler : la constitution de la capsule centrale et surtout l'existence d'un squelette absolument caractéristique à la fois par sa constitution chimique et par sa disposition géométrique. Parlons d'abord de ce dernier.

Squelette. - Le squelette est formé de vingt tigelles radiaires (sq.) qui partent exactement du centre de la capsule et vont surgir à la surface en des points déterminés. Le seul fait que ces baguettes sont radiaires et

Fig. 312 .

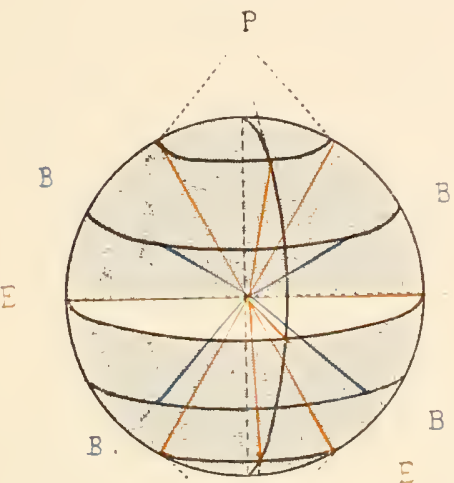

P.
Fig. 313 .

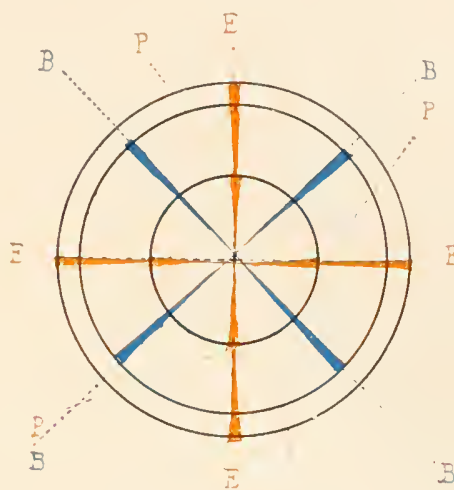

5
Acanthaires (Type morphologique)(Seh.). Constitution géométrique du squelette radiaire vu de profil. Les rayons en avant du plan de la figure ont été seuls représentés.

$\mathbf{B}$, rayous tropicaux; E, rayous équatoriaux ; $\mathbf{P}$, rayons politires.
Acanthaires (Type morphologique) (Sch.). Constilution géométrique du squelette radiaire vu par un des poles.

Pour lexplication des lettres voir la figure précédente.

se réunissent au centre où elles ont leur point de départ primitif est déjà hautement caractéristique, car jusqu'ici (et il en sera de mème dans les autres ordres) le squelette était essentiellement tangentiel ou formé de coques concentriques; les éléments radiaires $r$ étaient accessoires. De plus, quand ces coques arrivaient jusqu'au centre, ce qui était rare c'était secondairement, leur point de départ étant toujours à la surface, de la gelée primitive, c’est-à-dire en dehors de la capsule. Ici, au contraire, les vingt baguettes radiaires ont leur origine première au centre et se développent par un accroissement centrifuge (').

Clathrosphæra (Häckel) a ses coquilles doubles, formées de deux coques concentriques et lisses toutes les deux $(0,12$ à 0,18$)$;

Xanthiosphæra (Häckeh) de mìme, mais avec la coque externe épineuse en dehors 0,11 a 0,19$)$,

Ces genres forment la famille des CoLLospusarise [Collosphixida (J. Müller)] du sous-ordre des Sphxroida (Häckel), de l'ordre des sphxrellaria (Häckel).

${ }^{(2)}$ Elles arrivent au centre nième et pour cela leurs extrémités centrales sont taillées 
Elles sont formées, non de silice, mais d'une matière organique albumineuse qui semble ètre voisine de la vitelline, soluble dans les solutions salines un peu concentríes (curbonate de soude à $1 \%$ ou sel marin à 10 à $20 \%$ ), et que l'on a appelée acunthine.

Leur disposition géométrique est très régulière et suit une règle que l'on a baptisée du nom de loi de J. Mäller. Pour la comprendre, comparons l'animal au globe terrestre (fig. 312, 313). Traçons sur la splière l'équateur $(E E)$, et sur chaque hémisphère deux parallèles ayant à peu près la situation des tropiques $(B B)$, et des cercles polaires $(P P)$. Cela formera cinq cercles. Les vingt spicules forment cinq groupes ile quatre, Jout chacun part du centre et ra sortir, suivant un rayou, au niveau d'un de ces ciny cercles. Telle est leur disposition suivant les parallèles. Voici maintenant leur disposition suivant les méridiens. supposons deux plans méridiens perpendiculaires. Ils couperont les cercles aux degrés 0 et 180,90 et 270. Et bien, les spicules de l'équateur et des cercles polaires surgissent sur ces parallèles aux quatre points $(E P)$, où ces méridiens les coupent.Quant à ceux des cerclestropicaux $(B)$, ils sout exactement dans les plans bissecteurs des précédents, et par conséquent surgissent

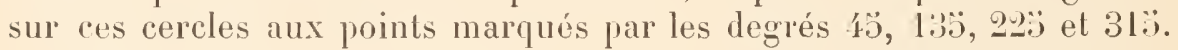

Gapsule et son contenu (fig.311). - La capsule centrale (caps. ctrl.) est sphérique et située au centre du corps. Elle a une paroi très mince qui, forcément, est percée par les vingt spicules du squelette. Cette paroi est criblée de très fins pores, mais ceux-ci ne sont pas, comme chez les Péripylaires, uniformément répartis sur toute sa surface. lls forment des groupes réguliers symétriques, réservant entre eux des liģnes polygonales ou des espaces réguliers.

Les noyaux $(\mathrm{N}$.) sont multiples el représentés par plusieurs petites masses qui se logent entre les spicules ('). Le protoplasma intracapsulaire présente une striation radiaire parfois assez nette.

en pyramides dont les faces s'appliquent les unes contre les autres, et dont les sommels se confondent en un point qui est le centre de la capsule.

(1) II est bien probable qu'il y a un stade jeune oủ le noyau est unique, mais on ne sait rien de positif à cet égar.l. Tous les auteurs décrivaient comme noyau unique (fig. 31's), un gros corps subcentral montrant cerlaines particularités lizarres. Du côté opposé au centre de la capsule où les spicules se joignent en dehors de lui, la membrane de ce prétendu noyau forme une invagination conique (in'g.) plissée, radiairement vers le sommet, circulairement plus bas, qui coiffe le prétendı nucléole (nct.), comme d'un bonnet de coton. Celui-ci différencie la partie ainsi coiffie en une substance plus claire. Plus tard cela disparaîtrait et la multiplication

Fig. 31 .

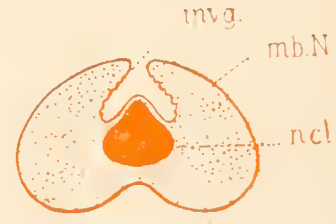

Prétendu noyau (coupe)

(Parasite acinétien) (Sch.).

inv.s., invagination; mb. N., nembrane nucliaire; n. c1., bourgeon inlerne. du prétendu noyau se prodıirait, commençant par celle du prétendu nuclèole et se continuant par des lobes profonds uqui découpent le soi-disant noyau.

Or Köprex [9千] a démontré que ce n'est aulre chose qu'un parasite Acinétien du genre Imabophrya Köppen) [.1. Acanthometre Köppen)]. Cet Acinélien se reproduit 
Organes extracapsulaires. - Le protoplasma extracapsuluire (cytop.), la gelée (gel.), ne présentent rien de lien particulier. Cetle dernière cependant se prolonge un peu le long des spicules en forme de manchons et ceux-ci ne sont libres que tout à fait à leur extrémité. lls sont donc plongés dans une sorte de gaine que leur forment les prolongements de la gelée. Or, dans celte gaine, se trouvent parfois, šinsérant à la paroi gélatineuse, une vingtaine de petits filaments protoplasmiques qui entourent le spicule, remontent le long de lui et viennent s'attacher à lui non loin de son extrémité. Ces filaments sont contractiles; ils ont reçu le nom de myophrisca et ont pour action de tirer sur la gaine gélatineuse du spicule, de la faire avancer plus loin sur lui et el ainsi de dilater le corps pour diminuer son poids spécifique et le faire flotter. Quand on les excite, its se contractent et produisent leur action sur la gaine. Nlais si l'excitation est trop forte, ils lichent prise sur les spicules et forment un bouquet de filaments épars que l'on avait appelés cils gélatineux (').

Les pseudopodes ( $p s d p$.) sont de deux sortes, les uns ordinaires, les autres immobiles el munis d'un filament axile comme chez les Héliozoaires. On nomme ces filaments axopodes. Enfin les Tunthelles offrent ceci le curieux, qu'elles sont pour la plupart intrarapsulaires. Elles sont situées, au nombre d’une vingtaine environ, tout contre la face interne de la capsule. On en trouve aussi quelques-unes éparses dans le réseau extracapsulaire. Elles sont constituées comme d'ordinaire, sauf peut-être une plus grande délicatesse de leur membrane.

La physiologie n'offre rien de spécial.

Nous diviserons les Acanthaires $\left(^{*}\right)$ en quatre sous-ordres caractérisés d’une manière bien nelle:

lo ACANThoridE, à vingl spicules disposés suivant la loi de Ilüller et subégaux, sans coque grillagée complète;

$2^{\circ}$ SP II ER OPIIRACTIID E, possélant tes vingt spicules régulier's subégaux et en outre, une coque grillagée sphérique;

$3^{\circ}$ Prevophactide, possédant l'es ringt spicules réguliers mais inégaux et, en outre, une coque grillagée elliptique ou discö̈le;

\{. ACTINELID E, à spicules radiaires en nombre non défini el à disposition irrégulière, privés aussi de coque grillagée.

comme Tokophrya par un bourgeon interne situé dans une cavité formée par une invagination de la surface et communic|uant avec elle par un col étroit. C'est ce bourgeon qui a été pris pour le nucléole et le parasite tout entier représente le prétendu noyau.

(1) Cela n'existe que chez les Acanthonidze.

() Ḧ̈скец les réunit en deux groupes d'importance supérieure, distincts par l'absence ou la présence d'une coque grillagée complète. Acanthomctra (J. Müller) comprenant les deux premiers les quatre groupes ci-dessous, et les Acanthophracta (i. Ilertwig) comprenant les deux derniers. 
$1^{\text {er SOUS-ORdre }}$

ACANTHONIDES. - ACANTHONHI.E

[ArANTHONIDA (Häckel)]

TYPE MORPHOLOGIOUE

(FIG. 315)

Caractérisé par ses vingt spicules subégaux et par l'absence de coque grillagée, il se confond avec le genre type Acanthometron.

Fig. 315.

\section{GENRES}

Acanthometron (J. Müller) (fig. 3I.̈) a les caractères du type morphologique de l'ordre des Acanthaires et, en outre, pour caractère générique, la forme de ses spicules, cylindriques sur la coupe transversale et entièrement dépourvus d'apophyse $\left(0,08\right.$ à $0,8\left({ }^{\circ}\right)$. Abondant dans toutes les mers chaudes) ( ${ }^{1}$ ).

(1) Genres voisins :

Zygacantha (J. Müller) est un Acanthomètre à spicules elliptiques ou losangiques sur la section transversale i 0,1 à 0,5$)$;

Acanthonia (Häckel) a ses spicules il section rectangulaire $(0,1$ ì 0,6$)$;

Lithophyllium (J. Hüller, au rontraire, a chacun de ses spicules muni de deux apophyses opposées simples $(0,02$ à 0,25$)$;

Phractacantha Häckel) est semblable, mais les apophyses de ses spicules sont ramifiées $(0,1$ à 0,15$)$;

Doracantha (Häcliel) de même, mais ses apophyses sont grillagées $(0,2)$;

Astrolonche Iläckel) ressemble il Lithophylinm mais la paire d'apophyses opposies se répète deux à quatre fois sur chaque spicule de manière i former deux rangíes longitudinales opposées $(0,2$ à 0,4$)$;

Xiphacantha (Häckel) a, sur charue spicule, łuatre épines en croix, simples c'est-à-dire non ramifiées $(0,1$ à 0,5$)$;

Stauracantha (Häckel) est de même, mais ses ipines sont ramifiées $(0,12$ a 0,5$)$;

Phatnacantha (Häckel) de même encore, mais ses épines sont grillagées $(0,12$ ì 0,18 ;

Pristacantha Häckelj a, comme Astrolonche, plusieurs épines disposies tout le long du spicule, mais ces épines forment quatre rangées longitudinales an lien de denx $(0,3$ à 0,6$)$.

Ces genres forment la famille des [Astrolorcmse (Astrolonchida) (Häcliel)]. 
Acanthostaurus (Häckel) (fig. 316) diffère d'Acanthometron par le fait que ses quatre épines équatoriales sont plus grandes et de forme autre que les seize autres. Les quatre spicules équatoriaux sont d'ailleurs égaux entre eux et les autres à peu près égaux aussi ou semblables entre eux. 0,2 à 1) (').

Amphilonche (IIäcliel) Fig. 316.

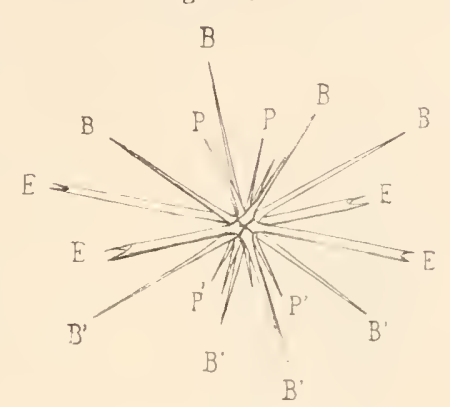

Acanthostaurus (Sch.).

$\mathbf{E}, \mathbf{E}, \mathbf{E}, . .$. spicules équatoriaux : $\mathbf{P}$. $\mathbf{P}$..

$\mathbf{P}+, \mathbf{P}+.$. spicules polaires; $\mathbf{B}, \mathbf{K}, \ldots$ 1B", B.. spicules tropicaux.
Fig. 317.

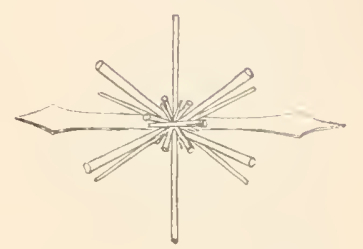

Amphilonche (im. IIickel).

se distingue par le

fail qu’il a deux de ses spicules (fig. 317), l’antérieur et le postérieur, notablement plus longs que les dix-huit autres $(0,1$ à 0,8$)\left({ }^{2}\right)$.

בe SOUS-ORdre

SPIIEROPIRACTIDES. - SPH. EROPHRACTID.E

[SPIIEROPHRACTA (Häckel)]

\section{TYPE MORPHOLOGIQUE}

(FIG. 318 ET 319)

Nous avons vu que souvent, dans les Acantuoners, les spicules, d'ailleurs égaux et disposés snivant la loi de Müller, portent des épines parfois ramifices, orientérs tangentiellement $(t)$. Ces épines, étant loutes

(1) Les genres ci-dessous ont aussi les qualre épines équatoriales différentes des antres et plus grandes mais, en outre, les spicules de mème catègorie peuvent présenter entre eux des différences.

Belonostaurus (Häckel) a les huit spicules tropicaux différents des huit polaires $(0,06$ ì 0,5$)$; Lonchostaurus (Häckel) a les deux spicules éfuatoriaux, antérieur el postérjeur, semblables entre eux, mais diffẻrents des deux latéraux $(0,24$ à 0,4$)$;

Zygostaurus (IIäckel) a, en outre, l'équatorial antérieur diffèrent du postérieur $\{0,4$ à 0,8$\}$; Quadrilonche (IIäcliel) est comme Acanthostaums, mais ses spicules sont munis d'aprophyses simples $(0,12$ a a 0,3$)$;

Xyphoptera(IIäckel) est de mème, mais ses apophyses sont ramifiées ou pinnées $(0,1$ à 0,26$)$. Lithoptera (J. Müller) de mème encore, mais ses apophyses sont grillagées $(0.2$ 't à 0,48$)$.

Ces genres forment la famille des QtadDRLoxchIXE [Quadrilonchida (Häckel)].

(2) Genres voisins :

Amphibelone (Häckel) est de mème et, en ontre, le spicule équatorial postérieur est plus long que l'antérieur $(0,1$ à 0,4$)$ :

Acantholonche (Häckel) est comme Amphilonche mais, tandis que chez celui-ci les huit spicules polaires sont semblables aux huit tropicaux, jei ils sont différents $(0,2$ ì. $0,0, \cdot)$.

Ces genres forment la famille des AmPILOxCnIs [Amphilonchida (Häckel)]. 
à mème distance du centre, sont disposées suivant la surface d'une sphère idéate ayant pour centre le centre du corps. Supposons que ces épines deviennent plus grandes, plus ramifiées, do manière à se joindre ot à se souder par l'extrémilé de leurs ramifirations; elles arriveront it former une coque grillagér, rontinue, splhérique, dont les spicules seront lesrayons. Cette coque grillagée est le caractère essentiel des genres de ce sous-ordre. Elle est, comme le

Fig. 318.

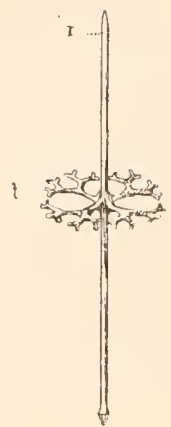

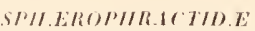

(Type morphologique) (Sch.). In spicule isolé.

r., tige radiale;

t., branches tangenlielles.
Fig. 319.

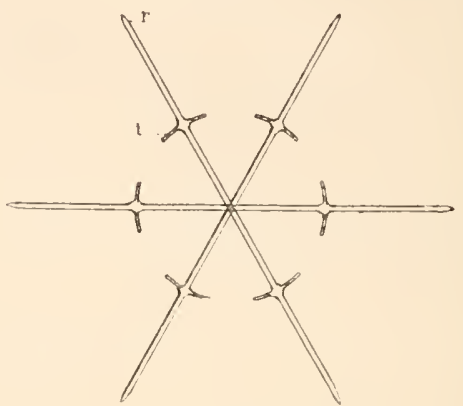

SPH.EROFIIR.1C77II.E

(Type morphologique) (Sch.).

r., spicules radiaires; t., branches langentielles formant par leur accroissement une sphere complete.

reste du squelette chez tous les Acanthaires, formée d'acanthine.

On romprent qu’il puisse se former ainsi plus l'une sphère grillagée, puisqu’il y a des Acanthonides ayant des épines à diverses hauteurs sur les spicules.

\section{GENRES}

Phractaspis (Ilïckel) (fig. 320) a sa coque grillagée ratlachée ì rhapue spicule radiaire seulement par deux tigelles, en sorte qu'on peut consilérer rettr coque comme dérivée de spirules à deux apophyses opposées 0.1 à 0,12$)\left({ }^{(}\right)$.

(1) Il en est de mème des genres ci-ılessous :

Pleuraspis (Häckel) est semblable mais a, en outre, des ipines accessoires $\langle 0,1$ a 0,15$\rangle$;

Dorataspis (Häckel) a, comme les suivants, les apophyses constimitives de la corfuille ilargies en lames percees chacune de deux trous vrais, sans compter les perforations suturales formées par deux encoches rapprochées de deux lames contiguës Phractaspis (im. Hiickel). $(0,11$ it 0,16$)$.

Diporaspis (Iäckel) est un Dorataspis avec des épines accessoires $(0,12$ a 0,16$)$;

Orophaspis (Iläckel) est un Dorotaspis muni, en dehors de la coque grillagée, d'une paire d’épines opposées sur la partie extérieure des spicules $(0,04$ i 0,08$)$ :

Ceriaspis IIächel) est un Dorataspis ì coque grillagée ornée d'un réseau de crètes $(0,1$ i 0,2 ; Hystrichaspis (Häckel) est un Ceriaspis avec des épines accessoires 0,12 i 0.25 ;

Coscinaspis (IIäekel) est un Dorataspis avec quatre i douze vrais trous dans chaque lame de la coque $(0,12$ is 1,15$)$;

Aconthaspis (IIäckel) est de mìme, mais il a, en plus, des épines accessoires $(0,13$ i 0,2$)$. 
Stauraspis (Häckel) a sa coque grillagée rattachée à chaque spicule radiaire par quatre tigelles, en sorte que l'on peut le consilérer comme dérivant des formes d'Acanthonides chez lesquelles les spicules portent quatre épines en croix à la même hauteur $(0,1$ à 0,15$)$ (').

Fig. 321.

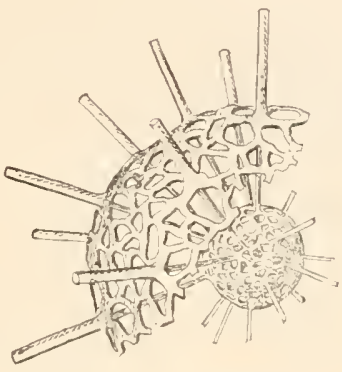

Phractopelta (im. Häickel).
Fig. 322.

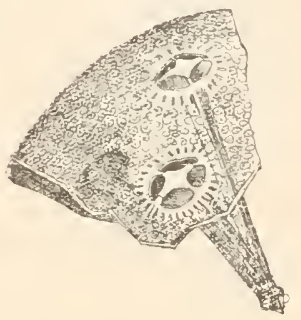

Spharocapsa (im. Häckel).

Sphærocapsa (Häckel) a, au contraire des précédents (fig. 322), la coque grillagée indépendante des spicules et formée d'une multitude de petites pièces indépendantes

(1) Il en est de mème les genres ci-dessous :

Echinaspis (Iläckel) est un Slauraspis i épines accessoires $(0,11$ i 0,15$)$;

Sonaspis (IÏ̈ckel) diffère de staurapsis par le fait que les épines ramifièes des quatre spicules équatoriaux, sans être aplaties en lames comme chez Dorataspis, forment quatre wrais trous, tandis que celles des autres spicules ne forment que des trous suturaux ; il a, en oulre, des épines accessoires $(0,11$ i 0,16$)$;

Dodecaspis (IIäckel) a aussi des épines accessoires et de rrais trous aux épines ramifiées de ses spicules équatoriaux et polaires $(0,12 ; 0,15)$;

Tessaraspis (Häckel) en a aux épines de tous ses spicules et n'a point d’épines accessoires $(0,08$ à 0,18$)$;

Lychnaspis (IIäckel) est un Tessaraspis à épines accessoires $(0,0 \ddot{3}$ ì 0,5$)$;

lcosaspis (IIäckel) est un Tessaraspis qui a aumoins huit trous vrais an système d'épines de cluarue spicule $(0,14$ i 0,1$)$ :

Hyalaspis (Häckel) est un Icosaspis it épines accessoires $\langle 0,18$ it 0,3$\rangle$.

Réunis aux genres Phractaspis. Stauraspis et à cenx qui dépendent l'eux, ces genres forment la famille des DORATASPIN.E [Dorastapida (lläckel)].

(Dans un autre mode de classification, le caractère des rrais trous est pris comme premier crilérium dans cette famille, et permet de la diviser en deux tribus : celles des Cladophracta (Häckel), où l'on réunit les formes qui n'ont de vrais trous à aucune ou a une partie senlement des apophyses de leurs spicules et celle des Peltophracta (Häckel) comprenant les formes qui en ont à tous leurs spicules).

() Genres voisins :

Pantopelta (Häckel) a, en plus, des apophyses sur la partie de ses spicules extérieure aux corpues $(0,08)$;

Octopelta (Häckel) n'en a qu'anx huit spicules tropicaux $(0,08$ ì 0,12$)$;

Dorypelta (Häckel) en a ì quatre de ses spicules polaires et aux huit spicules tropicaux $(0,08$ is 0,11$)$;

Stauropelta (Häckel) en a aux huit spicules tropicaux et aux huit polaires $(0,1$ a 0,12$)$.

Tous ces genres forment la famille des PHRACTOPELTIYE [Phractopeltida [Iäckel)]. 
percées chacune d'un trou. La coque se tient néanmoins, car ses pièces sont soudées bord à bord par un ciment organique. Les spicules sont juste assez grands pour atteindre la coque à laquelle ils se soudent. Mais, au point de soudure, la coque est percée d'un trou rond beaucoup plus granıl que les pores et le spicule est soudé aux bords de ce trou seulement par l'extrémité des crêles saillantes dont il est orné, en sorte qu'il reste, autour de chacun de ces points d'insertion, un assez large orifice découpé suivant la forme du spicule $(0,22$ à 0,3$)$ (').

$3^{\text {e Sous-Ordre }}$

\section{PRUNOPIIRACTIDES. - PRUNOPHRACTIDA \\ [PRUNOPIRACTA (lläckel)]}

\section{TYPE MORPHOLOGIQUE}

(FIG. 323)

11 peut se définir aisément en partant de celui des SEIIEROPIIRACTIDE (V. 1. 209) et en ajoutant aux caractères ile celui-ci que la capsule centrale et la coque grillagée ne sont plus sphériques, mais elliptiques ou lenticulaires ou discoïdes. La forme générale est, en outre, altérée par le fait que les spicules contenus dans le plan méridien sagittal (il y en a deux équatoriaux et quatre polaires) sont tous, ou en partie seulement, plus grands que les autres.

\section{GENRES}

Thoracaspis (llïckel) a la coque ellipsoïdale et les deux spicules équatoriaux sagiltaux seuls plus grands que les autres; en outre, il n'a d'autres vrais trous aux plaques constitutives de sa coque que cenx qui sont destinés au passage des spirules et sont divisées en deux par ceux-ci $(0,14$ à 0,2$)\left({ }^{*}\right)$.

Fig. 323.

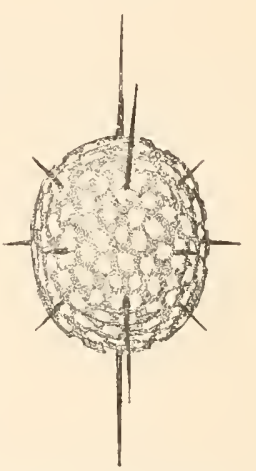

PRLNOPHRACTIDEE

(Type morphologique) (Sch.).

(1) Genres voisins:

Astrocapsa (Iäckel) a ses spicules plus longs et dépassant la coque qu'ils traversent par les orifices en question 0,25 à 0,36$)$;

Porocapsa (Häckel) a, au contraire, ses spicules plus courts et n'atteignant pas la corque qui n'en est pas moins percée d'orifices sul le prolongement des spicules $(0,28$ i $0,5 \%$;

Cannocapsa (Häckel) est de mème et a ses orifices prolongés extérieurement en tubes $(0,15$ it 0,24$)$;

Cenocapsa (Häckel) est comme Porocapsa, mais les spicules radiaires ont disparu $(0,2$ i 0,3 !.

Ces genres forment la famille des SPIIEROCAPSTE [Sphxrocapsida (Häckel)].

(2) Genres voisins:

Belonaspis (Häckel) a la mème structure et, en plus, des épines accessoires $(0,1$ ì 0,22$)$;

Dictyaspis (Häckel) n'a pas d'épines accessoires. mais a sa coque ornèe d'un réseau de crêtes saillantes $(0,12$ à 0,18$)$; 
Hexalaspis (Iläckel) (fig. 32k) a tous ses spicules du méridien sagittal (deux équatoriaux et qualre polaires) plus grands que les autres. Tous d'ailleurs dépassent la coquille $(0,11$ à $0,21)$ (").

Diploconus (Häckel) (fig. 325) n’a, comme Thoracaspis, que les deux spiculeśéquatoriaux sagittaux plus grands que les autres; mais ils sont énormément plus grands et entourés chacun d'une gaine de mème substance formant ainsi deux cônes tronqués opposés par le sommet. Ce sommet tronqué contient la coquille grillagée ordinaire avec les dix-huit autres spicules très petits el la capsule centrale ellipsoïle ou en

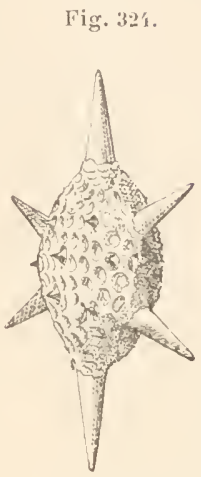

Ilexalaspis im. If iickel).
Fig. 325.

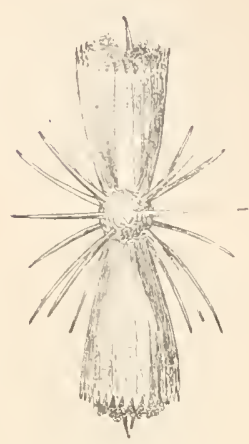

Diploconus (im. Hàckel).

forme de double cône comme la cơquille elle-mème 0,13 a 0,3$)\left({ }^{2}\right)\left(^{*}\right)$

4 SOUs-Ordre

ACTINELIIES. - ACTTIELHA.E

[ArTINELID.1 (Hïliel)]

TYPE MORPHOLOGIQUE

(FIG. 326

Il se caractérise par ses spicules égaux entre rux mais en nombre

Coleaspis (Häckel) rẻunit les épines accessoires et les crêtes des deux genres précédents $(0,10$ à 0,20$)$;

Phatnaspis (Iläckel) n'a ni épines, ni crêtes mais, en outre des trous pour le prassage de ses spicules, a sur chaque lame d'aulres srais trous dont le nombre depasse toujours deux et atteint souvent une centaine $(0,08$ à 0,32 .

(1) Genres roisins:

Hexaconus (IIäckel) est de même, mais a ses spicules tous ou seulement ceux du plan sagittal) entourès d'une gaine proéminente $(0,12$ à 0,2$)$; chez

Hexonaspis (IIäckel) les spicules du plan sagittal dépassent seuls le niveau de la corque; conme chez Hexalaspis ils sont sans gaîne proèminente $(0,11$ ì 0,2 ; ;

Hexacolpus (IIäckel), au contraire, a ces mèmes spicules seuls saillants hor's de la coquille, mais accompagnés d'une gaine comme celle d'Hexaconus $(0,1$ i 0,2$)$.

Ces genres, $y$ compris Thoracalpis et ceux qui se rattachent à lui, forment la famille des IIExatsPIXE [IIexalaspida (IIäcliel)].

(2) Genres voisins :

Diplocolpus (Häckel) est un Diploconus réduit à ses deux granrls spicules, les aulres

(·) Si nous conservions l'orientation que nous arons ordinairement appliquée aux animaux, nous ne pourrions figurer qu'une de ces épines et l'autre se projetterait sous la forme d'un gros point. Pour éviter cette dıfficulté, nons avons placé l'axe antéro-postérieur verticalement de mème que dans certaines des figures précédentes pour le même motif. 
indéfini très grand, et irrégulièrement disposés. Ainsi un des caractères essentiels du type général est ici perdu. Il serait impossible cependant de séparer des Acanthomètres ces formes qui s'en rapprochent par quelques transitions assez bien graduées et s'y rapportent par l'ensemble de leurs autres caractères.

\section{GENRES}

Actinelius (Häckel) est la représentation exacte du type morphologique $(0,2$ a 0,4$)$ (').

Litholophus (Häckel) (fig. 32T) n'a que dix ì vingt spicules. Il possède des myophrisca comme les Acanthomètres. Le corps et la capsule centrale sont coniques, les spicules étant tous dirigés du mème côté $(0,2$ à 0,5$)\left({ }^{2}\right)$.

Chiastolus (Häckel) a aussi trente-deux spicules, mais ceux qui sont opposés sur un mème diamètre se soudent au centre au lieu de s'y joindre seulement comme chez les Acanthomètres et tous les autres.

11 résulte de là qu'il n'y a plus que seize spicules diamétraux au lieu de trente-deux radiaires. Ces spicules se croisent près du centre en passant à côté les uns des autres $(0,3)\left({ }^{3}\right)$.

étant tout à fait ou partiellement atrophiés $(0,1$ a 0,2$)$.

Ces deux genres forment la famille des DIPLOCONITE [Diploconida (Häckel)].

(1) Genres voisins :

Astrolophus (Häckel), par l'inégalité de ses spicules, se sépare davantage encore du type normal $\{0,3$ a 0,5$\}$; mais

Actinastrum Häckel) s'en rapproche par ses spicules en nombre fixe, au nombre de trente-deux. réguliẻrement disposés en einq zones parallèles $(0,2$ à 0,3$)$.

Ces genres forment la famille des AstroLOPIIIN $E$ [A strolophida (Häekel)].

(2) Ce genre forme à lui seul la famille des LITHOLOPHINE [Litholophida (IIäckel)].

(3) Genre voisin :

Acanthochiasma (Krohn) (fig. 328) est semblable, mais n'a que dix spicules diamétraux, dérivant de vingt radiaires $(0.2$ à 1$)$.

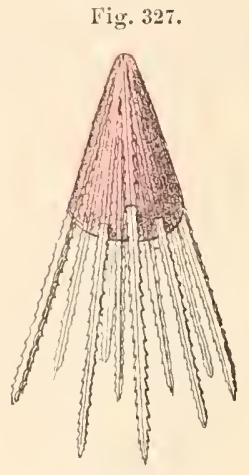

Litholophus im. Häckel).

Fig. 328.

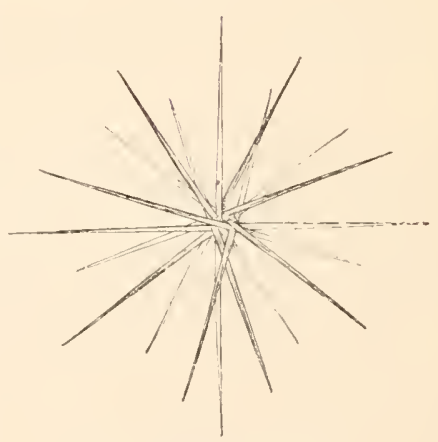

Acanthochiasma (Sch.).

Il forme, avec Chiastulus, la famille des ChIAstoLts:E [Chiastulida IIäekel)]. 


\section{MONOPYLAIRES. - MONOI'YLIIII}

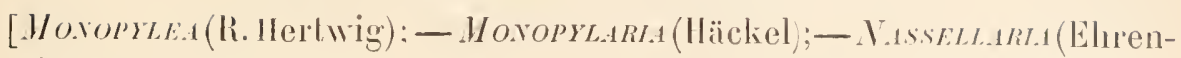

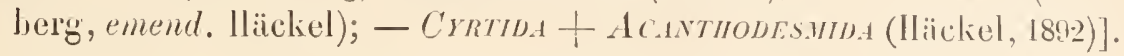

\section{TYPE MORPHOLOGIQUE}

(FIG. 329)

Le lype les Monopylaires diffère essentiellement de celui des aulres ordres par la constitution de sa capsule centrale et frar un squeletle

Fig. 329.

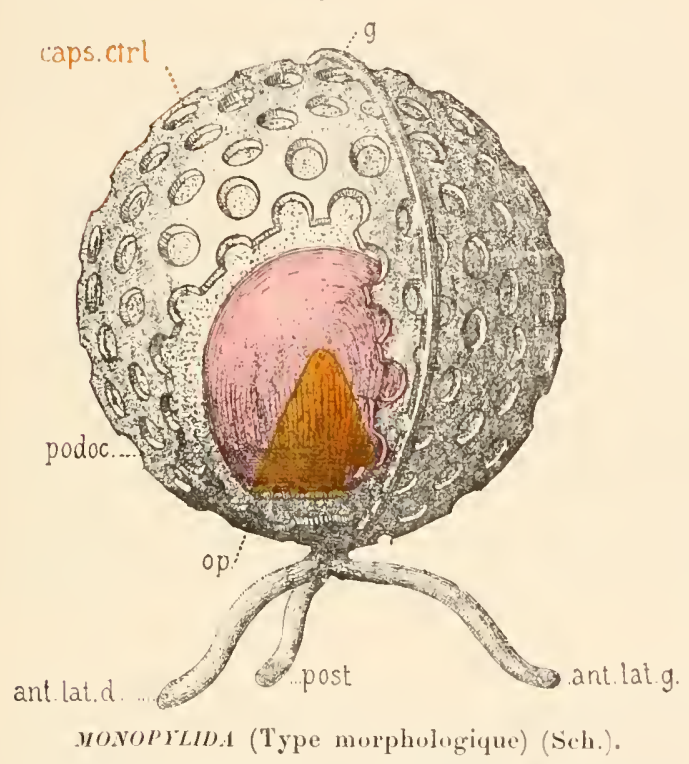

ant. lat. d., branche antéro-latérale droite; ant, lat. ar., branche antéro-latérale gatuche; post., branche postérieure; caps. ctrl., capsule centrale: w., grand cercle; op., opercule; porioc., podocone. dont la forme fondanentale est très caractéristique.

Gapsule centrale. - La caprsule centrale (caps. ctrl.) a la forme d'un ovoïde à grand axe vertical dont la base serait tronquée. Sur toule la parlie convexe, elle est entièrement déprourvue de pores. La hase plane, au contraire, constitue un large orifice, porte dacces unique de la cap'sule, d'où le nom donné

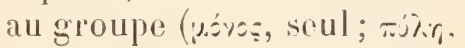
porte). Mlais ce large orifice n'est pas libre; il est fermé par un opercule (op.) percé lui-mème d'un assez grand nombre (une soixantaine peut-ètre) de pores très fins, en sorte que le large orifice est remplacé frar un groupe de petits. Cetopercule poreux est notablement plus épais que le reste de la membrane el, en outre. il se prolonge à l'intérieur de la capsule par un cône obtus au sommet et très élevé que l'on appelle le podocone (podoc.). Les fins canaux qui traversent l'opercule se continuent aussi à travers le podocone et mettent ainsi en communication les protoplasmas intra et extracapsulaires (").

Le noyau refoulé par la saillie du podocone est excentrique.

Organes extracapsulaires. - Le protoplasma extracapsulaire el les pseudopodes sont beaucoup plus abomlants en face de la région poreuse de la

(1) La constitution exacte de ce singulier organe est difficile a préciser el donne lieu 
capsule, à peu près conme chez les Foraminifères imperforés en face de la bouche de la coquille.

La gelée, les Zooxunthelles (ici extracapsulaires comme partout saut chez les Acantharia), n'offrent rien de particulier.

Squelette. - Le squelelte est formé de silice et les tigelles qui le constituent sont pleines. Il présente trois parties bien distinctes:

$1^{\circ}$ Le trépied formé de trois baguettes réunies en un point central commun et divergeant sous des angles égaux; il est orienté dans un plan perpendiculaire à l'axe vertical et situé immédiatement au-dessous de la capsule centrale qui s'appuie sur lui par sa base plane et poreuse; une de ses branches est postérieure (fig. 329, post.), les deux autres antéro-latérales (ant. lat.);

$2^{\circ}$ Le capitulum ou coque grillagée, sphérique ou ellipsoïde à grand axe vertical, renfermant la capsule plus une masse plıs ou moins grande de tissa extracapsulaire, fixé sur le milieu du trépied, au-dessus de lui;

$3^{\circ}$ Enfin, l'anneau $(g)$, cercle siliceux qui renforce la paroi de la coque grillagée dans le méridien sagittal.

La capsule centrale est en somme peu variable et se retrouve à peu près partout avec les caractères essentiels que nous venons de lui décrire; elle forme seulement des lobes parfois très saillants pour accompagner certaines déformations de la coquille.

Mais la coquille, elle, est extrèmement variable et n'existe peut-être nulle part, simple et complète à la fois, comme nous l'avons décrite. Non seulement toutes ses parties peuvent se compliquer d'apophyses, d'épines contournées, ramifiées de mille façons, mais elle peut disparaìtre, ou plus souvent se réduire à une ou deux de ses trois parlies essentielles, et res parties, surtout la coque grillagée, peuvent subir des variations lle forme extrèmement étendues que ra nous montrer l'étude des genres.

Lordre se divise en six sous-ordres:

NASSOIDE, entièrement dépourvus de squelette;

PLECTOIDE, à coque grillagée nulle ou incomplèle et toujours privés d'anneau, mais pourvus d'un trépied à trois branches ou plus;

STEPIIOIDE, à coque nulle ou incomplète et ordinairement privés de trépied, mais toujours pourvus d'un anneau simple ou inultiple;

CYRTOIDE, à coque grillagée complète, simple, sans constrictions vorticales;

SPYROIDE, à coque complète, mais divisée en deux loges par une constriction sagittale;

BOTRYOIDE, à coque complète, mais livisér en loges plus ou moins nombreuses par des constrictions verticales.

¿̀ des divergences d'interprétation. Il faut se représenter l'opercule comme un disque épais, criblé comme une écumoire de trous que remplissent de petits dés cylindriques (fig. 330, c' qui se teignent par le carmin plus fortement que le reste de la membrane ou de l'opercule. Le podocone est formé d'une substance qui se teint aussi par le car- 
$1^{\text {er Sous-Ordie }}$

\author{
NASSOÏDES. - NASSOIDA \\ [NASSOIDE (Här.kel)]
}

TYPE MORPHOLOGIQUE

(FIG. 330)

II suftit de retrancher son squelette au type morphologique que nous venons de décrire pour en faire celui des NASSOIDE.

\title{
GENRES
}

Cystidium (Häckel) est le genre principal ol presque unique du groupe. Il réalise le type morphologique. C'est un Actissa à capsule centrale monopylaire (Grand axe de la capsule 0,06 à 0,12 (').

Fig. 330.

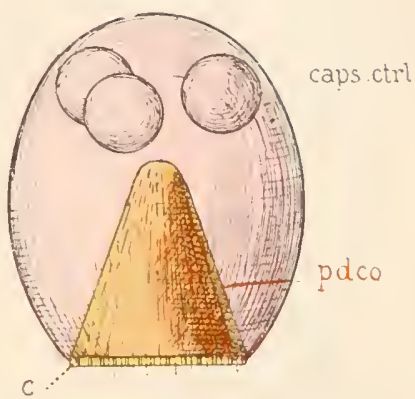

NASSOID.E(Type morphologique) (Cystidium) (Sch.).

$2^{\text {e SOUS-ORDRE }}$

$$
\text { PLECTOÏDES. - PLECTOIDA }
$$

[Plectoidea (Häickel); - PlagiaCantilda (R. Hertwig.emend.)]

\section{TYPE MORPHOLOGIQUE}

(FIG. 331)

C'est un Monopylaire chez lerjuel le squelette est réduit au trépied. Ce trépied est, en outre, aussi simple que possible, étant composé de trois branches

min. Ces dés sont traversés, chacun suivant son axe, d'un fin filament qui s'engage ensuite dans le cone et le (raverse dans toute sa hauteur. I)'après HenTwig: [79] qui a, le premier, bien étudié ces dètails, les petits des et le cône sont formés d'une substance analogue a celle de la membrane capsulaire, et les filaments qui les traversent sont des tractus protoplasmiques qui mettent en communication les plasmas intra et extracapsulaires et peuvent, par suite, être considérés comme les origines des pseudopodes. D'après IIÄCKeL [87] les petits dés seraient de petites soupapes pleines, non perforées, mais libres de jouer dans l'orifice 'ju'elles bouFig. 331 .

chent en se soulevant un peu. Les filaments du podocone seraient des myophrisea, insérés sur eux (sans les traverser) et destinés à les mouvoir. Les tractus protoplasmiques établissant la communication entre le dedans et le dehors de la capsule passeraient non dans ces pelits dés, mais entre eux et, en se soulevant, ils leur ouvriraient un passage plus large. Enfin le podocone ne serail qu'une masse de protoplasma intracapsulaire parcourue par les moplırisca. Tout cela attend de nouvelles recherches.

(1) Genre voisin :

Nassella (Häckel) est un Cystidium à grelée très alvéolaire $(0,1$ i 0,12 ). 
non ramifiées, droites, équidivergentes $(s q$.$) , disposées dans un plan$ horizontal et supportant une capsule centrale monopylaire (caps. ctrle). Des trois branches, une est postérieure, les deux autres antéro-latérales.

\section{GENRES}

Triplagia (Häckel) (fig. 33I) réalise, très exactement, le type morphologique $\{0,15$ à 0,25$)(")(*)$.

Triplecta (Häckel) (fig. 332) a aussi un trépied à trois branches horizontales, égales, équidivergentes, mais ces branches émettent des ramificalions latérales anastomosées en une sorte de tissu grillagé qui, sous une forme rudimentaire, incomplète, joue le rôle protecteur de la coque grillagée absente $\langle 0,14$ à 0,15$\left.\rangle)^{*}\right)$.

(1) Genres voisins :

Plagiacantha (Claparède) a les branches du trépied légèrement ascendantes $(0,12$ à 0,30$)$;

Tetraplagia (Häckel) a un tripied à quatre branclies disposées Fig. 3:2. dans deux plans rectangulaires et obliquement ascendantes $(0,12$ à 0,2$)$;

Plagoniscus (Häckel) a un trépied a quatre branches, dont trois horizontales et la quatrieme verticalement ascendante 0,1 a 0,3$)$;

Plagonidium (IIäckel) a quảtıe branches aussi, mais non iquidistantes, disposées en deux paires partant, non d'un point commun, mais les extrémités d'un petit bàtonnet horizontal et toutes semblables $\{0,16$; 0,18$)$;

Plagiocarpa (Häckel) est de même, mais l'une de ses branches est verticale et oppusée aux trois autres qui sont descendantes $(0,15$ à 0,27$)$;

Hexaplagia (Häekel) a un trépied à six branches $(0,12$ à 0,3$)$;

Plagonium (Häekel) a aussi six branches, mais disposées en deux groupes de trois $\langle 0,12$ à 0,2$\}$

Polyplagia (IIäckel) a beaucoup de branches (7 a 10 au moins) $(0,12$ à 0,26$)$.

Ces genres forment la famille des PLAGOrdNA [Plagonida (IIäckel)].

(2) Ce caractère se retrouve dans les genres ci-dessous:

Plectophora IIäckel) est un Triplecta à branches obliquement ascendantes $(0,12$ it 0,2$)$;

Tetraplecta (Häckel) a un trépied à quatre branches semblables $(0,15$ à 0,3 ;

Plectaniscus (Häckel) a quatre branches, dont une verticalement ascendante est opposée aux trois autres $(0,05$ à 0,32$)$;

Periplecta (IIäckel) a quatre blanches aussi, mais formant deux paires qui partent des extrémités d'un batonnet horizontal 0,25 à 0,3$)$;

Hexaplecta (lläckel a six branches partant d'un point central commun 0,22 i 0,25 ;

Plectanium IIäckel a six branches partant en deux groupes des extrémités d'un batomuet $(0,17$ iो 0,25$)$;

Polyplecta (IIäckel) a vept ì dix branches au moins $(0,05$ à 0,4$)$.

Ces genres forment la lamille des PLEeT.1 NTxE [Plectunida, IIäliel)].

(“) Ces dimeusions ainsi que les suivantes sont celles des épines. 
$3^{\text {e }}$ Sous-Ordre

\section{STÉPHOÏDES. - STEPHOII,E}
[STEPHOHEA (IIäckel); - STEPHIDA (Häckel): - ACANTHODESHDA (Bülschli, emend.)]

\section{TYPE MORPHOLOGIQUE}

(FIG. 333)

C'est notre Monopylaire typique, mais à squeletle sans coque grillagée, et réduit au trépied et à l'anneau sagittal. Cet anneau est soudé verticalement au centre du trépied et entoure la capsule qui, plus petite que lui, s'appuie sur sa hase. Les branches du trépied sont: une pos. lérieure et deux antéro-latérales $(0,07$ à 0,17$)\left({ }^{*}\right)$.

\section{GENRES}

Cortina (Häckel) (fig.334) est la réalisation de ce lỵje('). Archicircus (Häickel) (fig. 333̈) est un Cortina sans trépied. Son squelelte est donc réduil à un simple anneau verlical, sagitlal, r'égulier, parfois orné d'épines simples $(0,04$ à 0,15$)\left({ }^{2}\right)$.

Cortiniscus (Hïckel)

(fig. 336) est un

Cortina chez

lequel des ra-

mifications ,

arquées el

soudées en-

Ire elles, des

branches du

Fig. 33\%.

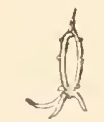

Cortinu

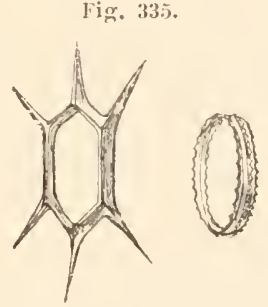

trépied ont

(im. Häckel). Archicircus in. Itackel
Fig., 333.

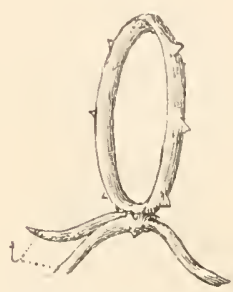

STEPIIOID.E

(Type morphologicue) (Sch.).

Fig. 336.

formé un deuxième anneau plus ou moins horizontal silué entre l'anneau typique verlical et le trépied $0,08^{\prime}$ à 0,18$\rangle\left({ }^{3}\right)$.

(1) Genre voisin :

Stephanium (IIäckel) est un Corlina dont le tripied a quatre branches, une antérieure, une postérieure et deux antéro-latérales $\{0,12$ ì 0,16$\}$.

(2) Genres voisins:

Lithocircus (J. Müller) est un Archicircus à anneau orné d'épines ramifiées $(0,07$ a 0,15$)$;

Zygocircus (Bütschli) est un Archicircus dont l'anneau est aplati en arrière $(0,04$ a 0,2$)$; Dendrocircus Häckel) est un Lithocircus avec la même modification $(0,04$ ì 0,11$)$.

Ces genres forment la famille des STEPHANINE [Stephanida (Häckel)].

(3) Genres voisins:

Stephaniscus (Häckel) est semblable, mais son trépied a quatre branches, une antérieure, une postérieure et deux antéro-latérales $\langle 0,08$ à 0,13 ;

(·) Ces nombres et les suivants indiquent le diametre de l'anneau. 
Semantis (IIäckel) (fig. 337) est un Cortiniscus mais sans trépied : il est réluit à ses deux anneaux. Mais, tandis que la eavité de l'anneau vertical est libre, celle de l'horizontal est découpée par la base du vertical en deux orifices, l'un droit, l'autre gauche $(0,06$ is 0,12$)$ (').

\section{Zygostephanus (Häckel) (fig. 338) ne} possède du squelette typique complet que l'anneau sagiltal, comme Archicircus. Mais il s'y ajoute un second anneau vertical perpendiculaire au premier et par conséquent coronal. Cela

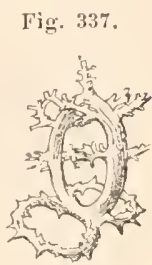

Semantis

(im. Häckel).
Fig. 338 .

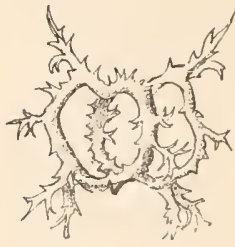

Zygostephanus

(im. Häckel). forme le cadre d'une coque sphérique communiquant arec le lehors par quatre énormes ouverlures, deux antéro-latérales et deux postérolatérales $(0,09$ à 0,20$)\left({ }^{*}\right)$.

Semantiscus (Häckel) est semblable encore, mais son trépied a six pieds, ceux du genre précédent, plus une paire postéro-latérale $(0,08$ i 0,12$)$.

(1) Genres voisins :

Semanthum (Häckel) a ses deux trous recoupés en quatre par deux tigelles qui les traversent $(0,08$ ì 0.1$)$;

Semantidium (IÏ̈ckel) les a recoupés en six par quatre tigelles $(0,08$ à 0,14);

Ch/athrocircus (Häckel) a les quatre trous basilaires du semanthum et, en outre, des trous diversement disposés mais toujours par paires symétriques le long de l'anneau vertical et léterminès par des apophyses de celui-ci $(0,08$ à $0,10 \%)$.

Réunis aux genres Cortiniscus. Semantis et à ceux qui dépendent d'eux, ces genres forment la famille des SEMANTIYE [Semantida (IIäckel)].

(2) Genres voisins :

Zygostephanium lläckel) a ses quatre grandes ouverlures en partie grillagèes par des apophyses ramifiées et anastomosées $(0,12$ à 0,18 ;

Coronidium (Häckel) a ses denx anneaux verticaux tronqués en bas et inserís sur un troisième anneau horizontal 0,14 is 0,18 ;

Acanthodesmia (J. Müller) est pareil, mais avec ses quatre ouvertures partiellement grillagées $(0.12$ à 0,18$)$;

Trissocircus (IIäckel) a ses deux anneaux verticanx complets, mais recoupes prar un anneau horizontal qui, au lieu d'être à leur base, est à l'équateur de la sphere; cela forme le cadre d'une sphère laissant huit larges ouver tures régulières $(0,08$ i 0,12$)$;

Trissocyclus (Häckel\, fig. 339) est pareil, mais ses huit ouvertures sont partiellement grillagées $(0,13$ à 0.16$)$;

Tristephanium (IIäckell est un Trissocircus dont l'anneau horizontal est au-dessous de l'équateur $(0,12$ ì 0.2$)$;

Tricyclidium [lläckel représente le précédent avec ses ouvertures partiellement grillagées 0,15 à 0,18 ;

Eucoronis IIärkel) a aussi trois anneaux, mais l'anneau horizontal, situé très bas, laisse le sagittal complet, tandis que le corona] se termine sur lni par ses extrémilés tronquies $(0,14$ i 0,23$)$ :

Fig. 339.

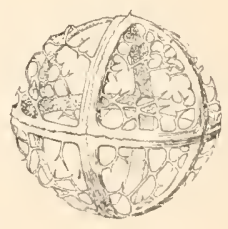

Trissocyclus (im. Häckel).

Plectocoronis (Ilärkel) est le précédent aver les ouvertures partiellement grillitgies $(0,13$ i 0,16 ; 
Protympanium (Iläcliel) (fig. 340) n'a du squelette typirjue que l’anneau sagittal; il s'y ajoute deux anneaux horizontaux, un inférieur basal, un supérieur apical qui sont complels et coupent l'anneau sagittal; il en résulte deux larges fenètres verlicales lalérales el quatre fenètres horizontales, deux en haut et deux en bas $(0,07$ a 0,15 (') ('

Podocoronis (Iläckel) représente Eucoronis augmenté de branches descendantes qui s'insèrent régulièrement sul l'anneau horizontal $(0,13$ à 0,2$)$.

Ces genres forment la famille des Conovire [Coronida

Fig. 340.

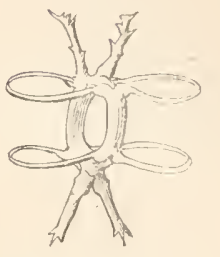

Protympanium

(im. Häckel). [Häckiel)].

(1) Genres voisins :

Acrocubus (Häckel) a, en plus, un anneau coronal et se trouve ainsi formé par deux anneaux parallèles réunis par deux anneaux se coupant à angle droit $(0,08$ à 0,21$)$; Toxarium (Häckel) possède, en plus, quatre ares verticaux, deux en haut, deux en bas, formés par des apophyses courbes soudées en cercle $(0,12$ ì $(0,2)$;

Microcubus (IIäckel) n'a point ces ares supplémentaires, mais possède un troisième anneau horizontal, équatorial $(0,12$ à 0,15$)$;

Octotympanum (Häcliel) a aussi ce troisième anneau, mais incomplet $(0,12$ à 0,2$)$;

Tympaniscus (Häckel) revient aux deux anneaux lorizontaux simples, mais coupés par trois anneaux verticaux $(0,09$ a 0,18$)$;

Tympanidium (Iläckel) les a coupés par quatre anneaux verticaux $(0,1$ ì 0,203$)$;

Parastephanus (Iläckel) est un Protympanium dont on aurait supprimé les parties de l'anneau sagiltal qui ne sont pas comprises entre les deux anneaux horizontaux. Ceux ei sont done simplement réunis par deux tigelles verticales daus le plan sagittal, vestiges d'un anneau sagittal incomplet $(0,08$ à 0,10$)$;

Prismatium (Häckel) a trois tigelles, restiges d'un anneau coronal et d'un deni-anneau sagittal 0,0 ' ì 0,09$)$;

Lithocubus (Häckel) a quatre tigelles, restes de deux anneaux complets $(0,05$ a 0,07$)$;

Pseudocubus (Häckel) est de même, mais les deux anneaux horizonlaux ne sont pas de mème taille, ce qui lui donne une forme générale tronconique $(0,05$ à 0,1$)$;

Eutympanium (Häckel) a six ou huit tigelles, restes de Irois a quatre anneaux $(0,08$ a 0,13$)$;

Circotympanum (IIäckel) est semblible, mais avec les deux anneaux horizontaux inégaux $(0,09$ à 0,12$)$;

Dystympanium (IÏ̈ckel) (fig. 341) a aussi six à huit tigelles ou même plus et ses anneaux inégaux, le supérieur plus petit, mais la fenêtre enclose par l'anneau supérieur est fermée par un tissu grillagé émanant de cet anneau $(0,09$ i 0,12$)$; Lithotympanum (Häckel) est de même, mais a ses anneaux égaux et ses deux fenêtres horizontales aussi obturées par du tissu grillagé 0,12 ¿̀ 0,13 ;

Fig. 341.

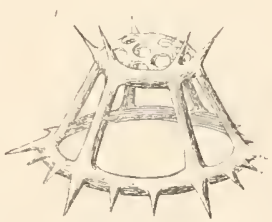

Dystympanium

(im. Häckel).
Fig. 342.

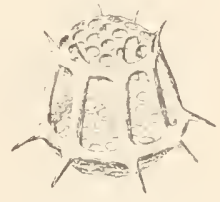

paratympanum

(im. Hïrel).

Paratympanum (Häckel) (fig. 342) est comme le précédenl, mais a l'anneau supérieur plus pelit que l'inférieur $(0, n 9$ à 0.1$)$.

Ces genres forment la famille des TrMPAXINE [Tympanida (Häckel)]. 
$4^{\text {e Sous-Ordre }}$

CYRTOÏDES. - CYRTOIDE

[CrRtoidea (Häckel); - Polycystina (Ehrenherg, p.p.); - Monodictya (Ehrenberg, $p \cdot p \cdot)]$

\section{TYPE MORPHOLOGIQUE}

(FIG. 343 ET 344 )

Comparé au type morphologique des Monopylaires, celui-ci s'en distingue par l'extrème réduction ou la disparition complète de l'anneau. Il comprend donc essentiellement une coque grillagée sphérique montée sur un pied à trois branches dont une postérieure et deux antéro-latérales. Dans cette coque, on observe souvent une sorte d'arc postérieur montant comme un demi-méridien de la base de la hranche impaire du pied jusqu'au sommet de la coque, oì souvent il se prolonge en une corne apicale.

Cette forme est la plus simple que puissent présenter les Cyrtoïdes. Mais souvent elle se complique d'une manière qui demande à ètre définie. Des branches du pied, qui sont obliquement descendantes, partent des apophyses ramifiées qui anastomosent leurs branches et forment, entre ces branches, un tissu grillagé tout semblable à celui qui forme la coque. De la sorte, naît un second compartiment de la coquille auquel on donne le nom de thorax (fig. 34 th.) et qui est séparé de la coque sphérique primitive, qui prend
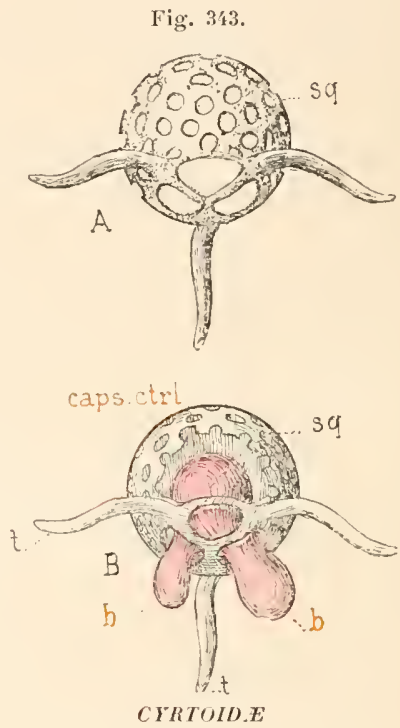

(Type morphologique) (Sch.). A. Coquille vide;

$B$. Coquille avec la capsule. le nom de tête $(c)$, par un étranglement cervical circulaire et transversal, la communication entre les cavités céphalique et thoracique étant ou libre ou rétrécie par un septum grillagí.

Pareille chose peut se renouveler une seconte fois et donner naissance au-dessous du thorax à un troisième compartiment, l'abdomen (ab.), séparé de celui-ci par un étranglement lombaire; et l'abdomen peut être lui-mème étranglé de distance en listance par des constrictions secondaires de moindre importance. L'abilomen, ou le thorax quand celui-ci manque, est normalement ouvert en lessous, mais un empiètement secondaire du tissu grillagé pariétal peut aussi le fermer.

La capsule centrale (fig. $343, B$ : caps. ctrl.) ne subit aucune modification quand la téte est assez grande pour la $\operatorname{loger}(A)$. Mais dans le cas 
contraire $(B)$, si elle est gènée par les branches du pierl, elle est obligée de former des lobes $(b, b)$ qui s'insinuent entre ces branches et pendent dans le thorax.

Ces trois éléments de variation : présence ou absence d'un thorax ou d'un abdomen, ce lernier étant segmenté ou non; absence ou présence

Fig. 3its.

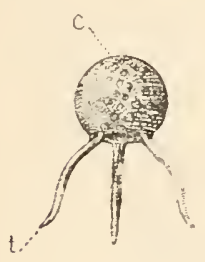

CIRTOID.E (Type morphologique) Sch.. Complication croissante de la coquille par l'addilion du thorax ot de l'abdomen.

al.. abdomen; als'., abdomen serondaire; c., tète; t., lranches du trípied; th., thorax. d'un pied et nomhre de ses branches; présence ou absence d'une lame grillagée pour fermer ou non le compartimentinférieur de la coquille : tout cela se combine de manière à permettre une subdivision du groupe en sections homogènes 'pui facilitent singulièrement la classification. Et cela

est d'autant plus à apprécier que ce groupe immense contient plus de onze cents espèces et que la plupart des formes fossiles lui appartiennent, certaines d'entre elles étanl assez riches en individus pour former des roches entiires.

Nous appellerons (') :

MoxocrRtö̈nes. MovorytroIDEA, ceux qui ont pour coque unesimple sphère sans étranglements ;

Dicyrtoünes. Micyrtome.1, ceux qui ont une tète et un thorax;

Tricritö̈des. Tricytardea, ceux qui ont une tète, un llorax et 1111 abdomen insegmenté: et

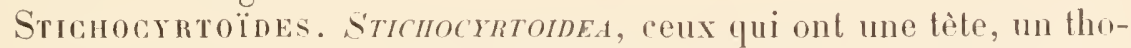
l'ax et un abdomen et dont l'abdomen est segmenté.

Et, dans rhacune de ces sections, nous allons trouver des croupes ayant soit trois branches an pied, soit quatre branches ou plus, soit point de pied.

Enfin, dans chacun des groupes de chacune de ces sections, nous trouverons des genres à corfuille librement ourerte en dessous ou fermée en cette région par un prolongement du tissu grillagé ou autrement, ce que l'on exprime en disant que la coquille est à bouche lilwement ouverte ou à bouche diaphragmée, en sorte que nous pourrons en quelques mots caractíriser le genre tỵpe de chaque série.

(1) Nous considérons ces subdivisions comme des simples groupes de genres, aussi leur donnons-nous la désinence en ea des groupes hors cadre. 


\section{GENRES}

\section{Monocyrtoïdes - Monocyrtoldea. \\ [MovocrRtid.1 (Häckel); - CrRTOIDEA MONothaL.1MA (Häckel)]}

Tripocalpis (Iläckel) (fig. 3łö) est un Monocyrtö̈le à pied pourvu de trois branches et à bouche librement ouverte. Il porte, en outre, une corne apicale et les branches de son pied se prolongent en une côte saillante jusqu'au sommel de la coquille $\quad 0,13$ à $0,27)(')$.

Archibursa (Häckel) (fig. 343) est un Monocyrtoïde à pied muni de trois branches et à bouche diaphragmée $(0,1)\left({ }^{2}\right)$.

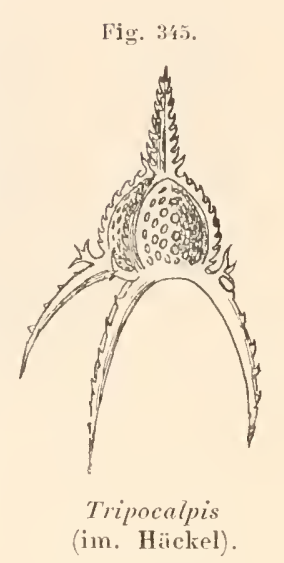

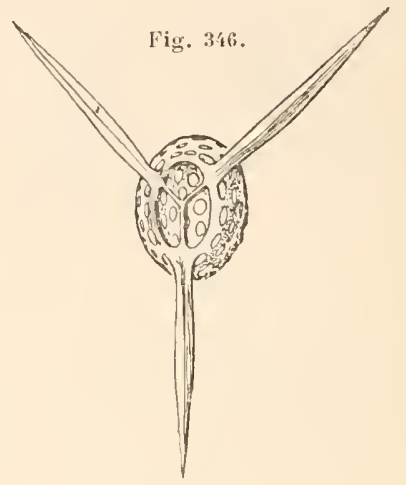

Archibursa (im. Häckel).

(1) Genres voisins :

Tripodonium (Häcliel) n’a pas de corne apicale $(0,1$ à 0,18$)$;

Archipilium Häckel) n'en a pas non plus et les lranches de son pied n'atteignent pas en bas le niveau de la bouche $(0,08$ a 0,09$)$;

Trissopilium (Häckel) est de même, mais a une corne $(0,18$ à 0,24$)$ :

Tripterocalpis (läckel) n'a pas de corne et est comme Archipilium, mais sa bouche est armée au horl libre de forles épines 0,18 à 0,2$)^{\prime}\left(^{*}\right)$;

Tripilidium (Häckel) a une corne, son pied ne dessine pas de cotes sur la coquille ef forme trois saillies libres $(0,12$ à 0,32$)$;

Tripodiscium (Häckel) est semblable, mais sans corne (1),08 à 0,22);

Tridictyopus (Häckel) n’a pas de corne, mais son pied est grillagé $(0,3$ à 0,4$)$.

(?) Genres voisins :

Peridium (Häckel) est semblable, avec une corne en plus $(0,14$ à 0,2$)$;

Archipera (Iäckel) semblable encore avec deux cornes ou plus $(0,1$ à 0,2$)$;

Euscenium Häckel) est un Archibursa muni d'une columelle c'est-à-dire d'une tige verticale qui part du centre du pied, monte dans l'axe de la coquille et se termine par une corne apicale $(0,15$ à 0,25$)$;

Cladoscenium (Häckel) est semblable, mais a sa columelle branchue $(0,15$ à 0,3$)$;

Pteroscenium Illärkel) diffère du précédent par trois ailes de tissu grillagé qui réunissent la corne aux trois branches du pied $(0,06$ à 0,1$)$ :

Archiscenium (Häckel) présente le mème caractère, mais sa columelle est simple $\{0,04$ à $0,05 \%$.

Réunis à Tripocalpis, à Archibursa et aux genres qui dépendent d'eux, ces genres forment la famille des TrIPOCaLPTIE [Tripocalpida (Häcliel)].

(•) La distinction entre les épines buccales et les prolongements des branches dn pied est quelque jeu arbitraire. 
Bathropyramis (Häckel) (fig. 347) est un Monocyrtoïde à pied muni de six à neuf branches ou plus, et à bouche librement ouverte. Les branches de son pied se prolongent en haut en côtes saillantes à l'intérieur de la coquille qui est de forme pyramidale et sans corne $(0,2$ a 0,4$)$ (").

Archiphæna (Häckel) (fig. 348) est un Monocyrtoïde à pied muni de six à neuf branches ou plus et à bouchediaphragmée 0,1 à $0,3\left(^{2}\right)$.

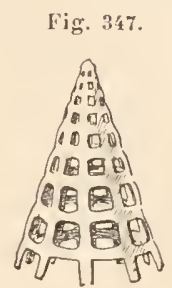

Bathropyramis (im. Häckel).
lFig. 3 १.

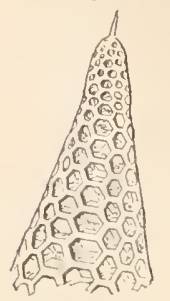

Cornutella (im. Häckel).

Cornutella (Ehrenberg) (fig. 349) a, en plus, une corne $\left(0,08\right.$ à 0,25) ( $\left.{ }^{3}\right)$. Archicapsa (Häckel) (fig. 330) est un Monocyrtö̈de sans pied à bouche diaphragmée $(0,09$ à 0,11$)\left({ }^{1}\right)$.

(1) Genres voisins :

Cinclopyramis (Häckel) est semblable, mais son tissu grillagé est louble, formant des mailles fines dans les grandes $(0,2$ à 0,4$)$;

Peripyramis (Häckel) est semblable aussi, mais son tissu est formé de deux couches grillagées superposées $(0,32$ à 0,45$)$;

Litharachnium (Häckel) diffère de Bathropyramis par la forme discoïdale ou campanulée aplatie de sa cơquille $(0,18$ à 1,12);

Fig. 350,

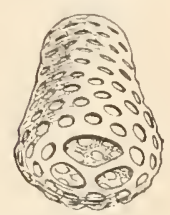

Archicapsa (im. Häckel).

Cladarachnium (Häckel) de même, mais ses còtes intèrieures sont ramifiées $(0,16)$; Cyrtophormis (Häckel) est un Bathropyramis de forme ovale ou liautement campanulée; sa bouche est rétrécie mais à bord simple $(0,09$ à 0,2$)$;

Archiphormis (Häckel) est semblalıle, mais à bouche armée d'épines $(0,08$ à 0,13 ;

Haliphormis (Ehrenberg) est comme le précédent avec une corne en plus $\{0,06$ à 0,12$\}$;

Carpocanistrum (Häcliel) diffère de Bathropyramis par l'absence des còtes intérieures saillantes, sa bouche est armée d'une couronne d'épines $(0,07$ à 0,31$)$;

Halicalyptra (Ehrenberg) est comme le précédent avec une corne en plus $\{0,06$ à 0,42$\}$;

Arachnocalpis (IIäckel) est un Carpocanistrum à tissu formé de deux couclıes grillagées superposées $(0,3$ à 0,46$)$.

(2) Genres voisins:

Calpophæna (Iläckel) a une corne en plus $(0,15$ à 0,40$)$;

Phænocalpis (IIäckel) est un Archiphæna avec une columelle simple $(0,11$ à 0,18$)$;

Phænoscenium (Häckel) est de mème, mais sa columelle est branchue $(0,09$ à 0,19$)$.

Cette série de genres forme avec la précédente la famille des PIIENOCALIXF [Phrenocalpina (IIäckel)].

$\left({ }^{3}\right)$ Genres voisins :

Cornutanna (Ï̈̈ckel) est un Monocyrtoïde sans pied et à bouche libre; sa coquille est conique $(0,1$ à 0,2$)$;

Cyrtocalpis (Häckel) est un Cornutama de forme ovale à bouche rétrécie $(0,08$ à 0,18$)$; Archicorys (IIäckel) est comme le précẻdent avec une corne en plus $(0,19$ à 0,23$)$; Mitrocalpis (IIäckel) diffère de Cyrtocalpis par son tissu grillagè à deux couches $(0,35)$; Spongocyrtis (Dunikovski) diffère du même par son tissu grillagé spongieux $(0,32$ à 0,35$)$.

(4) Genre voisin :

Halicapsa (Häckel) a, en plus, une corne $(0,17$ à 0,29$)$; 


\section{Dicyrtoïdes. - DicyrtoideA}

\section{[DICYRTIDA (Häckel); — CYRTOIDEA DITIIALAMIA (Häckel)]}

Sethopilium (Iläckel) (fig. 351) est un Dicyrtoüde. A ce titre il a donc sa coquille composée d'une téte et d'un thorax séparés par un étranglement. Son pied est composé de trois branches; mais ces trois branches sont ici noyées dans la paroi grillagée sur laquelle elles dessinent trois côtes parfois à peine visibles, et ne deviennent libres qu'en dessous. La bouche (c'est-à-dire l'orifice inférieur du thorax) est librement ouverte, tandis que l'orifice qui fait communiquer la tète et le thorax peut être plus ou moins diaphragmé $(0,09$ à 0,11$)\left({ }^{\prime}\right)$.

Fig. 351.

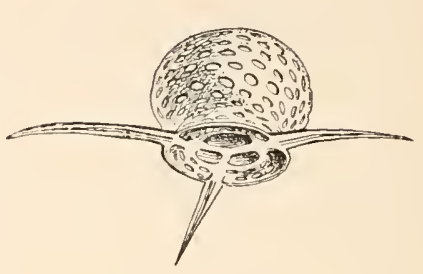

Sethopilum (im. Häckel).

Ces deux genres forment, avec la série précédente, la famille des CrRtocalpty.E [Cyrtocalpina (Häckel)].

(1) Genres voisins:

Dictyophimus (Ehrenberg) est conformé comme Sethopilium, mais il possède en plus une corne $(0,01$ à 0,66$)$;

Tripocyrtis (Häckel) de même, mais en outre le tissu grillagé s'étend jusqu'à la pointe de son pied $(0,15$ à 0,17$)$;

Psilomelissa (Häckel) a les trois còtes thoraciques prolongées latéralement en épines ou en ailes $(0,08$ à 0,12$)$;

Lithomelissa (Ehrenberg) est pareil, mais avec une corne $(0,08$ à 0,18$)$;

Spongomelissa (Häckel) pareil encore, mais à tissu grillagé spongieux $(0,08)$;

Chlathrocanium (Ehrenberg) a son tissu grillagé normal, mais percé de trois larges ouvertures entre les côtes $(0,09$ à 0,12$)$;

Lamprodiscus (Ehrenberg) a ses còtes complètement noyées dans la paroi au niveau du thorax qui est aplati $(0,1$ à 0,13$)$;

Lampromitra (Häckel) est un Lamprodiscus à bouche armée d'une couronne d'épines $(0,08$ à 0,19$)$;

Callimitra (Häckel) a une corne céphalique formée par le prolongement d'une columelle centrale et réunie par trois grandes ailes grillagées aux trois còtes noyées dans la paroi du thorax $(0,19$ à 0,22$)$;

Clathromitra (Häckel) a en plus une corne frontale $(0,05$ à 0,1$)$;

Clathrocorys (Häckel) diffère de Callimitra par trois larges ouvertures dans son tissu grillagé, entre les côtes $(0,17$ à 0,2$)$;

Eucecryphalus (Häckel) a les còtes pédieuses complètement détachées du thorax qui est libre en dedans d'elles $(0,03$ à 0,17$)$;

Amphiplecta (lläckel) a ses côtes pédieuses libres à l'intérieur du thorax et une large ouverture au sommet de la tête $\{0,09$ à 0,15$)$;

Lychnocanium (Ehrenberg) n'a plus de côtes, les trois branches de son pied naissent libres au-dessous du thorax $(0,08$ à 0,14$)$;

Lychnodictyum (Häckel) est pareil, mais ses branches pédieuses sont grillagées $\{0,08$ à 0,11 ). 
Sethopera (Häckel) (fig. 33̆2) est un Dicyrtoïde à pied formé de trois branches et la bouche operculée $(0,07$ à 0,12$)$ (").

Acanthocorys (Iläckel) (fig. 353) est un Dicyrtoöde à pied formé de nombreuses branches (neuf et plus) et à bouche libre. Les branches du pied forment sur le thorax des còtes épineuses et dépassent le thorax en autant de pointes libres; il y a ordinairement des cornes céphaliques $(0,07$ it 0,17$)\left({ }^{2}\right)$.

(1) Genres roisins:

Lithopera (Ehrenberg) diffère du précédlent par ses còtes pédieuses qui sont libres dans la cavité thoracique au lieu d'être noyées dans l'épaisseur de ses parois $(0,1$ à 0,19$)$;

Fig. 352.

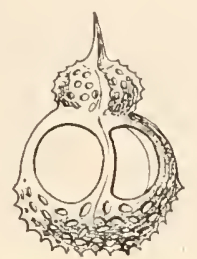

Sethopera

(im. Häckel).
Fig. 353 .

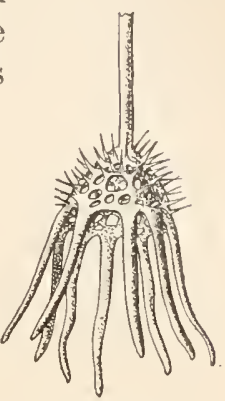

Acanthocorys (im. Häckel).

Peromelissa (Häckel) en diffère par ses côtes qui émettent trois épines latérales libres $(0,11$ à 0,14$)$;

Micromelissa (Häckel) est pareil avec une corne céphalique en plus $(0,1$ it 0,16$)$;

Sethomelissa (IIäckel) a, en place des épines du précédent, trois ailes grillagées (0, l'i);

Tetrahedrina (IIäckel) a les trois branches de son pied réduites ì leur partie terminale libre au bord de la bouche $(0,12$ ì 0,16$)$;

Sethochytris (IIäckel) est semblable, mais son pied est formé de tissu grillagé $(0,17$ it $0,2 \cdot 2)$;

Clathrolychnus (IIäckel) est pareil au précédent et a, en plus, une deuxième couche ì son tissu grillagé $(0,24$ à 0,26$)$.

Cette série de genres forme avec la précédente la famille des TRIPOCYRTIN [Tripocyrtida (IIäckel)].

${ }^{(2)}$ Genres voisins:

Arachnocorys (IIäckel) est semblable, mais a son tissu grillagí doublé en dehors d'une deuxième couche arachnoïde $(0,06$ à 0,29$)$;

Sethophormis (Häckel) diffëre d'Acanthocory's par ses côtes lisses et ne dépassant pas le bord inférieur du thorax, sa forme est campanuléc, surbaissée $(0,06$ a 0,48$)$;

Sethamphora (Häckel) est comme le précédent, mais sa forme est ovale et sa bouche rétrécie $(0,08$ ì 0,15$)$;

Sethopyramis (Häckel) a une forme plus haute encore, pyramidale $(0,16$ à 0,92$)$;

Plectopyramis (IIiickel) est semblable, mais a ses' mailles occupées par un second tissu grillagé plus fin $(0,21$ à 0,62$)$;

Spongopyramis (IIäckel) de même, mais le tissu de ses mailles est plus serré encore, spongieux $(0,22$ à 0,26$)$;

Acanthocyrtoma (IIäckel) n'a plus de còtes thoraciques, le pied, à six branches, est réduil ì la partie inférieure qui dépasse le bord libre du thorax; il a une corne apicale $(0,18$ à 0,26$)$;

Anthocyrtis (Ehrenberg) est semblable au genre précédent, mais son pied a neuf branches $(0,06$ it 0,24$)$;

Anthocyrtium (IIäckel) de même mais, au lieu d'avoir neuf branches au pied, il en a douze et plus $(0,06$ à 0,17$)$;

Anthocyrtidium (IIäckel) diffère du précédent par son pied qui devient libre un peu au-dessus de la bouche rétrécie $(0,07$ à 0,16$)$;

Carpocanium (Ehrenberg) dérive d'Anthocyrtium, mais n'a pas de corne, et sa tête rudimentaire est cachée dans la partie supérieure du thorax $(0,07$ i 0,14$)$. 
Clistophæna (Häckel) (fig. $3 \dddot{4}$ ) est un Dicyrloüde à pied formé de plusieurs branches (six ou plus) et à bouche diaphragmée $(0,1$ à 0,24$)$ ( $\left.{ }^{4}\right)$.

Sethoconus (Häckel) (fig. 355) est un Dicyrtoïde, sans pied et à bouche libre. Sa forme est conique ou campanulée $(0,06$ à 0,42$)\left({ }^{2}\right)$.

Dicolocapsa (Häckel) (fig. 356), est un Dicyrtoïde sans pied et à bouche diaphragmée $(0,12$ à 0,14$)\left({ }^{3}\right)$.

Fig. 35'.

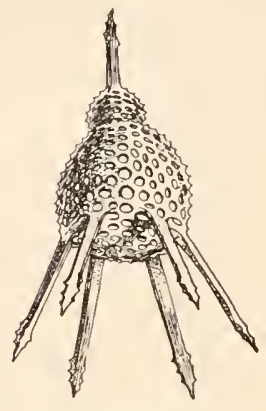

Clistophrna

(im. Häckel).
Fig. 355.

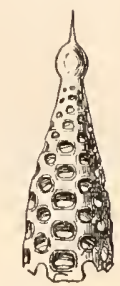

Sethoconus

(im. Häckel).
Fig. 356.

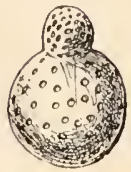

Dicolocapsa (im. IIäckel).

\section{Tricyrtoïdes. - tricyrtoideA \\ [T'RICYRTIDA (Häckel); — CYRTOIDEA TRITHaLAMIA (Häckel)]}

Theopodium (Häckel) (fig. 35̈7) est un Tricyrtoïde (c'est-à-dire a une coquille divisée par deux étranglements horizontaux en trois parties, lête, thorax, abdomen), à pied formé de trois branches et à bouche ouverte. Ici, les branches du pied forment des côtes sur le corps et ne se dégagent qu'au-dessous de l'ouverture de l'abdomen $(0,15$ à 0,18$)\left({ }^{2}\right)$.

\section{(1) Genre voisin :}

Sethophæna (IIäckel) est semblable, mais les branches de son pied sont développées en ailes ou en cornes latérales $(0,11$ à 0,2$)$.

Ces deux genres forment avec la série précédente la famille des ANTHOCYRTINA [Anthocyrtida (Häckel)].

$\left(^{2}\right)$ Genres voisins :

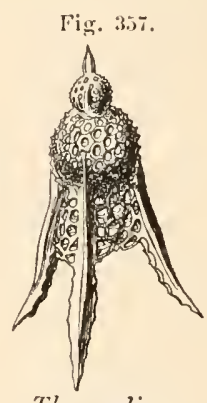

Theopodium (im. Häckel).

Periarachnium (Häckel) possède en plus un revêtement de lissu arachnoïde $(0,17)$;

Sethocephalus (Häckel) diffère de Sethoconus par sa forme déprimée $(0,08$ à 0,14$)$; Sethocyrtis (IIäckel) est un Sethocephalus armé d'une corne céphalique $(0,1$ à 0,19$)$;

Sethocorys (Häckel) est un Sethocyrtis à bouche tubuleuse $(0,08$ à 0,16$)$;

Lophophæna (Ehrenberg) est un Sethocyrtis à plusieurs cornes $(0,01$ i 0,14$)$;

Dictyocephalus (Ehrenberg), un Sethocorys sans cornes $(0,07$ ì 0,17$)$.

$\left({ }^{3}\right)$ Genres voisins :

Sethocapsa (Häckel) a, en plus, une corne $(0,07$ à 0,18$)$;

Cryptocapsa (Häckel) n'a pas de corne, mais sa tête est cachée dans la partie supérieure du thorax $(0,18$ à 0,29$)$.

Ces trois genres forment, avec la série précédente, la famille des SETHOCYRTIY E [Sethocyrtida (Häckel)].

(4) Genres voisins:

Pterocanium (Ehrenberg) en diffère par ses côtes et son pied grillagés $(0,08$ à 0,33$)$;

Pterocorys (IIäckel) a un pied qui n'atteint pas l'abdomen et forme au thorax trois saillies aliformes $(0,11$ a 0,38$)$; 
Theopera (Häckel) (fig. 3ว̈8) est un Tricyrtoïde à trois pieds et bouche diaphragmée. Les pieds forment trois côtes aliformes sur le thorax el l'abdomen $(0,16$ à 0,23$)(')$.
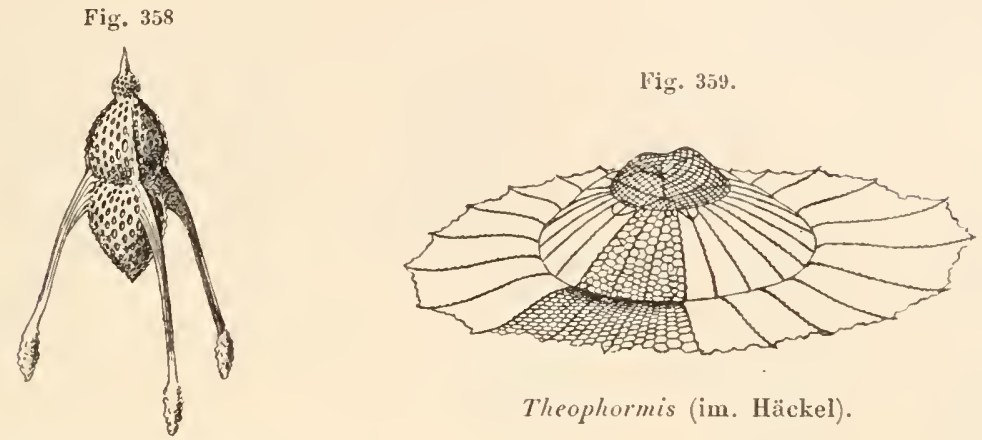

Theopera (im. Häckel).

Theophormis (Iläckel) (fig. 33̈9) est un Tricyrtö̈de à pieds nombreux et à bouche libre. Les pieds dessinent des còtes saillantes sur le thorax et

Theopilium (Häckel) de même, ef ses deux còtes ne forment aucune saillie extérieure $\langle 0,14$ à 0,17$\rangle$;

Pterocodon (Ehrenberg) est un Pterocoms dont les côtes aliformes se détachent au thorax sans atteindre l'abdomen, mais i la bouclıe on trouve un grand nombre d'appendices (épines ou dépendances du pied) $(0,1$ a 0,18$)$;

Dictyocodon (IIäckel) représente un Pterocorys it ailes et appendices huccaux grillagés $(0,08$ i 0,3$)$;

Corocalyptra (Häckel) est un Pterocorys dont les còtes aliformes se détachent du cou sans descendre sur le thorax $(0,1$ it 0,16$)$;

Dictyoceras (IIäckel) est un Pterocorys dont les ailes sont grillagies et ne se prolongent pas sur la tète 0,12 it 0,24$)$;

Pteropilium (Häckel) représente un Dictyoceras i ailes se prolongeant sur la tête $(0,16$ à 0,23$)$;

Pleuropodium (Iläckel) est un Theopodium dont les còtes pédieuses ne remontent pas plus hant que l'abdomen $(0,11$ i 0,14$)$;

Podocyrtis (Ehrenberg) est un Pleuropodium qui n'a mème plus de còtes abdominales; le pied est réduit à la partie libre infrabuccale $(0,07$ it 0,25$)$;

Thyrsocyrtis (Ehrenberg) est le précédent arec un pied ramifie $(0,12$ a 0,17$)$;

Dictyopodium (Ehrenberg) de mème, mais son pied est grillagẻ sans être ramifié $(0,14$ á 0,23$)$.

(1) Genres voisins :

Lithornithium (Ehrenberg) n'a d'ailes que sur le thorax 0,09 à 0,15 );

Sethornithium (Häckel) est de même, mais ses ailes sont grillagées $(0,18)$;

Rhopalocanium (Ehrenberg' est fusiforme et n'a d'ailes que sur l'abdomen (0,18 à 0,24);

Rhopalatractus (Häckel) est semblable, mais a une corne au pôle inférieur du corps $[0,18$ à 0,29 );

Lithochytris (Ehrenberg) est un Rhopalocanium de forme pyramidale et a les pieds dépassant librement en bas $(0,13$ à 0,2$)$.

Cette série de genres forme, avec la précedente, la famille des Poducretise [Podocyrtida (Häckel)]. 
sur l'abdomen; ce dernier, en outre, est largement évasé $(0,11$ à 0,14$)$ ('). Hexalatractus (Häckel) (fig. 360) est un Tricyrtoïde à pieds nombreux et à bouche diaphragmée. Les pieds forment six ailes latérales $(0,18$ à 0,19$)\left({ }^{*}\right)$.

Tricololampe (Häckel) (fig. 361) est un Tricyrtoïde sans pieds et à bouche libre. La forme générale est ici subcylindrique $(0,08$ à 0,28$)\left({ }^{3}\right)$.

Tricolocapsa (Häckel) (fig. 362) est un Tricyrtoïde sans pieds à bouche grillagée $(0,11$ à 0,21) (').

Fig. 360.

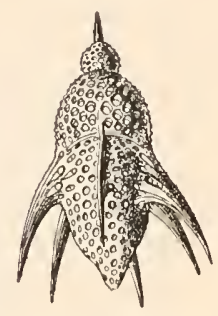

Hexalatractus (im. Häckel).
Fig. 361 .

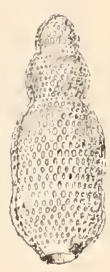

Trichololampe (im. IIäckel).
Fig. 362 .

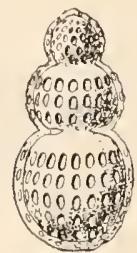

Tricholocapsa (im. Häckel).

(1) Genres voisins :

Phormocyrtis (Häckel) diffère du précédent par sa forme ovale ou cylindrique et sa bouche rétrécie $(0,18$ à 0,24$)$;

Cycladophora (Ehrenberg) n'a de côtes pédieuses que le long de l'abdomen $(0,15$ à 0,4$)$;

Alacorys (Häckel) de même, mais la bouche est entourée de proéminences libres qui sont leurs prolongements $(0,12$ à 0,32$)$;

Calocyclas (Ehrenberg) n'a plus du tout de côtes et n'a que ses proéminences buccales; son abdomen est ovoïde ou cylindrique $(0,13$ à 0,32$)$;

Clathrocyclas (IIäckel) de même, mais son abdomen est conique ou discoïde, dilaté $(0,08$ à 0,18$)$;

Lamprocyclas (Häckel) n'a aussi qu'une couronne buccale de prolongements, sans côtes pédieuses, mais cette couronne est double $(0,13$ à 0,16$)$;

Diplocyclas (Häckel) n'a aussi que ces deux couronnes, mais l'une d'elles est remontée au niveau de la constriction lombaire $(0,12$ à 0,14$)$.

(2) Genre voisin :

Theophæna (Häckel) est semblable, mais a neuf ailes pédieuses $\langle 0,3$ à 0,32$)$.

Ce genre, joint au précédent et à la série précédente, forme la famille des PHormoCYRTINA [Phormocyrtida (Häckel)].

(3) Genres voisins :

Theocyrtis (Häckel) est de même, avec une corne apicale en plus $(0,06$ à 0,25$)$;

Lophocyrtis (Häckel) de même, mais a deux cornes ou plus $(0,1$ a 0,21$)$;

Theosyringium (Häckel) est un Theocyrtis à abdomen tubuleux plus étroit que le thorax $(0,18$ à 0,24$)$;

Cecryphalium (IIäckel) a une forme générale déprimée et conique $(0,08$ à 0,12$)$;

Theocalyptra (Uäckel) de même avec une corne en plus $\{0,06$ a 0,14$)$;

Theoconus (Häckiel) est comme le précédent, mais en cône plus allongé $(0,07$ à 0,26$)$;

Lophoconus (IIäckel) est un Theoconus avec deux ou plusieurs cornes $(0,16$ à 0,21$)$;

Theocampe (Häckel) est un Tricololampe à abdomen ovoïde ou en cône renversé et à bouche rétrécie $(0,1$ à 0,2$)$;

Lophocorys (Häckel) est semblable avec deux cornes en plus $\langle 0,13$ à 0,21$)$;

Theocorys (IIäckel) semblable aussi avec une seule corne $(0,09$ à 0,32$)$;

Axocorys (Iäckel) est un Theocorys avec une columelle $\{0,21)$.

(4) Genres voisins :

Theocapsa (Häckel) est semblable avec une corne en plus $(0,13$ a 0,27$)$; 


\section{STichocirtoïdes. - S'TtCHOCYRTOIDEA}

[StichocyRtida (Häckel); — CYRTOIDEA POLYThaLAmia (Häckel)]

Stichopilium (Häckel) (fig. 363) est un Stichocyrtoïde (c'est-ì-dire un Tricyrtoïde dont l'abdomen est subdivisé par des étranglements secondaires) à trois pieds et à bouche libre. Ces pieds ont la forme d'épines se détachant du sommet de l'abdomen; il y a une corne céphalique $(0,12$ is 0,2$)(')$.

Stichopera (Häckel) (fig. 364) est un Stichocyrtoïde à trois pieds, à bouche grillagée; il a, en outre, une corne céphalique, et ses pieds forment trois còtes ou séries d'épines $(0,15$ à $0,20 \%)\left({ }^{*}\right)$.

Stichophormis (Häckel) (fig. 365) est un Sticho-

Fig. 363 .

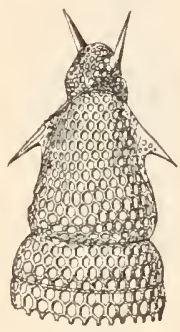

Stichopilium (im. Häckel) cyrtoïde à nombreux pieds et à bouche libre. Les pieds forment autant de côtes latérales prolongées en autant de pointes libres au delà de la bouche. La forme générale est conique ou pyramidale $(0,04$ a 0,24$)\left({ }^{3}\right)$.

Phrenocodon (Häckel) diffère des précédents par un septum grillagé complet entre le thorax et l'abdomen $(0,11)$.

Cette serrie de genres forme arec la précédente la famille des THEOCrRTINe [Theocyrtida (Häckel).

(1) Genres voisins:

Artopilium (Iläckel) est semblable, mais a ses trois apophyses pédieuses grillagées $(0,13$ à 0,3$)$;

Pteropilium (Häckel) de même, mais n’a pas de corne $(0,1$ ă à 0,2$)$;

Stichocampe (Häckel) a ses trois côtes pédieuses 'prolongées jusqu'au delá de la bouche en trois pointes libres $\{0,25$ à 0,3 ;

Stichopterium (Häckel) est semblable, mais a les pieds grillagés $(0,12$ a 0,22$)$;

Podocampe (IIäckel) a les trois pointes libres du Stichocampe, mais n'a pas les côtes pédieuses $(0,14$ à 0,2$)$;

Stichopodium (Häckel) est semblable au précédent, mais il a ses pointes pédieuses grillagées $(0,2)$.

(2) Genres voisins:

Cyrtopera (Häckel) est semblable, mais a ses côtes pédieuses grillagées $(0,17$ à $0,2: 3)$;

Artopera (Häckel) a l'abdomen rétréci en bas et terminé par une corne inférieure $(0,15$ a 0,18$)$.

Cette série de genres forme avec la précédente la famille des PODOCAMPINE [Podocampida (IÏ̈ckel)].

(3) Genres voisins:

Phor'mocampe (Häckel) est semblable, mais n'a pas les còtes pédieuses $(0,14$ à 0,22$)$; 
Artophæna (Häckel) (fig. 366) est un Stichocyrtoïde à pieds nombreux (six) sous forme de côtes aliformes ou d'épines, et à houche grillagée $(0,14$ à 0,2$)$ (').

Dictyomitra (Zittel) (fig. $367)$ est un Stichocyrtoïle sans pieds, à bouche libre. La forme est légèrement conique $\langle 0,08$ a $0,24)\left({ }^{2}\right)$.

Stichocapsa (Häckel) (fig. 368) est un Stichocyrtoïde sans pieds et à bouche grillagée $(0,1$ à $0,25)\left({ }^{3}\right)$.

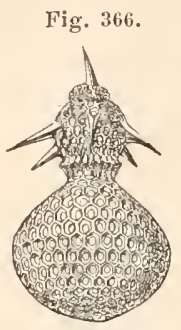

Arthophana (im. II ïckel).

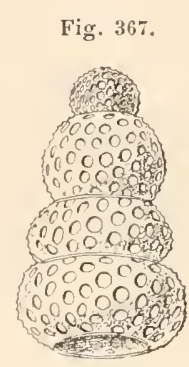

Dictyomitra im. Häckel).
Fig. 368 .

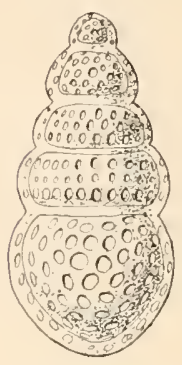

Stichocapsa (im. Häckel).

Artophormis (Häckel) est un Stichophormis ovoïde ou fusiforme, à bouche rétrécie $(0,16$ à 0,24$)$;

Cyrtophormis (Iläckel) est un Artophormis sans còtes pédieuses $(0,14$ à 0,3$)$.

(1) Genre voisin:

Stichophæna (Häckel) est un Arlophrena a neuf pieds au lieu de six $(0,20$ à 0,25$)$.

Ces deux genres forment avec la série précédente la famille des PIIORMOCAMPINE [Phormocampida (Häckiel)].

$\left({ }^{2}\right)$ Genres voisins :

Lithostrobus (Bütschli) est semblable, mais avec une corne en plus $(0,1$ à 0,32$)$;

Stichocorys (IIäcliel) est comme le précédent, mais de forme conique en haut, cylindrique en bas $(0,16$ à 0,24$)$;

Artostrobus (Häckel) de mème, mais subcylindrique dans toute sa hauteur $(0,1$ a 0,24$)$;

Lithomitra (Bütschli) est comme le précédent, mais sans corne $(0,06$ à 0,3$)$;

Lithocampe (Ehrenberg) est comme le précédent, mais ovoïde ou fusiforme $(0,08$ à 0,3$)$;

Siphocampe (IIäckel) est un Lithocampe avec un appendice tubuleux prolongeant la tète en haut $(0,14$ à 0,24$)$;

Eucyrtidium (Ehrenberg) est un Lithocampe avec une corne $(0,12$ à 0,26$)$;

Eusyringium (Häckel) est un Eucyrtidium prolongé en bas en tube $(0,2$ à 0,33$)$;

Spirocampe (Häckel) diffère des précédents par la disposition spirale des constrictions qui, ailleurs, sont annulaires et indépendantes $(0,10 \%$ a 0,2 ' $)$;

Spirocyrtis (IÏ̈ckel) est comme le précédent, mais arec une corne $(0,1$ à 0,25$)$.

(3) Genres voisins:

Cyrtocapsa (Häckel) est semblable, mais a une corne $(0,14$ à 0,23$\rangle$;

Artocapsa (Häckel) a, non seulement une corne céphalique comme le précédent, mais aussi une corne inférieure au pòle opposé $(0,17$ à 0,26$)$.

Ces trois genres forment, avec la série précédente, la famille des LITHociaptr.E [Lithocampida (IÏ̈kel)]. 


\section{$3^{\text {e SOUS-ORdre }}$ \\ SPYROÏDES. - SPYROID AE}

[SPYRIDINA (Ehrenberg, p.p.);-ZYGOCYRTID.4 (Häcliel, Bütschli); ACANTIOdesmida (R. Ilertwig); - SPYRoIdea (Häckel)]

\section{TYPE MORPHOLOGIQUE}

(FIG. 369)

Il ressemble tellement au type général des Monopylaires qu’il se confond presque avec lui. Il possède donc le pied à trois branches, une postérieure et deux antéro-latérales; la coquille grillagée complète et l'anneau sagittal (c). Ajoutons seulement, comme trait caractéristique, que l'anneau sagittal détermine une constriction de la coquille et subdivise sa cavité en deux loges, droite et gauche, plus ou moins distinctes, et que les trois branches du pied $(t)$, au lieu de partir d'un point central commun, s'insèrent souvent isolement sur la base de la coquille.

\section{GENRES}

Tristylospyris (Häckel) est la réalisation assez Fig. 369 .

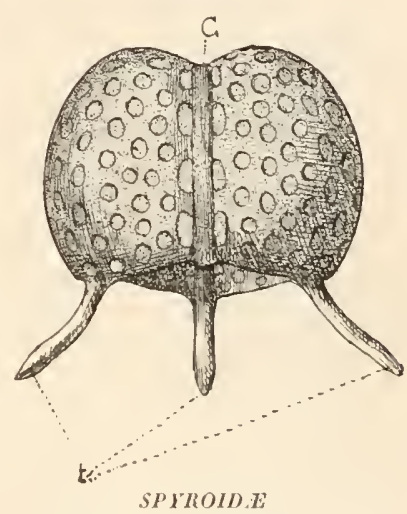

(Type morphologique) (Sch.). fidèle du type morphologique ci-dessus $(0,10$ i 0,38$)$ (').

(1) Genres voisins :

Tripospyris (IIäckel) a, en plus, une corne apicale au sommet de l'anneau $(0,10$ a 0,30$)$;

Triceraspyris (Häckel) a trois cornes apicales $\{0,15$ a 0,35$\rangle$;

Cephalospyris Häckel) n'a pas de cornes, mais deux larges orifices, de part et d'autre du bord supérieur de l'anneau, au sommet de la coque $(0,2$ à 0,32$)$;

Brachiospyris (Häckel) n'a pas de corne apicale comme Tripospyris, mais n'a au trèpied que les deux branches antéro-latérales $(0,3$ à 0,6$)$;

Dipospyris (Häckel) est pareil au précédent, mais avec une corne $(0,2$ a 0,6$)$;

Dendrospyris (Häckel) a une corne, et les deux branches de son pied ramifices $(0,22$ a 0,38$)$;

Dorcadaspyris (Häckel) a aussi une corne et les deux branches de son pied armées d'épines $(0,1$ aे 0,7$)$;

Gamospyris (Häckel) a une corne et les deux branches du pied arquies et soudees et un anneau $(0,33)$;

Stephanospyris (Häcliel) représente le précédent avec une sèrie d'épines latérales à l'anneau, pied en plus $(0,2$ à 0,4$)$;

Tessarospyris (Häckel) a au pied les deux branches latérales, une branche postérieure et une antérieure, et pas de corne apicale $(0,16$ à 0,31$)$;

Tetraspyris (Iäckel) est semblable, mais avec une corne $(0,02$ à 0,6$)$;

Cantharospyris (Häckel) a six branches au pied, savoir : deux latéro-postéricures en plus des quatre du précédent $(0,07$ à 0,22$)$; 
Tholospyris (IÏ̈ckel) (fig. 370) est un Tristylospyris muni, en plus, d'une sorte de casque fenestré qui surmonte le sommet de la coquille $(0,17$ à 0,26$)\left({ }^{\prime}\right)$.

Phormospyris (lläckel) (fig. 371) est un Tristylospyris muni, en plus, d'un prolongement grillagé de la coque qui s'étend, en dessous de celle-ci, entre les trois branches du pied et qui en est séparé par une constriction cipculaire horizontale. On désigne sous le nom de tête la coquille primitive, et cet appendice sous celui de thorax $(0,1$ à 0,14$)\left({ }^{2}\right)$.

Androspyris (IIäckel) (fig. 372) réunit les caractères des deux genres précédents: il a le thorax de Phormospyris et le casque de Tholospyris,

Hexaspyris (Iläckel) est semblable, mais a une corne $(0,11$ à 0,39$)$;

Liriospyris (IIäckel) est semblable encore, mais a trois cornes $(0,12$ i 0,22$)$;

Pentaspyris (IIäckel) a cinq branches au pied, qui sont celles de Cantharospyris, sauf que l'antérieure manque et il n'a pas de corne $(0,15$ à 0,28$)$;

Chlathrospyris (IIäckel) est semblable, mais a une corne $(0,08$ à 0,23$)$;

Egospyris (Häckel) est semblable encore, mais a trois cornes $(0,16$ à 0,28$)$;

Therospyris (Häckel) a quatre branches au pied, les deux antéro-latérales et les deux postéro-latérales, et point de cornes $(0,15$ à 0,17$)$;

Zygospyris (Häckel) est semblable, mais a une corne $(0,16$ a 0,18$)$;

Taurospyris (Häckel) semblable encore, mais avec deux cornes latèrales $(0,16$ à 0,38$)$;

Elaphospyris (Häckel) semblable encore, mais avec trois cornes, deux latérales et une médiane $(0,06$ à 0,3$)$.

Gorgospyris (IIäckel) a sept à douze branches au pied, et pas de corne $(0,07$ à 0,33$)$;

Petalospyris (Ehrenberg) est semblable, mais a une corne $(0,08$ a 0,31$)$;

Anthospyris (IIäckel) semblable encore, mais a trois cornes $(0,09$ a 0,44$)$;

Ceratospyris (Ehrenberg) semblable encore, mais a de nombreuses cornes $(0,09$ à 0,20$)$;

Dictyospyris (Erhenberg) n'a pas de pied ni de corne $(0,03$ à 0,14$)$;

Circospyris (Häckel) n'a pas de pied, mais a une corne $(0,06$ à 0,23$)$.

Ces genres forment la famille des ZYGOSPYRINE [Zjgospyrida (Häckel)].

(1) Genres voisins :

Lophospyris (Häckel) est un Tholospyris dont le pied n'a que les deux branches antérolatérales $(0,16$ à 0,17$)$;

Tiarospyris (Häckel) est, un Tholospyris dont le pied a six à neuf branches ou plus $\{0,01$ à $0,31)$;

Sepalospyris (Häckel) est de mème avec une corne sur le casque en plus $(0,19$ à 0,3 ;

Polospyris (IIäckel) a une corne comme le précédent, mais pas de pied $(0,07$ a 0,12$)$.

Ces genres forment la famille des THoLospyriy [Tholospyrida (Häckel)].

$\left({ }^{2}\right)$ Genres voisins:

Acrospyris (IIäckel) est un Phormospyris avec une corne apicale en plus $(0,07$ ì 0,22$)$; Desmospyris (IIäckel) n'a pas de corne, nrais a beaucoup de branches (neuf à douze ou plus) au pied $(0,08$ à 0,12$)$;

Patagospyris (Häckel) est de même, avec une corne en plus $(0,08$ à 0,15$)$; 
avec l'anneau sagittal et le pied à trois branches libres comme l'un et l'autre, et en plus une corne apicale $(0,2$ à 0,35$)$ (").

$6^{\mathrm{e}}$ Sous-Ordre

BOTRYOÏDES. - BOTRYOIDA

[Botryda vel Botryida (Häckel); - Polycyrtida (Häckel)]

TYPE MORPHOLOGIQUE

(FIG. 373)

La coque grillagée du type morphologique des Monopylaires est ici toujours présente et offre le caractère singulier d'être subdivisée par des constrictions verticales en lobes juxtaposés. Il n'y a pas ici seulement deux de ces lobes comme chez les Spyroïdes, mais trois au moins et ordinairement davantage. Le pied manque, mais, au-dessous de la coque lobée qui prendra ici, comme chez les Cyrtoidr, le nom de tête $(c)$, peuvent se trouver une ou deux constrictions horizontales séparant de la tête un thorax(th.) et mème un abdomen (ab.), simples compartiments de la coque, mais superposés. La capsule centrale, mal connue, semble se lober conformément à la coquille.

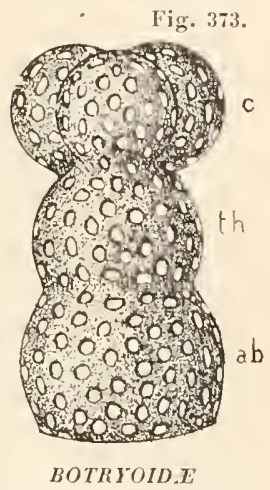

(Type morphologique) (Sch.).

\section{GENRES}

Botryoptera (Häckel) (fig. 374) a sa coquille réduite à la téte lobée; il n’y a point de constrictions transversales ni, par suite, de thorax ou d'abdomen $(0,0 \mathrm{z}$ à 0,09$)\left({ }^{2}\right)$.

Rhodospyris (Häckel) de même, mais avec trois cornes $(0,13$ il 0,18$)$.

Ces genres forment la famille des Pnorнospraxy [Phormospirida [IIäckel)].

(1) Genres voisins :

Lamprospyris (Iläckel) est semblable, mais a son tissu grillagé spongieux et la corne ordinairement branchue et fenestrée 0,3 a 0,6$)$;

Tricolospyris (Häckel) n'a plus de pied, et deux constrictions horizontales séparent le casque, la tète et le thorax $(0,12$ a 0,22$)$;

Fig. 37i.

Amphispyris (Häckel) est semblable, mais son tissu grillagé incomplet laisse de larges trous $(0,14$ à 0,24$)$;

Perispyris (Häckel) a son tissu grillagé formé de deux lames ou spongieux $(0,18$ à 0,24$)$; Sphærospyris (IIäckel) n'a ni pied ni étranglements circulaires, en sorte que sa coquille se réduit à une simple sphère grillagée avec un anneau sagittal, mais cet anneau est loin de remplir la coque, en sorte qu'il reste, au-dessus de lui, un dòme reprisentant le casque et une cage représentant le thorax $\{0,11$ a 0,26$\}$;

Nephrospyris (IÏckel) est de mème, mais à coque discoïde ou réniforme $(0,25$ à 0,6$)$.

Ces genres forment la famille des Avorospyrise [Androspyrida (Häckel)].

$\left.{ }^{2}\right)$ Genre voisin :

Cannobotrys (Häckel) est de mème, mais possède, en plus, des appendices tubuleux à parois poreuses $(0,05$ à 0,11$)$; 
Botryopyle (Häckel) (fig. 375) a, au-dessous de sa téte lobée, un thorax ouvert en bas $(0,08$ à 0,12$)(\mathbf{l})$.

Botryocyrtis (Ehrenberg) (fig. 376) a, audessous de sa téte lobée, un thorax et un abdomen séparés par deux constrictions horizontales. Le segment abdominal est librement ouvert en dessous $(0,09$ à 0,12$)\left({ }^{*}\right)$.

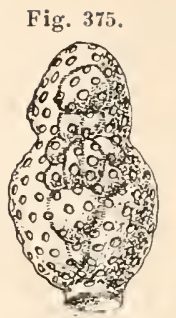

Botryopyle (im. Häckcl).
Fig. 376 .

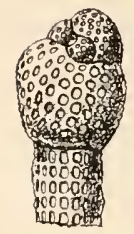

Botryocyrtis (im. Häckel).

\section{$4^{\mathrm{e}}$ Ordne

\author{
PHEODARIES. - PHAODARIDA \\ vel \\ CANNOPYLAIRES. - CANNOPYLIDA \\ [CANNopylea (Häckel); - PIIEOdaria (Häckel); \\ Tripylea (R. Hertwig); - Pansolenia (Häckel)]
} \\ TYPE MORPHOLOGIQUE \\ (FIG. 377)}

Le type morphologique des Cannopylaires se distingue de celui des Radiolaires en général (V. p. 169) en deux points essentiels : $1^{\circ}$ la constitution de la capsule centrale; $2^{\circ}$ la présence du phxodium.

Capsule centrale. - La capsule centrale (fig. 377, caps. ctrl. et passim, dans les figures de genres) a la forme d'un sphéroïde aplati aux pôles comme le globe terrestre, mais d'une manière beaucoup plus marquée (les pôles étant naturellement les points où la capsule est traversée par l'axe vertical du corps). Elle est pourvue de deux membranes, l'une externe plus épaisse, l'autre interne très mince et néanmoins assez résistante. Ces membranes sont formées, comme d'ordinaire, d'une substance qui présente les caractères physiques et plusieurs caractères chimiques de la chiline. Ces deux membranes sont appliquées l'une contre l'autre chez l'animal vivant. Mais, constamment, sous l'action les réactifs, elles s'écartent et nous les figurerons un peu écartées

Ces deux genres forment la famille des CaxoвotnRxe [Cannobothrida (Häckel)].

(1) Genres voisins :

Acrobotrys (Häckel) est de mème, mais posside, en plus, des tubes poreux (0,08 à 0,1:2). Botryocel/a (Iäckel) n'a pas ces tubes, mais le compartiment thoracique de sa coquille est fermé en bas par une plaque grillagée $(0,06$ à 0,10$)$;

Lithobotrys (Ehrenberg) est de mème, mais a, en plus, des tubes poreux $(0,08$ à 0,13$)$.

Ces genres forment la famille des LitповотилiN [Lithobothryda (IIäckel)].

(2) Genres voisins :

Pylobotrys (IIäckel) a, en plus, un certain nombre de tubes poreux $(0,11$ à 0,15$)$.

Botryocampe (Ehrenberg) n'a pas de ces tubes, mais son abdomen est fermé en dessous par deux lames grillagées $\{0,05$ à $0,15 \%$;

Phormobotrys (Häckel) est de mème, mais a, en plus, des tubes poreux $(0,1$ à 0,16$)$.

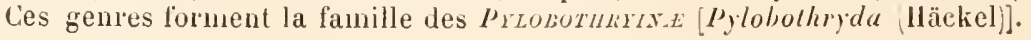


pour les distinguer plus aisément. La paroi est en général continue, sans pores et présente seulement trois ouvertures beaucoup plus larges

Fig. 377.

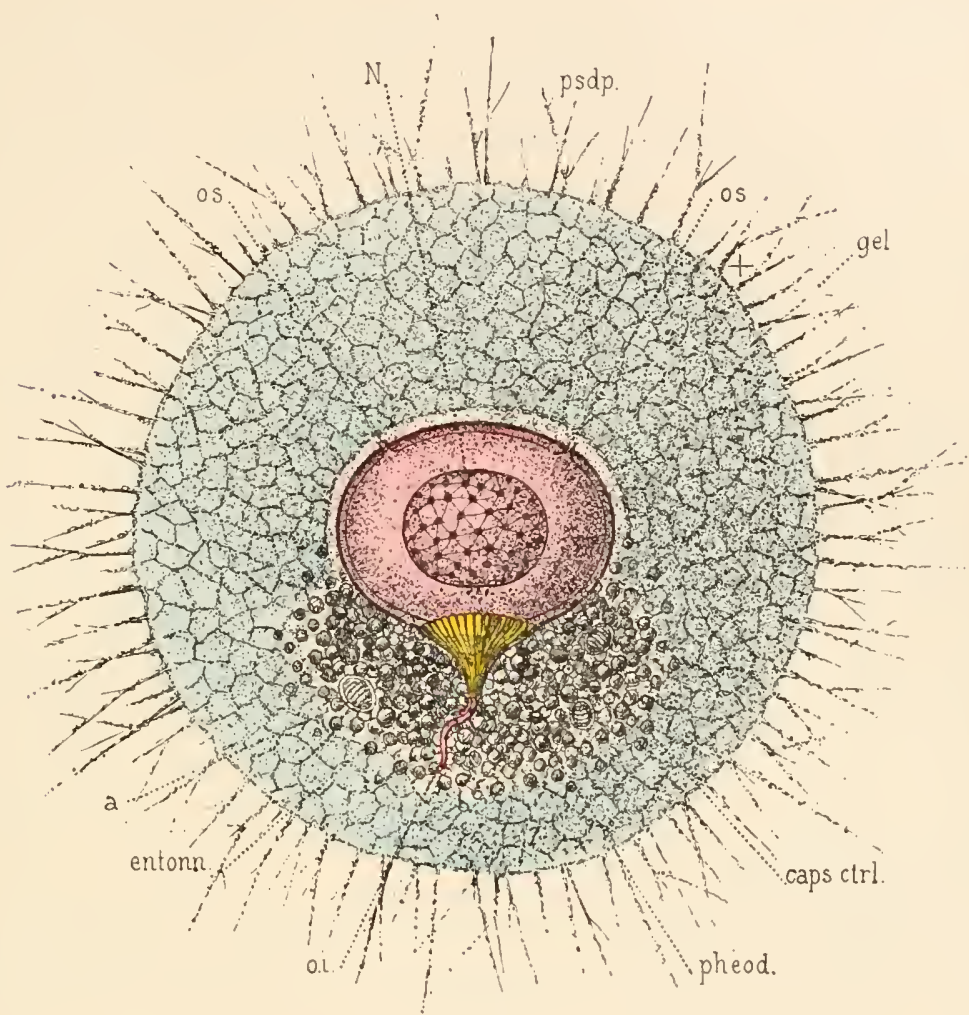

Cannopylaire ou Phæodarié (Type morphologique) (Sch.).

a, cellule; caps. etrl., capsule centrale; entonn., entonnoir; $\mathbf{~}$. , noyau; o..., orifice principal infërieur; o.s., orifices supérieurs; pheod., phæodium; psdp., pseudopodes.

que les pores des Péripylaires ou que ceux de l'opercule perforé des Monopylaires.

De ces trois ouvertures, une, l'orifice principal (o.i.), est percée au pôle inférieur même; les deux autres, orifices accessoires (o.s.), sont situées à droite et à gauche du pôle supérieur et par conséquent dans le plan coronal. Toutes les trois sont prolongées en un tube $\left(^{*}\right)$ d'abord conique, puis cylindrique, ouvert à son extrémité dans le plasma péricapsulaire qui forme ici une couche beaucoup plus épaisse que chez les autres

(•) C'est par allusion à cette particularité que Häckel a donné à ce groupe le nom de CAxxoPYLEA : de $x \dot{x} v \vee \alpha$, roseau, tubnleux comme un roseau; $\pi \dot{\prime} \lambda \gamma_{i}$, porte. Le nom de TRIPYLEA proposé par R. Ḣertwig semblerait meilleur, mais il y a assez souvent nne senle porte, la principale, et parfois plns de trois. 
Radiolaires. Les deux membranes prennent part à la formation de ces tubes et elles sont, à leur niveau, beaucoup plus solidement unies que dans le reste de leur étendue.

Les orifices accessoires ne présentent rien autre de particulier. Leurs tubes sont courts, rectilignes et, autour de leur base, la paroi a le même aspect que plus loin. L'orifice principal, au contraire, présente plusieurs particularités remarquables. Son tube est beaucoup plus long, onduleux et, autour de sa base, la paroi est modifiée dans sa forme et dans sa structure. Circulairement autour de lui, sur une surface d'un diamètre égal à la moitié environ de celui de la capsule, elle se soulève en cône surbaissé (entonn.) ou plutôt en forme de mamelle de femme dont la base conique du tube représenterait le mamelon. Toute la surface entre ce mamelon et le contour extérieur de l'éminence est marquée de grosses lignes radiaires, au nombre d'une soixantaine, tantôt plus tantôt moins, et très régulièrement disposées (').

La signification de ces parties n'est pas exactement connue. Pour Ḧ̈́ke ces lignes sont des stries d'épaississement de la membrane capsulaire externe; pour R. llertwig, elles appartiennent à la membrane interne. De fait, quand les membranes se séparent, le disque strié suit tantôt l'une tantôt l'autre, et l'aspect des figures permet de suggérer qu'il est formé d'une substance indépendante située entre les deux membranes ou même en dedans de l'interne. Il pourrait être formé soit, comme le suggère aussi IIäckel, par des filaments myophaniques destinés à dilater l'orifice, soit par une différenciation à déterminer du protoplasma intracapsulaire superficiel $\left({ }^{*}\right)$.

Le protoplasma intracapsulaire est remarquable par la présence, à son intérieur, de nombreuses vacuoles contenant chacune un ou plusieurs petits globules graisseux agités d'un mouvement brownien.

Le noyau $(N$.) est très gros, remplissant la moitié ou plus du volume de la capsule. 11 est de forme ellipsoïde, plus aplati que celle-ci et dans le même sens. Il contient de nombreux granules chromatiques disposés sur un réseau.

Phæodium. - Le protoplasma extracapsulaire est, avons-nous dit, beaucoup plus abondant que chez les autres Radiolaires. C'est surtout au niveau de l'orifice principal qu'il forme une masse épaisse, et là il conlient ce que Häckel a appelé le phxodium (de ọxós, brun). C'est un amas volumineux, plus gros souvent que la capsule centrale à demi noyée dans son intérieur, d'une couleur sombre variant du vert au brun

(1) Elles sont parfois ramifiées.

$\left({ }^{2}\right)$ En tout cas le nom d'opercule qu'on lui donne souvent ou la comparaison, souvent faite aussi, avec le couvercle d'une marmite ne peut que donner des idées fausses à son sujet, car il n'y a aucune interruption dans la continuité des parois à leur union a vec lui. Borgert [91] trouve que chez Castanidium la membrane externe des orifices accessoires se sépare du reste sous la forme d'une collerette et que le tout repose sur une sorte de bulbe. 
foncé, formé d'éléments assez disparates mais tous fortement pigmentés. On y trouve: $1^{\circ}$ de vraies cellules toutes comparables aux Zooxanthelles, avec une membrane, un noyau et un pigment vert brunàtre, mesurant 20 à $30 \mu$; $2^{\circ}$ des masses ou granules pigmentaires de toutes tailles, depuis celle des éléments précédents jusqu’à $1 \mu$, et qui forment la plus grande partie et souvent la totalité du phxodium; $3^{\circ}$ enfin parfois, quelques cellules $(a)$ semblables aux premières, mais atteignant 40 à $50 \mu$ et remarquables par une striation croisée en spirale qui rappelle celle de certains nématocystes avant leur éclatement (').

$\mathrm{Au}$ sujet de la signification morphologique et physiologique du phæodium, nous n’avons que des hypothèses. Les uns y voient des Algues spéciales symbiotiques remplaçant les Zooxanthelles absentes: mais alors que sont les grains non cellulaires? D'autres le considèrent comme un pigment formé par l'organisme mème comme celui des organes visuels rudimentaires et ayant peut-être des fonctions analogues : mais que sont alors les vraies cellules du phæodium? D’autres enfin pensent à un simple amas de résidus alimentaires ou de substances ayant un rôle dans l'assimilation (pigments biliaires peut-être) ou la désassimilation : cela semble bien improbable.

Le sujet réclame évidemment de nouvelles recherches $\left(^{(*)}\right.$.

Les autres parties extracapsulaires ne présentent rien de bien particulier. La gelée est fort abondante, le réseau intérieur ou superficiel du protoplasma et les pseudopodes ont ici les mèmes caractères qu'ailleurs. Il n'y a pas de Yanthelles $\left({ }^{\mathbf{3}}\right)$.

Les Phæodariés sont remarquables par leur grande taille. Ils mesurent à peu près autant de millimètres que les autres Radiolaires (sauf les Thalassicollides) mesurent de dixièmes de millimètres. La capsule centrale a jusqu'à un demi-millimètre et on connaît des espèces où le corps a jusqu'à trois centimètres de diamètre

(1) Remarquons que le phæodium est un caractère non pas seulement général, mais constant des Cannopylaires.

(2) Pendant la correction des épreuves, nous recevons le travail de KaRAvaleF [96] qui nie l'existence d'éléments vraiment cellulaires dans le phrodium. Il n'y trouve que du pigment, des granulations réfringentes non colorables et des conformations irrégulières, souvent en bàtonnets ou en capsules emboitées. Tout cela réuni forme de petites masses non cellulaires, les phrodelles. Entre les phæodelles se trouvent, parmi des éléments plus ou moins semblables à ceux qui les constituent, des restes de Diatomées digérées. Aussi l'auteur admet la fonction digestive du phaodium.

(3) La gelée (gel.) est fréquemment, mais non toujours vacuolaire. Il y a d'autant plus de chances pour qu'il y ait des vacuoles que l'animal est plus gros et a un squelette moins développé.

Il n'y a pas toujours un squelette et, quand il y en a un, il n'est pas, comme celui des Acanthomètres ou des Monopylaires, réductible á un type uniforme, aussi ne parlerons-nous de sa forme qu'en étudiant les sous-ordres. Mais nous devons indiquer ici les caractères histologique et chimique de ses tigelles constitutives, car ils sont, le premier très général, et le second probablement constant. La substance constitutive n'est ici ni organique pure comme chez les Acanthaires, ni siliceuse pure comme chez 
L'ordre des Cannopylaires ou Phæodariés se divise en quatre sousordres caractérisés par la constitution de leur squelette :

$1^{\circ}$ PII EOCYSTIDE, à squelette nul ou formé de pièces séparées;

$2 \circ$ PIIEOSPIIERIDE, à squelette formé d'une (ou plusieurs) coque grillagée simple;

$3^{\circ}$ PII EOGROMIDE, à squelette formé d'une coque grillagée percée en face de l'orifice principal de la capsule d'une large ouverture ordinairement épineuse; la capsule centrale est excentrique, placée près du pòle opposé à cette ouverture;

40 PHAOCONCIIDE, à squelette formé d'une coque grillagée bivalve à valves l'une dorsale l'autre ventrale, réunies ou non par une charnière.

\section{$1^{\text {er }}$ Sous-Ordre \\ PHEOCYSTIDES. - PHAOCYSTIDAE \\ [Pheocystina (R. Hertwig)]}

\section{TYPE MORPHOLOGIQUE}

(FIG. 378)

C'est presque identiquement le type que nous venons de décrire. Il

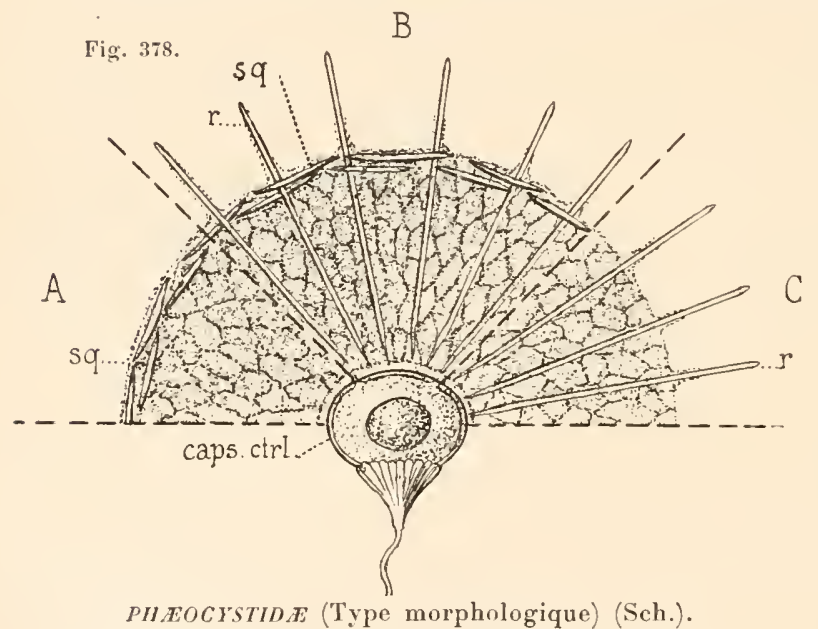

$A$, seeteur ne présentant que des spieules tangentiels so. $C$, secteur ne présentant que des spieules radiaires r. $B$, secteur présentant ees deux genres de spieules. caps. etrl., capsule centrale. suffit, pour le compléter, d'y ajouter deux traits, qui mème ne se présentent pas nécessairement, bien qu'ils soient très ordinaires :

1. La gelée est ordinairement vacuolaire, à la manière de celle des Thalassicolles; 2o il peut exister un squelette formé de pièces indépendantes lisposées, soit tangentiellement ( $s q$. dans le réseau superficiel de la gelée primaire, soit radialement $(r$.$) dans le réseau intéricur$ de la gelée.

les autres; partout oú on l'a analysie on y a trouvé de la silice et une substance organique. C'est sans doute un silicate organique. I'autre part, sauf quelques exceptions, Sagospherines, Castanellines, Concharines, il est formi de tigelles creuses dont la cavité est occupée par une gelée liquide et parfois par un filament axial. 


\section{GENRES}

Phæodina (Häckel) est un Phæocystide sans squelette. Sa capsule centrale est normale $(0,8$ à 1,5$)\left({ }^{*}\right)$.

Cannorraphis (Häckel) (fig. 379) est un Phæocystide à squelette formé de spicules en forme d'aiguilles ramifiées ou épineuses (sq.) disposées, tangentiellement à égale distance du centre $(10 \mathrm{~mm})\left({ }^{2}\right)$.

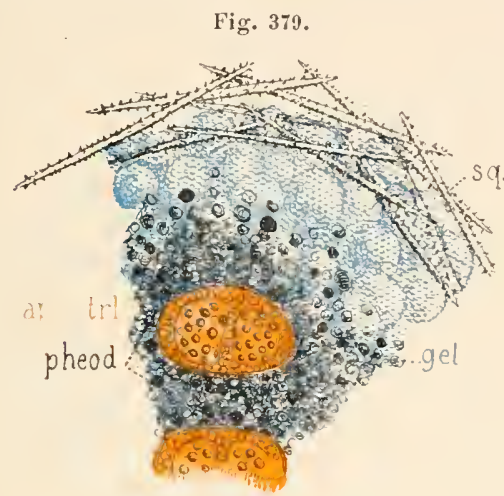

Cannorraphis im. Häckel).

caps. etrl., capsule centrale; gel., gelee; pheod., phacodium; sx., squelette.

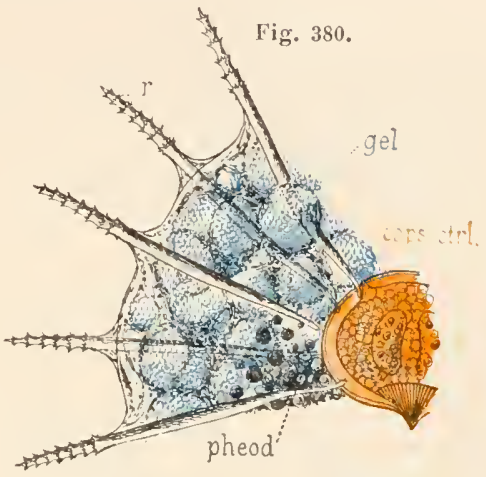

Aulactinium (im. Häckel).

eaps. etru., capsule centrale; gel., gelé, pheod., pheodium.

Aulactinium (Häckel) (fig. 380) a son squelette formé de spicules en forme

(1) Genre voisin:

Phæocolla (Häckel) (fig. 381) en diffẻre par sa capsule centrale Fig. 381, dépourvue d'orifices accessoires (1 à 1,2).

Ces deux genres forment la famille des PHEODIYINE [Phrodinida (Häckel)].

${ }^{(2)}$ Genres voisins :

Cannobelos (Häckel) en diffère par ses aiguilles simples et lisses (Spicules : 0,15 à 0,5 ) ;

Catinulus (Häckel) a des spicules non aciculés, mais en forme de petits chapeaux hémisphériques à parois non grillagées 0,6 à 1,2$)$.

Ces genres forment la famille des CANNOR-

Fig. 382. IHLAHINE [Cannorrhaphida (IIäckel)].

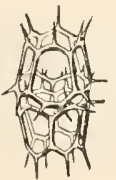

Distephanus (im. IIäckel).
Häckel ajoute à ces genres, sous le nom de DICTYOCHIDA (sous-famille) les quatre genres suivants qu'il caractérise ainsi :

Mesocena (Ehrenberg), Phæodarié ayant, en guise de squelette des sortes d'anneaux, siliceux (de 0,02 à 0,06 , assez rẻgulièrement distribués dans la couche périphérique de son corps; Dictyocha (Ehrenberg), semblable au précédent, mais ayant ses anneaux (de 0,02 à 0,03 ) surmontés de deux arcades perpendiculaires formant une sorte de petite charpente hémisphérique à jour; Distephanus (Stöhr) (fig. 382) ayant son squelette formé de deux anneaux parallèles réunis par des tigelles en une sorte de pyramide tronquée à jour $(0,02$ à 0,03$)$ 
d'épines simples disposées radiairement dans la gelée et atteignant la capsule centrale sur laquelle ils s'appuient par leur base $\left(10^{\mathrm{mm}}\right)\left({ }^{\prime}\right)$.

\author{
2 Sous-Ordne \\ PHEOSPHERIDES. - PHAEOSPHARID E \\ [PHAOSPHERIA (Häckel)]
}

TYPE MORPHOLOGIQUE

(FIG. 383)

Il se déduit aisément du type général des Phæodariés auquel il suffit d'ajouter une coquille formée d'une sphère grillagée régulière sécrétée par le réseau superficiel du protoplasma exlracapsulaire.

\title{
GENRES
}

Orona (Häckel) est l'expression simple de ce type morphologique (3 a 5,5$)\left({ }^{2}\right)$.

(souvent on trouve ces petites pyramides accouplées par deux); Cannopilus (Häckel) semblable au précédent, mais avec les tigelles de réunion des deux anneaux bifurquées de manière à former deux rangs de mailles $(0,02$ à 0,05$)$.

Mais Borgert [91], étudiant ces singulières formations squelettiques, en particulier chez Diste-

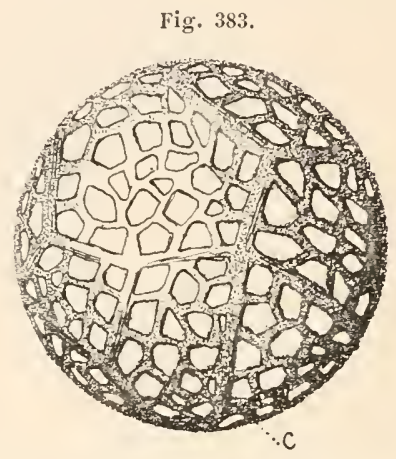

PIIEOSPHERID.E

('Type morphologique) (Sch.).

phanus, constate qu'on les trouve dans des Phæodariés d'autres genres, qu'on les rencontre dans des mers où il n'existe de Plıæodarié d'aucune sorte, et enfin qu'elles appartiennent à des êtres distincts virant en parasites ou en commensaux dans le corps de divers Radiolaires. Il en résulte que les quatre genres ci-dessus de Phæodariés doivent disparaître et que les êtres représentés par leurs parties molles retombent dans la famille des Phxodininæ ou Phæodariés sans squelette. Se confondent-ils avec les genres des Phæodininx ou doivent-ils recevoir de nouveaux noms? La question n'a pas été examinée. Quant aux noms de genres qu’ils possédaient avant, ils deviennent ceux des parasites que nous retrouverons plus loin en appendice aux Flagellés sous le nom de SILICOFLAGELLEA (Borgert).

(1) Genres voisins :

Aulacantha (Häckel) a, en plus, une couche d'aiguilles tangentielles (Spicules : 0,4 à 4,2); Aulographis (Häckel) a ses spicules radiaires terminés en dehors par un bouquet de branches simples $(0,4$ à 2,5$)$;

Auloceros (Häckel) les a terminées par un bouquet de branches ramifiées (Spicules : 0,6 à $3,5)$;

Autodendron (Häckel) a non seulement un bouquet terminal mais, dans la longueur des spicules, des épines éparses (Spicules : 0,7 à 3,6);

Aulospathis (IIäckel) de même, mais les épines latérales de ses spicules sont disposées en verticilles réguliers (Spicules : 0,15 à 0,25$)$;

Ces genres forment la famille des Avtıcısturse [Aulacanthida (IIäckel)].

(2) Genres voisins :

Orosphæra (IIäcliel) possède, en plıs, des épines radiales simples ou ramifiées (1 a 3,3 ; Oroscena (Häckel) a la surface de sa coquille parsemée de petites élévations coniques libres $(1,2$ à 3,2$)$; 
Sagena (Häckel) (fig. 384) a une coque grillagée formée de tigelles très délicates qui, par une exception assez rare chez les Cannopylaires, sont pleines et non creuses $(1,2$ à $2,5)(')$.

Aularia (Häckel) (fig. 38วั, 386) a une coquille sphérique grillagée simple (fig. 385) formée de tigelles

Fig. 384.

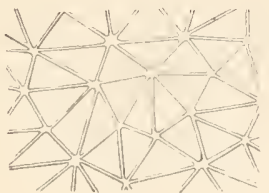

Sagena.

Partie du réseau (im. Häckel).
Fig. 385.

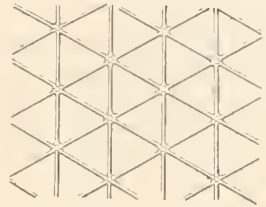

Aularia. Partie du réseau (im. IIäckel).
Fig. 386.

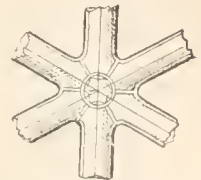

Aularia. Pièce stelliforme occupant les nœuds du réseau (im. Häckel).

creuses unies en un réseau à mailles triangulaires. Les tigelles s'unissent par six aux points nodaux, non en se soudant entre elles comme chez les précédents, mais en s'articulant par l'intermédiaire d'une petite pièce stelliforme (fig. 386) (1 à $3^{\mathrm{mm}}$ ) $\left(^{\mathrm{q}}\right)$.

Oroplegma (Häckel) est comme le précédent, mais ces petites élévations sont réunies entre elles, à leur sommet, par une deuxième enveloppe formée de tissu spongieux $(1,5$ a 3,3$)$.

Ces genres forment la famille des OrospIIERIN [Orosphrida (IÏ̈ckel)].

(1) Il en est de mème des genres ci-dessous.

Sagosphæra (Häckel) a, en outre, des épines radiales aux points nodaux ( 1 à $3 \mathrm{~mm}$ );

Sagoscena (IIäckel) a, au lieu de ces épines, de petites élévations pyramidales formées de faisceaux de tigelles $(1,7$ a 3,2$)$;

Sagenoscena (Häckel) est de même, mais l'axe de ses élévations pyramidales est occupé par un batonnet radiaile ( 1 à $4 \mathrm{~mm})$;

Sagenoarium (Borgert) est comme Sagoscena, mais les sommets des protubérances pyramidales sont réunis par destigelles qui constituent une deuxième coque ( $\mathrm{mm})$;

Sagmarium (läckel) a une coque simple, mais formee de tissu spongieux $(1,1$ à 2,4$)$;

Sagmidium (IÏ̈ckel) est de même, mais a, en outre, des épines radiales $(1,6$ a : $5 \mathrm{~mm})$;

Sagoplegma (IIäckel) est comme le précédent, mais a, en place des épines radiales, de petites élévations pyramidales surmontées d'une épine radiale ou d'un petit bouquet d'épines divergentes $(2 \mathrm{~mm}$ à $3, \breve{b})$.

Ces genres forment la famille des S.lGOsPILERIVA [Sagosphxrida (Häckel)].

${ }^{(2)}$ Genres voisins :

Aulosphæra (Häckel) a, en plus, des spicules radiaires aux nœuds du réseau (1 $1^{\mathrm{mm}}$ à 4,2);

Auloscena (Häckel) a ces mêmes spicules radiaux, mais au sommet d'éminences pyramidales (2 i $5 \mathrm{~mm})$;

Auloplegma (IIäckiel) a une coque formée d'après les mêmes prineipes qu'Aulosphara, mais spongieuse $(1$, ä i 3,2$)$;

Aulophacus (Häckel) est un Aulosphara à une coque déprimée dans le sens vertical (อ, כ̌ à 6 mm); Aulatractus (Hïckel) est un Aulosphxra à coque allongée dans le sens vertical (6 à $8^{\mathrm{mm}}$ ); Aulonia (Häckel) diffère de tous les précédents par la forme polygonale irrégulière de ses mailles, avec quatre tigelles seulement à chaque point nodal. Pour le reste, la

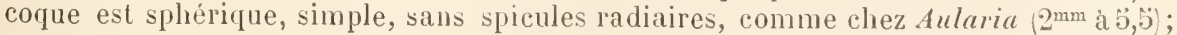
Aulastrum (Häckel) est de même avec des spicules comme ceux d'Aulosphæra (1,5̆ à 5ّm); Aulodictyum (IIackei) n'a pas de tubes radiaires, mais sa coquille est faite de tissu spongieux ( 2 à $\left.3^{\mathrm{mm}}\right)$.

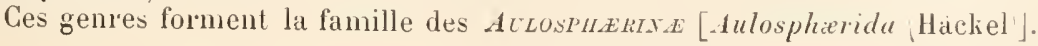


Cælacantha (R. Hertwig) a deux coques emboitées : une interne (mais extracapsulaire néanmoins), à ligelles soudées comme chez Orona el une externe à tigelles articulées comme chez Aularia (3 à 3,2) (").

\author{
$3^{\text {e }}$ Sous-Ordre \\ PHEOGROHIDES. - PII EOGROHIIIE \\ [PHEOGROMLA (Hïckel)]
}

\title{
TYPE MORPHOLOGIQUE
}

(FIG. 387)

Il dérive du lype morphologique des Cannopylaires, en ajoulant ce caractère essentiel qu'il existe une coquille formée d'une sphère grillagée pourvue d'une large ouverture, appelée bouche $(o)$, à la partie inférieure. La capsule centrale, construite sur le type ordinaire, n'est pas au centre de la coquille, mais plus près du pôle supérieur. La bouche est généralement armée d'apophyses dentiformes.

\section{GENRES}

Castanella (Häckel) (fig. 388) est un Phieogromide tout simple, à coque sphérique formée, non de spicules soudés en un grillage, mais d'une paroi compacte, percée de lrous. La coque est hérissée de petites épines. La bouche est relativement petite et entourée d'une couronne d'apophyses dentiformes $(0,22$ a $0,8)\left({ }^{2}\right)$.

(1) Genre voisin :

Cannosphæra(Iläckel)(fig.389) est de même, mais sa coque interne est continue, sans pores, el possède un grand orifice à

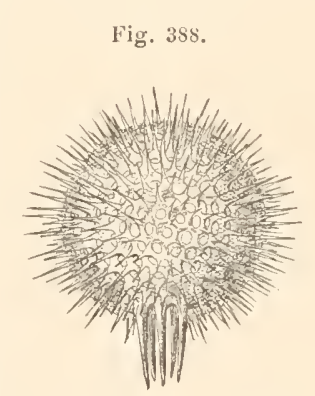

Castanella (im. Häckel).
Fig. 38 .

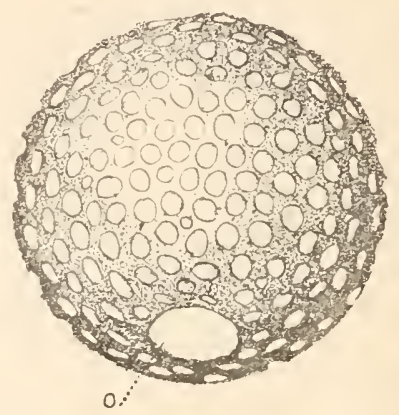

PHAOGROMIIDE

(Type mor'phologique) (Sch.). o., bouche.

la partie inférieure comme dans le sous-ordre suivant 0,5 a 2,5$)$.

Ces deux genres forment la famille des CANospHERTA [Camosplierida (IÏ̈ckel)].

$\left(^{2}\right)$ A part ces deux derniers qui sont génériques, ces caractères se retrouvent dans les genres ci-dessous :

Castanarium (Häckel) a la bouche dipourvue d'apophyses dentiformes 0,24 i 0, , iti ;

Castanidium (Häckel) a aussi la bouche inerme, mais la coquille armée de grandes épines simples, éparses parmi les petites $(0,22$ à 0,8$)$; 
Challengeria (J. Murray) (fig. 390) est remarquable par une structure très particulière de la coquille. Celle-ci est épaisse et formée, non de spicules, mais d'une substance compacle, homogène, et percée de fins canaux qui s'ouvient au dedans et au dehor's de la coquille par des pores si fins que l'on peut à peine s'assurer de leur existence. Mais, dans la partie morenne de leur trajet, ces canaux se dilatent en une petite chambre polygonale qui n'est séparée des voisines que par une mince paroi à la manière des alvéoles d'un gàteau l'abeilles. Quand on examine la coquille, ce sont ces cavités alvéolaires qui sautent aux yeux, et la coquille semble formée ou revètue d'une sorte de réseau polygonal. Mais ce n'est là qu'un aspect optique le la disposition que nous venons de dérrire. C'est ce que l'on appelle la structure diatoméenne à cause de sa ressemblance

Fig. 390 .

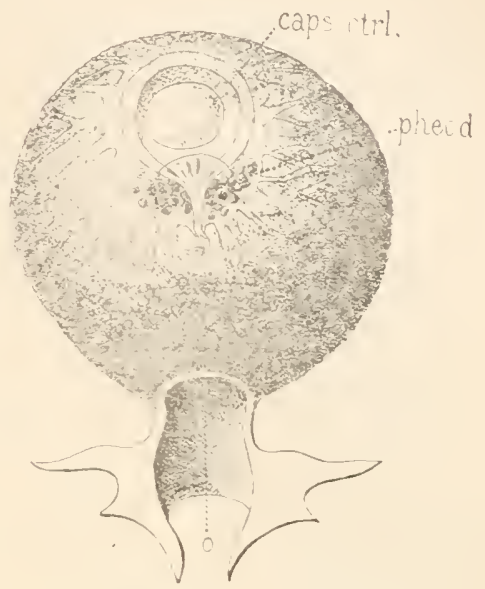

Challengeria (im. Häckel).

caps. ctrl., capsule cenlrale; pheod., pharodium: o., bouche.

avec celle des Diatomées. La capsule centrale n'a pas l'orifices accessoires $0,08$ à 0,8$\rangle\left({ }^{\prime}\right)$.

Cortinetta (Hïckel) (figg. 392) a une coquille extrèmement fragile, sphérique, surmontée d'une corne apicale et percée en lias d'une large bouche sans dents, mais entourée de trois longs pieds descendants, égaux, courhes, à concavité intérieure. La structure diffère de celle de Challengeria

Castanissa (Häckel) est semblable, mais a la bouche assez grande et armíe $\{0,28$ à 0,8$)$; Castanopsis (Häckel a la bouche petite et inerme: mais les grandes épines de la coquille ramifiées $(0,42$ i 0,7$)$;

Castanura (IIäckel) est semblable au précident, mais a la bonche armée $(0,28$ it 0,6 .

Ces genres forment la famille des FAstivelLIse [Castanellida [IÏ̈cliel)].

(1) Tous ces caractères se relrouvent dans les genres ci-rlessous; mais il n'en est pas de mème de la conformation de la bouche qui est fort variable et présente ici deux apophyses dentiformes:

Lithogromia (IIäckel) n'a pas ces dents buccales $(0,15$ à 0,27$)$;

Challengeron (\$. Murray' (fig. 391) les a, au contraire, et possede, en plus, une ou plusieurs épines 0,06 à 0,3$)$;

Pharynge/la (IIäckel), est comme Challengeria, mais possède, en plus, un tuhe qui part de la bouche et remonte en dedans $\{0,25$ i 0,36$\}$,

Entocannula Hickiel) est de mème, mais n'a pas de dents buccales $\{0,23$ it 0,36$)$;

Porcupinia (Häckel) de mème aussi, mais a des dents buccales et, en outre. des épines marginales $(0.2$ it 0,25$)$.

Ces genres forment la famille des CILALEXGERTYE [Challengerida (J. Muray)]. 
par le fait que les alvéoles $(B)$ sont très irréguliers et ne communiquent pas avec le dehors, mais seulement avec le dedans de la coquille, et encore n'a-t-on pu que rarement s'assurer de l'existence des trìs fins pores qui établissent cetle communication; la surface extérieure est entièrement continue. Ces alvéoles, très petits au sommet de la coquille, deviennent de plus en plus larges vers le bas et se continuent dans les pieds où ils forment une série unique axiale qui donne à ceux-ci un aspect articulé. Les alvéoles des piods communiquent entre eux par un petit canal saillant dans le compartiment distal par rapport à la cloison qui le porte. Tous les alvéoles sont remplis de gelée. Dans son ensemble l'animal ressemble à une petite Méduse $(0,14$ à 0,18$)$ (").

Circospathis (Häckel) (fig. 393) a une coquille polyédrique à quatorze faces, formée par de fines aiguilles pleines, tangenticlles, assez rares, empâtées dans une épaisse croûte de ciment opaque, porcelainé, qui forme, de beaucoup, la

Fig. 392.

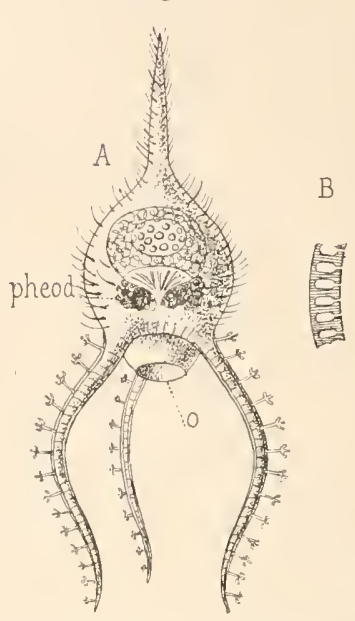

Cortinetta (im. Häckel).

$A$, lanimal entier; $B$, coupe do la paroi. pheod., phroodium; o., orifice. plus grande partie de la masse. Cette coque est gaufrée à sa surface

Fig. 393.

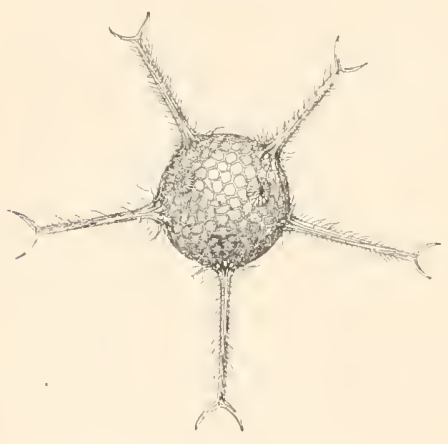

rircospathis (im. Häckel). externe de dépressions polygonales en cellules d'abeilles, séparées par des crêtes, et ces surfaces déprimées sont finement ponctuées. Ces ponctuations semblent être de fins pores qui traverseraient toute l'épaisseur de la coquille. Sur la coque ainsi constituée, s'insèrent, aux sommets du polyèdre, neuf grosses épines radiales dont la base est entourée d'un cercle de gros pores. Ces épines sont creuses et leur axe est occupé par un filament axial rattaché aux parois de la cavité centrale par des trabécules. La bouche de la coquille, relativement petite, est entourée de saillies dentiformes. La capsule centrale possède neuf ouver-

(1) Genres voisins :

Medusetta (Häckel) est semblable, mais a quatre pieds égaux $(0,05$ à 0,09$)$;

Euphysetta (Häckel) a trois petits pierls et un grand $(0,15$ à 0,16$)$;

Gazelletta J. Murray) n'a pas de corne, mais a six pieds $(0,02$ i 1,5$)$;

Gorgonetta (IÏckel) a les six pieds du précedent et, en outre, six pieds ascendants qui alternent avec les autres $(0,2$ à 0,5$)$;

Polypetta IIäckel) a dix (ou vingt ou plus) pieds descendants $(0,01$ à 0,12$)$.

Ces genres forment la famille des MEDL'SETTLNE [Medusettida (Iläckel)]. 
tures accessoires au lieu de deux et qui correspondent par leur position aux couronnes d'orifices qui entourent la base des épines radiales. Pour le reste, ce sont les caractères d'un Phægromide $\{0,5$ à 0,6$\}$ (").

Tuscarora (J. Murray) (fig. 394) a une coquille ovoïde, allongée dans le sens du grand axe et semblable à celle du genre précédent, c'està-dire formée d'une pâte porcelainée, opaque, arec des aiguilles tangentielles dans sa masse. Cette coquille est percée de nombreux petits pores et porte trois grandes épines radiales, équidistantes, creuses. Ces épines sont parcourues par un filament axile rattaché aux parois par des trabécules. Nais leur cavité communique avec celle de la coquille et, autour de leur base, sont percés aussi de gros pores qui débouchent dans la coquille et au dehors. En somme, la structure est très semblable à celle de Circospathis, mais la surface externe est lisse et non gaufrée d'alréoles. La capsule centrale semble avoir trois orifices accessoires

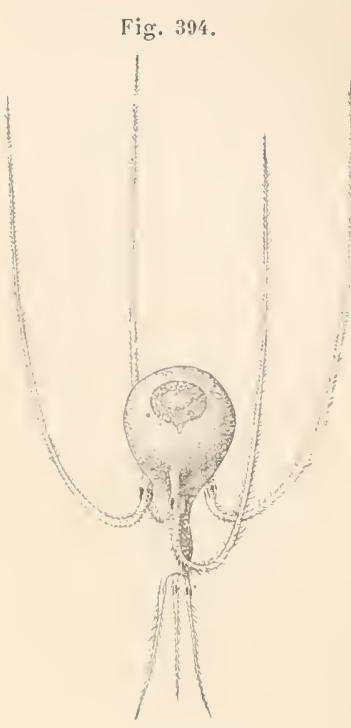

Tuscarora (im. Häckel). correspondant aux trois cercles de pores entourant la base des épines. La bouche est entourée de trois prolongements creux semblables aux épines radiales $(\mathbf{1}, 5$ à 2,5$)\left({ }^{*}\right)$.

\section{$4^{\text {e }}$ Sous-Ordre \\ PILEOCONCHIDES. - PHEOCONCHID.E \\ [PIIEOCONCHIA (Häckel)]}

\section{TYPE MORPHOLOGIQUE}

(FIG. 395)

En un seul point, le tỹpe de ce groupe diffère du type général des Radiolaires, mais ce point est essentiel et extrêmement remarquable. L'animal

(1) Genres voisins :

Circoporus (Häckel) est sphérique ou octaédrique et a seulement six épines $(0,16$ à $0,6 a$ b̆); Circogonia (Häckel) est icosaédrique et a douze épines $(0,6$ à 0,75$)$;

Circorrhegma (Häckel) est dodécaédrique avec vingt épines $(0.8)$;

Circostephanus (Häckel) est polyédrique it nombreuses faces, et compte vingt-quatre à quarante épines ou plus $(0,4$ i 0,8$)$;

Hæckeliana (J. Murray) est tout ì fait sphérique, les dépressions de sa surface sont de simples fossettes, et il a un nombre variable d'épines $\{0,25$ à 0,52$)$.

Ces genres forment la famille des CIRCOPORIsE [Circoporida (IIäckel)].

(2) Genres voisins :

Tuscarusa (Häckel) a quatre épines radiales au lieu de trois $(\mathbf{1}, 2)$;

Tuscaridium (IIäckel) n'en a qu'une, aborale $(3,2$ à 3,6).

Ces trois genres forment la famille des TUSCARORINA [Tuscarorida (Häeliel)]. 
possède, comme tant d'autres Radiolaires, une coquille grillagée plus ou moins sphérique formée de tigelles pleines, soudées en un réseau; mais celte coquille est formée de deux valves hémisphériques réunies en un point par un ligament élastique, absolument comme les valves d'un Lamellibranche, disons plutôt comme celles d'un Brachiopode, car ces deux valves sont l'une dorsale l'autre ventrale, la eapsule étant orientée à l'intérieur de telle façon que ses trois orifices (os. os. oi.) sont dans le plan coronal qui les sépare, en sorle que les tractus protoplasmiques qui en sortent sont directement en rapport arec l'ouverture de la coquille. Le ligament est situé au pôle supérieur de la coquille, vers lequel la capsule tourne ses orifices accessoires (os.), tandis que son ouverture principale (oi.) allonge son tube à travers le phaodium, vers le milieu de la ligne de déhiscence, au point opposé au ligament. La capsule est placée dans la coquille plus près du pôle supéricur que de l'inférieur (').

\section{GENRES}

Concharium (Hïckel) (fig. 396) est notre type morphologique mème, sauf l'absence de ligament: les deux valves sont entièrement indépendantes et simplement maintenues par la gelée qui les englobe $(0,2$ à 0,25$)\left({ }^{2}\right)$.

(1) Ces curieuses formes proviennent toutes des dragages du Challenger.

${ }^{(2)}$ Genres voisins :

Conchasma (IIäckel) est de même, mais a, en plus, deux cornes au pòle aboral, une sur chaque valve $\langle 0,16$ it 0,22$)$;

Conchelium (Häckel) est comme Concharium, mais ses deux valves s'engrènent par un bord denté $(0,06$ à 0,38$)$;

Conchidium (Häckel) est comme le précédent, mais avec deux cornes aborales $(0,24$ à 0,35$)$;

Conchonia (Häckel) a. non seulement ces deux cornes aborales, mais une dorsale $(0,21$ à 0,3$)$;

Conchopsis (IIäckel) n'a pas de cornes, mais est fortement comprimé de droite à gauche, et ses valves portent chacune une carìne sagittale $(0,55$ ì 0,8$)$;

Conchoceras (Häckel) est semblable au précédent, mais il a, en plus, deux cornes aborales $(0,1$ à 0,22$)$.

Ces genres forment la famille des CoxchaRtr. [Concharida (IIäckel)]. 
Cœlodendrum (IIiickel) (fig. 397) a sa coque bivalve très petite, entourant

Fig. 397.

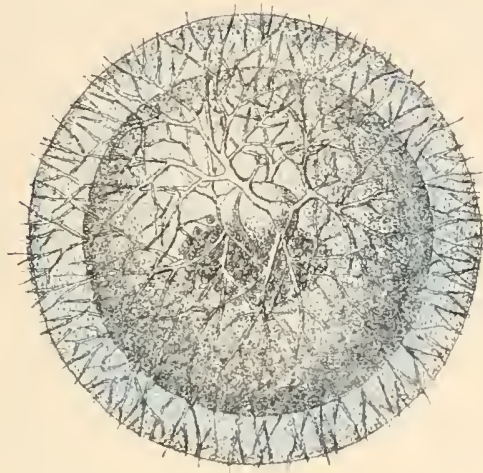

Coclodendrum (im. Häckel) immédiatement la capsule centrale. Ses valves sont étroitement jointes à leur ligne d'union. Elles sont percées de fins pores et très fragiles. Mais leur caractère le plus remarquable est qu'elles portent chacune, en leur centre (par conséquent l'une en avant l'autre en arrière), une sorte de proéminence en pyramide triangulaire, formée du mème tissu perforé. Chacune de ces éminences émet de longs prolongements en forme de tubes ramifiés extrèmement délicats dont les liranches ront former une sorte de buisson concentrique à la coquille primitive. Tout cela est noyé dans la gelée. Le phicodium, ne pouvant se loger dans la rraie coquille entièrement remplic par la capsule, occupe les deux couproles prramilales (dont la cavité communique par des perforations arec celle de la coquille, tandis qu'elle est entièrement séparée de celle des tubes), et se répand entre les branches des tubes rameux (1 is $3 \mathrm{~mm}$ ) (').

Cœloplegma (Hïckel) (fig. 398) nous montre, en mème temps que la dernière des formes types de la classe

(1) Genles voisins :

Cœlodoras (Iläckel) est semblable, mais plus simple, ses tubes n'etant pas ramifiés 0,16 à 0,2$\}$;

Cœlodrymus (Häckel) est comme Celodendrum el, en outre, les ramifications de ses tubes s'anastomosent entre elles en une sorle de réseau qui dessine une sphère creuse $\left(2\right.$ it $\left.3^{\mathrm{mm}}\right)$;

Cœlodasea (Häckel) est semblahle, mais

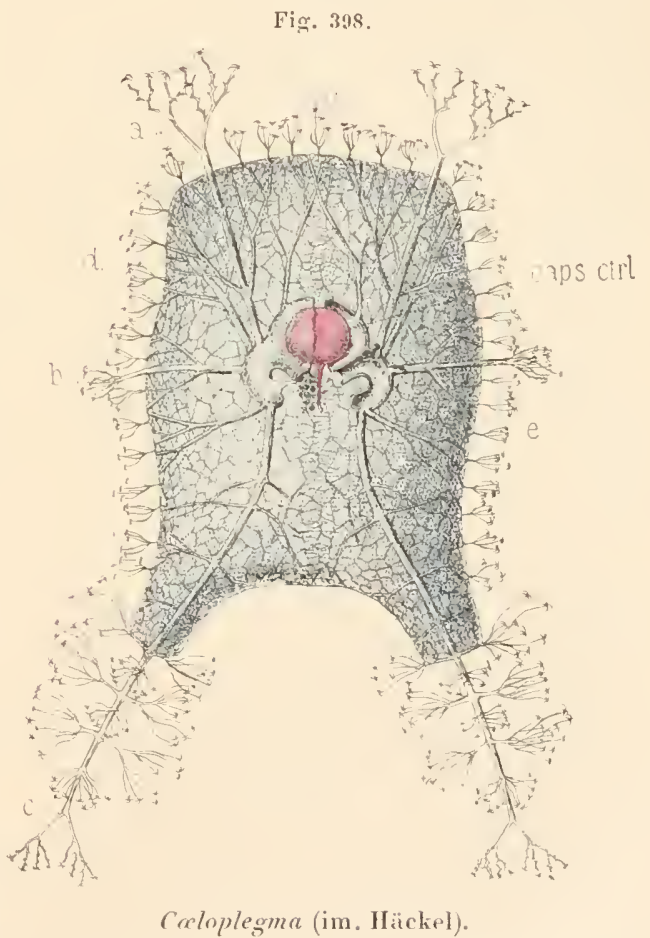
les ramifications de ses tubes se répandent, non seulement en surface, mais en épaisseur, de manière à former un tissu spongieux $(2 \mathrm{~mm}$ ì 2.3$)$.

Ces genres forment la famille des CaLODENDRTAE [Colodendrida (TIäckel)]. 
des Radiolaires, la forme la plus compliquée, et peut-être même le plus compliqué de tous les Protozoaires, quoique non pas le plus élevé en organisation. Comme dans le genre précédent, la coque sphérique $(d)$ est petite et formée d'un tissu délicat et percé de pores fins et irrégulièrement distribués. Elle n’entoure pas cependant aussi étroitement la capsule (caps. ctrl.), ses deux valves $(d, e)$ étant un peu écartées l'une de l'autre. Celles-ci portent l'une et l'autre, comme chez Colodendrum, une sorte de coupole d'où partent des tubes ramifiés, mais ici ces tubes sont parfaitement symétriques et leurs ramifications sont anastomosées en un réseau continu formant, à grande distance de la coque primitive, un buisson sphérique extrêmement délicat. En outre, les branches principales de ce buisson, au nombre de quatorzo (une impaire et six paires sur chaqur valve), se continuent en direction rarliale sans se ramifier et forment de grandes épines creuses $(a, b, c)$, plus ou moins chargées d'appendices latéraux verticillés et qui dépassent de beaucoup la surface buissonneuse. Mais le plus curieux, e'est que les deux coupoles émettent, en outre de ces tubes, chacune un prolongement creux ajpelé tube nasal (e) (rhinocanna) qui part de sa base, descend sur la paroi de la coque suivant le méridien sagittal correspondant (antérieur ou postérieur, selon la valve) et va s'ouvrir, en face de celui du côté opposé, juste au-lessous du point où se termine entre eux le tube de l'orifice principal de la capsule centrale. Ces tubes s'ouvrent d'autre part chacun dans la coupole d'où il part, tandis que la coupole ne communique, ni directement ni par des perforations, avec la coque ou avec les tubes qui forment le buisson de la coquille. Enfin, une tigelle squelettique, le frein, s'étend, de chaque côté, de la coupole à l'extrémité du tube nasal. La signification de ces complications extraordinaires est complètement inconnue. Le phæodium se loge dans les coupoles et dans les tubes nasaux et déborde en outre généralement au dehors. Toute la coquille est noyée dans la gelée, sauf les grandes épines radiaires qui la dépassent de beaucoup $(1,6$ à 3,2) (").

(1) Ces dimensions sont celles de la coquille sans les épines radiales : comme partout elles sont données en millimètres.

Cœlagalma (IIäcliel) est semblable, mais a seize grandes épines radiaires dépassant la gelée au lieu de quatorze $(5,4)$;

Cœlostylus (Häickel) n'en a, au contraire, que douze $(3,2$ a 4,2);

Colodecas (Häckel) n'en a que dix $(2,6$ à 3,6$\rangle$;

Cœlospathis (IIäckel) n'en a que huit (2 à $\left.3^{\mathrm{mm}}\right)$;

Cœlographis (Häckel) n'en a que six $(3,2$ a 6,4$)$;

Colotholus (Häckel) en a huit, mais leurs ramifications restent comme chez Colodendrum, indépendantes, et forment un buisson mais non un réseau $\left(2,5\right.$ ì $\left.4^{\mathrm{mm}}\right)$;

Cœlothauma (Häckel) en a douze avec le mème caractère $(3,5)$;

Cœlothamnus (Häckel), avec le mème caraclère, en a seize; avec les épines radiales, le diamètre atteint 33 centimètres chez une espèce. C'est le géant des Radiolaires $(1,8$ a 7,5$)$.

Cies genres forment la famille des CELOGRAPHITE [Calographida (IIickelj]. 
A P PENDICE

\section{TAXOPODES. - TAXOPODEA \\ [TAYOPODA (II. Fol)] \\ (FIG. 399 ET 400)}

Nous plaçons ici, en appendice à la classe des Rhizopodes, un être à affinités indécises que R. Hertwig [r] qui l'a découvert, rattachait aux Amibes faute de lui trouver une place meilleure, et ilont II. Fou [83] qui l'a le mieux étudié, faisait, parmi les Rhizopodes, un groupe intermédiaire aux Iléliozoaires et aux Radiolaires et de valeur égale, c’està-dire, pour lui un ordre, pour nous une sous-classe. Cet ètre est lo

Sticholonche (R. Ilertwig) comprenant une seule espèce, S. Zanclea (R. Hertwig). C'est un animal pélagique (Néditerranée) qui mesure environ $\mathrm{I}^{\mathrm{mm}}$. Il est formé d'une masse protoplasmique enfermée dans une coquille $(t)$ et émettant des sortes de pseudopodes (pspd.); il renferme un organe spécial, le corps réniforme (c. ren.) qui contient lui-mème un globule central (g.) et, à côté des corps réniformes, des productions énigmatiques interprétées, sans raisons suffisantes, comme corps reproducteurs.

La forme générale est une sorte d'ovoüle comprimé de manière à produire, parallèlement au grand axe, un Fig. 399 .

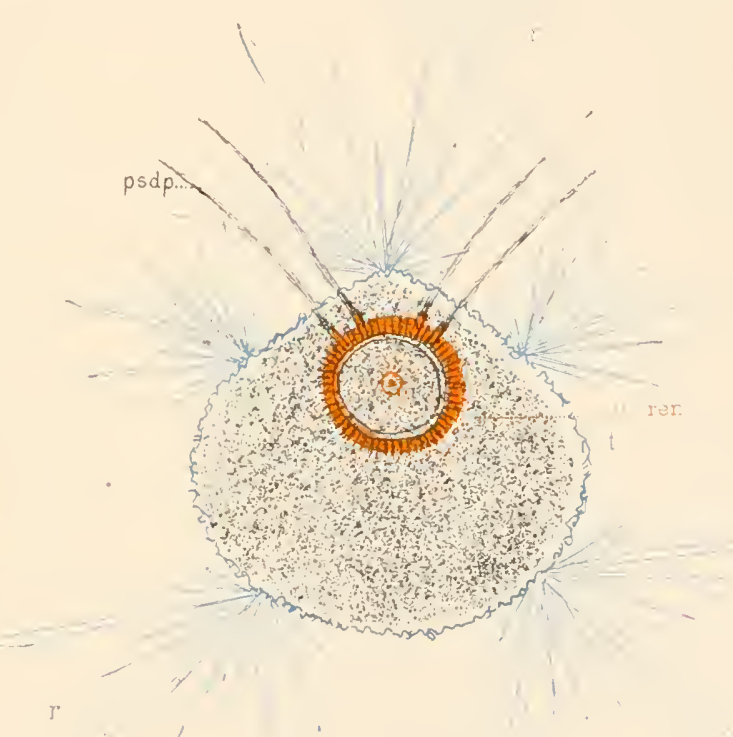
bord que nous appellerons dorsal et, opposée à ce bord, une face ventrale très bombée.

Le corps protoplasmique est revêtu d'une coquille formée d'un (feutrage de tubes très fins $(t)$ enchevètrés ensemble. Sur ce feutrage, sont insérés par bouquets, le longs spicules creux $(r)$ de taille si variée qu'il n'y en a pas deux semblables dans chaque bouquet. Le tout est formé 
d'une sulstance organique, plus ou moins semblable à la chitine, légè-

Fig. 400.

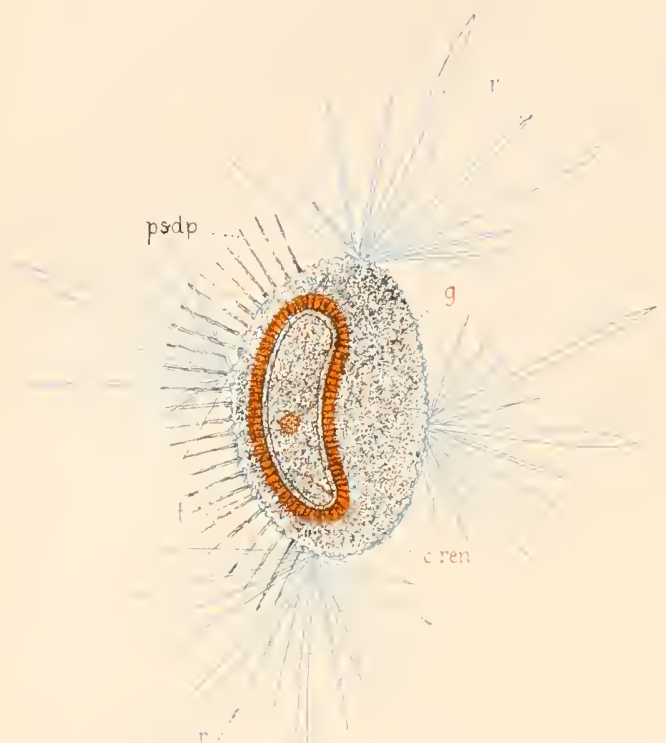

Sticholonche Zanclea. Coupe sagittale (Sch.) c.ren., corps réniforme; g., glolule chromatique; psip., pseudoporles; $\mathbf{r}$., spicules; t., corquille. rement minéralisée par un carbonate terrenx.

La masse protoplasmique ne présente rien autre chose de particulier que de très nombreux globules pâles.

Le corps réniforme a plutôt la forme d'un haricot que celle d'un rein. Il est orienté parallèlement au grand axe de l'animal, plus rapproché du bord lorsal de celui-ci, et tournant vers ce bord dorsal sa convexité. Il est entouré d'une membrane mince et contient, noyé dans un plasma d'apparence homogène, un globule chromatique $(g \cdot)$ qui contient luimème une granulation centrale.

Le corps réniforme est enveloppé dans une coque épaisse formée de petits prismes radiaires serrés les uns contre les autres. Ces prismes ne sont pas tous égaux. Le long du bord convexe du haricot, il y en a quatre rangées parallèles à ce borl, qui sont plus grandes que les autres.

Sur ces quatre rangées, s'insèrent quatre rangées longitudinales, verticales et légèrement divergentes de pseudopodes. Ces pseudopodes (pspd.) sont assez gros, pointus au bout, presque immobiles, peu anastomosables ou mème probablement pas du tout (').

On ne sait rien des fonctions de ces organes ni de la manière dont l'animal se nourrit $\left({ }^{2}\right)$.

(1) On les voit parfois battre ensemble du mème mouvement que les avirons d'une trirême, mais e'est quand l'animal, qui est d'une délicatesse extrème, est rétractè dans sa eoquille et plus ou moins détérioré. Ce sont des mouvements agoniques, sans doute eommuniqués par le eorps il ees appendices.

D’après Konotsef ils auraient un filament axile eomme eeux des ILéliozoaires et ee filament se prolongerait rlans la paroi du eorps réniforme et peut-être jusqu’ì son eentre.

${ }^{(2)}$ Chez la plupart des individus adultes, on trouve, dans la concavité du corps réniforme, soit de gros globules pàles, soit un corps spécial, le corps spiral, que Fol interprétail comme un spermatophore. Köppen [9: vient de démontrer que ee n'était qu un Aeinete parasite, Amobophyra sticholonchi, a bourgeonnemont interne comme 
Les affinités de ce singulier animal sont difficiles à saisir. La coquille ressemble plus à celle d'un Radiolaire qu'ì celle de tout autre Protozoaire. Le protoplasma du corps ne présente rien de particulier. Le corps réniforme est-il un noyau et contient-il un nucléole comme le veut FoL, ou est-il une capsule centrale et le globule qu'il contient est-il un noyau? Il ne ressemble nettement à aucun de ces organes. Plus difficiles encore à interpréter sont la coque de bàtonnets qui entoure le corps réniforme et les rapports de ces bâtonnets avec les singuliers pseudopodes.

Il n'y a rien à conclure, et l'on ne peut qu'exprimer des réserves en attendant de nouvelles études.

chez Dendrocometes ou Tokophrya. La portion interne n'est autre chose que le bourgeon de l'Acinète. Köppen a vu le jeune parasite pénétrer dans le col'ps de l'hôte. Rappelons qu'un parasite semblable avait été pris chez les Acanthaires pour le noyau (V. p. 206). 


\section{2e Chasse}

\section{SPOROZOAIRES. - SPOROZOARIA (*)}

\section{[SPORozoA (Leuckart)]}

Nous avons placé parmi les Rhizopodes tous les êtres qui émetlent par la surface nue de leur corps des pseudopodes, prolongements protoplasmiques dans lesquels le protoplasma superficiel se déplace, en sorte que ce n'est pas toujours la mème substance qui forme à chaque instant le mème point de la surface du corps. Les êtres que nous allons étudier maintenant se caractérisent au contraire par leur corps dépourvu d'appendices el muni d'une paroi fixe dont les éléments ne se mélangent pas entre eux. En outre, tous sont parasites et se reproduisent par des spores. C'est à ce dernier caraclère, que l'on a emprunté leur nom de SPOROZOAIRES.

\section{TYPE MORPHOLOGIQUE}

(FIG. 401)

Le type morphologique sera représenté par un organisme unicellulaire, parasite, de forme arrondie, formé d'une masse cytoplasmique

Fig. 401.
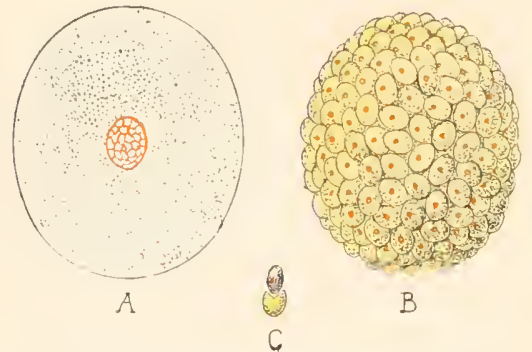

Sporozoatire (Type morphologique) (Sch.).

$A$, adulte; $\boldsymbol{B}$, sporulation; $C$, sporozoite sortant de la spore. renfermant un noyau el munie d'une paroi souple, mais fixe el absolument continue, sans bouche, anus, pore excréteur ou orifice quelconque. Il n'y a ni vacuole alimentaire, ni vésicule pulsatile, ni appendice (pseudopode, cil, flagellum). L'animal peut, à l'occasion, se reproduire par division, mais normalement, la reprorluction se fait par spor'ulation, c'est-à-dire que le protoplasma se divise en un cerlain nombre de parlies, les spores, pourvues chacune d'un noyau issu du noyau primitif el limitées chacune par une membrane protectrice. Mais ces spores ne donnent pas naissance directement à une forme semblable à celle de l'adulte : elles éclosent sous la forme d'un petit organite protoplasmique, nucléé, auquel on a donné le nom de sporozoïte el qui, en grandissant, devient l'animal adulte.

D'après les caractères de ce sporozoïte, on peut diviser les Sporozoaires en deux sous-classes :

$\left(^{*}\right)$ Nous exprimons ici tous nos lemercîments ì M. Labbé dont la compétence en matière de Sporozoaires est bien connue. M. Labbé a rédigé un résumé détaillé et fort bien fait qui nous a servi pour la rédaction et il a bien voulu revoir ensuite l'ensemble du chapitre. 
RIIABDOGENIE, à sporozoïte de forme définie, généralement arquée; AMOEBOGENLE, à sporozoïte amœboïle (").

\section{$1^{\text {re }}$ Sous-Cilasse \\ RHABDOGÉNIENS. - RHABDOGENIE \\ [CyTosporidies (Labbé)]}

\section{TYPE MORPHOLOGIQUE}

Il se déduit aisément du type général des Sporozoaires, en y ajoutant les caractéristiques indiquées ci-dessus, c’est-à-dire la forme fixe et généralement arquée du sporozoïte qui prend ici le nom de corpuscule falciforme. Les premiers stades de l'évolution se passent toujours dans l'intérieur d'une cellule de l'hòte.

Cette sous-classe se divise en deux ordres:

BracIIYCYSTIDA, chez lesquels la forme de l'adulte, à l'état de repos, dérive de la sphère;

DoLic II C YSTIDA, chez lesquels cette forme dérive d'un ovoïle allongé.

\section{L $^{\text {er ORDRE }}$ \\ BRACIIGYSTIDES. - BRACIIYCYSTIDA}

\section{TYPE MORPHOLOGIQUE}

Il est conforme au type rhabdogénique, en y ajoutant ce caractère de la forme sphérique de l'adulte à l'état de repos.

Les Brachycystides se divisent en quatre sous-ordres:

GrREARINIDE, libres, allongés, mobiles, pourvus de fibrilles contractiles dans l'épaisseur du tégument, et s'enkystant toujours hor's du tissu qui leur a donné asile pendant les premières phases de leur existence;

COCCIDIDE, non libres, sans fibrilles contractiles, s'enkystant dans le tissu où ils ont commencé leur évolution;

II EMOSPORIDIDE, libres et s'enkystant dans les globules sanguins;

GYYNOSPORIDIDE, à vie exclusivement intracellulaire; amoebiformes à l'état adulte et dépourvus d'enveloppe kystique.

(1) LABBÉ divise les Sporozoaires en Cytosporidies correspondant à nos Rhabdogéniens brachycystides et caractérisées par leur habitat intraceltulaire au moins au début de leur existence, et Histosporidies comprenant nos Rhabdogéniens dolichocystides et nos Amobogéniens, et caractérisées par leur labitat intercellulaire, dans le tissu de l'hôte. Mais les IIistosporidies des muscles sont souvent dans la cellule musculaire elle-même, et il nous a semblé avantageux de substituer à cette classification basée sur une caractéristique empruntée aux rappor's du parasite avec l'hôte, un groupement fondé sur des caractères anatomiques. Il nous a d'ailleurs aidé luimême à établir la classification adoptée ici. 


\section{1er SOUS-ORDRE \\ GRÉGARINIDES. - GREGARINID.E}

\section{TYPE MORPHOLOGIQUE}

(FIG. 402 A 414)

L'animal est un petit être blanchàtre, vermiforme, allongé, mesurant quelques dixièmes de millimètres de long sur une largeur deux ou trois fois moindre, et un peu aplati en outre dans le sens dor'so-ventral (fig. 402). Une des extrémités est arrondie, l'autre prolongée en une sorte de col qui se termine par un renflement armé de quelques crochets. Ce renfle-

Fig. 402.

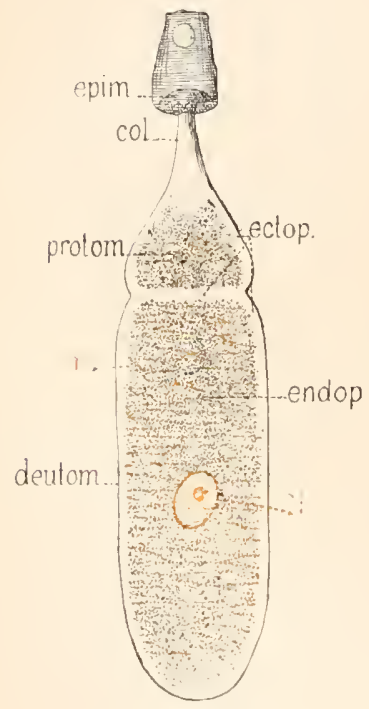

GREGALINID.

(Type morphologique) Stade céphalin fixé (Sch.).

col., eol; dentom., deutomérite; ectop., ectoplasma; endop., endoplasma; epim., épimérite, dans la cellule de l'hôte; m., myonèmes; N., noyau; protom., protomérite.

forme la plus grande partie de la masse du corps, l'autre plus ferme, l'ectoplasme (ectop.), disposé en une mince couche superficielle, sous

(1) La forme de cette parlie terminale et des organes fixateurs dont elle est pourvue est une des parties les plus variables dans le corps de l'animal. Elle fournil de bons caractères pour la délermination des genres.

En outre, cel aplatissement dorso-ventral n'est jas du tout constant chez tous les genres. de l'intestin et tout le reste du corps pend dans la cavilé digestive. Plus tard, quand l'animal sera devenu libre, c'est celte extrémité qui sera en avant dans la progression: elle est donc pour nous supérieure dans la position morphologique. Nous pouvons aussi distinguer, gràce au léger aplatissement, les faces dorsale ou ventrale des bords droit ou gauche, mais rien ne nous permet de dire laquelle des deux faces est dorsale ou ventrale, lequel des bords est gauche ou droit (').

\section{Structure.}

Constitution générale du corps. - L'ètre tout entier u'est qu'une cellule. Il se compose (fig. 402) d'un cytoplasme, d'une membrane et d'un noyau.

Le noyau $(N$.) est au centre du corps, un peu au-dessous du milieu; la membrane revèt toute la surface sans présenter d'orifice quelconque, bouche, anus ou pore excréteur. D'ailleurs, il n'y a ni tube digestif, ni vésicule pulsatile. Le cytoplasme se divise en deux parties, l'une centrale, fluide, l'endoplasme (endop.), qui

ment est fixé dans une des cellules épithéliales 
jacente à la membrane avec laquelle il constitue le tégument. L'ectoplasme tapisse la membrane dans toute son étendue el il forme, en outre, une cloison transversale qui sépare la cellule en deux parties, l'une inférieure contenant le noyau, lautre supérieure portant le col et son bouton terminal. Mais, comme le segment supérieur ne contient pas de noyau, celte cloison n'a nullement la signification d'une paroi cellulaire: elle n'est qu'une particularité interne de structure sans importance morphologique et le tout n'en reste pas moins une cellule unique. Elle permel de distinguer trois parties utiles à nommer pour les commodités de la description. Ce sont: 10 l'épimérite (épim.) formé par le col avec son bouton terminal; 2。 le protomérite (protom.) formé par le reste du segmenl supérieur; $3^{\circ}$ le deutomérite (deutom.) formé par le seggment inférieur contenant le noyau. Enfin, à la limite entre les deux couches du crloplasme, se trouve une couche de fibrilles contractiles ou myonimes $(m$.) appartenant à l'ectoplasme mais faisant saillie dans l'endoplasme (').

Membrane. - La membrane est formée d'une substance organique, protoélastine; elle n'est pas un produit de sécrétion, mais une différenciation de la partie superficielle du cytoplasma. Elle est assez épaisse et nettement délimitée par un double contour. Elle est striée longitudinalement (figg. 403, memb.), et cette striation est due à de petits sillons très étroits mais très profonds qui la seclionnent complètement, metlant l'ectoplasme à nu au fond des sillons. Il en résulte qu'elle est formée de lanières étroites placées de champ, côte à còte, tout autour de sa surface. Mais cette striation ne s'étend pas à l'épimérite. Vers la partie supérieure du corps, les sillons disparaissent peu à peu et l'épimérite est re-

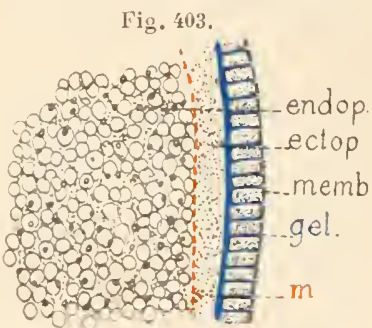

GRIGARIVID E

(Type morphologique)

d'ap. Cheviakof).

ectop., ectoplasma; endop., endoplasma; gel., couclie de gélatine; m., myonimes; memb., membrane. couvert d'une membrane continue et lisse. A l'extrémité inférieure, les lanières se soudent les unes aux autres par leurs extrémités à mesure

(1) Souvent, la cloison n existe pas et on ne distingue plus alor's ifue l'épimérite et le corps. La présence ou l'absence de celte cloison et par suite d'un protomérite, dont on faisail autrefois un caractère capital consacré par la distinction des Dicystides et des Polycystides, a si peu d'importance que, dans une mème espéce, on troure des individus a cloison et d'autres sans cloison. Tel est le cas, d'après Léger, pour Eirmocyrtis polymorpha et Clepsidrina Podure. Dans les associations de Porospora à trois individus, la cloison du troisième disparaît. II en est de mème pour celle du second dans les associations à deux que forme Didymophies. Gabriel a trouvé dans une Salicoque, Typton spongicola, une Grégarinide qui, jeune, u'a pas de septum et, adulte, en a plusieurs. Enfin, parlois, le noyau peut se trouver inclus dans le protomérite par suite d'unc migration tarlive arrétée par la formation précoce de la cloison, et l'animal n'est en rien troublé par cette modification de sa structure.

On a donné d’autres noms à ces diverses parties : on a appelé la membrane 
que le diamètre du corps diminue, en sorte que le nombre des sillons et

Fig. 404.

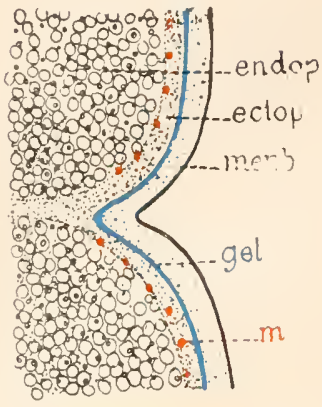

GREGARINIDE

(Type morphologique). Coupe longitudinale (d'ap. Cheviakof).

ectop., ectoplasma; endop., endoplasma; ger., couche de gélatine; m., myonc̀mes; memb., membrane. des lanières décroît progressivement jusqu'au pôle. A l'extrémité opposée, la membrane est plus épaisse sur le bouton de l'épimérite et surtout sur les crochets qui garnissent son contour.

Cytoplasma. - L'ectoplasme (fig. 403 et $\mathbf{4 0 4}$, ectop.) forme une couche d'épaisseur assez régulière et notablement plus forte que celle de la membrane. Seul il forme la cloison, seul il pénètre jusqu'au sommet de l'épimérite. Il est formé d'un protoplasma hyalin, homogène, sans granulations, où l'on a cru parfois distinguer un aspect rappelant la structure alvéolaire de Bütschli. 11 est ferme et suit, sans se déformer, toules les ondulations du corps dans les mouvements de celui-ci.

ll forme donc avec la membrane un vrai tégument pour l'animal.

L'endoplasme (endop.) est tellement granuleux qu'on ne lui distingue aucune structure. Il ne contient pas de vacuoles. Il semble formé d'une substance homogène presque fluide où flottent, indépendamment des microsomes protoplasmiques, de nombreux globules variés de taille et d'aspect. Quand on les a détruits à l'aide de certains réactifs, on croit lui reconnaitre une structure réticulée, mais rien ne prouve que ce ne soit pas là un effet des réactifs.

Les principales de ces inclusions sont réfringentes et montrent, avec les nicols croisés, une croix de polarisation comme l'amidon('). Elles paraissent formées d'une substance analogue à l'amidon et au glycogène, probablement le zooamylon de Maupas ou paraglycogène de Bütschli. D'autres globules sont de nature graisseuse. Les r.ns et les autres sont des éléments de réserve, et représentent une phase de l'évolution des substances alimentaires. On rencontre aussi quelques cristaux d'une substance protéique. Mlais on n'a pas trouvé de vrais grains d'excrétion.

La cloison entre le proto- el le deutomérite étant complète, il n'y a aucun passage des globules de l'un dans l'autre, même dans les contractions les plus énergiques du corps.

épicyte, l'ectoplasme sarcocyte, la couche des myonèmes mrocyte, l'endoplasme entocyte. Il nous paraît infiniment préférable de conserver les dénominations générales qui sont les mêmes pour tous les groupes et qui montrent immédiatement de quoi il s'agit.

(') Henneguy a montré, en outre, 'ru'à la lumière non polarisée ils présentent encore une croix brillante qui a une existence réelle puisqu'on peut la colorer avec le violet d'Ehrlich. 
A la limite entre l'ectoplasme et l'endoplasme, appartenant au premier mais faisant saillie dans le second, est une couche fibrillaire formée de myonèmes (fig. $403 \mathrm{et} \mathbf{1 0 4}, \mathrm{m}$.) tout à fait semblables à ceux que l'on a étudiés avec plus de détail chez les lnfusoires ciliés. Ce sont de longues fibrilles de lo. de large environ, circulaires sur la coupe et striées en échelle, comme si elles étaient formées de disques superposés. Les fibrilles sont logées chacune dans un canalicule d'un diamètre un peu supérieur au leur. Leur disposition générale est circulaire ou peut être héliçoïlale, autour du corps de l'animal, mais elles échangent de nombreuses anastomoses obliques, en sorte qu'elles deviennent en réalité un réseau. Vers les extrémités, le réseau se perd et cesse d'exister. Déjà sur le col de l'épimérite on ne le trouve plus. Etant sous-jacent à la cloison, il ne peut la franchir, aussi le système du deutomérite est-il discontinu avec celui du protomérite.

Dans certaines conditions, très fréquentes il est vrai, en particulier toutes les fois que la Grégarine est en marche, on observe, entre l'ectoplasme et la membrane, un étroit interstice occupé par une substance de consistance gélatineuse (gel.) qui est un produit de sécrétion accumulé là avant d'ètre évacué. Celte sécrétion forme donc au corps une enveloppe complète, c'est elle et non l'ectoplasme qui est à nu au fond des sillons de la membrane. On ne la trouve d'ailleurs que dans les points où ces sillons existent. Quand celte couche gélatineuse parait absente, on est autorisé à admettre qu'elle existe cependant, réduite à un vernis sans épaisseur. Nous allons voir bientôt quels sont ses usages.

Noyau. - Le noyau (fig. $40 \ddot{3}, \boldsymbol{N}$.) n'offre rien de particulier. Il est gros, arrondi et formé d'un réseau limité par une membrane et renfermant des grains chromatiques plus gros, que l'on appelle mucléoles sans pouvoir affirmer s'ils correspondent bien aux nucléoles vrais de la cellule typique.

\section{Physiologie.}

Habitat. - Notre Grégarine habite en parasite le tube digestif de quelque Articulé où elle est fixée, comme nous l'avons vu, par les crochets de son épimérite, dans une cellule épithéliale de l'intestin (').

Décapitation. - La première fonclion qu'elle ait à accomplir est de conquérir sa liberté. Pour cela, rlle se décapite (fig. 403̈), laissant son

(1) Dans certaines espèces de nombreux individus se groupent en masses plus ou moins volumineuses (Grex, gregis) d'oủ le nom donné au genre le plus ancien et à l'ordre. 
épimérite (épim.) dans la collule épithéliale, et tombe dans la cavité inlestinale. La plaie résultant de cette décapilation laisse échapper quelques granules d'endoplasme mais, très rapidement, se cicatrise et l'animal, réduit à son protomérite et à son deutomérite, commence sa vie libre. On le désigne alors sous le nom de sporadin. La phase où il était muni de son épimérite était le céphalin (').

On trouve pendant quelque temps un petit épaississement de la membrane au niveau de la cicatrice, mais ccla disparait assez vite et il ne reste aucune trace de l'épimérite disparu.

Mouvements. - La Grégarine est un animal assez inerle. Placée dans le chyle de l'hôte, dont elle fait sa nourriture, elle n'a aucun besoin de pourvoir à la recherche de ses aliments. Elle n’a qu'à éviter d'ètre entrainée au dehors avec les résidus de la digestion. Elle est cependant capable de mouvements et ceux-ci sont de trois sortes. Les uns sont des contractions péristaltiques, produites par un étranglement transversal qui se propage le long du corps. Les autres sont des inflexions brusques et assez énergiques dans un sens quelconque; souvent, elles alternent régulièrement de la face dorsale à la ventrale et inversement, produisant ce qu'on a appelé le mouvement de ressac. Le troisième est un glissement en avant, très lent ot qui se produil d'une manière inseusible sans aucune déformation ni contraction d'une partie queleonque; ce mouvement, dans lequel l'animal met plusieurs minutes à parcourir un millimètre, rappelle celui des Planaires glissant, le pied en haut, à la surface de l'eau, mais ici il n'y a pas, comme chez la Planaire, de cils vibratiles pour le déterminer.

Les mouvements de la première espèce s'expliquent à merveille par les fibrilles contractiles dont la disposition annulaire est exactement ce qu'elle doit ètre pour les produire.

Les seconds sont un peu moins aisés à comprendre. Des myonèmes longitudinaux seraient mieux disposés pour les engendrer. On a plusieurs fois cru trouver une couche de fibrilles dirigées en long, mais décidément il n'en existe pas. Ces inflexions peuvent cependant se comprendre comme résultal de contractions locales du sỵstème des myonèmes. (iràce aux anastomoses, ce système forme un réseau et si, en un point, les mailles du réseau se rétrécissent, le corps doit s’infléchir de ce côté $\left(^{2}\right)$.

Le troisième mouvement semble au contraire tout à fait inexplicable,

1) Dans quelipues cas, le céphalin se lígage de la eellule ipithéliale sans y laisser sa tête. Il se promène alors quelıue temps a l'ètat de ciphalin, mais bientôt, brusquement, son épimérite se détache et il passe à l'état le sporadin. Cela montre que la décapitation, dans le prenier cas, n’ilail pas du fout un traumatisme produit par les efforts de l'animal pour dégager sa lête. C'est un phénomine normal qui se produit au moment où un certain élat de maturite est atteint.

${ }^{(2)}$ Cependant il reste là quelque ehose d'inexpliqué, ear eclle inflexion aurait pour linite la réduction du eòlé eontracté à une surface plane, or l'animal peut s'incurver en eroissant. 
puisque rien ne se contracte dans l'animal pendant qu’il se produit. 11 s'explique cependant, mais non par une contraction active; il est dù à une sorte de déplacement régétalif que produit la poussée exercée par une sécrétion (").

Voici comment les choses se passent. L'ectoplasme (fig. 403 et 404 , ectop.) sécrète une sulıstance liquille (gel.) qui s’accumule au-dessous de la membrane formant ce que nous avons appelé la conche gélatineuse. Nous avons vu, d'autre part, que la culicule est fendue jusqu'au fond des sillons et met là cette sulıstance à nu. Dès lors celle-ri peut s'écouler par ces fentes. Mais elle ne s'épanche pas immédiatement à la surface. elle suit le fond des sillons et n'arrive au dehors que là où les sillons se terminent, c'est-à-dire à la partie inférienro du corps. Elle est filée en quelque sorte, comme celle qui sort des filières de l'Araignée. Comme cette dernière aussi, elle se solidifie dès qu'elle arrive au dehors. Tous ces fils solidifiés forment une sorte de rylindre, de manchon, dans lequel plongre l'extrémité inférieure du corps. Ce manchon s'accroìt saus cesse parl'addition de nouvelles quantités de substance à son extrémité supérieure, il pousse, en quelque sorte, comme une plante, et cliasse devant lui le corps de l'animal. Bien entendu, il y a une transition insensible entre la partie solidifiée du manchon que l'animal laisse derrière lui, et la partie molle en voie d'accroissement. La sulsstance se dépense plus vite qu’elle n’est sécrétée, aussi, après un certinin parcours, l’animal est obligí de s’arrèter jusqu'à ce qu'elle soil renouvelée. Cela explique pourquoi la couche gélatineuse est tantôt présente, tantôt absente entre la membrane et l'ectoplasme.

Alimentation. - L'animal se nourrit exclusivement par imbilition, par osmose, lu chyle au sein duquel il est plongé et, pas plus que le Ténia, n’a besoin d’organes quelconques pour cela.

Excrétion. - Nous venons d'en décrire une très active, mais on ne sait pas du tout si elle sert à expurger le corps de ses produits de désassimilation on şi cette fonction se prorluit par exosmose. En tont cas, il n'existe ni appareil excréteur spécial, ni grains d'excrétion dans l'endoplasme.

Association. - Souvent, delix ou plusieurs Grégarines s'unissent à la file, l'une se fixant par son protomérite à l'extrémité inféricure du deutomérite d'une autre (fig. 406); s’il y en a une troisième, elle se fixe de mème à la suite de la seconde et ainsi de suite $\left(^{*}\right)$.

(1) C'est a Cheviakof que l'on doit cette idée ainsi que l'explication suivante. Pour bizar're qu'elle paraisse, il faul bien l'accepter jusqu'à nouvel ordre, car elle repose sur des faits observés et on n'en connaît aucun autre ì lui substituer.

$\left.{ }^{2}\right)$ Ces associations sont très variables selon les espèces. Certaines Grégarines 
Reproduction. - L'animal se reproduit uniquement par spores. Par une exception remarquable et tout à fait caractéristique de toute la classe dont il est le type, il ne se multiplie pas par division simple. Il doit former des spores et pour cela s'enkyster.

Enkystement et sporulation. - L'animal s'enkyste (fig. 407), non pour résister à des conditions fàcheuses ou se reposer, mais parce qu'il est

Fig. 407 .
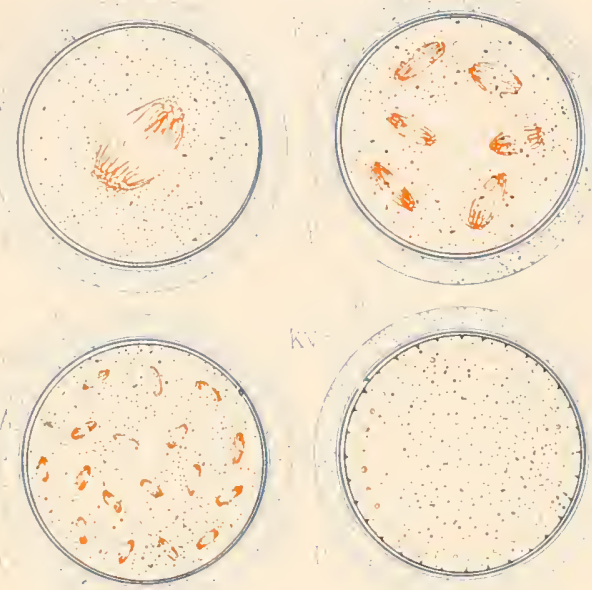

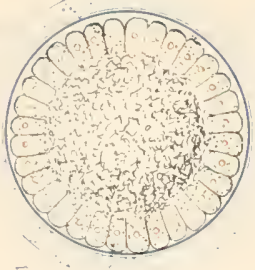

$\mathrm{sp}$

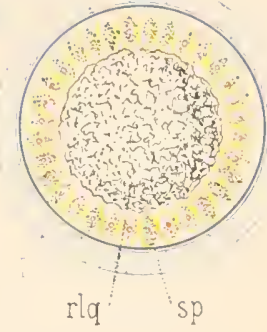

GREGARINID.E (Type molphologique).

Enkystement et sporulation (Sch.).

$A, B, C, D, E, F$, états successifs pendant la sporulation. e, conche interne du kyste; kys., couche externe du kyste; rlq., reliquat de segmentation; sp., spores. arrivé à la phase de son existence où il doit se reproduire. Il s'arrondit, devient immobile et se sécrète, dans le cas le plus complexe, deux enveloppes, une externe, épaisse, transparente et gélatineuse (kys.), l'autre interne, mince et résistante (e), et aussitôt il commence à former ses spores. Le phénomène de la sporulation est essentiellement nucléaire et a débuté, sans qu'aucun fait extérieur le manifestât, dès les premiers temps de la vie de l'animal. 11 se forme, aux dépens du noyau primitif, et par division mitosique ainsi que l'a montré Wolters, un grand nombre de noyauxfilles qui se portent à la périphérie (").

Ces noyaux s'adjoignent chacun une portion du cytoplasma ambiant (fig. 407, $D$ et $E$ ), et se transforment chacun en une spore $(F: s p$.$) . L'animal, dans$ lequel depuis quelque temps déjà l'ectoplasme et la cloison ont disparu, fondus sans doute dans le reste du cytoplasme, se présente alors comme une masse sphérique formée d'une couche périphérique de spores et d'une masse centrale $(r / q$.$) qui est le résilu$ inutilisé.

ne s'unissent guère, d'autres forment des groupes de rleux, ou des chaînes de plusieurs individus. Presque toujours, la chaine est simple. Parfois, cependant, elle se bifurque. Certaines de ces associations peuvent se défaire. D'autres sont solides et permanentes, par exemple chez Didymophyes.

(1) Lorsque notre Grégarine était toute petite, représentée par une simple masse 
Maturation des spores, formation des sporozoïtes. — Les spores n'ont pas encore achevé leur évolution.

Chacune sécrète deux membranes (fig. 40S), une endospore (endosp.) et une épispore (épisp.) et, sous ces membranes, divise son contenu ordinairement en huit petits corps, régulièrement disposés, les sporozoïtes $(s p$.$) dont chacun a$ reçu un huitième du noyau. II reste cependant un minime résidu inutilisé $(r l q$.$) (').$

protoplasmique, arrondie et nucléée, et encore contenue tout entière, comme nous le verrons bientòt, dans la cellule épithéliale à laquelle nous l'avons trouvée simplement accrochèe par son épinnérite, à ce moment, disons-nous, son noyau ne contenait qu'un seul globule chromatique. Ce globule s'est multiplie pendant ses phases d'accroissement et e'est ainsi que nous en avons trouvé un grand nombre dans le noyau de notre animal adulte. Pendant et après l'enkystement,

ling. 408.
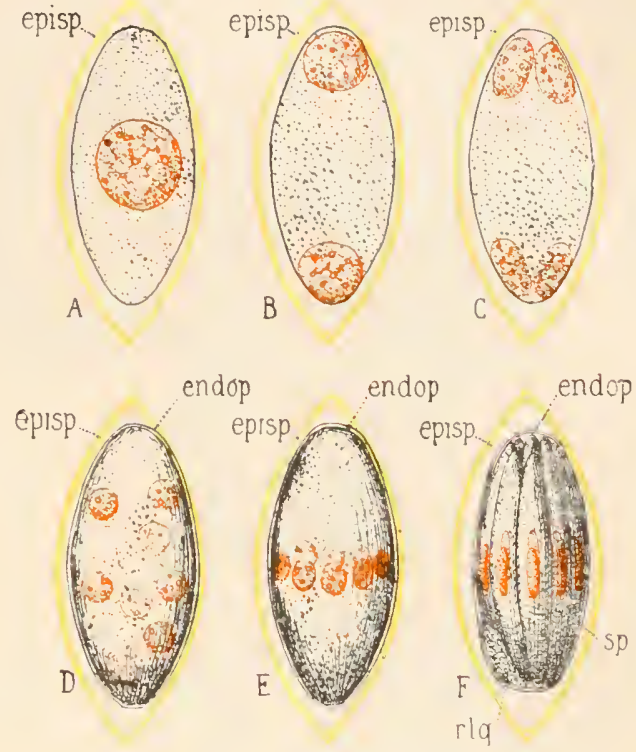

GREG.1RINID.E (Type morphologiquo). Maturation des spores (Seh.).

1, $B, C, D, E, F$, stades successifs de l'évolution endop., endospore; episp., épispore; rlq., reliquat; sp., sporozoïtes. ces globules continuent à se multiplier et finissent par être très nombreux.

M Arsilatl [93] a qui nous devons ces renseignements a décrit dans la multiplication des globules chromatiques des particularités remarquables (fig. 409), mais dont la signification et même la réalité sont assez douteuses. Lorsque le globule chromatique (il appelle toujours ces globules des nucléoles) unique du début s'est divisé en quatre, c'est un seul des quatre fou au plus deux) qui donnerait tous les suivants par un procédé de for-

Fig. 109.

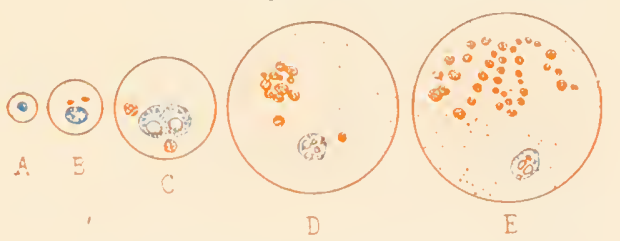

GREGARINID.E (Type morphologique).

$A$ à $E$, stades successifs.

Multiplieation des globules chromatiques

(im. Mar'shall).

mation endogène. Il grossirait et formerait à son intérieur de petites masses qui, sortant de sa cavité, iraient rejoindre celles produites antérieurement. Le ròle de ce nucléole formateur continuerait jusqu'a l'enkystement, mais alors les globules achèveraient de se multiplier par eux-mèmes, par une sorte de bourgeonnement. Alor's le noyau perdrait sa membrane et les globules chromatiques emporteraient chacun une partie de sa substance et émigreraient vers la surface du cytoplasme, où ils se disposeraient en une couche régulière de petits noyaux, contenant chacun un seul globule.

Mais, d'après Wouters [91] dont l'opinion semble mieux mériter confiance, il n'y aurait, au lieu de tout cela, qu'une multiplication par mitose.

(1) Pour Marshald, le globule chromatique qui, ici encore, semble être l'initia- 
Émission des spores. - Le kyste peut effectuer son évolution sans

Fig. 410 .

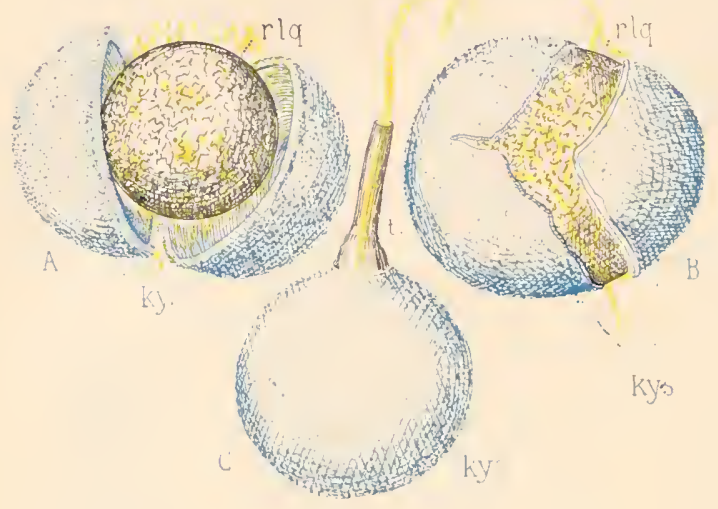

GREG.ARINID.E (Type morphologique).

Emission des spores (Sch.).

$A$, par éclatement simple; $B$, par gonflement du pseudokyste (rlq.);

$C$, par sporoductes. kys., paroi du kyste; riq., reliquat de segmentation; sp., spores; t., sporoductes. changer d'hòte; mais le plus souvent, sa maturation el sa déhisrence n’ont lieu qu'au dehors du tube digestif dans l'humidité, ce qui entraine un changement d'hôte. Lorsque samaturationestacherée, il faut qu’il émette ses spores au dehors. Le plus souvent (fig. 410, A), cela a lieu par simple éclatement de la paroi à maturité (').

Développement du sporozoïte, formation de la jeune Grégarine (fig. 111). - Voilà lonc les spores mûres et mises en liberté $(A)$. Dans le milieu extérieur, elles ne peuvent s'ouvir. Il faut pour cela qu'elles soient introduites de nouveau dans le tube digestif d'un individu de l'espèce où habite l'adulte. Elles sont donc avalées avec les aliments et, sous l'influence du suc gastrique, éclatent et mettent les huit sporozoïtes en liberté $(B, C)$. C'est d'abord l'épispore qui se fend $(B: e$.$) , puis l'endospore (C: e$.$) qui s'ouvre à son tour et permet$ aux sporozoïtes $\left(s p\right.$.) de sortir $\left({ }^{2}\right)$.

Ceux-ci $(I)$ se montrent sous la forme d'un petit bàtonnet arqué, légèrement renflé dans sa partie moyenne qui contient le noyau, et terminé en avant par une partie plus étroite, sorte de rostre très molile.

Le sporozoite est porté avec les aliments au contact de la muqueuse intestinale, il s'ouvre un passage dans une cellule épithéliale au moven de son rostre arec lequel il fouille énergiquement et pénètre tout entier dans la cellule où il se place entre la surface libre et le noyau $(E)$. Remarquons que ses faibles dimensions ( 8 ou 10 \% de long sur à

teur du mouvement, se serait multiplié pour cela par trois divisions directes successives.

(1) D'autres fois (fig. 410), c'est la masse résiduelle centrale appclée pseudolyste (parce qu'elle a l'aspect d'un kyste complet) qui se gonfle et fait éclater l'enveloppe $(B)$. Parfois enfin, il se forme des organes spéciaux de dissémination, les sporoductes $C$ ). Ces derniers ne se rencontrant que dans Clepsidrina et Gamocystis seront étudiés aree le premier de ces genres. On appelle parfois ces spores des pseudonaricelles et les kystes, kystes à pseudonavicelles.

(2) La forme des spores, leur mode de déhiscence sont très variables, mais partout le phénomène est essentiellement le même. 
peine $1 \mu$. de large) lui permettent celte effraction sans endommager sérieusement la cellule.

$\Lambda$ peine entré, il s'arronlit et apparaît dans la cellule comme une minime sphérule de protoplasma contenant un noyau central. Celle sphérule, se nourrissant aux dépens de la cellule, grossit, s'allonge et finit par faire effraction au delıors du còté de l’intestin $(E)$. La partie extracellulaire grandit et grossit rapidement, peu à peu le noyau descend dans son intérieur $(G)$, la cloison se forme el dessine le protomérite el le deutomérite $(I)$, la partie intracellulaire se transforme en épimérite, tous les détails de structure, membrane striée, myonèmes, granulations endoplasmiques se forment peu à peu. les grains chromaliques se multiplient dans le noyau, et l'on a enfin une Grégarine à l'étal de céphalin fixí, semblable à celle qui nous a servi de point de départ $(H)$.

Conjugaison. - Il semble que nous ayons tout dit puisque nous avons fermé le cycle évolutif. II reste cepentant encore un phínomène à étudier, celui de la conjugaison. Malhemeusement il est loin d'ètre bien connu.

Nous avons vu que notre Grégarine pouvail s'unir à une (ou à

Fig. 112

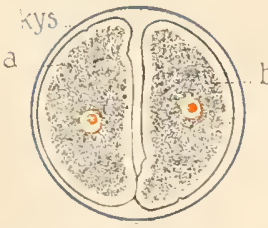

GREG.1RINID.E

(Type morphologique).

Enkystement de deux individus sans eonjugaison (Seh.).

a, b, Ies deux individus ; kys., kyste. plusieurs) de ses semblables par soudure de son protomérite à l'extrémilé du deutonérite de celle-ci. Il arrive parfois que, dans cel état, les deux arrivant ensemble à malurité, se renferment dans un kyste unique qu'elles sécrèlent en commun. Pour cela, elles se rabaltent l'une sur l'autre et se trouvent ainsi têtebèche, còte ì côte (fig. 412). Mais c'estlà un phénomène tout accessoire el qui n'a rien de commun avec une conjugaison. Les deux individus sporulent séparément, sans rien échanger, leur membrane de séparation étant encore intacte dans le kyste quand tous les pliénomènes essentiels sont déjà accomplis. Elle 
ne disparaît qu'au moment où les spores sont déjà disposées dans chacun d'eux en une couche superficielle autour d'un amas résiduel central.

Tout autre est le cas de la véritable conjugaison (fig. 413). Les deux

Fig. 413 .
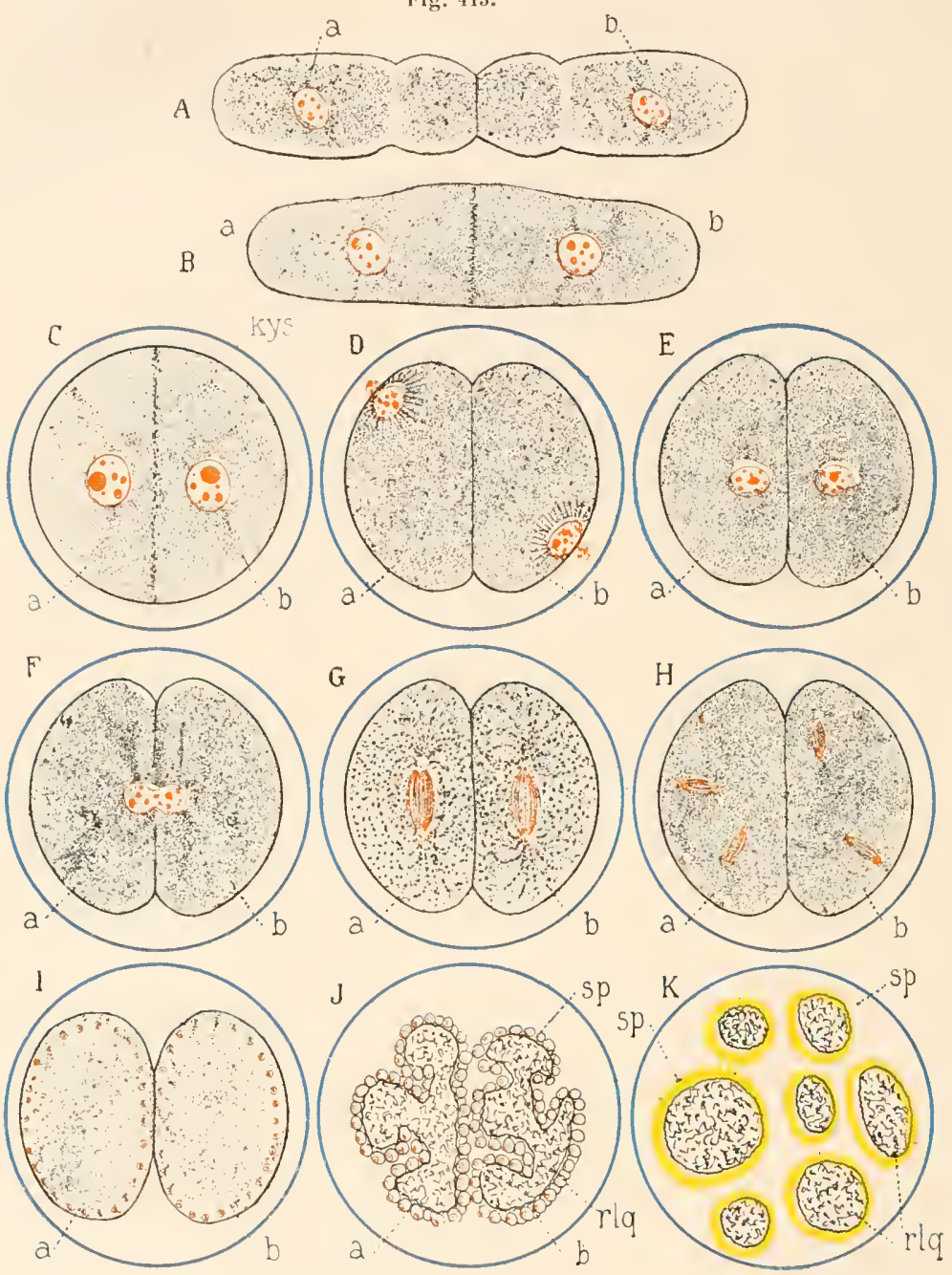

GREGARINID.E (Type morphologique). Conjugaison (Seh.).

$A$ à $K$, stades successifs. a, b, les deux individus: rlq., reliquat de segmentation; s1., spores.

individus se placent en opposition (A), c'est-à-dire tête contre tète ou, pour parler plus exactement, se soudent par leurs prolomérites en se plaçant sur le prolongement l'un de l'autre ('). Ici encore, ils s'enkystent

(1) A. Schneider [76] dont les beaux travaux sur les Grégarines nous ont appris la 
en commun $(C)$, mais les phénomènes consécutifs sont tout autres que dans l'enkystement ordinaire. D'après Wouters [91], dans chaque individu, le noyau sépare d'abord, par mitose, une moitié de sa substance qui se porte vers la surface et est éliminée à la manière d'un globule polaire (IN). Le demi-noyau restant se reconstitue, se porte vers celui de l'autre individu $(E)$, et s'unit à lui au centre du kyste $(F)$, la paroi de séparation des deux individus s'étant percée pour permettre leur réunion. Le noyau conjugué se divise alors (') en deux noyaux filles qui repassent chacun de son côté.

Les phénomènes ultérieurs se passent désormais séparément dans les deux moitiés. Ils consistent en une active prolifération mitosique de ces deux noyaux fécondés $(G)$, d’oì résultent les nombreux noyaux des spores, qui se portent à la périphérie $(I)$, laissant au centre de chaque individu un reliquat de segmentation abondant. Nais bientôt les spores deviennent trop nombreuses, pour rester à la périphérie; la couche qu'elles forment plonge vers le centre et découpe le reliquat en petites masses entourées chacune d'une couche de spores $(J)$, et toutes ces petites masses se mêlent, en sorte qu'on ne distingue plus celles qui appartiennent à l'un ou à l'autre des individus conjugués $(K)$. Dans chaque spore, le noyau se divise en huit pour former les sporozoïtes, mais ici on ne voit plus de mitose.

C'est donc, en somme, une conjugaison nucléaire oì les deux conjoints échangent un demi-noyau sans mèler leurs cytoplasmes.

Malhenreusement, tous ces phénomènes intéressants ont été vus par un seul observateur, Woutens [91] et fort incomplètement. Ils mériteraient confirmation.

En outre, on ne sait rien ici de tontes ces conditions si admirablement élucilées par Maupas chez les Infusoires, relatives à la dégénérescence sénile, à l'appétence sexuelle et au rajeunissement qui pourrait résulter de la conjugaison. Peut-ètre cette dernière a-t-elle lieu ici assez souvent pour que la première n'ait pas le temps de se produire, ni par conséquent la seconde de se manifester $\left({ }^{2}\right)$.

majeure partie de ce que l'on sait de ces animaux, appelle cela apposition et donne le nom d'opposition à la situation inverse.

Cela pourrait peut-être se soutenir étymologiquement, mais n'est point d'accord avec l'acception usuelle de ces mots.

(1) Toujours par mitose typique, avee disparition de la membrane nucléaire contrairement à ce qui a lieu d'ordinaire chez les Protozoaires.

$\left.{ }^{2}\right)$ Ces phénomènes ont été observés par [WoLters] chez Monocystis agilis et M. magna du testicule du Ver de terre. On ne peut dire encore dans quelle mesure il est légitime d'étendre cela aux autres Grégarines. Mais c'est là tout ce que l'on sait sur cette intéressante question.

Marshall appelle, comme tant d'autres, conjugaison l'enkystement à deux des Clepsidrines, mais ce n'en est pas une évidemment, puisque les conjoints n'échangent rien. 
Enkystement cœlomique.- Il semble que, gràce à cette série de phénomènes évolutifs, la propagation indéfinie de notre Grégarine soit assurée. L'Arthropode qui l'héberge éracue sans cesse des kystes dont les spores sont avalées et donnent de nouveaux kystes et ainsi de suite.

Ilais les choses ne sont pas toujours aussi simples.

Cet Arthropode est le plus souvent un Insecte et cet Insecte peut être un de ceux qui onl des métamorphoses profondes, de longue durée, comportant privation absolue d'aliments pendant toute la durée de la nymphose et parfois même lestruction histolytique du tube digestif. Les (irégarines ne pourront résister aux condilions nouvelles créées par ces changements, et toutes celles qui n'auront pas quitté l'hôte à l'état de kyste avant la nymphose vont se trouver condamnées à périr. Ces kystes, si leur's spores peuvenl attendre l'éciosion de l'imago, auront chance d'être avalés par celui-ci ot d'achever leur cycle, mais il faut pour cela que l'Insecte adulte ait le mème régime alimentaire que sa larve.

Entre formes dont le régime est très différent, ces échanges ne sauraient avoir lieu.

Si les spores ne peuvent achever leur évolution que dans le tube digestif de la larve, il faudra donc qu'elles soient assez bien protégées pour attendre l’apparition de la larve de la nouvelle génération. Cela peut avoir lieu dans certains cas, mais la Grégarine a trouvé un moyen bien plus simple de s'adapter à ce nouvel état de choses.

Pendant toute la jeunesse de la larve, elle s'enkyste dans le tube digestif, ses kystes sont expulsés, ses spores avalées et ainsi de suite pendant un bon nombre de générations. Mais, quand approche le mo-

Fig. 414.

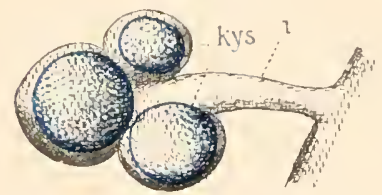

GREGARINII) E (Type morphologique)

Kystes coelomiques (im. Léger). ment de la nymphose, un changement se produil dans l'évolution. La jeune Grégarine intracellulaire, au lieu de faire effraction vers la cavité digestive, sort de la cellule du côlé opposé el arrive ainsi dans l'épaisseur de la paroi digestive; là, elle grossit, mais sans perdre sa forme sphérique (fig. 价位) et sans montrer d’aulre différenciation que-sa membrane, son endoplasma et son noyau. Il n'y a ni ectoplasma, ni fibrilles, ni épimérite, ni cloison. A mesure qu'elle grossit, elle fait saillie de plus en plus vers la cavité générale el finalement arrive à y tomber et s'y enkyste aussitôt sans perdre sa forme romle ni passer à l'état de (irégarine libre. Ce kysle sporule ('). Il faul que l'Insecle meure pour que ses spores soient mises en liberté par destrurlion des parois

(1) II y a quelques différences de taille entre les spores de ces kystes et celles des kystes intestinaux, mais elles sont sans importance et s'expliquent suffisamment par l'action directe des conditions nouvelles. 
du corps et puissent infecter d'autres larves en étant avalées par elles avec leurs aliments. Outre les avantages précédemment indiqués, cette modification adaptative de l'évolution a celui de mettre les ailes de l'Insecte adulte au service du parasite pour sa dissémination (").

Le sous-ordre des Grégarinides se divise en deux tribus:

CEPIALINa vel PoLycystina dont le corps a deux ou trois segments, et

ACEPHALIA vel Movocystixat dont le corps n'a qu'un seul segment $\left({ }^{\mathbf{z}}\right)$.

\author{
1re Tвlв U \\ CÉPHILINES. - CEPHALINA \\ vel \\ POLYCYSTINES. - POLYCYSTINA \\ [GREGarINES INTESTINALES]
}

Tous, sauf le genre Schneideria sont tricystidés, c’ost-à-dire pourvus l'un septum qui divise le corps en un protomérite et un deutomérite et munis d'un épimérite. Ils ne diffèrent entre eux que par des caractères assez secondaires relatifs surtout à la forme des spores et à celle de l'appareil de fixation de l'épimérite.

\title{
GENRES
}

Porospora (A. Schneider) (fig. 41:3). C'est la Grégarine géante du homard, Gregarina gigantea de Van Beneden. Son épimérite est lisse, inerme, en forme de petit bouton. Les adultes se trouvent associés deux à deux, le second étant beaucoup plus long que le premier. Les spores, par une exception unique, sont nues et formées par un nombre

(1) Cette double évolution n'est pas l'apanáge d'un groupe spécial,

Fig. 415.

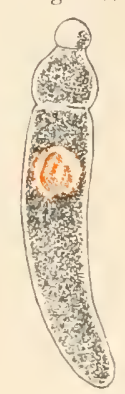
elle se rencontre chez certaines espèces de genres à évolution simple et normale, par exemple: Clepsidrina longa, Actinocephalus Tipulx (l'un et l'autre parasites de Tipula oleracea), Didymophies gigantea et D. rara (parisites, le prenier d'Oryctes nasicomis, le second de Ceotrupes stercorarius), enfin chez Lirmocystis polymorpha (parasite de la larve de Limmolia). Tous ces renseignements et bien d'autres encore dans cet article sont empruntés au beau travail de LÉger [?'́ ćlève de A. Schneider.

$\left.{ }^{(2}\right)$ Les termes en Gystides adoptés précédemment sont bien mauvais, pnisque les segments du corps ies prétendues Tricystides (épimérite, protomrérite el dentomérite ne sont que des parties d'une seule et même cellule. C'est pour cela que nous avons proposé ceux de rephalina et Acephalina. On pourrait aussi appeler les premiers Grégarimides intestinaux et les seconds Grégarimides calomiqnes. Chez les premiers, en effel, l'adulte habite toujours l'intestin pendant une partic au moins du cycle évolutif de l'espèce et tous passent par l'état que SuHxeider a appelé céphalin. Les seconds au contraire habitent le colome et n'ont pas de stade céphalin. 
considérable de très petits sporozoïtes disposés comme les rayons d'une sphère (Alteint $16 \mathrm{~mm}$ de long. Intestin de Ilomarus) (').

Toutes les autres Grégarines ont done des spores munies de leurs deux membranes. Toutes, sauf indication contraire, habitent le tube digestif des lnsectes.

Didymophyes (Stein) (fig. 416) est remarquable par le fait que les individus s'associent pardeux et que le second. après a voir plongé et soudé son protomérile entier dans le deutomérite du premier, résorbe son septum, en sorte que l'on croirait avoir une Grégarine extraordinaire formée d'un protomérite suivi de deux deulomérites nucléés. Mais ce n’est là qu'une apparence et l'animal est bien moins anormal qu'il ne parait.

Le kyste se rompt sans appareil spécial. L'épimérite est réduit à un petit lubercule terminé jar une pointe $\left(10^{\mathrm{mm}}\right.$ et plus. Intestin de la larve d'Oryctes nasicornis) $\left({ }^{2}\right)$.

Clepsidrina (Hammerschmidt) (fig. 417) a aussi un épimérite inerme en forme de bouton arrondi ou allongé, et les individus sont, aussi généralement, associés par deux, mais sans disparition du septum chez le second.

Son caractère principal réside dans nn appareil spécial servant à la dissémination des

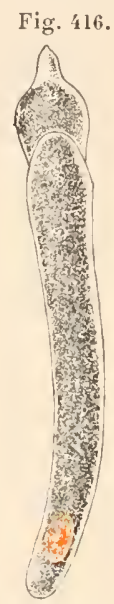

Didlymophyes

(D. gigantea) (d'ap. Léger).
Fig. 117

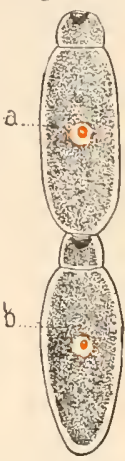

Clepsidrina (d'ap.

A. Schneider).

Etat jeune arant

lassociation.

spores. Cet appareil est constitué par des tubes appelés sporoductes, qui meltent la cavité du kyste en relation avec le dehors et par où sortent les spores soudées les unes aux autres en chapelets. Ces spores sont en forme de tonnelet (Intestin de beaucoup d'Insectes et de leurs larves; 0,07 à $1 \mathrm{~mm})\left({ }^{\mathbf{3}}\right)$.

(1) Parfois l'association comprend un troisième individu plus grand que les autres et dont le septum a disparu. VAx Bexedex 71 ] avait eru que cette Grégarine se développait au moyen d'amibes provenant des kystes et qui émettaient de longs pseudopodes, Psendofilaires, qui se détachaient el se transformaient en jeunes Grégarines.

LEger fait avec ce Porospora une famille caractérisée par des spores nues, spheriques: Grmaspontax [Gymnosporides (Léger)].

(2) C'est à ce genre que se réduit aujourd'hui la famille des DIDYMOPHYINE [Didymophyida Stein] que Stein avait cru beaucoup plus étendue.

(3) Voici comment se forment ces sporoductes d'aprẻs les recherches de Schneider [76] et de Bútschl fig. 418. Au lieu de rester à la surface de l'amas résidıel du liyste $(\boldsymbol{A})$, les spores se rassemblent au centre où elles forment une masse transparente $(\boldsymbol{B}: s p$.$) , laissant entre elles et la paroi du kyste une zone de proto-$ plasma granuleux $(\boldsymbol{B}: \boldsymbol{f}$ ) en même temps, une troisième membrane très minee se forme contre la paroi interne du kiyste. Dans la zone granuleuse périphérique, se dessinent six á huit traînées rayonnantes $(B: e)$ et, dans l'axe de chacune d'elles, se forme un tube qui se met tout aussitòt en rapport avec la nouvelle membrane sans que l'on puisse affirmer qu'il émane d'elle. Ce tube achève de se dessiner et bientòt on tonstate qu'il s'appuie par sa base élargie $(C: d)$ sur cette membrane, puis pré- 
Anthocephalus (Schneiler) se distingue des précédents par son épimérite

sente une partie courte, assez large, et se termine enfin par une partie cylindrique plus étroite qui se perd dans l'amas central de spores. Evidemment, mais sans qu'on voie le processus, la paroi du kyste s'ouvre à un moment à sa base, car on voit brusquement les sporoductes se dévaginer $(D$ : $s p d$.) et arriver loin au dehors de la membrane gélatineuse du kyste $(\boldsymbol{E}$ : $s p d$.). Cette dévagination a lieu, sans doute, par l'effet d'une pression croissante du liquide intérieur, et cette même pression pousse les chapelets de spores dans la lumière des sporoductes et les fait arriver au dehors ( $\boldsymbol{E}:$ sp.).

A la Clepsidrine se rattachent les genres suivants :

Gamocystis (Schneider), forme sans septum (pseudomonocystidée), où l'on rencontre encore des sporoductes. Mais c'est la seule, ces organes ne se retrouvant nulle part ailleurs (Intestin de Blatta et des larves d'Ephemera);

Conorhynchus (Greefl), remarquable par les vacuoles de l'endoplasme (Intestin d' $E$ chiurus);

Eirmocystis(Léger), chez lequel on trouve, dans une espèce, des individus sans septum associés à d'autres qui sont tricystidés (Intestin de Grillotalpa et des larves de Tipula et de Limnolia); Hyalospora (Schneider) rare (Intestin des Thysanoures);

Euspora (Schneider) (Intestin des Melolontlines).

Les genres précédents forment habituellement des associations tandis que les suivants sont toujours solitaires :

Cnemidospora (Schneider);

Stenocephalus (Schneider) (Intestin de Julus);

Sphærocystis (Léger) (fig. 419), pseudomonocystidé, c'est-à-dire dépourvu de septum (Intestin de la larve de Cyphon pallidus).

Tous ces genres forment la famille des CLEPSIDLIXIX [Clepsidrinides (Léger'].

Fig. 419.

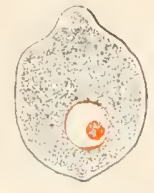

Sphierocystis (S. simplex) (d'ap. Léger) 
en forme d'urne, orné de fortes côtes, et par ses spores attachées par l'équateur pour former leurs chapelets ('). Dactylophora (Léger) (fig. 421) a un épimérite réduit à de courtes digitations disposées irrégulièrement autour d'un protomérite dilaté au sommet. Son kysle s'ouvre en deux valves égales par la poussée d'un pseudokyste latéral (fig. 420) (0,7 it 0,8. Tube digestif de Cryptops) ( $\left.{ }^{2}\right)$.

Stylorhynchus (Schneider) (fig. 122, 123) a un épimérite en forme de renflement inerme au bout d'un long col. Son kyste s'ouvre sous la pression d'un pseudokyste central et

Fig. 420.

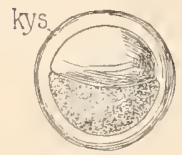

Dactylophora.

Kyste dont le reliquat de seg. mentation (pseudokyste) forme à lui seul un hémisphère (im. Léger). donne issue à des spores colorées, ovoüdes, attachées en chapelets par leurs pòles, et s'ouvrant Iongitudinalement suivant un demi-méridien, comme un porte-monnaie.

Lophorynchus (Schneider) diffère des précédents par l'épimérite armé d'une double courome de pointes, mais il se rat!ache à eux par le caractère de ses spores et de son kyste $\left({ }^{3}\right)$.

Pogonites (Léger) (fig. 424) est remarquable par son épimérite en forme de bouton autour duquel sinsèrent de longs filaments disposés en cercle. Les in-

(1) Il est a citer comme étant la seule Grégarine vivant chez un Arachnide, Palanginm. Il forme à lui seul la famille des ANTHOCEPIILLTXE [Authocéphalides (Léger)]. Lèger nous écrit qu'il est d'avis de supprimer cette fanille et de la rattacher aux Actinocéphalines.

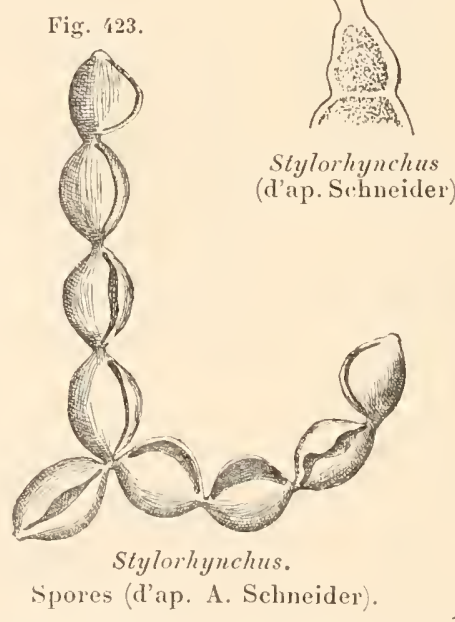

Fig. 122.

Fig. 421.

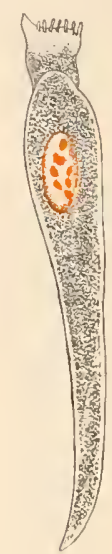

Dactylophora

D. robusta (d'ap. Léger).

Fig. 124.
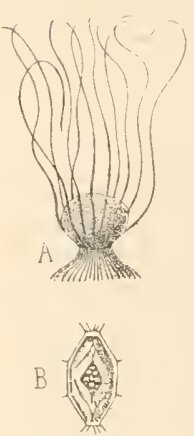

Pogonites

(P. capitatus)

(d'ap. Léger).

$A$, épimérite; $B$, spore.

(2) Genres voisins, n'en différant que par des caractères secondaires:

Echinocephalus (A. Schneider',

Pterocephalus (A. Schneider),

Trichorhynchus (A. Schneider).

Rhopalonia (Léger).

Tous ont prour hòtes des Myriapodes chiloporles et constituent la famille très naturelle des DACTYLOPIORLA [Dacty\%phorides, Léger)].

(3) Genres tries voisins le stylorhychus:

Cystocephalus (Léger)

Spherocephalus Léger)

Oocephalus (Liger).

Ils ont tous pour hôtes des Insectes carnassiers de la famille des Ténébrionines

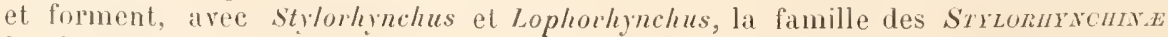
[Styloı/ymchides (Léger)]. 
dividus vivent solitaires et donnent des kystes qui s'ouvrent par simple ruplure sous l'effort du liquide central, sans appareil spécial disposé à cet effet. Les spores sont terminées en cône aux deux extrémilés et munies de pointes $(0,1$. Parasite dans les larves des Ifydrophilinit) (').

Supposons un Corycella, mais avec un col plus long et des spores en croissant, tout le reste étant semblable nous aurons

Menospora (Léger) (fig. 12马) (0,6 à 0,7. Intestin des larves d'Agrion);

Hoplorynchus (Schneider) est un genre voisin du précédent $\left({ }^{2}\right)$.

Supposons enfin qu'avec des caractères semblables encore de déhiscence du kyste, de vie isolée sans associations, mais avec des formes d'épimérites variables, les spores soient naviculaires, c'est-à-dire ovoïdes à extrémités pointues, nous aurons

Fig. 426.

Fig. 425

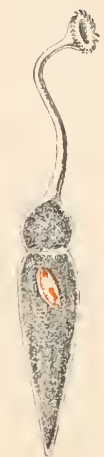

Actinocephalus

(A. Tipulix)

(d'ap. Léger).
Menospora

(I. polyacantha)

(d'ap. Léger).

Actinocephalus (Schneider) (fig. 426), chef d'une série qui termine celle des Polycystines ( $\left.{ }^{*}\right)$.

(1) Avec ces mêmes caractères du kyste et des spores, supprimons les filaments de l'épicyte et nous aurons le genre:

Acanthospora (Léger) $(0,3$ à 0,4 . Intestin de la larve des $C y$ stelina $)$.

Remplaçons ces filaments par une courome de crochets, recourbés en arrière et insérés au sommet de l'épimérite, et nous aurons les genres :

Corycella (Léger) (fig. 127) 0 ,3. Intestin de la larve de Gyrinus);

Ancyrophora (Léger) (Atteint près de $2 \mathrm{~mm}$. Intestin de ('arabus, Silpha, Dyliseus).

Syncystis (Schneider) se rattache aussi aux précédents.

Ces cinq genres réunis constituent la famille des A cinthosporinde [-1canthosporides (Léger)].

$\left({ }^{2}\right)$ Ces deux genres constituent la famille des MENosponta E[ Henosporides(Léger)].

(3) Celle série se compose des genres suivants :

Amphorella (Léger) et,

Pileocephalus (Schneider) qui ont l'épimérite en simple bouton sessile et inerme ;

Discocephalus (Léger), où le bouton est précédé d'un renflement basilaire;

Stephanophora (Léger) (tig. 428), où il forme une couronne ornée de còtes;

Fig. 427.

Fig. 428.

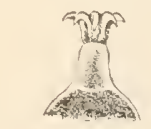

Corycella

(C. armata) Stephanophora

(d'ap. Lejger). (S.radiosa)

Fig. 430 .

Stephanophora
(S. radiosa)
(d ap. Léger).
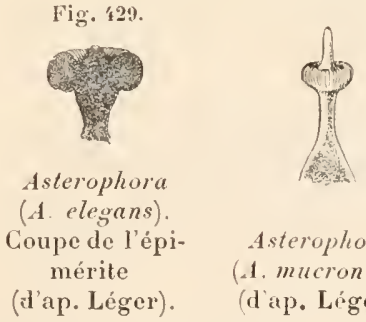

Fig. 431.

Asterophora

(1.) mucronata

(d'ap. Léger).
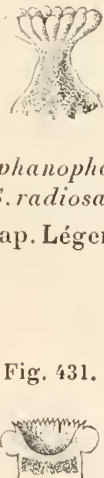

Phialis

(P. ornata)

(d'ap. Léger).

Asterophora (Léger) (fig. 429 et 430), où s'ajoute à la couronne précédente un prolongement conique central;

Phialis (Léger) (fig. 431), où il a la forme d'une cupule à bords dentés; 
$2^{\text {e TrIBU }}$

MCEPHALINES. - ACEPIIALINA
vel
MONOCYSTINES. - MONOCYSTINA
[GRÉGARINES COELOMIQUES]
TYPE MORPHOLOGIQUE
(FIG. 432)

Notre Monocystine, avec les caractères généraux de la Grégarine décrite au commencement de cet arlicle, présente trois particularités qui

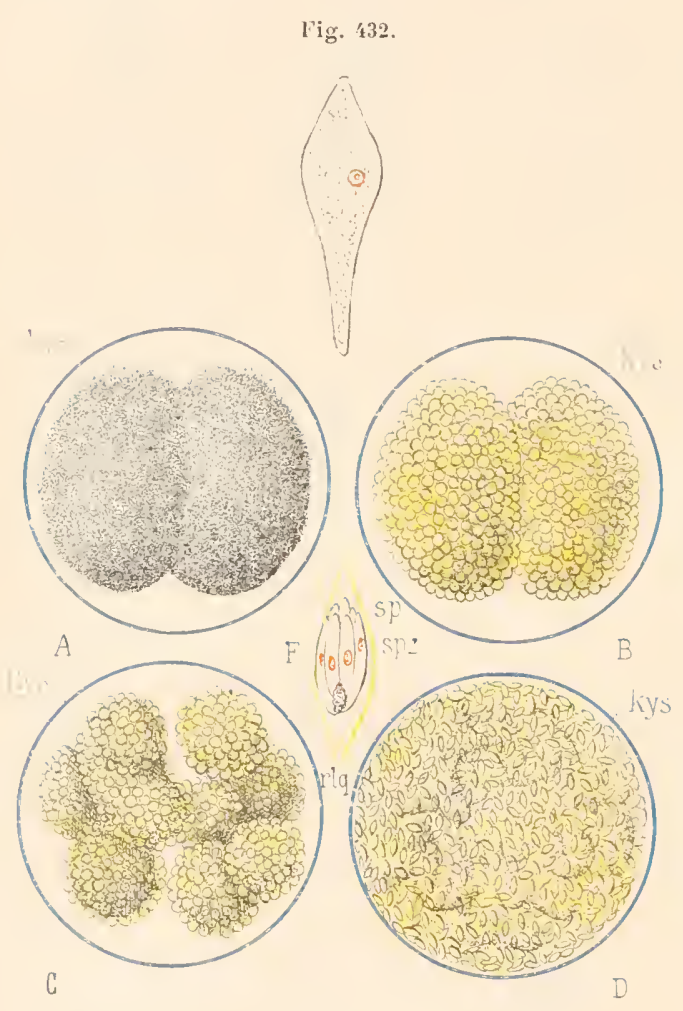

MONOCISTINA (Type morphologique) (Sch.).

L'adulte. les différents stades de la sporulation $A$ à $D$ et la spore $F$.

Kys., kyste; sp., spore; spz., sporozoïte a l'intérieur de la spore. en font un type à part: 10 il a pour hôte, non un Insecte ou quelque autre Articulé, mais un Ver (Annélide ou Géphyrien); $2^{\circ}$ il habite non l'intestin, mais la cavité générale; 30 il n'a pas d'épimérite et est par conséquent acéphale, puisqu'on nomme céphalins ceux de ces êtres qui ont un épimérite. En outre, il n'a pasde septum ni par conséquent de protomérite distinct d'un deutomérite; il redevient la cellule simple munie de sa membrane et de son noyau, sans appendices d'aucune sorte, mais allongée et mobile à la façon des Grégarines ordinaires, gràce aux myonèmes de son ectoplasme. Il vit là, se nourrissant sans doute des sulsstances albumineuses dissoutes dans le liquide cavitaire, grandit, arrive à maturité et s'enkyste. Ses kystes $(A)$ peuvent, selon le cas, ètre évacués par les organes segmentaires ou n’arriver au dehors qu'après la mort de l'hôte et la destruction de son

Pyxinia (Hammerschmidt) (fig. 433 à 435), où une pointe centrale naît en outre du centre de la cupule; 
corps. Là, dans l'eau, ils s'ouvrent par déhiscence simple, sans sporoductes ou pseudokyste ou autre disposition particulière quelconque, et les spores sont mises en liberté $(F)$. Elles sont avalées par des individus de l'espèce à laquelle appartenait leur hòte, s'ouvrent sous l'influence de leurs sucs digestifs et mettent leurs sporozoïtes (spz.) en liberté. Ceux-ci pénètrent dans une cellule de l'épithélium intestinal, mais ne s'y arrêtent pas (peut-être même passent-ils entre les cellules) et arrivent bien vite dans la cavité générale où ils n'ont qu'à grandir pour devenir la Grégarine monocystidée qui nous a servi de point de départ.

Geneiorhynchus (Schneider) (fig. 436), où il a la forme d'un petit bouton longuement pédonculé et armè d'une bande de crochets dirigès en bas;

Xyphorhynchus (Lèger) (fig. 437), où il est formé d'une couronne de crochets et d'une longue pointe conique;

Dufouria (Schneider), où l'on retrouve les longs filaments de Pogonites;

Bothriopsis (Schneider) (fig. 438), chez lequel l'épimérite est difficile à trouver et dont le protomérite en forme de large ventouse ressemble si bien, à un épimérite qu'on l'avait pris pour celui-ci et que l'on avait décrit l'animal comme manquant de protomérite;

Fig. 437 ,

Fig. 436.

Geneiorhynchus

(G. Monnieri)

(d'ap. A.

Schneider).
Fig. 433.

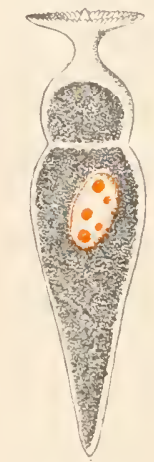

Pyxinia

(P. rubecula)

(d'ap. Léger).

Fig. 438 .

$X_{y p h o r h y n c h u s}$

(X. firmus)

(d'ap. Léger').

Bothriopsis

(B. histrio)

(d'ap. Léger).

schneideria (Léger) (fig. 439), qui a un épimérile en couronne côtelée avec une pointe au centre, se distingue des précédents et de tous les autres Polycystines jusqu'ici étudiés parce que seul il est, normalement et toujours, dicystidé, c'est-à-dire pourvu d'un épimérite à l'état jeune, mais dépourvu en tout temps de septum et par conséquent de protomérite;

Coleophora (Schneider) et

Doliocystis (Lėger) qui sont dans le mẻme cas.

Tous les genres de cette série habitent le tube digestif des Coléoptẻres carnassiers et forment avec Actinocephalus la famille des ACTLOCEPH.1LT:L [Actinocéphalides (Léger)]. 


\section{GENRES}

Monocystis (Stein) est la réalisation exacte du type morphologique ci-dessus décrit. Ce genre a contenu un moment toutes les formes de Monocystines vrais. Il ne contient plus aujourd'hui que les Monocystines de

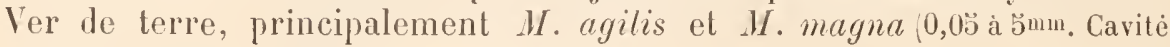
générale de Lumbricus ).

A ce genre se raltachent diverses formes mal déterminées qui ne sont lì que provisoirement ('), et quelques genres que l'on a séparés en se fondant sur la forme des spores et sur quelques autres caractères.

Savoir:

Zygocystis (Stein) (Testicule du Lombric);

Platycystis (Schneider) (Chez Sabella et Audouinia);

Gonospora (Schneider) (fig. 4ł0), à spores denticulées à un pòle (Chez Terebella, Glycera et autres Polychètes);

Fig. '1'tu.

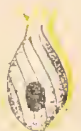

Gonospork.

Spore

(d'ap.Léger).

Ceratospora (Léger) (fig. 4.1), à spores munies de deux longs filaments divergents (Parasite de Glycera) ; et enfin

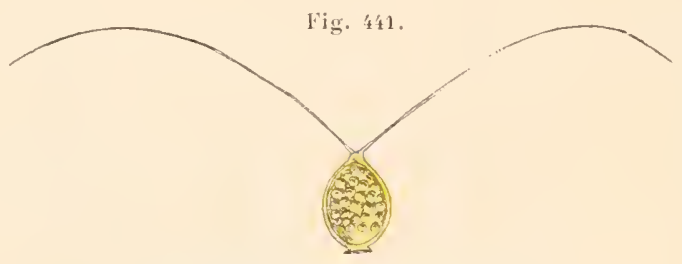

Fig. fiz.

Ceratospora. Spore (d'ap. Léger).

Urospora (Bütschli) (fig. 442), à spores munies d'un seul long filament (Testicules de Tubifex el cavité générale de divers Annelés: Nemertes, Sipun-

Urospora. Spore (d'ap. Léger). culus, Synapta.)

Kystes cœlomiques purs. - Certains de ces Urospor présentent dans leur développement une particularité du plus haut intérèt. Ceux de

(1) Telles sont:

Cytomorpha (Mingazzini) (Chez Diazona violacea);

Lecudina (Mingazzini) (Chez Nere is el Sagitta);

Köllikeria (Mingazzini) (Chez Stanroceplualus);

Ophioidina (Mingazzini) (Chez Lumbriconereis, Sapphirina, Neptlyss et Discocelis tigrina); Polyrabdina (Mingazzini) (Chez spio, Cirratulus et Serpula);

Esarabdina (Mingazzini) (Chez Terebella);

Nematoides (Mingazzini) (Chez Balanus);

Lankesteria (Mingazzini) Chez les Ascidies ;

Pleurozyga (Mingazzini) (Chez Clasellina);

Anchorina (Mingazzini) (Chez les Capilellieus).

Tous ces genres ont été créés yar llingazzini pour des Jonocystines qui ne sont guère déterminés que par leur habitat; leur's spores et leurs liystes n’ayant pas été rencontrés dans la majorité des cas. 
la Némerte et du Tubifex sont de vraies Grégarines, libres et mobiles dans le corps de leur hôte. Mais celui de la Synapte [U. Synaptæ (Léger)] met si longtemps à traverser la tunique intestinale de son hôle, qu'il est déjà adulte quand il arrive à la cavité générale. Aussi, bien qu'au moment où, se détachant de l'intestin, il tombe dans relte cavité, il n’ait encore aucune enveloppe liystique, il est déjà arrondi et immobile, comme pour s'enkyster, et il s'enkystera sans avoir pris forme de Grégarine ni manifesté la mobilité de ces animaux. Chez celui du grand Siponcle (U. Sipunculi) les clıoses vont encore plus loin, car l'enkystement a lieu pendant que l'animal est encore engagé dans les tuniques intestinales, et ce sont des kystes tout formés qui tombent dans

Fig. 143 .

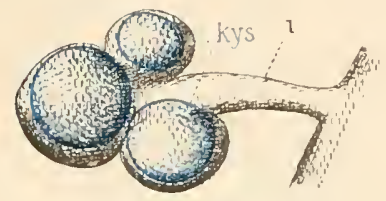

Kystes coelomiques

(im. Léger). la cavilé générale. La phase libre est supprimée. Nous arons rencontré une évolution semblable, à titre d'adaptation temporaire, chez certaines espèces de Polycystines (fig. 473) (V. p. 268). Mais chez elles la forme libre existait concurremment arec la forme kystique cœlomique, sinon au même moment, du moins avant chez le inême hôte, et après chez des hôles de la mềme espère. Ici, au contraire, la phase libre est supprimée pour l'espèce. C'est une espèce rérluile à des kiystes calomiques purs. Ce fait est surtout intéressant en ce qu'il nous met sous les yeux une forme qui se raltache aux Grégarines normales par des transitions insensibles et qui rependant ne diffère presque en rien d'une Coccidie, ainsi que nous allous le constater en éludiant cel ordre de Sporozoaires.

Lithocystis (Giard) est attribué par Cúxot aux Monocystines cœlomiques (Parasite des Oursins) (").

Distribution des Grégarinides. - D'une manière générale, on peut dire

(1) La elassification des Grégarines est rendue fort difficile par le fait que la variation de leurs caractères ne suit pas une marche parallèle. La forme et la structure de l'adulte, étant très uniformes, ne peuvent sérvir presque à rien. Nous avons vu que la présence ou l'absence du septum, sur laquelle se basait l'ancienne division en l'olycystides et Dicystides, n’a pas même une valeur spécifique absolue puisque des individus de même espèce penvent avoir un septum ou en manquer. La forme de l'épimérite en raison de sa fixité dans claque genre et de sa variabilité dans l'ordre, pourrait fouriir un critérium utile, mais c'est un organe caduc et lifficile à observer. Celle des spores a l'avantage de faire porter le critèrium sur un organe relativement facile à voir, mais elle n'est pas d'accord avec celle des épimérites, et ne respecte pas toujours les affinités réelles, comme le montre le cas de Monocystis qui, par ses spores, pourrait prendre place à côté de Pyxinia et de Dufouria. Le meilleur critẻrium semblerait devoir ètre l'évolution, mais elle dépend si étroitement de l'adaptation qu'elle établit des analogies entre les formes les plus disparates comme Clepsidriua longa et Urospora Sipunculi. Pour la succession des genres que nous venons d'étudier, nous avous combiné de notre mieux les divers eritériums, empruntant beaucoup à LÉgER et quelque peu aux autres, en attendant qu'on ait trouvé un classement satisfaisant à toutes les exigences. 
que les Grégarines polycystidées ont pour hôtes habituels les Arthropódes (sauf les Diptères, où elles sont très rares) les Hyménoptères et Lépidoptères, et les Arachnides. En revanche, les Colentérés, Echinodermes, tous les Vers, les Tuniciers, les Crustacés, abondent en Monocystines colomiques ou non. Les Vertébrés n’ont jamais de Grégarines, pas plus que les Mollusques (').

\section{Sous-Ordre \\ COCCIDIDES. - COCCIDID.E \\ [Coccidide (Leuckati); - Psorospermies oviformes] \\ TYPE MORPHOLOGIQUE \\ (FIG. 444 A 446)}

Notre Coccidie $\left({ }^{*}\right)$ typique est un petit être qui se présente, au starle de son évolution qui correspond le mieux à l'état alulte de la Grégarine, sous l'aspect d'une simple cellule, contenue, en qualité de parasite, lans une cellule intestinale de quelque hôte vertébré ou invertébré. Bien qu’elle soit très grosse par rapport à la cellule hospitalière qu'elle distend fortement, son volume se trouve limité par cette condition intracellulaire à 20 ou 30 ‥ Sa forme est sphérique.

(1) Amplioxus en possède peut-être une, décrile récemment par Pollard. Les Tuniciers (Salpes, Aseidies simples et composées) ont de nombreuses espèces de Monocystines. Les Vers abritent beaucoup de Monocystines, surtout les Annélides marins (Nereis, Lumbriconereis, Nepthys, Gl)cera, Spio, Serpula, Staurocephalus, divers

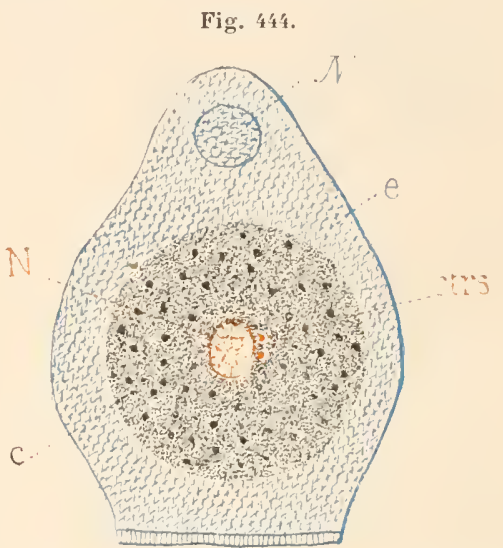

COCCIDIDE (Type morphologique) (Sch.).

c., la Coceidie; etrs., centrosomes; e., cellule de l'hôte; N., novau dı parasite; $N_{\text {. }}$ noyau de la cellule de l'hòte.

Capitelliens et Maldanniens. On en trouve aussi ehez les Oligochètes (Nä̈, Tubifex), les Géphyrierıs (Sipunculus, Phascolosouna, Echiurus), les Némertiens (Cirratulus, Borlasia), les Planaires (Discocelis, Planaria, Mesostomun), mème chez les Trématodes, les Cestodes et les Nématodes. Il en existe chez les Chætognathes (Sagitta). Enfin, les Echinodermes ont des Grégarines cœlomiques (Echinocaldium, Syuapta, Holothuria). Les Arthropodes possèdent surtout des Polycystines. Peripatus en héberge une. Les Crustacés n'ont que peu de ces parasites. Les Décapodes (Homarus, Carcinus), les Amphipodes (Gannarus), les Copépodes (Cyclops, Sapphirina) et les Cirripèdes (Balauus, Pollicipes) sont seuls infectés. Les Arachnides n'en ont pas. En revanclie, les Insectes (à la seule exception des Hyménoptères et Lépidoptères) et les Myriapodes possédent une énorme quantité de Grégarines.

L'aire de répartition des Grégarines est donc fort répandue. On peut remarquer que les Vertébrés et les Mollusques ne possèdent pas de Grégarines, mais seulement des Coceidies. Ce qui ne veut pas dire, du reste qu'il n'existe pas de Coccidies chez les Myriapodes et les Insectes, où ces parasites existent, au contraire, assez souvent.

(•) Ne pas confondre ce terme, pris souvent comme synonyme de Coccidides, avec le nom français du genre Coccidium. 


\section{Structure}

Elle a la constitution d'une cellule ordinaire $(c$.$) , sauf qu'elle est nue,$ ou du moins n’a que cette membrane protoplasmique, simple bordure hyaline, qui sépare du dehors le cytoplasme granuleux.

Son cytoplasme est finement vacuolaire et renferme, outre les microsomes, de grosses granulations qui représentent des produits plus ou moins arancés de l'assimilation (matériaux de réserve) ou de la désassimilation. Il n'y a pas d'ectoplasme distinct d'un endoplasme (').

Au centre du cytoplasme est un gros noyau ( $V^{T}$.) pourvu d'une membrane el dans lequel on distingue un réseau chromatique et un gros nucléole. A l'un de ses pôles sont, côte à côte, deux centrosomes (ctrs.) (Labbé).

\section{Physiologie.}

Nutrition. - En raison de sa situation intracellulaire et de l'absence le membrane, notre Coccidie n'a besoin d'aucun organe spécial de nutrition ou de désassimilation. Elle assimile et désassimile par simple osmose, dans son milieu ambiant formé par le cytoplasma vivant de la collule hospitalière. Elle n’a de mourements d'aucune sorte. Sa physiologie ne nous laisse donc à étudier que les phénomènes de son ç̣cle évolutif.

Division. - Bien qu'clle ne soit pas le mode principal de reproduction, la division n'en existe pas moins. L'animal peut, quand il ne remplit pas toute la cellule, se multiplier par ce processus. La division a lieu par milose (Labbé).

Enkystement. - Après avoir alleint sa taille définitive, la Coccidie s’enkyste sur place. Elle n’a pour cela qu’à sécréter son kỵste. Il est fait ici d'une mince membrane d'une subslance d'aspect chitinoïde (fig. 44ว, Kyst.) analogue à la membrane interne du kyste des Grégarines. Les granules plasmatiques disparaissent arant l'enkystement.

Sporulation (fig. 4\%). - Dès que l'enkystement est opéré $(A)$, l'animal se dispose à sporuler. La membrane nucléaire disparail $(B)$, le nucléole se résorlse, et il sort du noyau une masse chromatique qui se porte vers la surface de la cellule pour śéliminer et qui représente peut-ètre un globule polaire(Labbé). Mais cela n’est suivi đ'aucun phénomène sexuel, l'enkystement étant toujours solitaire. Le noỵau, resté au centre, se divise alors par mitose $(C)$ et donne successivement de petits noyaux qui se portent à la surface $(I)$ à peu près comme dans la segmentation centrolécithique des Insectes. $\Lambda$ la surface aussi s'est accumulé tout le plasma formatif du cy toplasme. Chaque noyau masse aulour de lui sa part proportionnelle de ce plasma et forme ainsi une petite masse nucléée. Ces petites masses sont d'abord

(1) LABBÉ distingue, selon leur aspect et leurs réactions des granules plasmatiques et des granules chromatoïdes plus petits. 
continues par leur base avec le cytoplasme sous-jacent, mais peu à peu elles s’individualisent, s'arrondissent et se séparent sous la forme de

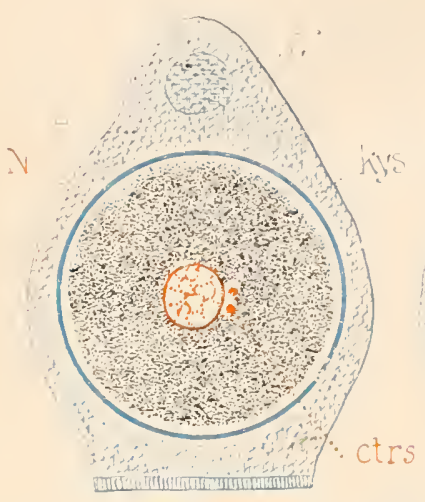

Fig. 445.
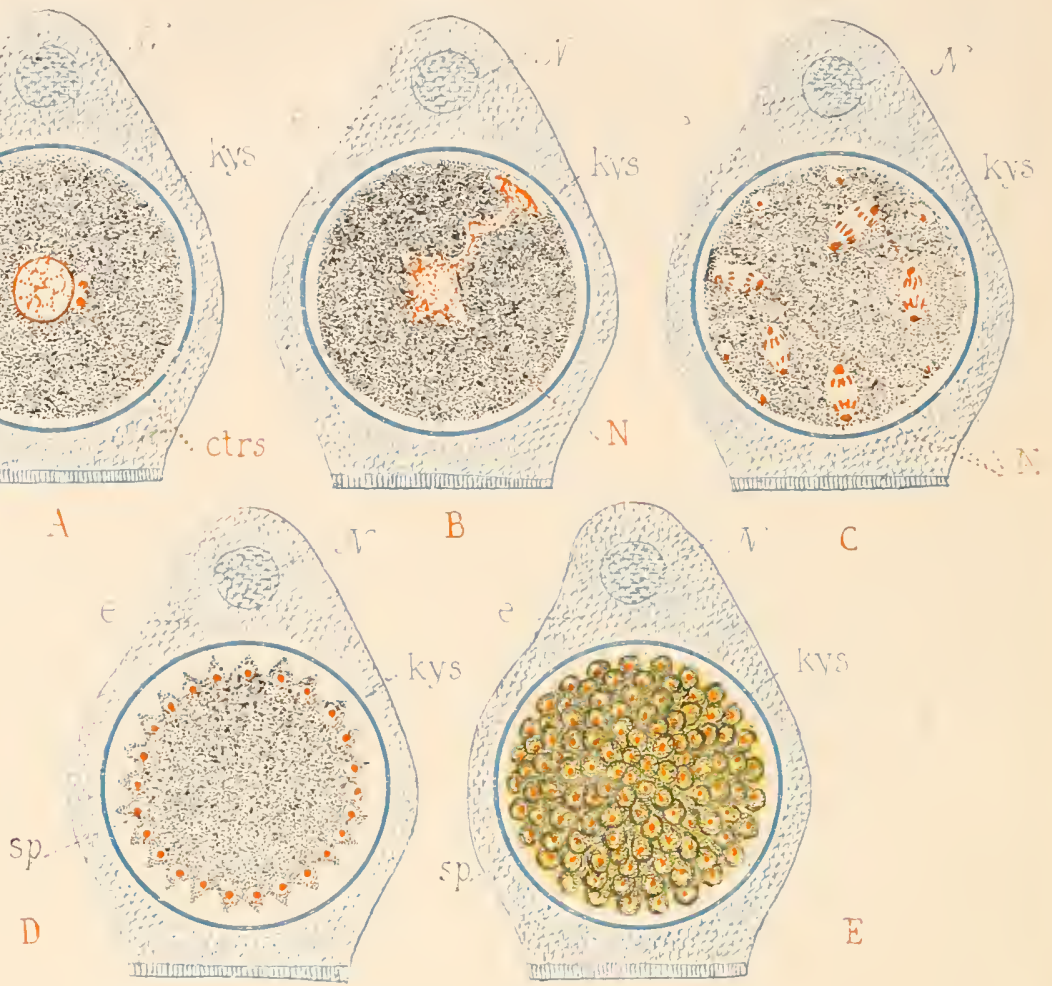

COCCIDID.E (Type morphologique). Sporulation (Sch.).

$A$ a $E$, Stades successifs. ctrs., centrosomes; lcys., enveloppe kystique; $N$., noyau de la cellule de l'hôte $(e)$; $\mathbf{x}$., noyau; sp., spores.

petites cellules nues, les sporollastes ('), du plasma formatif qui se retire au centre où il forme un volumineux reliquat résiduel qui ne sera pas utilisé.

Ces sporoblastes se transforment en spores par le fait qu'ils se sécrètent deux membranes, une exospore l'abord, une endospore ensuite.

Formation des sporozoites (fig. 446). - Après avoir formé ses membranes, la spore commence l'évolution intérieure qui conduit à la formation

(1) LABBÉ les appelle archéspores, mais nous préfẻrons conserver une dénomination tout aussi applicable, plus ancienne et d'une signification plus générale. Disons que c'est aux belles recherches de cet auteur que nous devons la connaissance des phénomènes réels de la sporulation. Sa description, beaucoup plus conforme aux lois générales de la cylologie, se substitue à celle que Schneider avait donnée pour Klossia octopiana et où il faisait dériver la chromatine des noureaux noyaux d'un bourgeonnement endogène du nucléole. 
des sporozoïtes. Pour cela, son noyau se divise el donne un petit nombre de noyaux entre lesquels le cytoplasma de la spore se partage, et ainsi se forment quelques sporozoïtes. Il reste cependant un petit résidu de cytoplasme inutilisễ. Pendant que ces phénomìnes s'accomplissent dans les spores, la cellule hospitalière est usée, détruite par le liyste qui forme dans son sein un énorme corps étranger incompatible avec une longue existence, et le kyste tombe dans la cavité intestinale. Là, deux éventualités peurent se présenter pour lui. Tantôt, il éclate dans l’intestin, laisse échapper les spores mûres, et celles-ci mettent en liberté les sporozoïtes qui attaquent immédiatement de nouvelles cellules du même hòte; tantôt, les Fig. 446.
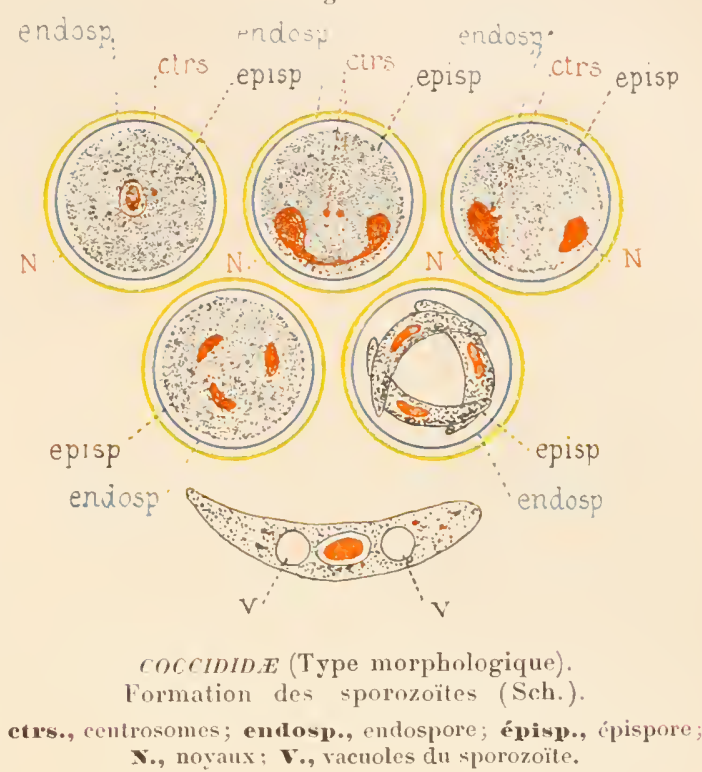

kysles sont éliminés arec

lés fèces, s'onvrent dans l'humidité, et les spores, aralées par le même hòle ou par ses congénères, mettent les sporozoïtes en liberté. Ceux-ci sont, comme chez les Grégarines, des corpuscules falciformes doués, ici aussi, de mouvements énergiques. Ils attaquent les cellules épithéliales de l’intestin, clıacun pénètre dans une d'elles, s'y arrondit et n'a qu'à grossir pour devenir la Coccidie adulte arec les caractères que nous lui avons tronvés en commençant cette description.

Les Coccilies ne sont donc, en somme, que des Grégarines qui restent intracellulaires jusqu’à l'enkystement ol sont, par conséquent, privées de vie lihre et de mourements. Mlais nous arons vu que, parmi les Grégarines colomiques, certaines (Jrospora Sipunculi) n'avaient pas non plus de vie libre. Il ne resterait done d'autre différence que lo fait que la Coccidie s'enkyste dans la cellule, tandis que le kyste colomique grégarinien s'enkyste hors de la cellule, dans le parenchyme de l'intestin. Mais cette différence elle-mème disparaìt, certaines Coccidies (Klossia) s'enkỵtant de la même manière, comme nous allous le roir dans un instant.

Le sous-ordre des Coccidides est fort homogène et nous n'allons trouver entre les genres que des différences secondaires portant surlout sur le nombre des spores dans le kyste et sur le nombre des sporozoïtes dans la spore. 


\section{GENRES}

Klossia (Schneider) représente à peu près le type morphologique el nous n’avons que quelques différences à signaler et quelques particularités à préciser. L'animal quitte la cellule hospitalière à l'élat de Coccidie et s'enkyste dans l'épaisseur des parois intestinales où il se forme mème un kyste adventice aux dépens du tissu conjonclif de cel organe. C'est plus tard, par destruction de la muqueuse, que les kystes sont mis en liberté dans l'intestin. Chaque kyste contient un nombre de spores très grand et non défini et chaque spore contient un nombre non défini de sporozoïtes (Bien visible à l'œil nu. Tube digestif de Sepia ou d'Octopus, principalement dans l'intestin spiral et rein d'Ifelix) (').

Pfeifferia (Lablı́) (fig. 147) est, comme Klossia, polysporé, c'est-à-dire qu'il produit dans son kyste un nombre grand et indéfini de sporoblastes, mais ici les sporoblastes se transforment directement chacun en un sporozoïte $B$ sans former de membrane autour le lui, c'esta-dire sans passer par l'état de spore. Le kyste émet lirectement les sporozoïtes quand it se rompt Parasite chez les Lapins, les Passereaux, les Gallinacés, le Triton et divers Poissons) $\left(^{2}\right)$.

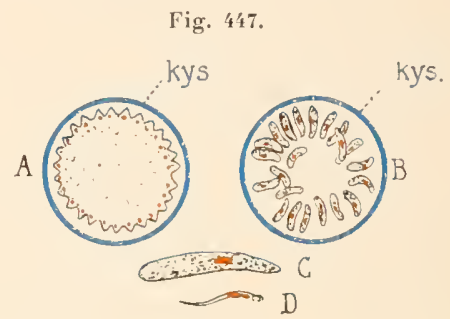

Pfeifferia (Sch.).

$A$, formation des sporozoïtes; $B$, sporozoïtes à l'intérieur du kyste; $C$, marrosporozoïte; $D$, microsporozoite. kys., kyste.

Adelea (Schneider) qui n'a que deux sporozoïtes Chez Litholius);

Barroussia (Schneider) qui n'en a qu'un (Chez Nepa cinerea).

Ces quelques genres constituent la tribu des Polyplastidés digéniques de Labbé : Polyplastides parce que les kystes contiennent beaucoup de spores, et digéniques parce que les sporozoïtes ne naissent pas directement des sporoblastes.

(2) Cette Coccidie présente encore deux particularités intèressantes. Premièrement, ses sporozoïtes sont de deux tailles selon les kystes. Il y a des kystes à macrosporozö̈tes $(14$ à $15 \mu)($ fig. $417 \mathrm{C})$ et des kystes à microsporozö̈tes (7 à $8 \mu)(D)$. La signification de cette diffèrence n'est pas connue; peut-être a-t-elle quelque elıose de sexuel. Il semble que les macrosporozoïtes doivent simplement differer des derniers par une plus grande richesse en matières nutritives, car ils ne laissent pas de reliquat résiduel. Or on sait que ce reliquat est formé, en génèral, de la partie nutritive du plasma. En second lieu, l'animal peut se multiplier une ou deux fois par division mitosique dans la cellule hospitalière, avant de s'enkyster. Elle produit alors des infections aiguës très graves pour l'hôte.

Avec le genre voisin

Eimeria (Schneider), à sporozoïtes en tonnelet (chez la Souris), il constitue pour LabBé, la tribu des Polyplastidés monogéniques dont la définition se comprend d'elle-même après ce qui a étè dit au sujet de Klossia.

C'est avec doute que nous plaçons ici les genres

Gymnospora (Moniez) trouvé par Moniez dans une Glienille et considéré par cet auteur comme appartenant aux Coccidies, malgré les sporoductes formés par son kyste. Le contenu du liyste se résout tout entier en nombreuses spores, mais qui donnent chacune plusieurs sporozoïtes. Le reste de l'évolution n'est pas connu; 
Coccidium (Leuckart) (fig. 448). Tout est conforme ì notre type morphologique chez Coccidium, sauf que le noyau du kyste ne se divise que deux fois et donne seulement quatre sporoblastes et par conséquent quatre spores : $(C: s p$.) celles-ci sont à l’intérieur du kyste et donnent, à leur tour, à leur intérieur, deux sporozoïtes disposés tête-bèche $(G)($ ').

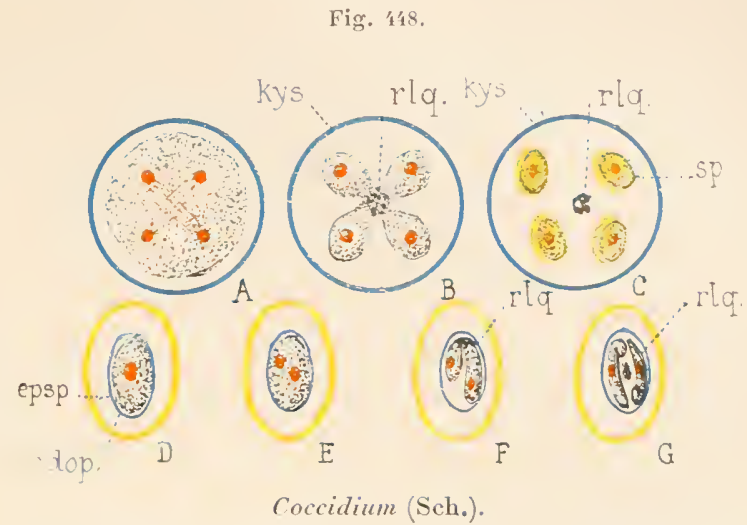

$A$ à $C$, ormation des spores dans le kivste; $D$ à $G$, formation des sporozoilles dans la spore; edon., endospore; epsp., épispore kys., kyste; rlq., reliquats de segmentation; sp., spores. cidides. - Les Coccillies se rencontrent dans toutes les classes des Vertébrés et chez quelques Invertébrés: (Céphalopodes (Octopus, Sepia), Gastéropodes (Limax, Helix, Succinea, Neritina), In yriapodes (Lithobius. Cilomeris), et peut-ètre dans une chenille de Lépidoptère $\left(^{2}\right)$.

Cretya (Mingazzini), genre incerlix sedis, qui se rapporte probablement ì Pfeifferia:

Gonobia (Mingazzini) qui se rapporte sans lloute à Eimeria;

Rhabdospora (Henneguy) qui se distingue des genres précédents par les sporozoites en forme d'épingle, la tête de l'épingle élant le noyau du sporozoïte (Trouvè par Laguesse et Thélohan dans diver's organes des l'oissons).

(') Les Coccidium sont les uns endogènes, les autres exogènes. Ils sont très répandus chez les Vertébrés supérieur's.

Citons : C. oviforme et $C$. perforens (Leuckart) du Lapin; $C$. Lenellum (Raillet) du Poulet; $C$. Delagei Labhé) des Tortues; $C$. proprium Schneider du Triton, etc.

Genres voisins :

Goussia Labbé), dont la spore s'ourre par leux valves Cliez les Poissons);

Cristallospora Labbél (Coccidium crystalloides de Thélolıan) à spore bipyramidale affectant une forme cristalline (Chez Motella);

Bananella (Labbé) à trois spores seulement;

Diplospora (Labbé) à deux spores qui ont chacune quatre sporozoïtes (Chez les Passereaux); Cyclospora (Schneider) et

Isospora (Schneider) sont également disporés.

Le genre Orthospora (Schneider) n'existe pas : c'estle Coccidium proprium du mème auteur.

$\left(^{2}\right)$ On a trouvé, chez l'llomme, plusieurs cas bien observés d'une roccidie voisine de $C$. perforans. Les Coccidies sont communes chez la plupart de nos animaux domestiques. 
$3^{\text {e SOUS-ORIRE }}$

IÉJOSPORIDES. - H.EMOSPORIDE

[HÉMosporides (Labbé)]

\title{
TYPE MORPHOLOGIQUE
}

\author{
(FIG.449 ET 450)
}

\section{Structure.}

Notre Hémosporidie (*) est un petit être mesurant 10 à I 5 y. de longueur, logé dans l'intérieur d'une hématie de quelque Reptile ou Amphibien. On peut le définir comme une petile Grégarino monocystidée dont

Fig. 449 .

A

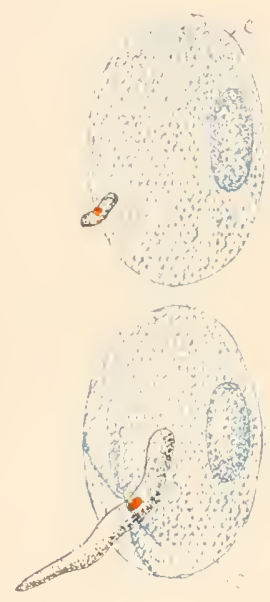

E
B
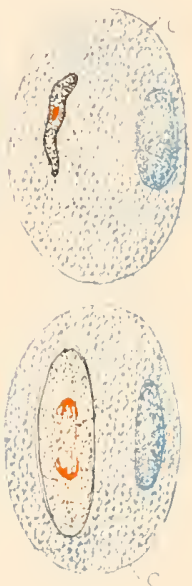

F
C.
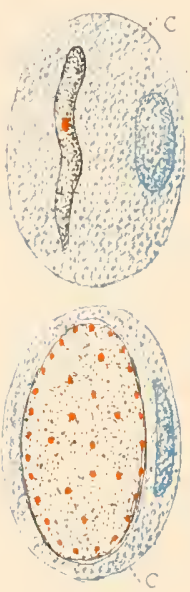

G
D
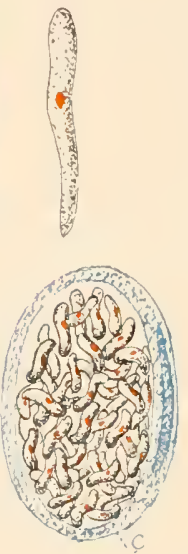

$\mathrm{H}$ la structure se serait quelque peu simplifiée en même lemps que sa taille se réduisait. Il est en forme d'ovoüde allongé. Un ectoplasmetrès mince forme, avecla minime membrane protoplasmique lont on ne peut guère le discerner, l'enveloppe tégumentaire de la cellule dans laquelle on apercoit cependant une différenciation fibrillaire longitudiuale qui représenle, sous une forme simplifiée, la couche à myonèmes des

Grégarines. L'endoplasme est, comme chez celles-ci, şarni de granulalions plasmaliques, amyloüdes, graisseuses, malériaux de réserve de l'alimentation, et conlient un noyau formé d'une mince membrane, et d'un contenu clair entourant une partie centrale chromatique.

$\left(^{*}\right)$ Ici encore, nous emploierons parfois dans le langage courant ce terme en place d'Hèmosporidide. Il désignerait un genre typiqne Hemosporilium qui d'ailleurs n'existe pas. 


\section{Physiologie.}

Nutrition. - Le petit parasile se nourrit de la substance du globule et grossit à ses dépens. Il n’avail guèreque $\because$ m quand il est entré dans la cellule $(A: c)$ et atteint jusqu’à $1 \partial ّ$ : quand il est ahulte. Aussi le globule est-il profondément atteint dans sa nutrition : il se décolore, son noyau, relégué à la périphérie, se déforme, se fragmente, le cytoplasme s’épuise rl, à la fin, il n’est plus représenté que par ses couches superficielles condensées en une enveloppe llétrie qui contient le parasile avec le (ou les) reste du noyau.

Enkystement. Formation des sporozoïtes. - ()uand il est mùr, le parasite s'enkyste, c'est-ì-dire se sécrète une inince cuticule, et entre en sporulation. Pour cela, son noyau se divise par mitose $(F)$, successivement plusieurs fois, et donne de petits noyaux qui se portent à la périphérie $(G)$, se parlagent le plasma formatif, el forment arec celui-ci de petiles masses cellulaires nues qui s’indivilualisent peu à peu et finalement se transforment en autant de sporozoïtes distincts $(I I)$ laissant un reliquat de plasma nutritif inutilisé. Il y a des kystes à macrosporozö̈tes et à microsporozoïles.

Les choses, on le roit, ont beaucoup d'analogie arec ce qui se passe chez la Coccidie Pfeifferiu. Ces sporozoïtes qui mesurent seulement quelques laquelle ils contribuent prar leur's mourements saccarlés. Libres dans le plasma sanguin, ils se répandent entre les globules $(a)$, les attaquent, les perçent et pénètrent daus leur intérieur $(A)$ où ils n’ont plus qu’à grossir pour recommencer la même série de phénomènes.

Vie à l'état libre - Leur crcle évolulil cependant ne se réduit pas tout ì fait à ce qui précède. Leur's mourements sont ordinairement peu actifs daus le globule el se réduisent à quelques secousses de loin en loin. Mais parfois, plus énergiques ou mieux lirigés, ils permettent aux sporozoïtes, surtout quand ceux-ci sont adultes, de sortir du globule(I)) et de s'agiter dans le plasma sanguin avec une énergie plus grande, jusqu’à ce qu'ils 'attaquent un autre globule pour se loger de nouveau à son intérieur $(E)$.

Conjugaison (fig. '́̉0). - Parfois, lorsque le parasite est devenu libre, il s'unit à un de ses con¿énères et se soude à son extrémilé postérieure par la partie correspondante $(a)$. Puis les deux conjoints, se rabattant l'un ver's l'autre $(b)$, arrivent à se placer còte à côte et, la soudure envahissant de bas en haut $(c)$, finissent par se fusionner complètement en un individu unique Fig. 4.50. (d) qui ne diffère des aulres que par son épaisscur plus grande. La conjonction s'étend aux noyaux qui se fusionnent l'un dans l'autre. Il y a 
donc là une vraie conjugaison totale. Cette conjugaison peut aussi avoir lieu entre individus intraglobulaires s'ils se rencontrent dans le même globule. Halheureusement, pas plus ici que chez les Grégarines, on ne sait rien de son influence sur le cycle évolutif.

\section{GENRES}

lls sont bien peu nombreux, puisque l'ordre n'en contient en tout que trois qui, étant lous très conformes au type morphologique ci-dessus, pourront être caractérisés en quelques mots.

Drepanidium (R. Lankester) (fig. 4.̈l, A), est de laille très petite, ne dépassant pas les trois quarts de celle du globule, auquel il ne parail guère nuire (Chez les Grenouilles (Rana esculenta) et chez quelques oiseaux).

Karyolysus (Labbé) (fig. 4öl, $B$ ) alteint la longueur du globule malgré l'hypertrophie que celui-ci subit sous son influence, et finit par le détruire (Chez les Lézards [Lacerta]).

Danilevskya (Labbé) (fig. 45̆l, C) est de taille très grande, double de celle du globule, dans lequel il est ployé en deux pour pouvoir y loger et exerce sur celui-ci une action mécanique nocive, mais insulfisante pour le détruire. Pas Fig. 451.

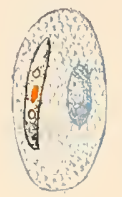

A

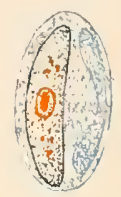

B

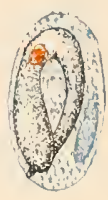

C

A, Drepanidium. B, Karyolysus. C, Danilevskya (im Labbé). de distinction entre microsporozoïtes et macrosporozoïtes; pas de conjugaison (Grenouille et divers Reptiles: Lacertiens, Ophidiens et Chéloniens.)

Distribution des Hémosporidides. - Les llémosporidies ont pour hôte les Reptiles el les Oiseaux. Aucune n’a été trouvée, ni chez les Mammifères ni chez les Poissons, ni chez les lnvertébrés.

4. SoUs-ORDRE

GYIINOSPORIDES. - GYHNOSPORID E

[Gruxosponidies (Labbé)]

TYPE MORPHOLOGIQQUE

(FIG. 452)

\section{Structure.}

Le type morphologique résumant en lui les caractères des Gymnosporidies (*) serait une toule petite masse proloplasmique de forme irrégulièrement arrondir, mesurant au plus 3 à 4 p. de diamètre, vivant en parasites daus les hématies d'un Vertébré à sang chaud.

Ce parasite a lous les caractères d'une amibe $(B)$, il en a la structure et les mouvements. Il est nu, sauf celte bordure continue qui constitue

(*) Mème observation qu'à la page 284 . 
la membrane proloplasmique sous sa forme la plus simple; il a un endoplasme légèrement vacuolaire, un ectoplasme où se trouvent de fines granulations, noires oubrunes, résultant de la dégénérescence pigmentaire de l'hémoglobine dont le pelit ètre s'est nourri; enfin il contient un noyau volumineux, bien rond, muni d'une fine membrane et contenant un beau nucléole excentrique noyé dans un plasmanucléaire hyalin(').

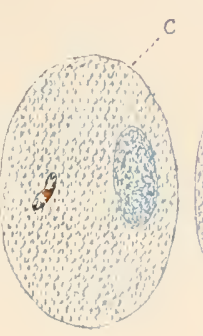

A
F'ig. 152

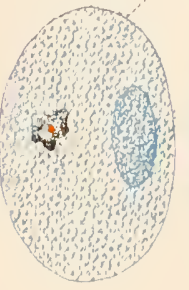

B

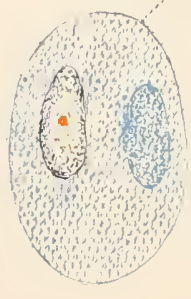

C

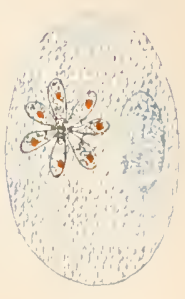

D

GYMNOSPORID.E. Type morpholugique (Sch.).

$A$, sporozoite ayant pénétré dans une cellule; $B$, mouvements amœboides du parasite; $C$, le parasite se prépare à se diviser; $D$, formation des sporozoites.

\section{Physiologie.}

Cetle amibe se nourrit par simple osmose dans le imilieu essentiellement nutritif où elle est plongée, et le globule, sous son influence, s'anémie et s’hypertrophie plus ou moins. Elle exécute des mouvements amibö̈les variés, émettant et rentrant ses pseudopodes. Elle peut se diviser. On la voit aussi parfois se conjugner avec un autre habitant du mème globule. Arrivée à l'élat adulte, ce qui ne demande que quelques jours, elle entre en sporulation, mais (et c'est là son caractère le plus remarquable, celui qui a valu son nom au groupe dont elle est te lype) sans s'enkyster. Elle s'arrondit, devient immobile $(C)$, mais ne sécrète aulour d'elle aucune membrane; son noyau se divise en un certain nombre (inléterminé mais pas très considérable) de petits noyaux, qui se portent à la périphérie, se partagent le cyloplasme et se trausforment, enfin, de la manière dont nous avons déjà vu tant d'exemples, en sporozö̈tes disposés à la surface d'un petil amas résiduel inulilisé $(D)$. Cés sporozoítes sorlent alors du globule et se répandent dans le plasma sanguin où ils atlaquent de nouveaux globules pour se loger à leur inlérieur.

\section{GENRES}

Hæmamœba (Grassi). La structure el l'évolulion de ce genre, au moins sous sa forme ordinaire (H. Laverani de Grassi), sont entièrement conformes au type ci-dessus étudié $\left({ }^{\boldsymbol{z}}\right)$.

(1) LABBÉ voit dans la partie claire une vacuole, le nucléole étant le vrai noyau.

(2) Mais il faut signaler quelques particularilés de polymorphisme chez ce parasite qui nous intéresse à un lıaut degré, parce qu'il est la cause de la malaria de l’homme.

Il a été découvert par Laverax à qui Grassi l'a dédié.

Sous une de ses formes, il produit la fièrre tierce. Il est alors remarquable par 
Un peu différent dans son évolution est

Halteridium (Labbé) qui, constitué au début comme les parasites de la malaria, sauf une forme plus allongée, montre, au moment de la sporulation, la particularité suivante. Son proloplasme s'accumule aux deux pôles el forme deux masses réunies par un isthme étroit. Le noyau s'est, pendant ce lemps, divisé en deux autres qui ont pris position chacun dans une de ces masses. L'isthme de réunion dégénère et derient plus tard un reliquat résiduel. Quant aux deux masses polaires, elles sporulent l'une et l'autre absolument comme un Hzmamoba ordinaire. La différence est donc en somme assez secondaire (Hématies des Oiseaux) (').

l'état lobé de ses pseudopodes el le petit nombre de ses sporozoïtes. Sous une autre forme, il produit la fière quarle el se distingue alors par des pseudopodes réticulés et des sporozoïtes plus nombreux.

On peul, a bon droit, considérer ces deux formes comme deux variétés distinctes, II. Laverani terliana et H. L. quartana. L'une et lautre reproduisent, quand on les injecte expérimentalement, le type de fièvre dont elles portent le nom. Et il n'est pas sans intérêt de remarquer que la variété tertiana complète son cycle en quarantehuit heures el la quartana en soixante-douze heures, c'est-à-dire dans le temps qui sépare deux accès consécutif́s.

Mais d'où vient la fièsre quotidienne? On a pensé pouvoir l'attribuer à une forme qui serait une troisième variété, le type en croissant el serait caractérisée par une forme en long ovoïde aroué, et par l'absence de mouvements ammboïdes. Pour le reste, elle ressemblerait aux autres, débutant far une petite amibe nobile el finissant par sporuler après s'ètre arrondie. Mais, on a beau les injecter, on ne reproduit pas pour cela la fièvre quolidienne et, d'autre part,on les rencontre aussi dans les types tierce et quarte. On en est done réduit à les considérer comme de simples étals de dimorphisme des variétés normales tertiana el quarfana. Quant à la fièvre quotidienne, elle ne serait peut-être qu'une double tierce ou une triple quarte á accès alternants de deux en deux ou de trois en trois jours. L'observation clinipue parle en faveur de cette hypothèse, car il est bien rare que les accès consécutifs de la quotidienne soient identiques entre eux.

Quand on observe le sang hor's des vaisseaux, on y trouve des amibes en tout semblables aux IIrmamcba, mais qui émettent trois ou quatre longs flagellums, souvent plus, que l'on voit sagiter vivement, puis se détacher. Laverax croyait que ces flagellums servaient à reproduire le parasite. Daxilevsky voyait en eux des êtres infusoriformes de nature spéciale, Polymitus (Danilevsky). LabBé a démontré que ce ne sont que des modifications agoniques des parasites normaux, se produisant sous l'influence de l'asphyxie. On ne les trouve, en effel, janrais dans le sang au moment du premier examen; ils se forment seulement après quelques minutes et parfois sous les yeux de l'observateur. Enfin, on peut retarder leur apparition en retardant ou accélérant la condition asphyxique par l'emploi de la chaleur, ou la hàter par l'addition d'un réducteur comme le pyrogallol.

Genres voisins :

Proteosoma (Labbé) qui produit une sorte de malaria (Chez les Passereaux);

Dactylosoma (Labbè) el

Cytamœba (Labbé), qui ne semblent pas nuire à leur hôte (Grenouille);

Acystis (Labbé), qui n'habile plus dans les globules, mais dans le cytoplasme ou le noyau de certaines cellules de tissu épithélium intestinal de la Salamandre et du Triton $(E)$.

(1) Malteridium évolue en six à sepl jour's sans paraitre gèner beaucoup son hòte. LABBE, à qui l'on doit nos meilleures connaissances sur tous ces ètres, considère ces 


\title{
$2^{\mathrm{e}}$ ORDRE \\ DOLICIOGYSTIDES. - DOLICIOCYYTIDA
}

\section{TYPE MORPHOLOGIQUE}

Ce type est représenté par celui des Sarcosporides, scul sous-ordre de cel ordre. Il se caractérise, par opposition arec celui des Brachycystides par sa forme allongée, ovoïde.

\author{
SOUS-ORDRE \\ SARCOSPORIDES. - SARCUSPORID E \\ [Sarcosporides (Balbiani); - Psorosperhies (*) des muscles; \\ Psorospermies utriculeuses]
}

TYPE MORPHOLOGIQUE

(FIG. 453)

Le parasile se rencontre dans le parenchyme musculaire ou conjonclif de quelque Manmifère, sous l'aspect de productions blanchàtres, ovoüdes,

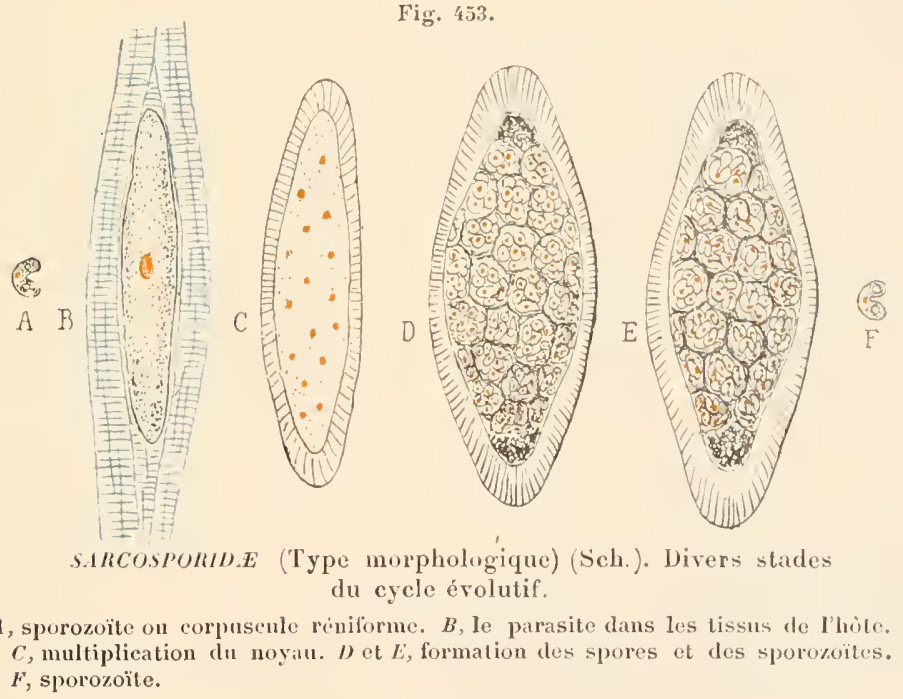

allongées, assez grosses pour être visibles à l'œil nu $(B)$. Chacune est formée d'une masse de protoplasma granuleux, contenant un noyau et renfermée dans une membrane qui semble ètre une sécrétion plutòt

deux masses comme des spores nues (ou plutòt sporoblastes), el voil là un déreloppenent digénique qüil oppose au développement monogénique d'Irxmamaba. L'assimilation est juste, mais la différence entre les deux développements n'est guère

(*) De $\pi \sigma \dot{\omega} \rho \alpha$, gale; $\sigma \pi \dot{\varepsilon} \rho \mu \alpha$, semence. 
qu'une membrane cellulaire. Peu à peu, celte masse grossit, arrive à mesurer plusieurs millimètres de long sur peut-être un millimètre de large, et bientôt se met à sporuler. Pour cela, le noyau se divise $(D)$, les noyaux filles se partagent le protoplasma, et tout le contenu de la membrane se trouve divisé en sphérules arrondies uninucléées qui sont des sporoblastes ou des spores nues. Dans chacune de celles-ci se forment de nombreux sporozoйtes $(E)$, appelés ici, d'ordinaire, les corpuscules réniformes $(F)$, en forme de bâtonnets arqués munis chacun d'un noyau central. On n’a pas observé leur mode de formation, mais il n'y a guère à douter qu'ils ne se forment par division du noyau de la spore et de son contenu protoplasmique suivant le procédé habituel. Ces sporozoïtes $(A$ et $F$ ) sont très actifs, se ployant en deux sur leur face concave et s'étendant par des contractions et des détentes énergiques. Malheureusement on ne sait rien de leur évolution ni du mode de propagation du parasite.

\section{GENRES}

Sarcocystis (R. Lankester) (fig. 40̈4) se distingue par sa forme trapue et l'épaisseur très grande de sa membrane qui est striée de lignes radiaires fines el serrées, représentant des pores canaliculaires ( 3 i $4 \mathrm{~mm}$. Nuscles de divers animaux domestiques: Bœuf, Houton, Cheval et surtout Cochon) (").

Miescheria (R. Blanchard) se distingue de Sarcocystis par sa membrane mince et anhiste, sa forme plus allongée et ses sporozoïtes fusiformes $\left({ }^{*}\right)$.

Balbiania (R. Blanchard) se distingue par ses kystes dans lesquels la sporulation détermine des alvéoles de deux tailles, les uns larges, à la périphérie, les antres, petits au centre. La sporulation est centrifuge, les alvéoles du centre étant pleins de sporozoïtes tandis que ceux de la périphérie sont encore à la phase de protoplasme granuleux (Tissu conjonctif, chez le Kanguroo et chez divers Oiseaux).

plus grande qu'entre Pfeifferia et Klossia clıez les Coccidies.

(1) Ces Sarcocystis (S. Miescheri), sont comnus aussi sous le nom de Tubes de Rainey, du nom du zoologiste qui les découvrit en 1858. Logés soit dans les fibres des muscles, soit entre elles, ils déterminent une myosite inter-

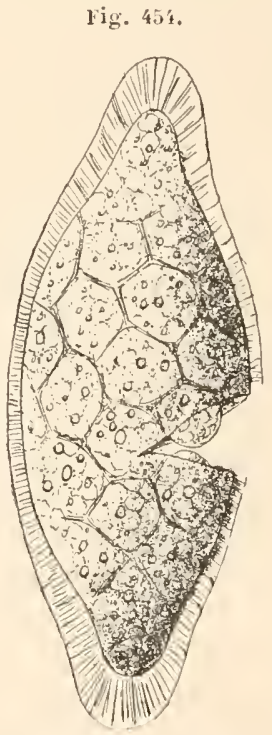

Sarcocystis d'ap. Manz). stitielle grave compliquée de symptômes généraux qui semb]ent dus à la sécrétion de toxines, car l'extrait, injecté à des Lapins, les plonge dans le collapsus. Les symptômes sont analogues à ceux de la trichinose. On n’a jamais observé que le parasite ait atteint l'Homme bien que celui-ci ait, sans doute bien des fois, ingéré les viandes infectées. Les sporozoiltes sont réniformes.

(2) On appelle aussi ces Miescheria, Tubes de Miescher. Ce sont les Sarcosporidies les plus anciennement connues, Miescher les ayant décrites dans les muscles de la Souris en 1843. On en a rencontré chez l'Otarie Huet), le Ghevreuil, le Mouton et même, semble-t-il, chez l'Homme (Baraban et Saint-liemy), dans les cordes rocales. 
$2^{\text {e Sous-Cilasse }}$

\section{AMCEBOGÉNIENS. - AMCEBOGENI.E}

Le type morphologique de cette sous-classe se confond avec celui de l'ordre unique qui la constitue.

ORDRE

\section{NEMATOCYSTIDES. - NEUATOCYSTIDA}

Le type morphologique, caractérisé comme ordre par sa forme allongée, se confond ici encore arec celui de l'unique sous-ordre qui le constitue.

SOUS-ORDRE

MYYOSPORIDES. - UYTOSPORID.E

[Mrxosponidies; - Psorospermes des polssons ; - Myxosporidia (Bütschli)]

\section{TYPE MORPHOLOGIQUE}

(FIG. 455 A 457)

Le Myxosporide n'est plus, comme les parasiles de l'orlle précédent, renfermé dans une cellule ou attaché à une cellule dont il est sorti. Il est logé en plein tissu ou libre à la surface interne des cavités naturelles, et son siège est assez variable. Il faut se le représenter comme une grosse amibe, pouvant atteindre $3 \mathrm{~mm}$, visible à l'œil nu sous l'aspect d'une petite tache laiteuse.

\section{Structure.}

Il a la constitution d'une amibe polynucléée (A), comme nous en avons rencontré parmi les Rhizopodes, sauf une différence capitale : comme tous les autres Sporozoaires sans exception, il est dépourvu de vésiFig. 455 .

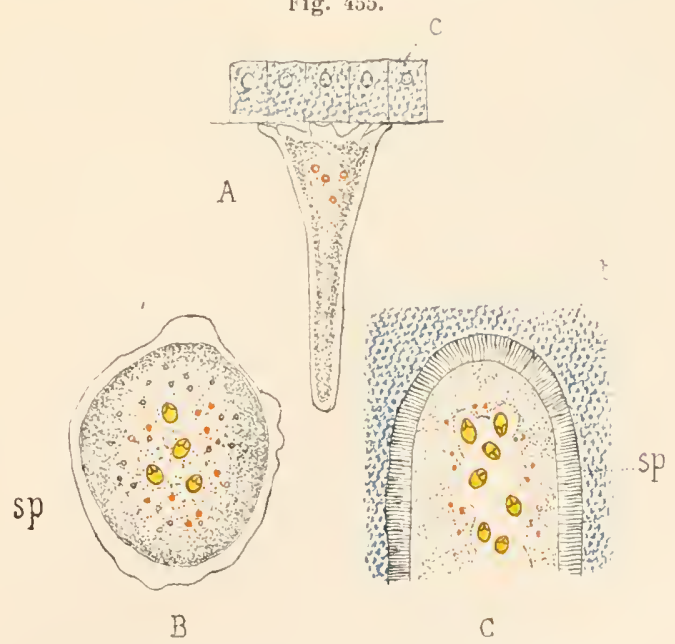

MYXOSPORIDE (Type morphologique) (Sch.).

$A$, l'amibe attachée à un épithélium de l'hòte $(c) ; B$, commen. cement de la sporulation; $C$, sporulation à un état plus avancé. t., tissus de l'hòte.

cule pulsatile. Son corps, tantôt en masse de forme irrégulière, tantùt 
plus ou moins découpé en lobes arrondis, se compose du cytoplasme et des noyaux.

Le cytoplasme laisse distinguer une hordure périphérique, l'ectoplasme, dont la surface est différenciée en une mince membrane protoplasmique un peu plus dense que les parties sous-jacentes. L'ecloplasme lui-même est, comme chez les vrais Amibes, formé de protoplasma hyalin où ne pénètrent pas les nombreuses granulations qui donnent à l'endoplasme un aspect beaucoup plus trouble et parfois une couleur caractéristique. Ces granulations endoplasmiques sont des dérivés divers des substances nutritives : on y distingue, entre autres, toujours des gouttelettes graisseuses el souvent des cristaux d'hémaloïdine dérivant du sang de l'hôte.

Les noyaux sont très nombreux, contenus exclusivement dans l'endoplasme el n'ont rien de particulier dans leur structure. Ces nombreux noyaux semblent rompre par leur multiplicilé l'uniformité que présentaient sous ce rapport les types des ordres précédents, loujours uninucléés. Mais ce n'est là qu'une apparence. A l’état jeune, le Myxosporide est binucléé, et si on lui trouve à l’àge adulte plusieurs noyaux, cela lient seulement à ce que la multiplicalion nucléaire qui est le premier phénomène de la sporulation, au lieu de s'accomplir, comme d'ordinaire, tardivement, rapidement, juste au moment de la formation des spores, se fait ici lentement, successivement et commence de bonne heure, pendant la phase d'accroissement du parasite. Cette mulliplication se fait par mitose.

\section{Physiologie.}

Habitat. - C'est chez les Poissons, les Crustacés ou les larves de certains Lépidoptères, qu'on a le plus de chances de rencontrer ce parasite. Il se trouve soit sous l'épiderme ou sous l'épithélium des branchies du Poisson, soit dans sa vessie urinaire ou sa vésicule hiliaire, libre à la surface muqueuse, soit dans les canalicules de son rein, soit enfin en plein tissu, au milieu des muscles ou du tissu conjonctif des organes les plus divers.

Nutrition. - Le parasite se nourrit par osmose, ef trouve ici toul autour de lui, dans les tissus de son hôle, les matériaux de son alimentalion. Il excrète sans vésicule pulsatile, aussi par simple osmose.

Mouvements. - Comme les Amibes, il esl doué de mouvements, émet de gros lohes pseudopodiques mousses, parfois mème de courts prolongements plus effilés el, par ce moyen, se déplace à la surface

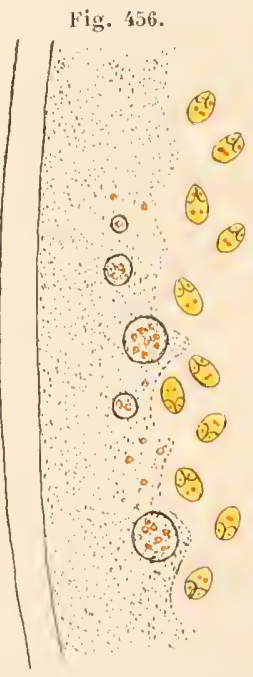

MYXOSPORID.E.

('Type morphologique). (Sch.).

Formation des sporoblastes et des spores. de la muqueuse ou dans l'épaisseur du lissu où il vit. Mais ces dépla- 
cements sont surtout actifs quand il est jeune. A mesure qu'il grandit, il devient plus inerte.

Sporulation. - Un caractirn essentiel (fig. 4.30 , $B$ et $C$ ), c'est que « la sporulation ne représente pas une phase particulière de l'existence, marquant la fin du cycle évolutif, comme cela s'observe chez d'autres Prolozoaires. On voit au contraire les corps reproducteurs se former de très bonne heure au sein te l'organisme qui n'en continue pras moins à se mouroir et à s'accroître». (Thélohan.)

Un noyau de l'endoplasme s'isole, s'entoure d'une petite quantité de protoplasma (fig. 4ว̈6). C'est le début d'un sporoblaste. Parfois le protoplasma se craquèle et forme des sporoblastes arrondis qui s'entourent d'une mince membrane; mais, autour de l'ensemble, il ne se forme pas de kyste (fig. $437, A)$. Les noyaux se multiplient dans chayue sporoblaste $(B$ et $C$ ) et donnent une dizaine de noyaux; le sporoblaste primitif se divise en deux parties (sporoblastes vrais) qui possèdent chacune trois noyaux $(D)$. Les noyaux restant constituent un reliquat.

Chaque sporoblaste forme alors une spore. Celle-ci se divise en trois parties : deux capsules polaires et une masse plasmique $(E)$, dont le noyau se divise de bonne heure $(F)$ pour donner deux noyaux ordinaires. Une membrane bivalve se forme autour de la spore. La masse plasmique binucléée est la partie essentielle qui, seule, servira à former l'amibe, stade initial du cycle évolutif. Les deux autres novaux s'entourent aussi chacun d'une petite masse de cytoplasme et constituent deux cellules nues qui vont se placer côte à côte an petit bout de lovoïde, et là vont se transformer chacune en un petit appareil très curieux qui servira à la dissémination du parasite et que l'on appelle capsule polaire $(G$ et $I I)$.

Les phénomènes de la formation de ces capsules élant les mèmes pour chacune d'elles, Fig. 457 .
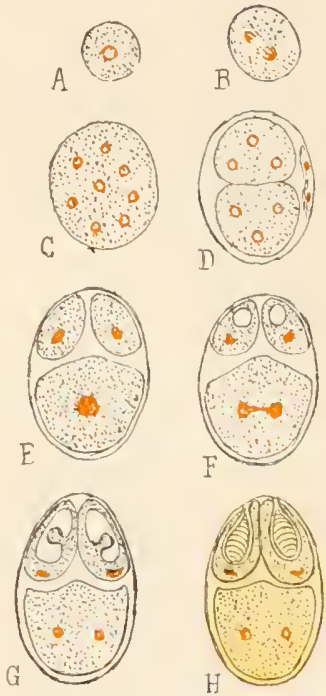

MYOSPORID.E.

(Type morphologique) (Sch.). Détail de la formation des spores. nous les décrirons pour une seule. Dans le cytoplasme de la cellule, se creuse une vacuole $(F)$ qui grandit rapidement. Bientôt une petite saillie protoplasmique se forme dans cette vacuole et grandit à son intérieur $(G)$; elle s'énuclée de plus en plus et finit par devenir libre dans la vacuole sous l'aspect d'une petite masse piriforme à queue allongée. La couche de cytoplasme qui limite la vacuole se condense et se transforme en une petite coque ovoïde qui contient la masse piriforme; celle-ci s'allonge beaucoup en se contournant et finit par se transformer $(I)$ en un long filament spiral ramassé sur lui-mème en 
tire-bouchon, tandis que, en dehors de la coque, le noyau et le reste du cytoplasma forment un petit amas résiluel destiné à disparaître. Les deux capsules polaires ressemblent singulièrement à des nématocystes de Cœlentérés et la ressemblance devient bien plus frappante si l'on songe que leur mode de formation et leur fonctionnement sont très semblables à ceux de ces organes.

La spore mûre mesur environ $10 \mu$ de longueur; elle se compose donc, en somme, des parties suivantes : $1^{\circ}$ une coque bivalve; $2^{\circ}$ une masse protoplasmique contenant une grosse vacuole (non pulsatile) centrale et deux petits noyaux situés à droite et à gauche de celle-ci; $3^{\circ}$ deux capsules polaires constituées comme des nématocystes et situées còte à côte au petit bout de l'ovoïde. Ces spores sont réunies par petits groupes arrondis en sporoblastes munis d'une mince membrane et ces sporoblastes groupés côte à côte occupent, dans les tissus ou à la surface de la muqueuse, la place de la grosse amibe nucléée qui a servi à les former.

Évolution des spores. - Dans les spores mûres et sous. l'influence d'excitations spéciales, chimiques ou mécaniques, les capsules polaires décochent leur filament qui sort par un orifice (Balbiani), s'étend sur une longueur dépassant plusieurs fois le diamètre de la spore et, terminé en pointe au bout, reste attaché par la base au sommet de la spore. C'est là évidemment un agent de dissémination. Les spores mises en liberté par la dissociation et l'ouverture des sporoblastes, soit après la mort de la victime quand elles sont en plein tissu, soit avant quand elles sont à la surface des muqueuses ou même peut-ètre sous-épithéliales, sont portées, sans doute par le hasard, au contact d'un nouvel hôte et s'accrochent à lui par leurs filaments dévaginés. Là, leurs valves s'entr'ouvrent, la masse protoplasmique intéricure sort et, grâce à ses mouvements amœboïdes, va prendre place au lieu qui lui convient, où elle n'a plus qu'à grandir aux dépens du tissu de l'hôte et à multiplier lentement ses noyaux pour arriver au stade où nous l'avons trouvée en commençant son histoire (").

La comparaison deviendrait aisée entre cette spore et celle des autres Sporozoaires, et les différences ne porteraient plus que sur des points secondaires si l'on admettait, ce qui semble assez légitime, que les capsules polaires sont les équivalents morphologiques de la masse plasmique de la spore. Masse plasmique et corpuscules polaires seraient les équivalents de trois sporozoïles, dont deux se seraient transformés en organes spéciaux chargés de favoriser la dissémination du troisième et son arrivée jusqu'au point où il pourra se développer. Ce troisième sporozoïte offre encore cette particularité, c'est qu'il a une forme arrondie et se déplace à la manière d'un Amibe, mais

(1) Cette dernière partie du cycle évolutif n'a guère étẻ observée d'une manière un peu complète mais, d'après ce qu'on en a vu, il n'y a guère place pour une autre évolution. 
c'est là un point secondaire. Il en est de mème du fait que les sporoblastes, an lieu de se transformer en une seule spore, en forment plusieurs à leur intérieur. Envisagé sous ce jour, le cycle évolutif des llyxosporides garde un faciès particulier, mais se laisse ramener cependant au schéma général commun à tous les Sporozoaires (").

Les IIyxosporides constituent un groupe sensiblement moins uniforme que les précédents. Bien des genres diffèrent sensillement de notre type morphologique. Il n'y a qu'un caractère essentiel : la constitution de la spore avec une ou plusieurs capsules polaires, munies d'un filament dévaginable. Les autres, habitat, forme de l'adulte, nombre des spores du sporoblaste, nombre et forme des capsules polaires, forme des enveloppes de la spore, donnent lieu à des variations étendues que nous allons maintenant examiner en étudiant les genres.

\section{GENRES}

Myxidium (Bütschli) (fig. 458) réalise, à très peu de chose près, notre type morphologique. Notons seulement que le sporoblaste forme ordinairement deux spores. Ces spores sont régulièrement fusiformes, avec deux capsules polaires. Il n'y a pas de vacuole dans la masse plasmique de la spore (Spore 10 à 12 .. Dans divers organes de Syngnatlus, Scorpiena, Esox, etc.) $\left({ }^{2}\right)$.

Fig. 458 .

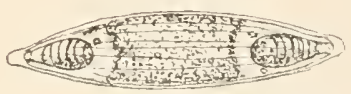

My.ridium (d'ap. Thélohan).

(1) G'est l'opinion de Mrngazzixi, mais non celle de Thélohax.

(2) My:xidium Lieberkülıni (Bütschli) vit à la surface de la muqueuse où il forme des taches jaunes. La couleur jaune de l'endoplasme est due a la présence de globules colorés; il y a de nombreux cristaux d'hématö̈dine (Vessie urinaire du Brochet $[$ Esox $]$; ;

Sphærospora (Théloban), a des spores sphériques (Rein et ovaire de Ginsterosteus);

Sphæromyxa (Thélohan), a des spores allongées, fusiformes, avec une capsule à chaque extrémité; la masse plasmique est en forme de disque ou de lentille biconvexe (Vésicule biliaire d'un Bufo brésilien);

Myxosoma (Thélohan) a des spores en fornte d'ovoïde aplati assez allongé (Spore 12 à $20 \mu$. Branchies dé Leuciscus; vessie urinaire de Lophius).

Ceratomyxa (Thélohan) (fig. 459), a des spores dont la forme est celle de deux cònes creux un peu recourbés, soudés par leurs bases; chaque valve est terminée par un prolongement aigu. La masse plasmique se trouve dans un de ces prolongements Vésicule biliaire de Motella, Crenilabrus, Alosa sardina, Scyllium, Loplius, Merlangus).

Leptotheca (Thélolıan', est caractérisé par des spores globuleuses ayant leur grand axe perpendiculaire au plan de suture (Rein de Scomber et de Rana).

Fig. 459.

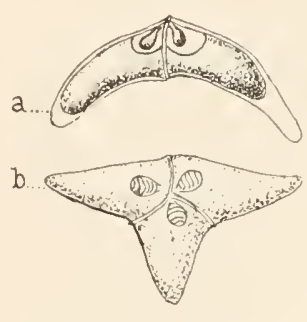

Ceratomy.xa

(d'ap. Thélohan).

a, spore normale; $\mathbf{b}$, spore anormale.

Tous ces genres constituent la famille rles $M_{Y \text { XIDINE }}$ [Myxididées (Thélohan)] ayant pour caractère des spores bi-capsulées i masse plasmique dépourvue de vacuole. 
Chloromyxum (Mingazzini) (fig. 460) se distingue par la présence de quatre capsules polaires lans la spore (Spore 6 it 8 p. Vésicule biliaire des Plagiostomes, rein de Syngnatus) (').

Henneguya (Thélohan) (fig. 46I), qui comprend les anciennes Psorospermies de J. Müller, possède une spore dont les valves allongées se prolongent en arrière en une sorte de queue, ce qui lui donne une vague ressemblance avec un spermatozoïde (Spore 10 a 40 i.. Branchies d'Esox et de Perca, rein et ovaire de Gasterosteus) ( $\left.{ }^{2}\right)$.

Myxobolus(Biitschli) possède une spore sans prolongementavec une ou deux capsules polaires (Spore 10 à 18 p... Divers organes des Cyprinoïdes) $\left({ }^{\mathbf{3}}\right)$.

Ces formes ne diffèrent pas essentielleFig. 460 .

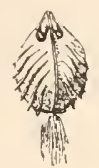

Chloromyxum. Spore
Fig. 461

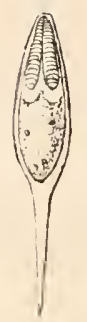

IIenneguya. Spore

(im. Thélohan). (im. Thélohan).

ment du type morphologique. Les suivantes s'en distinguent par des spores de très petite taille, arec une seule capsule à l'extrémité.

Thelohania (llenneguy) (fig. 462) forme, dans les faisceaux primitifs des muscles de son hôte, des îlots elliptiques ou très allongés qui écartent les fibres saines. Thélohan et HenneFig. 462. guy, qui les ont découverts, n’ont pas trouvé de phase amibö̈de. Le parasite se montre seulement à l'état de sporoblastes. Les plus jeunes de ces sporoblastes ont chacun un noyau. Ce novau se multiplie par mitose et dome naissance à huit noyaux qui sont ceux d'autant de spores très petites. Chacune a une capsule polaire avec filament dévaginable. C'est, en somme, un Myxosporide octosporé, unicapsulé, à phase amibe inconnue (Spore 2 à 6 p.. Crustacés divers: Palixmon, Crangon, Astacus) (').

Pleistophora(Gurley) ne liffère de Thelohania que par son caractère de Polys sporé (Spore 5.... Muscles de Cottus, Blennius, etc.).

(1) Le nom de ce genre est dì à ce que chez C. Leydigi (Mingazzini) des Plagiostomes, l'endoplasme présente une couleur jaune d'or ou jaune brun, due à la présence de globules colorés.

Ce genre forme à lui seul la famille des CHLOnomxtre [Chloro- (d'ap. Thélohan). myidées (Thélohan)].

(2) Le Myxosporide trouvé par Ryder dans Aphrododerns n'est sans doute qu'une espèce du précédent.

(3) M. Pfeifferia, une des nombreuses espèces du genre, produit, depuis quelques années, une épidémie très meurtrière sur les Barbus fluriatilis de la Moselle et de quelques rivières d'Allemagne (Pleiffer, Raillet). Chez Thymallus s'ulgaris (Omble Chevalier' ce parasite envahit mème le tissu nerveux (nerfs et cerveau) (L. Pfeiffer).

Ces deux genres forment la famille des MrxoboLINa [Myxobolidées (Thélohan)].

(4) Il se peut, comme le pense Thélohan, yue la phase amibe libre n'existe pas et que l'amibe issue de la spore se transforme immédiatement en sporoblaste sans phase d'accroissement libre. Les Crustacés atteints sont à demi paralysés; on les reconnaît a leur teinte laiteuse. Dans certaines localités, cette maladie a été très meurtrière pour les Ecrevisses. 
Gluǵea (Thélohan) (fig. 463) est aussi très semblable, mais il peut avoir une phase plasmique; il vit libre ou forme des tumeurs (Spore 4 a 5 . Mnscles, tissu conjonctif, foie de Gasterosteus, Barbus, Motella; cœur d'Alosa sardina; spermatoblastes d'dlcyonella.

Nosema (Niigeli). On faisait, tout récemment encore, du parasite qui produit la pébrine les Vers à soie, le tỵpe d'un ordre spécial de sporozoaires qu'on désignait sous le nom de Microsponmies (Balbiani). Mais, les spores de ces Microsporidies ayant absolument la mème constitution que celle de Ghigea, on est ol,ligé d'en faire aujourd'hui un simple genre du sous-ordre des Myxosporides, très voisin même de Glugea. Cela se justifie encore par la considération du cycle évolutif qui se réduit à ceci. Ces spores s'ouvrent dans le tube digestif de la Chenille, émeltent leur amilie; ces amibes traversent lentement la muqueuse digestive et se répandent dans tout l'organisme, se logeant principalement dans le tissu conjonclif interstitiel des organes. Là elles s'arrêtent, grandissent et finalement se mettent à sporuler. Leur noyau se multiplie et donne des sporoblastes dans chacun desquels se forment des spores en nombre indéfini, très pelites, unicorpusculées (').

(1) Les parasites qui engendrent la maladie les Vers ì soie ayant fait perdre à la France plus d'un milliard pendant les quelque vingt à vingt-cinq ans, où elle a le plus exercè ses ravages (surtout vers 1860 ), il n'est peut-ètre pas inutile d'ajouter quelques mots ilhistorique ì la description zoologique „jui prícède. La chenille, bien qu'affaiblie par la maladie, se transforme néanmoins en papillon, mais ces papillons restent rabougris, d'où le nom d'étisie, de maladie des petits qui fut d'abord donné i ce mal inconnu. Malheureusement, quoique malarle, le papillon peut pondre et ses oufs infectès, vendus sous le nom de grame, ont servi ì propager la maladie. Aussi PAstrun a-t-il indiqué le vrai remède pratique en conseillant de trier la graine et de détruire toute celle qui est alteinte. QuatheFages ayant remarqué sur des chenilles malades des taches semblables à des grains de poivre crut a un rapport entre ces laches et la maladie, et donna à celle-ci le nom de pébrine qui lui est resté. Mais il n’y a rien de commun entre ces taches inoffensives et, le parasite. Les spores, vraie cause du mal, furent découvertes par les savants italiens Conxagla, Philippi, mais ceux-ei les prirent pour des granulations pathologiques engendrées par la maladie à laquelle ils donnèrent le nom de gattina. C'est BALBLsi, qui reconnut la vraie nature de ces corpuscules de la pébrine et de la maladie corpusculeuse, démontra '] u'ils n'étaient 'que des spores parasites analogues a celles des Sporozoaires et les nomma Psorospermies des Articulés, et plus tard Microsporidies, dont il a fait un ordre spécial des Sporozoaires qui a persisté jusqu’aujourd'hui oì Thétohan, qui s'était déjà distingué par de remarquables travaux sur rette classe d'animaux, vient de le ramener à un simple genre des Myxosporidies. Quant au nom de Vosema il faut bien le conserver par respect pour la règle de priorité, bien qu'il consacre une erreur de Nägeli qui en faisait un genre de Schizomycetes. Les Nosema de diverses espèces sont très répandus chez les Insectes. On a trouvé aussi chez divers Crustacés et même chez des Reptiles et Batraciens des formes semblables, mais leur identité avec les précédents et mème leur nature microsporidienne n'est pas tout à fait démontrée. 
Distribution des Myxosporides. - On trouve surtout les Myxosporides chez les Poissons (sauf Amphioxus, les Ganoïdes, les Cyclostomes, et parmi les Téléostéens, les Pleuronectides et les Cycloptérides). Plusieurs espèces différentes peuvent du reste lıabiter le même organe du mème Poisson. Chez les Batraciens, on les a trouvés chez plusieurs Anoures et chez les Tritons (rein et vésicule biliaire). Parmi les Invertébrés, elles se rencontrent chez les Crustacés ( $P a$ lximon, Crangon, Astacus, Carcinus), chez les Vers (Naïs), chez les Bryozoaires (Alcyonella). Enfin, les Nicrosporidies, que nous avons vu n’être qu'une partie des Myxosporides, se trouvent chez tous les Articulés et aussi dans les muscles des Grenouilles, des Tortues et des Lézards (Danilersky el Pfeiffer).

\section{APPENDICE}

En appendice aux Sporozoaires, nous devons passer en revue un certain nombre de petits groupes à affinités indécises ou insuffisamment connus.

Tubes parasites des Articulés. - Il n’y a dans ce groupe qu'un seul genre : Amœbidium (Cienkovsky) (fig. 464), que nous devons décrire en lui-même puisqu il constitue l'unique genre du groupe. Il ne contient mème qu'une espèce ( $A$. parasiticum).

C'est au début un simple petit bâtonnet uninucléé (a), muni d'une paroi et fixé par une de ses extrémités sur les membres ou les branchies de quelque Entomostracé d'eau douce ou d'une larve aquatique d'Insecte, voire même sur le pédoncule d'une Vorticelle. Ce bâtonnet grandit, s'allonge en un tube, sa paroi devient plus forte et assez semblable à celle d'une cellule végétale, bien qu'elle n'ait pas les réactions de la cellulose et son noyau se multiplie et donne de nombreux noyaux filles élagés dans le tube en une file unique $(b, c, d)$. En cet état le tube peut atteindre jusqu'à un demi-millimètre de long. Bientòt son protoplasma se divise en autant de portions qu'il $y$ a de noyaux et forme ainsi autant de sporoblastes fusiformes disposés en hélice très allongée $(d)$. Dans chacun de ces corps fusiformes (e'est le nom sous Fig. 'tit.

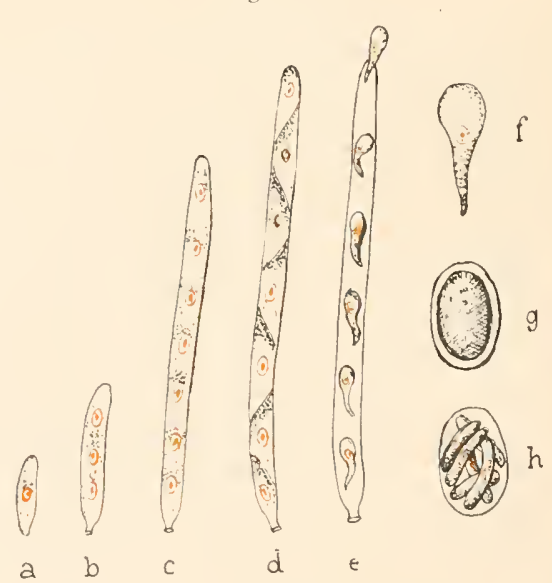

Amabidium (Sch.). Son cycle évolutif.

lequel on les désigne d'ordinaire) se forment, par division du noyau et du cytoplasme, un petit nombre d'amiles nucléées, mais nues et sans vésicule pulsatile, qui sortent par un trou qu'elles percent dans la paroi du tube et se disséminent par les mouvements de leurs pseudopodes $(e)$. Mais au bout de quelques heures, ces amibes, sans s'ètre nourries ni accrues, s'arrêtent (f), s'arrondissent, s'enkystent $(g)$ et sporulent. Tantòt la membrane est mince et l'enkystement est de courte durée, tantôt elle est épaisse et l'enkystement est long; mais cela ne change rien à la suite de 
l'évolution. Dans chacune se forment, par division du noyau et du cytoplasme, cinq à six sporozoïtes $(h)$ en forme de bâtonnets arqués, nus et munis d'un noyau. Ces sporozoites se fixent non loin du tube mère ou parfois sur lui et se développent en nouveaux tubes par formation d'une membrane, accroissement et multiplication de leurs noyaux.

N'ètait la phase amibe et si les kystes se formaient directement dans les sporoblastes fusiformes, il serait aisé de ranener cette évolution à celle d'un Sporozoaire, car les liystes seraient alors de simples spores formant des sporozoïtes à leur intérieur. On peut admettre cette assimilation et considèrer l'amibe comme un état spécial de la spore devenue mobile à un moment donné par adaptation, pour les besoins de la dissémination de l'espèce.

Cette nécessilé est rendue évidente par la considération de ce qui se passe en hiver où le cycle évolutif est abrégé par la suppression de l'amibe et du liyste. Les corps fusiformes sortent alors directement du tube et se développent directement en nouveaux tubes, représentant ainsi le sporoblaste, la spore et le sporozoïte condensés en un seul et même objet. Hais alors, faute d'être assez mobiles, ils se fixent toujours sur le tube maternel ou tout près de lui. Néanmoins, ces assimilations sont toujours un peu théoriques et on ne peut rien objecter de bien positif à Cienkovsky et à quelques autres qui placent Amobidium parmi les Algues ou les Champignons inférieurs. Le développement de ces singuliers parasiles comporte bien d'autres variations. Parfois, dans le cycle d'été, cliaque corps fusiforme se transforme directement en une seule amibe ou même les amibes se forment directement dans le tube sans passer par l'ètat de corps fusiformes. Il arrive aussi que les corps fusiformes sortent du tube avant de former les amibes à leur intérieur. Les causes de ces variations nous sont complétement inconnues.

Moniez fait d'Amobidium, une Algue, une Palmallacée répondant à Raphidium. polymorphum (Fresenius). Cette opinion est assez vraisemblable. Il ne paraît donc pas indiqué de créer pour lui un groupe entier de Sporozoaires comme l'ont fait quelques auteurs (Exosporides, Perrier).

Amœbosporidies (Aimé Schneider). - Ce groupe n’a qu'un genre :

Ophryocystis (Aimé Sclıneider) (fig. 46̈̈), sorte d'amibe polynucléée singulière rattachẻe avec doute par $\Lambda$. Schneider, qui l'a découverl et décrit, aux Myxosporides. Mais il n’a pas leur spore caractéristique. Il n’a aucun caractère des Infusoires, mais les phénomènes nucléaires de la conjugaison présentent un étonnant parallẻlisme avec ceux de la conjugaison de ces animaux.

Cet Ophryocystis comprend deux espèces qui vivent en parasite, l'une dans les tubes de Malpighi de Blaps, l'autre chez Akis. C'est une amibe mesurant 10 i $1:$ ph, sans vésicule pulsatile, mais polynucléée, le nombre de ses, noyaux pouvant atteindre une dizaine. Elle a des psendopodes irrẻgulier's, longs, déchiquetés, mais que l'on ne voit jamais reınuer, peut-être par suite de l'action du liquide artificiel où l'on est obligé d'examiner l'animal. Cette amibe polynucléée se divise-t-elle en autant

Fig. 465 ,

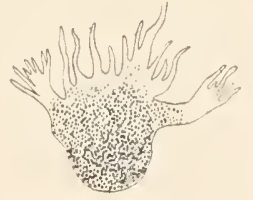

Ophryocystis

(d'ap. A. Schmeider). d'amibes qu'elle a de noyaux? On n'a pu l'observer, mais toujours est-il que ce sont exclusivement des individus plus petits et uninucléés qui servent de point de départ aux stades ultérieurs de l'évolution. Ces amibes à noyau unique s'associent et s'enkystent par deux, sous denombreuses enveloppes communes superposées. Le kyste présente une ligne - de déhiscence équatoriale. Leurs noyaux. en se multipliant, par division en donnent chacun trois. De ces trois noyaux, deux sont repoussés et seront éliminés avec une forte quantité de plasma résiduel. Les deux restants, appartenant chacın à l’un des conjoints, se fusionnent (accomplissant ainsi le phénomène essentiel d'une conjugaison nucléaire qui devient totale par le fait que les deux cytoplasmes se fusionnent aussi) en une spore unique flanquée de deux amas résiduels binucléés destinés à disparaître. Le noyau conjugué se divise alors en deux, puis quatre, puis sans doute huit qui devien- 
nent les centres de formation d'autant de sporozoïtes. Le reste de l'évolution n'est pas connu, mais se laisse aisément deviner si du moins il ne présente pas d'imprévu. Sans doute chaque sporozoile mis en liberté se transforme en une amibe.

Serumsporidies (Pfeiffer). - Ce petit groupe comprend le seul genre :

Serumsporidium (Pfeiffer) dans lequel Pfeiffer a réuni de petits parasites, connus depuis Leydig, du sang sans globules de divers Invertébrés inférieurs, principalement des Entomostracés et peut-être quelques Radiolaires, Dinoflagellés et Infusoires. Ce sont des amibes uninucléées dont la taille varie de 4 ou bै à 40 ou $50 \mu$, qui circulent dans le sang ou flottent dans le liquide cavilaire immobile. Arrivées à maturité, elles s'enkystent séparément et divisent leur contenu en petites masses nues uninucléées qui sont mises en liberté et se transforment chacune en une petite amibe.

Ces petits ètres ont des affinités évidentes avec les Gymnosporidies de Labbé, mais aussi avec les Chytridinées qui sont des Champignons.

Amœbiens de Sagitta. - Il semble bien qu'il faille rapporter aux Sporozoaires et non aux Rhizopodes deux Amœbiens que Grassi a decrits sous les noms de $\left.A m x^{2}\right) \boldsymbol{a}(\boldsymbol{A}$. Chrotognathi et 1 . pigmentifera). Ce sont de petits Amibes nucléés, à cytoplasma très chargé de granulations. Ils vivent dans le liquide cavitaire de leur hòte. Parfois ils s'unissent comme pour une conjugaison. En tout cas ils s'enkystent et sous ce kyste se divisent en nombreuses spores que la destruction du kyste met en liberté.

Parasites de la Vaccine, de la Variole, de la Varicelle, de l'Herpès zoster. Pfeiffer a trouvé dans le sang des malades atteints de ces maladies infectieuses des amibes qu'il croit tetre la cause de la maladie et qu'il faudrait sans doute rapporter aux Sporozoaires, bien qu'on ne sache rien de leur développement. Cependant certaines de ces amiles se montrent avec flagellum.

Parasites de la fièvre du Texas. - Suıth a trouvé dans les hématies des Chevaux atteints de ce mal une amibe voisine de celle de la malaria, dont il a fait le genre

Babesia (Smith). On ne connaît pas la sporulation. La maladie serait transmise d'un animal à l'autre par les Ixodes.

Parasites de l'hémoglobinurie des bestiaux. - Cette maladie des bestiaux de Roumanie serait produite d’après BaBEs par un Diplocoque, appelé

Pirosoma (Smith)(P. ligeminum), logé dans les hématies. On ne connait pas la sporulation.

Parasites du molluscum contagiosum (fig. 466). - On observe chez les Oiseaux, les Pigeons surtout, une infection speciale de l'éphithélium consistant dans une hypertrophie de la couche de Malpighi de l'épiderme. Chaque cellule de cette couche contient un corps qui, d'après les recherches de Pffiffer, semble bien être un parasite sporozoaire. Miviazzini a même trouvé de ces stades moruliformes de sporulation qui sont si caractéristiques chez ces êtres. Nersser a étendu ces résultats au molluscum de l'Homme. C'est surtout des Gymnosporidies qu'il faudrait rapprocher ces parasiles.

Parasites de la psorospermose folliculaire végétante ou maladie de Darier. - Dans les deux ou trois cas ou celte affection a été observée loujours chez l'Homme', les cellules de la couclie de Malpighi des follicules malades présentaient des formations élrangères très analogues à celles du molluscum.

Parasites de la maladie de Paget. - lest une affection du mamelon que Wickian et divers auteurs anglais rapportent à une Coccidie.

Fig. 466 .

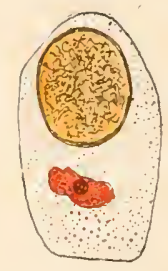

Parasite du Molluscum contagiosum (d'ap. Mingazzini).

Parasites trouvés dans certaines thoracentèses. - l)ans le produit d'une ponction de la cavité pleurale Küxstuen et Pitres ont trouvé de nombreuses spores contenant dix à vingt corpuscules falciformes accompagnés d’un reliquat. C'étaient là certainement des Sporozoaires, mais il est impossible de se prononcer sur leurs affinités spéciales, le reste du développement n'ayant pu ètre observé.

Parasites de certaines cirrhoses. - Poprissozkr a décrit sous le nom de

Karyophagus ('odwissozky) ( $\boldsymbol{K}$. hominis) un prétendu parasite nucléaire des cellules hépatiques qui semble bien n'être qu'une racuolarisation pathologique de leur noyau. 
Parasites des mélanosarcomes et des cirrhoses biliaires. - Stelshaus a décrit dans ces maladies des productions nucléaires qu'il scmble rapporter à des Coceidies karyophages et sur lesquclles il est actucllement impossible de se prononcer.

Parasites du Cancer. - Beaucoup plus importants au point de vue de l'homme, mais non moins obscurc, est la question des parasites du cancer. Dans divers carcinomes, en particulicr les épithéliomas, les cellules cancéreuses contiennent, outre leur noyau plus ou moins altéré, des productions d'aspect très variable. Tantôt, c'est une simple petite masse de forme assez régulière que l'on pourrait prendre pour une amibe contractéc. Elle n'a pas un noyau bien évident. Mais cependant les colorants de la chromatine colorent plus énergiquement certaines varties de son contenu. Ailleurs, on trouve une sphérule de forme analogue, mais présentant indubitablement des figures astéroïdes comme dans lesmitoses. D'autres fois enfin, et cela constitue une indication beaucoup plus précise, on observe, à l'intéricur de la sphérule parasite, une masse moruliforme semblable à celle qui caractérisc la plupart des Sporozoaires en train de sporulcr.

D’après nombre d'histologistes (Corxil, FABRE-Donergue, etc., ete.,) il n'y aurait là que des productions pathologiques non parasitaires, des bourgeonnements nucléaires. des mitoses anormales, des migrations de leucocrtes, des dégénírescences, des histotyses vitreuses ou colloüdes, ete.

l)'après d'autres auteurs (Madassez, Metchinikof, SAvtchenok, Nilssjöbrixir, SoldakEvitcl, etc., ce scrait là un parasite voisin des Coccidies. Korotref l'a nommé Rhopalocephalus (Korotnef) (R. curcinomatosus) (fig. 467) el lui a assignė un développement compliqué dans lequel il semble bien qu'il a dù comprendre des leucocytes migrateurs qui n'ont rien de commun avee la maladie. Le parasite formerait un kyste $(A)$, d'où pourraient sortir deux sortes de larves, les unes zooïdes ( $b$ ), se transformant en sortes de Grégarines monocystidées (C), les autres, sporozoïdes (D) se transformant en amibes $(E)$ qui développeraicnt en elles $(F)$ de nouveaux zooïdes et sporozoïdes.

Il semble bien d'après tout cela que : $1^{\circ}$ on a decrit

Fig. 16 i.
A
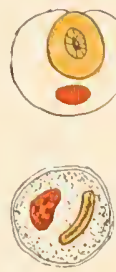

D
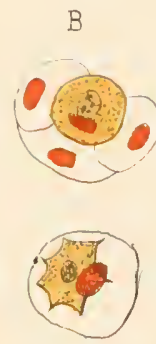

E
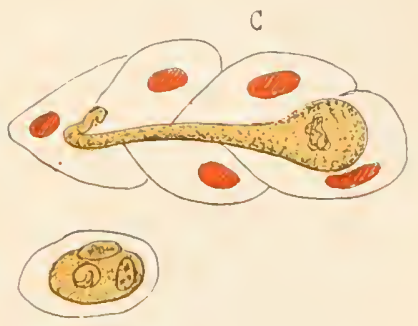

F

Rhopalocephalus (im. Korotnef).

comme parasite du cancer des formations pathologiques de la cellule altérée, mais qui n'ont rien de parasitaire; 20 il cxiste, dans les cellules cancéreuses, de vrais Sporozoaires à cycle évolutif encore indéterminé, mais on n'cst pas en état d'affirmer qu'elles y exislent constamment; $3^{\circ}$ la preuve reste a faire, que ces parasites soient la causc réelle de la maladie.

Le cancer est généralcment inoculable d'un point à un autrc de l'individu qui en est porteur. Il y a des cas incontestables d'inoculations d'un individu à un autre, même d'une cspèce à une autre, mais le résultat est alors très alèatoire. Enfin ces inoculations prouvent tout au plus la nature infecticuse de la maladie et nullement que le parasite sporozoaire soit l'agent de l'infection.

Cetle importante question attend de nouvelles études. 


\section{Sur le prétendu dimorphisme des Sporozoaires*}

« En 1891, le DrR. PfelfFkr, de Berlin, ayant trouvé dans l'intestin de jeunes Lapins une Coccidie à développement simple d'Eimeria, causant une infection grave de ces animaux, émit l'idée que cette Coccidie était une forme évolutive de Coccidium perforans, et qu'ainsi une même Coccidie pouvait avoir, suivant les circonstances, un double développement :

10 Un développement endogène simple (Eimeria) reproduisant l'infection chez le même individu par Schwärmersporencysten :

$2^{\circ}$ Un développement exogène à deux degrés (Coccidium) reproduisant l'infection chez d'autres individus par Dauersporencysten.

« Le Dr Ludwig Pfeiffer, de Weimar, étendit cette théorie à toutes les Coccidies d'abord, puis à tous les autres Sporozoaires, sauf aux Grégarines, bouleversant ainsi toutes les classifications. Chez la Salamandre, Karyophagus Salamandr $x$ de Steinhaus seraitle Schwärmerstadium de la Coccidie dont le Coccidium proprium de Schneider serait le Dauerstadium. Chez Lithobius, il y aurait une Eimeria, pour répondre à Adelea ovata qui serait le Danerstadium. La curieuse Coccidie trouvée par Podvissozky dans l'œuf de Poule serait le Schwärmerstadium de Coccidium tenellum. Chez les Sarcosporidies, la forme Dauercysten serait reprẻsentée par les tubes de Miescher.

"Plusieur's auteurs, Schuberg, Mixgazzisi, soutiennent cette theiorie.

«Dans une note précédemment publiée, nous avons ètabli plusieurs exemples d'infection double dans lesquels l'une des Coccidies était monogénique, l'autre étant digénique et à développement exogène.

"Sans entrer dans les détails des discussions, nous pouvons dire que partout l'évolution de ces Coccidies était diffẻrente de $\mathrm{A}$ jusqu'à Z; que le dèveloppement était indépendant chez toutes, qu'elles pouvaient être distinguées à tous les stades et qu'elles appartenaient certainement à des espèces différentes, sans qu'il fùt possible de dire qu'il y eut dimorphisme chez une seule espèce. De ce que deux parasites voisins se trouvent dans le même organe du même hòte, il n'en résulte pas nécessairement qu'ils dérivent l'un de l'autre; et, si leur structure et leur évolution diffèrent depuis les premier's stades jusqu'aux derniers, on ne peut admettre un dimorphisme et l'on doit conclure qu'ils appartiennent à des espèces différentes.

"Nous avons constaté qu'un tel dimorphisme n'existait pas davantage chez les Hémosporidies et les Gymnosporidies.

"Somme toute, nous ne savons pas s'il peut y avoir un dimorphisme évolutif chez les Sporozoaires, mais nous pouvons affirmer absolument que ce dimorphisme n'est pas prouvé dans les cas observés tant par nous que par divers auteurs, et que beaucoup de raisons s'opposent, au contraire, à ce qu'on l'admette. Provisoirement, nous pensons donc que les genres Pfeifferia, Eimeria, ete., sont parfaitement légitimes et ne représentent nullement des phases évolutives d'autres Coccidies ".

(*) Nous laissons la parole à MI. Labbé, sur cette importante question dont la solution intéresse la conception toute entiere de la classe des Sporozoaires. 


\section{3e CLASSE \\ FLAGELLÉS. - FLAGELLIA}

[Flageldates; - Fl.igellata (Ehrenberg); - Mastrgophora (Bülschli)]

\section{TYPE MORPHOLOGIQUE}

(FIG. 468 A 487 )

\section{Structure.}

Conformation générale. - Notre Flagellé est un petil être microscopique, unicellulaire. Son corps est ovoüde et formé d'une masse de cytoplasma contenant un noyau el une vésicule pulsatile. A une de ses extrémités, il est muni d'un prolongement filiforme, le flagellum. Ce flagellum est antérieur dans la progression et détermine par conséquent l'orientation longitudinale de l'animal. Nous appellerons donc supérieure l'extrémité flagellifère. Au-dessous du flagellum est une petite dépression infundibuliforme qui est le pharynx et dont l'orifice d'entrée est la bouche. La bouche détermine la face ventrale et compliele l'orientation morphologique. C'est à cela que se réduit l'énumération des organes de l'animal. Celui-ci est donc très simple.

Reprenons maintenant ces diverses parties pour les examiner avec plus de détail.

Cytoplasma. - Le cytoplasme offre un aspect très homogène. En dehor's des microsomes et si l'on met de côté les inclusions qui sont des parties surajoulées, on ne lui reconnaîl aucune structure bien définie el c'est à peine si on retrouve en lui de vagues indices de ces structures réticulée ou alvéolaire qui, ailleurs, ont donné lieu à tant de discussions. On peut lui distinguer cependant deux parties, une centrale, l'endoplasme, presque fluide et une périphérique, l'ectoplasme formant avec la membrane un mince revètement tégumentaire. Mais l'ecto-

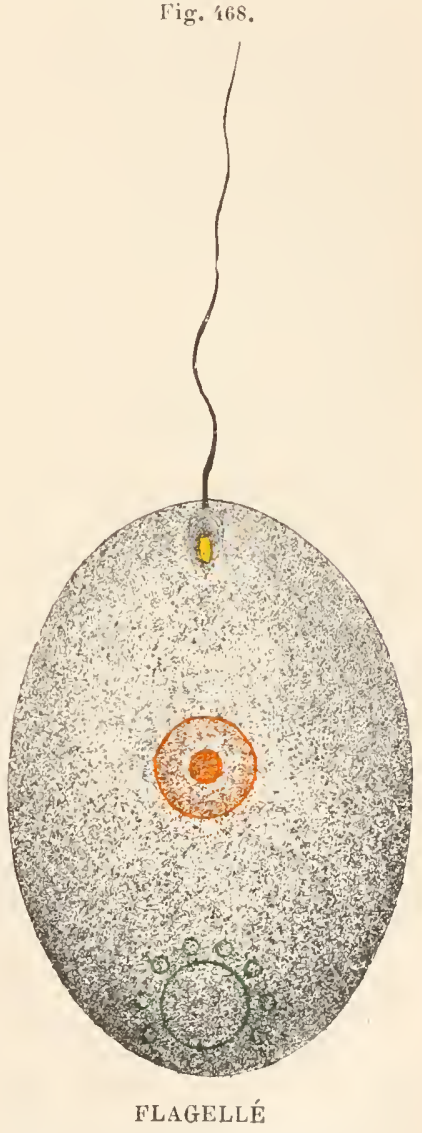

(Type morphologique) (Sch.). plasme passe en dedans à l'endoplasme par une transition graduelle et 
c'est un peu théoriquement que l'on désigne sous le nom de membrane sa couche périphérique plus dense et plus homogène. L'ectoplasme paraît n'être lui-même qu'un cytoplasma semblable à l'endoplasme, mais de structure plus ferme (').

Dans l'ectoplasme il n'y a pas de parties incluses ou différenciées. Dans l'endoplasme se montrent au contraire diverses inclusions, qui sont des produits d'assimilation ou de désassimilation plus ou moins avancés se présentant, les premicrs sous la forme de grains de paramylon $\left({ }^{2}\right)$, parfois de globules graisseux, les derniers sous relle de grains d'excrétion. On trouve, en outre, dans l'ectoplasme des substances alimentaires non encore digérées, contenues ou non dans des racuoles.

Pharynx. - L'infundibulum désigné sous le nom de pharynx n'est qu'un simple enfoncement très superficiel. La couche tégumentaire tout entière est déprimée à sois niveau mais, au fond, elle est interrompue et là se trouve une place où le cytoplasma est mou et facile à traverser pour les aliments. ll n'y a pas d'anus mais, sans doute, un point de moindre résistance à l'extrémité inférieure, car c'est en ce point que toujours les résidus alimentaires sont expulsés, sans laisser d'ailleurs après eux aucune trace de leur passage.

Flagellum. - Le flagellum est un appendice filiforme, plus long que le corps, en forme de cône extrèmement allongé. Il s'insère en ljas à la face dorsale de la dépression pharyngienne. Sa base non renflée ne mesure qu'une fraction de $\mu$ et, de là, il va en s'effilant progressivement jusqu'à la pointe $\left({ }^{3}\right)$.

Vésicule pulsatile. - La vésicule pulsatile est située tout contre l'ectoplasme; elle n’a pas de pore excréteur permanent, mais se met momentanément en rapport avec l'extérieur, à chaque systole, par une communication qui se referme aussitôt. Elle n’a pas de membrane propre; elle est entourée d'un cercle de petites vésicules formatrices dont nous verrons bientôt le fonctionnement.

Noyau. - Le noyau est bien rond, vésiculeux, pourvu d'une memlorane nette malgré sa minceur, et d'un nucléole. L'espace annulaire entre le nucléole et la membrane semble homogène comme s’il était formé

(1) L'ectoplasme et même la membrane sont des parties différenciées du cytoplasme. 11 en fautbien distinguer les enseloppes adlérentes au corps et les capsules situées à distance de lui el qui ne sont que des produits de sécrétion (V. p. 12 et suiv.). Ces productions sont en quelque sorte des parties surajoutées que nous n'arons pas cru devoir attribuer à notre type morphologique et qui seront décrites avec les genres.

(2) Isomère de l'amilon, mais ne bleuissant pas par l'iode et plus résistant aux divers réactifs, le paramylon se présente d'ordinaire sous la forme de bâtonnets courts, à structure stratifiée comme l'amidon. On ne le rencontre pas, tant s'en faut, chez toutes les espèces. Il en est de même des autres inclusions.

(3) Quelques auteur's assurent qu'il se termine par une extrémité tronquée et qu'il est de même largeur dans toute sa longueur. Cela est peut-être vrai chez certains genres, mais n'est certainement pas général. 
seulement de suc nucléaire, mais on arrive parfois à distinguer en lui un réseau délicat.

\section{Physiologie.}

Mouvements. - L'animal habite dans l'eau où il se meut avec activité et presque sans repos. Outre les mouvements actifs dus à l'action des parties contractiles, il existe ici, comme l'a montré Pferffer dans de remarquables expériences, des déplacements passifs dus à une attraction chimiotactique de ces ètres par les diverses substances. Cette attraction est élective, plus ou moins forte, positive ou négative selon les substances employées. Les mouvements actifs sont de deux sortes. Les uns sont dus à des contractions de son corps, les autres à l'action du flagellum.

Les contractions consistent en rétraction du corps qui, en mème temps, s'arrondit, se ramasse sur lui-même, ou en élongations avec amincissement corrélatif. Quand ces deux mouvements se succèdent régulièrement et que l'animal repose sur le sol, il en résulte une sorte de reptation analogue à celle du Ver de terre, mais cela est exceptionnel et ne dure jamais longtemps. D'autres fois, le mouvement consiste en inflexions latérales, plus ou moins brusques, plus ou moins accentuées dont l'animal use pour changer sa direction quand il rencontre un obstacle ou parfois sans motif apparent. Enfin, des contractions irrégulières peuvent produire des modifications temporaires très accusées de la forme génér'ale du corps. C'est ce qu'on appelle le mélabolisme ('). Le siège de ces contractions est évidemment le cytoplasme et très probablement l'ectoplasme, mais il n'y a point là de fibrilles musculö̈les différenciées, de myonèmes comparab̆les à ceux que nous rencontrerons chez les Infusoires $\left(^{2}\right)$.

Tous ces mouvements jouent un hien moins grand rôle dans la vie de l'animal que ceux de la seconde catégorie qui sont dus à l'activité du flagellum. Le flagellum, avons-nous vu, est situé en avant pendant la progression; il tire donc le corps à luí, à l'inverse de la queue du

(1) On sait que métabolisme signifie aussi mouvement nutritif d'assimilation et de désassimilation. Le mot a ici une acception tout autre.

(2) C'est sans doute un effet de la contractilité générale du protoplasme ou peutètre de quelque différenciation commençante moins avancée que celle qui engendre les myonẻmes et impossible à reconnaître avec nos moyens actuels d'investigation.

Kavkine [86] a cependant décrit chez les Euglènes des fibrilles longitudinales el d'autres circulaires limities à la partie supérieure du corps, et qui auraient pour siège l'ectoplasme; mais leur nature, sinon leur existence, reste sujelte à discussion, et en tout cas on n'a rien trouvé de semblable chez des formes plus contractiles encore que l'Euglène. Quant à certaines stries en relief qui ornent extérieurement la culicule de diverses espéces, elles n'ont certainement rien de commun avec la contractilité du corps. 
spermatozoïde ou de celle de l'Anguille (fig. 470) qui est en arrière, et pousse le corps devant lui. C'est là un fait général chez les Flagellés et nettement caractéristique de ces animaux (').

Il résulte de là que le mouvement du flagellum doit être autre que celui de la queue de l'Anguille, et il semble qu'au lieu de s'accomplir dans un plan il dessine une hélice, mais on ne sait rien de précis sur les particularités de ce mouvement. L'animal tourne sur un axe en même temps qu'il progresse en avant $\left(^{*}\right)$.

Bütschul croit avoir trouvé l'explication de ce mouvement et raisonne ainsi. Supposons que le flagellum prenne la forme d'une hélice allongée, comme l'indique la figure 469 , et que cette hélice se mette à tourner dans le sens des aiguilles d'une montre. En tout point tel que o la pression sur l'eau fics naitre une force ob normale au flagellum, et que l'on pourra décomposer en deux autres, l'une verticale oa déterminant la progression de l'animal en avant, l'autre horizontale $o c$ déterminant sa rotation autour de son axe. 11 est à remarquer que cette rotation est en sens inverse à celle du flagellum. Si l'animal faisait tourner son hélice en sens inverse, il reculerait. S'il la disposait en hélice sénestre, il y aurait également progression en avant pour un certain sens

Fig. 470 .

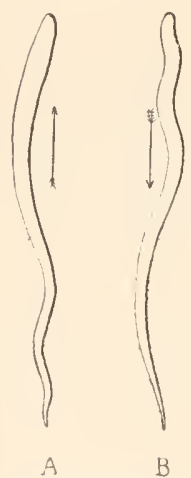

Ondulations de l'Anguille pendant sa progression (Seh.).

Les flèches indiquent le sens dans lequel l'animal se déplace. de la rotation et recul pour le sens inverse. - Tout cela est fort bien, mais Bütschli ne remarque pas que le mouvement qu'il décrit suppose un agencement qui se rencontre dans nos instruments de mécanique, mais jamais chez les êtres vivants. Pour que le corps et le flagellum puissent tourner indéfiniment en sens contraire autour du point $p$, il faudrait que le mode d'union entre eux fùt celui d'une épingle qui a

(1) Il I'y a d'exceptions que les deux suivantes : celle d'Oxyrrhis qui nage le flagellum en arrière el celle de Nephroselmis qui, étant plus large que long, se déplace dans le sens de son grand axe géométrique et, par suite, perpendiculairement à son axe morphologique. Tous les Flagellés peuvent aussi nager à reculons, mais e'est là un mouvement aceidentel et exceptionnel qui s'explique d'ailleur's sans difficulté par un changement dans le sens de la rotation. Les Choanoflagellés, quand par liasard ils nagent, vont aussi à reculons; ils y sont obligés par la présence de leur collerette.

(2) L'Anguille godille avec sa 'queue, c'est-ì-dire qu'elle imprime à eet organe des ondulations situées dans un plan. Le mouvement résulte de ce que la longueur de ces ondulations va en décroissant de la tête à la queue (fig. $470, A$ ). L’Anguille peut aussi reculer en renversant le sens des ondulations, e'est-à-dire en les disposant de manière que les plus grandes soient terminales $(\boldsymbol{B})$. Il ne semble pas qu'il en soit ainsi chez le Flagellé et un tel mouvement n'expliquerait pas la rotation de l'animal autour de son axe. 
percé une feuille de carton el peut tourner librement dans son trou, son union avec la carte étant assurée par la tête qui ne peut traverser le trou.

D’autre part, si l'on admel que le flagellum décrit un mourement de rotation, non autour de son axe à lui, mais autour de l'axe prolongé du corps, comme lorsque le bras tournant autour de l'épaule décrit un cône qui a celle-ci pour sommet (fig. 471), alors ce mouvement sera conciliable avec la constitution de l'organisme, mais il ne produira pas l'entraînement du corps.

Le mouvement d'entraînement déterminé par une hélice (à axe vertical, je suppose) résulte, cn effet, de ce que les différents segments de la courbe se meuvent obliquement dans l'eau el déterminent par conséquent une poussée oblique dont la réaction a une composante verticale. Si cette hélice pouvait tourner à la manière de celles de nos bateaux, celle obliquité serait en tous les points ascendante par rapport à la direction du mouvement rotatoire et la composante verticale de la réaclion serait aussi partout ascendante. Mais si l'hélice est animée d'un mourement comparable à celui du lıras autour de l'épaule (fig. 471), cette obliquilé conserve, pour chaque segment de la courhe, la mème direction absolue à toutes les phases du mouvement, tandis que la direction absolue du mouvement est de sens inverse pendant les deux moitiés d'un même tour; il en résulte que, pendant une moilié de chaque tour, l'obliquité des segments de la courbe est ascendante par rapport à la direclion du mouvement rotatoire, tandis que pendant l'autre moilié, cette obliquité est descendante. Par suile, chaque segment

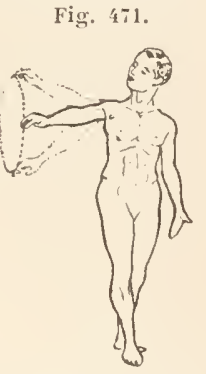

Mourement detrinslation conique sans rotation vraie. Le dos de la main est toujours tourné en haut. de l'hélice entraine l'animal on haut pendant une moitié de sa révolution

Fig. 472 .

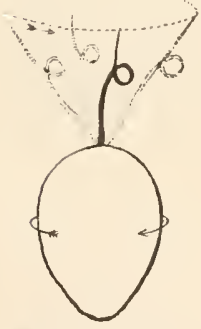

Mouvement de translation conique du flagellum

(Sch.). et en has pendant l'autre moitié, et ces deux actions s'annulent.

Il nous semble que la seule explication possible de la progression du Flagellé, consiste à admettre que l'animal fait tournoyer son flagellun de ce mouvement conique non rotatoire (fig. 472) que nous avons comparé à celui du bras autour de l'ćpaule, que ce mouvement fait tourner en sens inverse le système entier formé par le flagellum et par le corps autour de l'axe vertical de celui-ci, et que le flagellum, contourné une fois pour toutes en hélice, par suite de ce mouvement de rotation vraie, se visse en quelque sorte dans l'eau et entraine le corps à sa suite.

Pour donner une image de ce mouvement, nous supposerons un acrohate qui, se tenant sur la pointe d'un pied, décrirait au-dessus de sa tète un mouvement conique rapide avec un de ses bras 
(fig. 473). Si l'air était un milieu assez résistant, il imprimerait par cela seul à tout son corps un mouvement de pirouette continu et de sens inverse sur la pointe de son pied. Si la main du bras qui s'agite ainsi tenait une hélice, celleci, entraincé dans cette seconde rotation, lui imprimerait une force ascensionnelle qui pourrait l'enlever s'il était, comme le Flagellé, plongé dans un milieu de densité presque égale à la sienne, qui rendrait son poids presque nul (').

(1) Sans prétendre fournir la démonstration mathématique complète de ces assertions, nous pouvons leur donner ici un peu plus de précision.

Etablissons bien d'abord la différence des deux mouvements que nous distinguons. Dans le mouvement de tournoiement du bras, si le dos de la main est tourné, je suppose vers le eiel (fig.471), il gardera cette orientation, à quelques degrés près, pendant toute la durée du mourement, tandis que si le bras tournait à la manière d'une hélice de navire, la face dorsale regarderait successivement en haut, en arrière, en bas, en avant, etc., et la face palmaire tournée vers l'axe regarderait toujours cet axe, mouvement qui, nous le répétons, est im-

Fig. 474.

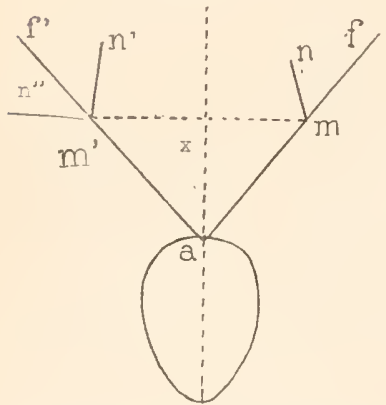

Schéma de la rotation conique et de la translation conique. possible chez les êtres organisés. Transportons ees notions chez le Flagellé armé de son flagellum (fig. 474). Si cet appendice $(a f)$ est linéaire et rectiligne il n'y aura aucune différence frappante entre les deux sortes de mouvements. Dans l'un comme dans l'autre, il se trouvera, après un demi-tour, en a $f^{\prime}$ symétrique de af par rapport à $a x$. Mais si $a f$ porte, d'un còté, un appendiee $m n$ ineliné sur af vers ax dans le plan $a f x$, dans le mouvement rotatoire vrai réalisé dans nos machines, amn tournera autour de af en même temps qu'autour de $a x$, ear il est lié au rayon vecteur $x m$ et, après un demi-tour, $m n$ aura la position $m n^{\prime}$ symétrique de $m n$ par rapport à $a x$; au contraire, dans le mouvement comparé à eelui du bras de l'homme, amn tournera autour de ax sans tourner autour de af, $m n$ restera toujour's tourné du même côté de l'espace el, après un demi-tour, aura la position $m n^{\prime}$ symétrique de $m n$ par rapport à $a f^{4}$. Pour les distinguer brièvement, nous appellerons ces deux sortes de mouve-

ments : la première, rotation conique, la seeonde, translation conique. Et nous allons maintenant montrer que la translation conique d'un flagellum héliçoïdal ne saurait produire un entraînement du système dans la direction verticale, landis qu'une rotation conique le ce même flagellum produirait cet entraînement.

Fig, 475 .

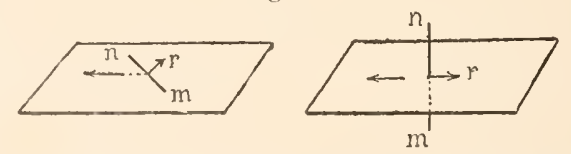

Les différents points du flagellum décrivant des cercles horizontaux, il faut, pour que ce mouvement puisse donner Réactions déterminées par le mouvement d'une droite horizontale ou vertieale. naissance à des composantes verticales, qu'il renferme des segments non horizontaux et formant avee la direction de la vitesse, e'est-à-dire avee les tangentes à la trajectoire un angle $>0$ et $<90^{\circ}$, ear un segment 
Alimentation. - Le courant d'eau que détermine l'animal avec son flagellum pendant sa progression est dirigé vers la base du flagellum,

mn horizontal (fig. 475, à gauche) ou vertical (fig. 475, à droite) ne donnerait lieu qu'ì des réactions $(r)$ horizontales.

Considérons done dans les deux sortes de mourements, les réactions développées par un élément $m n$ du flaggellum ayant l'obliquilé indiquée.

$1^{\circ}$ Cas de la translation conique (fig. 476 à 478). - Si l'on examine les positions que

Fig. 476 .

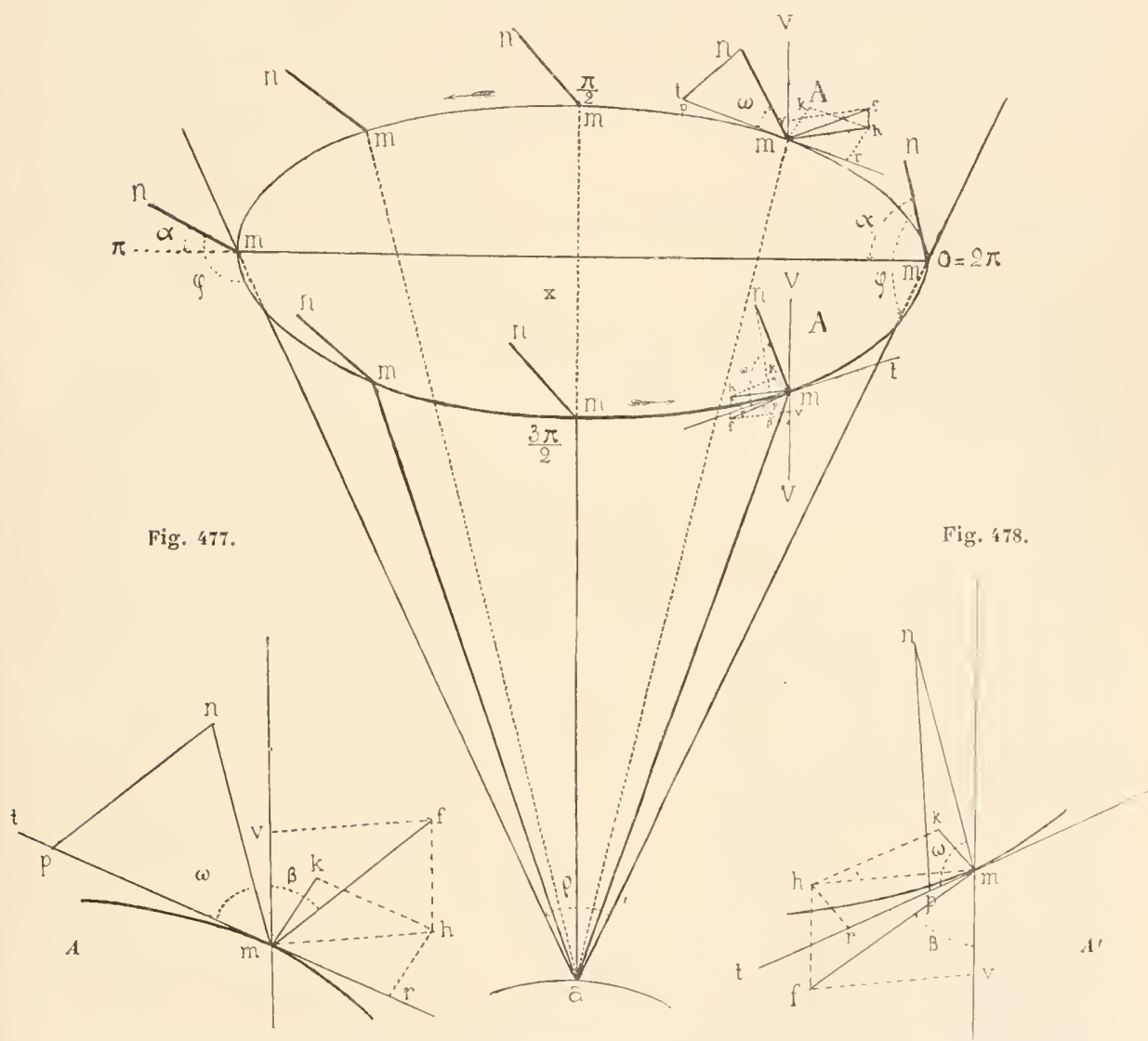

Détermination des forces développées par un segment $m n$ du flagellum dans le cas de la translation conique.

prend mn pendant un tour complet, on voit que, partant du point 0 où il est dans le plan du papier et fait un angle o avec la génératrice, il décrit sa trajectoire en maintenant l'angle o invariable. Son angle $\alpha$ avec le plan horizontal que dessine la trajectoire de $m$, diminue de 0 à $\pi$ où il prend la valeur de $\alpha$ - $\rho$ ( $\rho$ élant l'angle du cône) et augmente de $\pi$ à $2 \pi$ où il reprend la valeur $\alpha$. Par contre, l'angle (1) que fait $m n$ avec la direction de la vitesse en chaque point (Direction représentée par $m t$ tangente ì la circonférence en ce point) varie de la maniére suivante : aux points 0 et $\pi$ 
c'est-à-dire vers la bouche. C'est donc à cet orifice qu'arrivent naturellement les particules alimentaires. Mais, en outre de cela, l'animal peut imprimer à son flagellum un mouvement suffisant pour déterminer un courant d'eau alimentaire, sans entrainer le corps lui-même en avant. C'est, en tout cas, le flagellum qui est l'instrument de la capture des

il est de 90 , entre 0 et $\pi$ il diminue d'abord, puis augmente, entre $\pi$ et $\dot{2} \pi=0$ il augmente, puis diminue. Une courbe

Fig. 479.

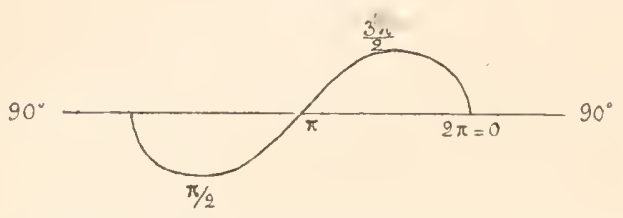

Variation de l'angle (1) pendant un tour complet. sinusoïdale ayant pour axe la cote 90 degrés donnerait une idée de sa variation (fig. $479 f$ ).

Sil'on examine qu'elle est la poussée de $m n$ sur l'eau aux différents points du mouvement (fig. 476, 477), on voit qu'en un point quelconque $A$, elle est proportionnelle en grandeur à $m n$ sin, (1); d'autre part, sa direction est donnée par une perpendiculaire élevée à $m n$ dans le plan $m n t$ que forme la droite avec la tangente. La réaction peut donc êlre représentée par cette perpendiculaire, placée sil'on veut au point $m$, et ayant pour longueur $m f=n p=m n \sin$. $\omega$. Cette force, élant perpendiculaire à $m$, est oblique comme elle par rapport à la verticale; elle a donc une composante verticale. Si l'on élève une verticale en $m$ cette composante sera $m$ v ayant pour valeur $m f \cos \beta, \beta$, en appelant $\beta$ l'angle de $m f$ avec $m$, et l'on aura $m \varphi=m n \sin . \omega \cos . \beta$.

Pour discuter cette formule il nous reste à examiner la variation de l'angle $\beta$ et pour cela il nous faut voir quelles sont les inclinaisons successives que prend la perpendiculaire $m f$.

Au point 0 , cette droite se confond avec la tangente $m t$ et est horizontale; il en est de même en $\pi$. Entre 0 et $\pi$ elle s'élève peu à peu sur l'horizontale, passe par un maximum vers $\pi / 2$, puis s'abaisse de nouveau jusqu'à l'horizontale. Entre $\pi$ et $2 \pi$ elle est située au-dessous de l'horizontale, formant avec le plan horizontal $m x$ un angle qui d'abord s'accroît, passe par un maximum vers $3 \pi / 2$ et diminue ensuite pour tomber dans ce plan en $2 \pi=0$.

Les valeur's successives de $\beta$ et de son cosinus sont donc:

$$
\begin{array}{ccc}
\text { en } 0: & \beta=90 & \cos \beta=0 . \\
\text { de } 0 \text { à } \pi: & \beta<90>0 & \cos \beta>0 . \\
\text { en } \pi: & \beta=90 & \cos \beta=0 . \\
\text { de } \pi \text { a } 2 \pi: & \beta>90<180 & \cos \beta<0 .
\end{array}
$$

La variation de $\beta$ peut être aussi figurée par une courbe de la forme de celle que représente la figure 479.

Mais tandis que $\omega$ passant par toutes les valeurs entre un minimum $>0^{\circ}$ et $<90^{\circ}$ et un maximum $>90^{\circ}$ et $<180^{\circ}, \sin$. $\omega$ a toujours une valeur positive, on voit que cos. $\beta$ est positif de 0 à $\pi$ et négatif rle $\pi$ à $2 \pi$.

Donc le produit $m$ sin. (1) cos. $\beta$ sera positif de 0 à $\pi$ et négatif de $\pi$ à $2 \pi$.

Cela veut dire que la composante verticale $m$ ' est ascendante pendant un demitour et descendante pendant le reste du tour, qu'elle tend à entraîner le système en haut pendant un demi-tour et en bas pendant le second demi-lour, à défaire pendant celui-ci ce qu'elle a commencé pendant celui-là.

En comparant deux à deux les positions de $m n$ entre 0 et $\pi$ avec leurs symétriques d'entre $\pi$ et $2 \pi$ par rapport, non au centre du cercle, mais au diamètre $0 \pi$, il est facile de voir que, $m n$ prenant des inclinaisons égales et symétriques de part et d'autre de ce diamètre, les valeur's absolues de $\sin$. $\omega$ et de $\cos$. $\beta$ sont les mêmes, et que, par suite, 
aliments. Ces aliments, généralement fort petits, Bactéries, parcelles quelconques, sont précipités dans le pharynx, trouvent au fond un pro-

les valeurs positives et négatives de $m$ s' s'annulent deux à deux. Donc le système n'aura aucun mouvement vertical continu $\left.{ }^{*}\right)$.

20 Cas de la rotation conique (fig. 480). - Dans ce cas, la construction au point quelconque $A$ est évidemment la mème et l'on a encore $m v=m n \sin$, (1) cos. $\beta$. Mais la variation des angles pendant la rotation est tout autre. Le segment $m$ n ayant quelque part (comme cela est forcé pour toute hélice) $\iota^{* *}$, par rapport à la verticale el à la direction de la vitesse donnée par la tangente, la double inclinaison indiquée, conserve pendant la révolution entière cette mème inclinaison, puisqu'elle est liée en mème temps que la tangente au rayon recteur $x m$ : en particulier, en aucun point elle ne pourra prendre une direction perpendiculaire à la langente comme dans le cas précédent aux points 0 et $\pi$, ni s'incliner au-dessous de l'horizontale comme dans le cas précédent entre $\pi$ et $2 \pi$. Il en résulte que cos. $\beta$ est toujours positif, que mv est toujours ascendant et que le système est entraìné vers le haut.

Tout cela d'ailleurs n'est que le développement de celte idée presque évidente a priori qu'une hélice ne saurait avancer sans tourner autour de son axe, qu'elle n'avancera pas en tournant autour d'un autre axe que le sien, si ce mouvement ne comporte aucune rotation continue autour de son axe à elle (ce qui est le cas du mouvement que nous arons appelé translation conique), et qu'enfin elle avancera, quel que soit le mouvement compliqué qu'on lui imprime, si ce mouvement comporte, entre autres éléments, une rotation autour de son axe à elle dans un sens constant, comme c'est le cas dans le mouvement que nous avons qualifié de rotation conique.

Il résulte de là que le seul mourement qui pourrait entrainer le Flagellé en avant eșt celui qui est incompatible avec sa structure.

Cependant, le Flagellé se meut, et l'observation montre qu'il avance en tournant et en faisant tournoyer son flagellum. L'analyse objective de son mourement vrai est à peu près impossible. Posons-nous done seulement la question suivante : imaginer un

$\left(^{*}\right)$ On pourrait croire, à première vue, que le système pourra recevoir une propulsion latérale des composantes horizontales $m k$ ou une rotation autour de l'axe $0 \pi$ de la part du couple $+m v$ (en $A$, fig. 476 et fig. 477 ) et $-m v$ (en $A^{\prime}$ fig. 476 et fig. 478 ). Nais en considérant une hélice entière au lieu du seul segment $m n$, on verra qu à chaque moment et pour chaque segment, ces forces sont détruites par les forces correspondantes déterminées par les segments situés dans le même plan vertical et qui ont au même moment une inclinaison inverse.

$\left({ }^{*}\right)$ C'est le contraire dans le cas de la translation conique. Tout segment $m m$ de l'hélice représentée par le flagellum au repos à la double inclinaison requise. Tandis que, dans la translation conique, lorsque ce flagellum s'incline et se met à tourner du mouvement indiqué, comme il reste toujours orienté du même côté de l'espace, il passe nécessairement par deux positions oì il est perpendiculaire à la tangente qui, elle, regarde successivement tous les azimuths. Dans notre figure 476. nous arons placé ces deux positions diamétralement opposées dans le plan du papier. 
toplasma nu et y pénètrent immédiatement. L'ingestion est si rapide que la particule a l'air d'avoir été entraînée dans les profondeurs du corps par un mouvement d'aspiration. Mais il est probable qu'il n'y a pas d'aspiration réelle.

L'endoplasme est animé d'un vague mouvement de cyclose ou plutôt d'oscillations irrégulières qui en trainent les particules alimentaires jusqu'à ce qu'elles soient digérées et expulsées à l'extrémité inférieure du corps.

Excrétion. - Celte fonction a pour organe la vésicule pulsatile. De l'eau entre sans cesse dans le corps par osmose, et aussi quelque peu avec les aliments, et est rythmiquement expulsée par la vésicule. En filtrant à travers le cytoplasme, elle s'est chargée des produits solubles de désassimilation et en débarrasse ainsi l'organisme. Elle peut servir aussi à la respiration, car elle entre chargée d'oxygène et peut dissoudre de l'acide carbonique. Mais cette fonction est certainement accessoire, car le corps du Flagellé est si petit qu'il respire suffisamment par les échanges osmotiques de sa surface, et la vésicule se rencontre aussi chez les formes pourvues de chlorophylle qui consomment intérieurement leur acide carbonique et fabriquent plus d'oxygène qu'il ne leur en faut.

Pour comprendre le jeu de la vésicule, prenons-la au moment où, étant en pleine diastole, elle va se contracter. On aperçoit alors autour d'elle une couronne de petites vésicules formatrices, simples vacuoles dues

mouvement du flagellum possible pour l'animal et capable de l'entraîner en avant.

Ce mouvement existe et il n'est autre que celui de translation conique que nous avons analysé. Nous venons de voir qu'il n'a aucun effet direct d'entraînement : montrons maintenant qu'il a cet effet d'une manière indirecte.

Reportons-nous aux figures 476, en $A$ ou 477, $e$. Nous a vons vu que la réaction oblique $m f$ a une composante verticale $m$ s. Mais celle-ci implique une composante horizontale $m h$ située à l'inter'section du plan horizontal $x m t$ et du plan fim déterminé par la résultante et par la première composante choisie. A son tour $m h$ peut ètre décomposée dans le plan horizontal en deux composantes dont une latérale qui n'a pas d'intérêt dans la question et une $m r$ suivant la tangènte, mais en sens inverse de la vitesse $m t$. Cette dernière force n'est autre chose que la réaction horizontale du mouvement. Elle alpour effet d'entraîner dans un mouvement de rotation pure autour de $a x$ l'ensemble du système formé par le flagellum et par le corps de l'animal. Il en résulte que le flagellum, en tournoyant d'un mouvement de translation conique autour de $a x$, prend appui sur le corps et le repousse dans un mouvement de rotation pure de sens inverse dans lequel il est lui-même entraìné.

Dès lors, si le flagellum se trouve, une fois pour toutes, contourné en hélice (hélice conique probablement), cette hélice, en tournant a utour de son axe, se déplacera le long de cet axe et entraînera le corps à sa suite. Pour changer le sens de son mouvement et reculer au lieu d'avancer, l'animal n'aurait qu'à changer, soit le sens d'enroulement de l'hélice de son flagellum, soit le sens dans lequel il fait tournoyer cet organe.

ll est à remarquer que, dans ce flagellum héliçö̈dal, tous les segments ont précisément cette obliquité que nous avons reconnue nécessaire à $m n$ pour engendrer les réactions décrites.

Disons enfin pour terminer que notre explication est non seulement possible mais probable, car elle correspond à ce que montre l'observation, savoir: un flagellum contourné en hélice, un mouvement gyratoire de ce flagellum et une rotation totale de l'animal en sens inverse de son flagellum, rotation affirmée par Bütschli lui-même. 
à l'accumulation de l'eau qui, filtrant à travers le cytoplasme, vient se rassembler autour de la vésicule pulsatile en gouttelettes indépendantes. Ces gouttelettes sont de simples interstices et n'ont pas de paroi propre. Quand la vésicule principale se contracte, elle force la mince paroi qui la sépare de l'extérieur et écoule au dehor's tout son contenu, ne laissant rien d'elle-mème. Derrière elle, le cytoplasma ambiant vient occuper, au fur et à mesure qu'elle diminue en se vidant, la place qu'elle laisse libre. Dès que la systole est achevée, les vésicules formatrices qui sont, elles, à ce moment, en pleine diastole se fondent les unes dans les autres et constituent, d'emblée, une nouvelle vésicule pulsatile à la place qu'occupait l'ancienne, mais complètement indépendante de celle-ci. En mème temps, l'eau qui continue à suinter vient reformer une nouvelle couronne de résicules formatrices et la mème série des phénomènes recommence indéfiniment.

Les systoles se succèdent rapidement. Il y en a de une à douze par minute, d'où résulte qu'en peu de temps l'or@̧anisme est traversé par un volume d'eau égal au sien. C'est donc là une fonction très active, un rinçage énergique et continu de l'organisme. Cette fonction est, comme toutes les autres, activée par la chaleur; elle a son maximum rers $40^{\circ}$ après quoi elle décline, et de 4.3 à 60 la mort survient.

Enkystement (fig. 481). - En général le Flagellé ne craint pas l'eau

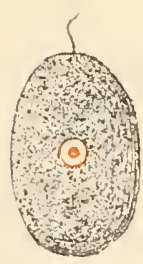

A

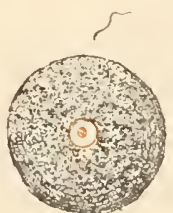

B

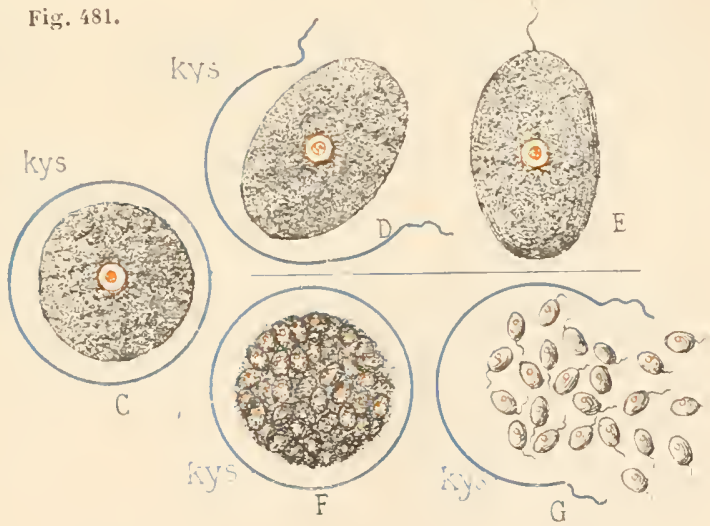

FLAGELLIA (Type morphologique). Enkystement (Sch.).

$A$ et $B$, l'animal se contracte et perd son eil ; $C$, il s'entoure d'un kyste (kys.); $D$, il sort de son kyste et reprend en $E$ sa forme primitive; $F$, il se divise; $G$, les produits de la division sont mis en liberté.

croupie, puisqüil se nourrit principalement des Bactéries qui y alıondent. Cependant, quand la putréfaction devient trop accentuée, il éprouve le besoin de s'abriter contre l'action des gaz qui en résultent. ll en est de mème lorsque l'eau se concentre et menace de disparaìtre par évaporation. Il mourrait infailliblement s’il se trouvait mis à sec. Dans ces conditions, il se contracte, s'arrondit $(B)$, perd son flagellum et se sécrète 
un kyste membraneux et gélatineux ( $C$ : liys.) sous lequel il reste à l'état de vie ralentie pendant un temps qui peut être fortlong. Au retour des conditions normales, il sort de son kyste $(D)$, reprend sa forme, fabrique un nouveau flagellum et reprend sa vie active $(E)$. Mais souvent il fait autrement et profite de cet enkystement pour remplir une autre fonction qu'il doit accomplir aussi de temps en temps, même quand les conditions restent favorables : il se divise sous son kyste en nombreux petits corps $(F)$ qui se développent rapidement au sortir du liyste en autant de petits Flagellés semblables à leur parent sauf la taille $(G)$. Nous aurons bientôt à revenir sur ces faits.

Division à l'état libre (fig. 482). - Le Flagellé se reproduit principalement

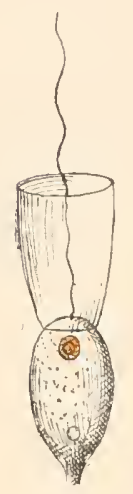

A

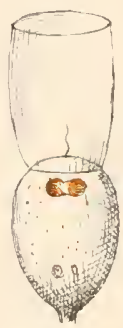

B

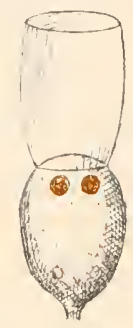

C
Fig. 482.

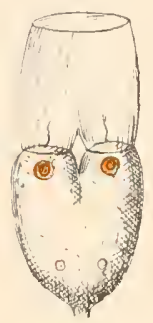

D

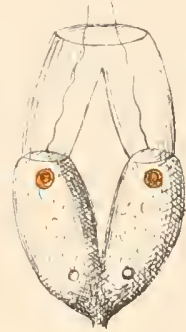

E

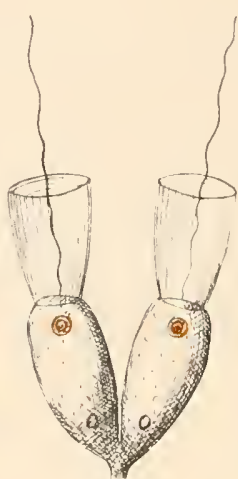

F

FLAGELLIA (Type morphologique).

Division à l'état libre (im. Blochmann).

par division longitudinale. Si l'on met à part les Chlamydomonadines et les Volvocines, qui sont presque des plantes et qui ont un mode spécial de division, on constate que la division est presque universellement longitudinale chez les Flagellés (").

La division longitudinale (fig. 482) commence par le

(1) On ne connaît qu'un petit nombre de divisions transversales vraies (Epipyxis. O.xyr-

Fig. 183.

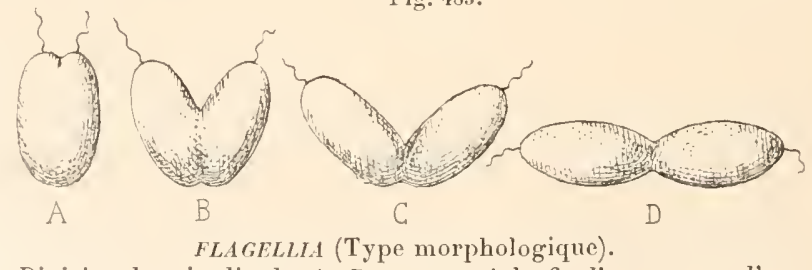

Division longitudinale $A, B$, prenant à la fin l'apparence d'une division transversale $C, D$ (Sch.).

rhis, Stylochrysalis et quelques autres Chrysomonadines, et enfin chez les Biliacines). On a souvent pris pour transversales des divisions longitudinales dans lesquelles les deux individus, n'étant plus attachés que par leur extrémité inférieure, s'étaient placés sur le prolongement l'un de l'autre (fig. 483). 
noyau. Celui-ci s'allonge transversalement et, peu à peu, se divise par un processus qui ressemble à la division directe, mais qui constitue en réalité une mitose plus ou moins simplifiée, car on y a reconnu une striation fibrillaire et les éléments d'un fuseau (").

Les deux nouveaux noyaux se placent à quelque distance l'un de l'autre dans un plan transversal. Pendant ce temps, une nouvelle bouche se forme à côté de l'ancienne, parallèlement à elle et un nouveau flagellum naît à côté du premier par une petite papille qui grandit rapidement. Une nouvelle résicule pulsatile se forme aussi à côté de l'ancienne et l'on a un individu dont tous les organes sont doubles. Bientôt une incision se forme à l'extrémité supérieure entre deux flagellums; cette fente s’approfondit progressivement et sépare peu à peu les deux individus qui restent unis en dernier lieu par leur extrémité inférieure arant de se séparer tout à fait $\left(^{*}\right)$.

(1) Blochmanx [9'] a récemment observé chez Euglena (fig. 484) un processus de division qui appartient nettement à 'la division indirecte, mais diffère de la mitose classique en quelques points importants. Le noyau au repos montre un corps central qui ressemble à un nucléole, mais qui est tout autre chose en réalité et, autour de ce nucléole, un semis de petits grains chromatiques $(B)$, qui sont des chromosomes. Le prétendu nucléole s'allonge en biscuit $(C)$, s'étire, et enfin se divise; les chromosomes s'allongent et se disposent en deux groupes autour des deux nucléoles filles $(F)$, sans que l'on puisse dire nettement s'ils subissent la division longiturlinale ou si, simplement, une moitié d'entre eux se sépare de l'autre moitié; enfin, les deux nucléoles s'écartent $(G, H)$, entraînent chacun un des groupes de chromosomes et la division s'achève ainsi ( $I$. La mitosique du noyau (chez Euglena) (d'ap. Blochmann). membrane nucléaire persiste tout le temps. Le prétendu nucléole, bien qu'il se comporte à peu près comme la chromatine, reste incolore en présence de certains colorants de cette substance qui teignent fortement les chromosomes. Il est donc formé d'une autre matière que la chromatine. En raison de sa situation, Blochmann incline à l'assimiler au fuseau central de Hermann, bien qu'il ne montre pas une structure fibrillaire. Blochmann a aussi observé à côté du noyau un à trois corps qui l'ont fait songer aux centrosomes, mais il faudrait avoir vu leur division pour que celte assimilation fùt légitime. On ne sait pas dans quelle mesure ces résultats pourraient être généralisés.

(2) Ce mode de division est le plus général de beaucoup; mais il souffre quelques exceptions. Tetramitus, Chlamydococcus divisent leur corps de bas en haut. On a parlé d'une division longitudinale du flagellum et de la vésicule, mais cela n'est pas réel. Cependant, chez Euglena, la portion de la vésicule appelée réservoir se doublerait, d'après Kifebs, par division. 
Division sous un kyste et sporulation (fig. 485 à 487). - Assez souvent, l'ani-

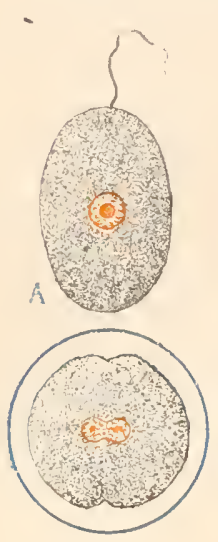

D
Fig. 18.).
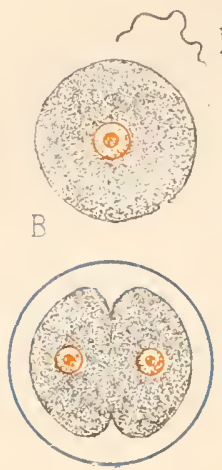

E
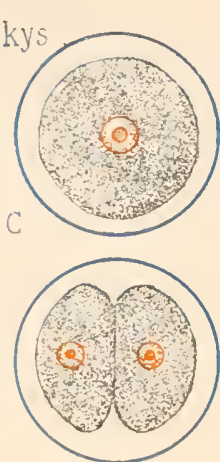

FLAGELLIA (Type morphologique). Division sous un kyste (Sch.). mal (fig. 4S:3, $A$ ) s'enkyste avant de se diviser. Il perd son flagellum $(B)$, s'arrondit, se sécrète un kyste ( $C$. liys.), et immédiatement se divise par un processus tout semblable à celui de la division à l'état libre $(D, E, F)$. Aussitôt formés, les deux jeunes sortent du kyste (fig. $486, G$ ), se forment chacun un flagellum et recommencent la vie libre (II). Nais parfois, au lieu de prendre leur liberté, les jeunes $(I)$ s'enkystent euxmêmes $(J)$ et se divisent de nouveau. D'autres fois

(fig. 4S7) l'animal, sous son liyste, se divise successivement, mais coup sur coup plusieurs fois, et donne huit, douze, seize, parfois un beaucoup plus grand nombre de petits globes que l'on pourrait appeler spores ct qui, mis en liberté, se munissent d'un flagellum et ac-
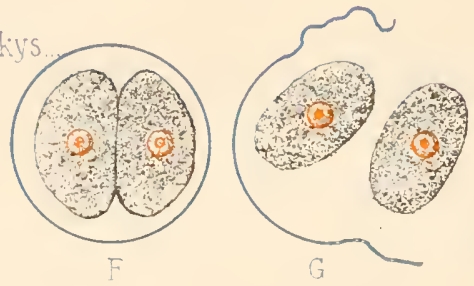
Fig. 496. quièrent rapidement les caractères de leurs parents. C'est à une sporu-

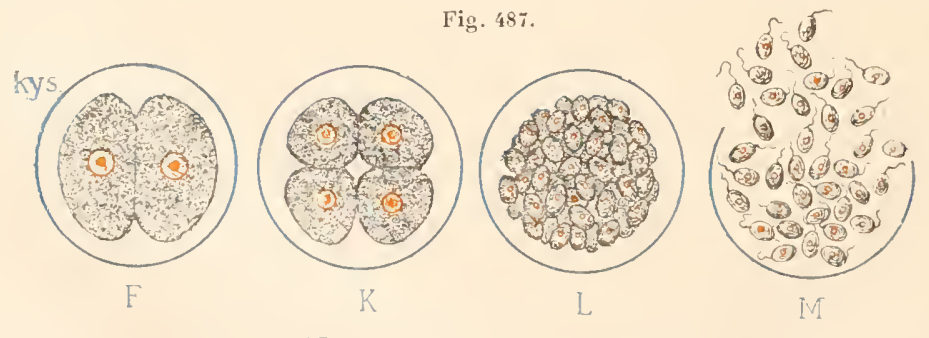

FLACELLIA (Type morphologique). Sporvlation (Sch.).

lation de ce genre qu'ahoutit parfois, comme nous l'arons vu plus hant, 
l'enkystement de protection commencé d'ahord pour un autre but ('). Conjugaison. - On n'a pas observé ici, comme chez les Ciliés, ainsi que nous le verrons plus tard, un phénomène de dégénérescence, d'épuisement organique produit par la succession indéfinie des générations agames. Mais il est possible que cette dégénérescence se produise sans se manifester par des caractères objeclifs bien évidents. Toujours est-il que, de loin en loin, une conjugaison s'intercale dans le cycle évolutif. Deux individus se joignent, se soudent, perdent leur flagellum, prennent un caractère plus ou moins amœboïde et, peu à peu, se fondent l'un dans l'autre. Les noyaux se fusionnent aussi. C'est une conjugaison totale. L'ètre résultant de la conjugaison s'enkyste et, sous ce kyste, se divise en nombreuses spores d'oì naissent aulant de petits Flagellés d'aspect ordinaire, mais qui ont eu deux parents au lieu d'un seul $\left({ }^{(2}\right)$.

Il s'en faut de beaucoup que les caractères assignés à notre type morphologique soient communs à tout le groupe. C'est une forme moyenne, rien de plus. Le corps peut être entièrement nu ou protégé par des enveloppes, des coques solides ou des capsules, petites logeltes sécrélées par l'animal pour s'abriter. Ces logettes peuvent être libres ou fixées, sessiles ou pédonculées, isolées ou réunies en colonies de formes variées. L'animal peut aussi former des colonies en restant nu ou en s'associant à ses semblables dans nne masse commune de gélaline. La bouche peut ètre notablement plus compliquée ou ne pas exister du tout. II y a toujours un flagellum, mais il peut y en avoir leux, trois, quatre, et jusqu'à huit. Ces flagellums peuvent ètre semblables ou différents. Toujours un au moins est dirigé en avant, mais l'autre ou les autres peuvent être dirigés en avant ou en arrière. Enfin, le cyloplasma renferme des chromoplastes chargés de chlorophylle ou de diatomine qui fonctionnent comme chez les plantes, et cela, joint à certaines particularités de reproduction et à la formation de colonies, arrive à constituer des ètres pour lesquels on est fort embarrassé de dire s’ils sont des animaux ou des plantes. Toutes ces variations vont nous occuper maintenant et passer sous nos yeux dans l'étude de la classification $\left({ }^{3}\right)$.

(1) Tous ces modes de division sous kyste peuvent se rencontrer dans la même espèce et il n'est pas abusif de les attribuer it notre type morphologique, mais d'ordinaire il n'en est pas ainsi ou du moins on n’a observé que les uns ou les autres.

$\left(^{2}\right)$ Ce n'est guère que chez les Chlamydomonadines et les Volvocines que l'on a observé ces phénomènes. Nous renvoyons pour le détail à ces groupes.

Ici, comme chez les Rhizopodes et les Sporozoaires, les plénomènes intimes de la conjugaison et surtout les relations exactes de la conjugaison avec le cycle évolutif ne sont que très imparfaitement connus comparativement à ce que l'on en sait chez les Ciliés. Il faudrait un autre Maupas pour débrouiller cette importante et difficile question.

(3) Le noyau est toujours unique, mais il arrive fréquemment qu'il y a deux, trois, jusqu'à cinq vésicules pulsatiles au lieu d'une et, au moins quand il n'y en a que deux, on a pu constater qu'elles se contractaient alternativement; leur situation 
Nous diviserons la classe des FlaterLés en cinq sous-classes :

ELFLAGELLIX, comprenant les Flagellés proprement dits;

Silir OFLAGELLIE, formes aberrantes parasites des Radiolaires;

DINOFLAGELLIE, formés des Péridiniens et des formes affines;

CYSTOFLAGELIA, qui sont les Noctiluques;

CATALLACTIE, contenant le seul genre Magosphara.

\section{$1^{\text {re }}$ Socs-Chasse}

\section{EUFLAGELLÉS. - EUFLAGELLIAE}

[MONADIDA (Bütschli) + ElGLENINA (Stein) + CIILoRomoxadiNa (Klebs) + CRYPTOMONADINA (Stein) +

Cillamyononadina (Bütschli) + Volvorina (Ehrenberg)]

Ce sont les Euflagellés, de beaucoup plus nombreux que les autres sous-classes, que représente surtout notre type morphologique. Pour les Dinoflagellés et les Cystoflagellés, nous aurons à le modifier plus ou moins, mais pour les Euflagellés, nous n'avous rien à lui ajouter ni à lui retrancher. Nous pouvons donc passer tout de suite à la classification de ce groupe.

Nous le diviserons en trois ordres :

MONADIDA, comprenant les Flagellés inférieurs. Ils ont souvent des déformations amœboïles et parfois mème des pseudopodes. Ils n’ont point de bouche, mais seulement un lieu d'élection pour l'ingestion des aliments ;

E $C_{G L E Y I D A}$, constituant, au contraire, le type le plus élevé et le plus normal des Flagellés. Ils ont une bouche et un pharynx parfaitement dessinés. La forme du corps est toujours nettement définie malgré les déformations temporaires que lui impriment ses contractions. Enfin, mème lorsqu'ils possèdent de la chlorophylle, ils n'en garlent pas moins un caractère franchement animal;

PIIYTOFLA GELLIDA, qui commencent, comme les Monadida, par des formes très inférieures et, en s'élevant en organisation, se rapprochent des végétaux, à la fois par leur structure, leur mode de vie et leur évolution (').

varie aussi par rapport à celle du noyau, mais ees particularités servent surtout à caractériser les genres, car elles n'ont guère de constance dans les groupes plus élevés.

(1) Nos Monadida sont les Monadina de Bütschli, emendate; nos Euglenida sont les Euglenina de Stein, emendatz; et nos Phytoflagellida [non Phytomonadina (Blochmann)] comprennent les Chloromonadina(Klebs) [ = Chrysomonadina (Stein, emend. Klebs) ], + les Cryptomonadina de Stein, + les Chlamydomonadina de Bütschli, + les Volvocina d'Ehrenberg. 


\section{$1^{\text {er ORDRE }}$ \\ MONADHDES. - MONADIDA \\ [Monadines; - MonadiNa (Bütschli) emend.]}

\section{TYPE MORPHOLOGIQUE}

(FIG. 488)

Il résulte de ce qui précède que nous pouvons, ici encore, définir en quelques mots notre type morphologique en partant de celui décrit pour l'ensemble des Flagellés. Nous n'avons, pour en faire un type moyen de Monadide, qu'à supprimer sa bouche et à rendre moins ferme, plus indécise, sa couche tégumentaire, pour rendre faciles les déformations amiboïdes qui lui sont habituelles.

Chez les Monalides, le nombre des fouets varic et il semblerait que ce caractère très saillant dùt ètre le meilleur guide dans la classification. Cela n'est pas tout à fait exact rar, dans des genres très voisins, on peut trouver ou non, à côté du fonct principal, un ou deux foucts accessoires généralement plus petits, mais parfois aussi grands que lui. Au contraire, ceux qui ont plus de trois fouets sont toujours éloignés des précédents par de nombreux caractères. D'autre part, il existe toute une série de formes où l'un des fouets accessoires devient beaucoup plus grand que le fouet principal, se dirige en bas et s’adapte à des usages spéciaux.

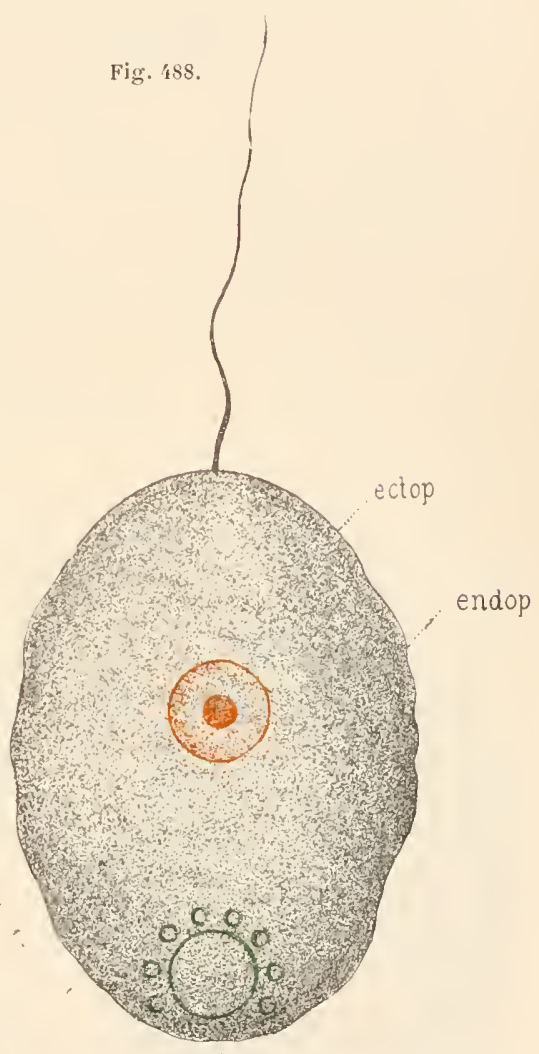

MONADIDA. Type morphologique (Sch.).

Cela nous autorise à distinguer dans l'ordre des Monadides trois sous-ordres :

OLIGOMASTIGIDE, ayant un fonet principal accompagné ou non de un ou deux fouets accessoires plus petits ou tout au plus égaux au fouet principal;

HETEROMASTIGIDE, ayant, outre le fouet principal, un ou deux grands fouets accessoires dirigés en bas;

POLYMASTIGIDE, ayant plus de trois fouets. 
$1^{\text {er }}$ SOUS-ORDRE

\section{OLIGOMASTIGIDES. - OLIGOHASTIGIDA}

Sans décrire pour eux de type morphologique, nous les diviserons en deux lriluus :

A CRASPEDINA sans collerette; et

CRASPEDINA munis d'une collerette (').

1re Tribu

ICRASPÉDINES. - ACRASPEDINA

TYPE MORPHOLOGIQUE

(FIG. 489 ET 490)

Il est inutile de nous étendre longuement sur la description du type morphologique de ce groupe. Nous aurions à répéter à peu près tout ce que nous avons dit

Fig. 189.

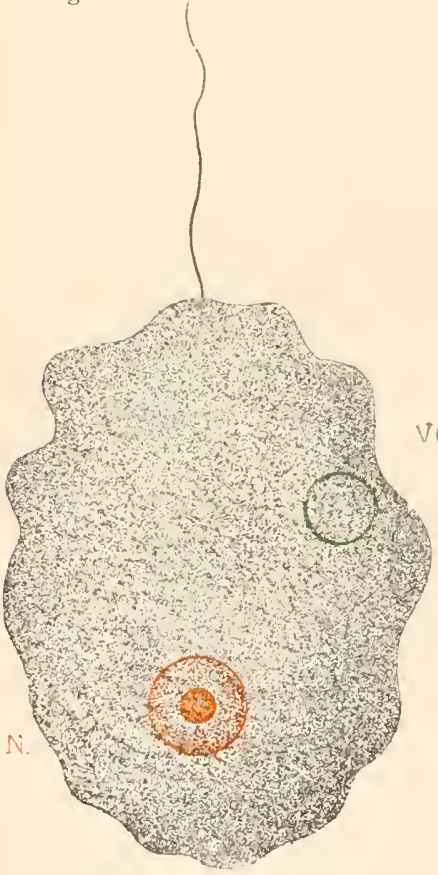

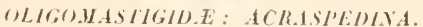

Type morphologique (Sch.)

s., noyau; Ve., vésicule pulsatile. du type général des Flagellés. Il ne diffère de ce dernier qu'en deux points, les téguments et la bouche, et, quand nous les aurons fait connaitre, il sera suffisamment décrit.

Les téguments ont toujours la mème constitution typique, mais leur's caractères pliysiques sont différents. La membrane est à peine indiøuée et n'a aucune frrmeté, et l'ectoplasme se dislingue, lui aussi, par une mollesse particulière. Il en résulte que le corps se prète à des déformations amœboüles plus ou moins étendues après lesquelles il reprend naturellement sa forme primitive : il est métabolique.

La bouche n'existe pas, en tant du moins que dépression infundibuliforme préformée. Il y a seulement un lieu d'élection pour l'entrée des aliments el ce lieu est situé à la base du flagellum. D'ordinaire, à cette place on ne remarque rien de particulier, surtout si l'animal n'est pas affamé. Mais si le fouet, dans son mourement, projette vers elle une particule alimentaire, colle-ci pénètre immédiatement dans le cytoplasme et est aussitôt englobér en mème temps qu'une petite masse l'eau qui forme autour d'elle une vacuolr alimentaire.

(1) Ces Craspedina correspondent aux Craspedomonudinu de Stein et aux Choanoflagellata de kient. 
Si l'animal, depuis quelque temps, n’a pas capturé de nourriture, on voit se former au lieu d'élection une vacuole, parfois si volumineuse qu'elle forme une gibbosité très saillante (fig. $490, A)$. C'estune vacuole alimentaire, mais vide, attendant un aliment. Dès qu'un globule assimilable vient à ètre projeté contre sa mince paroi, en un clin d'œil

0

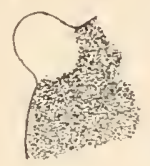

A

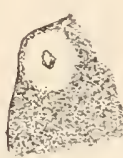

B
Fig. 490.

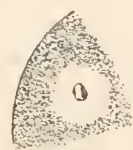

C

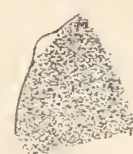

D

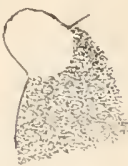

E

ACRASPEDINA (Type morphologique) (Sch.). Absorption d'une proie.

$A$, particule alimentaire arrivant à la vaeuole d'attente; $B$ et $C$, lit particule est englontie dans la vaeuole et la saillie de la paroi du eorps disparait; $D$ et $E$, une nouvelle vacuole d'attente se reforme.

celle-ci s'ourre, se referme sur lui et, s'enfonçant dans le corps $(B)$, l'entraine dans la profondeur $(C)$, landis qu'une nouvelle vacuole d'attenle $(D, E)$ vient prendre sa place à la base du flagellum. Ajoutons que la vésicule pulsatile occupe ici la partie supérieure du corps, tandis que le noyau est à la partic inférieure.

L'animal vil dans les infusions et les eaux croupissantes où abondent les Bactéries qui forment sa principale nourriture.

\section{GENRES}

Mastigamœba (F.-E.Schulze)(fig. 49I). Ce Flagellé se présente, quand il nage,

Fig. 491.

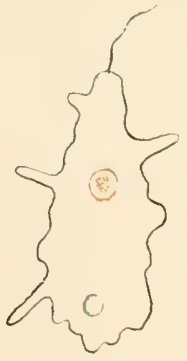

Mastigamaba (Sch.). sous un aspect très ordinaire, très normal pour un animal de cetle classe. Il a un seul fouel, et son corj's piriforme a un contour parfaitement lisse et régulier. On remar'que seulement qu'il ne capture point d'aliments. Puis, à un moment donné, on le voit ralentir sa marche, se déformer irrégulièrement, pousser de vrais pseudopodes irréguliers, ramifiés et se mettre à ramper absolument comme un Amibe. Non seulement il ressemble à un Amibe par sa forme, mais il agit comme lui, saisit des particules alimentaires arec ses, pseudopodes et les englobe en même temps qu'une petite quantité d'eau formant vacuole alimentaire autour de la particule ingérée. En un mot c'est un Amibe, sauf le flagellum qui persiste, quoique inerte, pendant cette métamorphose. Puis, au bout de quelque temps, il rentre ses pseudopodes, reprend sa forme régulière et se lance à la nage avec son fouet. C'est là sa vie : alternativement Rhizopode pour ramper et manger, Flagellé pour nager, il forme entre ces deux classes un lien des plus curieux (Atteint 0,1. Eau douce) (").

(1) Cela devient plus évident encore si l'on pense à certains Foraminilères qui ont un vrai pseudopode, comme Podostoma filigerum qui peut-ètre mème serait mieux à sa place ici. Cie Mastigamála a un beau noyau résiculeux en haut, une vésicule pulsatile en bas. On ne connait pas sa reproduction. 
Dimastigamœba (Blochmann) (fig. 492) est un genre voisin qui se distingue principalement par la présence de deux llagellums à peu près égaux dont l'un est souvent dirigé en Fig. 492. arrière (").

Dimorpha (Gruber, emend. Blochmann) (fig. 493) se distingue des précédents par le fail que, sous sa forme Rhizopode, il revêt l'aspect d'un Héliozoaire et fait netlement le passage à ce groupe. Les pseudopodes non seulement sont rayonnants, très fins, bien détachés du corps, mais possèdent un filament axial qui se prolonge jusqu’à un petit corps qui occupe, au centre du noyau, la position d'un nucléole. Les deux fouets sont permanents, même pendant l'état Héliozoaire, et se continuent aussi jusqu'au même point à l'intérieur du noyau. Pen-

Fig. 493 .

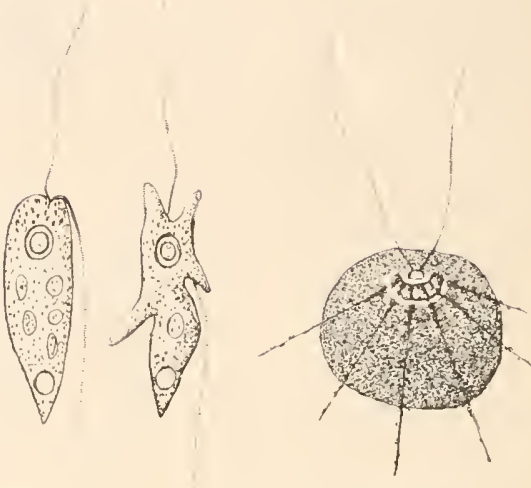

Dimorpha

(d'ap. Blochmann).

Dimastigamaba

(d'ap. Klebs). dant l'état Flagellé, on peut encore distinguer dans le corps des stries radiaires qui indiquent la persistance des filaments axiaux des pseudopodes. Les deux fouets sont toujour's dirigés en haut. 11 y a plusieur's vésicules contractiles (Eau douce).

Mastigophrys (Frenzel) peut être défini : un Ciliophys (V. p. 163) qui posséderait un flagellum unique, permanent, situé entre des pseudopodes ciliophrydiformes, permanents aussi. Il est entouré d'une mince couche gélatineuse protectrice. La présence de ce fouet permanent nous oblige, malgré ses caractères d'Héliozoaire, à faire de cet ètre un lilagellé $\left({ }^{*}\right)$.

Tripanosoma (Gruby) (fig. 494). Sa forme est d'ordinaire celle d'une languetle un peu épaisse, souvent arquée en croissant. Mais, en raison de sa mobilité, il est difficile de la

(1) L'animal se nourrit aussi pendant la marche à la manière d'une Monade (roir plus bas) el, pendant le repos, devient tout à fait amœboïde et se comporte comme un Mastigamaba. Il se divise comme l'Amibe a l'état de repos ou longitudinalement à l'état libre, et parfois se multiplie sous un liyste.

Il fait passage aux Heteromastigidx par son second fouet dirigé en bas.

Ces deux genres constituent la famille des RHizom-4stiaLA

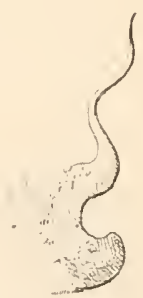

Tripanosoma (im. Lankester). [Rhizomastigina (Bütschli)] à laquelle Bürschli adjoint, à titre d'appendice, le curieux genre Mastigophrys (V. plus loin).

(2) Sans ce caractère il appartiendrait aux IIéliozoaires avec lesquels il a de grandes affinités. Au contraire, nous ayons laissé parmi les Héliozoaires les genres Ciliophry's et Actinomonas qui n'ont ce fouet que temporairement (V. p. 162). 
préciser. Le long de l'un des bords (le convexe s'il y en a un) règne une haute membrane ondulante qui se termine en haut par un flagellum. Ce flagellum peut disparaître par moments ainsi que la membrane et l'animal deviendrait alors amøboïde, mais la chose n'est pas absolument certaine (Jusqu'à $80 \mu$. Parasite dans le sang de diver's Vertébrés (Batraciens, Poissons, Tortues), et dans le tube digestif de divers Invertébrés (Ostrea, Tapes (sur la tige cristalline), Pontoldella, Pyxicola) ou Vertébrés (Poule, Oie, Canard, dans le cœcum). Cercomonas (Dujardin) (fig. 49:3) a la forme d'un ovoïde pointu aux deux bouts. L'extrémité supérieure porte le flagellum, l'inférieure se prolonge en un long appendice caudal. lci, tout redevient conforme à notre type morphologique en ce qui concerne la bouche et la capture des aliments; mais il reste une trace sensible du caractère amœboïde des formes précédentes dans le prolongement caudal et les parties voisines. Celles-ci prennent à certains moments $(B)$ le caractère de vrais pseudopodes. Cependant ce n'est là, ni une nécessité pour l'animal, ni mème une condition habituelle. C'est seulement au moment de la conjugaison qu'on le voit développer beaucoup cette partie amoboïde et s'en servir pour se souder à son conjoint $\{60 \mu$. Infusions d'eau douce et tube digestif Fig. 495 . de l'Ilomme, peut-être plus spécialement dans certaines diarrhées, mais seulement dans les points où la réaction est alcaline) (").

(1) Genres voisins :

Herpetomonas (Kent) (fig. 496), est dépourvu de prolongement caudal, à l'extrémité inférieure du corps, il est encore amœboïde, mais sensiblement moins; sa forme est très allongée;

Ancyromonas (Kent) (fig. 497), a les mêmes caractères, mais est piriforme $(6 \mu$. Mer);

Oikomonas (Kent) (fig. 498), est de même, mais presque sphérique et peut se fixer par son extrémité infẻrieure étirée en filaments ( 4 à 16 p. Eau stagnante et infusions);

Thylacomonas (Chevialiof) n'est plus du tout amøboïde et a son fouet implanté sur le còté latéral d'une légère excavation dont le fond n'est point une bouche (22 «.. Eau stagnante);

Phyllomonas (Klebs) (fig. 499) se distingue des précédents par sa forme singulière qui peut se comparer à une feuille triangulaire, à demi ployée et par son flagellum inséré et dirigé en arrière par rapport au sens de la progression ( 6 à $7 \mu$. Eau stagnante).

Ces êtres forment la famille des CELicoso
Fig. 498.

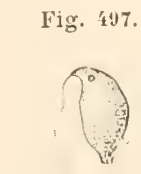

Ancyromonas (A. sigmoides) (d'ap. Kent).
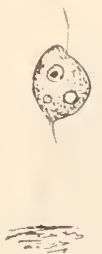

Oikomonas

(O. termo) (im. Kent).
Fig. 496.

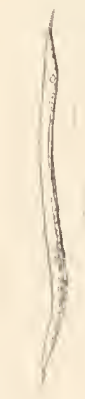

Phyllomonas (d'ap. Kent).

Herpetomonas

(II. musca-

domestica) (d'ap. Kent). 
Codonœca (J. Clark) (fig. らّ00) peut être comparé à un Oikomonas qui se serait sécrété une logette gélatineuse, en forme d'urne élégante, fixée par un pédoncule, el assez large pour que son corps ne la remplisse pas entièrement (14 $\mu$. Mer et eau douce) (').

Bicosœca (J. Clark) (fig. כ̈01) présente un caractèse noureau : c'est un prolongement aplati de l'extrémité supérieure, auquel on a donné le nom de péristome. Le flagellum part de la base du péristome et l'ingestion des aliments a lieu entre la base du llagellum el celle du péristome, ou

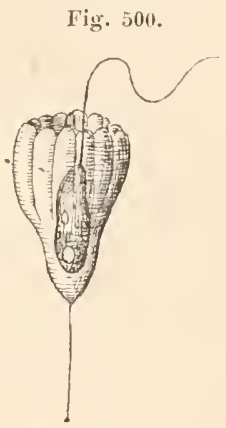

Codonaca (C. costata) (d'ap. Clark). à la face de celui-ci qui regarde le flagellum. L'animal se sécrète une capsule transparente, de forme ovoïde, juste suffisante pour le contenir. Il est fixé dans sa capsule par un pédoncule qui traverse celle-ci et se fixe au sol; il peut resserrer l'entrée de sa capsule lorsque, effrayé, il se rétracte à son intérieur (12 à $15 \%$ \%... Her et eau douce) $\left({ }^{2}\right)$.

Proteriodendron (Stein) (fig. 302) est un Bicoseca ì péristome beaucoup plus développé et qui forme des colonies résultant de ce que les jeunes restent fixés par leur pédoncule soit au pédoncule de leurs parents, soit à la paroi largement évasée de leur capsule $\left({ }^{\mathbf{3}}\right)$.

Monas (Ehrenberg, emend.) (fig. כ̋03)

Fig. 503.

nous ramène à une forme sans capsule el très voisine d'Oiliomonas par exemple; mais il s'en dis-

(1) Cette forme est fort incomplètement décrite.

Platytheca (Stein) (fig. 504) semble devoir prendre place auprès d'elle bien que son flagellum court et immobile n'ait guère le caractère de celui d'un Flagellé.

Ces deux genres for-

$$
\text { Fig. 504. }
$$

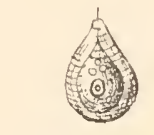

Platytheca

(P. micropora) (Stein).

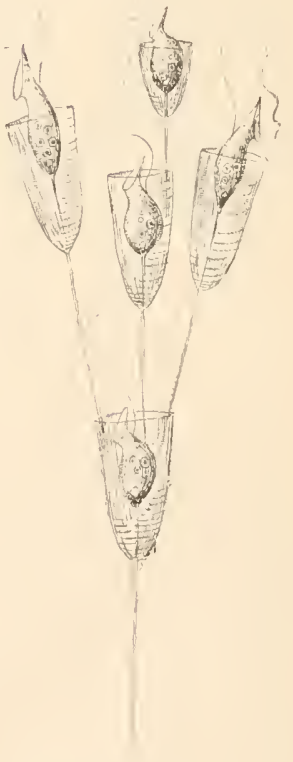

Fig. 502.

menl la famille des Codoractise [Codonacida (Kent)].

(2) Genre voisin :

Hedræophysa (Kent), à capsule sessile.

(3) Ces trois genres forment la famille des Butacta: [Bilicecina (Stein)]. 
tingue par la présence d'un ou deux petits fouets accessoires ì la lase du foutet principal ( $30 \mu$. Eau douce et peut-être mer) ( $\left.{ }^{2}\right)$.

Le genre libre Monas, est le chef de file d'une petite série de formes qui ont un fouet accessoire. mais sécrètent un pédoncule par lequel ils se fixent et s'associent en colonies (fig. $303 \ddot{)})$. Le fouel accessoire est inséré au pied du fouet principal et, au côté opposé de celui-ci, le corps se prolonge en une sorte de rostre assez accusé. Le lieu d’ingestion est à côté du fouet accessoire.

La colonie résulte toujours de la division d'un individu qui est lui-même fixé par un pédoncule $(A)$ et, en se divisant, se fend de haut en bas jusqu'à la

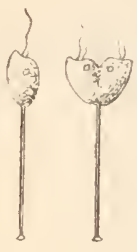

A $-B$
Fig. 505.

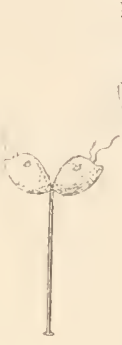

$\mathrm{C}$

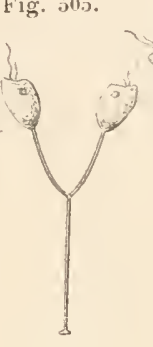

D

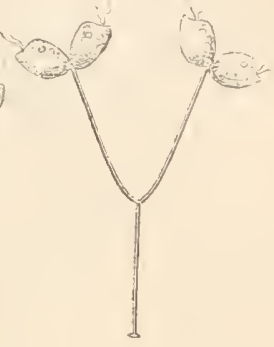

E

Formation de la colonie chez un Monadien colonial (Sch.). naissance de celui-ci $(B$ et $C$ ). Les deux individus ainsi formés allongent leur pédoncule $(D)$ et ainsi s'écartent l'un de l'autre, puis se divisent $(E)$, et ainsi de suite. On comprend que, suivant les rapports de vitesse de la division et de l'allongenent des pédoncules, les colonies prendront des formes variées. Ces formes permellent de distinguer trois genres:

Dendromonas (Stein) (fig. :̈06), qui forme des arbuscules régulièrement dichotomiques sur lesquels les individus sont situés côte à côte, tous à la mème hauteur (18 $\mu$. Eau douce).

Anthophysa (Bory de Saint-Vincent) (fig. 307 ), qui forme des capitules sphériques

(1) La Monade peut se déformer par des bosselures amoboïdes, mais cela ne va pas jusqu'à former des pseudopodes. Quelquefois, cependant, elle peut émettre un filament par lequel elle se fixe, et ce filament ne peut guère être interprété que comme un long pseudopode filiforme. Il existe, ici et chez quelques autres genres, une petite ligne brillante située

Fig. 506.

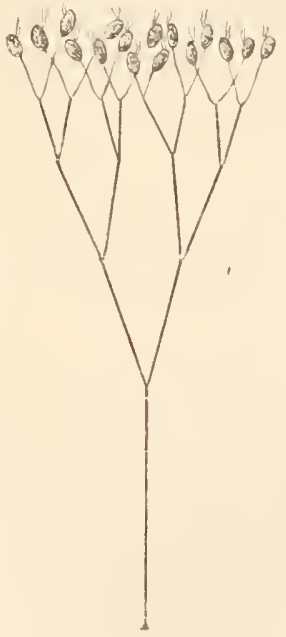

Dendromonas (Sch.). Anthopliysa (A. segetans) (im. Kent).

sous la base du fouet et que l'on appelle la bandelette buccale. Fraxzé a montré que ce n'est là qu'une rangée de grains d'amidon représentant morphologique- 
de cinquante à soixante individus sessiles au sommet de longs pédoncules dichotomiques dont les parties anciennes sont colorées en brun par de l'oxyde de fer $(30 \mu$. Eau douce); et

Cephalothamnium (Stein) (fig. כ08), qui forme des groupes d'importance différente d'individus sessiles au sommet de pédoncules raides et courts ramifiés une ou deux fois seulement (20 $\mu$. Eau douce) (').

Supposons un Monas chez lequel le fouet accessoire serait devenu égal au fouet principal, à tel point qu'on ne saurait l'en distinguer, ce sera

Amphimonas (Dujardin) (fig. 509) qui, en outre, est fixé par un pédoncule dont il peut se détacher à l'occasion pour nager librement ( $12 \mu$. Ner et eau douce) $\left({ }^{2}\right)$.

Imaginons maintenant que des Amphimonas sécrètent une substance gélatineuse capable de se solidifier autour d'eux de manière à les réunir en colonies et nous obtiendrons une petite série de genres, faciles à dis-

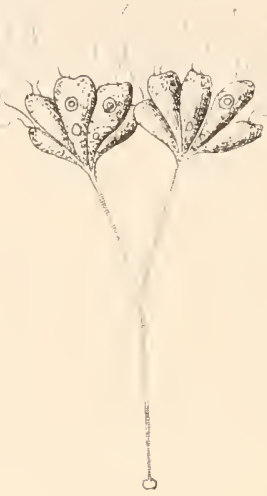

Cephalothamnium (d'ap. Kent).

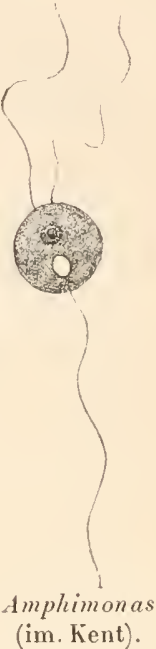

(im. Kent). tinguer d'ailleurs par la forme que prend cette sécrétion en se modifiant. Chez

Spongomonas (Stein) (fig. 510), c'est une masse gélatineuse commune dans laquelle les individus sont englués côte à côte, ne laissant sortir que leurs deux fouets $\left(^{\boldsymbol{3}}\right.$ ) Atteint $10 \mathrm{~mm}$. Eau douce). Chez

ment le stigma des Flagellès colorés, mais qui n’a ici aucun rôle dans la perception de la lumière ou de la chaleur. Genres voisins :

Sterromonas (Kent), qui est un peu douteux, et

Arhabdomonas (Fisch), qui n'a qu'un fout accessoire.

(1) Ces trois genres forment la famille bien naturelle des DEYDRowroxaDINE [Dendromonadina (Stein)].

Fig. 510.

(2) Il y a de plus cette différence que la vacuole nutritive peut se former en n'importe quel point du corps. Comme Monas, Amplimonas est un peu amoboïde. Les deux fouets sont parfois assez écartés l'un de l'autre. Le genre

Dinomonas (Kent) n'est peut-être pas très bien ici à sa place, car il paraît avoir une bouche préformée bien que non visible lorsqu'elle ne fonctionne pas.

Ce genre forme, avec Amphimonas, la famille des AMPHMONADINA [Amphimonadina (Kent)]. Mais on lui adjoint d'ordinaire diverses formes, Psendospora et autres que nous arons mises avec Ciliophrys et Actinomonas parmi les Héliozoaires (V.p. 163).

${ }^{(3)}$ Cette masse peut d'ailleurs revêtir les formes les plus diverses : sphérique, discoïde, cylindrique ou lobée de façons diverses. 
Cladomonas (Stein) (fig. 511 ), elle forme le petits tubes ramifiés et les individus sont logés au bout des branches où ils peuvent s'enfoncer plus ou moins ou sortir presque entièrement au dehors ( $75 \%$.. Lau douce). Chez

Fig. 511.

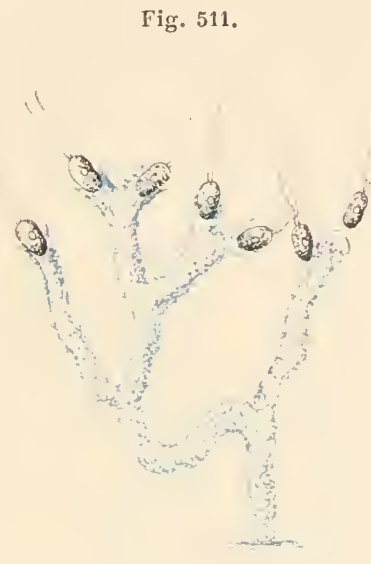

Cladomonas (C. fruticulosa) (im. Stein).

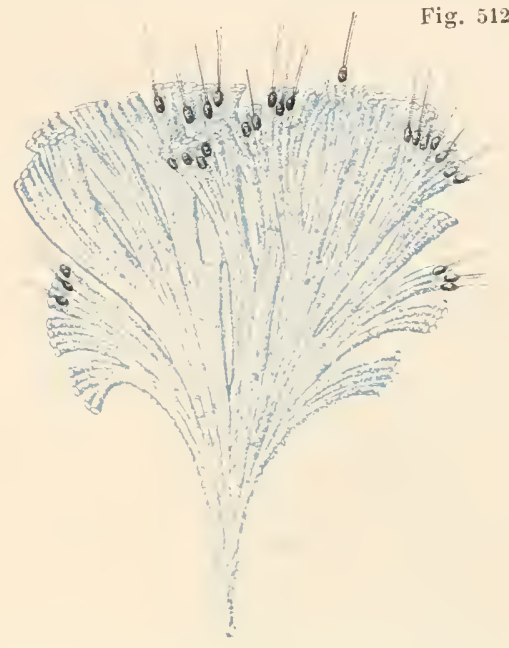

Rhipidodendron (R. splendidum) (im. Stein).

Rhipidodendron (Stein) (fig. 5̋l2), ce sont aussi des tubes, mais disposés côte à côte de manière à former une sorte d'éventail découpé (Eau douce. La colonie atteint jusqu'à $3^{\mathrm{mm}}$ de haut) (').

$$
\begin{aligned}
& 2 \text { Tribu } \\
& \text { CRASPÉDINES. - CRASIEDINA } \\
& \text { vel CIIOANO-FLAGELLÉS. - CIIOANO-FLAGELLINA } \\
& \text { [CRASPEDOMONADIA (Stein); - ChOANoflaGelLata (lient); } \\
& \text { CYLICOMastiges (Bütschli)] }
\end{aligned}
$$

\section{TYPE MORPHOLOGIQUE}

(FIG. 514 A 520 )

En tout ce qui concerne la constitution générale du corps, le Choano-flagellé est semblable au Flagellé ordinaire représenté par le type morphologique précédent. Bien qu'il n'y ait pas de mourements amoboïdes, les téguments sont si délicats qu’il est à peine permis de parler d'ectoplasme et de membrane; il n'y a guère qu'un léger raffermissement

(1) C'est avec doute que l'on place ici le genre Diplomita (Kent) (fig. b̈l3) que l'on peut définir : un individu de Spongomonas vivant isolé dans une loge de Bicosirca avec un pédoncule comme celui-ci (13 $\mu$. Eau douce).

Fig. 513.

Ces genres forment la famille des SPoxgomoxaDIxE [Spongomonodina (Stein)]. 
progressif de la surface du corps; le noyau est petit el silué en haut sous le flagellum; la vésicule pulsatile, souvent double, occupe au contraire

Fig. 514.

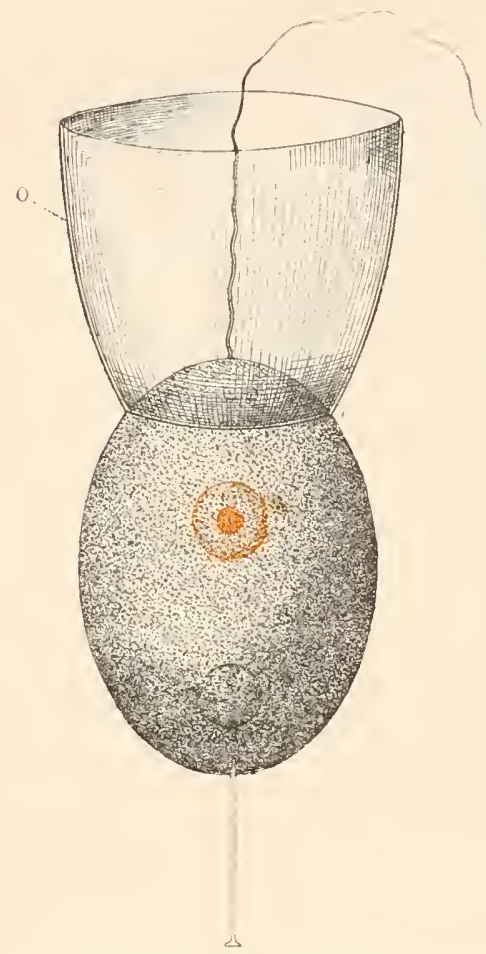

CRASPEDINA (Type morphologique) (Sch.). c., eollerette dilatée.
Fig. 515.

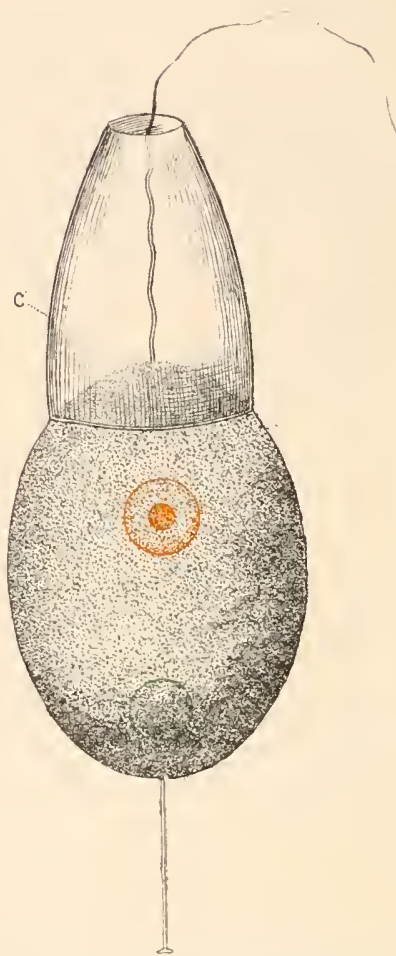

CRASPEDINA (Type morphologique) (Sch.). c., collerette eontraetée.

la région inférieure; en bas, le corps se prolonge en un pédoncule fixé à quelque support et qui est formé, non par une sécrétion, mais par une substance protoplasmique plus ou moins modifiée.

Mais ce sont là des particularités secondaires, de même nature que celles qui caractérisaient simplement les genres dans le groupe précédent. Ce qui donne aux Choano-flagellés un caractère à part et permet d'en faire une tribu ('), c'est un appareil singulier dont est munie l'extrémité supérieure de leur corps ; on l'appelle la collerette (c.). Que l'on se figure une sorte de cône tronqué dont la grande hase tournée en haut est libre, tandis que la petile base s'insìre à l'extrémité supérieure du corps, sur une ligne circulaire, à quelque dislance au-dessous du flagellum. Ce dernier est naturellement contenu dans la collerette,

(1) Bütschi en fait comme Kext un ordre, ce qui nous paraît exagéré et Klebs une simple famille ce qui nous semble insuffisant. 
mais il la dépasse de beaucoup, car il est deux ou trois fois plus long que le corps, landis que la collerette n'atteint que tout au plus la hauteurde celui-ci. La collerette est extrèmement mince et transparente; elle n'est pas une sécrétion inerte, mais une émanation protoplasmique du corps. Elle est mobile, non entraìnée, comme le croyail Kent, dans un mouve-

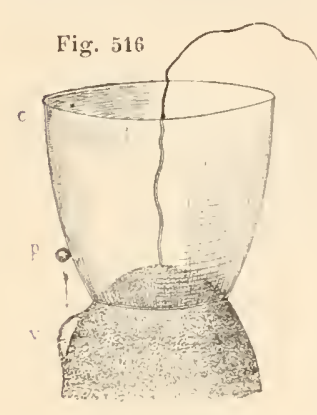

CR.ISPEIIIVI

(Type morphologique). Préhension des aliments (Sch.). ment tourbillonnaire, mais souvent animée d'un frémissement viluratoire et capable de s'étendre, de s'élargir (fig. $\breve{l} 4, c$ ) ou, au contraire, de se contracter et de se réduire à un petit tube conique (fig. $\ddot{l} \check{b}, c$ ) dont la partie étroite est tournée en haut. Elle peut mème, dans certaines conditions, rentrer entièrement dans le corps, comme un pseudopode. mais c'est là un fait exceptionnel qui ne se produit qu'à de rares intervalles (dans l'enkystement ou dans certains cas de division) et non à chaque instant et à volonté, comme la rétraction. L'animal fait tourbillonner l'eau arec son fouct et détermine un courant d'eau ascendant qui entraìne les particules situées au-dessous de la collerette et les précipite contre la face externe de cet organe oì elles se collent et sont peu à peu entraînées rers le bas (fig. 3̈l6). Là, se trouve une grosse vacuole d'attente (v.), vide par conséquent, qui semble faire incessamment le tour du corps un peu au-dessous de l'insertion de la collerette $(c$.$) . La particule alimentaire (p$. arrive nécessairement à la rencontrer et dès qu'elle l'a touchée, d'un mouvement brusque, elle est engloutie. La vacuole plonge alors et, devenue simple vacuole alimentaire, entraine la particule dans la profondeur du corps où elle est digérée, tandis qu'une nouvelle vacuole d'altente prend sa place à la base de la collerette.

La sortie des résidus de la digestion se ferait par l'extrémité supérieure, à l’intérieur de la collerette $\left({ }^{\mathbf{1}}\right)$.

La division longitudinale (fig. .51S), chez notre

(1) Kext croyait que les particules entraînėes dans le prétendu mourement tourbillonnaire de la collerette montaient d'abord sur la face externe, puis descendaient à la face interne pour être absorbées par un point de la partie du corps renfermé à son intérieur. Mais les observations nouvelles ont démenti cette interprétation.

II $y$ avait aussi une interprétation particulière de Extz, sur la nature vraie de la vésicule d'attente et nous n'en aurions peut-être pas parlé si tout récemment elle

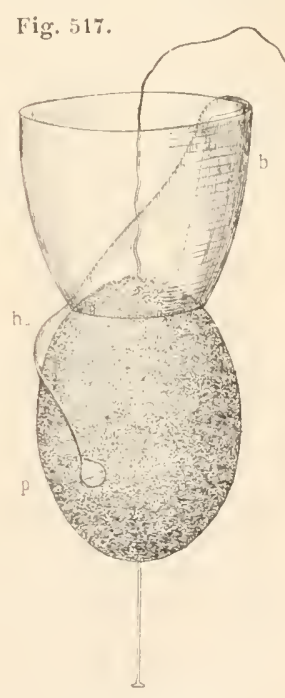

CRASPEDIX.A

(Type morphologique). Collerette en oublie (Sch.). n'avait èté reprise par Franzé [9i], et il n'est pas impossible que cette nouvelle manière de voir les choses soit la vraie et se substitue finalement à celle que nous arons 
Choano-flagellé, est quelque peu modifiée par les particularités de l'extrémité supérieure. L'animal commence par résorber son flagellum, puis il se fend à la partie supérieure de son corps, en sorte qu’à un certain moment, il a un corps simple en bas, bifide en haut, avec deux

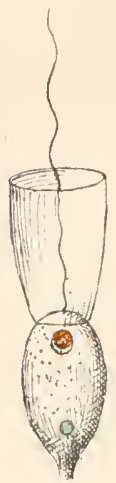

A

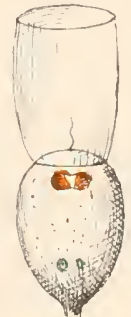

B

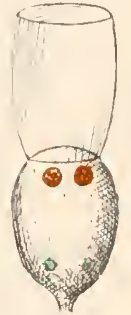

C
Fig. 518.

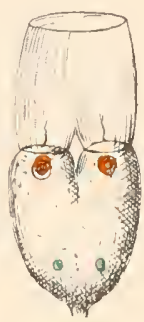

D

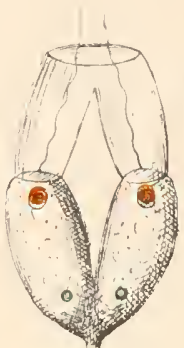

E

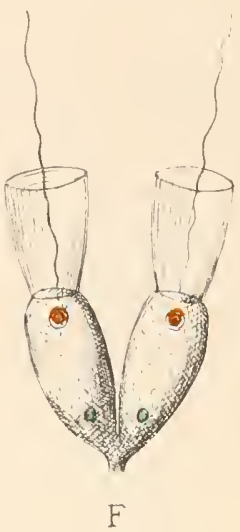

F

CRASPEDINA (Type morphologique). Division longitudinale (Sch.). $A$ à $F$, stades successif's de la division.

colleretles distinctes en bas, fusionnées en haut et ne présentant qu'un seul orifice. Comme les flagellums se sont régénérés à ce moment, on les voit sortir tous les deux par l'orifice commun. La division s'achève ensuite d'abord, pour la collerette, puis pour le corps, jusqu'au pédoncule.

La division transversale est moins rare ici que chez les Monadines sans collerette (').

exposée. La collerette ne serait pas fermée latéralement comme un vrai cône, mais fendue à la manière d'une oublie ou plutôt d'un cornet de papier (fig. 517), la partie qui double sur l'autre étant intimement appliquée sur celle-ci. $\Lambda$ la base, cette partie qui double sur l'autre, au lieu de s'arrêter à l'insertion sur le corps, s'étendrait vers le bas et c'est cette partie située au-dessous de la base d'insertion qui, à demi déroulée, formerait une saillie marginale $(h)$ et donnerait l'illusion de la vacuole d'attente. Cette partie saillante, formant une sorte de cuiller', recueillerait les particules alimentaires amenẻes sans doute jusqu'à elle par un mouvement gyratoire, el les conduirait par un prolongement de sa partie inférieure jusque vers la partie inférieure lu corps où une vacuole alimentaire $(p)$ les recevrait. Cette vacuole aurait souvent été vue, mais méconnue et prise pour une deuxième vésicule pulsatile. L'avenir dira ce qu'il faut croire de ces interprétations.

La sorlie des résidus de la digestion se ferait, non pas par l'extrémité supérieure, mais au contraire par un point quelconque de la surface, à l'exception de celui-là.

(i) Elle s'observe chez des formes sans pédoncule. L'individu supérieur garde la collerette ancienne ou parfois la régénère. L'inférieur s'en forme une nouvelle un peu à côté du pôle inférieur. 
L'enliystementse produit dans les conditions ordinaires (fig. つ̆l9), après disparition du flagellum et de la collerette. Il aboutit fréquemment à une

Fig. 519.
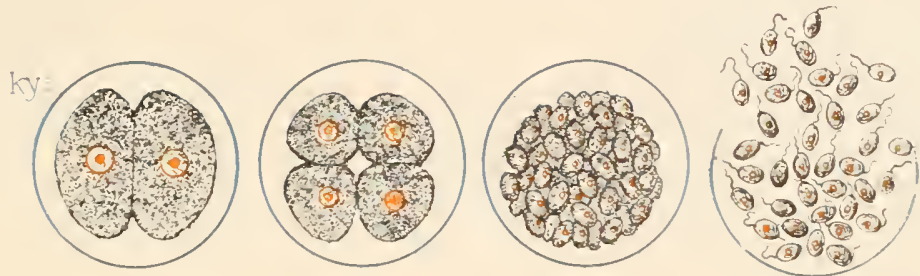

CRASPEDINA (Type morphologique).

Division dans un kyste et sporulation (Sch.).

$F$, première division dans le kyste, donnant naissance à deux masses cellulaires; $K$ à $L$, les deux masses filles continuent à se diviser;

$M$, sortie des produits de la division.

sporulation. Le contenu du krste se divise en nombreux fragments nucléés qui sortent, munis seulement d'un flagellum, puis se fixent, forment leur collerette et n'ont plus alors qu'ì grandir (").

On avait cru olserver une conjugaison dans laquelle un individu libre se serail soudé par sa base au flane d'un individu fixé (fig. 320 ). Nais, après vérification, il se trouve qu'il n'y a lì qu'un fait de division anormale qui cependant s'achève normalement, la ligne de séparation arrivant, à la fin, jusqu'au pédoncule.

Eufin, il arrive que l'animal peut quitter son pédoncule et nager quelque temps en liberté pour changer de place et se fixer ailleurs. Or on observe qu'en nageant, à l'inverse des autres Flagellés, il avance, le cil en arrière $\left({ }^{2}\right)$.

Fig. 520.

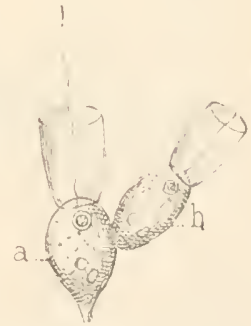

CRASPEDINA

(Type morphologique). Dirision anormale

(Sch.).

Tous ces caractères sont en somme fort constants dans le groupe

(1) Chez les formes pédonculées le kyste reste adhérent au sommet du pédoncule; chez celles qui ont une capsule, il reste dans la capsule.

$\left({ }^{2}\right)$ Ce n'est là, sans doute, que la régularisation d'un mode de natation que nous avons vu exister chez les autres Flagellés lorsqu'ils veulent reculer rlevant un obstacle. Ici l'obstacle est permanent, c'est la collerette qui serait extrêmement genante dans la progression en avant. L'animal cependant conserve à l'état de repos le même mouvement du flagellum qui le faisait, à l'état libre, progresser à reculons puisque nous avons vu que son courant d'eau alimentaire est dirigé de bas en haut, ce qui implique une réaction sur le corps en sens inverse. 
très naturel des Choano-flagellés. Il n'y a guère de variable que le pédoncule qui peut exister ou manquer, les cupules que la plupart sécrètent autour l'eux pour s'y abriter et la Fig. $521 . \quad$ Fig. 522. forme des colonies qui résultent, chez le plus grand nombre d'entre eux aussi, d'une séparation incomplète des individus nés de la division.

\section{GENRES}

Monosiga (Kent) (fig. 521). C'est exactement notre type morphologique. Il est donc solitaire, sans capsule, pédonculé 7 à 8 p. et 35 à $40 \mu$ y compris la collerette et le pédoncule moyennement développé. Ner et eau douce.)

Codosiga (Kent) (fig. 522) ne diffère du précédent que parce qu'il forme des colonies où les individus sont groupés par bouquets au sommet de longs et minces pédoncules (Colonies, 60 p.. Ner et eau douce) (').

Hirmidium (Perty) (fig. 523) est formé d’individus sans pédoncule, réunis côte à côte par le flanc en une série

Fig. 523.

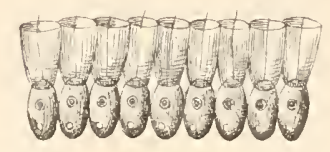

Hirmidium (im. Kent).

longitudinale. La colonie comprend une dizaine d'individus et se meut sous l'action synergique des fouets de ces individus (15 p... Ner et caudouce) $\left(^{2}\right)$.

(1) Genres roisins :

Astrosiga (Kent) ressemble absolument à un bouquet terminal de Codosiga détaché, et peutêtre n'est-il rien autre chose;

Codonocladium (Stein) (fig. 5224) peut être défini : une colonie de Codosiga réunis par les extrémités de leurs pédoncules sur un pédoncule commun (Colonie, 50 à 260 p.. Ner et eau douce).

$\left.{ }^{2}\right)$ Quand la colonie devient trop nombreuse, elle se scinde en deux.

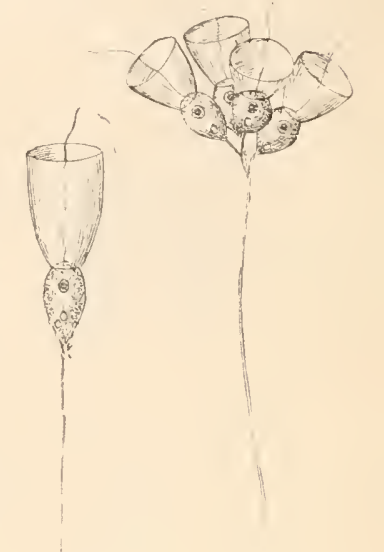

Monosiga Codosiga (im. Kent). (im. Kent).

Fig. 524.

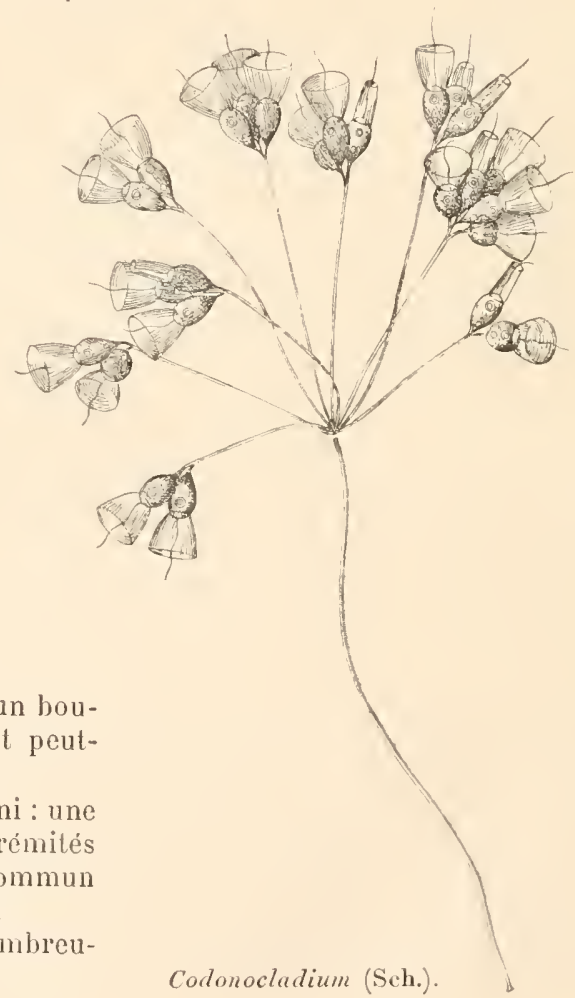


Sphærœca (Lauterborn) est formé d'individus sans loge, pédonculés, noyés, sans ètre unis par leurs pédoncules, dans une substance gélatineuse commune (Colonies, 12 à $200 \mu$. Etangs).

Diplosiga (Frenzel) (fig. 52つ) se distingue des précédents, auxquels il ressemble sous les autres rapports, par le caractère remarquable d'avoir deux collerettes concentriques (10 p.. Eau douce).

Protospongia (Kent) (fig. 526). Ce sont encore des individus semblables à ceux de Hono-

Fig. 525 .

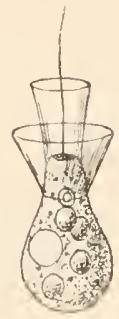

\section{Diplosiga}

(D. socialis)

(d'ap. Frenzel).
Fig. 526 .

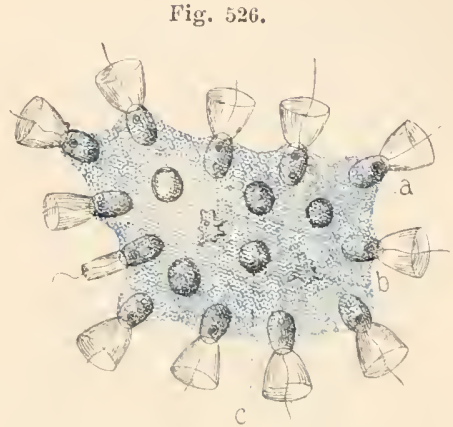

Protospongia (Sch.).

siga, mais sans pédoncule, et logés par 30 à 60 dans une masse commune d'une substance gélatineuse transparente qu'ils sécrètent /Colonie, 0,1. Eau douce) (").

Salpingœca (J. Clark) (fig. 527). L'animal ne diffère pas de ceux des genres précédents, mais il possède, en plus, une capsule fixée à quelque support par un pédoncule et dont la forme, variable el le plus sourent très élégante, se laisse ramener à une sorte d'urne renflée en bas avec un goulot létréci à sa naissance et évasé à sa terminaison. Il ne forme pas de colonies (Environ $30 \mu$ avec la capsule. Ner et eau douce! ( $\left.{ }^{2}\right)$.

Polyœca (Kent) (fig. ร̌2S) est un Sal-

Fig. 527.

Fig. 528.
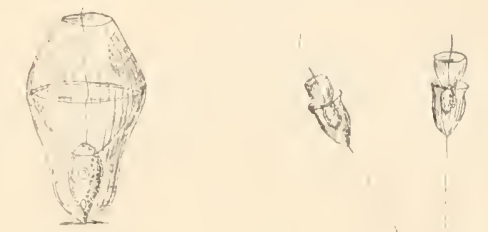

Salpingacia

(S. ampulla)

(d'ap. Kent).

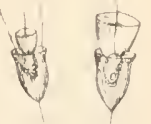

pingceca qui se distingue de celui-ci par le fait qu'il forme des colonies: les jeunes issus de la division se fixant par leur pédoncule aux bords de la capsule des individus anciens $\langle\mathrm{Mer}\rangle$.

(1) La colonie est discoïde et les individus, dont le nombre peut atteindre une soixantaine, $y$ sont irrégulièrement distribués et jouissent d'une certaine mobilité dans la masse. Ils peuvent passer à un état amoboïde et rentrer au centre de la colonie pour s'y enkyster et sporuler. Ce genre a une certaine célébrité parce qu’il avait été donnè comme fournissant le passage des Protozoaires aux Spongiaires, théorie abandonnée aujourd'hui.

$\left(^{2}\right)$ Mais les proportions du goulot par rapport à l'urne et sa forme établissent des diffẻrences spécifiques assez importantes. L'animal est d'ordinaire, mais pas toujour's, relie au fond de sa capsule par un ligament contractile. Il peut quelquefois se détacher et nager avec sa capsule en pleine 
La grande homogénéité de structure de toutes ces formes n'est un peu altérée que par un dernier genre,

Fig. 529.

\section{Phalansterium (Cienkovsky)}

(fig. 529) chez lequel la grande collerette mobile des autres types est remplacée par une étroite collerette tubuliforme fixe et semblable à ce que sont celles des autres genres dans leur état de rétraction maxima.

L'animal forme des colonies discoïdes ou arborescentes, constituées par des tubes gélatineux sécrétés par les individus et soudés entre eux de diverses façons, mais jamais ramifiés (Colonies, environ 0,13 p.. Eau douce) (').

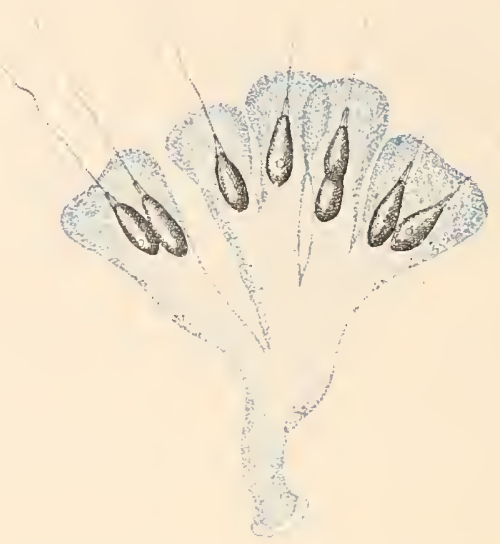

Phalansterium (P. digitatum) (d'ap. Stein).

2 Sous-Ordre

IIÉTÉROUASTIGIDES. - HETEROUASTIGID.E'

[BODONINA (Bütschli emend.)]

TYPE MORPHOLOGIQUE

(FIG. 530)

Revenons au type morphologique des Monadida (V.p. 319). Nous aurons, pour le transformer en type de ce groupe, à lui faire subir les mèmes modifications que pour obtenir le type des Acraspédines. C'est la même structure du corps, avec un tégument très délicat permettant des déformations presque amaboïdes, la même absence de bouche; le noyau et la vésicule pulsatile sont à la même place, le premier vers la partie inférieure, la seconde vers le haut. Le caractère essentiel réside dans la structure de l'extrémité supérieure et dans les fouets.

eau et l'on a voulu faire de cette forme libre un prétendu genre Lagencea (Kent).

Il se divise dans sa capsule, s'y enkyste, y spor'ule, en un mot ne s'en sépare jamais. On a observé chez certaines espèces une division transversale dans laquelle l'animal perd sa collerette et son flagellum el sépare de lui un individu amoboïde qui va plus loin se fixer et se transformer en un jeune salpingaca, tandis que l'autre individu garde la capsule et régénère un flagellum et une collerette.

(1) Quelques auteur's nient l'existence de cette collerette et placent ce genre parmi les Spongomonadines. 
Un peu au-dessous du pôle supérieur de l'animal, se trouve une encoche latérale, et du fond de cette encoche naissent deux fouets. L'un $(f l g . s$.$) est lirigé en haut, c'est le$ flagellum ordinaire. L'autre est donc le flagellum accessoire; il est cependant plus gros d'ordinaire que le principal et s'en distingue surtout en ce qu'il est dirigé vers le bas $(f l g . i$.$) . L'animal peut se servir de$ ce fouet accessoire pour se fixer temporairement: l'extrémité de cet appendice s'attachant à un support quelconque. Quand il nage lentement, il se sert seulement de son fouet principal, l'autre se laissant traîner inerte, et ne servant qu'à de rares intervalles à changer la direction au moyen de quelques secousses, à la manière d'un gouvernail ; mais quand il veut se hàlè les deux fouets contribuent également au mourement. Parfois aussi, il nage sous l'action de son fouet accessoire seulement. Il faut done que le mode d'action de ces deux fouets soit inverse, puisque l'un lire pendant que l'autre pousse. Le mode de

Fig. 530.

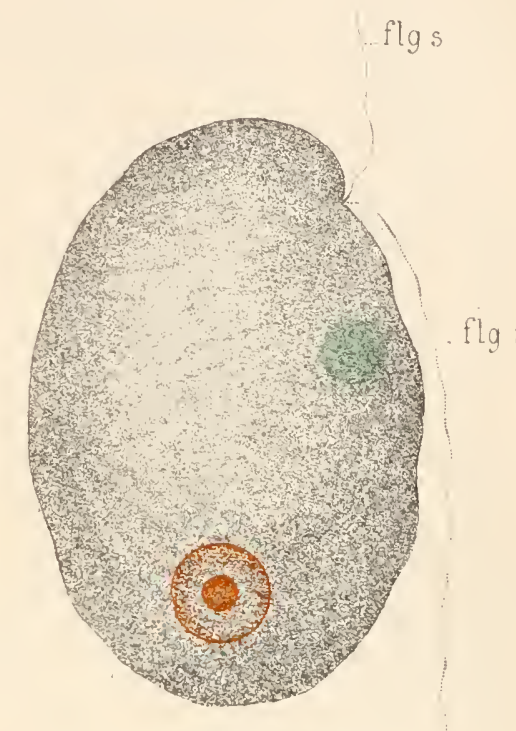

IIETERU.MASTIGID,E.

Type morphologique (Sch.).

11.. s., llagellum supérieur; $\mathbf{1 1}$ s. 1., flagellum inférieur. capture des proies est aussi très particulier. Le lieu d'élection pour l'absorption des aliments est exclusivement celle partie du corps qui surmonte les fouets à la manière d'un rostre. Là, la surface est glutineuse, molle, différenciée enfin de telle façon que les particules alimentaires qui viennent s'y coller sont retenues et aussitôt dégluties.

La division longitudinale, la sporulation sous un kyste ont élé observées ainsi que la conjugaison.

Fig. 531.

\section{GENRES}

Bodo (Stein) (fig. క33). C'est à peu près notre type morphologique. La parlie du corps située audessus des fouels est conformée en rostre et le noyau se trouve à peu près central $(20$ à 30 \%.. Eau douce et parlois mer (').

(1) Certaines formes peuvent passer à un état amœboïde. Certaines espèces vivent en parasites dans le tube

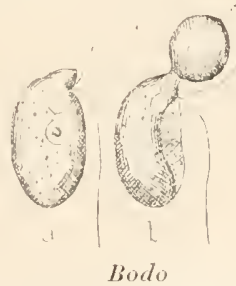

(B. edax) (d'ap. Klebs) a, un individu avec sou noyau et sa résicule pulsatile: b, un individu suegant une proie, 1\%. 
Phyllomitus (Stein) (fig. 3332) a ses deux fouets insérés dans une assez profonde fossette par laquelle a lieu la déglutition des aliments. C'est comme si le rostre de Bodo s'était élargi Fig. 532. et excavé (Environ 20 ... Eau douce).

Colponema (Stein) peut ètre considéré comme un Phyllomitus chez lequel un sillon, parti de la fossette buccale, parcourrail toute la face ventrale. Le fouel accessoire est logé dans ce sillon (30 p.. Eau douce).

C'est avec quelque hésitation que nous plaçons ici, avec Klebs, le genre si singulier

Oxyrrhis (Dujardin) (fig. 5333 ) conformé un peu comme Phyllomitus, mais chez lequel l'excavation buccale est

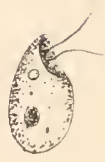

Phyllomitus (d'ap. Klebs).

très grande et s'enfonce en une sorte de pharynx dans le corps, presque comme chez les Euglènes dont il diffère d'ailleurs par Fig. 533.

Fig. 53't. ses téguments délicats. II se meut, le fouet en bas, à la manière d'un Dinoflagellé (30 p... Mer) (').

Trimastix (Kent) (fig. כ̈34). Le corps est piriforme, à extrémité supérieure très effilée. De cette pointe, part le fouet principal dirigé en haut.

Immédiatement au-dessous de celui-ci, naissent deux fouets accessoires, d'égale longueur, un peu plus pelits que le fouet principal, et dirigés l'un et l'autre en bas. Le côté droit du corps se développe en une sorte de membrane verticale, saillante en

Trimastix

(T. marina)

(im. Kent). avant, contre laquelle un des deux fouets accessoires se maintient

digestif de divers animaux (Lacerta, Grillotalpa et sa Fig. 535.

Fig. 536 . larve).

Pleuromonas (Perty) (fig. 533) est un Rodo réniforme dontles deux fouets sont assez écartés à leur base. chez

Rhinchomonas (Klebs) (fig. 536), le fouet principal est remplacé par une sorte de trompe très mobile. L'absorption de la nourriture se fait par la base de cette trompe, au-dessus du fouet accessoire.

(1) Les genres précédents, constituant la famille des Bodoxtre [Bodoniua Bülschli), Ueteromitide (Kent)],

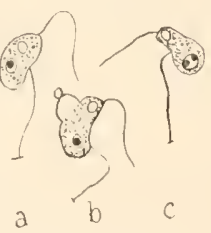

Pleuromonas (d'ap. Fisch).

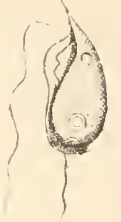
n'ont rju'un seul fouet accessoire.

Les suivants formant celle des ThIMASTIGLA [Trimastigina Blochmann] en ont deux. Its se distinguent absolument des Monades à deux fouets accessoires par le fait qu'ici, ces deux fouets sont diriges en bas, ce qui oblige à les incorporer aux Hétéromastigides. 
accolé. La vésicule pulsatile est placée très haut, imméFig. 537 . diatement au-dessous de la base des fouets (1\% $\mu$. Eau de mer croupie) (").

Il faut faire une place à part au genre

Costia (Leclercq) (fig. 537), chez lequel les trois fouets sont dirigés en bas et logés dans un sillon ventral pendant le repos, tandis que, pendant la natation, tous les trois sont libres et relevés vers le haut $(0,3$. Mer, eau douce, infusions) ( $(2)$.

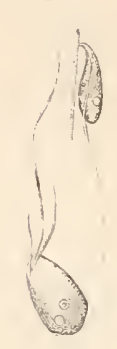

Costia

(C. necatrix) (d ap. Henneguy).

\section{POLYMASTIGIDES. - POLYHASTIGIDAE \\ [PoLymastigme (Bütschli, emend. Klebs)]}

\section{TYPE MORPHOLOGIQUE}

Il se caractérise uniquement par ses fouets, en nombre supérieur à trois. Les téguments sont délicats comme dans les groupes précédents, mais le noyau est souvent en haut, prenant la place de la vésicule qui se place à la partie inférieure. La position et la direction de ces fouets et les caractères de la bouche se montreront clairement dans les descriptions suivantes.

Fig. 538 .

Nous diviserons ce sous-ordre en quatre tribus :

AsTonINA, dépourvus de bouche;

MONOSTOMINA, avec une bouche unique;

DISTOMINA, à deux bouches;

TRICHONYMPIINA, formes aberrantes ayant des caractères de Ciliés.

(1) Genres voisins :

Dallingeria (Kent) (fig. 538), où il n'y a pas cette membrane et où les deux fouets accessoires s'insèrent plus latéralement et plus bas;

Elvirea (Parona).

$\left({ }^{2}\right)$ Il y en a un très grand et deux beaucoup plus petits. Le sillon, très dilatable, forme en haut une large excavation superficielle quand il est ouvert. Le noyau est au-dessus de la vésicule.

Le $C$. necatrix est le Bodo necator de Henneguy (20 à $30 \mu$ qui vit

$$
\begin{gathered}
\text { Dallingeria } \\
\text { (D. Dryselatii) } \\
\text { (d'ap. Dallinger } \\
\text { et Drysdale). }
\end{gathered}
$$
fixé sur l'épiderme des alevins de Truite (Trutta), chez lesquels il produit, quand il est abondant, une maladie mortelle. 
1 re Tribu

ASTOMINES. - ASTOMINA

[HOLOMASTIGINA (Lauterborn)]

TYPE MORPHOLOGIQUE

C'est un Polymastigide à nombreux flagellums et sans bouche préformée.

Ce groupe ne renferme qu'un petit nombre de genres placés auparavant en appendice aux Flagellés comme formes incertæ sedis el dont un seul est certain, le genre Multicilia.

\section{GENRES}

Multicilia (Cienkovslìy) (fig. 539) est un petit être incolore, de forme arrondie, ovoïde ou un peu irrégulière et très métabolique, qui porte, répartis sur toute la surface de son corps, de longs llagellums grêles qui s'agitent paresseusement et irrégulièrement, sans les foueltements énergiques el réguliers habituels à ces organes. Cela semble ètre dù d'ailleurs à leur longueur et à leur faiblesse plutôl qu'à une différence de nature. Parfois, entre eux, apparaissent de petits pseudopodes courts et obtus. Ce sont ces pseudopodes occasionnels et non les flagellums permanents qui servent à capturer la nourriture (consistant en Flagellés Fig. 539. de plus petite taille) qui est absorbée par un point quelconque du corps. Occasionnellement, ces pseudopodes peuvent aussi servir à la reptation. Il y a un (M. marina) ou plusicurs ( $M$. lacustris) noyaux, tantòt des vésicules pulsatiles (H. lacustris), lantôt point (M. marina). L'animal se reproduit par division (20 à 30 p.. Mer et eau douce) (').

1) A ce genre se rattachent un eertain nombre de formes douteuses :

Haliophrynella (Vejdovsky) que son auteur rapporte aux Héliozoaires, mais dont les longs appendices ressemblent plus à des flagellums qu'à des pseudopodes. Bütschli tendrait à y voir peut-être une larve de Bothriocéphale;

Grassia (Fiseh) (fig. 540 ) qui avait été déerit comme une forme autonome très semblable à la préeédente, s'en distinguerait par des flagellums plus nombreux et posséderail, outre le noyau, deux petites vésicules pulsatiles (A peine $气$ ఝ. Parasite dans le lube digestif de la Grenouille et dans le sang de la Rainette $[I Y / a]\}$.

Mais Schuberg [89] a démontré que la forme qui habite l'intestin

Multicilia

(d'ap. Cienkorsky). n'est autre ehose qu'une eellule épithéliale de la paroi digestive détaehée et déformée. Ce genre devra sans doute disparaitre. Cela est eneore plus eertain pour le prétendu

Asthmatos (Salisbury) qui vivrail en parasite sur les muqueuses oeulaire et respiratoire des personnes atteintes d'asthme et de coryza des foins et serait la eause de leur 
$2^{\text {e TRIB U }}$

MONOSTOMNES. - MONOSTOMLYA

[TETRAMITINA (Bütschli)]

\section{TYPE MORPHOLOGIQUE}

(FIG. 541)

Il peut être défini en quelques mots.

Il suffit de prendre notre type morphologique de Monadide (V.p. 319) el de placer à la partie supérieure de la face ventrale une large dépression peu profonde servant de lieu d'ingestion, puis d'insérer quatre ou Fig. Jisl. six fouets à la partie supérieure de cetle dépression.

\section{GENRES}

Collodictyon (Carter) (fig. 542) a la forme d'un ovö̈de aplati. La dépression buccale n'est pas bien marquée, mais il y a un sillon vertical assez accentué sur l'une de ses faces. Il y a quatre llagellums formant deux paires symétriques (33 $\mu$. Eau douce).

Tetramitus (Perly) (fig. 543 )

I'a pas de sillon ventral, mais la dépression buccale est très grande. Un des flagellums estordinairement dirigé en bas (50 „.. Mer et eau douce).

Monocercomonas (Grassi) diffère du précédent par sa forme effilée en bas et sa fossette buccale à peine indiquée (15 p. Parasite dans l'intestin de divers Insectes Fig. 5ィ3.

Collodictyon (C. sulcatus) (im. Stein). Fig. 5't2.
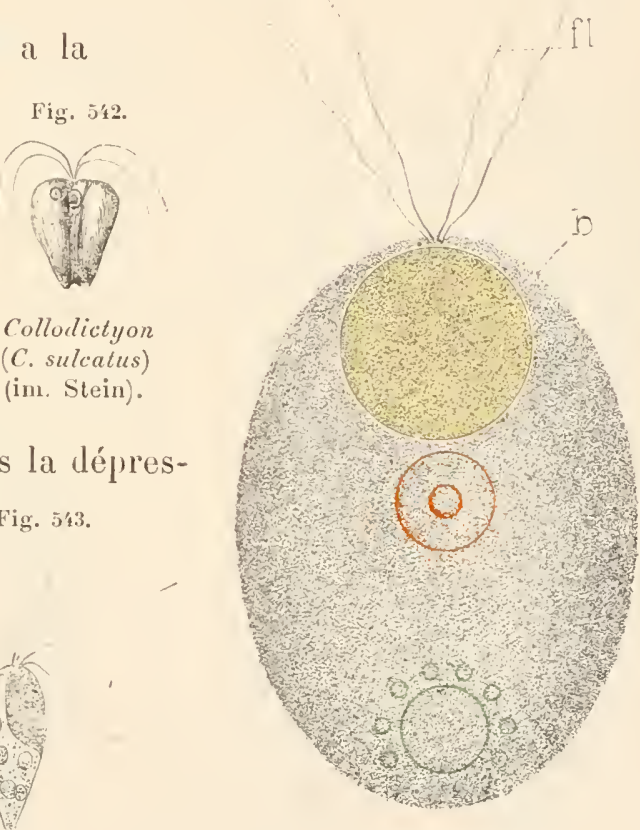

Tetramilus. (T. descissus) (d'ap. Klebs).

\section{MONOSTOMINA}

(Type morphologique) (Sch.). b., dépression buccale; fl., flagellums. et Reptiles et chez l'Homme dans certaines diarrhées) (').

affection. Ce n'est sans doute, comme l'a dit Leidy, que des cellules vibratiles plus ou moins altérées, détachées de l'épithélium nasal.

(1) Le genre.

Trichomastix (Blochmann) n'en diffère que par des caractères secondaires. 
Trichomonas (Donné) (fig. つ̌́t) a les mêmes caractères et ses trois flagellums ascendants sont normaux, mais le descendant est remplacé par une membrane ondulante ventrale. Le corps se termine ordinairement en pointe vers le bas. Il y a, en outre, une petite lame dorsale $(40 \mu$. Intestin de divers animaux: Rat, Souris, Chat, Cobaye, Canard, Limace, et dans le mucus vaginal de la Femme).

Megastoma (Grassi) a, au contraire, la dépression buccale beaucoup plus grande, bordée de deux ou trois paires de fouets insérés sur les bords et le corps terminé par une queue effilée qui porte une paire de longs fouets Intestin grèle de l'Homme, du Chat et de la Souris) (').

$3^{\circ}$ TriBu

Trichomonas

DISTOMINES. - DISTOMINA

(im. Stein).

[Distonata (Klebs)]

TYPE MORPHOLOGIQUE

(FIG. 545)

La constitution caractéristique des Polymastigidx, conservée en tout ce qui concerne les autres caractères, est modifiée par le fait que les fouets se sont séparés en deux groupes symétriques, situés l'un à droite l'autre à gauche, et à chacun desquels est annexée une grande dépression buccale $(b$. $)$ constituée d'ailleurs essentiellement comme la dépression unique de Tetramitus. Il y a quatre cils de chaque côté, dont trois ascendants $(c$. $)$ ou transversaux et un plus grand descendant $\left(c^{\prime}.\right)$. Tous s’insèrent à la partie supérieure de la dépression buccale et c'est surtout au-dessous de leur insertion que se fait l'ingestion des aliments.

(1) Genre voisin :

Polymastix (Bütschli) serait un Trichomonas armé de trichocystes. Mais la vraie nature de ces prétendus trichocystes n'est, paraît-il, rien moins que certaine. 


\section{GENRES}

Trigonomonas (Klebs) (fig. 546 ) est notre type morphologique avec une forme triangulaire et trois fouets seu-

Fig. 546.

lement à la partie supérieure de chaque bouche $(20$;.. Eau croupie).

Hexamitus(Dujardin) (lig. 54,5 1S). Pour faire de notre type un Hexamitus, il faut supposer que les deux bords de
Fig. 5i7.
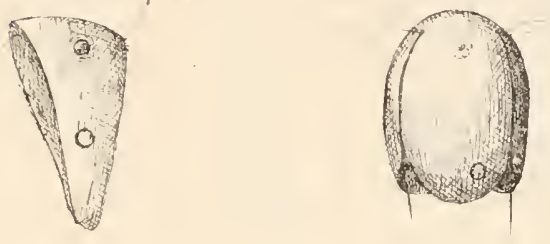

Fig. 548,

Trigonomonas

(T. compressa) (im. Klebs).

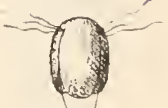

chaque bouche se sont élevés et arancés l'un vers l'autre de manière à dessiner de chaque côté un canal vertical et latéral, fendu le long de son bord

Mexamitus

(II. inflatus) (im. Klebs).

externe; mais, la fente est presque fermée dans la plus grande partie de sa longueur, et largement ouverte à son extrémité inférieure évasée. Les trois fouets supérieurs sont placés hors de la bouche et ne servent qu'à la locomotion; le fouct inférieur plus long el plus fort est au contraire resté engagé dans le canal et sort par son orifice inférieur. C'est lui seul qui, en tournoyant, détermine un courant alimentaire ascendant qui entre dans le canal buccal et y pousse les particules alimentaires qui sont aussitòt absorbées $(20$ it $30 \mu$. Eau douce crompie et intestin de quelques Amplibiens et de l'Huître)(").

Trepomonas (Dujardin) (fig. כั 9 ) peut aussi se comprendre aisément malgré sa forme singulière, en partant encore de notre type. Supposons qu'un seul des deux bords de chaque bouche se soit développé en une large expansion aliforme verticale, recourbée vers la bouche. Il en résultera que la bouche, au lieu de regarder latéralement, regardera directement en avant ou en arrière. Or, pour l'une des bouches, la lèvre est formée par le hord dorsal, pour l'autre, Fig. 549.

\footnotetext{
Hexamitus fixé par ses cils inférieurs
} (im. Stein). par le bord ventral, en sorte que l'une regarde en avant, l'autre en

$\left.{ }^{1}\right)$ L'animal nage en tournant sur lui-même. Il peut se fixer par l'extrémité de ses fonets inférieurs (fig. $5^{\prime} 18$ ) et se balancer ou tourner sur ce double pédoncule accidentel.

Urophagus (Klebs) est un genre voisin à extrémité inférieure formant une sorte de prolongement bilabié par où les aliments arrivent aux fentes buccales (Eau douce). 
arrière, sans qu'on puisse dire cependant où est le ventre et où est le dos puisque, en tournant de $180^{\circ}$ autour de son axe, l'animal ne change pas d'aspect. Vers le milieu de cette longue fosse buccale, s'insèrent (Jans chacune) trois à quatre flagellums, dont deux ou trois (appelés cils buccaux) assez courts, égaux, descendants, insérés dans la carité mème et un plus grand, inséré près du bord et dirigé vers le bas (20 ‥ Eau croupie) (").

Spironema (Klebs) (fig. 53̈0) offre une disposition analogue, mais les deux bouches sont olitenues par une simple excaration en gouttière de la partie supérieure du corps aux deux bords opposés des faces opposées, et les fouets, assez nombreux, sont insérés au hord externe libre de chacune iles deux bouches. Le corps, en outre, est effilé en pointe vers le bas (15 à 20 p.. Eaux stagnantes) $\left({ }^{2}\right)$.

Fig. 550 .

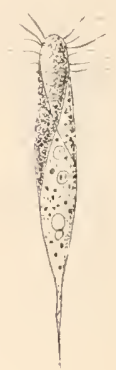

Spironema (S.multiciliatum) (d'ap. Klebs).

\section{4e Tribu}

\section{TRICHONYMPIINES. - TRICHONYMPHINA}

[Triciovympinda (Leidy, emend.)]

Nous plaçons ici comme tribu des Polymastigidx un petit groupe d'ètres dont la position n'est pas encore définitivement établie, mais qui semblent bien se rattacher ici par leurs affinités principales.

\section{TYPE MORPHOLOGIQUE}

(FIG. 551)

C'est un petit être ovoïlle mesurant 20 à $30 \mu$ et vivant en parasite tlans le rectum de quelque Orthoptère. Le corps est dépourvu d'appendices mobiles; de l'extrémité supérieure part une abondante touffe de longs flagellums

(1) Le corps est souvent métabolique, le noyau est vers le haut, la résicule a une position variable.

(2) Le genre

Gyromonas (Seligo) paraît voisin du précédent.

Assez douteuses sont les affinités de

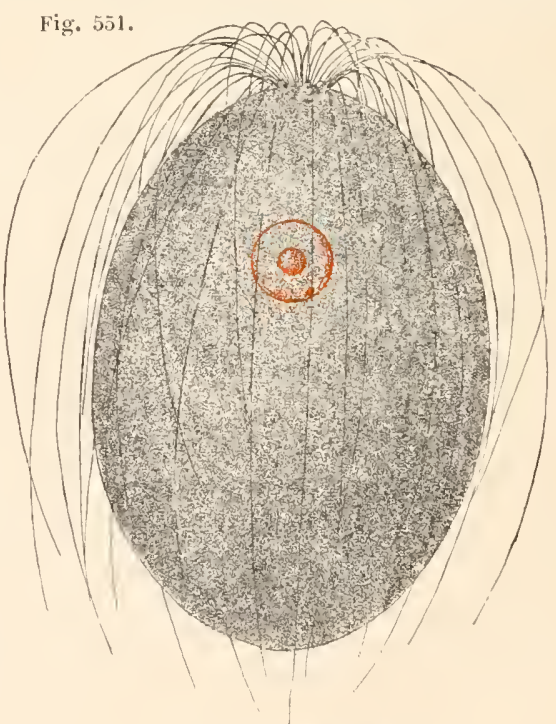

TRICHOXYMPHIN.1

(Type morphologique) (Sch.).

Pteridomonas (Pénard) parfois fixé par un filament inférieur et possérlant au haut une sorte d'excavation pharyngienne l'où sort un grand flagellum entouré ì sa base d'un cercle de 12 à 18 cils assez déreloppés. 
grè̀les qui s’agitent en ondulant sans énergie. A leur hase, se trouve un petit orifice buccal. Le corps est revètu d'une membrane. A l’intérieur, dans un cytoplasme sans différenciation en endoplasme et ectoplasme, se trouvent un noyau vésiculeux situé assez haut et de nombreuses particules alimentaires semblables aux résidus que contient le rectum de leur hôte. On ne connaìt pas la reproduction (').

\section{GENRES}

Lophomonas (Stcin) (fig. 5.52) ne diffère de notre type en rien d'essentiel. Les flagellums sont insérés sur une petite surface en fer à cheval située au sommet tronqué du corps $\left(0,03\right.$. Rectum de Periplaneta et peut-etre Grillotalpa) $\left.{ }^{(2}\right)$. Leidyonella (Frenzel) (fig. 533 ) diffère du précédent par son extrémité supérieure prolongée en

Fig. 552. Fig. 5i3.

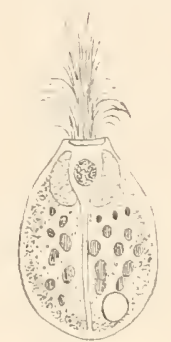

Lophomonas

(L. Blattarum)

(d'ap. Stein).

un col court, effilé, terminé par un bouton qui donne insertion aux flagellums. Il y a, en outre, une houppe de flagellums scmblables à l'extrémité inférieure $(0,2$ à $0,4:$. Rectum d'Eutermes $)\left({ }^{3}\right)$.

Trichonympha (Leilly) (fig. $30 ̈$ ' $)$ a l'extrémité supéricure du corps saillante, séparée du reste par un sillon transversal et semblable à une mamelle surmontéc de son mamelon. C'est sur cette partie que sont insérés les longs flagellums disposés sur trois cercles. Une quatrième rangée circulaire, insérée au voisinage de l'extrémité inférieure, repré-

(1) Lorsque l'animal devient très adulte, il a une tendance a perdre ses flagellums. Liexistence de la bouche n'est pas tout a fait certaine. Cependant elle est bien probable, car on est sùr, par la présence des parcelles alimentaires dans son intérieur, que l'animal avale de la nourriture solide et l'on a souvent cru voir, là et nulle part ailleurs, un petit orifice.

Ce que l'on a pris chez quelques genres comme une (L.Cordubensis)(d'ap. Frenzel). L'individu et le détail de sa cuticule.$$
\text { ail }
$$

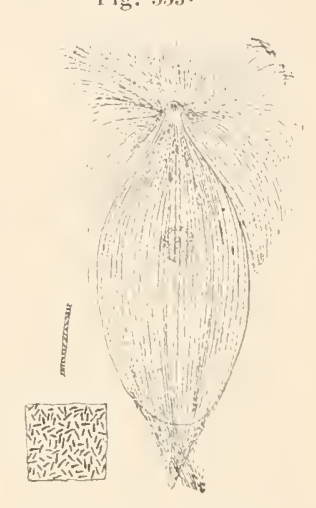


sente la houppe terminale du genre précédent 0,12 . Rectum de Termes). Jœnia (Grassi) ressemlılerait plutôt à Lophomonas, mais il en diffère par sa forme allongée, son extrémité supérieure plus largement tronquée, ses flagellums périphériques récumbants et la présence d'une garniture de courtes soies en forme de cils immobiles sur tout le corps (Rectum de Callotermes) (1).

\section{APPENDICE AUX TRYCIONYMPHINA}

Nous placerons ici, en appendice aux Trichonymphines, deux genres que Bütscul place dans leur groupe, mais qui mériteraient aussi bien d'ètre joints aux Infusoires holotriches comme l'indique Frexzel [91]. Ils diffèrent des premiers et de tous les Flagellés par un revìtement ciliaire général vrai, et se rapprochent par là des Ciliés, sauf l'ab)sence d'ectoplasme, de micronucléus el peut-ètre de bouche. Ils ont exactement l'habilat et les mœurs des Trichonymphides. Voici ces genres :

Pyrsonympha (Leidy) (fig. $3 \% 3$ ) a la forme d'une pointe allongée; l'extrémité obtuse, tournée vers le bas, porte un petit prolongement caudal et, tout le long d'un des bords, règne une sorte de membrane ondulante. Tout le corps est garni d'un revètement uniforme de vrais cils assez fins. On n'a pu découvrir de bouche bien que l'animal absorbe certainement de la nourriture solide que l'on retrouve dans son cytoplasme, tout comme chez les Trichonymphines $<0,1$. Rectum de Termes) $\left({ }^{2}\right)$.

Dinennympha (Leidy), (fig. 53̈6) en diffère par Fig. 555 . Fig. 556. sa forme rubanée, contournée en spirale et

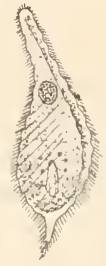

Pyrsonymplea (P. vertens) (d'ap. Leidy).

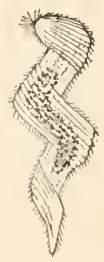

Dinennympha (D. gracilis) (d'ap. Leidy). peut-ètre par un petit bouquet de cils à l'extrémité supérieure 0,1 . Rectum de Termes) $\left({ }^{\boldsymbol{3}}\right)$.

Fig. 557.

(1) Il y a, en outre, au-dessous du noyau, un long processus strii comparable à un faisceau de queues de spermatozoỉdes, qui est tout à fait inigmatique.

(2) Parfois, on le rencontre tout à fait dépourvu de cils, mais ce n'est peut-être là qu'un phénomène secondaire comme chez les Trichonymphines vicux.

(3) Il faut sans doute ajouter ici quelques formes que Leidy donne comme des jeunes de Trichonympha (fig. 5ö7), mais dont les relations de parenté arec ce genre ne sont nullement démontrées et qui ont, comne les deux genres précédents, un revêtement ciliaire général.

Trichonympha

Forme jeune (?) (d'ap. Leidy). 


\section{ORDRE \\ EUGLENIDES. - ELGLENIDA \\ [EtGLENIDINA (Bütschli, emend.)]}

\section{TYPE MORPHOLOGIQUE}

(FIG. 558 ET 559)

Les Flagellés de cet ordre se distinguent de ceux de l'ordre précédent par deux caractères essentiels : $1^{\circ}$ leur tégument $(\mathrm{mb}$.$) est ferme, solide, bien$ délimité et, s'il permet encore les changements de forme, du moins s'opposet-il définitivement à toute espèce de mouvement amœboïde("); 20 la petite dépression buccale s'est transformée en un profond entonnoir $(p h$.) bien dessiné, s'ouvrant à l'extrémilé supérieure et un peu ventralement, et formé par un refoulement des téguments. Les parois de cet entonnoir ou pharynx ont donc la même structure que la surface externe lu corps, mais le fond laisse à nu l'endoplasme $(b$. $)$, et c'est là que se fait la pénétration des aliments. Le flagellum nait un peu aurdessus de ce point, à la face dorsale de l'entonnoir. La vésicule pulsatile $(\boldsymbol{V} c$.) se distingue par des connexions particulières. Elle est placée à quelque distance au-dessous de l'orifice interne du pharynx et se montre lí entourée d'une couronne de petites vésicules formatri$\operatorname{ces}\left(V^{\prime}.\right)$. Elle se vide, non au dehor's, mais dans une vésicule collectrice, parfois

(1) La structure de ce tégument est d'ailleurs assez mal déterminée. Il n'y a pas de

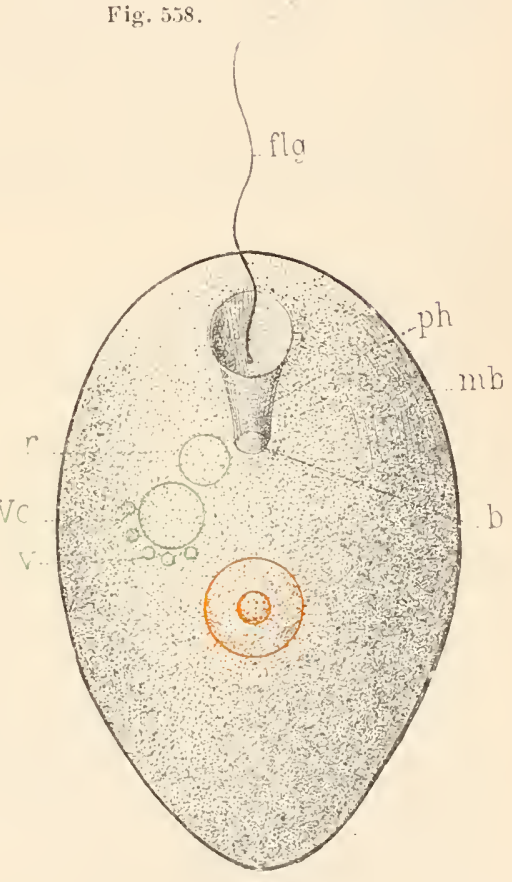

EUGLENID.A.

(Type morphologique) (Sch.).

b., orifiee buecal : flg., flagellum; mb., membrane; N., novau; ph., pharynx; r., réservoir; V'., vésicules formatrices; Ve., vésicule pulsatile.

distinction nette entre membrane et ectoplasme, comme si celle-ci s'était confondue avec la couche superficielle de ce dernier, imprégnée de substances non digestibles dans la pepsine et résistant à la putréfaction. Ce n’est cependant point de la cellulose. Au-dessous de cette couche, il n'y a pas d'ectoplasme mou; c'est directement l'endoplasme qui se rencontre là avec ses caractères ordinaires ef sa mobilitè très grande. Cependant, chez les formes contractiles, se trouvent, à la face profonde du tégument, des fibrilles, les unes longitudinales, les autres circulaires qui semblent bien ètre l'agent spécial de la contractilité. 
appelée réservoir (r.), qui s'ouvre elle-mème à l'orifice terminal du pharynx. Il n'y a pas là, sans doute, de conduit permanent, mais une simple communication qui s'établit au moment de la systole par ruplure de la mince couche de cytoplasme qui sépare la vésicule du fond du pharynx.

Le corps renferme de nombreux grains d'une substance amylacée, le paramylon (fig. 7399), ruii se présente sous la forme de Fig. 5.59. courts bâtonnets à structure stratifiée comme l'amidon. C'est un produit de réserve qui s'accumule quand la nourriture est abondante et s'épuise pendant la disette.

EUGLEN II) 1 ('Type morphologique) Paramylon.
L'ordre des Euglénides se divise naturellement en trois groupes auxquels nous préférons donner le nom de tribus que celui de sous-ordres pour ne pas attribuer aux différences qui les séparent une valeur exagérée :

ASTASINA, sans chlorophylle et ì pharynx presque fermé au fond ; EUGLEVINA, avec chlorophylle et à pharynx presque fermé au fond; PERANEMINA, sans chlorophylle et à pharynx largement ouvert au fond.

1re Tríu

ASTASINES. - ASTASINA

Fig. 560

\section{[Astasnd.l (Klebs non Bütschli)]}

\section{TYPE MORPHOLOGIOUE}

(FIG. 560)

Les Astasines se distinguent du type morphologique des Euglenida par un seul caractìre. Leur pharynx conique $(p h$.$) se termine presque en$ pointe au fond et ce sommet étroit est presque obturé par la base du flagellum $(f l g$.$) qui s’insère précisément là.$ Il reste cependant un petit espace libre $(b$.) et cet espace est indispensable pour l'ouverture de la vésicule collectrice, mais il est trop petit pour admettre de la nourriture solide. Il n'est même pas certain qu'il serve ì l'introduction de liquides alimentaires (').

L'animal se nourrit done par imbibition, par osmose, à travers son

(1) Cela est bien probable, cependant, d'après les observations de Kavkine [86]. Peutêtre même l'animal absorbe-t-il de temps à autre quelques minimes particules solides.

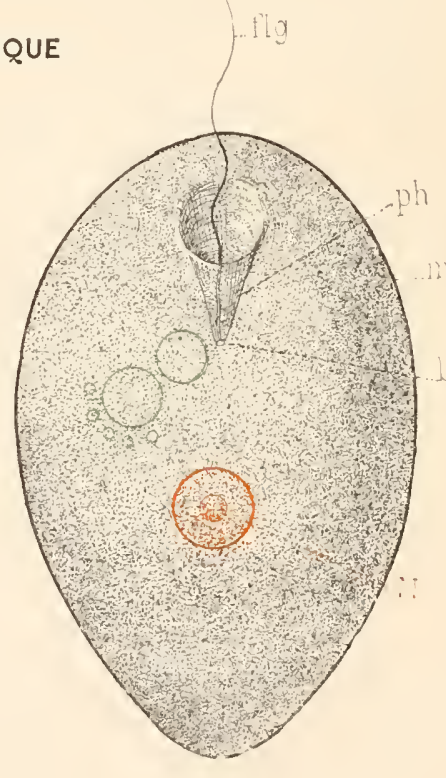

A.STASISH

(Type morphologique) (Sch.).

b., orifiee terminal du pharynx; $\mathbf{1 l g}$., flagellum; mb., membrane tégumentaire; $\mathbf{r}$, réservoir; V., vésieules formatrices; Vc., vésicule pulsatile. 
tégument, des éléments nutritifs contenus dans les liquides, à la manière d'une racine de plante dans le sol. C'est ce que l'on appelle l'alimentation saprophytique. Aussi ne peut-il vivre, ou du moins prospérer el se multiplier, que dans les eaux croupissantes riches en substances organiques dissoutes. Il se reproduit par division longitudinale à l'étal libre.

\section{GENRES}

Astasia (Dujarlin) (fiğ. 亏61, .562), forme allongée mais très métabolique, à cuticule finement striée en spirale. I'n seul flagellum $(0,1$ et plus. Eau douce) (').

Distigma (Ehrenberg) (fig. 363 , כ̋61) diffère du précédent uniquement par la possession d'un fouet accessoire, inséré à côté du principal et dirigé comme lui (fig. כ̋63) et, le plus souvent, par la présence de deux stigma noirs (taches oculiformes) à l'extrémité supérieure (fig. $564)$.

Menoidium (Perty) est un Astasia mais dont la forme du corps est fixe, non métabolique, un peu contournée i’10 p.. Eau douce) $\left({ }^{*}\right)$.

Sphenomonas (Stein) (fig. 563̈) est un I)istigma de forme fixe. Il a comme lui un fouet Fig. 561 . Fig. 562. accessoire, parfois deux, très petits $\left.(30 \mu \text {. Eau donce })^{(3}\right)$.

(1) Genres voisins :

Astasiopsis (Bütschli), Astasioides (Bütschli) et $(p, p)$, Cyclidium (Dujardin), ne sont guère que des espèces d'Astasia.

(2) Genre voisin :

Rhabdomonas (Fresenius) n'est qu'une espèce de Menoidium ou peut-être de Sphenomonas.

( ${ }^{3}$ ) Il se distingue en outre par la présence de une ou plusieurs carènes longitudinales et, dans l'intérieur du corps, par une grosse masse d'aspect gélatineux dont la signification est inconnue. Le genre

Atractonema (Stein) n'est qu'une espèce du précédent. 
2 e TRIBU

\author{
EUGLENINES. - EUGLENINA \\ [ElGLENIDA (Klebs), non E'GGLENINA (Stein)].
}

\title{
TYPE MORPHOLOGIQUE
}

(FIG. 566 ET 567)

Pour la structure du corps et des organes, c'est une Astasine. Mais il $\mathrm{y}$ a ici en plus deux organes: des grains de chlorophylle et une tache pigmentaire oculiforme ou stigma (').

Fig. 560 .

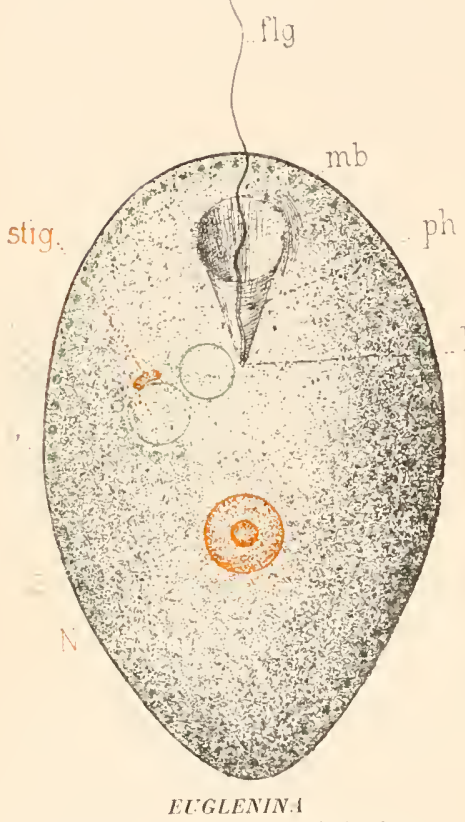

(Type morphologique) (Sch.).

b., bouche : c., grains de chlorophylle; flo., flagellum; mb., membrane; $\mathbf{N}$, noyau; ph., pharynx; 1 ., réservois: stig., stigma: v., résicules formatrices; Ve., vésicule pulsatile.
Les grains de chlorophylle $(c$.$) sont$ fort petits (2 à 4 \%) et généralement discoïles; ils sont logés sous le tégument et ne sont nullement des Nlgues parasites ou des particules ingérées : ce sont des organes de l'animal. Normalement, leur structure est la suivante. Au centre estle pyrénoïde, petite masse de protoplasma différencié, enveloppée d'une couche de paramylum qui prend la forme de deux hémisphères creux, formant une sorte de boìte sphérique qui renferme le globule protoplasmique. Tout cela est entouré d'une atmosphère de protoplasma coloré en rert par la chlorophylle. Mais souvent le pyrénoïle manque et le paramylum se forme sous la petite masse de chlorophylle, au contact du cytoplasma sous-jacent. ll y a, en outre, les grains de paramylum libres dans la profondeur du cytoplasma.

Le stigma (stig., fig. 567) a l'aspect d'un gros granule rouge situé contre la paroi de la vésicule collectrice. Il est formé d'une masse de protoplasma róliculé dont les mailles

sont occupées chacune par un globule d'une substance rouge, oléagineuse. Au centre de cette masse, se trouve un gros grain sphérique de paramylum formant lentille, et un petit nombre d'autres grains semblables, mais plus petits, sont situés plus superficiellement ou mème tout à fait à la surface, dans une disposition régulière. C'est bien là du para-

(1) Certaines Astasines (p. ex. Distigma) ont bien une tache pigmentaire comparable au stigma, mais par exception, landis qu ici le stigma est la régle.
Fig. 567 .

FIGLENIXI

(Type morphologique)

Stigma (im. Franzé).

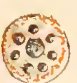


mylum car, lorsqu'il est soumis au jeûne, l'animal consomme ses cristallins en mème temps que ses autres réserves. Par son stigma l'animal sent la chaleur et reconnaît la lumière qu'il recherche avidement.

La chlorophylle fonctionne ici comme chez les plantes, décomposant l'acide carbonique de l'air, rejetant l'oxygène et fixant le carbone pour former du paramylum qui est consommé au fur et à mesure les besoins. Il en résulte que l'animal se nourrit à la manière des plantes. C'est ce que l'on appelle l'alimentation holophytique. Sans doute il peut absorber comme les Astasines des liquides nutritifs par la peau ou peut-c̀tre par le pharynx, mais c'est là un mode secondaire, accessoire ou accidentel. Dans une eau croupie et mal éclairée, il peut vivre longtemps de cette manière, mais il jaunit, s'étiole et ne peut se reproduire activement (').

Il jouit aussi d'une autre propriété que n'ont pas les Astasines, c’est celle de sécréter autour de lui une substance gélatineuse sous laquelle il peut s'arrondir et se mettre à l'abri après aroir perdu son flagellum. C'est une sorte d'enkystement rudimentaire dont il use toutes les fois que les conditions deviennent trop défarorables. Au retour des conditions normales, il sort de sa gélaline et reforme son flagellum. C'est toujours à l'état de repos sous sa gélatine qu'il se reproduit par division longitudinale, et c'est là une nouvelle différence qui le distingue les Aslasines. Cela ne l'empèche pas de former à l'occasion de véritables kystes.

\section{GENRES}

Fig. 568.

Euglena (Ehrenberg) (fig. 568 ) est notre type morphologique avec une forme très métabolique allongée, ordinairement ovoïle, effilée en bas, tronquée obliquement en haut et en avant $\left(100 \mu\right.$. Eau douce) $\left(^{2}\right)$.

(') Il faut bien que les Euglénines puissent absorber par la peau ou autrement quelques substances albumineuses, car ce n'est pas leur chlorophylle qui peut leur fournir l'azote dont elles ont besoin pour leur croissance. Ce qui le prouve, en outre, c'est la formation, bien observée par Karkixe [86], de grains de paramylum indépendamment des corps chlorophylliens chez les Astasines. Il y a quelques rares espèces et variétés de ce groupe qui sont incolores comme les Astasines.

(2) Les stries sous-tégumentaires sont bien visibles, les unes longitudinales, les autres obliques. L'animal rejette facilement son

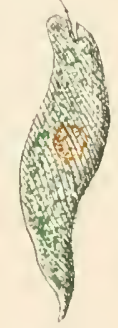

Euglena (d'ap. Kent). flagellum et le reforme. Il y a quelques variétés incolores. Chez beaucoup d'espíces, le fouet tombe facilement.

Il arrive fréquemment que les Euglènes s'enkystent et se divisent sous leur kyste. Les deux produits de la division, au lieu de quitter le kyste et de se transformer en Euglènes flagellées, s'enkystent, se divisent dans le liyste maternel, et la chose continue ainsi pendant plusieurs générations et donne lieu à de nombreux sýstémes de kystes emboités, tous contenus dans le kyste maternel primitif. Celui-ci se dilate progressivement au fur et à mesure de la multiplication des kystes contenus, se gélifie partiellement et se soude à ses voisins. Il résulte de là des nappes plus ou moins étendues flottant a la surface de l'eau ou gisant au fond. 
Colacium (Ehrenberg) (fig. ̈̈69) est une Euglène ıui, après avoir erré librement avec une constitution normale $(a)$, rejette son llagellum $(b)$, se fixe par l'extrémité cépha. lique sur quelque Copépode ou Rotifère, et sécrète un pédoncule gélatineux, gros et court $(c)$ et une enveloppe de la mème substance.

En se divisant en long $(c$ et $d$ ), elle forme de petites colonies (50 a 60 \%. Eau douce).

Fig. 569.

Fig. 570.

Eutreptia (Perty) (fig.570), est une Euglène à deux flagellums $\langle 50 \mu$. Eau douce). Ascoglena (Stein) (fig. 571), est une Euglène abritée dans une petite capsule fixée brunâtre, qu'elle a sécrétée ( $40 \mu$. Eau douce).

Fig. 571 .

Fig. 572.

Fig. 573

Trachelomonas (Ehrenberg) (fig. $572)$ a aussi une capsule, mais libre et ornée le plus souvent de sculptures ou d'épines. Le fouet est trois à quatre fois aussi long que le corps 20 à 30 p.. Ner et Eau douce).

Phacus (Nitzsch) (fig. 573). Le corps estici piriforme, aplati, prolongé en bas en une queue effilée, et plus ou moins as ymétrique et tor-

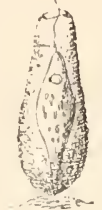

Ascoglen $\alpha$

(A. vaginicola) (d'ap. Stein).

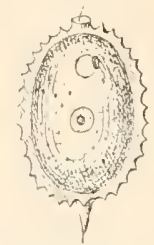

Trachelomonas

(im. Kent).

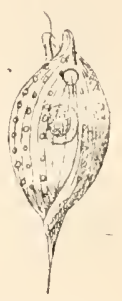

Phacus

(im. Franzé,

Stein). du sur son axe. La membrane est forte et la forme fixe, non métabolique. La bouche est rejetée un peu dorsalement (10 $\mu$. Eau douce) (").

Cryptoglena (Ehrenberg) (fig. 5774) est non métabolique, ovoïde, et a, sur les flancs, une paire de valves, d'une substance solide, sécrétée et appliquée immédiatement au corps. Sa chlorophylle forme deux rubans longitudinaux symétriques (clrmp.) (30 «. Lau douce!

(1) I'ordinaire le paramylum forme une ou deux fortes masses de formes diverses.

Lepocinclis (Perty) (fig. 575) est voisin.

Fig. 574.

Fig. 575.

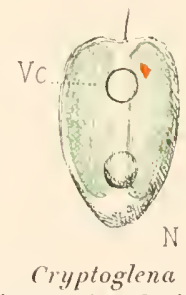

(im. Klebs, Stein).

chrmp., chromoplastes; stig., stigma; $\mathbf{N}$., noyau; Ve., vésicule pulsattile.

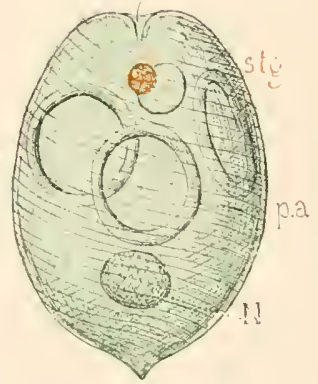

Lepocinclis (iin. Franzé, Bütschli). 
Xanthodiscus (Cheviakof) a son corps chlorophyllien formant une seule masse excavée et un gros pyrénoïde unique tout au haut (Eau douce, Australie).

\author{
3e TriBu
}

\title{
PÉRANÉJINES. - PERANEMINA \\ [PEranemina (Klebs) non Peranemina (Bütschli)]
}

\section{TYPE MORPHOLOGIQUE}

(FIG. 576)

L'animal est incolore comme une Astasine, mais son alimentation est animale au lieu d’être saprophytique. Il se nourrit de particules solides. qu'il introduit dans son cytoplasma par le moyen de son flagellum $(/ g$.$) . Pour$ cela, son pharynx $(p h$.$) est percé, au$ fond, d'un orifice suffisamment large mettant le cytoplasma à nu $(b)$.

En outre, le pharynx est fendu longitudinalement, en sorte que son orifice d'entrée n'est pas un trou rond comme chez les précédents, mais une fente verticale. Le flagellum est inséré, non au fond, mais sur la paroi dorsale de cette gouttière. Eufin, il existe une sorte d'appareil pharyngien $(c)$ formé de deux baguetles d'une substance protéique solidifiée qui convergent l'une vers l'autre ou mème se continuent l'une avec l'autre, disposées, en somme, comme les deux branches d'un diapason. Cet appareil, pris longtemps pour un oesophage, est plongé dans le cytoplasma et remonte vers le haut dans'la direction de la bouche pour venir faire saillie dans le pharynx en avant ou à còté de son orifice d'ingestion. Il peut s'avancer vers le dehors et doit aider probablement à l'ingestion des particules solides dont l'animal se nourrit.

Fig. 576.

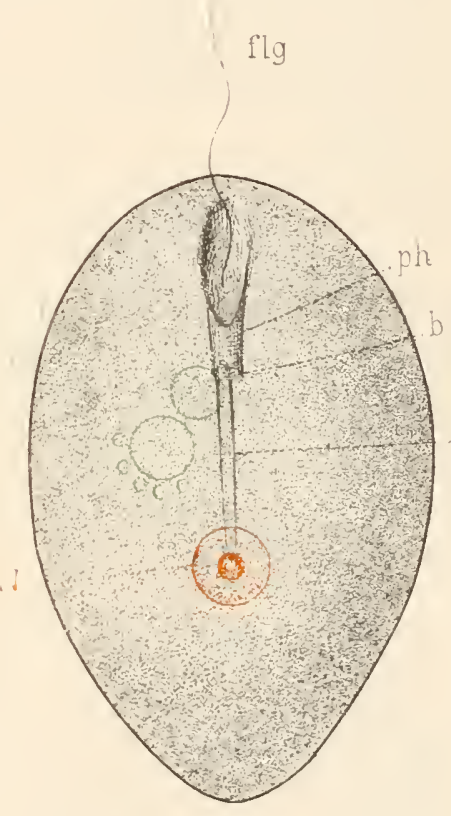

PARANENLNA

(Type morphologique) (Sch.).

b, orifice terminal du pharynx; e, appareil phary ugieu; fle., tlagellum; $\mathbf{x}$., noyau; ph., pharynx; $\mathbf{x}$. , réservoir; Ve., vacuole collectrice; v., vacuoles formatrices.

Malgré cette alimentation animale, il se forme du paramylon comme chez les Euglénines et Astasines.

\section{GENRES}

Le corps peut être de forme fixe ou métabolique; il peut $\mathrm{y}$ avoir deux fouts ou un seul. La combinaison de ces caractères deux à deux 
nous donne quatre caractéristiques qui permettent un groupement rationnel des genres : ceux qui sont métaboliques montrent une striation en hélice.

$1^{\circ}$ Corps métabolique, Fig. 577.

Jig. 578.

Fig. 579. un seul flagellum :

Euglenoplis (Klebs) (fig. 577) a une forme en fuseau, le pharynx fendu latéralement et point d'appareil pharyngien $\quad 20-30$ p. Eaux riches en matières végétales macérées);

Peranema (Dujardin) (fig. $578)$, au contraire, a un appareil pharyngien lien développé ; il possède aussi un anus fixe $(80 \mu$. Eau douce);

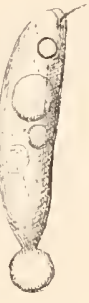

Euglenopsis

(E. $\operatorname{sor} a x)$

(d'ap. Klebs).

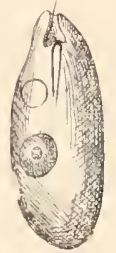

Peranema

(im. Klebs,

Bïtschli).

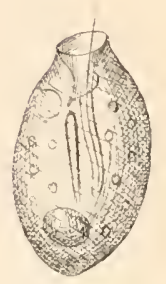

Ureeolus

(U. cyclostomils)

(im. Klebs).

Urceolus (Merechkovsky) (fig. J̈79) a le pharynx non fendu el très évasé en haut (50 p.. Eau doucej (').

2. Corps métabolique, deux flagellums:

Heteronema (Dujardin) (fig. 580 ) a le corps allongé, fusiforme ou globuleux, pas d'appareil pharyngien, le pharynx fendu verticalement et donnant insertion à deux fouets, un supérieur ascendant, gros et fort, et un inséré un peu plus bas, descendant et beaucoup plus petit (40-50 p.. Eau douce et mer);

Zygoselmis (Dujardin) est ovoïde à grosse extrémité inférieure $(0,1$. Eau douce);

Dinema (Perty) (fig. 5̊s) a la forme d'un boudin. Son pharynx est longuement fendu ventralement et possede un appareil pharyngien. Le fouet ascendant s'insère un peu latéralement. Un peu audessous de lui, est un fouet descendant beaucoup plus gros et plus long, qui se courbe en $U$ pour sortir du pharynx et dont la racine pénètre assez profondément dans le cytoplasme $(80 \mu$. Eau douce stagnante $\left({ }^{*}\right)$.

Fign. 380.

Fig. 5s1.

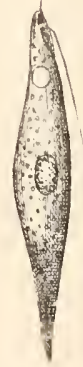

Heteronema

(II. acus)

im. Stein,

Klebs).

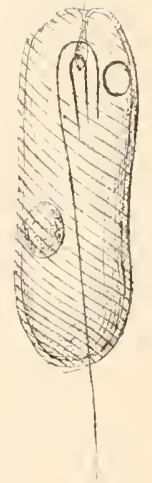

Dincma

(D. griseolum) (im. Klebs).

(1) Ces genres constituent la famille des PER_y emLy [Peranemina (Klebs)].

(2) Ces genres forment la famille des IIETERoyeunv. [Heteronemina (Klebs)]. 
$3^{\circ}$ Corps de forme fixe, un seul fouet:

Petalomonas (Stein) (fig. 582) a son fouet inséré latéralement dans le pharynx. Le corps porte des carènes ou des lohes qui lui donnent une forme bizarre. Le noyau est placé à la même hauteur que la vésicule pulsatile (20-30 $\mu$. Eau douce).

Scytomonas (Stein) (fig. S83) a, au contraire, une forme régulière ovoüle. 11 est très petil et se nourrit de Bactéries qu’il semble aspirer ("כà $6 \mu$. Eau douce) (').

to Corps de forme ordinairement fixe, deux fouets :

Anisonema (Dujardin) (fig. :58 caractérisé par la présence, sur la face ventrale, d'un sillon vertical assez profond. Il n'y a pas, à pro-

Fig. 582.

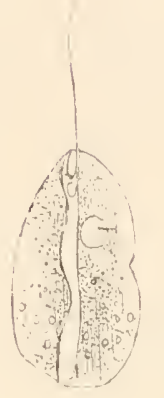

Petalomonas

(P. Steinii)

(d'ap. Klebs).
Fig. 584.

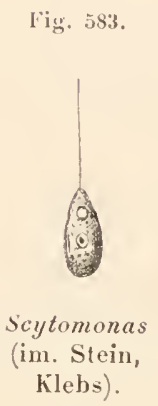

Anisonema

(im. Klebs, Stein). la partie supérieure du sillon précédent. Les deux fouets s'y insèrent à côté de l'orifice d'ingestion. Il ne parait pas y avoir d'appareil pharyngien. Des deux fouets, le plus petit est ascendant, le plus grand est descendant et inséré au-dessous de l'autre $13 \%$. Eau douce et peut-être aussi mer) $\left(^{2}\right)$.

Marsupiogaster (Chevialiof), avec ses deux fouets implantés au bord droit du péristome, nous semble devoir prendre place Fig. 58.;. ici (Eau douce).

Tropidoscyphus (Stein) (fig. $\check{83}$ ) est remarquable par huit fortes carènes longitudinales qui ornent son corps.

Entosiphon(Dujardin, emend.Stein)(fig..586) a le pharynx représenté par un lárge enfoncement termino-ventral, dans lequel fait saillic un appareil pharyngien protractile, et où s'insèrent les deux fouets dont l'inféricur est un peu plus grand et un peu rejeté de côté (20 it 30 p.. Her et eau douce) $\left({ }^{\mathbf{3}}\right)$.

(1) Ces deux genres forment la famille des PETAlomoradire [Petalomonadina (Bütschli)].

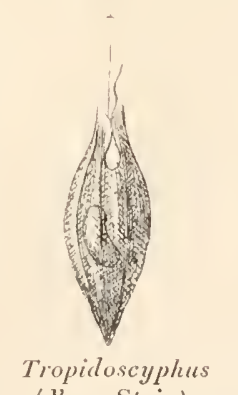

(d'ap. Stein).
Fig. 586 .

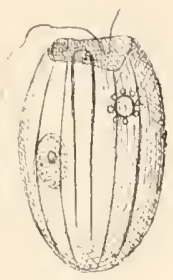

Entosiphon (im. Klebs, Kent).

(2) Klebs distingue un sous-genre

Metanema (Klebs) qui a tous les caractères d'Anisonema, mais dont le corps est nétabolique comme chez Heteroneminx, et dont les deux fouets sont subégaux.

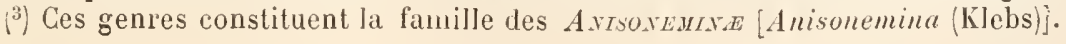




\section{$3^{\text {e }}$ ORDRE \\ PIITTOFLAGELLIDES. - PIIYTOFLAGELLIDA \\ [non Phytonovadisa (Blochmann)]}

Les représentants de cet ordre pour lequel il n'est guère aisé de constituer un type morphologique ont pour caractère commun une alimentation holophytique, identique à celle des régétaux colorés, en particulier des Algues, en suite de quoi la bouche et le pharynx disparaissent et l'animal se trouve ramené à une constitution très semblable à celle de certaines zoospores. Les formes les plus avancées de ce groupe arrivent à ressembler à tel point à des plantes qu'on les place souvent parmi les végétaux.

Nous diviserons l'ordre immédiatement en quatre tribus:

$1^{\circ}$ ChLOROMONADINA, à corps nu, possédant de la chlorophylle et pourvus d'une bouche et d'un pharynx ne servant pas à l'alimentation;

2。 CIR OMOMONADINA, à corps nu, possédant deux lames chromoplastiques jaunes, sans bouche ni pharynx;

$3^{\circ}$ CHLAMYDOMONADINA, à corps enfermé dans une capsule ferme, possédant de la chlorophylle, sans bouche, ni pharynx;

$4^{\circ}$ VOL VOCINA, à corps enfermé dans une substance gélatineuse commune à de nombreux individus formant une colonie, possédant de la chlorophylle, sans bouche ni pharynx.

\section{1re T'RIB U}

\section{CHLOROMONADINES. - CILOROMONIDINA \\ [CILOROMONADINA (Ḱlebs)]}

\section{TYPE MORPHOLOGIOUE}

(FIG. 587)

L'animal a un peu l'aspect d'une Euglène. Il en a la forme générale et la couleur verte, due comme chez elle à une couche de petits grains de chlorophylle disposés sur la surface du corps. Mais, au lieu du tégument solide et d'un dessin ferme de celle-ci $(c)$, il a un périplaste épais (ectop.), réfringent, homogène et très délicat, recouvert d'une très mince pellicule membraneuse. Aussi est-il très métabolique et même un peu amoboïde. Il n'a pas non plus les stries contractiles ni le stigma que l'on remarquait chez l'Euglène. Mais la différence capitale consiste dans la constitution de l'extrémité supérieure. En place du profond entonnoir pharyngien, on ne trouve qu'une petite dépression $(b$.$) dans laquelle$ s'insèrent deux flagellums subégaux ( $f l g . s$. et $f l g . i$.) et, du fond de la dépression, part un canal qui représente peut-être morphologiquement le pharynx des Euglènes, mais qui n'est actuellement que le canal excréleur d'une vaste vésicule collectrice par laquelle s'écoulent au dehors les produits de la vésicule pulsatile $\left(\boldsymbol{I}^{c} \mathrm{c}.\right)$, située un peu plus 
haut et entourée de sa couronne habituelle de petites vésicules formatrices (v.).

Plus bas que la vésicule, se trouve

le noyau $(\boldsymbol{N}$.). L'animal se reproduit par division sous une enveloppe de gélatine après perte des flagellums ef rétraction du corps, comme l'Euglène.

\section{GENRES}

Cœlomonas (Stein) (fig. כ̋SS). Ce serait exactement notre type morphologique si le fouet n’était unique (60 p.. Eau douce). Nais chez

Vacuolaria (Cienkovsky), au contraire, il y a bien les deux fouets, le second un peu plus petit et ramenélelong du corps (').

\section{Raphidomonas} (Stein) (fig. $589)$, se distingue de $\mathrm{C} e$ lomonas uniquement par une constitution plus ferme, par la possession éventuelle d'undeuFig. 588. Fig. $\quad$ 589.

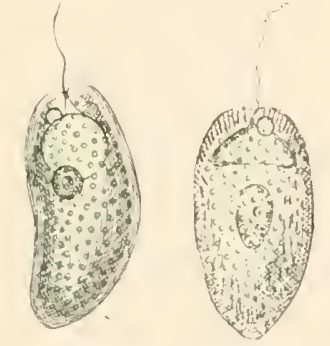

Colomonas (C. grandis) Raphidomonas (im. Stein).

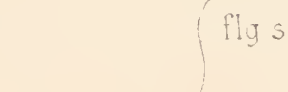

xième fouet, et la présence singulière de trichocystes dans l'ectoplasme. C'est le seul Flagellé qui en possède (50 … Eau douce).

2e T'R IB U

\section{CHRONONONADINES. - CHROMOMOMHDII \\ [CHROMOMONADINA (Klebs)]}

\section{TYPE MORPHOLOGIQUE}

(FIG. 590)

lci, les téguments ont la mème constitution délicate et homogène que chez les Chloromonadines, mais la bouche et le pharyux ont entièrement disparu. Il n'y a ni solution de continuité des téguments, ni mème

(1) Mais KLebs pense que ces deux genres n'en font qu'un et que Stein n'a pas vu le second fouet. Vacuolaria ne forme ni amidon, ni paramylum, mais de la graisse. C'est la forme que prennent, d'après K'lebs, ses réserves alimentaires. 
d'invagination en cul-de-sac. Les flagellums ( $f l g$.), au nombre de deux, égaux, šinsèrent simplement à la surface du corps. L'animal ne prend

Fig. 590.

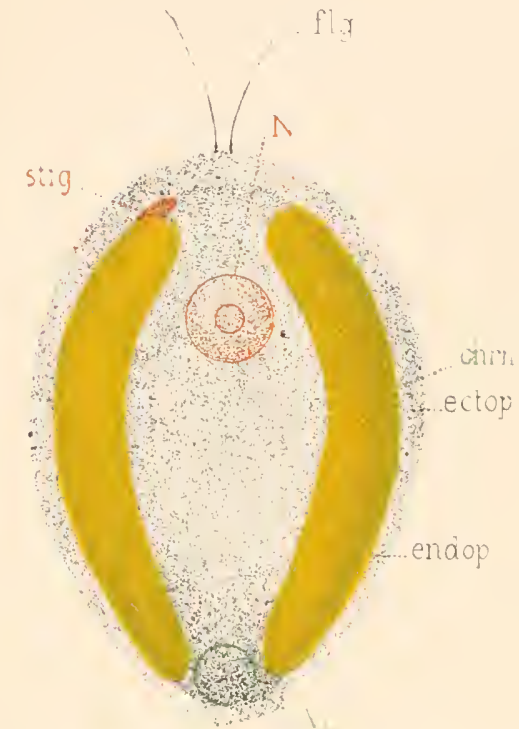

CIROMOMONADIN.1 (Type morphologique)(Sch.).

chrmp., chromoplastes : endop., endoplasma ;

eetop., ectoplasma; flg., flagellums; $\mathbf{N}$, noyau;

stig., stigma; V.e., vésiculc pulsatile. aucune nourriture solide renant du dehors ("). Il se nourrit à la manière des plantes au moyen de deux larges lames chromoplastiques (chrmp.) situées symétriquement à droite et à gauche sous la surface. Mais ces deux lames sont teintérs non en vert, mais en jaune plus ou moins rabattu de lorun par une substance, le chrysochrome (Klelss), voisine de la diatomine, et qui contient peut-être de la chlorophylle masquée par une teinte additionnelle, car en la traitant par l'alcool on la fait virer au vert comme si l'alcool dissolvant d'abord la couleur étrangère faisait apparaître la chlorophylle. Ces lames chromoplastiques ne conliennent pas de pyrénoüdes et la substance qu'elles élahorent au contact du cytoplasma n'est ni l'amidon ni le paramylum, mais une substance d'aspect graisseux et de nature protéique soluble dans l'eau, la leucosine (Klehs), qui se retrouve aussi chez les Hydrurées el les Phrosporées, et forme souvent dans le corps des accumulations considérables.

Accolé à l'une de ces lames, près de l'extrémité supérieure, est un stigma rouge (stig.).

Le noyau $(N$.$) est gros, vésiculeux, très pàle.$

La vésicule pulsatile (V.c.) simple ou multiple ( 1 à 5 ) est tantòt en haut tantòt en bas.

L'animal se reproduit par division longitudinale à l'état libre ou sous une enveloppe gélatineuse, et forme aussi, à l'occasion, des kystes de protection.

\section{GENRES}

Nous rencontrons d'abord deux genres notablement aberrants par rapport au type morphologique qui précède.

(1) Parfois, cependant, il absorbe quelques Bactéries, à la manière des Monades, au moyen d'une vacuole qui se forme vers l'extrémité supérieure. 
Cryptomonas (Ehrenberg) (fig. 591), en effet, possède un vaste infundihulum où s'insèrent les deux cils; il en part un tube qui plonge dans le corps et dessine un large pharynx, mais qui n'a peut-ètre pas la signification fonctionnelle de cet organe $(30 \mu$. . Mer et eau douce).

Chilomonas (Ehrenberg) se distingue du précédent par l'absence de plaques chromoplastiques et par une alimentation saprophrtique. Chose remarquable, il n'en forme pas moins de l'amidon (30 $\mu$. Infusions).

Cyathomonas (Fromentel, emend. K’nt) présente le même caractìre d'absence de chromoplastes, mais son alimentation est animale (25 $\mu$. Eau douce) (').

Les suivants sont au contraire conformes au type. Ils

Fig. 591 .

divisent en trois groupes selon que leur corps est nu ou protégé par une capsule ou une membrane adhérente au corps.

La première série de genres comprend ceux qui sont nus :

Chrysamœba (Klebs) (fig. 392 ) est aux Chromomonadines ce que sont aux Acraspédines les Mastigamibes. Quand il nage, il est conforme à notre type morphologique sauf qu'il ne portequ'un Fig. 593 . flagellum. Son corps nu est ile forme ovoïde et régulière. Mais par moment il s'arrête et se transforme en une amibe à vrais pseudopodes longs et fins,

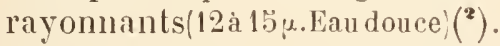
Chromulina(Cienkovsky)(fig.:593) n'est plus amoboürle que par places, surtout à l'extrémité inférieure; il est nu aussi et n'a également qu'un flagellum ("a à 40 p.. Eau douce) $\left({ }^{3}\right)$.

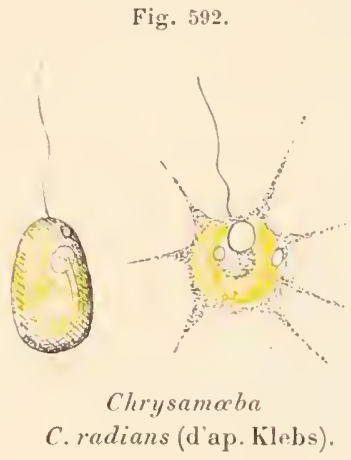

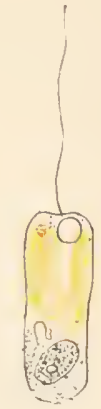

Chromulina (d’ap. Klebs).

(1) Ces trois genres forment à eux seuls la famille des CrYPTononADIx E [Cryptomonadina (Bütschli, emend. Klebs)]. D'après Dangeard, le pharynx ne serait pas infundibuliforme, mais formerait une simple gouttière ouverte en avant et tapissèe au fond de petits bitonnets d'une substance plasmatique. Les genres suivants de cette tribu constituent la famille beaucoup plus nombreuse des CnRrsonordDIx $[$ Chrysomonadina (Stein, emend. Klebs)] divisés, par leur auteur, en trois groupes: les nuda, nus ou enveloppés seulement, pendant l'état de repos, d'une couche de gèlatine; les loricata, abrités en tout temps dans une capsule mince et ferme, plus large que leur corps, et les membranata protẻgés par une enveloppe étroitement collée au corps.

(2) Cependant, mème dans cet état, il ne prendrait aucune nourriture solide, mais la chose est niée par certains auteurs. Ses lames de chrysochrome sont au nombre de deux à trois; il a deux à trois vésicules pulsatiles petites et une plus grande, et pas de stigma.

(3) Par une exception rare dans ce groupe, il absorbe aussi de la nourriture solide, en particulier des Diatomées. Dans l'état de repos, il se recouvre de gélatine. Il n'a parfois qu'une seule lame de chrysochrome. 
Ochromonas (Vyssotzki) (fig. 5้9') a encore des mouvements amœboïdes, parfois aussi une seule lame de chrysochrome, mais possède deux flagellums (15 à 20 . Eau douce) (').

Stylochrysalis (Stein) (fig. 595) a aussi deux cils, mais n'est plus amœboïde et est fixé par un pédoncule gélatineux. Il n'a pas de stigma (9 p.. Eau douce, fixés sur des Eudorina).

Les genres suivants ont le corps protégé par une capsule:

Chrysococcus (Klebs)

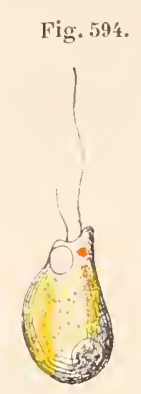

Ochromonas (d'ap. Klebs).
Fig. 595 .

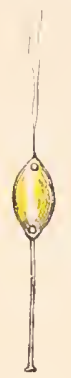

Stylochrysatis (S. parasitica) (d'ap. Stein).
Fig. 596.

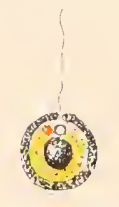

Chrysococcus (C.rufescens) (d'ap. Klebs).
Fig. 597.

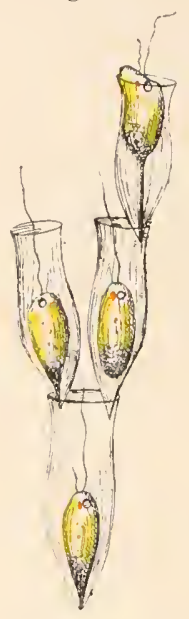

Dinobryon.

(D. sertularia) (im. Klebs, Stein).

Dinobryon (Ehrenberg) (fig. :397) a, au contraire, deux fouets, dont un très petit. Il est piriforme et se sécrète une capsule de forme analogue à la sienne, où il est très au large et fixé seulement au fond par l'extrémité effilée de son corps. Il nage librement en pleine eau, emportant sa capsule. Souvent les jeunes se fixent au bord interne de la capsule des parents el il en résulte des colonies arborescentes libres nageantes (1), I. Eau douce, au large dans les étangs) $\left({ }^{\boldsymbol{3}}\right)$.

Enfin, dans cetle dernière série de genres, l’animal est protégé, non par une capsule, mais par une memhrane adhérente au corps.

Hymenomonas (Stein) (fig. 598 ) représente aussi notre type morphologique, sauf qu'il a deux fouets et pas de stigma; son enveloppe est épaisse, molle, de couleur brunâtre (15 à $25 \mu$. Eau douce) $\left({ }^{1}\right)$.

(1) Il prend aussi de la nourriture solide.

(2) Il se divise dans sa coque et le jeune sort nu.

(3) La partie inférieure du corps contient une masse accumulée de leucosine. Il s'enkyste à l'occasion dans sa loge sous une épaisse coque siliceuse.

Genres voisins :

Epipyxis (Ehrenberg), solitaire, libre ou fixé par l'extrémité inférieure de sa capsule;

Chrysopyxis (Stein), fixé par deux filaments émanės de sa capsule. loin.

Blochmann place ici Nephroselmis que nous rencontrerons plus

(4) Il possède une accumulation de leucosine à l'extrémité inférieure du corps. 
Microglena (Ehrenberg) (fig. :̈99) rappelle le précédent, mais n’a qu’un foret; son enveloppe est beaucoup plus mince et son stigma peut ètre double ou triple ( $30 \mu$. Eau douce).

Mallomonas (Perty) (fig. 600) n'a aussi qu'un fouet, mais pas de stigma et son enveloppe est épaisse, réticulée et garnie de longues soies railes, dirigées en bas (20 à $25 \mu$ ).

Synura (Ehrenberg) (fig. 601, 602) ressemble au précédent, sauf qu’il a deux fouets, que sa coque est très mince, continue, et que les soies sont plus courtes, dirigées en haut et non constantes. Mais son caractère essentiel est qu'il forme des colonies sphériques, nées de la division longitudinale, dans lesquelles les individus, dont le nombre peut atteindre soixante, restent attachés ensemble au centre par leur extrémité inférieure effilée en un prolongement caudal. Ces colonies peuvent se diviser; elles peurent aussi se dissocier en mettant leurs membres en liberté

Fig. 599.

(33̈ u. environ et, par conséquent, 70 p. de diamèlre pour les colonies, Eau douce) (').

Fig. 601.

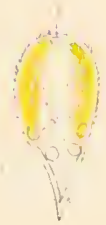

Syиura.

Un individu isolí

(d'ap. Klebs). lïg. 602 .

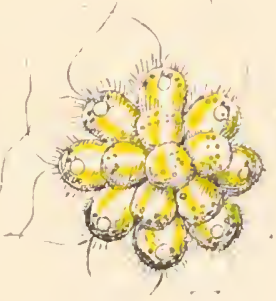

synura (s. uvella).

Colonio

(d'ap. Stein).
Fig. 603 .

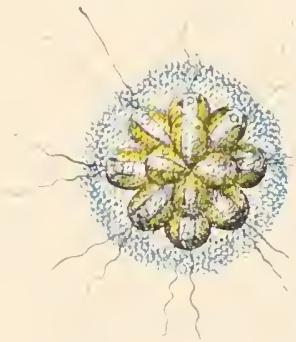

Synerypta. Colonie (d'ap. Stein).

Syncrypta (Ehrenberg) (fig. 603) représente une colonie de Synura, mais où les individus seraient nus ef la colonie, au contraire, pourvue d'une épaisse enveloppe gélatineuse qui ne laisse passer que les fouets (Colonie, 4ö p.. Eau douce).

Chlorodesmos (Phillips) est un genre voisin un peu douteux dont la colonic est en chaìne (Eau douce).

(1) Les individus contiennent une grosse accumulation de leucosine. 
Uroglena (Ehrenberg) (fig. 60') est formé d'individus nus avee deux fouels inégaux, réunis dans une épaisse masse gélatineuse sphérique. lls se prolongent tous vers le dedans en un filament caudal qui se ramifie et s'anastomose avec ceux des autres individus de manière à constituer un réseau qui va jusquau centre. Cela sert à donner plus de solidité à l'union des individus entre eux, car leur gélatine est extrêmement faible. Les colonies se divisent (Zacharias [94]) (Colonie, plus de 0,1. Eau douce).

Toutes ces formes ne sont point sans analogie avec les Volvocines, mais elles s'en distinguent nettement par leur matiòre colorante jaune et non verte.

3e TriBu

CHL.MYDOMONADINES. - CHLAMYDOMOMADINA

[CHLAMYDOMONADINA (Bütschli)]

TYPE MORPHOLOGIQUE

(FIG. 605 A 607)

Ici, le corps n'est plus, comme dans les Phytoflagellides précédents, revêtu d'un tégument délicat permettant des déformations variées. Il est emprisonné (fig. 603 ) dans une capsule ferme ( $m b$.), entièrement fermée et adhérente au corps, qui rappelle la membrane cellulosique épaissie d'une cellule végétale. Dans certains cas, on a constaté qu'elle était formée de cellulose. Cependant elle paraît correspondre plutôt à une capsule qu'à une vraie membrane cellulaire, car elle ne prend pas part à la division du corps, et parfois elle est ouverte et non adhérente au corps. Par elle, toute déformation du corps est empèchée.

Il n'y a pas trace de bouche ou de pharynx. L'extrénité supérieure régulièrement arronlie donne insertion à deux fouets égaux ( $/ l g$.). A l'intérieur, il n'y pas d'ectoplasme distinct. Le corps est occupé par une calotte le protoplasma chargé de chlorophylle qui l'embrasse tout entier sauf l'extrémité supérieure. Dans cette couche, immédiatement sousjacente à la membrane, sont plongés quelques pyrénoïdes épars $(p$.$) ,$ formés de leur petite masse rentrale de protoplasme et d'une enveloppe d'amidon, de vrai amidon végétal. Lu haut, est une vésicule pulsatile ( $V . c$.$) , et sur le côté un stigma (stig.) formé comme celui des Euglènes,$ 
sauf qu'il n’y a qu'un corps réfringent unique, central et formé d'un grain l'amidon.

L'ètre nage avec ses cils el se nourrit exactement comme une Algue véritable. Parfois il peut se mettre à l'abri sous un liyste. Pour se reproduire, au lieu de se diviser simplement en long comme un Flagellé ordinaire, il se comporte de la manière suivante (fig. 607). Sous la capsule qui reste intacte, il divise son corps protoplasmique en deux $(A)$, puis en quatre $(B)$, puis en huit $(C)$ et enfin en trentedeux $(I)$. Chose singulière, pendan t tout le temps de cette division, l'individu mère garde ses fouels et continue à se mouvoir, re qui

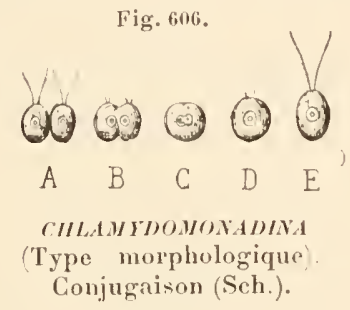

laisse supposer que ses fonets restent en relation avec quelqu'un des produits de la division. Enfin, les fouets tombent, la membranes'ouvie, les jeunes sortent munis de leur's deux fouets et se mettent à nager $(E)$. Alors ils se fusionnent par conjugaison totale (fig. 606)

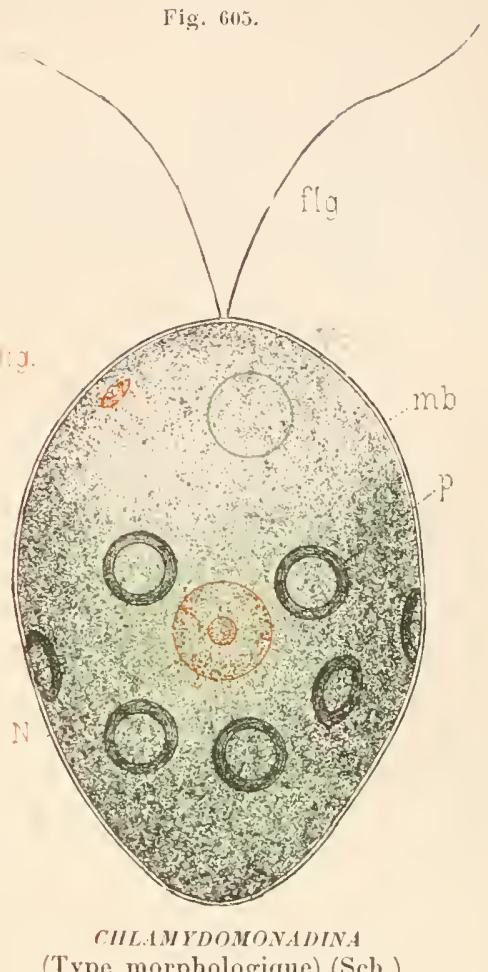

(Type morphologique) (Sch.).

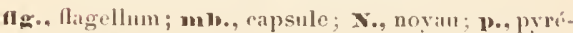
uoüdes: st is., stigma; V.e., visienl. pulsitili.

en un zygote arrondi et sans fouets. Mais bientôt les deux fouets se montrent, l'animal se met à nager et n'a plus qu'à grandir (').

(1) Ce cycle évolutif fort simple se complique dans la réalité par le fait que, 
Ces êtres, on le voil, ont beaucoup d'affinilés avec les Algues inférieures, et divers botanistes les réclament pour les placer à côté des Protococcus.

\section{GENRES}

Chlamydomonas (Ehrenberg) (fig. 608) est notre type morphologique, mais avec une forme cylindrique ou sphérique et une vésicule pulsatile double ( 40 p.. Eau douce et mer).

Chlorogonium (Ehrenberg) (fig. 609) s'en distingue, au contraire, par une forme très allongée et par la possession de nombreuses vésicules pulsatiles disséminées, ce qui est exceptionnel chez les Flagellés. Il y a une variété incolore (120 p. Eau douce).

Polytoma (Ehrenberg) ressemble encore plus à Chlamydomonas, bien qu’il ait un stigma, mais il est incolore. Aussi est-il saprophyte. Il forme de l'amidon aussi bien que s'il avail de la chlorophylle (Eau douce et infusions'.

Fig. 608 .

Hæmatococcus (Agardh) (fig. 610) ressemble à Chlamydomonas mais, sous sa capsule ovoïde, le corps protoplasmique est comme rétracté et ne tient à elle que par des brides rarliaires et par un prolongement au Fig. 610. Fig. 611. niveau des fouets. Pas de stigma (30 à 60 ‥ Mer, eau douce et neige des hautes montagnes et des régions polaires qu'il colore en rouge sang).

Carteria (Diesing) (fig. 611) est un Chlamydomonas à quatre foucts (20 p.. Eau douce).

Spondylomorum (Ehrenherg) est une colonie cylindrique de seize Carteria disposés en quatre couronnes superposées de quatre individus. Les

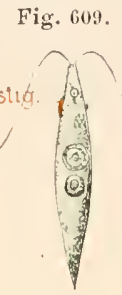

Chlorogonium (im. Franzé).
Chlamydumonas

(C. obtusa)

(d'ap. Franzé). colonies naissent ainsi toute formées de la division d'un mème inılividu

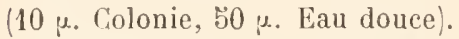

Chlorangium (Stein) (fig. 612) présente quelque chose de semblable, mais dérivant d'une forme à deux fouets et sans stigma. Les individus se forment sous la membrane maternelle, fixés par un péloncule qui part

après la division en quatre ou en huit, les quatre ou huit jeunes peuvent sortir de la capsule et mener quelque temps une vie libre avant d'achever leur division en huit ou en quatre pour arriver finalement toujours a trente-deux, après quoi is se conjuguent.

On a interprété comme macrogamètes et microgamètes ces différents gamètes dont la taille diffère naturellement par suite de celte particularité de la division. Mais le zygote se forme aussi bien par l'union de gamètes de même taille que par celle de gamètes différents.

Il n'y a donc là qu'un fait secondaire, nullement sexuel. 
de leur extrémité céphalique, en sorte qu’après la déhiscence de la capsule, ils forment un petit buisson $(F)$. Mais, à un moment donné, ils se détachent, forment une paire de fouets là où étail le pédoncule et nagent librement à la manière d'un Chlamydomonas. Puis, après un certain temps, ils perdentleurs fouets, se fixent parla tête $(B)$, développent un péloncule $(C)$ et se divisent sous leur membrane $(D$ et $E$ ) pour recommencer le même cycle (30 $\mu$. Eau douce).

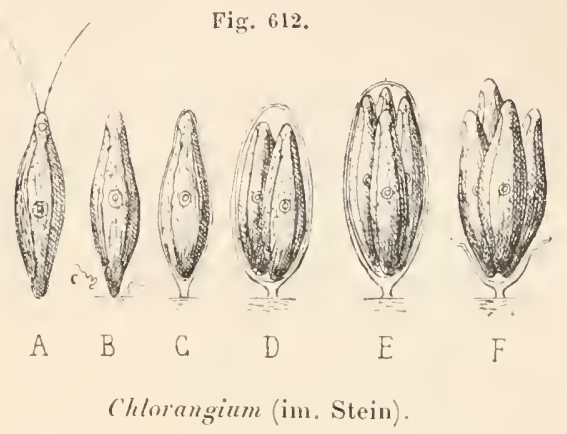

Coccomonas (Stein) (fig. 613) nous ramène à une forme isolée libre. Il ressemble fort à Hæmatococcus par sa capsule trop large pour son corps; mais cette capsule Fig. 613. Fig. 614. Fig. 613. Fig. 614. est percée au haut d'un petil orifice qui fait communiquer sa cavité avec le dehor's et par où sortent les deux fouets (20\%. Eau douce) ('). Mesostigma (Lauterjorn) est réniforme, muni de deux fouets un peu au-dessus du milieu de la face ventrale qui porte le hile. Sa capsule est délicate et ponctuée au bord (18 «. Eau douce stagnante).

Phacotus (Perty) (fig. 614) a une coque solide (peut-être silicieuse), lenticulaire, plus ou moins ornée de sculptures et bivalve (les deux valves étant simplement

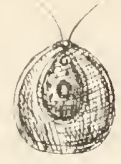

Coccomonas

(iim. Stein).

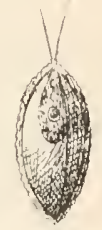

Phacolus

(P. lenticulaiis) (im. Stein) rapprochées et non soudées, et se détachant après la mort), mais sans ouverture large pour le passage des fouets. L'animal n'occupe qu'une partie de sa loge (25 p.. Eau douce) $\left(^{2}\right)$.

(1) On ignore s'il n'y a pas une seconde membrane
Fig. 615.

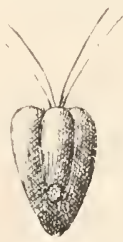

Pyramimonas (im. Stein).
Fig. 616. mince doublant le corps en dedans de la capsule.

${ }^{(2)}$ Bütschli place ici avec doute quelqueś formes dont les caractères ne se rapportent nettement à celle d'aucun groupe;

Tetratoma (Bütschli), qui serait un Carteria dont les quatre fouets s'inséreraient en quatre points séparés (Eau douce);

Pyramimonas (Schmarda) (fig. 615) a quatre fouets, mais rapprochés au sommet d'un corps conique muni de quatre côtes verticales et d'une mince enveloppe strièe ( 330 u. Eau douce);

Chloraster (Ehrenberg) (fig. 616), muni d'un cinquième fouet au milieu des quatre du genre précédent dont il ne diffère d'ailleurs en rien d'essentiel sous les autres rapports ( 33 p.. Mer et eau douce).

Blochmann les place dans une famille de Polyblepharidæx voisine des Chlamydomonadines dont il prend le type dans le genre 


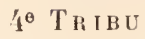

VOLVOCINES. - VOL VOCINA

[VoLVOCINA (Ehrenberg, emend)]

TYPE MORPHOLOGIOUE

(FIG. 617 ET 620 A 624)

\section{Structure.}

L’inclividu isolé est de structure très simple. Il diflère à peine de

Fig. 61 .

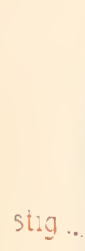

rolvoCIxA (Type morphologique) (Sch.).

Giel., gélatine; N., noyau; p., pyrénoïdes; stig., stigma. celui des Chlamydomonadines. C'est le même petit Flagellé piriforme, muni au gros bout de deux fouets, sans ectoplasme ni membrane distincts, dont le corps est coiffé d'une calotte de protoplasma coloré par de la chlorophylle où l'on distingue un ou deux gros pyrénoïdes $(p$.$) et de nombreux$ grains plus petits. Sous le flagellum, il montre deux petites vésicules pulsatiles. Son noyau $(N$.$) est gros et$ central. Un peu au-dessous les vésicules, il porte d'un côté un stigma rouge (stig.) formé d'un réticulum protoplasmique contenant dans ses mailles de nombreuses gouttelettes d'une huile rouge et, au centre, une lentille formée d'un grain l'amidon sphérique. Les

Polyblepharides (Dangeard) (fig. 618) à six ou huit foucts égaux, muni d'une délicate enveloppe de cellulose et coloré en vert vif. Sous le noyau, est un pyrénoïde arec enveloppe d'amidon. 11 a y un stigma et deux vésicules pulsatiles (10 à $14 \mu$. Eau douce).

Nous placerons ici avec Klebs le curieux

Nephroselmis (Stein) (fig. 619) réniforme, très aplati et à grand axe transversal. De l'échancrure tournée en haut naissent deux fouets subégaux. Toul le corps est entouré d'un long cordon arqué formé de substance chromatique brune avec un gros

Fig. 618 .

Fig. 619.

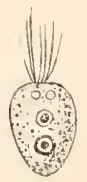

Polybleptarides

(P. singularis)

(d'ap. Dingeard). 
produits de réserve prennent, comme chez les Chlamylomonadines, la forme de grains d'amidon. Ce qui constitue ici le trait le plus frappant, c'est que l'animal (fig. 620) sécrète autour de lui une abondante substance gélatineuse Fig. 620 . (gel.) dans laquelle tous les individus nés de la division d'un mème individu mère sont enrobés côte à côte en une colonie sphérique ('). La colonie est permanente en ce sens que jamais les individus qui la composent n’ont élé et ne seront libres el isolés. Tous sont près de la surface et leur corps est complètement noyé dans la gélatine, mais leurs fouets émergent et mettent la colonie en mouvement.

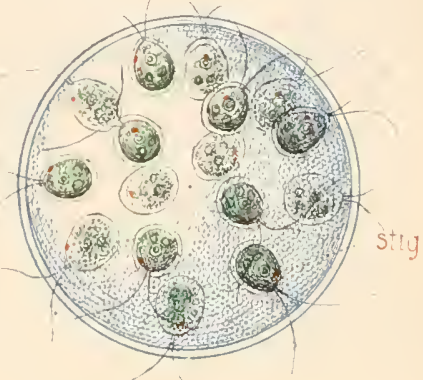

\section{Physiologie.}

Mouvements. Nutrition - La colonie se VOLVOCINA (Type morphologique). Colunie (Sch.). meut suivant une certaine direction, en touruant sur elle-mème. Les indivilus ne prennent aucune proie, leur alimentation est purement holophytique ef ces associations n'ont qu'un avantage défensif en formant les masses qui, par leur taille, résistent aux attaques des ennemis.

Reproduction - La colonie n'augmente pas le nombre de ses membres. Elle grossit seulement en se nourrissant et lorsqu'clle est bien adulte, elle commence à se reproduire d'abord par voie agane.

Division. - Les individus (fig. 621), les uns après les autres, au fur

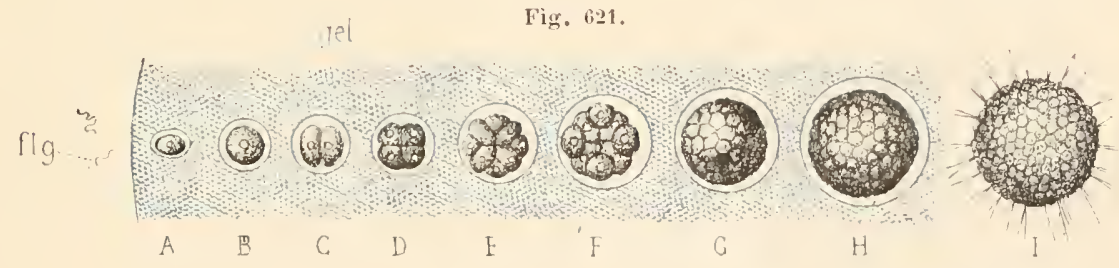

VOLVOCINA (Type morphologique). Reproduction par division (Sch.).

1. I'individu perd ses cils; $B$, il gague l'intérieur de la colonie; $C$ a $I$, stades successifs de la division

$I$, mise en liberté de la colonie fille. t1g., flagellums abandonnés; gel., gẻlatine de la colonie mére.

et à mesure qu'ils arrivent à maturité, perdent leurs fouets $(A: / g \cdot)$, rentrent dans l'intérieur de la gélatine $(B)$ et s'y divisent $(C$ à $I I)$. Cette division se fait d'une manière toute spéciale, par des plans rarliaires, en sortequ'au stade huit $(E)$, l'animal est découpé en huit segments, tous dans

grain d'amidon au milieu. Dans la concavité de l'are est la vésicule en haut, le noyau au-dessous. L'animal se meut transversalement dans la direction de son grand axe (18 p. Eau douce).

(i) Cette substance est considérée par quelques botanistes comme une membrane gélifiée. 
un même plan et convergeant vers le centre, comme une tarte découpée en huit parties. Mais à mesure que la division se poursuit, la lame s'incurve $(F)$ et finit par former une sphère creuse $(G)$ percée seulement d'un petit pore qui, lui-même, finit par disparaître $(H)$. Tous les inılividus sont à ce moment serrés les uns contre les autres, mais alors la petite colonie qui a, maintenant, son nombre de membres normal et définitif, sort de la colonie mère par destruction de la paroi de celle-ci $(I)$, sécrète sa gélatine qui écarte les individus les uns des autres et se met à nager, tournant en avant le pôle opposé au point où la sphère a achevé de se fermer. Elle n'a plus qu'à grossir. La colonie mère disparaît ainsi par transformation de tous ses membres en colonies nouvelles, mais sans que rien ne meure en elle, puisque tout, au contraire, prend un nouveau développement.

Gonjugaison. - Les choses se passent ainsi pendant toute la première

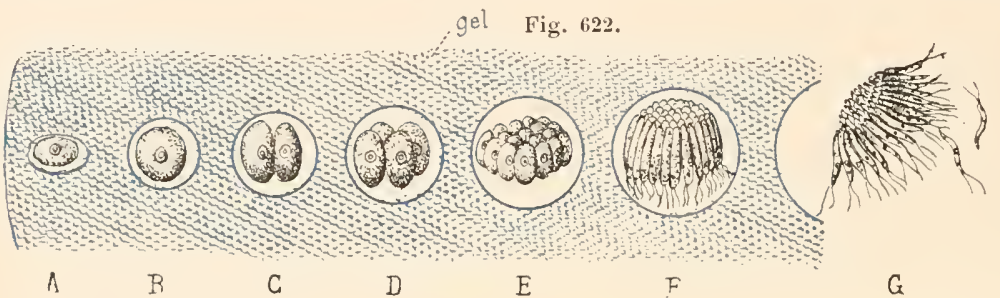

FOLIOCINA (Type morphologique). Formation des microgamètes (Sch.).

partie de l'année. Vers la fin de l'été (fig. 622), certains individus de

Fig. 623. la colonie se comportent comme précédemment ( $A$ à $E$ ); mais, dans les jeunes colonies auxquelles ils donnent naissance, toujour's par le mème procédé de division, les

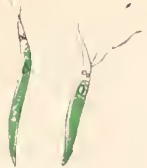

rOLVOCLNA

(Type morphol.).

Deux formes de microgamètes

(im. Stein). individus, au lieu de revètir la forme ordinaire, s'effilent beaucoup $(F$ et $G$ ) et deviemnent des microgamètes (fig. 62:3). Leur constitution interne est cependant au fond la même que celle des individus ordinaires; ils ont leur's deux vésicules, leur stigma, leur noyau, leur partie postérieure verte et leur pointe incolore et munie de deux fouets. Cette petite colonie màle sort de la mère, nage et, au moment voulu, se dissocie en ses membres qui vont léconder les macrogamètes. D'autres individus de la colonie maternelle, au contraire, ne se dirisent pas (fig. 621); ils deviennent des macrogamètes en grossissant sur place ou dans la profondeur de la gélatine où les microgamètes vont se conjuguer à eux (fig. 625). Après la conjugaison qui est totale, le

Fig. 62'.

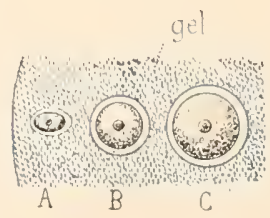

FULVUCIA

(Type morphologique). Formation des macrogamètes (Sch.). zygotes'enkyste(fig.625, $A$ ) sous une double membrane et mis en liberté par 
la destruction de ce qui reste de la colonie, tombe au fond de l'eau. Cela se passe à la fin de l'automne. Le kyste reste au fond le l'eau tout l'hiver, puis, dès le premier printemps, la membrane externe éclate, l'interne se gélifie et, sous cette enveloppe, le corps protoplasmique se divise. Cette division a lieu par le mème procédé que toutes les précédentes et donne naissance à une petite colonie sphérique (I) qui, dès qu'elle a acquis son nombre normald'individus, se munit de ses fouets et se met à nager, toujours en

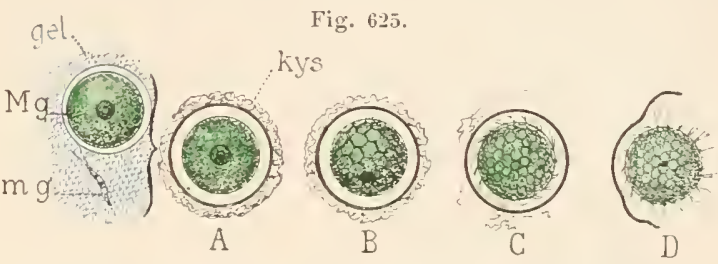

rOLFOCRA (Type morphologique). Conjugaison (Sch.). 1, zygote; $B$ et $C$, division à l'intérieur du kyste; $D$, mise en liberté de la eolonie; gel., gélatine; kys., kyste: Mg., macrogamete; mg., microgamète. toumant en avant le pòle opposé au point de fermeture de la sphère.

Notre type morphologique représente une forme moyenne, avec un cycle évolutif moyen. Nous allons voir ce cycle commencer par l'isogamie pure et arriver, dans les formes les plus élevées, à une véritable fécondation sexuelle et même à la séparation des sexes.

\section{GENRES}

Stephanosphæra (Cohn). La colonie est formée de huit individus disposés d'abord en anneau dans un plan, leurs foucts dirigés dans le plan (fig. 626) de l'anneau, et réunis par une gelée commune ( $\mathrm{gel}$.).

La colonic arrive ì la forme sphérique, mais non par le processus de notre type morphologique. Elle sécrète une masse sphérique le gélatine lont l'anneau primitif forme l’équateur. La surface de cette gelée se conlense en une membrane, les individús s’al-

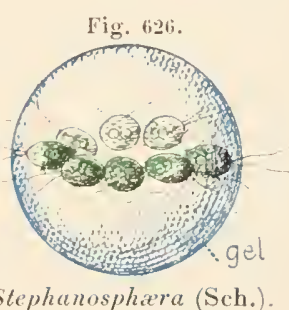

Stephanosphiera (Sch.). longent perpendiculairement au plan équatorial (fig. 627 7 ), et s'attachent

Fig. 627.

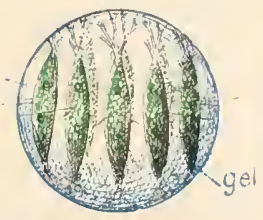

Stephanosphxra. Colonie (Sch.). à la membrane par leurs extrémités ramifiées. Les fouets n'en restent pas moins disposés suivant l'équateur et déterminent, en même temps que la translation, une grration autour de l'axe perpendiculaire à cet équateur.

La reproduction agame n'offre rien de particulier mais, dans la conjugaison, il n'y a pas distinclion en macrogamètes et microgamètes. Toutes les cellules de la colonie se divisent chacune en seize ou trente-deux gamètes semblables qui se conjuguent. Le zygote se comporte comme nous avons dit, mais 
au printemps, il donne d'abord par division quatre individus libres qui, chacun, donne naissance en se divisant à une colonie normale (Colonies 30 à 60 p. Eau douce, surtout eaux de pluie (').

Pandorina (Bory de Saint-Vincent) (fig. 628) a la forme d'une sphère pleine de 16 à 64 individus, à fouets orientés suivant les rayons de la sphère. La reproduction agame a lieu suivant le mode indiqué, sauf que la jeune colonie se recourbe d'emblée en sphère pleine et non creuse. Pour la conjugaison, les colonies nées par voie agame donnent naissance à de petites colonies de huit cellules qui deviennent chacune un gamète simplement en prenant leur liberté. Tous les gamètes sont égaux. Il y a donc isogamie comme chez le Stéphanosphère. Mais le zygote, au retour du printemps. donne directement naissance aux colonies comme dans notre type morphologique 60 -0. 0. . Ean douce. Colonies) $\left(^{(2}\right)$.

(1) Genres voisins :

Stephonoma (Werneck);

Gonium (0. F. Muller, emend.) (fig. 629). L'être forme des colonies de huit ou seize individus non réunis par des communications protoplasmingues, disposés Fig. 628.

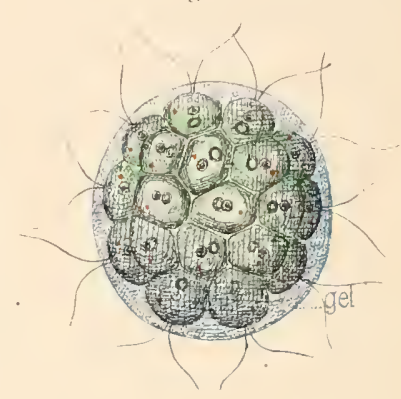

Panderina. Colonie sith aussi dans un plan, mais avec les fouets lournés ver's le haut, el animés dun tremblement saccadé et irrégulier. Dans certaines conditions, les cellules de la colonie se dissocient, perdent leurs cils et, après une période de repos durant laquelle elles se sont entourées d'une membrane de cellulose, se divisent pour constituer une nouvelle colonie de quatre cellules mobiles dont on n'a pas suivi le développement ultérieur. Le chromatophore n'est pas homogène, mais formé par la réunion de granulations chlorophylliennes rle $1 / 2$; de diamètre séparées par des espaces incolores (M1GULA).

La conjugaison n'est pas connue (Colonie, 90 ;. Eau douce).

Pectoralina (Bory de Saint-Vincent);

Glenogonium (Diesing).

G'est ici que nous semble devoir prendre place le genre

Stephanoon (Cheviakof) dont les colonies en forme d'ellipsoïde de révolution comprennent seize individus disposès en zigzag dans le plan équatorial (Eau douce, Australie).

Mastigosphæra (Cheviakof) se rapproche plutôt de Pando-

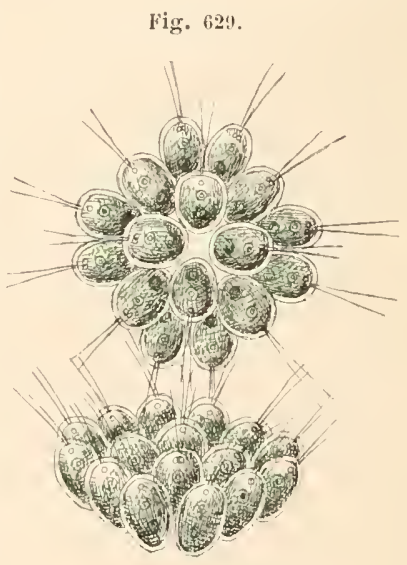

Gonium.

Colonie, en haut de face, en bas de profil (im. Stein). rina. C'est encore une colonie de seize indivirlus, mais formant une masse sphérique et disposée suivant les rayons de la sphère (Eau douce, Nouvelle-Zélande).

$\left({ }^{2}\right)$ Genres voisins :

Synaphia (Perly);

Diplodorina (Fromentel). 
Eudorina (Ehrenberg) (fig. 630)] forme des colonies de 16 à 32 individus, lisposés tout à fait comme dans notre type morphologique. La reproduction agame se fait aussi suivant le mode indiqué. Pour la conjugaison, il se forme des colonies qui, semblables au délıut aux autres, évoluent les unes en macrogamètes, les autres en microgamètes. Dans les premières tous les individus se transforment directement en macrogamètes par le seul fait qu'ils grossissent sans se liviser. Dans les secondes, tous les individus, au contraire, se divisent par le processus habituel et donnent de petites colonies non reployées en sphère, étalées en disque plan el formées de microgamètes confor-

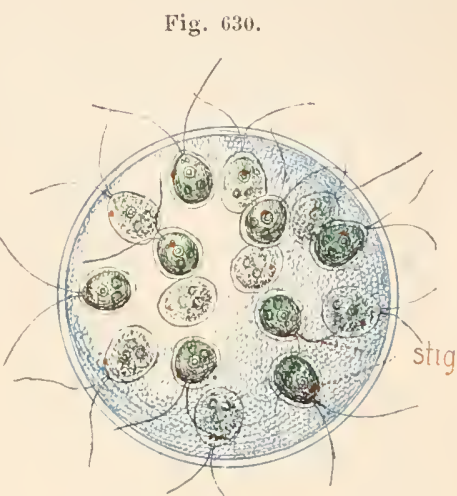

Eudorina. Colonie (Sch.). mes à ceux de notre type. Ces petites colonies planes nageut à la rencontre des colonies femelles, s'attachent à elles et se dissocient, alor's seulement, en leurs microgamètes constituants qui pénètrent dans la gélatine et se conjuguent aux macrogamètes: on pourrait dire les fécondent, car il y a là une vraie sexualité et mème, comme on le voit, séparation des sexes. Le reste le l'évolution est conforme au type morphologique (Colonie, 0,1. Eau douce).

Volvox (Linné, emend. Ehrenberg). Les colonies sphériques (lig. 631) sont formées d'un nombre considéralıle d'indivilus (jusqu'à 22,000). Tous sont réunis les uns aux autres par des communications protoplasmiques. La colonie est orientée par rapport au sens de son mouvement; c'est toujours le mème pôle qui regarde en avant et la rotation a lieu autour d'un axe passant par ce pôte ou tout à côté. Il résulte de là une différenciation intéressante. C'est que

Fig. 632.

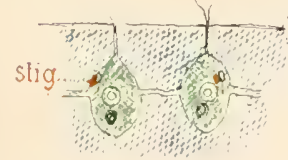

Volvox.

Deux individus du pòle antérieur avec leur's stigma (iim. Franzé).

stig., stigma; gel.. gélatine. lesindividus de l'hémisphère antérieur ont leur stigma bien développé (fig. 632); vers l'équateur, le stigma commence à se réduire; au delà, il s'alrophie de plus en plus el finit par disparaitre au pôle opposé. Tout cela donne à l'enscmble le faciès d'un ètre lendant ver's l'unité or'ganique et la pluricellularilé.

La différenciation s'étend plus loin, car les individus ne sont pas tous aptes à reproduire la colonie ni par voie agame ni autrement. Dans les colonies asexuées du printemps, il y a seulement 8 cellules, plus grosses que les autres et disposées sur l'hémisphère 
postérieur, qui soient aptes à reproduire la colonie. On les appelle les parthénogonidies. Il y a aussi 10 à 30 parthénogonidies abortives qui ont seulement une taille un peu plus élevée, sans avoir le pouvoir reproducteur et font le passage aux individus ordinaires. Quand ces 8 parthénogonidies ont donné, conformément à notre type morphologique, de nouvelles colonies, le reste de la colonie mère meurt.

Cette morl est un pliénomène nouveau que nous n'avions pas rencontré encore chez les Protozoaires et qui constitue une ressemblance nouvelle et non des moins remarquables entre les Métazoaires et le Volvox.

Dans les colonies sexuées de l'automne, nous trouvons la mème différenciation. Les macrogamètes et microgamètes, nous pourrions dire les wufs et les spermatozoïdes, se comportent tout à fait comme dans notre type, mais il n'y a que quelques colonies à microgamètes ou androgonidies (3 à 15 , ordinairement 5 ou 6 ) et une trentaine d'œufs par colonie mère, et ceux-ci n’apparaissent que lorsque les premières ont déjà quitté la colonie, ce qui constitue un hermaphroditisme protérandrique excluant la fécondation entre frères et sœurs (pas alssolument cependant). Le développement de l'œuf fécondé a lieu comme dans notre type morphologique.

Les Volvocines nous montrent done à la fois le passage des animaux aux plantes (des Flagellés aux Algues), celui des Protozoaires aux Métazoaires, et l'apparition de la sexualité vraie et complète, de la séparation des sexes et enfin de la distinction entre cellules somatiques et cellules reproductives chargées de plasma germinatif. Pour toutes ces raisons, elles oflrent un vif intérêt qui justifie l'extension que nous avons domnée à leur étude.

Nous laissons parmi les plantes les IIydrodictyejes immobiles. Nous ferons connaitre dans un chapitre spécial à la fin du volume sur quoi nous fondons notre distinction des animaux et des végétaux. 


\section{$2^{\text {e }}$ Sous-Chasse}

\section{SILICOFLAGELLÉS. - SILICOFLAGELLIA}

[SILICOFLAGELLATA (Borgert)]

Ce petit groupe, dont les affinités précises ne sont pas encore hien éclaircies, a été formé pour des ètres que l'on rangeait il y a quelques années parmi les Radiolaires dans lesquels ils vivent en parasites ou en commensaux, prenant leur squelette, seule partie que l'on connùt alor's de leur organisme, pour le squelette du Radiolaire lui-mème (V. p. 241).

\section{TYPE MORPHOLOGIOUE}

FIG. 633 ET 634)

Nous prendrons pour type le Distephamus (Stöhr) qui est le genre principal du groupe.

L'animal est formé d'une petite masse de protoplasma nu, mesurant environ $20 \%$, colorée en jaune par une substance qui semble ètre de la diatomine. Iu centre, est un noyau formé d'un gros corpss chromatique central entouré d'une couche de protoplasma vacuolaire (reticulum?), le tout renfermé dans une membrane nucléaire. Il n'y a point de vacuoles. Un flagellum part de la partie supérieure du corps et entraine l'animal en arant dans la natation. Il n'y a point de bouche. Ce qui donne i cet organisme, qui jusqu’ici ne présente rien de bien spécial, une place à part parmi les Flagellés, c'est la présence d'un squelette beaucoup moins semblable à celui d'un Flagellé qu'à celui d'un Radiolaire, en particulier à celui des TYMPANINE (parmi les Acanthaires) pour la forme, et à celui d'un PheOdarié pour la structure. Ce squelette se compose en effet de tigelles siliceuses creuses soudées en une

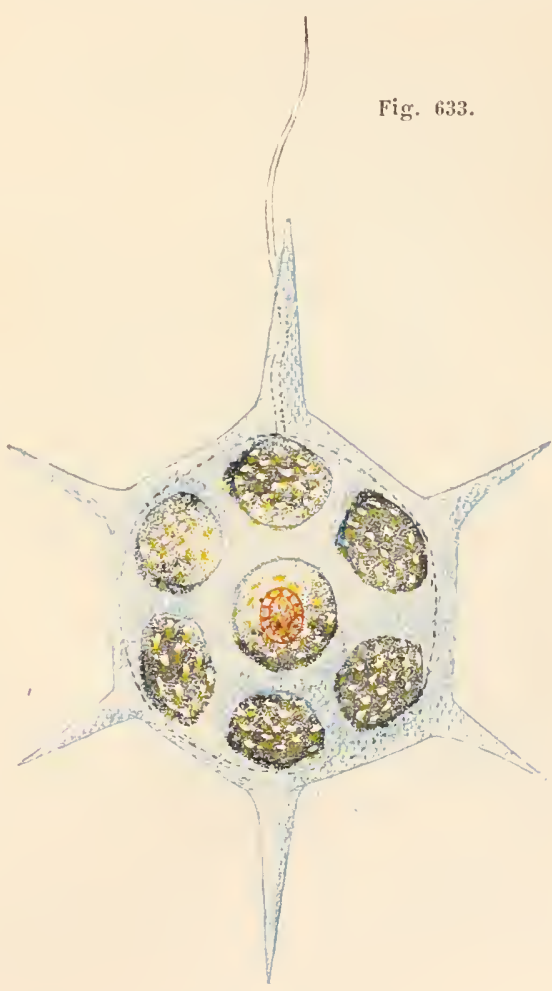

SILICOFLAGELLIE

(Type morphologique) (Distephanus)

L animal entier montrant le squelette, les parties molles et le flagellum petite coque grillagée. Il est formé ici de deux anneaux parallèles et de 
diamètre inégal réunis par des tigelles de manière à dessiner le cadre

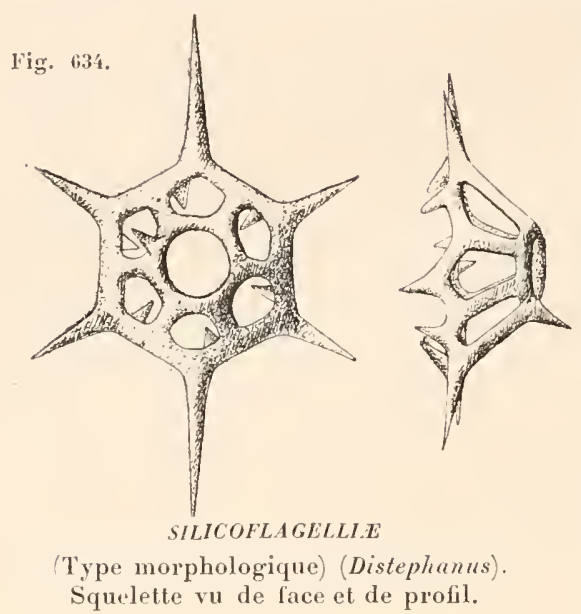
d'un tronc de cône ou de pyramide. Ce squelette est à la surface du corps, mais nullement renfermé dans une gelée. Ce fail, l'absence de vrais pseudopodes et la présence du flagellum sont les principaux caractères qui autorisent à séparer ces êtres des Radiolaires pour les réunir aux Flagellés.

On ne sait rien de la physiologie de l'animal, si ce n'est son mode de progression. On rencontre assez souvent des individus réunis à la manière de deux cònes arlossés par leurs

faces et l'on pense qu'il pourrait y avoir là un fait de conjugaison.

Ces êtres sont naturellement tous marins comme les Radiolaires qui les hébergent.

\section{GENRES}

Distephanus (Stöhr) (fig. 633 à 633) est le type mème ci-dessus décrit (').

(1) On remarquera que le grand axe de la coquille est horizontal. Cela est nécessité par la position du flagellum. Häcliel, au contraire, llaçait cet axe verticalement (V.p. 241).

Les autres genres du groupe ne se distinguent de Distephanus que par des caractères secondaires. Ce sont:

Mesocena (Ehremberg), ayant en guise de squelette des sortes d'anneaux siliceux de 0,02 à 0,05 , assez régulièrement distribués dans la couche Fig. 63i. périphérique de son corps;

Dyctiocha (Ehrenberg) semblable au précédent, mais ayant ses anneaux (de 0,02 à 0,03 ) surmontés de deux arcades perpendiculaires formant une sorte de petite charpente hémisphérique à jour ;

Cannopilus (Häckel), semblable au précédent, mais avec les tigelles de rẻunion des deux anneaux bifurquées de manière à former deux rangs de mailles $(0,02$ à $0,0.03)$;

Ebria (Borgert), qui s'en distingue par deux flagellums. 


\section{DINOFLAGELLESS. - DINOFLAGELLIE}

\section{[DINOFLAGELLATA (Bütschli)]}

\section{TYPE MORPHOLOGIQUE}

(FIG. 636 A 646)

Idée générale de l'animal. - L'animal a une forme ovoïde à grand axe vertical. Il mesure environ $0 \mathrm{mml}$; il est donc tout juste visible à l'œil nu. Son corpsest protégé par une épaisse culicule formée de plaques (fig. 636) réunies par des lignes suturales, ornées de dessins en relief et percées de fins pores (fig. 638 à 6 亿0). Il est partagé en leux parties à peu près égales par un sillon transversal en hélice sénestre qui fait un peu moins d'un tour $(p l$. s.) Ce sillon commence en haut sur la face ventrale, se porte à droite, c'est-à-dire vers le côté gauche de l'animal, traverse la face dorsale, reparaìt au bord droit et atteint de nouveau la face ventrale où il se termine à quelque listance au-dessous de son origine. Ce sillon, bien qu'assez creux, ne met pas le protoplasma à nu, car il estprotégé par une plaque en ceinture spéciale $(p l . s$.$) . In$ sillon longiudinal vertical mais pas toutà fait rectiligne, rejoint les deux extrémités du sillon transversal et s'é-

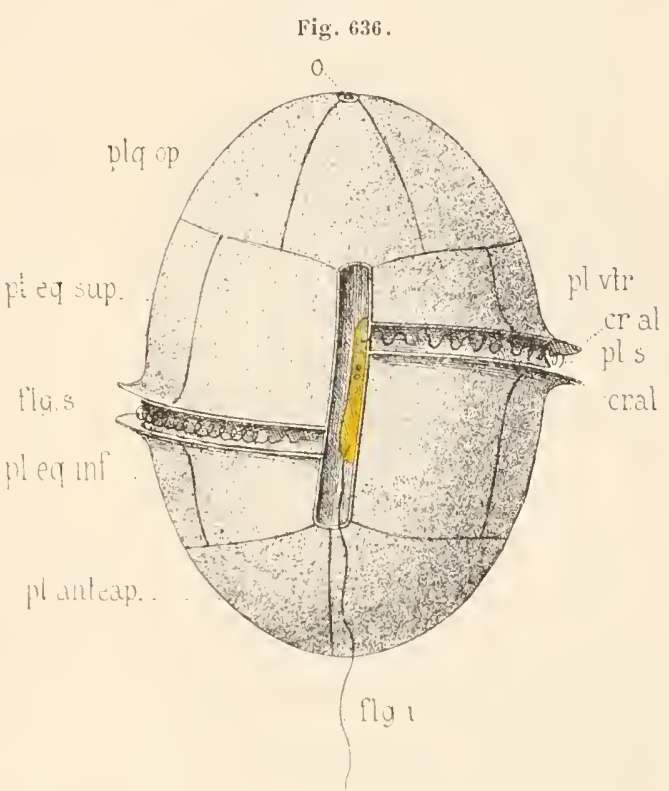

INOFLAGELLLE (Type morphologique) (Sch.).

Aspect extérienr montrant la dispusition des plaques do la cuticule, du sillon transversal et du sillon longitudinal avec sa fente flagellifère.

cr. al., crètes aliformes; flg. 1. , flagellum inférieur ou longitudinal; flg. s., flagellum supérieur ou transversal; plq. anteap., plaques antéapicales; pla. op., plaques apicales; p1. eq. sup., plaques équatoriales supérieures; pl. eq. inf., plaques équatoriales inférieures; pl.s., plaque en ceinture; pl. vir., plaque ventrale.

tend un peu au delà, en deśsus comme en dessous de lui. Il est, comme le transversal, en contre-bas de la surface générale, mais nemetpas davantage le cytoplasma à nu, protégé qu'il est par une plaque spéciale $(p l$. vtr.). Cependant, dans sa partie moyenne et le long de son bord gauche, 
cette plaque est percée d'un orifice fissiforme vertical, appelé la fente flagellifère, qui met à nu le cytoplasma sous-jacent. Il y a aussi un orifice au pôle supérieur du corps $(o$.$) . De la fente flagellifère, naissent$ deux flagellums ou fouets, un transversal et un longitudinal. Le flagellum transversal (/lg.s.) nait en face de l'origine du sillon transversal. Il se porte immédiatement dans ce sillon qu’il suit dans toute son étendue, logé dans sa cavité, décrivant comme lui un peu moins d'un tour d'hélice lescendante dextre. Il est contourné sur lui-même en ressort à boulin. Le flagellum longitudinal ( $/ g . i$.) naît un pen au-dessous du précédent, se

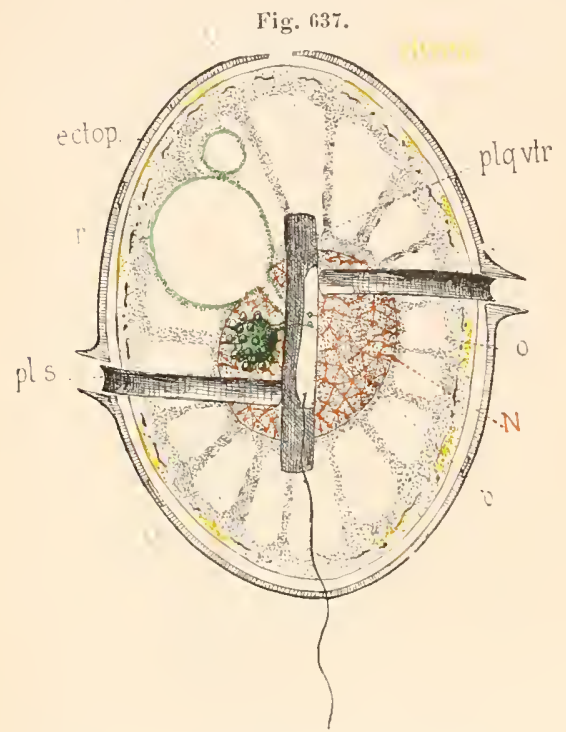

DINOFLAGELLIE (Type morphologique) (Sch.) Organisation interne.

chrmp., chromoplastes; ectop., ectoplasma N., noyau; o., orifice de la vésicule sac; $\mathbf{o}^{\prime}$, orifice de la vésieule pulsatile; p.,plaques de la cuticule; pl. s., plaque en ceinture; plx.vtr., plaque ventrale; r., vésicule sac; $\mathbf{V}$., vésicule accessoire; v'., vésicule pulsatile. porte en bas en snivant la portion du sillon longitudinal située andessous de lui, et s'étend bien au delà en formantune sorte de queue. Dans la fente buccale s'ouvrent, tout près l'un de l'autre, deux orifices $\left(o, o^{\prime}\right.$, fig. 637) qui sont ceux del'appareil pulsatile. Sous l'enveloppe cuticulaire est le cytoplasme dans lequel on peut distinguer un ectoplasme (ectop.) formant une couche assez épaisse où trouvent asile de nombreuses inclusions, chromoplastides (chrmp.), graisse, pigment, etc., et un endoplasme creusé d'une rangée de grandes vacuoles qui occupent presque tout l'espace annulaire qui reste entre l'ectoplasme et le noyau. Le noyau $(N$.) est subcentral el très grand; il est entouré d'une couche continue d'endoplasme. Enfin, dans l'endoplasme se trouve un appareil pulsatile volumineux et compliqué. ll y a d'abord une vésicule pulsatile proprement dite $\left(V^{\prime}\right)$, constituée sur le type ordinaire de cet organe, c'està-dire entourée d'une couche de petites vésicules formatrices el s'ourrant dans la fente buccale par un petit canal $\left(o^{\prime}\right.$.). Indépendamment d'elle, existe une vésicule beaucoup plus grande, la vésicule sac $(r)$ (Schïlt) dans laquelle s'ouvre une vésicule accessoire $(V$.$) (parfois plusieurs) et$ qui s'ouvre elle-même dans la fente buccale, indépendamment de la vésicule ordinaire, mais tout près d'elle (o.).

(1) Schürs [95] à qui l'on doit la description de cet appareil na que rarement suivi les deux canaux indépendamment jusqu’à leur orifice et l'on peut se demander si les connexions qu'il indique sont bien rigoureusement dėmontrées. 


\section{Structure.}

Naintenant que nous avons une idée générale le l'ètre, nous pouvons, sans crainte de nous égarer, aborder l'élude des détails de sa structure.

Enveloppe cuticulaire et organes extérieurs. - C'est une vraie cuticule (p., fig. 637), c'est-à-dire un produit de sécrétion formé en dehor's du cyloplasme et non par transformation de la couche superficielle (').

Elle est composée de cellulose ou d'une substance analogue à réactions un peu différentes. Elle est assez épaisse et assez rigide pour former une réritable coque qui se tient seule quand l'animal est mort ou qu'il s'est contracté pour' s'enkyster.

Les plaques (p.) qui la forment (fig. 638 à 640) s'unissent les unes aux autres par leurs hords en biseau dont l'un recouvrant et l'autre recouvert sont soudés par un ciment qui unit leurs faces en contact. Ces plaques sont polygonales et pourvues de còtes en relief suivant des

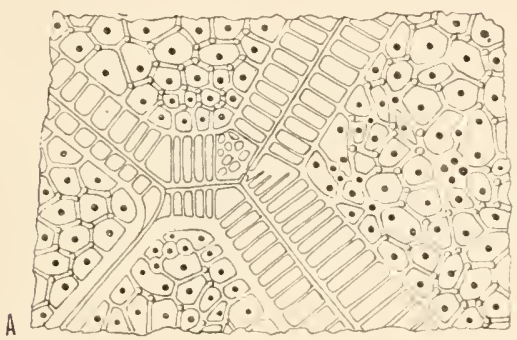

Fig. 638 .
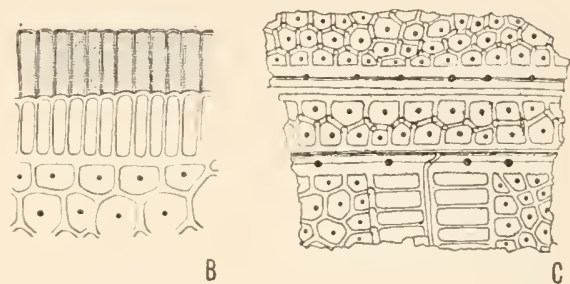

IINOFLAGELLIE (Type morphologique). Plaques de Peridinium divergens (d'ap. Schittl).

$A$, lieu de rúnion de 4 plaçues; $B$, biseau interne d'une plaque; $C$, région du sillon trancversal.

dessins variés et limitant des alvéoles en contre-bas. Cette disposition

Fig. 639.

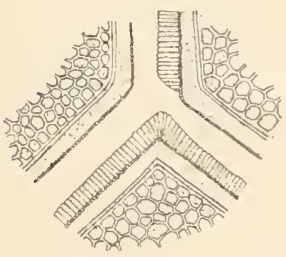

DINOFLA GELLIE

(Type morphologique)

Trois plaques de Peridinium oratum dissociées (d'ap. Schiitt). est destinée à alléger la coque en lui laissant une grande solidité. Dans le fond de ces alvéoles sont percés de petits pores très fins, mais qui traversent presque toute leur épaisseur, ne laissant qu'une minime pellicule au contact du cytoplasma.

On peut distinguer dans lesystème des plaques quatre groupes (fig. 636): deux équatoriaux, l'un supérieur (pl.eq. sup.), l'autre infërieur ( $p l . e q$. inf.), formant ceinture autour du corps,

(1) Mais il existe des formes nues chez lesquelles il y a, en place de cela, une simple membrane cellulaire ou périplaste,

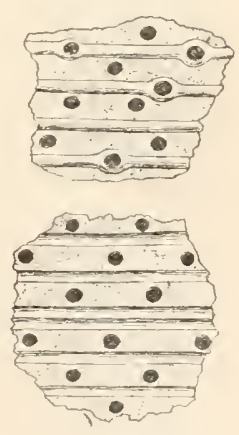

DINOFLAGELLLE (Type morpholog.). Plaques d'Oxytoxum scolopax

(d'ap. Schutt). formée par différenciation de la couche superficielle du cytoplasme, dans laquelle se sont déposées des substances additionnelles non digestibles dans la pepsine; celte 
l'un au-dessus, l'autre au-dessous du sillon transversal, un apical ( $p l q . o p$.) formé de plaques convergeant des équatoriales supérieures vers le pôle supérieur où elles réservent un orifice (o.) et un antapical disposé d'une manière semblable au pôle opposé (").

Les plaques n'empiètent pas sur les sillons qui sont fermés par des plaques spéciales.

Le sillon transversal est fermé par une mince plaque en ceinture $(p l . s$.$) en forme d'anneau en gouttière à concavité externe et formé de$ plusieurs pièces. Cet anneau n'est pas complet; il est interrompu en avant sur la largeur du sillon longitudinal aux deux bords duquel il s'arrête. Le sillon longitudinal est protégé lui aussi par une mince plaque ventrale $(p l q . v t r$.) de forme correspondante sauf au niveau de la fente buccale, où le cytoplasme est à nu. Les plaques limitantes du sillon transversal sont, aux bords de ce sillon, munies d'une crête horizontale de même nature que celles qui ornent le reste de la surface, mais beaucoup plus mince, tranchante mème au bord libre et beaucoup plus saillante. Il y a donc deux de ces crètes, une pour le hord supérieur du sillon, l'autre pour le borl inférieur; et elles suivent l'une et l'autre tout le trajet de ce sillon, en ligne continue, bien qu'elles soient formées d'autant de segments distincts qu'il y a de plaques limitrophes du sillon tant en dessus qu'au-dessous de celui-ci. Parfois, il y en a de pareilles pour le sillon longitudinal. On les appelle les crétes aliformes (cr.al.).

Nous n'arons rien à ajouter à ce que nous arons dit de la bouche et des fouets.

Cytoplasme. - L'ectoplasme et l'endoplasme ne diffèrent que par leur densité et la nature de leurs inclusions. Le premier n'est pas très ferme et le second est d'une ténuité extrême. Les vacuoles qu'il renferme (fig. 637) sont remplies d'un liquide arqueux contenant à peine quelques substances albumineuses en dissolution. Elles sont si grandes et disposées de telle façon qu'elles réduisent la partie protoplasnique de l'endoplasme à une couche sous-ectoplasmique et une couche périnucléaire, réunies par de minces lames radiaires qui sont les parois des vacuoles, ce qui donne à l'ensemble une grande analogie avec la disposition qui est si fréquente dans les cellules végétales.

Parmi les inclusions ectoplasmiques, au premier rang d’importance, viennent les chromoplastes (chrmp.). Ils ont la forme de plaquettes assez larges mais très minces et de forme extrèmement irrégulière situées parallèlement à la surface. Parfois, ils empiètent dans les travées endoplasmatiques intervacuolaires. Ils sont teintés en jaune brunàtre par le la

membrane n'en donne pas moins les réaclions de la cellulose ou d'une substance voisine. (Pour ces distinctions, voy. p. 12 à 14.)

(1) Cet orifice n'est pas constant et la disposition des reliefs, des alvéoles, des pores et des plaques elles-mèmes, ainsi que leur forme et leur nombre sont extrêmement variables. 
diatomine ou quelque substance analogue. Ils sont très sensibles et, sous l'influence de minimes excitations, ils se contractent, se fragmentent ou s'étendent et se soudent entre eux. Des grains d'amidon, formés sous leur influence, se trouvent au-dessous d'eux. On trouve, en outre, de petites lamelles de substance grasse qui senublent aroir aussi des plastides pour support, de la graisse en gouttelette, parfois du pigment jaume ou brun et diverses autres inclusions mal déterminées.

Le noyau $(N$.$) est pourvu d'une mince membrane et montre à l'inté-$ rieur un délicat réseau chromatique arec granulations aux points nodaux, et parfois un nucléole.

Dans l'appareil pulsatile, la vésicule sac $(r)$ est la plus granle et la plus facile à voir; elle semble posséder une membrane.

\section{Physiologie.}

Habitat. - Notre type est un être pélagique, vivant en pleine eau pure dans les lacs ou dans la mer'; il est parfois phosphorescent (").

Locomotion. - ll nage en portant en avant l'extrémité que nous avons appelée supérieure et en tournant autour de son axe. En général, il tourne dans le sens dextre, comme s'il vissait dans l'eau l'hélice de son sillon transversal. Mais il peut aussi, accilentellement, tourner en sens inverse et reculer. Pendant la natation, le fouet transversal, sans quitter la gouttière dans laquelle il est toujours couché, est agité d'un frémissement ondulatoire très vif. Les ondulations prennent naissance it son insertion et s'écoulent par son extrémité $\left(^{2}\right)$.

Il semble que ce mourement ait pour effet de déterminer la rotation du corps qui, par un elfet secondaire, progresse dans l'eau d'un mourement de vis. Le fouet longitudinal semble l'ordinaire immobile pendant la natation, donnant seulement quelques secousses de temp's à autre à la manière d'un gouvernail. Iais une obserration attentivemontre qu'il est souvent animé d'un mourement tourbillonnaire conique qui doit avoir une action sur la progression en arant. Ilais, ici comme ailleurs, le détail du mouvement et l'action mécanique précise des flagellums sont inconnus. L'animal est sensible à la lumière et la recherche.

Nutrition. - L'alimentation est certainement holophytique et identique à celle des Algues inférieures ou des Chloromonadines. L’animal pourrait, à ce qu'il semble, prendre de la nourriture solide avec son fouet longitudinal et l'absorber par son sillon buccal. On a vu quelque-

${ }^{1}$ ) Les formes d'eau douce ne sont jamais phosphorescentes. Parmi les formes marines, la phosphorescence a été constatée chez divers Ceratium (C. tripos. C. fusus, C. furca), chez Prorocentrum, Blepharocysta et peut-ètre chez Exuviclla. Diver's auteurs cependant la contestent absolument. En tout cas on ignore son siège.

$\left.i^{2}\right)$ Elles ont été longtemps interprétées comme l'expression optique du mouvement successif d'une couronne de cils, d'où le nom de Cílo-flagelés que ces êtres ont longtemps porté. 
fois des parlicules conduites ainsi jusqu'à la lıonche, mais la réalité de l'ingestion n'a jamais été démontréc (").

Excrétion. - Le fonclionnement de l'appareil pulsatile est fort mal connu. Il semble bien que la vésicule pulsatile avec ses vésicules formatrices devrait fonctionner comme chez les Euflagellés ou les Ciliés, mais le phénomène n’a pas la mème netteté. Quant à la vésicule sac et aux vésicules accessoires, leur rôle est tout à fait olıscur. Les pulsations ne sont pas régulièrement rythmées el semblent plus ou moins indépendantes les unes des autres. Bengn avait émis l'opinion que la vésicule sac (la seule qu’il connùt) étail un appareil d'aspiration pour une nourriture liquide. Elle représenterait alors un pharynx, mais son interprétalion est insuffisamment fondće.

Accroissement. - Il se fait par élargissement des plaques au niveau de leurs sutures. Sans doute, quand ces plaques s'accroissent en épaisseur, la mince lame qui se dépose à leur face profonde dépasse en tous sens légèrement la précédente et ainsi toute la coque s'élargit. Quant à l'épaississement des ornements extérieurs en relief, il suppose

Fig. 6il.

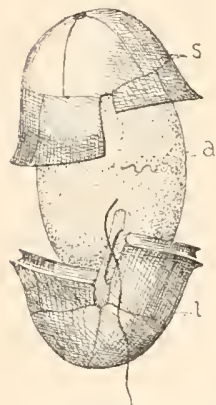

IINTOFLAGEILIE

(Type morphologique). Inue (Sch.) a, corps nu de l'aninual; s., moitié supérieure, +1 i., moilié inférieure de la coque mucip.

un accroissement concomitant par intussusception, tel qu'il a été démontré chez les cellules végétales.

Mue. - On roit parfois (fig. 641) l'animal s'étendre fortement en longueur, faire éclater sa cuticule qui se fend le long du sillon transversal, en rejeter les deux moitiés $(s$. el $i$.) et apparaitre nu $(a)$, mais pourvu de ses deux fouets qui se sont dégagés. En peu d'heures, il se revêt d'une nouvelle cuticule. Dans plusieurs cas, on a vu la nouvelle cuticule déjà formée sous l'ancienne, mais il est à croire que celle-ci s’était déjà légèrement écartée pour permeltre un certain accroissement. La nouvelle cuticule est d'aborl très mince et lisse, mais elle s'épaissit rapidement et forme ses relicfs el ses sculptures caractéristiques.

Si ce phénomène était régulier il expliquerait sans difficulté aucune tout le problème de l'accroissement. Mais il est trop rare pour qu'il n'y ait pas autre chose. P'eut-être se produil-il lorsque l'extensibilité de la cuticule a donné à l'accroissement par intussusception tout ce qu'elle pouvait.

Enkystement. - Dans des conditions mal

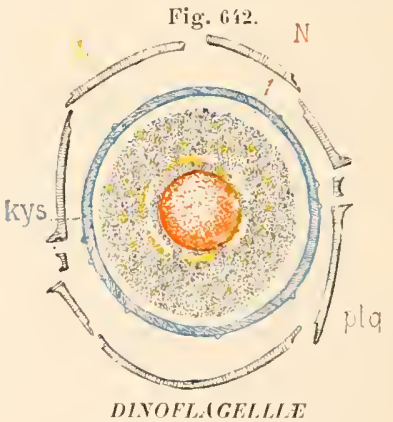

(Type morphologique).

Enkystement (Sch.).

„., globules de graisse; kys., membrane kystique; $\mathbf{x . , ~ n o y a r ; ~ p l q . . ~}$ plaques de la cuticule.

connues et sans doute assez rares, l'animal perd ses fouets (fig. 612),

(') Sauf pour quelques espèces ou genres, en particulier Polykrikos. 
efface ses sillons, s'arrondit et s'enkyste. Sous sa cuticule $(p l q$.$) qui se$ disloque, il se sécrite un kyste ( $K$ ys.) de forme analogue, mais plus voisine de l'ovoïde pur ou de la sphère ('). Ce liyste est assez épais, orné de très fines côtes correspondant aux anciennes sutures de la cuticule, et surtout très résistant aux agents chimiques. Il semble formé de cellulose imprégnée de silice. Sous le kyste, l’animal rétracté est séparé de la paroi par une mince couche liquide; les chromoplastes se sont ramassés au centre autour du novau et toule la surface est occupér par de très nombreux globules de graisse (g.) formés lans le corps pendant que l'enkystement se préparait. Ces kiystes passent l’hiver el, au printemps, l'animal en sort rlans le mème état qu'après une mue.

Reproduction. - Le Dinoflagellé se reprolluit uniquement par division simple à l'état libre ou enkysté.

Division à l'état libre. - Elle se fait (fig. 64:3) par un plan oblique de haut en bas el de droitr à gauche qui passe par la région buccale, attribuant à chaque individu fille deux moitiés dissemblables ‘le l'animal mère. Elle commence par le noyau qui se place dans ce plan et se divise suivant un processus intermédiaire à la mitose et à l'amitose qui ressemble à celui de la division du macronucléus des Infusoires ( $V$. phus loin) (²). L'animal, alors, s'allonge dans le sens perpendiculaire au futur plan de division et fait éclater sa cuticule suivant une ligne brisée qui suit les sutures les plus voisines du plan de division. Ainsi, l’individu supérieur gauche (a) conserve la moitié des plaques de son côlé et l’individı infórieur droit (l) garde les autres. Dans la fente

(1) Chez les formes qui ont des cornes, comme reratium, le kyste a aussi des cornes mais moins longues, et toute la forme est plus arrondie. On observe aussi parfois un prolongement en forme de corne sur le kyste de genres qui n'en ont pas. C'est alors un appendice du kyste qui semb]e destiné

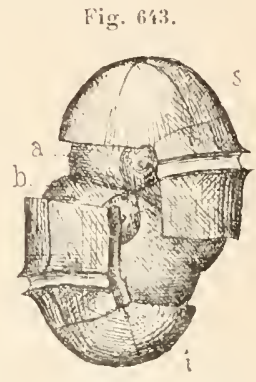

MINOFL.. GELLI.E(TYpe morphologiqui).

Dirision à l'état libre (Sich.).

a et $\mathbf{b}$, les deux individus résultant de la division; s., moitié supérieure de la cuticule; i., moitié intérieure.

a lixer celıi-ci.

* Ce processus a cité récennment étudié par LA ITERBOrx [95]. Le novau, à l'élat de repos, montre un réseau achromatique lypique arec grains te chromatine aux points nodaux (lig. 644. Ce réseau se coupe en fila ments qui s'orientent perpendiculairement au futur plan de division, puis
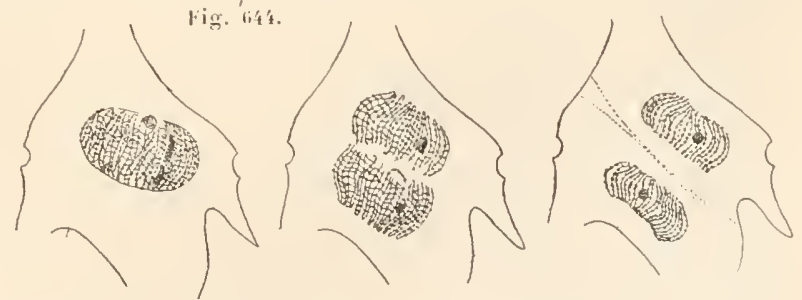

DINOFLAFELLIE (Type morphologique) Phènomènes nucléaires de la division (im. Lauterborn).

se coupent en leur milieu suivant ce plan, et les deux lots de chromosomes se rendent chacun dans la moitié correspondante du corps. Les aspects sont à peu près ceux d'une mitose, mais la différence est capitale par suite de l'absence de centrosome et de division longitudinale. Il y a aussi un ou deux nucléoles qui ne paraissent jouer aucun ròle actif. 
ainsi produite, le corps s'étire, s'étrangle circulairement et finalement se coupe. Les deux individus se séparent et bientôt se complètent (').

Division à l'état enkysté. - Elle est beaucoup plus fréquente que la précédente et constitue le procédé normal de reprorluclion de notre ani-

Fig. 615.

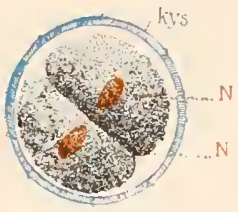

DINOFLAGEILIE (Type morphologique)

(Sch.).

Division sous un kyste

kys., membrane kystique; N., noyau. mal (fig. 6ł⿱艹)). Nous pouvons la définir en peu de mots : c'est une division semblable à celle de l'état libre, mais précédée d'un enkystement semblable à l'enkystement de protection. Souvent, il est impossible de dire si l'enkystement que nous avons décrit plus haut a pour but la protection simple ou une division. Il y a cependant quelques particularités utiles à ajouter. Le kyste n’est pas toujours cellulosique et siliceux; fréquemment, il est gélatineux. Sous le kyste, la division du noyau se fait comme dans le cas déjà décrit. Celle du corps a lieu suivant le mème plan oblique que si la coque cuticulaire devait y prendre part. Enfin, assez souvent, les deux individus se divisent une seconde fois, en sorte qu'il en sort du kyste quatre au lieu de deux, et ceux-ci, ayant reformi chacun leurs deux sillons etleurs deux fouets, n'ont plus qu’à sécréter leur cuticule $\left(^{2}\right)$.

Associations. - Parfois, la division à l'étal libre reste incomplète et les deux individus filles restent unis ensemble par une soudure étroite. Ils regardent du mème côté et la partie supéro-dorsale de l'individu inférieur est unie à la partie inféro-ventrale du supérieur. Parfois mème, ces divisions incomplèles se continuent et il se forme ainsi une chaîne de plusieurs individus (fig. $\left.6{ }^{16} 6\right)\left({ }^{3}\right)$. Ces associations n'ont rien de sexuel; elles ont la mème signification morphologique que les colonies non persistantes ilont nous

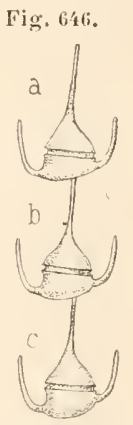

DINOFLA GELLIE (Type morphologique) (Sch.). Association chez Ceratium. cux individus. La lame du sillon longiludinal a été vue par P'ÉxA chez Ceratium, passer tout entière à l'individu inférieur Iroit, mais on ne sait pas du tout si cela est général. On ne sait pas comment les fouets se partagent. Peut-être se régénèrent-ils tous les deux sur chacun des deux individus filles. On ne sait rien non plus du sort de l'appareil pulsatile. Sur les individus encore incomplètement séparés, on voit se dessiner de part el d'autre de l'étranglement le rudiment des parties qui vont se régénérer. La portion manquante des sillons, les plaques absentes, tout cela commence à apparaitre, mais en raccourci el aura à prendre ses dimensions définitives après la séparation. Ivant la séparation complète les quatre fouets sont déjà parfois reformés. L'obliquité du plan de division est variable. Ordinairement at $45^{\circ}$, souvent plus ou moins, parfois disparaissant pour faire place a une division franchement longitudinale ou transversale.

$\left(^{2}\right.$ Iei de mème, la division est parfois longitudinale, parfois transversale.On a quelquefois observé, dans le plan de division, une plaque te petits grains qui semblent représenter la plaque équatoriale des végétaux.

(3) Ce phénomène n'est pas commun; il ne se rencontre que chez Glenodinium, Dinophysis el surtout Ceratium qui forment de véritables chaînes. 
avous trouvé l'exemple chez diverses Chlamydomonarlines, mais leur signification biologique est aussi obscure.

Conjugaison. - Si l'on met de côté les cas lle division inachevée ou de division normalement incomplète qui ont été souvent pris à tort pour des conjugaisons, il ne reste guère d'observations au sujet de ce phénomène. Il semble lien cependant que, dans certains cas, des individus jeunes, récemment issus d'une division mulliple sous kyste, a peine sortis se conjuguent et aussitôt s'enkỵstent en commun (Danysz) [86]. Nais toute la question réclame de nouvelles études.

Nous diviserons notre sous-classe en trois ordres:

ADINIDA, sans sillons :

DINIFERIDA, pourvus de deux sillons typiques; et

PoLYDINIDA, à nombreux sillons transversaux.

Le second est conforme à nolre type morphologique, les deux autres sont tout i fait aberrants.

\section{Jer ORDRE}

\section{ADINIIIES. - IIINID. \\ [A DINIDA (Bergh)]}

\section{TYPE MORPHOLOGIOUE}

En trois mots on peut le définir: c'est une Chromomonaline, dans l'enveloppe d'une Phacotus. Il est donc fort différent du type des Dinoflagellés: il n’a pas de sillons et ses flagellums très courts sont relégués à l'extrémilé supérieure. Cependant, le fait de josséder, arec des chromoplastes jaunes, une coque bivalre, surtout rette coque élant percée de pores, permel de raltacher cet èlre aux I)inollagellés, malgré l'alsence de sillons et la position des deux fouets.
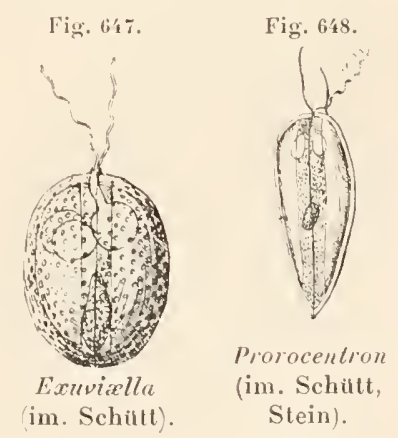

Prorocentron (Ehrenlierg) (fig.618). C'est un Exu-

\section{GENRES}

Exuviælla (Cienkorsky) (fig. 647). Le corps ovoüde lisse, sans sillons, est contenu dans une coque culiculaire hivalve crillée de pores: les deux fouets sortent entre les deux valves à l'extrémilé supérieure. Les chromoplastes jaunes sont tantôt isolés, tantôt soudés en deux plaquessymétriques ( $50 \mu$. Ver).

vixlla pointu en bas, et muni en haut d'un prolongement silué immédiatement en arrière des fouets et qui appartient soit à une seule valve, soit aux deux par moitié. Les chromoplastes offrent les mèmes variétés de disposition ( $(50 \mu$. Mer). 


\title{
DINIFERIDES. - DINIFERIDA
}

\author{
[DINIFERA (Berg)]
}

TYPE MORPHOLOGIQUE

C'est lui-mème que nous avons décrit comme type morphologique des Dinoflagellés (V. p. 373).

\section{GENRES}

Peridinium (Ehrenberg, emend. Stein) (fig. 638, 639 et 649). C'est à très peu de choses près la réalisation de notre type morphologique. Il est caractérisé génériquement par la disposition de ses plaques ( $150 \mu$. Mer et eau douce) (").

\section{(1) Genres voisins :}

Diplopsalis (Bergh) (fig. 650), de forme surbaissée, le pòle apical au sommet d'un petit prolongement, le sillon longitudinal descendant très bas $(40 \mu$. Mer $)$;

Amphidoma (Stein), (fig. 651) ovoïde, à grand axe vertical, à fente flagellifère réduite à un petit trou (Mer);

Protoceratium (Bergh) (fig.652), à plaques indistinctes, à cuticule semblant continue avec un réseau de crêtes saillantes (3\% [.. Mer);

Heterocapsa (Stein) (fig. 653), semblable, à hémisphère

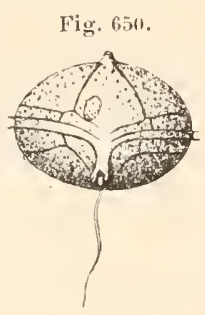

Diplopsalis (D. lenticula) (d'ap. Buitschli).

\begin{abstract}
Fig. 651.
\end{abstract}

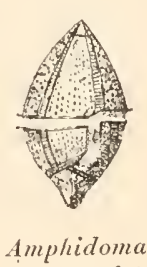

(A. nucula) (d'ap.

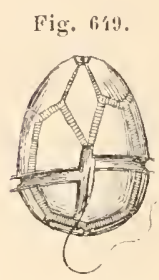

Peridinium (Sch.).

Fig. 6.,3.

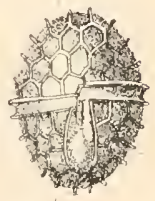

Protoceru-

tium

(I.reticulata)

(d'ap. Steiu).

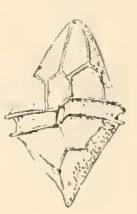

Heterocapsa (II. triquetra) (im. Schiitt). infẻrieur lisse $(20 \mu$. Mer $)$;

Blepharocysta (Ehrenberg) (fig. 6ら̆4), ovoïde, allongé, à sillons très superficiels, à fente flagellifère représentée par un petit trou placé très bas $(50$ [. Mer $)$;

Podolampas(Stein) (fig. 655), allongè en pointe vers le haut et muni à l'extrémité inférieure d'apophyses rappelant, avec une situation diamétralement opposée, celle de Prorocentrum (100 p.. Mer);

Goniodoma (Stein) (fig. 656), presque sphérique, à sillon buccal réduit à un petit orifice ovalaire ( $40 \%$. Mer);

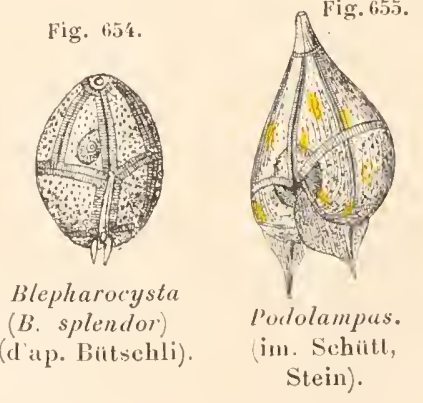

Fig. 656.

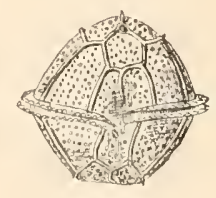

Goniodoma (Cr. acuminatum) (d'ap. Stein). 
Ceratium (Schrank, emend. Stein) (fig. 657) est remarquable par sa forme dérivant d'un ovoïde aplati dorso-ventralement et prolongé : en haut, en une longue corne ouverte au sommet, à laquelle participent les trois plaques apicales; en bas, en une à quatre cornes, le plus souvent deux, dirigées d'abord en dehors puis recourbées vers le haut. C'est dans ce genre qu'ont été olsservées les longues chaines (fig. 6ös) résultant de divisions incomplètes (30 a 40 p. Her et Fig. 6.59 . eau douce).

Glenodinium (Ehrenberg, emend. Stein) (fig. 659), se distingue moins parsa forme ovoïde aplatie dorso-ventralement ('), $\quad \begin{aligned} & \text { Glenodinium } \\ & \text { (im. Schutt). }\end{aligned}$

lig. 6 .

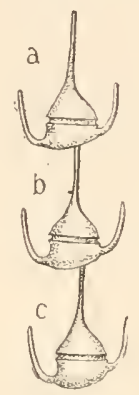

Ceratium

Association

(Sch.).

Pyrophacus (Stein) (fig. 660) de forme lenticulaire (Diametre horizontal 60 p. Mer);

Ptychodiscus (Stein', (fig. 661) de forme semblable, mais à sillon Iransversal remplacé par un mince ruban cuticulaire (Mer);

Gonyaulax (Diesing), (fig. 662', à sillon longitudinal s'ètendant dans loute la hauteur du corps avec la fente flagellifère réduite à

Fig.

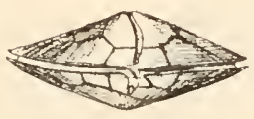

Pyropleacus (P. horologium) iin. Schult, Stein). ligg. 1561.

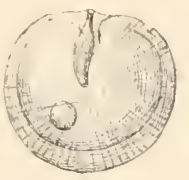

Ptychodiscus

(P. noctiluce) (im. Stcin).

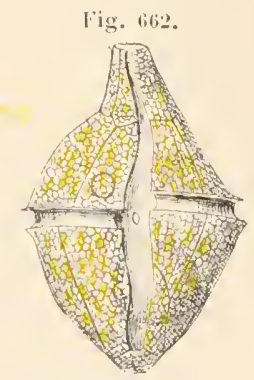

Gonyuulas

(G. polygramma) (int. Schüt). un petit orifice arrondi; souvent les deux pọles prolongés en pointes (20 «.. Mer); Steiniella (Schütt) (fig. 663), genre voisin $(100 \%$ is. Mer);

Oxytoxum (Stein) à sillon transversal situé très haut (fig. 640 et 664) (50 p.. Mer).

(l) Genre voi$\sin :$

Cladopyxis (Stein) (lig. 665), genre voisin mais douteux, caractérisé

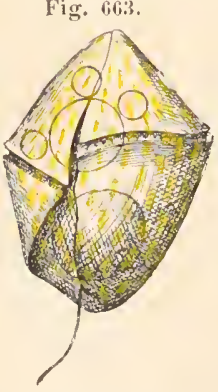

Steiniella

(S. /ragilis)

(im. Schütt).

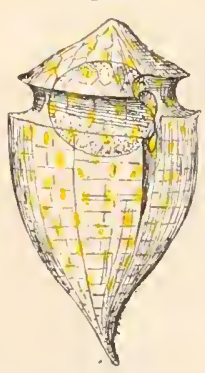

O.xyto.xum

(O. tessellatum) (im. Schutt).
Fig. 665.

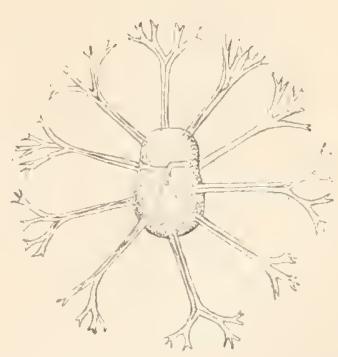

Cladopyis

(C. brachiolata) (Stein): 
que par sa cuticule très mince et anhiste (4⿳⺈.... Mer et eau douce). Gymnodinium (Stein) (fig. (666) est plus allongé que Gilenodinium et s'en distingue par l'alsence de cuticule. Il n'y a plus ici qu'une membrane cellulaire lisse, mince et dépressible. Selon les espèces, ou bien la forme est régulière, ou bien le sillon transversal décrit une hélice très allongée, ou bien en plus de cela, le corps se tord en liélice, ce qui contourne le sillon rertical. Plusieurs s'enkỵstent sous une épaisse enveloppe gélatineuse souvent munie de longues cornes. Certaines espèces ont un stigma oculiforme (50 à $150 \mu$; Mer et eau douce).

Hemidinium (Stein) (fig. 667) est un Gymnodinium pourvu d'une très délicate enveloppe et dont le sillon transversal est alssent à droite (2: م... Eau douce).

Pouchetia (Schiitt) (fig. 668) est nu comme les précédents, mais il a une forme bizarre et, au premier abord, incompréhensible, mais on arrive à la rendre claire en la ramenant a celle de certains Gymnodinium à sillon transversal en hélice allongée et i) corps contourné autour de son axe vertical. Si l'on suppose que le corps se tord d'un tour dans le même sens que le sillon transversal, celui-ci fera deux tours au lieu d'un et le sillon Fig. 667.

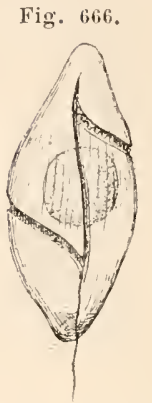

Gymnodinium (G. spirale) (im. Sch utt.). Fig. 668 . longitudinal, très long, décrira lui aussi un tour entre les extrémités, très éloignées en hauteur, du sillon transversal. En outre, l'animal possède un trìs gros ail formé d'une masse pigmentaire noire avec un cristallin sphérique, soluble dans l'acide acétique, ou de deux masses opposées l'une à l'autre, et déterminaul souvent une forte saillie (').

lar de longs prolongements ramifiés rayonnants; la constitution du sillon transversal n'est pas claire et il n'est pas même bien sûr que ce soit là un Dinoflagellé. En tout cas, par la ininceur de sa cuticule, il se rapprocherait de Glenodinium (60 $\mu$. Mer).

(1) Il faut attendre la publication de la fin du travail de Sснӥтт [93] pour décrire convenablement les trois nouveaux genres dont il donne seulement les figures:

Gymnaster (Ehrenberg, emend. Schütt),

Monaster (Schütt) (fig. 669), et

Amphitholus (Schütt) (fig. 670), caractérisés par un squelette intérieur qui rappelle celui des Radiolaires.

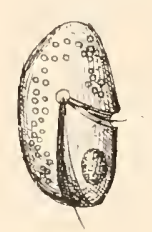

Memidinium

(II. nessutum) (d'ap. Stein).

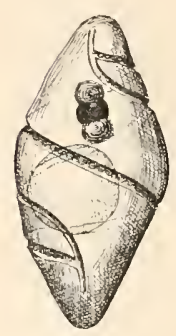

Pouchetia

(P. fusus)

(im. Schitt). 
Nous abordons maintenant une longue série de genres qui ont pour caractère commun la réduction externe de leur hémisphère supérieur, conséquence de la situation très élevée de leur sillon transversal. En outre, les crètes aliformes tant celles du sillon transversal que celles du longiludinal, sont très développées et arrivent à donner à ces ètres les physionomies les plus étranges (').

Phalachroma (Stein) (fig. 671) inaugure la série avec son sillon transversal déjà très rapproché du bord supérirur, en sorte que son hémisphère supérieur est réduit à une sorte de couvercle. Les crêtes aliformes des sillon horizontal et vertical sont assez élevées (80 … Ner).

Amphisolenia (Stein) (fig. 672) a des caractères semblables, mais avec un aspect tout différent dù à sa forme longuement lagéniforme, dilatée en bas, et a son orifice flagellifère placé très bas (Très grand. Mer).

Dinophysis (Ehrenberg) (fig. 673) est pres-

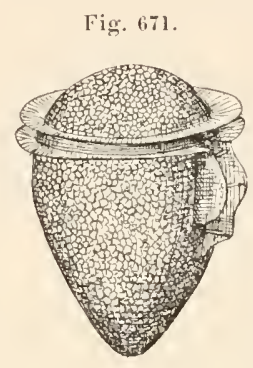

Palachroma (Sch.). que semblable à Palachroma, mais parfois orné de fortes pointes partant de l'hémisphère inférieur ( $80 \%$.. Mer).

Ceratocorys (Stein) (fig. 674) a les crètes aliformes du sillon transversal beaucoup plus saillantes encore et les pointes qui hérissent le corps constantes et harbelées (100 $\mu$. Mer).

Citharistes (Stein) (fig. 67:3) n’a pas ces pointes, mais la crête aliforme horizontalesupérieure est développée en une haute collerette infundibuliforme, dépassant de beaucoup le niveau de l'hémisphère supérieur très réduit ici comme dans tous ces

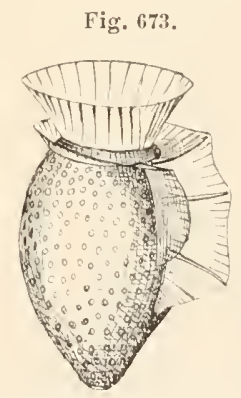

Dinophy'sis (Sch.).

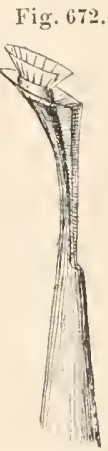

Amphisolenia (A. palmata). Parlic supérieure du corps (iim, Stein).

genres; son dos est muni d'une échancrure sul laquelle passent deux petites baguettes de manière ì former exactement l'anse d'une cruche; sa crète aliforme verticale gauche descend jusqu'en bas et, du côté droit, est une crète aliforme accessoire qui ferme de ce côté la cavité de l'anse et la transforme en une sorte de poche oì se loge une grappe de phxosomes (chromoplastes jaunes) (100 $\mu$. Mer).

(1) Ces genres forment la famille des DrvopIrisINe [Dinophysida (Bergh)].

Les précédents constituaient celle des PERIDINIx.E [Peri-

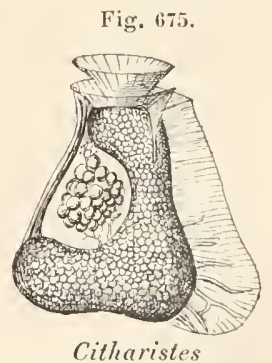

(im Schütt, Stein). 
Histioneis (Stein) (fig.676) a la collerette supérieure de Citharistes, mais encore plus haute et fendue en long, et la crête aliforme verticale gauche développée en une énorme expansion (70 $\mu$. Mer).

Ornithocercus (Stein) (fig. 677) a encore la mème collerelle supérieure, mais ici la crète aliforme horizontale inférieure s'élève elle aussi en entonnoir, presque aussi haut que l'autre, réduisant à une fente annulaire l'accès du dehors au sillon transversal compris entre les base des deux collerettes. Dans ce sillon s'accumulent des phrosomes; cn outre, la crète aliforme verticale gauche se léveloppe en un énorme appendice vertical soutenu par des côtes ornées de dessins variés (7ij) (... Ner).

Amphidinium (Claparède et Lachmann) (fig. 678) nous ramène à une apparence beaucoup plus simple. C'est un des genres précédents, caractérisé comme eux par la situation très élevée du sillon transversal et, par suite, par la réduction de l'hémisphère supérieur à une sorte d'opercule; mais la coque chitineuse, de forme si bizarre, a disparu et fait place à une mince culicule simple, lisse et à peine visible.

Fig. 676.

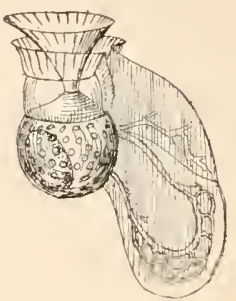

IIistioneis

(im. Schiatt, Stein). Fig. 677 .

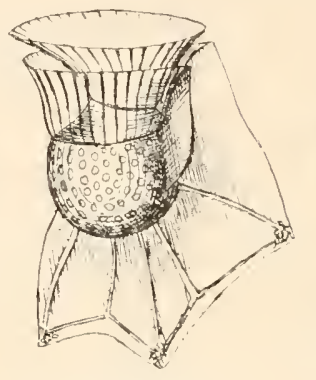

Ornithocercus (im Schiitt, Stein).
Amplidinium (im. Stein).

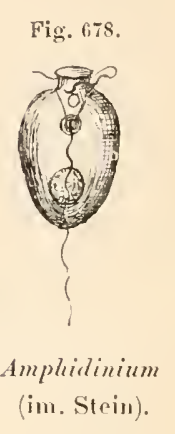

POLYDINIDES. - POLIDINIDA

[POLYDINIDA (Bütschli)]

Il contient le seul genre très remarquable Polykrıkos (Bütschli) (fig. 679), nu comme un Crymnordinium, en forme de boudin, avec huit sillons transversaux presque circulaires, leur extrémité gauche étant à peine plus élevée que la droite. Tous sont réunis par un unique sillon longitudinal commun, long ot étroit, parcourant toute la hauteur du corps. A l'extrémité inférieure de ce sillon, est un fouet longitudinal parfois double; chaque sillon transversal contient un fouet transversal. A l'intérieur sont quatre noyaux superposés, accompagné chacun d'un petit nombre de pelits novanx accessoires

dinida (Bütschli)]. Nous avons seulement fait passer le genre Cerrotocorys de la seconde à la première.

Fig. 679,

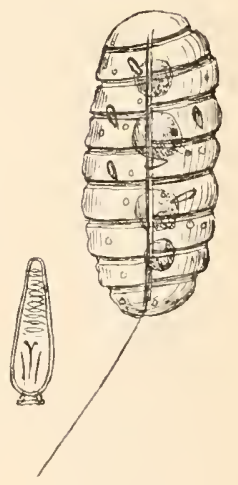

Polykrikos (P. auricularia) (d’ap. Bergh). A côté, un trichocyste plus grossi. 
que Bengı assimile au micronucléus des Ciliés, sans appuyer d'ailleurs son interprétation sur une comparaison détaillée des fonctions. Enfin, dans l'ectoplasme sont répandus des trichocystes semblables ì des nématocystes, moins le noyau. L’animal se reproduit par division transversale (100

\section{APPENDICE AUY DINOFLAGELLES.}

Erythropsis (R. Hertwig)(fig. 680, 681) est un animal dont les affinités sont tout à fait obscures.

Décrivons-le d'abord en lui-même sans employer à son égard aucune terminologie spéciale impliquant une opinion préconçue sur sa position taxonomique que nous discuterons ensuite.

Il a une forme irrégulièrement sphérique. Sur la face que tous s'accordent à considérer comme ventrale, se trouve un sillon vertical, profond et étroit au milieu, qui se perd en haut et en bas, en devenant de plus en plus large et superficiel. Dans sa partie moyenne, il esı limité à droite par une grosse protubérance arrondie, le talon (Sporenträger) qui se prolonge à son sommet en un petit appendice courbe appelé l'éperon (Sporn). A gauche et un peu plus haut, se trouve un gros ail, formé d'un beau cristallin à couches concentriques, logé dans une capsule qu'il ne remplit pas toute entière, et enchàssé à sa base dans une masse pigmentaire brune. La saillie de ces deux organes contribue

Fig. 680 .

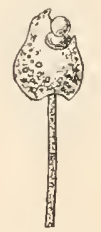

Erythropsis (E. agilis) (d'ap.

R. Hertwig). a augmenter, à leur niveau, la profondeur du sillon interposé. A l'extrémilé supérieure, se trouve la calotte (Deckel) formée simplement d'une partie un peu aplatie, débordant par ses bords le niveau général, de manière à déterminer au-dessous d'elle une goullière. Celte gouttière ne fait pas seulement le tour de la calotte, mais

Fig. 681.

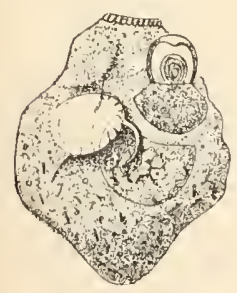

Erythropsis

(E. agilis).

L'animal plus grossi sans la quene (d'ap. R. Hertwig). monte sur sa face supérieure où elle se lermine. A la face interne du talon prend naissance un long filament contourné en ressort à boudin qui, de là, monte dans la partie supéricure du sillon. s'engage dans la gouttière et la parcourt jusqu'au bout. De l'extrémité inférieure du sillon vertical, part un gros appendice cylindrique, très mobile, très contractile, trois à quatre fois plus long que le corps, la queue (fig. 680). Le corps est recouvert d'une mince cuticule, rempli d'un protoplasma granuleux et renferme un très gros noyau subcentral.

On n'a pu observer, en tout, que deux exemplaires de cet animal.

R. Hertwir qui l'a découvert le considère comme un Protozoaire sans doute voisin des Infusoires. Il compare la calotte au disque des Vorticelles et l'appendice inférieur au pédoncule de celles-ci.

Метснnikof voit dans cet appendice un suçoir d'Acinète et rapporte l'animal aux suctoria.

C. Vogr, sans l'avoir vu, cherche à démontrer que c'est une vraie Vorticelle (dont il fait le genre Spasthostyla) détachée avec son pédoncule, et l'œil serait celui de quelque Méduse (Lizzia ou Nansilhoe) que l'animal aurait vainement cherché à engloutir et qui serait resté arrêté au passage, opinion renversée par le fait que cet œil se trouve aussi dans l'exemplaire de Metchnikof.

Il nous semble qu'il y a une autre manière de voir plus vraisemblable que les précédentes et que nous hasarderons tant elle nous semble probable, mais sous 
toutes réserves et sans méconnaître le danger qu'il y a à formuler une opinion sur un être que l'on n'a pu examiner.

Ce serait un Péridinien.

Son æeil, qui s'oppose à son admission chez les Vorticelles aussi bien que chez tout autre Cilié offre une ressemblance frappante avec celui de Pouchetia. Son sillon vertical est absolument celui d'un Péridinien. La gouttière représente le sillon transversal placé très haut comme cela arrive souvent. Le filament en ressort à boudin n'a rien de commun avec une zone aborale tandis qu'il représente exactement el fouet transversal d'un l'éridinien. Il faut cependant noter que Hertwig n'est pas très affirmatif sur la question de savoir si la portion qui occupe la gouttière est ou non continue avec celle qui est logée dans la partie supérieure du sillon longitudinal, et que ce fouel, par une exception unique, suivrait le sillon de gauche à droite. Lnfin, le gros appendice serait le fouet vertical. Il a les connexions et le mode d'action de cet appendice dont il ne diffère que par sa grosseur. C'est là certainement un caractère assez exceptionnel pour faire hèsiter, mais la différence n'est guère moindre avec un pédoncule de Vorticelle ou avec un suçoir d'Acinétien. Enfin remarquons que les diffẻrences peuvent peut-être s'expliquer aussi en partie par la très insuffisante connaissance que l'on a de ce curieux animal. (Celui de Hertwig perdit sa queue avant qu'on put le dessiner ou le fixer. Celui de Metchnikof n'avait pas d'éperon.)

(Taille non indiquée, semble peu différente d'un millimètre. Mer). 


\section{$4^{e}$ SOUS-CLASSE \\ CYSTOFLAGELLÉS. - CYSTOFLAGELLIE \\ [C'YSTOFLAGELLATA (Häckel)]}

Cette sous-classe ne contenant que deux genres, Noctiluca et Leptodiscus, nous prendrons pour type la Noctiluque qui est la plus commune et la mieux connue.

\section{TYPE MORPHOLOGIOQUE \\ (FIG. 682 A 687)}

La Noctiluque apparaît à l'œil nu comme une petite sphère molle, transparente, un peu jaunàtre qui l'a fait comparer à un grain de tapioca cuit. Elle en a aussi le diamètre, I millimètre environ.

\section{Structure}

Configuration extérieure. - L’animal (fig. 682) est à peu près sphérique, seulement un peu allongé dans le sens antéro-postérieur. En un point, qui sera pour nous le sommet de la face ventrale, se détache un flagellum à peine conique (/lg.), obtus au bout, aplati davant en arrière sur la section transversale, et d'une longueur à peu près égale au diamètre du corps. Au-dessous de ce flagellum se trouve une dépression méridienne verticale, comparable à celle qui sépare les deux moitiés d'un abricot, mais qui ne s'étend que sur une moitié à peine du contour total. On l'appelle le sillon ventral (sill.). En écartant les lèvres de ce sillon on constaterait qu’il s'enfonce peu à peu en descendant à partir de la hase du fouet, puis plonge brusquement dans une dépression profonde (perist.) au fond de laquelle est la fente buccale (f.b.). Delà, il regagne à pic le niveau

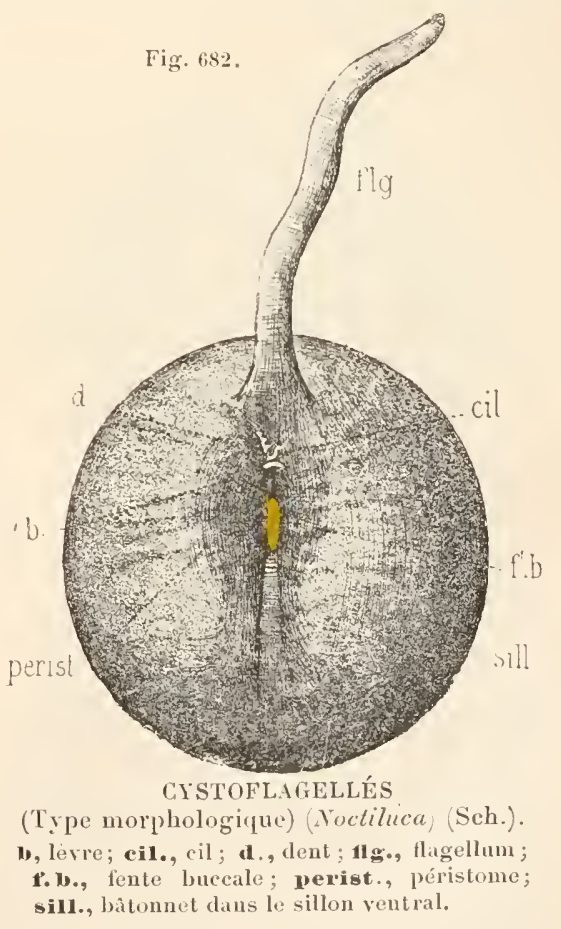
de la surface. A partir de ce point qui correspond à peu près au milieu de sa hauteur, le sillon continue très superficiellement jusqu’à sa termi- 
naison. Sur la partie peu profonde qui sépare la base du flagellum de la fente buccale, se trouve, un peu à droite de la ligne médiane, une petite lamelle saillante, insérée un peu obliquement, dont le bord libre est découpé en trois pointes, ce qui lui a fait donner, malgré sa mollesse, le nom de dent $(d$.$) . lmmédiatement au-dessous de l'extrémité inférieure$ de cette dent, surplombant la dépression brusque qui conduit à la fente buccale, se trouve une autre lamelle plus petite et aussi molle que la précédente, mais en forme de croissant et disposée transversalement, que l'on appelle la lève $(l$. $)$. Du point où la lèvre confine à la dent, naît un tout petit flagellum accessoire que l'on appelle le cil (cil.); il est si petit qu'il ne fait pas saillie hor's du sillon ventral. Tout cela est tapissé par la cuticule souple et très mince qui revêt le corps entier et c'est seulement au niveau de la fente buccale que le cytoplasma intérienr est à nu; les parois de linfundibulum ohlique qui conduit à cette fente sont elles-mèmes tapissées par celte cuticule. Dans sa moitić inféricure, le sillon ventral

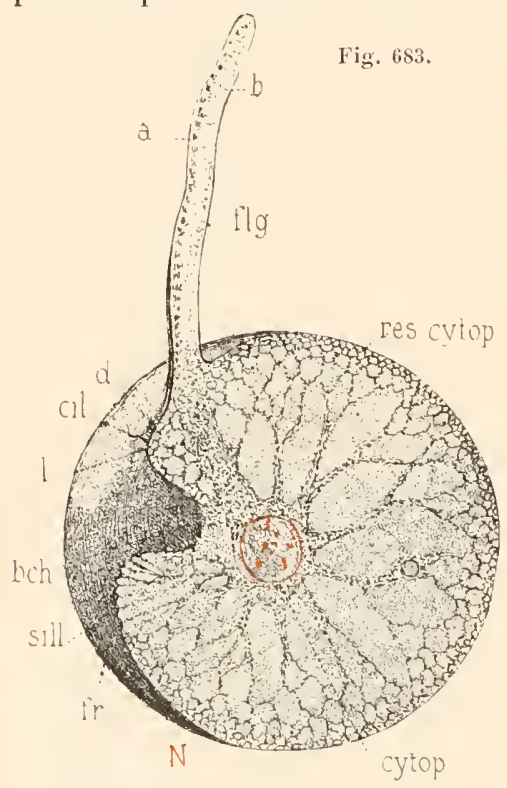

CYSTOFIAGELLÉS

(Type morphologique) (Noctiluea) (Sch.). Coupe sagittale.

a, partie ventrale du flagellum striée transversalement; $\mathbf{b}$, partie dorsale granuleuse; beh., fente bucrale; cil., cil; cytop., cytuplasma périnucléaire; d., dent ; fr., fronces dư bâtonnet; IIğ., flagellum; 1., levre; x., noyau; res., cytop., résean cy toplasmique tangentiel; sill., sillon ventral au nivean chi batonnet. est très peu profond et laisse apercevoir naturellement son fond bombé et froncé en travers (sill.). Cette partie froncée dessine donc une étroite surface linéaire verticale; à son niveau, la cuticule est non seulement froncée, mais sensiblement épaissie. Tout cela lui donne un aspect particulier qui lui a valu le nom de bîtonnet. Nais il faut bien se rendre compte que ce prétendu bàtonnet n'est point quelque organe saillant sur la surface: ce n'est simplement qu'une partic de la surface ellemème, logée mème au fond d'une dépression. On donne quelquefois le nom de péristome (périst.) à la partie du sillon ventral qui va du flagellum au batonnet. Le reste du corps est régulièrement arrondi et ne présente rien de particulier.

Conformation intérieure. - L'animal (fig. 683) est une simple cellule et en possède les organes, noyau, cytoplasme et membrane, mais tout cela avec des caractieres très particuliers. Il n'y a pas de vésicule pulsatile. La membrane forme la surface extérieure que nous venons de décrire. Elle semble, malgré sa mollesse et sa minceur $\left(I^{1} / 2 \%\right)$, participer aux caracteres d'une culicule puisque, ainsi que nous le verrons, elle 
peut ètre rejetée par une mue. Elle est partout continue, n’étant absente que dans le sillon buccal; elle n'est épaissie qu'au niveau du soi-disant bàtonnet (sill.); la levre et la dent sont revètues l'une membrane aussi mince que les parties roisines.

Le noyau $(N$.$) est situé à une faible distance en arrière de la fente lıuc-$ cale. Il est assez gros, rond, vésiculeux et contient une dizaine de petits corps chromatiques que nous verrons ètre des chromosomes épars au milieu d'une substance achromalique finement fibrillaire.

Le cytoplasme (cytop.) forme immédiatement derrière la fente buccale, i laquelle il confine, une importante accumulation massive dans laquelle est englobé le noyau. De cette masse centrale, partent de gros tractus qui se portent radiairement vers tous les points de la surface mais, dans ce trajet, se ramifient beaucoup à mesure qu'ils s'amincissent et anastomosent leurs ramifications de manière à former un réseau général. Les mailles de ce réseau constituent des vacuoles. Au roisinage de l'accumulation centrale du cytoplasma, elles sont larges et limitées par des parois épaisses; mais à mesure qu'elles se rapprochent de la surface, elles deviennent de plus en plus petites et serrées, jusqu’à n’avoir plus, sous la membrane, que 4 ou $\ddot{3} \mu$, leurs travées de séparation mesurant à peine $1 \%$. E. Elles se terminent sous la cuticule, non par une couche conlinue, mais par un réseau tangentiel (res. cytop.) formé par les dernières mailles du réseau général (").

En deux points seulement, le cytoplasme prend une disposition particulic̀re, c'est au niveau du bàtonnet et dans le llagrellum.

Vers le batonnet, la masse cytoplasmique centrale envoie un faisceau de filaments divergents qui s’insèrent aux saillies intérieures $(f r$. $)$ résultant du froncement de la cuticule à ce niveau. On ne sait rien de la signification de cette disposition, pas plus que de celle du bàtonnet. Ce n'est peut-être qu'une particularití insignifiante ou une partie plus rigide destinée à jouer un rôle de soutien.

Le flagellum (flg.) reçoit de la masse cytoplasmique centrale un prolongement qui, en pénétrant lans l'organe, subit une double différenciation. La partie tournée vers la face ventrale $(a)$ est, comme une fibre musculaire, striée transversalement et for'mée d'une succession de tranches alternativement claires el sombres. La partie tournée vers le dos $(b)$ est simplement granuleuse. Sans doute, la première est contractile et la deuxième simplement élastique pour jouer le rôle de ressort antagoniste.

Inclusions. - Dans les travées du réseau, on trouve, indépendamment des racuoles alimentaires dont il sera question plus loin, des inclusions de deux sortes : les unes sont des granulations protoplasmiques de couleur orangée qui donnent à l'animal la légère teinte jaunìtre dont nous avons parlé, les autres sont des gouttelettes graisseuses.

(1) Il y aurait en outre, d'après Bütschli, un réseau secondaire, très fin, dans les vacuoles qui occupent les grandes mailles. 


\section{Physiologie.}

Habitat. - Les Cystoflagellés sont exclusivement marins. Ils vivent dans presque toutes les mers des régions tropicales el tempérées. Ils se rencontrent parfois en si grande abondance que l'eau en est comme visquesise, surlout par un temps calme et une température élevée.

Locomotion. Mouvements. - L'animal flotte plutôt qu'il ne nage, en pleine eau, la fente buccale en bas. Cette attitude est un simple effet physique, le noyau et l'accumulation proloplasmique qui l'entoure étant les parties les plus lourdes de son corps. Les vacuoles au contraire sont remplies d'un liquide à peine additionné de quelques matières albumineuses et minérales et plus léger que l'eau de mer (").

Les mouvements du fouet ont peut-être pour effet de le déplacer quelque peu, mais ils sont trop faibles pour avoir une grande efficacité sous ce rapport; ils sont surlout destinés à l'alimentation. On olsserve aussi des contractions du corps et un mouvement inlérieur du cytoplasma dans lequel des mailles se coupent, d'autres se reforment et modifient sans cesse l'aspect du réseau. Mais il n'y a pas de vraie cyclose.

Alimentation. - L'animal se nourrit de petites proies qu'il capture avec son flagellum. Pour cela, il rabat brusquement cet organe sur le sillon ventral et les particules qui se trouvaient sous lui sont poussées vers la bouche. Pour le relever, il soulève d'abord la base, puis la pointe $\left({ }^{*}\right)$. Les proies sont englobées dans une vacuole alimentaire qui se forme en mème temps qu'elles pénètrent dans Je cytoplasma, et sont transportées avec cette vacuole de ci de là, un peu partout dans le corps, mais toujours dans l'épaisseur des Iravées, fortement renflées à leur niveau, et jamais dans les vacuoles intermédiaires. Elles sont ainsi peu à peu digérées et réduites à un résidu fécal qui est ramené à la bouche el expulsé par cet orifice.

L’absence de résicule pulsatile oblige à almettre que l'excrétion et la respiration se font par des échanges osmotiques superficiels.

La circulation des racuoles alimentaires s'explique par les mouvements du cytoplasma.

(1) İignal a montré qu'il peut subir un effet de ludion, c'est-à-dire qu'en vase clos, si on augmente la pression il plonge et remonte si on la diminue. Mais c'est là un effet brusque et sans doute passager. Götнanт et IIEINsius [9.3] ont constaté qu'en eau libre, il modifie sa łensité de manière à flotter toujours. En eau de mer à 1024, il pèse 101's. Si on réduit la densilé de l'eau de mer à 101', il prend celle de 1008 et continue ainsi, jusqu’à éclater lorsque la dilution devient trop forte.

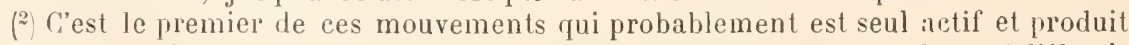
par la contraction de la couche striée, le soulèvement étant dì, sans loute, à l'élasticité de la couche granuleuse. On peut faire ingurgiter par l'animal des parcelles de jaune d'œuf cuit mêlées à son eau. Les proies qu'il absorbe sont parfois si volumineuses qu'elles le déforment el ont dù nécessiter une forte distension de la fente buccale. 
Mue. - Poucuet [90] a observé que parfois l'animal se débarrasse de sa membrane et en sort en conservant son fouet et tous ses organes. Sins doute alors, il n'en élimine que les parties superficielles cuticulaires.

Reproduction. - Il n'y a jamais d'enliystement. On a décrit un passage a l'étal de repos dans lequel l'animal s'arrondirait et perdrait ses organes extéricurs, tout comme avant la division, mais il n'est pas démontré qu’il y ait là autre chose qu'une préparation à la division ou à la sporulation.

L'animal se reproduit par division longitudinale et par sporulation. Les deux processus paraissent à peu près aussi fréquents l'un que l'autre.

Division (fig. 684). - Avant de se diviser, l'animal commence par subir les modifications dont nous parlions il y a un instant sous le nom de passage à l'état de repos. Il s'arrondit $(A)$, efface son sillon buccal, et résorbe tous ses organes externes, fouet, dent, lèvre, cil et bàtonnet: il se réduit à une simple sphère uniforme $(B)(')$. A l'intérieur, le réseau persiste, mais la plus grande parlie du eytoplasme se concentre dans la masse périnucléaire. Naturellement, l'animal est, dans cet état, complètement inerte.

La division est longitudinale et a lieu suivant un plan méridien qui suit le sillon venlral disparu. Le phénomène débute (lig. 684́, 1 ) par la division nucléaire qui se produit longitudinalement par un processus sans doute semblable à celui qui a été étudié avec plus de détail ì propos Fig. 68'.
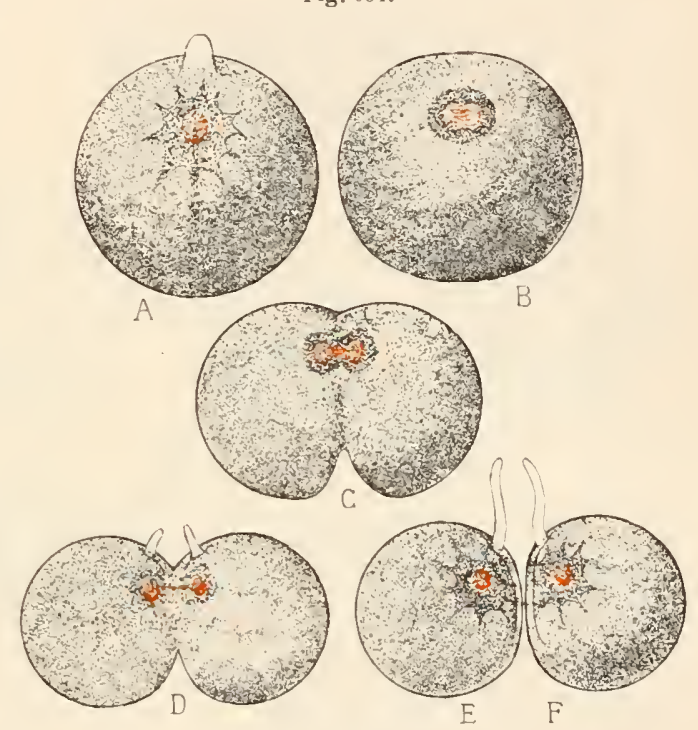

CISTOFLAGELLÉS

(Type morphologique) (Noctiluca) (Sch.).

$A$ is $D$, stades successifs de la division; $E$ et $F$, les deux individus filles.

de la sporulation et que nous décrirons en parlant de ce phénomène. Puis, le corps s'élargit et peu à peu s'étrangle suivant le plan de

(1) Il n'est pas certain, cependant, que la fente buccale devenue superficielle se ferme. Le cil, la lèrre et la dent rentrent simplement dans le corps, s'effacent comme un pli d'une étoffe que l'on tend et cela suffirait à prouver que ces deux derniers ne sont pas des apophyses cuticulaires. Pour le fouet, on a dit aussi qu'il tombait, mais il semble bien démontré aujourd'hui qu'il rentre peu à peu dans le corps. On le voit devenir d'heure en heure plus gros, plus court, plus mou et se réduire à un simple tubercule qui enfin disparait. Quant au batonnet, il s'efface simplement sans doute, par déplissement de ses fronces. 
division (fig. 684,C). La séparation marche plus vite en bas qu'en haut, en sorle que les deux individus filles sont allachés en dernier lieu par un isthme silué à la hauteur du flagellum $(D)$. Bien avant que la séparalion soit complète, les deux individus filles reforment leur sillon ventral et leur fouet et l'on constate alors qu'ils sont orientés perpendiculairement à l'individu mère, leur plan sagittal commun ayant, par rapprort à la mère, la position d'un plan coronal. Enfin la séjaration s'achève $(E, F)$ el les deux jeunes n’ont plus qu'à grossir.

Conjugaison. - La conjugaison (fig. 683̈) a lieu entre deux individus semblables qui se soudent par la région buccale et se fusionnent (A) peu

Fig. 685 .

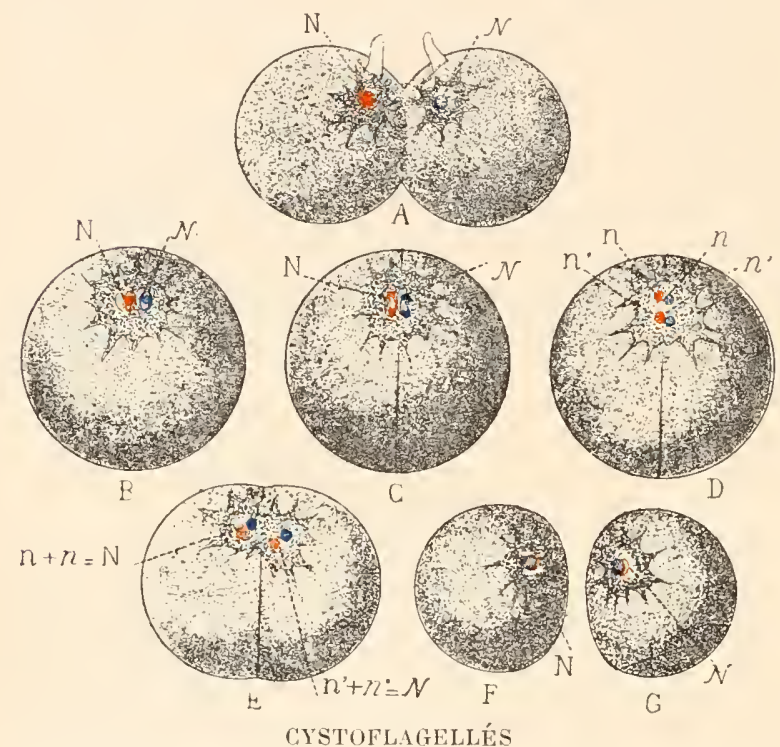

(Type morphologique) (Noctiluca) (Sch.). Conjugaison.

$A$ à $(i$, stades successifs depuis la conjugaison jusqu'à la formation des individus filles.

Les uoyaux appartenant it un des individus sont indiqués en lettres ordinaires et erinx de l'autre en italiques. à peu complètement, le manière à conslituer un individu unique $(B)$ ne différant en rien extérieurement d'un individu ordinaire de grande taille. Mais les noyaux ne se fusionnent pas, en sorte qu'il y a deux noyaux distincts dans une masse protoplasmique simple $\left(B: N\right.$. el $N^{\top}$.). Bientôt, l'animal se divise ì la manière ordinaire et les deux noyaux prennent part à la division. Des quatre noyaux filles (n., n'., $n ., n^{\prime}$.), deux passent dans chacun des deux individus filles, mais de telle façon que chacun ait un demi-noyau de chacun des parents et se constitue ainsi un appareil nucléaire d'origine double $(E)$. Toul aussitòt après, commence la sporulation dans larpuelle ainsi chacune des spores contiendra un peu de la substance des deux conjugués (').

(1) Ces observations remarquables sont dues à Ischikava [91] qui a malheureusement laissé dans l'ombre plusieurs points importants. L'auteur ne dit pas, mais la chose semble bien probable, que les deux demi-noyaux se fusionnent en un noyau unique avant la première division de sporulation. Il est peu explicite sur l'ètat des conjugués. Il semble d'après ses dessins plutôt que d'après son texte que les choses se passent de la manière suivante. Les deux conjugués sont des individus complets avec leur flagellum au moment où ils se joignent. Mais ils passent pendant la conju- 
Sporulation. - Les individus nés de cette division post-conjugale sont aptes à sporuler et le font soit imméliatement, soit quelque temps après. Pour cela, I'animal commence à passer à cet élat de sphère lisse (fig. $686, A$ ), sans appendices quelconques, que nous avons décrit comme précédant la division simple, clildivise son noyau suivant le processus d'une mitose parfaite, avec division longiludinale de chromosomes $(B$ et $C)$, el intervention de deux centrosomes arec leur sphère d'archoplasma. Mais l'où viennent ces centrosomes dont on ne royait pas trace près du noyau à l'état de repos? Sortent-ils du noyau, préexistaient-ils dans le cytoplasma amhiant? On ne sait. Celte division est suivie immédiatement d'une autre, puis d'une troisième et ainsi de suite jusqu'à huit ou neuf ce qui donne deux cent cinyuante-six ou cinq cenl douze noyaux filles (").

Fig. 686.
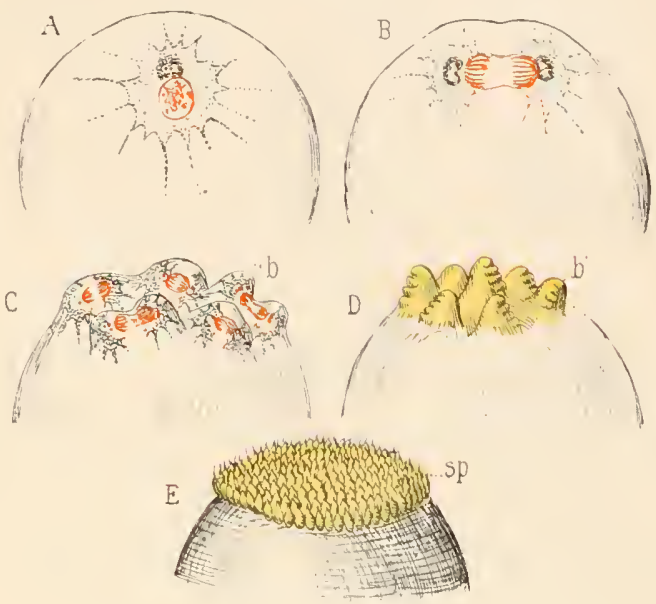

CXSTOFLAGELLÉS

(Type morphologique) (Noctiluca) (Sch.).

Sportation.

$A$ it $E$, states suceessils. b., pilpilles pour la formation des spores; sp., spores.

Penlant que ces phénomènes nucléaires s'accomplissent, le cyloplasma accumulé sous une région de la surface se divise progressivement, lui aussi, en autant de fragments uninucléés, mais par des plans qui d'abord n’entament pas toute son épaisseur, comme dans la segmentation superficielle discoïdale de certains Vertébrés. En mème temps, cette accumulation protoplasmique retirepeu à peu à elle, par sa face profonde, tout le reste du cytoplasma répandu dans le réseau, en sorte que la totalité du cytoplasme se trouve rassemblée en une sorle de gàteau sous une partie de la surface, laissant vide tout le reste de la cavité, sans mème une couche superficielle pour doubler la membrane. Les petites masses nucléées font saillie à la surface comme autant de papilles serrées les

gaison à l'état dit de repos dans lequel les fouets disparaissent ainsi que le sillon el tous les accessoires. L'animal unique issu de la conjugaison est done ì l'état de repos. Il se divise en eet état et sporule en cet élat. Mais lorsque quelque temps se passe entre la division post-conjugale et la sporulation, il reforme son fouet, son sillon, ete., repasse en un mot, momentanément, à l'état d'activité et reprend les allures d'un individu libre ordinaire, sauf qu'il a deux noyaux au lieu d'un.

(1) Ou quelque nombre intermédiaire, car il est bien possible que certains noyaux se divisent huit fois et d'autres neuf. 
unes contre les autres $(C: b)$; elles s'individualisent de plus en plus $(D: b)$ et finissent par former autant de corps indépendants qui se munissent d'un flagellum $(E: s p$.), se détachent et se dispersent, abandonnant le reste de la cuticule vide (').

Ces spores ont, lorsqu'elles sont entièrement formées, une très singulière conformation (fig. 687). Elles sont ovoïdes et comme sur-

Fig. 687 .

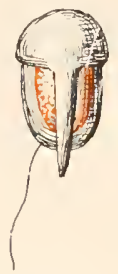

CYSTOFLAGELLÉS (Type morphologique) (Noctiluca) (d ap.Cienkovsky). Spore. montées d'un casque prolongé dorsalement vers le bas en un long appendice appelé le dard, expression absolument défectucuse. De la base du casque naissent, en avant, à l'opposé du dard, un fouet locomoteur assez long et une sorte d'appendice styliforme immobile. 0 n a avancé que le dard formait le bâtonnet de l'adulte, que l'appendice immobile se transformait en flagellum et que le fouet locomoteur devenait le petit cil prébuccal. Mais ce sont là des hypothèses, car l'évolution de ces spores n'a pas été suivie.

Phosphorescence. - La phosphorescence des Noctiluques est plus vive que celle d'aucun autre animalcule marin. Elle est due au cytoplasma ou du moins à une graisse qu'il contient $\left(^{2}\right)$. Elle est augmentée par la plupart des excitants chimiques ou physiques et par l'électricité. La lumière la contrarie. Portées du grand soleil à l'obscurité, les Noctiluques n'y deviennent lumineuses qu'après quelque temps. Massart [93] a constaté à leur sujet un phénomène très curieux d'infuence consécutive consistant en ce que, soumises à l'obscurité persistante ou à un éclairage permanent, elles continuent à éclairer davantage aux heures de nuit, comme lorsqu'elles étaient soumises aux alternatives du jour et de la nuit.

Régénération. - Les Noctiluques ont une puissance régénératrice remarquable. Elles réparent des portions considérables de leur corps excisées expérimentalement.

\section{GENRES}

Noctiluca (Suriray), vient d'ètre décrit comme type morphologique de la sous-classe $\left(1^{\mathrm{mm}}\right.$. Mer $)\left({ }^{3}\right)$.

(1) Nous suivons ici la version d'ICHIKAva [91] qui assure que tout le cytoplasma maternel est employé. D'ordinaire, on admetlait que la partie périphérique du réseau et la couche profonde de la région sporifère restaient inutilisées. On appelle ce mode de reproduction de la Noctiluque une Gemmation. Mais une gemmation dans laquelle la mère passe toute entière dans ses bourgeons, est une sporulation.

(2) D'après VIgxal, elle ne résulterait pas d'une oxydation de cette graisse car, dans l'eau bouillie, l'animal reste lumineux. Cela n'est pas très démonstratif, car elle peut résulter d'un déplacement d'oxygène combiné, dont la provision peut durer aussi longtemps que la vie, probablement fort courte, de l'animal en eau bouillie. Cela prouve seulement que ce n'est pas l'oxygène immédiatement absorbé par la respiration des tissus qui intervient.

${ }^{3}$ ) Il n'y a probablement qu'une espèce ( $N$. Miliaris), bien qu'on ait voulu en faire deux autres : $N$. homogenea el $N$. pacifica. 
Leptodiscus (R. Ilertwig) (fig. 68̄) a la forme d'un verre de montre, ou plutôt d'une lentille courbée de manière à ètre concave d'un côté, convexe de l'autre. Il a une membrane comme la Noctiluque. A l'intérieur, la structure est la mème, il a un noyau ( $\boldsymbol{I}^{\text {. }}$ ) au centre d'une accumulation protoplasmique qui occupe le milien de la face ventrale et. de cette masse, part un réticulum qui šétend dans toute la cavité du corps. Mais la conformation extérieure est fort différente de celle de la Noctiluque. Sur la face concave, qui est la dorsale, il n'y a rien de particulier, si ce n'est une fine ligne circulaire concentrique au bord formé d'une série de granulations sous-jacentes à la membrane. Sur la ventrale convexe, on trouve diver'ses parties dont la correspondance avec celles de la Noctiluque est assez incertaine. D'un côté, à une certaine distance du centre, se trouve une dépression (d.) qui s'enfonce obliquement vers le centre et vers la face ventrale, atteint presque celle-ci à peu de distance du centre et se perd là dans la masse protoplasmique centrale.

Un faisceau serré de fibrilles protoplasmiques partant de l'amas central

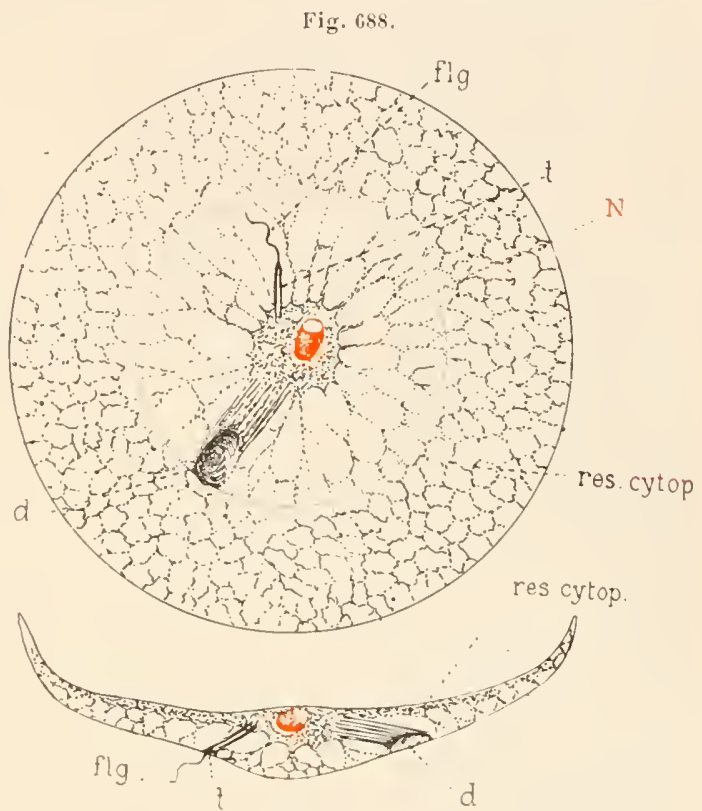

Leptodiscus (im. Hertwig).

Yu de face et en coupe sagittale.

d., dépression ; fly., hlagellum; res. eytop., résean eytoplasmique; $\mathbf{t}$, tube.

se dirige vers cette dépression et s'y' insère. Ilentwig voit là l'équivalent d'une bouche bien qu'il n'ait pu y trouver d'ouverture au fond. Bütscmi y voit l'homologue du bâtonnet. De l'autre côté du centre se trouve, toujours à la face dorsale, une autre dépression dirigée à peu près de même, mais beaucoup plus étroite $(t$.$) . Celle-là donne naissance$ à un petit flagellum $(f l g$.$) qui s'insère dans sa profondeur et s'étend$ assez loin au delà de son orifice. Bütschli y voit l'homologue de la fente buccale et du petit cil de la Noctiluque. On n'est pas sûr que le cytoplasma soit nu au fond de ce tube. On ne trouve dans le corps que de très fines particules alimentaires. 11 n'y a pas de gros fouet locomoteur (1,5. Mer)(').

(1) L'animal n'a été trouvé que dans la Méditerranée aux environs de Messine. 


\section{$\because)^{\circ} \operatorname{SOCS}-\operatorname{Classe}$ \\ CATALLACTES. - CATALLACTIA \\ [CATALLLCTA (IIäckel)]}

Les Catallactes ne renferment qu'un genre unique

Magosphæra (Iläckel) (fig. 689). A l'état adulte, la Magosphère est une petite sphère $(B)$ de $70 \mu$ de diamètre, transparente, incolore, qui nage en tournant au moyen de flagellums dont sa surface est garnie. Elle est formée de trente-rleux cellules disposées côte à côte en sphère creuse $(A)$. Ces cellules sont piriformes, leurs bases élargies, polyédriques par pression réciproque, sont tournées vers le dehors et portent un plateau sur lequel sont implantés quelques longs fouets; leurs queues se prolongent toutes jusqu'au centre où elles se rencontrent absolument comme des Symura. Elles sont noyées dans une substance gélatineuse qui comble tous les intervalles entre elles, mais ne recouvre pas leur surface externe. Elles mesurent $20 \%$ de large sur $3 \%$. de long y compris leur queue. Chacune renferme un gros noyau distal et une vésicule pulsatile située auilessous. Ces colonies ne semblent

Fig. 689.
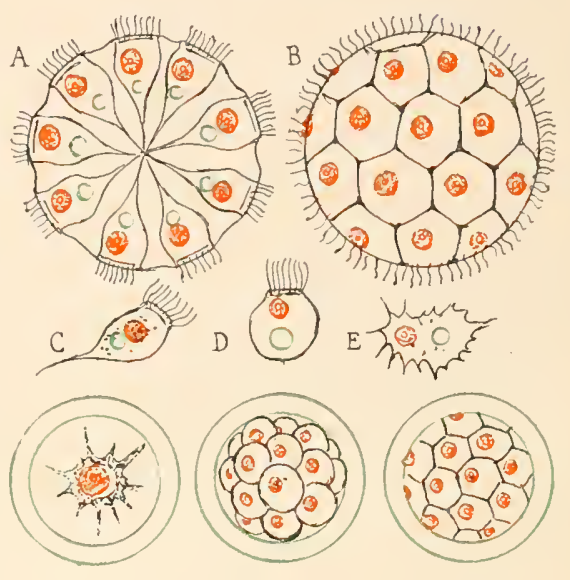

F

G

H

CATALLACTES (Magosphara) (im. Ilickel).

$A$, colonie en coupe optique; $B$, la mème en vue superfirielle; $C$, individu après la dispersion de la colonie ; $\mathrm{l}$, commencement de la transformation en amibe: $E$, stade amibe; $F$, enkrstement; $G$, division de l'individu enkysté; $I$, portion de la colonie fille. pas prendre de nourriture. Au bout de quelque temps, elles se dissocient en leurs éléments qui deviennent libres et se dispersent $(C)$. Ils nagent ainsi arec leurs fouets, se montrent très métaboliques et se nourrissent, absorbant des particules solides par leur disque flagellifère. Mais cet état dure très peu; au bout d'environ quatre heures, ils rétractent leur queue $(D)$, perient leurs fouets et se transforment en amilues $(E)$. Celles-ci rampent avec leurs pseudopodes, s'alimentent et grossissent probablement pendant un temps assez long. P'uis elles s'enkystent sous une épaisse membrane gélatineuse stratifiée $(F)$. Quand ces kystes sont mùrs, leur contenu se divise en deux, quatre... trente-deux cellules dahord arrondies et juxtaposées $(C$ ot $I I)$, qui se disposent à mesure qu'elles deviemnent plus nombreuses en sphèro creuse régulière. Leurs flagellums poussent comme de petits pseu- 
dopodes qui se régularisent et la sphère se met à tourner dans son enveloppe sous l'action de leurs battements. Enfin l'enveloppe se rompt et la sphère mise en liberté se trouve en l'état où nous l'avons trouvée en commençant cette description (').

Ce cycle érolutif est très simple et très clair, mais ce qui l'est moins, ce sont les affinités de ces ètres. Häcker les a nommés Catallactes

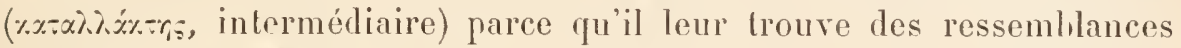
avec l'œuf des Métazoaires au stade kyste, arec l'œuf segmenté au stade de segmentation, avec une planula au stade de sphère ciliée enkystée, arec les Volvoces au stade de sphère ciliée libre, avec les Infusoires péritriches au stade flagellé dispersé et enfin arec les Rhizopodes au stade amibe. La Magosphère est une sphère magique

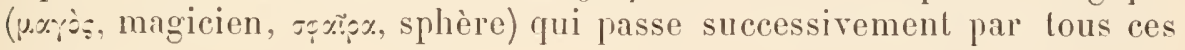
groupes.

Tout rela est incontestable à un certain point de vue, mais n’a pas la mème valeur au point le vue des affinités zoologiques. Trois hỵpothèses seulement sont permises : c'est un Rhizopode, un Flagellé ou un Infusoire. Nous rejettrons la première parce que l'amibe n'est pas à elle seule caractéristique du Rhizopode, vu que l'on trouve des stades annibes chez les Sporozoaires el les Flagellés, el que l'on ne troure an contraire jamais de Rhizopodes ayant des flagellums nombreux et bien définis. Les deux autres semblent pouvoir également se soutenir selon que l'on appellera cils ou flagellums les appendices vibratiles de nos animaux, la différence entre les deux n’ayant rien d'absolu. Bürschu penche vers la dernière et voit dans les Catallacles des lufusoires aberrants. Nous ne pourons accepter cette opinion, car on n’a jamais ru d'Infusoires passant par un stade amilie, ni se nourrissant sans bouche par absorption directe de particules solides en un point non différencié de sa surface. Parmi les Flagellés, au contraire, les pseudoporles ne sont point rares et de vraies phases amibes se rencontrent quelquefois (Mastigamoba et autres); le mode d'absorption de la nourriture au stade flagellé isolé est toul à fait caractéristique des Flagellés; enfin, ce n’est pas d'avoir une vingtaine de filaments vibratiles qui peut établir une barrière infranchissable quand on en tronve jusqu'à lıuit chez des Flagellés incontestables (Hexamitus). Ajoutons que la ressemblance de la forme adulte avec celle des. Symura est tout ì fait frappante. Nous en ferons donc une sous-classe des Flagellia ( $\left.{ }^{z}\right)$.

(1) Häckel n'a pas vu l'enkystement des amibes, aussi ne sait-on pas s'il y a entre le kyste et l'amibe quelque nouveau stade ou peut-être une conjugaison. On ne sait pas non plus ce qui détermine l'enkystement. En tout cas ce ne sont pas des kystes d'hivernage, car l'auteur les a vus éclore en septembre.

(1) Les Catallactes n'ont été observés que par Ḧ̈cker et par Parosa qui a décrit un M. Maggii des salines de Cagliari, mais sans rien ajouter d'important aux connaissances antérieures. 


\section{APPENDICE AUX FLAGELLÉS}

En appendice aux Flagellés et comme type intermédiaire entre eux et les Ciliés, nous placerons le genre aberrant:

Maupasia (Cheviakof). Cet ètre a une forme ovö̈de; mais il est fort contractile, allongé quand il nage, sphérique à l'état de contraction. Le quart supérieur du corps est revêtu de cils disposés sans ordre, courbés vers le haut. Le reste du corps est garni de filaments de diamètre uniforme ressemblant plutôt à des flagellums. A l'extrémité inférieure, est un flagellum fort long à la base duquel est un pore permanent qui est l'orifice du court canal excréteur d'une vésicule pulsatile située dans la partie inférieure du corps. Il y a une bouche ventrale supérieure, un court pharynx tubuleux, un noyau ovale d'aspect homogène Eau douce, Havaï).

Par la plupart de ces caractères, cette forme appartient aux Infusoires, mais en raison de ses flagellums et de l'alsence de micronucléus, son auteur le rattache aux Flagellés sans préciser sa position dans le groupe, c’est pourquoi nous la plaçons ici, bien que peut-être elle fùt aussi bien à sa place en appendice aux lnfusoires. 


\section{4e Chasse \\ INEUSOIRES. - INFUSORIA}

[INFUSORIA (Dujardin)]

Les Rhizopodes étaient caractérisés par le pseudopode, les Sporozoaires par l'absence de tout appendice et la continuité absolue de Ieur tégument, les Flagellés par le flagellum. Les Infusoires empruntent aussi au système appendiculaire leur caractère dominant. Ils sont revêtus de cils vibratiles. Un cil vilbratile isolé ne diffère pas d'un flagellum par des caractères bien importants. Il est plus petit, animé d'un mouvement uniforme de va-et-vient au lieu du mouvement conique précédemment décrit, mais il n'est jamais unique sur la cellule; il n'arrive guère non plus qu'on n'en trouve qu'un très petit nombre. Il y avait au plus quatre ou six flagellums chez les Flagellés. Ici, les cils se comptent au moins par dizaines et il y en a d'ordinaire des centaines et des milliers. Chez le Flagellé, les flagellums, même lorsqu'ils étaient multiples, gfardaient toujour's Ieur indépendance. Ici, au contraire, les cils souvent se soudent en organes moteurs plus complexes.

Nous définirons donc provisoirement l'Infusoire, un Protozoaire ayant pour appendices des cils vilbratiles nombreux, simples ou fusionnés en appendices coniques ou en membranes.

Comme presque toujours en pareil cas, notre caractéristique à peine établie se trouve en défaut. 11 existe des Infusoires dépourvus de cils. Comme ils se rattachent aux autres par toutes leurs affinités, nous devons les laisser dans Ie groupe, mais ils nous obligent à subdiviser aussitôt la classe des Infusoires en deux sous-classes:

$C_{I L I F,}$ pourvus de cils vibratiles, et les

TENTACULIE, dépourvus de cils et porteurs de tentacules.

$1^{\text {re }}$ Sous-Cílasse

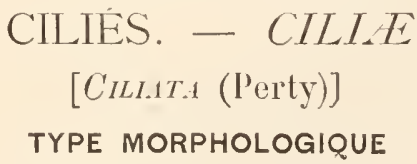

(FIG. 690 A 710 )

\section{Structure.}

Idée générale de l'animal. - Notre Cilié (fig. 690), étant une cellule, a les organes essentiels de toute cellule parfaite: une membrane, un cytoplasme et un appareil nucléaire. Mais Ia cellule est ici extrèmement 
compliquée: il s'est produit en elle des différenciations remarquables qui ont donné naissance à de nombreux organes.

Sa membrane est revètue de cils vilıraliles; elle est percée d'une bouche (b.) et d'un anus et, à la bouche, fait suite un pharynx (ph.). Mais tout le reste du tube digestif manque absolument au moins en tant qu’organe permanent et différencié. Son cytoplasme se divise en deux

Fig. 690 .

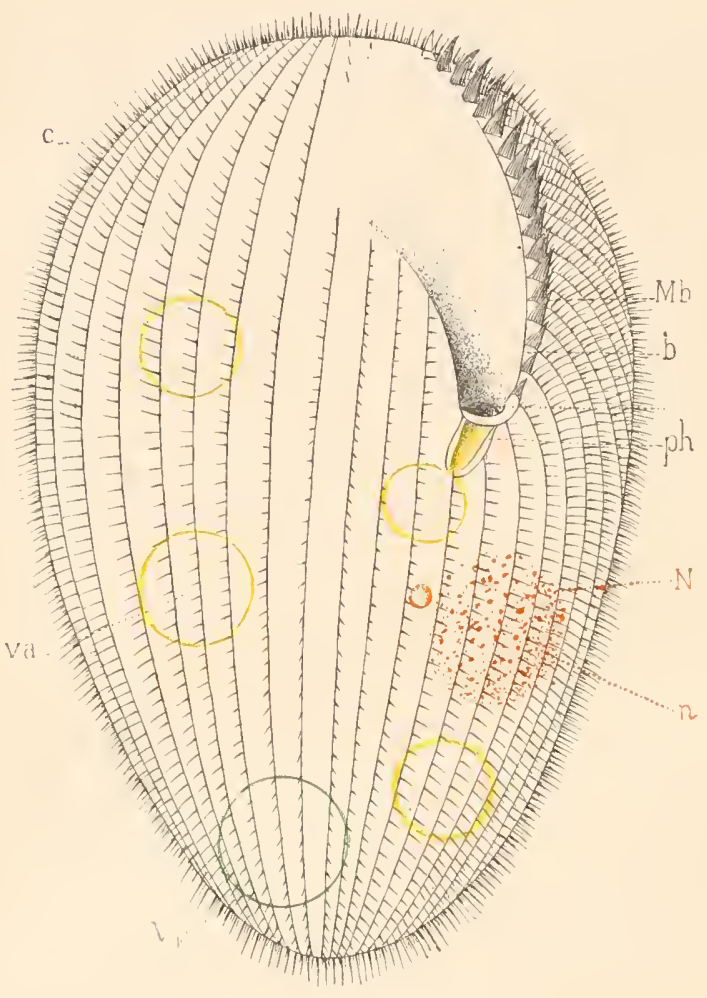

CILIES (Type morphologique) (Sch.).

b., bouche; mb., membranelles: $\mathbf{x}$, macronucléus; $\mathbf{n}$, micronucléus; 1., péristome; ph., pharynx; v. a., vacuole alimentaire; V. p., vésicule pulsıtile; $\boldsymbol{z}$. a., zone adorale. couches (fig. 691) : un ectoplasme (ectop.) très mince, formant avec la membrane un tégument ferme, et un endoplasme (endop.) diffluent qui constilue la plus grande partie de la masse. La couche superficielle de l'endoplasme est cependant assez ferme et mérite d'être distinguée sous le nom de plasma cortical (pl. cort.). Dans celte couche corticale se ramifient des canalicules excrétentrs lacunaires aboutissant à une vésicule pulsatile $(V \cdot p$.$) qui ex-$ pulse rhythmiquement au dehors les liquides excrétés, par l'intermédiaire d'un petit orifice, le pore excréteur. Dans l'endoplasme central, on trouve des vacuoles alimentaires (v.l.), cavités non permanentes qui se forment autour des particules alimentaires pour les digérer et conduire à l'anus

leurs résidus indigestes. On y trouve aussi des granulations diverses représentant, les unes des substances dérivant des aliments, les autres des excreta destinés à être éliminés. Enfin le corps nucléaire (fig. 690, $N$ et $n$ ) préscute ici un caractère lout à fait marquant. Tandis que, dans les cellules ordinaires, le noyau unique préside à la fois aux phénomines végélatifs et à la division de la cellule, ici ces deux fonctions se sont dissociées. Le noyau u'a point de centrosome, mais il s'est dédoublé en deux parties juxtaposées: chargée l'une des fonctions végétalives, le mucronucléus $(N)$; l'autre affectée à la reproduction, le micronucléus $(n)$. 
Maintenant que nous avons une idée générale de l'animal et de ses organes, nous allons reprendre une à une ses diffẻrentes parties pour les examiner en délail.

Extérieur. - Notre Cilié est un petit être de quelques dixièmes de millimètres (fig. 690), difficilement visible à l'œil nu dans l'eau où il se meut avec vivacité.

Sa forme est ovö̈le, variable et fixe à la fois : variable en ce qu'il est très contractile et se contourne, s'étend, se renfle de mille facons; fixe par le fait qu'au repos il reprend toujours le mème profil. Cela tient à ce qu’il est renfermé dans un tégument ferme et élastique à la fois (').

Quand il nage, il roule sur son axe, n’ayant de fixe que la direction de cet axe dont les extrémilés sont l'une en avant, l'autre en arrière. Mais parfois il rampe sur le sol et se tient alors sur une face un peu plus aplalie que l'opposée et qui se détermine par là comme ventrale. Ainsi se trouve fixée l'orientation morphologique de l'animal. Comme toujours, nous appellerons supérieure l'extrémité qui est en avant dans la progression el antérieure la face ventrale.

Sur la moitié supérieure de la face ventrale et non loin du bord gauche, se voit une dépression lonFig. 691 .

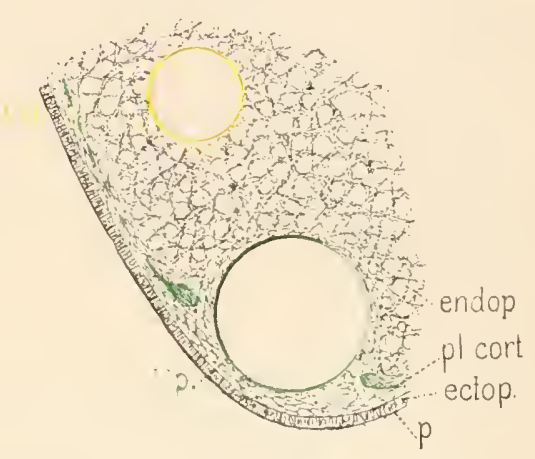

CiLiés (Type morphologique). Organisation interne. (Sch.)

ectop., ectoplasme; endop., endoplasme; p., membrane; pl. cort., plasme cortical; v. a.., vacuole allimentaire; V. p., vésicule pulsatile. gitudinale que l'on appelle le péristome $(P$.$) . Le péristome n'entame pas$ la continuité du tégument, c’est un simple enfoncement, une partie en contre-bas de la surface générale. 11 peut ètre décrit comme une goutlière qui, large et peu profonde en haut, près du bord supérieur et frontal, descend sur le côté gauche de la face ventrale en ilevenant de plus en plus creuse et étroite. Son bord gauche est nettement dessiné et taillé à pic $(Z . a ., f i g .690)$; le droil, au contraire, se perd invisiblement sur la surface générale. Sa direction exacte n’est ni rectiligne ni longitudinale, mais oblique et légèrement spirale: sa partie supérieure s’incurve vers la droite et l'on comprend que s'il continuait ainsi, il arriverait à former une courbe fermée plus ou moins arrondie $\left(^{(2)}\right.$.

(1) La forme des Ciliés est extraordinairement variée; mais l'ovoïde paraît être la forme simple d'où les autres sont dérivécs.

2) Beaucoup de Ciliés n'ont pas de péristome, mais quand il y en a un, cette forme est caractéristique. On peut faire dériver d'elle toutes les formes si variées que nous rencontrerons en étudiant la classification.

Le péristome des Stentor's lui-même cntre dans cette léfinition si l'on admet, comme on le doit, arec Schuberg, que chez ces animaux, la surface entourée par la 
A l'extrémité inférieure du péristome la dépression se transforme en un canal qui plonge assez brusquement dans les parties centrales du corps et, après un certain trajet, s'arrète brusquement, laissant l'endoplasme à nu au fond de sa cavité. Ce canal est le pharynx (ph.), son orifice d'entrée au fond du péristome est la bouche (b.).

La bouche est largement ouverte et toujours béante. Sa forme est ovalaire. Le pharynx a la forme d'un entonnoir allongé. Il est incliné obliquement en bas, en arrière el à droite, comme s'il se dirigeait vers le centre du corps. La bouche n'est donc point un orifice percé dans la paroi du corps, mais résulte, comme le pharynx, d'une invagination de la paroi arec toutes ses couches: membrane, ectoplasme et plasma cortical, et c'est seulement au fond lu pharynx que la paroi est vraiment percée et met à nu l'endoplasme (").

L'anus est beaucoup plus simple. C'est une simple incision traversant toute l'épaisseur des téguments, mais dont les bords sont exactement juxtaposés; et comme il n'y a aucune différenciation de son pourtour, on ne le voit qu'au moment où il s'entr'ouvre pour l'expulsion des fèces. Sa place normale est à la partie niarginale inférieure de la face dorsale $\left({ }^{2}\right)$.

Rappelons pour n’y plus revenir qu'entre l'anus et le fond du pharynx, il n'y a aucun tube digestif défini.

L'orifice excréteur est un tout petit pore arrondi, permanent, mais

zone adorale n'est pas le péristome vrai, mais un pseudostome, on pourrait dire plutit aire péristomienne ou champ frontal, homologue au front des Hypotrichides, et que le vrai péristome n'est que la partie qui avoisine le vestibule buceal. Il y aurait lieu, d'après cela, de distinguer toujours le péristome proprement dit et l'aire péristomienne, ou aire circonscrite par la zone adorale (V. plus loin). Mais d'ordinaire, nous nous contenterons, avec la plupart des auteurs, du mol péristome, le sens de la phrase indiquant suffisamment dans quelle acception il faut le comprendre.

(1) Cetle position de la bouche et du péristome est très fréquente, mais elle est sujette à des variations fort étendues fig. 692. Depuis l'exlrémité supérieure

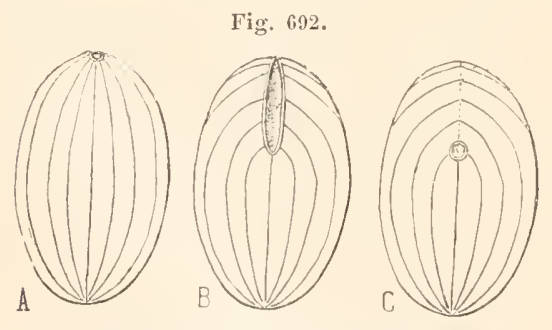

CILlÉs (Type morphologique).

Positions suceessives de la bouche (im. Bütschli). jusqu’à l'inférieure, il n'y a pas de point de la face ventrale que la bouche ne puisse occuper. Sa situation primitive $(A)$ semble être l'extrémité supérieure. l'our comprendre son déplacement il faut se représenter qu'elle s'est d'abord transformée en une fente s'étendant sur la face ventrale sans cesser d'avoir son origine au pôle supérieur $(B)$; puis que la partie de cette fente supérieure s'est fermée, suturée, laissant à sa place un raphé $(C)$. Cette soudure est démontrée par le fait que les lignes d'implantation des eils, au lieu de passer ininterrompues à ce niveau viennent buter lì, sans se continuer d'un còté à l'autre.

(2) C'est, en effet, sa position la plus habiluelle, mais on peut le rencontrer à peu près partout; souvent il est placé à la face ventrale ou même auprès de la bouclie Vorlicelles, Stentors;. 
difficile à voir, qui sert à l'expulsion du liquide de la vésicule pulsatile. Il vaudrait micux dire que c'est un court canal, traversant comme l'anus les deux couches légumentaires sans aller jusqu'à la vésicule elle-mème qui en reste séparée par une mince couche de plasma corlical, sauf au moment où elle se vide. Sa place est à l'extrémité inférieure, au voisinage de l'anus (').

La surface entière du corps est revètue de cils vibratiles (fig. 690) disposés le long de lignes longitudinales régulières. Ces lignes sont marquées par de fines stries dessinées sur la membrane. Ces stries suivent, comme direction générale, les méridiens de l’ovö̈de. Mais elles ont une tendance à se contourner en hélice très allongée el celles qui passent au voisinage du bord gauche du péristome se détournent pour plonger dans celle gouttière perpendiculairement à sa direction. Les cils d'une mème rangée sont plus rapprochés les uns des autres qu’ils ne sont de ceux des rangées voisines $\left(^{*}\right)$.

Les cils sont implantés obliquement, inclinés vers la partie inférieure qui est en arrière tans la progression. A leur base, ils traversent la membrane el se continuent arec la substance solide de l'ectoplasme dont ils sont une émanation.

Le long du bord gauche du péristome, les cils sont remplacés par des membranelles (mb.), sorte delamelles triangulaires, disposées transversalement etinsérées les unes au-dessous des autres. Leur ensemble forme ce que l'on a appelé la zone adorale( $Z$.a.).

Les membranelles ( $m b$.) ne s'insèrent pas seulement sur la membrane; elles la traversent (fig. 693) ainsi que l'ecloplasme et arrivent jusqu'ì l'endoplasme

Fig. 693.

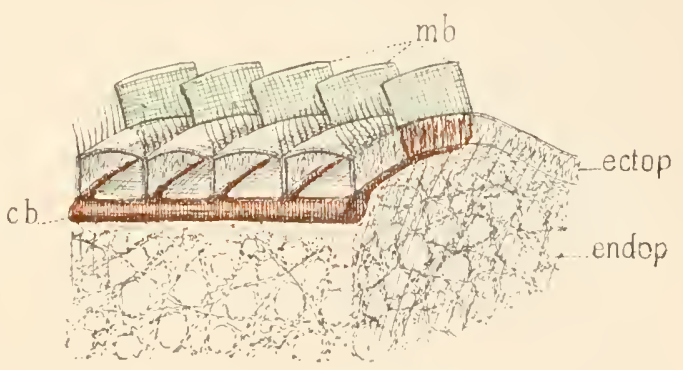

CILIÉs (Type morphologique). Constitution de la zone adorale (Sch.).

ch., appareil basal des membranelles; ectop., ectoplasme; endop., endoplasme; mo., membrandles formies par une portion des bandes ciliaires dont les cils sont agglutinés.

par une lame continue (Saum des auteurs allemands). Elles sont animées d'un mouvement monolone très vif qui a pour effet de faire tourbillonner l'eau et de la diriger vers le péristome el la bouche avec les particules élémentaires qu'elle contient $\left(^{\mathbf{3}}\right)$. Elles sont formées de cils

(1) Sa position est très variable aussi. Il est d'ordinaire terminal postérieur, plus souvent dorsal que ventral, parfois situé avec l'anus non loin de la bouche (Vorticelles, Stentors!.

(2) Ce n'est là, bien entendu, qu'une disposition fréquente. L'arrangement et la répartition sont sujets à des variations très considérables que nous indiquerons en ètudiant la classification.

(3) Les membranelles ne sont pas universelles. Les Ciliés de l'ordre des Holo- 
agghlutinés et que l'on peut séparer au moyen de réactifs convenables. Sur la face dorsale du corps, se voient, entremêlés aux cils ordinaires, des sortes de cils plus longs, raides, immobiles et qui servent, norì à la locomotion, mais au toucher. Ce sont les soies tactiles, simples cils modifiés et adaptés à une fonction spéciale (').

Cytoplasma. - Nous avons vu que le cytoplasma se compose de deux couches, une extérieure l'ectoplasme, l'autre intérieure l'endoplasme. Pareille chose existait chez beaucoup d'autres Protozoaires. Mais il n'y a de semblable que les noms, car l'ectoplasme est ici bien différent de ce qu'il était ailleurs. Toutes les couclies du cytoplasme (fig. 691) ont la mème constitution; elles ne diffèrent qu'en ceci, que la structure est plus fine, plus serrée, dans l'ectoplasme (ectop.) que dans l'endoplasme (endop.), en sorte que les déplacements étendus, habituels chez celui-ci, sont interdits à celui-là. L'endoplasme est très épais et forme la presque totalité de la masse du corps, tandis que l'ectoplasme forme une très mince couche superficielle qui double la membrane.

Endoplasma. - L'endoplasme (endop.) a la structure du cytoplasma des cellules ordinaires. Il est composé de deux substances, une relativement ferme dessinant un réseau continu, le hyaloplasma, l'autre à peu près liquide, le paraplasma, oceupant les mailles de la première. On y trouve aussi, comme toujours, l'innombrables petites granulations, les microsomes. En outre de ces éléments qui sont ceux de sa structure intime, on trouve dans sa substance de nombreuses vacuoles remplies de liquide. Ces vacuoles n'ont aucune constance dans leur taille ni dans leur répartition et varient sans cesse chez un mème indivilu. C'est daus la substance interposée entre elles que se troure les deux plasmas élé-

trichides n'en possèdent pas et chez beaucoup de genres dans les autres ordres elles sont aussi remplacées par de simples cils. Mais toujours les cils de la zone adorale sont plus longs que ceux du voisinage. On en trouve aussi parfois en d'autres endroits du corps que la zone adorale (couronne ciliaire des Vorticelles). Leur disposition sur le prolongement des stries ciliaires aboutissant au bord gauche du péristome est à remar'puer, leurs lignes d'insertions continuent chacune une strie ciliaire. Que l'on suppose les cils plantés beaucoup plus dru le long de ces lignes et ces cils soudés entre eux par leurs bords contigus etl'on aura les membranelles (fig. 693). Souvent les membranelles sont déchiquetées au bord libre; elles prennent alors le nom de pectinelles.

(1) Le fait que ees soies tactiles sont des cils modifiés est bien démontrẻ par l'observation du Stentor. On voit, chez cet Infusoire, les soies disparaitre brusifuement comme si elles rentraient dans le corps, puis reparaître non moins brusquement it une place voisine comme si elles étaient ressorties; et longtemps on a cru qu'elles exécutaient véritablement ces mouvements. Mais Johsson [93] a reconnu que c'est là une illusion due simplement à ce que, brusquement, à une place donnée, un cil cesse de vibrer, se raidit el fonctionne quelque temps comme soie tactile. Puis, brusquement, il se remet à vibrer comme ses voisins dont rien ne le distingue plus à partir de ce moment, tandis qu'à une autre place, un autre cil assume à son lour, pour un moment, ce ròle de soie tactile. Ici, soies et cils sont de mème longueur, et la différenciation des premiers n'est que temporaire, mais ordinairement les soies sont plus longues que les cils et leur différenciation est permanente. 
mentaires, hyaloplasma et paraplasma. On peut les considérer comme de rastes accumulations de paraplasma ou mème d'une sulistance encore plus liquide el moins essentielle (').

Ectoplasma. - L'ectoplasme (ectop.) a tout à fail la mème structure que l'endoplasme, mais les mailles y sont beaucoup plus étroites, plus serrées et disposées d'une façon plus régulière, perpendiculairement à la surface, souvent sur une seule rangée; en sorte qu'il a l'aspect d'une mince hande striée sous-jacente à la membrane $(p$.$) .$

Plasma cortical. - Entre l'ectoplasme el l'endoplasme, se trouve une couche dite de plasma cortical $(p l$. cort.) à structure intermédiaire, assez serrée pour ne pas prendre part au mouvement de cyclose et rester fixe sous l'ectoplasme, mais se rapprochant cependant de l'endoplasme par l'irrégularité de ses vacuoles el sa transition graduelle avec celui-ci, tandis que sa limite extérieure est neltement indiquée $\left({ }^{2}\right)$.

Membrane. - La membrane, souvent appelée cuticule ou encore pellicule $(p$.$) , est située à la surface externe de l'ectoplasme; elle est extrè-$ mement mince et anhiste, elle représente la membrane protoplasmique des cellules ordinaires : elle n'est point en effet une sécrétion cuticulaire distincte des substances protoplasmiques sous-jacentes, elle est simplement formée par la condensation de la couche la plus superficielle de l'ectoplasme, comme la couche cornée de l'épiderme est formée par la condensation des assises superficielles de celui-ci $\left(^{3}\right)$. Elle est ornée de stries longitudinales et un peu en hélice correspondant aux bases d'implantation des cils, comme nous l'avons vu plus haut (').

l'ectoplasme, uni à la membrane, forme une très mince couche superficielle it laquelle on peut donner le nom de tégument de l'Infusoire, car c'est elle, en effel, et non la membrane seule qui forme l'enveloppe dans laquelle est contenu l'endoplasma diffluent (").

(1) Au sujet de la structure intime de l'endoplasme se reproduisent ici les mêmes discussions qu'au sujet du cyloplasme en génèral ( $r$. p. 6 , nòte). Bütschli y voit de minuscules vacuoles, les al'réoles, à parois continues remplies d'un liquide qu'il appelle enchylema. Fabre-Domergue y voit un réseau de filaments contractiles qui rappelle la structure réticulée el les fibrilles de Fleumaxa. Enfin, récemment, Marian Przesmyck [9'] a retrouvé phez les Infusoires les granules de Altmans, non seulement dans l'ectoplasme, mais dans tous les organes, même les trichocystes.

(2) Les auteurs rattachent le plasma cortical, les uns i l'ectoplasme, les autres it l'endoplasme. La première opinion semble plus naturelle. Mais cela est une chose jurement conventionnelle.

(3) On peut se faire une juste idée du degré d'indépendance de cetle pellicule en se la représentant avec Bütschli (e'est hui qui lui donne ce nom) comme formée par l'ensemble des parois externes des alvéoles superfieicls, épaissis et soudés en une membrane continue.

(") Quand Ies cils disparaissent la striation disparait aussi. Minsi les Hypotrichides ne sont striés qu’à la face ventrale.

(5) Le plasma cortical donne asile aux trichocystes quand il y en a, mais comme ces appareils ne se rencontrent presqueque chez les Holotrichides (et encore pas chez tous), nous les décrirons seulement à propos de cet ordre (V. 1. 432). 
Inclusions du cytoplasme. - Dans l'ectoplasme, on ne trouve point d'inclusions proprement dites. car on ne peut lonner ce nom aux microsomes qui, ici comme partout, font partie de la structure intime du protoplasma ("). Nais dans l'endoplasme, on trouve des vacuoles alimentaires et des grains d'assimilation ou d'excrétion, etc.

Les aliments, en effet, ne sont point en contact avec l'endoplasme qui les renferme. Ils sont contenus dans de petits espaces sphériques entièrement clos et remplis de liquide qui sont les vacuoles alimentaires (fig. $690, v . a$. .). On trouve ces vacuoles en nombre trìs variable, éparses dans tous les points de l'endoplasme. Les aliments s'y trouvent à tous les états et celles où la digestion est le plus avancée méritent le nom de vacuoles à fèces.

Par contre, les produits de l'assimilation ne sont point contenus dans des racuoles; ils sont directement inclus dans l'endoplasme où ils constituent les grains d'assimilation (fig. 691). Ils sont formés d'une substance ternaire voisine le l'amidon et du glycogène que Maupas a appelée le zooamylum. Peut-ètre quelques-uns sont-ils de nature graisseuse. Enfin, les produits destinés à être excrétés se montrent inclus aussi directement dans l'endoplasme, sous la forme de grains d'excrétion de couleur grise ou verlàtre, plus connus ici, en raison de leur aspect optique, sous le nom de granules biréfringents. On a pensé qu'ils étaient formés de xanthine, de guanine, d'acide urique, il semble qu'ils ont plutôt pour base de l'urate de soude ou du phosphate bicalcique $\left(\mathrm{PO}^{4}\right)^{2} \mathrm{Ca}^{2} \mathrm{H}^{2}\left(^{2}\right)$.

(1) On trouve aussi quelquefois dans l'ectoplasme du pigment de couleurs variées, par exemple, le pigment bleu de stentor coruleus. Les myonemes appartiennent à l'ectoplasme mais, en raison de leur absence fréquente, nous n'avons pas cru devoir les introduire dans le schéma général de l'Infusoire. Leur description sera mieux placéeá l'occasion du stentor et des Torticelles, car en dehors des Péritrichides et Itétérotrichides, on n'en trouve guère que chez quelques rares Holotrichides (Holophrya, Prorodon) et chez certains Opalina. Il en est de même des trichocy.stes situés à moitié dans l'endoplasme, à moitié dans le plasma cortical. Nous les étudierons en parlant des Ilolotrichides.

(2) C'est Maupas qui admet l'urate de soude et Cheviakof qui semble avoir démontré le phosphate de chaux. Les grains d'excrétion ne se trouvent pas chez tous les Infusoires. Cela est moins étonnant qu'il ne semble au premier abord car, si les produits de dénutrition ne peuvent manquer, rien n'exige qu'ils revètent la forme solide.

On trouve assez souvent des Zoochlorelles immobiles dans le plasma cortical où parfois tombées dans l'endoplasme et entraînées dans sa cyclose. Mais elles y sont en qualité de parasites, parfois utiles sans doute, mais jamais nécessaires car, la plupart du temps, leur nombre, leur présence même, sont variables selon les individus. Maupas a vu que les Ciliés qui en possèdent normalement (Paramrcium lnursaria) pouvaient accomplir à l'obscurité tous les actes de leur évolution. Cependant ce mème Paramsecium, qui d'ordinaire recherche l'obscurité, va au contraire à la lumière lorsqu'il manque d'aliments. C'est sans doute pour tirer parti de l'amidon qui forment ses Zoochlorelles. Ces Algues sont mangées par l'lnfusoire et il se trouve qu'elles peuvent vivre dans son plasma au lieu d'être digérées par lui. On y trouve 
Vésicule pulsatile et canaux excréteurs. - Immédiatement sous l'ectoplasme, au niveau du pore excréteur, se troure une volumineuse vésicule (fig. 69l et fig. 69', I.p.) qui se contracte rhythmiquement pour expulser par ce pore le liquide qui s'amasse lentement dans sa cavité. Cette vésicule est contenue dans la couche du protoplasma cortical. Mais, comme son diamètre est très supérieur à l'épaisseur de cette couche, elle la soulève et fait fortement saillie dans l'endoplasme, sans cesser pour cela d'ètre revètue de plasma cortical.

Le liquide dont elle se remplit après s'ètre vidée lui arrive de tous les points du corps par un réseau de voies lacunaires creusées dans le plasma cortical (fig. 691). Ces roies dessinent, dans toute l'étendue de la couche sous-tégumentaire, un réseau de canalicules excréteurs (fig. 694, t.). Au voisinage de la vésicule, les branches de re réseau se groupent en quelques canalicules afférents volumineux qui convergent vers elle et se termine à son contact par autant de dilatations disposées en cercle autour d'elle (v.s.). Nous ne pourrons acquérir une illée complète de la constitution de cet appareil qu'en étudiant son fonctionnement au chapitre le la physiologie. Mais notons bien pour le moment que, dans tout ce système de canaux et de vésicules, nulle part il n'y a de parois propres ni même de fixité absolue lans la situation des parties. Des trajets peuvent se former en certains points et disparaitre complètement par soudure des parois, et d'autres s'ourrir dans le voisinage par Fig. 69'.

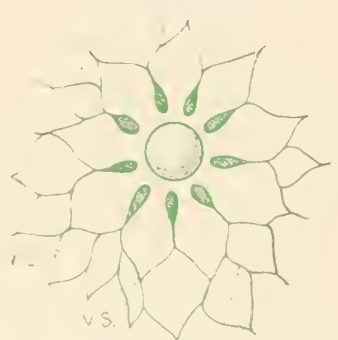

CILIÉs Type morphologique). Appareil excréteur vu de face dans la région de la risicule pulsatile. (Sch.).

t., trajets canaliculaires; v. p., vésicule pulsatile; v. s., vésicules formatrices.

simple écartement des parties, à la manière d'une bulle gazeuse qui se trace un chemin dans une substance sirupeuse, sans laisser lerrière elle aucune trace des voies qu'elle a parcourues. C'est ainsi que la communication de la vésicule pulsatile $\left(I^{\prime} . p\right.$.) avec les dilatations terminales du réseau ou arec le pore excréteur, n’élant pas permanente, s'établit chaque fois à nouveau, sans que l'on puisse dire que chaque communication nouvelle soit la réouverture de la communication précédente. Il n’y a d'absolument fixe que le pore excréteur, il vaudrait mieux dire le canal excréteur, qui traverse la membrane et l'ectoplasme, mais vient buter au fond contre le plasma cortical qui forme la paroi de la vésicule pulsatile, sans communiquer avec elle d'une manière permanente (').

aussi assez souvent divers parasites vrais: Bactéries, Chytridinées, parfois même des Infusoires acinètes appartenant aux genres spherophrya et Endosphxra.

(1) La vésicule pulsatile et les dilatations terminales du réseau sont toujours de taille à se voir aisément. Mais le réseau lui-même est si délicat qu'il faut, pour le reconnaître, un examen minutieux et de forts grossissements. FABRE-DONERGUE [87] a démontré son existence constance. L'aspect et la disposition des dilatations terminales des canalicules afférents sont extrèmement variables. D'ordinaire, ce sont de 
Corps nucléaire. - Le corps nucléaire se compose de deux noyaux le taille et le significalion différentes, situés côte à côle daus l'endoplasme, le macronucléus que nous désignons par la lettre $\mathrm{N}$ et le micronucléus que nous appellerons n (').

Macronucléus. - Le $\mathrm{N}$ a l'aspect ordinaire d'un noyau de cellule, mais il n'en a point la structure. Il est volumineux, de forme ronde ou allongée, immobile au sein de l'endoplasme (fig. 690, $N$ ). Il est muni d'une paroi et contient une substance d'apparence homogène, dans laquelle cependant on arrive à découvrir une vague indication d'un réseau chromatique el de granulations chromaliques plongées dans une substance colorable; mais on n'y trouve ni nucléole, ni chromosomes à aucun moment $\left({ }^{2}\right)$.

simples vacuoles irrégulières dont le diamètre varie sans cesse selon les états de systole ou de diastole, et qui dessinent une couronne autour de la vésicule pulsatile; on les nomme alors résicules formatrices (Ex.: Colpidium, Nictotherus, Prorodon, etc., ete.); ailleurs, elles sont plus fixes, plus régulières et se présentent comme autant de dilatations piriformes terminant chacune un canal excréteur facile à voir sur une certaine étendue et disposées autour de la vésicule comme les rayons d'une étoile. On pourrait les nommer résicales afférentes. Paranacinm avec sa double vésicule est un type bien connu de cette disposition. Ailleurs enfin, elles forment de longs canaux permanents dont le nombre et la disposition sont des plus divers. Chez stentor, il en est de même, moins le canal. Chez les Oxytrichiues, il y a deux canaux, un descendant et un ascendant qui va faire le tour du péristome; chez spirostomum, Climacostomum, il y a denx canaux ascendants; chez Urocentrum, quatre canaux dorso-ventraux et deux latéraux ascendants; chez ophryoglena, il y a jusqu’a trente canaux ramifiés, etc. Nous signalerons, en étudiant la classification, les dispositions les plus remarquables. Lorsqu'il y a des trichocystes, c'est en dedans d'eux que se trouve le système excréteur.

Les vésicules pulsatiles ne manquent que très exceptionnellement chez les Ciliés. Les seuls genres où on ne les ait pas trouvées sont les suivants: Opalina (toutes les espèces), Actinotrocha (1. saltans), Gonostomum (G. pediculiforme), IIolosticha (1I. Lacazei), Uronichia (U. transfuga), Diophrys (D. appendiculatus) et peut-être Strombidium (S. sulcatum et $S$. urceolare). Nais l'appareil excréteur ne manque pas pour cela, le réseau existe toujours. Fabre-Domeroue a reconnu son existence chez les Opalines en particulier, mais il n'a pu reconnaitre ni si ni comment il communiquait avec le dehors.

(1) Ces deux noyaux ont reçu bien des noms différents. On les a d'abord appelés noyau et nuclíole, dénominations à rejeter parce qu'ils n'ont ni la situation relative, ni la slructure, ni la signification physiologique du noyau et du nucléole des cellules ordinaires. Ituxley a proposé pour eux les noms d'endoplaste et endoplastule. On les a nommés aussi noyau principal et noyau accessoire, noyau secondaire et noyu primaire, noyan femelle et noyu mále, etc.

(2) La forme fondamentale est sphérique, mais elle peut s'allonger, soit en ovoïde, soit en bâtonnet rectiligne ou contourné, et ce bàtonnet peut même se scinder en fragments arrondis réunis par des tractus formés par la membrane seule, de manière ¿ prendre la forme d'un chapelet (stentor). Tout cela n’a aucune fixité et rarie d'une espèce il l'autre dans le même genre, ni aucune importance réelle et résulte de la nécessité de porter parlout l'influence du $\mathbf{N}$. Chez un Infusoire à corps très allongé, un $\mathbf{N}$ sphérique ne pourrait suffire à étendre l'influence nucléaire dans les parties éloignées du cytoplasma.

Le $\mathbf{N}$ montre dans divers genres des particularités de structure singulières 
Micronucléus. - Le n est extrèmement petit, mesurant au plus 3 à 4 : \%.. Il est sphérique et situé tout contre lo $\mathbf{N}$ ( $(i g .690, n)$. Il est muni a'une membrane et l'intérieur ne laisse apercevoir, et fort difficilement, qu'un semis de granulation plongées dans une substance plus claire (").

(fig. 695). Ce sont tantôt des condensations locales de substance, tantòt des inclusions rappelant le nucléole et qui ont reçu ce nom (Chilodon)(.1), ou bien il a l'air formé de deux masses accolées (Spirochona) (C). Ailleurs et plus souvent (Chlamydodon, D)steria, Nassula, Prorodon, Tionotus, stromlidinm et la plupart des Oxytrichines , on observe ce qu'on a appelé les noyanx it cloison ou noyaux it fente $(B)$. Dans le premier cas, le $\mathbf{N}$ est travelsé par une ligne sombre diamétrale, expression optique d'une séparation ėquatoriale entre deux moitiés hémisphẻriques; dans le second, cette séparation au lieu d'être représentée par un plan sans épaisseur, devient un espace rẻel lenticulaire dont la coupe optique est une ellipse qui se détache en clair et donne l'impression d'une fente de même forme (fig. 69:3, $B$ ). Toutes ces dispositions sont restées jusqu'à ces derniers temps complètement énigmatiques et il semblait qu'elles n'eussent pas grande

Fig. 695.

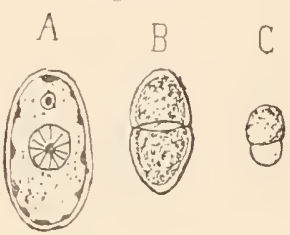

CILIÉS

Quelques formes du noyau (iim. Biilschli). importance, car elles se rencontraient ou manquaient dans les diverses espèces d'un mème genre. Mais un jour tout nouveau rient d'être jeté sur cette question par un récent travail de Balbians [95]. Cet observateur a trouré que, chez spirochona, l'apparence de cloison ou de fente résulte de ce que le $\mathrm{N}$ est formé de deux parties accolées représentant l'une la chromatine, l'autre la substance achromatique du Fig. 696. noyau (fig. 696. A). Ces sulistances affectent à divers moments les rapports les plus variés (B). Nime, un globule de substance chromatique peut s'isoler au centre de la
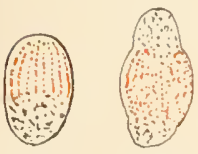

A

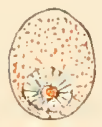

C

spirochona. Noyau (d'ap. Balbiani). substance acliromatique $C$ ) où il représente un nucléole ou plutôt un centrosome intranucléaire, jouant le mème ròle par rapport á la substance achromatique que le centrosome polaire des cellules ordinaires. Il est infiniment probable que ces observations peuvent ètre généralisées et que partout il $\mathbf{y}$ a dans le $\mathbf{N}$ des substances chromatiques et achromatiques qui, d'ordinaire, restent mélangées et, dans certains cas, forment des masses simplement juxtaposées ou incluses l'une dans l'autre.

(1) Bien que silué dans l'endoplasme, le $\mathbf{N}$, ne participe pas á la cyclose ou n'est agité que de faibles mouvements. Cela s'explique suffisamment par le seul fait que sa masse est trop forte pour obẻir it une force aussi faible. En outre, dans certains cas, ses extrémilès, lorsqu'il est très long, sont enchâssẻes dans le plasma cortical immobile. Enfin parfois (Zootricha), il est fixé par des sortes de tractus qui ront de sa surface au plasma cortical ambiant.

Normalement, il n'y a qu'un $\mathbf{N}$ et qu'un $n$. Mais assez fréquemment, on rencontre des exceptions à cetle règle. Lor'sque le $\mathbf{N}$ est très grand, il y a souvent de nombreux $n$. Ainsi chez stentor, on en trouve plusieurs associés à chacun des grains du $\mathbf{N}$ en chapelet. Loxodes rostrum a plusieurs $\mathrm{N}$ distincts à chacun desquels sont associes plusieurs $\mathbf{n}$. On est certain ici que ces $\mathbf{N}$ sont vraiment distincts, mais souvent il est difficile de distinguer un noyau en chapelet à grains réunis par des filaments très longs et très fins d'un $\mathbf{N}$ vraiment multiple. On a un bon critérium dans ce fait qu'au moment de la division. les $\mathbf{N}$ en cliapelet $(\mathrm{fig} .697, \mathcal{A}$ ) se condensent en une seule masse sphẻrique $(D)$, tandis que les $\mathbf{N}$ vraiment multiples restent indépendants. Il arrive parfois que l'on trouve un ou plusieurs $\mathbf{N}$ et pas de $\mathbf{n}$ et l'on interprete ces noyaux 


\section{Physiologie.}

Voyons maintenant comment, avec cette structure, notre Cilié va accomplir les diverses fonctions nécessaires à son existence.

Locomotion. - L'animal nage au moyen de ses cils qui battent l'eau d'un mouvement uniforme. Il s'avance, l'extrémité supérieure en avant, en tournant autour de son axe. Mais lorsqu'il se déplace en suivant une paroi, il se meut sur la face buccale sans tourner. Toujours en quête de nourriture, il est sans cesse en mouvement, ne s'arrêtant ni jour ni nuit. Cependant il peut arrèter le mouvement de ces cils et la distinction qu'on a voulu établir entre le flagellum soumis à la volonté el le cil automatique n’est pas absolue. Le Cilié peut rester immolile, parfois nager à reculons; il peut mème arrêter le mouvement de ses membranelles, mais cela est rare (").

La propulsion en avant est due sans doute à ce que les cils frappent l'eau plus énergiquement en se portant en arrière qu'en reprenant leur position en avant.

Contractions du corps. - L’animal peut se contracter, s`infléchir de côté et d'autre, gràce sans doute à une contractilité liffuse de son ectoplasma ou de son plasma cortical. Cela lui permet de s'insinuer dans les passages les plus contournés $\left({ }^{2}\right)$.

uniques ou multiples comme représentant un ou des $\mathrm{N}$. Nais cette interprétation n'est

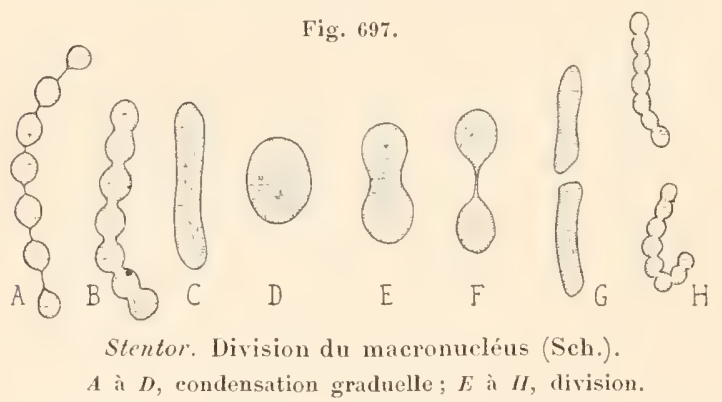
pas légitime. Il est plus naturel de penser que, dans ces cas, ils sont de vrais noyaux complets représentant $\mathbf{N}+\mathbf{n}$ non dissociès. Ce qui tend à le prourer, c'est qu'alors la division ressemble fort peu à celle des $\mathbf{n}$ ordinaires, pas du tout à celle des $\mathbf{N}$, et rappelle de très près celle des noyaux complets des cellules ordinaires (fig. 698). On a un exemple de ce fait dans les Opalines qui, à l'état jeune, ont un seul noyan et. ì l'état adulte, en ont de nombreux, tous identiques entre eux. Ces noyaux possèdent des nucléoles et, en se divisant, montrent de véritables chromosomes arec anses jumelles, plaque nucléaire, etc., etc..

(1) Cela dépend des sortes de Ciliés; ceux qui ont une ciliation uniforme (IIolotrichides) s'arrêtent peu, ceux au contraire qui ont des cirres it la face rentrale (IIy potrichides) s'arrîtent souvent et se meurent touFig. 698. jour's sur cette face lorsqu'ils marchent sur un sol au lieu de nager en pleine eau.

(2) Cette contractilité diffuse est indéniable, car beaucoup d'IIolotrichides et d'tlypo- 
Cyclose. - A l'intérieur du corps, l'endoplasma est perpéluellement entrainé dans un lent mouvement rotatoire auquel on a donné le nom de cyclose et qui rappelle le mouvement analogue que montre le protoplasma des cellules végétales. La cyclose ne s’arrète jamais. Le mouvement part de la bouche, se dirige d'abord vers le bas, puis se porte, en suivant le centre du corps, vers sa partie supérieure, l’atteint, et redescend alors de tous les côtés, le long des parois, en convergeant ver's l'anus d'où il revient vers le point de départ. Seul l'ectoplasme y prend part, l'endoplasme et le plasma cortical restent immobiles.

Alimentation. - Les membranelles qui entourent la bouche (fig. 690, $m l$.$) sont toujours en mouvement. Moins encore que les cils du corps,$ elles ne prennent de repos. Mème quand l'Infusoire est arrèté, sa zone adorale est en action. En lsattant l'eau, les membranelles déterminent un tourbillonnement actif qui entraine vers la bouche $(b$.$) tous les corps qui$ viennent à passer dans sa sphère d'action. Ces particules sont précipitées dans la déjression péristomienne $(P$.) et la parcourent de haut en bas pour arriver à la bouche. Là, elles traversent le pharynx $\left(p h_{\text {. }}\right.$ ) el arrivent en contact arec l'endoplasme qui en occupe le fond. Sous la pression de l'eau poussée par les meml,ranclles en mème lemps que les particules alimentaires, l'endoplasme est refoulé el, au fond du pharynx, se forme une goutte, dans laquelle sont contenues les particules alimentaires. La goutte grossit lentement à mesure que de nouvelles yuantités d'eau arrivent et, à un moment, lorsqu'elle est assez grosse, on la voit s'ébranler sous la poussée de la cyclose de l'endoplasme où elle plonge, et finalement se délacher. Elle est ainsi transformée en une vacuole alimentaire (v.a.) qui s'éloigne lentement du pharynx, pendant qu'une nouvelle goulte commence a se former.

Aiusi sont introduites peu à peu dans le corps des vacuoles alimentaires. Quand l'alinentation est active, le corps est bourré de ces vacuoles qui circulent sans cesse, participant au mourement de cyclose de l'endoplasme où elles sont plongées.

Digestion. - Le liquide de la vacuole est d'abord simplement de l'eau. Mais des échanges osmotiques, avec le liquide plasmatique le transforment peu à peu en une linueur acide apte à digérer les particules incluses. Celles-ci sont en effet lentement dissoutes el réduites à un résidu fécal, tandis que les substances nutrilives dissoutes repassent dans le cyloplasme avec la majeure partie du liquide vacuolaire (').

trichides sont de contractiles sans posséder myonèmes. Mais dans les Ciliés munis de myonèmes, la contractilité ayant un organe différencié devient plus facile et plus énergique. Les Stentor et les Vorticelles en sont les principaux exemples.

(1) LE DANTEC [91] cherche à expliquer cette sécrétion d'acide aux dépens d'un protoplasma alcalin en imaginant que ce protoplasma contient des sels neutres dont l'acide diffuse plus vite que la base. Il est plus probable que cela résulte d'actions chimiques comparables à celles qui permettent aux cellules des glandes stomacales des animaux supérieurs de fournir un suc acide aux dépens du sang alcalin. Ce suc 
Il n'y a pas de choix d'orlinaire dans les aliments. La particule qui se présente est acceplée sans examen. On peut faire absorber à l'animal des poudres inertes qu'il doit rejeter comme fèces sans en avoir rien extrait. On peut mème l'empoisonner par des substances nocives. Parfois cependant, on voit des particules entrainées par le tourl,illon jusqu'à la bouche ressortir sans avoir été almises (').

Défécation. - Quand les vacuoles alimentaires sont réduites à l'état de vacuoles à fèces, elles sont peu à peu entrainées vers l'anus; souvent deux ou un plus grand nombre se fusionnent en éclatant l'une dans l'autre et mêlent leur contenu. Quand une de ces grosses vacuoles est arrivée en face de l'anus, elle est poussée par une contraction de l'endoplasme ambiant, écarte le plasma cortical, entr'ouvre la fente préexistante de l'ectoplasme et de la membrane, et est expulsée au dehors avec une certaine énergie. L'anus se referme immédiatement derrière elle par accolement intime de ses parois.

Excrétion. - L'excrétion a lieu par le moyen de la vésicule pulsatile et de ses annexes. L'alimentation introlluit de l'eau dans le corps en masse beaucoup plus considérable que les aliments solides dont elle est le véhicule. Cette eau n’est que partiellement expulsée au moment de la défécation. D'autre part, de l'eau pénètre par osmose à traver's le tégument. Cela est prouvé par le fait que quelques Ciliés privés de bouche, comme certaines Opalinides (Anoplophrya, IIoplitophrya, par exemple), n'en expulsent pas moins beaucoup d'eau par leur vésicule. Toute cette eau qui pénètre sans cesse dans le corps doit nécessairement en sortir. La vésicule pulsatile lui en fournit le moyen. Nais en traversant l'organisme, l'eau s'est chargée de tous les produits de désassimilation et les entraine ainsi au dehors. On n’a pu démontrer par l'analyse la présence de ces produits dans le liquide expulsé. Mlais cela résulte de l'examen des phénomènes. Un être aussi actif que notre Cilié, en mourement nuit et jour pendant toute sa vie et qui absorbe des quantités formidables de nourriture, a nécessairement beaucoup de produits de désassimilation à rejeter et s'il n'utilisait pas pour cela la voie si naturelle de la vésicule pulsatile, on ne voit pas par où il pourrait s'en débarrasser. Ces produits de désassimilation nous apparaissent assez souvent précipilés à l'élat solide sous la forme de grains d'excrétion. Or ces grains ne sont pas évacués par l'anus. Cependant ils disparaissent peu à peu lorsque l'animal est soumis au jeûne. Il faut donc qu'ils aient été dissous et expulsés à l'état liquide : il n’y a que la résicule gui puisse remplir cette fonction. Sans doute, l'urate de soude, s'il y en a dans les grains d'excrétion est lentement dissous en nature, quant au phosphate bicalcique $\left(\mathrm{PO}^{4}\right)^{2} \mathrm{Ca}^{2} \mathrm{H}^{2}$

acide digère les substances albumineuses. Il atlaque la chlorophylle, l'amidon, mais ne semble pas les digérer.

$\left.{ }^{1}\right)$ Ce n'est guère que chez les Torticelles que l'on a observẻ ce triage. 
insoluble, il doit passer à l'état de phosphate monocalcique soluble $\left(\mathrm{PO}^{4}\right)^{2} \mathrm{CaH}^{4}$.

Respiration. - Mais l'eau qui traverse ainsi l'organisme est chargée d'oxygène à son entrée. L'animal troure donc tout naturellement en elle l'élément nécessaire à sa respiration, et si l'on analysait l'cau expulsée par la vésicule on y trouverail sans doute $\mathrm{CO}^{2}$ en proportion plus grande que dans le liquide ambiant. La vésicule est done à la fois l'organe de l'excrétion et celui de la respiration (").

Fonctionnement de la vésicule pulsatile et des canaux sécréteurs. - Voyons maintenant comment la vésicule fonctionne (fig.691). Prenons le phénomène au moment où, venant de se vider, elle a disparu. Il n'y a alors autre chose que les dilatations terminales $(v . s$.) des canalicules afférents déjà assez fortement distendues par l'afflux incessant du liquide qui leur arrive du réseau $(t)$. Ces dilatations n'ayant plus devant elles qu'un espace libre occupé par du plasma cortical et se vidant ensemble dans cet espace, reconstituent d'emblée une résicule pulsatile (V. p. 409) en état de demi-diastole et de forme plus ou moins irrégulière.

Cette vésicule est alor's creusée dans une forte masse de plasma cortical et séparée du pore excréteur par une épaisse lame de cette substance (V. P. 103, fig. 691). Hais elle continue à recevoir du liquide des mêmes dilatations terminales qui lui en ont déjà fourni ; elle grossit et peu à peu amincit la lame qui la sépare de son orifice en mème temps qu'elle s'arrondit et se dessine avec un contour plus accusé. Il semble qu'à mesure qu'elle se gonfle, elle éprouve plus de peine à recevoir d'autre liquide comme si sa dilatation excitait la contractilité du plasma qui forme ses parois. Toujour's est-il que, lorstu'elle est bien remplie, il y a comme une courte pause et, brusquement, la mince lame le plasma cortical qui la séparait encore du pore excréteur se rompt et le liquide s'échappe par ce pore $\left({ }^{*}\right)$.

(1) Büтschur croit que la vésicule ne sert qu’à la respiration et nie la fonction excrétrice. Mais Maupas fait remarquer avec raison la nécessité physiologique de l'exerétion et invoque le fait de la présence d'une vésicule pulsatile chez certaines zoospores vertes qui, ayant de la chlorophylle, n'ont pas besoin de l'oxygène extérieur. Cependant certaines Opalinides (Opalina) n'ont pas de vésicule et si, sans son aide, elles éracuent leur's produits d'excrétion, les autres Ciliés devraient pouvoir en faire autant. Cela dépend peut-être du degré de solubilité de ces produits. II se prourrait que, dans certains cas, étant très solubles, ils soient éliminés par simple exosmose tandis que d'ordinaire ils auraient besoin du courant incessant déterminé par la vésicule.

(2) Bürschu a émis l’idée que la systole de la vésicule est due à une simple force physique, la tension superficielle qui, en raison de la forte courbure de la résicule est supérieure à celle du liquide extéricur. La vésicule se viderait dans ce liquide dés que la liume de plasma cortical qui l'en sépare a disparu, comme une pelite goutte d'eau se vide dans une grosse quand elle arrive à son contact sur une surface qu'elles ne mouilleat pas et qui leur permet de conserver leur forme sphérípue. Il est possible que cette force intervienne, mais, au début de la systole tout au moins, Ia contractilité du plasma eortical intervient aussi, car nous avons vu que la systole com- 
A mesure que la vésicule se vide, sa paroi conflue de tous les points vers le pore et sa cavité disparaît jeu à peu comme celle d'une bulle de savon dont on aspire le contenu par la pipette qui a servi à la gonfler, et quand la systole est achevée, il n'y a plus trace de sa cavité : celle-ci s'est évanouie, confondue avec l'espace extérieur. La cavité de la nouvelle vésicule n'a donc rien de commun avec celle de l'ancienne : elle se forme à nouveau, par irruption d'une nouvelle masse de liquide dans la masse de plasma corlical qui est venue occuper la place de la vésicule

Fig. 699 .

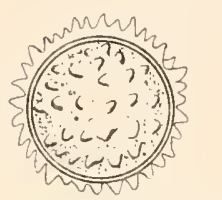

CILIÉs (Type morphologique). Formes diverses de kystes.

ancienne à mesure que celle-ci disparaissait (').

La vésicule se contracte toutes les deux à trois minutes et, comme son volume représente environ $1 / 10^{\circ}$ à $1 / 15^{\circ}$ de celui lu corps, il en résulte qu'en moins d'une demi-heure, l'animal a évacué un volume d'eau égal au sien. Cela montre l'activité extrème de cet appareil $\left({ }^{*}\right)$.

Enkystement. - Notre Cilié est capable de s'enkyster, c'est-à-dire de sécréter autour de lui une membrane chitineuse derrière laquelle il s'abrite (fig. 699).

mence après un moment d'arrêt de la diastole et lorsque la lame de plasma cortical a encore une certaine épaisseur. Cela suppose une contraction active des parois. I'autre part, la contractilité du plasma cortical est démontrée par l'expulsion des fèces puisque, d'ordinaire, l'anus est absolument fermé. Dès lors, pourquoi n'interviendrait-elle pas dans le jeu de la vésicule?

(1) Cette description s'applique surtout a un cas moyen comme celui de Paramrcium. Quand les dilatations terminales des canalicules afférents sont moins fixes et qu'elles effectuent la forme de racuoles formatrices irrégulières, celles-ci, au lieu de former une vésicule pulsatile nouvelle par injection de leur contenu, se fondent ensemble elles-mèmes pour former cette vésicule, et le liquide qui continue à suinter des canalicules afférents forme autour de la vésicule de nouvelles vacuoles formatrices. La vésicule ainsi constituée est d'abord très large et irrégulière. Elle s'arrondit peu à peu, se régularise, se concentre et se trouve arrivée à l'état qui précède immédiatement la systole sans avoir reçu des nouvelles vacuoles formatrices, avec lesquelles d'ailleurs elle ne communique pas, de nouvelles quantités de liquide. Elle se contracte alors, se vide et le phénomène recommence. Ce cas montre bien combien est faible l'indépendance de cet appareil. Ce n'est en somme qu'un système de voies que le liquide lui-même se fraye dans la substance sirupeuse du plasma cortical. Là où il coule sans cesse sans avoir a modifier son cours, ses voies ont une certaine permanence; il en est ainsi généralement dans le réseau. Mais dans la région de la vésicule, où il est soumis à des oscillations continuelles, il se fraye chaque fois de nouveaux chemins dans le plasma, et la limite entre la partie permanente et celle qui ne l'est pas varie selon les types d'organisation. Enfin, lorsque la vésicule est en rapport avec un petit nombre de larges canaux, ceux-ci se comportent comme la vésicule afférente de Paranixcium.

(2) La fréquence des contractions varie, selon les espèces, d'une demi-minute à quinze ou vingt minutes; elle est augmentée par les alcalis dilués, par divers alcaloïdes, diminuće par les acides dilués, le sucre de canne, le sel marin. Ce dernier fait explique la lenteur générale des contractions de la résicule cliez les formes marines. Il semble que la privation d'oxygène (eau bouillie) el l'excès d'acide carbonique augmentent leur fréquence, ce qui parlerait en faveur du rôle respiratoire, mais les expériences ne sont pas très concordantes sur ces points. 
Il use de celte faculté dans diverses circonstances :

$1^{\circ}$ Pour se diviser. Il peul d'ailleurs se diviser sans enkrstement préalahle el nous éludierons ce cas parliculier à propos de la reproduction;

20 Pour se reposer et digérer à l'aise quand il s'est si bien repu, qu'il ne pourrait plus rien absorber, cas un peu exceptionnel;

$3^{\circ}$ Enfin, et c'est là la circonstance la plus habituelle, pour se mettre à l'abri des influences nocives du milieu ambiant, lorsque l'eau qu’il habite se condense, se putréfie, se dessèche. Dans ce cas, son enkystement peut ètre de longue durée d'où le nom de liystes durables (Dauercysten) donné aux kystes qu'il produit alors. C'est si bien la qualité de l'eau ambiante qui détermine l'enkyslement que celui-ci peut s'arrèter et se défaire si l'eau redevient pure lorsqu’il n’est encore que commencé.

Pour s'enkyster, l'animal s'arrète, s'arrondit; ses cils et membranelles se résorbent, son péristome s'efface, sa bouche disparait; il éracue ses grains d'excrétion et sécréte autour de lui une substance gélatineuse qui peu à peu se durcit et se montre avec les caractères de la chitine. Seule, sa vésicule pulsatile fonctionne encore quelque temps après que toute apparence de vie a disparu; elle expulse encore de l'eau qui s'accumule entre le corps et la paroi du kyste; mais bientôt ses mouvements se ralentissent, s'arrêtent et clle-mème disparait. On ne trouve plus alors dans le kyste qu'un protoplasma condensé, concentré par soustraction d'eau, sans distinction de couches diverses, dans lequel le $\mathbf{N}$ et le $\mathrm{n}$ persistent seuls, un peu condensés mais en somme inaltérés. Sous cel état, l'animal peut suljir une dessiccalion très forte. L'évaporation lui soustrait encore de l'eau et, semblable à un grain de poussière, il est enlevé par le rent el entrainé avec les autres poussières de l'air'. Il peut supporter celte dessiccation et cette mort apparente pendant des années, et s'il vient à tomber dans une eau suffisamment pure, s’imbiber à travers la paroi perméable de son enveloppe, reprendre peu à peu son aspect normal, reformer en quelques heures ses organes, faire éclater son liyste et s'élancer dans l'eau en quète de nourriture. Certaines espèces communes sont toujour's représentées dans les poussières de l'air et tombent sans cesse dans les rases d'ęu non courorts. Elles y éclosent et, si le vase contient un liyuide nutritif, elles s'y multiplient et foisonnent. De là était née l’idée de la génération spontanée de ces ètres (").

Reproduction. - Le Cilié se reproduit miquement par ilivision. Mais cette division peut se faire à l'état libre ou dans un ky̧ste; elle peut succéder à une conjugraison. Nous aurons lonc à ćtudicr ces trois phé-

(1) Les liystes sont d'ordinaire simples et arrondis, mais chez certaines espèces ils peurent posséder deux (Torticelles, Oxytrichines, liursuria) ou mime trois (Colpoda) enveloppes séparées par des couches d'eau, ou présenter des formes diverses, ou ètre ornés de dessins variés. Parfois (Colpoda), il y a dans la paroi du liyste un petit trou spécial par où l'animal sort à la tin de l'enkystement. Chez ce mème Colpode on trouve des kystes emboîtés résultant de ce que les individus nés de la division dans le liyste se sont enliystés dans le liyste maternel. 
nomènes : $1^{\circ}$ division simple, $2^{\circ}$ division dans un kyste, $3^{\circ}$ conjugraison. Division simple. - La reprorluction par division n'est autre chose qu'une division cellulaire compliquée de ce fait que, les deux moitiés n'étant pas identiques, il faut que chacune régénère ce qui lui manque pour former un individu complet. La division est transversale et se fait suivant le plan équatorial de l'ovoïde. La moitié supérieure emporte la bouche et le péristome, l'inférieure garde l'anus et la vésicule (").

Examinons d'abord les phénomènes nucléaires.

Macromeléus. - Le $\mathrm{N}$ se double par division directe. Il se concentre et prend une forme tout à fait sphérique et se place dans le futur plan de division. Puis il s'étrangle au milieu, s'élire en biscuit (fig. 700) et se coupe dans sa partie moyenne. La membrane n'a pas cessé un instant d'exister (').

Fig. 700 .

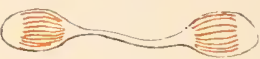

CILIÉS

(Type morphologique). Division directe du noyau (Sch.).

(1) La division est fréquemment un peu oblique, mais ce n'est ‘u'une variétè sans importance de la division transversale. Quant aux prétendues divisions longitudinales, sauf le cas des Péritrichides qui será expliqué quand nous parlerons de ces animaux, ce sont des conjugaisons mal interprétées.

(2) Il y a ici celte particularité que la partie moyenne qui réunit les extrémités renflées est beaucoup plus longue que dans les amitoses ordinaires (fig. 700). Il résulte de cela que, dès avant la fin de la division, les deux parties renflèes représentent les deux $\mathbf{N}$ filles occupant déjà leur position définitive dans les deux futures moitiés. A l'intérieur, se dessine une striation onduleuse entortillée fui indique un stade spirème, mais le phènomène ne va pas plus loin, en sorte que nous avons là plutôt une mitose incomplète, avortée, qu'une amitose réelle. Romper. [95] a signalé chez Kentrochona (fig. 701, A) la présence d'un centrosome donnant à la division du $\mathbf{N}$ quelques caractères d'une milose; et il est a remar fuer que, dans ce cas, le $\mathbf{n}$ était situé loin du $\mathbf{N}$, ce qui contredirait l'assimilation quelquefois proposée du $\mathbf{n}$ des Ciliés

A
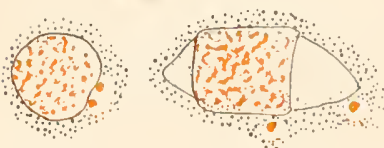

Fig. 701

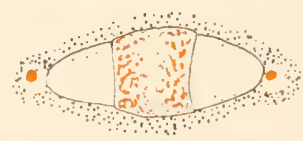

B

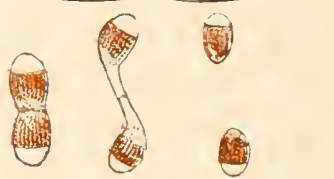

Division nucléaire. $A$, chez Réntroetiona (d'ap. Rompel); $B$, chez Sprirochona (d'ap. Balbiani). avec le centrosome des cellules ordinaires. Mais Balbiaxl [95] (fig. 701, B) a récemment trouvé chez spirochona des phénomènes qui se rapprochent beaucoup plus d'une milose vraie avec substances achromatiques disposées aux pôles et même avec intervention de petits globules chromatiques jouant le rôle de centrosomes, et assure que les noyaux donnés prar Rompel comme en état de division sont au repos, les prétenrlus centrosomes n'étaient sans doute que des Fig. 702 . micronucléus (V. p. 481, 182).

Chez les opalinides où il $y$ a, comme nous l'avons vu, de nombreux noyaux 
Micronucléus. - A l'inverse du $\mathrm{N}$, le $\mathrm{n}$ se divise par mitose mais sans centrosome. Au repos (fig. 703, A) il ne montre d'autre structure qu'un fin pointillé chromatique et de petites stries achromatiques. Mais bientòt il commence à se gonfler et son contenu prend une structure entortillée qui rappelle un stade spirème $(B)$.

Il se gonfle ainsi jusqu'à doubler presque de diamètre et, à la fin de ce stade d'accroissement, montre ses filaments orientés netlement d'un pòle à l'autre. C'est sans doute le stade de peloton segmenté. Puis ces filaments se raccourcissent el se disposent dansla région équatoriale, dessinant là une épaisse plaque nucliaire $\left(C^{\prime}\right)$ où cependant on ne distingue pas d'anses jumelles bien nettes. La substance achromatique occupe les deux pòles d'où elle Fig. 703 .

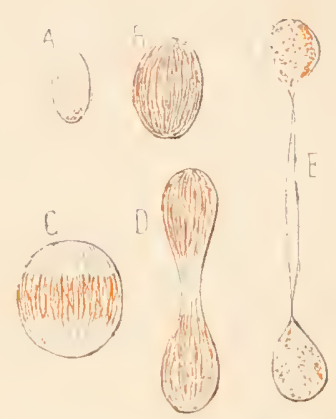

CILIÉs (Type morpholog.). Division du mieronucléus (d'ap. Maupas).

rayonne des points que devraient occuper les centrosomes absents. A ce stade de métaliynèse succède un stade diaster $(D)$ dans lequel les filaments chromaliques occupent les deux pòles, tandis que l'espace intermédiaire est occupé par des filanents connectifs de substance achromatique. Enfin, la partie moyenne s'étire en un cordon qui s’allonge considérablement $(E)$ el est formé par la membrane nucléaire (qui ne disparait à aucun moment) et par quelques stries achromatiques, tandis que, aux deux bouts, sont les deux n filles où la chromatine repasse à l'état le ‘lispirème, pruis de noyau au repos. Le corlon qui réunit les deux n filles sert à les liriger respectivement vers la place qu'ils

représentant chacun un $\mathbf{N}+\mathbf{n}$ non dissociés, et qui, par là, s'éloignent du type normal des Ciliés, ces noyaux se divisent suivant la mitose typique avec anses jumelles bien distinctes et tous les stades habituels (fig. 702), sauf cette particularité qu'il n'y a pas le centrosome et que la nrembrane nucléaire est persistante.

Le cordon qui réunit les deux parties renflées est formé uniquement par la membrane et par quel,fues stries achromatiques. Quand le noyau est en ehapelet, comme par exemple chez stentor (fig. 70' lous les grains du chapelet $(.1)$
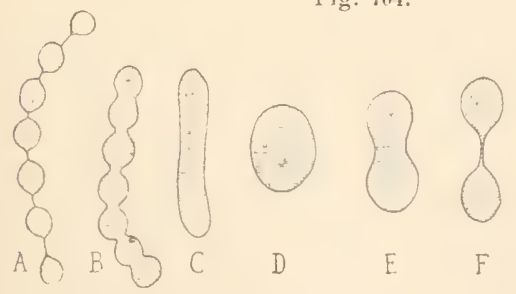

D

E

F

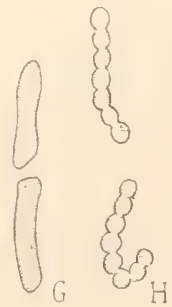

Division du macronucléus chez stentor (Sch.). en un bàtonnet allongè et de ramener celui-ci à une forme sphérique $(D)$. Alors se produit une sorte de pétrissage, puis le $\mathbf{N}$ s'allonge de nouveau $(\boldsymbol{E})$, se sectionne et les deux $\mathbf{N}$ filles prennent la forme de bâtonnets $(G)$, puis de chapelels $(\boldsymbol{I})$. Lorsquau contraire il $\mathbf{y}$ a, non un $\mathbf{N}$ dissocié, mais vraiment plusieurs $\mathrm{N}$ comme chez Loxodes, alor's les $\mathbf{N}$ ne se réunissent pas en un seul pour se diviser. 
doivent occuper, d'où le nom de gubernaculum qu'on lui donne quelquefois. Ce gubernaculum, après avoir accompli sa fonction, s'amincit, se coupe à ses poinls d'altache arec les n filles, el finit par se résorber.

Phénomènes extérieurs. - Pendant que ces phénomènes nucléaires s'accomplissent, le corps ne resle pas inactif. Il commence par s'allonger; puis un sillon équatorial étroit se dessine (fig. 7030). Ce sillon s'appro-

liig. 705 .

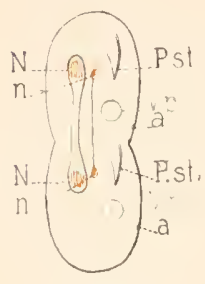

CILIES

('Type morpholog.). Division (Sch.).

a., anus; $\mathbf{x}$, macrowucléus; $\mathbf{n}$, micronucléus; P.st., péristome; v.p., vésiculo pulsutile. fondit plus rapidement qu’il ne s'élargit et finil par couper l'Infusoire en deux.

Nous royons bien comment charue individu fille possède un $\mathrm{N}$, un $\mathrm{n}$, un tégument, un endoplasme, un revètement ciliaire; mais comment vont se former les organes uniques: péristome, bouche, pharynx, anus, vésicule pulsatile, etc.?

Pour la bouche el ses annexes, la chose a élé nellement observée. Déjà avant que la division nucléaire ait commencé, on voil, au-dessous de l'équaleur, se dessiner sur la paroi ventrale un nouveau péristome (P.st.). La membrane se fend, mel à nu l'ectoplasme qui pousse une série de membranelles; à gauche des membranelles, se creuse un sillon péristomien; à l'extrémité inférieure du sillon, se perce la bouche, le pharynx se dessine et l'on a un individu à deux bouches qui aurait l'air d'une forme tératologique si, entre les deux bouches, ne se formait le sillon de sépraration. Pendant ce temps les $\mathbf{N}$ et $\mathrm{n}$ se sont divisés, ont envoyé un $\mathbf{N}$ et un $\mathrm{n}$ filles dans clracune des moiliés, el la séparation s'achève. La nouvelle vésicule el le nouvel anus se forment à leur place normale dans l'individu supérieur, les cils de la zone de section qui s'étaient résorbés se régénèrent sur chaque indivilu suivant le type qui convient à l'espèce et l'on a enfin deux individus libres el complets. La durée du phénomène est d'environ vingl-ruatre heures (').

Division dans un kyste. - Il y a peu à dire sur ce mode de reproduction un peu exceptionnel. L'animal s'arrondit et s'enkyste comme pour se reposer ou s’abriter, mais le kyste est mince et peu résistant, car il doil durer peu de lemps. Sous ce kyste, il résorbe ses cils, sa bouche et son plrarynx, mais garde sa vésicule qui continue à fonctionner sans interruption. I'uis, au bout de quelifue temps, sa substance se tivise en deux ou quatre masses plus petites qui se revêtent de cils et se munissent des organes ordinaires. Le kyste alors se rompt el les jeunes

(1) Souvent, les deux individus issus de la division ne sont pas de taille identique. Lor'sque celte rlifférence est très forte et surtout lorsque le petit individu nait d'une partie d'abord très petite et qui grossit aux dépens de la grosse avant de se séparer, cela devient tu bourgeonnement. Les phénomènes nucléaires sont les mèmes, il n’y a pas là une différence essentielle. Ce cas élant assez rare nous nous contenterons de le signaler quand il se présentera, par exemple chez śpirochona. 
individus sortent et nagent, n'ayant plus qu'a s'alimenter pour grossir (').

Phénomènes consécutifs à la division. Dégénérescence sénile. - L'lnfusoire a une puissance reproductrice considérable. Bien nourri, il peut se diviser, si la température est suffisamment tiède, denx ou trois fois par vingt-quatre heures $\left(^{2}\right)$.

Au moment de leur naissance, les deux individus filles ne diffèrent de leur parent que par une taille moindre et, en se nourrissant, ils atteignent bien vite une taille égale. Il en est ainsi pendant de nombreuses générations. Nais après 1300 ou 200 le ces générations agames, on constate que les noureau-nés n’atteignent pas on grandissant la taille normale de l'espèce; ils restent un peu plus petits, ol ce phénomène s'accentue sur les générations suivantes, de telle façon yu'à la fin, vers la $300^{\circ}$ génération, on n’a plus que des avortons ayant à peine le tiers de la taille normale. Nais bien avant d'en ètre arrivés à ce point, d'autres signes de dégénérescence s’ajoutent successivement à celui-ci : c’est le péristome qui se rétrécit, so déforme, c’est la zono arlorale qui se róluit et montre des anomaliss plus ou moins accentuées; la vésicule se réduil, devient moins active; le $\mathrm{N}$ se llétrit, s'appaurrit en chromatine; enfin, ce qui est plus grave, le n lui-mème s'atrophic peu à peu et, à partir de ce moment, on commence ì trouver des individus de plus ell plus nombreux dépourvus de n. Quoi que l'on fasse, la colonie est condamnée à mort. Un seul phónomène peul lui rendre la vie, c’est la conjugaison $\left({ }^{3}\right)$.

Conjugaison. - Cette dégénérescence sénile développe. en s’accentuant, un appétit sexuel de plus en plus vif. Les individus se cherchent, se tìtent, se prennent, se lìchent, en cherchent d'autres arec une activité fébrile et finalement arrivent à se conjuguer deux ì deux. Chose singulière, le n que nous allons roir joner le principal ròle dans la conjugaison n’est pas l'organe de ces sensations, carles individus qui, arrivés au der-

1 Parfois, il semble que les cils ne disparaissent pas. Le nombre des individus formés dans le kyste est ordinairement 2 ou 4, mais parfois il atteint 8, 16, 32. l'arfois, ce nombre est beaucoup plus grand (certains liystes des Colpodes), mais alors cela devient une véritable sporulation: aussi les modifications de l'individu enkysté sont-elles beaucoup plus profondes? Toute trace cle l'organisation antérieure a disparu en lui. La division dans un livste ne se rencontre guère que cliez les formes inférieures des Holotrichides. Sauf chez Colpoda, tui se reproduit toujours à l'état enkysté, ce mode de division coexiste toujours avec la division à l'état libre.

(2) Cela dépend surtout, naturellement, des espèces. Certaines Paramxcium bursaria, Stentor, ne se divisent que tous les deux ou trois jours; d'aulres, comme Lencophrys patula, peuvent se diviser toutes les trois heures. Nous prenons toujours pour notre type morphologique les nombres les plus ordinaires.

$\left.{ }^{3}\right)$ Le fait de la dégénérescence sénile est absolument général. Matpas qui l'a découvert dans ses admirables expériences de culture l'a rencontré ehez tous les Ciliés qu'il a étudiés. Mais elle est plus ou moins prompte suirant les espèces. Les termes extrèmes obserrés par Haupas sont fournis par stylonichia pustulata qui s'éteint après 2 lö générations et Leucophrys patula qui n'est détruit qu'ì la 660e. Le nombre d'environ 300 peut etre considéré comme moyen. 
nier terme de la dégénérescence n’ont plus de n, n’en sont pas moins actifs dans cette recherche. Ils se conjuguent mème, mais sans résullat et meurent sans postérité.

Les condilions d'une conjugaison fertile ont été déterminées par Maupas et sont au nombre de trois : $1^{\circ}$ un état suffisamment arancé de dégénérescence sénile, mais pas assez avancé pour que le $\mathbf{n}$ soil atteint; $2^{\circ}$ un étal d’inanition relative résultant de la pénurie d’aliments, les colonies très bien nourries mourant de dégénérescence sans que l’instinct sexuel se développe en elles; $3^{\circ}$ une généalogie ancestrale différente, les individus issus d'un même conjuğué ne pouvant prorluire que des unions stériles et devant, pour que leur conjugaison soit fertile, être eux-nièmes descendants de conjugués différents.

Il est à remarquer que ces conditions doivent se rencontrer souvent. Un vase exposé à l'air', ensemencé naturellement par les poussières de l'air ou par quelques gouttes d'eau rhargée d'lnfusoires, conliendra généralement plusieurs individus non parents et d'une mème espèce. En se multipliant, ces individus consomment les substances nutritives du liquide avec une rapidité qui croìt avec leur nombre. En sorte que la

Fig. 706 .

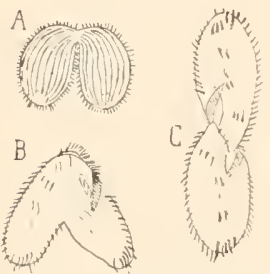

CILIES (Type morphologique).

Divers modes de conjugaison (Sch.). pénurie d'aliments arrive pour tous en mème temps. Si elle n'arrive qu'assez tard, les individus se trouvent ensemble à la période de dógénérescence el de nombreux légénérés non parents trouvent à s'accoupler entre eux. De là ces épidémies de conjugaison dont on ne comprenait pas tout d'abord la raison (").

Après ces préliminaires, éludions les phénomènes de la conjugaison entre deux individus aptes ì former une union fertile.

Les deux conjugués (fig. 706), après s'ètre définitivement saisis, se placent bouche contre bouche et se soudent par la surface située au-dessus de la bouche $\left({ }^{\mathbf{2}}\right)$.

(1) I,e fait que les unions d'individus issus d'un mème conjugué sont stériles donne un singulier appui à l'idée de Wersmax, que la génération sexuelle a pour but la variation par le mélange de plasmas germinatifs diffẻrents et, par suite, doués d'aptitudes évolutives différentes dans les limites de la variation physiologique de l'espère. Il faut remarquer, en cffet, que les dégénérés issus d'un mème conjugué ont un plasma germinatif identique, bien ‘fu'ils ne soient parents qu'au 300e degré, parce qu'ils sont nés de divisions agames et que la fẻcondation seule modifie brusquement la constitution du plasma germinatif $Y$. pour l'ètude de ces questions, Y. Delage [95]).

(2) Il résulte de cette position que les deux faces rentrales ne sont pas symétriquement superposées. Les conjoints ètant unis par la partie gauche de leur face ventrale, débordent par la partie droite de cette face. La bouche, étant libre au-rlessous de la soudure, peut continuer à fonctionner pendant les premières phases de la conjugaison et elle recommence aussi a fonctionner vers la fin.

Mais cela n'a rien d'absolu. La bouche est souvent comprise dans la sourlure et les pronucléus peuvent se servir de cette voie pour passer d'un individu à l'autre Paramxcium). Quand elle est terminale, c'est toujours par elle que la soudure se fait et les conjoints ont alors leur axe longitudinal sur le prolongement l'un le l'autre. 
La partie du péristome comprise dans la soudure perd naturellement toute structure, ses membranelles tombent el, plus tard, quand les

Fig. 707.

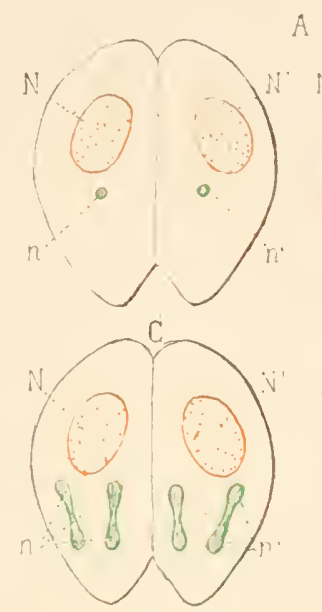

A
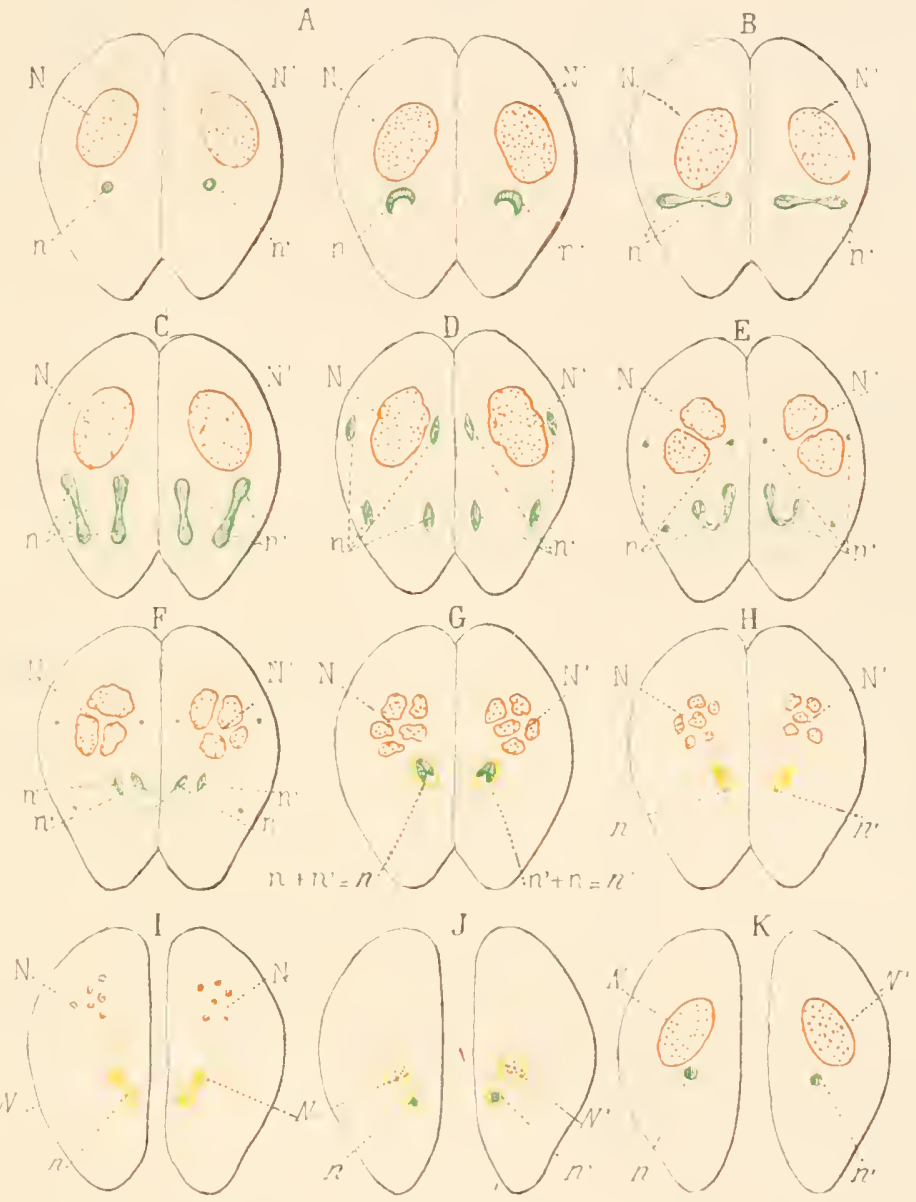

ClulÉs Type morphologique).

Phínomènes présentés par lo macronucléns et le mieronucléus pendaut la conjugaison. (Sch.). $A$ ì $K$, stades successifs. $\mathbf{N}$, macroucléus, of $\mathbf{n}$, microucléus du premier individu; $\mathbf{N}$, macronucléus, et $\mathbf{n}^{\prime}$, micronucléus (lu sceond individu; $n\left(=\mathbf{n}+\mathbf{n}^{\prime}\right)$ micronucléus du prenier individu aprés la conjugaison de son micronurléus femelle arec le micronuelẻus mâle du secont ; $n^{\prime}$ (=n' $+n^{\prime}$, mieronucléus du second individu après la conjugaison de son micronucléus femclle avec le micronucléus mâle du premier: $x$, macronuclèus de nouvelle formation résultant de la division dn micronucléus conjuguẻ du premier individu: $N^{\prime}$., idem du second.

Cette position est probablement primitive comme aussi la position terminale de la bouche. Quand la bouche est ventrale, les deux conjoints se rabattent l'un sur l'autre par la face ventrale, et le fait qu'ils se soudent par la partie située au-dessus de la bouche s'explique, si l'on se rappelle que la bouche ventrale dérive d'une bouche longitudinale partant de l'extrèmitć supérieure et s'étendant sur la face ventrale, dont la partie superieure sest suturée laissant à sa place un raphè (1. 1. 404). 
conjoints se sépareront, ils auront à reformer les parties détruites. Cette soudure est tout à fait complète. Les membranes se détruisent sur les surfaces accolées, les deux ectoplasmes se fusionnent en une lame unique et, un peu plus lard, cette lame se perce pour laisser passer les produits à échanger, établissant entre les deux cytoplasmes une libre communication. Les leux conjoints lombent dans un état d'apathie profonde gisant au fond de l'eau presque sans mouvements.

Les phénomènes intérieurs de la conjugaison sont surtout nucléaires ("). Nous allons examiner successirement ce qui se passe dans le $\mathbf{n}$ et dans le $\mathbf{N}$.

Disons d'abord que ces phénomènes comprennent de nombreuses divisions et que ces livisions se font comme celles dont nous avons éludié le type à propos de la reproduction par division ( $\mathrm{V} . \mathrm{p} .4 \mathrm{lS}$ ); cela nous permettra d’alı́écer beaucoup la description.

Phénomènes micronucléares. - Le n (fig. 707, n et n' et fig. 712) grossit $(A)$, puis se divise en deux $(B)$, puis chacun des deux $\mathrm{n}$ filles se divise encore en deux $(C)$, ce qui donne quatre $\mathbf{n}$ petites-filles $(D)\left({ }^{*}\right)$.

Ces quatre $\mathrm{n}$ sont, en apparence du moins, identiques entre eux; cependant leur sort est bien différent. Celui des quatre qui se trouve le plus près de la surface de soulure persiste seul (E); les trois autres

Fig. 708 .

n

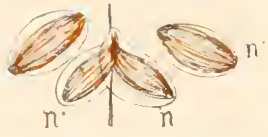

CILIÉS

(Type norphologique). Eehange des pronucléus ơ (d'ap. Maupas). s'atrophient peu à peu, une vacuole se forme aulour d'eux et ils sont traités par l'endoplasme comme de simples particules alimentaires.

Le n destiné à survivre se divise encore une fois et donne deux n filles ( $E$ et $F$ ) que nous appellerons l'un le pronucléus ơ (e, fig. 712) l'autre le pronucléus of ( $s$, fig. 712).

Ces deux pronucléus ne paraissent d'ailleurs différer en rien. Le ost le plus voisin de l’orifice de communication, le q est le plus enfoncé dans l'endoplasme (fig. 708) (: $\left.{ }^{*}\right)$. Les deux $\mathbf{n}_{\sigma^{*}}$ franchissent cet orifice et se portent vers le $\mathrm{n}$ o de l'autre

(1) Le cytoplasma devient trouble par l'apparition de nombreuses granulations (zooamylum ou grains d'excrétion?) qui sont l'indice d'une activité métabolique très grande. Il joue un ròle sans doute, mais qui consiste probablement en phénomènes chimirues et en échanges osmotiques invisibles.

(2) Chez Paramiecium, que l'on prend souvent pour exemple, pendant la phase d'aceroissement qui précède sa première division, le $\mathbf{n}$ prend des formes bizarres en croissant spiral (fig. 709. On ne connait pas la signification de re phénomène qui, d'ailleurs, n'est pas du tout général el paraît sans grande importance.

${ }^{3}$ ) Dans la division lu $\mathrm{n}$ en deux pronucléus le gubcrnaculum joue comme d'ordinaire un rôle actif. D'une part, il pousse le pronucléus o vers loorifice de communication entre les deux conjoints et, d'autre part, en se recourbant en are, maintient le pronucléus o à peu de distance de cet orifice, toutes choses qui facilitent les phéFig. 70!.

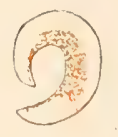

Micronucléus en croissant de Paramixcium (d'ap. Мraupas). nomènes consécutifs. Mais à ce moment il se détruit et les autres mourements s'accomplissent sans lui. 
conjoint pour se fusionner avec lui (fig. 707, F, 710 et 712 ). Pour cela, les deux membranes, qui comme on sait ne disparaissent point dans la division, se soudent, s'ouvrent l'me dans l'autre et les deux substances intérieures se joignent sous une membrane commune donnant ainsi naissance à un n conjugué (fig.707, G, 711 et $712, c)$.

Dans chacun des deux conjoints (fig. 707, $H$ ), ce n conjugué se divise en deux autres qui sont identiques d'abord en apparence $(I)$, mais dont l'm garlant ses caraclères primitifs devient le $\mathrm{n}$ définitif $\left(J: n\right.$ el $\left.n^{\prime}\right)$, tandis que l'autre grossil beaucoup et derient le nouveau $\left(J: N^{\top}\right.$ et $\left.N^{\prime}\right)(')$.

Après s’être séparés, les ex-conjugués reslent encore quelque temps inertes au fond de l'eau. Mais peu à peu ils régénèrent leurs mem-

(1) Rèduite à ces termes, la description schématique

$n+n^{\prime}=$

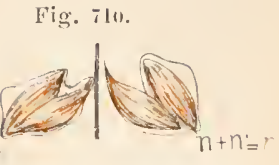

CILIẺS (Type morphologique). Fusion des micronucléus

(d'ap. Maupas).

st à la fois simple et rlaire. Mais il faut dire que, dans la rẻalité, elle se complique par le fait fule les premicres livisions consécutives à la conjugaison se préparent lans l'appareil nucléaire avant que les conjoints se soient séparés et qu'au lieu de se faire sur les $\mathbf{N}$ et $\mathbf{n}$ nouveaux après leur différenciation, elles se produisent sur le noyau conjugue qui se divise une ou plusieurs fois en noyaux représentant chacun un $\mathbf{N}$ plus un $\mathbf{n}$ encore confondus ensemble. Le cas réel le plus simple est celui re Colpoda. Ia simple inspection du schéma ci-contre (fig. 712), montre que le noyau

Fig. 712.

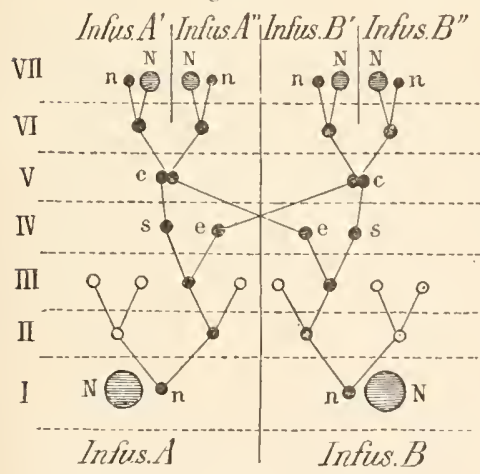

CILIES (Type morphologique).

Diagramme montrant l'évolution des noyaux daus la conjugaison (im. Maupas). conjugué $(c$.) se divise r'abord dans chaque conjoint en deux autres récllement identiques entre eux et destinès chacun à l'un des prorluits de la division qui se prépare, puis ces deux noyaux se divisent encore chacun en deux illentiques entre eux en apparence et qui sont l'un un futur $\mathrm{N}$, l'autre un futur n encore non lifférenciés. En sorte qu'i ce montent chaque conjoint contient puatre n. Mais ils sont dejjà groupès en deux paires éloignées l'une de l'autre. Bientôt, tans chaque paire, l'un reste semblable à lui-même et devient le $n$, l'autre grossit et devient le $\mathbf{N}$ et la division se produit. Les conjoints, bien qu'ils n'aient rien à faire ensemble après la formation des deux noyaux conjugués, ne se séparent cependant que quelque temps après, lorsque la première division a commencé à se préparer dans l'appareil nucleaire.

Chez d'autres formes, la complication va encore plus loin, mais c'est toujours par le même processus d'anticipation rles phénomènes de division post-conjugale qui s'intercalent avant la fin de la conjugaison, ou par le fait de la multiplicité des noyaux. Le schéma ci-contre se comprend sans explication.

Nous décrirons à propos des Péritrichides les phénomènes encore plus compliqués qui se passent chez ces animaux.

Bien qu'on n'ait pu s'assurer ici formellement de la chose, comme on l'a fait pour Ascaris megalocephala, il est bien évident que la division du noyau conjugué en 
Iranelles détruiles, réparent leur péristome et toutes les parties endommagées par la soudure et reprement quelque activité (').

Dès lors, ils recommencent à absorber de la nourriture, grossissent, et l'élat normal est reconstitué. Bientòt ils se divisent, et c’est là le point de départ d’une nouvelle sćrie de générations agames.

Phénomines macronucléaires. - Qu'est devenu pendant ce temps le $\mathrm{N}$ ancien? Il s'est dítruit (fig. $7\left(17, \mathrm{~N}\right.$ et $\mathrm{N}^{\prime}$ ), et le $\mathbf{N}$ nouveau est destiné à le remplacer. Dès le délıut, il a commencé à se flétrir, à se déformer, à s'appauvrir en chromatine et, ver's le milieu de la conjugaison, on le voit se fragmenter en nombreux petils corps ( $E$ à $G: \mathrm{N}$ et $\mathrm{N}^{\prime}$ ), qui lentement dégénèrent $(I I$ et $I$ ) et sont traités par I'endoplasme comme de simples aliments qu'il digère et dont il rejette par l'anus les résidus inutilisables.

Mais cette disparition est assez longue à s'opérer et souvent, dans les produits de la deuxième division, après la conjugaison, on en retrouve encore quelques fragments. Il est possible qu'il y ait pendant la conjugaison échange par diffusion de quelques substances cytoplasmiques entre les conjoints. Mais on ne sait rien l'un pareil phénomène el, jusqu'à plus ample informé, la conjugaison des Ciliés doit ètre considérée comme une conjugaison nucléaire $\left({ }^{2}\right)$.

Phénomènes consécutifs à la conjugaison. - Nous arons vu que les divisions agames trop longtemps continuées engendraient la dégénérescence sénile. La conjugraison a pour effet de mettre un terme à cette dégénérescence. Sous linfluence de l'appareil nucléaire renouvelé, les produits de la première division post-conjugale atteignent en grandissant la taille maxima de l'espèce et tous leurs organes, péristome, cils, membranelles, etc., reprennent une conformation irréprochable. Ces acquisitions se maintiennent ensuite pendant de nombreuses générations agames, après quoi la dégénérescence sénile reparaît peu à peu et la série de phénomènes décrits se reproduit de nouveau. Il y a alternance régulière entre la conjugaison et une série de livisions agames ahoulissant à la dégénérescence.

Mais là se borne le rôle de la conjugaison et tout ce que l'on a dit

leux antres qui seront ceux des deux produits de la première division de l'Infusoire, ne sépare pas les substances micronucléaires des deux conjoints, réunies pour le former, et que chaque produit de la division emporte une moitié de la substance micronucléaire des deux parents. La conjugaison a donc pour effet, ici comme partout, la constitution d'un nouvel individu avec participation des substances de deux autres.

(1) Cette régénération va quelquefois très loin, Ghez beaucoup d'Ilypotrichides les cirres tombent jusqu'à une assez grande distance de la soudure et c'est l'oceasion d’une rénovation presque générale de l’appareil locomoteur.

2) Les cas de conjugaison totale observés par Engelnann chez les Stylonichia qui se fusionneraient entierement l'un dans l'autre méritent d'être confirmés et leur interprétation n'est rien moins que cerlaine. Par contre chez les Vorticelles il y a une conjugaison totale, mais nous l'examinerons en parlant des l'éritrichides. 
Ilu coup de foucl donné par elle à la reproduction agame a ćlé infirmé par Marps. Les produits d'une conjugaison récente ne se diviseut ni plus ni moins vile que les dégénérés prèts à se conjuguer de nouveau. La conjugaison a donc pour effel, non de réveiller une puissance reproductrice languissante, mais de réparer les délériorations conséculives à une rie trop longlemps entrelenue par des générations agames(').

Mérotomie. Régénération. - L'étude de la physiologie normale de notre type morphologique est maintenant terminéc. Mais nous devons encore parler d'un phénomène pathologique intéressant en ce quil nous renseigne sur les fonclions de son appareil nucléaire. Il s'agit le la régénération. Si on coupe le Cilié en deux parties dont l'une contiendra le $\mathrm{N}$ (et aussi forcément le n qui est accolé au premier), ce fragment nucléé ne lartera pas à régénérer toul ce qui manque el à reformer un animal entier. Mème si ce fragment est plus pelit que l'autre, mème s'il ne porte pas la bouche, il peut reformer l’individu complel. L'autre fragment au contraire peut continuer à vivre quelque temps, ses mouvements, sa nulrition ne sont pas brusquement abolis, mais il meurl fatalement, aussi incapable de se régénérer que de se reproduire $\left({ }^{2}\right)$. Cela nous montre que le $\mathrm{N}$ est nécessaire aux fonctions régétatives, el comme nous sarons qưil ne prend pas part à la reproduclion, nous pouvons ajouler que le $\mathrm{n}$ est l'organe exclusif de la division $\left({ }^{\mathbf{3}}\right)$.

Babmaxi [93] tire de ses célìhres expériences de mérotomie les conclusions suivantes :

Le noyau el le cytoplasma ne sont pas antagonistes, ils ont des fonctions, les unes différentes, les autres communes. Le plasma dirige

(1) Chez les Métazoaires, dans la succession des générations, les parents meurent laissant après eux des enfants qui représentent seulement une partie de leur subslance dont la masse s'est accrue par la nutrition. Chez les Ciliés, comme chez tous les Prolozoaires I'ailleurs (nais c'est surtout a propos des Infusoires que la question s'est posée), les parents ne meurent point, puisqu'en se divisant ils répartissent entre leurs deux descendants la totalité de leur substance et que celte substance continue tout entière a virre en eux. D'oń celte proposition de WEIsmans que l'Infusoire est immortel. Ce n'est pas ici le lieu d'entrer dans les longues discussions qui ont été soulevées à ce sujel ( $V$. Yres Delage [95]). Mais nous devons nous demander si la découverte de la dégénérescence sénile n'infirme pas l'idée d'immortalité de ces animaux. C'est l'avis de Matpas, mais cetle idée n'est pas juste. L'Infusoire est immortel puisqu'il a un moyen le ne pas mourir et que ce moyen n'est que la réalisation d'un phénomène plysiologique. II n’y a rien de semblable pour le Métazoaire pour lequel il n'existe aucun moyen de sauver de la mort la partie de son êlre qui n'est pas élément sexuel. Cela n'empêche pas l'Infusoire comme le llétazoaire de mourir très fréquemment d'accident, mais ce n'est pas là ce qui est en question.

${ }^{(2)}$ Le pouvoir de régénération est très variable chez les Cíliés, très fort chez stentor qui a servi à presque toutes les expériences, faible chez Paramacium, nul chez loxodes.

(3) Jubıx a tiré parti de ces faits pour chercher à donner la raison plýsiologique des phénomènes successifs de la conjugaison. Si le jeùne développe l'appétit sexuel c'est parce que le $\mathrm{N}$ est le premier atteint par la consomption qui en résulte, dès lors il perd son action sur la cellule qui tombe sous l'empire du n ou noyau reproducteur. 
les mouvements du corps, des cils, la préhension des aliments, l'évacuation des fíces, la contraction de la vésicule pulsatile, la division du corps dans la scission. Les fragments non nucléés sont, en effet, capables de tous ces actes. Mais le noyau est nécessaire pour la sécrétion, la régénération et la division. Pour ces fonctions, le cytoplasma agit, mais a besoin de l'influence du noyau (').

Lorsque le $n$ s'est divise en quatre, c'est simplement le commencement d'une reproduction scissipare. Mais comme le $\mathbf{N}$ n'est plus là pour diriger les phénomènes cytoplasmiques qui devraient l'achever, cette scission ne se prorluit pas et les trois $n$ inuliles se détruisent. Ciest par la mème raison que les premières divisions postconjugales ne s'achèvent dans le cytoplasma que longlemps après s'être produites dans l'appareil nucléaire. Elles ne peuvent s'achever, en effet, que lorsque les $\mathbf{N}$ se sont différenciés parmi les produits de la division du noyau conjugué. Tout cela est un peu hypothélique, mais assez suggestif.

(1) Historique. - Tous ces phẻnomènes de la conjugaison des Ciliés qui semblent si clairs, aujourd'hui qu'ils sont bien connus, ont été extrêmement difficiles à débrouiller. Il a fallu des générations de travailleurs intrépides pour y arriver. Bien des fausses voies ont èté suivies avant que l'on trourât enfin la bonne, et ce n'est qu'en 1889 que l'interpritation définitive a ėté enfin donnẻe par les admirables découvertes d'un chercheur hors ligne, IIAupas. Il n'est pas sans intérêl de retracer à grands traits l'historique de cette importante question et, par la même occasion, rle l'ensemble de nos connaissances sur les Infusoires cilies.

Les Infusoires ont étẻ décourerts à la fin du vue siècle par Leuwenhozk. Mais les procédés d'étude étaient trop grossiers à cette éporue pour que l'on pût se rendre compte le leur organisation. Un siècle plus tard, O.-F. MüLler les ètudiait aussi, avec plus de délail et découvrait en particulier la conjugaison. Nais, faute de microscope suffisant, les détails d'organisation échappaient encore à ses yeux. Il faut arriver à 1836 et à Ehrenberg pour trourer des éludes vraiment détaillées sur ces petits êtres.

Comme 0.-F. Müller, Ehrenberg considèrait comme Infusoires tous les animalcules microscopiques qu'il rencontrait dans les infusions ou dans les liquides naturels. S'il ne leur avait adjoint de ce chef que des Flagellés et des lihizopodes, il n'y aurait eu que demi-mal, mais il y fit entrer aussi les Dialomées, des Algues, des Champignons et même les Rolifères. Il fit de ces derniers une étude approfondie, leur découvrit un tube digestif, un appareil circulatoire et des organes génitaux, qu'ils possèdent réellement puisque ce sont des Vers et, les considérant comme le type des Infusoires, fut conduit à rechercher dans les vrais Infusoires tous les organes qu'il trouvait chez eux. Aussi, areuglé par ses idées préconcues, ne manqua-t-il pas de les trouver, sinon arec les yeux, du moins avec l'imagination. I'ailleurs, l'erreur est excusable. Les Infusoires ont une bouche, un anus. Il est naturel de penser que la portion moyenne du tube digestif n'est pas absente. Chez beaucoup d'entre eux, la vacuole qui contient les particules alimentaires n'est pas sans quelque ressemblance avec un estomac et a èté prise par Ehrenberg pour un estomac veritable. Lorsque plusieurs de ces vacuoles sont disposées à la file, il semble donc y avoir plusieurs loges stomacales. 1)e là ces fameux Infusoires polygastriques qui ont fait tant de bruil. Pour Ehrenberg, le nuclèus était un testicule, et il élait en rapport avec la vésicule contractile qui lui servait de résicule séminale. Les nombreuses granulalions arrondies que l'on rencontre dans le corjs étaient des a ufs. Enfin rien ne manquait aux Infusoires pour ètre des organismes parfaits.

Ces idées furent universellement acceptẻes par les savants et, il y a quelques années, étaient enrore enseignées par quelques professeur's. Dirarnis seul eut le mérite de ne pas se laisser éblouir et s'efforģa de démontrer que l'Infusoire n'est 
Tels sont les caractères et la physiologie de la forme moyenne que représente notre type morphologique. Dans un groupe aussi vaste et aussi varié que celui des Ciliés il s'y ajoute nécessairement bien des complications et bien des modifications. La classification va nous les faire connaître.

qu'une cellule et ne contient que du sarcode plus ou moins différencié, mais point d'organes proprement dits. Ces idées furent d'abord très mal accueillies, surtont en Allemagne, mais aujourd'luu ' fu'elles sont universellement acceptees on s'efforce de lui en ravir la priorilé pour la donner à Ton Siebold.

Après que les idèes de Ehrenberg eurent été dèmontrées fausses par Dujardin, puis par Claparède et Lachuans, la croyance à la présence d'une foule d'organes différenciés chez l'Infusoire, fut définitivement abandonnée, mais la doctrine de leur unicellularité ne fut pas encore établie pour cela. Car il restait un élément de doute: l'Infusoire paraissant contenir au moins des testicules et des œufs.

C'est Stern surtout qui, daus ses volumineuses publications, s'efforça d'établir cette idée. Selon lui, le noyau, élait sinon un osaire. du moins un organe reproducteur et le nucléole rtait un véritable testicule. Voici l'origine de ces opinions :

Quelque temps avant Stein, I. MïLler avait observé des filırilles dans le $\mathbf{n}$ et, sans se prononcer formellement sur leur nature, avait émis l'ilée que ce pouvaient être des spermatozö̈des. C'étaient, comme Balbiani le prouva plus tard, simplement des Bactèries. Stein réussit aussi à voir ces fibrilles nuclcolaires et affirma leur nature spermatique. Dès lors le nucléole élait un testicule. Il lui fallait un ovaire, il le trouva dans le noyau qui, en effet, se présente dans certaines conditions comme s'il donnait naissance à des oufs.

Stein décrivit ainsi l'évolution des produits sexuels. Deux Infusoires se montrent unis par la bouche. L'un et l'autre ont des filaments spermatiques dans le testicule et un corps reproducteur intact. Comme le volume des testicules s'oppose ì toute supposition d'échange des capsules séminales, il faut de toute néeessité que la fécondation ait lieu dans chaque individu par lui-même. Ce n'est qu'après la fécondation que le corps reproducteur se divise en partieules ayant l'apparence d'axufs. Mais ce ne sont done pas des oufs, car des œuf́s seraient fécondès eux-mèmes après leur formation. Il les appelle des sphères germinales. Queliue temps après la séparation, on trouve dans l'Infusoire une masse de petits bourgeons mobiles ayant une certaine ressemblance avee le petits Acinètes. Stein conclut de lá que du corps (nucléus) fécondé par les spermatozoïdes étaient issues des splères germinales qui s’ètaient lèveloppées en embryons. Ces observations avaient portees sur les Paramecium et les Vorticelles (Epistylis). Chez les premiers il se contente de constater une certaine ressemblance entre ces embryons et des Acinètes, mais chéz les seconds il reconnaît en eux de vrais Acinetes el, renversant une opinion dèja ancienne qu'il avait émise, considère les Aeinèles comme des larves de Vorticelles. Quant i la conjugaison, puisqu'il n'y a pas cchange, elle produit seulement une excitation réciproque qui provoque la maturation des produits sexuels.

BaLB1ANı, en 1860, eut le grand mérite de débrouiller ce fouillis compliquè d'observations vraies et de sèluisantes erreurs, et montra que ces prétendues larves de Paramiecium et d'Epistylis ne sont que de vrais Acinetes vivant en parasites dans le corps des autres Infusoires et appartenant a un genre créé par Claparède et Lachmann, le genre Sphrophry. Tout son travail montre une observation admirable. Malheureusement la technique n'était pas encore assez parfaite pour permettre une interprétation exacte de toutes les particularités et il tomba dans certaines erreurs. Il observa nettement la conjugaison des Paramxeies, reconnut l'échange des nucléoles et l'interpréta avec raison comme une fécondation réciproque. Mais il vit, malheureusement trop tòt pour les comprendre, les filaments protoplasmiques du $\mathbf{n}$ en voie de 
Nous diviserons la sous-classe des Ciliés en quatre ordres:

IIOLOTRICHIDA, dont les cils sont uniformes sur le corps el ne forment point autour de la bouche une zone adorale;

II ETEROTRICHIDA, dont les cilssont uniformes sur le corps et forment autour de la région buccale une zone adorale, c'est-à-dire une série courbe de cils plus forts soudés eu pelites lames appelées membranelles;

IIYPOTRICHID.1, dont les cils, absents sur le dos, sont différenciés sous le ventre en appendices plus gros appelés cirres, et forment autour de la bouche une zone adorale;

I'ERITRICHIDA, n'ayant point de cils sur le corps, sauf ceux qui forment la zone adorale et parfois une simple rangée circulaire vers l’extrémité inférieure.

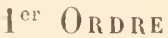

\section{HOLOTRLCHIOES. - IIOLOTRICIIIT.J}

[HoLotriciles; - HoLotricha (stein)]

Les Ilolotrichides sont des Ciliés à cils tous à peu près semblables entre eux. Ils peurent avoir des cils plus longs autour de la houche, mais ces cils ne sont jamais transformés en membranelles et ne conslituent pas une zone adorale distiucle.

Ils se divisent naturellement en deux sous-ordres:

(YYMNOSTOMIDE, à bouche ordinairement fermée quand elle ne fonctionne pas et dépourvue de membrane ondulante;

IIYMENOSTOMIDE, à bouche toujours ouverte et pourvue d'une membrane ondulante.

division et les prit pour des spermatozoïdes. Il vit les produits de la fragmentation du $\mathbf{N}$ après la conjugaison, les prit pour des oufs et crut que ces aul's élaient fécondes par les spermatozoüles issus des capsules spermatiques érlangées.

Englimann, en 1873 , repoussant toute ilée d'ovaire el de testicule, vit la fragmentation du $\mathbf{N}$, la division du $\mathrm{n}$, l'échange des fragments du $\mathrm{n}$, mais il crut que les $\mathrm{n}$ échangés servaient à féconder les fragments du $\mathbf{N}$ et interpréta. ces phénomènes, non comme une féconilation par spermatozolides et oeufs, mais par les fragments du noyau sexué.

Bütschu, le premier, en 1876, comprit bien la signification des fibrilles du nucléole. Il reconnut que le $\mathbf{N}$ se divise en fragments qui se détruisent el que tout lappareil nucléaire nouveau provient $d \mathbf{u} \mathbf{n}$, mais il assimila la destruction du $\mathbf{N}$ à une évacuation de globules polaires, et ne connut rien du sort réel et de l'évolution des produits de la division du $\mathrm{N}$.

Enfin, c’est Macpas qui, en 1889, a définitivement élucidé tous les points les plus difficiles de la conjugaison, fait connaitre l'évolution du $\mathrm{n}$, dícouvert la drgènérescence sénile et le ròle de la conjugaison pour en réparer les effets.

l"est sur sa lescription que nous nous sommes presque constamment guidés clans notre exposé. 
Ler SOUS-ORDRE

GYHNOSTOMIDES. - GYMIOSTO HIIU.E

[Grmostomes; - Grunostomata (Büstchli)]

TYPE MORPHOLOGIQUE

(FIG. 713 A 716)

Notre Gymnostomide est de taille modérée, mesurant environ 1/3 le millimètre de long. Sa forme est ovoüde, assez régulière. Il porte un

Fig. 713.

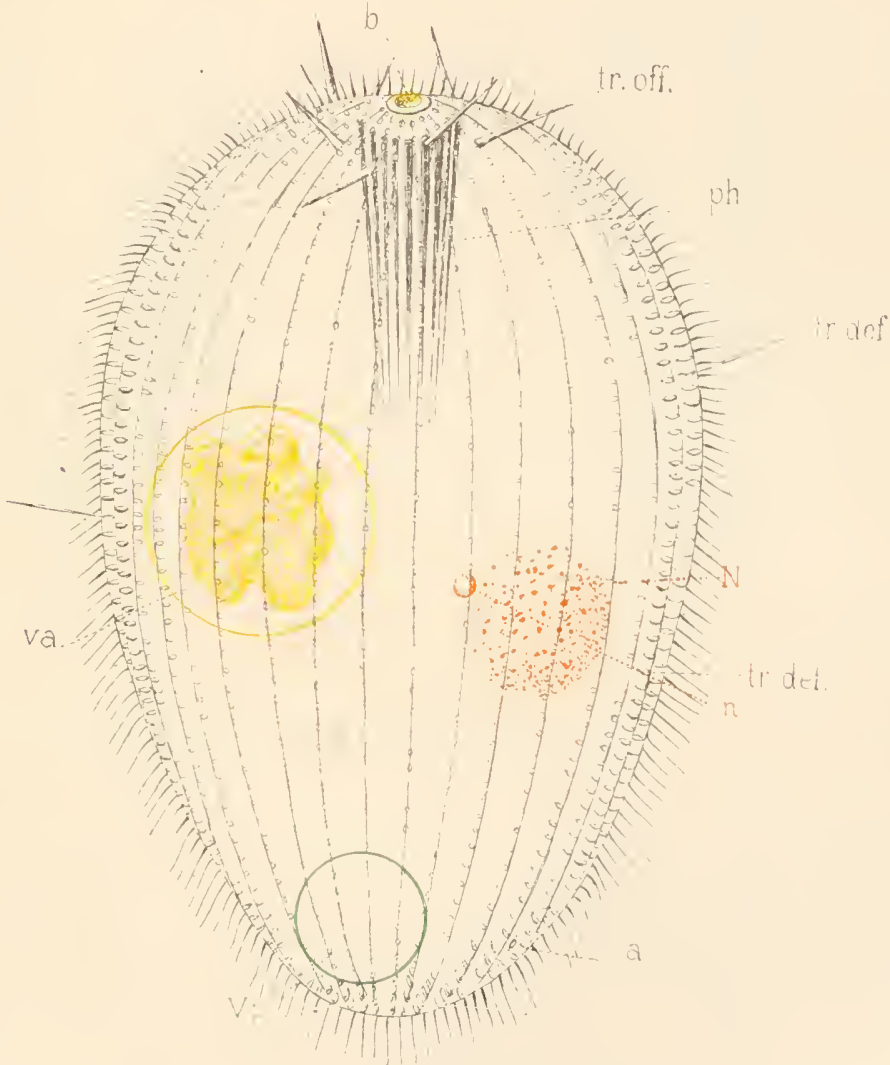

GYYNOSTO.HID.E Type morphologique) (Sch.)

a., anus; b., bonche; $\mathbf{x}$, macronucléus; $\mathbf{n}$, micronucléus; plz., pharrns;

tr. déf., trichocrstes defensifs : tr. off., trichoeystes oflensif́; : va., vacuole alimentaire: V. p., vésienle pulsatile.

revètement général de cils miformes et ceux qui entourent la bouche ne se distinguent des autres ni par leur forme ni par leur arrangement, ni mème par leur longueur. 
La bouche $(b$.$) est dans sa situation primitive, c'est-à-dire terminale.$ Elle n'est point pourvue d'un péristome et s'ouvre simplement à la surface du tégument. A l'état de repos, elle est fermée et n’apparaît que comme un petit pertuis (fig. 714), mais quand elle fonctionne elle se dilate considérablement (fig. 715 ). Il résulte de cette absence de péristome, de zone adorale, d'appentices buccaux quelconques, qui justifie le nom de Gymnostome, que l'animal s'alimente par un tout autre procédé que les autres Ciliés et que le type morphologique décrit précédemment. Il ne produit pas de tourbillon alimentaire pour absorber au hasard les

Fig. 71't

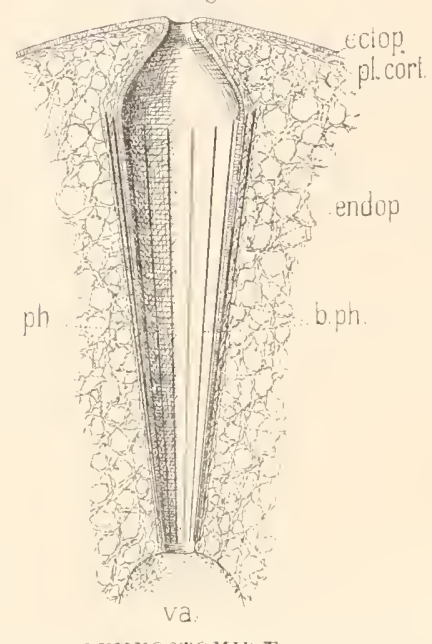

GYMYOSTOMLID.E.

Bouche et appareil pharyngien contractes (Sch.).
Fig. 715.

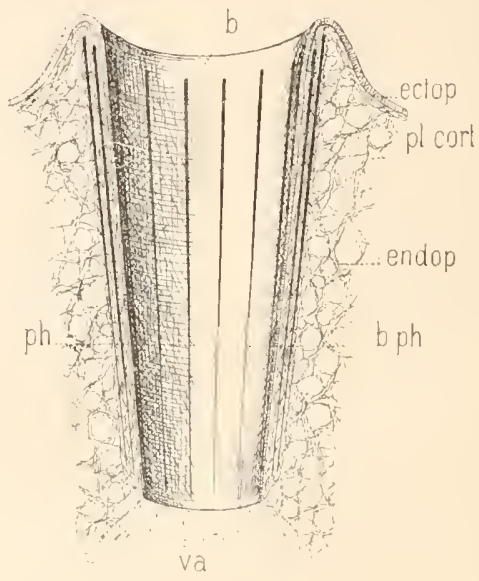

(i) UNOSTOMID.E.

Bouche et appareil pharyngien dilatés (Sch.).

b., bouche; 1. pla., bagueltes pharyngiennes; ectop., cetoplasme; endop., cndoplasme; ph., nasse pharyngienne; pl. cort., plasma cortical; v. a., vacuole alimentaire.

particules que re tourbillon pourrait entrainer. Il doit chasser sa proie, la poursuirre, l’atteindre. la luer et la déglutir par un acte particulier. Mais on ne chasse pas de celle manière une poussière nutritive, on ne peut altaquer ainsi que des proies relativement volumineuses. C'est ce yui a lieu en effel. Nolre chasseur se nourrit de Prolozoaires inférieurs ou mème d'Infusoires parfois presque aussi gros que lui. Celi explique la nécessité de cette bouche si dilatable.

En sa qualité de chasseur et de carnassier, il a des armes d'atlaque, ou, à l'occasion, de défense, et est pourvu d'une armature pharyngienne spéciale. Ces armes sont les trichocystes, cette armature est la nasse pharyngienne.

Les trichocystes sont de petils organes acérés, logés sous le légument, a moitié dans l'ecloplasme, à moilié dans le plasma cortical, en dehors 
des canalicules excréteurs. Ils sont de deux sortes. Les uns destinés à I'attaque (fig. $713, t r$. off.), les autres défensifs (fig. $713, t r$. déf.).

Les trichocystes offensifs sont de petits dards acérés, disséminés autour de la bouche ou dans ses parois, que l'animal projette comme des flèches à une assez bonne distance de lui par une simple contraction qui a son origine hors du trichocyste, en sorte que celui-ci ne subit pas de modification dans sa structure quand il est projeté. On le retrouve hors du corps a vec le mème aspect qu'il avait sous le tégument. Lorsque l'animal en chasse a rencontré une proie, souvent un Infusoire plus gros que lui, il lıi décoche ces petites flèches et le paralyse, gràce sans doute à quelque venin spécial dont elles sont mouillées. Toujours est-il que l’ètre atteint est non pas tué, mais paralysé, car sa vésicule pulsatile continue à battre lentement; il devient inerte el peut c̀tre dévoré d'une pièce ou déchiqueté par son ennemi. On voit parfois celui-ci revenir à la charge plusieurs fois sur la même victime, la heurtant de sa tète et lui décochant chaque fois une nouvelle volée de traits.

Les trichocystes défensifs sont disposés en couche régulière sur toute la surface du corps. Ils ont la forme d'un ovoïde très allongé dont la grosse extrémité est surmontée d'une petite pointe couique qui, sans doute, sert à transmettre l'excitation qui détermine l'explosion. Car c'est une sorte d'explosion qui se produit, par laquelle le trichocyste se développe avec la rapidité de l'éctair en une aiguille fine et acérée d'une lougueur dix fois supérieure à la sienne (fig. 716). Cette aiguille, en raison mème de sa longueur, dépasse de beaucoup la surface et peut blesser l'ennemi. Elle est elle-mème entraìnée d'ordinaire hors des téguments et tombe à côté de l'animal.

Les trichocystes des deux sortes semblent formés d'une substance plasmatique durcic (").

(1) On ne sail pas au juste quelle est cette substance et on ne sait pas du tout par quel moyen les trichocystes défensifs subissent la transformation soudaine que nous venons de décrire. On a pensé à un filament spiral bandé sous une membrane qui éclaterait à un moment donné. Mais on ne retrouve rien de celte prétendue membrane et l'on n'a jamais vu ce prétendu spiral. C'est une élongation brusque d'un ovöile en une aiguille. Au bout de la pointe du trichocyste défensif, on retrouve une petite masse (fig. 716) qui serait peutêtre un reste de l'appareil, entraîné là par l'explosion.

Les Gymmostomidx n'ont pas tous des trichocystes, les Enchelinx n'en ont souvent pas, Lox'odes et d'autres encore en sont dépourvus. Pour l'ensemble des Holotrichides voici, d'après Maupas, la liste des genres où on en rencontre, et encore pas toujours dans toutes les espèces. Paranxcinm, Cyrtostonum, Tillina, Nassula. Ophryoglena, Plenroneula, Prorodon. Enchelys, Lacrymaria, Lagynus, Didininm, Fig. 716. Amphileptus, Dileptus, Trachelius, Loxophyllun, Urocentrum. Parmi

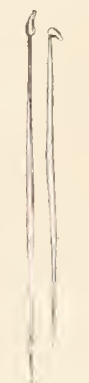
les autres ordres, on en trouve seulement chez un Hétérolrichide, Stroubidium (S. sulcatum el S.urceolare) et chez un P'éritrichide, Epistylis (E.umbellaria). Enfin on en trouve, parmi les autres classes, chez deux Polykrikos qui sont des Dinoflagellés et 
Le pharynx(fig. 71', 715้) est très léveloppé, plonge profondément dans l'endoplasme (endop.) et est entouré d'une armature de bagueltes (b.ph.) que l'on a comparée, en raison de sa forme, à une nasse de pêche et nommée pour cela la nasse pharyngienne. Le pharynx lui-mème ne présente rien de particulier; mais la couche de plasma cortical ( $p l$. cort.) invaginé qui l'entoure est parliculièrement épaisse. Dans cette couche et à une certaine distance de l'ectoplasme pharyngien, sont logées des luaguettes prismatiques disposées parallèlement à son axe, côte à côte autour de lui, de manière à lui former une sorte de garniture externe. Ces baguettes $(b . p h$.$) se terminent en haut par une extrémité coupée à pic, à$ quelque distance au-dessous de la bouche. En bas, elles se perdent insensiblement en pointe, loin au delà de l'extrémité inférieure du pharynx. Cette armature n'est rien autre chose qu'un squelette servant à donner une certaine rigilité au pharynx et à lui permettre d'obéir par des mouvements d'ensemble aux contractions du protoplasma ambiant. Gràce à elle, le pharynx peut ètre partiellement projeté hors de la bouche et, là, dilater son ouverture pour saisir la proie. Après qu'il l'a saisie, il peut se refermer sur elle, rentrer dans le corps et peu à peu la faire cheminer jusqu'à son orifice inférieur. Ces baguettes sont formées d'une substance albumineuse condensée, car la pepsine les digère (').

Tel est le type morphologique des fiymnostomides. Pour le reste : structure du cytoplasme, vésicule pulsatile, corps nucléaire, il ne diffère en rien du type précédemment décrit.

Le caractère principal dont la variation va nous permettre de classer les Gymnostomides est la position de la bouche qui, de sa situation terminale primitive, va peu à peu descendre sur la face ventrale et, dans les derniers types du sous-ordre, nous montrera une vague ébauche de péristome et de zone adorale. Comme caractère secondaire, nous aurons à envisager les modifications de l'appareil ciliaire qui, au lieu de rester uniforme, va disparaître à certaines places, se modifier à certaines autres. Enfin, des caractères empruntés sans ordre aux autres organes nous permettront de définir les principaux genres.

chez un Raphidomonas qui est un Euflagellé. Il y en a aussi chez un Tentaculifère, opleriodendron.

(1) Il ne faut pas confondre avec l'armature pharyngienne vraie ou nasse, l'appareil de bâtonnets que l'on observe chez les genres Enchelyodon, Pseudosporodon, spathidium, Trachelophyllum, Lacrymaria et autres. Ces bàtonnets, malgré leur disposition assez régulière autour du pharynx, ne lui sont pas liès à titre d'appareil squelettique et ce pharynx n'est pas protraclile; ils peuvent être projetés par l'animal et ne sont au fond que des trichocystes d'altaque. Bloghmaxx les désigne quelquefois sous le nom de trichites. Ils ne nous paraissent lifférer en rien d'essentiel de ce que nous avons appelé. avec Maupas, trichocystes offensifs.

Celte forme intermédiaire donne à pensel que les bàtonnets de l'armature pharyngienne et les trichocystes ne sont peut-être au fond qu'une mème production morphologique, lantòt différenciée pour l'attaque ou la défense, tantôt fixe et adaptée à un rôle de soulien. 


\section{GENRES}

Nous trouvons d'abord une longue série de genres chez lesquels la bouche est terminale ou à peine prolongée vers la face ventrale ("). Holophrya (Ehrenberg) (fig. 717), par la simplicité de sa structure et ses caractères un peu négatifs, peut servir de point de départ. Il n’a pas de trichocystes et son armature pharyngienne est à peine indiquée $\left(0,4\right.$. Ner et eau douce) $\left({ }^{2}\right)$.

Enchelys (Ehrenberg) (fig. 7 IS) s'en distingue par son corps étiré vers le haut en forme de cou. Malgré sa petite taille et gràce à ses trichocystes d'attaque très développés, il est beaucoup plus carnassier que le précédent et ne craint pas de s'attaquer ì des Paramécies qui sontiles géants auprès de lui $(0,02$ à 0,2 . Her et eau douce $)\left({ }^{\mathbf{3}}\right)$.

Prorodon (Ehrenberg) (fig. 719) n'a, inversement, que peu de trichocystes, mais son armature pharyngienne est très développée (1 ${ }^{\mathrm{mm}}$ et plus. Eau doure) (").

(1) Cette série qui s'étend jusqu'au genre Pompholixia inclus (V. P. 439) constitue la famille des ExcIIELIY.L [Enchelina (Ehrenberg, emend., Stein)].

(2) Pour le reste, par sa forme simple, sa eiliature uniforme, sa bouche terminale, sa vacuole terminale et voisine de l'anus, etc., etc., il est bien conforme au type morphologique.

Genre voisin :

Ichthyophthirius (Fouquet) ( 45 p. Parasite sous la peau de certains Poissons l'eau douce).

Ici semblent devoir prendre place:

Perispira (Stein) qui ne s'en distingue rque par la forme spirale de ses stries ciliaires (b̆0 à $60 \mu$. Eau douce stagnante); et

Blepharostoma (Cheviakof lmalgré ses cils péribuccaux deux fois plus longs que les autres et l'absence de pharynx (1: $\mu$. Eau douce).

(3) Genres voisins :

Enchelyodon (Claparède et Lachmann), qui a autour du pharynx des trichites simulant une armature pharyngienne (0,3. Eau douce, stagnante);

Pseudosporodon(Blochmann), qui est de forme cylindrique $(0,45$. Eau douce);

Spathidium (Dujardin) (fig. 720), obliquement tronqué en haut au niveau de la bouche $(0,4$. Eau douce);

Chænia (Quennerstedt), qui est pourvu d'une ceinture supérieure de cils plus grands $(0,2$ ว. Mer $)$;

Cephalorhynchus (Diesing) n’est qu'une espèce du précédent.

(4) Genres voisins :

Cranotheridium (Cheviakiof) a son armature ressemblant à celle de Nas-

Fig. 719.

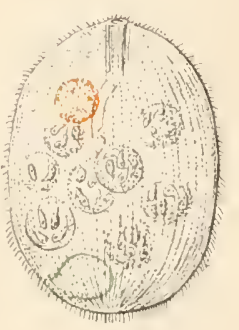

Prorodon

(P. teres)

(im. Bütschli).

Fig. 720.

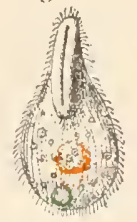

Spathdium

(s. Lieberkühnii)

(d'ap. Bütschli). sula (V. plus loin). Il est remarquable par son anus et sa vésicule pulsatile termi naux, et par ses n multiples annexés à un $\mathbf{N}$ unique (0,17. Eau douce);

Urotricha (Claparède el Lachmann) se distingue de Prorodon par une soie à l'extrémité inférieure $(0,04$. Eau douce); 
Lacrymaria (Ehrenberg, s. lat.) est un ancien grand genre caractérisé par sa forme en bouteille et par une ceinture de longs cils au-dessous de la bouche (Atteint 0,8. Eau douce) (').

Actinobolus (Stein), par sa forme en toupie, sa ciliature uniforme, sa bouche terminale munie d'une petite armature pharyngienne, se rattache aux précédents; mais il mérile une description spéciale en raison d'un caractère très particulier. Quand on l'examine pendant qu'il nage (fig. 721), on ne lui trouve rien de spécial. Mais on remarque que son corps est garni de trichocystes de 10 u. de long, en forme d'épingles qui seraient fichées dans le corps parleurs pointes et saillantes hors de la membrane par

Fig. 721.

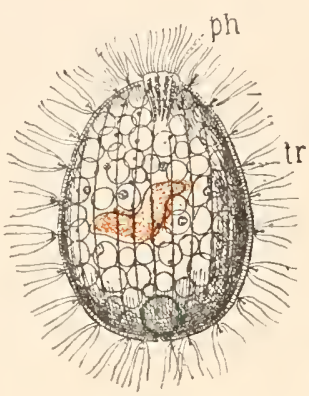

Actinobolus

(A.radians)(d'ap. Erlanger). Aspect de l'animal pendant la natation.

wh.,pharynx; tr., trichocystes rétractés.
Fig, 722.

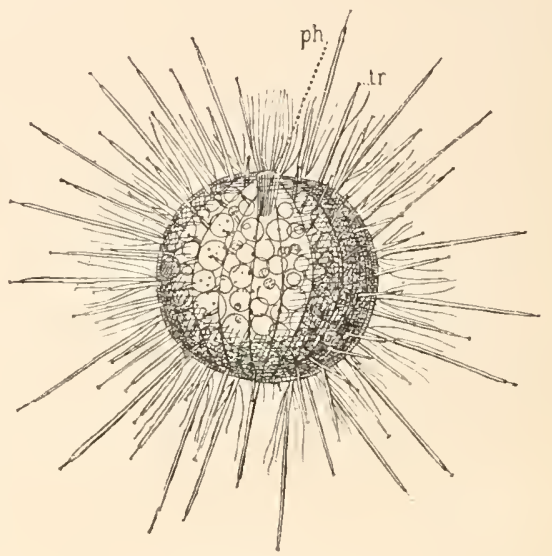

Actinobolus

(A. radians) (d'ap. Erlanger).

L'animal ì l'état de repos.

ph., pharynx; tro, trichocystes à l'extrémité des prolongements pseudopodiformes. rêter (fig. 722), on voit des trichocystes $(t r$.$) sortir peu à peu du corps,$ portés au bout d'un long el fin prolongement que l'on a assimilé à un pseudopode. Ces sortes de pseudopodes sont plus longs que le corps, un peu élargis à la base, cylindriques dans le reste de leur étendue. Les trichocystes occupent leur extrémité. On ne les a pas vus éclater naturel-

Balanitozoon (Stokes) est un genre douteux se rattachant au précédent (14 p.. Eau douce); Dinophrya (Bütschli) a sa bouche terminale portée sur un còne sans cils et entourée à sa base d'une couronne d'environ vingt groupes de cils (0,1. Eau donce).

${ }^{1}{ }^{1}$ Il a été dédoublè en plusieurs genres et sous-genres :

Lacrymaria (Ehrenberg, s. str.), en bouteille à long col, à rangées de cils spirales 0,8 . Mer et eau douce);

Phyalina (Ehrenberg) qui n'est qu'un sous-genre du précédent;

Lagynus (Quennerstedt), en bouteille à cou court, à pharynx entouré de trichites $(0,16$ à 0,18 . Ner et eau douce);

Trachelophyllum (Claparède et Lachmann) qui, un peu plus aplati, n'est qu'un sous-genre du précédent ( 0 2. Mer et eau douce);

Trachelocerca (Ehrenberg, cmend. Cohn), très allongé, à bouche quadrilobėe (atteint $3 \mathrm{~mm}$. Nerl;

Vasicola (Tatem) 0,13 . Eau douce) et

Metacystis (Cohn) (30 p. Eau douce), sont des formes douteuses, sans doute voisines. 
lement mais, sous l'action des réactifs, ils font jaillir au delà de leur tête terminale un petit acicule très acéré en continuité de substance avec le corps du trichocyste. Quand l'animal veut de nouveau se mettre en marche, il rétracte lentement ces pseudopodes, les trichocystes s'enfoncent d'abord dans le bout de leur pseudoporle, puis ceux-ci se retirent dans le cytoplasma, ne laissant passer que la tête du trichocyste. D'ailleurs, ces pseudopodes, en se rétractant, semblent se fondre entièrement dans le cytoplasma comme s’ils n'avaient aucune individualité persistante. A la base des pseudopodes, l'ectoplasme est interrompu, ce qui fait qu'ils semblent émaner de l'endoplasme $(0,1$. Eau donce $)$ (").

Actinobolus est quelque peu isolé dans la série naturelle de ces formes. On lui rattache cependant une autre forme aberrante, c'est le genre

Ileonema (Stokes) (fig. 723). Il a la forme d'une bouteille dont le col porterail la bouche au sommet et renfermerait le pharynx muni d'une armature bien développée. Près de la bouche, naît une sorte de gros tentacule formé d'une partie basilaire contournée en vis et d'un filament terminal plus mince. Le tout est mobile, mais n'a pas de mourements réguliers et semble servir plutòt à fixer temporairement l'animal. Le filament est aisément rétractile dans la partie contournée et celle-ci peut aussi rentrer daus le corps (0,2. Eau douce, Amérique).

Nous allons maintenant rencontrer des formes qui ne sont plus uniformément ciliées sur toute la surface du corps. Dans le genre Bütschlia (Schuherg) (fig. 729), les cils paraissent se réduire à une couronne péribuccale, à queliues touffes formant au milieu du corps une ceinture incomplète et à un petit houquet situé à l'extrémité inférieure. En réalití, la ciliature est complète, mais les autres cils sont très petits, assez espacés, en sorte qu’il est difficile de la voir. La réduction ne porte donc ici que sur la taille des cils généraux. On n'est pas sùr qu’il y ait une vésicule pulsatile et, dans la partie supérieure du corps, se trouve, près de la surface, une accumulation de particules colorées, brillantes dont la signification est assez peu claire. Serait-ce des grains d'excrétion? (0,06. Panse des Ruminants en compagnie des Isotrichines et des Ophryoscolécides) (V. plus loin) ${ }^{\boldsymbol{*}}$ ).

(1) Parfois cependant, on voit deux lignes fines continuer vers l'intérieur le tentacule à demi rétracté.

C'est cette absence d'individualité qui a permis de comparer ces sortes de tentacules à des pseudopodes. Mais s'ils ne sont pas de vrais tentacules il faut reconnaìtre qu'ils diffèrent aussi beaucoup des pseudopodes des Rhizopodes. On a cherché aussi à les assimiler aux tentacules des Acinètes, on a mème tenté d'établir ici un groupe de Cilio-tentaculifères. Mais celte assimilation n'a rien de réel.

(2) C'est Eberleix [95] qui tout récemment a reconnu que Bütschlia a ce revêtement 
Stephanopogon (Entz) (fig. 723̈), est de forme un peu allongée, beaucoup

Fig. 725 .

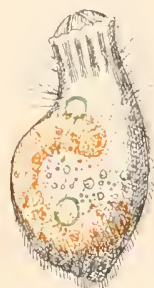

Stephanopogon

(S. colpoda)

(d'ap. Entz). moins épais que large, aplati à la face ventrale, bomlsé sur le dos, rendu asymétrique par le fait que le noyau en fer à cheval détermine une forte voussure du bord droit. La face ventrale est seule ciliée et ses cils sont dirigés suivant des lignes légèrement spirales. La bouche, allongée en forme de fente, est bordée par quatre saillies dentiformes formées en réalité par des cils soudés et qui, tantôt sont immobiles et tantôt vibrent rapidement. Il y a sur le corps quelques soies plus fortes que les cils ordinaires. Le pharynx est entouré de lignes sombres représentant sans doute une armature pharyngienne peu développée. Il y a deux vésicules contractiles $(0,07$. Mer).

Coleps (Nitzsch) (fig. 726), est une forme bien remarquable par la présence d'une carapace fort compliquée. Le corps, cylindrique, obtus à l'extrémité inférieure, est tronqué en haut par la bouche qui occupe toute la largeur de cette extrémité. Il est revêtu de quatre verticilles superposés de pièces squelettiques $(p$.$) . Ces$ pièces sont allongées, rectilignes d'un côté, dentées en scie de l'autre. Il résulte de cette disposition que le corps est à nu entre ces dents et c'est par ces orifices ménagés entre elles que sortent les cils longs et rares. L'extrémité inférieure est abritée par une calotte de pièces plus petites, laissant au pôle même une ouverture pour le pore excréteur qui se trouve là et pour l'anus situé tout à côté. Les pièces du verticille supérieur se terminent par une dent acérée tournée vers la bouche. La bouche s'ouvre au centre de cette couronne de dents et porte, en outre, une couronne de cils. Le Coleps est très carnassier, attaque les plus grros Infusoires en les déchirant au moyen de

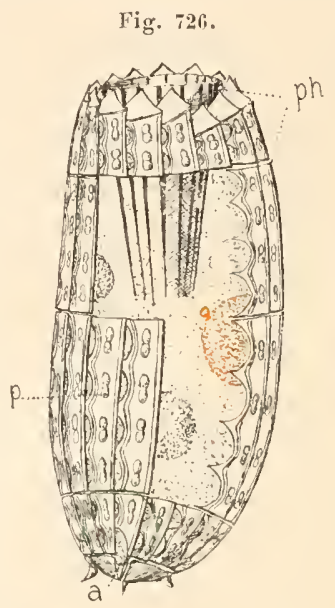

Coleps (im. Maupas).

a., anus; p., plaques du test; ph., pharynx. ses dents qui, par le jeu des pièces qui les portent, peurent s'écarter ou converger vers le centre de la bouche comme celles d'un Oursin. La carapace est hyaline, formée d'une matière organique durcie, sans éléments minéraux et, en somme, pas très résistante. Elle est un produit de sécrétion (0,0弓. Eau douce) (").

uniforme de très petits eils assez espacés sur des lignes un peu spirales. Avant celte constatation, re genre aurait dì prentre place entre Didinium et Mesodinium.

(1) Maupas à qui sont empruntés la plupart de ces détails, trouve le $\mathbf{n}$ sous la membrane du $\mathbf{N}$. Mais est-ce bien le $\mathbf{n}$ ?

A ces deux formes principales se rattachent encore les genres

Plagicpogon (Stein) sans carapace (Eau douce) et

Tiarina (R.-S. liergh), cuirassé comme Coleps (Mer`. 
Bien plus réduite encore et plus singulièrement disposée est la ciliaLure dans les genres suivants qui sont derniers de cette série:

Didinium (Stein) (fig. 797) dont le corps est cylindrique, trapu et se terminant en haut par un col conique, au sommet duquel est la bouche aussi remarquable par son extrème étroitesse que par son étonnante dilatabilité qui permet à l'animal d'engloutir des proies presque aussi grosses que lui (fig. 728). Didinium est d'ailleurs armé pour les chasser de trichocystes offensifs dont il larde sa victime à distance. Sa ciliature est réduite à quelques rangées annulaires perpendiculaires à l'axe (Moins de 0,2. Eau douce) (').

Mesodinium (Stein), chez lequel la réduction

Fig, 727.

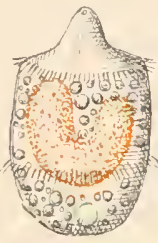

Didinium.

(D. Balbianit) (d'ap, Balbiani). (d'ap. Balbiani).

des cils est poussée à sos dernières limites, puisqu'il n'y en a plus qu'une seule couronne. Le corps est ì peu près sphérique et surmonté d'un prolongement conique presque aussi gros que lui, rétractile et portant la bouche à son sommet. L'unique couronne de cils est à l'union du corps et de ce prolongement; mais ces cils sont longs, coniques et très gros. Quatre d'entre eux, insérés un peu plus en dedans que les autres, sont relevés vers la bouche. Les autres, plus nombreux, sont étalés ou rabattus vers le has. Des bords de la bouche, partent quatre courts tentacules rétractiles non constants que l'on a comparés à des pseudopodes. C'est par leur moyen sans doute que l'animal peut se fixer ( $40 \mu$. Mer et eau douce) $\left(^{*}\right)$.

(1) On a décrit et dessiné une trompe à cel animal, mais MAUPAS a montré rqu'elle n'existait pas et que son apparence était due à des trainées de l'endoplasıne de la victime que Didinium entraine lorsqu'il bondit en arrière après une attarfue.

Monodinium(Fabre-Domergue) n'est qu'un sous-genre du précédent (40 «. Mer et eau douce).

(2) Genre voisin :

Askenasia (Bloclımann) ( $50 \mu$. Eau douce stagnante).

Il existe dans la cavité générale des Siponculides (Siponcles, Phascolosomes) de petits organites que divers auteurs considèrent comme un Péritrichide parasite:

Pompholixia (Fabre-Domergue). Le corps a l'apparence d'une grande vésicule transparente, ayant la forme d'une sphère tronquée à la partie inférieure (fig. 729, $\boldsymbol{A})$. Cette partie tronquée est fermée par une lame épaisse borlée d'un cercle de

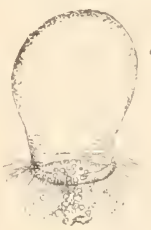

A

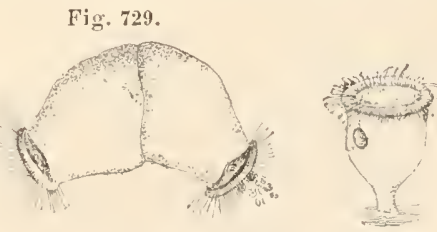

B

P'ompholixia $A$ et $B$ (d'ap. Fabre-Domergue). cils puissants et bombée au centre sous la forme d'une papille saillante dans la vésicule. On n'a point vu de bouche. La division a été observée $(B)$ ' $90 \mu$. Mer).

Cette strueture ne se laisse guère ramener à celle d'un Infusoire. D'autre part, divers observateurs assurent que ces prétendus parasites sont des organes de l'hòte. Ce seraient des entonnoir's ciliés (urnes), fixés sur le péritoine par un pédoneule creux $(C)$ et faisant communiquer la cavité générale avee le schisocèle sous-jacent 
Ici commence une nouvelle série de genres où la bouche prend la forme d'une fente partant de l'extrémité supérieure et s'élevant sur la face ventrale, ou même se porte tout entière à la face ventrale et reste séparée de l'extrémité supérieure par un lobe frontal plus ou moins développé. On peut considérer ce second cas comme dérivant du premier par le faitque la partie supérieure de la fente buccale se serait suturée, sur une certaine étendue('). Amphileptus (Ehrenberg) (fig. 730) a la bouche encore peu ventrale (Atteint 0,2 . Mer et eau douce).

Cet orifice ne descend guère plus bas chez Loxophyllum (Dujardin) (fig. 731), mais la partie supérieure de la fente se ferme et la portion du corps qui surmonte la bouche se développe en un lobe frontal. Il n'y a de cils que du côté droit $(0,04$. Ner et eau douce).

Cette disposition s'accentue bien plus dans les genres Lionotus (V'rzesniovski), semblable à Amphileptus, mais à trompe beancoup plus longue et aplatie $\{0,4$. Mer et eau douce); Trachelius (Claparèle et Lachmann), de forme plus ramassée et pourvu ordinairement, à la base de la trompe, outre sa bouche, d'une dépression infundibuliforme située vers le milieu du corps, et que l'on a prise pour la bouche avec laquelle elle n'a rien de commun $(0,4$. Eau douce); et

Dileptus (Dujardin) (fig. 732). Ce dernier est très étroit par rapport à sa longueur. Son corps ovoüde, allongé, se prolonge en haut en un très long lobe frontal appelé souvent la trompe. Cette trompe est entierement mobile et peut se contourner en tous sens. Elle est garnie le long de la ligne ventrale d'une bande de trichocystes et, de chaque côté de cette bande, d'une rangée de cils plus longs et plus forts que ceux du corps et dessinant une sorte de zone adorale. Ces deux rangées, en effet, descendent jusqu'à

au péritoine. Ce seraient des appareils destinés à passer le sang (Cuenot).

Il est à remarquer que chez les Synaptes on a observé des appareils tout semblables qui servent à faire passer les globules de la cavité générale dans le Schisocèle.

(1) Cette série, jusqu'à Loxodes inclusivement, constitue la famille des TRACHELINA [Trachelina Ehrenberg, emend. Stein)].

Avec Bütschli, nous avons placé dans la famille

Fig. 730 .

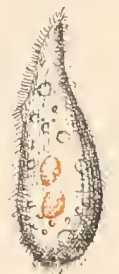

A. Claparedi) (d'ap. Entz).
Fig. 731 .

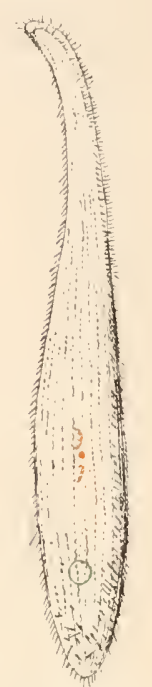

Loxophyllum

(L. duplostria(um)

(d'ap. Maupas).

Fig. 732 .
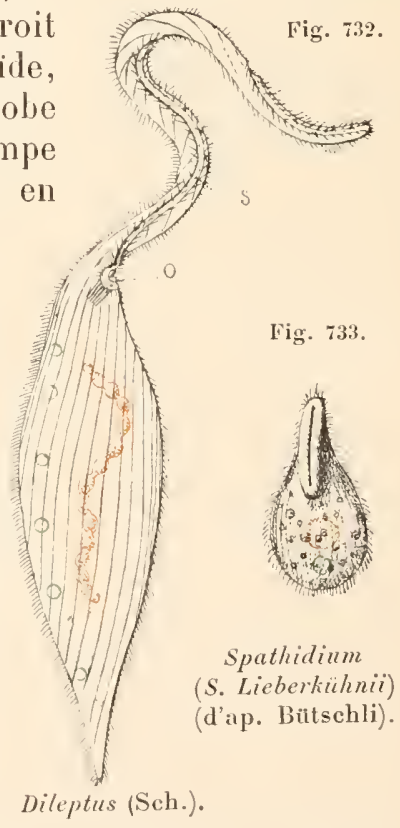

Dileptus (Sch.). précédente le genre spathidium (fig. 733) qui serait peut-être mieux ì sa place ici 
la bouche, l'entourent et se jettent l'une dans l'autre, au-dessous d'elle. Le pharynx a une armature bien développée. Toute la surface du corps est garnie de cils uniformes et parsemée de trichocystes. Le $\mathrm{N}$ est long et en chapelet et d'assez nombreux n sont annexés à ses renflements ( $1 \mathrm{~mm}$. Her et eau douce).

Chez toutes ces formes la trompe était rejetée en arrière; il en est autrement dans le genre

Loxodes (Claparède et Lachmann)(fig. 731), forme remarquable à plusieurs égards. Le corps est fusiforme, allongé, aplati et garni de cils sur la face ventrale, convexe et pourvu seulement de soies tactiles sur le dos. Les bords, que l'animal porte d'ordinaire relevés sur le dos, sont, en outre, garnis d'une rangée de fines soies. L'extrémité supérieure est recourbée en crochet vers la ganche. Au niveau de la base de ce crochet, au côté gauche de la face ventrale, est creusé un court péristome vertical qui s’approfondit en bas où il plonge pour former la bouche et le pharynx. Le bord inférieur et le còté gauche de ce péristome donnent insertion à une membrane qui s’appuie sur le bord droit, mais sans se souder à lui, de manière à fermer l'entrée du péristome, tout en lui laissant la facilité de s'ouvrir. L'endoplasme est creusé de Fig. 73t.

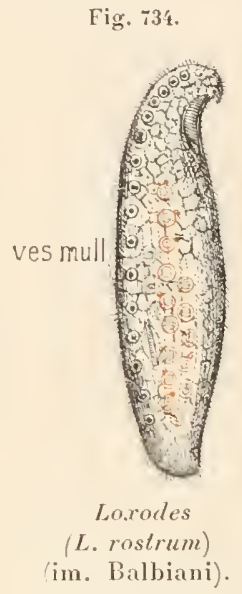

Ves. mu11., Vúsicules de Muller. vacuoles si nombreuses et si grosses qu il prend l'aspect d’un réticulum à grosses mailles. Mais aucune de ces vacuoles n'est pulsatile. Peut-ètre l'anus, qui est subtermino-dorsal, snffit-il à l'expulsion des liquides. Le long du bord droit, dans le plasma cortical, est une rangée de petites vacuoles bien rondes contenant chacune un ou deux grains d'excrétion. On les nomme les vésicules de .Müller. Enfin, par une exception peut-ètre unique, on trouve ici de nombreux $\mathbf{N}$ qui sont bien de vrais $\mathbf{N}$, car on trouve en outre d'incontestables n en général associés chacun à un $\mathbf{N}(0,:$. Eau douce).

Ici, commence une troisième et dernière série de genres où la bouche est franchement ventrale, en ce sens que la partie qui la sépare de l'extrémité supérieure n'est plus seulement une portion rétrécie en trompe, mais une partie aussi large que le reste(').

Nous allons d'ailleurs voir cet orifice descendre progressivement sur la face ventrale jusqu'à atteindre toul à fait la partie inférieure du corps.

Nassula (Ehrenberg) (fig. $73 \ddot{3}$ ), est en forme de cylindre,

et, tout au moins, fait la transition aux Trachelines par sa

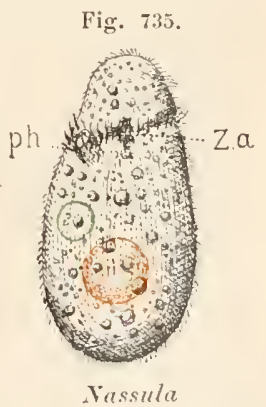

(N. aurea)(im. Bütschli). longue bouche fusiforme s'étendant sur la face ventrale.

(1) Ces genres, jusqu'à la fin du sous-ordre constituent la famille des CIILAMYDo. DOVTIXE [Chlamydodonta (Bütschli)]. 
un peu aplati dorso-ventralement et arrondi aux deux bouls. L'extrémité supérieure est ordinairement un peu inclinée sur le côté gauche. La bouche est assez has sur la face ventrale; elle est entourée de quelques gros cils qui partent de son bord droit, passent au-dessous d'elle puis, de là, se portent sur le côté gauche du corps, le contournent, passent à la face dorsale et s'y lerminent à peu près à l'opposé de la bouche, formant ainsi une sorte de demi-ceinture. On peut y voir une vague indication de zone adorale. Le reste du corps est partout revêtu de cils uniformes. La bouche dont nous venons de voir la position ne conduit pas directement dans le pharynx. C'est l'entrée d'un petit vestibule dont le fond convexe porte, au centre, l'orifice pharyngien. Le pharynx est garni d'une armature en nasse très développée. Le corps est pourvu d'une couche générale de trichocystes. L'anus est assez haut sur la face ventrale. Il y a tantôt une seule vésicule pulsatile à la place ordinaire, tantôt trois ou quatre disséminées plus haut $(0,03$. Ver et eau donce $)$ (').

Nous venons de voir que, chez Nassula, la ciliature étail générale et uniforme. Peut-être chez

Orthodon (Gruber) y a-t-il encore des cils partout, mais en tout cas ceux du dos sont beaucoup plus fins que les autres $(0,26$. Ner et eau douce).

Chez les genres suivants, ils ont tout à fait lisparu de la face dorsale. Tel est

Chilodon (Ehrenberg) (fig. 736). Ce Cilié a une forme oroïde comprimée dorso-ventralement; il est plat et cilié en avant, bombé et nu en arrière, du moins dans sa moitié inférieure; l'extrémité supéricure est inclinée sur le côté gauche comme chez la Nassule, mais pas assez pour déterminer, comme parfois chez celle-ci, un pli latéral. De la bouche, située vers le quart supérieur de la face ventrale, part une rangée de cils à peine plus forts que les autres, constituant une sorte de zone adorale. Cette rangée de cils se dirige en ondulant de la bouche vers le sommet morphologique du corps qui est rejeté à gauche. L'armature pharyngienne est puissante. L'anus est ter-

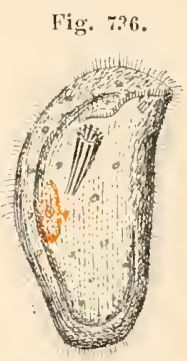

Chilodon (C. cucullus) (im. Stein). mino-ventral, les résicules pulsatiles sont petites et nombreuses; le $\mathrm{N}$

(1) L'endoplasme est très vacuolaire et ses vacuoles sont remplies d'un liquide bleu qui paraît provenir des Oscillaires dont ce Cilié fait sa nourriture (plycochrome). A leur intérieur, on peut trouver des granulations d'un bleu intense, sans doute graisseuses. On trouve, au bord gauche de la face dorsale, sous le sillon qui va à la bouche, une tache pigmentaire bleue très nette; on a voulu $y$ voir un foie ou un oil. Mais ce n'est qu'un groupe permanent de ces vacuoles à suc bleu dont parfois quelques-unes sont détachées par la cyelose et entraînées dans l'endoplasme. Au noyau unique sont associés un ou plusieurs petits nucléoles. Nassula oblonga a un $\mathbf{N}$ à cloison (V.p. 409).

Genres voisins:

Cyclogramma (Perty), à bouche pourvue de trois groupes de cils membranelliformes. (70 p.. Eau douce);

Chilodontopsis (Blochmann), très aplati dorso-ventralement $72 \mu$. Eau douce). 
est ovoïde avec un petit corps central qu'il ne faut pas confondre avec le $n$ situé près de lui, mais en dehors de sa membrane $(0,3$. Her et eau douce) ("). Enfin, la série des Gymmostomides se termine par quelques formes qui ont beancoup d'analogie avec les genres précédents, mais qui s'en distinguent par la présence d'une queue. Prenons pour exemple le genre Onichodactylus (Entz) (fig. 737) qui rappelle le Chilodon. La forme lu corps, la zone adorale, la forme de la bouche sont assez semblables; ses cils sont de mème réluits à ceux de la face ventrale. Mais ce corps porte à la partie inférieure un petit appendice conique en forme de queue, qui n'est rien autre chose que l'extrémité terminale du corps, rétrécie el ramenée en ce point. L'anus en effet s'ouvre sur ce prolongement 0,2 . Mer) $\left({ }^{2}\right)$. Le genre

AEgyria (Claparède et Lachmann) (fig. 738), dérive du précédent par le fait que l'animal a l'habitude de se plier en deux, longitudinalement sur sa face ventrale de

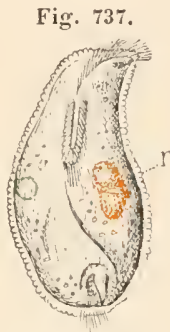

Onichodactylus

(O. acrobates)

(d'ap. Entz).
Fig. 738 .

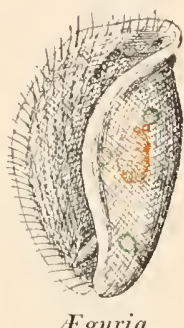

(im. Claparède et Lachmann). telle sorte que la partie visible de cette face est réduite à une étroite goutlière longitudinale. C'est la seule région qui porte des cils $\left(0,15 . \operatorname{Mer}\left({ }^{\mathbf{3}}\right)\right.$.

(1) Les genres suivants:

Phyllotrichum (Ehrenberg-Bütschli), trouvé par ce dernier dans les cartons du premier, semble être un Chilodon à dos 11 et s'élevant en crête sur la parlie qui surmonte la bouche $(0,13$. Mer $)$;

Chlamydodon (Ehrenberg), semblable au précédent, mais plus obtus vers le haut et portant, du côté dorsal, un sillon strié au fond, qui fait tout le tour du corps parallèlement aux bords 0,12 . Mer);

Plascolodon (Stein), à dos nu, très bombé, à face ventrale ciliẻe, large cn haut, rétrécie en bas en une sorte de queue $(0,09$. Eau douce);

Scaphidiodon(Stein), à peine distinet du précédent $(0,1$. Mer); Fig. 739 .

Odontochlamys, Certes) et

Ehrenbergius (Ormancey), peut-être non distinct de Scaphidiodon:

Opisthodon (Stein) fig. 739), s'en distinguant seulement par la situation très basse de la bouche $(0,18$. Eau douce);

Gastronauta (Bütschli) (fig. 740) semble devoir prendre place ici mais, en raison de sa large bouche transversale bordée de deux lèvres et de diver's autres caractères, il mériterait peut-être de devenir le type d'une série spéciale $(0,13$. Mer $)$.

(2) Ajoutons que le dos est recouvert d'une plaque gélatineuse qui fait fonction de carapace. Le $\mathbf{N}$ est de la nature de ceux que l'on appelait noyaux it cloison (V. p. 409, note), le $\mathbf{n}$ est à côté de lui, la vésicule

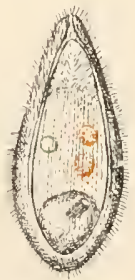

Opisthodon

O. Niemeccensis) (d'ap. Stein).
Fig. I'to.

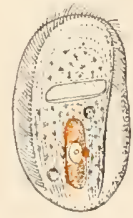

Gastroncula

(G. membrana-

ceus) (d'ap.

Blochmann). pulsatile est dans la partie dorsale droite de la région moyenne du corps.

( $\left.{ }^{3}\right)$ Genres voisins

Trochilia (Stein) à quene bien développée, mais à face ventrale ciliẻe réduite à une bande assez large, concave vers la gauche $(0,03$. Mer et eau douce); 
2e SOUS-ORDRE

HYMÉNOSTOMIDES. - IIYMEYOSTOMIDLE

[TRICHOSTOHATA (Bütschli)]

TYPE MORPHOLOGIQUE

(FIG. 741)

Notre type d'Holotrichide gymnostomide différait du type général des

Fig. 741.

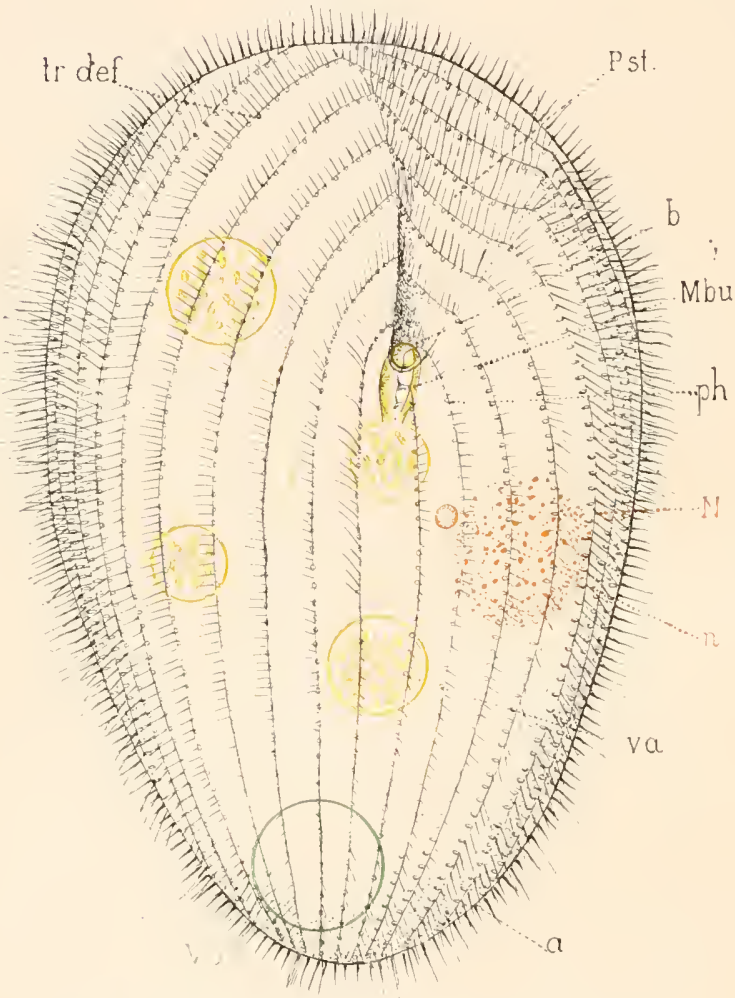

IIMEXOSTOMID.E (Type morphologique) (Sch.).

a., anus; b., bouche; Mb.u., membrane ondnlante; $\mathbf{N}$, macronncléns ; n, micronucléus; pl.., pharynx; P.st., péristome; tr.def., trichocystes défensifs ; v. a., vacuoles alimentaires; v. p., vésicule pulsatile.
Ciliés par sa bouche ordinairement fer mée et très dilatable et par ses mœurs de bète de proie. Chez les Hyménostomes et chez tous les Ciliés qui nous restent à étudier, la bouche prend la forme et les caractères que nous avons attribués au type général, c'est-ìdire qu'elle est toujours ouverte et absorbe, automatiquement et sans interruption, toutes les fines particules que lui amène le tourbillon. Ainsi, plus de grosses proies, plus de chasse active et par suite plus de trichocystes d'attaque, plus d'armature pharyngienne destinée à faciliter la déglutition de bols volumineux. alimentaire, puisqu'il

Dysteria (Iluxley) qui peut être défini un Egyria qui serait d'une façon fixe et permanente dans l'état où est celui-ci pendant la contraction de sa face ventrale $\left\langle 0,15 . \mathrm{Mel}^{*}\right.$ et eau douce);

Iduna (Claparède et Lachmann) n'est qu'un sous-genre reposant sur des distinctions artificielles. 
n’y a ici (c’est le caractère général des Holotrichides) ni membranelles ni mème de zone adorale formée de cils vraiment assez forts et assez actifs pour en tenir lieu?

Ce qui le détermine ce sont des membranes ondulantes $(M b . u$.) situées à l'entrée du pharynx $(p h$.$) ou dans la cavité de cet organe.$

Notre Ilyménostomide sera donc un Cilié semblable au type général de Gymnostomide que nous avons décrit plus haut, c'est-à-dire qu'il est pourvu d'un revêtement ciliaire uniforme, a son $\mathrm{N}(\boldsymbol{N})$, son $\mathrm{n}(n)$, sa vésicule pulsatile $(V \cdot r$.) constitués comme chez celui-ci. La place de son anus $(a$. $)$ et de son pore excréteur sont les mèmes. Mais il n'y a que des trichocystes de défense ( $t r$. def.) et son appareil bucco-pharyngien est tout autrement construit.

La bouche $(b$.$) est vers le milieu de la face ventrale et toujours large-$ ment ouverte. Le pharynx ( $p h$.) est entièrement dépourvu d'armature en nasse, mais il est muni d'une membrane ondulante ( $(K b . u$.) qui commence au bord de la bouche et se prolonge dans le pharynx, insérée à son bord dorsal. Au fond du pharynx, se forment sans cesse des vacuoles alimentaires $(v \cdot a$.$) , qui se remplissent sous l'effort de l'eau poussée par$ ces membranes et se détachent successivement pour tomber dans l'endoplasnie qui les emporte dans son mouvement de cyclose.

D'ailleurs, ces caractères ne sont pas absolus et nous allons voir la bouche se déplacer de plus en plus vers le bas et se munir de lèvres ondulantes ou d'un sillon péristomien de plus en plus développé, ou disparaitre dans les formes parasites; enfin, la ciliature du corps subit des réductions et localisations plus ou moins accentuées. C'est la variation de ces divers caractères qui va llous servir de guide pour mettre en ordre les genres qui sont fort nombreux.

\section{GENRES}

Nous trouvons d'abord une série de formes à ciliature uniforme et sans péristome. Une des plus connues est

Colpoda (0.-F. MüHer) (fig. 742), qui foisonne dans toutes les infusions. Il est tordu à droite à sa partie supérieure, comprimé latéralement, ce qui fait qu'on le voit le plus souvent de profil. Vu ainsi, il parait réniforme parce que sa bouche $(b$.) est au fond d'une petite dépression. Cette bouche conduit directement dans un court pharynx qui porte une membrane ondulante insérée le long de son corps dorsal. Mais quand on le voit de face, on constate que

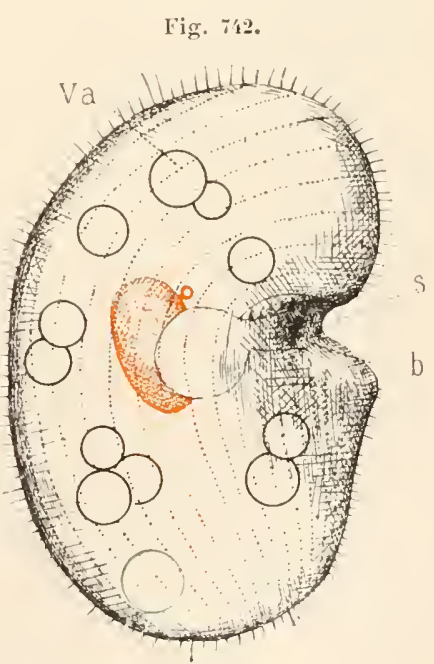

Colpoda (im. Maupas). la dépression prébuccale est un large sillon (s.) qui traverse de haut 
en bas et de droite à gauche toute la face ventrale et que la bouche est à l'extrémité droite de ce sillon, position un peu exceptionnelle. La figure 742 montre la situation et les caractères de ses autres organes, $\mathbf{N}, \mathrm{n}$, vésicule pulsatile et anus (0,2. Eau douce, infusions) (').

Colpidium (Stein) (fig. 743) est très semblable au Colpode: la taille, l'habitat, la forme et la structure du corps et des organes sont à peu près les mêmes, mais la région frontale est nue, la dépression transversale où est logée la bouche est moins étendue, et celle-ci a un aspect sensiblement différent. La membrane pharyngienne, en effet, au lieu de s'arrêter à l'orifice buccal se prolonge le long de son bord droit et forme là une lèvre vibrante. Au bord gauche de la bouche s'en trouve une autre semblable et ces deux lèrres prennent une part aclive à la formation du tourbillon alimentaire $(0,12$. Her et eau douce, infusions). Chez

Glaucoma (Ehrenherg), la conformation de la bouche se modifie sensiblement: les lèvres deviennent plus saillanles et suppléent entièrement la membrane ondulante pharyngienne qui, au contraire, a disparu (0,1. Eau douce) $\left(^{(2}\right)$. Le pharynx, en même temps qu’il perdait sa membrane ondulante, devenait peu indiqué chez Crlaucoma. Chez

Stegochilum (Chevialiof), le pharynx disparait tout à fait. La membrane labiale, bien développée au contraire, fait tout le tour de la bouche sauf en bas $\left(0,07\right.$. Eau douce, Australie) $\left.{ }^{(\boldsymbol{3}}\right)$.

(1) Le Colpode se reproduit uniquement par division à l'état enkysté. Les kystes ont trois membranes concentriques el sont pourvus d'un orifice par où l'animal sort à la fin de l'enkystement. Souvent les jeunes s'enkystent à nouveau dans le kyste maternel, on a alors des liystes emboìtés.

Les genres suivants se rapportent à ce type de structure :

Monachilum (Clıeviakof), en forme de cylindre allongé, élargi en haut, et à bouche ventrale ; Ophryoglena (Ehrenberg), à bouche située plus haut et dépourvue de sillon transversal qui y conduise $(0,5$. Lau douce);

Leucophrys(Stein), à bouche encore plus élevée, en forme de longue fente arrivant jusqu'à l'extrémité supérieure $(0,25$. Eau douce);

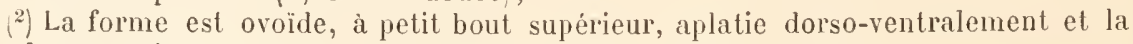
bouche en croissant est assez rapproclıée de l'extrémité supérieure.

Ce développement des membranes labiales aux dépens de la membrane pharyngienne disparue se retrouve dans les genres voisins:

Dallasia (Stokes), qui ne diffère du précédent que par sa forme bizarre, concave sur le dos, convexe sur le ventre $(0,1 \%$. Eau douce $)$;

Frontonia (Claparède et Lachmann), de forme allongèe, à bouche située plus bas et bordée d'un sillon cilié qui se prolonge loin au-dessous d'elle (0,35. Ner et eau douce);

Chiasmatosoma (Engelmann), semblable aux précédențs, mais à bouche située à peine audessus du milieu de la face ventrale 00,05 . Eau douce).

(3) La forme est ovoïde, mais à gros bout supérieur.

Les genres suivants présentent des caractères analogues:

Dichilum (Cheviakof), it bouche pourvue seulement d'une lèvre droite et d'une"gauche, cette derière moins développée (0,03. Lau douce, Australie); 
Uronema (Dujardin) (fig. 7ł'4) se distingue par une longue soie insérée à l'extrémilé inféricure du corps et, caractère plus important, par le fait que la bouche est précédée d'un sillon péristomien peu déreloppé, mais cependant très net, qui annonce les péristomes si développés que nous allons bientôt rencontrer $\{0,07$. Mer et eau douce $\rangle$ (').

Jusqu’ici le revêtement ciliaire étail uniforme. Il cesse de l'ètre chez

Urozona (Chevialiof), où la partie moyenne du corps est seule garnie de cils qui forment une large ceinture occupant environ le tier's de la hauteur $\left(0,03\right.$ à 0,0 ' . Eau douce) $\left(^{2}\right)$. Dans le genre

Urocentrum (Nilzsch) (fig. 745), le corps, en forme de cylindre étranglé au milieu el arrondi aux extrémités, esi aussi (im. Maupas). dépourvu de cils aux deux pòles, mais la bande ciliée moyenne est siluée plus haul et il existe une seconde bande ciliée étroite

Fig. i'ts.

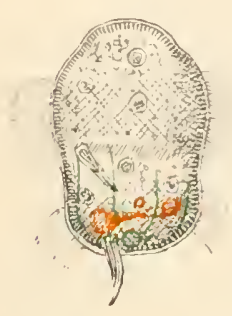

Crocentrum (U. turbo) (d'ap. Bütschli). au-dessous de la première, séparée de celle-ci par un espace annulaire nu. La soie caudale d'Lrozona est remplacée par un fort pinceau de cils agghulinés, mais qui se séparent assez facilement. La bouche est siluée au bord inférieur de la ceinture ciliaire supérieure, au milieu d'une élroite bande circulaire de cils délicals.lille n'a pas de lèrres, mais est pourvue d'une sorte de sillon péristomien qui, au lieu de se porter en haut, se dirige ver's le bas. Jans l'épaisse couche de plisma cortical se trouve une couche uniforme de trichocystes. Le $\mathrm{N}$ en fer ì cheval est couché horizontalement dans la partie inférieure du corps; le n est au-dessus de sa partie movenne; enfin la vésicule pulsatile est siluée au pòle inférieur el vers elle convergent quatre canaux ascendants très nets qui se terminent à son contact par des extrémités renflées $\left(0,1\right.$. Mer et eau douce $\left({ }^{*}\right)$.

Celle situation singulière du péristome, au-dessous de la bouche,

Plagiocampa (Cheviakof), à bouche en forme de fente arquée oblique, bordée d'une lèvre au bord gauche seulement el munie a droite d'une bordure de eils labiaux 0,0 '. Lau douce, Australie).

1) La forme est ovale, comprimée, un peu convexe sur le dos, plane sur le ventre et un peu excavéc dans la région buccale.

Loxocephalus (Kient), voisin d'Uronema présente le caraclère de la soie anale, mais non celui de rudiment de péristome (0,0̈. Eau douce).

${ }^{(2}$ La forme générale est conique, la bouche est au milieu de la face ventrale ef une soie tactile est présente à l'extrẻmité inféricure de la face ventrale.

Tous les genres précédents forment avec quelques formes douteuses:

Plagiopyla (Stein),

Pleurochilidium (Stein),

la famille des CHILIFERTE [Chilifera (Bütschli)].

$\left({ }^{3}\right)$ Ce genre forme a lui seul la famille des UnocExtrLx.E [Vrocentina Bütschli]. 
se retrouve dans un certain nombre de formes dont le type peut être emprunté à

Cinetochilum (Perty) (fig. 746), en forme d'ovoüde aplati transversalement, dont la grosse extrémité, tournée en bas, est

Fig. 746 .

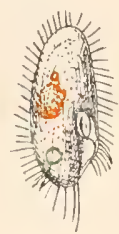

Cinetochilum

(C. margaritaceum)

(d’ap. Bütschli). munie de quelques grandes soies et creusée d'un sillon péristomien assez accusé, au haut duquel est la bouche bordée de deux lèrres vibrantes, la droite plus forte que la gauche. Sur le reste du corps, la ciliature est uniforme. Si l'animal était retourné de haut en bas, tous ses rapports redeviendraient normaux, en sorte que l'on a le droit de se demander si ce qui a été changé en lui n'est pas plutôt le sens de la natation. Ce serait alors un Cilié normal nageant à reculons $(0,04$. Eau douce) (').

Cette interprétation semble encore plus naturelle pour un petit groupe comprenant seulement deux genres qui vivent l'un et l'autre en parasites dans la panse des Ruminants en compagnie des Ophryoscolecinx (V.plus loin) et y jouent le même rôle phy̧siologique. Ce sont les genres Iasytricha et Isotricha. - Chez

Isotricha (Stein) (fig. 747), non seulement la bouche est tout à fait inférieure par rapport au sens dans lequel se meut l'animal, mais l'anus est à l'extrémité supérieure. Cependant les auteurs qui se sont occupés de ces formes (Schuberg [88], Eberbein [95]) consilèrent les dispositions morphologiques comme normales et attribuent le renversement aux habitudes physiologiques: ils décrivent l'animal comme ayant la bouche en haut, l'anus en bas et se mouvant à reculons $\left(^{2}\right)$.

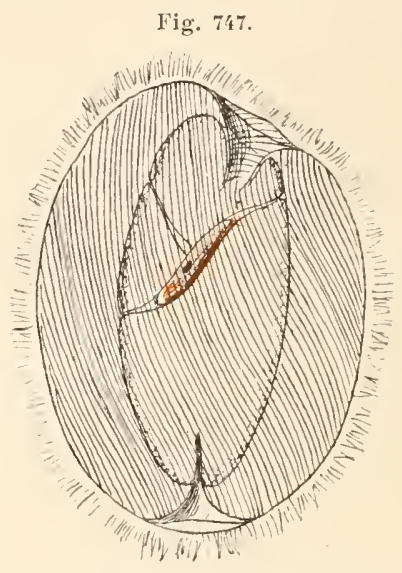

Isotricha (I. prostoma)

(d'ap. Eberlein).

(1) Genres voisins :

Microthorax (Engelmann), semblable à Cinetochilum, à bord dorsal convexe et à bord ventral droit (la bouche clant rejetée sur le côte droit ordinairement interprété comme ventral) (0,06. Eau douce);

Ptychostomum (Stein), de forme sub-triangulaire à base inférieure, pour le reste à peu près semblable au précédent $(0,1$. Intestin des Oligochètes);

Ancistrum (Maupas), de forme ovoüde ou en massue à grosse extrémité supérieure avec une série de cils plus longs au bord ventral (vague indication d'une zone adorale) (0,07. Mer', cavité palléale de quelrues Lamellibranches);

Hysterocineta (Diesing), forme douteuse peut-ĉtre identique au précédent;

Drepanomonas (Fresenius), animal de forme bizarre, en croissant, ì caractères discutés, qui paraît avoir au-dessous de la bouche une sorte de sillon péristomien ascendant que d'autres disent descendant et placé au-dessus d'elle (0,07. Eau douce);

Ces genres constituent avec Cinelochilum la famille des MICROTHOR.1CLY [Microthoracina Bütschli)].

(2) Comme chez les Ophryoscolécines, la structure intérieure préseute d’étonnantes 
Nous n’avons pas encore jusqu’ici rencontré de péristome normal el bien développé, la plupart des formes précédentes en élaient entièrement dépourvues. La fossette transversale des Colpodes n'est guère assimilable à un péristome et le sillon intrabuccal d'Urocentrum el de Cinetochilum doit ètre mis à part en raison de sa situation renversée. Seul, le genre Uronema nous a montré un sillon prébuccal qui, par sa siluation, pouvait être considéré comme un rudiment de péristome. Nous allons maintenant rencontrer des formes munies de vrais péristomes bien développés. La première est

Paramæcium (Stein) (fig. 748), tỵpe classique de Cilié, que l’on trouve très communément. La forme est celle d'un cylindre très allongé, un peu aplati dorso-ventralement, à extrémités un peu rétrécies el arrondies. La surface du corps est uniformément revètue de cils assez longs, implantés chacun au milieu de l'un des petils champs polygonaux déterminés par le croisement des stries de la membrane. Du bord gauche de l'extrémité supérieure jusqu’à la bouche située un peu au-dessous du milieu de la face ventrale, s'étend un long péristome oblique au fond duquel s'ouvre la bouche. De celle-ci, part un pharynx arqué muni sur sa face dorsale d'une membrane ondulante. Sous toute la surface du corps s'étend une couche continue de trichoeystes défensifs. Le $\mathbf{N}$ ovö̈le, auquel est annexé un $\mathrm{n}$ arrondi, est dans la partie moyenne du corps. Il y a deux vésicules pulsatiles situées à égale distance, l'une de l'extrémité supérieure, l’autre de l'extrémité inférieure el entourées chacune d'une étoile de petits canaux excréteurs qui

Fig. 7is.

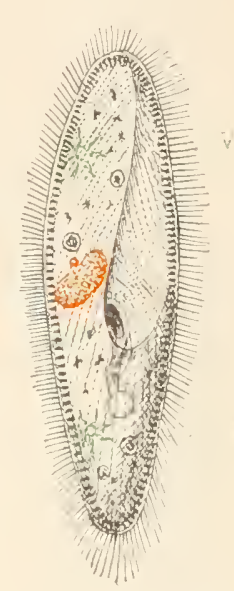

Paramacium (Sch.) commencent à son contact par une extrémité dilatée et, de là, rayonnent dans le plasma cortical. Les grains d'excrétion sont très évidents $(0,2 \%$. Mer et eau douce) (').

farticularités difficiles à coneilier avec le plan général de structure des Giliés. Ici, le noyau est suspendu dans l'endoplasme par des cordons qui semblent formés par la réflexion d'une membrane limitante interposée à l'endoplasme el à l'ectoplasme, en sorte rue la situation morphologique du noyau (obtenue en supposant ces invaginations dévaginées) serait ectoplasmique, comme chez les ophryoscolécides. Mais, pour les uns comme pour les autres de tous ces hòtes de la panse des Ruminants, nous croyons utile de faire quelques réserves en altendant une interprétation définitive de ces particularilés.

Dastyricha (Schuberg) diffère dı précédent par son noyau sans ligaments suspenseurs, par ses stries ciliaires spirales el par l'absence d'anus $(0,1)$.

Ces deux genres forment la famille des 1sotrichx E [Isotrichina (Bütschli)].

(1) Le nombre des $\mathbf{n}$ et des vésicules pulsatíles n'est pas constant. Il peut y aroir deux $\mathrm{n}$ et une seule vésicule. Les trichocystes sont aussi variables et peuvent manquer. Tout cela dépend des espèces.

Le genre Paramacium constitue a lui seul la fanille des P'ARAMECTAE [Parama- 
Ici prenment place quelques genres qui se rattachent aussi bien à Uronema et aux formes voisines qu'à Paramxcium et à Lembadion dont nous allons parler. Le principal de ces genres est: Pleuronema (l)ujardin), qui n'est qu'un Uronema à péristome élargi et muni du côté droit d'une lèvre ondulante très développée; il possède la même soie terminale, mais ses cils sont très longs $(0,03$. Ner et eau douce) (').

Dans les genres voisins $\left({ }^{2}\right)$, le péristome s'agrandit, sa lèvre devient plus saillante, une deuxième lèvre se montre au bord opposé et l'on arrive enfin au type le plus accentué de la série, qui est

Lembadion (Perty) (fig. 749), petit Cilié chez lequel le péristome occupe toute la face ventrale. Le corps a la forme d'un ovoïde, un peu aplati dorso-ventralement, à grosse extrémité supérieure; il est muni d'un revêtement ciliair'e uniforme, sauf quelques cils plus longs à l'extrémité inférieure. Le péristome forme une profonde excavation qui occupe presque toute la largeur de la face ventrale. Au lieu de s'approfondir vers le bas et de conduire en ce point à la bouche, il est au contraire plus superficiel aux extrémités qu'au milieu. C'est là, au milieu de sa lıauteur, derrière le bord gauche du péristome, qu’est la bouche, représentée par une simple surface longitudinale où le tégument manque. II n'y a donc pas de pharynx. L'excavation péristomienne est recouverte par deux grandes lèvres rig. 750.

cina (Bütsclıli)].

Nous y ajouterons le genre voisin :

Philaster (Fabre-Domergue) se dislinguant du précédeut par un long cil rigide à l'extrémité inférieure $(0,1$. Mer).

(1) Il est de forme lenticulaire, comprimé latéralement, à bord ventral rectiligne et bord dorsal convexe.

${ }^{(2)}$ Ces genres sont les suivants:

Cyclidium (Claparėde et Lachmann) (fig. 7.50), simple sous-genre du précédent;

Calyptotricha (Phillips), semblable, mais abrité dans une capsule ovoïde ou lagéniforme lixée à ruelıue oljjet $(0,03)$;

Lembus (Cohn), cylindrique, allongé, vermiforme à extrémité supérieure prolongée en un col, à bouche à peu près médio-ventrale $(0,1$. Mer et eau douce);

Proboscilla (Kent) n'est qu'une espèce de Lembus:

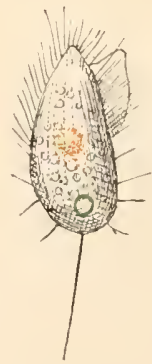

Cyclidium (C. Glaucoma) (im. Gourret et Rinser).

Anophrys (Cohn) est un genre douteux paraissant trouver place plutòt ici cu'ailleurs. 
membraneuses, une gauche $\left(m b^{1}\right.$.) qui s’insère tout le long du bord gauche, recouvre la lèvre droite et s'étend jusqu'au bord droit et une droite $\left(m l^{2}.\right)$, moins large, qui s'insère tout le long du bord droit et s'élend en largeur jusqu'au milieu de la distance qui le sépare du bord gauche. Enfin, dans l’intérieur mème du péristome, un peu à droite, existe une troisième membrane ondulante $\left(m l^{3}\right.$.). Il y a un $\mathrm{N}$ réniforme arec un n près de son liile et une vésicule pulsatile située assez haut, mais iqui communique par un long canal avec un pore excréteur situé au pồle inférieur $\left\{0,14\right.$. Eau douce) $\left({ }^{1}\right)$.

L'ordre des Holotrichides se termine par un petil groupe d'ètres quii sont bien Hololrichides, mais dont on ne saurait dire s'ils sont plutôt Gymnostomes ou Ilyménostomes puisqu'ils n'ont pas de bouche. Cependant c'est ì ces derniers qu'on les rattache de préférence $\left(^{*}\right)$.

L'absence de bouche n'est pas ici une condition de simplicité primilive. C'est un effet secondaire du parasitisme. Ces ètres vivent, en effet, en parasiles dans le tube digestif des Vers ou des Amphibiens. Le type le plus normal de ce groupe anormal est Anoplophrya (Stein) (fig. 731, 73̈3). Il est ovoïde, allongé, comme un peu lordu sur son axe, uniformément cilié, muni d'un gros $\mathrm{N}$ ovö̈le, d'un petit n et de plusieurs vésicules pulsatiles étagées le lonğ d'un de ses bords latéraux. Il ne présente aucune trace de houche mi de pharynx $(0,1$ i 0,9 . Tube ligestif de divers Annélides ou Gastéropodes; sang de Crustacés arquatiques).

Bien qu'il ait douné son nom à ce groupe, le genre

Opalina (Purkinje etValentin) (fig. 752) est beaucoup plus aberrant par l'absence de vésicule contractile (mais le réseau des canalicules excréteurs est présent), et la présence de nombreux noyaux qui ne sont ni des $\mathrm{N}$ ni des $\mathrm{n}$, mais de vrais noyaux tels que ceux des cellules des llétazoaires, c'est-ì-

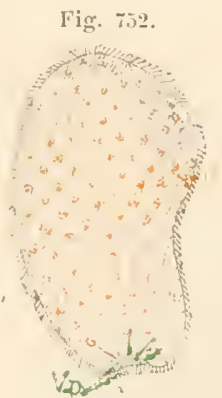

Opalinu (d'ap. Zeller)
Fig. 751.

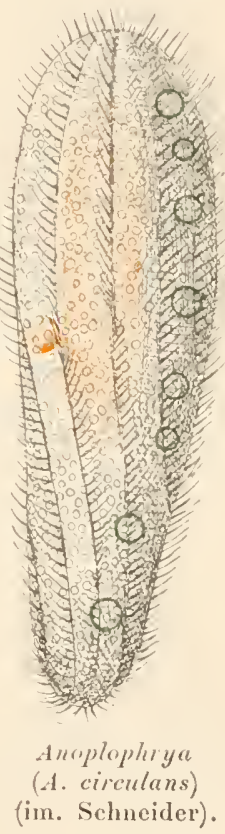
dire sans dédoublement en deux parties chargées de fonctions différentes $\left(0,1\right.$. Tube digestif ou, plus rarement, ressie urinaire de la firenouille $\left({ }^{\mathbf{3}}\right)$.

(1) La série de formes précédentes constitue la famille des PLETRoxEMTYE [Pleuronemina (BütschLi)].

(2) Ils constituent la famille des OP.ILtNTSE [Opalinina (Stein)].

(3) Il est comprimé latéralenent. Pour ses autres caractères il est conforme au genre précédent. Genre voisin :

Opalinopsis (Föttinger) (fig. 756) (0,12 à 0,15. Foie et appendices veineux urinaires de divers Céphalopodes!. 
Enfin, le parasitisme accentue encore ses effets dans les deux derniers genres de la famille: - chez

Discophrya (Stein) (figg. 733), l'extrémité supérieure du corps est excavée à la face ventrale en une capsule arrondie formant ventouse (2mm. Tube digestif de divers Planaires et Amphibiens) ('); - chez

Hoplitophrya (Stein) (fig. 754, 757), la même excavation existe, moins développée, il est vrai, et incapable d'agir comme ventouse, mais de son fond naissent deux crochets fixateurs. Ces crochets sont formés par les deux branches divergentes d'une petite pince qui se prolonge par une

Fig. 753.

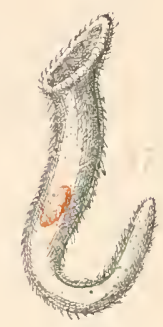

Discophrya

(D. Planariarum) (d'ap. Büıchli).
Fig. 75'.

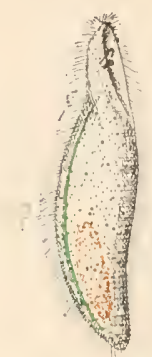

IIoplitophrya

(II. uncinata) (d ap. Butschli). vp., vésicule puIsatile. paisseur du tégument dont elle estune production. Parfois, cette troisième branche n'existe pas $\{0,1$ à 0,9 . Intestin de Planaires et de diver's Oligochètes) ( $\left.{ }^{2}\right)$.

A partir de maintenant, nous allons trouver chez tous les Ciliés une zone adorale formée de membranelles $\left(^{3}\right)$.

(1) L'animal, de forme allongée, rétrécie vers le bas, se distingue encore par sa vésicule pulsatile ( $Y p$.) qui prend la forme d'un long vaisseau dorsal contractile. Il a un revêtement ciliaire uniforme.

(2) Moitié plus petile que la précédente, cette forme lui ressemble par ses autres caractères. Chez certaines espèces, on trouve des vésicules pulsatiles ordinaires; chez d'autres, un vaisseau dorsal $\left(V_{P}\right)$ les remplace comme chez Discophrya.

Bourgeonnement. - Dans la plupart des genres de la famille des opalinines, certaines espèces présentent, en outre, un caractère intéressant à signaler: la division y est très inégale, les deux produits de la division ne se séparent que tardivement et, avant qu'ils se soient séparés, de nouvelles divisions interviennent. Il en résulte la formation de chaines qui rappellent celles de certains Vers, exemple: Anoplophry nodulata (fig. 755), Opalinopsis elegans (fig. 756) des organes urinaires des Céphalopodes, Hoplitophrya Lumbrici (fig. 757).

Citons pour terminer le genre:

Mitophora (Perty) dont la place mème parmi les Holotrichides est sujette à caution.

${ }^{(3)}$ Tous les Ciliés qui nous restent à étudier, c'est-ì-dire les trois ordres des Ifétérotrichides, IIjpotrichides ct Péritrichides, sont réunis par

Fig. 757.

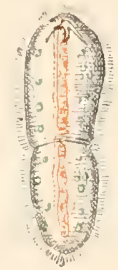

Hoplitoplerya (H. Lumbrici) (d'ap. Stein).

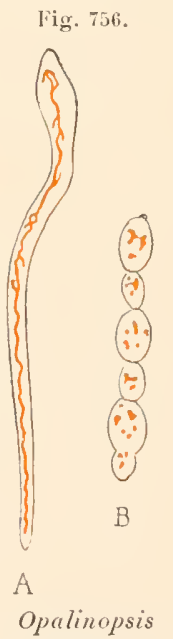

(o. elegans) (im. Fötinger).
Fig. 755 .

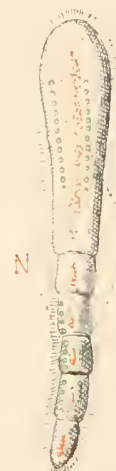

Anoplophrya (A. nodulata) (d'ap. Claparede et Lachmann).

v., noyau;

v. p., vésicules pulsatiles.

bëtscint dans un mème sous-ordre des spirotriches, éest-à-dire des Ciliés numis d'une 


\section{ORDRE \\ IIETEROTRICIIIES. - IIETEROTRICIIIDA \\ [Hétérotriches; - Heterotricha (Stein, emend.)] \\ TYPE MORPHOLOGIQUE \\ (FIG. 758 A 770 )}

Le type morphologique des llétérotrichides est un Cilié de taille relativement grande. Nous

lui donnerons $1^{\mathrm{mm}}$ environ de longueur. Son corps est ovoïlle: c'est comme toujour's la forme fondamentale initiale, etcomme toujours le gros bout de l'ovoüde est tourné en haut. Hais ici nous observons en plus que ce grosbout est tronqué de liais en avant, de manière à constituer une surface oblique, dont on ne saurait dire, au premier coup d'oil, si elle appartient à la face ventrale ou à l'exlrémilésupérieure, puisqu'elle est intermédiaire à l'une et à l'autre. Mais, en réalité, elle appartient à la première, et c'est son point le plus élevé, situé du côté dorsal,

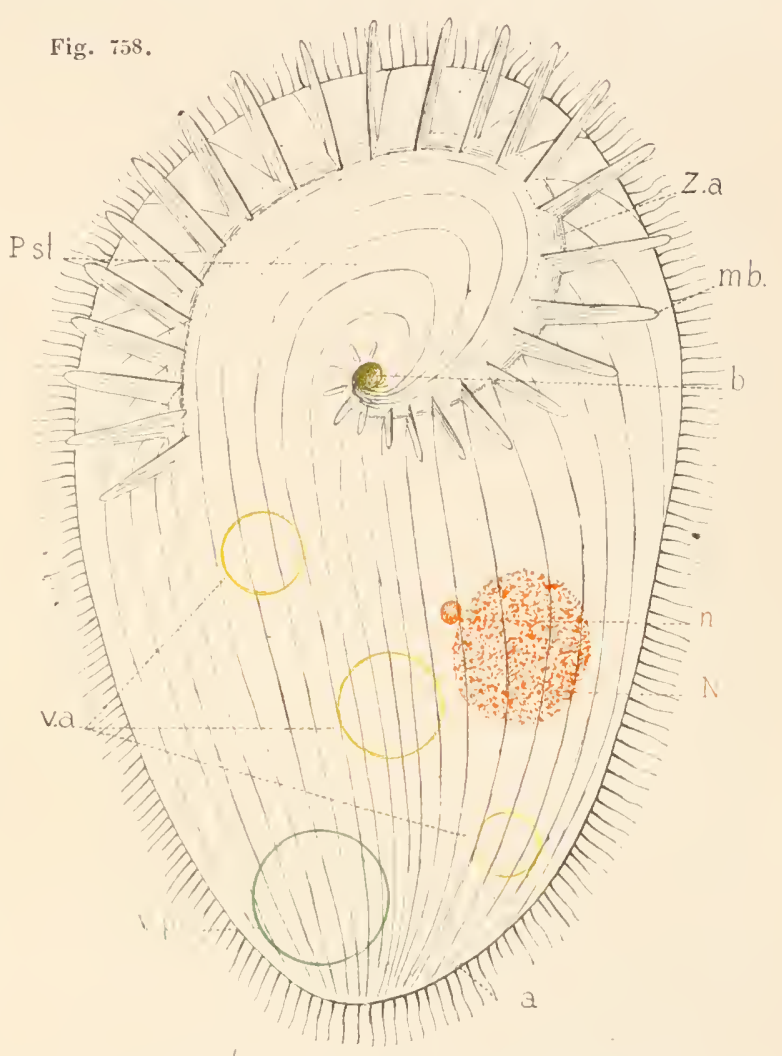

HETEROTRICHIDA (Type morphologique).

a., anus; $\mathbf{b . ,}$ bouehe; mb., membranelles; $\mathbf{N}$, macronucléus : $\mathbf{n}$, mieronucléus ; 1'st., pseudostome ; va., vacuoles alimentaires; Vp., Vésieule pulsatile; Za., Zone adorale.

zone adorale spirale, dont nos ordres ne sont que des subdivisions.

Voici la correspondance des deux classifications :

$\begin{array}{ccc} & \text { Bütschli } \\ \text { Ordres } & \text { Sous-ordres Seetions }\end{array}$

Gymnostomes..............

Trichostomes $\left\{\begin{array}{l}\text { Aspirotriches } \\ \text { Spirotriches }\left\{\begin{array}{l}\text { Ilétérotriches } \\ \text { Oligotriches } \\ \text { Ilypotriches } \\ \text { Péritriches }\end{array}\right.\end{array}\right.$
Nobis

$$
\text { Sons-ordres }
$$

Ordres

Gymnostomides ) Hyménostomides\} Polytrichides Oligotrichides

Ilolotrichides

IIétérotrichides

Iy potrichides Péritrichides 
qui représente le pôle supérieur de l'animal. Celte surface oblique est excavée et constitue le péristome ou pseudostome (Pst.), ou mieux encore, champ frontal (V. p. 403, note 2). Ce champ frontal n'est pas tout à fail circulaire, il est allongé obliquement de gauche à droite et de haut en bas. Il est limité par une bordure de hautes membranelles (mb.) constituant une zone adorale très évidente $(Z a$. $)$. La zone adorale ne forme pas une courbe fermée; elle commence à la parlie la plus déclive, située à droile et en avant, suit tout son contour en montant d'abord le long de son bord droil, puis redescend le long de son bord gauche el, arrivée à quelque distance à gauche du point de départ, se contourne en hélice sénestre, de plus en plus serrée, pour plonger dans la bouche (b) (").

La bouche, en effel, se trouve là el l'excavation frontale, très superficielle à droite et en haut, va en s'approfondissant de plus en plus de manière à déterminer en ce point une sorte d'entonnoir au fond duquel est la bouche. De la bouche, part un pharynx tubuleux et la zone adorale non seulement plonge en spirale dans la bouche, mais continue jusqu'au fond du pharynx. Cette constilution de la région buccale est le Irail caractéristique des Hélérotrichides. Elle comporte une zone adorale de

(1) La définition des expressions dextre el sénestre est presque toujours donnée d'une manière insuffisante. Et comme elles reviendront bien des fois dans cet ouvrage, il n'est pas inutile de bien fixer, une fois pour toutes, les idées sur ce point.

Une spire n'est ni dextre, ni sénestre en elle-même. Cela dépend de la manière dont on la place. Prenez le ressort spiral d'une montre et mettez-le sur une table devant vous; il aura, je suppose, la rlisposition suivante (fig. 759); retournez-le sens dessus dessous, il prendra la disposition (fig. 760) qui est évidemment l'inverse de la première: il n'y a donc pas les ressorts spiraux dextres et des sénestres.

Ce spiral est la projection horizontale d'une hélice conique. Examinons maintenant la projection verticale de cette hélice. Les deux hélices coniques $A$ et $B$ (fig. 761) sont évidemment l'inverse l'une de l'autre, leur projection verticale est cependant celle qu'indique la figure 762 pour l'une comme pour l'autre; et, si on les suppose indéfinies, il est impossible de les distinguer. Cela montre que la notion dextre, sénestre ne peut se définir par les éléments d'un plan. Il faut les trois dimensions de l'espace pour en contenir le concept.

Plaçons-nous done clans l'esprace et examinons de nouveau la question: voici deux hélices coniques ( $D$ et $S$, fig. 763) qui sont l'inverse

Fig. 763.
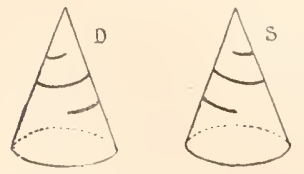
l'une de l'autre, et il n'y a aucune manière de les amener en coïncidence. Quoi que vous fasFig. 761.

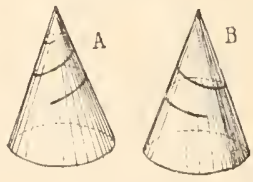

Fig. 762.

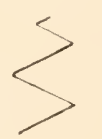
siez, si vous considérez la portion de la courbe qui est tournée vers vous, la seule que vous puissiez voir sur le còne opa(que, la courbe va de gauche a droite en montant pour $I$ ) et de droite à gauche en montant pour $S$. Et cela est vrai aussi bien si vous tournez le cône devant derrière, ou si vous le placez la pointe en bas, comme on peut s'en assurer en regardant ce dessin à l'envers. C'est d'après cela que les bolanistes ont défini le sens de giration des tiges volubles. l'our eux, l'hélice I) est dextre, l'hélice s'sénestre. Si l'on s'en te- 
membranelles courbes et circonscrivant lans sa cavilé une portion de la surface ventrale du corps. Cetle porlion est appelée souvent péristome, mais à torl, car le péristome vrai, s’il peul contenir comme nous le verrons plus tard quelques lignes de cils spéciaux, n’est jamais uniformément cilié. Il vaudrait mieux l'appeler pseudostome, comme l'on dil quelquefois, ou mieux encore champ frontal, comme nous avons fail plus haul. Le péristome vrai de notre tỹpe n'est que celte parlie situće en dedans de la zone adorale, aux environs de la bouche, qui précède celle-ci sous la forme d'une dépression de plus en plus creuse où les cils n'ont point accès. Il se continue d'ailleurs insensiblement avec la sur-

nait à cela, il faudrait dire que la spire du Stentor est dextre, car en effet elle monte it droite, comme le montre le croquis ci-contre (fig. 764).

Fig. 764.

Cette définition est applicable aux formes simples qu'étudient les botanistes. Hais chez les animaux où des invaginations, des contournements de toutes sortes interviennent, elle se montre bientôt insuffisante. Supposons en effet que, chezle Stentor', la bouche, au lieu d'être au-dessous de lorigine de la zone adorale sur le corps, remonte aulessus de celle-ei et devienne le point le plus élevé lu corps, ce qui est sa vraie position morphologique, la zone deviendra alors sénestre d'après la définition des botanistes. On voit bien, cependant, qu'elle n'a pas changé de sens. Les deux qualifications inverses se trouveront ainsi appliquées à deux dispositions fondamentalement identiques.

Pour sortir de cet embarras, il suffirait de considerer la bouche comme étant toujours le sommet morpliologique du corps et d'envisager seulement la portion extrapharyngienne de la zone. Mais il y a une manière bien plus scientifique de considérer les choses et qui supprime d'un coup toutes les difficultés. C'est de considérer la courbe non dans ses aspects par rapport à un observateur qui la regarde de dehor's, mais dans ses rapports avec un observateur qui se mouvrait sur elle. Supposons que la zone soit un sentier parcouru par un petit promeneur qui viendrait du fond du plarynx et se dirigerait vers la terminaison extérieure de la courbe; supposons aussi, naturellement, que ce promeneur ait son axe parallèle à celui de l'Infusoire et sa tète tournèe comme lui en haut. La zone sera dite

Fig. 765.

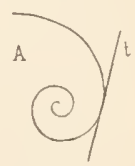

Fig. 766 .

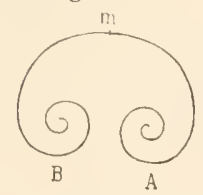

sénestre tant que, en chaque point, pour asancer, le promeneur se dirigera vers la gauche de la tangente $t\rangle$ en ce point (fig. 765 ), ce qui revient a dire, tant que pour avancer il tournera vers sa gauche. On dit quelquefois que la courbe est sẻnestre si l'observateur a l'axe à sa gauche. Cette définition est bonne en général, mais plus sujette à se trouver ell défaut à l'occasion de déformations secondaires comme il s'en rencontre quelquefois, par exemple chez spirochona, Epistylis umbellaria, ete. Ainsi, chez spirochona, la courbe $A$ (fig. 766, $A$ ) est sẻnestre d'après les deux définitions. Mais supposons (fig. 766, $B^{\prime}$ ) que, au point $m$, elle s'enroule autour d'un nouvel axe : la courbe $B$ n'est que la continuation de la courbe $A$ et on ne doit pas dire qu'elle est devenue dextre, puisque, en fait, elle n'a pas changé de sens; cependant l'axe de $A$ est en certains points a la droite du promeneur parcourant $B$. Par rapport a ses tangentes, au contraire, la courbe n'a point clıangé, ce qui tient à ce qu'elles se déplacent avec elle et suivent ses variations. Même une courbe telle que celle de la figure 767 resterait sénestre dans toutes ses

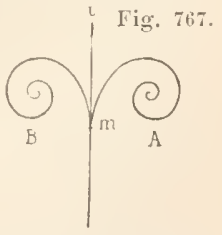
parties par rapport à notre dẻfinition, car au point $m$, le promeneur n'a qu'à tourner 
face ciliée générale, comme d'ordinaire, par une transition insensible du côté opposé à la bouche et à la zone adorale (").

Toute la surface du corps est revêtue de cils uniformes.

Il n'y a poinl de trichocystes.

Le $\mathbf{N}$, le $\mathrm{n}$, la vésicule pulsatile ne présentent rien de particulier $\left.{ }^{(\boldsymbol{}}\right)$. Les myonèmes (fig. $\mathbf{7} 68, m$.) correspondent aux stries ciliaires et ont,

Fig. 768.

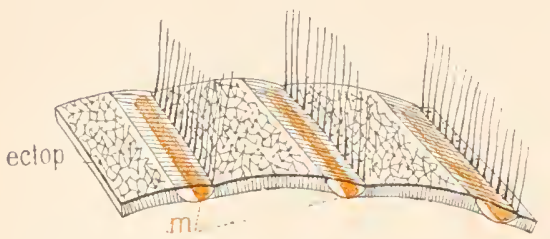

HETEROTRICHIDA (Type morphologique). Disposition des myonèmes dans l'ectoplasme (Sch.).

m., myonèmes; ectop., ectoplasme. comme celles-ci, une orientation générale méridienne. $\Lambda$ côté de chacune des stries ciliaires, se trouve, sous la membrane, un petit canal au niveau duquel l'ectoplasme est absent, et, au fond de ce canal, sur la paroi qui le sépare de l'endoplasme, est couchée une fibrille, de section elliptique et striée transversalement comme si elle était formée de petits disques empilés. Il semblerait que l'ectoplasme (ectop.) qui devrait occuper la place de

sur ses talons, sans asancer, pour prendre par rapport à la tangente commune ( $t$.) la position qui lui permet de parcourir le segment $B$ sans cesser de rester á la gauche des tangentes.

Par contre dans la figure 769 , le segment $B$ est bien rẻellement de sens inverse à $A$, car il lui est superposable, mais le promeneur, en le parcourant, fait comme s'il revenait sur ses pas sur la courbe $A$. Or l'on voit bien qu'en effet il passe à la droite des tangentes.

(1) La figure 770 montre la série des modifications qui relient le péristome normal aux formes les plus aberrantes de pseudostome.

Partant du type morphologique $(A)$, on le roit se fermer en s'allongeant chez $\mathrm{Ba}$ lantidium $(B)$ et chez Condylostomum $(C)$, se contourner chez Climacostomum $(D)$, s'élargir en cercle chez Stentor $(E)$, et enfin, chez Folliculina $(F)$, se diviser en deux lobes Fig. 770 .
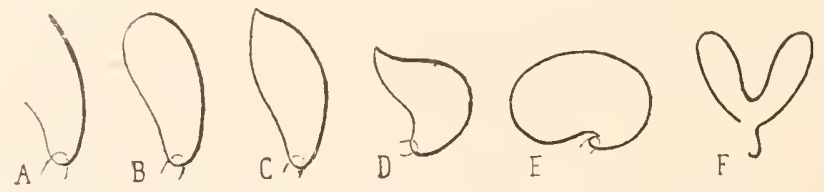

Diagramme indiquant la manière dont les péristomes de divers Péritrichides derivent du type morphologique (im. Johnson).

$A$, type morphologique; $B$, Balantidium; $r$. Condylostomum; $D$, Climacostomum; E, Stentor; F, Folliculina.

aliformes situés de part et d'autre de la bouche. L'idée de cetle dérivation est empruntée à Johnson.

(2) Chez un petit nombre de genres: Conchophthirus, Metoputs, Spirostomum, ce ne sont pas des membranelles, mais de forts cils qui se forment sur la zone adorale. Mais le corps est très contractile et possède des fibrilles appelẻes myonèmes qui sont les agents de cette contractilité.

On trouve des formations analogues chez quelques Iolotrichides, mais moins développées; les IIypotrichides n'en ont pas: quand aux Péritrichides, ils ont un appareil musculaire bien plus développé et qui sera décrit au moment opportun. 
ce canal se soit condensé en cette fibrille, laissant par suite de cette condensation un espace vide qui serait la cavité du canal ( $\left.{ }^{\mathbf{1}}\right)$.

Bien entendu, ces caractères du type morphologique ne se retrouvent pas dans tous les genres réels. Deux éléments principaux de variation vont nous servir à la classification de ceux -ci :

$1^{\circ}$ Le péristome, que nous allons voir commencer par une fente étroite et verticale sur la face ventrale, puis s'élargir peu à peu, jusqu'à devenir très grand et très ouvert, ou rester élroit et se relever sur une surface oblique de plus en plus voisine de l'horizontale et s'incurver en cercle;

20 La ciliature qui cessera d’ètre générale pour se limiter à certains points du corps.

Quant aux autres caractères empruntés à la forme générale, au $\mathbf{N}$ et au $\mathrm{n}$, à la résicule, etc., ils sont très variables aussi, mais non d'une façon continue et ne peuvent caractériser que les genres isolés ou les espèces.

Nous diviserons les Ilétérotrichides en deux sous-ordres:

Polytrichide, pourvus d'un revètement ciliaire général.

OLIGOTRICIIIDE, qui n'ont de cils sur le corps qu'à certaines places déterminées.

$$
\begin{gathered}
\text { fer Sous-Ordre } \\
\text { POLITRICIIDES. - POLYThICHID.E } \\
\text { [Polytriciles; - Heterotrichi (Stein)] }
\end{gathered}
$$

\section{TYPE MORPHOLOGIQUE}

Il sera suffisamment défini en disant qu’il a tous les caractères du type général les llélérotrichides, y compris celui d'aroir outre sa zone adorale, un revètement ciliaire général sur tout le corps.

Les genres sont faciles à classer en prenaut pour critérium la rariation progressive du péristome, à laquelle nous venons de faire allusion.

(1) On a pensé que les cils se prolongeaient jusqu'au myonème, s'altachaient sur lui et recevaient de lui le mouvement. Mais il n'en est rien. Les cils sont automobiles el le myonème sert à la contraction générale du corps. Comme une contractilité générale existe dans bien des cas où on n'a pas trouvé de myonèmes, il convient d'admettre rue l'ectoplasme est naturellement contractile et que les myonèmes ne sont qu'une différenciation plus avancée, rendant la fonetion plus parfaite.

C'est surtout chez le Stentor 'jue l'on a observé celle constitution des myonèmes. Comme les tissus contractiles des animaux plus élevés en organisation, les myonèmes sont anisotropes, c'est-à-dire ont la double réfraction. Il en est de mème de ceux des Vorticelles. 


\section{GENRES}

Conchophthirus (Stcin) (fig. 771) nous montre le premier degré de ces transformations. Le péristome est représenté par une simple fosselte infundibuliforme et la zone adorale, réduite à quelques cils plus longs situés aux bords supérieur el inférieur de celle fossette, ne montre aucune tendance à la forme spirale. De la bouche, part un pharynx cilié $(0,2$. Dans le mucus de divers $A$ céphales d'eau douce ou la cavité générale de diverses Actinies) ('). - Dans le genre

Plagiotoma (Dujardin) (fig. 772), le péristome s'accuse, il devient long el étroit, situé entièrement sur la face ventrale lont il suit la ligne médiane en direction franchement verticale. Il aboutil en loas à la bouche d'où part un pharynx bien dessiné. Son bord gauche

Fig. $7 i 1$.

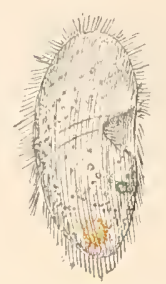

Conchophthirus

(C. Anodontx)

(d'ap. Engelmann).
Fig. 772.

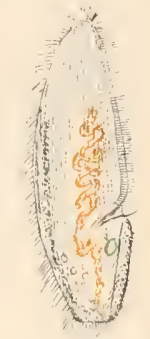

Plagiotoma

(P. Lumbrici) (d'ap. Stein). donne insertion à une zone adorale de membranelles qui se continuent jusqu'au fond du pharynx en suivant son bord dorsal, mais sans contournement spiral (0, 亿. Parasite dans le tube digestif des Vers de terre) $\left({ }^{*}\right)$. - Chez

Spirostomum (Ehrenberg) (fig. 773 et 77 亿), la conformation est à peu près la mème, mais le corps est plus allongé el l'animal a l'habitude de se contourner très fortement en vis. Le

(1) La vésicule pulsatile est variable, les $\mathbf{N}$ et les $\mathbf{n}$ ne présentent rien de bien intéressant à noter. Le genre

Helicostoma (Colin) est une forme douteuse se rattachant à la précédente.

(2) La bouche est située au-rlessous du milieu du corps. Le pharynx donne insertion, le long du bord ventral, en face des membranelles, à une membrane ondulante. La vésicule pulsatile est au-dessous du pharynx. Le $\mathbf{N}$ est long et très contourné. Le corps est comprimé latèralement. - Genres voisins:

Nyctotherus (Leidy), qui n'est qu'un sous-genre du précédent (Parasite dans l'intestin de divers Anoures, Insectes et Myriapodes); et

Blepharisma (Perty), libre au contraire, mais n'en différant aussi que très peu (Mer et eau douce).

Divers auleurs placent ici le genre Peritromus (fig. 775े), qui ne diffère du précédent que par son péristome irrégulièrement demi-circulaire et parallèle au bord frontal, tandis que d'autres, considérant ses cils ventraux comme

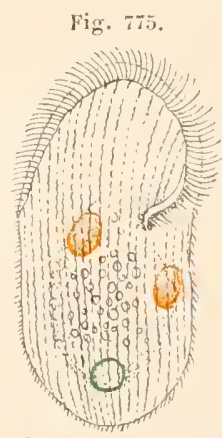

Peritromus P. Emmor

(dap. Butlschli).
Fig. 77'.

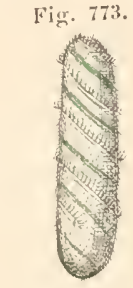

Spirostomum

contracté

(d'ap. Stein). de petits cirres, le joignent aux Iypotrichides. Nous arons suivi ces derniers (V.p. 473).

Il possède deux $\mathbf{N}$ avec chacun un $\mathbf{n}$ associé à lui. Il est en forme de laricot, très aplati sur la face ventrale, et ne porte sur le dos que des soies tactiles en place de 
péristome arrive alor's à faire plusieurs fois le tour du corps $(3 \mathrm{~mm}$. Mer et eau douce) (').

Metopus (Claparèı et Lachmann) (fig. 776), est, lui aussi, conformé comme les précédents, mais son péristome est, à l'état de repos et par conséquent d'une façon presque permanente, phus ou moins contourné en spirale sénestre, et ce contournement est plus ou moins accentué selon les variétés: il varie depuis une faible léviation jusqu’à un tour complet $\left(0,3\right.$. Mer et eau douce) $\left(^{*}\right)$.

Thylakidium (Cheriakof) a un long péristome ventral, assez étroit, incurvé, à concavité regardant à droite, bordé à gauche de membranelles qui, en haut, se continuent avec les cils du corps, en bas, plongent dans le pharynx. Les cils du corps ne pénètrent pas dans le péristome (Eau douce,

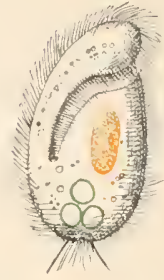

Metopus

(1I. sigmoides)

(d'ap. Gourret

et Roser.) Australie) $\left({ }^{\mathbf{3}}\right)$. - Dans le genre

Balantidiopsis (Bütschli), peu différent par ses autres caractères du genre Plagiotoma, le péristome s'élargit un peu $(0,15$. Intestin de Rana esculenla). - Il s'élargit davantage encore chez

Balantidium (Claparède et Lachmann) (fig. 777), surtout vers le haut, et prend une forme un peu triangulaire. En mème temps, la zone adorale s'étend au bord frontal et à une partie du bord droit du péristome, enfermant

cils. En raison de ces deux derniers caractères, Stern le plaçail parmi les IIypotrichides dans sa famille des Peritromina.

(1) Ce Spirostomum est remarquable, en outre des caractires cidessus indiqués, par son $\mathbf{N}$ long et ordinairenent en cliapelet, ses n nombreux, sa vésicule pulsatile vaste, située à l'extrémité infèrieure et se continuant en un long canal dorsal. L'anus est terminal, il n'y a pas de membrane ondulante dans le pharynx. La zone adorale est forme de simples eils et non de membranelles.

(2) Metopus est de forme beaucoup plus courte que spirostomum. La zone adorale est formée aussi de simples cils. Son $\mathbf{N}$ est arrondi, il a un seul $n$, sa vésicule est à l'extrémité infèricure, mais n'a

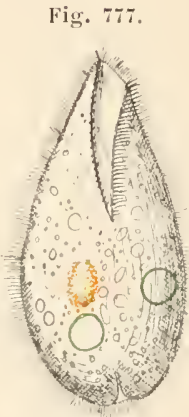

Bulantidium (d'ap. Claparède et Lachmani1). pas de canal dorsal; une touffe de soies tactiles plus longues orne l'extrémité inférieure. Ordinairement, dans la partie supérieure du corps, il y a une taclie pigmentaire volumineuse. Nous avons vu que plusieurs des genres précédents ont la zone arlorale formée, non de membranelles, mais de simples cils et l'on parait vouloir, en raison de ce fait, les rattacher aux Ilolotrichides. Pour cerlains d'entre eux la chose ne serait pas impossible, mais comme ils sont reliés entre eux par des affinités réelles el qu'une bordure de cils bien développés ressemble plus à une zone de membranelles qu'aux quelques cils péribuccaux de certains Holotrichides, il est, à tout prendre, moins choquant de les laisser parmi les Ilétérotrichides que de les joindre aux Holotrichides.

Ces divers genres constituent la famille des PLatotomxa [Plagiotomina Claparède et Lachmann ].

Dicella (Elırenberg), est un genre douteux, et l'on n'est pas bien sùr si c'est ici qu'il doit prendre place.

(3) L'endoplasme contient des Zoochlorelles. 
de plus en plus la surface péristomienne dans sa cavité, mais elle semble encore formée de cils forts et non de véritables membranelles. L'intérieur du péristome n’est pas cilié. La lèvre gauche du péristome se prolonge en bas en une courte lèvre membraneuse $(0,:$. Parasite dans la cavité générale de certains Annélides ou dans le gros intestin de divers Batraciens et Mammifères, même de l'Ilomme) ('). — Si nous passons à

Condylostoma (Dujardin) (fig. 778), le péristome devient tout à fait triangulaire en mème temps qu’il s'élargit beaucoup et son bord droit donne attache à une large lèvre oudulante qui le recouvre en entier. La surface du péristome n'est pas ciliée 0,5 . Mer et eau douce). - Enfin nous arrivons au genre

Bursaria (Claparède et Lachmann) (fig. 779) qui nous montre le degré le plus élevé de celte série de formes. Ici, le péristome devient une énorme excaration qui occupe presque toute la hauteur de la face rentrale et une grande parlie de sa largeur. Il a la forme d'un triangle courbe dont la base tournée en haut occupe toute la largeur du bord frontal, tandis que le sommet se recourbe à gauche et se termine

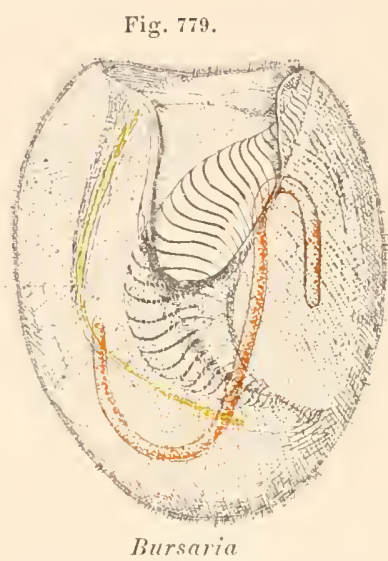

(B. truncatella) (d'ap. Brauer). b., bouche.

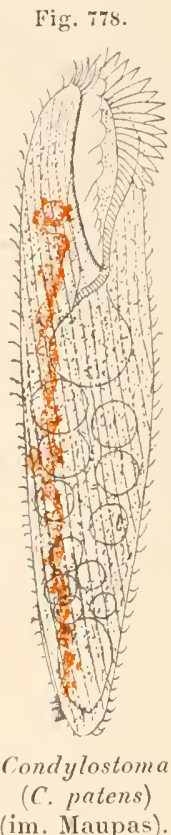

(im. Maupas).

dans la profondeur de la partie inférieure gauche du corps. En haul, il est peu profond et ouvert sur toule sa largeur. Mais en descendant, son lıord droit se soulève en une épaisse lèvre, de plus en plus saillante, qui détermine sous elle une goultière ouverte à gauche, et de plus en plus profonde à mesure que l'on s'avance vers le las. Le bord gauche forme aussi une saillie, mais plus épaisse et moins accentuée. Enfin, un peu au-dessous du milieu, les deux hords sont réunis par une lame qui rétablit de l'une à l'autre la continuilé de la surface. Mais derrière cette lame la cavité péristomienne existe toujours. Cetle cavité a donc, en somme, la forme d'une excavation de plus en plus profonde de haut en bas, creusée en profonde gouttière à gauche, et se terminant en un entonnoir entièrement fermé en avant. Le long de l'épaississement du lıord gauche, règne une rangée de larges membranelles qui descend en diminuant progressivement de largeur jusqu'au fond du péristome.

(1) La forme est ovoüde à grosse extrémité supérieure, l'anus esl termino-dorsal, les vésicules pulsatiles sont multiples, situées le long des borrls; le $\mathbf{N}$ est simple, ovoïde ou en fer à cheval contenant le $\mathbf{n}$ dans sa concavité. 
La bouche occupe le sommet inférieur de l'entonnoir péristomien, mais elle se continue, en outre, sous la forme d'une fente des téguments, dans presque toute la hauleur du péristome, en suivant d'abord le fond de la gouttière que détermine la saillie du bord droit, puis en se délournant à gauche le long de l'origine de ce bord. Le fond du péristome n'est pas cilié; ses lıords ne portent pas de lèrres ondulantes, mais ils sont soutenus en différents points par une sorte de cordon (la bande péristomienne) formé par un bourrelet de l'ectoplasme saillant à sa face profonde el qui semble jouer le ròle d'un lien élastique (1,.ّ. Eau douce) (").

Nous avons jusqu'ici rencontré des péristomes de plus en plus larges, et des zones adorales de plus en plus parfaites et formées de membranelles de plus en plus accentuées. Mais le péristome est resté toujour's exclusivement ventral. Nous allons mainlenant roir le péristome se relever comme dans notre type morphologique et occuper finalement une position lout à fait horizontale a la face supérieure du corps.

Climacostomum (Stein) (fig. 780) représente ì peu près un Balantidium chez lequel la portion de la face ventrale qui porte le péristome se serait relevée de manière ¿ prenclre une direction oblique en bas et en avant. Sur celte face oblique, le péristome, allongé et très

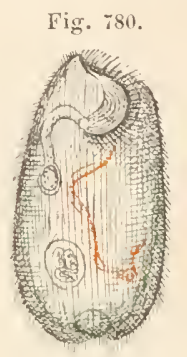

Climacostomum

(C. virens)

(d'ap. Stein). étroit, dessine une courl,ure à concavilé tournéc en bas et à droite. L'espace inscrit dans cette courbure est garni de cils comme le reste du corjs et constilue le pseudostome ou champ frontal 0,36 . Eiu douce) $\left({ }^{2}\right)$.

Ce mourement de relerement de la surface qui porte le péristome devient complet chez

Stentor (Oken) (fig. 781). lci, le corps a la forme l'une trompette, c'est-i-dire qu'il est longuement conique el que sa base tournée en haul est érasée; c'est celle hase, perpendiculaire ì l'axe du corps, qui constilue le champ frontal et porte la zone adorale. Ce champ' frontal est légèrement excavé et ses lignes de plus grande pente se dirigent toutes vers un point silué en avant et un peu à gauche, qui se déprime en in-

1) L'animal est de forme ovoïde, se meut en tournant sur lui-même. Il possède un long $\mathbf{N}$ rubané aurjuel sont annexés plusicurs n. Les résicules pulsatiles paraissent tantôt nombreuses, tantôt absentes.

Ces divers genres constituent la famille des BLTSAIRTE [Bursarina (Bütschili)].

(2) Le pharynx est long et coudé, cilié. La vésicule pulsatile émet deux canaux qui remontent le long des bords de l'animal. Elle s'ourre, comme l'anus, à l'extrémité inférieure du corps. 
fundibulum et constitue un vestibule buccal au fond duquel s'ouvre le pharynx. Son pourtour est saillant et porte une zone adorale formée de hautes et très étroites membranelles qui ressemblent à de simples gros cils. En dedans de ces membranelles, est une rangée de cils (comparables aux cils paroraux). La zone adorale n'est pas circulaire. Elle commence sur la ligne médiane antérieure, au point le plus élevé de l'aire frontale, part de là pour suivre tout le contour du pseudostome mais, un peu avant d’atteindre le point de départ, elle se détourne en arrière pour suivre le bord de l'infundibulum buccal, plonge à son intérieur el suit le pharynx jusqu'au fond en dessinant une spire allongée. Toute la surface du pseudostome est parsemée de cils fins disposés sur des lignes parallèles au contour extérieur. Le péristome proprement dit n'est que celte partie de l'aire frontale qui se déprime à l'approche de la bouche. Il est limité en dehors par la partie gauche de la zone adorale, tandis qu'en dedans et en arrière il se continue insensiblement avec l'aire frontale ciliée ( $1 \mathrm{~mm}$ et plus. Eau douce) (").

(1) Nous adoptons ici la manière de voir de Schuberg. Mais il faut dire que la plupart des naturalistes, au moins avant la publication du mémoire de cet auteur, comprenaient autrentent les choses. Ils considéraient ce que nous avons appelé aire frontale ou champ frontal comme étant le pẻristome et correspondant à l'excavation péristomienne de Balantidium et de Condylostoma devenue plus large encore et circulaire. Mais le fait qu'elle est cilieje montre que la surface enclose par la ligne des membranelles appartient au corps et non au péristome. Elle représente le champ frontal des Ilypotrichides (V. plus loin), Quant au vrai péristome, ce n'est que l'étroite dépression qui précède immédiatement la bouche.

Le Stentor est un type si remarquable par sa grande taille et les particularités de sa constitution anatomirue et de sa biologie qu'il convient de s'étendre un peu sur lui.

Il mesure jusqu'à $4 \mathrm{~mm}$ de long lorsqu'il est entièrement étendu et vit fixé à quelques

Fig. 782.

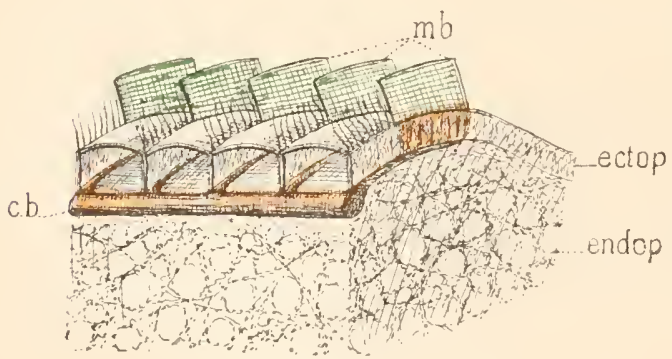

CILIÉs (Type morphologique).

Constitution de la zone adorale (Sch.).

cb., appareil basal des membranelles; cctop., ectoplasme; endop., endoplasme; mb., membranelles formées par une portion des bandes eiliaires dont les cils sont agglutinés. brindilles par son extrémité inférieure au niveau de laquelle la membrane inanque et le cytoplasma sous-jacent émet de vèritables pseudopodes qui servent à la fixation. 11 peut, à volonté, faire cesser cette adhérence et nager à la recherche d'une nouvelle place. Il ne manque pas de le faire toutes les fois que l'eau devient malsaine ou la nourriture trop rare. Son corps est uniformément revêtu de cils disposés sur des lignes longitudinales. A ces lignes de cils correspondent, dans l'ectoplasma sous-jacent, des myonèmes que nous avons décrits à l'occasion du type des Ilétérotrichides.

Les membranelles ont la forme de petits sacs triangulaires et se prolongent à travers les téguments jusqu'à l'endoplasme dans lequel elles plongent (figr. 7ð2). Elles s'y prolongent même par un fila- 
Folliculina (Lamark) (fig. 783) peut ètre considéré comme dérivant d'un Stentor dont le pseudostome se serait développé en deux ailes latérales très élendues, dressées, tandis que les parties médianes antérieure et postérieure seraient restées au même niveau. Il en résulte que le péristome a pris la forme d'un profond entonnoir fendu en arant et en arrière. La zone adorale suit tout son bord libre, commence en avant, suit le bord libre de l'aile droite, descend au fond du sinus postérieur un peu moins profond que l'antérieur, remonte sur l'aile gauche et redescend enfin vers le point de lépart; mais, au lieu de l'atteindre, elle plonge en

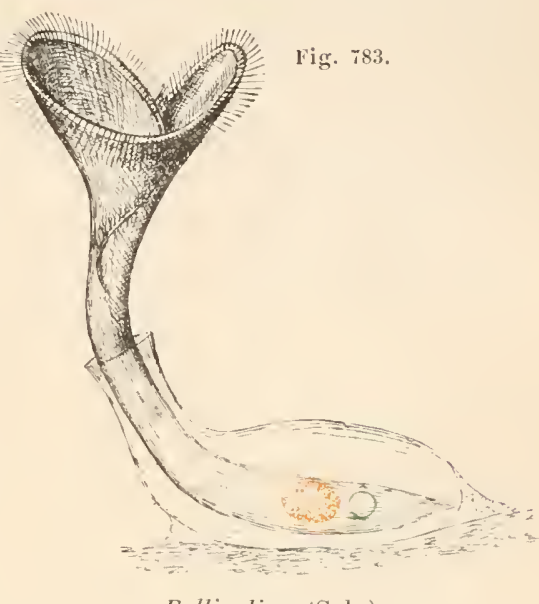

Folliculina (Sch.). spirale dans le vestibule buccal où elle fait un tour et demi et entre enfin

ment radiculairc et les extrémilés de tous ces filaments radiculaires sont relices par un cordon circulaire qui suil le bord du péristome dans l'endoplasme et qui, n'étant pas un myonème, pourrait bien être dc nature nerveuse.

Le bord du péristome se prolonge en avant de l'infundibulum en une sorte de mcmbrane mince forméc par deux lames tégumentaires sans endoplasme interposé et que l'on appelle l'hypostome. Dans l'ectoplasme interposé aux stries ciliaires est, dans l'espèce la plus commune ( $ミ$ ca'ruleus), un pigment bleu abondant. Le $\mathbf{N}$ est très long, en cliapelet; à chacun de ses grains sont associẻs de nombreux n. Il y a une grosse vésicule pulsatile située immédiatement au-dessous du péristome. Elle émet deux canaux, l'un inférieur qui descend le long du bord gauche du corps, l'autre supérieur qui suit le bord du péristome au-dessous de la zone adorale. Ce dernier, cependant, qui doit son origine au processus de la division, s'atrophie (dans certaines
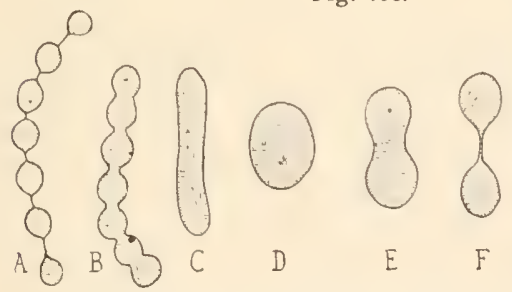

D
Fig. 784.

Division du macronuchéus chez Stentor (Sch.). espèces sinon dans toutes) chez l'adulte. L'anus est situé du côté dorsal à la hauteur de la vésicule.

Le phénomène de la division a été particulièrement bien étudié chez le Stentor. Là mieux qu'ailleurs, on voit (fig. 785) la nouvelle bouche et le nouveau péristome (Pst.) se former de toutes pièces sur un point de la paroi ventrale immédiatement audessous de la future ligne de division que rien n'indique encore. Ce noureau péristome se formc par une simple fente des téguments par laquelle poussent des membranelles. Cette fente est d'abord rectiligne et verticale 1 . Elle prend peu à peu sa forme courbe $B$ et $C$ ) el sa situation horizontale sur la lisce supérieure du corps $(D, E)$.

Le $\mathbf{N}$ donne un excellent exemple de concentration avant la division fig. 784 . On 
dans la bouche. Toute la face interne des ailes, représentant la surface du péristome, est finement ciliće $1 \mathrm{~mm}$. Mer et eau douce (').

Fabrea (Ilenneguy) est voisin du précédent. Il en diffère par son pseudos-

voit le chapelet resserrer ses grains, se transformer en un long bâtonnet et enfin en une masse sphérique puis, après la division qui a lieu par amitose comme toujours, les deux moitićs s'allonger de plus en plus, se diviser en grains rattachés les uns aux autres par d'étroits jédoncules formés par la membrane nucléaire seule et reprendre cn un mot la constitution primitive.

La nouvelle vésicule pulsatile (fig. $783, V p^{\prime}$.) se forme par une dilatation de la

Fig. 785.
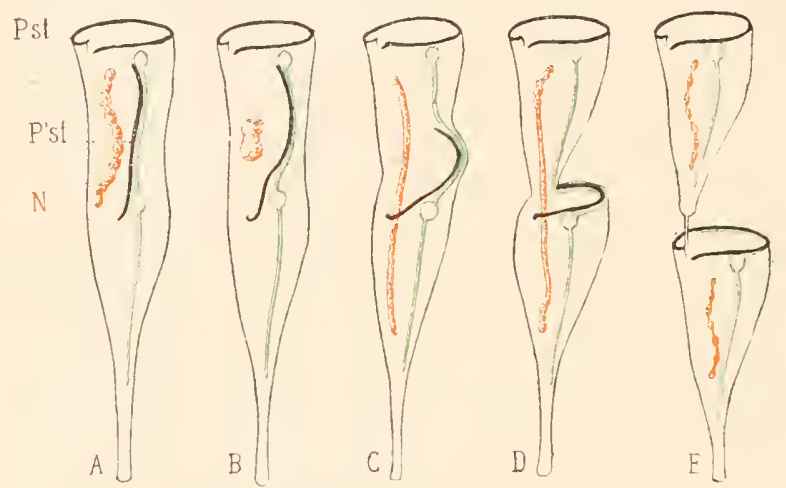

Division ehez le Stentor (Sch.)

1. st,. péristome ancien; $\mathbf{P}^{1}$.st., péristome nouveau; V.p., résicule pulsatile ancienne; $\mathbf{v} \cdot \mathbf{p .}$, vésicule pulsatile nouvelle.

supérieur est fixé par son pied dans le péristome de l'inférieur.

Un autrc phénomène bielı remarquable dans la biologie du Stentor sont les rénov' tions successives du péristome. Ce phénomène appelé à tort régénération consiste en ceci quc, sur un individu dont le péristome est entièrement normal, on voit se former un nouveau péristome, tout comme pour une division. Mais cctte division ne se produit pas et, par un phénomène d'accroissenent inégal, le nouveau péristome, d'abord rectiligne et vertical, prend peu à peu la forme et la place de l'ancien tandis quc celui-ci recule et s'atrophie devant son remplaçant. ll se pourrait bien que ce phénomène dont le but nous échappe dérivât d'une division qui se serait réduite à l'un des phénomènes qui la constituent.

Enfin, rappelons que c'est surtout sur le Stentor qu'ont élè exécutées les expériences de mérotomie, c'est-à-dire de division artificielle qui ont fait connaître le rôle remarquable du $\mathbf{N}$ comme agent nécessaire de la régénération. Dans ces cxpériences, le péristome cnlevé ne se rẻgénère pas précisément, il est remplacé par un autre né à cóté tout comme dans la division ou la rénovation. Si l'on coupe seulement quelques membranelles, elles ne sc régénèrent pas.

Le Stentor se nourrit d'Algues, de liotifères et d'auires Ciliés jlus petils que lui.

(1) L'anus est ì la face externe de l'aile gauche, près de sa base; la vésicule pulsatile cst au milieu de la hauteur du corps; le $\mathbf{N}$ est subcentral, ovale; le $\mathbf{n}$ est inconnu; la forme est indiquée sur la figure. L'animal vit dans un tube chitineux qu'il se sécrète el qu'il fixe par un ciment chitineux sur les Algues ou dans lcs coquilles vides des Mollusques. 
tome divisé par une côte saillante, en deux parties droite et gauche dont la dernic̀re seule est entourée par la zone adorale. Sur le pseudostome, est une tache pigmentaire qui permet sans doute à l'animal de distinguer la lumière qu'il recherche avidement. L'anus est inférieur'; il n'y a pas de vésicule pulsatile. La fonction de ce dernier organe est sans doute remplie par les vacuoles à fèces où l'on trouve des grains d'excrétion (').

\section{2e SOUS-ORDRE \\ OLIGOTRICHIDES. - OLIGOTRICHID.E \\ [Oligotriches; - Oligotricha (Bülschli)]}

\section{TYPE MORPHOLOGIQUE}

Les Hélérotrichides polytrichides avaient, outre leur zone adorale, un revêtement ciliaire uniforme sur tout le corps. lci, la ciliature générale est plus ou moins réduite, ou même tout à fait absente $\left(^{*}\right)$.

\section{GENRES}

Strombidium (Claparède et Lachmann) (fig. 7S6) peut ètre considéré comme le type le plus simple de cette série de formes. Le corps, plus ou moins conique, est nu sauf quelques cils ì la face ventrale. Le péristome occupe la base tournée en liaut. Sa surface n’est pas excarée, et porte au contraire une proéminence centrale; mais sa partie gauche se prolonge sur la face ventrale en un sillon très prononcé au fond duquel est la bouche. Ce sillon représente l'infundibulum luccal du Stentor qui se serait ourert le long de sa face antérieure. La zone adorale formée de longues membranelles, commence à droite de ce sillon, fait tout le tour du péristome et descend le long de la lèvre gauche du Fig. 786.

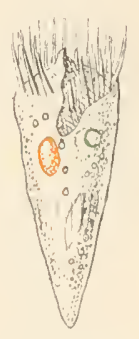

Strombilium (S. typicum) (d'ap. Bütschli). sillon jusqu’à la bouche. La surface du péristome n’est pas ciliée, mais il y a quelques cils épars sur la face ventrale $\left(0,0\right.$ '. Mer et eau douce) $\left({ }^{3}\right)$. Halteria (Dujardin), très peu diffèrent du précédent, s'en distingue d'ordinaire par une forme plus arrondie et par de longues soies tarliles dispo-

(1) Ces genres forment la famille des Stextorise [Stentorina (Stein, emend. Bütschlil].

$\left(^{2}\right)$ Quand la réduction porte sur l'existence des cils, comme chez Strombidium, le caractère est très net. 11 l'est moins lor'squ'elle porte sur leur taille comme chez Meseres.

(3) Le pharynx est à peine indiqué. Au bord droit du péristome, les trois cils plus gros sont des cirres au moyen desquels l'animal peut se fixer. Le $\mathbf{N}$ est ovale, la vésicule placée un peu haut. Par une exception unique dans un ordre autre que les Holotrichides, il y a des trichocystes défensifs disposés ordinairement en une ceinture au-dessous du milieu du corps 0,4. Mer et eau douce). Souvent il existe des grains chlorophylliens. Strobilidium (Cheviakof) est un strombidium sans aucune autre production ciliaire que sa zone adorale; il est fixé par son extrémité inférieure (Lau douce).

Meseres (Cheviakof) a, au contraire, de courts et fins cils sur tout le corps (Lau douce). 
sées en un cercle équatorial. L'animal se tient immobile, faisant activement tourbillonner l'eau avec ses membranelles, puis, d'un bond, s'élance à une place voisine où il reprend son immobilité (0,0's. Eau douce) (").

Tintinnopsis (Stein) (fig. 787) ressemble beaucoup à Strombidium; il en diffère cependant par plusieurs caractères. Le bord de son péristome est saillant et forme une circonférence complètement fermée, sans gouttière descendant sur la face ventrale; la zone adorale est formée de deux parties : une rangée externe de longues membranelles, et une rangée interne de cils appelés les cils paroraux implantés au pied des membranelles; elle forme un cercle complet fermé, et se prolonge néanmoins dans l'infundibulum buccal situé dans le péristome, en avant et à gauche, à l'intérieur du cercle adoral, comme chez le Stentor; le centre du péristome s'élève en une saillie comparable à celle de Strombidium, mais beaucoup plus développée et très mobile. L'anus est dans le péristome, à gauche de la bouche. La surface du péristome est nue. La ciliature du corps

Tintinnopsis (Sch.).

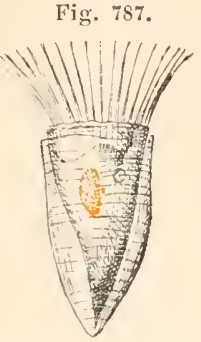

est réduite à quatre bandes de cils qui partent de la zone adorale et descendent en suivant des lignes verticales un peu courbées en spirale. $\Lambda$ ces caractères s'en ajoute un autre bien remarquable : l'animal a la partie inférieure du corps étirée en un pied par le moyen duquel il se fixe au fond d'une coquille en forme d'urne, chitineuse, mince, agglutinant des corps étrangers 0,2 . Mer', pélagique) $\left({ }^{2}\right)$. - Chez

(1) On a cru longtemps que ces soies étaient l'instrument de ce mouvement et on les nommait soies saltatrices avec Claparède et Lachmann. Mais il n'en est rien. MaUPas a montré qu'elles servent uniquement d'appareils sensitifs, et le mouvement est produit sans doute par des cils qui leur sont mêlés.

Ces deux genres constituent la famille des H.ILTERT.ve [Halterina (Claparède et Lachmann)].

Ici, sans doute, doivent ètre placées quelques formes prises par Van Beneden pour de jeunes Stentors (fig. 788) et rui sont incontestablement autonomes. Ce sont de pelits Infusoires, subsphériques, porlant sur l'hémisphère supérieur, parfois aplati, une zone adorale spirale. Le péristome est cilié on non; le corps est revêtu de cils plus ou moins rares et porte parfois une rangée de cils plus forts comparables aux cils transversaux des Hypotrichides. Mais ces formes sont trop mal connues pour que l'on ait pu les diviser en genres. Bütschli en fait une famille que nous appellerons LIEBERKLIINTV.L [Lieberkülnnina Bütschti], mais il n'ose pas leur attribuer des noms de genre.

(2) Au genre type Tintinnopsis s'en rattachent quelques autres qui diffèrent de lui principalement par les caractères de leur coquille. - Chez

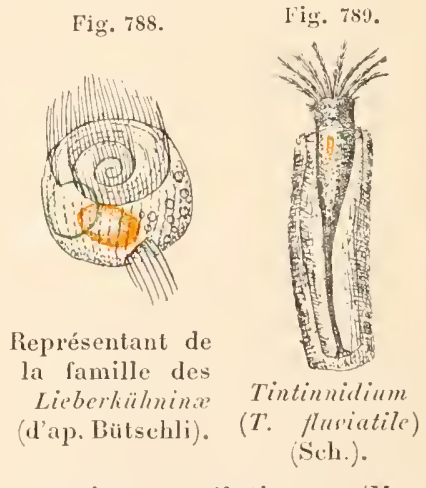

Tintinnidium (Kent) (fig. 789), cette coujuille est cylindrique, épaisse et gélatineuse (Mer et eau douce); - chez 
Codonella (Häckel) (fig. 790), la coquille a la forme d'un petit pot à orifice évasé ; elle est ornée de dessins hexagonaux et possède un appareil de fermeture $(0,1$. Mer et eau douce).

\section{Enfin chez}

Dictyocysta (Erhenberg), la coquille possède aussi un appareil de fermeture et, en outre, est ajourée; elle rappelle singulièrement celle de certains Radiolaires $(0,1$. Mer $)\left({ }^{2}\right)$.

Les caractères de Strombidium reparaissent dans une petile série de genres qui vivent en parasites en nombre immense dans l'estomac des Ruminants el semblent, d'après les recherches récentes d'EberLeix [95], ètre utile à ces animaux en transformant la cellulose de leurs aliments en

Fig. 790 .

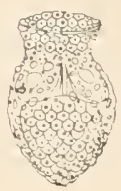

Codonclla (C. lagenula) (d'ap. Entz). une substance plus assimilable. On peut prendre pour type de ces ìtres Ophryoscolex (Stein) (fig. 791, 792). L'extrémité supérieure du corps est horizontalement tronquée el excavée en un entonnoir qui est l'entrée du pharynx. Les membranelles buccales sont insérées dans un sillon sur le bord de cet entonnoir et décrivent une spirale qui part du bord ventral, passe à gauche, en arrière, à droite et enfin plonge dans le pharynx. Les hords de l'entonnoir peuvent se replier en dedans et abriter les membranelles. En outre, il existe sur le corps, un peu plus bas, une deuxième rangée spirale de hautes membranelles insérées dans un sillon et dessinant un peu moins de deux tours de spire. C'est du còté ventral qu'est l'interruption.

Fig. 791.

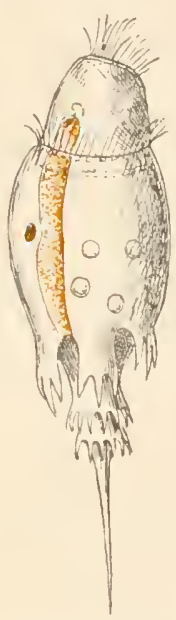

Ophryosiolex

(d'ap. Eberlein).
Fig. 792.

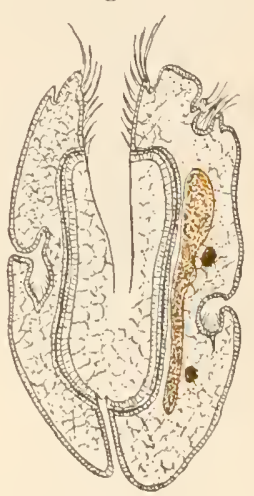

Ophryoscolex

Coupe longitudinale

(im. Eberlein).

Le reste du corps est entièrement dípourvu de toute production ciliaire. L'anus est au pôle opposé à la bouche 0,1 ì 0,3$)$.

Selon les espèces, le corps est, en bas, ou arrondi ou prolongé en pointes, les unes terminales, les autres disposées en rangées circulaires superposées.

Il en est de mème sous ce rapport clıcz

Tintinnus (Schrank, emend. Fol) (fig. 793), elle est chitincuse, plus on moins épaisse, sans colps étrangers $(0,3$. Mcr $)$;

Cittarocyclis (Fol) est à peine un sous-genre du précédent.

(1) Ces divers genres sont tous pèlagiques dans la mer ou dans les

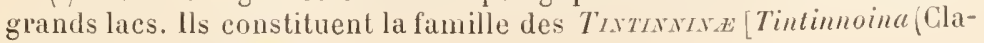
parède ef Lachmann)?

Fig. 793.

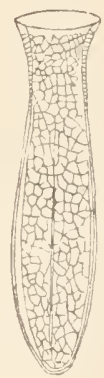

Tinlimulus (T. amphora)

(d'ap. Biitschli). 
Entodinium (Stein) (fig. 79ł) chez qui, en outre, les membranelles du corps ont disparu $(0,03$ a 0,12$)$.

Diplodinium (Schuberg) est très semblable au précédent, mais la zone adorale, après avoir formé sa spirale à l'entrée du pharynx, s'échappe par la tangente du côté gauche et va former une seconde spirale, toujours sénestre, dans un petit enfoncement en cul-de-sac qui se trouve situé dorsalement en arrière de la bouche (').

En appendice aux Hétérotrichides oligotrichides, il nous reste à mentionner deux formes aberrantes : ce sont les genres Gyrocorys et Maryna.

Gyrocorys (Stein) (fig. 793) a une apparence au premier

Fig. 794.

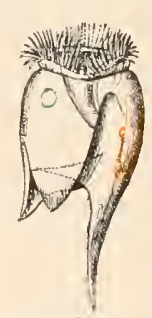

Entodinium

(E. caudatum)

(d'ap. Schuberg). abord inexplicable. Que l'on se figure une sorte de petite Méduse dont le manubrium serait si épais à la base qu'il réduirait la cavité sous-ombrellaire à un simple sillon et se prolongerait en pointe

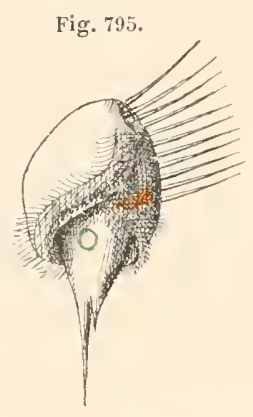

Gyrocorys (Sch.). conique hor's de l'ombrelle.Ce manubrium représente en réalité le corps du Cilié et l'ombrelle n'est rien autre chose qu'un vaste péristome dont le fond s'est fortement bombé en voùte, laissant le bord fort audessous de lui. Sur la face ventrale de ce péristome bombé, est une goultière verticale bordée à gauche de deux rangées parallèles de cils très forts,s'étendant de l'apex du péristome jusqu'à son bord ventral. On croirait que la bouche devrait se trouver au fond : il n'en est rien. Cetle gouttière se continue en un long sillon spiral qui court en dessous du bord du péristome, par conséquent entre le bord de l'omlirelle et le manubrium, passe à droile, en arrière, puis à gauche, en

(1) Ces trois genres, qui constituent la famille des OPHexoscolectse [Ophryoscolecina (Stein)], présentent de très remarquables particularités d'organisation. Partant de l'extérieur (fig. 792) on rencontre les parties suivantes : $1^{\circ}$ une membrane limitante externe; $2^{\circ}$ une couche alvéolaire externe; $3^{\circ}$ une épaisse couche de substance cytoplasmique contenant le noyau; 40 une couche alvéolaire interne; $5^{0}$ une membrane limitante interne; $6^{\circ}$ enfin, une grande cavité centrale dans laquelle plonge le pharynx et qui est occupée par une substance ayant l'aspect de protoplasma.

Schuberg et surtout Eberleis considèrent cette partie centrale comme représentant l'endoplasme, et tout le r'este comme constituant l'ectoplasme formé d'une masse épaisse comprise entre deux couches alvéolaires limitées chacune par une membrane el qui représenteraient l'une la pellicule externe ordinaire, l'autre une couche limitante spéciale interposée à l'ectoplasme et à l'endoplasme. Le pharynx se jetterait comme d'ordinaire dans l'endoplasme, mais l'ectoplasme aurait une épaisseur et une structure tout à fait inusitées et renfermerait le noyau.

Toute cette description est si extraordinaire, elle admet une structure et des rapports entre les parties essentielles si peu conciliables avec la conformation habituelle des Infusoires que nous éprouvons une grande répugnance à l'accepter et accueillons avec empressement une interprétation que ScHuberg a émise, mais d'ail- 
suivant une hélice descendante et se jette enfin dans la bouche située ventralement, après avoir fait un peu moins d'un tour. Ce sillon étant la seule séparation entre l'ombrelle et le manubrium, il existe donc une étroite région où le profil vertical du corps est continu et, en ce point, la distinction du corps et du manubrium n'existe pas. Ce sillon est bordé de deux rangées parallèles d'organes moteurs : une rangée de petites membranelles immédiatement au-dessus de lui, et une rangée de longs cils un peu au-rlessus des membranelles. Cils, membranolles et sillon suivent parallèlement le mème trajet héliçö̈dal depuis la gouttière péristomienne jusqu’à la bouche. De la bouche, part un pharynx ascendant. L'anus n'est pas connu. Le $\mathrm{N}$ est bi ou quadritobé; un $\mathbf{n}$ lui est associé; il existe une grosse vésicule pulsatile.

L'animal se meut en tournant rapidement sous l'impulsion des longs cils silués à gauche du sillon péristomien. Les cils el les membranelles du sillon préluuccal servent à déterminer le courant alimentaire. Les auteur's ne se prononcent pas nettement sur les assimilations de ces organes, mais il semble difficile de voir autre chose que la zone adorale dans la rangée des membranelles, et une bordure de cils paroraux dans la rangée ciliaire parallèle. Quant aux cils bordant la goultière verticale, ils ne peuvent appartenir qu’à la ciliature du péristome, si vraiment toute la surface bombée est formée par une voussure du péristome. Mais il se pourrait que la surface située à droite de cette gouttière appartint seule au péristome et, dans ce cas, ces cils moteurs appartiendraient au revètement général du corps. Il n’y a d'ailleur's pas d'autres cils sur le corps (0mm1. Ner ou eau douce). - Dans le genre

leurs sans la préférer à l'autre, retenu surtout par la grande ressemblance de la substance centrale avec du protoplasme.

Cette interprétation consiste à considérer la masse centrale, non comme de l'endoplasme mais comme une sorte de chyme formé par les matières ingérées par le pharynx, à demi digérées et destinées à être en partie absorbées à travers la paroi, en partie rejetées par l'anus qui s'ouvre dans cette même cavité. L'ensemble ne serait qu'un estomac ou plutòt un vrai tube digestif complet. Les couche limitante spéciale et alvéolaire interne formeraient a ce tube digestif une paroi comparable à la paroi pharyngienne des autres Ciliés' et n'en différant que par le fait qu'elle s'étendrait sans interruption de la bouche à l'anus. Dès lors, le reste devient conforme à ce que l'on trouve chez tous les Ciliés. Les couches limitante externe et alvéolaire externe constitueraient le tégument ordinaire avec ses deux couches, la couche alvéolaire interne formerait l'endoplasme contenant le noyau.

Les choses ne seraient-elles pas même encore plus simples et n'aurait-on pas le droit de considérer ce prétendu tube digestif comme une portion de la surface du corps invaginée, quelque chose comme ce que l'on obtiendrait chez Bursaria en prolongeant le fond de l'infundibulum jusqu'à ce qu'il vienne s'ouvrir à l'extrèmité inférieure. Le prétendu amus ne serait que ce second orifice et les prétendus bouche et pharynx ne seraient que l'infundibulum péristomien. Bien entendu, nous ne proposons cela que comme une simple suggestion destinée à provoruer de nouvelles observations et, en particulier, la recherche d'une fente buccale quelque part sur la paroi du soi-disant estomac. 
Maryna (Gruber) (fig. 796, 797) le corps, recouvert sur toute sa surface de cils fins, a la forme d'un ovoüde dont le quart supérieur tronqué, évasé et excavé formerait le péristome. Ce péristome est interrompu en avant par une gouttière triangulaire qui descend sur la ligne médiane el conduit à la louche; une zone adorale de cils à peine plus développés que ceux du corps orne son bord libre. Du centre, s'élève une haute papille appeléel'entonnoir, qui rappelle la forme du péristome lui-même. Elle est, en effet, comme celui-ci, excavée, munie sur son bord libre de cils, mais ici très grands, et se prolonge sur la face ventrale en une gouttière verticale. L'entonnoir est immobile, mais les cils sont très actifs. Les aliments sont attirés par le tourbillon

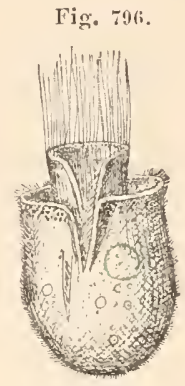

Maryna

(M. socialis) (d'ap. Gruber). Individu isolé. que déterminent les cils de l'entonnoir dans le sillon entre l'entonnoir et le corps, et sont conduits par là à la bouche.

L'animal sécrète un tulıe gélatineux jaunâtre (fig. 797). Quand il se divise, les individus filles continuent ce tube en produisant une ramification dicholomique; ces tubes ramifiés portent à leur extrémilé une petite excavation où se loge l’individu qui a sécrété le tube. Cette forme n'est pas sans quelques resseml,lances avec les Vorticelles $(0,15$. Eau douce).

$3^{\text {e }}$ ORDRE

\section{IIYPOTRICIIDES. - IIYPOTRICIIDA}

[Hypotriciles; - Hypotricha (Stein)]

\section{TYPE MORPHOLOGIQUE}

(FIG. 798)

Le corps de taille moyenne (environ $0 \mathrm{~mm} 3$ ) est ovoüde, à grosse extrémité supérieure, plat sur la face ventrale, bombé sur le dos. $\Lambda$ la partie supérieure gauche de la face ventrale, se trouve un large péristome en forme de triangle curviligne, très accusé et de constitution assez compliquée. ll part de l'extrémité supérieure, suil le bord frontal du corps de droite à gauche, et de là descend sur la face ventrale où il aboulit à sa partie moyenne après un trajel oblique et curviligne à concavilé tournée à droile. Ce péristome est profondément excavé, de plus en plus creux ver's le bas où il conduit à la bouche que continue un pharynx peu développé. La lèvre gauche est large el donne insertion à une rangée d'actives membranelles ( $m b$.) constituant la zone adorale ( $Z$.a.). Les mem- 
branelles se continuent le long du bord frontal, toujours en suivant la lèvre péristomienne gauche, devenue dorsale à ce niveau. Le bord droit du péristome, au contraire, est mince. Il se prolonge en une membrane préorale ( $\mathbf{H L} 6 . \mathrm{u}$. pre.) qui se rabat sur le péristome et le recouvre presque dans toule sa largeur.

Derrière la membrane préorale, est une rangée de cils préoraux (c.pre.) quisuit le mème trajet. Un peu plus en dedans, au fond de la gouttière péristomienne mais un peu à droile, est une membrane endorale ( $\mathbf{I} \mathrm{l} . u$. end.) disposée aussi parallèlement à l'axe du péristome et qui, à son extrémité inférieure, plonge dans le pharynx. Puis vient, tout au fond de la gouttière péristomienne, encore une rangée verticale de cils dits endoraux, qui se continuent aussi

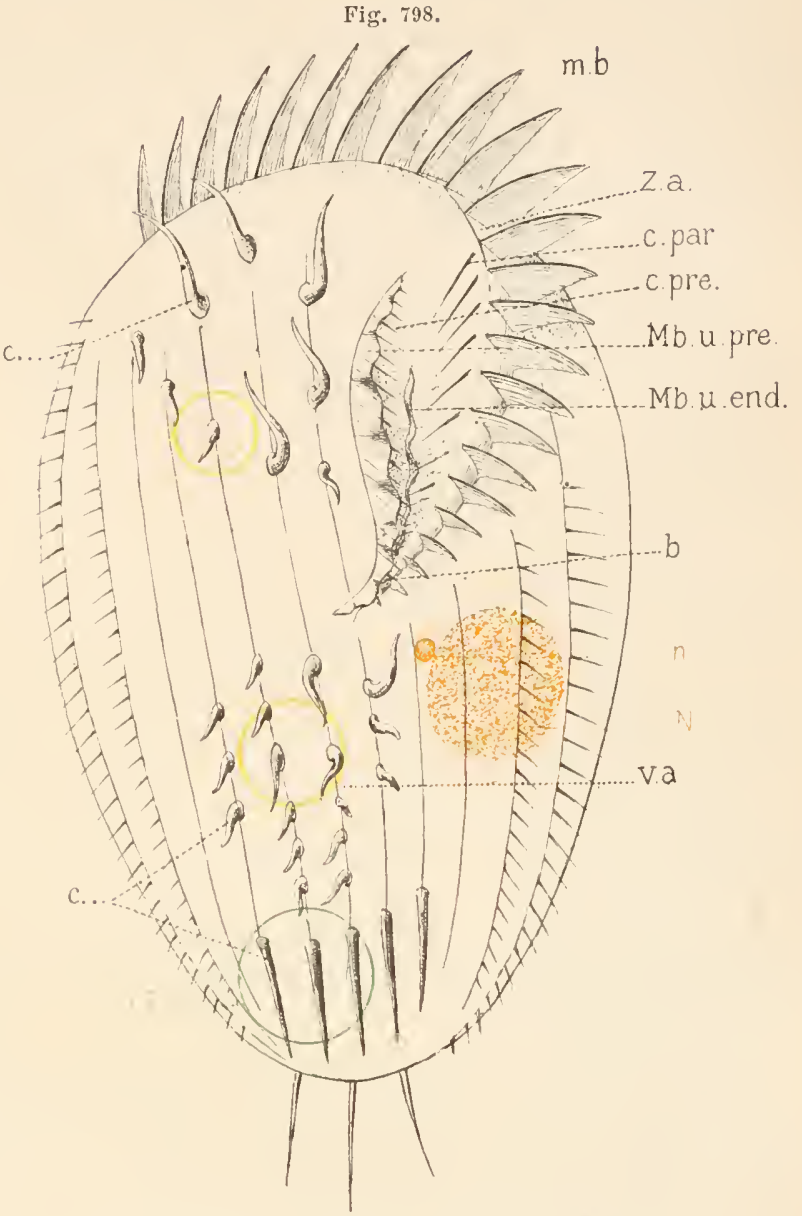

HFPOTRICHIDA (Type morphologique) (Sch.).

b., bouehe; c., cirres; c. par., cils paroraux; c. pre., eils préoraux ; mb., membranelles; Mb. u. end., membrane ondulante endorale; Iu. u. pre., membrane ondulante préorale; $\mathbf{N}$, macronucléus; $\mathbf{n}$, micronucléus; va., vacuole alimentaire; v. p., vésicule pulsatile; z. a., Zone adorale.

dans le pharynx; enfin, le long de la zone adorale, est une rangée de cils dits paroraux (c.par.) en mème nombre que les membranelles et disposés chacun à droite de la membranelle correspondante (').

Sur toute la face dorsale, le revètement ciliaire est très modifié : il n'y a plus de cils, mais des soies tactiles, raides et immobiles dispo-

(1) Il s'en faut de beaucoup que le péristome soit toujours ainsi constitué, il est parfois plus, souvent moins compliqué. Les membranelles sont typiques, la membrane préorale ne manque que rarement, les autres parties sont plus souvent absentes. 
sées comme des cils sur des lignes longitudinales, mais plus espacées.

Sur la surface ventrale, tous les cils sont transformés en cirres (c.), c’est-à-dire en petit pinceau conique de cils agglutinés, se mouvant selon les hesoins comme les pattes d'un animal supérieur et non agités d'une vibration monotone comme ceux des types précédents (').

Les cirres $(c$. sont disposés comme les cils ordinaires en série longitudinale, mais ces séries ne sont ici ni régulières, ni complètes; en bien des points elles sont interrompues par des lacunes et les cirres restants deviennent alors beaucoup plus gros.

Dans la région frontale, s'étendant du bord supérieur à la bouche, ils manquent tous, sauf un petit groupe d'une dizaine situé à droite de la bouche el que l'on appelle les cirres frontaux. Dans la région moyenne ou abdominale qui va jusqu'aux cirres transversaux, on distingue deux rangées marginales et plusieurs rangées moyennes. Les deux marginales sont complètes, formées de cirres petits el bien semblables; elles dépassent la région moyenne et s'étendent presque jusqu'aux extrémités. Dans les rangées moyennes, beaucoup de séries sont incomplètes, mais les cirres restants sont développés. Ces cirres plus développés occupent des hauteurs diverses dans les différentes séries, ce qui fait qu’ils onl l'air d'ètre irrégulièrement disposés. Dans la région inférieure ou caudale, on trouve une rangée de cirres transversaux appelés souvent cirres anaux (c., au bas de la figure), parce que l'on croyait à tort que l'anus s'ouvrait au-dessous d'eux. Ces cirres forment une rangée transversale ou plutôt un peu oblique à droite et en bas. Ils sont glutineux et peuvent servir à fixer l'animal. Enfin, à l'extrémité inférieure du corps, se trouve un groupe de soies homologues aux cirres, mais qui sont des soies tactiles raides et immobiles $\left(^{2}\right)$.

Il faut bien comprendre que toute cette irrégularité de la ciliature ventrale repose uniquement sur l'absence de quelques cirres et le développement de certains autres et que, si l'on comblait les lacunes laissées par les absents, on retrouverait des séries longitudinales régulières et complètes, mais formées de cirres les uns petits, les autres très grands $\left({ }^{\mathbf{3}}\right)$.

(1) Bien qu'ils soient certainement formés de cils agglutinés comme le prouve l'artion de certains réactil's permetlant de les dissocier', les cirres ne se forment pas ontogéniquement d'un pinceau de cils. Comme les membranelles, ils naissent formés tout d'une pièce. La distinetion entre un cil et un eirre très petit est un peu artificielle. Chez les formes les plus inférieures d'llypotrichides, Peritromus par exemple, on peut aussi bien appeler cils que cirres les appendices de la face ventrale. Plus ils se réduisent en nombre, plus ils deviennent différents des cils ordinaires. Les gros cirres subissent parfois des différenciations plus avancées, en crochets par exemple. Les anciens zoologistes distinguaient les cirres, styles, cornicules, crochets, etc.

$\left(^{2}\right)$ Parfois elles servent aussi au saut (Styloplotes, Uronichia).

(3) Chez les Ilypotrichides inférieurs le revêtement des cirres de la face ventrale est complet et uniforme et l'on voit peu à peu se constituer, en parcourant la série des genres, l'état que nous avons décrit et d'autres oủ la réduction et la différenciation sont poussées encore plus loin. 
Il n'y a pas de trichocystes. L'anus est.situé à la face dorsale, un peu à gauche, à quelque distance au-dessus du niveau des soies transversales. La vésicule pulsatile et son pore excréteur sont situés un peu audessus de lui ('). Le $\mathbf{N}$ et le $\mathrm{n}$ n'ont rien de particulier.

L'animal ne se meut plus de ce mouvement monotone des Holotrichides ou des llétérotrichides: il marche véritablement avec ses cirres sur la face ventrale, comme un animal supérieur avec ses pattes, se mouvant dans des directions déterminées et variées, comme si ce mourement était déterminé par sa volonté. Souvent, il reste en repos; seules ses membranelles sont toujours actives pour déterminer le tourbillon alimentaire. En outre des mouvements et des déformations déterminées par les cils, il est très contractile : tout se passe comme si sa face ventrale était parcourue par des myonèmes longitudinaux, et cependant il n'y en a pas trace $\left({ }^{2}\right)$.

\section{GENRES}

Tous ces caractères ne se rencontrent pas d'emblée dans les genres. Il n'y a guère de général que la situation du péristome et les grands traits de sa constitution, l'aplatissement de la face ventrale, la transformation des cils dorsaux en soies tactiles et celle des cils ventraux en cirres. C'est surtout la différenciation progressive de la ciliature ventrale qui va nous servir de guide dans la classification.

La forme que l'ón peut considérer comme l'origine de toutes ces séries de transformations nous semble être le genre

Peritromus (Stein) (fig. 799). C'est absolument le type morphologique ci-dessus décrit, sauf que le revètement ciliaire ventral est entièrement uniforme. Ce sont des cirres tous semblables, peu différents des cils

Fig. 799.

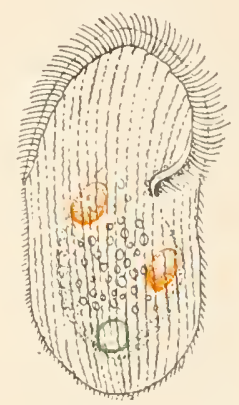

Peritromus (P. Emma)

(d'ap. Bütschli). d'un Holotrichide, disposés en rangées verticales $(0,1$. Mer $\rangle\left(^{3}\right)$.

Lapremière petite différencialion apparaìt dans le genre Kerona (Ehrenberg) (fig. S00) chez lequel la ciliature abdominale nous montre, en dedans d'une bordure régulière formée par les ileux rangées marginales, six à sept rangées obliques de petits cirres à peine différents des cils ordinaires et tous semblables entre eux. C'est à peine si les cinq derniers de la dernière rangée à droite montrent un léger accroissement de taille et un arrangement plus

(1) Tout cela, bien entendu, est variable suivant les genres. L'anus peut mème être rentral, chez les Euplotines par exemple.

Fig. 800,

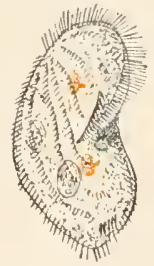

Kerona

(K. pediculus) (d'ap. Stein).

$\left({ }^{2}\right)$ Chez beaucoup d'Hypotrichides il n'y a pas d'ectoplasme distinct. Le protoplasma se raffermit graduellement a la surface du corps sans changer de caractère.

${ }^{(3)}$ Nous avons vu (p. 458) qu'en raison de ce fait divers auteurs le placent parmi les Hétérotrichides. 
régulier qui fait deviner en eux les futurs cirres transversaux 0 , 15. Eau douce, parasite sur les Ilydres) (').

Partant de là, nous pouvons suivre deux séries de variations. La première est très courte et porte immédiatement sur la différenciation des cirres sans entamer le nombre de leurs séries; elle nous conduit au genre

Urostyla (Ehrenberg) (fig. 801) chez lequel la face ventrale est garnie de rangées longitudinales de cirres à peine différents des cils ordinaires, mais qui présentent une différenciation très nette des cirres frontaux et des cirres transversaux $(0,3$. Her et eau douce).

La seconde série des variations nous conduit au genre Epiclintes (Stein) chez lequel nous trouvons les séries ventrales de cirres réduites à cing ou six, y compris les deux marginales qui ne sont pas distinctes des autres. Il n'y a aucune différenciation des cirres frontaux ou transver-

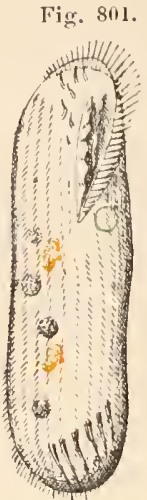

Lrostyla (Sch.). saux (0,3. Mer). - Ce nombre subit une nouvelle réduction dans le genre Stichotricha (Perty) où les séries deviennent fortement spirales $\left(0,1\right.$. Mer et eau douce) $\left(^{*}\right)$. - Chez

Holosticha (Vrzesniovski) (fig. 802), les cirres transversaux commencent à se montrer, mais il n'y a pas de cirres frontaux $(0,3 . \mathrm{Ner})$. - Chez

Strongilidium (Sterki), ce sont les frontaux qui apparaissent, mais les transversaux manquent (Eau douce). - Il en est de mème chez

Uroleptus (Ehrenberg) (fig. 803) où les cirres frontaux deviennent très forls (0,5. Mer et eau douce) $\left({ }^{\mathbf{3}}\right)$.

Tetrastyla (Cheviakof) possède les uns et

( ${ }^{1}$ L'animal a la forme d'un haricot, son $\mathrm{N}$ est double, cloisonné.

Il y a bien une forme plus primitive encore peut-être, le genre :

Trichogaster (Sterki) chez lequel les cirres ont encore le caractère de cils, sauf quelques-uns différenciés en vrais cirres dans la région frontale et au-dessous du péristome, mais ce genre est mal connu et n'a pas été figuré.

(²) L'extrémitè supérieure est étirée en une

Fig. 803 .

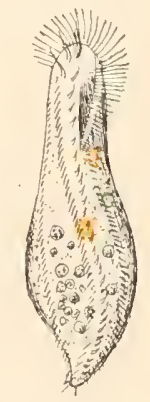

Uroleptus

(U. musculus)

(d'ap. Stein).
Fig. 812.

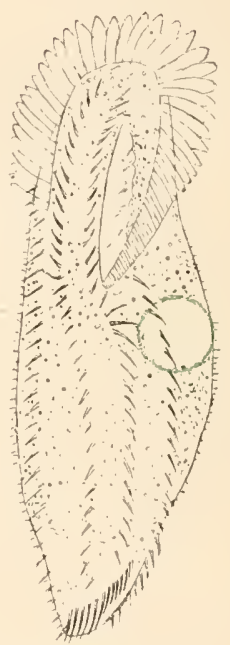

Holosticha

(II. mutinucleata) (d'ap. Maupas).

trompe très mobile sur laquelle se prolonge la zone adorale.

Sparotricha (Entz) est un genre voisin chez lequel la zone adorale ne dépasse pas le milieu de la trompe $(0,1$. Etangs salés).

Peut-ètre est-ce ici ru'il faut placer les genres douteux et insuffisamment décrits : Drepanidium (Ehrenberg) et

Mitra (Quennerstedt).

( ${ }^{3}$ Les deux rangées marginales s'écartent des rangées ventrales, celles-ci sont réduites à deux et la grande est formée de cirres marcheurs bien réveloppés. - Ghez Stylonetes (Sterki), genre douteux, il semble en être de même. 
les autres car il a quatre cirres frontaux et quatre cirres transversaux bien développés et trois rangées abdominales complètes (Eau douee, Nouvelle-Zélande) ('). - Enfin dans le genre

Amphisia (Sterki) (fig. S04) nous avons aussi les uns et les autres et désormais ils ne manqueront plus $(0,04)$.

Dans les formes précédentes, les rangées ventrales de cirres pouvaient se restreindre à un petit nombre, mais du moins étaient-elles complètes; elles ne montraient pas de discontinuité dans leur longueur. Nous allons rencontrer maintenant une série de formes où, non seulement les cirres des régions frontale et ablominale inférieures sont différents de ceux des rangées ventrales dont ils dérivent, mais où, en outre, ces rangées ventrales vont se disloquer; certains de leurs cirres disparaissant tandis que d'autres deviennent plus développés, au point qu’il finit par devenir impossible de reconstituer par la pensée les séries longitudinales auxquelles appartiennent ces éléments épars. Les deux rangées marginales de cirres ront cependant encore garder ici leur individualité complète. Pleurotricha (Stein) (fig. 80:3) peut ètre considéré comme le type de cette série de formes. En dedans de sa bordure de cirres marginanx, il montre huit ì dix rangées ventrales dont les plus externes ne sont point modifiées et se montrent composées de cirres uniformes et régulièrement ¿lisposés, tandis que les cinq rangées moyennes incomplètes et irrégulières se trourent réduites à un groupe de huit cirres fronlaux étagés sur trois rangs, à un groupe de cinq cirres transversaux el à un petit nombre de cirres ventraux dont cinq sont particulièrement développés $(0,4$. Eau douce $)\left({ }^{2}\right)$.

Cette réduction s'accentue de plus en plus dans les divers autres genres de cette série $\left({ }^{3}\right)$.

(1) Mais son auteur ne mentionne pas les rangées marginales.

Fig. 801

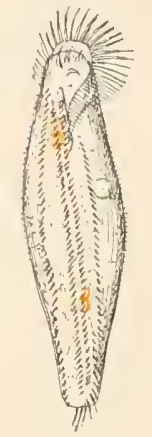

Amphisia (A. Kessleri) (d'ap. Virzesniorski).

- Le genre

Fig. 805 .

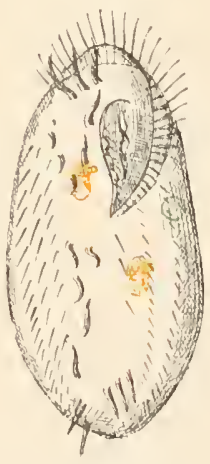

Pleurotricha

(im. Stein).

S'il faut interpréter comme telles les deux abdominales latérales, cela réduit à une la série des abrlominales vraies.

() L'animal a un péristome régulièrement eonformé, deux $\mathrm{N}$ il cloison avee chaeun un $\mathbf{n}$ annexé à lui et une vésicule pulsatile située assez haut, à gauehe de la bouche.

(3) Voiei ees gellres :

Onichodromus (Stein), à trois rangées de eirres frontaux et trois à quatre cirres abdominaux (0,33̈. Lau douce);

Allotricha (Sterki), insuffisamment connu, paraissant se rattaeher au préeédent (Eau douce); Gastrostyla (Engelmann), à einq à six eirres frontaux et une rangée abdominale $(0,32$. Eau douce);

Gonostomum (Sterli), à deux cirres abdominaux seulement et einq transversaux $(0,2$. Mer et eau douce); 
On peut avoir une idée de ce à quoi elle aboutit par l'examen du genre

Stylonichia (Stein) (fig. S06) chez lequel, en dedans de la bordure de petits cirres marginaux, on ne trouve plus que de gros cirres subulés, les uns frontaux, d'autres transversaux, les autres ventraux (0,4. Eau douce) (").

Une mention spéciale est nécessaire pour le genre remarquable Actinotricha (Cohn) qui diffère sous plusieurs rapporls des types de la série précédente. Le péristome est rérluit à une fente si étroite qu'on le distingue à peine. La lèvre gauche porte de très larges membranelles disposées en éventail. Celles de la région frontale de la fente péristomienne sont beaucoup plus grandes encore et plus divergentes que les autres. Toutes ces membranelles, par une exception singulière, sont immobiles et sans doute ne mancurrent que lorsque l'animal est lui-mème en mouvement pour chercher sa nourriture. Dès qu'il

Fig. 807 .

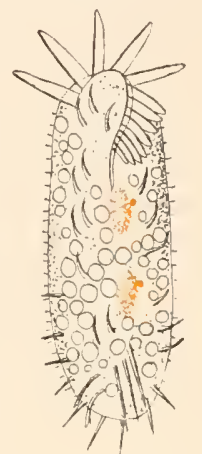

Actimotricha

(A. saltans)

(d'ap. Maupas).
Fig. 806.

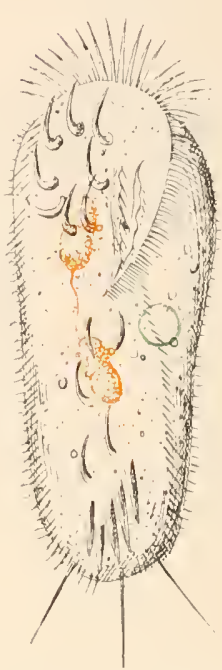

Stylonichia (im. Stein). s'arrête, ses membranelles s'arrêtent aussi. Les cirres frontaux, transversaux, ventraux ne présentent rien de particulier, mais les marginaux sont ģros, subulés, très espacés comme s'ils commençaient à se disloquer et à prendre les caractères de cenx de la face ventrale $\left(0,1\right.$. Mer) $\left(^{\mathbf{2}}\right)$.

Citons aussi les genres aberrants

Balladina (Kovalevsky), remarquable par la disparition des cirres frontaux, la grande réduction de nombre des cirres ventraux et la grande longueur des marginaux $(0,04$. Lau douce); et

Psilotricha (Stein) où la ciliature se réduit à des cirres épars sur la face ventrale sans distinction des groupes frontal, abdominal, transversal; la série marginale elle-mème ne se reconnaìt plus $\left(0,1\right.$. Eau douce) $\left(^{3}\right)$.

Urosoma (Kovalevsliy), à huit cirres abdominaux et cinq transversaux $(0,24$. Mer et eau douce);

Oxytricha (Sterki), à cinq cirres abdominaux, sans cirres caudaux $(0,2$. Ner et eau douce); Histrio (Sterki), à cinq cirres abdominaux, les deux rangées marginales continues d'un còté et d'autre (Eau douce).

(1) Il y a, en outre, trois soies terminales. Le $\mathbf{N}$ est doublé, formé de deux lobes réunis par un long filament mince; chacun des deux lobes est cloisonné et un n lui est annexé.

() Le $\mathbf{N}$ est semblable à celui du genre précédent.

(3) On peul sans doute adjoindre ici

Stylocoma (Gruber) qui n'est qu'un genre douteux.

Toute cette nombreuse série constitue la première grande famille d'llypotrichides 
Dans les deux genres précédents, la réduction des cirres portail irrégulièrement sur tous et tendait à effacer leurs différences plutôt qu'à les accuser. Il en est autrement dans la petite série de genres suivants, chez lesquels elle porte sur les rangées latérales et sur le groupe abdominal, mettant en relief les groupes frontal el transversal qui arrivent à absorber la totalité de la ciliature. En outre, le corps se distingue par une fermeté particulière.

Le type de celte série est le genre

Euplotes (Ehrenberg) (fig. S08). Il montre nettement six ou sept cirres frontaux, une bande très accusée de cinq cirres transversaux qui sortent du fond d'un sillon transversal; on voit aussi très bien quatre cirres terminaux qui se laissent assez aisément interpréter comme les derniers d'une série marginale disparue. Mais il existe, en outre, sur la face ventrale, deux ou trois cirres dont l'interprétation est assez difficile, car on peut aussi bien les appeler abdominaux en raison de leur situation entre les frontaux et les transversaux que frontaux en raison de ce qu'ils sont situés à droite du péristome. Le péristome descent, en effet, très bas et les cirres transversaux sont situés très haut, en sorte qu'il ne reste guère de place pour une région abdominale propre-

Fig. 808 .

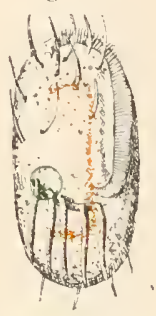

Euplotes

(im. Claparide et Lachmann). ment dite, et c'est là un des principaux caractères du genre $(0,2$. Her et eau douce) (').

Tous ces caractères s'exagèrent dans le genre

Diophrys (Dujardin) où le péristome descend plus bas et arrive presque au contact des cirres transversaux qui sont énormes 0,15 . Mer) $\left({ }^{*}\right)$, et chez Uronichia (Stein), où en outre les cirres frontaux disparaissent $(0,1$. Mer).

Ėnfin cette réduction de la région abdominale arrive à son maximum dans le genre

Aspidisca (Ehrenberg) (fig. S09). Le corps est si raccourci qu'il prend un contour ovale et presque rond et que la forme se rapproche

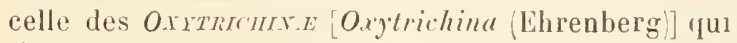
s'oppose a une seconde grande famille, celle des EIT'LotTiE [Euplotina (Ehrenberg)] dont nous allons maintenant parler.

Entre ces deux familles, mais se rattachant plutôt à la première, se place le genre

Certesia (Fabre-Domergue) caractérisé par une consistance

Fig. 809 .

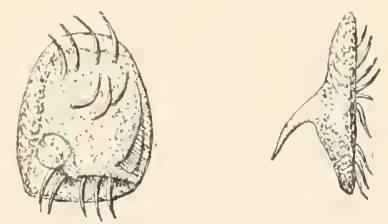

Aspidisca (A. turrita)

(d'ap. Claparède et Lachmann).

ferme et par la présence de cirres marginaux d'un senl còté (à gauche), en outre destuels il $y$ a seulement neuf cirres frontaux, un abdominal et cing transversaux très développés (Mer).

(1) Ajoutons que le péristome s’ètend tout le long du bord frontal, que l'anus et la vésicule sont situés dorsalement à droite, à la hauteur des cirres transversaux et que le $\mathbf{n}$ est très long, rubané en forme de C, mais à concavité tournće it droite.

( $^{2}$ Il y a, en outre, trois soies saltatrices termino-dorsales de remarquable forme. Planiplotes (Andrussova) est un genre douteux voisin. 
de celle d'une lentille plan convexe. Le bord droit régulièrement arrondi se continue avec le bord frontal. Le péristome abandonne tout à fait le bord frontal, descend le long du côté gauche et prend la forme d'une étroite fente verticale qui s'étend vers le bas jusqu'au delà du milieu du corps. Le bord droit du péristome forme une grande lèvre saillante qui recouvre le péristome et n'en laisse guère qu'une située à gauche. Le bord gauche du corps est séparé en haut du hord frontal par une profonde encoche, se prolonge en bas en une pointe plus ou moins accentuée et se continue à droite, sur la face ventrale, en une lame qui détermine en dessous d'elle un profond sillon. C'est du fond de ce sillon que partent cinq cirres transversaux. La région abdominale a donc tout à fait disparu. Sur la large région frontale située à droite du péristome, s'insèrent sept gros cirres et c'est à ces douze cirres que se réduit toute la ciliature de l'animal. L'anus est à droite, immédiatement au-dessous des cirres transversaux; la vésicule pulsatile est auprès de lui; le $\mathrm{n}$ est unique et le $\mathbf{N}$ a la forme d'un anneau presque fermé, parallèle au contour du corps. L'animal se meut rapidement le plus souvent en cercle 0,07 . Mer et eau douce).

Nous avons décrit avec quelques détails cette forme extrème pour montrer combien nous sommes, par des transitions insensibles, arrivés loin de l'Infusoire typique qui nous a servi de point de départ (").

\section{$4^{e}$ ORDRE}

\section{PÉRITRICHIDES. - PERITRICIIIDA [Péritriches; - Perithicha (Stein)]}

Les Péritrichides ont tous la zone adorale contournée en hélice. Mais chez les uns la courje est sénestre comme chez les Hétérotrichides, et comme d'ailleurs chez tous les lnfusoires étudiés jusqu'ici; chez les autres elle est dextre. Cela constitue deux types de structure que l'on peut faire dériver l'un de l'autre par des contournements plus ou moins vraisemblables, mais qui n’en sont pas moins très différents et doivent ètre étudiés séparément $\left({ }^{*}\right)$.

(1) Aspidisca est le type unique peut-être de la famille des AspiDLsctse [Aspidis. cina (Stein)]. Le genre

Onichaspis (Stein) semble n'être qu'une espèce du précédent. C'est ici que semble devoil' prendre place le genre douteux :

Rhabdotricha (Greeff). Nous ne ferons que citer les noms des genres:

Discocephatus Ehrenberg),

Turpinius (Ormancey),

Gervasius Ormancey), dont la place mème parmi les IIypotrichides est douteuse.

(2) Pour la définition exacte des termes dextre el sénestre (voyez p. 40̈4). 
Nous diviserons done cet ordre en deux sous-ordres:

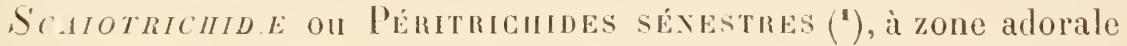
sénestre;

Dexiotrichide ou Péritricindes dextres $\left({ }^{*}\right)$, à zone adorale dextre.

$$
\text { L er Sous-Ordre }
$$

\section{SCAIOTRICHIDES. - SCAIOTRICHID.E}

ou

\section{I'ÉRITRICHIDES SÉNESTRES}

\section{[LICNOPHORINA (Bütschli) + SPIRocIIONINA (Bütschli)]}

Comme il n'y a ici que deux genres, il est inutile de les ramener à un type morphologique, mieux raut les étudier directement.

Licnophora (Claparède) (fig. 810). L'animal a l'aspect d'une massue. Il est formé d'un corps renflé, ovoüde, et d'un pédoncule terminé en bas par une ventouse circulaire par laquelle, étant parasite, il se fixe sur son lıôte. Celte ventouse est renforcée d'un anneau qui représente, sous une forme très réluite, l'appareil que nous rencontrerons bientòt si développé chez Trichodina. Le bord externe de la ventouse donne insertion à une couronne de cils qu'il faut nommer paree que nous la rencontrerons souvent chez les Péritrichides, ce sera Ia couronne ciliaire inférieure.

Sur la face antérieure de la portion renflée se trouve un vaste péristome qui n'est nullement excavé et représente simplement la portion de la surface gé-

Fig. 810.

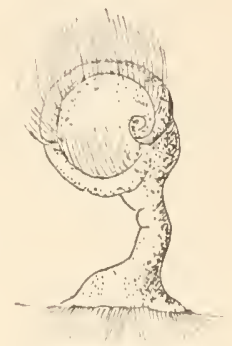

Licnophora (im. Claparède). nérale qui est entourée par la zone adorale formée d’une rangée de simples cils. En dehors de celle-ci, on observe une seconde rangée de cils. La zone commence assez bas au côté droit de la face ventrale, suit à faible distance tout le contour du corps, redescend le long du côté gauche et, de là, se porte vers la bouche qu'elle entoure d'un tour de spire. La bouche est située un peu an-dessous et à gauche, au milieu du corps. Toute la surface du corps est entièrement dépourvue de cils : il n'y a pas d'autre production ciliaire que la zone adorale et la couronne inférieure $(0,12$. Parasite sur divers Invertébrés, marins : Echinoderınes, Annélides, Méduses, Opisthobranches) $\left(^{3}\right)$.

1) de $\Sigma \% x$ :ós, sénestre.

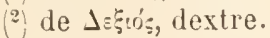

(3) Il y a un long noyau en chapelet à grains dissociés et une vésicule situėe à gatuche de la bouche.

Bütschli erée pour ce seul genre une famille: LICxopronswe Licnophorima [Bütschli] . 
Spirochona (Stein) (fig. S11, S12). Tout autre est la forme de Spirochona. Celui-ci a plutòl la physionomie d'un Stentor. Sa forme

Fig. 811.

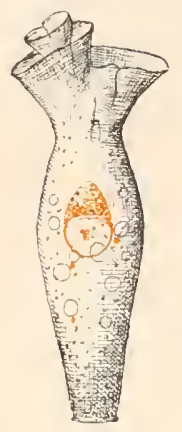

Spirochona (im. Hertwig). générale est celle d'un cône allongé dont le sommet largement tronqué est dirigé en bas et sert de surface de fixation. Cette surface tronquée est, en effet, transformée en un disque adhésif circulaire. Ce disque présente quelques stries radiaires formées par la membrane. Pour comprendre la forme très compliquée du péristome, représentons-nous celui d'un Stentor (fig.\$12,B) qui nous servira de point de départ. Comme chez le Stentor, la surface péristomienne est horizontale et occupe la base supérieure du cône. La zone adorale commence, comme celle du Stentor, par une courbe spirale qui fait un tour presque complet avant de plonger dans la bouche située dans la boucle formée par son extrémité gauche. Mais ici, l'extrémité aborale de la courbe, au lieu de s'arrêter à quelque distance de l'extrémité orale, la rejoint, lui devient tangente et, continuant à tourner dans le même sens, fait encore un tour dans l'intérieur du péristome du côté droit (fig. S12, A). Chrez le Stentor, le rebord du péristome est légèrement saillant; ici, il l'est aussi, mais beaucoup plus, il s'élève en un entonnoir très développé ou plutôt en cornet d'oublie (fig. S11). Ce cornet est situé immédiatement en dehors de la zone adorale, en sorte que celle-ci reste au fond lu péristome, au pied de l'entonnoir. Enfin ce comet ne s'arrète pas, comme la zone adorale, après avoir formé un tour à droite en

Fig. 812.

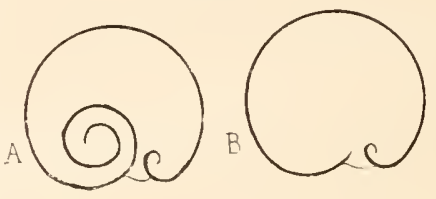

Disposition de la zone adorale (Sch.).

A, chez Spirochona; $B$, ehez Stentor. dedans du premier tour, il continue à s'enrouler sur lui-même et forme encore presque un tour, en sorte qu'il fait en tout près de deux tours en dedans du tour extérieur; mais, dans son dernier tour, il n'est pas accompagné par la zone. Enfin, pour se faire une idée complète de la chose, il faut se représenter encore que le bord supérieur du cornet n'est pas dans un plan, mais que les spires intérieures du côté droit s'élèvent de plus en plus à mesure qu'elles tournent, en sorte que la plus interne est la plus saillante et que les autres forment des échelons successifs. Les cils de la zone adorale sont les seuls que possède l'animal. Son péristome n'est pas cilié, son corps est nu et il n'a pas de couronne ciliaire inférieure 0,12. Branchies ou poils des pattes de petits Crustacés marins et d'eau douce: Nebalia, Limnoria, Gammarns) ('). 
A côté de Spirochona, longtemps considéré comme une forme isolée, se place un genre intéressant récemment découvert.

fente ou à cloisons et que BaLbian [95] a montlé (fig. 813) que cette apparence était due à une simple particularité de la distribution des substances chromatiques et achromatiques dans le noyau (V. p. 410).

L'animal se reproduit exclusivement par bourgeons et ce phénomène mérite de nous arrêter un peu, tant à cause de l'intérêt qu'il présente en lui-mème que parce qu'il nous permetlra de suivre sur le bourgeon les transformations par lesquelles Spirochona dérive de Licnophora. Au bord antérieur de l'entonnoir, sur le premier tour, un peu à gauche de la bouche, se trouve un pli vertical. Au-dessous de ce pli, le corps forme une voussure rui est l'origine du bourgeon et qui reçoit un $\mathbf{N}$ et un $\mathbf{n}$ produits par la division des $\mathbf{N}$ et $\mathbf{n}$ de la mère comme d'lıabitude (fig. 814, 1). Le pli du Fig. 813.

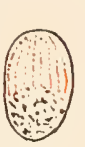

A

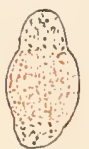

B

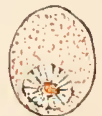

C

Spirochona. Noyau (d'ap. Balbiani). péristome maternel se prolonge à la base dans le bourgeon et y pénèlre entrainant

Fig. 814.
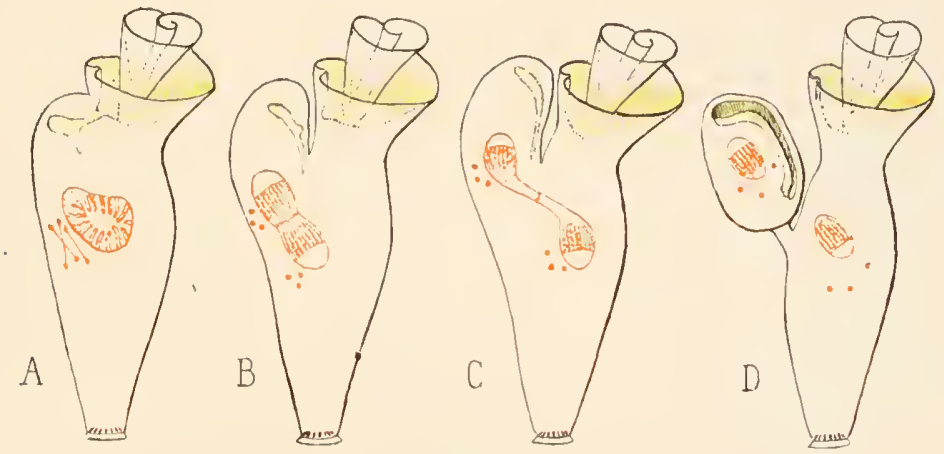

Spirochona. Division (Seh.).

A a $D$, stades suecessifs de fi division; en $D$, le bourgeon n'est plus rattaché à l'animal mère que par un pédicule.

une portion de zone adorale et introduisant dans son intérieur un diverticule inva-

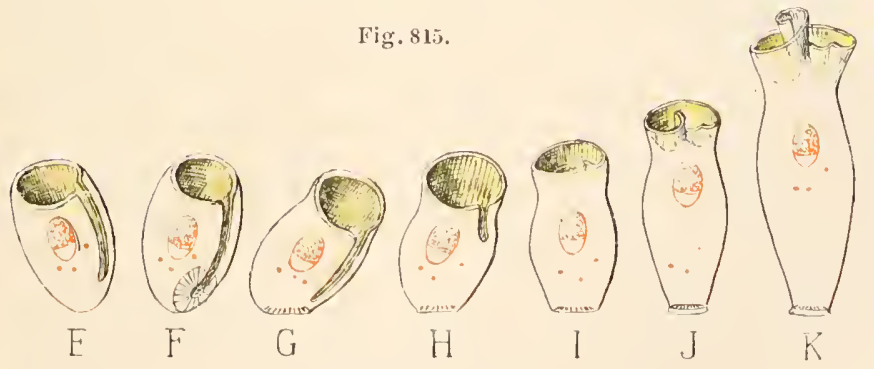

Spirochona. Division (suite) (Sch.).

$E$ à $K$, stades successifs de l'évolıtion de lindividu fille après qu’il s'est détaché de la mère. des deux individus est fixée dès le début par le plan de division, landis que le jeune bourgeon continue à grossir par accroissement avant de se séparer de la mère et ainsi tous les éléments du péristome.

On pourrait se demander pourquoi on décrit ces phénomènes sous le nom de bourgeonnement quand on pourrait aussi bien les interpréter comme division inégale. La différence gìt en ceci que, dans la division inégale, la grosseur

giné qui contient 
Kentrochona (Rompel) (fig. 816). L'animal a la forme d'un ovoïde aplati dorso-ventralement et surmonté d'un large entonnoir aplati dans le même sens qui représente celui du Spirochone avec celte différence qu'il n'est pas spiral et se ferme sur lui-mème comme un vrai entonnoir. Deux paires de baguettes rigides servent à le soutenir, une dorsale et une ventrale, cette dernière plus forte. Dans l'entonnoir est une zone de membranelles qui, après en avoir fait le tour, plonge ventralement dans le pharynx. Celui-ci part du fond de l'entonnoir, s'enfonce dans l'endoplasme en obliquant à droite et s'y perd $(0,04$. Parasite sur les lames epi- et exopodiales de Nebalia)(').

Fig. 816.

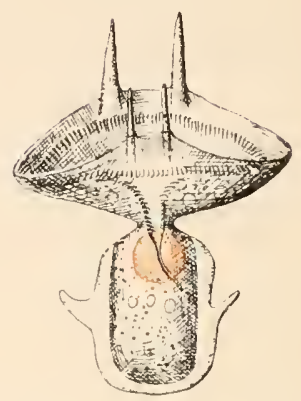

Kentrochona (d'ap. Rompel).

sans déplacement de la ligne de séparation, par augmentation de volume aux dépens de matériaux fournis par la mère. Or c'est ainsi que les choses se passent iei fig. 814, $B, C, D$. D'ailleurs, le jeune bourgeon arrive après séparation (fig. $815, E$ ) i la taille normale $(K)$ et ne constitue pas un mierogamèle. La conjugaison a lieu entre petits individus de taille égale.

Revenons à notre description. Le bourgeon se sépare de plus en plus, finit par se délacher et se montre alors (fig. $815, E$ ) sous une forme très différente de la mère. Il a l'aspect d'un ovoïde dont la partie supérieure est tronquée et excavée en un péristome. Ce péristome est circulaire, mais est interrompu en avant et là se prolonge très bas en gouttière sur la face ventrale. La zone adorale part du bord gauche de la portion horizontale du péristome, suit le contour du péristome du côté dorsal et descend jusqu'au fond de la gouttière, le long de son hord droit. A son extrémité inférieure, la gouttière ventrale circonscrit une petite surface arrondie qui se transforme en disque adhésif $(\boldsymbol{k})$, et aussitôt la gouttiẻre ventrale abandonne ce point $(G)$, recule vers le haut $\langle I\rangle$, alteint le bord antérieur du péristome et le dépasse mème en formant un petit sinus rentrant $\langle\boldsymbol{I}\rangle$. La bouche est à gauche de ce petit sinus, en dedans, juste au point où commençaient les cils de la zone adorale au stade précédent. A partir de là, les transformations sont très simples. Le bord droit du sinus continue à s'invaginer en s'enroulant sur lui-même $(J)$ et, en même temps, s'accroît en hauteur en dehor's de la zone adorale, de manière à constituer l'entomnoir. Cela montre bien que le péristome horizontal apical de l'adulte dérive d'un péristome rertical et ventral analogue à celui de Licnophora, el qu'ainsi ces deux formes se rattachent l'une à l'autre.

Lor`sque la mères'est épuisée par une longue suite de bourgeonnements successifs, elle subit une sorte de rénośation par le fait que son noyau émigre dans la partie supérieure du corps qui se détache du reste et régénère un individu complet. C'est comme un bourgeonnement dans lequel le bourgeon accaparerait la totalité des organes essentiels de la mère.

(1): Il est collé par la face ventrale sur ces lames, au moyen d'une sécrétion gélatineuse, qui déborde souvent sur les côtés en prolongements plus ou moins accentués, mais parait absente ou très réduite sur le dos. $\mathbf{A}$ l'intérieur, le $\mathbf{N}$ est sous la base de l'entomnoir et le $\mathbf{n}$ serait (exception rare) silué loin de lui, vers l'extrémité inférieure. A la place du n, c'est-à-dire dans une excavation du $\mathbf{N}$ se trouve (lig. 818)un globule pale qui se comporterait absolument comme un centrosome et devia en recevoir le nom. C"est le seul exemple cité d'un centrosome chez les Ciliés. Quand le $\mathbf{N}$ se divise, le centrosome se diviserait aussi en deux autres dont l'un resterait à sa place, tandis que 
2. Sous-ORDRE

PÉRITRICIIDES DEXTRES.-DEXIOTRICHIDES.-DE.TOTRICHID.E' [Vorticellina (Ehrenberg, emend. Bütschli)]

\section{TYPE MORPHOLOGIQUE}

(FIG. 817 ET 819 A 822)

\section{Structure}

L'animal a la forme générale d'un cône à pointe arrondie. La base tournée en haut constitue le péristome (P.st.) et porte la zone adorale $(Z a$.$) ;$ un peu au-dessus de l'extrémité inférieure se trouve une rangée annulaire de cils, c'est la couronne ciliaire postérieure (cour.). Le corps et la surface du péristome sontentièrementuus. $\mathrm{Au}$ côté gauche de l'animal, à l'union du péristome avec la face ventrale, est un large orifice que l'on croirait ètre la bouche, mais qui est, en réalité, l'entrée d'un large vestilule qui sert d'antichambre à la bouche. C'est bien un vestibule, en effet, c'est-à-dire une portion de la surface du c.par. P.st Fig. 817 .

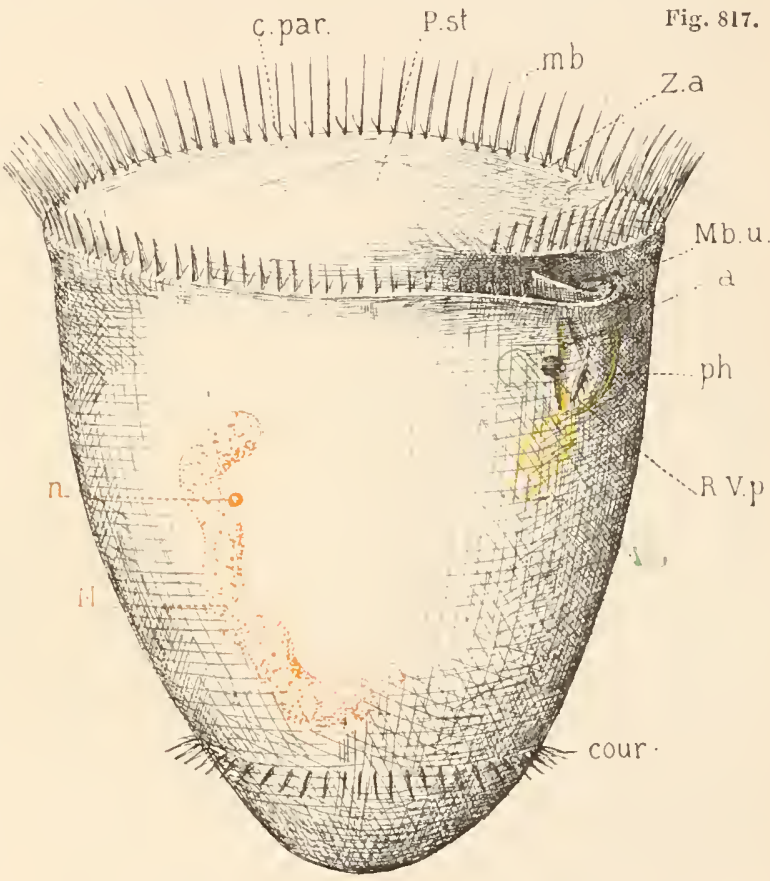

DEXTOTRICIID.E (Type morphologique) (Sch.).

a., anus; cour., couronne ciliaire postérieure; c. par., cils paroraux ; mb., membranelles; Mb. n., membrane ondulante; $\mathbf{x}$, macronucléus; n., mieronucléus; ph., pharynx; P.st., péristome; R. V. p., réservoir de la vésicule pulsatile; v. p., vésicule pulsatile; $\boldsymbol{z}$. a., zone aclorale.

corps invaginée, car on trouve à son intérieur des parties qui, morpholoFig. 818.

l'aulre se transporterait an pòle opposé, et l'on observerait un cône, sinon achromatique du moins fait d'une substance peu colorable entre ces centrosomes et la substance chroma-
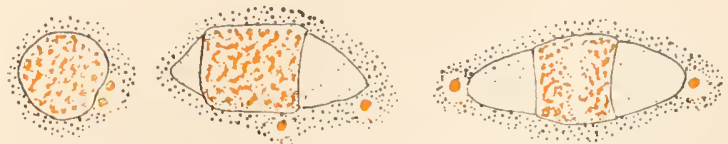

Division nucléaire chez Kentrochona (d'ap. Rompel). tique interposée. Celle-ci se diviserait en deux masses par un plan équatorial et chaque 
giquement, appartiennent à la surface externe, savoir: l'anus (a.) et le pore excréteur, le premier à peu près au milieu de sa hauteur, le second un peu au-dessous. L'un et l'autre du côté tourné vers le centre du corps. Au fond de ce vestibule s'ouvre la vraie bouche conduisant dans un pharynx $(p h$.$) bien déreloppé.$

Le $\mathbf{N}$ est grand $\left(\Lambda^{\top}\right)$, en forme de bâtonnet arqué, le $\mathbf{n}$ est unique et fort petit $(n$.$) et annexé au N. La vésicule pulsatile ne s'ouvre pas direc-$ tement au pore excréteur. Elle s'ouvre dans une cavité nouvelle, le réservoir (R.V.p.) qui lui-même s'ouvre dans le vestibule. Ce réservoir est strié à sa surface de lignes se coupant en losange et qui sont probablement l'indice de filaments contractiles, car il se contracte énergiquement pour se vider dans le vestibule. Il n'est autre chose qu'une dépendance du vestibule, c'est-à-dire une seconde invagination de la surface dans l'invagination vestibulaire. Aussi est-il en communication permanente avec le vestibule, tandis qu'il n’a, avec la vésicule, qu’une communication temporaire, comme celle des vésicules des autres Ciliés avec la surface du corps. Le vrai pore excréteur morphologique est cet orifice non permanent entre la vésicule et le réservoir.

Il ne nous reste à décrire, pour bien comprendre notre type, que son péristome et sa zone adorale. Nous les avons gardés pour la fin, car ici surgissent iles complications inattendues. La face supérieure du corps horizontale et circulaire constituant le péristome $(P . s t$.) est bordée par la zone adorale $(Z . a$.$) . Mais cette zone, au lieu de tourner dans le même$ sens que chez le Stentor, tourne en sens inverse; en allant de la bouche vers l'extérieur, elle passe à droite de sa tangente: elle est donc dextre (V.p.43\%). Elle part du bord inférieur de l'orifice du vestibule, parcourt successivement les bords antérieur, droit, postérieur et gauche du péristome en montant légèrement et, revenant ainsi un peu au-dessus de son point de départ, passe au-dessus de l'orifice vestiluulaire et continue encore son trajet vers la droite pendant environ un quart de tour. Elle est formée de hautes et étroites membranelles ( $m b$.) à chacune desquelles est annexé en dedans un cil paroral (c.par.).

En arrivant au vestilule, les membranelles se transforment brusquement en une membrane $(\boldsymbol{M b} . u$.) de la nature des membranes ondu-

moitié reformerait avec le centrosome adjacent un noyau complet. Ce serait donc une sorte de mitose, mais sans chromosomes ni vrais filaments achromatiques. Mais nous avons vu que BaLbiani a infirmé ces interprétations par ses observations sur Spirochona (V.p. 418).

L'animal se reproduit par bourgeons qui naissent sur la partie moyenne du corps sans emporter, comme chez spirochona, une partic de l'entonnoir. Des deux noyaux issus de la division, l'un reste dans la mère, l'autre passe dans le bourgeon.

Certaines espèces, cependant, possèdent quelques soies sensitives au bord libre de l'entonnoir. On a voulu les élever à la dignité de gemre et on a fait le Slylochona (Kent). - Le genre

Heliochona (Plate) n'est guère mieux justifié.

Ces genres forment la famille des SPThochostre [Spirochonina (Stein)]. 
lantes pharyngiennes, mais qui est ici immobile (ou du moins non vibrante et ne se mouvant qu'occasionnellement au moment de la déglutition) et qui continue leur trajet. Cette membrane plonge en hélice dans le restibule qu'elle suit presque jusqu'à l'orifice buccal en décrivant un tour et demi; très haule à son origine à l'entrée du vestilıule, elle diminue progressivement de hauteur et se termine en pointe. Sa partie terminale sépare le vestibule en deux couloirs, un plus large qui sert au passage des aliments, et un plus étroil où se trourent le pore excréteur et l'anus, en sorte que les matières rejetées se trouvent séparées des substances ingérées. La série des cils paroraux se continue aussi dans le vestibule, y décrit aussi une hélice mais, au lieu de s'arrêter comme la membrane ondulante avant la bouche, s'étend presque jusqu'au fond du pharynx. Les cils sont, dans le vestibule, inclinés vers l'orifice d'entrée et conservent l'orientation correspondante dans la partie extérieure de la zone adorale(").

(1) La constitution de la zone et du péristome est aisée à comprendre, mais ce qui l'est moins c'est de quelle manière cette disposition, exactement inverse de celle des autres Ciliés, a pu prendre naissance. Voici l'explication proposée par Bütschli.

Partons de Licnophora fig. 819\%. Ce Péritrichide a un péristome vertical ventral et une zone adorale sénestre comme les Ciliés ordinaires. La couronne ciliaire inférieure est horizontale et perpendiculaire à l'axe du corps. Mais nous avons vu que dans le genre Spirochona où elle a la mème disposition chez l'adulte, elle appartient nettement chez le jeune à la face ventrale. Nous pouvons donc admettre que chez le prototype du Péritrichide cette couronne appartenait à la partie inférieure de la face ventrale et qu'elle était dans le mème plan que le péristome $(A)$. Supposons que la couronne ciliaire devienne plus saillante et que la zone adorale, s'étendant vers le bas par son extrémité aborale, fasse le tour de la couronne ciliaire de manière à l'enfermer dans sa cavité, à remonter jusqu'à la bouche et a la dépasser même en passant dorsalement par rapport à elle $(B)$. Supposons enfin que la couronne ciliaire

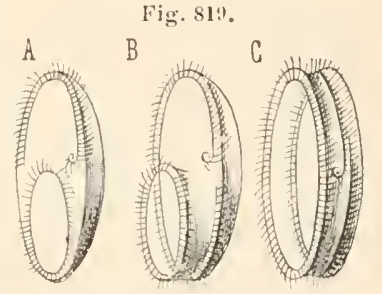

Diagramme montrant conment les DETTOTRTIIIDE dérivent des SCATOTRTCIIDE

(d'ap. Bütschli.) s'étende de manièreà occuper toute la face ventrale, tandis que la face dorsale s'aplatit, et plaçons lanimal comme si son axe était perpenticulaire au centre de sa couronne ciliaire au lieu de lui être parallèle. Nous aurons alors un ètre $(C)$ qui ne différera de notre type en rien d'essentiel, qui, au point de vue descriptif, aura comme lui un péristome et une couronne ciliaire horizontaux et perpendiculaires à l'axe du corps, et une zone adorale dextre, tandis qu'au point de vue morphologique, sa face supérieure devra ètre considérée comme dorsale, l'inférieure comme ventrale et son axe vertical comme un axe antéro-postérieur, le vrai axe morphologique du corps étant parallèle à ses faces horizontales. Cela explique en mème temps le renversement du sens de l'hélice adorale. Ce renversement n'est qu'apparent : il vient de ce que l'on voit cette courbe par derrière lorsqu'on la regarde par la face péristomienne, puisqu'en réalité cette face est dorsale. Pour la voir dans sa position morphologique, il faudrait la regarder en plaçant devant soi la face pédieuse de l'animal (représentée par la couronne ciliaire); on la verrait alor's dans le sens normal.

Nous ferons remarquer que ce mode de dérivation n'est pas du tout démontré ef qu'il ne donne même pas satisfaction aux exigences de l'esprit. Sans entrer dans le détail de la discussion d'une théorie aussi dénuée de base, faisons remarquer que, dans 


\section{Physiologie}

Locomotion. Alimentation. Excrétion. - Notre Péritrichide nage au moyen de sa couronne ciliaire et peut se reposer en se fixant momentanément par l'extrémité inférieure du corps. Il s'alimente au moyen d'un tourbillon déterminé par la zone adorale et il y a ici une sorte de choix des aliments, car bien des parcelles solides précipitées dans le vestibule en ressortent sans en avoir dépassé le fond. Le pharynx se remplit peu à peu de particules alimentaires. Quand il est plein, tout ce bol est avalé d'un coup avec une certaine quantité d'eau et forme une vacuole alimentaire. La membrane vestibulaire semble aider à ce mouvement de déglutilion.

Nous avons vu les modifications bien superficielles apportées au fonctionnement de l'appareil excréteur par la présence du réservoir contractile. Il nous reste à décrire les phénomènes de la reproduction.

Division. - La division a lieu ici longitudinalement par un plan sagittal qui laisse la bouche et le vestibule à l'individu de gauche (').

La manière dont se forme le nouveau péristome est très remarquable (fig. 820). A l'opposé du vestibule ancien (A), se forme un nouveau vestiluule et il se détache de la zone adorale une nouvelle courbe spirale

Fig. 820.

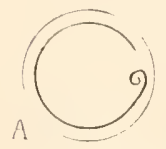

DEXIOTRICIIID.E

(Type morphologique).

Division du péristome (Sch.).

A, Péristome avant la division; $B$, après sa division. qui continue, avec un rayon beaucoup plus petit, la courbure primitive, et plonge dans ce nouveau vestibule. Puis, la portion moyenne de la zone adorale ancienne se détruil, et il reste deux portions semblables de zone adorale représentées l'une el l'autre par une extrémilé vestibulaire $(B)$. Ces deux portions se complètent et l'on a alors une Vorticelle à deux vestibules et deux zones adorales semblables. Déjà l'animal a commencé à s'élargir transversalement el son péristome a pris une forme ovalaire. Après la division, chaque individu se trouvera avoir une zone et un péristome complets.

Les phénomènes nucléaires n'offrent rien à signaler.

Conjugaison. - La dégénérescence sénile produit ici ses effets ordinaires, mais elle n'altère pas progressivement la taille de tous les indi-

l'hypothèse de Bütschli, le pharynx rlevrait, à partir de l'orifice buccal, remonter vers la surface du péristome au lieu de plonger dans la profondeur.

${ }^{1}{ }^{1}$ Si vraiment le péristome était morphologiquement dorsal, cetle exception ì la règle de division transversale deviendrait plus apparente que réelle, car alors le plan transversal couperait le péristome suivant un diamètre vrai. A vrai dire, il faudrait que la bouche fùt rigoureusement antérieure pour que le plan de division tel qu'il est placé soit réellement transversal. Or, nous avons vu qu'elle est ì la partie gauche de la face ventrale. Mais nous savons qu'une certaine obliquité du plan de division est fréquente. 
vidus, comme à l'ordinaire. Tous gardent d'abord leur taille normale; mais, au moment de la maturité sexuelle, certains se divisent deux fois successivement et si rapidement que l'on trouve des stades où les quatre individus nouveaux sont encore unis en une rosette ('). Ces quatre individus finissent cependant par se séparer. Ils ne grossissent pas et constituent les microgamètes, tandis que les individus non divisés qui ont gardé la taille normale jouent le rôle de macrogamètes.

Le microgamète (fig. $821, A: m g$.) nage à la recherche d'un macro-

Fig. 821 .
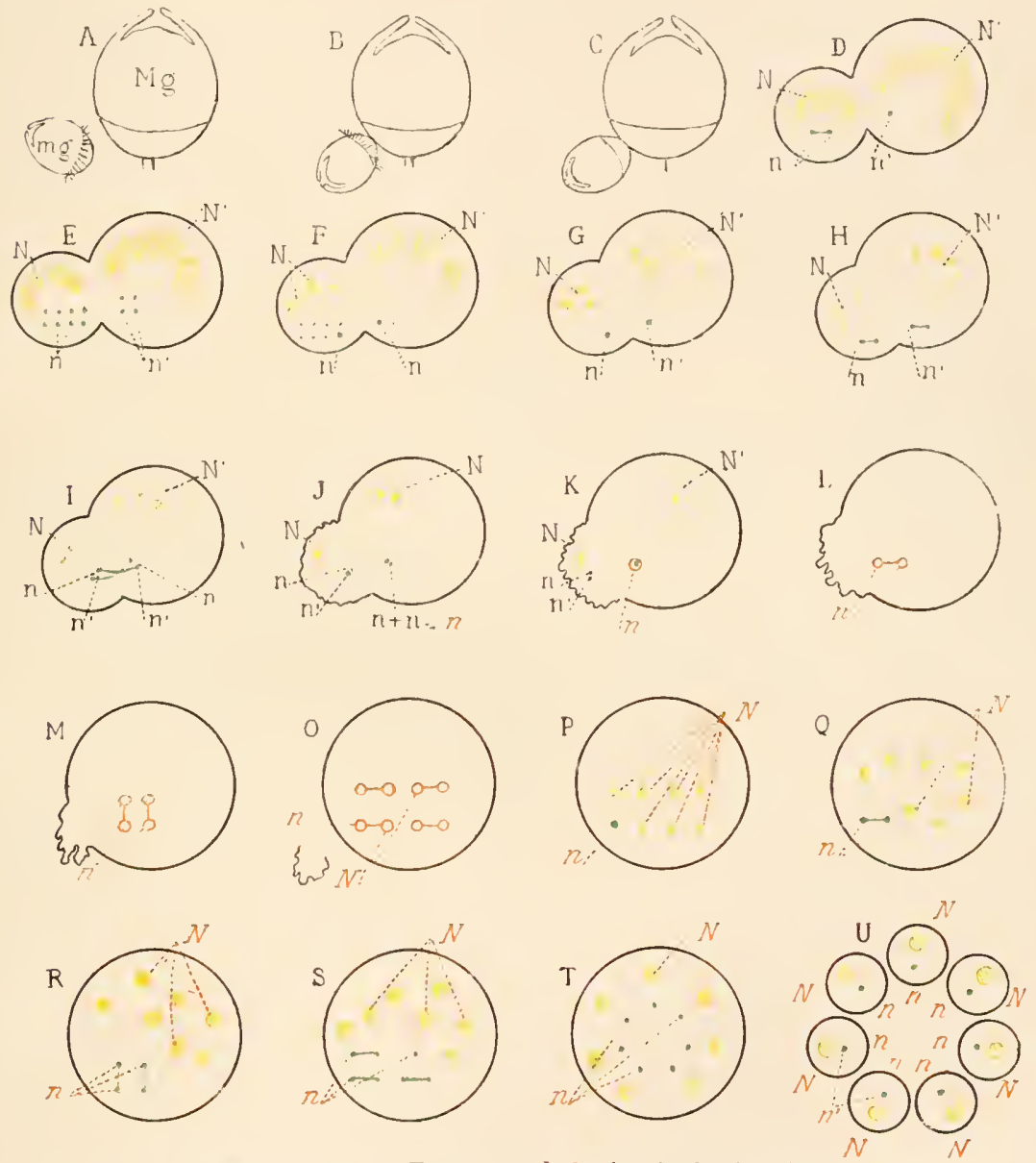

DEX゙IOTRICHID.E (Type morphologique). Conjugaison.

$A$ à $\boldsymbol{U}$, stades successifs de la conjugaison; $\mathbf{M} \mathbf{m} .$, macrogaméte; mo., microgaméte; $\mathbf{x}, \mathbf{n}$, macroet mícronucléus du microgamete; $\mathbf{N}^{\prime}, \mathbf{n}$, macro- et micronucléus du macrogamète; $N, n$, macroet micronucléus de nouvelle formation.

gamète $(\boldsymbol{A}: \boldsymbol{M g}$.$) apte à se conjuguer, le poursuit, l'atteint et s'attache$

(1) Souvent il n'y a qu'une division en deux, parfois il y en a trois donnant huit indivi- 
à lui un peu au-dessous de la couronne ciliaire, par son extrémité postérieure $(B)$, el les deux couronnes ciliaires ne tardent pas à disparaître $(C)$. Dans les deux individus, le $\mathbf{N}$ se fragmente et les fragments se résorluent leutement à la manière ordinaire $\left(N\right.$. et $N^{\prime \prime}$.). Nous n'aurons plus à nous en occuper.

Les phénomènes micronucléaires sont d'abord normaux. Chacun-des deux $\mathrm{n}$ se divise en quatre dont trois disparaissent et le quatrième se divise en deux, un pronucléus $\sigma^{*}$ et un pronucléus $q$, et le pronucléus de chacun des deux conjoints se porte vers le pronucléus $q$ de l'autre resté immobile ("). Mais ici le n montre une différence importante : au lieu que, des deux côtés, les couples de pronucléus se fusionnent, cela n'a lieu que dans le macrogamète. Son pronucléus t et le pronucléus \& que lui a envoyé le microgamète $(I)$ se fusionnent comme d'ordinaire en un n conjugué $\left(J: n+n^{\prime}=n\right)$, tandis que dans le macrogamète, les deux pronucléus, après s'être rapprochés, s'arrêtent sans se joindre et bientôt se résorbent. Cela s'explique tout naturellement par le fait que le microgamète, au lieu de se séparer comme d'ordinaire après l'échange des pronucléus, achève au contraire de se fondre dans le macrogamète $(J, K, L, M)$. Son cytoplasme passe peu à peu dans celui

dus. Dans d'autres cas (Vorticella macrotoma, Lagenophrys), il y a division des individus ordinaires en deux autres très inégaux dont le gros deviendra un macroga-

Fig. 8⒉

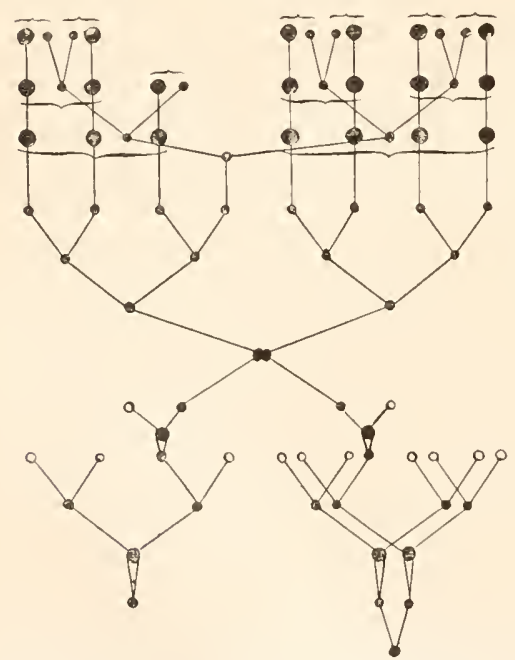

DEXIOTRICHINE (Type morphologique). Diagramme de la conjugaison (d'ap. Mlaupas). mète et le petit un microgamète. Dans le genre Zoothamnium il y a des macrogamètes spéciaux prédestinés, situés sur les rameaux de premier ordre de la colonie à l'aisselle des rameaux de deuxième ordre.

(1) En réalité les choses se passent un peu autrement: le $\mathbf{n}$ du microgamète se divise d'abord en deux $(D: n)$. Ces deux grossissent et se comportent chacun à la manière ordinaire, c'est-à-dire se divisent chacun en quatre par deux bipartitions successives $(E: n)$. Mais les quatre descendants de l'un d'eux se résorbent, tandis que, de l'autre, trois seulement subissent ce sort $\left(\boldsymbol{F}^{\prime}: n\right)$, en sorte, qu'il ne reste plus, comme d'ordinaire, qu'un seul $\mathbf{n}(G: n)$ qui va se diviser en un pronucléus ot un pronucléus $q(I I)$. Le seul effet persistant de cette particularité c'est que le $n$ survivant chez le microgamète représente seulement un huitième de la substance du n primitif, au lieu d'en représenter un quart comme chez le macrogamète et chez les autres Ciliés. On peut exprimer encore cela en disant que le microgamète expulse sept globules polaires au lieu de trois, ou trois au lieu de deux, selon que l'on comple ou non les descendants des $\mathbf{n}$ abortifs.

Le diagramme ci-dessus (fig. 822) montre clairement cette série de phénomènes. 
du macrogamète et sa membrane, vidée et flétrie, après ètre restée quelque temps appendue au point de soudure, finit par tomber et se perdre $(O)$. Il ne reste donc plus qu'un individu, et un seul n conjugué $(K: n)$ lui suffit. A partir de ce moment, les phénomènes ne diffèrent plus de ceux du cas typique que par un détail un peu secondaire qui est celui-ci. Le n conjugué, au lieu de se diviser en deux dont l'un sera le $\mathbf{N}$ et l'autre le $\mathrm{n}$ définitifs, se divise en huit dont sept grossissent et deviennent autant de $\mathrm{N}(P: N)$, tandis que le huitième reste petit et se divise successivement en sept $(P, Q, R, S: n)$ ('). De là résultent sept couples formés chacun d'un $\mathbf{N}$ et un $\mathbf{n}$ que des bipartitions successives répartiront lientôt en sept individus régulièrement constitués $(U)$.

La conjugaison est donc totale et non simplement nucléaire.

\section{GENRES}

Trichodina (Stein) (fig. \$ュ3, \$ュ4). La Trichodine est presque la réalisation de notre type morphologique. Elle n'en diffère essentiellement que par la transformation de l'extrémité inférieure en une ventouse (fig. S24). Sa forme est celle d'un disque peu élevé dont les deux bases seraientévasées, làsupérieure plus que l’inférieure. La base inférieure est entourée par le

Fig. 823.

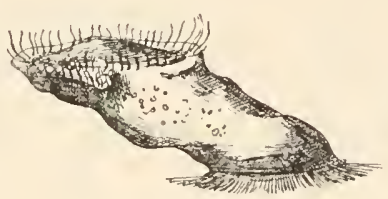

Trichodina

(d'ap. Fabre-Domergue).
Fig. 82f.

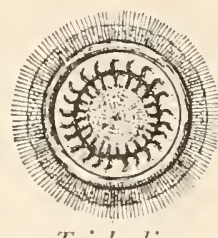

Trichodina

Disque adhésif (d'ap. Fabre-Domergue). cercle des cils, la couronne ciliaire inférieure représente par conséquent la portion du corps situće dans cette couronne; chez notre type morphologique elle est excavée et transformée en un appareil adhésif. Pour cela, la membrane qui tapisse la cavité porte des épaisissements en forme de baguettes et le crampons qui servent, on ne sait trop comment, à la faire adhérer au support. On trouve d'abord un anneau périphérique strié et portant une couronne de 20 à 203 crochels, puis, en dedans de ceux-ci, des baguettes radiaires en nombre égal qui partent de la base des crochets et vont jusqu'au centre. Ces productions sont de nême nature chimique que la membrane, mais d'une constitution physique plus dense et plus résistante, elles sous-tendent des parties membraneuses dont les figures indiquent la disposition et qui concourent sans doute au résultat sans que l’on sache bien comment. Immédiatement au-dessus de la couronne ciliaire, insérée à la périphérie du disque adhésif, se trouve une membrane péripédieuse circulaire qui s'étale sur les cils de la couronne et recouvre leur moitié proximale. Le

(1) Il y a trois bipartitions successives qui devraient donner naissance à huit noyaux, mais l'une de celles de la deuxième génération ne se produit pas. 
corps est tout à fait dépourvu de cils. Le péristome et l'organisation intérieure sont entièrement conformes ì notre type morphologique, seulement l'entrée du vestibule est siluée franchement sur la surface verticale du corps et c'est seulement après un certain parcours ascendant que la zone adorale atteint le bord du péristome horizontal et achève alors son trajet en suivant ce bord ( $1 \mathrm{~mm}$. Parasite sur divers animaux marins et d'eau douce: Ilydres, Eponges, Planaires, Acéphales, Batraciens, Poissons) (').

Nous allons maintenant rencontrer une longue série de genres qui vont dériver les uns des autres par une série de complications progressives portant sur quatre points : $1^{\circ}$ formation d'un rebord contractile autour du péristome; $2^{\circ}$ production d'un pédoncule pour se fixer; $3{ }^{\circ}$ sécrétion de logettes pour s'ahriter; $4^{\circ}$ enfin, formation de colonies arborescentes par division avec séparation incomplète.

La Vorticelle, genre principal du groupe, va nous montrer d'un coup l'apparition des deux premiers caractères.

Vorticella (Linné, emend. Ehrenberg) (fig. \$23 à 829). L'animal a la forme d'un còne ì hase tournée en haut (fig. \$פכ̌) et dont le sommet, légèrement tronqué, donne naissance à un long et mince pédoncule $(p d$.) par lequel il se fixe à quelque objet immergé. Ce pédoncule n'est pas une sécrétion, c'est une dépendance du corps, c'est la partie inférieure du corps elle-même qui s'est étirée en un long et et mince prolongement. Nous verrons quelle est sa structure en décrivant la musculature. La portion située au-dessus de lui est divisée en deux autres par un étroit sillon transversal circu-

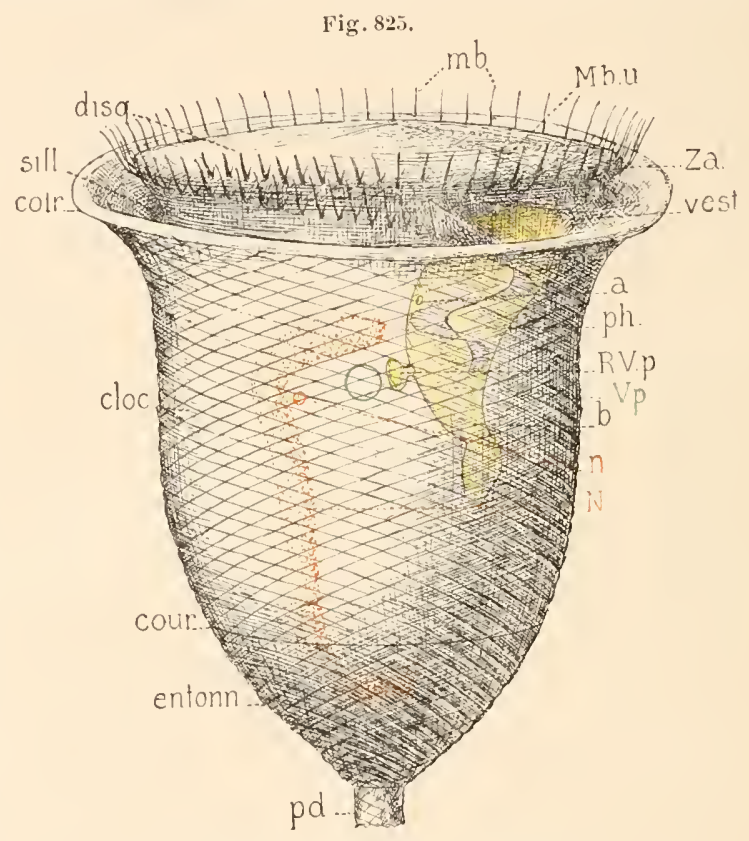

Vorticella en état d'extension (Sch.).

a., anus; b., bouche ; cloc., cloche; colr., collerette; cour., conronne ciliaire; disq., disque; entonu., entonnoir; m1)., membranelles; Mu.u., membrane ondulante; $\mathbf{N}$, macronucléus; n, mieronucléus; pd., commeneement du pédoncule; ph., pharynx; R.v.p., réservoir de la vésieule pulsatile; sill., sillon; rest., vestibule; vp., vésicule pulsatile; Za., zone adorale.

laire au fond duquel la membrane ahsente laisse l'ectoplasme à nu. Ce

(1) Quand l'animal adhère au support, sa membrane péripédieuse est rabattue sur les cils de la couronne ciliaire qui sont immobiles; pour se déplacer, 
sillon occupe exactement la place de la couronne ciliaire absente et nous verrons bientôt que, dans certaines conditions, cette couronne reparait exactement dans ce sillon. Sauf ce cas et sauf bien entendu la zone adorale, il n'y a nulle part de cils ni de productions ciliaires quelconques. On a appelé entonnoir (entonn.) la partie du corps située entre ce silton et le pédoncule, et cloche (cloc.) tout ce qui est au-dessus. Les parois de la cloche montent vers le péristome qui occupe, comme d'ordinaire, la base supérieure; mais, au lieu de se jeter simplement sur lui de manière à ce que le bord supérieur des parois verticales se confonde avec le bord externe de la base horizontale, elles s'en écartent, le dépassent et forment tout autour de lui une forte collerette (colr.), séparée de lui par un sillon circulaire (sill.). Cette collerette souvent décrite comme la portion périphérique du péristome est, en réalité, tout à fait indépendante de lui et a une origine à part.

On donne souvent le nom de disque au péristome vrai (disq.), e’est$\grave{d}$-dire à tout ce qui est au dedans du sillon. Comme la collerette est trìs contractile, elle peut se fermer au-dessus de lui comme une bourse dont on tire les corlons, de manière à protéger les parties sous-jacentes. C'est là son principal ròle, mais elle sert aussi à déterminer, entre sa base interne et le péristome, le sillon circulaire (sill.) que suivent les aliments pour arriver ì la houche. Ce sillon n'a pas partout une profondeur égale. Au niveau du hord droit, il est peu profond, mais il se creuse et s'élargit en contournant le disque en avant et en arrière, pour atteindre à gauche et un peu en avant son maximum de largeur et de profondeur. Là, il aboutit à un large orifice (vest.) qui est celui du vestibule.

Le péristome ou le disque (disq.), si l'on veut, a la forme d'un large plateau horizontal porté sur un large et court pédoncule, formé par la lèvre interne du sillon qui le sépare de la collerette. Ce pédoncule est naturellement plus élevé lu côté gauche où est le vestibule, que du côté droit où le sillon est le moins profond. Il est bordé d'une zone adorale $(Z . a$.$) qui tourne autour de lui en lıélice et plonge dans le vesti-$ bule. La zone commence, parson extrémité adorale, au bord antérieur du disque ou mème un peu à droite, passe successivement à gauche, en arrière et de nouveau à droite en descendant peu à peu sur le pédoncule du disque de manière à se trouver, après un tour complet, un peu au-dessous de son point de départ. De là elle continue à tourner en descendant et arrive ainsi $(M / . u$. $)$ à l'entrée du vestibule où elle plonge toujours en tournant en hélice.

il décolle sa membrane, agite ses cils et se meut en glissant sur son support sans se séparer de lui. Il peut aussi se détacher tout à fait et nager en pleine eau en tournant.

Sa couronne ciliaire est seule active dans tous ces mouvements. - Genres voisins : Anhymenia (Fabre-Domergue), sans membrane péripédieuse, simple sous-genre; Cyclochæta (Jackson), arec une couronne de hautes soies dressées remplaçant cette membrane, se subdivisant en deux sous-genres: Leiotrocha (Fabre-Domergue), à anneau du disque adhésif denticulé, et 
La forme et la disposition du vestibule, l'anus $(a$.$) , la vésicule pulsa-$ tile (Y.p.) avec son réservoir (R.Y.p.), la constitution de la zone adorale $(Z . a$.$) , la continuation de la membrane ondulante ( M b . u$.) qui fait suite aux membranelles $(m b$.) dans le vestibule, celle des cils paroraux jusqu’au fond du pharynx, le macronucléus (N.), le micronucléus (n.), tout cela est entierement conforme à notre type morphologique. Il n'y a qu'à reporter ici ce que nous avons dit à ce moment (').

Cyclocyrrha (Fabre-Domergue), à anneau du disque adhèsif non denticulé;

Hastatella (Erlanger), libre, it soies fortes et nombreuses ( $40 \mu$. Eau douce, stagnante);

Trichodinopsis (Claparède et Lachmann), à péristome très rétréci 0,13 . Intestin et poumon de Cyclostoma elegans). - Ici se rattache avec doute,

Hemispeira (Fabre-Domergue), genre à zone dextre et ì affinités multiples et indécises.

Ces divers genres forment la famille des URCEOLARINE [Urceolarina (Stein)].

(1) 11 ne nous reste pour compléter cette description anatomique qu'à parler maintenant du système musculaire de l'animal, système extrêmement dẻveloppé et compliqué. Il a èté étudié arec beaucoup de détails par Exтz [91].

Le tégument se compose, comme d'ordinaire, de la membrane et de l'ectoplasme. La membrane a ici une individualité bien plus marquée qu'à l'ordinaire; elle est limitée par un double contour très net; vue de face, elle paraît ornée de dessins losangiques rẻguliers qui, sur la coupe optique, montrent un certain relief comme s'ils résultaient d'écailles imbriquées. L'ectoplasme est formé de deux couches, une superficielle constituée par les plans musculaires que nous allons bientòt décrire et une profonde de structure très particulière.

Cette couche profonde est formèe d'une assise unique de petits corpuscules que l'on serait tentẻ d'assimiler à de minimes cellule (fig. 826). Chacun est formé, en effet, d'une petite masse protoplasmique figurant le cytoplasme et d'une partie centrale plus dense, plus chromophile, figurant le noyau. Evidemment, ce ne sont pas de vraies cellules, mais des condensations locales régulières de cytoplasme. Pour rappeler ces analogies, on a appelè la masse totale cytophane et le grain central karyophane. On distingue parfois une sorte de filament spiral s'étendant du karyophane à la surface du cytophane en décrivant un ou deux tours. Cette particularité appuyée sur quelques considérations théoriques a fait attribuer à ces petits organes des fonctions nerveuses, mais il n'y a là rien de positif. L'endoplasme se montre, lui aussi, formé de cytophanes au moins dans ses couches superficielles.

La couche superficielle de l'ectoplasme est formée de deux assises de myonèmes (fig. 827), une externe et une interne, comprenant chacune deux couches de fibrilles, une circulaire externe et une longitudinale interne. On trouve donc de dehors en dedans :

1. Une couche circulaire externe (mc. ext.) formée par une seule immense fibre héliçoïdale qui monte, en tour's serrés, de la

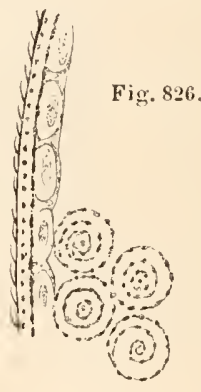

Vorticelia. Structure de l'ectoplasme montrant les cytophanes et les karyophanes (d'ap. Entz). base du pédoncule jusqu'au centre du disque, en garnissant toute la paroi sans interruption; elle se révèle au dehors par une fine striation transversale;

$2^{\circ}$ Une couche longitudinale externe (ml. ext.) formée de fibrilles qui vont aussi, serrées les unes contre les autres, de l'extrémité inférieure du pédoncule jusqu'au centre du disque, garnissant, elles aussi, toute la surface du corps; sur le disque, ces fibrilles deviennent radiaires. Au niveau de la collerette, les fibrilles de ces deux couches suivent ce repli, montant dans sa paroi externe, redescendant sous sa paroi interne, passent sous le fond du sillon et arrivent au disque par son pédoncule;

$3^{\circ}$ Une couche circulaire interne (mc. int.) très incomplète, absente sur le pédon. 
La division des Vorticelles se fait suivant un plan vertical antéropostérieur, non médian : les deux individus filles sont donc inégaux. L'un conserve la bouche et le pédoncule, l'autre se forme une bouche nouvelle, à la manière décrite à propos du type morphologique, et est dépourvu

cule, sur la cloche et sur le disque, et formant seulement quelques tours de spire à la base de l'entonnoir et un fort sphincter dans le bord libre de la collerette. C'est ce sphincter qui sert à fermer la colleretle comme une bourse au-dessus du disque;

40 Enfin, une couche longitudinale interne (ml. int.) qui se comporte comme la longitudinale externe, mais est formée de fibrilles beaucoup moins nombreuses, plus espacées et, arrivée au sommet du pédoncule, quitte la paroi et se jette sur le cordon central du pédoncule pour former le spasmonème dont nous allons bientòt parler.

En outre de ces myonèmes ectoplasmiques, il existe un gros faisceau central, contenu dans l'endoplasme, e'est le Fig. 827 . rétracteur du disque. Il s'attache à la base du disque el se porte, de là, ver's le pharynx pour's'attaclier, sans doute, a sa membrane par ses fibrilles dissociées. 11 sert à rétracter le disque lorsque la collerette se ferme au-dessus de lui.

Le pédoncule (fig. 827, 828) est formé d'une paroi et d'un cordon central baigné dans un liquide qui occupe l'espace intermédiaire.

La paroi n'est autre chose que le prolongement de celle de l'entonnoir. Elle a done là mème structure: on y trouve la membrane, puis les deux couches musculaires externes (les deux internes manquent, puisque la circulaire interne fait défaut là comme sur la cloclie et que la longitudinale interne s'est séparée de la paroi pour se joindre au cordon axial), puis l'assise eytophanique de la couche profonde de l'ecloplasme. Ver's le bas du pédoncule, toutes ces couches deviennent indistinctes, et la membrane perd sa structure. Le liquide n'est ‘u'un élément de remplissage.

Reste a décrire le cordon axial.

Ce cordon axial a pour paroi une gaine anhiste qui sépare son contenu du liquide précédent. Ce contenu comprend deux parties juxta-

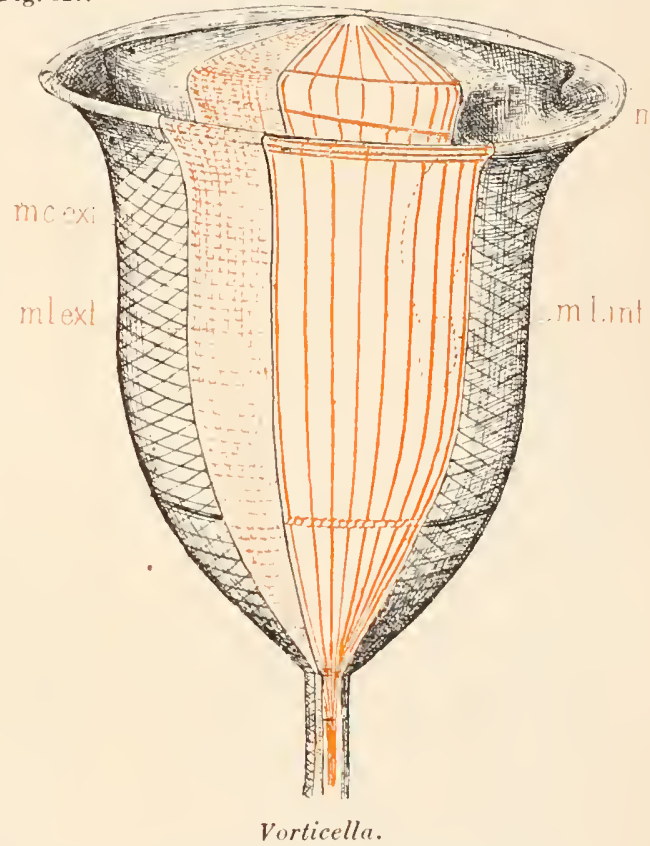

Disposition des deux couches de myonèmes ectoplasmiques (Sch.).

Dans un des secteurs, la membrane superficielle est enlevee pour montrer la couche externe des myonémes composée de fibres circulaires me. ext. et de fibres longitudinales mi. ext. Dans lautre secteur, on a enlevé en outre la couche externe des myonémes pour montrer la couche interne composée de fibres circulaires me. int. et de fibres longitudinales m1. int. posées (fig. 828): le spasmonème et le cordon plasmatique.

Le spasmonème (spas.) forme un corton rectiligne ou irrégulièrement onduleux, occupant dans la gaîne une position excentrique. 11 est formé uniquement de myonèmes longitudinaux qui ne sont autres que ceux de la couche longitudinale interne de l'entonnoir groupés en un faisceau massif.

Le cordon plasmatique se compose de deux parties: a) l'axoneme (axo.), cordon central, rectiligne, parallèle au spasmonème et formé de gros cytophanes reliés par 
de pédoncule. Par là, la division se rapproche du bourgeonnement puisque l'on peut distinguer un individu mère et un individu fille. Ce dernier ne

de nombreux et fins tractus longitudinaux, b) le spironème (spir.) qui décrit une hélice dextre à tours serrés autour de l'axonème. Ce spironème est lui-même formé de trois

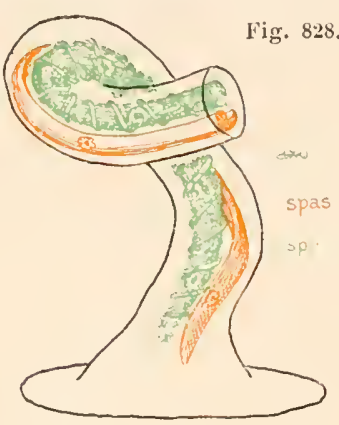

Vorticella.

Structure du pédoncule (im. Entz)

Le dessin représente l'extrémité inférieure avec le disque adhésif. axo., axonème; spas., spasmonème spir., spironème. difficile. Ouand la Vorticelle est inquiétée, brusquement, elle se contracte (fig. 829), retire son disque (disq.), ferme sa collerette (colr.), prend une forme sphérique et, en même temps, son pédoncule ( $p d$.) se raccourcit en se tortillant en tire-bouchon dans le même sens que le spironème. Or cet état, qui semblerait être le résultat de la contraction active des éléments du pédoncule, est au contraire un etat passif, car les Vorticelles mortes ou enliystées ont leur pédoncule entortillé. C'est la distention rectiligne qui est l'étal actif. D'autre part, quand par la putréfaction les parties molles du pédoncule ont étè détruites et que la membrane reste seule, le pẻdoncule s'ètend de nouveau. L'èlasticité de la membrane tend donc à l'èlendre, ce n'est donc pas elle qui l'enroule, et il faut qu'il y ait, parmi les autres éléments du pẻdoncule, une partie qui joue le rôle de ressort élastique passif, plus fort parties concentriques : $\alpha$ ) un axe central (contournè en hẻlice comme le reste du spironème), rappelant en petit l'axonème et formé comme lui d'une seule file de cytophanes reliés par un filament comme les grains d'un cliapelet; $\beta$ ) une enveloppe anhiste entourant le reste; $\gamma$ enfin une couche de myonèmes longitudinaux disposés entre l'axe et la gaîne. Comme toujours, tous ces organes contractiles sont anisotropes, c'est-a-dire biréfringents.

Le fonctionnement de toutes ces parties n'est pas aisé à définir. Mettons de côté les cytophanes et par conséquent l'axonėme qui en est formé, car, qu'ils soient ou non des éléments nerveux, ils ne sont évidemment pas les agents mécaniques de la contraction. L'action des myonèmes du corps se laisse aisèment définir, surtout pour le sphincter de la collerette et le rétracteur du disque. Dans le corps, les éléments longitudinaux doivent servir à ouvrir la collerette et à ètaler l'animal, tandis que les élèments circulaires servent peut-ètre à faire saillir le asme. Mais c'est pour le pédoncule que la chose est

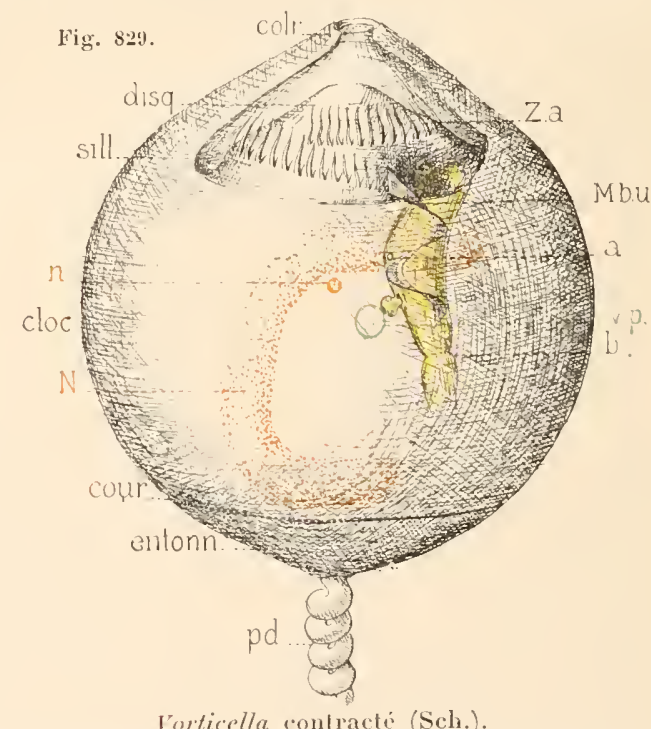

a., anus; b., bouche; eloc., clorhe; colr., collerette; cour., couronne; disq., disque; entonn., entonnoir; $\mathbf{u b . u . , ~ m e m - ~}$ brane ondulante; $\mathbf{N}$, macronucléus; $\mathbf{n}$, mieronucléus; od., commencement du pédoncule; sill., sillon; V.p., vésicule pulsatile; z.a., zone adorale.

que la membrane et déterminant l'enroulement. 
forme pas tout de suite un pédoncule. Il se munit d'abord d'une couronne ciliaire à l'endroit correspondant au sillon qui sépare la cloche de l'entonnoir, couronne formée, en réalité, de petites membranelles. Il nage, l'extrémité inférieure en avant, à la recherche d'une place pour se fixer. Quand il l'a trouvée, il se fixe par cette extrémité et développe son pédoncule. A la place de la couronne ciliaire disparue, on trouve le sillon circulaire qui persiste chez l'adulte.

Quand les conditions deviennent mauvaises, en particulier quand l'eau se putréfie, l'adulte développe une couronne ciliaire de membranelles qui sortent de son sillon, il rompt son attache à son pédoncule, et se lance à la nage. Il nage comme le jeune, la couronne ciliaire en avant et la collerette complètement fermée par-dessus son péristome. Quand il a trouvé une place qui lui convient, il se fixe, résorbe les membranelles de sa couronne ciliaire, ouvre sa collerette, recommence à manger et, peu à peu, reforme son pédoncule.

Il en est de mème au sortir d'un enkystement, car la Vorticelle s'enkyste au bout de son pédoncule, mais bientôt le kyste tombe et, au sortir du kyste, l'animal est libre.

La longueur de ces détails se justifie par l'importance de celle forme et sa remarquable différenciation 0,2 , sans le pédoncule. Mer et eau douce).

Avant la Vorticelle que nous avons cru devoir décrire la première pour donner tout de suite une idée des parlicularités du péristome, nous aurions dù placer, si nous avions voulu suivre l'ordre taxonomique régulier un petit nombre de formes dépourvues de pédoncule. Il est aisé de les définir en quelques mots.

Que l'on suppose une Vorticelle de forme cylindrique, sans pédoncule, se terminant à la partie inférieure par un disque adhésif analogue à celui de la Trichodine, mais sans vélum ni couronne ciliaire, et l'on aura l'un des trois genres:

Mais quel est cet élément? Exтz à qui nous empruntons la descriplion de tout ce système veut que ce soit le spasmonème. Mais on ne voit pas comment il déterminerait le tortillement du pédoncule en tire-bouchon. Il nous semble plus naturel d'admettre que c'est la gaine du spironeme et peut-c̀tre son axe et l'axonème qui jouent ce rôle. Les myonènes en hélice de la paroi dı pédoneule et ceux du spironème seraient les agents de l'allongement car, en se contractant, ils tendent à se rapprocher de la direction rectiligne et par conséquent à détordre le pédoncule et à bander le ressort spiral. Le spasmonème servirait a donner plus de vivacité au mouvement de rétraction en tirant suivant une des composantes du mouvement de retrail. Les fibrilles héliçö̈dales de la paroi semblent bien faibles pour lutter contre la tension du ressort spiral. Mais nous ne savons rien de la force de celui-ci. G'est peut-itre pour reposer leurs muscles extenseurs que l'on voit souvent les Vorticelles se contracter brusquement sans cause appréciable, comme nous clignons de l'œil pour reposer le releveur de la paupière. Au surplus la question réclame de nouvelles études. 
Scyphidia (Lachmann) (fig. 830) qui, quoique dépourvu d'anneau adhésif, vit fixé $(0,12$. Her el d'eau douce, sur des Gastéropodes ).

Gerda (Claparède et Lachmann) (fig. 831), qui s'en distingue par une forme plus effilée vers le haut $(0,2$. Eau douce);

Astylozoon (Engelmann) qui se caractérise par une vie libre et par son extrémité inférieure effilée, munie de deux soies saltatrices $(0,1$. Eau douce $)$ (').

Revenant maintenant à la Vorticelle, nous trouvons une série de genres qui en dérivent d'une manière très naturelle.

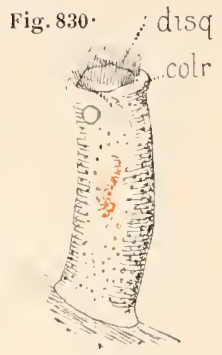

Scyphidia

(im. Glaparède et Lachmann).
Fig. 831.

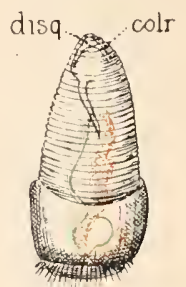

Gerda (G. glans) (d'ap. Claparède et Lachmann).

Carchesium (Ehrenberg) (fig. 832) est une Vorticelle chez laquelle, dans la division, l'individu qui ne garde pas le pédoncule maternel, au lieu de se séparer tout à fait et d'aller se fixer ailleurs, reste attaché à la base de celui-ci et se sécrète là un nouveau pédoncule. Il en résulte que les cordons contractiles despédoncules ne sont pas continus et que les individus peuvent se contracter isolément ou par groupes. L'ensemble forme un petit arbuscule dichotomique La colonie peut atteindre $4^{\text {ma }}$ de haut. L'animal lui-mème n'a que $1 \mathrm{~mm}$. Le pédoncule commun est fixé sur les plantes d'eau douce). - Chez

Zoothamnium (Ehrenberg) (fig. \$33), au contraire, la division

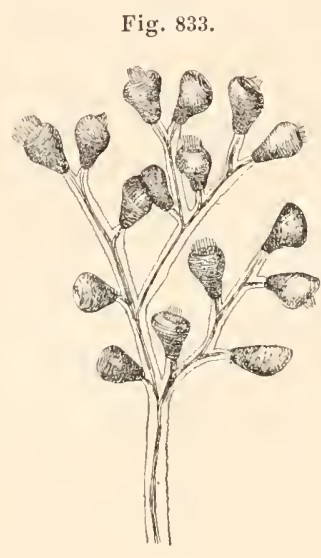

Zoothamnium ( $Z$. alternans) (d'ap. Claparède et Lachm.).
Fig. 832 .

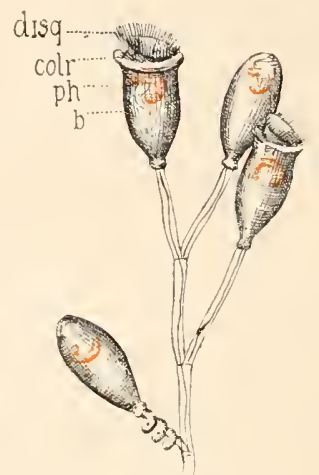

$p d$
Carchesium (C. epistylis) (im. Claparède et Lachmann). b., bouche; colr., collerette; disp.. disque; plı., pharynx.

se prolonge jusque sur le pédoncule dont chaque individu fille reçoit une moitié longitudinale sur une certaine longueur. Il en résulte que le système contractile du pédoncule est unique et que la colonie se contracte en bor, tous les individus à la fois $(0,08$. Les colonies atteignent plusieurs millimètres et se rencontrent dans la mer et l'eau douce) $\left(^{*}\right)$.

${ }^{1}$ Ces trois formes constituent pour Bütschli un petit groupe des Scyphidina.

$\left.{ }^{(2}\right)$ Des individus plus gros $(0,12)$ situés sur les rameaux de premier ordre à l'aisselle de ceux de deuxième ordre constituent des macrogonidies.

Ces deux genres forment, pour Bütschli, avec la Vorticelle, le groupe des Contractilia (Bütschli). 
Dans les deux formes que nous venons de décrire le pédoncule est histologiquement constitué comme chez la Vorticelle; il est contractile comme chez elles. Dans celles que nous allons décrire maintenant, il est semblable extérieurement à celui des genres précédents, mais il ne contient pas de filament axile et n'est pas contractile(').

Glossatella (Bütschli) (fig. 834) est isolé, a un pédoncule si court, qu'on pourrait le dire sessile; il est remarquable par l'énorme développement de sa membrane ondulante (mb.u.) (0,04. Eau douce). Chez.

Rhabdostyla (Kent), le pédoncule reprend le développement qu'il avait chez la Yorticelle, mais l'animal ne forme pas non plus une colonie (90 p. Eau douce, sur des larves de Diptères $\left({ }^{\mathbf{2}}\right)$. Epistylis (Erhenberg) (fig. 833̈), au contraire, est colonial comme Carchesium 40,8 , coFig. 83 . lonies $4 \mathrm{~mm}$. Ner el parfois eau douce). - On a créé le genre :

Campanella (Goldfuss) (fig. 836 à S38) pour une espèce d'Epistylis (E. umbellaria), remarquable par l'énorme développement de sazone adorale qui se prolonge à son extrémité aborale et décrit cinq tours complets sur le disque (fig. S37). Cette forme est encore remar quable par le fait que, seule

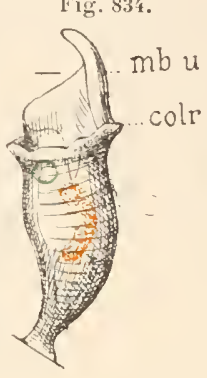

Glossatella

(G. tintinnabulum) (d'ap. Kent).

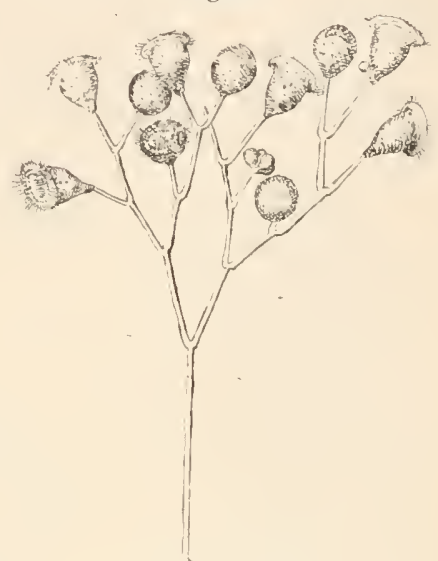

Epistylis (Sch.).

Fig. 836.

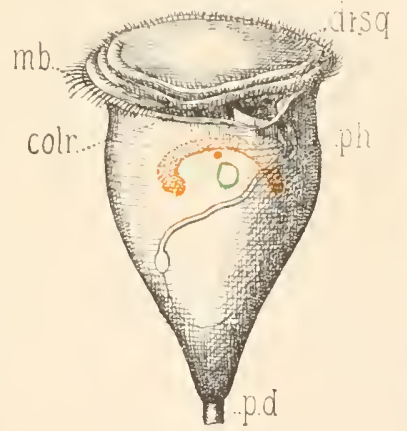

Campanella (Sch.).
Fig. 837.

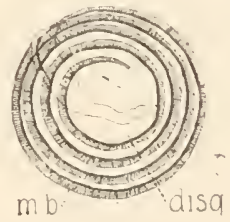

Campanella.

Disque (disq.) vu

de dessus

(d'ap. Bütschli).

mls., membranelles.
Fig. 838.

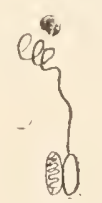

Campanella.

Deux nématocystes dont un présente son filament déroulé (d'ap. Biitschli).

colr., collerette; aisq., disque; pa., pédoncule ; ph., pharyux; mb., membranelles. parmi les Péritrichicles, elle possède des trichocystes (tig. 838), très gros (35 p.) au nombre d'une trentaine, épars parallèlement à la surface. Ces

(1) On trouve à sa partie supérieure un rudiment de spasmonème mais qui se termine presque aussitôt en pointe.

${ }^{(2)}$ Ce n'est peut-être que le jeune de certains Epistylis. - Le genre opisthostyla (Stokes) n’est guère qu'un sous-genre du précédent. 
trichocystes, par une exception unique chez les Ciliés, sont comme les nématocystes des Coelentérés, formés d'un filament spiral qui se dévagine à l'explosion. Cette explosion est facile à provoquer artificiellement, et elle doit avoir lieu nalurellement, mais on ne l'a jamais observée $(0,15$, colonie $4 \mathrm{~mm}$. Eau douce $)$.

Opercularia (Stein) (figg. 839) peut ètre défini comme un Epistylis de forme plus rétrécie en haut, à vestibule très élargi d'où sort une énorme membrane ondulante. Cet élargissement du vestibule entraìne un rétrécissement du pédoncule du disque qui prend l'aspect d'un opereule muni d'un manche étroit et fonctionne comme tel, en se rabattant $(0,25$. Eau douce $)$ (").

Ophrydium (Bory de Saint-Vincent) (fig. 840 ) est comme un Operculaire lont le vestibule serait moins large et le disque plus trapu tout en gardant les caractères d'un opercule. Les indiviłus d'une mème colonie sécrètent une substance gélatineuse commune au-dessus de laquelle ils peuvent s'élever en s'épanouissant, mais où ils se retirent dès qu’ils sont inquiétés, chacun dans une logette particulière $\left(0,4\right.$. Eau douce) $\left(^{2}\right)$.

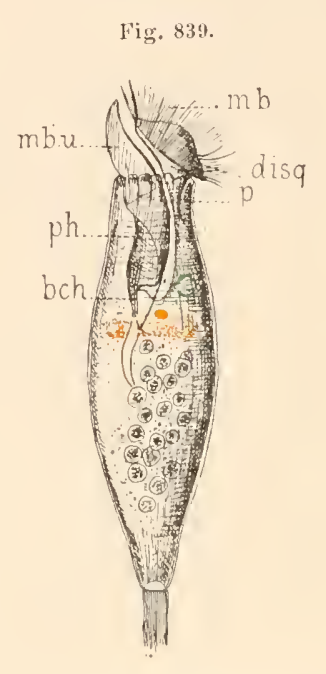

Opercularia (O. articulatu) (im. Bütschli).

bel., bouche; disq., disque mb., membranelles; mb.u., membrane oudulante; p., pédoncule du disque; ph., pharynx.
Fig. 810 .

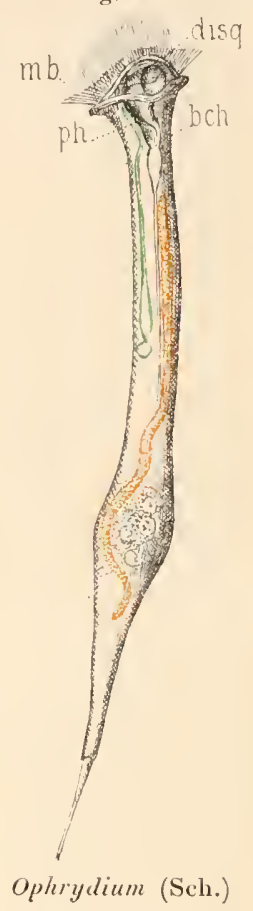

Toutes les formes que nous venons de décrire, pédonculées ou non, étaient nues. Nous allons maintenant rencontrer un perfectionnement nouveau, c'est la formation de petites logettes permanentes sécrétées par l'animal autour de lui pour s'abriter.

Vaginicola (Claparède et Lachmann) (fig. S41) peut être défini comme une Vorticelle qui aurait perdu son pérloncule et pris la forme d'un urinal de ma-

(1) Genre voisin :

Pyxidium (Kent), forme douteuse qui en différerait par l'absence de colonie (50 p.. Eau douce, sur des Cypris.

(2) Remarquer en outre la forme du corps allongé, un peu renflè en bas, la longueur et l'étroitesse du pharynx, el l'énorme développement du réservoir de la vésicule pulsatile. Quand les colonies deviennent très grosses (elles atteignent alors plusieurs pouces de diamétre) il peut se développer dans la masse gélatineuses des bulles gazeuses qui la détachent et la font flotter.

Ces genres constituent pour Bütschli un petit groupe des Acontractilia Bütschli). 
larle. Il habite une petite logette chitineuse de même forme à laquelle elle est altachée par toute sa face ventrale. La tète sort par le goulot. La coquille elle-mème est fixée sur les plantes (0,1. Eau douce). - Chez Cothurnia (Claparède et Lachmann) (fig. S42) la logette est plus ou moins cylindriyue, verticale, et l'animal, fixé au fond par un court pédoncule, n'a pas d'autre attache avec elle; mais il porte sous le bord de son entonnoir une sorte de bourrelet saillant qui ferme l'entrée de la logette quand il se contracte 0,4 . Her et eau douce) ("). - Enfin dans le genre

Lagenophrys (Stein) (fig. 843), l'animal, de forme arrondie, habite une coquille grélatineuse de mème forme, munie d'un orifice étroit; il est fixé par les bords de sa face supérieure à cel orifice, en sorte que son disque cilié, longuement pédonculé, peut seul sortir. Ce disque ne porte pas de clapet, mais les bords de l'orifice de la coquille peuvent se rabattre en dedans pour le fermer $\langle 0,07$. Eau douce, sur les branchies ou les poils des petits (rustacés) $\left({ }^{*}\right)$.

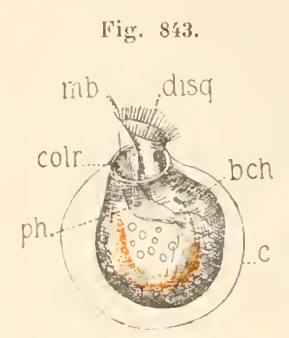

Lagenophrys (Sch.).

bel., bouche; c., loge: colr., eollerette; disq., discue; ph., pharynx.

(1) Parfois, il existe au lieu de l'appareil précédent, un opercule chitineux spécial (op., fig. 842), indépendant du corps, attaché par une partie de son bord à la partie supérieure du tube et relić au fond de celui-ci par une mince membrane en gouttière $(m$.$) . Cette partie n'a aucune contractilité propre, mais$

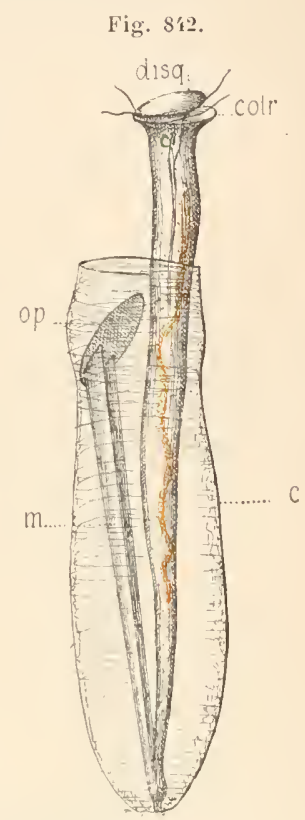

Cothurnia (Sch.).

c., logette; colr., eollerette; disq., disque; m., membrane en gouttiere; op., opercule. quand l'animal rentre dans son tube il force la membrane à se courber et par suite à tirer vers le bas l'opercule qui ainsi lerme le tube. Pyxicola (Kent),

Pachycola (Kent),

Pachytrocha (Kenl),

Thuricola (Kent), sont plutôt des sous-genres caractérisés par diverses particularités du mode de fermeture. - Genre voisin :

Cothurniopsis (Entz), commensal d'animaux aquatiques, à $\mathbf{N}$ courbe au lieu d'être rubané (IIer et eau douce).

Ces quelques formes constituent pour Bütschli le petit groupe des Cothurnina.

Depuis et y compris Vorticclla. celte longue série de genres constitue la famille des VortTCELLINE [Vorticellina (Ehrenberg, emend. Bütschli]].

$\left(^{2}\right)$ Pour se diviser, l'animal rompt ses adhérences à sa coquille et se retire au fond; un des individus filles garde l'ancienne coquille, l'autre sort et s'en reforme une nouvelle. Cette forme constitue presque à elle seule la famille des LAGESOPHRTLY.E [Lagenophryina (Bütschlii)].

stylohedra (Kellicott) n'est qu'un sous-genre dlu précédent.

Quelques auteur's rattachent aux Vorlicelles ou aux Infusoires tentaculifères le genre encore mal connu Erythropsis (R. Ilertwig!, que nous avons placé en appendice aux Dinoflagellés (V. p. 387). 


\title{
$2^{\text {e }}$ Sous-Chasse
}

\section{TENTACULIFERES. - TENTACULIFERIA vel}

\section{SUCEURS. - SUCTORIE}

\author{
[Acinetina (auct.); - Tentaculifera (Iluxley, Kent); - \\ SUctoria (Kent, Bütschli)]
}

Si nous nous étions astreints à décrire les groupes en suivant l'ordre de supériorité organique, nous aurions dù placer les Tentaculifères avant les Ciliés, car il leur sont incontestablement inférieurs. Nous avons préféré les décrire après ceux-ci, parce qu'ils peuvent être facilement étudiés par comparaison avec le type des Ciliés, tandis que l’inverse n’eût

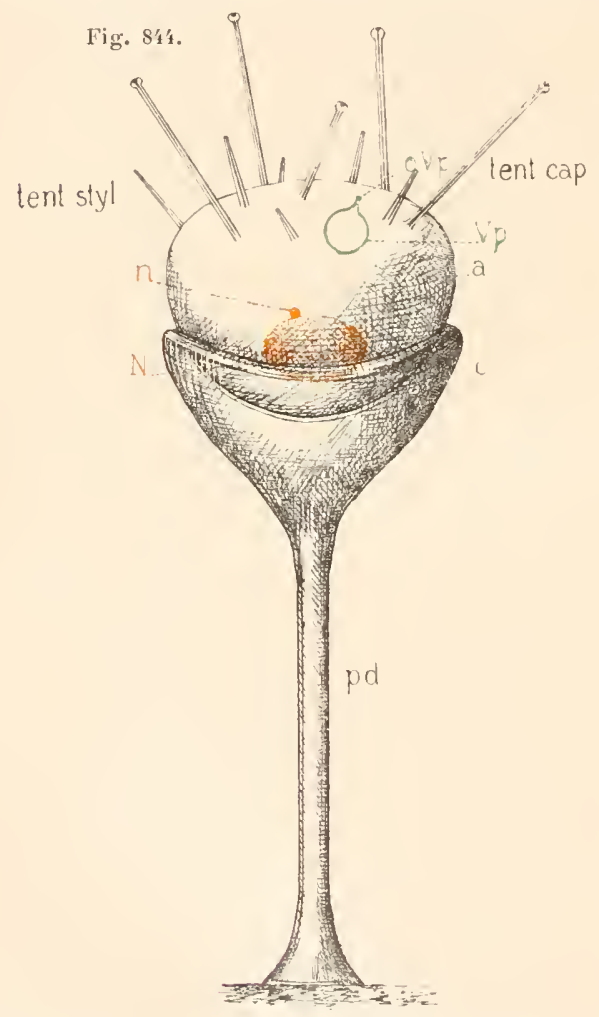

TENTACULIFERLE (Type morphologique) (Sch.).

a., l'animal; c., partie cupuliforme du pédoncule; $\mathbf{s}$, macronucléus; n, micronucléus; ovp., orifice exrréteur; tent. cap., tentacule capité; tent. styl., tentacule styliforme; $\mathbf{V}$ p., Vésicule pulsatile. guère été possible; et, d'autre part, nous avons le droit de suivre cet ordre parce que les Tentaculifères, s'ils sont inférieurs aux Ciliés, ne sont pas pour cela plus rapprochés du type ancestral. Il est probable qu'ils en dérivent au contraire par modification régressive, comme semble l'indiquer le fait qu'ils ont, à l'état embryonnaire, des cils qu'ils perdent plus tard (').

\section{TYPE MORPHOLOGIQUE}

(FIG. 844 A 850 )

\section{Structure.}

Idée générale de l'être. - Nolıe Tentaculifère est un animalcule plus pelit que le Cilié, ne mesurant guère qu'un dixième de millimètre au lieu d'un tiers de millimètre, comme celui-ci. Il est fixé au sommet d'un long pédoncule $(p d$.$) , mince mais rigide$ el dilaté au sommet en coupe (c.)

(1) Bürschli considère les tentacules des Tentaculifères comme homologues à la bouche des Ciliés qui se serait multipliée et placée au bout d'un tentacule. Celte opinion semble à peine soutenable. 
pour le recevoir. Dans celte excavation terminale, est l'animal (a.), en forme d'ovoüle trapu, reposant dans la coupe par le petit bout. Son corps est entièrement dépourvu de cils. On y trouve, vers la parlie supérieure, un petit pore excréleur ( $\mathrm{V} p$.), semblable à celui d'un Cilié, mais il n'y a ni bouche, ni anus. En place de cils, la surface porte de nombreux prolongements tentaculiformes (tent. cap., tent. styl.) qui ont valu son nom à ce groupe d'ètres. L’organisation inlérieure (fig. Sł引̈) est, en gros, relle d'un Cilié. Il y a une membrane, un ectoplasme (ectop.), un endoplasme (endop.), avec des inclusions variées, un $\mathrm{N}$ et un $\mathrm{n}$, silués vers la parlie inférieure du corps, et enfin une vésicule pulsatile $\left(I_{p}\right.$.) , à côlé du pore excréteur.

Pédoncule. - Le pérloncule (fig. S4ł, pd.) n'est pas, comme celui des Vorlicelles, une partie du corps devenue filiforme. C'est une parlie surajoutée, une simple sécrétion comparable à ces logelles chilineuses que nous avons maintes fois trourées chez d'autres Ciliés. La seule différence, c'est que l'animal est soudé dans une excavation terminale de sa logelte au lieu d'ètre contenu à son intérieur. Ce pédoncule se compose de trois parlies : une moyenne, cylindrique, une inférieure,

Fig. S'ts.

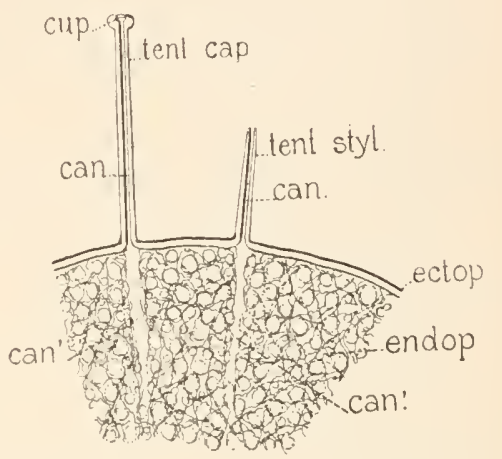

TENTACELIFERIE (Type molphologique). Structure (Sch.).

can., canal du tentacule; can'., prolongement endoplasmique du canal: cup., cupule du tentacule capiti: ectop., ectoplasme; endop.. endoplasme; tent.cap., tentacule capite: tent. styr., tentacule styliforme. un peu élargie, fixée par une sécrétion durcie à quelque objel immergé, et une supérieure dilatée el excavée $(c$.). Le tout est creux et formé d'une paroi chitineuse et d'un contenu liquile; la membrane chitineuse qui forme le fond excaré de la capsule est continue, et il n'y a aucune communication entre le corps de l'Infusoire et le liquide sous-jacent(').

Tégument. Cytoplasme. - L'endoplasme (fig. 840 , endop.) a la mème structure que chez les Ciliés. Il u'y a pas de plasma corlical distinct.

(1) Cette paroi devrait laisser distinguer trois couches, de haut en bas : 10 l'ectoplasme, $2^{\circ}$ la membrane appartenant au corps de l'Infusoire, $3^{\circ}$ la paroi chitineuse du pédoncule. En réalité, on ne distingue, le plus souvent, qu'une paroi, comme si les trois couches se confondaient là en une simple cloison de séparation. Mais la paroi pédonculaire existe certainement, car lorsque l'animal meurt et se décompose, cette paroi résiste et persiste lorsque toute trace du corps a disparu. D'autre part, il semble bien peu probable que l'endoplasme soit à nu au contaci d'un produit de sécrétion.

Le pédoncule n'existe pas toujours. Bon nombre de Tentaculifères sont sessiles et dépourvus de toute enveloppe sécrétée. Quelques-uns sont tout à fait libres. D'autres fois, au contraire, le corps est dans une vraie logette ouverte seulement en un ou quelques points. 
L'ectoplasme (ectop.) a ici un aspect homogène el on ne lui trouve plus aussi nettemenl la striation radiaire qu'il présentait habituellement chez. les Ciliés. La membrane existe mais peu accusée(").

Inclusions du cytoplasme. - L'ectoplasme ne renferme point d'éléments différenciés. Il n'y a ni myonèmes ni trichocystes $\left({ }^{*}\right)$.

Dans l'endoplasme, on trouve les vacuoles ordinaires faisant partie de sa struclure, mais pas de vacuoles alimentaires. En fait d'inclusions solides, on ne trouve pas de grains d'excrétion, mais on rencontre en grande abondance des grains incolores réfringents qui sont des réserves nutritives, car le jeùne les fail disparaître. Certains d'entre eux, mais pas tous, sont de nature graisseuse $\left({ }^{\mathbf{3}}\right)$.

Appareil nucléaire. - Le $\mathrm{N}$, ovoüde, est disposé transversalement à la partie inférieure du corps. Tout contre lui, est un n. Ils ont l'un et l'autre la même structure que chez notre type morphologique de Cilié.

Vésicule pulsatile. - Il y a une (parfois plusieurs) vésicule pulsatile du type de celles à vésicules formatrices en cercle autour de la vésicule centrale.

Elle est placée sous l'ectoplasme dans la partie supérieure du corps, en face du point où le pore excréteur traverse celui-ci. Tout l'appareil est constitué comme chez les Ciliés, mais on ne voit point de réseau de canalicules ('). Cela d'ailleur's n'a pas grande importance. Qu'il y ait ou non des chemins tracés d'arance dans la substance, l'eau n'en suit pas moins le mème cours, entrant dans le corps par osmose en tous points el convergeant vers la vésicule chargée de l'expulser.

On le voit, notre Tentaculifère est, sous tous ces rapports, entièrement conforme aux Ciliés et, malgré l'absence de bouche (les Opalines sont dans le mème cas), malgré mème l'absence de cils, nous en aurions

(1) Il y a de grandes discussions au sujet des couches tégumentaires. Les uns décrivent une membrane sans ectoplasme, les autres un ectoplasme sans membrane; d'autres enfin, un ectoplasme et une membrane. La première opinion semble peu soutenable, car cetle membrane serait bien épaisse et formerait à elle seule la paroi des tentacules et, d'autre part, on peut affirmer ì priori que, s'il y a un ectoplasme ayant la structure fondamentale du cytoplasma, même à éléments aussi fins que l'on voudra, il y a toujours, à la surface au moins, ce liséré continu que nous avons défini comme ètant la membrane protoplasmique sous sa forme la plus simple. Or, la membrane des Infusoires est une membrane protoplasmique et non un exsudat, même lorsqu'elle est le plus apparente. Récemment, Cheviakof a représentė chez un Trichophrya un ectoplasme avec la structure typique qu'il a chez les Ciliés.

(2) Le genre Ophriodendron seul fait exception; il possède des formations qui semblent bien être des trichocystes.

(3) Dans quelques genres, on a rencontré des grains d'excrétion. On a trouvé parfois des granulations pigmentaires, variant du jaune au brun rouge. Enfin, on a donné le nom de grains chromophiles à des particules qui se rencontrent quelquefois, mais qui ne constituent probablenent pas une catégorie spéciale, car elles ne sont, selon toute apparence, que des débris du $\mathrm{N}$ fragmenté après la conjugaison et en voie de résorption.

(4) Dans quelques cas cependant on a trouvé les premières voies de ce réseau lacunaire. 
fait tout au plus un ordre de ces derniers, s’il n'en différait par un caractère capital. Ce caraclère, c'est le tentacule que nous allons maintenant décrire.

Tentacules. - Toute la surface du corps, principalement vers la partie supérieure, est hérissée de prolongements assez fins et dont la longueur est à peu près égale au diamètre du corps, ce sont les tentacules. Les tentacules sont de deux sortes; les uns (fig. 844) sont graduellement effilés, puis légèrement tronqués au hout (tent. styl.), les autres terminés par un petit renflement eu tête d'épingle (tent.cap.). On les distingue souvent sous les noms de ravisseurs et suceurs, il raudrait encore mieux les appeler styliformes et capités car, sous le rapport des fonctions, il n'y a entre eux aucune différence réelle (").

Les uns et les autres sont creux et leur canal (fig. S librement ouvert, d'un côté dans l'endoplasme, de l'autre au dehors. Dans les styliformes, le canal s'ouvre simplement à la pointe tronquée du tentacule; dans les capilés, il s'ouvre au fond d'une pelite dépression cupuliforme (cup.) qui constitue une sorte de ventouse au sommet du renflement terminal. A leur base, les tentacules s'insèrent à angle vif sur la surface du corps. Leur membrane se continue avec la membrane, leur ectoplasme avec l'ectoplasme et leur canal central s'ouvre en plein endoplasme. Ils sont done formés par un prolongement de la couche légumentaire $\left(^{\mathbf{2}}\right)$.

Quand ils rentrent dans le corps leur paroi se fusionne avec celle du corps à leur base et leur caual se perd dans la cavité virtuelle qui sépare l'ectoplasme de l'endoplasme.

\section{Physiologie.}

Mouvements. - L'animal étant fixé sur un pédoncule non musculeux, ćlant dépourvu de cils vibratiles, est condamné à l'immolilité. Il n'y a de mohile en lui que ses tenlacules, mais ils le sont à un haut degré el par eux le corps est susceptible de quelques mourements. Les tentacules peurent se contracter en se contournant en vis, comme si le mouvement était produit par une contraction de l'ectoplasme suivant une ligne héliçoïdale, ou s'étendre en reprenant leur aspect primitif; ils peuvent, en tous sens, se courber ou, restant droits, s'infléchir à partir de leur

(1) La preuve en est que bien des Tentaculifères n'ont que des tentacules dits ravisseurs et, cependant, se nourrissent tout comme les autres par suecion. Les longs tentacules ravisseurs n'ont pas été observés jusqu'ici chez les formes d'eau douce qui n’ont que des tentacules capités ou coniques à tête, mais très courts.

(2) La distinction des deux couches tégumentaires dans leur paroi est encore plus théorique que pour le reste du corps. En réalité, on ne voit que quatre lignes, deux limitant le canal et deux limitant le tentacule. Dans quelifues cas, on roit le canal central se continuer assez avant dans l'endoplasme (fig. 845, cam'), mais ce n'est là qu'un trajet sans parois spéciales autres que l'endoplasme commun et cela ne prouve point, comme quelques-uns le prétendent, que le tentacule lui-mème s'enfonce dans le corps en se rétractant. 
hase; ils peuvent enfin se rétracter en rentrant dans le corps, partiellement ou lout à fait, et disparaître alors entièrement pour se reformer plus tard. Ces divers mouvements sont très lents mais fort étendus, aussi, quand on ne regarde l'animal qu'un instant, on le croit inerte, mais si on le regarde de nouveau quelque temps après, on voit que la disposition de ses tentacules est complètement modifiée. Il faut trois ou qualre heures à un tentacule pour se rétracter entièrement, un peu moins pour se reformer.

Alimentation. - Quand un animalcule (c'est généralement un Cilié souvent plus gros que notre Tentaculifère) vient en nageant à rencontrer un tentacule, il est tout l'abord arrêté, car le tentacule est glutineux ef se colle à lui. Cependant il se débarrasserait bientôt d'une si faible attache, mais les tentacules voisins convergent vers lui et, s'attachanl aussi sur la proie, la maintiennent solidement: les tentacules capités s'attachent par leur venfouse, les autres simplement par leur pointe. D'ailleurs, il semble qu'un venin spécial émane des tentacules, car la victime avant d'ètre mécaniquement endommagée semble paralysée : si elle est ciliée, ses cils s'arrêlent et elle devient incapable de se défendre (').

Les tentacules exercent alors une succion. Tout d'abord, on n'en voit pas les effets, mais sous son influence la membrane et l'ectoplasme du Cilié capturé finissent par crever et l'endoplasme alors pénètre dans le canal tentaculaire, le parcourt, et arrive à l'endoplasme de l'agresseur dans lequel il s'écoule d'un mouvement continu. Quand la victime est complètement vidée, sa dépouille est abandonnée par les tentacules qui làchent prise et s'écartent lentement.

La cause mécanique de cette succion n'est pas très nettement élucidée. On a cru d'abord à un mouvement de pompe, exercé par les contractions alternatives des tentacules, mais en réalité ce mouvement n’a pas lieu. Tout est immobile dans l'Infusoire pendant qu'il suce avec énergie ; tout, sauf naturellement la vésicule pulsatile. Aussi est-ce à elle qu'Eısıono [90], attribue le rôle actif dans ce phénomène. Expulsant sans cesse de l'eau qui occupait dans le corps une certaine plare, elle produit un vide relatif qui tend à faire affluer dans son intérieur toutes les substances mobiles auxquelles la voie est ouverte pour y entrer $\left(^{\boldsymbol{2}}\right)$.

Enkystement. - Lanimal s'enkyste rarement pour se soustraire à des influences nocives et jamais pour se diviser. Pour s'enkyster, il

(1) Claparède et Lachmann ont vu cependant une fois un Stylonichia captmré se diviser rapidement et ne laisser au ravisseur qu'une de ses moitiés. C'est un mode d'autotomie défensive assez original.

$\left({ }^{2}\right)$ Quand l'Infusoire ne mange pas, cette tendance aspiratrice est satisfaite par l'eau qui pénètre par osmose à travers les téguments. Or, cette cau continue à pénétrer quand l'Infusoire mange. Il est vrai que l'aspiration doit se partager entre la surface du corps et les orifices des tentacules, mais étant proportionnelle à la surface, elle doit être bien minime dans ceux-ci. Si la vésicule se contractait plus vite pendant la succion, l'hypothèse d'Eismond se trouverait presque démontrée, mais l'auteur ne dit pas qu'il en soit ainsi. 
rétracte complètement tous ses tentacules, s'arrondit et se sécrète une coque de chitine au sommet de son pédoncule. On peut appliquer ici tout ce que nous avons dit de l'enkystement défensif chez notre type morphologique de Cilié.

Reproduction. - Notre Tentaculifère se reproduit uniquement par division transversale. Mais cette division, égale dans le principe, assume les caractères d'un bourgeonnement lorsque l'individu fille supérieur est plus pelit que l'inférieur; ce bourgeonnement peut devenir interne lorsque le bourgeon s'invagine dans une cavité incubatrice pour se lévelopper; enfin, au lieu d'un bourgeon, il peut y en avoir plusieurs, soit cxternes, soit internes. Cela fait autant de cas qu'il nous faut examiner successivement.

Division égale ou presque égale (fig. 8 46). - Elle se fait, comme d'ordinaire chez les Ciliés, par un plan transversal. Les phénomènes cytoplasmiques et nucléaires, la formation d'une seconde vésicule pulsatile, ne présentent aucune différence importante avec ce qui se passe chez ceux-ci. Mais voici oì la chose devient intéressante. Avant de se diviser, l'animal

Fig. 84ti.
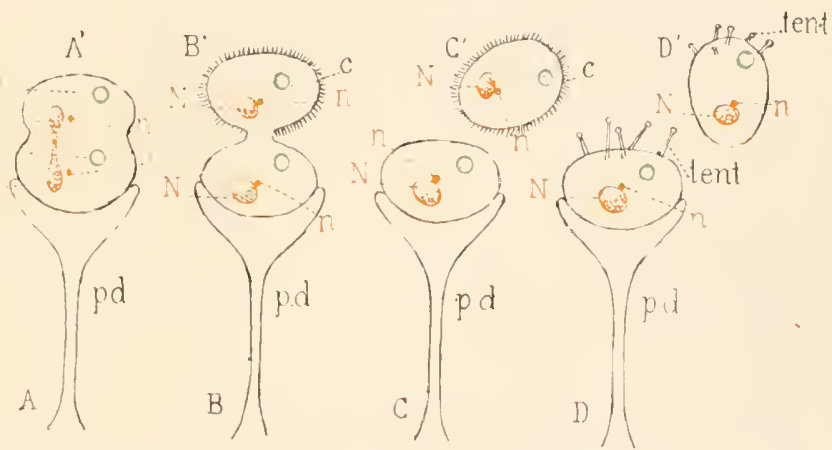

TEvTretelket Type morphologique). Division (Sch.).

$A$ à $D$, stades successifs de la division: $A^{\prime}$ à $D^{\prime}$ produit de la division correspondant à ehacun de ces stades; $\mathbf{c . ,}$ cils : $\mathbf{N}$, macronucléns: $\mathbf{n}$, micronucléus; pd., pédoneule; Vp., veisirules pulsatiles.

rétracte complètement tous ses tentacules $(A)$; après la division, l'individu inférieur garde le pédoncule $(p d$.) et il n’a qu’à pousser de nouveaux tentacules pour être de nouveau complet $(I)$ ). Mais que va faire l'individu supérieur $\left(A^{\prime}\right)$ ? On le voit, arant que la séparation soit achevéc, se courrir de cils $\left(B^{\prime}: c\right.$. ) comme un Cilié, et avec ces cils il nage à la recherche d'une place pour se fixer $\left(C^{\prime}\right)$. Pendant tout ce temps, il ne diffère en rien d'essentiel d'une Opaline par exemple. Quand il a trouvé un support propice, il s'attache à lui par un point de sa surface, résorbe ses cils, pousse des tentacules $\left(D^{\prime}\right)$, sécrète un petit pédoncule qui grandit lentement et, peu à peu, revêt ainsi les caractères d'un Tentaculifère normal (').

Bourgeonnement externe simple. - Il n'y a d'autre différence entre

(1) Ce mode de division se rencontre chez les Podophrya, Sphxrophrya, Umula. Metacineta. Chez spherophrya l'individu libre présente des tentacules dès sa séparation. 
ce cas et le précédent qu'une forte infériorilé de taille de l’individu supéricur $\left(A^{\prime}\right)$. Celui-ci se préscnte comme un simple mamelon au pôle supérieur du corps.

Bourgeonnement externe multiple (fig. S17). - L’Infusoire s’étant pré-

Fig. 847.

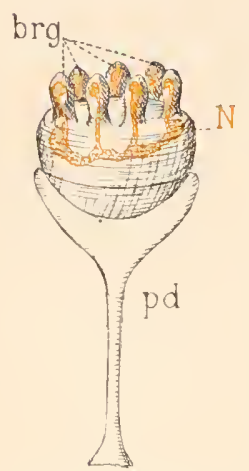

TEVTARULIFERIE

(Type morphologique). Bourgeon nement externe mulliple (Sch.).

brg., hourgeons : $\mathbf{N}$ macronucléus; pal., pédoncule. paré comme pour une division égale ou un bourgeonnement simple, c'est-à-dire ayant rétracté ses tentacules, forme à sa partie supéricure un certain nombre de mamelons saillants juxtaposés (brg.); le $\mathbf{N}$ se ramific et envoic une branche dans chacun de ses bourgeons; le $\mathrm{n}$, sans doute, se divise aytant de fois qu'il est nécessaire, les bourgeons se détachent et tout s'achève pour chacun d'cux comme dans le cas précédent.

Bourgeonnement interne simple (fig. S S S ). - Le phénomène commence comme pour le bourgeonnement externe simple mais, dès que le bourgeon $(A: \operatorname{lrg}$.) commence à se dessiner comme un pelit mamelon superficicl, on le voit s'enfonecr dans le corps de la mère $(B)$. Un sillon circulaire se creuse autour de lui et, par l'approfondissement graduel de ce sillon, il s'inragine à mesure qu'il grandit $(C)$. Il se trouve de la sorte formé, comme à l'ordinaire, d'une protubérance bicn accusée, mais cette protubérance est logée au fond d'une cavité incubatrice dont les parois remontent autour d'elle et se rejoignent au-dessus sans arriver tout it fait au contact et en laissant un petit orifice de communication arec le dehors. Les phénomènes nucléaires se passent comme à l'orlinaire et, en outre de son $\mathbf{N}$, de son $\mathrm{n}$ et de sa vésicule, le hourgeon se munit d'une ceinture verticale de cils. Quand tout cela est préparé, le bourgeon continue à grandir, achève de s'énuclécr, dilate l'orifice de la cavité inculbatrice et apparait libre au dehors $(I): \operatorname{lrg}$. $)$. Ou bien, tout en restant 
cncore attaché à la mère, il se dévagine $\left(C^{\prime}\right)$, la cavité incubatrice s'efface autour de lui et il ressemble à un bourgeon externe; mais il finit enfin par se détacher et derient libre également $\left(D^{\prime}: \operatorname{lrg}.\right)$. Avec sa ceinture ciliaire qui s'est complétée, il nage à la recherche d'un support convenable et, quand iI l'a trouvé, il se fixe par un point voisin de sa ceinture ciliaire. Tout s'achève alors comme pour Ies bourgeons externes $(E, F, G)$.

Bourgeonnement interne multiple. - Qu'il ait lieu par bourgeons séparés dans autant de cavités distinctes ou par plusieurs bourgeons logés dans une cavité incubatrice commune, la différence est toute contingente et le processus se comprend sans plus ample description (').

Conjugaison. - La conjugaison (fig. S19) a lieu ici comme chez les Ciliés. Deux individus roisins s'accrochent par quelques tentacules, se rapprochent, se soudent, et entre eux se produit toute la série de divisions d'échanges, de transformations dont nous arons décrit Ia succession à propos de notre type morphologique de Cilié.

Ici, mahheurcusement, quelques stades n'ont pu ètre observés, mais leur existence résulte d'une induction très légitime. C’est encore Mlıups

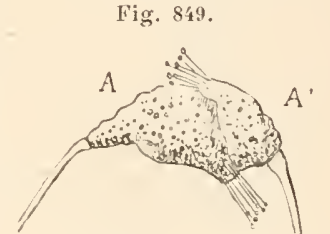

TEVTARULIFERIE

(Type morph.).Conjugaison (d'ap. Fraipont). qui les a observés et bien des auteurs moins prudents se seraient contentés de ce qu'il a vu, car il a reconnu le plus grand nombre de stades et en parliculier les plus importants. Il est à remarquer que la

(1) Bien entendu, ces divers modes ne se rencontrent pas à la fois dans la même espèce. Chaque forme a le sien. La division égale est rare et ne se rencontre que dans des formes inférieures : Iypocoma, sphierophrya, Podophrya. Elle devient un peu inégale et fait le passage au bourgeonnement chez certaines espèces d'Urmula, Metacineta, Acineta. Le bourgeonnement simple ne se rencontre guère que chez les spharophrya et les petites formes a bourgeonmement multiple. Ce dernier mode est le plus habituel chez les Tentaculifères. Ephelota gemmipara en fournit un exemple bien counu. Il se rencontre aussi chez la plupart des Icinétines et ailleurs.

Le bourgeonnement interne simple se rencontre principalement chez Dendrocometes, Tokophrya et quelques espèces d'Acineta. Dendrosoma possèlle des chambres incubatrices multiples contenant chacune un seul bourgeon; enfin, on troure plusieurs bourgeons dans une mème chambre incubatrice chez certains Acinètes, A. tuberosa, A. cucullus et chez Ophryodendron.

Les cils du bourgeon n'ont souvent pas achevé de disparaitre lorsque les lentacules se montrent déjà; d'autre part, ceux-ci ne disparaissent pas toujours, au moins complètement, dans la division, en sorte que la coexistence momentanée de ces deux sortes d'appendices n'est pas rare.

Dans ces divisions et bourgeonnements, le ròle du n n'a pu être nettement observé, mais il n'esł guère douteux qu'il ne soit le même que chez les Ciliés, et ne consiste en une division simple ou multiple.

La répartition des cils sur le bourgeon est très variable : tantôt les cils sont uniformément répartis, tantôt ils n'occupent que la face ventrale, ou dessinent des spires, ou se réduisent à une simple couronne. De là, la distinction des bourgeons holotriches, hypotriches, hétérotriches. Mais ces expressions n'ont qu'une valeur descrip- 
série semble ici plus simple que chez les Ciliés. Elle est conforme à ce

Fig. 850

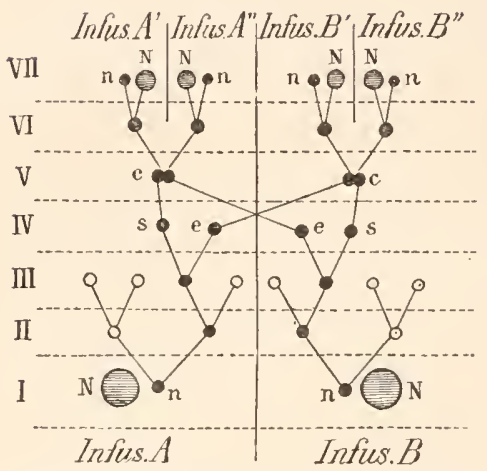

TENTACULIFERLE (Type morpholog.). Diagramme montrant l'érolution des noyaux dans la conjugaison (im. Maupas). que nous avons décrit chez le type morphologique, c'est-à-dire que le n conjugué donne immédiatement par division un $\mathbf{N}$ et un $\mathbf{n}$, sans se diviser au préalable en les noyaux conjugués des deux individus filles qui doivent naître de la première division post-conjugale. Le schéma réel est donc celui que nous avons donné pour notre type de Cilié et que nous reprorluisons ici (fig. 8500 ).

\section{GENRES}

De tous ces caractìres, les plus rariables sont la présence ou l'absence du pédoncule, le nombre, la forme et la disposition des tentacules. Partant des Acinétines qui représentent la forme moyenne, nous suivrons deux séries de transformations, l'une descendante où nous verrons le pédoncule disparaître, les tentacules diminuer de nombre, et enfin des cils se montrer; l'autre ascendante où le pédoncule disparaîtra encore, mais où la forme va se compliquer par des prolongements variés au sommet desquels les tentacules vont se localiser. Quant à la présence de logettes protectrices, aux variations secondaires des tentacules, du noyau, de la vésicule, au mode de bourgeonnement, tout cela ne suit aucune marche régulière et nous servira à caractériser les genres.

Acineta (Erhenberg, emend. Bütschli) (fig. S.̈̈). C'est

live, car nulle part on ne trouve les cirres ou la zone adorale caractéristiques des deux derniers ordres et, si ces êtres etaient des adultes, on les classerait tous parmi les Holotrichides. La disposition des cils en ceinture annulaire chez les bourgeons internes de Tolophrya a un intérêt particulier, parce qu'elle permet de fixer l'orientation du bourgeon par rapport à

Fig. 851 .

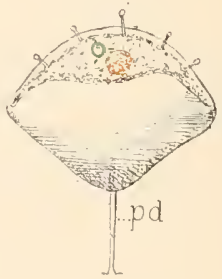

Acineta

(A. emaciata)

(d'ap. Maupas).

l'animal. En effet, cet anneau ciliaire étant vertical et médian sur le bourgeon il faut le considérer comme traçant le plan sagittal, et le bourgeon se fixant par un point voisin de cet anneau prend, sùrement dans un sens et très probablement dans les deux, la même orientation que la mère.

On observe dans quelques genres: Podophrya, Metacineta, Dendrocometes, un singulier phénomène qui s'explique par une modification de la division. L'animal abandonne son pédoncule, ou, s'il n'en a pas, son point de fixation, se munit de cils et se met à mener une vie libre. Ce n'est pas ici cette simple mutation de condition commune chez les Vorticelles, mais quelque chose de comparable à la rénosation totale de Spirochona (V.p. 482). L'animal, en effet, se comporte comme pour' se diviser, mais il fait passer toute sa substance nucléaire et la presque totalité de son cytoplasma, y compris la vésicule, dans l'indiridu supérieur qui se détache. It n'abandonne qu'une minime partie de sa substance sous la forme d'un résidu minime, incapable 
notre type morphologique, sauf que les tentacules sont tous capités $(0,2$. Mer et eau douce) ("). - Chez

Metacineta (Bütschli) (fig. S:j2), la cupule terminale se développe en une logette qui enveloppe entièrement le corps, sauf six fentes verticales partant de la partie supérieure et régulièrement espacées, par lesquelles sortent les tentacules disposés en six groupes. Mais la cupule est libre ainsi que la cavité du pédoncule : il n'y a pas, comme chez l'Acinète, un liquide spécial maintenu par une cloison (0,7. Eau douce) $\left({ }^{2}\right)$. - Chez

Solenophrya (Claparède et Lachmann ) (fig. S3̈3), le pédoncule disparaìt et il n’y a plus que la cupule terminale, mais largement ouverte à sa partie supérieure $\left(0,16\right.$. Eau douce) $\left({ }^{3}\right)$. - Chez

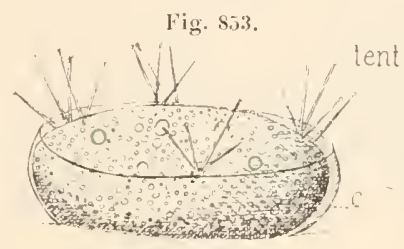

Solenophrya (S. crassa)

(d'ap. Claparède et Lachmann).

Tokophrya (Bütschli) (fig. S5̋), au contraire, c'est la cupule terminile qui disparait et il ne reste qu'un pédoncule directement inséré au pôle inférieur du corps $\left(0,24\right.$. Ner el ean dlouce) $\left({ }^{\mathbf{A}}\right)$. Il en est de mème chez

Podophrya (Erhenberg, emend. Bütschli), chez qui, en outre, les tentacules deviennent de longueur inégale $(0,07$. Lau douce et probablement mer).-De même encore, chez Ephelota (Wright, non Kent) (fig. 85̈3),

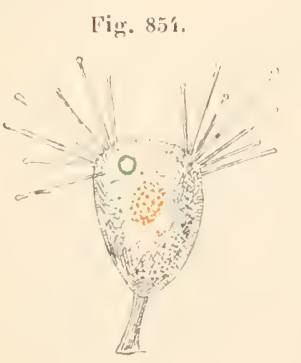

Tokophrya (d'ap. Cheviakof).
Fig. 852.

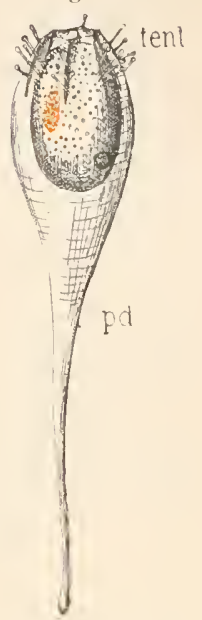

Metacineta (Sch.).

de vivre, mais représentant morphologiquement l'individu inférieur d'une bipartition incomplète, réduit à un volume dérisoire.

(1) Le bourgeonnement est interne. La longueur du pédoneule et la hauteur de la partie du corps abritée dans sa cupule terminale sont très variables avec les espèces. Dans certaines, les tentacules tendent en outre à se grouper par petits bouquets. Aussi les genres caractérisés d'après le développement du pédoncule ou de ses parties, ou par la disposition des tentacules sont-ils un peu secondaires.

Acinetopsis (Robin) n'a sans doute que la valeur d'une espèce du genre Acineta.

$\left(^{2}\right)$ Aussi faut-il sans doute considèrer cela comme une logette analogue à celle que nous trouverons chez Urnula par exemple, et munie d'un prolongement pédonculaire, plutôt que comme un vrai pédoncule semblable à celui du type morphologique. Mais la distinction est un peu subtile et ni ce caractère ni les autres ne nous semblent autoriser pour ce genre la création d'une famille des Metacinetina Bütschli).

(3) C'est encore, ici comme dans le cas précédent, une vraie logette. Actinocyathus (Kent) n'est qu'un genre douteux voisin du précédent (0,0't. Mer).

(4) Genre voisin :

Discophrya (Lachmann) qui n'est guère qu'un sous-genre du précédent (Mer et eau douce). 
mais ici les tentacules sont, non seulement de longueur inégale, mais de nature différente : les longs sont styliformes, les courts sont capités (0,2. Mer, sur divers Hydraires, Bryozoaires el Crustacés (').

Certaines espèces de Podophrya ( $P$. libera) peuvent se délacher de leur pédoncule et mener une vie libre. Elles établissent par là la transition avec le genre

Sphærophrya (Claparède et Lachmann) (fig. 85̋6, 85̋7). Ici, toute trace de Fig. 856.

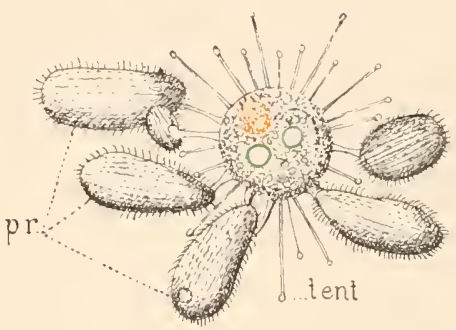
pédoncule ou de logelte disparaît. L'animal devient entièrement libre et son corps, à peu près sphérique, est garni sur toute sa surface de longs tentacules capités (fig. Sö6, tent.). Quand un Cilié ( $p r$.) vient à passer au contact d'un de ses tentacules, ce dernier le saisit, se fixe à lui, le paralyse et suce son endoplasme, puis rejette la dépouille et atlend une autre proie. Mais il n'y a que les grosses espèces qui se comportent Spharophrya

(S. Magna) (d'ap. Maupas). pr., Ciliés capluré par les tentaeules. aiusi (S. magna, S. sol). Les petites procèdent autrement (fig. Sร̃7) ('). Chez S. pusilla par exemple, le jeune bourgeon libre el cilié, capable de nager par conséquent el déjà muni de quel-

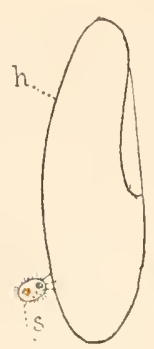

A

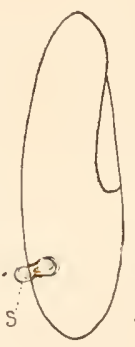

B

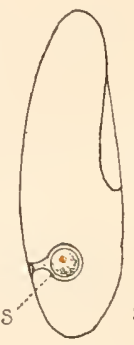

C

Fig. 857 .
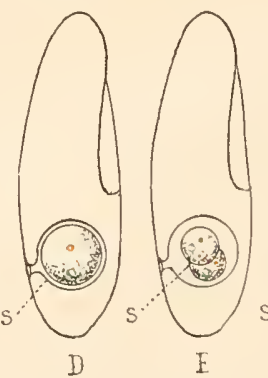

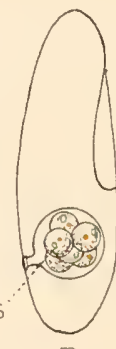

F

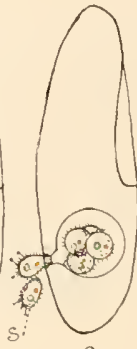

G

Spharophrya. Stades successifs de l'évolution à l'intérieur d'une Paramécie (Sch.).

I., silhouelle de la Paramécie. A el $B$, pénélralion; $C$ et $D$, aecroissement;

$E$ et $F$, bourgeonnement; $G$, sorlie des embryons eiliés du spherophrya (s.)

ques tentacules capités, atteint une Paramécie et se fixe à elle par ses

Ne pas confondre avec le genre homonyme Discophrya (Stein), Cilié voisin des Opalines que nous avons déerit plus haut (V. p. 45\%2).

Ces genres forment la famille des ICTNETIX $[$ Acinetina (Bütsclıli), à l'exception de Metacineta].

(1) En outre, le $\mathbf{N}$ est long et courbé en fer à cheval. Il se ramifie pour les besoins du bourgeonnement qui est externe et multiple. - Le genre

Podocyathus (Kent) possède, en plus, une logette terminale où il abrite son corps $(0,0$ '́. Mer, sur des Bryozoaires et llydraires).

$\left.{ }^{2}\right)$ Ces espèces ne sont pent-ètre que des àges divers d'une mème forme. Mais cela n'a point d'intérèt dans la question. 
tentacules (A). Il perd ses cils, s'enfonce dans le corps de l'hôte $(B)$ et finalement se trouve logé au centre de celui-ci, dans une profonde dépression communiquant avec le dehors par un canal plus étroit $(C)$. Pendant ce temps, il a perdu ses tentacules et, en se nourrissant sans doute par imbibition, a beaucoup grossi. Il a donc l'aspect d'une sphère entièrement lisse $(D)$, munie d'un $\mathbf{N}$, d'un $\mathbf{n}$ et d'une vésicule pulsatile. Il se divise en deux $(E)$, qui se divisent à leur tour et ainsi multiplient et occupent, dans leur chambre agrandie, une bonne partie de la cavité de la Paramécie $\left(F^{\top}\right)$. Peu à peu, cette division se transforme en bourgeonnement externe. Les bourgeons naissent munis de cils et de quelques tentacules, ressortent par l'orifice d'entrée $(G)$ et ront à la recherche d'un nouvel hòte. C'est alors que Stein les avait pris pour les jeunes de la Paramécie.

A la fin, les derniers individus prennent aussi les caractères de bourgeons et se comportent comme les précédents. Ces formes parasites sont très petites. Elles atteignent les Paramécies, les Stylonichies et les Stentors (0,08. Eau douce).

Endosphæra (Engelmann) (fig. S3̈S), ne diffère du précédent que par son mode de bourgeonnement qui est interne et par ses bourgeons ciliés, mais dépourvus de tentacules (Endoparasite chez diverses Vorticellines et chez des Tentaculifères). - Ici se place aussi le genre

Amæbophrya (Köppen), parasite interue soit des Acantlomètres (A.acanthometrx), soit de Sticholonche (A. Sticholonchx). Dans l'un comme dans l'autre de ses hôtes, il a été méconnu et pris comme un organe de ceux-ci. Dans les Acanthomètres, on l'a décrit comme un noyau de forme spéciale arec une portion invaginée (fig. 310) qui n'est autre chose en réalité qu'un bourgeon interne analogue à celui du Tokophrya, et dans le Siicholonche on l'a pris pour un organe spécial, le corps spiral (V. p. 206 et $23 \mathrm{l}$ ) (").

Fig. 858.

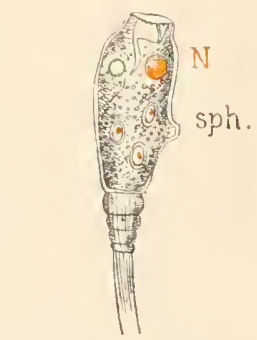

Endosphara (spli.) dans un Epistylis (d'ap. Claparède et Lachmann).

Avec les trois formes suivantes, nous tombons aux dermiers degrés de dégradation des appareils qui caractérisaient les formes élevées dont nous sommes partis.

Urnula (Claparède et Lachmann) (fig. \$59) qui, vivant fixé sur le pédoncule des Épistylis, a été pris à une époque pour le jeune de ce Cilié, est un petit être de forme irrégulièrement ovoïde, fixé par un très court pédoncule dans une logette chitineuse fixée elle-mème par un court prolongement pédonculaire sur le pédoncule de l'hòte. On trouve un $\mathrm{N}$ et deux résicules pulsatiles avec leur's caractères habituels, mais l'appareil tentaculaire est réduit a deux ou trois (prarfois un seul, rare-

Fig. 8.59 .

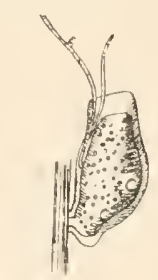

Crnula

(It. Epistylidis) (d'ap. Claparède et Lachmiann).

(1) Ces formes constituent la famille des PODOPHRYIrL [Podophryina (Bütschli)]. 
ment jusqu'à cinq) lentacules d'aspect si singulier qu'on les a pris un moment pour des pseudopodes de Rhizopodes.

Ils sont, en effet, hérissés de petites saillies irrégulièresqui semblent se mouvoir à leur surface comme des granulations protoplasmiques et montrent parfois comme un commencement de ramification. Mais une observation attentive a montré que cet aspect est dù aux plissements que provoquent les contractions, car tout cela s'efface quand le tentacule est lout à fait étendu $(0,08$. Eau douce). - Chez

Rhyncheta (Zenker) (fig. 860), il n'y a plus qu'un seul tentacule styliforme, mais bien formé et très actif, qui prolonge le corps à la manière d'une trompe $(0,09$. Eau douce; fixé, la trompe en Fig. 860 . arrière, entre les pattes d'un Cyclops)('). - Enfin dans le genre

Hypocoma (Gruber) (fig. 861), il n'y a aussi qu'un tentacule, mais capité et qui semble se prolonger en dedans dans le corps. Ce tentacule (tent.) nait de la partie supérieure de la face ventrale, et tout le reste de cette face est garni de cils, sauf une étroite bordure qui en est dépourvue. On pourrait voir là une transition entre les Tentaculifères et

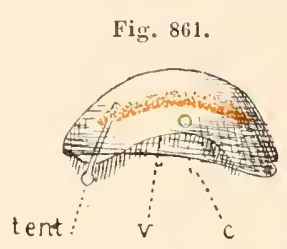

Hypocoma (II. parasitica) (d'ap. Plate). les Ciliés, mais c'est plus probablement la persistance d'une condition larvaire $\langle 0,046$. Mer, sur le pédoncule de diverses Vorticellines) $\left({ }^{2}\right)$.

Nous plaçons ici avec doute deux formes de Tentaculifères qui, bien plus que la précédente, feraient le passage aux Ciliés si leurs particularités d'organisation étaient mieux connues et plus solidement interprétées. La première est le genre

Suctorella (Frenzel) (fig. 862) qui a l'apparence d'un Toloplriya el possède, en plus, une petite ouverture fissiforme garnie de cils très fins. Si l'animal avait contenu un bourgeon interne, l'interprétation de cet orifice cilié eût été simple, et sa présence n’eût rien eu de lien remarquable, mais il n'y avait rien de tel, en sorte que l'on se demande si ce ne serait pas le reste, physiologiquement inactif (car les cils sont bien trop fins pour jouer une fonction alimentaire), d'une bouche $(0,04$. Eau douce). - Le second est le

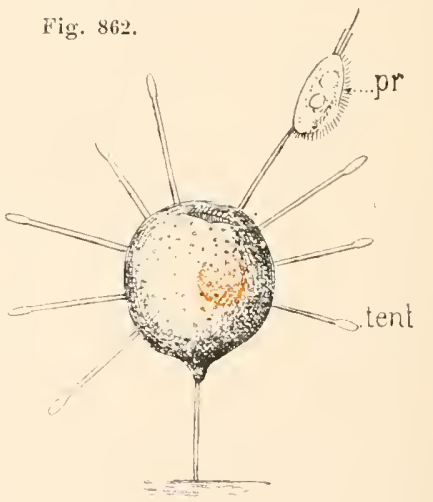

Suctorella (d'ap. Frenzel).

(1) Ces deux genres constituent la famille des Urvolise [Uruulina (Bütschli)].

(2) L'animal est de forme ovalaire, mais avec la face ventrale excavée. Il con- 
Peitiada (Frenzel) (fig. S63), il a une forme ovoïde, mais se prolonge en haut en deux cols qui, l'un et l'autre, se terminent par un tentacule capité. A la base de chaque col, est une sorte de soie raide et tout le corps est revètu de cils fins $\{0,075$. Dans une petite lagune) (").

Revenons maintenant aux Acinètes, aux Tolophrya, à ces formes typiques qui nous ont servi de point de départ.

Nous allons suivre une nourelle série de modifications qui va nous conduire aux formes les plus étranges qu'il soit possible d'imaginer. Ces modifications ont pour origine principale la formation de lobes sur lesquels les tentacules viennent se grouper.

Trichophrya (Claparède et Lachmann) (fig. S6 t) ne diffère

Fig. 863 ,

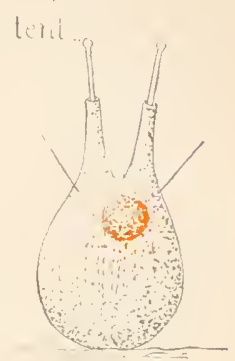

Peitiada

(d'ap. Frenzel). presque de Tokophrya que par l'absence de pédoncule. Que l'on suppose un T'oliophrya libre, sans pédoncule, arec les tentacules groupés, comme ils le sont dans quelques espèces de ce genre, en bouquets sur des parties du corps légèrement saillantes et l'on aura un Trichophrya $(0,24$. Ner el eau douce $)\left({ }^{2}\right)$.

Fig. 865 .

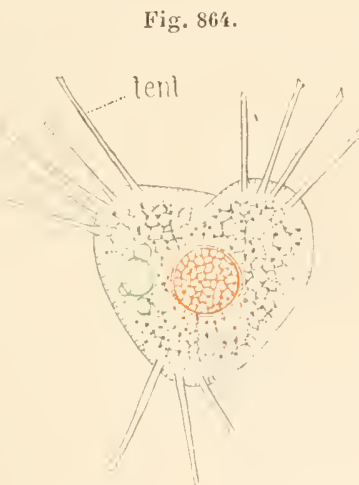

Trichophrya (T. cordiformis) (d'ap. Cheviakof).

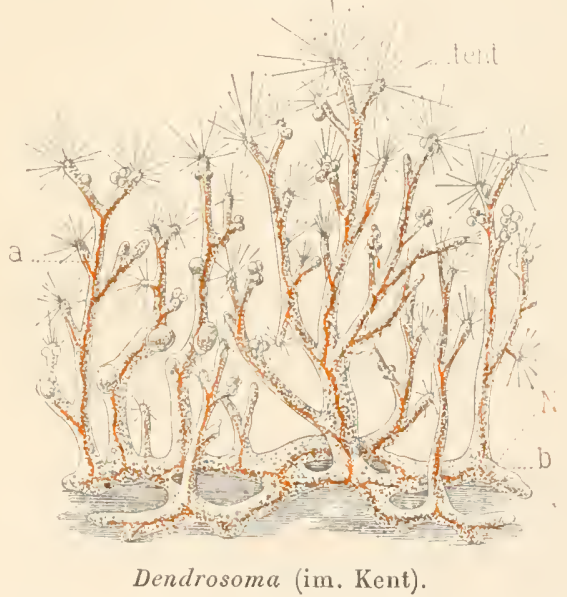

Dendrosoma (Ehrenberg) (fig. 863̈) est d'une taille colossale pour un Ten-

tient un long $\mathbf{N}$, concave aussi en bas et une vésicule pulsatile située dans la concavité du $\mathrm{N}$. Ce genre constitue la famille des Нуросомпж [Hypocomina (Bütschli)].

(1) Frenzel a trouvé ces deux formes dans la République argentine. La première vivail en compagnie d'autres Acinètes. Il a vu les tentacules fonctionner. C'est done sùrement un Tentaculifère. Il a trouvé le $\mathbf{N}$, deux vésicules pulsatiles, mais pas de $\mathbf{n}$. Un individu ne montrait aucune trace de cette bouche. La seconde est moins sùrement un Tentaculifère, car l'auteur n'a pas vu les tentacules en action.

(2) Cette forme vit sur divers animaux marins et d'eau douce (pédoncule d'Epis- 
taculifère, puisqu'il alteint $2 \mathrm{~mm} / / 2$. On croirait, au premier abord, avoir devant les yeux une petite colonie d'Ilydraires. Ce sont en effet comme des stolons ramifiés dans un plan horizontal, sur lesquels auraient poussé des individus dressés côte à côte, ramifiés et portant au sommet de chacune de leurs ramifications un petil bouquet de tentacules terminés en boule au sommet. Eh bien, malgré cet aspect, ce n'est rien qu'un Tentaculifère à tentacules capités, et encore n’est-il pas colonial, car une olservation attentive montre que tout le système est parcouru par un énorme $\mathrm{N}$ continu, mais ramifié et envoyant une branche dans chacun des rameaux dressés (2,5. Eau douce) ( $\left.{ }^{1}\right)$.

Dans ces deux genres les tentacules étaientnettementcapités. Dansle genre Dendrocometes (Stein) (fig. S66), ils sont styliformes, très courts et invaginables au bout. Le corps, en forme d'hémisphère allongé, est fixé par sa face Fig. 866 .

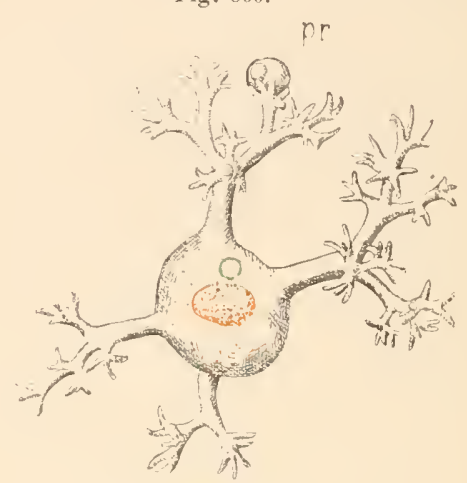

Dendrocumetes (D. paradoxus). pr., proie (d'ap. Vrzesniovski). plane et émet par sa surface convexe trois à quatre (parfois deux, d'autres

$t$-lis, cavité branchiale de divers Tuniciers, surface du corps de certains Entomostracés et même sur les branchies de quelques Poissons. L'une d'elles $\boldsymbol{T}$. cordiformis qui vit entre les fourches caudales de cyclops phaleratus a élé prise pour une valve operculaire, sorte de telson, ılestiné à protéger l'anus. Le bourgeonnement est endogène el tout semblable à celui de Tokophrya.

Nous placerons ici, l'auteur n'ayant pas discuté ses affinités, le curieux genre

Staurophrya (Zacharias) (fig. 867), forme libre, comparable à une sphère munie de six protubérances, deux aux pôles supérieur et inférieur, déterminant le grand axe el quatre dans le plan équatorial. Ces protubérances sont grosses, très obtuses et portent chacune quinze à vingt tentacules non capitís, très rétractiles bien que lents it se mouvoir. Sur ces tentacules, on voit parfois se former des vésicules comme s'ils avaient une membrane. l'ar une exception rare, l'animal est capable d'absorber de petils fragments solides que l'on refrouve dans son endoplasme $\{0,05$. Eau douce).

(1) Il y a de nombreuses vésicules pulsatiles éparses dans tout le corps. Aux extrémités de certains rameaux, se voient des bourgeons exlernes el, plus bas sur la con-
Fig. $86 \%$.

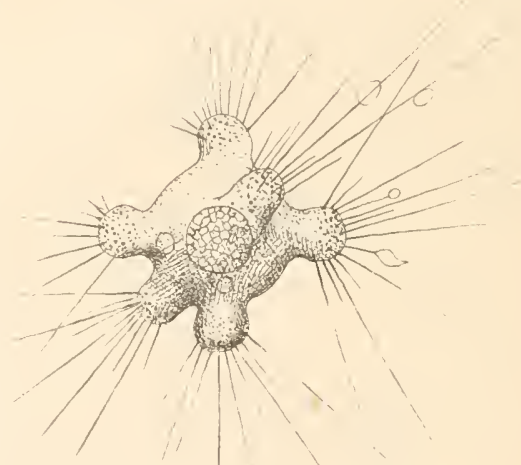

Staurophrya (d'ap. Zacharias). 
fois cinq ou six) gros prolongements qui se ramifient deux ou trois fois, et chaque fois en trois ljranches, mais irrégulièrement. Chaque branche se termine par un petit bouquet de trois à quatre tentacules très courts, en forme de cône fortement tronqué, dans lesquels le canal et ses parois sont très évidents et qui sont très rétractiles et peut-ètre invaginables à leur extrémité.

Les canaux des différents tentacules se continuent indépendamment les uns des autres jusque dans l'intérieur du corps, ce qui permet d'interpréter les bras tentaculifères comme formés simplement par les tentacules soudés jusqu'auprès de leur extrémité. La lobation du corps est donc plus apparente que réelle (0,1. Eau douce, sur les branchies des Gammarus) ('). - Chez

Stylocometes (Stein), les tentacules, semblables en eux-mèmes à ceux du précédent, resteut indépendants jusqu'au bout, en sorte qu’il n'y a plus du lout de lobation, ni réelle, ni apparente $(0,1$. Eau douce, sur les branchies d'Asellus et sur les bouquets d'Ophrydium) ( $\left.{ }^{\mathbf{2}}\right)$.

Il ne nous reste plus à présenter qu'un genre, mais le plus singulier de tous, incontestablement. C'est le genre

Ophryodendron (Claparède et Lachmann) (fig. 868 à 870$)$. L'animal (fig. 868), de forme ovoïde allongée, est fixé par l'extrémité inférieure de son corps rétrécie en pédoncule. A la partie supérieure, il porte un à quatre gros et très longs prolongements coniques, dressés, très rétractiles, armés vers l'extrémité de petits tentacules styliformes. L'animal produit par bourgeonnement interne de petites larves munies d'une ceinture de cils (fig. 869), qui se fixent et se transforment à la manière ordinaire en individus semblables au parent.

Mais il naît aussi, à la base lu (ou des) prolongement tentaculifère, des bourgeons externes (fig. $870, A: a)$ qui se détachent

tinuité des rameaux, des sortes de tumeurs déterminées par des bourgeons endogènes.

Ces deux genres constituent la famille des DENDROSOMINA [Dendrosomina (Bütschli)].

(1) L'animal possède un $\mathrm{N}$ auquel est accolé la vésicule pulsatile. Celle-ci est done profondément située dans le corps, aussi est-elle munie d'un long canal excréteur très évident. Le bourgeonnement est endogène

Fig. 869.

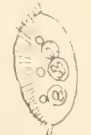

Ophryodendron. (O. abietinum). Larve (d'ap. Claparide et Lachmann).
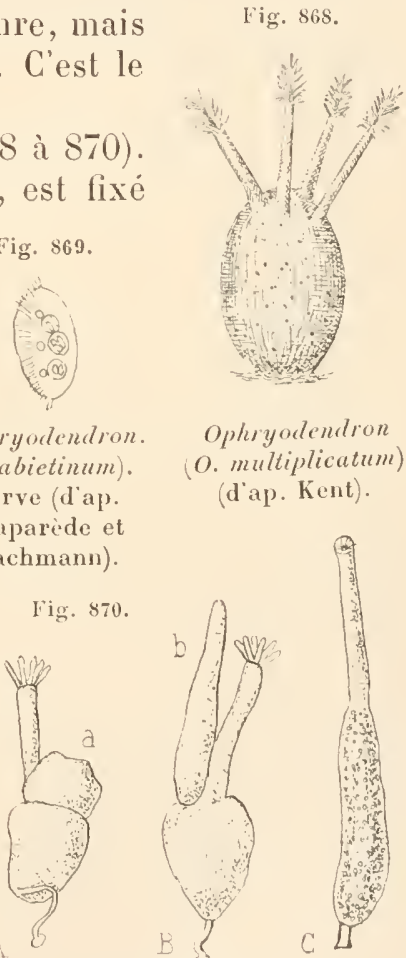

ophryodendron (O. multiplicatum) (d'ap. Kent).

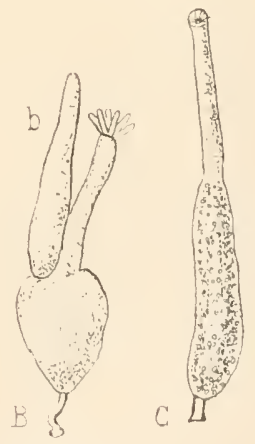

ophryodendron.

Formation de lindividu vermiforme.

$A, B(O$. pedicellatum $) ; \mathbf{a}, \mathbf{b}$, bourgeons (d'ap. Koch); $C$, individu vermiforme d'O. trinacrium (d'ap. Gruber).

${ }^{(2)}$ Ces deux genres forment la famille des DENDROCOMETLY [Dendrocometina (Stein)]. 
$(B: b)$ et se transforment en individus dits vermiformes $(C)$ qui sont lout autrement conformés que le parent. Leur forme est plus allongée et leur corps se termine par un simple prolongement en col de liouteille muni d'un orifice à son extrémité. lis sont fixés auprès des individus de la première forme ou mème sur eux.

On ne sait rien de certain sur les relations biologiques de ces deux formes.

On s'est demandé si la seconde ne représentait pas simplement une phase jeune de certaines larves, ou si elle n’était pas destinée à s'unir à l'autre par conjugaison $(0,13$. Mer) (').

(1) Les espèces, assez nombreuses, ont des formes très diflẻrentes et on a souvent fait des genres avec des différences moindres. Chez une espèce, $O$. abietinum, on trouve des trichocystes. Nais comme ces trichocystes manquent quelquefois ef qu'ils sont tout à fait semblables aux nématocystes de la Campanulaire sur laquelle vit l'animal, on est peut-être fondé à se demander s'ils lui appartiennent bien en propre.

Ce genre constitue à lui seul, et à bon droit, la famille des OPIIRTODEVDRINE [Ophryodendrina (Stein)]. 


\title{
LES PROTOZOAIRES
}

\author{
CONSIDERES DANS LEUR ENSEUBLE
}

Après avoir étulié indépendamment les uns des autres les divers groupes des Protozoaires, nous devons maintenant examiner l'embranchement dans son ensemble pour le délimiler, faire ressortir ses caractères généraux, établir un lien entre ses parties constitutives, montrer la variation des fonclions et des organes, enfin résumer l'ensemble de sa classification. C'est à cela que sont destiués les deux chapitres qui suivent et les tableaux synoptiques qui terminent l'ouvrage.

\section{Garactères distinctifs des animaux et des plantes.}

C'est seulement à propos des Protozoaires que se pose la question indiquée dans ce titre. Chez les ètres plus hautement organisés, le caractère animal ou végétal s'exprime si nettement que la confusion n'est pas possible.

D'ailleurs la question u’est pas si grave qu'elle le paraîl. Au point de vue théorique, posée d'une certaine façon, elle n'existe pas, envisagée de l'autre, elle est insoluble. Si l'on demande, en effet, de répartir les ìtres vivants en deux groupes distincts comprenant l'un les plantes, l'autre les animaux, la question u'a pas de sens, car l'animal et la plante sont des concepts qui n’ont aucune réalité objective; dans la nature, il n’y a que des individus. Si, prenant les ètres que nous considérons comme des animaux ou des végétaux incontestables, nous cherchons à remonter leur lignée phylogénétique, décidés à mettre dans la mème catégorie tout ce qui dépend de la mème lignée, nous sommes sùrs de n’arriver à rien. Les tentatives de ce genre sont toujour's restées infructueuses. En outre, qui nous dit que les animaux et les plantes peuvent 
se ramener à denx séries convergentes seulement? Il est fort probable qu'il n'en est pas ainsi.

Par contre. la question a une utilité pratique indéniable comme toutes nos classifications et, nous plaçant à ce point de vue, nous pourons nous demander quelles formes, parmi celles qui sont indécises, doivent être classées parmi les animaux et à quels caractères on peut les distinguer de celles qu'il vaut mieux ranger parmi les plantes, tout en reconnaissant que ces caractères n'ont rien d'absolu.

Nalhenreusement, tous les caractères que l'on cherche à invoquer romme critérium distinctif se montrent en défaut ici ou là.

$1^{\circ}$ En général, les enveloppes fermes que sécrète la cellule pour se protéger sont formées de cellulose chez les plantes, de chitine chez les animaux, ou de substances analogues à l'une ou à l'autre.

Mais sans parler de la tunicine qui forme la tunique des Ascidies et qui est plus voisine de la cellulose que de la chiline, on a constaté la présence de la cellulose dans les kystes ou dans les capsules de divers Protozoaires qui, par tous leurs autres caractères, se rattachent aux animaux, et celle de la chitine chez les Champignons (Gilson [95]);

20 Même chose arrive pour la chlorophylle.

On sait que les Champignons en sont dépourvus et que, chez divers animaux, on la rencontre, non sous formes d'Algues commensales, mais appartenant en propre à l'organisme où elle se trouve. Ainsi, chez les Bonellies, Rietscin [86] a montré que la substance verte est diffuse dans les cellules, sans ìtre mème sous forme de grains. Son spectre ne diffère de celui de la chlorophylle en rien d'essentiel et tous les auteurs (Gottuer, Schuarda, Schenk), s'accordent à la considérer, sinon comme de la chlorophylle véritable, lu moins comme une substance extrèmement voisine (').

Il existe un Infusoire, Vorticella campanula, qui a des grains de chlorophylle. Enfin, les Euglènes ont de la chlorophylle en grains, avec pyrénoïde et amidon. Bien des auteurs, il est vrai, considèrent les Euglènes comme des plantes, mais cette opinion est inacceptable, car les Euglènes sont inséparables des Péranémines dont elles sont la copie exacte jusque dans les minimes détails. Or les Péranémines avec leur molilité, leur vésicule pulsatile, leur houche et leur pharynx lien dessinés, absorbant des proies solides, etc., etc., sont des animaux au mème degré que les Infusoires.

$3^{\circ}$ Les animaux capturent des proies solides, les végétaux ne se nourrissent que des liquirles qui les baignent (Dangearo).

Ce caractère a plus de valeur que les précédents, mais il souffre des exceptions. Nombre de Flagellés et tous les Sporozoaires se nourrissent,

(1) Rietsch, il est vrai, n'a pu obtenir que des traces d'oxygène, mais il déclare luimême que ses expériences nont pas été faites dans des conditions où elles pussent donner des résultats concluants. 
sous ce rapport, comme les végétaux. Pour les Sporozoaires, leur parasitisme pourrait expliquer la chose : un Ténia n’est pas un végétal ljien qu’il se nourrisse par imbibition. Nais, pour les Flagrellés, cre caractère obligerait à mettre parmi les plantes ceux qui, comme les Astasines par exemple, se nourrissent uniquement de substances dissoutes el dont l'alimentation est caractérisée par les termes expressifs de holophylique ou saprophytique et dont plusieurs cependant ont une bouche tout comme ceux à nutrition animale, mais une bouche sans fonctions.

$4^{\circ}$ A l'étul adulte, les animaux ont des mouvements de locomotion, les végétaux sont immoliles ou n’ont que des mouvements locaux sans déplacement de l'ensemble.

C'est là, à notre avis, le meilleur des critériums. Chez les Algues le plus franchement végétales, l'anthérozoïde est mohile; nombre de végétaux inférieurs ont des zoospores aussi moliles que des Flagellés. Hais cela n’infirme point la valeur du caractère, car il s'agit là d'éléments reproducteurs ou de stades jeunes où la mobilité est rendue nécessaire pour les besoins de la dissémination. A l'état adulte, il n’arrive presque jamais que le végétal, tel qu'on le conçoit d'un commun accord, soit capalıle, nous ne disons pas de certains mouvements locaux, mais de déplacements d'ensemble, de locomotion.

Nous disons presque jamais, car en ces matières, il n'y a rien d'alsolu. Ainsi les Bactéries, les I)iatomées, les Oscillaires surtout, sont susceptibles the vrais déplacements d'ensemble. Mais si l'on analyse le phénomène on roit qu’il y a une réelle différence entre ces mourements dus à des résultats indirects de la contractilití générale du protoplasme qui est commune à tous les ètres virants, et la locomotion par pseudopodes, cils ou flagellums propres aux animaux seuls à l'étal adulte.

C'est done sur ce caractère d'abord, et secondairement sur les autres que nous nous sommes principalement appuyés, dans cel ouvrage, pour trancher la question dans les cas difficiles. C'est par lui que nous arons laissé : parmi les animaux, les Myxomycètes, les Lahırinthulés, les Vampyrelles, les Euglenes, les Astasines, les Dinoflagellés, les Volvocimes, etc., etc., considérés par divers au teurs comme des plantes; et parmi les végétaux, les Chytridinées qui ont une phase amibe, mobile, et un cycle évolutif, parfois très comparable à celui des Protozoaires, les Ilydrodyctiées, etc.

Mais pas plus que les autres, ce caractère n'a de valeur absolue, et dans divers cas nous arons dù nous laisser guider par le sentiment des affinilés pour allribuer certaines formes, soil aux plantes, soit aux animaux, en dépit de ce critérium. C'est ainsi que les Coccidies, malgré leur immobilité à l'état adulte, ont été laissées dans le Règne animal, à cause des Grégarines, qui sont incontestablement des animaux; que nous avons laissé les Oscillaires avec les Algues dont il est impossible de les séparer. Par contre, nous n'avons pas craint de joindre aux 
animaux certaines formes ordinairement considérées comme végétales, et de démembrer ainsi le groupe botanique auquel elles appartenaient, lorsqu'il s'est trouvé quelque groupe zoologique auquel on puisse le joindre. C'est ainsi qu'en attribuant le Plasmodiophora, par exemple, aux Protéomyxés, nous ne nous sommes pas cru ohligés de prendre avec lui les autres Chytridinées. Chez le premier, en effet, la spore en germant engendre une amibe munie de courts prolongements comparables à des pseudopodes, tandis que chez les vraies Chytridinées les plus voisines, ces prolongements sont très longs, filiformes, fixes, et représentent un mycelium ( ${ }^{2}$ ).

En somme, dans l'appréciation de la nature animale ou végétale d'une forme inférieure à affinilés discutables, nous nous sommes laissé guider par un ensemble de caraclères et de considérations dont aucun n’a et ne peut avoir de valeur absolue, mais qui, dans leur ensemble, permettent, la plupart du temps de se décider sans trop de difficulté.

(1) Il existe deux autres caractères distinctifs, qui ne peuvent servir de critérium dans les cas difficiles, vu qu'ils ne se rencontrent que chez les êtres à cellules nombreuses organisées ell tissu, chez lesquels la nature animale ou végétale est toujours netlement exprimée.

$1^{\circ}$ Dès que la cellule se divise prour donner naissance à des tissus, un caractère distinctif remarquable apparaît dans le mode de cloisonnement qui a tendance à se faire, chez la plante, dans une direction prédominante de manière à former des filaments, et s il se produit plus tard des lames ou des formes massives, e'est par association de files cellulaires plus ou moins parallèles. La file longitudinale se reconnaît presque toujours dans les organes massifs des végétaux. Chez l'animal, au contraire, le cloisonnement se fait dans les trois directions et il en résulte une forme massive d'emblée.

20 Les invaginations, reploiements de feuillels si communs chez les animaux ne se rencontrent pas chez les végétaux. Chez ceux-ci l'accroissement est toujours centrifuge et les rapports le conliguité entre les cellules voisines sont toujours primitifs; chez ceux-là au contraire il arrive très fréquemment que des lames cellulaires s'invaginent et viennent établir des rapports de contiguïté secondaires entre des cellules nées à bonne distance les unes des aulres.

C'est Nägeli qui a le premier mis en lumière la première de ces deux différences; quant à la seconde, elle est inédite et provient d'une remarque d'un autre botaniste, le docteur Poirault.

Rappelons enfin que les plantes se nourrissent de substances ternaires et fabriquent avec elles seules les composès quaternaires de leur organisme, tandis rue les animaux ont besoin pour vivre des substances quaternaires élaborées par les plantes. Mais ici encore, la différence n'existe que chez les formes supérieures. Les champignons se nourrissent, sous ce rapport, à la manière des animaux et nous avons déjà indiqué que certains Flagellés ont une alimentation saprophytique ou même holopletique. 


\section{Garactères généraux des Protozoaires.}

Le Protozoaire est essentiellement un être unicellulaire. Lorsque plusieurs cellules s'unissent pour former l'individu, ces celiules sont toutes homologues et homodynames et cette individualité polycellulaire n'est guère qu'une colonie d'intlividualités unicellulaires loutes complètes en elles-mèmes et capables de vie indépendante. Dans certaines formes cependant, che\% les Volvocines, par exemple, on observe un commencement de différenciation des individus de la colonie en des sens différents mais, en tous cas, ces différenciations portent sur des cellules isolées ou des groupes cellulaires massifs et non sur les assises disposées en membranes concentriques et comparables aux feuillets des Mélazoaires (').

La cellule unique qui forme le corps de tous les Protozoaires possède les parties essentielles de toute cellule, le cytoplasma el un noyau $\left({ }^{\boldsymbol{2}}\right)$. Le cytoplasma a la structure normale du protoplasma et montre plus ou moins nettement les divers aspects que l'on a décrits dans cette substance. Ce sont d'ordinaire les structures alvéolaires et granulaires qui se dessinent. La couche superficielle est, le plus souvent, différenciée en une mince lame limitante que l'on appelle ectoplasme par opposition à la partie centrale qui devient l'endoplasme. D'ordinaire, la structure est la mème dans ces deux couches, mais dans l'ectoplasma tous les éléments sont plus fins. A la surface de l'ectoplasme, se dessine toujours au moins une memlırane cellulaire protoplasmique, souvent différenciée en une pellicule plus ferme qui, arec l'ectoplasme, forme une sorte de tégument. Le noyau laisse reconnaître d'ordinaire une memlnane el un suc nucléaire, renfermant des grains chromatiques appelés souvent nucléoles, bien qu'ils correspondent plutòt sans doute à des chromosomes. Assez souvent, on peut distinguer un réseau plus ou moins net, mais ce n'est que toul à fait exceptionellement que l'on a reconnu l'existence de véritables centrosomes.

Mème lor'squ'elle est réluite à ces parties essentielles la cellule unique qui forme le corps n'en sait pas moins accomplir toutes les fonctions nécessaires à la vie; mais, le plus souvent, elle se différencie plus ou moins, et crée à son intérieur de véritables organes, en tout comparables à ceux des Métazoaires, mais qui en diffèrent en ce qu’ils sont des parties

(1) Ici comme partout, cependant, il y a des formes de transition qui font échouer toutes nos tentatives de délimitation nette. L'un de nous, Delage [96], a montré dans la Salinella une de ces formes de transition.

${ }^{(2)}$ Pour la question des Monères ou Protozoaires sans noyau, voyez p. 65. 
de cellule appropriées à une fonction et non des groupes de cellules associées en un appareil.

Le.protoplasma simple, non différencié, contient le germe des fonctions les plus compliquées. Aucune de ces dernières n’est une création absolument nouvelle prenant naissance à quelque moment dans la série des ètres : toutes commencent dès la cellule, toutes y sont représentées sous une forme plus ou moins rudimentaire. Les plus simples des Mlonères, les êtres que nous avons placés à la base des Protéomyxés, sont absolument dépourvus d'organes. Ils se meuvent néanmoins, sans appendice, se nourrissent sans bouche ni tube digestif, respirent, excrètent, se reproduisent, réagissent aux excitations, par la totalité de leur protoplasma.

Mlais dès que l'on s'élève un peu dans la série des Protozoaires, on voit se former pour chaque fonction des organes spéciaux qui, chez quelques-uns, arrivent même à un degré remarquable de complication. Nous allons rapidement passer en revue ces organes en nous plaçant au point de vué de la fonction qu'ils ont à remplir.

Mouvements. Locomotion. - Seuls, certains parasites, comme les Coccidies par exemple, se montrent entièrement immobiles pendant leur état adulte.

Chez tous les Sponozouries, le corps est entièrement dépourvu d'appendices moljiles, mais certains d'entre eux n'en sont pas moins mobiles, soit par de vagues contractions d'ensemble de leur protoplasma, soit au moyen de vraies fibrilles contractiles, les myonèmes, formées d'une différenciation de la couche profonde de leur ectoplasma.

Chez les Rurzopones, le corps forme des prolongements mobiles de sa couche superficielle, les pseudopodes, qui sont, ou obtus et non anastomosables (Амовиекs), ou effilés et anastomosés en réseau, réticulés (Foraminifères, Radiolares), parfois munis d'un filament axile central de nature protoplasmique qui leur sert de soutien (Héuozoarres).

Chez les Fagrclés, les pseudopodes font place au flagellum, appendice contractile le forme et de position fixes, simple ou multiple, mais jamais très nombreux (souvent deux ou trois, mais jamais plus de six à huit), qui entrainent le corps à leur suite, gràce au mouvement ondnlatoire dont ils sont doués.

Enfin chez les Ciutis, le corps est en partie ou en totalité recouvert d'appendices analogues aux flagellums, mais plus petits, plus nombreux, el qui sont animés d'une vibration monotone rarement interrompue. Ils agissent comme te petites rames ('). Ces cils, chez les Infsomes nypo-

(1) Les Tentaculifères sont dépourvus de cils, mais par une régression secondaire, car leurs larves en sont pourvues. Ils ont en place rle cela de longs appendices digitiformes, creux, faisant fonction de sucoirs et incapables de produire une vraie locomotion. Aussi la plupart sont-ils fixés. 
Tricindes se soudent en pinceaux et forment les cirres qui se meuvent à volontí comme les pattes d'un animal supérieur.

Une locomotion sans appendices, par des mouvements du corps ou des contractions non locomotrices se rencontrent quelquefois, par exemple chez les Grégarinides parmi les Sporozoarres el chez les Hétérotrichides ou les Péritricindes parmi les Ciliés.

Alimentation. - Il faut distinguer, dans l'accomplissement de cette fonction, trois stades : la capture et l'ingestion des aliments, leur digestion et le rejet des résidus ou défécation.

Nous arons déjà vu que les Sponozonres se nourrissent par imbibition. Ils n'ont donc pour aucune de ces fonctions hesoin d'appareil spécial. Tout se borne chez eux à des phénomènes d'osmose. Il en est de même chez les Fuagelcés à alimentation holophytique ou saprophytique. Mais dans tous les autres groupes il $\mathrm{y}$ a des appareils plus ou moins différenciés.

La capture et l'ingestion des aliments ont lieu, chez les Ruzopopes, le plus souvent par les pseuriopodes, parfois directement par des points de la surface du corps, les pseudopodes étant réservés à la locomotion. Dans ce dernier cas ou lorsqu'il s’agit de pseudopodes loliés, la surface du corps se soulève tout autour de la particule à saisir el se refermo peu à peu au-dessus d'elle ("). La particule esl englolvée avec une gouttelette d'eau qui constitue autour d'elle une vacuole alimenlaire. Quand il s'agit de pseudopodes réticulés, ceux-ci étendent leur réseau autour de la particule qui se trouve enfermée dans une maille et est digérée sur place ou entraînée dans le corps. En aucun cas il n’y a de vraie bouche, tout au plus ohserve-t-on un lieu d'élection pour l'ingestion des aliments et encore est-il très vaguement limité.

Chez les Fugeldés et les Ciliés, c'est le mouvement tourbillonnaire du (ou des) flagellum ou des cils préribuccaux, souvent soudésen groupes aplatis, les membranelles, qui entraine les particules vers le lieu d'ingestion. Cependant quelques Cilués, les Ilozotrichides no zostomides saisissent leur proie avec leur bouche et l'avalent par un vrai mourement de déglution. Les Textaculafères collent sur la proie leurs suçoirs et ahsorbent peu à peu son contenu. Les F́tagelés ont, soit une vraie bouche suivie d'un petit pharynx, soit tout au moins, à la hase du flagellum, un lieu d'élection nettement limité pour l’ingestion des aliments. Les CuLı́s ont une vraie bouche suivie le plus souvent d'un pharynx. Les Tentaculirères n'ont d'autre lieu d'ingestion que les orifices terminaux de leurs suçoirs.

La digestion se fait partout directement dans l'endoplasme, sans intermédiaire d'un tuhe digestif (sauf chez les Ophryoscolécines). Partout où la nourriture est solide, elle est contenue dans une vacuole alimen-

(1) D'autres disent qu'elle se déprime en face de la particule, ce qui, pour le résultat, revient au:même. 
taire. Le liquide de cette vacuole qui est d'abord de l'eau, se transforme, par échanges osmotiques avec les sucs du cyloplasme, en un suc spécial qui digère la particule et dissout ses portions assimilables qui sont absorbées par osmose.

La défécation a lieu par expulsion de la vacuole ci-devant alimentaire, maintenant fécale. Celte expulsion a lien, soit en un point quelconque ou très vaguement déterminé (Ruzopodes), soit en un poinl défini, ordinairement l'extrémité aborale, sans anus préformé (la plupart des Flagellés), soit par un anus préformé quoique non visible et ne s'ouvrant qu'au moment où il fonctionne (Cisıés).

Excrétion. - Elle a lieu souvent par simple osmose. C'est le cas chez tous les Sporozonires, chez bon nombre de Ruzopodes et de Fuagellés inféricurs et, chose curieuse, chez le plus grand nombre des formes marines mème dans le cas où les formes voisines d'eau douce ont un appareil excréteur spécial. C'est pour cela sans doute que les Radolinies, tous marins, en sont dépourvus. tandis que les Hébızosıres, qui leur sont trìs inférieurs, mais qui sont ordinairement d'eau douce, ont un apparcil bien caractérisé.

Cet appareil, partout où il existe, consiste en une vésicule pulsatile située près de la surface. Chez les lvfusolres et beaucoup de Flageldés il existe un pore ot un petit canal excréteurs permanents, mais en tout cas il n'y a pas de communicalion permanente entre la vésicule el ce canal. Celle-ci s’établit à chaque systole de la vésicule par rupture du cytoplasma qui en forme la paroi.

Chez tous les Protozoaires l'eau entre en masse daus le corps soit par imbibition générale, soit avec les aliments. Elle se rassemble dans la vésicule, soit par des interstices non préformés (Rmzopodes, litigelLÉs), soit par un réseau de canalicules sous-tégumentaires (CiLiés) et est expulsée par la systole.

Les produits excrémentitiels sont tantôt invisibles, sans doute parce qu’ils sont liquides ou trop finement précipités, tantôt précipités sous la forme de grains d'excrétion visibles. Ceux-ci sont sans doule lentement dissous par le courant d'eau qui traverse l'organisme sous l'action de la vésicule.

Respiration. - Nulle part il n'y a d'organes spéciaux pour la respiration; mais il est bien à croire que le courant d'eau déterminé par la vésicule fournit au corps de l'oxygène en même temps qu'il entraine ses excreta. L'eau qui entre dans le corps par osmose esl, en effet, chargée d'oxygine.

Protection du corps. - Le corps est parfois nu ou ì peine revèlu de la plus mince membrane cellulaire. C'est le cas des Ruzopodes inférieurs, de divers Hélozonines, de quelques Radolanes et de la plupart des Flageldés. Ailleurs il y a un tégument un peu plus ferme formé par une pellicule membraneuse unie ì la couche ectoplasmique du corps, c'est le cas des CiLiés. 
Chez les Sponozoures on troure d'ordinaire une vraie membrane ferme arec une cuticule relativement solide.

A cette protection directe et immédiate s'en joint sourent une autre due à des capsules ou à des coquilles formées autour du corps, tout contre lui ou à quelque distance de lui. Telles sont les coquilles des Fontmiviferes, les capsules des divers Fldgellés, les carapaces de certains Ixfrsornes. Au mème ordre de production se rattachent les pédoncules, non celui de la Vorticelle qui est un prolongement du corps, mais ceux des Acinètrs, des Flagellés coloniaux, etc.

Le squelette des Radiolares mérite une mention à part par le fait qu'il n'entoure pas seulement le corps, mais souvent le pénètre et parfois jusqu'à son centre.

Colonies. - La formation de colonies a lieu de deux façons essentiellement différentes, c'est prarfois une réunion secondaire et souvent temporaire d'individus primitivement distincts. C'est le cas des Grégarines, de certains IIÉLozourres, des Myxomycètes, etc. Cela constitue à parler plus proprement des associations. Plus souvent, la colonie est due à un phénomène de multiplication arec séparation incomplète des individus filles, e'est le cas pour les Flagelés qui forment ces élégants arbuscules semblable à des Hydraires en miniature.

Enkystement. - C'est aussi à la fonction de protection qu'il faut rattacher l'enkystement. Il se présente dans tous les groupes et peut se produire daus direrses circonstances. Tantôt, il est provoqué par une circonstance éventuelle, dessiccation, putréfaction de l'eau, besoin de repos pour digérer à l'aise après une alimentation trop abondante; tantôt, c'est un phénomène évolutif, préambule d'une acte r'eproducteur. Pour s'enkyster, l'animal s'arrondit, rétracte ou élimine ses appendices, flagellum, cils ou pseudopordes, ferme sa bouche et ses autres orifices en établissant à leur niveau la continuité de sa paroi, efface la plupart des différenciations intérieures qu'il pouvait posséder et se sécrète une ou plusieurs enveloppes concentriques de chitine, de cellulose ou de substances analogues.

Le degré de cette régression est très variable selon les groupes; il est moins avancé pour les enkystements éventuels dont l’animal doit sortir sans s'ètre modifié; il est maximum dans ceux qui aboutissent à une sporulation.

Non moins variable est la durée de l'enkystement; elle est relativement fixe dans ceux qui sont le prélude d'un acte reproducteur; mais dans ceux qui sont éventuels, elle peut varier de quelques heures à tout un hiver ou mème plus, selon les circonstances.

Reproduction. - Le Protozoane se reproduit essentiellement par division, cependant chez les Sporozoures, les Radolures el les Fonamirières à coruilles dures ce procédé, sans faire défaul, est moins ordinaire et est remplacé normalement par la sporulation. La division a lieu par amitose pure dans les formes inférieures, par mitose parfaite dans les plus élevées, 
mais, le plus souvent, par un processus intermédiaire tenant plus ou moins de l'une ou de l'autre suivant les cas. Le centrosome a été rarement observé, mais les chromosomes et leur division ne sont pas rares.

Quant aux organes du cytoplasme (bouche, péristome, flagellum, vésicule pulsatile, etc.), tantòt ils sont partagés en deux (surtout dans la division longitudinale), tantôt ils se reforment chez l'individu qui en est privé. Mème chose arrive pour les squelettes et coquilles.

Sporulation. - La sporulation a lieu normalement sous un kyste. Le noyau se divise en un grand nombre de petits noyaux qui se partagent le cytoplasme el forment ainsi autant de petites spores nuclées. Tautôt, ces spores sont nues et munies d'un ou deux flagellums (zoospores), tantòt elles sont munies d'une enveloppe et en sortent à la germination, le plus souvent sous une forme amœbö̈de. Ces zoospores ou ces amibes reproduisent la forme adulte à la suite d'une évolution trop souvent inconnue.

Conjugaison. - Dans des cas chaque jour plus nombreux, mais qui sont loin de s'étendre encore à l'ensemble des Protozoaires, on a observé la conjugaison. Ce phénomène consiste dans la fusion de deux individus en un seul. Quand la fusion est temporaire, elle ne constilue pas une vraie conjugaison et relève plutôt de ce que nous avons appeléassociation. Quand elle est permanente et ne s'étend qu'au cytoplasme, plastogamie, elle ne paraît pas non plus aroir beaucoup d'influence sur le cycle évolutif. La vraie conjugaison doit s'étendre aux noyaux, liaryogamie, elle est alors un acte reproducteur, comparable à la fécondation des llétazoaires, mais avec celte différence qu'il n'y a pas d'ordinaire de distinctions sexuelles entre les deux individus conjugués. Parfois cependant une différence de ce genre existe, par exemple chez les Vorticelles où l'un des individus, microgamète, est plus petit et se porte plus activement à la recherche de l'autre qui est le macrogamète. Parfois, la conjugaison a lieu entre des spores nues dont souvent l'une est plus petite, microspore, et l'autre plus grande, macrospore. ll semble que l'on soit fondé dans ces cas à assimiler le plus petit des conjoints au mâle et le plus gros à la femelle.

Il semble que la conjugaison, qui est le contraire de la reproduction puisqu'elle diminue le nombre des individus, soil nécessaire pour remédier à une dégénérescence de l'espèce qui se produit à la suite d'une multiplication agame trop longtemps continuée. Mais ce phénomène, appelé dégénérescence sénile par Muvpas, n’a été constaté que chez les Ciuiés et c'est par induction seulement que l'on suppose que, chez les autres Protozoaires où elle se rencontre, la conjugaison doit avoir un effet de même ordre.

Rapports avec la nature et avec l'homme. - Les Protozoaires se rencontrent partout. Les Flageldés, les Ixfusorres sont absolument cosmopolites el abondants en lous lieux. Quelques-uns seulement sont plus cantonnés: les Radiolares dans la mer, les Sporozoarires, tous parasites, 
dansles hôtes qui les hébergent. La plupart sont sans intérêt pourl'homme et sans grande importance dans la nature. Mais quelques-uns jouent un rôle remarquable. Les Forunniféres forment comme l'on sait presque entièrement certaines annexes géologiques; divers Sporozonrres attaquent l'homme (Hémosporidie de la malaria) ou les animaux domestiques (Coccidie du Lapin); enfin les Flagellés et les Infosolres rendent leur pureté aux eaux croupies en dévorant les Bactéries qui y pullulent, après que celles-ci ont détruit, en se les assimilant, les matières organiques qui les corrompaient.

\section{III}

\section{Tableaux synoptiques de la classification des Protozoaires.}

Dans ces tableaux nous n'arons pas indiqué la valeur des groupes successifs. Cela nous en̂t empêché de les faire tenir dans une page et eût beaucoup nui à leur clarté. Mais il est facile de reconnaitre les groupes en se rappelant que:

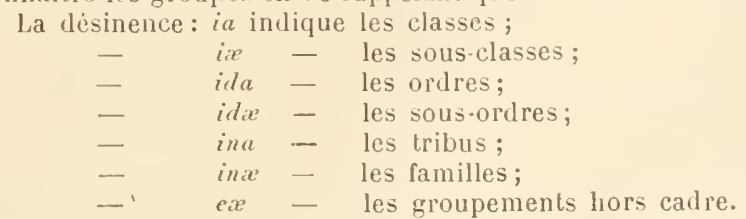

Nous n'avons pas jugé utile de donner les familles qui d'ailleurs dans tout l'ourrage sont reléguées au second plan.

La lernière colonne indique seulement quelques genres donnés comme exemples. 
$1^{\text {re }}$ CLASSE. - RIIZOPODIA

Corps émettant par sa surface des prolongements protoplasmiques mobiles appelés pseudopodes.

(1. ACYSTOSPORID.A se reproduisant par simple division sans kystes ni spores. Y Protogenes. $-$

2. IZOOSPORIDA se reproduisant par des kystes dou sortent des jeunes qui । rumpyrella.

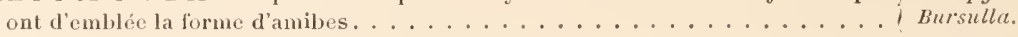

3. ZOOSPORIIDA se reproduisant par des kystes d'ou sortent des zoospores qui f protomonas.

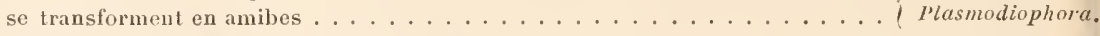

1. PSEUDOPLASMODIDA (Acrasiés). Amibes se groupant sans se souder f Guttulina. (pseudoplasmode $\ldots \ldots \ldots \ldots \ldots \ldots \ldots \ldots \ldots \ldots \ldots \ldots$ A . . . . . . . . . . . . . .

2. FILOPLASMODIDA (Labyrinthulés). Amibes se soudant par leurs pseu- | Labyrinthula. dopodes seulement (plasmode filamenteux) ............. Chiamydomyxu.

3. EUPLASMODIDAA Myxomycètes). Amibes se soudant par leur corps f Fuligo.

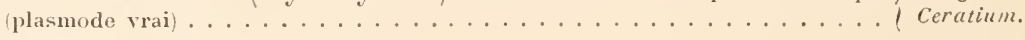

1. IYMNAMOELIDA. Amœbiens nus.....

2. TIIECAMOEBHD A. Amøbiens à carapace ou à coquille.......... Arcella.

2. Diff ugia.

1. GROMID.E. Coquille continue, ehitineuse. $\left\{\begin{array}{l}\text { Gromia. } \\ \text { Amphitrema. }\end{array}\right.$

MILILola.

1. IMPERFORIDA. Coquille non percée de pores et ne communiquant avec le dehors que par une on deux bouches.

2. MILIOLID E. Coquille continue, calcaire.. Irbitolites.

\begin{tabular}{|c|c|c|}
\hline $\begin{array}{l}\text { 3. ARENACID E. } \\
\text { Coquille dis- }\end{array}$ & $\begin{array}{l}\text { 1. ASTRORIHIINA. Co- } \\
\text { quille simple, asymé- } \\
\text { trique, ordinairement } \\
\text { monothalame. }\end{array}$ & $\begin{array}{l}\text { Astrorhiza. } \\
\text { Saccammina. }\end{array}$ \\
\hline $\begin{array}{l}\text { mée de grains } \\
\text { de sable. . . }\end{array}$ & $\begin{array}{l}\text { 2. LITUOLINA. Coquille } \\
\text { régulière, symétri - } \\
\text { que, ordinairement } \\
\text { polythalame. }\end{array}$ & $\begin{array}{l}\text { Lituola. } \\
\text { Loftusia. }\end{array}$ \\
\hline
\end{tabular}

1. LAGENID E. Pores fins, pas d'enroulement f Lagena. vrai................... Nodosaria.

2. CHILOSTOMELLIDKE. Pores fins, enroule- Chilostomella. ment de Miliolide (2 loges par tour). . . . Ellipsoidina.

2. PERFORIDA. Coquille percéc de nombreux et fins pores par où sortent les pseudopodes..............

3. TEXTULARID E.Pores fins, enroulement en Textularia. hélice........................ Bulimina.

4. GLOBIGERINID E. Pores larges, enroule- f Globigerina. ment en spire obscure. . . . . . . . Hastigerina.

5. ROTALIDE. Eores larges, enroulement spi- $\{$ spirillina. ral, régulier, symétrique ...... Rotalia.

6. NUMMULITID E. Pores fins, enroulement f Polystomella. spiral, régulier, symétrique....... Nummulites.

Appendice.

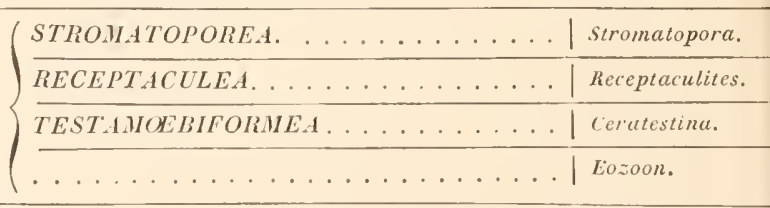

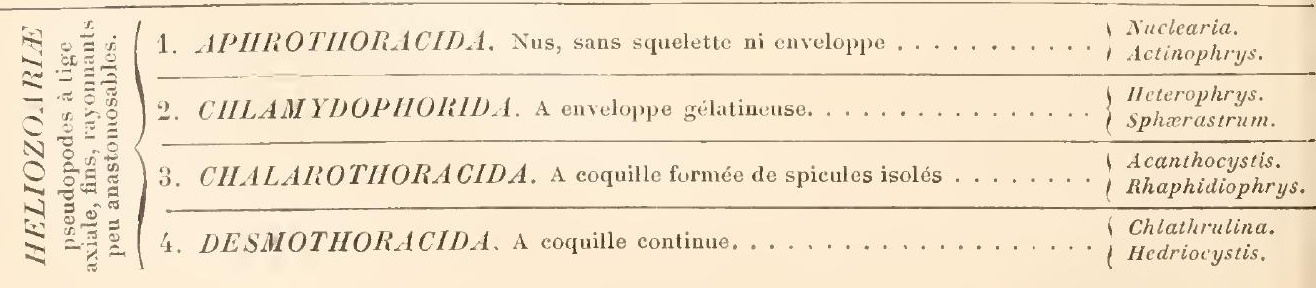




\section{2 e CLASSE. - SPOROZOARIA}

Corps sans appendiees mobiles, revêtu d'une membrane sans orifices; reproduetion par spores d'ou sortent des sporozoïtes.

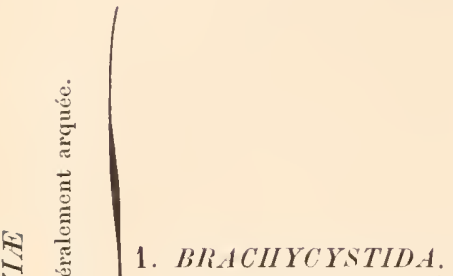

forme de l'adulte dérivant de la sphére.....
1. GREGARINID.E. Une forme libre, allongée, mobile, pourve de fibrilles contractiles; s'enkystant hors du tissu oi elle est née. .....

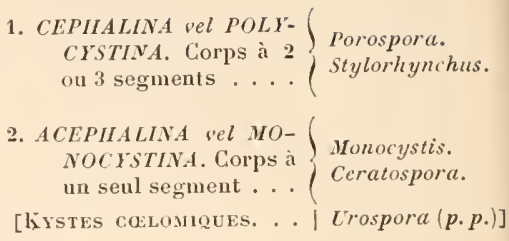

2. COCCIDID.E. Pas de phase libre, pas de fibrilles contractiles; parasite s'enkystant dans le tissu Klossia. où il est né.................. Coccidium.

3. IIEMOSPORIDA. Une phase libre; parasite ( Drepanidium. s'enkystant dans les globules sanguins.... Karyolysus.

4. GYINOSPORID E. Vic exclusivement intracel- I IIemamaba. lulaire, pas d'enveloppe kystique. ....... IIalteridium.

2. DOLICIIOCYSTIDA. Forme de l'adulte déri-
vant d'un ovoüle allongé.

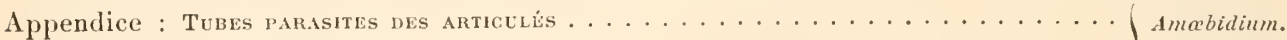

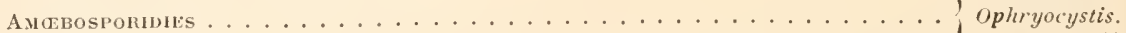

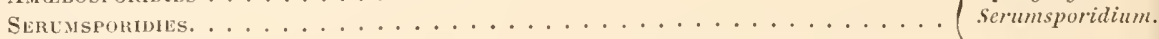

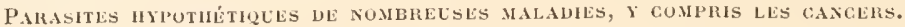


$4^{\mathrm{e}}$ CLASSE. - CILILE

Ayant pour organes locomoteurs des eils vibratiles nombremx on des tentaeule succurs.

1. HOLOTRICIIIDA. Cils à peu pris uniformes; pas de zone adorale..... 2
1 GYMNOSTOMID. E. Bouche sans membrane on- $\left\{\begin{array}{l}\text { Irolophrya. } \\ \text { Prorodon. } \\ \text { dulante, ferméc à l'état de repos. . . . . . } \\ \text { Nassula. }\end{array}\right.$

2. IYYMNOSTOMHDE. Bouche pourvue d'une ( Colpoda. membane ondute, loujours Anoplophyra. action ........................... Opalina.
2. IIETEROTRICIIIDA. Cils du corps uniformes; une zone adorale de membranelles.........

2. OLIGOTRICIID.E, n'ayant de cils sur le eorps qu'à certaines places détermiuées ........ Ophryoscolex. Entodinium. Stentor.

3. HYPOTRICIIID A. Cils remplacés sur le dos par quelques soies tactiles, et sur le ventre par des cirres; une zone adorale.

Livostyla. Stylonichia.

1. POLYTRICHID E, pourvus diun revetement (Plagiotoma. Busaria.

4. PERITRICIIIDA. Sans cils sur le corps, parfois une ecinture circulaire; une zone adorale de membranelles styliformes...

1. SCAIOTRICIIID E. Zone adorale senestre .. (Licnophora. spirostomum.

II TENTACULIE. Pas de cils vibratiles, des tentaeules suceurs. . . . . . . . . . . Podophrya. 2. DEXIOTRICIID E. Zonc adorale dextre.... $\begin{aligned} & \text { Trichodina. } \\ & \text { Vorticella. } \\ & \text { Epistylis. }\end{aligned}$ 


\section{INIEX BIBLIOGRAPIIQUE}

Pour l'orthographe des noms d'auteurs des pays où l'alphabet est diflèrent du nôtre, eomme la Russie, nons arons lraduit les noms directement en françis sans passer. comme on le fait sans raison, par l'intermediaire de l'ortlographe allemande. Ainsi nous écrivons: Cheriakof el non Sehewiakofl, Kovalevsky et non kowalewsky, ete.

\section{LA CELLULE}

Pour la bibliographie complete, voir: Flemming (82), Hertwig (92), Delage (95) et Henneguy (96).

Altmax (R.). - Die Elementarorganismen und ihre Beziehungen zu den Zellen. Leipzig.

Balbiani (E.-G.). - Sur la structure du noyau des rellules salivaires chez les larves

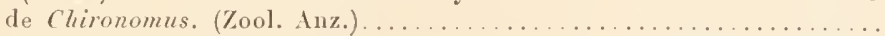

Centrosome et “ Dotterkern ». (Journ. de l'Anat. et de la Phys., val. 29)........ BÉcirAup (A.). - Les microzymas, dans leurs rapports arec l'hétérogénie, la physiologie et la pathologie, etc., xxxvir-992 p., 5 pl. Paris.

Bienedex (Ed. Van). - La maturation de l'uenf, la fécondation et les premières phases du développement embryonnaire des mammifères d'après des reeherches faites chez le Lapin. (Bull. Acad. Roy. Belgique, ee sér., vol. 11).

Recherches sur la maturation de l'œuf, la fécondation et la division cellulaire. Gand, Leipzig et Paris.

Bertiold (G.). - Studien über Protoplasma-Mekinik. I.eipzig, 332 p., 7 pl....... Boveri (Th.). - Zellen-Studien. (Jen. Zeit. f. Nat., vol. 21, p. 423-515, pl. 25-28).... Id. Ibid. Vol. 22, p. 685-852).

Id. (Ibid. Yol. 21, p. 314-101, pl. 11-13

Brauer (A.). - Zur Kenntniss der lieifung des parthenogenetiseh sich entwickelnden Eies von Artemia Salina. (Arch. f. mikr. Anat., vol. 13, p. 162-222, pl. 8-11)...

Bírscili (O.). - Ueber den sogenannten Centrallörper der Zelle und ihre Bedeutung. (Verh. nat. med. Ver. Ileidelberg, vol. 4, p. 335-338)..

Untersuchungen iiber mikroskopische Schäume und das Protoplasma. Versuche und Beobachtungen zur Lösung der Frage nach den physicalischen Bedingungen der Lebeiserseheinung. Leipzig, 234 p., 23 fig., $6 \mathrm{pl} \ldots \ldots \ldots \ldots \ldots \ldots$

Garvor (J.-B.). - La biologie cellulaire.

Cineviáof (W.). - Ueber die karyokinetische Kerntheilung der Euglypha alveolata. (Morph. Jahrb., vol. 13, p. 193-258).

DAxilevsk (A.-J.). - La substance fondamentale du protoplasma et ses modifications par la vie. (Communic. au congr. de médec. de Rome).

Daxtec (F. Le), - Recherches sur la digestion intracellulaire chez les Protozoaires. (Thèse. Paris). .

Delage (Yves) - - La structure du protoplasma et les théories sur l'hérédité et le

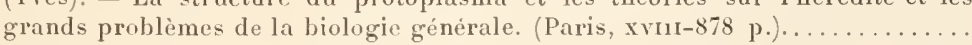

Eismond (J.). - Einige Beiträge zur Kenntniss der Attractionssphären und der Centrosomen. (Anat. Anz., vol, 10)..

Ueber die Verhältnisse des Kerns zum Zelleibe und über die Zelltheilung. (Sitz. d.

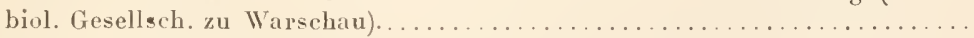

Flemmang (V.). - Zellsubstanz, Kern- und Zelltheilung. Leipzig..............

Ueber Theilung und Kernformen bei Leucocyten und ïber deren Attractionssphären. (Arch. f. milir. A nat., vol. 37, p. 249-298)... . . . . . . . . . . . .

- Neue Beiträge zur Kenntniss der Zelle. (Arch. f. mikr. Anat, rol. 37, p. 685-751.)

Fol (H.). - Le quadrille des centres, un épisode nouveau dans l'histoire de la fécondation. (Arch. sc. phys. et nat. Genève, $3^{e}$ période, XXV, no du 15 aviril).....

Fromaxx (C.). - Zur Lehre von der Struktur der Zellen. (Jen. Zeit. f. Med., vol. 9)...

1894

1881

1893

1883

1875

1883

1886

1887

1888

1890

1893

1892

1892

1884

1887

1894

1891

1895

1894

1890

1882

1891

1891

1891

1875 
Gautier (A.). - La chimie de la cellule vivante. Paris

Gruber (August). - Ueber Kern nnd Kerntheilung bei den Protuzoen. (Zeit. f. wiss. Zool., vol. 40, p. 121-153, pl. 8 et 9)

Guignard (L.). - Nouvelles études sur la fécondation. (Ann. des sc. nat. (liot.), 7e ser., p. 163-296, vol. 15, pl. y-17).

1891

IÏ̈ckeß (V.). - Die Keimbläschen, seine Elemente und Lageveränderungen. I. (Arch. f. mikr'. Anat., vol. 41) ...

- Id., Il (Ibid. vol. 42).

IIallez (P.). - Pourquoi nous ressemblons à nos parents? 32 p. Paris

Hexking (H.). - Künstliche Nachbildung von Kerntheilungsfiguren. (Arch. f. mikr.

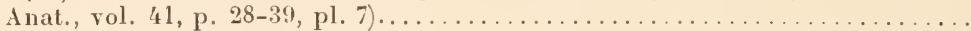

Hennegur (L.-F.). - Nouvelles recherches sur la division cellulaire indirecte. (Journ. de l'Anat. et de la Physiol., vol. 27, p. 392-423). . . . . . . . . . . . . . .

Lecons sur la cellule. Paris........

IEmtzanin (C.). - Untersuchungen iiber Protoplasma. (Wiener Sitzungsb. Math.-nat. Classe Lxvi1)...

Hermann (F.). - Beitrag zur Lehre von der Entstehung der karyokinetischen Spindel. (Arch. f. mikr. Anat, vol. 37, p. 569-586.).

IIERTwig (O.). - Welchen Einfluss übt die Schwerkraft auf die Theilung der Zellen. 30 p., 1 pl. Jena

Die Zelle und Gewebe. Jena.

Beiträge zur Kenntniss der Bildung, Befruchtung und Theilung des thierischen Eies. (Horphol. Jahrb.).............. Vol. 1, 1875; 2, 1877; 3,

Das Problem der Befruchtung und der Isotropie des Eies. Eine Theorie der Vererbung. (Jen. Zeit. f. Naturw., vol. 18).

— Vergleich der Ei- und Samenbildung bei Nematoden. Eine Grundlage für celluläre Zeitfragen. (Arch. f. mikr. Anat., vol. 36, p. 1-138, pl. 1-4.

Kossel. - Untersuchungen iaber die Nucleine und ihre Spaltungsprodukte. Strassburg.

Zur Chemie des Zellkerus. (Hoppe Serler's Zeit. f. physiol. Chemie, vol. 12....

Krasser (Fridolin), - Ueber das angebliche Vorkommen eines Zellkerns in den IIcfezeller. (Esterreichische botanische Zeitung, p. 373-377) . . . .

Künstrer (J.). - De la constitution du protoplasma. (Bull. sc. du Nord et de la Belgique, $2^{\mathrm{e}} \operatorname{sér}^{\mathrm{r}}$, $5^{\mathrm{e}}$ année).

KuppFer (C.). - Ueber Differenzirung des Protoplasmas an den Zellen thierischer Gewebe. (Schr. d. naturw. Vereins f. Schleswig-lIolstein. I) . . . . . . . . . .

Levdig (F.). — Zelle und Gewebe. Gr. in-8, vi-219 p., 6 pl. Bonn $\ldots \ldots \ldots \ldots \ldots$

LiLienfELD (L.). - Wahlverwandtschaft der Zellelemente zu gewissen Farbstoffen. (Verhandl. d. physiol. Gesellsch. in Berlin)

MAGGI (L.). - I plastiduli nei ciliati, plastiduli liberamente viventi. (Atti della soc. ital. di sc. natur. Milano).

Maupas (E.). - Recherches expérimentales sur la multiplication des Infusoires ciliés. (Areh. de Zool. exp. et gen. 2e sér., vol. 6, p. 165-277, pl. 9-12) . . . . . .

Le rajeunissement karyoganique chez les Ciliés. (Ibid.. 2e série, vol. 7. p. 150 à 517$, pl. 9 i 23.$)$

Meves (F.). - Ueber amitotische Kerntheilung in den Spermatogonien des Salamanders und Verhalten der Attractionssphäre bei derselben. (Anat. Anz., rol. 6,

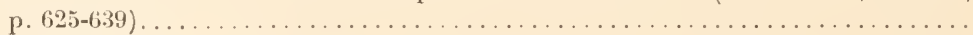

Mitrophanor (P. J.). - Contribution a la division cellulaire indirecte chez les Sćlaciens. (Journ. intern. d'Anat, et de Phys., vol. 11).

— Ueber Zellgranulationen. (Biolog. Centralbl., vol. 9).. .

Prenant (A.). - Contribution à l'étude de la division cellulaire. (Arch. de Phys. norm. et path. 5 e sér. 4)...

Quncke (G.). - Ueber Protoplasmabewegung. (Biulog. Centr., vol. 8, p. 499-506).

RabL (C.). - Ueber Zelltheilung. (Iorphol. Jahrb., vol. 10, p. 214-330).

Ueber Zelltheilung. (Anat. Anz., vol. 4, p. 2l-30).

Ranvier. - Traité d'IIistologie. Paris

Ratr (O. vom). - Ueber die Konstanz der Chromosomenzahl bei Thieren. (Biol. Centralbl., vol. 14).

Reinke und Rodewald (II.). - Studien über das Protoplasma. (Untersuch. aus dem bot. Inst. der Univ, Göttingen).

R̈̈́кеrt (J.). - Die Ghromatinteduktion bei der Reifung der Sexualzellen. (Ergeb. d. Anat. und Entw, ron Merkel und Bonnet. III. Litt. 1893). 
Sabitier (A.). - Contribution à l'ritude des globules polaires et des éléments élimines

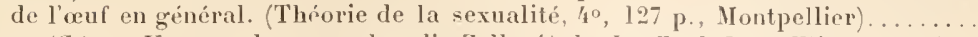

Schneider (C.). - Untersuchungen über die Zelle. (Arb. des Zool. Inst. Wien, vol. 9) ...

1584 1891

Strasburger (E.). - Ueber den Theilungsvorgang der Zellkerne und das Verhältniss der Kerntheilung zur Zelltheilung. (Arch. f. mikr. Anat., vol. 21, p. 476-490). .

1882

Die Kontroversen der indirekten Kerntheilung. (Arch. f. mikr. Anat., vol. 22, p. 246-30't).

Ueber periodisehe Reduktion der Chromosomenzahl im Entwickelungsvorgang

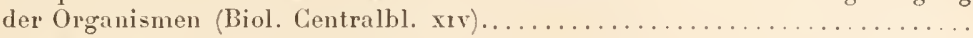

VERworn (Max). - Biologische Protisten-Studien. (Zeit. f. wiss. Zool., vol. 50, 1. 44l-468. pl. 18).

Bewegung der lebendigen Substanz. Eine vergleichend-physiologische Untersuchung der Contractionserscheinungen. (Iena, 1035 p., 10 fig.)

Watasé (S.). - Ilomology of the Centresome. (Joum. of. Morphol., vol 3, p. 433.443).

Weismaxx (A.). - Ueber die Zahl der Richtungskörper und über ibre Bedeutung für die Vererbung. $8^{\circ}$, Jena.

Das Keimplasma. Eine Theorie der Vererbung. So, vol. I8, p. 628, pl. 24, Abb. im Text. Jena. . . . . . . . . . . . . . . . . . . . . . . . . .

Amphimixis oder die Vermisehung der Individuen. so $^{\circ}$ Jena, 176 p., 12 fig. .... Zagianis. - Ueber Eiweiss, Nuclein und Plastin. (Bot. Zeit. p. 281-329)....

Zimermaxn (A.). - Beitrïge zur Morphologie und Physiologie der Pflanzenzelle. Tübingen.....

1884

1894

1890

1892

1893

1887

1892

1891

1883

1890

\section{PROTOZOAIRES EN GÉNERAL}

Pour la bibliographic complète antérieure à 1850, voir : Bïtschli [82].

Blavgiard (R.). - Traité de Zoologie médicale. Paris.

Buochians (F.). - Kleine Mitteilungen iber Protozoen. (Biologisches Centralblatt, vol. 14, P. $82-91,3$ fig.)

1886

1891

Bütschli (O.). - Bronn's Klassen und Ordnungen des Thierreichs. I, Protozoa... 1880-1882

Chaparét. (Edouard) et Lachuaxi (Johannès). - Etudes smr les Infusoires et les Rhizopodes. (Mémoires de l'Institut national genirois, vol. 5 et 6). . . . . 1857-1858

Delage (Ives). - Lat conception polyzoïque des ètres. Revue scientifique, 28 mai et 20 juin.).

Dreyer (Friedrich). - Die Pylombildungen in vergleichend anatomischer nnd entwicklungsgeschichtlicher Beziehung bei Radiolarien und bei Protisten überhaupt. (Jen. Zeit. f. Naturw., vol, 23, p. 77-21/4, pl. 6-11).

Dumanin (F.). - Sur les Organismes inférieurs. (Anmales des Sciences Natur., 2e série, rol. 4, p. 3'i3-38'i).

Recherches sur les organismes inférieurs. (Ann, des Sciences Natur., 2e série, vol. 5 , p. 193 ì 206 )...

_- Histoire naturelle des Zoophytes Infusoires. Paris.

EHrexberg (Chr. G.). - Die Infusionsthierchen als volkommene Organismen. Berlin 11. Leipzig. .

Frenzel (Joh.). - Untersuchungen iber die mikroskopische Fauna Argentiniens. (Arch. f. mikr. Anat., vol. 38, p. 1 à 2't, pl. 1).

Ueber einige merkwürdige Protozoen Argentiniens. (Zeit. f. wiss. Zool, rol. 53, p. $334-360$, pl. 17) ...

Fronentel E. de). - Etudes sur les llicrozoaires ou Infusoires prop. dits. Paris...

1896

1889

1835

1836

1841

1838

1891

1892

1874

Gruber (August). - Untersuchungen iber einige Protozoen. (Zeit. f. wiss. Zool.,

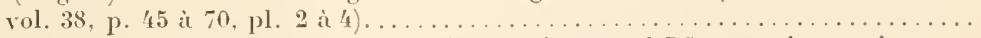

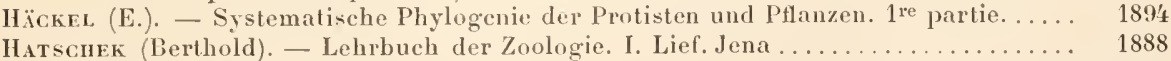

Kfant (Sar.) — Manual of Infusoria. London. . . . . . . . . . . . . . . . . . . . . . $1880-1882$

Lanessan (G. L. de). - Traite de Zoologie. Protozoaires. I. Paris............... 1882

LANG (A.) - Lehrbuch der vergleichenden Anatomie. I. Abth. Jena. . . . . . . . . . . 1888

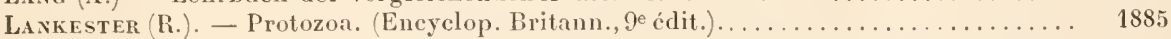

Merechkorsky (C. von). - Studien über Protozoen des nördlichen Russlands. (Areh. f.

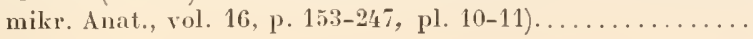


Osborx (II.-L.). - The Protozoa, a Phylum of the animal kingdom considered Biologically. (Microscopical Journal, vol. 13, p. 233 à 243 )..............

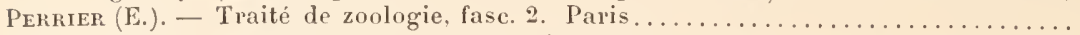

Pfeiffer (L.). - Die Protozoen als Krankheitserreger. 2e édit. Jena, 216 p., 21 fig....

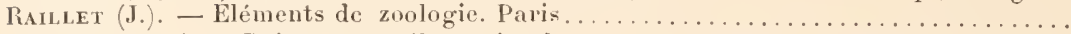

Scureidek (A.). - Beiträge zur Kenntniss der Protozoen. (Zeit. f. wiss. Zool., vol. 30,

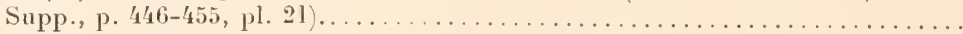

Siebold (Th.). - Ueber einzellige Pflanzen und Thiere. (Zeit. f. wiss. Zool., rol. 1, p. $270-294)$.

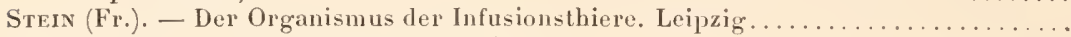

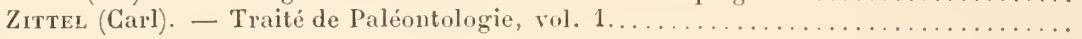

1892

1893

1891

1886

1879

1849

1878

1883

\section{RHIZOPODES}

Pour la bibliographie complete antérieure à 1880, voir : Bütschli [80-82].

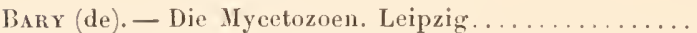

Blaxe (H.).- La Gromia Brunnerii, un nouveau Foraminifère. Recueil zool. suisse. Vol. 4, no 4, p. 497-513, pl. 2'i).

Les Difflugies de la faune profonde du lac Léman. Extrait du Recueil inaugural de l'université de Lausanne, p. 11, pl. 2) .....................

Les Difflugies de la faune profonde du lac Léman. (Extrait du Recueil inaugural de l'Université de Lausanne, p. 1-10, pl. 2, Lausanne ..................

Bradr (II.-B.). - On the reticul. and radiol. Rhizopoda of the Northpols expedit. of

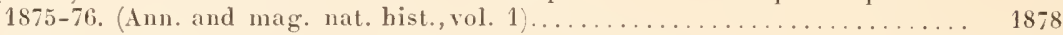

Foraminifera. (Report on the Se. Results of the Challenger) ........... 1873-1876

Braxdt (Karl). - Fauna und Flora des Golfes von Neapel. (Koloniebildende Rarliolarien Sphärozoacen.)...

Brauer (A.). - Ueber die Eneystirung von Actinospharium Eichhorni (Ehrbg.). (Zeit.

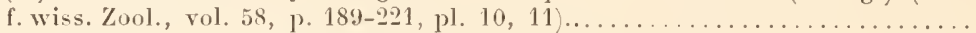

Brefeld. - Dictyostelium mucoroüdes. (Ahhandl. der Sächs. Naturf.-Gesellsch., vol. 7, Francfort)...

Broeck (van der). - Étude sur le Dimorphisme des Foraminifires. (Bull. soc. helge de Géol., p. 6, Bruxelles).

Brurne (C. de). - Monadines et Chytridiacées, parasites des Algues du golfe de Naples.

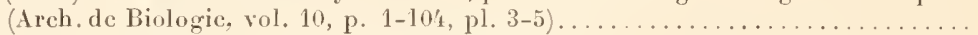

Butschu (O.). - Zur Kenntniss der fortpflanzung bei Arcella rulgaris (Ehrh.). (Areh. mikr. Anat., vol. 11, p. 459-467, pl. 25).

Bronn's Classen und Ordnungen des Thier-Reichs I, Protozoa.

Carpexter (W.). - On the mieroscop. struet. of Nummulines, Orbitolites and Orhitoïdes. (Quart. journ. geol. ser., vol. 6, p. 22) .

Chevikof (W.). - Ueber die karyokinetische Kerntheilung der Euglypha alveolata. (Iorphologisehes Jahrbuch, vol. 13, p. 193-255, pl.6-7) . . . . . . . . . .

Cienkorski. - Zur Entwicklungsgeschichte der Myxomyceten. (Jahrb. f. wiss.

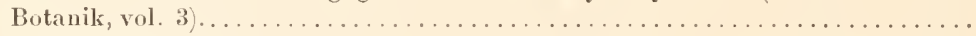

Ueher einige Rbizopoden und verwandte Organismen. (Arch. f. mikrosk. Anat.,

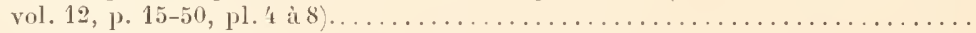

Ueher einige Rhizopoden und verwandte Organismen. (Areh. f. mikr. Anat.,

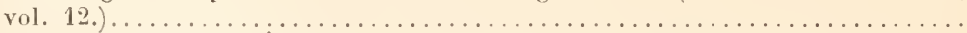

Claparède et Lagumann. - Étude sur les Infusoires et les Rhizopodes. Genève......

De la IIARPe. - Sur l'importance de la loge centrale chez les Nummulites. (Bull. soc.

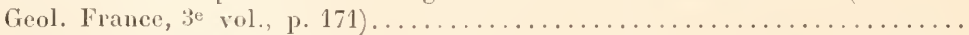

Dreyer. - Die Pylombildungen in vergleichend-anatomischer und entwickelungsgeschlechtlicher Beziehung, bei Protisten iberhaupt, nehst System und Beschreihung und der bis jetzt bekimnten pylomatischen Spumellarien. (Jen.

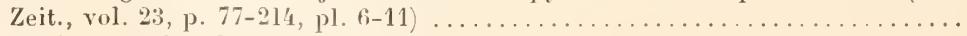

Dujardin (F.). - Recherches sur les organismes inférieurs. (Ann. des se. nat., 2e sér., vol. 4 , p. 343 )

Observations sur les lihizopodes et les Infusoires. Compt. rend. Ac. des Se. Paris, $n^{\circ} 111$, p. $202-203$.

Iistoirc naturelle des Zoophytes Infusoires. Paris. 
Ehrenberg (Chr.-G.). - Die Infusionsthierehen als vollkommene Organismen. Berlin

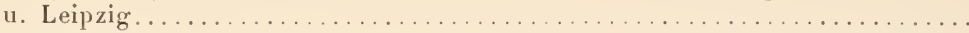

Ents (Geza). - Ueber die Rhizopoden des Salzteiches zu Szamosfalva. (Naturbist. Ilefte des Nat. Museums zu Budapest, 1. lleft).

Frexzel (J.). - Ueber einige merkwiirlige Protozoen Argentiniens. (Zeit. f. wiss. Zool. 53, p. 334-360, pl. 17) .......

Gould (Lilian J.). - Note on the minute strueture of Pelomyxa palustris Greefl) (Quart. Journ. Mierose. Scienee. New serie, vol. 36, p. 296-306, pl. 20-21). . .

Greefr (Riehard). - Pelomyxa palustris (Pelubius), ein amöbenartiger Organismus des siissen Wassers. (Arch. f. mikr. Anat., vol. 10, p. 51 i 73).

Н̈̈скеL E.). - Die Radiolarien (Rhizopoda radiara). Eine Monographie.

thickelungszustände von Magosphiera planula. (Jen. Zeit. f. Naturw., vol. 6, p. 1-21, pl. 1).

Nachträge zur Monographie der Moneren. (Jen. Zeit. f. Naturw, vol. 6, p. 23-42).

Report on the seientific results of the Exploring royage of II. M. S. Challenger, vol. 18

Hallez (Paul). - Sur un nouveau Rbizopode Arcuothrix Balbianii nop. gen., nos. sp. (Mémoires de la Société des Scienees de Lille, vol. 14, le série).

Hertivig (R.) et Lesser (E.). - Ueber Rhizopoden und demselben nahestehende Organismen. (Arch. f. mikr. Anat., vol. 10, p. 35-2't3, pl. 2 a 6 ) .

Kext (Saville). - Étude sur les Physémaires. (Arch. de zool. exp. (Notes et revues), vol. 8, p. 8 i 11)

Korotner (A.). - Etudes sur les Rhizopodes. (Arch. de Zool. exp., vol. 8, p. 467-482,

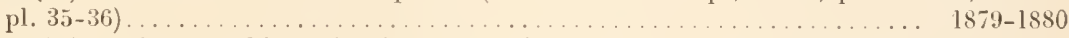

Kunstler (F.). - Sur un lihizopode. (Compt. rendus Aead des seiences Paris, vol. 99, p. $337-338)$.

$188^{\prime}$

LAMARCK (J.-B. de). - llistoire naturelle des animaux sans rertèbres. (Paris, rol. 7, 2. édition).

LE Daxtec (F.). - Reeherehes sur la digestion intraeellulaire ehez Ies Protozoaires. (Tlièse de Paris).

Lister (J.-J.). - Contribution to the life history of the Foraminifera. (Philos. trans. vol. I86, p. $401-453$, pl. 6-!)

Moxiez (R.). - Note sur une nouvelle forme de Sareodine, le Schizogenes parasiticus. (Journ. Auat. Physiol., vol. 12, p. 5lis-523, pl. 16.) Nov.-díe.

Müller (G. W.). - Ueber Schizogenes parasiticus Moniez). 2 vol. Anz., p. 395, 396 ...

Munier-Cilamas et Schlumbercier. - Nouvelles observations sur le dimorphisme des Foraminiferes. (Compt. rendus Acad. des Seiences, p. 862-866, 't fig.).

1895

Nouvelles observations sur le dimorphisme des Foraminiferes. Bulletin de la Société zoologique de France, vol. 8, 3e série, p. 300, pl. 862-866).

Note sur les Miliolidées Trématophorées. Extrait du Bulletin de la Société géologique de liranee, 3e série, rol. 13, p. 273-323, pl." 13 et 14 bis).

Orbigxy (A. d'). - Article : Foraminifères. Dict. llist. Nat. de Gh. d'Orbigny, vol. 5, p. 662 ).

Péxard (Eug.). - Notes sur quelques lléliozoaires. Areh. des Seicnces phys, et nat., troisième période, vol. 22, n० 12 , p. 523-539

Études sur quelques lléliozoaires d'eau douce. Arch. de Biol., vol. 9, p. 419472, pl. 30 a 32 ).

1883

1883

1885

1844

1889

1889

Études sur quelques lléliozoaires d'eau douce. (Areh. de Biol., vol. 9, pl. 9 a 11$)$. .

Rhizopodes deau douce. (Mém. de la Soe. de Phys. et d'Hist. nat. de Genève, vol. 31, p. 1 i 230, pl. 1 à 11).

Rostafixiz. - Versuch eines Systems der Myeetozoen. Strasbourg

Rumber (von L.). - Beiträge zur Kenntniss der lihizopoden, 1. Ueber Entstehung und sekundaires Wachsthum der Gehäuse einiger süsswasserrhizopoden. (Zeit. f. wiss. Zool., vol. 52, p. 515 à 550, pl. 32 ).

Die IJerkunft des Globigerina. - Einschlusse bei Orbulina unisersa (d'Orbigny). Vorläuf. Mitth, (Zool. Anz., vol. 17, p. 196-202, l fig.)

Beiträge zur Kenntnis del Rhi\%opoden. Zeit. f. wiss. Zool., rol. 57, p. 433-586, pl. 21 à 24 )..

Beiträge zur Kenntuis der Rhizopoden. (Zeit f. wiss. Zool., vol. 57, p. 587-617, pl. 25). 
Sassaki (Chiujeo). - Untersuchungen über Gymnosplatra albida, eine neue marine Heliozoe. (Jen. Zeit. f. Naturw., vol. 28. p. $45-52$, pl. 2) ................

Sinaudinx (F.). - Untersuchungen an Foraminiferen, I. Calciluba polymorplu. (Zeit. f. wiss. Zool., vol. 59, p. 191-233, pl. 1't-25)....................

Ueber Kerntheilung mit nachfolgender Körpertheilung bei Amaba crystalligera (Gruber). (Sitz. Ber. K. Preuss. Akad d. Wiss., vol. 38, p. 1029-1036, fig.)....

Camptonema nutans $n$. g., $n$. sp., ein neuer marine Rlizopode. (Sitz. Ber. K. Preuss, Akad d. Wiss., vol. 52. p. 1277-1286, pl. 7) ...................

- Ucber die Theilung ron Amceba binucleata Gruber). (Sitz. Ber. Ges. Naturf. Fr. Berlin, p. 130-141)

Ileliozoa (Das Thierreich, herausg. v. d. deutsch. zool. Gesellsch.) ..............

Ueber die Copulation ron Actinophrys sol (Ehrbg.). (Sitzungsb. der Kün. Preuss. Akad. der Wiss. zu Berlin)

Schlumberger (C.). - Note sur quelques foraminifères nouveaux ou peu connus du golfe de Gascogne. Campagne du Travaillcur. Feuille des jeunes naturalistes, p. 1-8, pl. 2 et 3$)$.

1895

$18 \%$

1894

1895

1896

$189 \%$

Sur un nouveau Pentellina. Association francaise pour l'arancement des sciences, congrìs de la Ruchelle).

Note sur le genre runeolina. (Bulletin de la Société géologique de France, 3e série, vol. 11, p. $272-273$ ).

Sur le Biloculina depressa (d Orb.) au point de vue du dimorphisme des Foraminifères. Association française pour l'avancement des sciences, Congrès de Rouen, 1883, p. 520-527)..

Sur l'Orbulina unisersa. (Bulletin de la Socićté zoologique de France, 3e série, rol. 8, p. 300).

Note sur le genre Adelosina. (Bulletin de la Société zoologique de France, vol. 11, p. $91-104$, pl. 16) .

Note sur le genre Planispirina. Bullctin de la Société zoologique de France, vol. 12, p. 475-488, pl. 7 )..

Note sur les Biloculina bulloides (d Orb.) et Biloculina ringens (Lamk.). (Bulletin de la Société géologique de France, 3 e série, vol. 15. p. 139-147, pl. 15).

Sur la reproduction des Furaminifères. Bulletin de la Société zool, de Frauce, vol. 13, p. 135-137, pl. 222)

Deseription of a New Species of Fabularia. From the Transactions of the Royal Society South-Australia, p. 346-349, pl. 13)

Note sur un Foraminifire nouveau de la cote d Afrique. (Extrait des mémoires de la Société zoologique de France, vol. 3, p. 211-21't, pl. 7).

Note sur l'Adelosina polygonia. (Bulletin de la Sociéli zoologique de France, vol. 15, p. 139-147).

- Revision des Biloculines des grands fonds. (lémoires de la Société zoologique de lirance, vol. 4, p. 542-580, pl. 19-22

Note sur le Ramulina Grimaldi. Mémoires de la Société zoologique de France, p. 509-512, pl. 5 ).

Nole préliminaire sur les Foraminifères dragués par $\mathrm{S}$. A. le prince Albert de Monaco. Mémoires de la Société zoologique de France, vol. 5, p. 193-198. p1. 8).

Note sur les genres Trillina et Linderina. (Bulletin de la Societé géologique de France, vol. 21, 3e série, p. 118-123.pl. 3

Monographie des Miliolidées du golfe de Marseille. Mémoires de la Société zoologique de France, vol. 6, p. 199-228, pl. 5)

Note sur Lacasina Wichmanni (Schlumberger, n. sp.). (Bulletin de la Société géol. de France, vol. 22, 3e série, p. 295-298, pl. 12). .

Note sur les foraminiferes des mers aretiques russes. (Bulletin de la Société zoologique de France, vol. 7, p. 237-243, pl. 3)

1880

1882

1883

1883

1884

1886

1887

1887

1888

1891

1890

1890

1891

1891

1892

1893

1893

$189^{\prime} t$

1894

185 '

Schultze (Mlax). - Ueber den Organismus der Polythalamien Foraminiferen. Leipzig.

Schulze (Franz Eilhard). - Rhizopodenstudien. (Arch. f. mikrosk. Anat., vol. 10, p. 377400, pl. 26-27)

Rhizopodenstudien. (Arch. f. mikiosk. Anat., vol. 11, p. 329-353. pl. 18-19).

Rhizopodenstudien. (Arch. f. mikrosk. Anat., vol. 11, p. 583-596, pl. 35-36)

1875

Rhizopodenstudien : Mastigamaba aspera n.g., n. s. (Arch. f. mikr. Anat., rol. 11, p. 583-596, pl. 35 et 36$)$. .

1875

1875

Sorokix. - Bursulla crystallina. (Ann. se, nat. Botanique, 6e série. p. 3) ........ 1876 
Stuart (Alex.). - Ueber Coscinosphara ciliosa, eine neue Radiolarie. (Zeit. f. wiss.

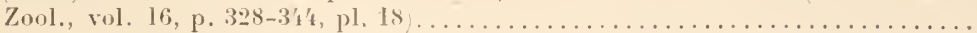

Topsext. - Deseription de Pontomypa flusa, Rhizopode marin, type multiuuelée des

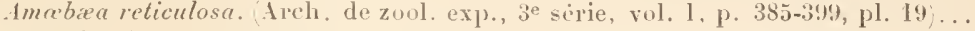

VAN Tieghen (Th.). - Sur quelques Myxnmycetes a plasmode agrige. liull. de la

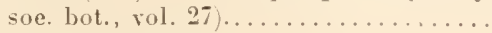

Traité de Botanique.

Vonosix. - Plasmodiophora Brassicue. (Jahrbücher. f. wiss. Botanik, vol, 11, p. 548).

Wanrlici (W.). - Anatomisebe Eigenthümlichkeit einer Vumpyrella. Berichte der Deutseh. botan. Gesellsch., iol. 7, p. 277-279. pl. 10)

1878

Zacharias (Otto). - Experimentelle Lntersuchungen iber Pseudopodien-bildung. (Biolog. Centralbl., vol. 5, p. 259-262 .................... $1885-1886$

Zorf (IV.). - Ueber einen neuen Sebleimpilz im Sehweinekörper. (Biolog. Centrabl.,

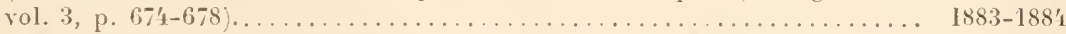

Zur Morphologie und Biologie der niederen Pilzthiere Monadinien). Zugleieh ein Beitrag zur Phytopathologie, p. 1-15, pl. 1-5.

1885

Die Pilzthiere oder Sehleimpilze, nach dem neuesten Standpunkte bearbeitet, p. $1-174$

Zur Kenntniss der Labyrinthuleen, einer Familie der Hyeetozoen. Beitrïge zur Physiologie und Morphologie niederer Organismen, p. 36-48, pl. 4-5).......

Ein in Saccamminagehäusen vorkommender Myxomyeet. Zeit. f. wiss. Zool.,

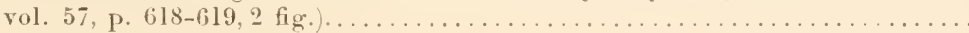

1885

1892

$1899^{\prime}$

\section{SPOROZOAIRES -}

Pour la bibliographie spéciale s'adresser aux ouvrages généraux de Balbiaui, Bülselli, Labbé, Mingazrini, Pfeiller, silhneider, Thẻohan.

Bılbixi, - Étude sur les maladies psorospermiques des lers à soie. Journ. Anat. et Phys., vol. 4, p. 26i3-276).

Baraban et Saxt-Remy. - Le parasitisme des Sarcosporidies chez l'homme. (Bibliogr.

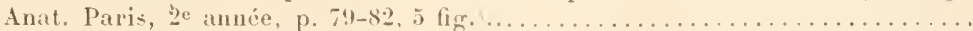

Lecons sur les Sporozoatres. Paris, Jouru. mirro., se anne ..............

BExEDen (Van). - Reeherehes sur l'ćvolution des fregarines. Bullet. Ae. roy. de Belgique, 2e sér., vol. 31, p. 325-59, l pl.)

Blaximano (h.). - Sur un nouveau type de Sareosporidies. (C. R. Ac. des sr. de

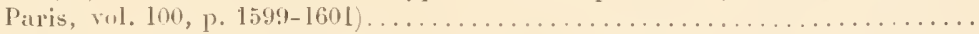

Note sur les Sarcosporidies et sur un rssai de elassificalion de ees Sporozoaires.

Bull. de la Soe. zool. de France, vol. 10, p. 2'1').

Borrf.. Évolution eellulaire et parasitisme dans l'épithelioma. (Thise de Montpellier).

Bütscrn. - Beiträge zur henntniss der Fiseh-Psorospermien. (Zeit f. wiss. Zool.,

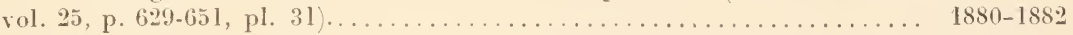

Cuevikof Wladimir). - Ueber einige Ekto- und Entoparasitische Protozoen der Cyclopiden. Extrait du Bull. de la soe. imp. des Naturalistes de Moseou, $1^{\circ} 1$,

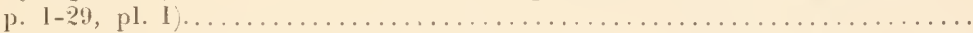

- Teber die Ursaehe der fortschreitenden Bewegung der Gregarinen. (Zeit. f. wiss. Zool., rol. 58, p. 340 à 334, pl. 20 et 21 ).

Crenkorsix L.). - Ueber parasitaire Sehläuche auf Crustaceen und einige Insectenlarven (Amabidium parasiticum). (Botan. Zeitung, p. 169-179, pl, 8).......

Daxilevsí (B.). - Zur Parasitologie des Blutes (Biolog. Centralb. vol. 5, p. 529$537)$

Parasitologie comparée du sang. Karliof, vol. I et 2.

- Ueber dei Hyoparasiten der Amphibien und Reptilien. (Centralbl. f. Bakt. u.

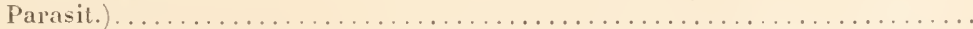

Eımer (Th.). - Ueber die ei- und kugelförmigen Psorospermien der Wirbelthiere.

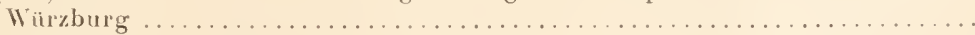

Fabre-Domergue. - Pseudococeidies des cancer's épithéliaux, Soc. de Biol., vol. 4. p. $33 \overline{7}-339$

FoA. - Parasites du eancer. (Riforma medica)

Frexzel (1.). - Ueber einige in Seethieren lebende Gregarinen. (Areh. f. mikr. Anat., vol. 2't, p. 5't5-58s, vol. $25-26$, pl. $25-2(i)$. 
Gabrıel. - Zur Classifieation der Gregarinen. (Zool. Anz., vol. 3, p. 569-572)

Garbint. - Contributo alla conoseenza dei Sareosporidi. (Rind. del. R. Aead. dei Lineei Roma, vol. 3, p. 151-153)

GÉard (A.). - Sur une nouvelle espèee de Psorospermie (Lithocystis Schneideri) parasite de I'Echinocardium cordatum. Compt. rend. Ac. des se., Paris, vol. 82, p. 1208 ).

Gukley (B.). - On the Classifieation of the Myxosporidies. Bull. U. S. Fisch-Comm. for 1894 , rol. 11, p. $40 \overline{7}-420$..

Hennegur (P.) et Tú́conax (P.). - Myxosporidies parasites des museles ehez quelques Crustacés décapodes. Ann. de Micrographie, vol. 4, p. 617-641, pl. 4)......

KLoss. - Ueber Parasiten in der Niere von llelix. (Abhandl. der Nenekenberg. naturf. Gesellseh., vol. 1, p. 189-213, pl. 15-16i).

LonotNefF (A.). - My.xosporidium bryozoides. (Zeit. f. Wiss., vol. 53, p. 591 a 596 , pl. 24). Rhopalocephalus carcinomatosus $(n . g$. und $s p$.) (Krebsparasit). (Centralbl. f. liakterund Parasitenkunde, vol. 13, p. 373 a 380,15 fig.) . . . . . . . . . . . . .

Sporozoen als Krankheitserreger. (Untersueh. üb. den Parasitismus des Careinoms, p. 1 à 33,4 pl.)

Labié (A.). - Coccidium Delagei. Coeeidie nouvelle parasite des Tortues d'eau douee. (Areh. de zoologie exp., 3e série, vol. 1, p. 267-280, p]. 17) . . . . . . . .

Bananella Lacazei, genre nouveau de Coecidie oligosporée. Areh. de zool. exp., notes et revue, p. 15 ).

Sur deux Coccidies nouvelles parasites des Poissons. Bulletin de la Société zoologique de Franee, vol. 18, p. 202-204)..

Sur les Coceidies des Oiseaux, C. R. Aead des se. 5 juin a 18 septembre)

Sur la coexistenee ehez le mème hòte d'une Coccidie monosporée et d'une Coccidie polysporée. Cumpt. rend. Acad. des sc., Paris, 24 sept.

Reeherches zoologiques et biologiques sur les parasites endoglobnlaires du sang des Vertébrés. Areh. de zool. expér. et génér., fase. 1-2, p. 56-25s, pl. 1-10).

Sur la morphologie et la classifreation des Coecidies. Compt. rend. Acad. des se., Paris)

Recherches zoologiques, cytologiques et biologiques sur les Coceidies. (Arch. de zool. expér. et gén., 3e série, vol. 4

LAverax. - L'llémato\%oaire du paludisme. Paris.

Lécrer (L.). - Recherches sur les Grégarines. Thèse de Paris).....

L'évolution des Grégarines intestinales des Vers marins. Compt. rend. Ae. des se., Paris, p. 20't-206)

Leuckart (B.). - Die Parasiten des Menschen.

Lieberkërx (IV.). - Evolution des Grégarines. Mem. des sar. étr. de l’.le. de Belg., vol. 26$)$

Maxiaberg (J.). - Die Malariaparasiten. Wien

Maxy (W.). - Beitrag zur Kenntniss der Nlieseher'schen Sehläuche. (Areh, f, mikr. Anat., vol. 3, p. 345-366, pl. 20).

Malsmall-Stanley (W.). - Beiträge zur Kenntniss der Gregarinen. (Areh. f. Naturgeschichte, vol. 1, p. $25-44$, pl. 2 )

Mrescher (F.). - Ueber eigenthümliche Schläuehe in den Muskeln einer Inasunas. (Berichte über die Tcrhandl. d. nat. Ges. in Basel, vul. 5, p. 198-202)

Mricazzin (P.). - La Parentela dei Coceidi colle Gregarine. Bollet. soc. Nat. Napoli, p. $151-159)$.

Classificazione dei Cuccidi e delle Gregarine. Atti d. Real. Acad. d. Lincei, ser 5., vol. 1, p. $68-75$ )

Le Gregarine delle Oloturie. Rendic. del real. Acad. d. Lincei.

Contributo alla eonoseenza degli Sporozoi. Rome.

Moxiez (R.). - Observation pour la revision des Myxosporidies. Compt. rend. Acad. des se., Paris, p. 1312-1313

Note sur le genre Gymnospora, type nouvaru de Sporozoaires. Extrait du liulletin de lat Société zoologique de France, vol. 11, p. 8

Pastelr L.). - Etude sur la maladie des Vers a soie. Ze vol. Paris

PFenfer L.). - Beiträge zur Kenntniss der pathogen. Gregarinen. Zeit. i. Hyg. Leipzig, vol. 4.)

Ueber einge nere lormen von Ilicschers'chen Schliuchen mit mikro-, myxound sarkosporidien Inhalt. (Virehow's Archiv, vol. 122, p. 552-573, pl. 19)...

- Die Coceidienkrankheit der Kaninchen, Berlin.

1880

1891

1876

1894

1892

1855

1892

1893

1893

1893

1895

1893

1893

1594

1894

1895

1896

1891

1892

1893

1579

1855

1893

I 867

1893

1843

1890

1890

1890

18.45

1887

1886

1870

1888

$18: 10$

$18 \% 2$ 
Pfenfer (L.). - Die Protozoen als Krankheitserreger. 2e éd . . . . . . . . . . . . 1891

_. Untersuchungen über den kirebs. Jena . . . . . . . . . . . . . . . . . . . . . . . . . 1893

"Die Serumsporidien ». Ueber Blutparasiten bei blutkörperehenfreien niederen

Thieren. Corresp. Blätt. des allgem. ärtz. Ver. $\nabla$. Thüringen. ...........

1875

Plate (Ludwig). - Untersuchungen einiger an den Kiemenblätern des Gammarus pulex lehenden Ektoparasiten. (Zeit. f. wiss. Kool., vol. 43. p. 175-240)...... 1886;

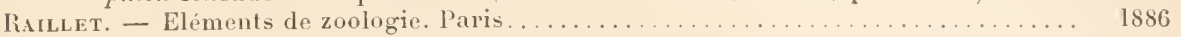

Rallet et Lccet. - Observations sur quelques Coecidies intestinales. Compt. rend.

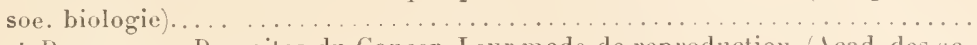

1890

Ruffer et Plimer. - Parasites du Caneer. Leur mode de reproduction. (Acad. des sc., avil 1893, et Journ. of pathol. and baeteriol. . . . . . . . . . . . . . . . . . . 1893

Scuxemer A. - Contributions a l'histoipe des Grégarines des Invertebrés de Paris
et de Roscoff. (Areh. de zuol. expér, et gén., vol. 4. p. 493-60'4) . . . . . . . .

Sur les Psorospermies oriformes ou Coccidies. Espèces nonvelles ou peu eonnues. (Archives de zoologie expér. et gén., vol. 9, p. 357-402, pl. 12)

Nouvelles observations sur la sporulation du Klossia octopiana. Arehives de zoologie expérimentale, 2e série, vol. I, p. 77-103, pl. 9) . . . . . . . . . .

Ophryocystis Bütschlii Sporozoaire d'un noureau type. Archives de zuologie expérimentale, 2e série, vol. 2, p. 111-126, pl. 6). .

ophryocystis Francisci. (Tableties zoologiques, rol. 1, p. 1-3, pl. 1).

Grégarines nouvelles ou peu connues. (Tablettes zool. vol. 1, p. 25-30, pl. 23).

Grégarines nouvelles ou peu comues (2e article). (Tablettes zoologiques, vol. 1, p. $90-103 . \mathrm{pl} .23-28)$

Coecidies nouvelles ou peu ennnues. Ibid., rol. 1 .

Scutberg (A.). - Ueber Coceidien der Mäusedarmen. Sitzber. Würzhurg, Is März.)

Sodakwicir. - Recherches sur les parasites du eancer. (Ann. de I Inst. Pasteur, 6 e année. p. 1'15-157. pl. 5-7) ....

Sterx (Fr.). - Ueber die Natur der Gregarinen. (Areh. f. Anat. u. Phys., p. 182-223)..

Trílonax P.). - Sur quelques Coccidies nonvelles, parasites des poissons. (Journal de lAnatomie et de la Physiologie, rol. 28, p. $151-162$, pl. 12. fig. 1-25)

Sur des Sporozoaires indèterminés parasites des Poissons. (Journal de l'Anatomie et de la Physiologie, vol. 28, p. 163-172, pl. 12, fig. 25-32) . . . . . .

Nouvelles reeherehes sur les Coceidies. Arch. de zool. exp. et gen. p. 541-573, pl. 23$\rangle$.

Sur la présenee d'une capsule a filament dans les spores des Iyxosporidies. (Compt. rend. soc. Biol. Paris). .

Reeherehes sur les Ilyxusporidies. (Bull. se. du nord de la France et de la Bel-

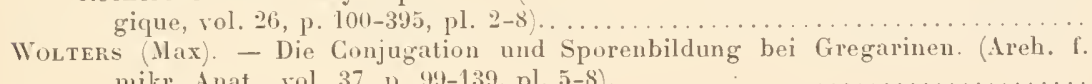
mikr. Anat., rol. 37, p. 99-139, pl. 5-8)..

1875

1881

1883

1885

1886

1886

1856

1886

1892

1892

1846

1892

1892

$1894_{1}^{\prime}$

1894

1895 1891

\section{FL I GELLÉS}

Pour la bibliographie complete antérieure à 1883, voir : Biitschli [83-87].

Blocimaxx (L.).- Zur Kenntniss von Dimorpha nutans (Grub.). (Biol. Centrabl., vol. 14,

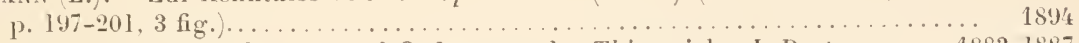

Bütscul (O.) - Bron's Classen und Ordnungen des Thierreichs. I, Protozoa. . 1883-1887

Certes (A.). - Note sur les miero-organismes de la panse des Ruminants. (Bull. de la Soeiété zool. de Franee, vol. 14, p. 70-73)..

Sur le Trypanosoma Balbianii. (Bull. de la Soeiété zool. de France, vol. 16, p. 95 et $130-131)$...

Gienkorsky (L.). - Beiträge zur Kenntniss der Mlonaden. (Areh. f. mikr. Anat., vol. 1, p. 203-232, pl. 12-14).

Ueber Palmellaeen und einige Flagellaten. (Areh. f. mikr. Anat, vol. 6, p. 421-436, pl. 23-24).

Cons (Ferdinand). - Ueber eine neue Gattung aus der Familie der lolvoeinen. (Zeit.

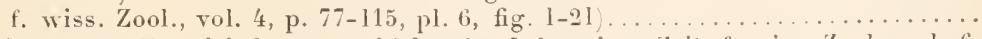

Beiträge zur Entwickelungsgeschichte der Infusorien. (Zeit. f. wiss. Zool., vol. 6, p. $253-281)$ 
Dangeard (P.). - Les Péridiniens et leurs parasites. (Journal de Botanique. 2e année,

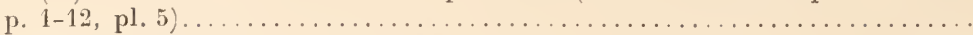

Davane (G.). - Monadiens. (Dietionnaire des sciences médicales, vol. 9. 2e série, p. $115-130)$.

Dujardin (M. F.). - Sur les Infusoires munis d'un double filament locomoteur. (Ann.

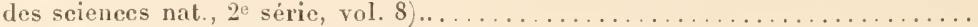

Sur les Monades à filaments multiples. (Ann. des seiences nat., 2 e série, vol. 10, p. $17-35)$.

Ebertu (Jos.). - Ueber ein neues Infusorium im Darm verschiedener Vögel. (Zeit.

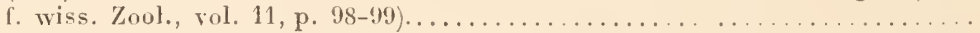

Fisci (C.). - Untersuchungen über einige Flagellaten und verwandte Organismen. (Zeit. f. wiss. Zuol., vol. 42, p. 47-125, pl. 1-6).

Fraxzé (Rudolf). - Zur Morphologie und Physiologie der Stigmata der Mastigophoren. (Zeit. f. wiss. Zool., vol. 56, p. 139-162). . . . .

Grassi (B.). - Intorno ad alcuni protisti endoparasitici. (Atti soc. ital. d. se. nat.

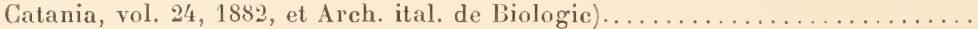

GrUBY. - Sur une nouvelle espèce d'Ilématozoaires. (Compt. rend. 17, 1843, et Ann. des sc.nat., p. 104-107, pl. 1)..

Hennegur (L.-F.). - Sur un Infusoire Flagellé ectoparasite de la Truite. (Arch. de zool. expérim., 2e série, vol. 2, p. 405 à 411).

IIERtwig (O.). - Erythropsis agilis. Einc neue Protozoe (Morph. Jahrb., vol. I0, p. 204-212, pl. 6)....

- Erythropsis agilis, cine langgerissene spasthostyla Sertulariarum? (Zool. Anz., vol. 8, p. 108-112). .

IschiкAwA (C.). - Torläufige Mittheilungen iber die Conjugationserseheinungen bei den Noetiluccen. (Zool. Anz., 14e année, p. 12 a 15).

Ueber dic Kerutheilung bei Noctiluca miliaris. (Berichte der Naturforsehenden Gesellschaft zu Freiburg, p. 1 à 12, pl. 3).

Kent (Sar.). - A Manual of Infusoria. London.....

Knawkine (W.). - Recherehes biologiques sur l'Astasia ocellata $n$. s. et l'Euglena viridis. (Ann. des se. nat., 7e série, vol. 1, p. 319 à 375, pl. 16). . . . . . . .

KLers (Georg). - Flagellatenstudien. (Zeit. f. wiss. Zool., vol. 55, p. 265-445, pl. 17 et 18).

KLEIN (Ludwig). - Neue Beiträge zur Kenntniss der Gattung Volvox. (Berichte der Deutseh. Botan. Gesellseh.. vul. 7, p. 42-53, pl. 3

Krassulstscin (von J.). - Ueber eine neue Flagellate Cercobodo laciniegerens n. o. et n. sp. (Zool. Anzeiger, vol. 9, p. 365-36! et 39'-399). . .

Zur Entwickehungsgeschichte und Systematik der Gattung Polytoma. (Zool. Anz., vol. 5, p. 426 à 429 ).

línstler (J.). - Contribution à l'étude des Flagellés. Bull. soc. zool. de France, p. 112, pl. 3). .

Nouvelles contributions à l'étude des Flagellés. (Bull. soe. zool. de Francc, p. $230-236)$.

Lauterborn (R.). - Protozoenstudien I, Kern- und Zelltheilung von Ceratium hirnndinella (O. F. M.). (Zeit. f. wiss. Zool., vol. 59, p. 167-191, pl. 12 et 13).

Maupas (E.). - Sur la position systématique des Volvocinées et sur les limites du régne régétal et animal. (Compt. rend. Ac, des sc., Paris, vol. 88, p. 1274-77).

Mетспхлоғ (E.). - Zur Streitfrage iber Erythropsis agilis. (Zool. Anz., vol. 8, p. 433, 434.).

Mitropinnof (V.). - Beiträge zur Kenntniss des IIxmatozoa. (Biol. Centralbl., vol. 3, P. $\left.35-4 \prime^{\prime}\right)$. . .

Möвıus (K.). - Trypanosuma Balbianii(Certes) im Krystallstiel sebleswig-holsteinischer Austern. (Zool. Anz, vol. 6, p. 148-152) ....................

Penand (Eug.). - Contributions ì l'étude des Dinoflagellés. Recherches sur le Ceratium macroceros avee observations sur le Ceratimm cornutum. (Dissertation présentéc à la Faculté des seiences de l'Unirersité de Crenève, p. 1-13,

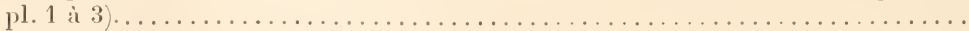

Les Peridiniacés du Léman. (6e Pulletin rles travaux de la Soeiété botanique de

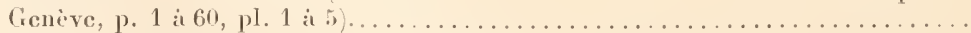

Poucnet (G.). - Nouvelle contribution a lisistoire des Péridiniens marins. Journal de l'Anatomie et de la Physiologic, p. 28 à $88, \mathrm{pl} .2$ a 4 ). 
Pouchet (G.). - Cinquième contribution à l’histoire des Péridiniens. (Peridinium pseu-

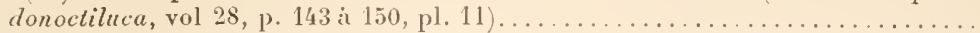

Contribution à l'bistoire des Cilio-Flagellés. (Journal de l'Anatomic et de la Physiologie, p. 1 à 57, pl. 19 à 22). .

Sur Gymnodinium Polyphemus. (Comptes rendus Acad. des se, vol. 95. p. 79'4-796)

Contribution à l'histoire des Noetiluques. (Journal de l'Anatomie et de la Physiologie, p. 104 à 125, pl. 4)

Schüt (Franz). - Ueber Organisationsverhältnisse des Plasmaleibes der Peridinien. (Sitzungsb. der König. Preus. Akad. der Wiss. zu Berlin, p. 377-38't, pl. 2

Die Peridinen der Plankton-Expedition

Srex (Fr.). - Der Organismus der Infusionsthiere. IlI. Der Organismus der Flageilaten. Leipzig. .........................

Vogt (Carl). — Ueber Erythropsis (R. Hertw.). (Tatur, vol. 3't, p. 183-187, . . .

Zacharias (Otto). - Ueber den Bau der Monaden und Familienstöcke von Eroglena volvox. (Zool. Anz, vol. 17, p. 353 à 357 ).

1884

1882

1890

1892

1895

1878

1885

1894

\section{CILIÉS}

Pour la bibliographie complète antérieure à 1885, voir : Bütschli [83-87].

Balbiaxi (G.). - Évolution des mieroorganismes anim. et vég. pirasites. (Journ. de Mlierographie) .................... vol. 10, 1886; vol. 11

Rechercbes expérimentales sur la mérotomie des Infusoires riliés. Rev. zool. suisse, 1859, et Annales de Nicrographie, p. 1-25. '19-8't, 113-137,3 frg. pl. 1-2). $1892-1893$

Sur la strueture et la division du noyau chez le Spirochona gemmipara. (Ann. de micrographie, vol. 7, p. 1-41, pl. 2)..

Brauer (A.). - Bursaria truncatella unter Berucksichtigung anderer Heterotrichen und der Forticellinen. (Jen. Zeit. f. Naturw, vol. 19, p. 489-519, pl. 12.) 1885

Bütschl. (O.). - Bronn's Classen und Ordnungen des Thierreichs. I. Protozoa .. 1883-1887

Ciarrí̇Re (von J.). - Trichodince (sp.). Schmarotzer im Seitencanal von Cothus gobio. (Areh. f. mikr. Anat, vol, 33, p. '102-115, pl. 2'

1887

Gertes (A.). - Note sur deux Infusoires nouveaux des environs de Paris. Mémoires de la Société zoologrique de France, vol. 4, p. 536-5't1, pL. 7)

Cuexkorsir. - Ueber Cystenbildung bei Infusorien. Zeit. f. wiss. Zool., vol. 6, p. 301 à $305, \mathrm{pl} .10$ et $1 \mathrm{l}) \ldots \ldots \ldots$. . . .

Ciaparène et Lacumaxx. - Étude sur les Infusoires et les Rhizopodes. Genève

Cons (Ferdinand). - Beiträge tur Entwickelungsgeschichte der Infusorien. Zeit. f. wiss. Zool., vol. 4, p. $253-280$, pl. 133

Neue Infusorien im Seeaquarium. Zeit. f. wiss. Zool., vol. 16, p. 253-302, pl. 1't et 15$)$.

Dnoar (Eng.). - Ein kleiner Beitrag zur Kenntniss der Infusorien-Fauna des Golles von Neapel. (Mitt. Zool. Stat. Neapel, vul.6, p. 481-493, pl. 25)

Eberlers (R.). - Ueber die im Wiederkäuermagen rorkommenden ciliaten Infusorien. (Zeit. f. wiss. Zool., vol. 59, p. 233-305, pl. 16-18). .

Ecker (A.). - Zur Entwicklungsgeschichte der Infusoricn. (Zeit. für wiss. Zool., vol. 3, P. 412-417, pl. 13, fig. 1-4).

Eneneng (G.). - Beitrag zur Kentniss der Organisation der Infusorien und ihrer geographischen Verbreitung. (Abh. Berl. Akad.)

Entz (Geza). - Beiträge zur Kenntniss der Infusorien. Zeit. 1. wiss. Zool., vol. 38 ,

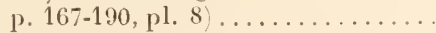

Ueber die Infusorien des Golfes ron Neapel. Mittheil. Zool. Stat. zu Neapel, vol. 5 , p. 289-4'1, pl. 20-25).

Die clastiseben und contratilen Elemente der Vorticellinen. Mathem-naturwissensehaftliche Beriehte aus Ungarn, p. 1-48, p1. 1-3)

Elilanger (R. V.). - Zur Kenntniss einiger Infusorien. (Zeit. f. wiss. Zool., vol. 49, p. $649-661, \mathrm{pl} .29) \ldots$

Fabre-Donergue. - Sur les Corpuscules de la Cavité générale du Siponele. Bull. scient. du Nord, vol. 9, p. 359-360).

Fol (II.). - Contributions à l'étude des Tintinnoïdiens. (Rec. de zool. suisse, vol. 1,

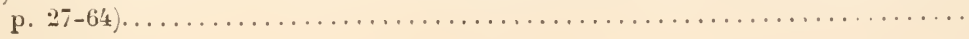


Fol (II.). - Sur lc Sticholonche Zanclea et un nouvel ordre de Rhizopodes. (Ilćmoires de l'Institut national genèvois, vol. 15, p. 1 à 31).

Fratpont Julien). - Recherches sur les Acinétiniens de la côte d'Ostende. (Bull. de

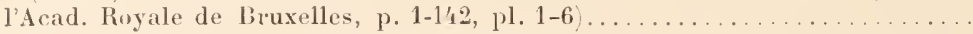

Gourret (P). et Reser (P). - Description de deux Infusoires du port de Bastia. (Journ.

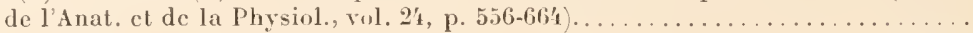

Gruber (August). - Neue Infusorien. (Zeit. f. wiss. Zool., vol. 33, p. 439-465, pl. $25-26)$. . . .

— Untersuchungen über einige Protozoen. (Zcit. f. wiss. Zool. 38, p. 45-70, pl. 2-3).

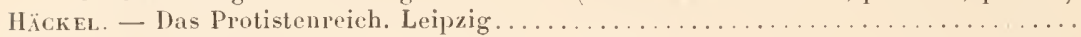

Hexnegur (L. F.). - Xote sur un nouvel Infusoire cilié (Ascobius lentus.) (Arch. de

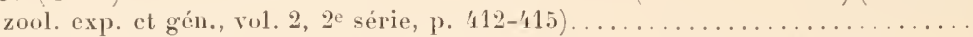

- Anoplophrya circulans. (Tabletles zonlogiques, vol. 1, p. 31-80, pl. 12 à 17.).....

- Fragments sur les Infusoires. Rajeunissement dans Dendrocimetes. (Tablettes zoologiques, vol. 1, p. $82-87$, pl. 19 et 20$)$.

IIEktwig (Richard). - Ueber den Bau und die Entwicklung der Spirechona gemmipara. (Jen. Zeit für Nat., vol. 11, p. 149-18'4, pl. 10-12).

Jessie (A.) et SALLiTt. - On the chlorophyll Corpuscles of some Infusoria. Quarterly journal of Nicroscopical Science, vol. 1'4, p. 166-170, pl. 13 et 14) . . . . . 1884

KEXT (Saville). - A manual of the Infusoria. London ................... 1880-1882

Köppex (Nicolas). - Amabophrya stycholonche nor. gen. now. sp. (Kool. Anz., vol. 17,

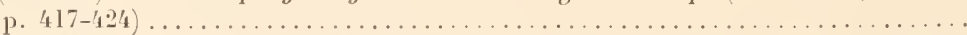

lionotxefF (A.). - Zoologische Paradoxen, p. 612-628 Sticholoncle Zanclea. (Zeit. f. wiss. Zool., vol. 51, p. 622-626, fig. $20-27$, pl. 30 à 32$) \ldots \ldots \ldots \ldots \ldots \ldots$

1880

1882

1878

1884

1886

1886

1887

1884

1894

1891

Maupas (E.). - Contribution à l'étude des Acincticns. (Arch. de Zool. cxp. et gén., vol. 9 , p. 299-368).

Contribution à l'étude morphologique et anatomique des Infusoires ciliés. (Arch.

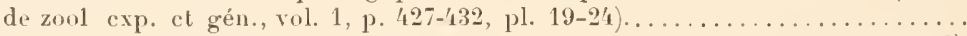

- Sur Coleps Hirtus (Ehrenberg). (Arch. de zool.exp. et gén., 2e sćrie, vol. 3, p. 337-366).

- Recherrhes expérimentales sur la multiplication des Infusoires ciliés. (Arch. de

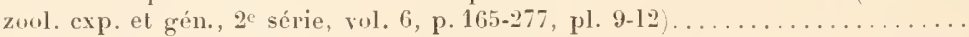

- Le rajeunissement karyogamique chez les Ciliés. (Arch. de zool. exp. et gén.,

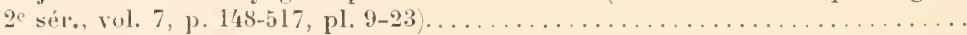

Ries (von J.). - Ueber cinige Fälle von Parasitismus bei Infusorien. (Zcit. f. wiss.

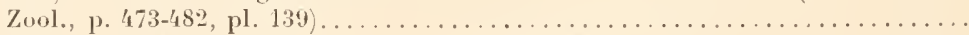

Schietder (A.). - Anoploplerya circulans. (Tablettes zuologiques, vol. 1, p. 32-80,

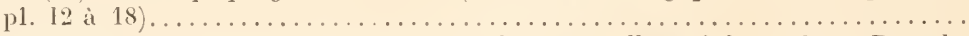

Schubekg (von A.). - Ueber den Bau der Bursaria truncatella; mit besonderer Berücksichtigung der protoplasmatischen Strukturen. (Morph. Jahrb., vol. 12,

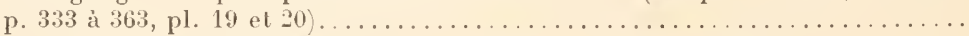

Ueber Grassia ranarum (Fisch). (Biol. Centralbl., vol. 9, p. 284-287) ...........

Ueber einige Organisationsverhältnissc der Infusorien des Wiederkäuermagens,

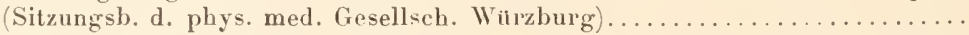

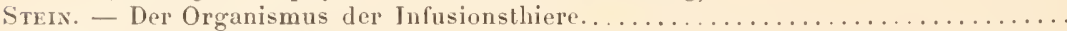

Sterki. - Beiträigc zur Morphologie der Oxytrichinen. (Zeit.f. wiss. Zool., vol.30, p. 31).

VRzesriorski (Iugust). - Beiträge zur Naturgeschichte des Infusorien. (\%cit. f.

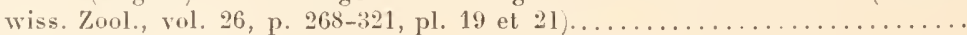

Zachakias (0.). - Infusorien als Hautparasiten bei Fischen. (Zeit. f. Fischerei, no 4, p. 153-161)

Zeleer (Ernst). - Untersuchungen uber die Fortpflanzung und die Entwicklung in unseren Batrachiern schmarotzenden Opalinen. (Zeit. f. wiss. Zool., vol. 29, p. $353-378$, pl. 23-21) 


\title{
TABLE DES MOTS TECHNIQUES
}

\author{
ET INDIGATIONS DIVERSES
}

Abdomen, 222.

Acantline, 175, 206.

Accroissement cellnlaire, 1 s, 19.

Acetabularia, 42.

Achromatique (réseau), \%.

Achromatiques (filaments), 30 .

$$
1 \%
$$
(substances),

Arénine, ¿1.

Adorale (zone), 405.

Arsimilation, 26 .

Albumineuses (substances), 16.

Albuminoïdes (substances), 16.

Algues calcaires, Lis3.

Alvéolaire (tléorie), 7.

$$
\text { - (structure), } 7 \text {. }
$$

Alvéoles, 7, 375.

Anidon (grains $\mathrm{ll}^{\prime}$ ), $(i 1$.

Amitose, 27.

Amphiaster, 31.

Amphipyrénine, $15,16$.

Anaphase, 27, 32, 3t.

Androgronidies, 370 .

Animal pôle), 37.

Anisospores, 19ז.

Inisotropes (organes), 49'.

Ammeau (des Monopylaires), 216.

Anses chromatiques, 29, 31 .

Auses jumelles, 32, 33, 36.

Apposition (des Grégarines), 267.

Archésjore, 280.

Archoplasma, 11, 13.

Aréolaire (théorie), s.

Aléoles, 8.

Iscaris, 5'.

Assimilation, 1s, 19.

Association (des Héliozoaires), 160.

Ister, 6, 11, 30 .

Attraction à distance, ว̀l.

Attraction sexuelle, 51.

Attractive (sphère), 11.

Ixonème, 493.

Axopodes pseudopodes), 171, $20 \%$.

\section{B}

Bacillus amylobacter, io.

Bactéries, $6 \tilde{\text { Jे. }}$

ballast, 40 .

Bandelette buceale des Monades), 325.

Bâtonnet, 391.

liotactisme, 2?.

Biréfringents (graumles), ‘0s.

lilastogenese, 1'́.5.

Bothridinm, 42 .

louche, 101.

Burrgoonnement des Opalines, 4.5.

- les Hetérotriclides, 5)7.

- des Irpotrichides, „or.

- des IIolotrichides, 507.

Brillants (corps), 91, 98, 100 .

\section{C}

Calcium (chlorures et phosphates, 15.

Calotte, 3ิs\%.

Calymna, 1:0.

Canaux des Foraminifères, $49,51,147$.

- du test, 151 .

- convergents, 14\%.

- méridiens, 147 .

- spirats, 151.

Cancer, 301.

Capillitium, ś.

Capités (teutacules), 50.3.

Capitulum des Monopylaires, 216.

Capsule cellulaire, 14 .

- contrale, 15\%, 170.

- nucléaire, $15 \%$

- polaire, 293.

Capsules, 304.

- (sécrétion des), 13, 1', 211.
Capture d'une proie (Héliozoaires), 159.

Carapace, 20.

Cellulaire (corps), 17.

- (membrane), 13.

Cellule, 3.

(plaque), 34.

Cellulose, 15.

Centrosome, 5, 11, 31, 46, 52, 279.

Céphalin, 260.

Champ frontal, 45', 461.

- polaire, 9, 32.

Clitine, 15, 75.

Clilorophylle, 92, 360, 518 .

Chlorures, 15.

Cholestérine, 15, 16, 17, 20.

Chromatine, s, 10, 15, 16, 17.

Chromatique (réduction), $4 \dot{4}$.

Chromatoïdes (granules), 279.

Chromophiles (grains), 502.

Chromoplastes, $61,376$.

Chromoplastiques (lames) 356 .

Cliromosomes, 9, 29, 31, 46.

- (permanence des) 36.

- rapports avoc les filaments) 29,36 .

Chrysochrome, 356.

Chylema, S.

Cils endoraux, $\$ 71$.

Cils gélatineux, 207 .

Cils paroraltx, 460, 471

Cirres, 430, 472.

Cirrhose, 300, 301.

Closterium, 42.

Coccolithes, 68.

Coccosphères, 68 .

Collerette, 328, 491,

Columelle, 87, 224.

Cônes antipodes, 31,32 .

- d'attraction, 31, 32, 51 .

Conique (mouvements de), translation et de rotation), 308.

Conjonetive (fibre), 20.

Conjugaison, 4, 40, 41.

Conjugaison (épidémies de), \{2. 
Conjugaison mucléaire, 41, Dispermie, 54. 43.

Conjngaison partielle, 41, 43.

Conjugaison totale, 41.

Connectifs (filaments), 33.

Contractiles (fibrilles), 92.

Copulation, 41.

Coque, 13, 84, 175.

Coquille, 13.

- des Foraminifères, 14.

Cordon, 28.

Cordon dorsal, 151.

Cornicules, 472.

Corpuscules réniformes, 290.

Corpusculeuse (maladie), 297.

Corps celluleuse (division), 36. - intermédiaire de Flemming, 34 .

Corps réfringents, 99 .

Coryza des foins, 339.

Couche corticale, 30.

Coupole des Cœeloplegma, 250.

Crête aliforme, 376 .

Cristaux, 171, 173, 198.

Crochets, 472.

Croissant (formes en), 288.

Cuticule, 13, 14, 92.

Cuticules (sécrétion des), 20.

Cyclose, 22.

Cytes, 48, 49.

Cytodes, 10.

Cytologie, 4.

Cytophane, 492.

Cytoplasma, 5, 17, 46, 59, 89.

- lomogène, 6 .

- péricapsulaire,171.

- (protectiondu), 6

Cytoplasmiques (modifica-

tions), 49.

\section{D}

Darier (maladie de), 300.

Dauercysten (Ciliés), 417.

Dauersporencysten (Sporozoaires) 302.

Dégénérescence sénile, 40.

Dermatoplasma, 14.

Désassimilation, 18, 19.

Deutolécithe, 20.

Deutomérite, 257.

Dextre (spire), $45 \dot{y}^{\prime}$.

Diaphragmée (bouche), 223.

Diarrhées, 323, 339.

Diatoméenne (strueture), 245.

Dictyota, 42.

Dimorphisme des Foramifẻres, 118, 141, 150, 158,

Directeurs (globules), 5 . .

Direction du plan de division (loi de), 36.
Dispirème, 33,34 .

Disque des Trichodines, 491.

Division, 19, 26, 103, 104 .

- cellulaire (théories sur la), 38.

- directe, 27, 37.

- indirecte, $2 \%$.

- réductrice, 45.

- simple à l'état libre, 64 .

- directe et indirecte (relation entre les), 3 .

\section{$\mathbf{E}$}

Echinodermes, 54 .

Eetocarpus, 42.

Ectoplasma, 13, 60.

Egesta, 25.

Elastique (fibre), 20.

Emission du globule polaire, 58.

Encliylema, ?.

Endoplasma, 60.

Endoplaste, 410.

Endoplastule, 410

Endospore. 263.

Entocyte, 258.

Entonnoir des Trichodines, 491.

Enveloppes, 30 i. - gélatineuses des Protozoaires, 11.

Eperon, 387.

Epicyte, 258.

Epimérite, 256.

Epispore, 263.

Eponges, 153.

Eponges (spicules d'), 130 .

Epuration chromatique, 58. nucléaire, 55.

Etisie, 297.

Etranglement cervical, 222.

Excitabilité, 19.

lombaire, 2?2.

Excreta, 12, 21, 90.

Excrétion (produits d') externes, 19.

(produits d') internes, 19.

Extracapsulaires (corps), 199.

\section{F}

Falciformes (corps), 281.

Fèces, 92.

l'écondation, 4, 44, 61.

Fer (combinaison organique), 15.

Ferments, 20.
Fibres conjonctives, 20.

- élastiques, 30.

Fibrillaire (structure), 6.

Fibrilles, 5.

- contractiles, 92.

Fievre tierce, $28 \%$.

Fièvre quarte, 288.

- quotidienne, 288.

- du Texas, 300.

Filaire (substance), 6 .

Filament axile (Iéliozoaires), 158.

45.

(du spermatozoïde),

Filaments achromatiques, 30 .

- connectifs, 33

- unissants, 31 .

Filarsubstanz, 6.

Filoplasmodium, 79.

Flagellum, 303.

Fleurs du tan, 87.

Fragmentation nucléaire, 37.

Frein des Cœloplegma, 250.

Fucus, 43.

F'nsean, 6.

- central, 30, 31, 36 .

- (origine des tilaments (iii), 35.

\section{G}

Gaîne protoplasmique (spermatozoïde), 45.

Galles des plantes aquatiques, 76

Gamètes, 41 .

Gastrique (suc), 20.

Gattina, 29\%.

Gélatinense (couche), 14.

Gélatineux (cils), $20 \%$.

Gelée, 171.

Germinales (cellules), 45.

Girandia, 42 .

Glanzkörperchen, 91.

Glas funèbre de la cellule, 38.

Globules abortifs, 55 .

- directeurs, 55 .

- graisseux, 90

- polaires, 46, 47, 162 .

- polaires (théoriorles), 55.

Globulines, 15, 16.

Glycogène, 20.

Gonies, 48 .

Gouttelettes huileuses, 61 .

Grains d'assimilation, 61 .

- de chlorophylle, 92

- d'excrétion, $61,90,96$.

- réfringents, 113.

Granulaire (structure), 7 .

Granulations, $\%$. 
Granulations élémentaires, 90 .

Granules, 7, 10, 15.

- chromatoïles, 279.

- graisseux, .6 .

Guanine, 21. plasmatiques, 279.

Gubernaculum, 420 .

\section{$\mathbf{H}$}

Hémoglobine, 20.

Hémoglobinurie, 300.

IIermaphroditisme protérandrique, 370.

Hernie du chou, 76 .

Herpes zoster, 300.

Hétérogamie, 11

Hétéroplastides, 41, 57 .

Holophytique (alimentation), 349.

Homogène (structure), 6 .

Homoplastides, 41,57 .

ILouppe postérieure, 9'́.

Huiles, 20.

IIyaloplasma, $5,(;, 15), 17,406$.

Hydrocorallines, 153.

Hypostome, 463.3.

\section{I， J}

Imago, 268.

Inclusions endosplasmati yues, 90.

Ingesta, 25.

Incubatrice (cavité), $500^{3}$.

Intermédiaire (corps), 3 i. (plaque), 34.

Intersquelette des Foraminifèros, 145 .

Intracellulaire (squelette), 20.

Intussusception, 1', 26 .

Isogamie, 41, 368.

Isospores, 198 .

\section{$\mathbf{K}$}

Karyogamie (des Hẻliozoaires), 162.

Karyokynèse, 27, 109, 120.

Kítryophane, 492.

Kératine, 15, 16.

Kinoplasma, 6, 13, 49, 50, 5́.

Kystes, 70.

- colomiques, 2i\%.

- de protection, 70 .

- de repos, 70.

- durable, 72.

Kystique (nembrane), 14 .

\section{L}

Labyrinthique (tissu), 13'.

Lahyrinthiques (chambros), 132.

Larmes, 20.

Lécithine, 15, 16, 17, 20.

Lécithiques (substances), 20.

Leucosine, 356 .

Liliacées, 39.

Limite de taille cellulaire, IS.

Linine, 9, 15, 16, 17.

Lizzia, 387.

Loge initiale, 158.

Logettes (des Protozoaires), 1 í.

Loi de Müller, 206.

\section{M}

Macroganietes, $362,366,487$. Macronncléus, 110 .

Macrospores, 42. 199.

- (des Radiolaires),

Macrosporozoïtes des Coccidies, 28.2 . des IIémosporidies, 285.

Magnésium chlorures et phosphates), 15.

Malaria, $28 \%$.

Mèduse, 3i\%.

Megasphère, 118.

Mélanosarcome, 301

Nembrane cellulaire, i, $\lfloor 2$, 13.

- endorale, 471.

-_ liystique des Protozoaires, 1 i.

- nucléaire, 8 .

- préorale, 4i.

- (sécrétion de la), 20.

vitelline, 1't, 15, is.

Nembranelles, 405, 454, 462.

Mérotomie, 427, 464 .

Mésoplasma, 92.

Mésostomum, 278.

Métabolisme, 305.

Métakynése, 31 .

Métapliase, 2\%, 31 .

Microgamétes, 362, 360, 487.

Micronucléus, 410.

Mlierosomes, 90, 406 .

Microsplière, 113.

Microspores (des Radiolaires), 199.

Microsporozoïtes des Coreidies, 28:?
Microsporozoïtes des Hémosporidies, 285.

Miescher (tubes de), 290.

Mitom, 6.

Mitôme, 6.

Mitose, 27, 418.

- chez Englypha, 109.

Molluserm, 300 .

Monères (question des), 65.

Monothalame, 118.

Motilité, 18.

Mouvements cellulaires, 23.

(production des), 19.

Mucus nasal, 20.

Miuller (loi de), 206.

- (vésicules de), 441.

Iusculine, 20.

Myéline, 20 .

Myocyte, 258.

Myonèmes (Ciliés), 456.

(Grégarines), $25 \%$.

Myophrisca, 207, 214, 217.

\section{$\mathbf{N}$}

Nasse pharyngienne, 432, 434.

Nausithoe, 387.

Neige rouge (voir Hrematococcus), 36?.

Noyau, 5, 7, 17, 39.

- à cloison, 411 .

- à fente, 411.

- (contractions du), 41 .

- de Rabl, 9.

- de segmentation, 53.

- (division du), 418, 463 (voir ausi Karyokynèse).

en biscuit, 37 .

- loi de position du , 8 , 30 .

Nucléaire (fragmentation), 37.

- (membrane), 5.

- (segments), 29.

- (sue), 17.

Nucléine, 17.

Nucléiniens (corps); 10.

Nucléique (acide), 17.

Nuclóo-albumines, 15, 16, 17.

Nucléolaire (corps), 10.

Nucléole, 8, 10, 15, 17, 263.

Nucléo-microsomes, 10.

Nucléoplasma, 5.

Nutrition cellulaire, 18, 24 .

- par approximations successives, 16.

(Euf, 46.

Ondulante (nembrane), 耂钅 
Opercule des Monopylaires, 215.

- des Cannopylaires, 238.

Opposition (des Grégarines), 267.

Ovocentre, 53, $27 \%$.

Ovocytes de $1^{\text {er ordre, }} 46$. de $2^{\mathrm{e}}$ ordre, 46.

Orogénèse, 46.

Ovogonies, 46.

Orules, 46.

Ovules abortifs, 5.5.

\section{$\mathbf{P}$}

Paget (maladie de), 300.

Parachromatine, 15, 17.

Paraglycogène, 258.

Paramylon, 61, 346.

Paranucléine, 15, 16.

Paraplasma, 6, 15, 406.

Parthénogénèse, 49.

Parthénogonidies, 370.

Pébrine, $29 \%$.

Pellicule, 13.

Peloton, 13

(des Ciliés), 13, 407.

- làche, 2S, 33.

- segmenté, 29.

- serré, 28.

Périplaste, 13.

Péristome des Ciliés, 403. de Bicosteca, 324.

Péristomienne (aire), 404.

Phreodelles, 239.

Phreodium, 236, 239

Phaeosomes, 38.5.

Phosphates, 15.

Phosplorescence (Noctilu ques), 396.

- (Péridiniens), $37 \%$.

- Radiolaires, 174.

Phycochrome, 442.

Pigments, 20,61 .

Plaque cellulaire, 34.

- en ceinture, $3 \%$.

- équatoriale, 32 .

- intermédiaire, 34 .

- nucléaire, 32.

Plasma cortical, 92, 406.

Plasmatiques (granules), 279.

Plasmodium filamentenx, 77, 79.

vrai, 77.

Plastines, 16, $1 \%$.

Plastiques (granules), 279.

Plastogamie, 162.

Podocone, 215.

Pôle, 31.

- animal, 36 .
Polaire (champ), 9,32 .

- (globule).

- (globule) des Héliozoaires, 16 ?

Polyoastriques (Infusoires), 428.

Polynucléaire (capsule), 196.

Polythalame, 118.

Pores, 135, 375.

Position du noyau (loi de), 8, 36.

Potassium (chlorures et phosphates de), 15.

Production des mouvements (voir mouvements).

Produits cellulaires, 21, 23.

Pronucléus, 52.

Prophase, 22, 27.

Protéiques (substances), 17.

Protérandrique (hermaphroditisme), 370.

Protistes, 65.

Protoélastine, 2.5\%.

Protolécithe, 20.

Protomérite. 25\%.

Protoplasma (circulation du), 22.

Pseudotilaire, 270.

Pseudokyste, 204.

Pseudonavicelles, 264.

Pseudonucléoles, 10.

Psendoplasmodium, 77.

Psendopodes, 22, 59, 60, 61, $89,92$.

lohés, 61 .

- $\quad$ Articulés, 62

I'seudopodienmutterboden, 171.

Pseudostome, 454. 461 .

Psorospermies, 291.

- des Avticulés, 297.

Psorospermose, 300 . folliculaire végé-

I'ylôme, 1 \3. tante, 300 .

Pyrénine, 15, 16, 17.

P'yrénoïdes, :348, 360 .

Pyrogallol, 28s.

Pyxides, st.

\section{Q}

Quadrille des centres, 53.

Quaterne (groupe), 48.

Quene du spermatozoïde, 45.

\section{$\mathbf{R}$}

Rabl (noyaux de), 9.

Rainey tubes le), 290.
Ravisseurs (tentacules), 503.

Réfringents (corps), 99.

Reconstitution du noyau, 33.

Réduction chromatique, 4,48 .

Régénèration, 128, 464.

Reliquat de segmen tation, 267.

Réniformes (corps), 252.

(corpuscules), 290.

Rénovation, 482.

du péristome, 464.

Reproduction cellulaire, 19,26. parthénogénéti que, 58.

Réseau au repos, 33 .

Ressac (nouvement de), 261 .

Réserves nutritives, 12.

Réservoir, 484.

Respiration cellulaire, 18, 19, 21.

Réticulaire (structure), 6.

Rhabrlolithes, 68.

Rhablosphères, 6s.

Rhinocamna, 250.

Rhizidium, 102.

Rotation conique, 30 ?.

\section{$\mathbf{S}$}

Salive, 20.

Sapropliytique (alimentation) $31 \%$

Sarcoblastes, 20 .

Sarcocyte, 258.

Saum, 405.

Schwärmersporencysten, 302 .

Secreta, 21.

Sécrétion (produits de) externes, 19, 20.

- (produits de) internes, 19, 20).

Segment intermédiaire, 45.

Seguentation Iongitudinale, 29.

Sénestre (spire), 454 .

Sexuels (produits), 44.

Sida, 39.

Sociétéde consommation, 160 .

Sodium (chlorures et phosphates), 15.

Spasmonème, 493.

sipermatides, 45.

Spermatocytes de ler ordre, 45.

- $\quad-2^{\mathrm{e}}-45$

Spermatogénèse, 45.

Spermatogonie, 45.

Spermatozoïde, 位.

Spermocentre, 5?.

spiredextre ou sénestre, 454.

Spirème, 28.

Spirogyra, 42.

spironème, 194.

Spirophora, 102 . 
Spongioplasma, 5, 6, 15 .

Sporadin, 260.

Sporenträiger, 387.

Spores a cristaux, 173, 198.

Sporoblaste, 280.

Sporocystes, 73.

Sporoductes, 264 .

Sporozoite, 263.

Sporulation, si.

Squelettes intracellulaires, 20 Stigma, 340, 348, 360, 364.

Styles, 4 iz.

Styliformes (tentacules), 503.

Substance de rebut, 56. - tilaile, 6 .

Suc gastrique, 20.

- nucléaire, 8, 9, 15.

Suceurs (tentacules), 503.

Sucre, 21.

Suturales (perforations), 210.

Syncytium, 41.

\section{$\mathrm{T}$}

Tableau comparatif des clas sifications de Bütschli et cet ouvrage pour les Infusoires ciliés, 35.3 .

Tactiles (soies), 406.

Tactismes, 22.

Tentacules, 503.
Test (canaux du), $15 \%$.

Tête, 222.

- du spermatozoïrle, 45.

Thoracentèse, 301.

Thorax, 2?2.

Translation conique, 309.

Travail cellulaire, 19.

Trépieddes Monopylaires,216.

Trichites, 43'.

Trichocystes, 43?.

Trophoplasma, 6, 7, 50.5'.

Trous vrais, 210.

Tubes de Miescher, 290.

- parasites des insectes, 300.

- de Rainey, 290.

\section{U}

Ulothria, 42.

Urée, 21.

Urnes des Siponcles, 439.

\section{V}

Vaccines, 300.

Vacuoles, 612

- $\quad$ a gaz, 90.

- alimentaires, 61, 62, 90.

d'attente, $32 ?$.
Vacuoles fécales, 61.

- simples, 61.

Varicelle, 300 .

Variole, 300.

Vermiformes (individus), 515.

Vésicule attractive, 30 .

- directrice, 31.

- pulsatile (contraction), 61, 63, 90, $91,15 \%$.

Vierergruppe, 48. germinative, 46.

Vitelline (membrane), 14, 15.

\section{$\mathrm{X}$}

Xanthelles, 172, 207.

\section{$\mathrm{Y}, \mathrm{Z}$}

Zanardinia, 42.

Zone adorale, 405.

Zooamylon, 408.

Zoochlorelles, 408.

Zoocystes, 73 .

Zooïdes, 301.

Zoospores, 73 .

7ooxanthelles, 171, 173, 216.

Zygogonium, 42.

Zygote, 362, 366 . 


\section{LISTE DES HÖTES DES PARASITES}

A

Acanthomètre, 206, 511.

Acéphales d'eau douce (mucus), 458.

Actinies (cavité généralo), 458.

Agrion (intestin), 273.

Alkis, 299.

Aleyonella, 297.

Alosa-sardima, 295, 297.

Amphibiens (voir aussi Anoures), 341.

Amphioxus, 278.

Amphipodes, 278.

Anas (voir canard).

Annelés (cavité génèrale des), 276.

Annélides, 479.

- (cavité générale), 460.

(tube digestif), 451.

marines, 278.

- polychètes (voir polychètes).

Anoures (intestin), 4.8.

Anser (voir Oie).

Aphroloderus, 296.

Arachnides, 278.

Araignées (voir Arachnides).

Arthropodes, 278.

Ascaris (cultures d'), 101.

Ascidies, 276.

- composées, 278.

- simples, 278.

Asellus (branchies), 515.

Astacus, 296.

Audruinia, 276.

\section{B}

Balanus, 276, 278.

Barbeall (voir Barbus flurir(ilis).

Barbus fluvialilis, 296, 297.

Batraciens, 323 .

460

(gros intestin),

Baudroie (voir Lophius).

Bivalves (voir Acéplıales).
Blaps, 299.

Blalla (intestin), 2\%l.

Blemius, 299.

Boeuf, 290.

Bulasia, 2is.

liovis (voir Boenf).

Brussica, 76.

Brochet (voir aussi Esox), 295.

Bryozoaires, 510.

Bufi, 295.

\section{C}

Callotermes, 34t.

Campanulaire, 516.

Canard, 323. 340.

Capitelliens, 276, 278.

('urabus (intestin), 273.

Carcimus, 278.

('uulerpa, 76.

Céphalopodes, 283. - (foie et appendices veineux urinaires), 4.1.

Cervus rapienlus (voir chovreuil).

Cestodes, 278.

Chat, 340 .

Ellamidomonas, rí.

Ch:etognathes, 278.

Chéloniens (voir aussi Tortues), 286.

Cheval, 290.

Chevreuil, 290.

Chou, 76.

Cirraluhus, 276, 278.

Cirripèdes, 278.

Clavellina, 276.

Cobaye, 340.

Cochon, 290.

Cochon d'Inde (voir Cobave).

Coelentérés, 278.

Colcochare, \%5.

Copéporles, 2ix, 3.00.

I'ollux, 296.

Crabe (roir C'areinus).

Crangon, 296.

Crapaud (voir Bufo).

Crenilubrus, 295.

Crottin de cheval, TR, 82.
Crustacés, 2\%8, 510.

- aquatiques (sang) 451.

(branchies et poils), 499.

Cryplops (tube digestif), 272.

Cyclops, 278, 512.

Cycloys phaleralus, 514.

Cyclostoma eleyrns: (intestin et poumon), 492.

Cyphom pallirlus (intestin de la larve de), 271.

Cyprinoïdes, 296.

rypris, 498.

Cystelina' (intestin des larves (les), 273.

D

Décapodes, 2\%.

Diatomées, 80.

Jirzona violacea, 2:6.

Dinoflagellés, 300 .

Diptères, 278.

Diptères (larves), 497.

lliseacelis tigrina, 276, 2is.

Dytique (voir Dyliseus).

Dyliscus (intestin), 273.

E

Exhinocardium, 2\%8.

Echinodermes, 2is, 479.

Echimus, 271, 278.

Ecrevisse (voir 1stecus).

Entomostracés, 298, 300.

Ejhemera (larves), 27.

Epinoche (voir Gusterosteus).

Epistylis, 511.

Equuls (voir Cheval).

Esnr (voir aussi Brochet), 29), 296.

Eutorina, 358.

Eulermes, 343.

\section{F}

Felis domestica (roir Chat).

Femme (vagin), 340. 
Gallinacés，282.

Gillmmirus, $27 R$.

$$
\text { - } \begin{gathered}
\text { (branchies), } 515 . \\
\text { poils), } 480 .
\end{gathered}
$$

Gastéropodes, 283, 496.

(tube digestif) 451.

Cirasterosteus. $295,296,297$.

Cientrupes stercurarius, 269

Géphyriens, 278.

Glomeris, 283.

lilyeere, 276, 278.

(iomphumemu, 71.

Grenouille, 286, 288, 338, 451 .

(irillotalpu, 336, 343, 221.

Ciyrimus (intestin des larves), 273.

\section{$\mathrm{H}$}

II lix, 283.

- (intestin spiral et rein), 282.

Hexopodes (voir Insectes).

Ifolotherin. 278.

Homard (intestin), 269, 27\%.

Hommins, 278.

Homme, 287, 290, 300, 340.

- (cavité pleurale), 300 , 301 .

- (diarhées), 323, 339.

- (intestin), 98.

- (gros intestin), 400 .

- (tube digestif), 323.

Huitre, 3 il.

Hydraires, 510.

IIydre, 674.

IIyelrophiline (larves), 273.

IIyla, 338.

Hyménoptères, 2iR.

\section{I, $\mathbf{K}$}

Infusoires, 300 .

Insectes, 339, 458.

Invertébrés marius, 4i?.

Iulus (intestin), 271.

Ixorles, 300 .

Fienguran, 290.

\section{L}

Lacerta, 286, 336 .

Lacertiens, 286.

Lamellibranches (voir Acéphales).

Lapin, 282, 283.

Lépidoptères, 278, 283.

Lepus cuniculus (voir Lapin).

Leuciscus, 295.
Lézard (voir aussi Lacerta), 286.

Liyidium, 112.

Limace (voir aussi Limux), 340.

Limaçon (voir Helix).

Limax (voir aussi Limace), 283.

Limnobic (larves), 269, 271.

Limnoria (branchies et poils), 480.

Lithobius, 282, 283.

Lombric (testicule), 226.

Lophine, 295 .

Lumbricnnereis, 276, 278.

Lumbricus (cavité générale), 276.

\section{M}

Maldaniens, 2\%8.

Mammifères (gros intestin), 460.

Maqnereau (voir Scomber").

Méduses, 4is.

Melolonthines (intestin), 2i1.

Merítum! us, 295.

Mesustrmulum, 278.

Molll", 283, 295, 297.

Mouches (voir Diptères).

Douton, 290.

I/us decumemus (voir Rat).

Mus meseutus (voir Souris).

Myriapodes, 278, 283, 458.

\section{$\mathbf{N}$}

Neris, 228.

Nebrlic (branchies et poils), 480, 482.

Nématodes, 278.

Vemertes, 276.

Némertiens, 278.

Nepu cinereu, 282.

Neplleys, 276, 278

vereis, 2ib, 278.

Veretinu, 283.

Octrinus, 282, 283.

Oie, 32:3.

Oiseanx, 286, 288, 290.

Oligochètes, 2is.

(intestin), 452.

Omble chevalier(voir Thymallus rulyaris).

Ophidiens, 286.

Ophrydium, 515.

Opisthobranches, 479.

Oryctes nasicornis, 269, 270.

Ostren, 323 (voir aussi Huître).
Otarie, 290.

Oursins, 277.

Oris (voir Mouton).

\section{P}

Pale'mon, 296.

Papillons (voir Lépidoptéres).

Paramécies, 5l1.

Passereanx, 282, 288.

Perca, 296.

Perehe (voir Perca).

Peripatus, 278.

Periplunela, 343.

Phascolosoma, 278.

Pisces (voir Poissons).

Piscicolu, 323.

Plagiostomes, 296 .

Planaires, 278.

Planaires (intestin), 452.

Poissons, 282, 283, 323, 435.

I'olliciues, 278.

Polychètes, 276.

Pontobdell", 323.

Poule, 323.

Poulet, 283.

Poulpe (voir Octoprus).

$\mathbf{R}$

Radiolaires, 300 .

Rainette (voir //ylu).

liuna (voir anssi Grenouille), 295.

Lana esculenta, 286.

Rat, 340.

Reptiles, 339 .

Rotifères, 350 .

Ruminants (panse des), 13\%, $439,448,449,467$.

\section{$\mathbf{S}$}

Subellu. 276.

Suyillu, 276, 278, 300 (voir aussi Chretoguathes).

Salamandre, 288.

Salicoque (voir Typton), 257.

Salpes, 278.

Sapphirina, 276, 278.

scomber, 295.

Scoriuena, 295.

scyllium, 295.

Soiche (voir Sepia).

Sepria, 282, 283.

Serpents (voir Ophidiens).

Serpula, $276,278$.

Silpha (intestin), 273.

sipunculus, 276, 278 .

Souris, 290, 340.

spio. $276,278$. 


\section{LISTE DES HOOTES DES PARASITES}

spiragyra, 80, 81.

Spirula, 75.

Stanirocephalus, $276,278$.

Silentor, 511.

Sicholonche, 252, 511 .

Stylonichies, 511.

Succinea, 283.

Suceurs (voir Tentaculifères).

Sus (voir Cochon).

Synapta, 276, 278.

Syngnothus, 295, 296.

\section{$\mathbf{T}$}

Tan, 87.

Tapes, 323 .
Taupe-grillon (voir Grillotalpa).

Tenebrionines, 2\%2.

Tentaculifères, 511 .

Terebella, 276.

Termes, 344.

Thymallus vulgaris (voir Omble chevalier), 296.

Thysanoures intestin), 271.

Tipula oleracea, 269, 271.

Tortues (voir aussi Chéloniens), $283,323$.

Trématodes, 278.

Triton, 282, 283, 288.

Truite, $33 \%$.

Tizulla, $33 \%$.
Tubifex, 276, 278.

Tuniciers (voir aussi Ascidies), 278.

Typtom spongicola, $25 \%$.

\section{V}

Ver de farine (voir Tenebrionines).

- de terre (tube digestif), 458.

- (testicule), 267, 276.

Vers, 2 i8.

Vertèbres, 283.

Vorticellines, 511, 512. 


\section{INDEX GENERIQUE \\ DES PROTOZOAIRES}

CONTENANT LLS PRINCIPIUX SINONIUES ET L'INDICITION DES PARASITES

Les noms de groupes sont en gros caractères, les noms de genres en petits caractères, les synonymes entre parentheses, les parasites sont marqués d'un astérisque.

\section{A}

(Acantharia, Häckel) $=$

Acantharida vel Actipilida 176, $204=$

(Acantharia, Iläckel; Actipylea,

Häckel; Acanthometrea, Ilertwig Acanthochiasma 214

Acanthocorys 227

Acanthocyrtoma 227

Acanthocystis 162,167

Acanthodesmia 220

(Acanthodesmida, Hertwig) $=$ SpyAcantholonche 209 [roidie

(Icantholonchida, Ilïckel)= Ican[tholonchine

(Acanthometra, $A u \in t.)=$ Acanthometron

(Acanthometrea, Hertwig) $=$ Acan[tharida Acanthometron $208=$ Acanthometra, Auct.; Acanthonia 208 [Astrolithiun, Iläckel)

(Acanthonila, Iiickel $)=$ Acantho[nidie

Acanthonide207,208= Acanthonida, [Häckel]

$\Lambda$ canthosphæra $182=$ (Rhaphidococcus, lläckel; Rhaphidosphæra, lläckel)

* Acanthospora 273

*Acanthosporina $273=($ Acantlospo-

Acanthostaurus 209 [rides, Léger)

$($ Acarella, Cohn $)=$ Mesodinium

${ }^{*}$ Acephalina vel Monocystina 269, 274

(Acervulina, M. Schultze $)=$ Planorbulina

(Acidophorus, Stein) $=$ Nassula

(Acineria, Maupas) $=\Lambda$ mphileptus

Acineta $508=($ Autacineta, Iräckel $)$

Acinetina, auct. = Tentaculiferix Acinetina $510=(p . p$. Acinetina,
(Acinetoides, Plate $)=I$ Iypocoma Acinetopsis 509

(Acomia, lujardin) =Glaucoma Acontaspis 210

Acontractilia 198

(Acotrypus, Rïist $)=$ Podocampe

${ }^{*}$ Acrasis $\pi 8$

Acraspedina 320

Acrobotrys $236^{\circ}$

Acrocubus 221

Acrosphera $203=$ (Pyrosolenia, Ehrenberg)

Acrospyris 234

Actinastrum 214

(Actinelida, Iläckel) $=$

Actinelide $207,213=$ (Actinelida, Actinelius 214

[Häckel)

Actinobolus $436=$ (Ciliotentaculifères) *Actinocephalina $275=$ (Actinoce*Actinocephalus 273, 269 [phalides, Léger)

Actinocyathus 509

Actinocyclina 152

Actinolophus 165

Actinomma 183

Actinomonas $16 \mathbf{b}^{4}$

Actinophrys $15 \%, 162,164$

Actinosphæridium 165

Actinosphærium 157, 165

Actinotricha 470

(Actipvlea (IIäckel) $=$

Actipylyda vel Acantharida 176, 204 Actissa lis [Voir Acantharida)

*Acystis $288=$ (Karyophagus, Steinhaus; Cyto[phagus, Steinhaus) Acystosporida $66=$ (Amobaa reti[culosa, Bütschli) (Acyttaria, Häckel $)=p \cdot p$. Rhi${ }^{*}$ Adelea 282 [zopodia $($ Adelocyrtis, Pantanelli $)=$ Dicolocapsa didelosina 123 
Adinida 381

Lgospyris $23 \hat{i}=$ (Ceratospyris, Ehrenberg;

['T'riospyris, lläcliel)

Egyria $4\{3=$ (? Trichopus, Claparède et Lach[mann; Glenotrochilia, Diesing)

(Ethalium, Link) $=$ Fuligo.

Ethaliopsis 86

(Agathistegia, d'Orbigny) = Milio[lide

( glenophrya, liesing $)=$ Frontonia

Alacorys 230

(Ilastor, P'erty) $=$ Kerona

(Alderia, Pritchard) = Ephelota

(Allodorina, Fromentel $)=11$ ematococcus Allomorphina 138

Allotricha 475

Alveolina $118,126=$ (Borelis, Montfort; Clausulus, Montfort; Melonia, Lamarck; Melonites, Lamarck; Mliliolites, Montforl);

Alveolinina 127

(Alyscum, 1)ujardin) $=$ Cyclitium

(Amastiga, Diesing $)=$ Cilix

Amaurochete $8 \gamma$

( 1 mblyophis, Ehrenberg = Euglena

(Amiba, Bory $)=$ Dileptus

Ammodiseus 133

Amceba $98=$ (Corycia, Dujardin; Trichamceba, Fromentel; Lithamoba, Lanliester; Ouramceba, Leidy; Proteus, (Amoloxa, auct.) $=\quad$ Rösel; Amobix $89=$ (Amobxa, auct.; Amœbilæ, Häckel; Amœbina, Dujardin ; Gymnomoneres, Hä$($ Amœbidæ, Häckel $)=\quad[$ cke] $)$ (Amøbina, Dujardin) $=$ Amobix *Amœbidium $298=$ (Raphidium, Fresenius; [Exosporidies, Perrier

"Amobogenia 255, 291

* Amoebophrya 206, 252, 511

Amocbosporidies 299

Imphiactura 188

Amphibelone 209

I mphibrachium 18?

Amphicoryne 137

Amplicraspedım 189

Amphicyclia 188

Implidinium 386

Amplidloma 382

Amphileptus $440=$ (Acineria, Maupas)

Amphilonche 209

(Amphilonchida, Iläckel) $=$ Amphilonchina $209=$ Amphilon[chida, Iliickel

Amphimonadina, Kent) $=$
Amphimonadinæ $326=$ (Amphimo[nadina, Kent)

Amphimonas $326=$ (Deltomonas, Kent)

Amplimorphina 137

Amphiplecta 226

Amphipyle 193

Amphipylonium 193

Amphirrhopalum $\mathbf{1 8 9}$

Amphisia $475=$ (Eschaneustyla, Stolies)

Amphisolenia 385

Amphisphera 180

Imphispyris, 235

Amplistegina 136,151

(Amphistomata, Hertwig et Lesser) $[=$ Amphistomine

(Amphistomina, Bütschli) $=$ Amphistomine $116=$ Amphistomata, Hertwig et Lesser; AmphistoAmphistylus 180

Amplitholonium 193

A mphitholus (Radiolaire) 193

Amphitholus (Flagellè) 384

Imphitrema 116

Amphizonella 100

* Imphorella 273

( $\mathrm{m}$ mphorina, d'Orbigny) = lagena

Amphymenium $189=$ (0)matogramma. Ehrenberg)

(Amygdalina, Seguenza $)=$ Lagena

* Ancliorina 276

Ancistrum 448

Ancyromonas 323

* Ancyrophora 273

(Andromedes, Montfort) $=$ Polystomella

(Androspyrida, Häckel) $=$

Androspyrina $235=$ (Androspy-

Androspyris 231

Anhymenia 491

[rida, Iläckel)

Anisonema $353=$ (Plœotia, Dujardin)

(Anisonemina, Kent, Klebs) $=$ Anisonemin:e $353=$ Anisonemina,

Inomalina 144

[Kent, Klebs)

* Anophrys 450

(Anopisthia, Ehrenberg) = Dexio-

* Inoploplirya 451, 452

[trichidae

*Anthocephalinie 272

* Anthocephalus 271

(Anthocorys, llïckel) $=$ Phormocampe

Antlocyrlidium 227

Inthocyrtis 227

Anthocyrtium 227

Anthophysa $325=$ (Dimastix, Diesing; Ster[reonema, Kützing; IVella, Lhrenberg), Anthospyris 234

(Anthusa, Montfort) $=$ Polymorphina 
(Apgaria, Slokes $)=$ Blepharisma

*Aphelidium 75

Aphrosina 145

(Aphrothoraca, Ilertwig) $=$

Aphrothoracida 16?, 163= Aphrotho-

[raca, Ilertwig]

(Aphthonia, Perly) $=$ Pleuronema

(Apiopterina, Zborzewslii) $=$ Lagena

(Apiosoma, Wandollek $)=$ Pirosoma

(Apiosoma, Blanchard) = Rhabdostyla

(Aporea, Bailey $)=$ Rhipidodendron

(Arachnidium, lient) = Mesodinium

Irachnocalpis 22.5

Arachnocorys 227

Arachnopegma $18: 3$

Arachnopila 183

(Arachnopilium, Häckel) = Pteropilium Arachnosphæra 183

A rachnula 163

Arcella 103

$\triangle$ rcellina $106 ;$

Archadiscus $152=($ Archoodiscus, Brady $)$

Archrocyathellus 154

Archaocyathus 154

(Archatiscus, Brady) $=$ Irchadiscus Archeospherina 15\%

(Archais, Montfort) = Orliculina

Archerina 6.5, 163

A reliacina 126 ;

Archibursa 224

Archicapsa 225

Archicircus 219

Archicorys 22:)

Archidiscus $18 \mathrm{~s}$

(Irchimedes, IIudson) = Stichotricha

Archipera 221

Archiphana 225

A rehiphormis 2:5

Alchipilium 224

Archiscenium 224

Arcuothrix $[01$

A reyria 86

Arhabdomonas $320 ;$

(A risterigina, d'orbigny) = Rotalia

(Aristerospira, lihrenberg) = Truncalulina

(Arthronia, Ilill) $=$ Oxytrichine Articulina 124

Artiscus 18.;

Arlocapsa $2: 32$

[thocampinæ

(Artocapidie, Häckel $)=p \cdot p$. Li(Artocorida, Häickel $)=p \cdot p$. LithoArtodiscus 168

Arlopera 231

Artophana 232

Artophorm is 232

Artopilium 2:31=(Makropyrgus, Iäckel ; StyArtostrobus 232

Aschemonella 131
(Ascobius, Ilenneguy $)=$ Folliculina Ascoglena 350

(Asellicolla, Plate $)=$ Stylocomètes Askenasia 439

Aspidisca $477=$ (Tribulina, Bory; Monostylus, [Pereiaslavzeva; Ratulus, Bory)

(Aspidiscina, Stein) $=$

Ispidiscinse, $478=$ (Aspilliscina,

(Aspidomma, Iläckel $)=$ Astrolonche

(Aspidospira, Fhrenberg) = Truncatulina

(Aspirotricha, Bütschli) $=$ Para-

Assilina 136,151

Assulina 112

Astasia 347

(Istasiida, Klebs) $=$

Astasina $346=$ (Astasiida, Klebs)

Astasioides 347

Astasiopsis 347

(Isteracites, Schlotheim) $=0$ rbitoïdes

Asterigerina 1't

Isterocyclina 152

* Asterophora 273

* Asthmathos 338

$$
\text { (Astoma, Siebold) = Flagellia }
$$

Astomina, 337, 338= Holomastigini ,

Istractura IsS

Astrocapsa 212

[Lauterhorn

(? Astrococcus, Greeff) $=$ Sphærastrum Astrocyclia 188

Astrodisculus 166

(Istrodiscus, F. E. Schulze) $=$ Astrorhiza

(Istrolithium, IIäcliel) = Acanthometron

Istrolonche $208=($ Aspidomma, Irickel)

(Astrolonchida, Hä̈ckel) $=$

Astrolonchinæ $208=$ Astrolonchi[da, Hïckel)

(Astrolophida, Itïkel) $=$

Astrolophinæ $214=$ Astrolophida, Astrolophus 214

[lläckel)

(Astromma, Ehrenberg) = Cypassis

Astrophacus 188 = (Chilomma, Ehrenberg)

(Astrophormis, Ifitckel) = Sethoformis

Astrorhiza $128=$ Astrodiscus, F. F. Schulze

[Ireckelina, Bessels)

(Astrorhizina, Brady) I28 [Brady) Astrorhizinie $129=$ Astrorhizina,

Istrosestrum 188

Astrosiga 332

Astrosphara $18 \%$

(Astrosphærida, Häckel) $=$

Astrosphærinæ I83 $=$ Astrosphe-

Astylozoon 496 [rida, Häckel)

(Atactodiscus, IIäckel $)=$ Porodiscus

(Atractolina, Schlich $\mathrm{t})=$ Polymorphina 
Atractonema 347

Aulacantha 242

(Aulacanthida, Iläckel) $=$

Aulacanthina 242 = Aulacanthi-

Aulactinium 211 [da, IIiicliel)

Aularia 243

Aulastrum 243

Aulatractus $2: 3$

Auloceros 242

Aulodendron 242

Aulodictyum 243

Aulographis 242

Aulonia 243

Aulophacus 243

Auloplegma 243

Auloscena 243

Aulospathis 242

Aulosphæra 243

(Aulosphærida, Häckel) $=$

Aulospharin: 24:)= (Nulosphæri[da IIäckel)

(Aulostomella, Alth $)=$ Polymorphina

(Autacineta, lläcliel $)=$ Acineta

(Autochloë, Joseph) =Zoothamnium

Axellipsis 184

Axocorys 230

Axodiscus 189

Axoprunum 184

(Azoosporea, Zopf) $=$

Azoosporida $69=$ (Azoosporea, Zopf)

\section{B}

${ }^{*}$ Babesia 300

Badhamia 86

Balanitozoon 436

(Balantidion, Eberhard) = Enchelys

${ }^{*}$ Balantidiopsis 459

${ }^{*}$ Balantidium 459

*Balbiania 290

Balladina $4 \% 6$

+ Bananella 283

${ }^{*}$ Barroussia 282

Bathropyramis 225

Bathybius 65, 68

Bathysiphon 130

(Baum, Eichhorn) = Carchesium

Bdelloïdina 132

Belonaspis 212

Belonostaurus 209

Belonozoum 202

(Benedenia, Föttinger) $=$ Opalinopsis

(Benedenia, Aimé Schncider $)=$ lílossia

Bicosœca 324

Bifarina 140

Bigenerina 139

(Bikcecina, Stein) $=$

Bikocina $324=$ Bikacina, Stein)
Biloculina, 118, 123 = (Lagenula, Flemming; [Pyrgo, Defrance]

(Blanchardia, Vierzejski) = Serumspori[dium

Blcpharisma $458=$ (Apgaria, Stokes; T'richomecium, Fromentel = I psistoma, Bory; Plagiotricha, Bory; Porpostoma, Möbius)

Blepharocysta 382

Blepharostoma, 435

Boderia 67

${ }^{*}$ Bodo $335=$ (Diplomastix, lient; Heteromita, Dujardin; Isomita, Diesing; Spiromonas,

Perty

(Bodonina, Bütschli emend.) $=\mathrm{He}-$ [teromastigidie

Bodoninæ $336=$ (Bodonina, Bülschli; [Ileteromitide, Kent)

Bolivina 140

(Borelıs, Montfort) $=$ Mlveolina

Botellina 131

(Botellus, Moniez) = Serumsporidium

${ }^{*}$ Bothriopsis 275

(Bothrostoma, Stokes) $=$ Pleuronema

Botryocam pe 230

Botryocella 236

(Botryocortys, Ehrenberg) $=$ Pylobotrys

Botryocyrtis, 236

(Botryila, Häckel) $=$ Botryoüdæ

$($ Botryda, Iläckel $)=$ Botryoïdœ

(Botryoidea, Häckel $)=$

Botryoüdse 216, $235=$ (Botryida, Iläckel; Botryda, Häckel; Polycyrtida, Iläckel; Botryoidlea, Iläckel)

Botryoptera 235

Botryopyle 230

Brachiospyris 233

${ }^{*}$ Brachycystida $255=$ (Cytosporidia, Bradyina 134

Brœckella 126

Brøeclina 126

Buccinosphæra 203

Bulimina 140

Buliminae 140

Bursaria 460

(Bursarina, Perty, Bütschli) $=$ Bursarinae $461=$ (Bursarina, Perty,

${ }^{*}$ Bursulla 71

${ }^{*}$ Bütschlia 437

[Bütschli

\section{C}

(Cadarachnium, IIäckel $)=$ Sethoconus (Cadium, Bailey) = Lithogromia (Crenomorpha, Perty) = Gyrocorys (Calcanthus, Montfort) = Polystomella (Calcaria, Gruber) = Gyrocorys

Calcarina 1 行 = (Pleurotrema, Elirenberg; 
Siderolina, d'Orbigny; Siderolithes, Lamarck; Siderospira, Ehrenberg

(Calceolus, Diesing $=$ Urocentrum

Calcituba 121

(Calia, Werneck) = Phalansterium

Calix, Fraipont $)=$ Solenophrya

Callimitra 226

Calocylas 230

Calpophina 225

Calyptotricha 450

(Camerina, Bruguière $)=$ Nummulites

Caminosphæra 203

Campanella 497

(Campanelle, Colombo $)=$ Vorticella

Campascus 112

Camptonema 165

Candeina 1 t3

Cannartidium 186

Cannartiscus $186^{\circ}$

Cannartus $186^{\circ}$

Cannobelos 241

(Cannobotryida Iläckel =

Cammobotrynae $236=$ Cannobo-

Cannobotrys 235] [tryida, Häckel)

Cannocapsa 212

Cannopylea, Häckel =

Cannopylida 176, 236= Cannopylea, Ilïckel: Phieodaria, Häckel; Tripylea, R. Hertwig; Pansolenia, ${ }^{*}$ Cannopilus 242, 372

(Cannor'rhaphirla; Iläckel) =

Canmorrhaphinae $241=$ CannorrhaCannorrhaphis 2'1

Cannosplicera 2:í

Cannosphirida, Iläcliel -

Cannospharrina $241=$ Cannosphir-

Cantharospyris 23:3 Lridia, IIiickel

Cantharus, Montfort $=$ Polymorphina

Capitellina 137

"Carchesium $4 \%=$ (Baum, Eichhorn)

Carpenteria 1'́t = (Raphidodendron, Nöbius

Carpocanistrum $225=$ Lithocarpium, Stöhr

Carpocanium $227=$ (Crytoprora, lihrenberg)

Carposplicera 180

Carteria $362=$ Polyselmis, Dujardin)

Carteria, Brally = Saccamina

Carterina $1: 3 g$

(Caryolithis. Erhenber $\mathrm{q}$ ) = Prunulum

Caryomma 18:3

Caryosphiera 181

Caryostylus I\&0

Cassidulina 1 io

Cassiduline $140=$ Cassidulina,

Castanarium 21.

[Braily
Castanissa $24 \overline{3}$

Castanopsis 245

Castanura 245

Catallacta, Hïckel $=\quad$ L'kel)

Catallactice 31 s, $398=$ Callacta, II ï-

(Catharia, leidy) = Iyalosphenia

Catinulus 211

Caunopora 153

Cecryphalium 230

Cenchridium, Ehrenberg) = lagena

Cenellipsis 184

Cenocapsa 212

(Cenodiscida, Häckel) $=$

Cenodiscinæ $187=$ Cenodiscida, Hä-

Cenodiscus 187

Cenolarcus 192

Cenosphara $179=($ Cyrtidosphwra, Häckel)

Centrocubus 183

(Centropyxis, Stein $=$ Difflugia

Centrospira, lläckel $)=$ Porodiscus

Cephalina 269

(Cephalopyramis, Iläckel $)=$ Sethopyramis

Cephalorhynchus 435

Cephalospyris $2: 33$

Cephalothamnium 326

Ceratestina 151

Ceratium (Iyxomycete) si [Diesing); Ceratium Flagellé) $383=$ Dimastigoaulax,

(llirundinelld, Bory de $s^{t}$ Vincent)

Ceratocorys 38. ,

Ceratocyrtis, Bütschli $)=$ Sethoconus

Ceratomyxa 29.5

Ceratophorus, lliesing) = I'eridinium

(Ceratospirulina, Elirenberg) $=$ Vertebra-

Ceratospora 276

Ilina

Ceratospyris, Lhrenberg) = Aegospyris

Cercobodo, hirassilstschili) = Dimastiga-

[moeba

Cercomadina, lient emend.) -

Cercomonalina: $323=$ Cercomona-

Cercomonas $323 \quad$ [lina, Kent emend.)

Ceriaspis 210

(Ceriosphera, Ehrenherg) $=$ Ifeliosphara

Certesia $47 \%$

Chænia 435

(Chetoglena, Ehrenberg) = Trachelomonas Chetomonas, Ehrenberg) = IJexamitus

Chietophlya, Ehrenherg) = Trachelomonas

Chætoproteus $99=($ Dinamœba, Leidy)

Chetospira, Lachmann) = Stichotricha

Chalarothoraca, Hertwig et Lesser) = Chalarothoracida $162,167=$ Chala-

[rotharaca, Ilertwig et Lesser)

Challengeria $245=$ (I'rotocysiis, Wallich)

Challengeron 245

Chiasmatostoma $\mathbf{4} 40$

Castanidium 238, 244 
(Chiastolida, Häckel) $=$

Chiastoline $214=$ Chiastolida, IläChiastolus 214

(Chilifera, Bütschli) $=$

Chiliferinæ $447=$ Chilifera, Büitschli) (Chilocineta, Diesing) $=$ Iypotri[chida

Chilodon $442=$ (Euodon, Ehrenberg)

Chilodontopsis 442

(Chilomma, Ehrenberg) $=\Lambda$ strophacus

Chilomonas $35 \%=$ (Plagiomastix, Diesing)

Chilostomella 138

Chilostomellida $136,138=$ Ciryptos-

Chitonastrum 189] [tegia, Reuss

(Chlamydococeus, Braun) = Ilematoccus

Chlamydodon 443

(Chlamydodonta, Bütschli) $=$

(Chlamydorlontide, Kent) $=$

Chlamydodontinx $411=$ Chlamydodonta, Bütschli; Chlamydo[dontidæ, lient

Chlamydomonarlina 354,360

Chlamydomonas $362=$ (Allodorina, Fromen[tel; Diselmis, Dujardin)

(Chlamydomonas, Cohn) $=$ Polyloma

Chlamydomyxa 82

(Chlamydophora, Archer) =

Chlamydophorida 162, $166=$ (Chla-

[mydophora, Archer)

(Chlamydophrys, Cienkovsky) $=$ Platoum Chlorangium 362

Chloraster 363

Chlorodesmos 359 [nomorum, Ehrenberg)

Chlorogonium $362=$ (Dyas, Ehrenberg; Gle-

Chloromonadina 354

*hloromyxina $296=$ Chloromy-

${ }^{*}$ Chloromyxum 296 [xides, Thelohan]

(Chloropeltis, Stein) = Lepocynclis

(Choanoflagellata, kent) $=$

Choano-flagellina vel Craspedina 320, $327=$ Choanollagellata, Kent; Discostomatagymnozoïda, Kent; Craspedomonadina, Stein ; Cylicomastiges, Bütschli)

(Chonia, Gourret et Roser) = Chienia

Chøenicosphiera, 203

Chondrioderma 87

Chondropus 166

(Chonemonas, Perty) $=$ Trachelemonas

Chromatella 65,100

[phthirius

(Chromatophagus, lierbert) $=$ Ichthyo-
Chromomonadina 354,355

(Chromophyton, Voronin) = Chromulina Chromulina $35 \%=$ (Chromophyton, Voronin ; Chrysomonas, Stein)

Clırysalidina 139,140

Chrysamieba $35 \%$

Chrysococeus 358

(Chrysolus, Mlontfort $)=$ Nonionina

(Chrysomonadina, Stein) $=$ Chry[somonadina

Chrysomonadina $357=$ (Chrysomo[nadina, Stein)

Chrysomonas, stein) $=$ Chromulina

Chrysopyxis 358

*(Chydridema, Ioniez $)=$ Serumsporidium (Cibicides, Ehrenberg) = Truncatulina

Cienkorskya $16 \%$

[liata, Perty) Cilie $40 \mathrm{I}=($ Amastiga, Diesing; $\mathrm{Ci}$ (Ciliata, Perty) = Cilix

Cilicomasliges, Bütschli $=$ ChoaCiliophrys 163 [no-flagellina

Ciliolentaculiferes) $=$ Actinobolus

Cimsenomonas, Grassi $)=$ Trichomonas

Cinclopyramis 225

Cinetochilum 148

Circogonia 2y

Circoporida, Häckel $=$ [Häickel Circoporina $247=$ Circoporida, Cireoporus 217

Circorrhegma 247

Circospathis 246

Circospyris 231

Circostephanus 247

Circotympanum 2:21

(Citharina, d'Orbigny) = Vaginulina

Citharistes 385

Cittarocyclis 467

Cladarachnium 22.5 $=$ (Cystophormis, Hickel)

Cladococeus 182

Cladomonas 327

$($ Cladonema, Kent $)=$ Dendromonas $[$ pince

Cladophracta 211$)=p p$. DoratasCladopyxis $383=$ (Xanthidium, Ehrenberg)

Cladoscenium 224

Claparedia, Diesing) $=$ Epiclintes

Clathrocanium $2: 26$

Clathrocircus 220

Clathrocorys 226

Clathrocyclas 230

(Clathrocysta, Stein $)=$ Protoceratium

Clathrodictyum 153

Clathrolychnus 227

Clathromitra 226

Clathroptychium 85

Clathrosphara 205

Clathrospyris 231 
Clathrulina $168=$ (Podosphæra, Archer)

(Clausulus, Montfort) $=$ Alveolina

Clavulina 140

*Clepsidrina 270, 269 [Légrer

(Clidostomum, Elurenter

Climacammina 140

Climacostomum 461

Clistophæna $2: 28$

${ }^{*}$ Cnemidospora 271

$($ Cobalina, Perty $)=$ opalina + Leuco[phrys + lierona + I'lagioloma

(Coccidiidie, Leuckart) $=$

"Coccidida 255, $278=$ Coccidiidie, Leuckart, $p . p$. Gregarinida,

[Bütschli

*Coceidium 283 = (Cytospermiun, Rivolta; [Orthospora, Schneider)

(Coccochloris, Sprengel) $=$ Ophrydium

Coccocyclia 188

(Coccodiscida, Iläckel $)=$

Coccodiscinie $189=$ Coccodiscila,

Coccodiscus 188

Coccolarcus 192

Coccomonas 363

(Coccudina, Bory) $=$ Euplotes

Cochliopodium 102= Cyphidium, Ehrenberg

Codonella $467=$ Petalotricha, lienI: Cyttaro-

Codonocladium 332

[ryclis, I)aday)

Codonodesmus 332

Codonceca 32'

(Codonocila, Kent $)=$

Codonoecine $324=$ Codonocida,

(Codonosiga, Stein $)=$ Codosiga

Codosiga $332=$ Colonosiga, Kent)

[Kent)

Culacantlıa 244

Colodasea 249

Colodccas 250

(Cœlodendrida, IIäckel) =

Coelollendrine $249=$ (Coelodendrida, Iläckel)

Cœlodendrum 249

Cœlodoras 219

Colodrymus 2i?

Calogalma 250

Cœlographida, Häckel $=$ Colographins

Cœlographins $250=$ (Colographi(la, lläckel)

Coelographis 250

Coelomonas 350

Coloplegma 249

Culospatliis 250
Cœlostylus 250

Ccelothamnus 250

Cœlothauma 250

Colotholus 250

${ }^{*}$ Cononia 78

(Coenostroma, Nichulson et Murie) = Stromatopora

Colacium 350

Coleaspis 213

${ }^{*}$ Coleophora 275

Coleps $438=$ Diceratella, Bory; Craterina, Bory ; Cricocoleps, Diesing; Pinacoleps, Diesing; Dictyocoleps, Diesing)

(Collodaria, Häckel) = Thalassicollidie + Collozoida + Thalassospharidae + Spharoida

Collodictyon 339

Collodiscus 202

Colloidea 202

Colloprunum 202

Collosphiera 203

(Collospharida, J. Müller, Brandt) = Collospherina

Collosphicridæ $203=$ Collospharidea, J. Miïller, Brandt emend.; Sphreroidea, Häckel)

(Collosphieridea, J. Müller), Brandt emend. = Collospharidic

Collosphærinæe $205=$ (Collosphierida, J. Müller)

Collozoida, Iläckel $)=$

Collozoïdæ $201=$ Collozoida, Ïӓckiel

Collozoum 201

Colpidium $4 \dot{4} 0=$ Doyerius, Ormancey; Tillina, Stokes)

Colpoda $t^{\prime \prime}=$ Kolpoda, Still

*Colpodella if, iti

Colponema $3: 36$

Comatricha 87

(Condrachnium, Iläckel) - Sethoconus

Concharida, Häckel $)=$

Concharine $248=$ Concharida, Häckel

Concharium 248

Conchasma 248

Conchellium 248

Conchidium 2 :8

Conchoceras 248

Conchonia 2 is

${ }^{*}$ Conchophthirus $458=$ (Peripheres, Carus)

Conchopsis 248

Condylostoma $460=$ hondyliostoma, Bory)

Coniocyclis, $\mathbf{f u l}=$ Tintinnopsis 
${ }^{*}$ Conorhynclius 2i1

Conosphæra 182

Contraclilia 496

Conulina 137

(Conulites, Carter) $=$ Patellina

${ }^{*}$ Copromyxa 78

Corethria, Pritchard $)=0$ phryodendron

Cornuspira 126

Cornutanna 225

Cornutella 225

Cornuvia 80

Corocalyptra 229

\section{Coronida, Häickel $)=$ Coroninie}

Coronidium 220

Coronince 2:21 $=$ Coronida, Iäckel

Coronosphara 204

Cortina 219

Cortinelta 245

Cortiniscus 219

*Corycella 273

(Corycia, Dujardin) $=$ ? Amreba

Coscinaspis 210

Coscinomma 182

Coscinospira, Stuart $=$ Globigerina

Coscinopora, Ehrenberg) = Peneroplis

Coskinolina 132

${ }^{*}$ Costia 337

Cothurnia $499=$ Stylocola, Fromentel; Thuricolopsis, Stolies; Limnias, Goldfuss;

Cotlutrnina $499 \quad$ [Planicola, Fromentel)

Cothurniopsis $499=$ Tubularia, schrank

Cranotherielium 43.)

Craspedarium, Ilill $)=$ Vorticella

Craspedina vel Choano-llagellida 316 , $327 \quad$ Crasperlomonadina, Stein; Cylicomastiges, Bütschli; Choano-llagellata, Stein; Discosomala grmmozoida, kent

Crasperlomonadina, Stein $=$ CrasCraterina, Bors $=$ Coleps $\quad$ [pedina

Craterium 86

Crateromorpha, P'ereiaslavzeva) = Euplo-

+ Cretya 283

Cribraria 85

Cribrospira 14 í

Cricocoleps, biesing) $=$ Coleps

* Ciristallospora 283

Cristellaria $137=$ Hemirobulina, Staclıe; Lenticulina, Lamarck; Lenticulites, La marck; Nummularia, Sorby; Saracenaria,

[Defrance Hemicristellaria, Stache) Crommyomma 183

Cromostylus, Ilickel = Stylocromyum

Cromyatractus $1 \$ 5$

Cromyechinus $18 ; 3$

Uromyocarpus 185

Cromyodruppa 185

Cromyodrymus 183
Cromyosphæra 180

Cromyostaurus 181

Cromyostylus 180

Crucidiscus, Häckel 187

(Cruciloculina, d'Orbigny) = Triloculina

(Crumenula, Dujardin) = Euglena

Cryptocapsa 228

(Cryptocephalus, IIichel) = Eucyrtidium

(Cryplochilum, Maupas) $=$ Lronema

Cryptoglena 350

(Cryplomonadina, Bütschli emend. Cryptononadina $357=$ Cryptomonadina, Bütschli emend. Klelss)

Cryplomonas 357

(Cryploprora, lihrenberg) = Carpocanium

(Cryplostegia, Reuss) $=$ Chilostomellidie

(Ctedoctema, Stokes) $=$ Cyclidium

Cubaxonium 182

Cubosphiera 182

Cubospharida, Häckel) $=$

Cubospherine 182 $=$ (Cubospherida, IÏ̈ckel)

Cubotholonium 19'

Cubotholus 1\%'

Cuneolina 139

Cupulites, d'orbigny) = Orbitolites

Cyathomonas $357=$ Goniomonos, Stein)

Cycladophora 230 (Santerna, Bury)

Cyclammina 134

Cycleorlictyina $154=$ Tapinia, Perty

Cyclirlium 317

Cyclidium $100=$ Alyseum, I)ujardin; Citedoctema, Stokes; Districha, Fromentel)

Cyclochicta 491

Cyeloclypeinie 15\%

Cycloclypeus 151

Civelocrinus 15.t

Cycloeyrrha 492

Cyclogramma 4 \&2

Cyclolina, d'Orbigny) $=$ Orbitolites

(Cyclosiphon, Ehrenberg) = Orbitoïles

+ Cyclospora 2E3

[pedina 327

Crlicomastiges, Bütschli $=$ CrasCymbalopora 1't $=$ (liosalina, d'Orbigny)

Cypassis $186=$ (Astromma, Ehrenberg)

Cyphanta $180^{\circ}$

Cyphidium, Ehrenberg) = Cochlyopodium

Cyphinida, Iläckel = Cyphinina Cyphinidium $18 f=0 \mathrm{mmatospyris,} \mathrm{lihren-}$ [herg) Cyphinince $186=$ (Cyphinida, II:̈[ckel) Cyphinus $186=$ (0mmatospyris, Ehrenberg) Cyphocolpus 186

Cypholeria $112=$ Lagynis, M. Schultze 
Cyphonium $186^{\circ}=$ (Didymocyrtis, IIäckel)

(Cypridium, Ken $\mathrm{t}$ ) = Dysteria

(Cyrtiłosphara, Häckel) = Cenosphæra

(Cyrtocalpida, Häckel) =

Cyrtocalpine $226=$ Cyrtocalpida,

Cyrtocalpis 225

Cyrtocapsa 232

(Häckel)

[ckel)

Cyrtoide 216, 222 = Cyrtoilea, Ilä-

(Cyrtoilea dithalamia, Häckel) $=$ [Dicyrtoidea

(Cyrtoidea trithalamia, Häckel $)=$ [Tricyrtoidea

(Cyrtoidea polythalamia, II:̈ckel) $=$ [Stichocyrtoidea

(Cyrtolophosis, Stokes) = Lembus

Cyrtopera 231

Cyrtophormis 225, 232

(Cyrtostomum, Stein) $=$ Frontonia Cystidium 217

(Cystobia. Mingazzini) $=$ Urospora * Cystocephalus 272

(Cystoflagellata, Iläcliel) $=$

Cystoflagclliae $318,389=$ Cystoflagel[lata, Ilickiel)

(Cystophormis, Iläckel) = Claılarachnium (Cystophrys, Archer 116) = Microgromia

* Cytamoula 28s

(Cytodiscus, Lütz) = Spharomyxa ${ }^{*}$ Cylomorplia 276

(Cytophagus, Steinhaus $)=$ Acystis

(Cytospermium, Rivolta) = Coccidium

(Cytosporidia, Labbé) = Brachy[cystida

(Cyltarocyclis, Dadlay) $=$ Codonella

\section{D}

Dactylophora, 2\%:

'Dactylophorinæ 272 = (Dactylopho[rides, Léger)

(Dactylopora et les Dactyloporides) ne sont

${ }^{*}$ Dactylosaccus 130

Dactylosoma 288

Dactylosphiera, 99

Dactylospherium 99

Dallasia $460=$ (Diplomastax, Stokes; Diplo-

Dallingeria 337

[mestoma, Stolies)

*Danilevsliya, $286=$ (Ilemogregarina, Lani-

*Dasytricha $\$ 49$

(Daucina, Bornemann) $=$ Lingulina

(Decteria, Perty) $=$ Trachelinæ

(Deltomonas, Kent) $=$ Amplimonas
(Dendrella, Bory) = Zoothamnium

(Uendritina, d'Orbigny) = Peneroplis

Dendrocircus 219

${ }^{*}$ Dendrocometes 514

(Dendrocomelina, Stein) $=$

Dendrocometinæ $515=$ (Dendroco-

[metina, Stein)

(Dendromonadina, Stein) $=$

Dendromonadine $326=$ (Dendro[monadina, Stein)

Dendromonas $325=($ Cladonema, Kent $)$

Dendrophrya 129

Dendrosoma 513

(Dendrosomina, Bütschli) $=$

Dendrosomine $515=$ (Dendroso-

Dend rospy ris $2: 33$

Dentalina 1303,137

[mina, Bütschli)

Dentalinopsis 137

${ }^{*}$ Dentrotuba 130

(Desmarella, Kient $)=$ Ilirmidium

Desmartus 187

Desmocampe 186

Desmospyris 23'

ser) $=$

(Desmothoraca, Ilertwig et Les-

Desmothoracila $164,168=($ Desmo[thoraca, Hertwig et Lesser)

Dexiotricha, Stolies $=$ loxocephalus

Dexiotrichidæ $479,483=$ (Anospisthia,

Ehrenberg; Ophrydina, Ehren-

berg + Vorticellina, Ehrenberg)

Diachea 87

Diaphoropodon 115

Dicella 459

(? Diceras, Éberharl) = spathidium

(Diceratella, Bory), = Coleps

(Dicercomonas, Grassi) = Ilexamitus

Dichilum 4 i6

Dicolocapsa $228=$ (Alelocyrtis, Pantanelli)

Dicranastrum 190

Dictyaspis 212

Dictyastrum 189

Dictydium 85

Dictyocephalus 228

Dictyoceras 229

*Dictyocha 241, 372

Dictyocodon 229

(Dictyocoleps, Diesing) $=$ Coleps

Dictyocoryne 191

Dictyocrinus $15 \mathrm{t}$

Dictyocysta 467

Dictyomitra 232

Tictyophimus $226=$ (Lamprotripus, Häclel;

(Dictyoplegma, IIäckel) = Spongodyction

Dictyopodium 229

(Diclyoprora, IIäckel) = Encyrtidium 
(Dictyosoma, J. Müller) = Spongodyction

Dictyospyris 231

${ }^{*}$ Dictyostelium 78

Dictyostroma 153

\section{(Dicyrtida, Häckel $)=$}

Dicyrtoidea $223,226=$ (Dicyrtida, Ilïckel; Cyrtoidea Dithalamia, Ilä-

[ckel)

Didinium 433, 439, = (Ecclissa, schrank; WaDidymium 87 [gneria, Alenitzin)

(Didymocyrtis, Iläckel) = Cyplınium

Didymophies 269, 270

*(Didymophyida, Stein) $=$

*Didymophyina $270 \overline{\overline{ }}$ (Didymo-
[phyida, Stein)

Difflugia $104=$ (Centropyxis, Stein ; Echino-

[pyxis, Claparède et Lachmann)

(Digitalina, Bory) $=$ Epistylis

(Digitophrya, Fraipont $)=$ stylocometes

Dileptus 433, $440=$ (Amiba, Bory; Phragel[liorhynchus, Herrick)

Dimastigamoba $322=$ (Cercobodo, liras-

(Dimastix, Diesing) $=$ Anthophysa [silstschik)

(Dimastigoaulax, Diesing) = Ceratium

Dimorpha 32:

Dimorphina $137=$ orthoceratium, soldani ;

(Dimorphus, Grassi) = Megastoma

(Dinamcba, Leidy) $=$ Chætoproteus

Dinema 352

*Dinennympha 344

(Dinifera, Bergh) $=$

Diniferida 381, 382=(Dinifera, Bergh)

Dinobryon 358

(Dinoflagellata, Bütschli) $=$

Dinoflagellix $318,373=$ (Dinoflagel-

Dinomonas 326

[lata, Büitschli)

Dinophrya $436=$ (Siagonophorus, Eberhard)

(Dinophysida, Bergh $)=[$ Bergh)

Dinophysinæ $385=$ Dinophysida,

Dinophysis 385

(Dinopyxis, Stein) $=$ Exuvixlla

Diophrys $477=$ (Styloplotes, Stein; Schizopus, Claparade et Lachmann

Diplactura 188

(Diplagiotricha, Bory) $=$ Epiclintes

Diplocolpus 213

[nine

(Diploconida, Häckel $=$ Iiploco-

Diploconinæ $214=$ Diploconida,

Diploconus 213

Diplocyclas 230

[Ilïckel]

Diplocystis 168

Diplodinium 468

Diplodorina 368

(Diplomastax, Stokes $)=$ Dallasia
(Diplomastix, Kent) $=$ Bodo

(Diplomestoma, Stokes $)=$ Dallasia

Diplomita 327

${ }^{*}$ Diplophrys 81, 116

*Diplophysalis 74

Diplopsalis 382

Diplosiga 333

Diplosphera 183

*Diplospora 283

Diporaspis 210

Dipospyris 2:33

* Discella 112

Discoida $177,187=$

(D) iscoidea, lläckel $)=$ Discoidae

(Discerea, Morren) = Iæmatococcus

${ }^{*}$ Discocephalus, Sporozoaires 273

Discocephalus, Ciliés 478

Discocyclina 152

Discophrya, Suct. $509=$ (Lada, Veydorsky

${ }^{*}$ Discophrya, Cil. $452=$ (Haptophrya, Stein)

Discopyle 190

Discorbina $14^{\prime} t=$ (Discorbites, d'Orbigny)

(I)iscorbites, d'Orbigny = Discorbina

(Discospira, $p \cdot 1$.Stöhır $)=$ Perichlamydium

(Discostomata-gymnozoida, Kent) - Choanoflagellina

Discozonium 190

(Diselmis, Dujardin) = Chlamydomonas

(Disoma, Ehrenberg) = Enchelys

${ }^{\star}$ Distephanus 241, 372

Distigma 347

(Distomata, lilebs) $=$

Distomina $337,340=$ (Distomata,

Distriactis 187

(Districha, Fromentel) $=$ Cyclidium

Ditrema 116

Dizonium 193

Dodecaspis 211

*Dolichocystida 255, 289

*Doliocrstis, $2 \pi$.

Doracantha 208

(Dorataspida, Iläckel) $=$

Dorataspinie $211=($ Dorataspila,

Dorataspis 210

[Häckel)

(Dorbignyina, IIagen) = Ilaplophragmium

Dórcadospyris 233

Dorypelta 211

Doyerius, Ormancey) $=$ Colpidium

*I)repanidium (Sporoz.) $286=$ =(llwogregarina,

Drepanidium (Cilié) 174

[Danilevsky)

(1)repanoceras, Stein) $=$ Drepanomonas

Drepanomonas $4 \mathbf{4}=$ (Drepanoceras, Stein)

(Drepanostoma, Engelmann) $=$ Loxodes

Drimosplicera 183

Druppatractus 185

Druppocarpus 184

Druppula 184 
(Druppulida, lläckel) $=$

Druppulinie $185=$ (Druppulida,

Orymospliara 183

* Dufouria 275

[Häckel)

(Dujardinius, (Ormancey = P'hacus)

(I)yas, lihrenberg) = Chlorogonium

Dysteria $4 \dot{t}=$ (Cypridium, Kent; Ervilia, I)ujardin; Gasterochoeta, Dujardin; Hux-

l)ystympanium 221

[leya, Claparede et Lachmann)

\section{E}

* Ebria 372

$[-$ Vorticella

(Ecclissa, Schrank $)=$ Didinium + Stentor (lichaneustyla, stokes) $=\Delta$ mphisia

Echinactura 189

Echinaspis 211

(Echinella, Agardlı $=$ Ophrydium

* Echinocephalus 272

Echinomma 183

(Echinopyxis, Claparèle et Lachmann) =

${ }^{*}$ Ectobiella 76

[Difflugia

Ehrenbergia 140

Ehrenbergius 443

likenia 100

* Limeria 282

${ }^{*}$ Eirmocystis 271, 26?

Elarorhanis 65,166

Elaphococcus 182

Elaphospyris 23' $=$ (Girattospyris, IIäckel

Elaster 168

Elatomma 182

Ellipsactinia 153

(Ellipsida, Iläckel) = Ellipsinæe

Ellipsidium 184

Ellipsinie $184=($ Ellipsida, Häckel $)$

Ellipsoidina 138

Ellipsostylus 18t

Ellipsoxiplius 184

(Elphidium, Montfort = Polystomella

Elvirea 337

(Enallostegia, d'Orbigny) $=$ Textularidae [rostomida $p . p$.

(Enantiotreta, Ehrenberg) = Gym(Encheliua, Ehrb. emend. Stein)= Enclielina $435=$ Enchelina, EhrenEnchelyodon $435 \quad$ [berg emend. Stein)

Enchelys 435, 433 = Balantilion, Eberhard ;

*Endosphara 511

[Disoma, Ehrenberg)

(Endosporés) 87

Endothyra 133

Eudothyrima $1: 33$

*Endyonema 71

Enerthenema s7

(Enneaphormis, Iläckel) = Sethophormis
Enteridium 85

Enteromyxa 72

(Enterozoon, Häckel) = Haliphysema

Entocannula 245

Entodinium 468

(Entomostegia, d'Orbigny), voir

Entosiphon 353

Entosolenia 137

[Orljiculina

Eophyllum, Hahn 15.) $=$ Eozoon

Eozoon $155=$ Eophyllum, Hahn

Ephelota $509=$ (Alderia, Pritehard; Ilemiophrya, lient)

[giotricha, liory)

Epiclintes $474=($ Claperedia, Diesing; Dipla-

(Epitricha, Ehrenberg) = Cycli[dium + Peridinium

Epipyxis $3 \pi 8$

[Bory; Ilyrtilina, Bory)

Epistylis $497=$ (Wigitalina, Bory; Mespilina,

(Evilia, Dujardin) $=$ Dysteria

Erythropsis $387,499=$ (Fpastostyla, $($ Entz)

*Esarabdina, 276

Estrella 164

Ethmosphara 780

Eucecryphalus 226

Euchitonia $189=$ (Pteractis, Ehrenberg)

Eucoronis 220

Eucyrtidium 232 = Dictyoprora, Häckel; Cryptocephalus, Häckel; (Eucyrtis, Rüst; 'theocorys, Iläckel)

(Eucyrtis, Rüst) = Eucyrtidium

Eudorina 369

Euflagelliæ $318=$ (Monalina, Bülschli; Euglenina, Stein; Chloromonadina, Ḱlebs; Cryptomonadina, Stein; Chlamydomonadina, Bütschli; Volvocina, Ehrenberg)

Euglena $305,315,349=$ (Furcocerca. Lamarck; Crumenula, Dujardin; = Amblyophis, lihrenberg; Microcystis, Kützing; Lacrimatolia, Bory) [Bütschli emend.)

Euglenida 318, $345=$ Euglinidina, (Euglenida, Klebs) = Euglenina

(Euglenidina, Bütschli) = Eugle[nida

(Euglenina, Stein $)=$ Euflagellic Euglenina $346,348=$ Euglinida, Euglenoplis 352

Euglypha 110

[Klebs)

(Euglyphina, Bütschli) =

Euglyphinie $112=$ Euglyphina,

[Bütschli)

(Euodon, Ehrenberg) = Chilodon

Euphysetta 246

Euplasmodida 83

(Euploea, Ehrenberg) = Euplotes 
Euplotes $47 \%=$ (Ploesconia,Bory; Crateromorpha, Pereiaslavzeva; Coccudina, Bory; Euploea, Ehrenberg; ?IImantophorus, Ehrenberg)

(Euplotina, Ehrenberg) $=$

Euplotinde $477=$ Euplotina, Ehren-

Euscenium 221

*Euspora 271

Eusyringium 232

Eutreptia 350

Eutympanium 221

(Exosporés) 87

Exuviælla $381=($ Dinopyxis, Stein; Pyxidi[cula, Ehrenberg)

\section{$\mathbf{F}$}

Fabrea 464

Fabularia 118, 123

(Faujasina, dOrbigny $)=$ Polystomella

(Filigera, Perty) $=$ Flagellia

Filoplasmodida, 79

Fissurina 137

Flabellina $13 \%$

Flabelliporus $147=$ (Iyogypsina, de Amicis)

(Flageliata, Ehrenberg) $=$

Flagellia, $303=$ Flagellata, Ehren-

berg; Mastigophora, Bülschli;

Astoma, Siebolil; Filigera, Perty)

(Florilus, Montfort) = Nonionina

(Flustrella, Ehrenberg) $=$ Porodiseus

Folliculina $463=$ Ascobius, Henneguy; Freia, Claparède et Lachmann; Lagotia, [Wright; Pebrilla, Giard)

(Foraminifera, d'Orbigny emend.)= Foraminiferiæ $107=$ Foramini[fera, d'Orbiguy emend.]

(Freia, Claparède et Lachmann) = Follicu[lina

Frondicularia $137=$ (Ilucronina, d'Orbigny) Frontonia $446=$ (l'anophrys, Dujardin; Igle-

[nophrya, Diesing; Cyrtostomum, Stein)

(Frumentaria, soldani $)=$ Miliola

Fuligo $86=$ (Ethalium, Link)

(Furcocerca, Lamarck $)=$ Euglena

Fusulina 147

Fusulinella 148

\section{G}

\section{${ }^{*}$ Gamocystis 271}

Gamospyris 233

(Gasterochæta, Dujardin $)=$ Dysteria

Gastronauta 443

Gastrostyla 475

Gaudryina, 14
Gazellelta 246

Gemmulina 139

${ }^{*}$ Geneiorhynchus 275

(Geophonus, Montfort) = Polystomella

Geoponus, Ehrenberg) = Nonionina

Gerda 496

Gervasius 478

(Giraffospyris, Iläckel) = Elaphospyris

Glandulina 137

Glaucoma $446=$ Acomia, Dujardin; Ptyxi-

Glenodinium 3\$3

Glenogonium 368

[dium, Perty)

(Glenomorum, Ehrenberg) = Chlorogium

(Glenopanophrys, Diesing) $=$ Ophryoglena

(ilenopolytoma, Jiesing) = Polytoma

(Glenotrochilia, Diesing) $={ }_{\text {EEgria }}$

(Glenouvella, Diesing) = Synura

Globigerina $14=($ Coscinospira, Stuart; Polydexia, Ehrenberg; Rhynchospira,

[Ehrenberg)

(Globigerinide, 136, 141

(?Globularia, WedI) $=$ Trypanosoma

(Globulina, d'Orbigny) = Polymorphina

Gloidium 65, 99

Glossatella 497

*Glugea 297

(Glyphidium, Fresenius) $=$ Oxyrrhis Goniodoma 382

Coniomonas, Stein) = Cyathomonas

Gonium $368=$ (Tetrabæna, Dujardin)

${ }^{*}$ Gonobia 283

${ }^{*}$ Gonospera 276

Gonostomum 475

Gonyaulax $383=$ ("? Roulea, Gourret)

(Gonyostomum, Diesing) = liaphidomonas

Gorgonetta $246=$ (l'orospathis, Häckel)

Gorgospyris 231

* Goussia 283

(Grammobotrys, Ehrenberg) = Virgulina Grammostomum $139=$ (Vulvulina, d'Orbigny) ${ }^{*}$ Grassia 338

(Gregarina, bufour) $=$

${ }^{*}$ Gregarinida 255, 256 = (Gregarinida, $p \cdot p$. Bütschli; Sporadina + Gre[garinaria, stein

Gregarinida, Bütschli) = GregaGringa $65,99 \quad$ [rinide + Coccidida Gromia 112

Gromida 109

(Gromidea, Claparède et Lachmann) $[=p \cdot p \cdot$ Gromidic

Gromine $116=$ (Gromidea, Clap. et [Lachm.)

(Grymra, Fresenius $)=$ Trepomonas

${ }^{*}$ Guttulina 75

(Guttulina, d'Orbigny) $=$ Polymorphina

(iymnamubie, Ilertwig) = 
Gymnamœbida $89=\langle$ Gymnamœbæ Gymnastes 384

[Hertwig

Gymnococcea is

(iymnococcinie 75

*Gymnococcus 75

Gymnodinium 381

Lbia

(Gymnomoneres, Iiickel $)=\Lambda$ mœ-

(Gymnopharynx, Diesing $)=$ Prorodon

fiymnophrys 65,67

Gymnosphera 15, 165

${ }^{*}$ Gymnospora 282

[rides, Léger

Gymnosporina $270=$ Gymnospo-

(Gymnosporidies, Lahbé) $=$

*Gymnosporidie 255, 286= Gymnos-

[poridies, Lablé)

(iymnostomata, Bülschli) $=$

Gymnostomidie $431=$ liymnosto-

Gypsina 140

Lmata, Büitschli

fiyrocorys $468=$ Calcaria, Gruber; Cono[morpha, stein

(Gyroidina, d'Orbigny) $=$ I'lanorbulina

Ciyromonas 342

\section{$\mathbf{H}$}

(IIabrodon, Perty) $=$ Spathidium

Ilreckeliana $2: \%$

(Ilieckelina, Bessels) $=$ Istrorhiza

II eckelina 65, 165

*IIæmamelsa 287

IIrmatococcus $362=$ (Ilorlorina, Fromentel

Discerea, Morren; Chlamydococcus,

[Braun)

(IIimatomonas, Mitrophanof) = Trypa[nosoma

(IIæmogregarina, Danilevsky) = Drepanidium + Danilesliya + Karyolysus

Ilæmosporilie 255,284

Ilagiastrum 190

IIalicalyptra $22^{5}$

IIalicapsa 22;

Ilaliomma 182

(Ialiommatidium, II äcliel) = Lychnaspis + Haliophrynella 33.

Haliphormis 225

Ilaliphysema $131=($ Enterozoon, Häckel $)$

Ilalteria 465

${ }^{*}$ Ilalteridium 288

(IIalterina, Clap. et Lachm.) = Ilalterinæ $466=$ (Ilalterina, Clapar.

*IIaplococeus 71 [el Lachm.)

IIaplophragmium $132=$ (Proteonina, Will; Ilaplosticlıe 132

[d'Orbignyina, Hagen]

(IIaptophrya, Stein) $=$ Discophrya

(Ilarmodirus, I'erty) $=$ Trachelius llastatella 492

Ilastigerina 143

(Ilauerina, Brady) =

Ilauerinæ $124=$ (Ilauerina, Brarly)

Hedraophysa 324

IJerriocystis 168

(Ielenis, Montfort) = Orbiculina

IIeleopera $106^{\circ}$

(Ielicites, Soldani) = Nummulites

IIelicostoma 4.5

IIeliochona 484

IJeliodiseus 187

Heliodrymus 188

(Ieliophrys, GreeIf) $=$ Nuclearia

Heliosestrum 187

Heliosoma 182

Heliosphara $182=$ (Ceriosphxra, Ehren(Ileliozoa) $=$

[berg)

Heliozoario $156=($ Ileliozoa, IÏ̈ckel)

Hemiareyria 86; [+ Cristellaria

(Itemicristellaria, Stache) = Marginulina

Ilemicycliostyla, Stokes) $=$ Trostyla

(Iemicyclidum, Eberhard) = Microtlorax

Ilemidinium 384

Ilemifusulina 1 is

(Ilemiophrya, Kent) = Ephelota

(Itemirobulina, Stache) = Cristellaria

Hemispeira 492

IIemistegina 152

*Hemosporidæ $284=$ (Ilemosporidies,

*Henneguya 296

[Labbé)

IIerpetomonas $323=($ Leptomonas, Kent; Mo-

Ileterocapsa $3 \times 2$ [nomita, Grassi)

Heteromastigoda, $p \cdot p \cdot$.Bütschli) $=$

Heteromastigidx $319,334=$ (Iletero-

mastigida, IIäckel; Borlonina, [Bütschli emend.

Heteromastigodie, Kent) $=$ IIeteromastix, $5 \Upsilon_{1}=$ (Heteromastigodie, (IIeteromita, Dujardin) = Bodo [Kent) (Icteromitidæ, Kent) = Bodonina Ileteronema 352

Ileterophrys 166

IIeterostegina 151

(Heterotricha,Stein $=$ Polytrichida Heterotrichida $430,453=p \cdot p$. (lleteIlexacaryum 182

Ilexacolpus 213 [rotricha, Stein)

llexacontarium 182

Ilexacontium 182

Ilexaconus 213

Hexacromyum 182

Ilexadendron $18 \%$

Ilexadoras 18\%

llexadoridium 182 
(Hexalaspida, Häckel) $=$

Hexalaspinæ $213=$ Hexalaspida,

Ilexalaspis 213

Hexalastrum 190

Ilexalatractus 230

Ilexaloncharium 182

Ilexalonche 182

Hexalonchidium 182

(Hexamita, Dujardin) $=$ Hexamitus

*Ilexamitus $341=$ (Dicercomonas, Grassi ; Hexamita, Dujardin; Chetomonas, ElırenHexancistra 182

(Ilexaphormis, Häckel) = sethophormis

Ilexaplagia 218

Hexaplecta 218

IJexapyle 190

Ilexaspyris 234

IIexastylarium 181

Ilexastylidium 182

Ilexastylus 181

Ilexinastrum 190

IIexonaspis 213

(?IImantophorus, Ehrenberg) $=$ OnychoHippocrepina 133 [dromus + Euplotes IIrmidium $332=$ Desmarella, Kent; Codono(lesmus, Stein)

ratium

(Ilirundinella, Bory de St-Vincent) $=$ Ce-

(IIisterobalantidium,Stokes) $=$ Pleuronema Ilistiastrum 190

Histioneis 386

(Histosporidies, Labbé) = SarcosIIstrio $4 \% 6$ [poridies + Myxosporidies

Holocladina, 15.5

(Holomastigine, Lauterborn =

IIolophrya 435

Holosticha 474

[Astomina.

(Holotricha, Stein $)=$

Holotrichida $430=$ Holotricha,

*Iloplitophrya 452

*Iloplorhynchus 273

[Stein)

IIormosina 133

(IIuxleya, Claparède et Lachmann) = DysIlyalaspis 211

Ilyalodiscus 99

(IIyalolampe, Greeff $)=$ Pompholyxoplirys

Hyalopus 113,120

Hyalosphenia $104=$ (Catharia, Leidy)

*Hyalospora 271

Ilymenactura 189

IIymeniastrum, $189=($ Stylactis, Stöhr $)=$ [(IIymenocyclus, Bronn $)=0$ rbitoïdes IIymenomonas 358

(IIymenostoma, Stokes) = Lembadion

Ilymenostomidie $430,444=$ (TrichosHynoniastrum 189

IIyperammina 131
${ }^{*}$ Iypocoma $512=($ Acinetoides, Plate $)$

(Hypocomina, Bütschli) =

IIypocominx, $513=$ (IIypocomina, (Hypotricha, Stein $)=$ [Bütschli) Iypotrichida $430,470=$ (Hypotricha, Ilysterocineta 448

Ilystrichaspis 210

[Stein)

\section{I}

*Ichtlyophthirius $435=$ (Chromatophagus, Idalina 124

Iduna 444

[lierlert)

Ileonema 437

(Ilotes, Montfort) $=$ Orbiculina

Imperforida $107=$

(Imperforata, Carpenter $)=$ Imper-

Infusoria 401

Involutina 133

[forida

Ischadites, 154

(Isomita, Diesing) = Bodo

Isoscaspis 211

* Isospora 283

* Isotricha 4 敌

[chinie

(Isotrichina, Bülschli) = IsotriIsotrichine $449=$ (Isotrichina,

\section{J}

[liütschli)

Jaculella 130

*Joenia 34 't

\section{K}

*Karyolysus $286=$ (IIrmogregarina, DaniKaryopliagus 300 [levsky)

(Karyophagus, Steinhaus) $=$ Acystis.

* Iientrochona 482

Keramospluara 127

(Keramospharina, Brady) $=$

Keramosphærinæ $127=$ Keramo[sphærina, Brady)

(Kerobalana, Bory) = Vorticella

* lierona $473=($ lastor, Perty)

${ }^{*}$ Klossia $282=$ (Benedenia, Aime Schneider)

* Kollikeria 270

(Lolpoda, still) = Colpoda

(Kondyliostoma, Bory) $=$ Londylostoma

(Kïnclielia, Künstler) $=$ Cercaire

\section{L}

Labechia 153

${ }^{*}$ Labyrinthula 79,81

[rinthulida

(Labyrinthulea, Ilïckel) $=$ Laby- 
Labyrinthulida $79=$ (Labyrinthulea,

${ }^{*}$ Lacazina 118,124

[Hiickel)

Lathnobolus 86;

(Lacrimatoria, Bory) = Euglena et Lacry-

Lacrymaria 436

[naria

(Lada, Vejdovsky) $=$ Discophrya

Lagena $\mathbf{1 3 6}=$ Amphorina, d'Orbigny; Amygdalina, Seguenza; Apiopterina, Zborzewsky; Cenchridium, Ehrenberg; Tetragonulina, Seguenza; Trigonulina, Seguenza; Obliquina, Seguenza; Oolina, d'orbigny; (Frelina, Ehrenberg; Phialina,

[Costa)

(Lagenella, Ehrenberg) $=$ Trachelomonas

Lagenidie 136

Lagenina 137

$($ Lagenœea, Kent $)=$ Salpingœca

(Lagenophryina, Bütschli) = La[genophryinae

Lagenophryinæ $499=$ (Lagenophry-

${ }^{*}$ Lagenophrys 499 [ina, Bütschli)

(Lagenula, Flemming $)=$ Biloculina

$($ Lagotia, Wright $)=$ Folliculina

(Lagynis, II. Schultze) = Cyphoderia

Lagynus 436

Lampoxanthium 179

Lamprocyclas 230

Lamproderma $s 7$

Lamprodiscus 226

Lampromitra 226

Lamprospyris 235

(Lamprotripus, Hiickel) = Dictyophimus

* Lankesteria $2 \%$

(Lanterna, Bury) = Cycladophora

(Larcarida, Ilïckel) $=$

Larcarins $192=$ (Larcarila, Häckel)

Larcarium 19\%

Larcidium 192

Larcoidie 177, $191=$

(Larcoidea, Hïckel $)=$ Larcoidæ

Larcopyle 192

(Larcopylida, Dreyer) $=$

Larcopylinx $192=$ (Larcophlida,

Larcospira 194

[Hreyer)

Larnacalpis 192

Larnacantha 192

(Larnacida, Häckel) = Larnacinæ

Larnacidium 192

Larnacilla 192

Larnacinx $192=$ (Larnacidir, IÏ̈-

Larnacoma 192

[ckel)
(Laverania, Grassi) $=$ Hamamoba $p . p$. $[+$ lactylosoma + IIaltericlium

Lecquereusia 100

* Lecudina 276

Lecythia $11 \%$

Lecythium $115=$ (Phonergates, Buch)

* Leidyonella 343

Leiotrocha 491

Lembadion $400=$ (Hymenostoma, Stokes)

Lembus $400=$ (Cyrtolophosis, Stokes)

Lenticulina, Lamarck) = Cristellaria

Lenticulites, Lamarck $=$ Cristellaria

(Lenticuliles, Schlotheins) $=$ Nummulites

Leocarpus 86

Lepidocyclina 152

Lepidoderma 87

Lepocinclis $350=$ (Chloropeltis, Stein)

Leptodiscus 397

(Leptomonas, Kent) =IIerpetomonas

* Leptophrys 70

${ }^{*}$ Leptosphæra 182

Leptotheca 295

* Leucophrys 446

(Liehenopora, Reuss) $=$ Polyphragma

Lichnaspis 211

lichnosphæra 183

Licnophora 479

(Licnophorina, Bütschli) $=$

Licnophorinæ $479=$ (Licnopho-

Lieberkühnia 65, 113 [rina, Bütschli)

(Lieberkühnina, Bütschli) $=$

Lieberkuhnina $466=$ (Lieberküh-

Limbladia $85 \quad$ [nina, Bütschli)

(Limnias, Goldfus) $=$ Cothurnia

(Linckia, Wiggers) $=0$ phrydium

Linderina 123

Lingulina $137=($ Daucina, Bornemann $)$

Lingulinopsis 137

(Linza, Schrank $)=$ Stentor

Lionotus 440

(Liosiphon, Ehrenberg) = Nassula

Liosphæra 180

(Liospharida, Iläckel) $=$

Liosphærinæ $181=$ Liospharida,

Liriospyris 234 [IÏ̈ickel

(Litham oba, Lankester $)=$ Amoba

Lithapium 184

Litharachnium 225

Lithatractus 185

(Lithelida, Iläckel) $=$

Lithelinæ $195=$ (Lithelida, Häckel)

Lithelius 194

(Lithobotryida, IIäckel) $=$

Lithobotrynæ $236=$ (Lithobotryida, [Hiickel)

Lithobotrys $236=($ Salpingocapsa, Rüst) 
Lithocampe 232

(Lithocampida, Häcliel) $=$

Lithocampina $232=$ (Lithocam[pida, Häckel)

(Lithocampium, Rïst) $=$ Lithomitra

(Lithocarpium, Stöhr) $=$ Carpocanistrum

Lithochytris $229=($ ? Podocapsa, Rüst)

Lithocircus $219=$ (Monostephus, IIäckel)

Lithocolia 65,166

Lithocubus 221

Lithocyclia $188=($ Stephanopyxis, Bury)

${ }^{*}$ Lithocystis 277

Lithogromia $245=($ Cadium, Bailey $)$

(Litholophida, Häckel) $=$

Litholophinæ $214=$ (Litholophida,

Litholophus 214

Jithomelissa 226

[Häckel)

Lithomespilus 184

Lithomitra $232=$ (Lithocampium, Stöhr)

Lithopera $227=($ Pylospyris, Häckel; Spirido-

Lithophyllium 208

Lithoptera 209

[botrys]

Lithornithium 229

Lithosphærella 166

Lithostrobus 232

Lithotympanum 221

(Litonotus, Vrzesniovsky) $=$ Amphileptus

Lituola 132

Lituolina 128, 132

Lituolinæ 132

(Lobatula, Flemming) $=$ Truncatulina

Loftusia 134

Lonchostaurus 209

Lophoconus 230

Lophocorys 230

Lophocyrtis 230

* Lophomonas 343

Lophophana 228

${ }^{*}$ Lophorhynchus 272

Lophospyris 234

Loxocephalus $447=$ (Dexiotricha, Stokes)

Loxodes $441=$ (Drepanostoma, Engelmann, [Pelecida, Perty)

Loxophyllum $410=$ (Stomophyllum, Lieber-

[kühn)

(Loxostomum, Ehrenberg) $=$ Textularia

Lycea 85

Lychnaspis $211=($ Haliommatidium, Häckel $)$

Lychnocanium 226

Lychnodictyum 226

Lychnosphæra 183

Lycogala 86

(Lycophrys, Montfort $)=$ Nummulites

\section{M}

(Hacrocercus, Hill $)=$ Vorticella

( ladre porites, Deluc) $=$ Orbitolites

Jagosphæra 398
(Makropyrgus, Häckel) $=$ Artopilium Mallomonas 359

[lites)

(Narginopora, Quoy el Gaymard = OrbitoMarginulina $136=$ Hemicristellaria, stache. Marsipella 130

Marsupiogaster 353

Varyna 470

Massilina 123

[zomonas, Kent)

Mastigamœba $321=$ Reptomonas, Kent; Rhi-

(Nastigophora, Bütschli $=$ Fla-

Mastigophrys 322

Mastigosphiera 368

Maupasia 400

llazosphæra 203

lledusetta 246

(Medusettida, Iläckel $)=[$ ckel $)$

Medusettinie 246 (Meduseltida, Hii-

${ }^{*}$ Megastoma $340=$ (Dimorphus, Grassi)

(Ilegatricha, Perty) $=$ Mesodinium

(Melonia, Lamarck) $=$ Alveolina

(Melonis, Montfort) $=$ Moniona

(Melonites, Lamarck) $=$ Alveolina

(Meniscostomum, Kent $)=$ Ophryoglena

Menoidium 347

*Menospora 273

Uenosporinis [Léger)

(Merotricha, Merejkovsky) $=$ Raphido-

Neseres 465

* Nesocmena 241,372

Mesodinium $439=($ Acarella, Cohn: Arachnidium, Kent; Megatricha, Perty)

Mesostigma 303

(Mespilina, Bory) $=$ Epistylis

Metabolica, Perty $)=$ Lacrymaria + Metacincta 509

Trachelocerca

(Metacinetina, Bütschli $)=$ Metaci-

Metacystis 436

Nletanema 353.

(Metopides, Quennerstedt) = Metopus

Metopus $459=$ (Ietopides, Ouennerstedt)

Microcubus 221

(Microcystis, Kü̈zing) $=$ (?) Euglena

Mlicroglena 359

Nicrogromia $113=$ (Cystophrys, Archer)

Micromelissa 227

(Microsporilia, Balbiani $=p \cdot p$. [Myxosporidie

(Microthoracina, Bütschli) $=$ Microthoracine $448=$ (Microthora[cina, Bülschli)

Microthorax $448=$ (Ilemicyclidum, Eber-

${ }^{*}$ Niesclieria, 290

Nikrocometes 143 [hard)

Viliola $122=$ Frumentar [culum, Montagu

(Miliolida, Carpenter emend.) = 
Miliolide $117=$ Agathistegia, d'Orbigny; Miliolida, Carpenter Miliolina 120,124

Milioline 122

Miliolites, Montfort $=$ Alveolina

(Millepora, Pallas) $=$ Polytrema

(Milleporites, Lamarcli $=$ Orbitolites

(Misilus, Montfort $=$ Polymorphina

Nitophora 4.52

Mitra 4 í

Mitrocalpis 225

Monachilum 440

Monadida 318,319

(IIonadina, Bülschli) = Euflagellix

(Ionadina zoosporea, $\mathrm{Zop} f)=\mathrm{Zoo}$ *Monadopsis il

[sporida

Monas $324=$ P'aramonas, Kent; PliysomoMonaster 384

[nas, Kent)

(Monères 49)

Monobia 65, 164

${ }^{*}$ Honocercomonas 339) $=$ Protomyxomyces, Cunningham ; schedoocercomonas, Grassi

[loidea

(Ionocrrtida, Häckel) $=$ MonocyrMonocyrtoidea 223, 224 = (Monocyrtida, Iläckel; Cyrtoidea monothalamia, Häickel) [stina

(Ionocystida, Bü̈lschli = Monocy(Monocystidea, Stein) $=$ Monocy[stina

${ }^{*}$ Ionocystina 269, 274= Acephalina ; Grégarines colomiques; Monocystilie, Bütschli; Monocystidea,

* Monocystis 276

(Monocyttaria, Häckel) $=$

[Stein)

Häckel)

Honocyttarea $176=$ (Ionocrllaria, Monodinium 439

[toilia

(Monodyctia, Ehrbg. $=p \cdot p$.) Cyr-

(Monomita, Grassi) $=$ Herpetomonas

(Monopylaria, Häckel = Monopy-

(Honopylea, R. Hertwig) $=$

[lida

Monopylida $176,215=$ Monopylea, R. Ilertwig; Monopylaria, II ïckel; Nasellaria, Ehrenlere, emend. Bütschli; Osculosa, IIäckel ; Cyrtida + Acanthodesmila, Iiackel)
Monosiga 332

[feria

(Ilonosporea, Schneider) = l'feifMonostegia, d'Orl. = Imperforida

Monostephus, Häckel) = Lithocircus

IIonostomina $337,334=$ Tetrami-

[tina, Bütschli

(Ionostylus, Pereiaslavzeva $=\Lambda$ spidiscus

(Ionothalamia, Schultze) $=p \cdot p$. Monozonium 192 [Foraminiferix

(Mucronina, dorbigny) = Frondicularia

Iulticilia 338

Mycetomyxa 65, 101

Mycelozoarice ir = Myxomvcètes, [auct. + Labyrinthulida

Myelastrum 190

Myrtilina, Bory $=$ Epistylis

Myogypsina, de Amicis) = Flabelliporus Myxastrum i2, 86

*Mxidina $295=$ Myxididées, Thé* Myxidium 295

[lolian)

Myxobolinie $296=$ Myxobolidées, * Myxobolus 296

[Thélohan)

Myxobrachia, Häcliel $=$ Thallassophysa

Myxodictyum 65, 68

[plasmodirla

Itrxomycètes, auct. $75,83=$ Eu-

Myxosoma 295

Nyxosphara 2012

(Mrxosporidia, Bütschli $)=$

Mrxosporide $291=$ Myxosporidia, [Bülschli)

\section{$\mathbf{N}$}

Nassella 217

(Nasselaria, Iläckel) = Ilonopylyda Nassoidie 216, 217

Nassula $441=$ (Liosiphon, Ebrenberg; AciYebela 106

Nematocrslida 291

*Nematoides $2 \pi 0$

Tephroselmis $305,358,364$

Nephrospyris 235

Noctiluca $389,396=$ (Slabberia, Oken)

Nodosaria $136=$ Orthocera, Lamarch; Ortho-

Nodosarina 137

(Nodosarina, Carpenter) $=$

Norlosarina $137=$ Nodosarina, Car-

Todosinella 133

[penter)

Noniona, Hontfort $=$ Nonionina

Nonionina $150=$ Chrysolus, Montfort; Floribus, Montfort; Noniona, Montfort. Placentula, Lamarck; Pulvillus, Lamarck; 
Chrysolus, Montfort; Geoponus, Ehren[berg; Lenticulina, Lamarck)

${ }^{*}$ Yosema $297=$ (Panhystophylon, Lebert)

(Nothopleurotricha, Diesing $)=$ Gastrotricha Nubecularia 122

Nubecularina 122 [discus, Ehrenberg) Nuclearia $163=$ IIeliophrys, Greeff; ? Tricho-

Nummularia, $p . p$. Sowerby $)=$ Cristellaria $[+$ Nummulites

(Nummulella, Carus) $=$ Trichodina

Nummulina 151

Nummulinida, Carpenter emend. [Brady $=$ Nummulitida

Nummulites $136,150=$ (Camerina, Bruguière;

Ilelicites, Soldani; Lycophrys, Montfort; ()robias, Eichwald; Rotaliles, Montfort; Lenticulites, Schlotheim; Nummularia, [Sowerby)

Nummulitida 136, $147=($ NummuliLnida, Carpenter, emend., Brady) Nummulitinie 152

*Nyctotherus 458

\section{0}

(Obliquina, Seguenza) = Lagena

Ochromonas 358

Oetodendron 183

()etopelta 211

(Octophormis, lläckel) $=$ Sethophormis

Oetopyle 193

Oclotympanum 221

Odontochlam ys, 443

[sula

(Odontoholotricha, Diesing $)=$ Nas$[p \cdot p .=$ Gymnostomidæ

(Odontohypotricha, Diesing) $=$ Odontosphara 203

Oiliomonas $323=$ (Spumella, Bütschli)

Oligomastigida $319,320 \quad$ [cididie

(Oligoplastida, Labbé $=p \cdot p$. Coc-

Oligosporea, Schneider) $=$ Cocci-

Oligotricha, Biilschli $=\quad$ [dium Oligotrichidæ, $457,465=$ Oligotricha,

Ommatartus 186

Ommatocampe 186

Ommatodiscus 189

[Bütschli)

(Ommatospyris, Ehrenberg $)=$ Cyphinus +

[Cyphinidium

(Omphalocyclus, Bronn $)=$ Orbitolites

Onychaspis 478

Onychodactylus 443

Onychodromopsis, Stolies) = P'leurotricha Onychodromus $475=$ (? IIimantophorus, *ocephalus 272

[Ehrenberg
(Oolis, Phillips) = Spirillina

${ }^{*}$ Opalina 451

Opalinina, Stein) $=$

Opilinina $451=$ (Opalinina, Stein)

${ }^{*}$ Opalinopsis $45 \mathrm{l}=$ (Benedenia, Föttinger)

Opercularia $498=$ (Valvularia, Goldfuss $)$

Operculina 151

Ophioidina 276

* Ophiotuba 130

(Ophrydia, Bory) $=$ Vorticella

Ophrydina, Ehrenberg $=p \cdot p$. De-

[xiotrichidie

Ophrydium $498=$ (Coccochloris, Sprengel;

Echinella, Igardh; Linckia, Wigger's;

Raphanella, Bory; Tremella, Linnė)

(Ophryocerca, Ehrenberg) = Trachelius

*Ophryocystis, 299

(Ophryodendrina, Stein) $=$

Ophryodendrinæ $516=$ Ophryoden[drina, Stein)

*ophryodendron $515=$ (Corethria, Pritchard)

Ophryoglena $446=$ (Glenopanophrys, Die-

sing; Meniscostomum, Kent; Otostoma, Carter; Sisyriodon, Eberhard)

(Ophryoscolecina, Stein) $=$

*phryoscolecine $468=$ (Ophryos- Ophryoscolex 467 [colecina, Stein)

Ophthalmidium 124

Opisthodon 443

0 pisthostyla 497

Orbiculina $125=($ Archais, Nontfort; Ilelenis,

[Montfort; Ilotes, Montfort)

Orbitolites 118, 120, 125 = (Cupulites, d'Orbigny; Cyclolina, d'orbigny; Nadreporites, Deluc; Marginopora, Quoy et Gaimard; Milleporites, Lamarck; Omphalocyclus, Bronn; Orbulites, Lamarcki)

Orbitoides $152=($ Asteracites, Schlotheim; Cyclosiphon, Ehrenberg; Iymenocyclus, Bronn; Orbitulites, auct.)

(Orbitulites, $a u c l.)=$ Orbitoides

Orbulina $141=$ (Sphærula, Soldani)

Orbulinella 168

(Orbulites, Lamarck $)=$ Orbitolites

(Orcula, Weisse $)=$ Podophrya

Ornithocercus 386

(Orobias, Eichwald $)=$ Nummulites

Orona 242

Orophaspis 210

Oroplegina 243

Oroscena 212

Orosphæra 242

(Orosphærida, Häckel $)=[$ ckel $)$ Orospherine $243=$ Orospærida, Ilä-

(Orthocera, Lamarck $)=$ Nodosaria

Orthoceras, Gualtieri) = Nodosaria Orthoceratium, Soldani) = Dimorphina 
Orthocerina $137=($ Triplasia, Reuss)

orthodon 4 ' $2=$ (Rhabdodon, Entz)

Ortloplecta, 140

(ortlıspora Schneider) $=$ Coccidium

(Osculosa, Ilïckel) = Monopylida + Phicodarida

Otosphæera 204

(Otostoma, Carter $)=$ Ophryoglena

(Ouramoba, Leidy) = Amœeba

(Ovulina, Ehrenberg) = Lagena

(Oxitricha, Bory) = Uroleptus [senius)

Oxyrrhis $305,314,336=$ Glyphidium, FreOxytoxum, $383=$ Pyrgidium, stein)

Oxytricha $476=$ Nteinia, Diesing; Tachy-

(Oxytrichina,Ehrl,.) $=[$ soma, Stolies)

()xytrichinie, $477=($ Arthronia, Hill $)$

\section{$\mathbf{P}$}

Pachycola 499

l'achym mхa 9?

(Pachysoma, Mingazini $)=$ Urospora

Pachystroma 153

Pachytrocha 499

Pamphagus $11 \%$

l'anarium 180

Panartus 186

(Panartida, llïckel $)=$ Panartinie

Panartinæ $186=$ Panarlida, IÏ̈-

Pandorina 102, 368

(l'anhystophyton, Lebert) $=$ Nosema

Panicium 186

(Panophrys, Dujardin) $=$ Frontonia

(Pansolenia, IIäckel =l'harodarida

Pantopelta 211

(Pantutricha, Ehrenlerg) = Trotricha

(Paramecina, Bülschli $)=$

Paramecinæ $449=$ Paramæcina,

Bülschli; Aspirolricha, Bütschli)

(Paramacioides, Grassi) = Trypanosoma

Paramiecium 4i?

(l'aramonas, Kent) $=$ Monas

Parastephanus 22]

Paratympanum 221

Parlieria 134

(1'arrocelia, Gourret) $=$ I'odolampas

Pasceolus 154

Patagospyris 234

Patellina $145=$ (Conulites, Carter)

Paulinella 112

Pavonina 140

(Pebrilla, Giard) = Folliculina

Pectoralina 368

Jeitiada 513

(Pelecida, Perty) $=$ Loxodes

(?Pelekydion, Eherhard) = spathidium

(Pelobius, Greeff) = Pelomyxa
Pelomyxa $100=$ (Pelolsius, Greefr)

(Pelorus, Montfort) $=$ Polystomella

Pelosina 128

Peltierius, Ormancey) $=$ spirostomum

Pellophracta) $211=p$. p. Dor'a(Peneroplida, Brady) $=[$ laspina Peneroplinie $124=$ Peneroplida,

[Brady)

Peneroplis $124=$ Coscinopora, Ehrenberg;

Dendritina, d'Orbigny; Spirolina, d'Or'-

Pentactura 188

Pentalastrum 190

[bigny)

(Pentaphormis, Häckel) = Sethoplormis

Pentas pyyris 2:34

Pentellina 118, 123

Pentinastrum 190

Pentophiastrum 190

Jeranema 352

(Peranemina, Klebs) $=$

Peranemina $346,352=$ Peranemina, (Perforata, Carpenter $)=[$ Klebs $)$ Perforida $135=$ (Perforata, CarPeriarachnium 228

Perichana 86

[penter)

Perichlamydium $189=($ Discospira, $p \cdot p$. Stöhr $)$ Pericometes, Schneider) = Stylocometes Peridinida, Bütschli $=\quad[$ schli Perilininx $385=$ (Peridinida, BütPeridinium $382=$ Ceratophorus, Diesing; Protoperidinium, Bergh)

(Peridiniopsis, Clarlie) $=$ Urocentrum Peridium 224

Periloculina 1:4

Peripanarium $186^{\circ}$

Peripanartus 186

Peripanicium $180^{\circ}$

Periphiena 187

(Peripheres, Carus) $=$ Conchophtirus

Periplecta 218

(Peripylaria, Häckel $)=$ Peripylida (Peripylea, llertwig) $=$

Peripylila $176=$ (Peripylaria, Iliicliel; Peripylea, Ilertwig; spu-

Peripyramis 225 [mellaria, Häckel)

Perispira 435

('erispongidium, Rüst) $=$ Porodiscus

Perispyris 235

(Peritricha, Stein) $=$

Peritrichida $430,478=$ (Peritricha,

(Peritromina, Stein) $=$ Peritromus

Peritromus 45, 473

Perizona 188

Peromelissa 227

(Petalomonadina, Bütschli $)=$ 
Petalomonadina $353=$ (PetalomoPetalomonas 353 Petalopus 100

Pelalospyris 234

(Petalotricha, Kent) = Codonella

*PfeifTeria 282

Phacelomonas, Stein $=$ Spondylomorum (Phacoliscirla, Häckel) =

Phacodiscina $18 s$ - Phacodiscida, Phacodiscus 188

Phacopyle 188

Phacostaurus 188

Phacostylus 188

Phacotus 363

Phacus $350=$ (Dıjardinius, Ormancey)

(Phrnocalpida, IÏickel) =

Phanocalpinx 225 - PhenocalPhenocalpis 225

Phænoscenium 225

Phrocolla 241

(Phicoconchia, Hïckel) --

Phicoconchidie 240, 247 = (PhreoconPheocystidie $240=$ [chia, Hïckel)

Phaocrstina, R. Hertwig = Phao(Phicodaria, Itïckel = [cystidie) Phoodarida 176,236 = Cannopylea, Iïckel; Phæodaria, IIäckel; Pansolenia, Iäckel; Tripylea, R. HertPhicodina 241

(Phcodinida, Häickel $)=$ [Ilïckel) Phæodininæ $241=$ Phrodinida,

(Phrogromia, Häckel) -

Phrogromida 240, $244=$ Phacogro[mia, Häckel)

Phicospharia, Iläckel) =

Phæosphæridæ 240, 242 = (PhæosPhalachroma 385 [phrria, Häcliel)

Phalansterina, kent $=$ Phalansterium

Phalansterium $334=$ (Calia, Werneck)

Pharyngella 24.)

Pharyngosphara 203

P'hascolodon 443

Phatnacantha 208

Phatnaspis 213

('hialina, Costa) $=$ Lagena

* Phialis 273

(Phialonema, Stein $)=$ Urceolus

Philaster 4, $0=$ Haliommalidium, Häckel (Phlebarachnium, Hïckel) = Rethoconus (Phonergates, liuck) = Lecythium

l'hormobotrys 236 ;

Phormocampe $231=$ (Anthocorys, lläckel

(Phormocampida, Iläcke) =
Phormocampine $232=$ Phormo[campida, Häckel)

(Phormocyrlida, lläckel) $=$

Phormocyrtine $230=$ (PhormoPhormocyrtis 230 [cyrtida, Häckel)

Phormospyrida, Häckel) $=$

Phormospyrina 235 = (PhormosPhormospyris 234 [pyrida, Härkel)

(Phorticida, Itïckel) $=$

Phorticina 194 $=$ (Phorticila, lläckel $)$ Phorticium 194

Phractacantha 208

Phractaspis 210

Phractopelta 211

(Phractopeltida, Iräckel) $=$

Phractopeltina 211 = Phracto[peltida, Itäckel)

(Phragelliorhynchus, Ilerreck) $=$ Iileptus phrenocodon 231

Phyalina 436

Phyllomitus 336

Phyllomonas 323

Phylotrichum 443

Physarum 86

(Physémaires, Iläckel) voy. IlalyPhysematium 179

(Physomonas, Kent) = Monas

Phvtoflagellida $318,354=$

Phytomonadina, Blochmann) $=$

* Pileocephalus $2: 3$

[Phytoflagellida

I'ilulina 12 !

Pinaciophora 168

('inacoleps, lliesing) $=$ Coleps

Pinacocystis 168

Pipetta 185

Pipettaria 185

Pipetella 154

${ }^{*}$ T'irosoma $300=$ Apiosoma, Tandollek; PyroPityomma 183 [soma, Smith)

(Placentula, Lamarck) $=$ Nonionina

Placocysta 112

Placopsilina 133

P'lacus 447

P'lagiacantha 218

Plagiacanthida, Hertwig) $=$ PlecPlagiocampa $4 \mathbf{4}$ [toidte Plagiocarpa 218

('lagiomastix, Diesing $)=$ Chilomonas

Plagiophrys 112

Plagiopogon 4.38

Plagiopyla 417

*Plagiotoma 45s

$[\operatorname{mann})=$

Plagiotomina, Claparède ef Lach- 
Plagiotominie $459=$ Plagiotomina [Clap. et Lach.)

Plagiotricha, Bory) = Blepharisma

Plagonidium 218

Plagonium 218

Plagoniscus 218

Plakopus 100

(Planicola, Fromentel) $=$ Cothurnia

Planiplotes 477

Planispirina 123

Planorluulina 14t $=$ Acervulina. Schultze; Gyroidina, d’orbigny; Siphonia, Reuss)

l'lanularia 137

Planulina 14́t

* Plasmodiophora $7 \%$

(Plasmodiophoree, Zopf $=$ Plasmodiophorinie $76=$ Plasmo[diophoreat, Zopf

Platoum 115 = Chlamydophrys, Cienkorsky;

(Platycola, Kent $)=$ Vaginicola [Troglody tes, (iabriel)

${ }^{*}$ Platycystis 276

Platytheca 324

(Platytrichotus, Stokes $=$ Uroleptus

I'lecanium 139

(Plectanida, Iläckel) $=$

Plectanina $218=$ I'lectanida, lläckel

Plectaniseus 218

Plectanium 218

Plectina 140

Plectocoronis 220

Plectoidir 216, $217=$ Plectoidea, [llickel; Plagiacanthida, Ilertwig. Plectoilea, IIäckel = I'lectoille

I'lectophora 218

l'lectophrys 11.;

Plectopyramis $22 \%=$ ('yramis, Bory)

l'legmosphar'a 180

* Pleislophora $29 \%$

P'leuraspis 210

(Pleurites, lihrenberg) = I'olymorphina

l'leurochilidium 4 i\%

Pleuromonas $: 336$

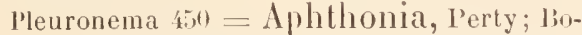
[throstoma, stokes ; Histerobalanti(lium, stokes)

(Pleuronemina, Bütschli $=$ I'leu[ronemina

Pleuroneminie $151=$ (Pleuromina, [Biitschli

(Pleurophrys auct. $)=$ Pseudodiflugia

Pleuropodium 229

Pleurostomella 1 :0

Pleurotrema, Ehrenberg) = Calcirina

Pleurotricha $4 \%=$ (Onychodromopsis,Stokes)

*Pleurozyga 276

(Ploeotia, I)ujardin) $=$ Inisonema
(Ploesconia, Bory) = Euplotes

Podocampe $231=($ Acotrypus, Rüst $)$

Podocampida, lläckel) =

P'odocampinie $231=$ (Podocampida,

[Häickel)

(Pod ocapsa, Rü̈st) $=$ (Lithochytris, Iläcliel)

Podocoronis 221

P'odocyathus 510

Podocyrtida, Iäckel) $=$

Podocyrtinie $229=$ Podlocyrtida,

Podocyrlis 22?

[lläckel)

Podolampas $382=($ Parrocelia, Gourrel $)$

Podophrya $509=$ (Orcule, W'eisse)

(Podophryina, Bülschli) $=$

Podophryina $511=$ Podophryina, [Builschli)

(Podosphæra, Archer) = Clathrulina

Podostoma 101

*Pogonites 2\%?

Polospyris 23'

(Polyblepharidae, Blochmann) 363 l'olyblepharides 361

Polycyrtida, Iackel = Botryoida

('olycystidea, Schneider) =

Polycystina $269=$ Polycystidea, [Schneider)

Polycittarea 176, 195= Polyeyttaria, [llickel; Spherozoidea, Brandi) ('olycyltaria, Iläckel) = Polycyl-

l'olyilexia, Elırb.) = Globigerina [iarea Polydinida :381, 386

Polyoeca 333

Polygonosphierites 15́

Polykrikios 381

Polymastigine, Bütschli $=$

Polymastigidae $337=$ ('olymasti[gin:e, Büitschli, emend. Klebs) Polymastix 310

Polymorphina $137=$ Anthusa, Montforl; Atractolina, Schlicht; Iulostomella, Alth.; Cantharus, Montfort; Elobulina, d'Orbigny; Gultulina, d'Urbigny; Misilus, Montfort; Pleurites, Ehrenlerg; l'olymorphium, Šoldani; Pyrulina, dorbigny; liaphanulina, Zborzevsliy; lienoidea, Brown; Rostrolina, schlicht; strophocoPolvmorphina I3s [nus, Elırenberg) (Polymorphinine, Brady) = Poly[morphinie

(Polymorphium, soldani) $=$ Polymorphina Polyoeca 333

l'olypetta 246

Polyphragma $133=$ (Lichenopora, Reuss) 
Polyplagia 218

(Polyplastida, Labbé) $=p \cdot p$. Cocci-

Polyplecta 218

* Polyrabdina 276

('olyselmis, Dujarlin) = Carteria

* Polysphondylium 78 [mella

(Polystomatium, Elırenberg) $=$ (Polysto-

Polystomella 119, 136, I48 = Andromedes, Montfort; Calcanthus, Montfort; Elphidium, Montfort; Farjasina, d'Orbigny; Geophonus, Montfort; Pelorus, Montfort; Polystomatium, liluenberg; Sporilus, Montfort; Themeon, Montfort; Vorticialis, Polystomelline 150 [B!ainville) (Polythalamia, Schultze) $=p \cdot p$. [Foraminiferia Polytoma $362=$ Chlamydomonas, Diesing Glenopolytoma, Cohn) [ria, Gray) Polytrema 146 = Millepora, Pallas; P'ustulaPolytrichide $457=$ (lleterotricha, [Stein)

(Polyxenes, Elurenberg) = Truncatulina

* Pompholyxia 439

Pompholyxophrys $168=$ (Hyalolampe, Greefl) Pontomyxa 67

Porcupinia 245

Porocapsa 212

(Porodiscida, Iläckel $=$ [Häckel P'orodiscinæ $190=$ (Porodoscida, 'orodiscus I89 = (Itactodiscus, Häckel; Centrospira, Häckel; Flustrella, Ehrenberg; Perispongidium, Rïist; Trematodiscus, [Häcliel]

Forospathis, Häcliel) $=$ Gorgonelta

* Porospora 269

(Porpostoma, Möbius) = Blepharisma

(Postprorocentrum, Gourret) = Prorocentrum

Poteriodendron 32i $=$ Stylobryon, FroPouchetia 384

Prismatium 221

Pristacantha 208

Proboscilla 450

Prorocentrum 381

Prorodon $435=$ Gymnopharynx, 1)iesing)

(Proroporus, Elirenberg) = Textularia

Protamoba 65,99

(Proteomyxa, Lankester) $=$

Proteomyxie $66=$ Protomyxa, Lan-

[liester)

Proteonina, $\mu . \mu$. Will $)=$ Haplophragmium *Proteosoma 288

(Proteus, Rösel) $=\Lambda \mathrm{m} \infty \mathrm{ba}$

Protobatlybius 65,68

I'rotoceratium $382=$ Clathrocysta, stein

I'rotocyathus $15 \dot{4}$

Protocystis, Wallich $)=$ Clallengeria
Protogenes 65,67

*Protomonas 74

*Protomyxa 7\%

[cercomonas

(Protomyxomyces, Cunningham) = Mono-

(Protoperidinium, Bergh) = Peridinium

Protospongia 333

Protympanium 221

Prunocarpus 185

Prunoida: $177,184=$

(Prunoidea, Häckel) = Prunoidie (Prunophracta, Iläckel) $=$

Prumophractidie 207, 212 = (PrunoI'runopyle 183 [phracta, Häckel) Prunulum 184 = Caryolithis, Ehrenberg)

(Psammosphara, Schultze $)=$ Saccammina Psecadium 137

${ }^{*}$ Pseudamphimonas 76

Pseudocubus 221

Pseudochlamys 103

Pseudodifflugia $115=$ ('leurophrys, auct.)

Pseudoplasmodila $77=$ (Acrasiées, ${ }^{*}$ Pseudospora 7'

[Van Tieghem)

(I'seudospora, /opf) = Pseudospo-

${ }^{*} P$ 'seudosporidium 75

rillie

Pseudosporinie $74=$ Pseudospor:e,

P'seudosporodon 435

I'silomelissa 226

Psilotricha 476 [\%opf)

*(Psorospermies oviformes) $=$ Coc-

*(I'sorospermies utriculeuses) $=$

[Sarcosporidie

(Pteractis, Ehrenber $r$ ) Euchitonia

Pteridomonas 342

Pterocanium 228

*Plerocephalus 272

Pterocodon 229

Pterocorys 2:8 = (P'terocyrtidium, Bütschli) (Pterocyrtidium, Bütschli $)=$ Pterocorys

Pteropilium, Iläckel 188I, $229=$ Arachnopilium, Häcliel)

(Pteropilium, lläckel $188 \%)=$ Rhopalocyrtis

Pteroscenium 224

Ptychodiscus 383

*Ptychostomum 448

(I’tyxidium, Perty) = Glaucoma

Pullenia 143

Pulvillus, Elsenberg) $=$ Nonionina

Pulvinulina 144

(Pustularia, Gray) = l'olytrema

(Pvlobothryda, Häckel) $=$

Pylobothryina $236=$ Pylobothrya, [Häckel)

Pylobotrys 236 $=$ (Botriocortys, Ehrenberg) (P'lodiscida, Iläckel) $=[$ ckel) Pylodiscinæ $190=$ Pylodiscida, Hä- 
Pylodiscus 190

Pylolena 190

(l'ylonida, Häckel) =

Pylonina $193=$ (Pylonida, Iläckel)

Pylonium 193

Pylospira 194

(Pylospyris, Häckel $)=$ Lithopera

Pylozonium 193

(Pyramidomonas, Stein $)=$ Pyramimonas

Pyramimonas $363=$ Pyramidomonas

(Pyramis, Bury) = Plectopyramis

(Pyrgo, Defrance $)=$ Biloculina

(Pyrgidium, Stein) $=$ Oxytoxum

(Py rosolenia, Lhrenberg) $=$ Icrosphara

(Pyrosoma, smith) = Pirosoma

'P'yrsonympha 3ít

P'yrophacus 383

(Pyrulina, d'urbigny) = Polymorphina

P'ythelios, 163.

P'yxicola 499

(Pyxidicula, Elırenberg) = lixuviæella

*Pxidium 498

l'yxidula 102

*Pyxinia 274

Quadrilonelic 209

(Quadrilonchida, Iläckel) =

Quadrilonchince $209=$ (Quadrilon-

Quadrula lo5

Quinqueloculina $118,12:$

\section{$\mathbf{R}$}

(Radiolaria, IÏickel $)=$ Radiolarice Radiolarie $169=$ (Radiolaria,

liamulina 137

[Häckel)

Ramulinie $138=$

(Ramulinines, Brady) = Ramulinie

(Raphanella, Bory) =0phrydium

(Raphanulina, Zborzevsky) = I'olymor-

Raphidiophrys, 167

(Raphidium, Fresenius) $=\Lambda$ mobidium

(Raphidodendron, Möbius) = Carpenteria

Raphidomonas $355=$ Merotricha, Merej-

(Ratulus, Bory) $=A$ spidisea

[liovsliy

Receptaculea $1.53=$ (Receptaculi-

lieceptaculites 15 í [tida, Römer) (Receptaculilidæ, Römer) $=$ Re[ceptaculea

(Renoidea, brown = Polymorphina

(Remulina, Blainville) = Vcrtebralina

(Renulites, Lamarck) = Vertebralina

(lieptomonas, kent) = Mastigamolua

lieticularia $80^{\circ}$

Reussia, 139?

lihabdammina 130
(Rhabdodon, Entz) $=$ Orthodon

*Rhabdogeniæ 255

Rhabdamminæ $130=$

(Rhaldamminina, Brady)=Rlıab-

Rhabdogonium 137 [damminie

(Rhabdolithis, Ehrenberg) = Spliærostylus

Rhabdomonas 346

* Rhabdospora 283

Rhabdostyla $497=($ piosoma, Blanchard $)$

Rhabdotricha 478

(Rhaphanella, Bory) $=$ Ophrydium

Rhaphidiophrys $167^{\circ}$

(Rhaphidocoecus, Häckel $)=$ Acan thosphæra

(Hhaphidosphæra, Ilïckel) $=$ Acanthosphæra

Rhaphidozoum 202

Rheophax 132

Phinchomonas 336

Rhipidocyclina 152

Rhipidodendron $327=$ (Iporea, Bailey)

Ihizammina 131

(Rhizomastigina, Bütschli $)=p \cdot p$. Rhizomastigina $322=$ Rhizomastigina, Bütschli + Mastigophrys)

Rhizomonas, lient) = Mastigameba. Rhizoplegma 183

(Rhizopoda, Dujardin $)=$

Rhizopodia $59=$ Amœbidie, Häckel ; Rhizopoda, Dujardin ; SarRhizosphæra 183 [kodina, Bütschli) Rhodosphiera 180

lihodospyris 2:35

Rhopalastrum 18?

Rhopalatractus $22 ?$

Rhopalocaniun 2:?

* Rhopalocephalus 301

lihopalocyrtis 583=(Iteropilium, lläickel 1887)

lihopalodictyum $191=$ ('Triactinosphara,

* lilopalonia 272

* Rliynclieta 5L2

* Riliynchogromia 130

Rilınchoplecta, Lhrenberg) = Textularia * Rliynchosaccus 130

(lihynchospira, Ehrenberg) $=$ Globigerina

Rimulina 137

(Rinella, Bory) = Vorticella

Robertina 140

Robulina 137

(Rosalina, d'Orbigny) = Cymbalopora

(Rostrolina, Schlicht) = Polymorphina

Rotalia $136,144=$ (Aristerigina, d'Orbigny)

(Rotalina, Brady) $=$

Rotalina $115=$ (Rotalina, Brady).

Rotalidie 143

(Rotalites, Montfort) $=$ Nummulites

(Roulea, Gourret) = Gonyaulax

Rupertia 145 


\section{S}

Saccammina $129=$ (Carteria, liady; Psam[mosphiera, schultze)

Saccaminie $130=$ Saccamininie,

[Brady

(Saccaminilla, Brady) $=$ Sacca-

Sagena 243

[minx

Sagenella 131

Sagenoarium 243

Sagenoscena 243

Sagmarium 243

Sagmidium 243

Sagoplegna 243

Sagoscena 243

Sagosphara 243

(Sagosphicrida, Iläckel) =

Sagospherina $243=$ SagospheriSagrina 138 [da, lläckel)

(Salpingocapsa, Rüst) = Lithobotrys

Salpingoca $333=$ (Lagenoca, Kent)

Salpistes, Wright

Saltonella 100

(Saprophilus, Stokes) $=$ Uronema

(Saracenaria, Defrance) $=$ Cristellaria

(Sarcodina, Bütschli) Voy. Sarko- Sarcocystis 290

[dina

"Sarcosporidie $289=$ Psorospermies utriculeuses; Sarcosporidia,

(Sarcosporidia, Bütschli) [Bütschli)

(Sarkodina, Bütschli)=Rhizopodia

Saturnalis 180

Saturninus 181)

(Saturnodoras, Häcliel) = Staurodoras

Saturnulus 180

Scaiotrichidie $479=$ Licnophorina, Bütschli + Spirochonina, Büt.-

Scaphidiodon 4 43 [schli)

(Scelasius, Hill) $=p \cdot p \cdot$ Oxylrichinæ

(Schedoacercomonas, Grassi)= llonocercomonas

${ }^{*}$ Schizogenes $65,68,583$.

(Schizomma, Ehrenberg) = Tetrapyle

Schizophora 139

(Schizopus, Claparede et Lachmann) $=$ Diophrys

Schizosiphon, Kent) = Stichotricha

* Schneideria 275

Schwaggerina 148

* Seyphidia 496

Scyphidina (Bütschli $)=p \cdot p$. Vorti[cellina
Scytomonas 353

(Semantida, Häckel) $=$ Seman-

Semantidium 220

[tinie.

Semantinæ $220=$ (Semantida, Ilä-

Semantis 220

Semantiscus 220

Semantrum 220= Stephanolitis (Bütschli)

Sepalospyris 234

* Serumsporidium $300=$ (Blanchardia, Vierzejski; Botellus, Moniez; Chydridema,

[Moniez)

Sethamphora, 227.

Sethocapsa 228

Sethocephalus 228

Sethochytris, $22 \%$.

Sethoconus $228=$ (Cadarachnium, Hiickel); Ceratocyrtis, Bütschli; Conarachnium, [lläckel; Phlebarachnium, Häckel).

Sethocorys 228.

(Sethocyrtila, Häckel) $=$

Sethocyrtinæ $228=$ Sethocyrtida,

Sethocyrlis 228

[lläckel)

Sethodiscus 187

Sethomelissa 227

Sethopera 227

Sethophæna 22s

Sethophenida (Iläckel) +

(Sethophormida) (Häckel) $=$ An-

[thocyrtin:e

Sethophormis $227=($ Astrophormis, Häckel $)$;

Hexaphormis, Häckel; Octophormis,

(Häckel); Pentaphormis, Häckel; TetraSethopilium 220

[plıromis, Häckel)

Sethopyramis $227=$ Cephalopyramis, Häckel)

Sethornithium 229

Sethosphæra 180

Sethostaurus 187

Sethostylus 187

Shepheardella 116

(Siagonophorus, Eberhard $\mathbf{t})=$ Dinophrya

Siderolina, d'(Orbigny) $=$ Calcarina

Siderolithes, lamarcki) = Calcarina

(Siderospira, Ehrenberg) = Calcarina

sigmoilina 124

(Silicoflagellata, Borgert) $=$

Silicoflagelliae $242,371=$ Silicoflagel-

Siphocampc $23 ?$

[lata, Borgert

Siphogenerina 136,139

(Siphonia, Reuss) $=$ Planorbulina

Siphonosphæra 203

(Sisyriodon, Eberhard) $=$ ?Ophryoglena

(Slabberia, Oken) $=$ Noctiluca.

Solenoplirya $509=$ (Calix, Fraipont)

Solenosphiera $203=$ (Tetrasolenia, Ehrensoreuma 195 [berg; Trisolenia, Lhrenberg) 
(Soreumida, Häckel)=Soreuminæe Soreuminæ $195=$ Soreumida, Iläckel Sorites 125

Sorolarcus 195

Sorosphwera 130

Sparotricha $4 \pi$ f

(Spasthostyla, Entz) = Vorticella

(Spastostyla, Entz) Vogt) = Erythropsis

Spathidium 435, $440=$ (Diceras, Eberhard; ? Pelekydion, Eberhard; ? Ilabrodon, [Perty)

Spharastrum $160=($ ? Astrococcus, Greeff $)$ Sphærocapsa 211

(Sphaerocapsida, Häckel) = Spha[rocajosine Spharocapsina $=$ Sphierocapsida, * Sphrocystis $2 \% 1$

Sphxerøea 333

Spharoida 177, $179=p \cdot p$.

Spharoidea, Iläckel = Sphae-

Sphæroidina 143

[roida + sphierozoidie

${ }^{*}$ Sphæromyxa $295=$ (Cyrtodiscus, lütz).

(Spharophracta, IIäckel) $=$

Sphicrophractidie $207,209=$ Spliaro*Sphærophrya 510

Sphæropyle 183

(Sphicropvlida, Dreyer) $=$

Spharepylina $185=$ Sphieropvlida, [Diever

(Spharosira, Lhrenberg $)=$ Volvox

Spharospongia 154

* Sphærospora 29.5

sphitrospyris 235

Spherostylus $180=$ (Rtuabdolithis, Ehrenberg) śphærozoida $202=p . p$. Sphieroidie, Häckel) Sphierozoida 201, 202 $=p \cdot p$. (Sphieloidea, Iläckel ; p. p. spharo[zoidea, lirandt)

Spharozoidea, Brandt non Iläckel [E Sphacrozoidil + Collozoida spharozoum 202

(Spharula, Soldani) $=$ Orbulina

sphenoderia 112

Sphenomonas 347

*Spherocephalus 272

Spirema 194

(Spiridobotrys, Häckel) $=$ Lithopera

Spirillina $144=$ Oolis, Phillips)

Spirilline $14 t=$

(Spirillinina, Brady) $=$ Spirillinæ

Spirocampe 2:32

*Spirochona 490

(Spiroctronina, Stein) $=$
Spirochonina $484=$ (Spirochonina, Stein)

Spirocyrtis 232

(Spirolina, d'Orbigny) $=$ Peneroplis

(Spirolina, Brown) = Vaginulina

Spiroloculina 123

(Spiromonas, Perty) $=$ Bodo

Spironema 342

Spironium 19'

Spiroplecta 140

Spirostomum $458=$ (Peltierius, Ormancey)

(Spirotricha, Bütschli) = Heterotrichida + Hypotrichida + Peri-

[trichida

Spondylomorum $362=$ (Phacelomonas, Stein $)$ Spongaster 191

Spongasteriscus 191

Spongatractus 185

Spongechinus 183

Spongellipsis 185

Spongiomma 183

Spongobrachium 191 = (Spongocyclia, 1 äckel $)$ Spongocore 185

Spongocyclia lläckel) $=$ Spongobrachium Spongocyrtis 225

Spongodictyon $180=$ (Dictyoplegma, Häckel; [Dictyosoma, J. Müller)

(Spongodiscida, Häckel) $=$

spongodiscine 192 $=$ (Spongodisci[da, Häckel)

Spongodiscus $191=$ ('ipongospira, Häckel)

Spongodruppa 185

Spongodrymus 183

Spongolarcus 192

Spongolena $19 \mathrm{~L}$

Spongoliva 185

Spongolonche 181

Spongolonche 191

(Spongolonchis, Iräckel) = Spongostylus Spongomelissa $226^{\circ}$

Spongomonadina, Stein) $=$ Spongomonadinæ $326=$ SpongoSpongononas, $320^{\circ}$ Spongophacus 191 [monadina, Stein Spongophortis 194 Spongopila 183 Spongoplegma 180 Spongoprunum 185 Spongopyle 192

Spongopyramis 227

Spongosphæra 183

(Spongospira, Häckel) $=$ Spongodicus

Spongostaurus 191

Spongostylidium 181

Spongostylus $181=$ (Spongolonchis, Häckel)

Spongothamnus 183

Spongotripus 191 
Spongotrochus 191

Spongoxiphus 185

(Spongurida, Häckel $)=[$ Häckel $)$ =Spongurinæ $185=$ Spongurida, Spongurus $185 \quad$ [rinidæe

(Sporadina, Stein $)=p \cdot p \cdot$ Grega(Sporilus, Montfort) $=$ Polystomella

(Sporozoa, Leuckart) $=\quad$ [ckart

${ }^{*}$ Sporozoaria $254=$ (Sporozoa, LeuSpumaria 87

(Spumella, Bütschli) = 0ikomonas

(Spumellaria, Häckel $=$ Peripylida Spyroidæ 216, 233= (Acanthodesmida, Hertwig; Sproidea, Häckel; Zygocyrtida, Iläckel, Bütschli) (Spyroidea, Häckel) = Spyroida (Spyridina) 233

Squamulina 122

Stacheya 134

Stauracantha 208

Stauracontium 181

Stauractura 188

Stauralastrum 190

Staurancistra 181

Stauraspis 211

Staurocaryum 181

Staurocromyum 181

Staurocyclia 188

Staurodictya 189

Staurodoras $181=$ Saturnodoras, Häckel

Staurolonche 181

Staurolonchidium 181

Stauropelta 211

Staurophrya 514

Staurosphiera 181

(Staurosphærila, Iläckel) $=$

Staurospherinæ $181=$ Stauros-

Staurostylus 181 [pherida, Iläckel)
Staurotholonium 193 (phes

Staurotholus 193

Stauroxiphos 181

Stegochylum $\mathbf{4 4 6}$

(Steinia, Diesing $)=$ Oxytricha

Steiniella 383

Stemonitis 87

*Stenocephalus 271

Stentor $461=$ (Ecclissa, Schrank; Linza, [Schrank; Salpistes, Wright)

(Stentorina, Stein) $=$

Stentorina $465=$ Stentorina (Stuin) Stephanastrum 190

(Stephanida, Iäckel) $=$ -

Stephanince $219=$ (Stephanida, Ḧ̈-

Stephanisciss 219

Stephanium 219
(Stephanolithis, Bütschli) = Semantrum Stephonoma 368

Stephanoon 368

${ }^{*}$ Stephanophora 273

Stephanopogon 438

(Stephanopyxis, Bury) = Lithocyclia

Stephanosphæra 367

Stephanospyris 233

(Stephida, Häckel) $=$

Stephoidæ216, 219=(Acanthodesmida, Bütschli; Stephida, Häckel : Stephoidea, Häckel)

(Stephoidea, Häckel) $=$ Stephoidæe

(Sterreonema, Kutzing) $=$ Anthophysa

Sterromonas 326

Stichocampe 231

Stichocapsa $232=($ Tetracapsa, Rüst $)$

(Stichocapsida, Häckel) $=p \cdot p \cdot$ Li-

[thocampinæ

(Stichochæta, Claparède et Lachmann) = [Stichotricha

(Stichocorida, Häckel) $=p \cdot p$. Li[thocampinæ

Stichocorys 232

(Stichocyrtida, Häckel) $=$

Stichocyrtoidea 223, 231 = Stichocyr-

tida, IIäckel; Cyrtoidea polytha-

Sticholonche 251

[lamia, Iläıkel

Stichopera 231

Stichophæna 232

Stichophormis 231

Stichopilium 231

Stichopodium 231

Stichopterium 231

Stichotricha $474=$ (Archimedes, IIudson ; Chætospira, Lachmann; Schizosiphon,

Kent; stichocheta, Claparède et LachStigmosphitra 179

Stomatodiscus 189

Stomatosphæra 183

(Stomophyllum, Lieberkühn) $=$ LoxophylStorthosplizera 129

Streblacantha 194

Streblonia 194

(Streblonida, IIäckel)=Strebloninæ Streblonince $194=$ (Streblonida,

Streblopyle 194

Strobilidium 405

Stromatocerium 153

[Häckel)

Stromatopora $153=$ Coenostroma, Nicholson

Stromatoporea $153=$

(Stromatoporida, Nicholson et Mu$[$ rie $)=$ Stromatoporea

(Strombidinopsis, lient) $=($ ?) Tintinnidium 
Strombidium $465=$ (Tortaqualella, Lankester) Strongylidium 47 ' [phina

(Strophoconus, Ehrenberg) = Polymor(stychopterygium, IIäckel) $=\Lambda$ rtopilium

(Stylactis, Stöhr) = IIymeniastrum

Stylamoeba 100

Stylartus 185

Stylatractus $18 j$

(Stylobryon, Fromentel) = PoteriodenStylochlamydium 189

[dron

Stylochona 484

* Stylochrysalis 358

(Stylocola, Fromentel) $=$ Cothurnia

Stylocoma 476

Stylocometes 515 $=$ (Asellicola, Plate; Digitophrya, Fraipont; Pericometes, Schnei-

Stylocromium $180=$ (Cromostylus, Ifiekel) [der

Styloeyclia 188

Stylodictya $189=$ (Stylospira, IIäekel)

Stylodyction $153=$ (Syringosiroma, Bütsclıli $)$ Stylodiseus 187

Stylohedra 499

Stylonetes 474

Stylonychia 476

(Styloplotes, Stein $)=$ Jiophrys

*Stylorhynchine, $272=$ (Stylorhyn-

${ }^{*}$ Stylorhynchus 272

Stylosphara 180

[chides, Léger)

(Slylosphærida, Iläckel) $=$

Stylosphærinæe, $181=$ Stylosphæ[rida, Häckel)

(Stylospira, IIäckel) = Stylodictia

(Stylospongia, Häckel) = Stylotrochus

(Stylospongidium, Häckel) $=$ Stylotrochus Stylostaurus 181

Stylotrochus I91=(Stylospongia, Häckel; StyStypolarcus 192 [lospongidium, IIäckel)

Styptosphæra 180

Suctorella 512

\section{(Suctoria, Kent) $=$}

Suctori: vel Tentaculiferix, $500=$ (Acinetina, auct.; Suctoria, Kent; Tentaculifera, Iluxley, Kent) Sycydium 137

Synaphia 368

Synerypta $359=$ (? Uvella, Fromentel)

* Syneystis 273

Synura $359=$ (Frlenouvella, Diesing)

Syringammina, 129

(Syringostroma, Bütsehli) = Stylodyetion

\section{$\mathbf{T}$}

(Tachysoma, Stoles) = Oxytricha

(Tapinia, Perty) = Cyclidium

Taurospyris 234
(Taxopoda, Fol) $=$

Taxopodea $251=$ (Taxoporla, $\mathrm{Fol}$ )

Technitella 129

(Telotrochidium, lient $)=$ Vorticella

(Tentaculifera, Kent, Iluxley $\Longrightarrow$ Tentaculiferia vel Suctoria $500=$ (Acinelina, auct.; Sucloria, lient, Bütschli; Tentaculifera, Huxley,

Tessaraspis 211

Tesserastrum 190

Tessarospyris 233

Testamceliformea $154=$

(Testamobiformia, Carter) - Test[amobiformea

(Tetrabrena, Iujardin) = Gonium

(Tetracapsa, Rüst) = Śtichocapsa

Tetragonis $\mathbf{1 5 4}$

(Tetragonulina, Seguenza $)=$ Lagena

Tetrahedrina 227

Tetrachytris

(Tetramitina, Bütschli) $=$ Monos-

Tetramitus 339

*Tetramyxa 76 [lomina

(Tetraphormis, Häckel) = Sethophormis 'Tetraplagia 218

Tetraplecta 218

Tetrapyle $193=$ (Schizomma, Ehrenberg) T'etrapylonium 193

(Tetrasolenia, Ehrenberg) = Solenosphæra Telraspyris 233

Tetrastyla 47 '

Tetrataxis 140

Tetratoma 363

(Textilaria, Defrance $)=$ Textularia

Textularia $139=$ (Clidostomum, Ehrenberg; Loxostomum, Ehrenberg; Proroporus, Ehrenberg ; Rhynchoplecta, Ehrenberg; [Textilaria, Defrance)

Textularida 139

Textularinæ $136,140=$ Textularidæ, Carpenter; Enallostegia, d'Or[bigny

(Thalamophora, Hertwig) $=$ TheThalamopora 147 Thalassicolla 177

[camœbina

(Thalassicollida, Häckel) $=$

Thalassicollidæ $177=$ (Thalassicol[lida, Häckel)

Thalassicollinæ $178=p \cdot p$. (ThalassiThalassolampe 178 [collida, Häckel) Thalassophysa $178=($ Myxobrachia, Häckel) Thalassopila 178 
Thalassoplancta 179

Thalassosphæra 178

(Thalassosphærida, Häckel) $=$

Thalassosphæridæ $177,178=$ (Tha[lassosphærida, Häckel)

Thalassosphærinæ $179=p \cdot p$. (Tha[lassosphærida, Häckel)

Thalassoxanthium 178

Thecamobina $10 \mathbf{l}=$ (Thalamophora,

Thecosphara 180

(Themeon, Montfort) $=$ Polystomella

${ }^{*}$ Thelohania 296

Theocalyptra 230

Theocampe 230

Theocapsa $230=($ Urocyrtis, Pantanelli $)$

Theoconus 230

Theocorys, 230

(Theocyrtida, Häckel) =

Theocyrtinæ 23ı = (Theocyrtida,

Theocyrtis 230

Theodiscus 187

Theopera 229

Theophæna 230

Theopilium 229

Theophormis 229

Theopodium 228

Therospyris 234

Theosyringium 230

Tholartus 193

Tholocubus 193

Tholodes 193

Tholoma 193

(Tholonida, Häckel) =

Tholoninie $194=$ (Tholonida, Häc-

Tholonium 193

Tholospira 194

(Tholospyrida, Häckel) $=$

Tholospyrinæ $234=$ (Tholospyrida,

Tholospyris 234

Tholostaurus 193

[lläckel)

Thoracaspis 212

Thurammina 133

Thuramminopsis 133

Thuricola 499

(Thuricolopsis) = Cothurnia

Thylacomonas 323

Thylakidium 459

Thyrsocyrtis $2: 9$

Tiarina 438

Tiarospyris 234

(Tillina, Stokes $)=$ Colpidium

Tilmadoche 86

Tinoporinæ 147

Tinoporus 145

Tintinnidium 466 (9) (Strombitinent)
Tintinninæ $467=$

(Tintinnoina, Claparède et Lach$[$ mann $)=$ Tintinninæ

Tintinnopsis $466=$ (Conicyelis, Fol)

Tintinnus $46 \%=$ (Undella, Daday)

Tokophrya $509=($ Volverelia, Bory $)$

(Torquatella, Lankester $)=$ Strombidium

(Torquatina, Gros) = Trichodina

Toxarium 2:1

Trachelinæ $440=($ Decteria, Perty; [Trachelinea, Diesing)

$($ Trachelinea, Diesing $)=$ Trachelinæ Trachelius $40=$ (Harmodirus, Perty)

Trachelocerca 436

Trachelomonas $350=$ Chætoglena,Ehrenberg; Chætophlya, Ehrenberg; Chonemonas, Perty; Lagenella, Ehrenberg; Trypemo[nas, Perty)

Trachelophyllum 436

(Trematodiscus, lläckel $)=$ Porodiscus

(Tremetla, Linnè) = Ophrydium

Trepomonas $3 \nmid 1=$ (Grynæa, Fresenius)

(Triactinosphæra, Dunikovsky) = Rhopalo-

Triactiseus 187 [dyetium

Tribonosphæra 2013

(Tribulina, Bory) $=$ Aspidisca

Triceraspyris 233

(Trichamœba, Fromentel) $=$ Amœba

Trichia 86

${ }^{*}$ Trichodina $489=$ (Nummulella, Carus; Tor[quatina, Gros; Urceolaria, Lamarck)

${ }^{*} T$ richodinopsis 492

(Trichodiscus, Ehrenberg) = Nuclearia

Trichogaster 474

(Tricholeptus, Fromentel) $=$ Uroleptus Trichomastix 339

(Trichomecium, Fromentel $)=$ Blepharisma ${ }^{*}$ Trichomonas $340=$ Cimænomonas, Grassi)

${ }^{*}$ Trichonympha 343

(Trichonymphida, Leidy) $=$

Trichonymphina $337,342=$ (Tricho-

*Trichophrya 513 [nymphida, Leidy)

(Trichopus, Clap. et Lachm.) = Egyria

Trichorhynchus 272

*'Trichorhynchus 4 it

Trichosphærium 99

(Trichostomata, Bütschli) = IIyme-

Tricolocampe 230 nostomidx

Tricolocapsa 230

Tricolospyris 235

Tricyelidium 220

(Tricyrtida, IIäckel) $=$

Tricyrtoidea 223, 228 = (Tricyrtida,

Ilïckel; Cyrtoidea trithalamia)

Tridictyopus 224 
Trigonactura 188

Trigonastrum 190

Trigonocyclia 188

Trigonomonas 341

(Trigonulina, Seguenza) = Lagena

Trillina, 118, 123

Triolena, 190

$[\mathrm{gny})$

Triloculina 118, $123=$ Cruciloculina, dOrbi(Trimastigina, Blochmann) $=$ Trimastiginæ $336=$ (Trimastigina, Trimastix 330 ;

Trinema 112 [Blochmann)

\section{Triodiscus 190}

Triolena 190

Triopyle 190

(Triospyris, Häcliel) = Egospyris

(Triostephanium, IIäckel $)=$ Acanthodesmia Tripilidium 22:

Triplagia 218

(Triplasia, Reuss) $=$ Orthocerina

Triplecta 218

(Tripocalpida, Ilïckel) $=$

Tripocalpinæ $224=$ Tripocalpila, Tripocalpis 224

(Tripocyrtida, lläckel) $=$

Tripocyrtine $227=$ (Tripocyrtida, 'Tripocyrtis $226^{\circ}$

Tripodictya 189

[Häcliel

Tripodiscium $22 \dot{i}=$ (Tripodiscus, Rüst)

(Tripodiscus, Rüist) $=$ Tripodiscium

Tripodonium 22'

Tripospyris 233

Tripterocalpis 224

$($ Tripylea, IIäckel $)=$ Phæodarida

(Trisolenia, Ehrenberg) = Solenosphara

Trissocircus 220

'Trissocyclus 220

'Trissopilium 22'

Tristephanium 220

Tristylospyris 233

Tritaxia 13!

Trizonium 193

Trochanımina 133

Trochammine $133=$

(Trochamminine, Brady) $=$ TroTrochilia 4 43

Trochodiscus 187

(Troglodytes, Gabriel) = Platoum

Tropidoscyphus 353

Truncatulina, $136,14:=$ (Aspirlospira, Elrenberg; Aristerospira, Ehrenberg; Cibicirles, Ehrenberg; Lobatula, Flemming; [Polyxenes, Ehrenberg)

*Trypanosoma $322=$ (Glolublaria, Wedl; Hæmatomonas, Mitrophanof; Undulina, LanTrypanosphiera 203

[liester]

(Trypemonas, Perty) = Trachelomonas

('Tubularia, Schrank) $=$ Cothurniopsis
Tubulina 85

(Turbinella, Bory) $=$ Urocentrum

Turpinius 48

Tuscaridium 247

Tuscarora 247

(Tuscarorida, IIäckel) = Tuscarorinæ $=$ (Tuscarorida, Häckel) Tuscarusa 247

(Tympanida, Häckel) $=$ Tympaninæ $221=$ Tympanida,

Tympanidium 221

Tympaniscus 221

[Häckel)

\section{$\mathrm{U}$}

(Undella, Daday) = Tintinnus

('ndulina, Lankester) = Trypanosoma.

Uniloculina 123

(Lrceolaria, Lamarck) $=$ Trichodina

Urceolus $3 \% 2=$ (Phialonema, Stein)

Irnula 511

(Urnulina, Bütschli $)=$

Urnulinæ $512=($ Urnulina, Bülschli) (Urocentrina, Bütschli $)=$

Urocentrinæ $447=$ (Urocentrina, [Bütschli)

Urocentrum 447 $=$ (Calceolus, Diesing; Peridiniopsis, Clarke; Turbinella, Bory)

(Urocyrtis, Pantanelli) = Theocapsa

Iroglena 360

Iroleptus $4 \%=$ (Platytrichotus, Stokes; Tricholeptus, Fromentel)

Uronema $447=$ (Cryptochilum, Maupas; Saprophilus, stokes)

Uronychia 477

Urophagus 341

Urosoma $470^{\circ}$

[chysoma, Mingazzini)

${ }^{*}$ Lrospora $276=$ Cystobia, Mingazzini; PaLrostyla $474=$ (Hemicycliostyla, Stokes)

Urotricha $435=$ (Pantotricha, Ehrenberg $)$

Urozona ity

[pliysa

(Uvella, Ehremberg) = Synura + Antho-

(Uvella, Fromentel) $=($ ?) Syncrypta

Uvigerina 138

\section{V}

Vacuolaria 355

Vaginicola $498=($ Platycola, Kent $)$

Vaginulina $137=$ Cilharina, d'Orbigny); [Spirolina, Brown)

(Valvularia, Goldfuss) $=$ Opercularia

Valvulina 13!

*Vampyrella 70

* Vampyrellidium 71

(Vasia, Nilne Edwards) = Vorticella

Vasicola 436

Venilla I3? 
(Vermiculum, Montaigu) $=$ Miliola

Verneuilina 139

Vertebralina $124=$ (Ceratospirulina, Ehrenberg;? Renulina, Blainville; Renulites, [Lamarck)

Virgulina $140=$ (Grammobotrys, Ehrenberg) (Volverella, Bory) $=$ Tokophrya

Volvocina 354,364

Volvox $369=$ (Sphærosira, Ehrenberg)

Vorticella $490=($ Campenella, Colombo; Craspedarium,Hill; Ecclissa, Schranl; Kerobalana, Bory; Macrocereus, Hill ; Ophrydia, Bory; Rinella, Bory; Spasthostyla, Entz; Telotrochidium, Kent; Vasia, Milne Edwards)

[Ehrenberg)

Vorticellinæ $499=($ Vorticellina $p \cdot p$. (Vorticellina, Ehrenberg) $=p \cdot p$. [Dexiotrichida

$($ Vorticialis, Blainville $)=$ Polystomella

(Vulvulina, d'Orbigny) $=$ Grammostomum

\section{W}

Wagnerella 168

(Wagneria, Alenitzin) $=$ Didinium Webbina 133

\section{$\mathbf{X}$}

(Xanthidium, Ehrenberg) $=$ Cladopyxis Xanthiosphera 205

Xanthodiscus, 351

Xiphacantha 208

Xiphatractus 185

Xiphodictya 189

Xiphoptera 209

${ }^{7}$ Xorhynchus 275
Xiphosphæra 180

Xiphostylus 180

$\mathbf{Y}$

(Ypsistoma, Bory $)=$ Blepharisma

\section{$\mathbf{Z}$}

('\%onarida, Iläckel) $=$

Zonarina $194=$ (Zonarida, Häckel) Zonarium 194

Zonaspis 211

Zonodiscus 187

(Zoocladium, Ehrenberg) = Zoothamnium Zoosporida $72=$ (Monadina zoosZooteira 165

[porea, Zopf)

(Zoothamnia, Bory) $=$ Zoothamnium

Zoothamnium 496=(Autochloe, Joseph; Dendrella, Bory; Zoocladium, Ehrenberg;

Zygacantha 208

('yggartida, IIäckel) =

[Zoothamnia, Bory)

Zygartina $187=$ (Zygartida, IÏ̈-

Zygartus 187

Zygocampe 187

Zygocircus 219

${ }^{*}$ Zygocystis 276

Zygoselmis 352

(Zygospyrida, Häckel) $=$

Zygospirina 234 = (Zygospyrida,

Zygospyris 234

Zygostaurus 209

Zygostephanium 220

Zygostephanus 220

[Häckel 


\section{CORRIGENDA ET ADDENDA}

\section{CORRIGENDA}

Dans l'indication des lignes, la lettre $n$ signifie que la ligne à corriger appartient aux notes; la lettre $r$ indique qu'il faut compter les ligues en remontant.

\begin{tabular}{|c|c|c|c|}
\hline Page & Ligne & Au lieu de : & Lire : \\
\hline $55 \ldots$. & 1. & Théorie.. & Théories. \\
\hline$\ldots$ & $6, n \ldots \ldots \ldots$ & Pouchet. ........... & Moniez. \\
\hline ... & titre ....... & Monadinazoosporea ............ & Monadina zoosporca. \\
\hline 74 & $10, r \ldots \ldots$ & Chlamidomonas................. & Clamydomonas. \\
\hline$\cdots$ & $1, n ., r \ldots \ldots$ & OEthaliopsis ......... & Ethaliopsis. \\
\hline ... & $4, n ., r \ldots .$. & Astinocyclina......... & Actinocyclina. \\
\hline & $5, n ., r \ldots$. & Phipidocyclina ....... & Rhipidocyclina. \\
\hline & $9, r \ldots \ldots \ldots$ & Chlathrodictyon ..... & Clathrodictyum. \\
\hline . . & $3, n, r \ldots \ldots$ & Archaospharina. & Archaospharina. \\
\hline$\ldots$ & $1 \ldots \ldots \ldots$ & Chlathrulina ........ & Clathrulina. \\
\hline . & $5, n ., r \ldots \ldots$ & Sphxropylynæ............. & Spharopylina. \\
\hline$\cdots$ & $5, n . ., r . \ldots$ & Artrosphærinæ [Artrosphærida. & Astrosphærinæ [Astrospharida \\
\hline . & $10 \ldots$ & Artrosphara .............. & Astrosphara. \\
\hline . & $22, n \ldots \ldots \ldots$ & I'epipanicium............ & Peripanicium. \\
\hline . & $5, n ., r \ldots \ldots$ & Iynoniastrum ......... & Hуmeniastrum. \\
\hline & 16. $n \ldots \ldots \ldots$ & Pentrisastrum .......... & Pentinastrum. \\
\hline & $1, n ., r \ldots \ldots$ & Aconthaspis.... & Acontaspis. \\
\hline & $3, n \ldots \ldots \ldots$ & Sonaspis.. & Zonaspis. \\
\hline 211 & $15, n_{.}, r_{\ldots} \ldots$ & Dorastapida............ & Dorataspida. \\
\hline 2 & $3 \ldots$ & [Nassoidae, Häckel].... & [Nassoidea, IIäckel]. \\
\hline 220 & $4, n$. & Semanthum..... & Semantrum. \\
\hline 2 & $7, n$. & Chlathrocircus... & Clathrocircus. \\
\hline & $9, n$. & Cyrtophormis........... & Cystophormis (1). \\
\hline . & $2, n$. & Cyrtocalpina .... & Cyrtocalpida \\
\hline & $12, n \ldots \ldots \ldots$ & Chlathrocanium. & Clathrocanium. \\
\hline & $5, n$. & Chlathrospyris... & Clathrospyris. \\
\hline & titre & 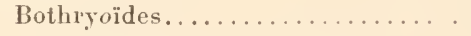 & Botryoïdes \\
\hline & $1, n, \ldots \ldots$ & Cannobothrinz, Cannobothricla. & Cannobotrinx, Cannobotryda. \\
\hline & $7, n \ldots \ldots \ldots$ & Lithobothrinx, Lithobotryda. . & Lithobotrinæ. Lithobotryda. \\
\hline & $1, n$. & Pylobothryina, Pylobothryda. & Pylobotrina, Pylobotryda. \\
\hline & $8, n$. & Mesocena....... & Mesoczna. \\
\hline & $3 \ldots$ & Cannorraphis.... & Cannorrhaphis. \\
\hline & $17, n$ & Silicoflagellea... & Silicoflagellix. \\
\hline & titre & Phaospharides... & Phxosphérides. \\
\hline & $1 \ldots$ & Calacantha..... & Coelacantha \\
\hline & $1, r \ldots \ldots$ & 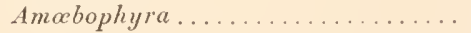 & Amabophrya. \\
\hline
\end{tabular}

(1) Häckel dit bien Cyrtophormis dans le texte de son ouvrage, mais daus la table il eorrige et met Cystophormis, s'étant apereu saus doute, à ce moment, du double emploi d'un même nom. D'autres doubles emplois analogues se retrouvent aussi ailleurs dans ce mème ouvrage, par exemple : Pteropilium, spongolonehe, ete. 


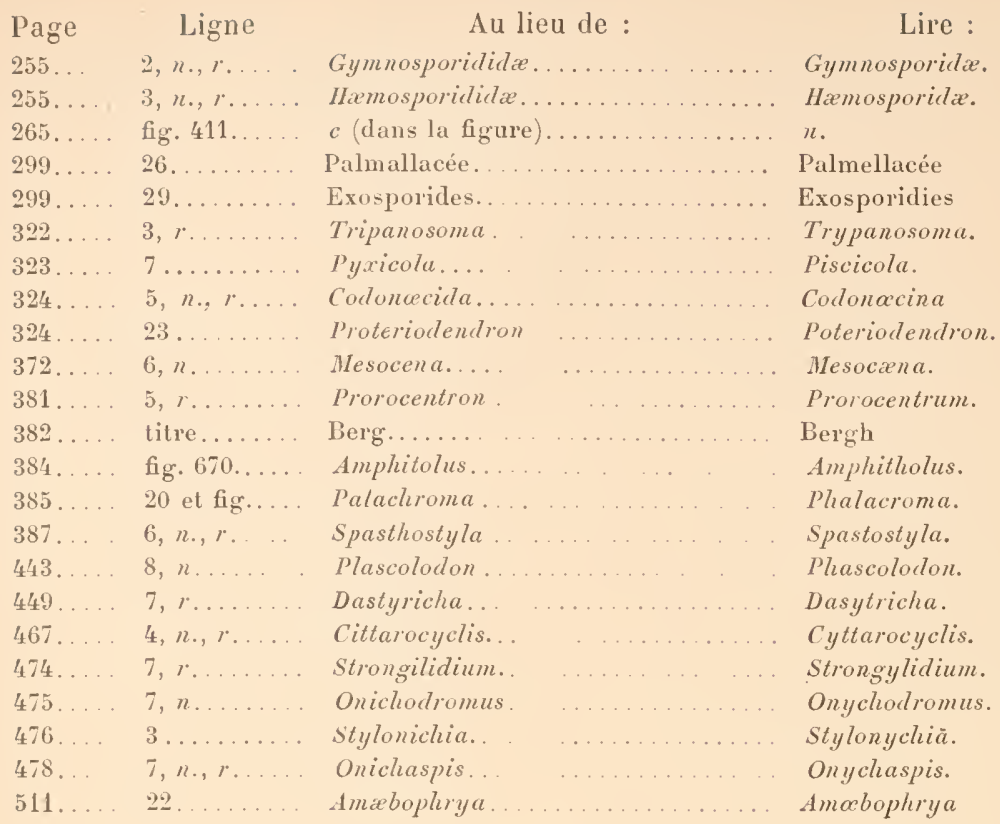

\section{A D DENDA}

59.... A la synonymie de Rhizopoclia ajoutez Sarkodina (Bütschli).

67. A la fin du troisième alinéa des notes, ajoutez : G. V. MǘLLer [95] a démontré récemment que ces prétendus Scliizogenes n'étaient autre chose que des gouttelettes de la sécrétion de la glande coquillèrc. Cependant, le Sclizogones ayant été rencontré chez des hòtes dépourvus de glande coquillère, il reste encore quelques doutes à son sujet.

103.... ligne 5, en remontant: Après le mot membraneux, ajoutez: (Eau douce).

231 . Häckel a créé le genre Pteropilium (lläckel) en 1887 sans sc rappeler qu’il cn avait créé un autre du même nom en 1881 (voir page 229) et sans savoir que sous le nom de

Rhopalocyrtis (Bütschli), Bütschli, en 1881, l'avait déjà observé et nommé.

$233 \ldots$ A A près le genre Metanema ajouter ce qui suit :

Heteromastix (Clark), forme ì affinités douteuses et insuffisamment décrite.

$255 . .$. Mettre Cystrsporidies (Labbé) comme synonyme non de Rlabdogcnia mais de Brachycysticla. 





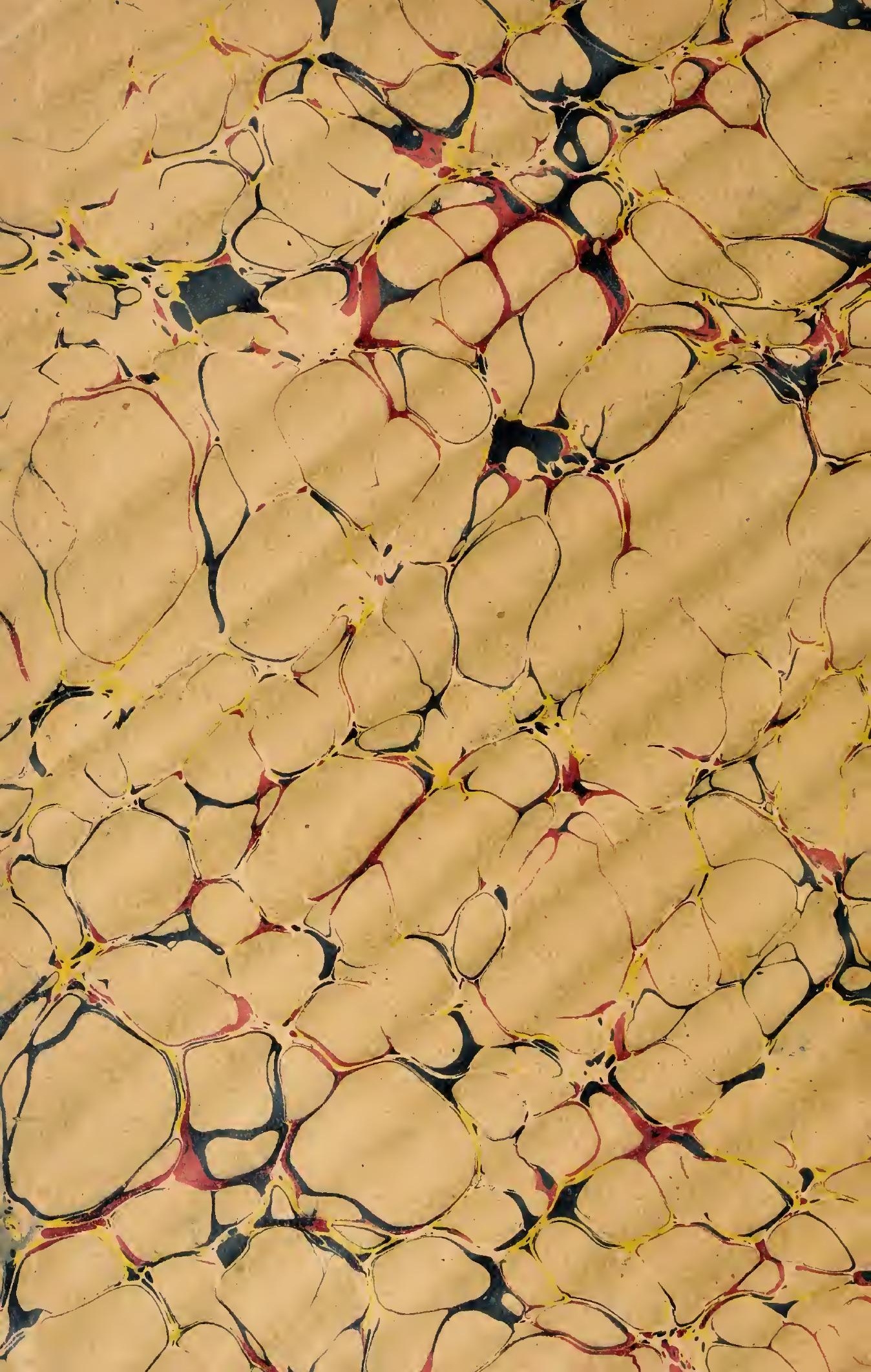




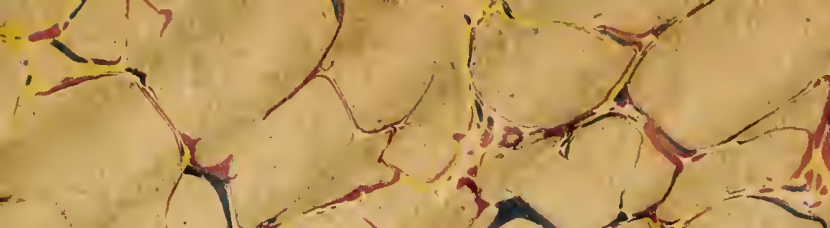

$x^{2} x^{2}+2$
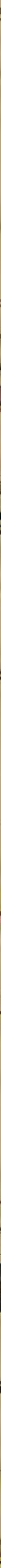

$1 / 1=0$

$x^{2}=$ 
(

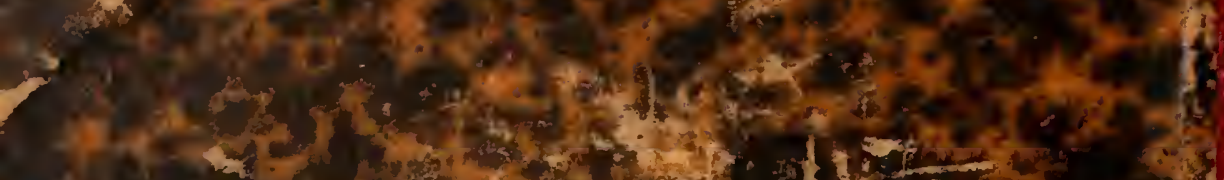
$42000+15=$

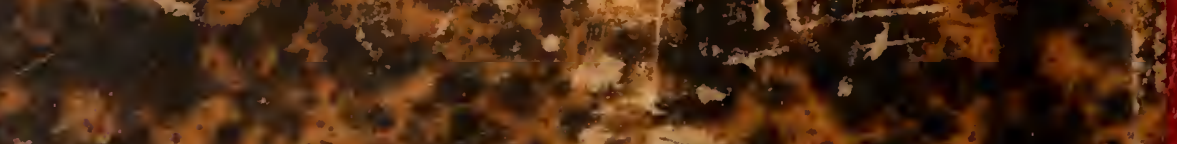

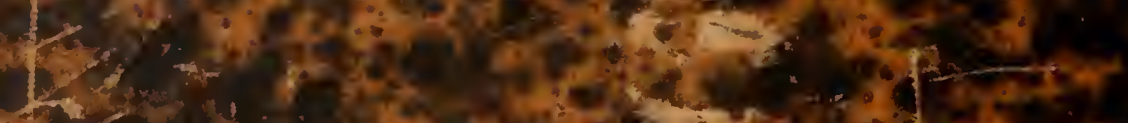

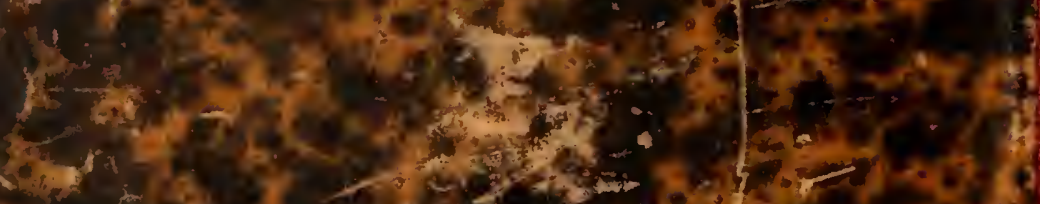

1)

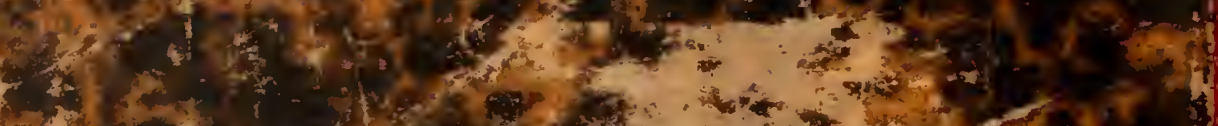

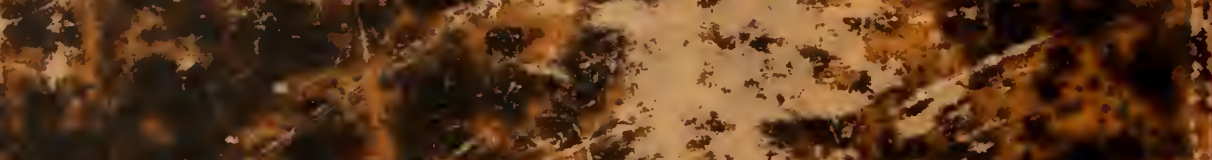

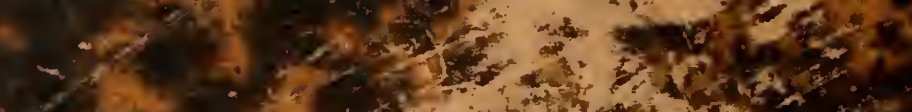

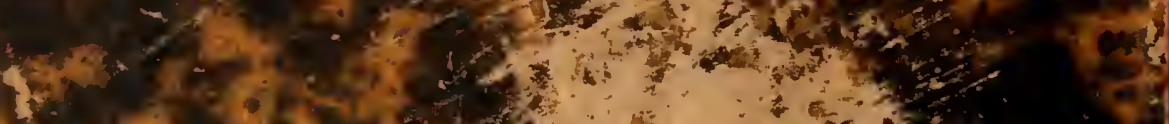
(1)

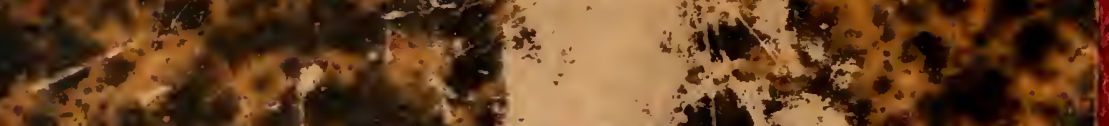
-

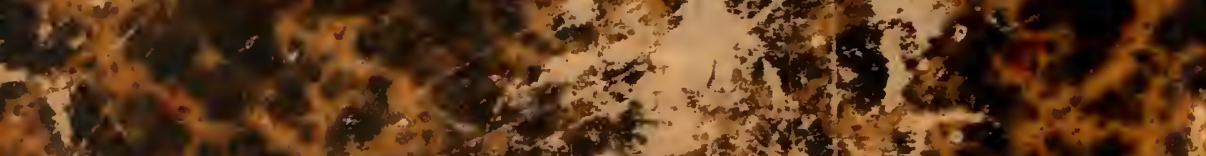

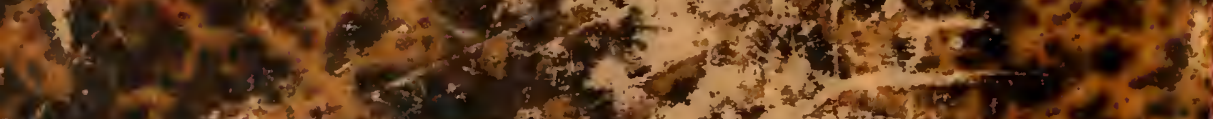

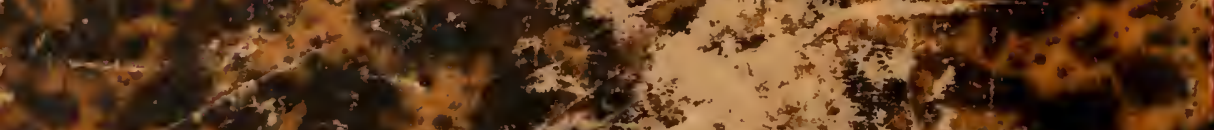

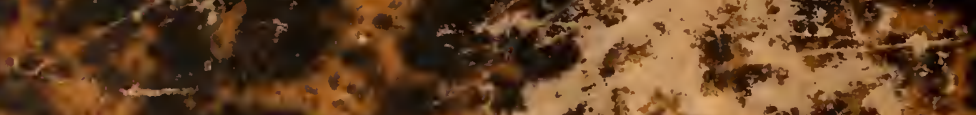

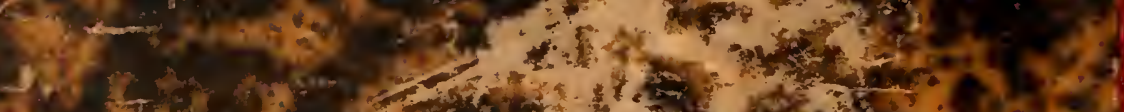

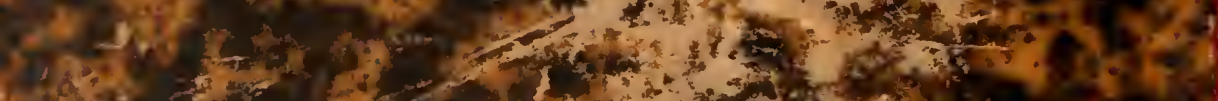

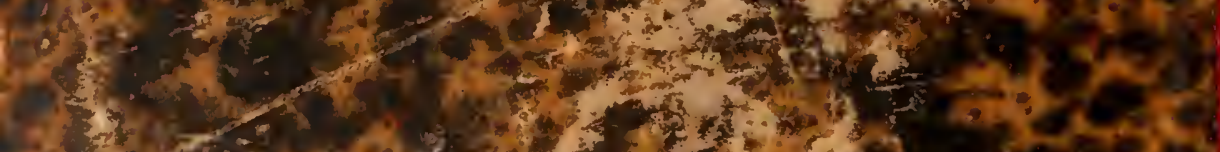

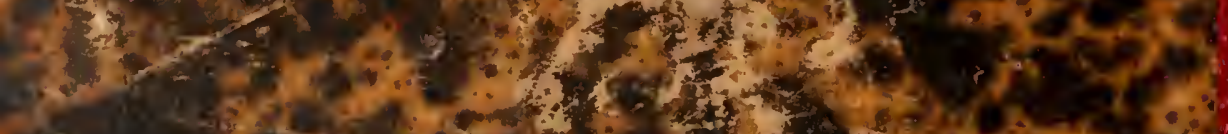

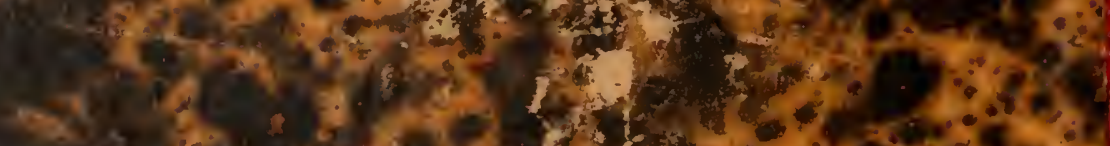

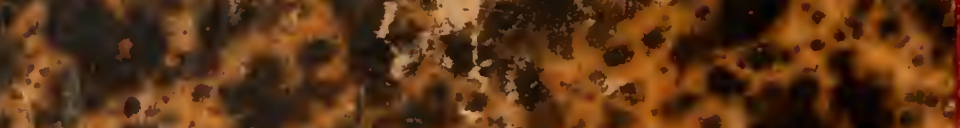

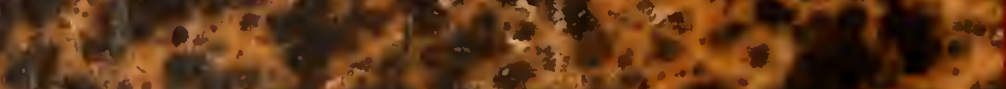

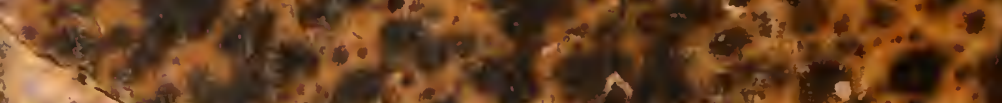

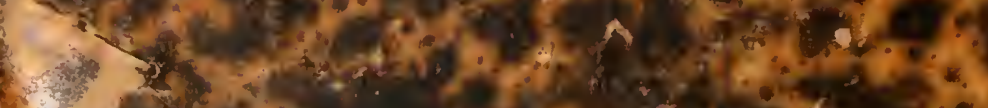

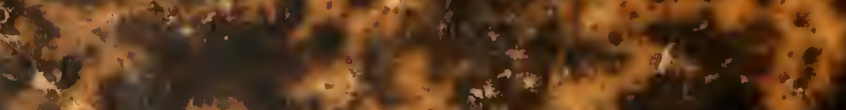
$(8,3)$ 